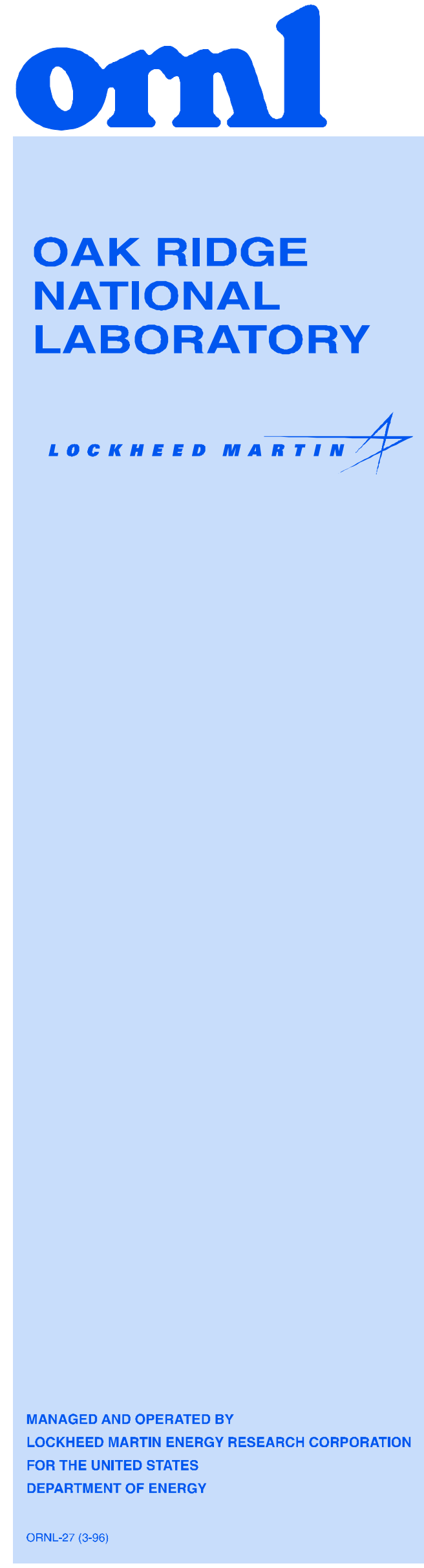

ORNL/TM-1999/299

\title{
Neutronics Benchmarks of Mixed-Oxide Fuels using the SCALE/CENTRM Sequence
}

\author{
D. F. Hollenbach
}

P. B. Fox 
This report has been reproduced directly from the best available copy.

Available to DOE and DOE contractors form the Office of Scientific and Technical Information, P.O. Box 62, Oak Ridge, TN 37831; prices available from (615) 576-8401.

Available to the public from the National Technical Information Service, U.S. Department of Commerce, 5285 Port Royal Rd., Springfield, VA 22161.

This report was prepared as an account of work sponsored by an agency of the United States Government. Neither the United States nor any agency thereof, nor any of their employees, makes any warranty, express or implied, or assumes any legal liability or responsibility for the accuracy, completeness, or usefulness of any information, apparatus, product, or process disclosed, or represents that its use would not infringe privately owned rights. Reference herein to any specific commercial product, process, or service by trade name, trademark, manufacturer, or otherwise, does not necessarily constitute or imply its endorsement, recommendation, or favoring by the United States Government or any agency thereof. The views and opinions of authors expressed herein do not necessarily state or reflect those of the United States Government or any agency thereof. 
Computational Physics and Engineering Division

\section{Neutronics Benchmarks of Mixed-Oxide Fuels using the SCALE/CENTRM Sequence}

D. F. Hollenbach

P. B. Fox

Date Published: February 2000

Prepared by the

OAK RIDGE NATIONAL LABORATORY

Oak Ridge, Tennessee 37831-6370

managed by

LOCKHEED MARTIN ENERGY RESEARCH CORP.

for the

U.S. DEPARTMENT OF ENERGY

under contract DE-AC05-96OR22464 



\section{CONTENTS}

Page

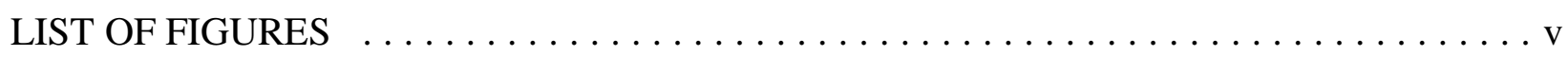

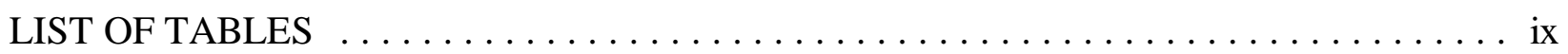

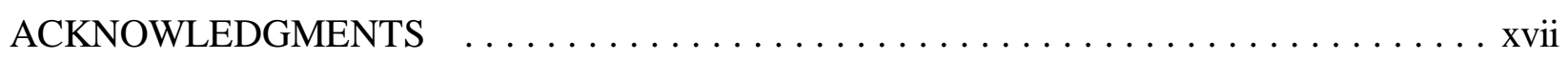

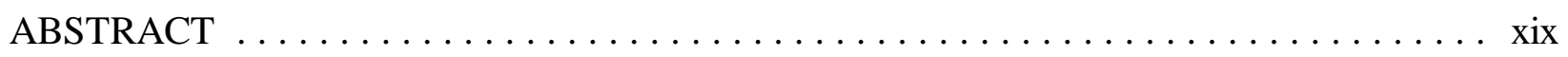

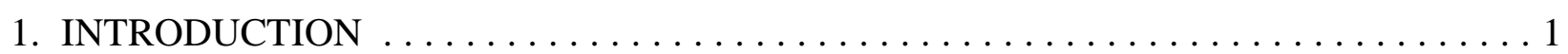

1.1 THE SCALE 5.0 SEQUENCES $\ldots \ldots \ldots \ldots \ldots \ldots \ldots \ldots \ldots \ldots \ldots \ldots \ldots$

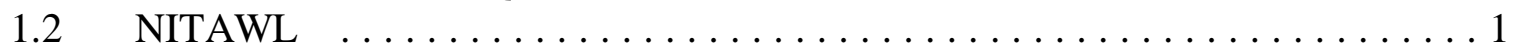

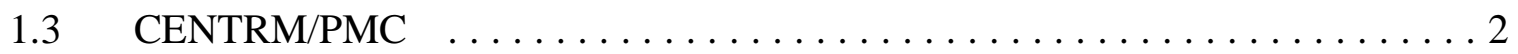

2. MIX-COMP-THERM-2 (PNL-30 to PNL-35) $\ldots \ldots \ldots \ldots \ldots \ldots \ldots \ldots \ldots \ldots \ldots$

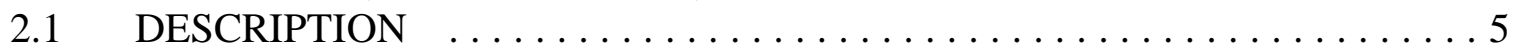

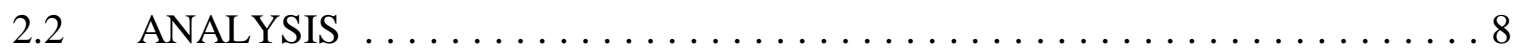

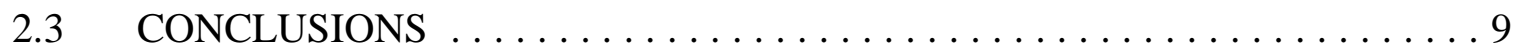

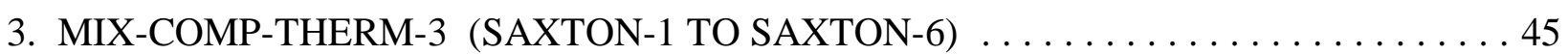

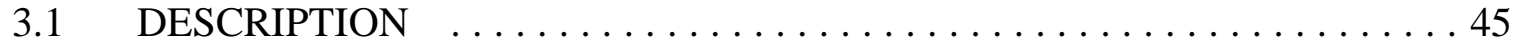

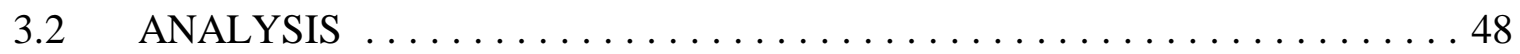

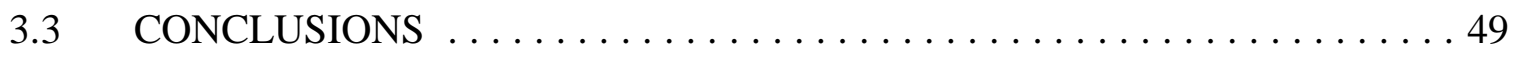

4. MIX-COMP-THERM-4 (TCA-1 TO TCA-11) . . . . . . . . . . . . . . . . 81

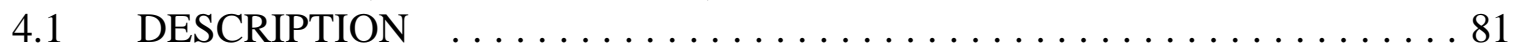

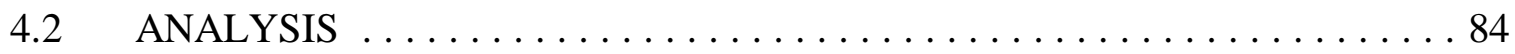

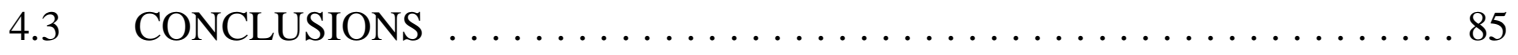

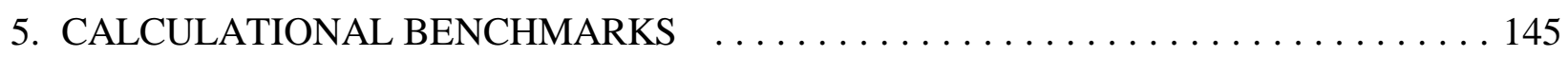

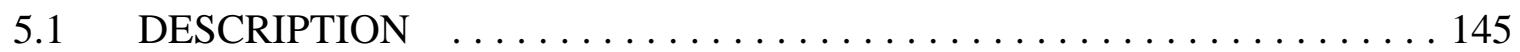

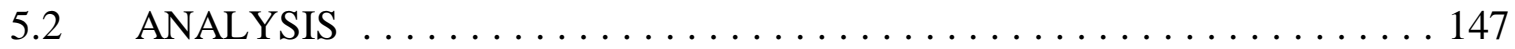

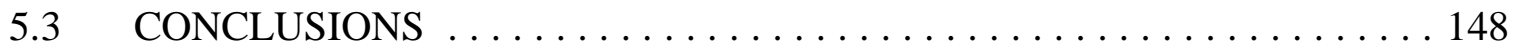

6. REFERENCES ......................................... 153

APPENDIX A: SCALE INPUT OF MIX-COMP-THERM-2 PROBLEM SET . . . . . . 155

APPENDIX B: SCALE INPUT OF MIX-COMP-THERM-3 PROBLEM SET $\ldots \ldots \ldots 207$ 


\section{CONTENTS (continued)}

APPENDIX C: SCALE INPUT OF MIX-COMP-THERM-4 PROBLEM SET $\ldots \ldots \ldots .241$

APPENDIX D: SCALE INPUT OF VARIANTS PROBLEM SET ... . . . . . . . . . . 295 


\section{LIST OF FIGURES}

\begin{tabular}{|c|c|}
\hline e & Page \\
\hline 2.1 & Schematic of the PNL fuel rods with reflector \\
\hline 2.2 & Pin layout of benchmark case PNL-30 \\
\hline 2.3 & Pin layout of benchmark cases PNL-31 and PNL-33 \\
\hline 2.4 & Pin layout of benchmark case PNL-32 $\ldots \ldots \ldots$ \\
\hline 2.5 & Pin layout of benchmark case PNL-34 \\
\hline 2.6 & Pin layout of benchmark case PNL-35 \\
\hline $2.7 \mathrm{a}$ & $\begin{array}{l}\text { Pin-power distribution for CENTRM benchmark PNL-30. Value in } \\
\text { parentheses is the percent standard deviation. } \ldots \ldots \ldots \ldots \ldots \ldots\end{array}$ \\
\hline $2.7 \mathrm{~b}$ & $\begin{array}{l}\text { Pin-power distribution for NITAWL benchmark PNL-30. Value in } \\
\text { parentheses is the percent standard deviation. . . . . . . . . }\end{array}$ \\
\hline $2.8 \mathrm{a}$ & $\begin{array}{l}\text { Pin-power distribution for CENTRM benchmark PNL-31. Value in } \\
\text { parentheses is the percent standard deviation. . . . . . . . . . . }\end{array}$ \\
\hline $2.8 \mathrm{~b}$ & $\begin{array}{l}\text { Pin-power distribution for NITAWL benchmark PNL-31. Value in } \\
\text { parentheses is the percent standard deviation. . . . . . . . . . . }\end{array}$ \\
\hline $2.9 \mathrm{a}$ & $\begin{array}{l}\text { Pin-power distribution for CENTRM benchmark PNL-32 } \\
\text { with standard deviation between } 0.59 \text { and } 0.87 \% \text { of the value }\end{array}$ \\
\hline $2.9 \mathrm{~b}$ & $\begin{array}{l}\text { Pin-power distribution for NITAWL benchmark PNL-32 } \\
\text { with standard deviation between } 0.57 \text { and } 0.90 \% \text { of the value. }\end{array}$ \\
\hline $2.10 \mathrm{a}$ & $\begin{array}{l}\text { Pin-power distribution for CENTRM benchmark PNL-33. Value in } \\
\text { parentheses is the percent standard deviation. . . . . . . . . . }\end{array}$ \\
\hline $2.10 \mathrm{~b}$ & $\begin{array}{l}\text { Pin-power distribution for NITAWL benchmark PNL-33. Value in } \\
\text { parentheses is the percent standard deviation. . . . . . . . . . }\end{array}$ \\
\hline $2.11 \mathrm{a}$ & $\begin{array}{l}\text { Pin-power distribution for CENTRM benchmark PNL-34. Value in } \\
\text { parentheses is the percent standard deviation. . . . . . . . . . . }\end{array}$ \\
\hline $2.11 \mathrm{~b}$ & $\begin{array}{l}\text { Pin-power distribution for NITAWL benchmark PNL-34. Value in } \\
\text { parentheses is the percent standard deviation. . . . . . . . . . . }\end{array}$ \\
\hline $2.12 \mathrm{a}$ & $\begin{array}{l}\text { Pin-power distribution for CENTRM benchmark PNL-35. Value in } \\
\text { parentheses is the percent standard deviation. . . . . . . . . . }\end{array}$ \\
\hline $2.12 b$ & $\begin{array}{l}\text { Pin-power distribution for NITAWL benchmark PNL-35. Value in } \\
\text { parentheses is the percent standard deviation. . . . . . . . . . }\end{array}$ \\
\hline 3.1 & Schematic of the SAXTON fuel rods with reflector \\
\hline 3.2 & Pin layout of benchmark case SAXTON-1 \\
\hline 3.3 & Pin layout of benchmark case SAXTON-2 \\
\hline 3.4 & Pin layout of benchmark case SAXTON-3 \\
\hline 3.5 & Pin layout of benchmark case SAXTON-4 \\
\hline 3.6 & Pin layout of benchmark cases SAXTON-5 and 6 \\
\hline $3.7 \mathrm{a}$ & $\begin{array}{l}\text { Pin-power distribution for CENTRM benchmark SAXTON-1. Value in } \\
\text { parentheses is the percent standard deviation. . . . . . . . . . . . }\end{array}$ \\
\hline
\end{tabular}




\section{LIST OF FIGURES (continued)}

\begin{tabular}{|c|c|}
\hline $3.7 \mathrm{~b}$ & $\begin{array}{l}\text { Pin-power distribution for NITAWL benchmark SAXTON-1. Value in } \\
\text { parentheses is the percent standard deviation. . . . . . . . . . . . }\end{array}$ \\
\hline $3.8 \mathrm{a}$ & $\begin{array}{l}\text { Pin-power distribution for CENTRM benchmark SAXTON-2. Value in } \\
\text { parentheses is the percent standard deviation. . . . . . . . . . . . . . }\end{array}$ \\
\hline $3.8 \mathrm{~b}$ & 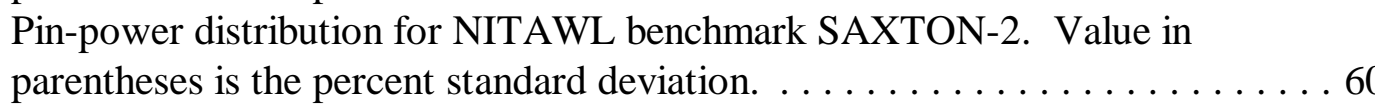 \\
\hline $3.9 \mathrm{a}$ & 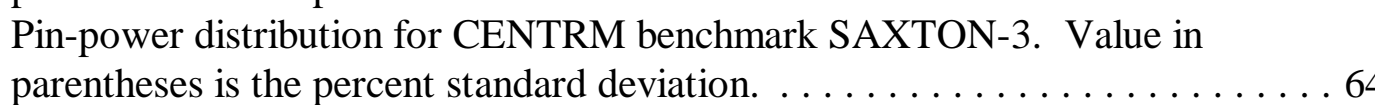 \\
\hline $3.9 \mathrm{~b}$ & $\begin{array}{l}\text { Pin-power distribution for NITAWL benchmark SAXTON-3. Value in } \\
\text { parentheses is the percent standard deviation. . . . . . . . . . . . }\end{array}$ \\
\hline $3.10 \mathrm{a}$ & 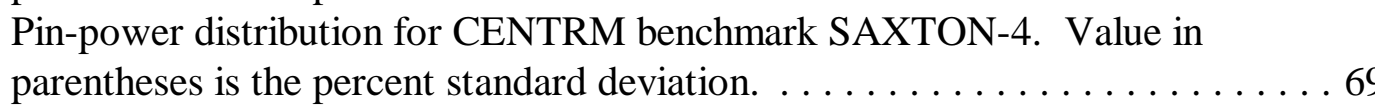 \\
\hline $3.10 \mathrm{~b}$ & 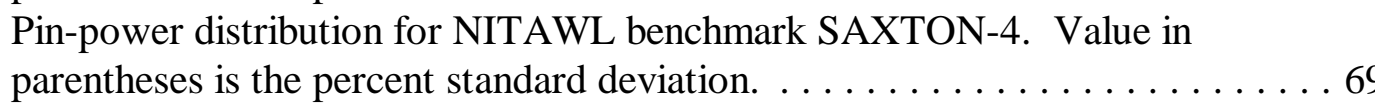 \\
\hline $3.11 \mathrm{a}$ & 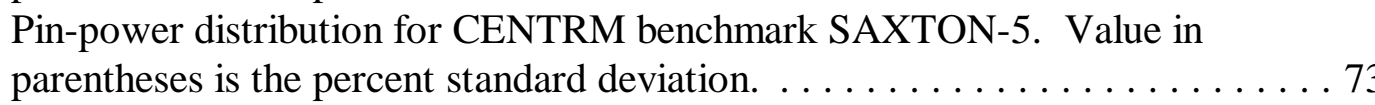 \\
\hline $3.11 \mathrm{~b}$ & $\begin{array}{l}\text { Pin-power distribution for NITAWL Benchmark SAXTON-5. Value in } \\
\text { parentheses is the percent standard deviation. . . . . . . . . . . . . . . }\end{array}$ \\
\hline $3.12 \mathrm{a}$ & $\begin{array}{l}\text { Pin-power distribution for CENTRM benchmark SAXTON-6. Value in } \\
\text { parentheses is the percent standard deviation. . . . . . . . . . . }\end{array}$ \\
\hline $3.12 \mathrm{~b}$ & $\begin{array}{l}\text { Pin-power distribution for NITAWL benchmark SAXTON-6. Value in } \\
\text { parentheses is the percent standard deviation. } \ldots \ldots \ldots \ldots \ldots \ldots \ldots\end{array}$ \\
\hline 4.1 & Schematic of fuel rods and bottom reflector \\
\hline 4.2 & Pin layout of benchmark cases TCA-1, TCA-2, and TCA-3 \\
\hline 4.3 & Pin lavout of benchmark cases TCA-4, TCA- 5 ,TCA- 6 , TCA-10, and TCA-11. 88 \\
\hline 4.4 & Pin lavout of benchmark cases TCA-7, TCA- 8 , and TCA- 9 \\
\hline $4.5 \mathrm{a}$ & $\begin{array}{l}\text { Pin-power distribution for CENTRM benchmark TCA-1. Value in } \\
\text { parentheses is the percent standard deviation. } \ldots \ldots \ldots \ldots \ldots \text {. }\end{array}$ \\
\hline $4.5 \mathrm{~b}$ & $\begin{array}{l}\text { Pin-power distribution for NITAWL benchmark TCA-1. Value in } \\
\text { parentheses is the percent standard deviation. . . . . . . . . . }\end{array}$ \\
\hline $4.6 \mathrm{a}$ & $\begin{array}{l}\text { Pin-power distribution for CENTRM benchmark TCA-2. Value in } \\
\text { parentheses is the percent standard deviation. . . . . . . . . . }\end{array}$ \\
\hline $4.6 \mathrm{~b}$ & 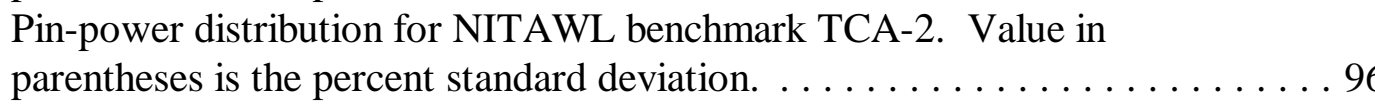 \\
\hline $4.7 \mathrm{a}$ & $\begin{array}{l}\text { Pin-power distribution for CENTRM benchmark TCA-3. Value in } \\
\text { parentheses is the percent standard deviation. . . . . . . . . . . . }\end{array}$ \\
\hline $4.7 \mathrm{~b}$ & $\begin{array}{l}\text { Pin-power distribution for NITAWL benchmark TCA-3. Value in } \\
\text { parentheses is the percent standard deviation. . . . . . . . . }\end{array}$ \\
\hline
\end{tabular}




\section{LIST OF FIGURES (continued)}

\begin{tabular}{|c|c|}
\hline $4.8 \mathrm{a}$ & $\begin{array}{l}\text { Pin-power distribution for CENTRM benchmark TCA-4. Value in } \\
\text { parentheses is the percent standard deviation. . . . . . . . . . . }\end{array}$ \\
\hline $4.8 \mathrm{~b}$ & $\begin{array}{l}\text { Pin-power distribution for NITAWL benchmark TCA-4. Value in } \\
\text { parentheses is the percent standard deviation. . . . . . . . . . }\end{array}$ \\
\hline $4.9 \mathrm{a}$ & $\begin{array}{l}\text { Pin-power distribution for CENTRM benchmark TCA-5. Value in } \\
\text { parentheses is the percent standard deviation. . . . . . . . . . . }\end{array}$ \\
\hline $4.9 \mathrm{~b}$ & $\begin{array}{l}\text { Pin-power distribution for NITAWL benchmark TCA-5. Value in } \\
\text { parentheses is the percent standard deviation. . . . . . . . . . }\end{array}$ \\
\hline $4.10 \mathrm{a}$ & $\begin{array}{l}\text { Pin-power distribution for CENTRM benchmark TCA-6. Value in } \\
\text { parentheses is the percent standard deviation. . . . . . . . . . . }\end{array}$ \\
\hline $4.10 \mathrm{~b}$ & $\begin{array}{l}\text { Pin-power distribution for NITAWL benchmark TCA-6. Value in } \\
\text { parentheses is the percent standard deviation. . . . . . . . . . . . }\end{array}$ \\
\hline $4.11 \mathrm{a}$ & $\begin{array}{l}\text { Pin-power distribution for CENTRM benchmark TCA-7. Value in } \\
\text { parentheses is the percent standard deviation. . . . . . . . . . . }\end{array}$ \\
\hline $4.11 \mathrm{~b}$ & $\begin{array}{l}\text { Pin-power distribution for NITAWL benchmark TCA-7. Value in } \\
\text { parentheses is the percent standard deviation. . . . . . . . . . }\end{array}$ \\
\hline $4.12 \mathrm{a}$ & $\begin{array}{l}\text { Pin-power distribution for CENTRM benchmark TCA-8. Value in } \\
\text { parentheses is the percent standard deviation. . . . . . . . . . }\end{array}$ \\
\hline $4.12 b$ & $\begin{array}{l}\text { Pin-power distribution for NITAWL benchmark TCA-8. Value in } \\
\text { parentheses is the percent standard deviation. . . . . . . . . . }\end{array}$ \\
\hline $4.13 a$ & $\begin{array}{l}\text { Pin-power distribution for CENTRM benchmark TCA-9. Value in } \\
\text { parentheses is the percent standard deviation. . . . . . . . . . }\end{array}$ \\
\hline $4.13 b$ & $\begin{array}{l}\text { Pin-power distribution for NITAWL benchmark TCA-9. Value in } \\
\text { parentheses is the percent standard deviation. . . . . . . . . . . . }\end{array}$ \\
\hline $4.14 \mathrm{a}$ & $\begin{array}{l}\text { Pin-power distribution for CENTRM benchmark TCA-10. Value in } \\
\text { parentheses is the percent standard deviation. . . . . . . . . . }\end{array}$ \\
\hline $4.14 \mathrm{~b}$ & $\begin{array}{l}\text { Pin-power distribution for NITAWL benchmark TCA-10. Value in } \\
\text { parentheses is the percent standard deviation. . . . . . . . . . }\end{array}$ \\
\hline $4.15 a$ & $\begin{array}{l}\text { Pin-power distribution for CENTRM benchmark TCA-11. Value in } \\
\text { parentheses is the percent standard deviation. . . . . . . . . . . }\end{array}$ \\
\hline $4.15 b$ & $\begin{array}{l}\text { Pin-power distribution for NITAWL benchmark TCA-11. Value in } \\
\text { parentheses is the percent standard deviation. . . . . . . . . . . }\end{array}$ \\
\hline
\end{tabular}





\section{LIST OF TABLES}

$\underline{\text { Table }}$

Page

2.1

2.2

2.3

2.4

2.5

2.6

2.7

2.8

2.9

2.10

2.11

2.12

2.13

2.14

2.15

2.16

2.17

2.18

2.19

2.20

2.21

2.22

2.23

2.24

2.25

2.26

2.27

2.28

2.29

2.30

2.31

2.32

2.33

2.34

2.35

2.36

2.37

Lattice description for benchmarks $\ldots \ldots \ldots \ldots \ldots \ldots \ldots \ldots \ldots \ldots$

Constant benchmark atom densities $\ldots \ldots \ldots \ldots \ldots \ldots \ldots \ldots \ldots \ldots$

Moderator atom densities . . . . . . . . . . . . . . . . . . . 8

Comparison of $k_{\text {off }}$ and EALCF from CENTRM and NITAWL $\ldots \ldots \ldots \ldots 10$

PNL-30 CENTRM reaction rates and fluxes $\ldots \ldots \ldots \ldots \ldots \ldots \ldots \ldots$

PNL-30 NITAWL reaction rates and fluxes $\ldots \ldots \ldots \ldots \ldots \ldots \ldots \ldots$

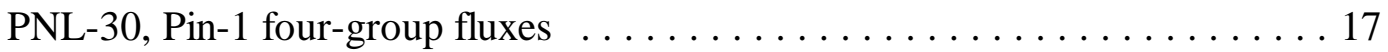

PNL-30, Pin-1 four-group U-235 cross sections $\ldots \ldots \ldots \ldots \ldots \ldots \ldots \ldots$

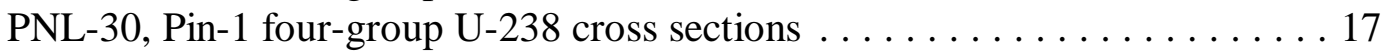

PNL-30, Pin-1 four-group Pu-239 cross sections $\ldots \ldots \ldots \ldots \ldots \ldots \ldots 17$

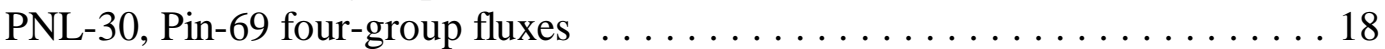

PNL-30, Pin-69 four-group U-235 cross sections $\ldots \ldots \ldots \ldots \ldots \ldots \ldots$

PNL-30, Pin-69 four-group U-238 cross sections . . . . . . . . . . . . 18

PNL-30, Pin-691 four-group Pu-239 cross sections $\ldots \ldots \ldots \ldots \ldots \ldots . . \ldots$

PNL-31 CENTRM reaction rates and fluxes $\ldots \ldots \ldots \ldots \ldots \ldots \ldots \ldots 21$

PNL-31 NITAWL reaction rates and fluxes $\ldots \ldots \ldots \ldots \ldots \ldots \ldots \ldots 21$

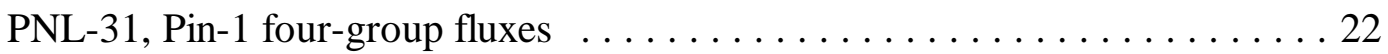

PNL-31, Pin-1 four-group U-235 cross sections $\ldots \ldots \ldots \ldots \ldots \ldots \ldots \ldots 22$

PNL-31, Pin-1 four-group U-238 cross sections . . . . . . . . . . . . 22

PNL-31, Pin-1 four-group Pu-239 cross sections $\ldots \ldots \ldots \ldots \ldots \ldots \ldots 22$

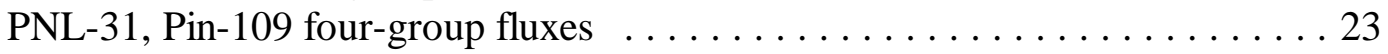

PNL-31, Pin-109 four-group U-235 cross sections . . . . . . . . . . . . 23

PNL-31, Pin-109 four-group U-238 cross sections . . . . . . . . . . . . 23

PNL-31, Pin-109 four-group Pu-239 cross sections $\ldots \ldots \ldots \ldots \ldots \ldots 23$

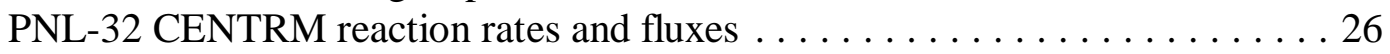

PNL-32 NITAWL reaction rates and fluxes $\ldots \ldots \ldots \ldots \ldots \ldots \ldots \ldots . \ldots \ldots$

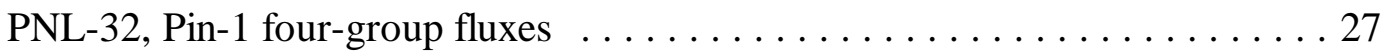

PNL-32, Pin-1 four-group U-235 cross sections $\ldots \ldots \ldots \ldots \ldots \ldots \ldots \ldots 27$

PNL-32, Pin-1 four-group U-238 cross sections . . . . . . . . . . . . . 27

PNL-32, Pin-1 four-group Pu-239 cross sections . . . . . . . . . . . . 27

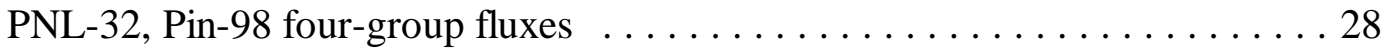

PNL-32, Pin-98 four-group U-235 cross sections . . . . . . . . . . . . 28

PNL-32, Pin-98 four-group U-238 cross sections . ................. 28

PNL-32, Pin-98 four-group Pu-239 cross sections . . . . . . . . . . . . . 28

PNL-33 CENTRM reaction rates and fluxes $\ldots \ldots \ldots \ldots \ldots \ldots \ldots \ldots \ldots \ldots$

PNL-33 NITAWL reaction rates and fluxes $\ldots \ldots \ldots \ldots \ldots \ldots \ldots \ldots \ldots \ldots$

PNL-33, Pin-1 four-group fluxes $\ldots \ldots \ldots \ldots \ldots \ldots \ldots \ldots \ldots \ldots \ldots \ldots \ldots \ldots$

ix 


\section{LIST OF TABLES (continued)}

\begin{tabular}{|c|c|}
\hline 2.38 & PNL-33, Pin-1 four-group U-235 cross sections \\
\hline 2.39 & PNL-33, Pin-1 four-group U-238 cross sections \\
\hline 2.40 & PNL-33, Pin-1 four-group Pu-239 cross sections \\
\hline 2.41 & PNL-33, Pin-109 four-group fluxes \\
\hline 2.42 & PNL-33, Pin-109 four-group U-235 cross sections \\
\hline 2.43 & PNL-33, Pin-109 four-group U-238 cross sections \\
\hline 2.44 & PNL-33, Pin-109 four-group Pu-239 cross sections \\
\hline 2.45 & PNL-34 CENTRM reaction rates and fluxes .... \\
\hline 2.46 & PNL-34 CENTRM reaction rates and fluxes \\
\hline 2.47 & PNL-34, Pin-1 four-group fluxes \\
\hline 2.48 & PNL-34, Pin-1 four-group U-235 cross sections \\
\hline 2.49 & PNL-34, Pin-1 four-group U-238 cross sections \\
\hline 2.50 & PNL-34, Pin-1 four-group Pu-239 cross sections \\
\hline 2.51 & PNL-34, Pin-27 four-group fluxes \\
\hline 2.52 & PNL-34, Pin-27 four-group U-235 cross sections \\
\hline 2.53 & PNL-34, Pin-27 four-group U-238 cross sections \\
\hline 2.54 & PNL-34, Pin-27 four-group Pu-239 cross sections \\
\hline 2.55 & PNL-35 CENTRM reaction rates and fluxes \\
\hline 2.56 & PNL-35 NITAWL reaction rates and fluxes \\
\hline 2.57 & PNL-35, Pin-1 four-group fluxes \\
\hline 2.58 & PNL-35, Pin- 1 four-group U-235 cross sections \\
\hline 2.59 & PNL-35, Pin-1 four-group U-238 cross sections \\
\hline 2.60 & PNL-35, Pin-1 four-group Pu-239 cross sections \\
\hline 2.61 & PNL-35, Pin-99 four-group fluxes \\
\hline 2.62 & PNL-35, Pin-99 four-group U-235 cross sections \\
\hline 2.63 & PNL-35, Pin-99 four-group U-238 cross sections \\
\hline 2.64 & PNL-35, Pin-99 four-group Pu-239 cross sections \\
\hline 3.1 & Lattice description for SAXTON benchmark cases \\
\hline 3.2 & Constant benchmark atom densities \\
\hline 3.3 & Moderator atom densities \\
\hline 3.4 & Comparison of $k_{o f f}$ and EALCF from CENTRM and NITAWL \\
\hline 3.5 & SAXTON-1 CENTRM reaction rates and fluxes \\
\hline 3.6 & SAXTON-1 NITAWL reaction rates and fluxes \\
\hline 3.7 & SAXTON-1, Pin-1 four-group fluxes \\
\hline 3.8 & SAXTON-1, Pin-1 four-group U-235 cross sections \\
\hline 3.9 & SAXTON-1, Pin-1 four-group U-238 cross sections \\
\hline 3.10 & SAXTON-1, Pin-1 four-group Pu-239 cross sections \\
\hline 3.11 & SAXTON-1, Pin-132 four-group fluxes. \\
\hline 3.12 & SAXTON-1, Pin-132 four-group U-235 cross \\
\hline
\end{tabular}




\section{LIST OF TABLES (continued)}

\begin{tabular}{|c|c|}
\hline 3.13 & SAXTON-1, Pin-132 four-grou \\
\hline 3.14 & SAXTON-1, Pin-132 four-group Pu-239 cross sections \\
\hline 3.15 & SAXTON-2 CENTRM reaction rates and fluxes \\
\hline 3.16 & SAXTON-2 NITAWL reaction rates and fluxes \\
\hline 3.17 & SAXTON-2, Pin-1 four-group fluxes \\
\hline 3.18 & SAXTON-2, Pin-1 four-group U-235 cross sections \\
\hline 3.19 & SAXTON-2, Pin-1 four-group U-238 cross sections \\
\hline 3.20 & SAXTON-2, Pin-1 four-group Pu-239 cross sections \\
\hline 3.21 & SAXTON-2, Pin-55 four-group fluxes \\
\hline 3.22 & SAXTON-2, Pin-55 four-group U-235 cross sections \\
\hline 3.23 & SAXTON-2, Pin-55 four-group U-238 cross sections \\
\hline 3.24 & SAXTON-2, Pin-55 four-group $\mathrm{Pu}-239$ cross sections \\
\hline 3.25 & SAXTON-3 CENTRM reaction rates and fluxes \\
\hline 3.26 & SAXTON-3 NITAWL reaction rates and fluxes \\
\hline 3.27 & SAXTON-3, Pin-1 four-group fluxes \\
\hline 3.28 & SAXTON-3, Pin-1 four-group U-235 cross sections \\
\hline 3.29 & SAXTON-3, Pin-1 four-group U-239 cross sections \\
\hline 3.30 & SAXTON-3, Pin-1 four-group Pu-239 cross sections \\
\hline 3.31 & SAXTON-3, Pin-66 four-group fluxes \\
\hline 3.32 & SAXTON-3, Pin-66 four-group U-235 cross sections \\
\hline 3.33 & SAXTON-3, Pin-66 four-group U-238 cross sections \\
\hline 3.34 & SAXTON-3, Pin-66 four-group $\mathrm{Pu}-239$ cross sections \\
\hline 3.35 & SAXTON-4 CENTRM reaction rates and fluxes \\
\hline 3.36 & SAXTON-4 NITAWL reaction rates and fluxes \\
\hline 3.37 & SAXTON-4, Pin-1 four-group fluxes \\
\hline 3.38 & SAXTON-4, Pin-1 four-group U-235 cross sections \\
\hline 3.39 & SAXTON-4, Pin-1 four-group U-238 cross sections \\
\hline 3.40 & SAXTON-4, Pin-1 four-group Pu-239 cross sections \\
\hline 3.41 & SAXTON-4, Pin-28 four-group fluxes \\
\hline 3.42 & SAXTON-4, Pin-28 four-group U-235 cross sections \\
\hline 3.43 & SAXTON-4, Pin-28 four-group U-239 cross sections \\
\hline 3.44 & SAXTON-4, Pin-28 four-gro \\
\hline 3.45 & SAXTON-5 CENTRM reaction rates and fluxes \\
\hline 3.46 & SAXTON-5 NITAWL reaction rates and fluxes \\
\hline 3.47 & SAXTON-5, Pin-1 four-group fluxes \\
\hline 3.48 & SAXTON-5, Pin-1 four-group U-235 cross sections \\
\hline 3.49 & SAXTON-5, Pin-1 four-group U-239 cross sections \\
\hline 3.50 & SAXTON-5, Pin-1 four-group Pu-239 cross sections \\
\hline 3.51 & SAXTON-5, Pin-21 four-group fluxes \\
\hline
\end{tabular}




\section{LIST OF TABLES (continued)}

\begin{tabular}{|c|c|}
\hline 3.52 & SAXTON-5, Pin-21 four-group U-235 cross se \\
\hline 3.53 & SAXTON-5, Pin-21 four-group U-238 cross sections \\
\hline 3.54 & SAXTON-5, Pin-21 four-group Pu-239 cross sections \\
\hline 3.55 & SAXTON-6 CENTRM reaction rates and fluxes \\
\hline 3.56 & SAXTON-6 NITAWL reaction rates and fluxes \\
\hline 3.57 & SAXTON-6, Pin-1 four-group fluxes \\
\hline 3.58 & SAXTON-6, Pin-1 four-group U-235 cross secti \\
\hline 3.59 & SAXTON-6, Pin-1 four-group U-238 cross sections \\
\hline 3.60 & SAXTON-6, Pin-1 four-group Pu-239 cross sections \\
\hline 3.61 & SAXTON-6, Pin-21 four-group fluxes \\
\hline 3.62 & SAXTON-6, Pin-21 four-group U-235 cross sections \\
\hline 3.63 & SAXTON-6, Pin-21 four-group U-239 cross sections \\
\hline 3.64 & SAXTON-6, Pin-21 four-group Pu-239 cross se \\
\hline 4.1 & Lattice description for benchmarks \\
\hline 4.2 & Benchmark atom densities \\
\hline 4.3 & $\mathrm{Pu}-241$ and $\mathrm{Am}-241$ atom densities \\
\hline 4.4 & Comparison of $k_{\text {off }}$ and EALCF from CENTRM and NITAWL \\
\hline 4.5 & Selected reaction rates for CENTRM case TCA-1 \\
\hline 4.6 & Selected reaction rates for NITAWL case TCA-1 \\
\hline 4.7 & TCA-1, Pin-1 four-group fluxes \\
\hline 4.8 & TCA-1, Pin-1 four-group U-235 cross sections \\
\hline 4.9 & TCA-1, Pin-1 four-group U-238 cross sections \\
\hline 4.10 & TCA-1, Pin-1 four-group Pu-239 cross sections \\
\hline 4.11 & TCA-1, Pin-78 four-group fluxes \\
\hline 4.12 & TCA-1, Pin-78 four-group U-235 cross sections \\
\hline 4.13 & TCA-1, Pin-78 four-group U-238 cross sections \\
\hline 4.14 & TCA-1, Pin-78 four-group $\mathrm{Pu}-239$ cross sections \\
\hline 4.15 & Selected reaction rates for CENTRM case TCA-2 \\
\hline 4.16 & Selected reaction rates for NITAWL case TCA- 2 \\
\hline 4.17 & TCA-2, Pin-1 four-group fluxes \\
\hline 4.18 & TCA-2, Pin-1 four-group U-235 cross sections \\
\hline 4.19 & TCA-2, Pin-1 four-group U-238 cross sections \\
\hline 4.20 & TCA-2, Pin-1 four-group Pu-239 cross sections \\
\hline 4.21 & TCA-2, Pin-78 four-group fluxes \\
\hline 4.22 & TCA-2, Pin-78 four-group U-235 cross sections \\
\hline 4.23 & TCA-2, Pin-78 four-group U-238 cross sections \\
\hline 4.24 & TCA-2, Pin-78 four-group Pu- 239 cross sections \\
\hline 4.25 & Selected reaction rates for CENTRM case TCA-3 \\
\hline
\end{tabular}




\section{LIST OF TABLES (continued)}

\begin{tabular}{|c|c|c|}
\hline 4.26 & Selected reaction rates for NITAWL case TCA-3 & \\
\hline 4.27 & TCA-3, Pin-1 four-group fluxes & 10 \\
\hline 4.28 & TCA-3, Pin-1 four-group U-235 cross sections & 10 \\
\hline 4.29 & TCA-3, Pin-1 four-group U-238 cross sections & 1 \\
\hline 4.30 & TCA-3, Pin-1 four-group $\mathrm{Pu}-239$ cross sections & \\
\hline 4.31 & TCA-3, Pin-78 four-group fluxes & \\
\hline 4.32 & TCA-3, Pin-78 four-group U-235 cross sections & \\
\hline 4.33 & TCA-3, Pin-78 four-group U-238 cross sections & \\
\hline 4.34 & TCA-3, Pin-78 four-group Pu-239 cross sections & \\
\hline 4.35 & Selected reaction rates for CENTRM case TCA- 4 & 1( \\
\hline 4.36 & Selected reaction rates for NITAWL case TCA-4 & \\
\hline 4.37 & TCA-4, Pin-1 four-group fluxes & \\
\hline 4.38 & TCA-4, Pin- 1 four-group U-235 cross sections & \\
\hline 4.39 & TCA-4, Pin-1 four-group U-238 cross sections & \\
\hline 4.40 & TCA-4, Pin-1 four-group $\mathrm{Pu}-239$ cross sections & \\
\hline 4.41 & TCA-4, Pin-66 four-group fluxes & \\
\hline 4.42 & TCA-4, Pin-66 four-group U-235 cross sections & 1 \\
\hline 4.43 & TCA-4, Pin-66 four-group U-238 cross sections & \\
\hline 4.44 & TCA-4, Pin- 66 four-group $\mathrm{Pu}-239$ cross sections & \\
\hline 4.45 & Selected reaction rates for CENTRM case TCA-5 & 1 \\
\hline 4.46 & Selected reaction rates for NITAWL case TCA-5 & 1 \\
\hline 4.47 & TCA-5, Pin-1 four-group fluxes & 1 \\
\hline 4.48 & TCA-5, Pin-1 four-group U-235 cross sections & 1 \\
\hline 4.49 & TCA-5, Pin-1 four-group U-238 cross sections & 1 \\
\hline 4.50 & TCA-5, Pin-1 four-group Pu-239 cross sections & \\
\hline 4.51 & TCA-5, Pin-66 four-group fluxes & \\
\hline 4.52 & TCA-5, Pin- 66 four-group U-235 cross sections & 1 \\
\hline 4.53 & TCA-5, Pin- 66 four-group U-238 cross sections & 1 \\
\hline 4.54 & TCA-5, Pin-66 four-group Pu-239 cross sections & 1 \\
\hline 4.55 & Selected reaction rates for CENTRM case TCA-6 & 1 \\
\hline 4.56 & Selected reaction rates for NITAWL case TCA-6 & 1 \\
\hline 4.57 & TCA-6, Pin-1 four-group fluxes & \\
\hline 4.58 & TCA-6, Pin-1 four-group U-235 cross sections & 1 \\
\hline 4.59 & TCA-6, Pin-1 four-group U-238 cross sections & 1 \\
\hline 4.60 & TCA-6, Pin- 1 four-group $\mathrm{Pu}-239$ cross sections & 11. \\
\hline 4.61 & TCA-6, Pin-66 four-group fluxes & 1 \\
\hline 4.62 & TCA-6, Pin-66 four-group U-235 cross sections & \\
\hline 4.63 & TCA-6, Pin-66 four-group U-238 cross sections. & \\
\hline 4.64 & TCA-6, Pin- 66 four-group Pu- 239 cross sections & \\
\hline
\end{tabular}




\section{LIST OF TABLES (continued)}

\begin{tabular}{|c|c|c|}
\hline 4.65 & Selected reaction rates for CENTRM case TCA-7 & \\
\hline 4.66 & Selected reaction rates for NITAWL case TCA-7 & 122 \\
\hline 4.67 & TCA-7, Pin-1 four-group fluxes & 123 \\
\hline 4.68 & TCA-7, Pin-1 four-group U-235 cross sections & 123 \\
\hline 4.69 & TCA-7, Pin-1 four-group U-238 cross sections. & 123 \\
\hline 4.70 & TCA-7, Pin-1 four-group $\mathrm{Pu}-239$ cross sections & 123 \\
\hline 4.71 & TCA-7, Pin-55 four-group fluxes & 124 \\
\hline 4.72 & TCA-7, Pin-55 four-group U-235 cross sections & 124 \\
\hline 4.73 & TCA-7, Pin-55 four-group U-238 cross sections & 124 \\
\hline 4.74 & TCA-7, Pin-55 four-group Pu-239 cross sections & 124 \\
\hline 4.75 & Selected reaction rates for CENTRM case TCA-8 & 127 \\
\hline 4.76 & Selected reaction rates for NITAWL case TCA- 8 & 127 \\
\hline 4.77 & TCA-8, Pin-1 four-group fluxes & 128 \\
\hline 4.78 & TCA-8, Pin-1 four-group U-235 cross sections & 128 \\
\hline 4.79 & TCA-8, Pin-1 four-group U-238 cross sections & 128 \\
\hline 4.80 & TCA-8, Pin-1 four-group Pu-239 cross sections & 128 \\
\hline 4.81 & TCA-8, Pin-55 four-group fluxes & 129 \\
\hline 4.82 & TCA-8, Pin-55 four-group U-235 cross sections & 129 \\
\hline 4.83 & TCA- 8 , Pin-55 four-group U-238 cross sections & 129 \\
\hline 4.84 & TCA- 8 , Pin-55 four-group $\mathrm{Pu}-239$ cross sections & 129 \\
\hline 4.85 & Selected reaction rates for CENTRM case TCA-9 & 132 \\
\hline 4.86 & Selected reaction rates for NITAWL case TCA-9 & 132 \\
\hline 4.87 & TCA-9, Pin-1 four-group fluxes & 133 \\
\hline 4.88 & TCA-9, Pin-1 four-group U-235 cross sections & 133 \\
\hline 4.89 & TCA-9, Pin-1 four-group U-238 cross sections & 133 \\
\hline 4.90 & TCA-9, Pin-1 four-group $\mathrm{Pu}-239$ cross sections & 133 \\
\hline 4.91 & TCA-9, Pin-55 four-group fluxes & 134 \\
\hline 4.92 & TCA-9, Pin-55 four-group U-235 cross sections & 134 \\
\hline 4.93 & TCA-9, Pin-55 four-group U-238 cross sections & 134 \\
\hline 4.94 & TCA-9, Pin-55 four-group Pu- 239 cross sections & 134 \\
\hline 4.95 & Selected reaction rates for CENTRM case TCA-10 & 137 \\
\hline 4.96 & Selected reaction rates for NITAWL case TCA-10 & 137 \\
\hline 4.97 & TCA-10, Pin-1 four-group fluxes & 138 \\
\hline 4.98 & TCA-10, Pin-1 four-group U-235 cross sections & 138 \\
\hline 4.99 & TCA-10, Pin-1 four-group U-238 cross sections & 138 \\
\hline 4.100 & TCA-10, Pin-1 four-group Pu-239 cross sections & 138 \\
\hline 4.101 & TCA-10, Pin-66 four-group fluxes & 139 \\
\hline 4.102 & TCA-10, Pin-66 four-group U-235 cross se & 139 \\
\hline
\end{tabular}




\section{LIST OF TABLES (continued)}

\begin{tabular}{|c|c|}
\hline 4.103 & TCA-10, Pin-66 four-group U-238 cross sections \\
\hline 4.104 & TCA-10, Pin-66 four-group Pu-239 cross sections \\
\hline 4.105 & Selected reaction rates for CENTRM case TCA-11 \\
\hline 4.106 & Selected reaction rates for NITAWL case TCA-11 \\
\hline 4.107 & TCA-11, Pin-1 four-group fluxes \\
\hline 4.108 & TCA-11, Pin-1 four-group U-235 cross sections \\
\hline 4.109 & TCA-11, Pin-1 four-group U-238 cross sections \\
\hline 4.110 & TCA-11, Pin-1 four-group Pu-239 cross sections \\
\hline 4.111 & TCA-11, Pin-66 four-group fluxes \\
\hline 4.112 & TCA-11, Pin-66 four-group U-235 cross sections \\
\hline 4.113 & TCA-11, Pin-66 four-group U-238 cross sections \\
\hline 4.114 & TCA-11, Pin-66 four-group Pu-239 cross sections \\
\hline 5.1 & State parameters for the calculational benchmark variants \\
\hline 5.2 & Basic fuel isotopic composition for each computational benchmark variant ... 146 \\
\hline 5.3 & Nonfuel material isotopic composition \\
\hline $5.4 \mathrm{a}$ & $k_{\Lambda}$ and EALCF values and differences for the va \\
\hline $5.4 \mathrm{~b}$ & $k_{\Lambda}$ and EALCF values and differences for the variant cases (cont.) \\
\hline $5.5 \mathrm{a}$ & Buckled $k_{\triangleleft}$ and EALCF values and differences for the variant cases \\
\hline $5.5 \mathrm{~b}$ & Buckled $k_{4}$ and EALCF values and differences for the variant cases (cont.) \\
\hline
\end{tabular}





\section{ACKNOWLEDGMENTS}

This work is supported jointly by the DOE Nuclear Criticality Safety Program and the DOE Fissile Material Disposition Program.

The author would like to thank the following individuals for their contributions: M. L. Williams, Louisiana State University, who wrote CENTRM and was available whenever problems were encountered with the code; L. M. Petrie for his assistance in implementing CENTRM into the SCALE sequence; W. C. Jordan and R. Q. Wright for testing and often breaking the code; M. E. Dunn and N. M. Greene for help creating the point cross-section library used by CENTRM; and J. B. Anderson for preparing this report. 



\section{ABSTRACT}

The purpose of this study is to determine and document the reactor physics parameters (multiplication factors, spatially dependent flux ratios, and spacially dependent reaction rates ) for several distinct sets of problems using two distinct resonance cross-section processing techniques. In SCALE, by default, resonances are processed using NITAWL, which utilizes the Nordheim Integral Treatment. The results produced using this sequence are considered to be the base results. A second set of results are produced by replacing NITAWL with CENTRM/PMC. CENTRM produces point-wise fluxes for a given geometry configuration and set of isotopes. Using these fluxes, PMC produces problem-dependent self-shielding cross sections. Both sequences use ENDF/B-V cross-section data. 



\section{INTRODUCTION}

This report examines four sets of problems that are used to evaluate the differences between the NITAWL resonance processor and the CENTRM/PMC resonance processor. All the problems are predominantly thermal, with the energy of the average lethargy causing fission (EALCF) being below $1 \mathrm{eV}$. They are all water-moderated fuel rod lattices of various dimensions and fuel materials. The objective of this report is to compare the differences between the NITAWL and CENTRM/PMC resolved-resonance processors for the class of problems presented in this report. The existence of severe resonance overlap when mixing uranium and plutonium could cause a significant problem with the NITAWL results. CENTRM/PMC does not contain this potential problem, which is a limitation of the Nordheim Integral Treatment.

All problem were processed using SCALE5.0, which allows one to choose which resolved resonance processor will be used to self-shield the nuclides, NITAWL or CENTRM/PMC. SCALE5.0 is a developmental version of the SCALE code system. The current version of SCALE, SCALE4.4, does not contain CENTRM but is essentially the same in all other aspects to SCALE5.0. SCALE5.0 has the ability to use either NITAWL or CENTRM/PMC as the resolved-resonance processor. All problems were run first using CENTRM/PMC and then using NITAWL. The results were then analyzed and compared.1,2

\subsection{THE SCALE 5.0 SEQUENCES}

SCALE 5.0 contains two distinct sequences used for processing cross-section data. The original sequence consisting of the codes CSAS, BONAMI, and NITAWL process all resolved resonance data in NITAWL. The new computational sequence added to SCALE5.0 uses the following sequence to process the cross sections: CSAS, BONAMI, NITAWL, CENTRM, PMC, WORKER. CENTRM/PMC process the resolved resonances cross sections in the unit cell. All other resolved resonance cross sections are processed using NITAWL. To calculate the desired physics parameters, the three experimental benchmark sets then use KENO-V.a and the set of computational benchmarks uses XSDRNPM. Many of the physics parameters are compared with direct experimental data and others are compared only with the same parameters calculated using difference codes and cross sections.

\subsection{NITAWL}

NITAWL performs problem-dependent resonance self-shielding using the Nordheim Integral Treatment, which involves solving for the energy dependence of the neutron flux in a material region containing a resonance absorber and a maximum of two moderating materials. The resonance selfshielding is made with reference to infinite dilution cross-section values. The material region is either infinite or a one-dimensional (1-D) slab, cylinder, or sphere surrounded by moderator. In the moderator, the neutron flux is assumed to be spatially flat and slowly varying with energy. The presence of more than one fuel region in the moderator, such as a lattice, is accounted for using a Dancoff factor. 
There are many problems where the Nordheim Integral Treatment may not be appropriate because of the following assumptions:

1. Each resonance nuclide can be treated without consideration of other resonance nuclides present in the system.

2. The neutron flux is spatially uniform in the absorber and moderator regions.

3. Neutron transport into and out of the absorber region can be treated with first-flight escape probabilities.

4. The presence of other absorber lumps in the system can be accounted for with a Dancoff factor which corresponds to the first-flight transmission probability across the moderator. A Dancoff factor of zero corresponds to a single lump in the moderator. A Dancoff factor of 1 corresponds to an infinite medium of the absorber material with no moderator outside the absorber lump.

\subsection{CENTRM/PMC}

The new resolved-resonance processor is composed of two codes that are executed sequentially. The first code, CENTRM, is a discrete-ordinance code that produces a point-wise continuous-energy flux spectrum for a given infinite or 1-D problem. Subsequently, PMC uses this point flux spectrum to collapse point cross sections to a problem-dependent multigroup cross-section set. To generate the flux profile and produce new group cross sections, the two codes use a pointwise continuous cross-section library that contain all the nuclides present in the selected multigroup cross-section library. All the cases in this report were analyzed using the 238-group ENDF/B-V multigroup cross-section library.

CENTRM has several options that determine the range over which the flux spectrum will be generated and the parameters used for generating the flux spectrum. Although pointwise continuous in energy, the flux spectrum is generated on a specified 1-D spacial mesh. For all cases in this report, the spatial mesh is over an infinite cylinder that is automatically generated by the code. The flux spectrum is generated from $20 \mathrm{MeV}$ to $1.0 \mathrm{E}-5 \mathrm{eV}$ over the spatial mesh using a default quadrature of 8. CENTRM uses the specified quadrature to generate Legendre coefficients for the scattering component of the pointwise flux.

CENTRM is capable of using a fixed or fission input source to generate the flux profile. For simplicity and because the benchmarks are fission problems, a spacially flat fission source is assumed as an initial guess. Two other parameters that can affect the flux are the point convergence criterion and the tolerance used for thinning the pointwise cross sections. These parameters are both set to default values of 0.0001 for the convergence criterion and 0.0025 for the thinning tolerance.

In order for CENTRM to generate the flux profile it first combines the pointwise continuous-energy cross sections of all nuclides in each zone. Each zone contains only one material but is divided into many intervals, with each interval being a mesh point where the entire energy spectrum of the flux is calculated. There is no code limit on the number of materials and 1-D zones. However, for large problems involving many materials and zones, computer memory and CPU speed may be a limiting factor.

By default, the flux profile is generated using an $S_{N}$ calculation over the entire spectrum. The option exists to perform several other types of calculations independently over the fast, thermal, and 
pointwise ranges. Over all energy ranges, scattering for the pointwise continuous cross sections is assumed to be elastic and isotropic in the center of mass system. Consequently, no scattering data are contained in the point library, only 1-D cross sections.

PMC replaces the cross sections for specified nuclides and energy groups in the problem-dependent multigroup library. PMC reads the pointwise flux file, the pointwise continuous cross sections, and the problem-dependent multigroup library; generates new multigroup data from the point data; and finally rewrites the multigroup library with the new multigroup data. PMC can produce multigroup cross sections over three ranges for all nuclides: the entire point library range, the resolved resonance range, or the pointwise flux range. For the problems, in this report PMC produces multigroup cross sections over the resolved resonance range of each nuclide in the unit cell data.

PMC has three methods for computing the scattering component of the new multigroup cross sections. The $\mathrm{P}_{\mathrm{N}}$ components of the original multigroup scattering cross sections can be renormalized to the new 1-D scattering cross sections. The $\mathrm{P}_{\mathrm{N}}$ components of the original multigroup scattering cross sections can be renormalized using the scalar flux. Finally, new $\mathrm{P}_{\mathrm{N}}$ components for the scattering cross section can be computed using the pointwise flux moments. For the problems in this report, the first option of renormalizing the $\mathrm{P}_{\mathrm{N}}$ components using the new 1-D cross sections is used.

A set of pointwise continuous cross sections are available for use by CENTRM and PMC. The point cross sections used in the report were generated using the AMPX code system from the ENDF/B-V nuclear data library. The library contains all the nuclides present in both the 238-group and 44-group ENDF/B-V multigroup libraries. The library contains the total, elastic scattering, and radiative capture cross sections for each nuclide. If a nuclide is fissionable, the fission cross section is also present. Also, if a nuclide contains inelastic data at higher energies, inelastic data are also included in the library. The pointwise continuous library is divided up into files, with each file containing all the cross-section data for one nuclide. The ENDF/B-V pointwise library was generated to be used with the ENDF/B-V multigroup libraries. Using it with any other type of library, such as one generated using ENDF/B-VI data, could produce incorrect results. 



\section{MIX-COMP-THERM-2 (PNL-30 to PNL-35)}

\subsection{DESCRIPTION}

This section describes a set of six critical experiments, each consisting of a square-pitched array of mixed plutonium-uranium fuel rods submerged in water surrounded by a water reflector. The water contains boron concentrations from 0.9 to $767.2 \mathrm{ppm}$. This set of experiments is contained in the International Handbook of Evaluated Criticality Safety Benchmark Experiments. 3

This set of experiments was performed between December 1975 and March 1976 at the Plutonium Recycle Critical Facility at Pacific Northwest Laboratory. The benchmark experiments are light-water-moderated critical assemblies consisting of a core array supported by upper and lower lattice plates. The lattice plates are $2.54 \mathrm{~cm}$ thick and located at the top and bottom of the fuel region - The reactor is brought to critical by raising the water level in the tank, thus avoiding the use of control rods. The fuel rods sit on a support plate above the bottom of the tank. The tank is wide enough to assume an infinite moderator on the sides and bottom $(\sim 30 \mathrm{~cm}$ of water).

All fuel rods have the same physical dimensions. A schematic diagram of the fuel rods and bottom reflector is given in Fig. 2.1. Each fuel rod has an active fuel length of $86.6775 \mathrm{~cm}, 0.5 \mathrm{~cm}$ of $\mathrm{UO}_{2}$ at the bottom of the fuel, a $0.6985-\mathrm{cm}$ cladding plug on the bottom and a 0.8255 -cm cladding plug on the top. The fuel has a radius of $0.64135 \mathrm{~cm}$. The cladding has an outside radius of 0.71755 $\mathrm{cm}$. A $0.3175-\mathrm{cm}$ water gap is located between the top of the aluminum support plate and the bottom of the lower lattice plate. The aluminum support plate is $2.8575 \mathrm{~cm}$ thick. There is a $0.889-\mathrm{cm}$ water gap between the bottom of the lead plate and the top of the upper lattice plate. The lead plate is $0.9525 \mathrm{~cm}$ thick. The top reflector varies depending on the water level for the particular problem.

The primary differences between the six benchmarks are lattice pitch, number of rods in the lattice, water level, and boron concentrations. All other benchmark characteristics are constant. There are three different lattice pitches, with each pitch used in two problems: $1.778 \mathrm{~cm}, 2.20914 \mathrm{~cm}$, and $2.51447 \mathrm{~cm}$. The fuel is arranged in a square-pitched lattice. The characteristics of each of the four lattices are given in Table 2.1. Table 2.2 contains the atom densities for all the materials in the problem except B-10 and B-11. The atom densities in Table 2.2 are constant for all benchmarks. Table 2.3 contain the atom densities of B-10 and B-11 for each benchmark. All material temperatures are assumed to be $23^{\circ} \mathrm{C}(295 \mathrm{~K})$.

As shown in Table 2.1, the six benchmark problems change by moderator boron concentration, number of fuel rods, lattice pitch and upper-reflector thickness. To simplify the accumulation of power densities, advantage was taken in the symmetry of the problem whenever possible. The entire problem was explicitly modeled, but instead of having a separate unit for each pin, 1/8th symmetry was used for every problem except PNL-32. Due to an irregular placement of outer rods, this problem needed to be modeled using a unit for each pin. The lattice is then filled from the 1/8th section by inserting additional pins in a mirror image. Figures 2.2 through 2.6 show the lattice map for each problem. Figure 2.4 shows a full-lattice mapping; the other figures contain only 1/8th lattices. The remaining lattice can be extrapolated from these $1 / 8$ th sections. 


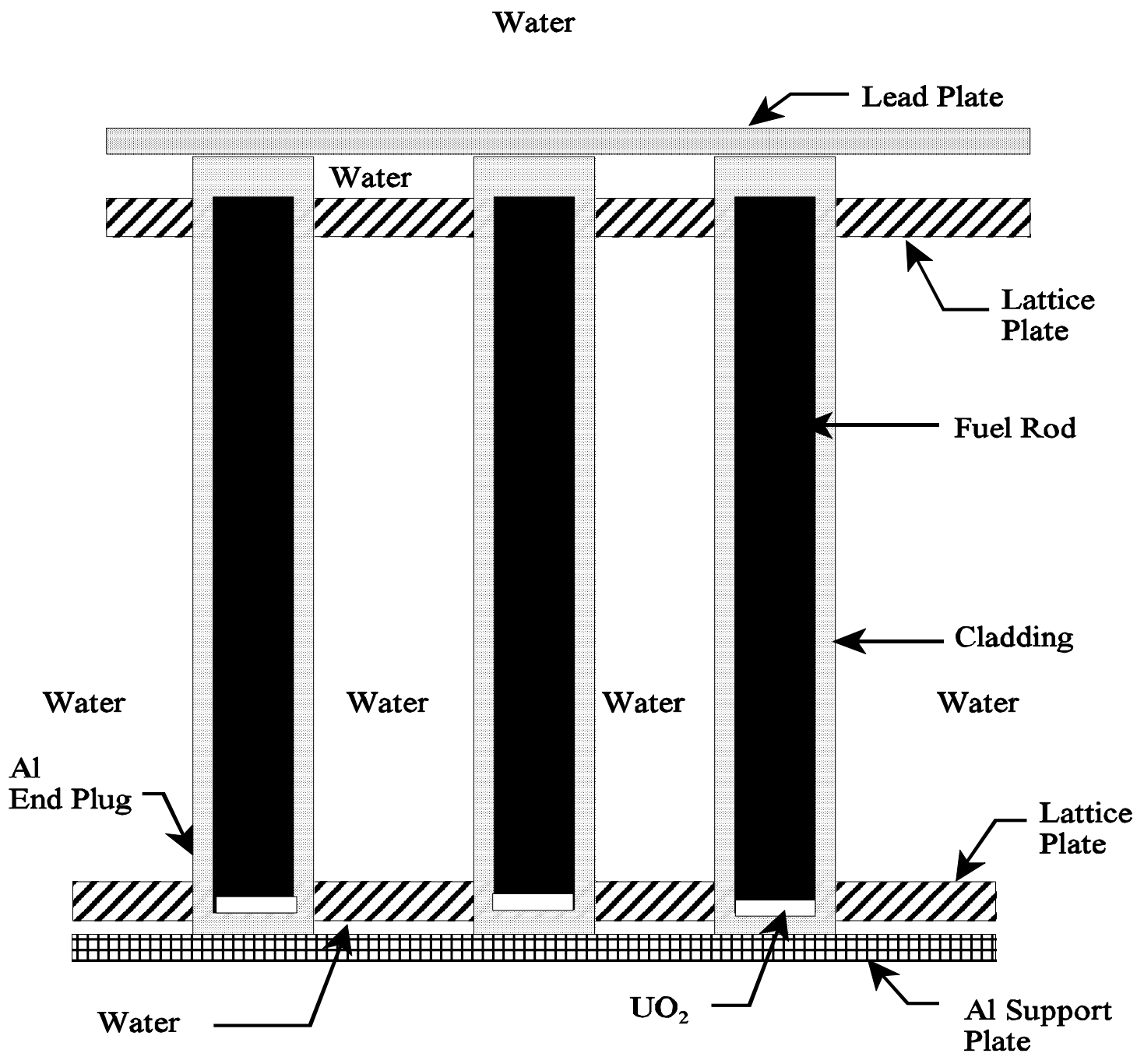

Water

Fig. 2.1. Schematic of the PNL fuel rods with reflector. 
Table 2.1. Lattice description for benchmarks

\begin{tabular}{ccccc}
\hline $\begin{array}{c}\text { Benchmark } \\
\text { No. }\end{array}$ & $\begin{array}{c}\text { Boron con. } \\
(\mathrm{ppm})\end{array}$ & $\begin{array}{c}\text { Critical } \\
\text { No. rods }\end{array}$ & $\begin{array}{c}\text { Lattice } \\
\text { pitch } \\
(\mathrm{cm})\end{array}$ & $\begin{array}{c}\text { Water level } \\
\text { above lead } \\
(\mathrm{cm})\end{array}$ \\
\hline 30 & 1.7 & 469 & 1.778 & 13.462 \\
31 & 687.9 & 761 & 1.778 & 13.462 \\
32 & 0.9 & 195 & 2.20914 & 3.937 \\
33 & 1090.4 & 761 & 2.20914 & 13.462 \\
34 & 1.6 & 160 & 2.51447 & 0.508 \\
35 & 767.2 & 689 & 2.51447 & 13.462 \\
\hline
\end{tabular}

Table 2.2. Constant benchmark atom densities

\begin{tabular}{cccccc}
\hline Material & Isotope & $\begin{array}{c}\text { Atom density } \\
\text { (atoms/barn-cm) }\end{array}$ & Material & Isotope & $\begin{array}{c}\text { Atom density } \\
\text { (atoms/barn-cm) }\end{array}$ \\
\hline Fuel & $\mathrm{U}-234$ & $1.2458 \times 10^{-6}$ & Lattice plate & $\mathrm{Si}$ & $1.3742 \times 10^{-2}$ \\
$\mathrm{UO}_{2}-\mathrm{PuO}_{2}$ & $\mathrm{U}-235$ & $1.4886 \times 10^{-4}$ & Support plate & $\mathrm{Fe}$ & $4.5919 \times 10^{-2}$ \\
$(9.54 \mathrm{~g} / \mathrm{cc})$ & $\mathrm{U}-236$ & $2.0936 \times 10^{-9}$ & & $\mathrm{Cu}$ & $1.1532 \times 10^{-4}$ \\
& $\mathrm{U}-238$ & $2.0936 \times 10^{-2}$ & & $\mathrm{Mn}$ & $9.6395 \times 10^{-4}$ \\
& $\mathrm{Pu}-238$ & $3.8836 \times 10^{-8}$ & & $\mathrm{Mg}$ & $1.2388 \times 10^{-4}$ \\
& $\mathrm{Pu}-239$ & $3.9462 \times 10^{-4}$ & & $\mathrm{Cr}$ & $1.7409 \times 10^{-3}$ \\
& $\mathrm{Pu}-240$ & $3.3206 \times 10^{-5}$ & & $\mathrm{Zn}$ & $1.6617 \times 10^{-2}$ \\
& $\mathrm{Pu}-241$ & $1.6081 \times 10^{-6}$ & & $\mathrm{Ti}$ & $4.6052 \times 10^{-4}$ \\
& $\mathrm{Pu}-242$ & $1.1882 \times 10^{-7}$ & & $\mathrm{Al}$ & $1.5025 \times 10^{-3}$ \\
& $\mathrm{Am}-241$ & $1.4954 \times 10^{-6}$ & & & \\
& $\mathrm{O}-16$ & $4.3779 \times 10^{-2}$ & & & \\
& $\mathrm{U}-234$ & $1.2406 \times 10^{-6}$ & Cladding & $\mathrm{Sn}$ & $4.8328 \times 10^{-4}$ \\
Natural UO $\mathrm{UO}_{2}$ & $\mathrm{U}-235$ & $1.4824 \times 10^{-4}$ & & $\mathrm{Fe}$ & $9.5642 \times 10^{-5}$ \\
& $\mathrm{U}-236$ & $2.0848 \times 10^{-9}$ & & $\mathrm{Cr}$ & $7.6093 \times 10^{-5}$ \\
& $\mathrm{U}-238$ & $2.0525 \times 10^{-2}$ & & $\mathrm{Ni}$ & $3.0336 \times 10^{-5}$ \\
& $\mathrm{O}-16$ & $4.1943 \times 10^{-2}$ & & $\mathrm{Zr}$ & $4.2621 \times 10^{-2}$ \\
& & & & $\mathrm{~Pb}$ & $3.2174 \times 10^{-2}$ \\
& & & & \\
\hline
\end{tabular}


Table 2.3. Moderator atom densities

\begin{tabular}{ccccc}
\hline $\begin{array}{c}\text { Benchmark } \\
\text { No. }\end{array}$ & \multicolumn{4}{c}{ Atom densities ( atoms/barn-cm ) } \\
\cline { 2 - 5 } & $\mathrm{H}$ & $\mathrm{O}$ & $\mathrm{B}-10$ & $\mathrm{~B}-11$ \\
\hline PNL-30 & $6.6706 \times 10^{-2}$ & $3.3353 \times 10^{-2}$ & $1.8706 \times 10^{-8}$ & $7.5770 \times 10^{-8}$ \\
PNL-31 & $6.6605 \times 10^{-2}$ & $3.3400 \times 10^{-2}$ & $7.5838 \times 10^{-6}$ & $3.0718 \times 10^{-5}$ \\
PNL-32 & $6.6706 \times 10^{-2}$ & $3.3353 \times 10^{-2}$ & $9.9034 \times 10^{-9}$ & $4.0114 \times 10^{-8}$ \\
PNL-33 & $6.6672 \times 10^{-2}$ & $3.3427 \times 10^{-2}$ & $1.2034 \times 10^{-5}$ & $4.8746 \times 10^{-5}$ \\
PNL-34 & $6.6706 \times 10^{-2}$ & $3.3353 \times 10^{-2}$ & $1.7606 \times 10^{-8}$ & $7.1313 \times 10^{-8}$ \\
PNL-35 & $6.6682 \times 10^{-2}$ & $3.3405 \times 10^{-2}$ & $8.4597 \times 10^{-5}$ & $3.4266 \times 10^{-5}$ \\
\hline
\end{tabular}

\subsection{ANALYSIS}

All six computational benchmarks in this section were processed twice using SCALE 5.0. The set labeled NITAWL uses the NITAWL resonance processor to self-shield the resolved resonance region for all nuclides in the fuel and clad. An identical set, labeled CENTRM, replaces NITAWL with the CENTRM/PMC code sequence for the nuclides in the unit cell. The NITAWL and CENTRM results are then compared.

Table 2.4 contains the $k_{e f f}$ and energy of the average lethargy causing fission (EALCF). The $k_{e f f}$ values for all the benchmark cases are close to 1.0: the worst NITAWL benchmark is $0.90 \%$ high, and the worst CENTRM case is $0.68 \%$ high. A small negative bias does appear between NITAWL and CENTRM, with the CENTRM cases about $0.25 \%$ lower on average than the NITAWL cases.

The EALCF is also shown in this table. The EALCF values listed are from the CENTRM cases. EALCF is the energy of the average lethargy causing fission and is calculated by summing the lethargies of the neutrons that undego fission, dividing the value by the number of fissions, and converting this value to energy. For all cases, the difference between this value for CENTRM and NITAWL was less than $0.1 \%$. Both CENTRM and NITAWL produce excellent and consistent results for the $k_{\text {eff }}$ and fission energy for this set of problems.

Additional parameters calculated for each problem include: pin-power distributions; absortption, $\mathrm{G}_{\mathrm{f}}$, and fission reaction rates and fluxes in the pin fuel, clad, and moderator; and four group cross sections and fluxes for a corner outside pin and the innermost pin. Each of these sets of data are calculated using both CENTRM and NITAWL. All the data for each case are contained in Figs. 2.7a through $2.12 \mathrm{~b}$ and Tables 2.5 through 2.64 .

The pin-power distributions for this problem assumed 1/8th core symmetry with a surrounding reflector for all benchmark cases except PNL-32. Because of a lack of symmetry in two outer pins the entire assembly needed to be modeled. The pin-power data consist of a value and a standard deviation for each pin. The values for the pin powers are actually in units of fissions per $\mathrm{cm}^{3}$-s-source particle $\times 10^{-5}$. The value in parentheses is the percent standard deviation of the pin-power value.

In most cases the CENTRM and NITAWL results for each benchmark case fall within 2 standard deviations. The peak-to-low power changes with change in pitch and boron concentration. 
The peak-to-low power ratio changed from a low of 1.86 for the smallest pitch with no boron added to a high of 4.19 at the largest pitch with added boron. The addition of boron significantly shifts the flux profile to the center of the assembly by depressing the thermal flux in the moderator.

The reaction rates, total fluxes, and flux ratios for the CENTRM and NITAWL cases of each benchmark case are also in good agreement, seldom varying by more than $1 \%$. These values are included for a corner pin and the center pin for each case. All the reaction rates and fluxes increase as the pins approach the center of the assembly if the outer two layers of the arrays are excluded. The lowest power pin occurs in either the outer layer or the next inner layer of the array. The flux ratios over the fuel, clad, and moderator are relatively constant for a given pitch and boron concentration. However, as the pitch increases, the proportion of the total pin flux in the moderator increases.

Finally, four-group fluxes and cross sections were calculated for selected nuclides in the fuel region of the same corner and center pins. The macroscopic cross sections listed include the radiative capture, fission, and nu*fission cross sections for U-235, U-238, and Pu-239. The four groups are collapsed from the 238-group multigroup cross-section set using the flux profile calculated in KENOV.a as follows: group 1 is from $20 \mathrm{Mev}$ to $9.5 \mathrm{keV}$, group 2 is from $9.5 \mathrm{keV}$ to $3.0 \mathrm{eV}$, group 3 is from $3 \mathrm{eV}$ to $0.4 \mathrm{eV}$, and group 4 is from $0.4 \mathrm{eV}$ to $10^{-5} \mathrm{eV}$. The $0.4 \mathrm{eV}$ was chosen as a boundary because it is the cadmium cutoff energy. Groups 2 and 3 contain the resolved resonance regions for most of the nuclides used in these cases. Most of the cross-section data for the CENTRM and NITAWL cases of a given benchmark case agree within $1 \%$.

\subsection{CONCLUSIONS}

For this set of benchmark cases, using either NITAWL and CENTRM as the resonance region processor produces acceptable results of the $k_{\text {eff }}$ values. All other values produced using CENTRM and NITAWL are also consistent with each. For all cases, the $k_{\text {eff }}$ 's produced using CENTRM are slightly lower, $\sim 0.25 \%$, than those produced using NITAWL to do the resonance self-shielding. As a result, the average $k_{\text {eff }}$ of all the CENTRM cases is closer to 1.0 than the average $k_{\text {eff }}$ of all the NITAWL cases for this set of benchmarks. The pin-power distributions, fluxes, reactions rates, and macroscopic cross sections all agree to approximately $1 \%$ between CENTRM and NITAWL.

The presence of boron in the moderator depresses the fraction of the flux in the moderator, thus creating a larger difference between the highest- and lowest-power pins. No significant differences were identified between the results produced by NITAWL and CENTRM for these benchmark cases. 
Table 2.4. Comparison of $k_{\text {eff }}$ and EALCF from CENTRM and NITAWL

\begin{tabular}{ccccc}
\hline $\begin{array}{c}\text { CASE } \\
\text { MIX-COMP-THERM- }\end{array}$ & $\begin{array}{c}\text { NITAWL } \\
k_{\text {eff }}( \pm \mathrm{F})\end{array}$ & $\begin{array}{c}\text { CENTRM } \\
k_{\text {eff }}( \pm \mathrm{F})\end{array}$ & $\%$ DIFF & $\begin{array}{c}\text { EALCF } \\
(\mathrm{eV})\end{array}$ \\
\hline PNL-30 & $1.000(0.0005)^{a}$ & $0.9966(0.0004)$ & -0.34 & 0.575 \\
PNL-31 & $1.0026(0.0004)$ & $0.9987(0.0003)$ & -0.39 & 0.768 \\
PNL-32 & $1.0021(0.0004)$ & $1.0000(0.0004)$ & -0.21 & 0.193 \\
PNL-33 & $1.0090(0.0003)$ & $1.0064(0.0004)$ & -0.26 & 0.282 \\
PNL-34 & $1.0046(0.0004)$ & $1.0020(0.0005)$ & -0.26 & 0.138 \\
PNL-35 & $1.0079(0.0004)$ & $1.0068(0.0004)$ & -0.11 & 0.182 \\
\hline
\end{tabular}

${ }^{a}$ Values in parentheses is the percent standard deviation.

\begin{tabular}{|c|c|c|c|c|c|c|c|c|c|c|c|}
\hline & & & & & & & & & & & 69 \\
\hline & & & & & & & & & & 67 & 68 \\
\hline & & & & & & & & & 64 & 65 & 66 \\
\hline & & & & & & & & 60 & 61 & 62 & 63 \\
\hline & & & & & & & 55 & 56 & 57 & 58 & 59 \\
\hline & & & & & & 49 & 50 & 51 & 52 & 53 & 54 \\
\hline & & & & & 42 & 43 & 44 & 45 & 46 & 47 & 48 \\
\hline & & & & 34 & 35 & 36 & 37 & 38 & 39 & 40 & 41 \\
\hline & & & 25 & 26 & 27 & 28 & 29 & 30 & 31 & 32 & 33 \\
\hline & & $\mathrm{M}$ & 16 & 17 & 18 & 19 & 20 & 21 & 22 & 23 & 24 \\
\hline & $\mathrm{M}$ & $\mathrm{M}$ & $\mathrm{M}$ & 8 & 9 & 10 & 11 & 12 & 13 & 14 & 15 \\
\hline $\mathrm{M}$ & $\mathrm{M}$ & $\mathrm{M}$ & $\mathrm{M}$ & $\mathrm{M}$ & 1 & 2 & 3 & 4 & 5 & 6 & 7 \\
\hline
\end{tabular}

Fig. 2.2. Pin layout of benchmark case PNL-30. 


\begin{tabular}{|c|c|c|c|c|c|c|c|c|c|c|c|}
\hline & & & & & & & & & & & 109 \\
\hline & & & & & & & & & & 107 & 108 \\
\hline & & & & & & & & & 104 & 105 & 106 \\
\hline & & & & & & & & 100 & 101 & 102 & 103 \\
\hline & & & & & & & 95 & 96 & 97 & 98 & 99 \\
\hline & & & & & & 89 & 90 & 91 & 92 & 93 & 94 \\
\hline & & & & & 82 & 83 & 84 & 85 & 86 & 87 & 88 \\
\hline & & & & 74 & 75 & 76 & 77 & 78 & 79 & 80 & 81 \\
\hline & & & 65 & 66 & 67 & 68 & 69 & 70 & 71 & 72 & 73 \\
\hline & & 55 & 56 & 57 & 58 & 59 & 60 & 61 & 62 & 63 & 64 \\
\hline & 44 & 45 & 46 & 47 & 48 & 49 & 50 & 51 & 52 & 53 & 54 \\
\hline 32 & 33 & 34 & 35 & 36 & 37 & 38 & 39 & 40 & 41 & 42 & 43 \\
\hline $\mathrm{M}$ & 21 & 22 & 23 & 24 & 25 & 26 & 27 & 28 & 29 & 30 & 31 \\
\hline $\mathrm{M}$ & $\mathrm{M}$ & $\mathrm{M}$ & 12 & 13 & 14 & 15 & 16 & 17 & 18 & 19 & 20 \\
\hline M & $\mathrm{M}$ & $\mathrm{M}$ & $\mathrm{M}$ & $\mathrm{M}$ & 5 & 6 & 7 & 8 & 9 & 10 & 11 \\
\hline $\mathrm{M}$ & $\mathrm{M}$ & $\mathrm{M}$ & $\mathrm{M}$ & $\mathrm{M}$ & $\mathrm{M}$ & $\mathrm{M}$ & $\mathrm{M}$ & 1 & 2 & 3 & 4 \\
\hline
\end{tabular}

Fig. 2.3. Pin layout of benchmark cases PNL-31 and PNL-33. 


\begin{tabular}{|l|l|l|l|l|l|l|l|l|l|l|l|l|l|l|l|l|}
\hline $\mathrm{M}$ & $\mathrm{M}$ & $\mathrm{M}$ & $\mathrm{M}$ & $\mathrm{M}$ & $\mathrm{M}$ & $\mathrm{M}$ & 193 & 194 & 195 & $\mathrm{M}$ & $\mathrm{M}$ & $\mathrm{M}$ & $\mathrm{M}$ & $\mathrm{M}$ & $\mathrm{M}$ & $\mathrm{M}$ \\
\hline $\mathrm{M}$ & $\mathrm{M}$ & $\mathrm{M}$ & $\mathrm{M}$ & $\mathrm{M}$ & $\mathrm{M}$ & 187 & 188 & 189 & 190 & 191 & 192 & $\mathrm{M}$ & $\mathrm{M}$ & $\mathrm{M}$ & $\mathrm{M}$ & $\mathrm{M}$ \\
\hline $\mathrm{M}$ & $\mathrm{M}$ & $\mathrm{M}$ & $\mathrm{M}$ & 178 & 179 & 180 & 181 & 182 & 183 & 184 & 185 & 186 & $\mathrm{M}$ & $\mathrm{M}$ & $\mathrm{M}$ & $\mathrm{M}$ \\
\hline $\mathrm{M}$ & $\mathrm{M}$ & $\mathrm{M}$ & 167 & 168 & 169 & 170 & 171 & 172 & 173 & 174 & 175 & 176 & 177 & $\mathrm{M}$ & $\mathrm{M}$ & $\mathrm{M}$ \\
\hline $\mathrm{M}$ & $\mathrm{M}$ & 154 & 155 & 156 & 157 & 158 & 159 & 160 & 161 & 162 & 163 & 164 & 165 & 166 & $\mathrm{M}$ & $\mathrm{M}$ \\
\hline $\mathrm{M}$ & 139 & 140 & 141 & 142 & 143 & 144 & 145 & 146 & 147 & 148 & 149 & 150 & 151 & 152 & 153 & $\mathrm{M}$ \\
\hline $\mathrm{M}$ & 124 & 125 & 126 & 127 & 128 & 129 & 130 & 131 & 132 & 133 & 134 & 135 & 136 & 137 & 138 & $\mathrm{M}$ \\
\hline 107 & 108 & 109 & 110 & 111 & 112 & 113 & 114 & 115 & 116 & 117 & 118 & 119 & 120 & 121 & 122 & 123 \\
\hline 90 & 91 & 92 & 93 & 94 & 95 & 96 & 97 & 98 & 99 & 100 & 101 & 102 & 103 & 104 & 105 & 106 \\
\hline 73 & 74 & 75 & 76 & 77 & 78 & 79 & 80 & 81 & 82 & 83 & 84 & 85 & 86 & 87 & 88 & 89 \\
\hline $\mathrm{M}$ & 58 & 59 & 60 & 61 & 62 & 63 & 64 & 65 & 66 & 67 & 68 & 69 & 70 & 71 & 72 & $\mathrm{M}$ \\
\hline $\mathrm{M}$ & 43 & 44 & 45 & 46 & 47 & 48 & 49 & 50 & 51 & 52 & 53 & 54 & 55 & 56 & 57 & $\mathrm{M}$ \\
\hline $\mathrm{M}$ & $\mathrm{M}$ & 30 & 31 & 32 & 33 & 34 & 35 & 36 & 37 & 38 & 39 & 40 & 41 & 42 & $\mathrm{M}$ & $\mathrm{M}$ \\
\hline $\mathrm{M}$ & $\mathrm{M}$ & $\mathrm{M}$ & 19 & 20 & 21 & 22 & 23 & 24 & 25 & 26 & 27 & 28 & 29 & $\mathrm{M}$ & $\mathrm{M}$ & $\mathrm{M}$ \\
\hline $\mathrm{M}$ & $\mathrm{M}$ & $\mathrm{M}$ & $\mathrm{M}$ & 10 & 11 & 12 & 13 & 14 & 15 & 16 & 17 & 18 & $\mathrm{M}$ & $\mathrm{M}$ & $\mathrm{M}$ & $\mathrm{M}$ \\
\hline $\mathrm{M}$ & $\mathrm{M}$ & $\mathrm{M}$ & $\mathrm{M}$ & $\mathrm{M}$ & 4 & 5 & 6 & 7 & 8 & 9 & $\mathrm{M}$ & $\mathrm{M}$ & $\mathrm{M}$ & $\mathrm{M}$ & $\mathrm{M}$ & $\mathrm{M}$ \\
\hline $\mathrm{M}$ & $\mathrm{M}$ & $\mathrm{M}$ & $\mathrm{M}$ & $\mathrm{M}$ & $\mathrm{M}$ & $\mathrm{M}$ & 1 & 2 & 3 & $\mathrm{M}$ & $\mathrm{M}$ & $\mathrm{M}$ & $\mathrm{M}$ & $\mathrm{M}$ & $\mathrm{M}$ & $\mathrm{M}$ \\
\hline
\end{tabular}

Fig. 2.4. Pin layout of benchmark case PNL-32.

\begin{tabular}{|c|c|c|c|c|c|c|c|c|}
\hline & & & & & & & & 27 \\
\hline & & & & & & & 25 & 26 \\
\hline & & & & & & 22 & 23 & 24 \\
\hline & & & & & 18 & 19 & 20 & 21 \\
\hline & & & & 13 & 14 & 15 & 16 & 17 \\
\hline & & & $\mathrm{M}$ & 8 & 9 & 10 & 11 & 12 \\
\hline & & $\mathrm{M}$ & $\mathrm{M}$ & $\mathrm{M}$ & 4 & 5 & 6 & 7 \\
\hline & $\mathrm{M}$ & $\mathrm{M}$ & $\mathrm{M}$ & $\mathrm{M}$ & $\mathrm{M}$ & $\mathrm{M}$ & 2 & 3 \\
\hline $\mathrm{M}$ & $\mathrm{M}$ & $\mathrm{M}$ & $\mathrm{M}$ & $\mathrm{M}$ & $\mathrm{M}$ & $\mathrm{M}$ & $\mathrm{M}$ & 1 \\
\hline
\end{tabular}

Fig. 2.5. Pin layout of benchmark case PNL-34. 


\begin{tabular}{|c|c|c|c|c|c|c|c|c|c|c|}
\hline & & & & & & & & & & 99 \\
\hline & & & & & & & & & 97 & 98 \\
\hline & & & & & & & & 94 & 95 & 96 \\
\hline & & & & & & & 90 & 91 & 92 & 93 \\
\hline & & & & & & 85 & 86 & 87 & 88 & 89 \\
\hline & & & & & 79 & 80 & 81 & 82 & 83 & 84 \\
\hline & & & & 72 & 73 & 74 & 75 & 76 & 77 & 78 \\
\hline & & & 64 & 65 & 66 & 67 & 68 & 69 & 70 & 71 \\
\hline & & 55 & 56 & 57 & 58 & 59 & 60 & 61 & 62 & 63 \\
\hline & 45 & 46 & 47 & 48 & 49 & 50 & 51 & 52 & 53 & 54 \\
\hline 34 & 35 & 36 & 37 & 38 & 39 & 40 & 41 & 42 & 43 & 44 \\
\hline $\mathrm{M}$ & 24 & 25 & 26 & 27 & 28 & 29 & 30 & 31 & 32 & 33 \\
\hline $\mathrm{M}$ & $\mathrm{M}$ & 15 & 16 & 17 & 18 & 19 & 20 & 21 & 22 & 23 \\
\hline $\mathrm{M}$ & $\mathrm{M}$ & $\mathrm{M}$ & 7 & 8 & 9 & 10 & 11 & 12 & 13 & 14 \\
\hline $\mathrm{M}$ & M & $\mathrm{M}$ & $\mathrm{M}$ & $\mathrm{M}$ & 1 & 2 & 3 & 4 & 5 & 6 \\
\hline
\end{tabular}

Fig. 2.6. Pin layout of benchmark case PNL-35. 


\begin{tabular}{|c|c|c|c|c|c|c|c|c|c|c|c|}
\hline & & & & & & & & & & & $\begin{array}{r}2.475 \\
(0.91)\end{array}$ \\
\hline & & & & & & & & & & $\begin{array}{l}2.458 \\
(0.46)\end{array}$ & $\begin{array}{r}2.460 \\
(0.46)\end{array}$ \\
\hline & & & & & & & & & $\begin{array}{l}2.413 \\
(0.48)\end{array}$ & $\begin{array}{c}2.427 \\
(0.31)\end{array}$ & $\begin{array}{r}2.423 \\
(0.44)\end{array}$ \\
\hline & & & & & & & & $\begin{array}{r}2.235 \\
(0.48)\end{array}$ & $\begin{array}{l}2.337 \\
(0.35)\end{array}$ & $\begin{array}{c}2.354 \\
(0.35)\end{array}$ & $\begin{array}{r}2.386 \\
(0.43)\end{array}$ \\
\hline & & & & & & & $\begin{array}{r}2.095 \\
(0.46)\end{array}$ & $\begin{array}{c}2.183 \\
(0.35)\end{array}$ & $\begin{array}{l}2.220 \\
(0.32)\end{array}$ & $\begin{array}{c}2.275 \\
(0.33)\end{array}$ & $\begin{array}{c}2.293 \\
(0.46)\end{array}$ \\
\hline & & & & & & $\begin{array}{r}1.883 \\
(0.57)\end{array}$ & $\begin{array}{l}1.982 \\
(0.35)\end{array}$ & $\begin{array}{l}2.074 \\
(0.32)\end{array}$ & $\begin{array}{l}2.127 \\
(0.36)\end{array}$ & $\begin{array}{c}2.150 \\
(0.35)\end{array}$ & $\begin{array}{c}2.168 \\
(0.49)\end{array}$ \\
\hline & & & & & $\begin{array}{l}1.636 \\
(0.58)\end{array}$ & $\begin{array}{c}1.761 \\
(0.41)\end{array}$ & $\begin{array}{l}1.860 \\
(0.35)\end{array}$ & $\begin{array}{l}1.947 \\
(0.35)\end{array}$ & $\begin{array}{l}1.999 \\
(0.36)\end{array}$ & $\begin{array}{l}2.033 \\
(0.35)\end{array}$ & $\begin{array}{c}2.042 \\
(0.49)\end{array}$ \\
\hline & & & & $\begin{array}{l}1.423 \\
(0.62)\end{array}$ & $\begin{array}{c}1.534 \\
(0.40)\end{array}$ & $\begin{array}{r}1.616 \\
(0.35)\end{array}$ & $\begin{array}{r}1.707 \\
(0.41)\end{array}$ & $\begin{array}{r}1.792 \\
(0.38)\end{array}$ & $\begin{array}{l}1.833 \\
(0.35)\end{array}$ & $\begin{array}{l}1.874 \\
(0.35)\end{array}$ & $\begin{array}{c}1.877 \\
(0.50)\end{array}$ \\
\hline & & & $\begin{array}{l}1.530 \\
(0.56)\end{array}$ & $\begin{array}{l}1.381 \\
(0.46)\end{array}$ & $\begin{array}{r}1.416 \\
(0.43)\end{array}$ & $\begin{array}{l}1.483 \\
(0.40)\end{array}$ & $\begin{array}{l}1.557 \\
(0.42)\end{array}$ & $\begin{array}{l}1.624 \\
(0.38)\end{array}$ & $\begin{array}{r}1.670 \\
(0.33)\end{array}$ & $\begin{array}{l}1.710 \\
(0.38)\end{array}$ & $\begin{array}{c}1.714 \\
(0.55)\end{array}$ \\
\hline & & 0 & $\begin{array}{l}2.213 \\
(0.35)\end{array}$ & $\begin{array}{l}1.510 \\
(0.44)\end{array}$ & $\begin{array}{c}1.358 \\
(0.41)\end{array}$ & $\begin{array}{l}1.367 \\
(0.43)\end{array}$ & $\begin{array}{r}1.423 \\
(0.42)\end{array}$ & $\begin{array}{l}1.493 \\
(0.39)\end{array}$ & $\begin{array}{l}1.530 \\
0.43)\end{array}$ & $\begin{array}{l}1.566 \\
(0.39)\end{array}$ & $\begin{array}{c}1.566 \\
(0.55)\end{array}$ \\
\hline & 0 & 0 & 0 & $\begin{array}{l}2.160 \\
(0.37)\end{array}$ & $\begin{array}{l}1.519 \\
(0.43)\end{array}$ & $\begin{array}{l}1.391 \\
(0.43)\end{array}$ & $\begin{array}{l}1.437 \\
(0.44)\end{array}$ & $\begin{array}{l}1.480 \\
(0.39)\end{array}$ & $\begin{array}{l}1.539 \\
(0.40)\end{array}$ & $\begin{array}{l}1.566 \\
(0.39)\end{array}$ & $\begin{array}{r}1.573 \\
(0.54)\end{array}$ \\
\hline 0 & 0 & 0 & 0 & 0 & $\begin{array}{l}2.311 \\
(0.40)\end{array}$ & $\begin{array}{l}2.001 \\
(0.43)\end{array}$ & $\begin{array}{c}2.035 \\
(0.39)\end{array}$ & $\begin{array}{l}2.124 \\
(0.40)\end{array}$ & $\begin{array}{r}2.197 \\
(0.36)\end{array}$ & $\begin{array}{l}2.225 \\
(0.39)\end{array}$ & $\begin{array}{c}2.254 \\
(0.57)\end{array}$ \\
\hline
\end{tabular}

Fig. 2.7a. Pin-power distribution for CENTRM benchmark PNL-30. Value in parentheses is the percent standard deviation. 


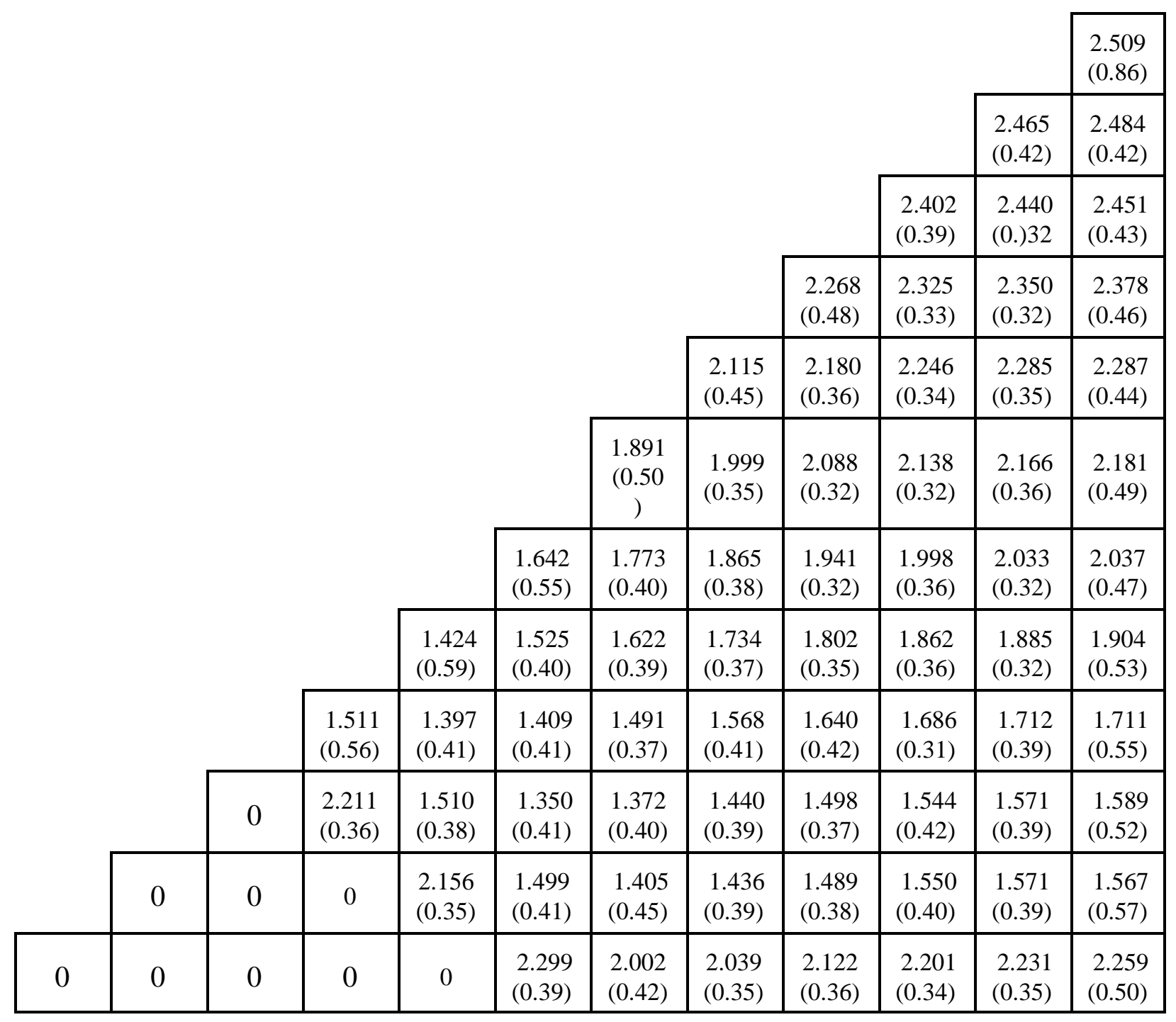

Fig. 2.7b. Pin-power distribution for NITAWL benchmark PNL-30. Value in parentheses is the percent standard deviation. 
Table 2.5. PNL-30 CENTRM reaction rates and fluxes

\begin{tabular}{|c|c|c|c|c|c|c|}
\hline Pin & Region & $\begin{array}{c}\mathrm{G}_{\mathrm{a}} \mathrm{M} \\
\left(\mathrm{cm}^{-3}-\mathrm{s}^{-1}\right)\end{array}$ & $\begin{array}{c}\mathcal{G}_{\mathrm{f}} \mathrm{M} \\
\left(\mathrm{cm}^{-3}-\mathrm{s}^{-1}\right)\end{array}$ & $\begin{array}{c}\mathrm{G}_{\mathrm{f}} \mathrm{M} \\
\left(\mathrm{cm}^{-3}-\mathrm{s}^{-1}\right)\end{array}$ & $\begin{array}{c}M \\
\left({\left.\mathrm{~cm}-\mathrm{s}^{-1}\right)}^{-1}\right.\end{array}$ & $M / M_{t}$ \\
\hline \multirow{3}{*}{1} & Fuel & $1.417 \mathrm{E}-05$ & $2.311 \mathrm{E}-05$ & $8.244 \mathrm{E}-06$ & $1.385 \mathrm{E}-2$ & 0.412 \\
\hline & Clad & $2.864 \mathrm{E}-07$ & 0.0 & 0.0 & $2.672 \mathrm{E}-3$ & 0.079 \\
\hline & Mod. & $5.922 \mathrm{E}-07$ & 0.0 & 0.0 & $1.710 \mathrm{E}-2$ & 0.509 \\
\hline \multirow{3}{*}{69} & Fuel & $1.809 \mathrm{E}-05$ & $2.453 \mathrm{E}-05$ & 8.727E-06 & $3.412 \mathrm{E}-2$ & 0.410 \\
\hline & Clad & $4.701 \mathrm{E}-07$ & 0.0 & 0.0 & $6.638 \mathrm{E}-3$ & 0.080 \\
\hline & Mod. & $5.476 \mathrm{E}-07$ & 0.0 & 0.0 & $4.237 \mathrm{E}-2$ & 0.510 \\
\hline
\end{tabular}

Table 2.6. PNL-30 NITAWL reaction rates and fluxes

\begin{tabular}{ccccccc}
\hline Pin & Region & $\begin{array}{c}\mathrm{G}_{\mathrm{a}} \mathrm{M} \\
\left(\mathrm{cm}^{-3}-\mathrm{s}^{-1}\right)\end{array}$ & $\begin{array}{c}\mathrm{G}_{\mathrm{f}} \mathrm{M} \\
\left(\mathrm{cm}^{-3}-\mathrm{s}^{-1}\right)\end{array}$ & $\begin{array}{c}\mathrm{G}_{\mathrm{f}} \mathrm{M} \\
\left(\mathrm{cm}^{-3}-\mathrm{s}^{-1}\right)\end{array}$ & $\begin{array}{c}\mathrm{M} \\
\left(\mathrm{cm} \mathrm{-} \mathrm{s}^{-1}\right)\end{array}$ & $\mathrm{MM}_{\mathrm{t}}$ \\
\hline \multirow{2}{*}{1} & Fuel & $1.407 \mathrm{E}-05$ & $2.297 \mathrm{E}-05$ & $8.192 \mathrm{E}-06$ & $1.384 \mathrm{E}-2$ & 0.412 \\
& Clad & $2.802 \mathrm{E}-07$ & 0.0 & 0.0 & $2.660 \mathrm{E}-3$ & 0.079 \\
& Mod. & $5.895 \mathrm{E}-07$ & 0.0 & 0.0 & $1.707 \mathrm{E}-2$ & 0.509 \\
\hline \multirow{2}{*}{69} & Fuel & $1.835 \mathrm{E}-05$ & $2.515 \mathrm{E}-05$ & $8.948 \mathrm{E}-06$ & $3.395 \mathrm{E}-2$ & 0.410 \\
& Clad & $4.678 \mathrm{E}-07$ & 0.0 & 0.0 & $6.629 \mathrm{E}-3$ & 0.080 \\
& Mod. & $5.644 \mathrm{E}-07$ & 0.0 & 0.0 & $4.232 \mathrm{E}-2$ & 0.510 \\
\hline
\end{tabular}


Table 2.7. PNL-30, Pin-1 four-group fluxes

\begin{tabular}{ccc}
\hline Group & $\begin{array}{c}\text { CENTRM M } \\
\left(\mathrm{cm}^{-2}-\mathrm{s}^{-1}\right)\end{array}$ & $\begin{array}{c}\text { NITAWL M } \\
\left(\mathrm{cm}^{-2}-\mathrm{s}^{-1}\right)\end{array}$ \\
\hline 1 & $7.308 \mathrm{E}-05$ & $7.296 \mathrm{E}-05$ \\
2 & $2.292 \mathrm{E}-05$ & $2.291 \mathrm{E}-05$ \\
3 & $5.145 \mathrm{E}-06$ & $5.269 \mathrm{E}-06$ \\
4 & $2.251 \mathrm{E}-05$ & $2.239 \mathrm{E}-05$ \\
\hline
\end{tabular}

Table 2.8. PNL-30, Pin-1 four-group U-235 cross sections

\begin{tabular}{cccccccc}
\hline Group & \multicolumn{3}{c}{ CENTRM cross sections $\left(\mathrm{cm}^{-1}\right)$} & \multicolumn{2}{c}{ NITAWL cross sections $\left(\mathrm{cm}^{-1}\right)$} \\
\hline & $\frac{\mathrm{G}_{\mathrm{a}}}{2.31102 \mathrm{E}-04}$ & $\frac{\mathrm{G}_{\mathrm{f}}}{5.17496 \mathrm{E}-04}$ & $\frac{\mathrm{G}_{\mathrm{f}}}{2.00508 \mathrm{E}-04}$ & $\frac{\mathrm{G}_{\mathrm{a}}}{2.30990 \mathrm{E}-04}$ & $\frac{\mathrm{G}_{\mathrm{f}}}{5.17470 \mathrm{E}-04}$ & $\frac{\mathrm{G}_{\mathrm{f}}}{2.00433 \mathrm{E}-04}$ \\
1 & $5.24167 \mathrm{E}-03$ & $8.10227 \mathrm{E}-03$ & $3.32510 \mathrm{E}-03$ & $5.22152 \mathrm{E}-03$ & $8.04138 \mathrm{E}-03$ & $3.30010 \mathrm{E}-03$ \\
2 & $8.11652 \mathrm{E}-03$ & $1.64870 \mathrm{E}-02$ & $6.76610 \mathrm{E}-03$ & $8.06149 \mathrm{E}-03$ & $1.64181 \mathrm{E}-02$ & $6.73784 \mathrm{E}-03$ \\
3 & $7.34638 \mathrm{E}-02$ & $1.52885 \mathrm{E}-01$ & $6.27423 \mathrm{E}-02$ & $7.34430 \mathrm{E}-02$ & $1.52842 \mathrm{E}-01$ & $6.27253 \mathrm{E}-02$ \\
\hline
\end{tabular}

Table 2.9. PNL-30, Pin-1 four-group U-238 cross sections

\begin{tabular}{cccccccc}
\hline Group & \multicolumn{3}{c}{ CENTRM cross sections $\left(\mathrm{cm}^{-1}\right)$} & \multicolumn{3}{c}{ NITAWL cross sections $\left(\mathrm{cm}^{-1}\right)$} \\
\hline & $\frac{\mathrm{G}_{\mathrm{a}}}{}$ & $\frac{\mathrm{G}_{\mathrm{f}}}{1.78395 \mathrm{E}-03}$ & $\frac{1.18456 \mathrm{E}-02}{4.21512 \mathrm{E}-03}$ & $\frac{\mathrm{G}_{\mathrm{f}}}{4.78829 \mathrm{E}-03}$ & $\frac{\mathrm{G}_{\mathrm{f}}}{1.18801 \mathrm{E}-02}$ & $\overline{\mathrm{G}}_{\mathrm{f}}$ \\
\hline & $4.22404 \mathrm{E}-03$ \\
3 & $1.10308 \mathrm{E}-02$ & $3.66348 \mathrm{E}-06$ & $2.34636 \mathrm{E}-06$ & $4.43898 \mathrm{E}-02$ & $5.54963 \mathrm{E}-06$ & $2.29918 \mathrm{E}-06$ \\
4 & $4.21801 \mathrm{E}-02$ & $1.98117 \mathrm{E}-07$ & $8.20835 \mathrm{E}-08$ & $4.21730 \mathrm{E}-02$ & $1.98085 \mathrm{E}-07$ & $8.20706 \mathrm{E}-08$ \\
\hline
\end{tabular}

Table 2.10. PNL-30, Pin-1 four-group Pu-239 cross sections

\begin{tabular}{cccccccc}
\hline Group & \multicolumn{3}{c}{ CENTRM cross sections $\left(\mathrm{cm}^{-1}\right)$} & \multicolumn{3}{c}{ NITAWL cross sections $\left(\mathrm{cm}^{-1}\right)$} \\
\hline & $\frac{\mathrm{G}_{\mathrm{a}}}{7.31247 \mathrm{E}-04}$ & $\frac{\mathrm{G}_{\mathrm{f}}}{2.10623 \mathrm{E}-03}$ & $\frac{\mathrm{G}_{\mathrm{f}}}{6.81955 \mathrm{E}-04}$ & $\frac{\mathrm{G}_{\mathrm{a}}}{7.31013 \mathrm{E}-04}$ & $\frac{\mathrm{G}_{\mathrm{f}}}{2.10628 \mathrm{E}-03}$ & $\frac{\mathrm{G}_{\mathrm{f}}}{6.81768 \mathrm{E}-04}$ \\
1 & $1.46556 \mathrm{E}-02$ & $2.42259 \mathrm{E}-02$ & $8.41230 \mathrm{E}-03$ & $1.42800 \mathrm{E}-02$ & $2.35796 \mathrm{E}-02$ & $8.18791 \mathrm{E}-03$ \\
2 & $4.46091 \mathrm{E}-02$ & $8.80453 \mathrm{E}-02$ & $3.05734 \mathrm{E}-02$ & $4.45318 \mathrm{E}-02$ & $8.79178 \mathrm{E}-02$ & $3.05291 \mathrm{E}-02$ \\
3 & $3.81701 \mathrm{E}-01$ & $7.65540 \mathrm{E}-01$ & $2.64839 \mathrm{E}-01$ & $3.80931 \mathrm{E}-01$ & $7.64203 \mathrm{E}-01$ & $2.64376 \mathrm{E}-01$ \\
\hline
\end{tabular}


Table 2.11. PNL-30, Pin-69 four-group fluxes

\begin{tabular}{ccc}
\hline Group & $\begin{array}{c}\text { CENTRM M } \\
\left(\mathrm{cm}^{-2}-\mathrm{s}^{-1}\right)\end{array}$ & $\begin{array}{c}\text { NITAWL M } \\
\left(\mathrm{cm}^{-2}-\mathrm{s}^{-1}\right)\end{array}$ \\
\hline 1 & $1.978 \mathrm{E}-04$ & $1.958 \mathrm{E}-04$ \\
2 & $7.342 \mathrm{E}-05$ & $7.309 \mathrm{E}-05$ \\
3 & $1.445 \mathrm{E}-05$ & $1.458 \mathrm{E}-05$ \\
4 & $1.901 \mathrm{E}-05$ & $1.966 \mathrm{E}-05$ \\
\hline
\end{tabular}

Table 2.12. PNL-30, Pin-69 four-group U-235 cross sections

\begin{tabular}{cccccccc}
\hline Group & \multicolumn{3}{c}{ CENTRM cross sections $\left(\mathrm{cm}^{-1}\right)$} & \multicolumn{3}{c}{ NITAWL cross sections $\left(\mathrm{cm}^{-1}\right)$} \\
\hline & $\frac{\mathrm{G}_{\mathrm{a}}}{2.40583 \mathrm{E}-04}$ & $\frac{\mathrm{G}_{\mathrm{f}}}{5.25394 \mathrm{E}-04}$ & $\frac{\mathrm{G}_{\mathrm{f}}}{2.05274 \mathrm{E}-04}$ & $\frac{\mathrm{G}_{\mathrm{a}}}{2.40289 \mathrm{E}-04}$ & $\frac{\mathrm{G}_{\mathrm{f}}}{5.25082 \mathrm{E}-04}$ & $\frac{\mathrm{G}_{\mathrm{f}}}{2.05125 \mathrm{E}-04}$ \\
1 & $5.09194 \mathrm{E}-03$ & $7.87946 \mathrm{E}-03$ & $3.23366 \mathrm{E}-03$ & $4.90525 \mathrm{E}-03$ & $7.60379 \mathrm{E}-03$ & $3.12052 \mathrm{E}-03$ \\
2 & $8.03087 \mathrm{E}-03$ & $1.62800 \mathrm{E}-02$ & $6.68118 \mathrm{E}-03$ & $8.07572 \mathrm{E}-03$ & $1.64173 \mathrm{E}-02$ & $6.73752 \mathrm{E}-03$ \\
3 & $6.44140 \mathrm{E}-02$ & $1.33756 \mathrm{E}-01$ & $5.48922 \mathrm{E}-02$ & $6.49323 \mathrm{E}-02$ & $1.34838 \mathrm{E}-01$ & $5.53362 \mathrm{E}-02$ \\
\hline
\end{tabular}

Table 2.13. PNL-30, Pin-69 four-group U-238 cross sections

\begin{tabular}{|c|c|c|c|c|c|c|}
\hline \multirow[t]{2}{*}{ Group } & \multicolumn{3}{|c|}{ CENTRM cross sections $\left(\mathrm{cm}^{-1}\right)$} & \multicolumn{3}{|c|}{ NITAWL cross sections $\left(\mathrm{cm}^{-1}\right)$} \\
\hline & $\mathrm{G}_{\mathrm{a}}$ & $\mathrm{G}_{\mathrm{f}}$ & $\mathrm{G}_{\mathrm{f}}$ & $\mathrm{G}_{\mathrm{a}}$ & $\mathrm{G}_{\mathrm{f}}$ & $\mathrm{G}_{\mathrm{f}}$ \\
\hline 1 & $6.48013 \mathrm{E}-03$ & $1.00639 \mathrm{E}-02$ & $3.58425 \mathrm{E}-03$ & $6 . \overline{50153 \mathrm{E}-03}$ & $\overline{1.01355 \mathrm{E}-02}$ & $\overline{3.612} \overline{66 \mathrm{E}-03}$ \\
\hline 2 & 4.33496E-02 & $5.86890 \mathrm{E}-06$ & $2.43146 \mathrm{E}-06$ & 4.25035E-02 & $5.67595 \mathrm{E}-06$ & $2.35151 \mathrm{E}-06$ \\
\hline 3 & $1.10028 \mathrm{E}-02$ & $3.78364 \mathrm{E}-08$ & $1.56763 \mathrm{E}-08$ & $1.10397 \mathrm{E}-02$ & $3.81740 \mathrm{E}-08$ & $1.58162 \mathrm{E}-08$ \\
\hline 4 & $3.74989 \mathrm{E}-02$ & $1.75656 \mathrm{E}-07$ & $7.27780 \mathrm{E}-08$ & 3.77530E-02 & $1.76873 \mathrm{E}-07$ & 7.32818E-08 \\
\hline
\end{tabular}

Table 2.14. PNL-30, Pin-69 four-group Pu-239 cross sections

\begin{tabular}{cccccccc}
\hline Group & \multicolumn{3}{c}{ CENTRM cross sections $\left(\mathrm{cm}^{-1}\right)$} & \multicolumn{3}{c}{ NITAWL cross sections $\left(\mathrm{cm}^{-1}\right)$} \\
\hline & $\frac{\mathrm{G}_{\mathrm{a}}}{7.34312 \mathrm{E}-04}$ & $\frac{\mathrm{G}_{\mathrm{f}}}{2.06900 \mathrm{E}-03}$ & $\frac{\mathrm{G}_{\mathrm{f}}}{6.75303 \mathrm{E}-04}$ & $\frac{\mathrm{G}_{\mathrm{a}}}{7.34377 \mathrm{E}-04}$ & $\frac{\mathrm{G}_{\mathrm{f}}}{2.07025 \mathrm{E}-03}$ & $\frac{\mathrm{G}_{\mathrm{f}}}{6.75577 \mathrm{E}-04}$ \\
1 & $1.41486 \mathrm{E}-02$ & $2.33093 \mathrm{E}-02$ & $8.09402 \mathrm{E}-03$ & $1.38472 \mathrm{E}-02$ & $2.29011 \mathrm{E}-02$ & $7.95226 \mathrm{E}-03$ \\
2 & $4.37500 \mathrm{E}-02$ & $8.64152 \mathrm{E}-02$ & $3.00072 \mathrm{E}-02$ & $4.41986 \mathrm{E}-02$ & $8.73169 \mathrm{E}-02$ & $3.03204 \mathrm{E}-02$ \\
3 & $4.24647 \mathrm{E}-01$ & $8.20289 \mathrm{E}-01$ & $2.83994 \mathrm{E}-01$ & $4.24708 \mathrm{E}-01$ & $8.21267 \mathrm{E}-01$ & $2.84326 \mathrm{E}-01$ \\
\hline
\end{tabular}




\begin{tabular}{|c|c|c|c|c|c|c|c|c|c|c|c|}
\hline & & & & & & & & & & & $\begin{array}{c}1.824 \\
(0.90)\end{array}$ \\
\hline & & & & & & & & & & $\begin{array}{l}1.821 \\
(0.58)\end{array}$ & $\begin{array}{l}1.818 \\
(0.53)\end{array}$ \\
\hline & & & & & & & & & $\begin{array}{c}1.780 \\
(0.50)\end{array}$ & $\begin{array}{l}1.785 \\
(0.32)\end{array}$ & $\begin{array}{l}1.817 \\
(0.48)\end{array}$ \\
\hline & & & & & & & & $\begin{array}{l}1.693 \\
(0.56)\end{array}$ & $\begin{array}{r}1.725 \\
(038 .)\end{array}$ & $\begin{array}{l}1.759 \\
(0.36)\end{array}$ & $\begin{array}{r}1.764 \\
(0.47)\end{array}$ \\
\hline & & & & & & & $\begin{array}{l}1.611 \\
(055 .)\end{array}$ & $\begin{array}{r}1.650 \\
(0.34)\end{array}$ & $\begin{array}{l}1.683 \\
(0.35)\end{array}$ & $\begin{array}{c}1.706 \\
(0.36)\end{array}$ & $\begin{array}{l}1.687 \\
(0.50)\end{array}$ \\
\hline & & & & & & $\begin{array}{l}1.492 \\
(0.52)\end{array}$ & $\begin{array}{l}1.535 \\
(0.38)\end{array}$ & $\begin{array}{c}1.601 \\
(0.35)\end{array}$ & $\begin{array}{c}1.624 \\
(0.40)\end{array}$ & $\begin{array}{l}1.633 \\
(0.38)\end{array}$ & $\begin{array}{c}1.634 \\
(052 .)\end{array}$ \\
\hline & & & & & $\begin{array}{c}1.338 \\
(0.58)\end{array}$ & $\begin{array}{l}1.419 \\
(0.41)\end{array}$ & $\begin{array}{c}1.476 \\
(0.41)\end{array}$ & $\begin{array}{r}1.509 \\
(0.35)\end{array}$ & $\begin{array}{r}1.550 \\
(0.37)\end{array}$ & $\begin{array}{r}1.562 \\
(0.41)\end{array}$ & $\begin{array}{l}1.570 \\
(0.57)\end{array}$ \\
\hline & & & & $\begin{array}{l}1.202 \\
(064 .)\end{array}$ & $\begin{array}{c}1.271 \\
(0.42)\end{array}$ & $\begin{array}{l}1.332 \\
(0.39)\end{array}$ & $\begin{array}{r}1.385 \\
(0.40)\end{array}$ & $\begin{array}{l}1.437 \\
(0.36)\end{array}$ & $\begin{array}{l}1.460 \\
(0.44)\end{array}$ & $\begin{array}{l}1.488 \\
(0.40)\end{array}$ & $\begin{array}{c}1.500 \\
(0.51)\end{array}$ \\
\hline & & & $\begin{array}{l}1.018 \\
(0.65)\end{array}$ & $\begin{array}{l}1.098 \\
(0.46)\end{array}$ & $\begin{array}{l}1.175 \\
(0.42)\end{array}$ & $\begin{array}{l}1.245 \\
(0.48)\end{array}$ & $\begin{array}{l}1.304 \\
(0.38)\end{array}$ & $\begin{array}{l}1.346 \\
(0.40)\end{array}$ & $\begin{array}{l}1.359 \\
(0.40)\end{array}$ & $\begin{array}{c}1.393 \\
(0.40)\end{array}$ & $\begin{array}{c}1.393 \\
(0.59)\end{array}$ \\
\hline & & $\begin{array}{l}0.861 \\
(0.74)\end{array}$ & $\begin{array}{l}0.930 \\
(0.45)\end{array}$ & $\begin{array}{l}1.016 \\
(0.48)\end{array}$ & $\begin{array}{l}1.078 \\
(0.45)\end{array}$ & $\begin{array}{l}1.139 \\
(0.45)\end{array}$ & $\begin{array}{c}1.195 \\
(0.46)\end{array}$ & $\begin{array}{r}1.228 \\
(0.40)\end{array}$ & $\begin{array}{l}1.261 \\
(0.41)\end{array}$ & $\begin{array}{l}1.282 \\
(0.45)\end{array}$ & $\begin{array}{r}1.288 \\
(0.58)\end{array}$ \\
\hline & $\begin{array}{c}0.719 \\
(0.74)\end{array}$ & $\begin{array}{c}0.777 \\
(0.57)\end{array}$ & $\begin{array}{l}0.849 \\
(0.55)\end{array}$ & $\begin{array}{c}0.919 \\
(0.46)\end{array}$ & $\begin{array}{l}0.978 \\
(0.53)\end{array}$ & $\begin{array}{l}1.026 \\
(0.43)\end{array}$ & $\begin{array}{l}1.088 \\
(0.43)\end{array}$ & $\begin{array}{c}1.121 \\
(0.49)\end{array}$ & $\begin{array}{l}1.157 \\
(0.44)\end{array}$ & $\begin{array}{l}1.164 \\
(0.45)\end{array}$ & $\begin{array}{r}1.181 \\
(0.66)\end{array}$ \\
\hline $\begin{array}{l}0.924 \\
(0.72)\end{array}$ & $\begin{array}{r}0.737 \\
(0.59)\end{array}$ & $\begin{array}{c}0.737 \\
(0.54)\end{array}$ & $\begin{array}{l}0.771 \\
(0.57)\end{array}$ & $\begin{array}{r}0.822 \\
(0.52)\end{array}$ & $\begin{array}{c}0.872 \\
(0.47)\end{array}$ & $\begin{array}{l}0.918 \\
(0.48)\end{array}$ & $\begin{array}{c}0.965 \\
(0.46)\end{array}$ & $\begin{array}{l}1.011 \\
(0.51)\end{array}$ & $\begin{array}{r}1.043 \\
(0.47)\end{array}$ & $\begin{array}{c}1.054 \\
(0.45)\end{array}$ & $\begin{array}{r}1.056 \\
(0.64)\end{array}$ \\
\hline 0 & $\begin{array}{r}0.985 \\
(0.52)\end{array}$ & $\begin{array}{c}0.860 \\
(0.51)\end{array}$ & $\begin{array}{l}0.772 \\
(0.55)\end{array}$ & $\begin{array}{c}0.756 \\
(0.56)\end{array}$ & $\begin{array}{c}0.787 \\
(0.57)\end{array}$ & $\begin{array}{l}0.818 \\
(0.53)\end{array}$ & $\begin{array}{l}0.862 \\
(0.51)\end{array}$ & $\begin{array}{c}0.888 \\
(0.48)\end{array}$ & $\begin{array}{c}0.916 \\
(0.53)\end{array}$ & $\begin{array}{l}0.932 \\
(0.42)\end{array}$ & $\begin{array}{r}0.944 \\
(0.67)\end{array}$ \\
\hline 0 & 0 & 0 & $\begin{array}{c}1.046 \\
(0.49)\end{array}$ & $\begin{array}{c}0.900 \\
(0.48)\end{array}$ & $\begin{array}{c}0.789 \\
(0.52)\end{array}$ & $\begin{array}{c}0.782 \\
(0.57)\end{array}$ & $\begin{array}{l}0.792 \\
(0.56)\end{array}$ & $\begin{array}{r}0.788 \\
(0.56)\end{array}$ & $\begin{array}{r}0.803 \\
(0.49)\end{array}$ & $\begin{array}{r}0.820 \\
(0.51)\end{array}$ & $\begin{array}{c}0.821 \\
(0.70)\end{array}$ \\
\hline 0 & 0 & 0 & 0 & 0 & $\begin{array}{r}1.065 \\
(0.45)\end{array}$ & $\begin{array}{c}0.946 \\
(0.50)\end{array}$ & $\begin{array}{l}0.890 \\
(0.55)\end{array}$ & $\begin{array}{l}0.781 \\
(0.59)\end{array}$ & $\begin{array}{l}0.745 \\
(0.55)\end{array}$ & $\begin{array}{r}0.751 \\
(0.60)\end{array}$ & $\begin{array}{l}0.764 \\
(0.79)\end{array}$ \\
\hline 0 & 0 & 0 & 0 & 0 & 0 & 0 & 0 & $\begin{array}{l}1.051 \\
(0.45)\end{array}$ & $\begin{array}{l}0.917 \\
(0.54)\end{array}$ & $\begin{array}{l}0.911 \\
(0.50)\end{array}$ & $\begin{array}{r}0.903 \\
(0.77)\end{array}$ \\
\hline
\end{tabular}

Fig. 2.8a. Pin-power distribution for CENTRM benchmark PNL-31. Value in parentheses is percent standard deviation. 


\begin{tabular}{|c|c|c|c|c|c|c|c|c|c|c|c|}
\hline & & & & & & & & & & & $\begin{array}{l}1.829 \\
(1.01)\end{array}$ \\
\hline & & & & & & & & & & $\begin{array}{l}1.827 \\
(0.51)\end{array}$ & $\begin{array}{r}1.832 \\
(0.53)\end{array}$ \\
\hline & & & & & & & & & $\begin{array}{l}1.775 \\
(0.50)\end{array}$ & $\begin{array}{c}1.798 \\
(0.35)\end{array}$ & $\begin{array}{l}1.794 \\
(0.52)\end{array}$ \\
\hline & & & & & & & & $\begin{array}{r}1.722 \\
(0.57)\end{array}$ & $\begin{array}{c}1.742 \\
(0.35)\end{array}$ & $\begin{array}{l}1.776 \\
(0.37)\end{array}$ & $\begin{array}{r}1.785 \\
(0.51)\end{array}$ \\
\hline & & & & & & & $\begin{array}{l}1.612 \\
(0.53)\end{array}$ & $\begin{array}{r}1.666 \\
(0.36)\end{array}$ & $\begin{array}{l}1.698 \\
(0.38)\end{array}$ & $\begin{array}{l}1.716 \\
(0.35)\end{array}$ & $\begin{array}{l}1.724 \\
(0.50)\end{array}$ \\
\hline & & & & & & $\begin{array}{r}1.502 \\
(0.53)\end{array}$ & $\begin{array}{r}1.549 \\
(0.38)\end{array}$ & $\begin{array}{l}1.593 \\
(0.40)\end{array}$ & $\begin{array}{c}1.640 \\
(0.35)\end{array}$ & $\begin{array}{l}1.644 \\
(0.37)\end{array}$ & $\begin{array}{l}1.646 \\
(0.56)\end{array}$ \\
\hline & & & & & $\begin{array}{l}1.353 \\
(0.57)\end{array}$ & $\begin{array}{c}1.418 \\
(0.43)\end{array}$ & $\begin{array}{r}1.474 \\
(0.37)\end{array}$ & $\begin{array}{l}1.525 \\
(0.38)\end{array}$ & $\begin{array}{l}1.566 \\
(0.38)\end{array}$ & $\begin{array}{c}1.580 \\
(0.38)\end{array}$ & $\begin{array}{c}1.572 \\
(0.51)\end{array}$ \\
\hline & & & & $\begin{array}{l}1.192 \\
(0.61)\end{array}$ & $\begin{array}{c}1.274 \\
(0.44)\end{array}$ & $\begin{array}{l}1.343 \\
(0.39)\end{array}$ & $\begin{array}{l}1.402 \\
(0.41)\end{array}$ & $\begin{array}{r}1.435 \\
(0.38)\end{array}$ & $\begin{array}{c}1.478 \\
(0.41)\end{array}$ & $\begin{array}{l}1.493 \\
(0.37)\end{array}$ & $\begin{array}{r}1.486 \\
(0.58)\end{array}$ \\
\hline & & & $\begin{array}{l}1.017 \\
(0.66)\end{array}$ & $\begin{array}{l}1.107 \\
(0.47)\end{array}$ & $\begin{array}{l}1.177 \\
(0.43)\end{array}$ & $\begin{array}{l}1.252 \\
(0.40)\end{array}$ & $\begin{array}{l}1.299 \\
(0.39)\end{array}$ & $\begin{array}{l}1.359 \\
(0.42)\end{array}$ & $\begin{array}{l}1.381 \\
(0.44)\end{array}$ & $\begin{array}{l}1.384 \\
(0.40)\end{array}$ & $\begin{array}{l}1.399 \\
(0.55)\end{array}$ \\
\hline & & $\begin{array}{r}0.860 \\
(0.75)\end{array}$ & $\begin{array}{l}0.929 \\
(0.51)\end{array}$ & $\begin{array}{l}1.010 \\
(0.46)\end{array}$ & $\begin{array}{l}1.079 \\
(0.46)\end{array}$ & $\begin{array}{l}1.145 \\
(0.45)\end{array}$ & $\begin{array}{l}1.202 \\
(0.41)\end{array}$ & $\begin{array}{r}1.245 \\
(0.43)\end{array}$ & $\begin{array}{l}1.272 \\
(0.39)\end{array}$ & $\begin{array}{l}1.295 \\
(0.43)\end{array}$ & $\begin{array}{l}1.297 \\
(0.59)\end{array}$ \\
\hline & $\begin{array}{l}0.722 \\
(0.79)\end{array}$ & $\begin{array}{l}0.771 \\
(0.55)\end{array}$ & $\begin{array}{l}0.841 \\
(0.55)\end{array}$ & $\begin{array}{l}0.915 \\
(0.51)\end{array}$ & $\begin{array}{l}0.977 \\
(0.43)\end{array}$ & $\begin{array}{l}1.041 \\
(0.47)\end{array}$ & $\begin{array}{l}1.106 \\
(0.47)\end{array}$ & $\begin{array}{l}1.131 \\
(0.43)\end{array}$ & $\begin{array}{c}1.162 \\
(0.43)\end{array}$ & $\begin{array}{r}1.179 \\
(0.48)\end{array}$ & $\begin{array}{c}1.181 \\
(0.60)\end{array}$ \\
\hline $\begin{array}{l}0.937 \\
(0.68)\end{array}$ & $\begin{array}{c}0.736 \\
(0.61)\end{array}$ & $\begin{array}{c}0.728 \\
(0.54)\end{array}$ & $\begin{array}{l}0.766 \\
(0.58)\end{array}$ & $\begin{array}{c}0.825 \\
(0.55)\end{array}$ & $\begin{array}{l}0.880 \\
(0.47)\end{array}$ & $\begin{array}{l}0.917 \\
(0.49)\end{array}$ & $\begin{array}{c}0.982 \\
(0.47)\end{array}$ & $\begin{array}{l}1.013 \\
(0.45)\end{array}$ & $\begin{array}{l}1.031 \\
(0.46)\end{array}$ & $\begin{array}{l}1.049 \\
(0.45)\end{array}$ & $\begin{array}{r}1.064 \\
(0.66)\end{array}$ \\
\hline 0 & $\begin{array}{r}0.991 \\
(0.51)\end{array}$ & $\begin{array}{l}0.853 \\
(0.52)\end{array}$ & $\begin{array}{l}0.774 \\
(0.57)\end{array}$ & $\begin{array}{c}0.771 \\
(0.53)\end{array}$ & $\begin{array}{l}0.795 \\
(0.53)\end{array}$ & $\begin{array}{l}0.827 \\
(0.53)\end{array}$ & $\begin{array}{l}0.866 \\
(0.48)\end{array}$ & $\begin{array}{l}0.891 \\
(0.52)\end{array}$ & $\begin{array}{l}0.918 \\
(0.52)\end{array}$ & $\begin{array}{l}0.925 \\
(0.44)\end{array}$ & $\begin{array}{c}0.936 \\
(0.67)\end{array}$ \\
\hline 0 & 0 & 0 & $\begin{array}{r}1.039 \\
(0.54)\end{array}$ & $\begin{array}{c}0.888 \\
(0.51)\end{array}$ & $\begin{array}{l}0.787 \\
(0.50)\end{array}$ & $\begin{array}{l}0.778 \\
(0.47)\end{array}$ & $\begin{array}{l}0.787 \\
(0.60)\end{array}$ & $\begin{array}{l}0.798 \\
(0.55)\end{array}$ & $\begin{array}{c}0.803 \\
(0.52)\end{array}$ & $\begin{array}{c}0.811 \\
(0.51)\end{array}$ & $\begin{array}{r}0.817 \\
(0.72)\end{array}$ \\
\hline 0 & 0 & 0 & 0 & 0 & $\begin{array}{l}1.064 \\
(0.49)\end{array}$ & $\begin{array}{c}0.952 \\
(0.45)\end{array}$ & $\begin{array}{c}0.896 \\
(0.51)\end{array}$ & $\begin{array}{l}0.782 \\
(0.53)\end{array}$ & $\begin{array}{l}0.751 \\
(0.55)\end{array}$ & $\begin{array}{r}0.757 \\
(0.55)\end{array}$ & $\begin{array}{l}0.744 \\
(0.80)\end{array}$ \\
\hline 0 & 0 & 0 & 0 & 0 & 0 & 0 & 0 & $\begin{array}{r}1.052 \\
(0.48)\end{array}$ & $\begin{array}{l}0.917 \\
(0.52)\end{array}$ & $\begin{array}{c}0.905 \\
(0.49)\end{array}$ & $\begin{array}{c}0.914 \\
(0.71)\end{array}$ \\
\hline
\end{tabular}

Fig. 2.8b. Pin power distribution for NITAWL benchmark PNL-31. Value in parentheses is percent standard deviation. 
Table 2.15. PNL-31 CENTRM reaction rates and fluxes

\begin{tabular}{|c|c|c|c|c|c|c|}
\hline Pin & Region & $\begin{array}{c}\mathrm{G}_{\mathrm{a}} \mathrm{M} \\
\left(\mathrm{cm}^{-3}-\mathrm{s}^{-1}\right)\end{array}$ & $\begin{array}{c}\mathcal{G}_{\mathrm{f}} \mathrm{M} \\
\left(\mathrm{cm}^{-3}-\mathrm{s}^{-1}\right)\end{array}$ & $\begin{array}{c}\mathrm{G}_{\mathrm{f}} \mathrm{M} \\
\left(\mathrm{cm}^{-3}-\mathrm{s}^{-1}\right)\end{array}$ & $\begin{array}{c}\mathrm{M} \\
\left(\mathrm{cm}-\mathrm{s}^{-1}\right)\end{array}$ & $\mathrm{M}_{\mathrm{t}}$ \\
\hline \multirow{3}{*}{1} & Fuel & $6.732 \mathrm{E}-06$ & $1.0493 \mathrm{E}-05$ & $3.738 \mathrm{E}-06$ & $7.748 \mathrm{E}-3$ & 0.412 \\
\hline & Clad & $1.407 \mathrm{E}-07$ & 0.0 & 0.0 & $1.494 \mathrm{E}-3$ & 0.079 \\
\hline & Mod. & 5.801E-07 & 0.0 & 0.0 & $9.563 \mathrm{E}-3$ & 0.509 \\
\hline \multirow{3}{*}{109} & Fuel & $1.352 \mathrm{E}-05$ & $1.821 \mathrm{E}-05$ & 6.477E-06 & $2.577 \mathrm{E}-2$ & 0.410 \\
\hline & Clad & 3.513E-07 & 0.0 & 0.0 & $5.039 \mathrm{E}-3$ & 0.081 \\
\hline & Mod. & 8.806E-07 & 0.0 & 0.0 & $3.197 \mathrm{E}-2$ & 0.509 \\
\hline
\end{tabular}

Table 2.16. PNL-31 NITAWL reaction rates and fluxes

\begin{tabular}{|c|c|c|c|c|c|c|}
\hline Pin & Region & $\begin{array}{c}\mathrm{G}_{\mathrm{a}} \mathrm{M} \\
\left(\mathrm{cm}^{-3}-\mathrm{s}^{-1}\right)\end{array}$ & $\begin{array}{c}\mathcal{G}_{\mathrm{f}} \mathrm{M} \\
\left(\mathrm{cm}^{-3}-\mathrm{s}^{-1}\right)\end{array}$ & $\begin{array}{c}\mathrm{G}_{\mathrm{f}} \mathrm{M} \\
\left(\mathrm{cm}^{-3}-\mathrm{s}^{-1}\right)\end{array}$ & $\begin{array}{c}\mathrm{M} \\
\left(\mathrm{cm}-\mathrm{s}^{-1}\right)\end{array}$ & $\mathrm{M} / \mathrm{M}$ \\
\hline \multirow{3}{*}{1} & Fuel & $6.745 \mathrm{E}-06$ & $1.049 \mathrm{E}-05$ & $3.740 \mathrm{E}-06$ & $7.754 \mathrm{E}-3$ & 0.412 \\
\hline & Clad & $1.404 \mathrm{E}-07$ & 0.0 & 0.0 & $1.500 \mathrm{E}-3$ & 0.079 \\
\hline & Mod. & $5.820 \mathrm{E}-07$ & 0.0 & 0.0 & $9.588 \mathrm{E}-3$ & 0.509 \\
\hline \multirow{3}{*}{109} & Fuel & $1.362 \mathrm{E}-05$ & $1.838 \mathrm{E}-05$ & 6.538E-06 & $2.583 \mathrm{E}-2$ & 0.411 \\
\hline & Clad & $3.478 \mathrm{E}-07$ & 0.0 & 0.0 & $5.033 \mathrm{E}-3$ & 0.080 \\
\hline & Mod. & $8.960 \mathrm{E}-07$ & 0.0 & 0.0 & $3.200 \mathrm{E}-2$ & 0.509 \\
\hline
\end{tabular}


Table 2.17. PNL-31, Pin-1 four-group fluxes

\begin{tabular}{ccc}
\hline Group & $\begin{array}{c}\text { CENTRM M } \\
\left(\mathrm{cm}^{-2}-\mathrm{s}^{-1}\right)\end{array}$ & $\begin{array}{c}\text { NITAWL M } \\
\left(\mathrm{cm}^{-2}-\mathrm{s}^{-1}\right)\end{array}$ \\
\hline 1 & $4.151 \mathrm{E}-05$ & $4.145 \mathrm{E}-05$ \\
2 & $1.455 \mathrm{E}-05$ & $1.468 \mathrm{E}-05$ \\
3 & $3.350 \mathrm{E}-06$ & $3.351 \mathrm{E}-06$ \\
4 & $9.754 \mathrm{E}-06$ & $9.744 \mathrm{E}-06$ \\
\hline
\end{tabular}

Table 2.18. PNL-31, Pin-1 four-group U-235 cross sections

\begin{tabular}{cccccccc}
\hline Group & \multicolumn{3}{c}{ CENTRM cross sections $\left(\mathrm{cm}^{-1}\right)$} & \multicolumn{3}{c}{ NITAWL cross sections $\left(\mathrm{cm}^{-1}\right)$} \\
\hline & $\frac{\mathrm{G}_{\mathrm{a}}}{2.34277 \mathrm{E}-04}$ & $\frac{\mathrm{G}_{\mathrm{f}}}{5.20559 \mathrm{E}-04}$ & $\frac{\mathrm{G}_{\mathrm{f}}}{2.02137 \mathrm{E}-04}$ & $\frac{\mathrm{G}_{\mathrm{a}}}{2.34433 \mathrm{E}-04}$ & $\frac{\mathrm{G}_{\mathrm{f}}}{5.20890 \mathrm{E}-04}$ & $\frac{\mathrm{G}_{\mathrm{f}}}{2.02275 \mathrm{E}-04}$ \\
1 & $5.31166 \mathrm{E}-03$ & $8.16900 \mathrm{E}-03$ & $3.35248 \mathrm{E}-03$ & $5.27939 \mathrm{E}-03$ & $8.08910 \mathrm{E}-03$ & $3.31969 \mathrm{E}-03$ \\
2 & $8.11675 \mathrm{E}-03$ & $1.65287 \mathrm{E}-02$ & $6.78319 \mathrm{E}-03$ & $8.06329 \mathrm{E}-03$ & $1.64082 \mathrm{E}-02$ & $6.73381 \mathrm{E}-03$ \\
3 & $7.08071 \mathrm{E}-02$ & $1.47273 \mathrm{E}-01$ & $6.04396 \mathrm{E}-02$ & $7.10855 \mathrm{E}-02$ & $1.47857 \mathrm{E}-01$ & $6.06792 \mathrm{E}-02$ \\
\hline
\end{tabular}

Table 2.19. PNL-31, Pin-1 four-group U-238 cross sections

\begin{tabular}{|c|c|c|c|c|c|c|}
\hline Group & \multicolumn{3}{|c|}{ CENTRM cross sections $\left(\mathrm{cm}^{-1}\right)$} & \multicolumn{3}{|c|}{ NITAWL cross sections $\left(\mathrm{cm}^{-1}\right)$} \\
\hline & $\mathrm{G}_{\mathrm{a}}$ & $<\mathrm{G}_{\mathrm{f}}$ & $\mathrm{G}_{\mathrm{f}}$ & $\mathrm{G}_{\mathrm{a}}$ & $\mathrm{G}_{\mathrm{f}}$ & $\mathrm{G}_{\mathrm{f}}$ \\
\hline 1 & $6.72455 \mathrm{E}-03$ & $\overline{1.13885 \mathrm{E}-02}$ & $\overline{4.04980 \mathrm{E}-03}$ & $6 . \overline{74729 \mathrm{E}-03}$ & $\overline{1.14467 \mathrm{E}-02}$ & $\overline{4.07385 \mathrm{E}-03}$ \\
\hline 2 & $4.56936 \mathrm{E}-02$ & $5.60056 \mathrm{E}-06$ & 2.32029E-06 & 4.55851E-02 & $5.56831 \mathrm{E}-06$ & 2.30692E-06 \\
\hline 3 & $1.10516 \mathrm{E}-02$ & 3.83036E-08 & $1.58698 \mathrm{E}-08$ & 1.10407E-02 & $3.82095 \mathrm{E}-08$ & $1.58309 \mathrm{E}-08$ \\
\hline 4 & $4.08111 \mathrm{E}-02$ & $1.91551 \mathrm{E}-07$ & 7.93633E-08 & 4.09499E-02 & $1.92215 \mathrm{E}-07$ & $7.96381 \mathrm{E}-08$ \\
\hline
\end{tabular}

Table 2.20. PNL-31, Pin-1 four-group Pu-239 cross sections

\begin{tabular}{cccccccc}
\hline Group & \multicolumn{3}{c}{ CENTRM cross sections $\left(\mathrm{cm}^{-1}\right)$} & \multicolumn{3}{c}{ NITAWL cross sections $\left(\mathrm{cm}^{-1}\right)$} \\
\hline & $\frac{\mathrm{G}_{\mathrm{a}}}{7.32515 \mathrm{E}-04}$ & $\frac{\mathrm{G}_{\mathrm{f}}}{2.09589 \mathrm{E}-03}$ & $\frac{\mathrm{G}_{\mathrm{f}}}{6.79949 \mathrm{E}-04}$ & $\frac{7.32859 \mathrm{E}-04}{2.09690 \mathrm{E}-03}$ & $\frac{\mathrm{G}_{\mathrm{a}}}{6.80273 \mathrm{E}-04}$ \\
1 & $1.48948 \mathrm{E}-02$ & $2.45228 \mathrm{E}-02$ & $8.51540 \mathrm{E}-03$ & $1.45238 \mathrm{E}-02$ & $2.40236 \mathrm{E}-02$ & $8.34210 \mathrm{E}-03$ \\
2 & $4.46260 \mathrm{E}-02$ & $8.81024 \mathrm{E}-02$ & $3.05931 \mathrm{E}-02$ & $4.49138 \mathrm{E}-02$ & $8.86079 \mathrm{E}-02$ & $3.07687 \mathrm{E}-02$ \\
3 & $3.92873 \mathrm{E}-01$ & $7.79195 \mathrm{E}-01$ & $2.69624 \mathrm{E}-01$ & $3.93098 \mathrm{E}-01$ & $7.80112 \mathrm{E}-01$ & $2.69936 \mathrm{E}-01$ \\
\hline
\end{tabular}


Table 2.21. PNL-31, Pin-109 four-group fluxes

\begin{tabular}{ccc}
\hline Group & $\begin{array}{c}\text { CENTRM M } \\
\left(\mathrm{cm}^{-2}-\mathrm{s}^{-1}\right)\end{array}$ & $\begin{array}{c}\text { NITAWL M } \\
\left(\mathrm{cm}^{-2}-\mathrm{s}^{-1}\right)\end{array}$ \\
\hline 1 & $1.485 \mathrm{E}-04$ & $1.486 \mathrm{E}-04$ \\
2 & $5.674 \mathrm{E}-05$ & $5.667 \mathrm{E}-05$ \\
3 & $1.105 \mathrm{E}-05$ & $1.126 \mathrm{E}-05$ \\
4 & $1.379 \mathrm{E}-05$ & $1.412 \mathrm{E}-05$ \\
\hline
\end{tabular}

Table 2.22. PNL-31, Pin-109 four-group U-235 cross sections

\begin{tabular}{ccccccc}
\hline Group & \multicolumn{2}{c}{ CENTRM cross sections $\left(\mathrm{cm}^{-1}\right)$} & \multicolumn{2}{c}{ NITAWL cross sections $\left(\mathrm{cm}^{-1}\right)$} \\
\hline & $\frac{\mathrm{G}_{\mathrm{a}}}{2.40011 \mathrm{E}-04}$ & $\frac{\mathrm{G}_{\mathrm{f}}}{5.25238 \mathrm{E}-04}$ & $\frac{\mathrm{G}_{\mathrm{f}}}{2.05020 \mathrm{E}-04}$ & $\frac{\mathrm{G}_{\mathrm{a}}}{2.41044 \mathrm{E}-04}$ & $\frac{\mathrm{G}_{\mathrm{f}}}{5.25948 \mathrm{E}-04}$ & $\frac{\mathrm{G}_{\mathrm{f}}}{2.05500 \mathrm{E}-04}$ \\
1 & $5.08433 \mathrm{E}-03$ & $7.90435 \mathrm{E}-03$ & $3.24387 \mathrm{E}-03$ & $5.05763 \mathrm{E}-03$ & $7.83928 \mathrm{E}-03$ & $3.21717 \mathrm{E}-03$ \\
2 & $7.87603 \mathrm{E}-03$ & $1.60055 \mathrm{E}-02$ & $6.56854 \mathrm{E}-03$ & $7.93289 \mathrm{E}-03$ & $1.60894 \mathrm{E}-02$ & $6.60295 \mathrm{E}-03$ \\
3 & $6.39052 \mathrm{E}-02$ & $1.32666 \mathrm{E}-01$ & $5.44450 \mathrm{E}-02$ & $6.44026 \mathrm{E}-02$ & $1.33734 \mathrm{E}-01$ & $5.48830 \mathrm{E}-02$ \\
\hline
\end{tabular}

Table 2.23. PNL-31, Pin-109 four-group U-238 cross sections

\begin{tabular}{|c|c|c|c|c|c|c|}
\hline \multirow[t]{2}{*}{ Group } & \multicolumn{3}{|c|}{ CENTRM cross sections $\left(\mathrm{cm}^{-1}\right)$} & \multicolumn{3}{|c|}{ NITAWL cross sections $\left(\mathrm{cm}^{-1}\right)$} \\
\hline & $\mathrm{G}_{\mathrm{a}}$ & $<\mathrm{G}_{\mathrm{f}}$ & $\mathrm{G}_{\mathrm{f}}$ & $\mathrm{G}_{\mathrm{a}}$ & $\mathrm{G}_{\mathrm{f}}$ & $\mathrm{G}_{\mathrm{f}}$ \\
\hline 1 & $6 . \overline{52904 \mathrm{E}-03}$ & $\overline{1.02652 \mathrm{E}-02}$ & $\overline{3.65253 \mathrm{E}-03}$ & $6 . \overline{48723 \mathrm{E}-03}$ & $\overline{1.00441 \mathrm{E}-02}$ & $\overline{3.57473 \mathrm{E}-03}$ \\
\hline 2 & 4.26228E-02 & 5.78745E-06 & 2.39771E-06 & 4.25107E-02 & 5.49843E-06 & 2.27798E-06 \\
\hline 3 & $1.10023 \mathrm{E}-02$ & $3.78561 \mathrm{E}-08$ & $1.56844 \mathrm{E}-08$ & $1.09805 \mathrm{E}-02$ & $3.77877 \mathrm{E}-08$ & $1.56562 \mathrm{E}-08$ \\
\hline 4 & $3.72074 \mathrm{E}-02$ & $1.74257 \mathrm{E}-07$ & 7.21979E-08 & 3.74882E-02 & $1.75595 \mathrm{E}-07$ & 7.27521E-08 \\
\hline
\end{tabular}

Table 2.24. PNL-31, Pin-109 four-group Pu-239 cross sections

\begin{tabular}{cccccccc}
\hline Group & \multicolumn{3}{c}{ CENTRM cross sections $\left(\mathrm{cm}^{-1}\right)$} & \multicolumn{3}{c}{ NITAWL cross sections $\left(\mathrm{cm}^{-1}\right)$} \\
\hline & $\frac{\mathrm{G}_{\mathrm{a}}}{7.34579 \mathrm{E}-04}$ & $\frac{\mathrm{G}_{\mathrm{f}}}{2.07331 \mathrm{E}-03}$ & $\frac{\mathrm{G}_{\mathrm{f}}}{6.76047 \mathrm{E}-04}$ & $\frac{\mathrm{G}_{\mathrm{a}}}{7.34616 \mathrm{E}-04}$ & $\frac{\mathrm{G}_{\mathrm{f}}}{2.06809 \mathrm{E}-03}$ & $\frac{\mathrm{G}_{\mathrm{f}}}{6.74966 \mathrm{E}-04}$ \\
1 & $1.42795 \mathrm{E}-02$ & $2.36502 \mathrm{E}-02$ & $8.21241 \mathrm{E}-03$ & $1.41529 \mathrm{E}-02$ & $2.32927 \mathrm{E}-02$ & $8.08827 \mathrm{E}-03$ \\
3 & $4.25902 \mathrm{E}-02$ & $8.43169 \mathrm{E}-02$ & $2.92786 \mathrm{E}-02$ & $4.25863 \mathrm{E}-02$ & $8.42498 \mathrm{E}-02$ & $2.92554 \mathrm{E}-02$ \\
4 & $4.32807 \mathrm{E}-01$ & $8.32922 \mathrm{E}-01$ & $2.88390 \mathrm{E}-01$ & $4.26617 \mathrm{E}-01$ & $8.23669 \mathrm{E}-01$ & $2.85166 \mathrm{E}-01$ \\
\hline
\end{tabular}




\begin{tabular}{|c|c|c|c|c|c|c|c|c|c|c|c|c|c|c|c|c|}
\hline 0 & 0 & 0 & 0 & 0 & 0 & 0 & 4.104 & 3.592 & 3.967 & 0 & 0 & 0 & 0 & 0 & 0 & 0 \\
\hline 0 & 0 & 0 & 0 & 0 & 0 & 4.526 & 3.498 & 3.291 & 3.407 & 3.866 & 4.466 & 0 & 0 & 0 & 0 & 0 \\
\hline 0 & 0 & 0 & 0 & 4.931 & 4.345 & 3.900 & 3.723 & 3.716 & 3.703 & 3.685 & 3.685 & 4.587 & 0 & 0 & 0 & 0 \\
\hline 0 & 0 & 0 & 4.714 & 3.978 & 4.001 & 4.116 & 4.282 & 4.343 & 4.308 & 4.142 & 3.941 & 3.917 & 4.729 & 0 & 0 & 0 \\
\hline 0 & 0 & 4.627 & 3.919 & 4.040 & 4.348 & 4.602 & 4.824 & 4.909 & 4.867 & 4.690 & 4.406 & 4.053 & 3.954 & 4.586 & 0 & 0 \\
\hline 0 & 4.571 & 3.828 & 3.932 & 4.394 & 4.732 & 5.084 & 5.314 & 5.470 & 5.314 & 5.046 & 4.789 & 4.388 & 3.947 & 3.705 & 4.529 & 0 \\
\hline 0 & 3.922 & 3.663 & 4.307 & 4.687 & 5.147 & 5.525 & 5.761 & 5.808 & 5.730 & 5.474 & 5.132 & 4.724 & 4.133 & 3.716 & 3.894 & 0 \\
\hline 4.157 & 3.430 & 3.833 & 4.318 & 4.889 & 5.371 & 5.758 & 5.973 & 6.000 & 5.910 & 5.721 & 5.336 & 4.926 & 4.334 & 3.776 & 3.452 & 4.097 \\
\hline 3.718 & 3.346 & 3.822 & 4.366 & 4.891 & 5.430 & 5.794 & 6.009 & 6.086 & 6.014 & 5.818 & 5.526 & 4.983 & 4.389 & 3.801 & 3.329 & 3.647 \\
\hline 4.092 & 3.451 & 3.831 & 4.371 & 4.841 & 5.391 & 5.711 & 5.923 & 5.990 & 5.928 & 5.707 & 5.408 & 4.879 & 4.329 & 3.765 & 3.512 & 4.149 \\
\hline 0 & 3.884 & 3.703 & 4.188 & 4.675 & 5.207 & 5.526 & 5.712 & 5.786 & 5.739 & 5.492 & 5.143 & 4.681 & 4.107 & 3.701 & 3.953 & 0 \\
\hline 0 & 4.583 & 3.803 & 4.002 & 4.472 & 4.861 & 5.180 & 5.299 & 5.429 & 5.395 & 5.173 & 4.764 & 4.385 & 3.921 & 3.733 & 4.522 & 0 \\
\hline 0 & 0 & 4.695 & 3.920 & 4.100 & 4.404 & 4.715 & 4.886 & 4.992 & 4.915 & 4.709 & 4.402 & 4.043 & 3.925 & 4.545 & 0 & 0 \\
\hline 0 & 0 & 0 & 4.721 & 3.921 & 3.954 & 4.219 & 4.362 & 4.377 & 4.325 & 4.198 & 3.946 & 3.951 & 4.748 & 0 & 0 & 0 \\
\hline 0 & 0 & 0 & 0 & 4.639 & 3.762 & 3.701 & 3.821 & 3.812 & 3.789 & 3.926 & 4.415 & 4.865 & 0 & 0 & 0 & 0 \\
\hline 0 & 0 & 0 & 0 & 0 & 4.516 & 3.925 & 3.438 & 3.393 & 3.471 & 4.512 & 0 & 0 & 0 & 0 & 0 & 0 \\
\hline 0 & 0 & 0 & 0 & 0 & 0 & 0 & 4.100 & 3.642 & 4.175 & 0 & 0 & 0 & 0 & 0 & 0 & 0 \\
\hline
\end{tabular}

Fig. 2.9a. Pin-power distribution for CENTRM benchmark PNL-32 with standard deviation between 0.59 and $0.87 \%$ of the value. 


\begin{tabular}{|c|c|c|c|c|c|c|c|c|c|c|c|c|c|c|c|c|}
\hline 0 & 0 & 0 & 0 & 0 & 0 & 0 & 4.193 & 3.696 & 4.139 & 0 & 0 & 0 & 0 & 0 & 0 & 0 \\
\hline 0 & 0 & 0 & 0 & 0 & 0 & 4.568 & 3.565 & 3.354 & 3.466 & 3.987 & 4.530 & 0 & 0 & 0 & 0 & 0 \\
\hline 0 & 0 & 0 & 0 & 4.902 & 4.409 & 3.981 & 3.841 & 3.804 & 3.753 & 3.706 & 3.767 & 4.624 & 0 & 0 & 0 & 0 \\
\hline 0 & 0 & 0 & 4.739 & 3.971 & 4.071 & 4.190 & 4.304 & 4.352 & 4.301 & 4.177 & 3.952 & 3.930 & 4.705 & 0 & 0 & 0 \\
\hline 0 & 0 & 4.625 & 3.956 & 4.080 & 4.417 & 4.773 & 4.884 & 4.914 & 4.907 & 4.720 & 4.427 & 4.010 & 3.991 & 4.684 & 0 & 0 \\
\hline 0 & 4.612 & 3.769 & 4.039 & 4.470 & 4.913 & 5.191 & 5.404 & 5.433 & 5.357 & 5.142 & 4.790 & 4.319 & 3.940 & 3.779 & 4.489 & 0 \\
\hline 0 & 3.884 & 3.700 & 4.150 & 4.792 & 5.200 & 5.595 & 5.759 & 5.763 & 5.729 & 5.484 & 5.192 & 4.683 & 4.164 & 3.727 & 3.874 & 0 \\
\hline 4.153 & 3.500 & 3.842 & 4.455 & 4.989 & 5.431 & 5.783 & 5.974 & 5.974 & 5.953 & 5.726 & 5.346 & 4.840 & 4.324 & 3.784 & 3.470 & 4.059 \\
\hline 3.735 & 3.352 & 3.825 & 4.417 & 4.978 & 5.524 & 5.756 & 6.026 & 6.099 & 5.988 & 5.801 & 5.425 & 4.969 & 4.412 & 3.818 & 3.329 & 3.711 \\
\hline 4.211 & 3.533 & 3.823 & 4.416 & 4.969 & 5.395 & 5.781 & 5.929 & 5.958 & 5.856 & 5.731 & 5.360 & 4.817 & 4.315 & 3.757 & 3.404 & 4.099 \\
\hline 0 & 3.894 & 3.705 & 4.182 & 4.755 & 5.208 & 5.492 & 5.682 & 5.749 & 5.595 & 5.475 & 5.095 & 4.677 & 4.078 & 3.680 & 3.876 & 0 \\
\hline 0 & 4.558 & 3.742 & 4.015 & 4.413 & 4.846 & 5.195 & 5.336 & 5.383 & 5.265 & 5.059 & 4.737 & 4.377 & 3.944 & 3.712 & 4.465 & 0 \\
\hline 0 & 0 & 4.614 & 3.980 & 4.088 & 4.380 & 4.731 & 4.941 & 4.880 & 4.853 & 4.659 & 4.358 & 4.024 & 3.882 & 4.574 & 0 & 0 \\
\hline 0 & 0 & 0 & 4.789 & 3.910 & 3.973 & 4.142 & 4.350 & 4.423 & 4.348 & 4.130 & 3.973 & 3.904 & 4.683 & 0 & 0 & 0 \\
\hline 0 & 0 & 0 & 0 & 4.625 & 3.768 & 3.664 & 3.837 & 3.803 & 3.735 & 3.920 & 4.301 & 4.912 & 0 & 0 & 0 & 0 \\
\hline 0 & 0 & 0 & 0 & 0 & 4.516 & 3.907 & 3.442 & 3.317 & 3.414 & 4.463 & 0 & 0 & 0 & 0 & 0 & 0 \\
\hline 0 & 0 & 0 & 0 & 0 & 0 & 0 & 4.045 & 3.626 & 4.106 & 0 & 0 & 0 & 0 & 0 & 0 & 0 \\
\hline
\end{tabular}

Fig. 2.9b. Pin-power distribution for NITAWL benchmark PNL-32 with standard deviation between 0.57 and $0.90 \%$ of the values. 
Table 2.25. PNL-32 CENTRM reaction rates and fluxes

\begin{tabular}{|c|c|c|c|c|c|c|}
\hline Pin & Region & $\begin{array}{c}\mathrm{G}_{\mathrm{a}} \mathrm{M} \\
\left(\mathrm{cm}^{-3}-\mathrm{s}^{-1}\right)\end{array}$ & $\begin{array}{c}\mathcal{G}_{\mathrm{f}} \mathrm{M} \\
\left(\mathrm{cm}^{-3}-\mathrm{s}^{-1}\right)\end{array}$ & $\begin{array}{c}\mathrm{G}_{\mathrm{f}} \mathrm{M} \\
\left(\mathrm{cm}^{-3}-\mathrm{s}^{-1}\right)\end{array}$ & $\begin{array}{c}\mathrm{M} \\
\left(\mathrm{cm}-\mathrm{s}^{-1}\right)\end{array}$ & $\mathrm{M} M$ \\
\hline \multirow{3}{*}{1} & Fuel & $2.451 \mathrm{E}-05$ & $4.086 \mathrm{E}-05$ & $1.457 \mathrm{E}-05$ & $2.060 \mathrm{E}-2$ & 0.267 \\
\hline & Clad & 4.627E-07 & 0.0 & 0.0 & $3.943 \mathrm{E}-3$ & 0.051 \\
\hline & Mod. & $1.135 \mathrm{E}-06$ & 0.0 & 0.0 & $5.259 \mathrm{E}-2$ & 0.682 \\
\hline \multirow{3}{*}{98} & Fuel & $3.946 \mathrm{E}-05$ & $6.076 \mathrm{E}-05$ & $2.164 \mathrm{E}-05$ & $4.729 \mathrm{E}-2$ & 0.266 \\
\hline & Clad & $8.113 \mathrm{E}-07$ & 0.0 & 0.0 & $9.173 \mathrm{E}-3$ & 0.052 \\
\hline & Mod. & $1.536 \mathrm{E}-06$ & 0.0 & 0.0 & $1.121 \mathrm{E}-2$ & 0.682 \\
\hline
\end{tabular}

Table 2.26. PNL-32 NITAWL reaction rates and fluxes

\begin{tabular}{|c|c|c|c|c|c|c|}
\hline Pin & Region & $\begin{array}{c}\mathrm{G}_{\mathrm{a}} \mathrm{M} \\
\left(\mathrm{cm}^{-3}-\mathrm{s}^{-1}\right)\end{array}$ & $\begin{array}{c}\mathcal{G}_{\mathrm{f}} \mathrm{M} \\
\left(\mathrm{cm}^{-3}-\mathrm{s}^{-1}\right)\end{array}$ & $\begin{array}{c}\mathrm{G}_{\mathrm{f}} \mathrm{M} \\
\left(\mathrm{cm}^{-3}-\mathrm{s}^{-1}\right)\end{array}$ & $\begin{array}{c}\mathrm{M} \\
\left(\mathrm{cm}-\mathrm{s}^{-1}\right)\end{array}$ & $\mathrm{MVM}_{\mathrm{t}}$ \\
\hline \multirow{3}{*}{1} & Fuel & $2.429 \mathrm{E}-05$ & 4.063E-05 & $1.449 \mathrm{E}-05$ & $2.033 \mathrm{E}-2$ & 0.266 \\
\hline & Clad & $4.624 \mathrm{E}-07$ & 0.0 & 0.0 & $3.930 \mathrm{E}-3$ & 0.051 \\
\hline & Mod. & $1.136 \mathrm{E}-06$ & 0.0 & 0.0 & $5.223 \mathrm{E}-2$ & 0.683 \\
\hline \multirow{3}{*}{98} & Fuel & $3.963 \mathrm{E}-05$ & $6.092 \mathrm{E}-05$ & $2.169 \mathrm{E}-05$ & $4.753 \mathrm{E}-2$ & 0.267 \\
\hline & Clad & 8.007E-07 & 0.0 & 0.0 & $9.195 \mathrm{E}-3$ & 0.052 \\
\hline & Mod. & $1.550 \mathrm{E}-06$ & 0.0 & 0.0 & $1.214 \mathrm{E}-1$ & 0.681 \\
\hline
\end{tabular}


Table 2.27. PNL-32, Pin-1 four-group fluxes

\begin{tabular}{ccc}
\hline Group & $\begin{array}{c}\text { CENTRM M } \\
\left(\mathrm{cm}^{-2}-\mathrm{s}^{-1}\right)\end{array}$ & $\begin{array}{c}\text { NITAWL M } \\
\left(\mathrm{cm}^{-2}-\mathrm{s}^{-1}\right)\end{array}$ \\
\hline 1 & $1.048 \mathrm{E}-04$ & $1.029 \mathrm{E}-04$ \\
2 & $3.087 \mathrm{E}-05$ & $3.073 \mathrm{E}-05$ \\
3 & $7.654 \mathrm{E}-06$ & $7.310 \mathrm{E}-06$ \\
4 & $4.050 \mathrm{E}-05$ & $4.057 \mathrm{E}-05$ \\
\hline
\end{tabular}

Table 2.28. PNL-32, Pin-1 four-group U-235 cross sections

\begin{tabular}{cccccccc}
\hline Group & \multicolumn{3}{c}{ CENTRM cross sections $\left(\mathrm{cm}^{-1}\right)$} & \multicolumn{3}{c}{ NITAWL cross sections $\left(\mathrm{cm}^{-1}\right)$} \\
\hline & $\frac{\mathrm{G}_{\mathrm{a}}}{4.28226 \mathrm{E}-04}$ & $\frac{\leftarrow_{\mathrm{f}}}{5.15645 \mathrm{E}-04}$ & $\frac{\mathrm{G}_{\mathrm{f}}}{1.99145 \mathrm{E}-04}$ & $\frac{\mathrm{G}_{\mathrm{a}}}{2.28198 \mathrm{E}-04}$ & $\frac{\mathrm{G}_{\mathrm{f}}}{5.16018 \mathrm{E}-04}$ & $\frac{\mathrm{G}_{\mathrm{f}}}{1.99123 \mathrm{E}-04}$ \\
1 & $5.29819 \mathrm{E}-03$ & $8.08102 \mathrm{E}-03$ & $3.31639 \mathrm{E}-03$ & $5.44312 \mathrm{E}-03$ & $8.33186 \mathrm{E}-03$ & $3.41933 \mathrm{E}-03$ \\
2 & $8.13381 \mathrm{E}-03$ & $1.64731 \mathrm{E}-02$ & $6.76041 \mathrm{E}-03$ & $8.07192 \mathrm{E}-03$ & $1.63713 \mathrm{E}-02$ & $6.71862 \mathrm{E}-03$ \\
3 & $7.46578 \mathrm{E}-02$ & $1.55396 \mathrm{E}-01$ & $6.37731 \mathrm{E}-02$ & $7.43303 \mathrm{E}-02$ & $1.54714 \mathrm{E}-01$ & $6.34932 \mathrm{E}-02$ \\
\hline
\end{tabular}

Table 2.29. PNL-32, Pin-1 four-group U-238 cross sections

\begin{tabular}{|c|c|c|c|c|c|c|}
\hline \multirow[t]{2}{*}{ Group } & \multicolumn{3}{|c|}{ CENTRM cross sections $\left(\mathrm{cm}^{-1}\right)$} & \multicolumn{3}{|c|}{ NITAWL cross sections $\left(\mathrm{cm}^{-1}\right)$} \\
\hline & $\mathrm{G}_{\mathrm{a}}$ & $<\mathrm{G}_{\mathrm{f}}$ & $\mathrm{G}_{\mathrm{f}}$ & $\mathrm{G}_{\mathrm{a}}$ & $<\mathrm{G}_{\mathrm{f}}$ & $\mathrm{G}_{\mathrm{f}}$ \\
\hline 1 & $6.95603 \mathrm{E}-03$ & $1.26008 \mathrm{E}-02$ & $\overline{4.48409 \mathrm{E}-03}$ & $6.96190 \mathrm{E}-03$ & $1.26902 \mathrm{E}-02$ & $\overline{4.50424 \mathrm{E}-03}$ \\
\hline 2 & 4.76213E-02 & $5.91756 \mathrm{E}-06$ & $2.45162 \mathrm{E}-06$ & 4.49401E-02 & $5.50696 \mathrm{E}-06$ & $2.28152 \mathrm{E}-06$ \\
\hline 3 & 1.10199E-02 & 3.80020E-08 & $1.57448 \mathrm{E}-08$ & $1.10245 \mathrm{E}-02$ & $3.79821 \mathrm{E}-08$ & $1.57367 \mathrm{E}-08$ \\
\hline 4 & 4.27798E-02 & 2.00991E-07 & 8.32743E-08 & 4.26310E-02 & $2.00282 \mathrm{E}-07$ & 8.29805E-08 \\
\hline
\end{tabular}

Table 2.30. PNL-32, Pin-1 four-group Pu-239 cross sections

\begin{tabular}{cccccccc}
\hline Group & \multicolumn{3}{c}{ CENTRM cross sections $\left(\mathrm{cm}^{-1}\right)$} & \multicolumn{3}{c}{ NITAWL cross sections $\left(\mathrm{cm}^{-1}\right)$} \\
\hline & $\frac{\mathrm{G}_{\mathrm{a}}}{\mathrm{7.30986 \textrm {E } - 0 4}}$ & $\frac{\mathrm{G}_{\mathrm{f}}}{2.12139 \mathrm{E}-03}$ & $\frac{\mathrm{G}_{\mathrm{f}}}{6.84695 \mathrm{E}-04}$ & $\overline{7.30230 \mathrm{E}-04}$ & $\frac{\mathrm{G}_{\mathrm{f}}}{2.12081 \mathrm{E}-03}$ & $\frac{\mathrm{G}_{\mathrm{f}}}{6.84023 \mathrm{E}-04}$ \\
1 & $1.53809 \mathrm{E}-02$ & $2.53859 \mathrm{E}-02$ & $8.81516 \mathrm{E}-03$ & $1.48494 \mathrm{E}-02$ & $2.44809 \mathrm{E}-02$ & $8.50088 \mathrm{E}-03$ \\
2 & $4.46591 \mathrm{E}-02$ & $8.80834 \mathrm{E}-02$ & $3.05866 \mathrm{E}-02$ & $4.50509 \mathrm{E}-02$ & $8.87866 \mathrm{E}-02$ & $3.08308 \mathrm{E}-02$ \\
3 & $3.79625 \mathrm{E}-01$ & $7.64299 \mathrm{E}-01$ & $2.64388 \mathrm{E}-01$ & $3.77128 \mathrm{E}-01$ & $7.59495 \mathrm{E}-01$ & $2.62726 \mathrm{E}-01$ \\
\hline
\end{tabular}


Table 2.31. PNL-32, Pin-98 four-group fluxes

\begin{tabular}{ccc}
\hline Group & $\begin{array}{c}\text { CENTRM M } \\
\left(\mathrm{cm}^{-2}-\mathrm{s}^{-1}\right)\end{array}$ & $\begin{array}{c}\text { NITAWL M } \\
\left(\mathrm{cm}^{-2}-\mathrm{s}^{-1}\right)\end{array}$ \\
\hline 1 & $2.586 \mathrm{E}-04$ & $2.586 \mathrm{E}-04$ \\
2 & $8.866 \mathrm{E}-05$ & $8.976 \mathrm{E}-05$ \\
3 & $1.956 \mathrm{E}-05$ & $2.042 \mathrm{E}-05$ \\
4 & $5.545 \mathrm{E}-05$ & $5.552 \mathrm{E}-05$ \\
\hline
\end{tabular}

Table 2.32. PNL-32, Pin-98 four-group U-235 cross sections

\begin{tabular}{cccccccc}
\hline Group & \multicolumn{2}{c}{ CENTRM cross sections $\left(\mathrm{cm}^{-1}\right)$} & \multicolumn{3}{c}{ NITAWL cross sections $\left(\mathrm{cm}^{-1}\right)$} \\
\hline & $\frac{\mathrm{G}_{\mathrm{a}}}{4.34086 \mathrm{E}-04}$ & $\frac{\mathrm{G}_{\mathrm{f}}}{5.20599 \mathrm{E}-04}$ & $\frac{\mathrm{G}_{\mathrm{f}}}{2.02092 \mathrm{E}-04}$ & $2.34329 \mathrm{E}-04$ & $\frac{\mathrm{G}_{\mathrm{f}}}{5.21021 \mathrm{E}-04}$ & $\frac{\mathrm{G}_{\mathrm{f}}}{2.02251 \mathrm{E}-04}$ \\
1 & $5.15153 \mathrm{E}-03$ & $7.96071 \mathrm{E}-03$ & $3.26701 \mathrm{E}-03$ & $5.21152 \mathrm{E}-03$ & $7.99933 \mathrm{E}-03$ & $3.28285 \mathrm{E}-03$ \\
2 & $8.14944 \mathrm{E}-03$ & $1.65985 \mathrm{E}-02$ & $6.81186 \mathrm{E}-03$ & $8.16239 \mathrm{E}-03$ & $1.66200 \mathrm{E}-02$ & $6.82071 \mathrm{E}-03$ \\
3 & $7.06334 \mathrm{E}-02$ & $1.46886 \mathrm{E}-01$ & $6.02804 \mathrm{E}-02$ & $7.03727 \mathrm{E}-02$ & $1.46337 \mathrm{E}-01$ & $6.00555 \mathrm{E}-02$ \\
\hline
\end{tabular}

Table 2.33. PNL-32, Pin-98 four-group U-238 cross sections

\begin{tabular}{cccccccc}
\hline Group & \multicolumn{3}{c}{ CENTRM cross sections $\left(\mathrm{cm}^{-1}\right)$} & \multicolumn{3}{c}{ NITAWL cross sections $\left(\mathrm{cm}^{-1}\right)$} \\
\hline & $\frac{\mathrm{G}_{\mathrm{a}}}{}$ & $\frac{\mathrm{G}_{\mathrm{f}}}{1.75406 \mathrm{E}-03}$ & $\frac{1.14805 \mathrm{E}-02}{4}$ & $\frac{\mathrm{G}_{\mathrm{f}}}{4.08322 \mathrm{E}-03}$ & $\frac{6.78241 \mathrm{E}-03}{1.15393 \mathrm{E}-02}$ & $\frac{\mathrm{G}_{\mathrm{f}}}{4.10572 \mathrm{E}-03}$ \\
2 & $4.75024 \mathrm{E}-02$ & $5.74765 \mathrm{E}-06$ & $2.38123 \mathrm{E}-06$ & $4.63517 \mathrm{E}-02$ & $5.42737 \mathrm{E}-06$ & $2.24854 \mathrm{E}-06$ \\
3 & $1.10577 \mathrm{E}-02$ & $3.83380 \mathrm{E}-08$ & $1.58842 \mathrm{E}-08$ & $1.10567 \mathrm{E}-02$ & $3.83610 \mathrm{E}-08$ & $1.58937 \mathrm{E}-08$ \\
4 & $4.07001 \mathrm{E}-02$ & $1.91006 \mathrm{E}-07$ & $7.91378 \mathrm{E}-08$ & $4.05670 \mathrm{E}-02$ & $1.90373 \mathrm{E}-07$ & $7.88750 \mathrm{E}-08$ \\
\hline
\end{tabular}

Table 2.34. PNL-32, Pin-98 four-group Pu-239 cross sections

\begin{tabular}{cccccccc}
\hline Group & \multicolumn{3}{c}{ CENTRM cross sections $\left(\mathrm{cm}^{-1}\right)$} & \multicolumn{3}{c}{ NITAWL cross sections $\left(\mathrm{cm}^{-1}\right)$} \\
\hline & $\frac{\mathrm{G}_{\mathrm{a}}}{7.32645 \mathrm{E}-04}$ & $\frac{\mathrm{G}_{\mathrm{f}}}{2.09793 \mathrm{E}-03}$ & $\frac{\mathrm{G}_{\mathrm{f}}}{6.80410 \mathrm{E}-04}$ & $\frac{\mathrm{G}_{\mathrm{a}}}{7.32954 \mathrm{E}-04}$ & $\frac{\mathrm{G}_{\mathrm{f}}}{2.09852 \mathrm{E}-03}$ & $\frac{\mathrm{G}_{\mathrm{f}}}{6.80530 \mathrm{E}-04}$ \\
1 & $1.46368 \mathrm{E}-02$ & $2.41261 \mathrm{E}-02$ & $8.37768 \mathrm{E}-03$ & $1.46065 \mathrm{E}-02$ & $2.41771 \mathrm{E}-02$ & $8.39539 \mathrm{E}-03$ \\
2 & $4.63098 \mathrm{E}-02$ & $9.11738 \mathrm{E}-02$ & $3.16598 \mathrm{E}-02$ & $4.55958 \mathrm{E}-02$ & $8.98849 \mathrm{E}-02$ & $3.12122 \mathrm{E}-02$ \\
3 & $3.98803 \mathrm{E}-01$ & $7.88748 \mathrm{E}-01$ & $2.72941 \mathrm{E}-01$ & $3.99072 \mathrm{E}-01$ & $7.88716 \mathrm{E}-01$ & $2.72936 \mathrm{E}-01$ \\
\hline
\end{tabular}




\begin{tabular}{|c|c|c|c|c|c|c|c|c|c|c|c|}
\hline & & & & & & & & & & & $\begin{array}{l}2.024 \\
(1.15)\end{array}$ \\
\hline & & & & & & & & & & $\begin{array}{l}2.037 \\
(0.53)\end{array}$ & $\begin{array}{l}2.025 \\
(0.53)\end{array}$ \\
\hline & & & & & & & & & $\begin{array}{l}1.958 \\
(0.50)\end{array}$ & $\begin{array}{c}2.001 \\
(0.40)\end{array}$ & $\begin{array}{l}2.009 \\
(0.55)\end{array}$ \\
\hline & & & & & & & & $\begin{array}{l}1.872 \\
(0.50)\end{array}$ & $\begin{array}{r}1.935 \\
(0.32)\end{array}$ & $\begin{array}{r}1.955 \\
(0.40)\end{array}$ & $\begin{array}{r}1.973 \\
(0.54)\end{array}$ \\
\hline & & & & & & & $\begin{array}{l}1.783 \\
(0.57)\end{array}$ & $\begin{array}{l}1.848 \\
(0.41)\end{array}$ & $\begin{array}{c}1.869 \\
(0.37)\end{array}$ & $\begin{array}{c}1.905 \\
(0.40)\end{array}$ & $\begin{array}{l}1.904 \\
(0.51)\end{array}$ \\
\hline & & & & & & $\begin{array}{l}1.626 \\
(0.54)\end{array}$ & $\begin{array}{c}1.702 \\
(0.39)\end{array}$ & $\begin{array}{l}1.760 \\
(0.37)\end{array}$ & $\begin{array}{c}1.801 \\
(0.37)\end{array}$ & $\begin{array}{l}1.832 \\
(0.41)\end{array}$ & $\begin{array}{l}1.848 \\
(0.47)\end{array}$ \\
\hline & & & & & $\begin{array}{l}1.461 \\
(0.56)\end{array}$ & $\begin{array}{l}1.539 \\
(0.42)\end{array}$ & $\begin{array}{c}1.624 \\
(0.37)\end{array}$ & $\begin{array}{l}1.661 \\
(0.37)\end{array}$ & $\begin{array}{c}1.707 \\
(0.41)\end{array}$ & $\begin{array}{l}1.721 \\
(0.37)\end{array}$ & $\begin{array}{l}1.746 \\
(0.55)\end{array}$ \\
\hline & & & & $\begin{array}{l}1.275 \\
(0.64)\end{array}$ & $\begin{array}{c}1.368 \\
(0.47)\end{array}$ & $\begin{array}{c}1.450 \\
(0.43)\end{array}$ & $\begin{array}{c}1.509 \\
(0.45)\end{array}$ & $\begin{array}{l}1.564 \\
(0.43)\end{array}$ & $\begin{array}{c}1.599 \\
(0.37)\end{array}$ & $\begin{array}{l}1.636 \\
(0.40)\end{array}$ & $\begin{array}{l}1.638 \\
(0.55)\end{array}$ \\
\hline & & & $\begin{array}{l}1.065 \\
(0.68)\end{array}$ & $\begin{array}{l}1.155 \\
(0.47)\end{array}$ & $\begin{array}{l}1.251 \\
(0.50)\end{array}$ & $\begin{array}{l}1.341 \\
(0.45)\end{array}$ & $\begin{array}{l}1.403 \\
(0.43)\end{array}$ & $\begin{array}{l}1.448 \\
(0.39)\end{array}$ & $\begin{array}{l}1.485 \\
(0.43)\end{array}$ & $\begin{array}{l}1.512 \\
(0.41)\end{array}$ & $\begin{array}{l}1.518 \\
(0.58)\end{array}$ \\
\hline & & $\begin{array}{c}0.850 \\
(0.79)\end{array}$ & $\begin{array}{c}0.954 \\
(0.52)\end{array}$ & $\begin{array}{l}1.040 \\
(0.51)\end{array}$ & $\begin{array}{c}1.130 \\
(051 .)\end{array}$ & $\begin{array}{c}1.204 \\
(0.45)\end{array}$ & $\begin{array}{c}1.258 \\
(0.45)\end{array}$ & $\begin{array}{l}1.323 \\
(0.45)\end{array}$ & $\begin{array}{c}1.358 \\
(0.43)\end{array}$ & $\begin{array}{l}1.373 \\
(0.42)\end{array}$ & $\begin{array}{l}1.386 \\
(0.60)\end{array}$ \\
\hline & $\begin{array}{c}0.639 \\
(0.83)\end{array}$ & $\begin{array}{c}0.738 \\
(0.57)\end{array}$ & $\begin{array}{c}0.846 \\
(0.54)\end{array}$ & $\begin{array}{c}0.910 \\
(0.58)\end{array}$ & $\begin{array}{c}1.008 \\
(0.49)\end{array}$ & $\begin{array}{c}1.077 \\
(0.48)\end{array}$ & $\begin{array}{l}1.137 \\
(0.49)\end{array}$ & $\begin{array}{l}1.182 \\
(0.53)\end{array}$ & $\begin{array}{c}1.213 \\
(0.50)\end{array}$ & $\begin{array}{c}1.231 \\
(0.48)\end{array}$ & $\begin{array}{l}1.240 \\
(0.72)\end{array}$ \\
\hline $\begin{array}{c}0.564 \\
(1.07)\end{array}$ & $\begin{array}{c}0.554 \\
(0.78)\end{array}$ & $\begin{array}{c}0.633 \\
(0.62)\end{array}$ & $\begin{array}{c}0.712 \\
(0.59)\end{array}$ & $\begin{array}{c}0.793 \\
(0.58)\end{array}$ & $\begin{array}{r}0.866 \\
(0.60)\end{array}$ & $\begin{array}{c}0.931 \\
(0.48)\end{array}$ & $\begin{array}{l}0.991 \\
(0.45)\end{array}$ & $\begin{array}{l}1.039 \\
(0.53)\end{array}$ & $\begin{array}{c}1.078 \\
(0.48)\end{array}$ & $\begin{array}{l}1.092 \\
(0.54)\end{array}$ & $\begin{array}{l}1.099 \\
(0.62)\end{array}$ \\
\hline 0 & $\begin{array}{c}0.565 \\
(0.69)\end{array}$ & $\begin{array}{c}0.594 \\
(0.73)\end{array}$ & $\begin{array}{c}0.618 \\
(0.63)\end{array}$ & $\begin{array}{c}0.663 \\
(0.64)\end{array}$ & $\begin{array}{c}0.731 \\
(0.57)\end{array}$ & $\begin{array}{c}0.786 \\
(0.54)\end{array}$ & $\begin{array}{c}0.838 \\
(0.57)\end{array}$ & $\begin{array}{l}0.883 \\
(0.53)\end{array}$ & $\begin{array}{r}0.932 \\
(0.52)\end{array}$ & $\begin{array}{r}0.942 \\
(0.51)\end{array}$ & $\begin{array}{l}0.937 \\
(0.74)\end{array}$ \\
\hline 0 & 0 & 0 & $\begin{array}{c}0.621 \\
(0.69)\end{array}$ & $\begin{array}{c}0.628 \\
(0.59)\end{array}$ & $\begin{array}{c}0.628 \\
(0.52)\end{array}$ & $\begin{array}{c}0.658 \\
(0.61)\end{array}$ & $\begin{array}{c}0.702 \\
(0.63)\end{array}$ & $\begin{array}{l}0.735 \\
(0.62)\end{array}$ & $\begin{array}{c}0.761 \\
(0.54)\end{array}$ & $\begin{array}{l}0.783 \\
(0.62)\end{array}$ & $\begin{array}{l}0.783 \\
(0.86)\end{array}$ \\
\hline 0 & 0 & 0 & 0 & 0 & $\begin{array}{l}0.622 \\
(0.64)\end{array}$ & $\begin{array}{l}0.618 \\
(0.65)\end{array}$ & $\begin{array}{l}0.639 \\
(0.63)\end{array}$ & $\begin{array}{c}0.619 \\
(0.63)\end{array}$ & $\begin{array}{l}0.623 \\
(0.66)\end{array}$ & $\begin{array}{l}0.641 \\
(0.63)\end{array}$ & $\begin{array}{c}0.659 \\
(0.97)\end{array}$ \\
\hline 0 & 0 & 0 & 0 & 0 & 0 & 0 & 0 & $\begin{array}{l}0.610 \\
(0.66)\end{array}$ & $\begin{array}{l}0.570 \\
(0.66)\end{array}$ & $\begin{array}{l}0.585 \\
(0.75)\end{array}$ & $\begin{array}{c}0.604 \\
(0.90)\end{array}$ \\
\hline
\end{tabular}

Fig. 2.10a. Pin-power distribution for CENTRM benchmark PNL-33. Value in parentheses is percent standard deviation. 


\begin{tabular}{|c|c|c|c|c|c|c|c|c|c|c|c|}
\hline & & & & & & & & & & & $\begin{array}{l}2.037 \\
(0.95)\end{array}$ \\
\hline & & & & & & & & & & $\begin{array}{c}2.052 \\
(0.57)\end{array}$ & $\begin{array}{l}2.057 \\
(0.51)\end{array}$ \\
\hline & & & & & & & & & $\begin{array}{c}2.004 \\
(0.50)\end{array}$ & $\begin{array}{l}2.007 \\
(0.40)\end{array}$ & $\begin{array}{r}2.018 \\
(0.53)\end{array}$ \\
\hline & & & & & & & & $\begin{array}{l}1.884 \\
(0.57)\end{array}$ & $\begin{array}{l}1.950 \\
(0.36)\end{array}$ & $\begin{array}{l}1.981 \\
(0.38)\end{array}$ & $\begin{array}{l}1.981 \\
(0.51)\end{array}$ \\
\hline & & & & & & & $\begin{array}{l}1.783 \\
(0.53)\end{array}$ & $\begin{array}{l}1.833 \\
(0.41)\end{array}$ & $\begin{array}{l}1.883 \\
(0.35)\end{array}$ & $\begin{array}{l}1.917 \\
(0.38)\end{array}$ & $\begin{array}{l}1.917 \\
(0.54)\end{array}$ \\
\hline & & & & & & $\begin{array}{l}1.646 \\
(0.55)\end{array}$ & $\begin{array}{l}1.711 \\
(0.38)\end{array}$ & $\begin{array}{l}1.772 \\
(0.37)\end{array}$ & $\begin{array}{l}1.804 \\
(0.40)\end{array}$ & $\begin{array}{l}1.823 \\
(0.40)\end{array}$ & $\begin{array}{l}1.827 \\
(0.49)\end{array}$ \\
\hline & & & & & $\begin{array}{l}1.466 \\
(0.59)\end{array}$ & $\begin{array}{l}1.553 \\
(0.39)\end{array}$ & $\begin{array}{l}1.610 \\
(0.40)\end{array}$ & $\begin{array}{l}1.670 \\
(0.43)\end{array}$ & $\begin{array}{l}1.718 \\
(0.41)\end{array}$ & $\begin{array}{l}1.736 \\
(0.37)\end{array}$ & $\begin{array}{l}1.751 \\
(0.50)\end{array}$ \\
\hline & & & & $\begin{array}{l}1.245 \\
(0.60)\end{array}$ & $\begin{array}{l}1.362 \\
(0.43)\end{array}$ & $\begin{array}{l}1.456 \\
(0.39)\end{array}$ & $\begin{array}{l}1.527 \\
(0.40)\end{array}$ & $\begin{array}{l}1.572 \\
(0.41)\end{array}$ & $\begin{array}{l}1.604 \\
(0.41)\end{array}$ & $\begin{array}{l}1.643 \\
(0.40)\end{array}$ & $\begin{array}{l}1.638 \\
(0.54)\end{array}$ \\
\hline & & & $\begin{array}{l}1.067 \\
(0.72)\end{array}$ & $\begin{array}{l}1.155 \\
(0.45)\end{array}$ & $\begin{array}{l}1.257 \\
(0.46)\end{array}$ & $\begin{array}{l}1.330 \\
(0.48)\end{array}$ & $\begin{array}{l}1.403 \\
(0.43)\end{array}$ & $\begin{array}{l}1.447 \\
(0.43)\end{array}$ & $\begin{array}{l}1.491 \\
(0.43)\end{array}$ & $\begin{array}{l}1.509 \\
(0.42)\end{array}$ & $\begin{array}{l}1.528 \\
(0.55)\end{array}$ \\
\hline & & $\begin{array}{l}0.839 \\
(0.75)\end{array}$ & $\begin{array}{c}0.943 \\
(0.60)\end{array}$ & $\begin{array}{l}1.050 \\
(0.52)\end{array}$ & $\begin{array}{l}1.135 \\
(0.48)\end{array}$ & $\begin{array}{l}1.220 \\
(0.47)\end{array}$ & $\begin{array}{l}1.283 \\
(0.45)\end{array}$ & $\begin{array}{c}1.321 \\
(0.48)\end{array}$ & $\begin{array}{l}1.371 \\
(0.47)\end{array}$ & $\begin{array}{c}1.393 \\
(0.45)\end{array}$ & $\begin{array}{c}1.391 \\
(0.60)\end{array}$ \\
\hline & $\begin{array}{l}0.632 \\
(0.82)\end{array}$ & $\begin{array}{c}0.731 \\
(0.65)\end{array}$ & $\begin{array}{l}0.824 \\
(0.57)\end{array}$ & $\begin{array}{l}0.919 \\
(0.50)\end{array}$ & $\begin{array}{l}1.004 \\
(0.50)\end{array}$ & $\begin{array}{l}1.069 \\
(0.49)\end{array}$ & $\begin{array}{l}1.141 \\
(0.47)\end{array}$ & $\begin{array}{l}1.181 \\
(0.52)\end{array}$ & $\begin{array}{l}1.220 \\
(0.44)\end{array}$ & $\begin{array}{l}1.247 \\
(0.44)\end{array}$ & $\begin{array}{l}1.249 \\
(063 .)\end{array}$ \\
\hline $\begin{array}{l}0.565 \\
(0.98)\end{array}$ & $\begin{array}{l}0.561 \\
(0.63)\end{array}$ & $\begin{array}{c}0.631 \\
(0.62)\end{array}$ & $\begin{array}{l}0.703 \\
(0.54)\end{array}$ & $\begin{array}{l}0.788 \\
(0.53)\end{array}$ & $\begin{array}{l}0.880 \\
(0.54)\end{array}$ & $\begin{array}{l}0.938 \\
(0.51)\end{array}$ & $\begin{array}{l}0.990 \\
(0.53)\end{array}$ & $\begin{array}{l}1.042 \\
(0.53)\end{array}$ & $\begin{array}{l}1.071 \\
(0.53)\end{array}$ & $\begin{array}{c}1.099 \\
(0.50)\end{array}$ & $\begin{array}{l}1.088 \\
(0.73)\end{array}$ \\
\hline 0 & $\begin{array}{l}0.566 \\
(0.64)\end{array}$ & $\begin{array}{c}0.586 \\
(0.69)\end{array}$ & $\begin{array}{l}0.617 \\
(0.62)\end{array}$ & $\begin{array}{r}0.672 \\
(0.68)\end{array}$ & $\begin{array}{l}0.729 \\
(0.58)\end{array}$ & $\begin{array}{l}0.795 \\
(0.53)\end{array}$ & $\begin{array}{c}0.845 \\
(0.55)\end{array}$ & $\begin{array}{l}0.886 \\
(0.53)\end{array}$ & $\begin{array}{l}0.922 \\
(0.55)\end{array}$ & $\begin{array}{r}0.941 \\
(0.55)\end{array}$ & $\begin{array}{r}0.947 \\
(0.78)\end{array}$ \\
\hline 0 & 0 & 0 & $\begin{array}{c}0.619 \\
(0.69)\end{array}$ & $\begin{array}{c}0.618 \\
(0.67)\end{array}$ & $\begin{array}{r}0.625 \\
(0.68)\end{array}$ & $\begin{array}{l}0.665 \\
(0.63)\end{array}$ & $\begin{array}{l}0.708 \\
(0.54)\end{array}$ & $\begin{array}{l}0.737 \\
(0.61)\end{array}$ & $\begin{array}{l}0.763 \\
(0.54)\end{array}$ & $\begin{array}{c}0.776 \\
(0.62)\end{array}$ & $\begin{array}{l}0.797 \\
(0.82)\end{array}$ \\
\hline 0 & 0 & 0 & 0 & 0 & $\begin{array}{l}0.621 \\
(0.67)\end{array}$ & $\begin{array}{c}0.619 \\
(0.68)\end{array}$ & $\begin{array}{l}0.637 \\
(0.67)\end{array}$ & $\begin{array}{l}0.618 \\
(0.65)\end{array}$ & $\begin{array}{r}0.629 \\
(0.68)\end{array}$ & $\begin{array}{l}0.634 \\
(0.69)\end{array}$ & $\begin{array}{l}0.642 \\
(0.97)\end{array}$ \\
\hline 0 & 0 & 0 & 0 & 0 & 0 & 0 & 0 & $\begin{array}{l}0.608 \\
(0.65)\end{array}$ & $\begin{array}{l}0.579 \\
(0.62)\end{array}$ & $\begin{array}{l}0.588 \\
(0.72)\end{array}$ & $\begin{array}{l}0.590 \\
(0.96)\end{array}$ \\
\hline
\end{tabular}

Fig. 2.10b. Pin-power distribution for NITAWL benchmark PNL-33. Value in parentheses is percent standard deviation. 
Table 2.35. PNL-33 CENTRM reaction rates and fluxes

\begin{tabular}{ccccccc}
\hline Pin & Region & $\begin{array}{c}\mathrm{G}_{\mathrm{a}} \mathrm{M} \\
\left(\mathrm{cm}^{-3}-\mathrm{s}^{-1}\right)\end{array}$ & $\begin{array}{c}\mathrm{G}_{\mathrm{f}} \mathrm{M} \\
\left(\mathrm{cm}^{-3}-\mathrm{s}^{-1}\right)\end{array}$ & $\begin{array}{c}\mathrm{G}_{\mathrm{f}} \mathrm{M} \\
\left(\mathrm{cm}^{-3}-\mathrm{s}^{-1}\right)\end{array}$ & $\begin{array}{c}\mathrm{M} \\
\left(\mathrm{cm}^{-1}\right)\end{array}$ & $\mathrm{M}_{\mathrm{t}}$ \\
\hline \multirow{2}{*}{1} & Fuel & $3.818 \mathrm{E}-06$ & $6.043 \mathrm{E}-06$ & $2.152 \mathrm{E}-06$ & $3.853 \mathrm{E}-3$ & 0.267 \\
& Clad & $7.376 \mathrm{E}-08$ & 0.0 & 0.0 & $7.423 \mathrm{E}-4$ & 0.051 \\
& Mod. & $4.770 \mathrm{E}-07$ & 0.0 & 0.0 & $9.836 \mathrm{E}-3$ & 0.682 \\
\hline \multirow{2}{*}{109} & Fuel & $1.360 \mathrm{E}-05$ & $2.034 \mathrm{E}-05$ & $7.243 \mathrm{E}-06$ & $1.705 \mathrm{E}-2$ & 0.265 \\
& Clad & $2.788 \mathrm{E}-07$ & 0.0 & 0.0 & $3.401 \mathrm{E}-3$ & 0.051 \\
& Mod. & $1.498 \mathrm{E}-06$ & 0.0 & 0.0 & $4.516 \mathrm{E}-2$ & 0.684 \\
\hline
\end{tabular}

Table 2.36. PNL-33 NITAWL reaction rates and fluxes

\begin{tabular}{ccccccc}
\hline Pin & Region & $\begin{array}{c}\mathrm{G}_{\mathrm{a}} \mathrm{M} \\
\left(\mathrm{cm}^{-3}-\mathrm{s}^{-1}\right)\end{array}$ & $\begin{array}{c}\mathrm{G}_{\mathrm{f}} \mathrm{M} \\
\left(\mathrm{cm}^{-3}-\mathrm{s}^{-1}\right)\end{array}$ & $\begin{array}{c}\mathrm{G}_{\mathrm{f}} \mathrm{M} \\
\left(\mathrm{cm}^{-3}-\mathrm{s}^{-1}\right)\end{array}$ & $\begin{array}{c}\mathrm{M} \\
\left(\mathrm{cm}^{-1}\right)\end{array}$ & $\mathrm{M}_{\mathrm{t}}$ \\
\hline \multirow{2}{*}{1} & Fuel & $3.812 \mathrm{E}-06$ & $6.048 \mathrm{E}-06$ & $2.155 \mathrm{E}-06$ & $3.867 \mathrm{E}-3$ & 0.267 \\
& Clad & $7.253 \mathrm{E}-08$ & 0.0 & 0.0 & $7.426 \mathrm{E}-4$ & 0.052 \\
& Mod. & $4.787 \mathrm{E}-07$ & 0.0 & 0.0 & $9.858 \mathrm{E}-3$ & 0.681 \\
\hline \multirow{2}{*}{109} & Fuel & $1.375 \mathrm{E}-05$ & $2.067 \mathrm{E}-05$ & $7.357 \mathrm{E}-06$ & $1.779 \mathrm{E}-2$ & 0.268 \\
& Clad & $2.783 \mathrm{E}-07$ & 0.0 & 0.0 & $3.442 \mathrm{E}-3$ & 0.052 \\
& Mod. & $1.505 \mathrm{E}-06$ & 0.0 & 0.0 & $4.521 \mathrm{E}-2$ & 0.680 \\
\hline
\end{tabular}


Table 2.37. PNL-33, Pin-1 four-group fluxes

\begin{tabular}{ccc}
\hline Group & $\begin{array}{c}\text { CENTRM M } \\
\left(\mathrm{cm}^{-2}-\mathrm{s}^{-1}\right)\end{array}$ & $\begin{array}{c}\text { NITAWL M } \\
\left(\mathrm{cm}^{-2}-\mathrm{s}^{-1}\right)\end{array}$ \\
\hline 1 & $2.014 \mathrm{E}-05$ & $2.016 \mathrm{E}-05$ \\
2 & $6.869 \mathrm{E}-06$ & $6.908 \mathrm{E}-06$ \\
3 & $1.672 \mathrm{E}-06$ & $1.719 \mathrm{E}-06$ \\
4 & $5.718 \mathrm{E}-06$ & $5.745 \mathrm{E}-06$ \\
\hline
\end{tabular}

Table 2.38. PNL-33, Pin-1 four-group U-235 cross sections

\begin{tabular}{ccccccc}
\hline Group & \multicolumn{2}{c}{ CENTRM cross sections $\left(\mathrm{cm}^{-1}\right)$} & \multicolumn{3}{c}{ NITAWL cross sections $\left(\mathrm{cm}^{-1}\right)$} \\
\hline & $\frac{\mathrm{G}_{\mathrm{a}}}{4.31933 \mathrm{E}-04}$ & $\frac{\leftarrow_{\mathrm{f}}}{5.20055 \mathrm{E}-04}$ & $\frac{\mathrm{G}_{\mathrm{f}}}{2.01129 \mathrm{E}-04}$ & $\frac{\mathrm{G}_{\mathrm{a}}}{2.31159 \mathrm{E}-04}$ & $\frac{\mathrm{G}_{\mathrm{f}}}{5.18686 \mathrm{E}-04}$ & $\frac{\mathrm{G}_{\mathrm{f}}}{2.00672 \mathrm{E}-04}$ \\
1 & $5.35943 \mathrm{E}-03$ & $8.22495 \mathrm{E}-03$ & $3.37546 \mathrm{E}-03$ & $5.34155 \mathrm{E}-03$ & $8.22719 \mathrm{E}-03$ & $3.37636 \mathrm{E}-03$ \\
2 & $8.26154 \mathrm{E}-03$ & $1.68721 \mathrm{E}-02$ & $6.92416 \mathrm{E}-03$ & $8.25026 \mathrm{E}-03$ & $1.68098 \mathrm{E}-02$ & $6.89860 \mathrm{E}-03$ \\
3 & $7.10700 \mathrm{E}-02$ & $1.47828 \mathrm{E}-01$ & $6.06675 \mathrm{E}-02$ & $7.10641 \mathrm{E}-02$ & $1.47819 \mathrm{E}-01$ & $6.06639 \mathrm{E}-02$ \\
\hline
\end{tabular}

Table 2.39. PNL-33, Pin-1 four-group U-238 cross sections

\begin{tabular}{|c|c|c|c|c|c|c|}
\hline \multirow[t]{2}{*}{ Group } & \multicolumn{3}{|c|}{ CENTRM cross sections $\left(\mathrm{cm}^{-1}\right)$} & \multicolumn{3}{|c|}{ NITAWL cross sections $\left(\mathrm{cm}^{-1}\right)$} \\
\hline & $\mathrm{G}_{\mathrm{a}}$ & $<\mathrm{G}_{\mathrm{f}}$ & $\mathrm{G}_{\mathrm{f}}$ & $\mathrm{G}_{\mathrm{a}}$ & $<\mathrm{G}_{\mathrm{f}}$ & $\mathrm{G}_{\mathrm{f}}$ \\
\hline 1 & $6.93024 \mathrm{E}-03$ & $1.22507 \mathrm{E}-02$ & $\overline{4.34474 \mathrm{E}-03}$ & $6.91791 \mathrm{E}-03$ & $\overline{1.22576 \mathrm{E}-02}$ & $\overline{4.35689 \mathrm{E}-03}$ \\
\hline 2 & 4.93469E-02 & $5.63616 \mathrm{E}-06$ & $2.33504 \mathrm{E}-06$ & 4.72956E-02 & 5.71788E-06 & 2.36889E-06 \\
\hline 3 & $1.10965 \mathrm{E}-02$ & 3.87293E-08 & $1.60462 \mathrm{E}-08$ & $1.10697 \mathrm{E}-02$ & 3.84758E-08 & $1.59412 \mathrm{E}-08$ \\
\hline 4 & 4.09384E-02 & $1.92170 \mathrm{E}-07$ & 7.96200E-08 & 4.09467E-02 & 1.92207E-07 & 7.96350E-08 \\
\hline
\end{tabular}

Table 2.40. PNL-33, Pin-1 four-group Pu-239 cross sections

\begin{tabular}{cccccccc}
\hline Group & \multicolumn{3}{c}{ CENTRM cross sections $\left(\mathrm{cm}^{-1}\right)$} & \multicolumn{3}{c}{ NITAWL cross sections $\left(\mathrm{cm}^{-1}\right)$} \\
\hline & $\frac{\mathrm{G}_{\mathrm{a}}}{7.32468 \mathrm{E}-04}$ & $\frac{\mathrm{G}_{\mathrm{f}}}{2.11213 \mathrm{E}-03}$ & $\frac{\mathrm{G}_{\mathrm{f}}}{6.82512 \mathrm{E}-04}$ & $\frac{\mathrm{G}_{\mathrm{a}}}{7.31890 \mathrm{E}-04}$ & $\frac{\mathrm{G}_{\mathrm{f}}}{2.11186 \mathrm{E}-03}$ & $\frac{\mathrm{G}_{\mathrm{f}}}{6.82669 \mathrm{E}-04}$ \\
1 & $1.55242 \mathrm{E}-02$ & $2.53875 \mathrm{E}-02$ & $8.81569 \mathrm{E}-03$ & $1.47320 \mathrm{E}-02$ & $2.43541 \mathrm{E}-02$ & $8.45686 \mathrm{E}-03$ \\
2 & $4.75761 \mathrm{E}-02$ & $9.35353 \mathrm{E}-02$ & $3.24798 \mathrm{E}-02$ & $4.58342 \mathrm{E}-02$ & $9.03415 \mathrm{E}-02$ & $3.13708 \mathrm{E}-02$ \\
3 & $3.92576 \mathrm{E}-01$ & $7.79160 \mathrm{E}-01$ & $2.69612 \mathrm{E}-01$ & $3.91025 \mathrm{E}-01$ & $7.76590 \mathrm{E}-01$ & $2.68717 \mathrm{E}-01$ \\
\hline
\end{tabular}


Table 2.41. PNL-33, Pin-109 four-group fluxes

\begin{tabular}{ccc}
\hline Group & $\begin{array}{c}\text { CENTRM M } \\
\left(\mathrm{cm}^{-2}-\mathrm{s}^{-1}\right)\end{array}$ & $\begin{array}{c}\text { NITAWL M } \\
\left(\mathrm{cm}^{-2}-\mathrm{s}^{-1}\right)\end{array}$ \\
\hline 1 & $9.567 \mathrm{E}-05$ & $9.796 \mathrm{E}-05$ \\
2 & $3.454 \mathrm{E}-05$ & $3.486 \mathrm{E}-05$ \\
3 & $8.083 \mathrm{E}-06$ & $7.903 \mathrm{E}-06$ \\
4 & $1.794 \mathrm{E}-05$ & $1.809 \mathrm{E}-05$ \\
\hline
\end{tabular}

Table 2.42. PNL-33, Pin-109 four-group U-235 cross sections

\begin{tabular}{ccccccc}
\hline Group & \multicolumn{2}{c}{ CENTRM cross sections $\left(\mathrm{cm}^{-1}\right)$} & \multicolumn{3}{c}{ NITAWL cross sections $\left(\mathrm{cm}^{-1}\right)$} \\
\hline & $\frac{\mathrm{G}_{\mathrm{a}}}{4.35819 \mathrm{E}-04}$ & $\frac{\leftarrow_{\mathrm{f}}}{5.23015 \mathrm{E}-04}$ & $\frac{\mathrm{G}_{\mathrm{f}}}{2.03090 \mathrm{E}-04}$ & $\frac{\mathrm{G}_{\mathrm{a}}}{2.36391 \mathrm{E}-04}$ & $\frac{\mathrm{G}_{\mathrm{f}}}{5.23032 \mathrm{E}-04}$ & $\frac{\mathrm{G}_{\mathrm{f}}}{2.03334 \mathrm{E}-04}$ \\
1 & $5.23295 \mathrm{E}-03$ & $8.08842 \mathrm{E}-03$ & $3.31943 \mathrm{E}-03$ & $5.36724 \mathrm{E}-03$ & $8.21076 \mathrm{E}-03$ & $3.36962 \mathrm{E}-03$ \\
2 & $8.20877 \mathrm{E}-03$ & $1.67738 \mathrm{E}-02$ & $6.88382 \mathrm{E}-03$ & $8.26627 \mathrm{E}-03$ & $1.68900 \mathrm{E}-02$ & $6.93152 \mathrm{E}-03$ \\
3 & $6.90920 \mathrm{E}-02$ & $1.43635 \mathrm{E}-01$ & $5.89465 \mathrm{E}-02$ & $6.84549 \mathrm{E}-02$ & $1.42274 \mathrm{E}-01$ & $5.83881 \mathrm{E}-02$ \\
\hline
\end{tabular}

Table 2.43. PNL-33, Pin-109 four-group U-238 cross sections

\begin{tabular}{|c|c|c|c|c|c|c|}
\hline \multirow[t]{2}{*}{ Group } & \multicolumn{3}{|c|}{ CENTRM cross sections $\left(\mathrm{cm}^{-1}\right)$} & \multicolumn{3}{|c|}{ NITAWL cross sections $\left(\mathrm{cm}^{-1}\right)$} \\
\hline & $\mathrm{G}_{\mathrm{a}}$ & $<\mathrm{G}_{\mathrm{f}}$ & $\mathrm{G}_{\mathrm{f}}$ & $\mathrm{G}_{\mathrm{a}}$ & $<\mathrm{G}_{\mathrm{f}}$ & $\mathrm{G}_{\mathrm{f}}$ \\
\hline 1 & $6.78959 \mathrm{E}-03$ & $\overline{1.14474 \mathrm{E}-02}$ & $\overline{4.06739 \mathrm{E}-03}$ & $6.75365 \mathrm{E}-03$ & $1.12640 \mathrm{E}-02$ & $\overline{4.00886 \mathrm{E}-03}$ \\
\hline 2 & 4.77122E-02 & $5.87328 \mathrm{E}-06$ & $2.43327 \mathrm{E}-06$ & 4.72083E-02 & 5.94071E-06 & $2.46121 \mathrm{E}-06$ \\
\hline 3 & 1.10759E-02 & $3.86960 \mathrm{E}-08$ & $1.60324 \mathrm{E}-08$ & $1.11162 \mathrm{E}-02$ & 3.88436E-08 & $1.60936 \mathrm{E}-08$ \\
\hline 4 & 3.99038E-02 & $1.87192 \mathrm{E}-07$ & $7.75574 \mathrm{E}-08$ & $3.95563 \mathrm{E}-02$ & $1.85520 \mathrm{E}-07$ & $7.68646 \mathrm{E}-08$ \\
\hline
\end{tabular}

Table 2.44. PNL-33, Pin-109 four-group Pu-239 cross sections

\begin{tabular}{cccccccc}
\hline Group & \multicolumn{3}{c}{ CENTRM cross sections $\left(\mathrm{cm}^{-1}\right)$} & \multicolumn{3}{c}{ NITAWL cross sections $\left(\mathrm{cm}^{-1}\right)$} \\
\hline & $\frac{\mathrm{G}_{\mathrm{a}}}{7.33828 \mathrm{E}-04}$ & $\frac{\mathrm{G}_{\mathrm{f}}}{2.09652 \mathrm{E}-03}$ & $\frac{\mathrm{G}_{\mathrm{f}}}{6.79997 \mathrm{E}-04}$ & $\frac{\mathrm{G}_{\mathrm{a}}}{7.33983 \mathrm{E}-04}$ & $\frac{\mathrm{G}_{\mathrm{f}}}{2.09254 \mathrm{E}-03}$ & $\frac{\mathrm{G}_{\mathrm{f}}}{6.79479 \mathrm{E}-04}$ \\
1 & $1.54018 \mathrm{E}-02$ & $2.52039 \mathrm{E}-02$ & $8.75191 \mathrm{E}-03$ & $1.50735 \mathrm{E}-02$ & $2.48369 \mathrm{E}-02$ & $8.62447 \mathrm{E}-03$ \\
2 & $4.51535 \mathrm{E}-02$ & $8.91184 \mathrm{E}-02$ & $3.09459 \mathrm{E}-02$ & $4.89102 \mathrm{E}-02$ & $9.59904 \mathrm{E}-02$ & $3.33323 \mathrm{E}-02$ \\
3 & $4.06251 \mathrm{E}-01$ & $7.98367 \mathrm{E}-01$ & $2.76308 \mathrm{E}-01$ & $4.12182 \mathrm{E}-01$ & $8.07020 \mathrm{E}-01$ & $2.79323 \mathrm{E}-01$ \\
\hline
\end{tabular}




\begin{tabular}{|c|c|c|c|c|c|c|c|c|}
\hline & & & & & & & & $\begin{array}{c}7.799 \\
(0.66)\end{array}$ \\
\hline & & & & & & & $\begin{array}{l}7.518 \\
(0.37)\end{array}$ & $\begin{array}{l}7.649 \\
(0.35)\end{array}$ \\
\hline & & & & & & $\begin{array}{l}6.835 \\
(0.40)\end{array}$ & $\begin{array}{l}7.190 \\
(0.27)\end{array}$ & $\begin{array}{r}7.306 \\
(0.37)\end{array}$ \\
\hline & & & & & $\begin{array}{l}5.772 \\
(0.44)\end{array}$ & $\begin{array}{l}6.293 \\
(0.30)\end{array}$ & $\begin{array}{l}6.617 \\
(0.26)\end{array}$ & $\begin{array}{c}6.772 \\
(0.35)\end{array}$ \\
\hline & & & & $\begin{array}{l}4.766 \\
(0.47)\end{array}$ & $\begin{array}{l}5.125 \\
(0.31)\end{array}$ & $\begin{array}{l}5.558 \\
(0.30)\end{array}$ & $\begin{array}{l}5.878 \\
(0.29)\end{array}$ & $\begin{array}{l}5.991 \\
(0.39)\end{array}$ \\
\hline & & & 0 & $\begin{array}{l}5.095 \\
(0.29)\end{array}$ & $\begin{array}{l}4.633 \\
(0.33)\end{array}$ & $\begin{array}{l}4.852 \\
(0.31)\end{array}$ & $\begin{array}{l}5.064 \\
(0.30)\end{array}$ & $\begin{array}{l}5.107 \\
(0.46)\end{array}$ \\
\hline & & 0 & 0 & 0 & $\begin{array}{l}5.015 \\
(0.27)\end{array}$ & $\begin{array}{l}4.688 \\
(0.34)\end{array}$ & $\begin{array}{l}4.382 \\
(0.33)\end{array}$ & $\begin{array}{l}4.282 \\
(0.47)\end{array}$ \\
\hline & 0 & 0 & 0 & 0 & 0 & 0 & $\begin{array}{l}4.431 \\
(0.34)\end{array}$ & $\begin{array}{l}3.764 \\
(0.52)\end{array}$ \\
\hline 0 & 0 & 0 & 0 & 0 & 0 & 0 & 0 & $\begin{array}{l}4.082 \\
(0.48)\end{array}$ \\
\hline
\end{tabular}

Fig. 2.11a. Pin-power distribution for CENTRM benchmark PNL-34. Value in parentheses is percent standard deviation. 


\begin{tabular}{|c|c|c|c|c|c|c|c|c|}
\hline & & & & & & & & $\begin{array}{l}7.789 \\
(0.58)\end{array}$ \\
\hline & & & & & & & $\begin{array}{l}7.559 \\
(0.29)\end{array}$ & $\begin{array}{l}7.692 \\
(0.25)\end{array}$ \\
\hline & & & & & & $\begin{array}{l}6.856 \\
(0.30) \\
\end{array}$ & $\begin{array}{l}7.211 \\
(0.20) \\
\end{array}$ & $\begin{array}{l}7.342 \\
(0.28) \\
\end{array}$ \\
\hline & & & & & $\begin{array}{l}5.778 \\
(0.31)\end{array}$ & $\begin{array}{l}6.319 \\
(0.21)\end{array}$ & $\begin{array}{l}6.661 \\
(0.21)\end{array}$ & $\begin{array}{c}6.794 \\
(0.32)\end{array}$ \\
\hline & & & & $\begin{array}{l}4.790 \\
(0.33)\end{array}$ & $\begin{array}{l}5.142 \\
(0.25)\end{array}$ & $\begin{array}{l}5.607 \\
(0.23)\end{array}$ & $\begin{array}{c}5.928 \\
(0.22)\end{array}$ & $\begin{array}{l}5.991 \\
(0.32)\end{array}$ \\
\hline & & & 0 & $\begin{array}{l}5.108 \\
(0.24)\end{array}$ & $\begin{array}{l}4.634 \\
(0.25)\end{array}$ & $\begin{array}{l}4.865 \\
(0.21)\end{array}$ & $\begin{array}{l}5.047 \\
(0.23)\end{array}$ & $\begin{array}{l}5.134 \\
(0.33)\end{array}$ \\
\hline & & 0 & 0 & 0 & $\begin{array}{l}5.009 \\
(0.25)\end{array}$ & $\begin{array}{l}4.689 \\
(0.24)\end{array}$ & $\begin{array}{l}4.367 \\
(0.26)\end{array}$ & $\begin{array}{l}4.295 \\
(0.36)\end{array}$ \\
\hline & 0 & 0 & 0 & 0 & 0 & 0 & $\begin{array}{l}4.412 \\
(0.26)\end{array}$ & $\begin{array}{l}3.775 \\
(0.40)\end{array}$ \\
\hline 0 & 0 & 0 & 0 & 0 & 0 & 0 & 0 & $\begin{array}{l}4.055 \\
(0.41)\end{array}$ \\
\hline
\end{tabular}

Fig. 2.11b. Pin-power distribution for NITAWL benchmark PNL-34. Value in parentheses is percent standard deviation. 
Table 2.45. PNL-34 CENTRM reaction rates and fluxes

\begin{tabular}{|c|c|c|c|c|c|c|}
\hline Pin & Region & $\begin{array}{c}\mathrm{G}_{\mathrm{a}} \mathrm{M} \\
\left(\mathrm{cm}^{-3}-\mathrm{s}^{-1}\right)\end{array}$ & $\begin{array}{c}\mathcal{G}_{\mathrm{f}} \mathrm{M} \\
\left(\mathrm{cm}^{-3}-\mathrm{s}^{-1}\right)\end{array}$ & $\begin{array}{c}\mathrm{G}_{\mathrm{f}} \mathrm{M} \\
\left(\mathrm{cm}^{-3}-\mathrm{s}^{-1}\right)\end{array}$ & $\begin{array}{c}\text { M } \\
\left(\mathrm{cm}-\mathrm{s}^{-1}\right)\end{array}$ & $\mathrm{MVM}_{\mathrm{t}}$ \\
\hline \multirow{3}{*}{1} & Fuel & 2.378E-05 & 4.072E-05 & $1.453 \mathrm{E}-05$ & $1.638 \mathrm{E}-2$ & 0.205 \\
\hline & Clad & 4.283E-07 & 0.0 & 0.0 & $3.115 \mathrm{E}-3$ & 0.039 \\
\hline & Mod. & $1.208 \mathrm{E}-06$ & 0.0 & 0.0 & $6.042 \mathrm{E}-2$ & 0.756 \\
\hline \multirow{3}{*}{27} & Fuel & 4.850E-05 & 7.778E-05 & $2.772 \mathrm{E}-05$ & $4.812 \mathrm{E}-2$ & 0.206 \\
\hline & Clad & $9.350 \mathrm{E}-07$ & 0.0 & 0.0 & $9.315 \mathrm{E}-3$ & 0.039 \\
\hline & Mod. & $2.117 \mathrm{E}-06$ & 0.0 & 0.0 & $1.767 \mathrm{E}-1$ & 0.755 \\
\hline
\end{tabular}

Table 2.46. PNL-34 NITAWL reaction rates and fluxes

\begin{tabular}{|c|c|c|c|c|c|c|}
\hline Pin & Region & $\begin{array}{c}\mathrm{G}_{\mathrm{a}} \mathrm{M} \\
\left(\mathrm{cm}^{-3}-\mathrm{s}^{-1}\right)\end{array}$ & $\begin{array}{c}\mathcal{G}_{\mathrm{f}} \mathrm{M} \\
\left(\mathrm{cm}^{-3}-\mathrm{s}^{-1}\right)\end{array}$ & $\begin{array}{c}\mathrm{G}_{\mathrm{f}} \mathrm{M} \\
\left(\mathrm{cm}^{-3}-\mathrm{s}^{-1}\right)\end{array}$ & $\begin{array}{c}\mathrm{M} \\
\left(\mathrm{cm}-\mathrm{s}^{-1}\right)\end{array}$ & $\mathrm{M} / \mathrm{M}_{\mathrm{t}}$ \\
\hline \multirow{3}{*}{1} & Fuel & 2.359E-05 & $4.048 \mathrm{E}-05$ & $1.444 \mathrm{E}-05$ & $1.632 \mathrm{E}-2$ & 0.205 \\
\hline & Clad & 4.263E-07 & 0.0 & 0.0 & $3.103 \mathrm{E}-3$ & 0.039 \\
\hline & Mod. & 1.204E-06 & 0.0 & 0.0 & $6.010 \mathrm{E}-2$ & 0.756 \\
\hline \multirow{3}{*}{27} & Fuel & 4.865E-05 & $7.795 \mathrm{E}-5$ & $2.778 \mathrm{E}-05$ & $4.833 \mathrm{E}-2$ & 0.206 \\
\hline & Clad & $9.241 \mathrm{E}-07$ & 0.0 & 0.0 & $9.314 \mathrm{E}-3$ & 0.040 \\
\hline & Mod. & 2.122E-06 & 0.0 & 0.0 & $1.768 \mathrm{E}-1$ & 0.754 \\
\hline
\end{tabular}


Table 2.47. PNL-34, Pin-1 four-group fluxes

\begin{tabular}{ccc}
\hline Group & $\begin{array}{c}\text { CENTRM M } \\
\left(\mathrm{cm}^{-2}-\mathrm{s}^{-1}\right)\end{array}$ & $\begin{array}{c}\text { NITAWL M } \\
\left(\mathrm{cm}^{-2}-\mathrm{s}^{-1}\right)\end{array}$ \\
\hline 1 & $7.853 \mathrm{E}-05$ & $7.833 \mathrm{E}-05$ \\
2 & $2.067 \mathrm{E}-05$ & $2.085 \mathrm{E}-05$ \\
3 & $5.292 \mathrm{E}-06$ & $5.285 \mathrm{E}-06$ \\
4 & $4.175 \mathrm{E}-05$ & $4.127 \mathrm{E}-05$ \\
\hline
\end{tabular}

Table 2.48. PNL-34, Pin-1 four-group U-235 cross sections

\begin{tabular}{|c|c|c|c|c|c|c|}
\hline \multirow[t]{2}{*}{ Group } & \multicolumn{3}{|c|}{ CENTRM cross sections $\left(\mathrm{cm}^{-1}\right)$} & \multicolumn{3}{|c|}{ NITAWL cross sections $\left(\mathrm{cm}^{-1}\right)$} \\
\hline & G & $\varangle$ & $\mathrm{G}_{\mathrm{f}}$ & G & $\varangle_{.}$ & $\mathrm{G}_{\mathrm{f}}$ \\
\hline 1 & $2 . \overline{24131 \mathrm{E}-04}$ & $\overline{5.12577 \mathrm{E}-04}$ & $1.97109 \mathrm{E}-04$ & $2.23704 \mathrm{E}-04$ & $\overline{5.11854 \mathrm{E}-04}$ & $1.96816 \mathrm{E}-04$ \\
\hline 2 & $5.42283 \mathrm{E}-03$ & $8.35085 \mathrm{E}-03$ & $3.42710 \mathrm{E}-03$ & $5.42556 \mathrm{E}-03$ & $8.28643 \mathrm{E}-03$ & $3.40068 \mathrm{E}-03$ \\
\hline 3 & $8.31740 \mathrm{E}-03$ & $1.69753 \mathrm{E}-02$ & $6.96653 \mathrm{E}-03$ & $8.31298 \mathrm{E}-03$ & $1.69590 \mathrm{E}-02$ & $6.95981 \mathrm{E}-03$ \\
\hline 4 & 7.54771E-02 & $1.57143 \mathrm{E}-01$ & $6.44898 \mathrm{E}-02$ & $7.58120 \mathrm{E}-02$ & $1.57838 \mathrm{E}-01$ & $6.47754 \mathrm{E}-02$ \\
\hline
\end{tabular}

Table 2.49. PNL-34, Pin-1 four-group U-238 cross sections

\begin{tabular}{cccccccc}
\hline Group & \multicolumn{3}{c}{ CENTRM cross sections $\left(\mathrm{cm}^{-1}\right)$} & \multicolumn{3}{c}{ NITAWL cross sections $\left(\mathrm{cm}^{-1}\right)$} \\
\hline & $\frac{\mathrm{G}_{\mathrm{a}}}{}$ & $7.08275 \mathrm{E}-03$ & $\frac{\mathrm{G}_{\mathrm{f}}}{1.34026 \mathrm{E}-02}$ & $\frac{\mathrm{G}_{\mathrm{f}}}{4.75965 \mathrm{E}-03}$ & $\frac{\mathrm{G}_{\mathrm{a}}}{7.06095 \mathrm{E}-03}$ & $\frac{\varangle_{\mathrm{f}}}{1.33747 \mathrm{E}-02}$ & $\frac{\mathrm{G}_{\mathrm{f}}}{4.74910 \mathrm{E}-03}$ \\
2 & $5.08244 \mathrm{E}-02$ & $6.00968 \mathrm{E}-06$ & $2.48979 \mathrm{E}-06$ & $4.81132 \mathrm{E}-02$ & $5.51775 \mathrm{E}-06$ & $2.28598 \mathrm{E}-06$ \\
3 & $1.11012 \mathrm{E}-02$ & $3.87466 \mathrm{E}-08$ & $1.60534 \mathrm{E}-08$ & $1.11191 \mathrm{E}-02$ & $3.89584 \mathrm{E}-08$ & $1.61412 \mathrm{E}-08$ \\
4 & $4.32220 \mathrm{E}-02$ & $2.03118 \mathrm{E}-07$ & $8.41557 \mathrm{E}-08$ & $4.33792 \mathrm{E}-02$ & $2.03865 \mathrm{E}-07$ & $8.44652 \mathrm{E}-08$ \\
\hline
\end{tabular}

Table 2.50. PNL-34, Pin-1 four-group Pu-239 cross sections

\begin{tabular}{cccccccc}
\hline Group & \multicolumn{3}{c}{ CENTRM cross sections $\left(\mathrm{cm}^{-1}\right)$} & \multicolumn{3}{c}{ NITAWL cross sections $\left(\mathrm{cm}^{-1}\right)$} \\
\hline & $\frac{\mathrm{G}_{\mathrm{a}}}{}$ & $7.29345 \mathrm{E}-04$ & $\frac{\mathrm{G}_{\mathrm{f}}}{2.13767 \mathrm{E}-03}$ & $\frac{\mathrm{G}_{\mathrm{f}}}{6.87363 \mathrm{E}-04}$ & $\frac{\mathrm{G}_{\mathrm{a}}}{7.28644 \mathrm{E}-04}$ & $\frac{\varangle_{\mathrm{f}}}{2.13663 \mathrm{E}-03}$ & $\frac{\mathrm{G}_{\mathrm{f}}}{6.86999 \mathrm{E}-04}$ \\
2 & $1.53106 \mathrm{E}-02$ & $2.52664 \mathrm{E}-02$ & $8.77363 \mathrm{E}-03$ & $1.54151 \mathrm{E}-02$ & $2.54295 \mathrm{E}-02$ & $8.83026 \mathrm{E}-03$ \\
3 & $4.67356 \mathrm{E}-02$ & $9.20261 \mathrm{E}-02$ & $3.19556 \mathrm{E}-02$ & $4.76683 \mathrm{E}-02$ & $9.37614 \mathrm{E}-02$ & $3.25584 \mathrm{E}-02$ \\
4 & $3.72031 \mathrm{E}-01$ & $7.53188 \mathrm{E}-01$ & $2.60517 \mathrm{E}-01$ & $3.73795 \mathrm{E}-01$ & $7.56765 \mathrm{E}-01$ & $2.61754 \mathrm{E}-01$ \\
\hline
\end{tabular}


Table 2.51. PNL-34, Pin-27 four-group fluxes

\begin{tabular}{ccc}
\hline Group & $\begin{array}{c}\text { CENTRM M } \\
\left(\mathrm{cm}^{-2}-\mathrm{s}^{-1}\right)\end{array}$ & $\begin{array}{c}\text { NITAWL M } \\
\left(\mathrm{cm}^{-2}-\mathrm{s}^{-1}\right)\end{array}$ \\
\hline 1 & $2.549 \mathrm{E}-04$ & $2.550 \mathrm{E}-04$ \\
2 & $8.111 \mathrm{E}-05$ & $8.287 \mathrm{E}-05$ \\
3 & $1.947 \mathrm{E}-05$ & $1.947 \mathrm{E}-05$ \\
4 & $7.406 \mathrm{E}-05$ & $7.415 \mathrm{E}-05$ \\
\hline
\end{tabular}

Table 2.52. PNL-34, Pin-27 four-group U-235 cross sections

\begin{tabular}{cccccccc}
\hline Group & \multicolumn{3}{c}{ CENTRM cross sections $\left(\mathrm{cm}^{-1}\right)$} & \multicolumn{3}{c}{ NITAWL cross sections $\left(\mathrm{cm}^{-1}\right)$} \\
\hline & $\frac{\mathrm{G}_{\mathrm{a}}}{2.32268 \mathrm{E}-04}$ & $\frac{\mathrm{G}_{\mathrm{f}}}{5.19584 \mathrm{E}-04}$ & $\frac{\mathrm{G}_{\mathrm{f}}}{2.01251 \mathrm{E}-04}$ & $\frac{\mathrm{G}_{\mathrm{a}}}{2.31678 \mathrm{E}-04}$ & $\frac{\mathrm{G}_{\mathrm{f}}}{5.19278 \mathrm{E}-04}$ & $\frac{\mathrm{G}_{\mathrm{f}}}{2.00931 \mathrm{E}-04}$ \\
1 & $5.33129 \mathrm{E}-03$ & $8.13383 \mathrm{E}-03$ & $3.33805 \mathrm{E}-03$ & $5.40039 \mathrm{E}-03$ & $8.25888 \mathrm{E}-03$ & $3.38937 \mathrm{E}-03$ \\
2 & $8.21787 \mathrm{E}-03$ & $1.67382 \mathrm{E}-02$ & $6.86918 \mathrm{E}-03$ & $8.24787 \mathrm{E}-03$ & $1.68440 \mathrm{E}-02$ & $6.91263 \mathrm{E}-03$ \\
3 & $7.26200 \mathrm{E}-02$ & $1.51090 \mathrm{E}-01$ & $6.20057 \mathrm{E}-02$ & $7.26900 \mathrm{E}-02$ & $1.51227 \mathrm{E}-01$ & $6.20624 \mathrm{E}-02$ \\
\hline
\end{tabular}

Table 2.53. PNL-34, Pin-27 four-group U-238 cross sections

\begin{tabular}{cccccccc}
\hline Group & \multicolumn{3}{c}{ CENTRM cross sections $\left(\mathrm{cm}^{-1}\right)$} & \multicolumn{3}{c}{ NITAWL cross sections $\left(\mathrm{cm}^{-1}\right)$} \\
\hline & $\frac{\mathrm{G}_{\mathrm{a}}}{6.87156 \mathrm{E}-03}$ & $\frac{\mathrm{G}_{\mathrm{f}}}{1.19877 \mathrm{E}-02}$ & $\frac{\mathrm{G}_{\mathrm{f}}}{4.26261 \mathrm{E}-03}$ & $\frac{6.88984 \mathrm{E}-03}{1.21250 \mathrm{E}-02}$ & $\frac{\mathrm{G}_{\mathrm{a}}}{4.30392 \mathrm{E}-03}$ \\
1 & $4.88942 \mathrm{E}-02$ & $5.93992 \mathrm{E}-06$ & $2.46088 \mathrm{E}-06$ & $4.94199 \mathrm{E}-02$ & $5.55323 \mathrm{E}-06$ & $2.30068 \mathrm{E}-06$ \\
2 & $1.10643 \mathrm{E}-02$ & $3.83905 \mathrm{E}-08$ & $1.59059 \mathrm{E}-08$ & $1.11102 \mathrm{E}-02$ & $3.88583 \mathrm{E}-08$ & $1.60997 \mathrm{E}-08$ \\
3 & $4.17166 \mathrm{E}-02$ & $1.95887 \mathrm{E}-07$ & $8.11600 \mathrm{E}-08$ & $4.17541 \mathrm{E}-02$ & $1.96065 \mathrm{E}-07$ & $8.12334 \mathrm{E}-08$ \\
\hline
\end{tabular}

Table 2.54. PNL-34, Pin-27 four-group Pu-239 cross sections

\begin{tabular}{cccccccc}
\hline Group & \multicolumn{3}{c}{ CENTRM cross sections $\left(\mathrm{cm}^{-1}\right)$} & \multicolumn{3}{c}{ NITAWL cross sections $\left(\mathrm{cm}^{-1}\right)$} \\
\hline & $\frac{\mathrm{G}_{\mathrm{a}}}{7.32585 \mathrm{E}-04}$ & $\frac{\mathrm{G}_{\mathrm{f}}}{2.10845 \mathrm{E}-03}$ & $\frac{\mathrm{G}_{\mathrm{f}}}{6.82303 \mathrm{E}-04}$ & $\frac{\mathrm{G}_{\mathrm{a}}}{7.32086 \mathrm{E}-04}$ & $\frac{\mathrm{G}_{\mathrm{f}}}{2.11047 \mathrm{E}-03}$ & $\frac{\mathrm{G}_{\mathrm{f}}}{6.82341 \mathrm{E}-04}$ \\
1 & $1.52932 \mathrm{E}-02$ & $2.51849 \mathrm{E}-02$ & $8.74535 \mathrm{E}-03$ & $1.50659 \mathrm{E}-02$ & $2.45838 \mathrm{E}-02$ & $8.53662 \mathrm{E}-03$ \\
2 & $4.71772 \mathrm{E}-02$ & $9.27560 \mathrm{E}-02$ & $3.22091 \mathrm{E}-02$ & $4.72429 \mathrm{E}-02$ & $9.29723 \mathrm{E}-02$ & $3.22843 \mathrm{E}-02$ \\
3 & $3.90512 \mathrm{E}-01$ & $7.78732 \mathrm{E}-01$ & $2.69434 \mathrm{E}-01$ & $3.90550 \mathrm{E}-01$ & $7.78815 \mathrm{E}-01$ & $2.69459 \mathrm{E}-01$ \\
\hline
\end{tabular}




\begin{tabular}{|c|c|c|c|c|c|c|c|c|c|c|}
\hline & & & & & & & & & & $\begin{array}{l}2.316 \\
(1.24)\end{array}$ \\
\hline & & & & & & & & & $\begin{array}{l}2.296 \\
(0.53)\end{array}$ & $\begin{array}{l}2.287 \\
(0.52)\end{array}$ \\
\hline & & & & & & & & $\begin{array}{l}2.231 \\
(0.55)\end{array}$ & $\begin{array}{l}2.256 \\
(0.39)\end{array}$ & $\begin{array}{l}2.273 \\
(0.58)\end{array}$ \\
\hline & & & & & & & $\begin{array}{c}2.099 \\
(0.52)\end{array}$ & $\begin{array}{l}2.175 \\
(0.40)\end{array}$ & $\begin{array}{l}2.223 \\
(0.43)\end{array}$ & $\begin{array}{l}2.208 \\
(0.54)\end{array}$ \\
\hline & & & & & & $\begin{array}{l}1.961 \\
(0.55)\end{array}$ & $\begin{array}{c}2.034 \\
(0.41)\end{array}$ & $\begin{array}{l}2.098 \\
(0.41)\end{array}$ & $\begin{array}{l}2.134 \\
(0.38)\end{array}$ & $\begin{array}{l}2.135 \\
(0.61)\end{array}$ \\
\hline & & & & & $\begin{array}{r}1.756 \\
(0.64)\end{array}$ & $\begin{array}{l}1.872 \\
(0.43)\end{array}$ & $\begin{array}{l}1.941 \\
(0.42)\end{array}$ & $\begin{array}{l}1.996 \\
(0.39)\end{array}$ & $\begin{array}{l}2.057 \\
(0.38)\end{array}$ & $\begin{array}{l}2.047 \\
(0.55)\end{array}$ \\
\hline & & & & $\begin{array}{l}1.554 \\
(0.67)\end{array}$ & $\begin{array}{l}1.667 \\
(0.45)\end{array}$ & $\begin{array}{l}1.768 \\
(0.42)\end{array}$ & $\begin{array}{l}1.831 \\
(0.46)\end{array}$ & $\begin{array}{c}1.888 \\
(0.45)\end{array}$ & $\begin{array}{l}1.915 \\
(0.41)\end{array}$ & $\begin{array}{l}1.936 \\
(0.60)\end{array}$ \\
\hline & & & $\begin{array}{l}1.322 \\
(0.73)\end{array}$ & $\begin{array}{c}1.441 \\
(0.50)\end{array}$ & $\begin{array}{c}1.550 \\
(0.43)\end{array}$ & $\begin{array}{l}1.640 \\
(0.45)\end{array}$ & $\begin{array}{c}1.704 \\
(0.46)\end{array}$ & $\begin{array}{c}1.742 \\
(0.40)\end{array}$ & $\begin{array}{c}1.797 \\
(0.43)\end{array}$ & $\begin{array}{l}1.788 \\
(0.57)\end{array}$ \\
\hline & & $\begin{array}{c}1.050 \\
(0.80)\end{array}$ & $\begin{array}{l}1.196 \\
(0.58)\end{array}$ & $\begin{array}{l}1.301 \\
(0.46)\end{array}$ & $\begin{array}{c}1.414 \\
(0.47)\end{array}$ & $\begin{array}{c}1.498 \\
(0.52)\end{array}$ & $\begin{array}{l}1.567 \\
(0.46)\end{array}$ & $\begin{array}{l}1.606 \\
(0.46)\end{array}$ & $\begin{array}{l}1.640 \\
(0.43)\end{array}$ & $\begin{array}{l}1.640 \\
(0.61)\end{array}$ \\
\hline & $\begin{array}{c}0.798 \\
(0.83)\end{array}$ & $\begin{array}{c}0.924 \\
(0.60)\end{array}$ & $\begin{array}{c}1.047 \\
(0.60)\end{array}$ & $\begin{array}{c}1.159 \\
(0.53)\end{array}$ & $\begin{array}{c}1.254 \\
(0.49)\end{array}$ & $\begin{array}{l}1.331 \\
(0.51)\end{array}$ & $\begin{array}{r}1.400 \\
(0.49)\end{array}$ & $\begin{array}{l}1.441 \\
(0.49)\end{array}$ & $\begin{array}{c}1.475 \\
(0.45)\end{array}$ & $\begin{array}{l}1.488 \\
(0.64)\end{array}$ \\
\hline $\begin{array}{l}0.657 \\
(0.98)\end{array}$ & $\begin{array}{r}0.680 \\
(0.66)\end{array}$ & $\begin{array}{c}0.780 \\
(0.67)\end{array}$ & $\begin{array}{l}0.888 \\
(0.60)\end{array}$ & $\begin{array}{c}1.002 \\
(0.60)\end{array}$ & $\begin{array}{c}1.099 \\
(0.58)\end{array}$ & $\begin{array}{l}1.163 \\
(0.52)\end{array}$ & $\begin{array}{l}1.236 \\
(0.55)\end{array}$ & $\begin{array}{l}1.270 \\
(0.55)\end{array}$ & $\begin{array}{c}1.299 \\
(0.48)\end{array}$ & $\begin{array}{l}1.291 \\
(0.76)\end{array}$ \\
\hline 0 & $\begin{array}{c}0.645 \\
(0.64)\end{array}$ & $\begin{array}{r}0.667 \\
(0.70)\end{array}$ & $\begin{array}{l}0.755 \\
(0.71)\end{array}$ & $\begin{array}{l}0.838 \\
(0.66)\end{array}$ & $\begin{array}{c}0.926 \\
(0.58)\end{array}$ & $\begin{array}{l}1.004 \\
(0.57)\end{array}$ & $\begin{array}{c}1.052 \\
(0.58)\end{array}$ & $\begin{array}{l}1.091 \\
(0.50)\end{array}$ & $\begin{array}{c}1.122 \\
(0.56)\end{array}$ & $\begin{array}{l}1.114 \\
(0.73)\end{array}$ \\
\hline 0 & 0 & $\begin{array}{r}0.605 \\
(0.73)\end{array}$ & $\begin{array}{c}0.620 \\
(0.71)\end{array}$ & $\begin{array}{l}0.685 \\
(0.66)\end{array}$ & $\begin{array}{c}0.759 \\
(0.64)\end{array}$ & $\begin{array}{l}0.821 \\
(0.68)\end{array}$ & $\begin{array}{l}0.868 \\
(0.53)\end{array}$ & $\begin{array}{r}0.902 \\
(0.62)\end{array}$ & $\begin{array}{l}0.924 \\
(0.56)\end{array}$ & $\begin{array}{l}0.929 \\
(0.81)\end{array}$ \\
\hline 0 & 0 & 0 & $\begin{array}{l}0.562 \\
(0.81)\end{array}$ & $\begin{array}{l}0.591 \\
(0.78)\end{array}$ & $\begin{array}{r}0.617 \\
(0.75)\end{array}$ & $\begin{array}{c}0.649 \\
(0.79)\end{array}$ & $\begin{array}{l}0.696 \\
(0.66)\end{array}$ & $\begin{array}{l}0.736 \\
(0.69)\end{array}$ & $\begin{array}{l}0.753 \\
(0.64)\end{array}$ & $\begin{array}{l}0.760 \\
(0.98)\end{array}$ \\
\hline 0 & 0 & 0 & 0 & 0 & $\begin{array}{c}0.553 \\
(0.78)\end{array}$ & $\begin{array}{c}0.558 \\
(0.83)\end{array}$ & $\begin{array}{l}0.591 \\
(0.70)\end{array}$ & $\begin{array}{l}0.619 \\
(0.71)\end{array}$ & $\begin{array}{l}0.642 \\
(0.75)\end{array}$ & $\begin{array}{l}0.645 \\
(1.12)\end{array}$ \\
\hline
\end{tabular}

Fig. 2.12a. Pin-power distribution for CENTRM benchmark PNL-35. Value in parentheses is percent standard deviation. 


\begin{tabular}{|c|c|c|c|c|c|c|c|c|c|c|}
\hline & & & & & & & & & & $\begin{array}{l}2.333 \\
(0.98)\end{array}$ \\
\hline & & & & & & & & & $\begin{array}{l}2.308 \\
(0.50)\end{array}$ & $\begin{array}{l}2.324 \\
(0.46)\end{array}$ \\
\hline & & & & & & & & $\begin{array}{l}2.251 \\
(0.49)\end{array}$ & $\begin{array}{l}2.275 \\
(0.36)\end{array}$ & $\begin{array}{l}2.283 \\
(0.54)\end{array}$ \\
\hline & & & & & & & $\begin{array}{l}2.133 \\
(0.50)\end{array}$ & $\begin{array}{l}2.193 \\
(0 .) 36\end{array}$ & $\begin{array}{l}2.202 \\
(0.36)\end{array}$ & $\begin{array}{l}2.210 \\
(0.48)\end{array}$ \\
\hline & & & & & & $\begin{array}{c}1.973 \\
(0.50)\end{array}$ & $\begin{array}{l}2.044 \\
(0.36)\end{array}$ & $\begin{array}{l}2.110 \\
(0.35)\end{array}$ & $\begin{array}{l}2.131 \\
(0.37)\end{array}$ & $\begin{array}{l}2.142 \\
(0.54)\end{array}$ \\
\hline & & & & & $\begin{array}{l}1.790 \\
(0.55)\end{array}$ & $\begin{array}{c}1.887 \\
(0.38)\end{array}$ & $\begin{array}{r}1.950 \\
(0.40)\end{array}$ & $\begin{array}{c}2.004 \\
(0.36)\end{array}$ & $\begin{array}{l}2.038 \\
(0.35)\end{array}$ & $\begin{array}{l}2.050 \\
(0.48)\end{array}$ \\
\hline & & & & $\begin{array}{c}1.583 \\
(0.60)\end{array}$ & $\begin{array}{c}1.688 \\
(0.41)\end{array}$ & $\begin{array}{c}1.765 \\
(0.40)\end{array}$ & $\begin{array}{l}1.827 \\
(0.40)\end{array}$ & $\begin{array}{l}1.890 \\
(0.38)\end{array}$ & $\begin{array}{c}1.916 \\
(0.37)\end{array}$ & $\begin{array}{l}1.941 \\
(0.52)\end{array}$ \\
\hline & & & $\begin{array}{c}1.329 \\
(0.66)\end{array}$ & $\begin{array}{r}1.460 \\
(0.45)\end{array}$ & $\begin{array}{l}1.552 \\
(0.42)\end{array}$ & $\begin{array}{c}1.636 \\
(0.38)\end{array}$ & $\begin{array}{c}1.692 \\
(0.38)\end{array}$ & $\begin{array}{c}1.766 \\
(0.39)\end{array}$ & $\begin{array}{c}1.772 \\
(0.40)\end{array}$ & $\begin{array}{c}1.802 \\
(0.60)\end{array}$ \\
\hline & & $\begin{array}{l}1.065 \\
(0.73)\end{array}$ & $\begin{array}{c}1.212 \\
(0.48)\end{array}$ & $\begin{array}{c}1.315 \\
(0.50)\end{array}$ & $\begin{array}{c}1.409 \\
(0.45)\end{array}$ & $\begin{array}{c}1.482 \\
(0.42)\end{array}$ & $\begin{array}{l}1.566 \\
(0.44)\end{array}$ & $\begin{array}{l}1.603 \\
(0.42)\end{array}$ & $\begin{array}{l}1.622 \\
(0.42)\end{array}$ & $\begin{array}{l}1.645 \\
(0.59)\end{array}$ \\
\hline & $\begin{array}{l}8.130 \\
(0.80)\end{array}$ & $\begin{array}{r}9.340 \\
(0.58)\end{array}$ & $\begin{array}{c}1.060 \\
(0.54)\end{array}$ & $\begin{array}{c}1.164 \\
(0.47)\end{array}$ & $\begin{array}{l}1.252 \\
(0.50)\end{array}$ & $\begin{array}{c}1.329 \\
(0.47)\end{array}$ & $\begin{array}{c}1.391 \\
(0.45)\end{array}$ & $\begin{array}{l}1.433 \\
(0.44)\end{array}$ & $\begin{array}{c}1.479 \\
(0.41)\end{array}$ & $\begin{array}{l}1.488 \\
(0.61)\end{array}$ \\
\hline $\begin{array}{l}6.750 \\
(0.89)\end{array}$ & $\begin{array}{l}6.790 \\
(0.62)\end{array}$ & $\begin{array}{c}7.980 \\
(0.63)\end{array}$ & $\begin{array}{c}9.02 \\
(0.55)\end{array}$ & $\begin{array}{c}1.011 \\
(0.53)\end{array}$ & $\begin{array}{l}1.095 \\
(0.48)\end{array}$ & $\begin{array}{c}1.159 \\
(0.50)\end{array}$ & $\begin{array}{l}1.220 \\
(0.46)\end{array}$ & $\begin{array}{l}1.258 \\
(0.46)\end{array}$ & $\begin{array}{c}1.301 \\
(0.47)\end{array}$ & $\begin{array}{l}1.315 \\
(0.61)\end{array}$ \\
\hline 0 & $\begin{array}{r}0.651 \\
(0.64)\end{array}$ & $\begin{array}{l}0.663 \\
(0.67)\end{array}$ & $\begin{array}{c}0.742 \\
(0.60)\end{array}$ & $\begin{array}{l}0.837 \\
(0.60)\end{array}$ & $\begin{array}{l}0.927 \\
(0.54)\end{array}$ & $\begin{array}{c}0.994 \\
(0.54)\end{array}$ & $\begin{array}{l}1.033 \\
(0.52)\end{array}$ & $\begin{array}{l}1.087 \\
(0.53)\end{array}$ & $\begin{array}{c}1.101 \\
(0.49)\end{array}$ & $\begin{array}{l}1.123 \\
(0.72)\end{array}$ \\
\hline 0 & 0 & $\begin{array}{l}0.607 \\
(0.74)\end{array}$ & $\begin{array}{l}0.616 \\
(0.66)\end{array}$ & $\begin{array}{r}0.677 \\
(0.65)\end{array}$ & $\begin{array}{l}0.765 \\
(0.57)\end{array}$ & $\begin{array}{r}0.819 \\
(0.56)\end{array}$ & $\begin{array}{c}0.870 \\
(0.59)\end{array}$ & $\begin{array}{l}0.900 \\
(0.53)\end{array}$ & $\begin{array}{l}0.920 \\
(0.59)\end{array}$ & $\begin{array}{l}0.921 \\
(0.77)\end{array}$ \\
\hline 0 & 0 & 0 & $\begin{array}{c}0.565 \\
(0.68)\end{array}$ & $\begin{array}{l}0.591 \\
(0.65)\end{array}$ & $\begin{array}{c}0.606 \\
(0.66)\end{array}$ & $\begin{array}{r}0.653 \\
(0.63)\end{array}$ & $\begin{array}{c}0.686 \\
(0.66)\end{array}$ & $\begin{array}{l}0.717 \\
(0.59)\end{array}$ & $\begin{array}{c}0.744 \\
(0.59)\end{array}$ & $\begin{array}{l}0.755 \\
(0.87)\end{array}$ \\
\hline 0 & 0 & 0 & 0 & 0 & $\begin{array}{r}0.557 \\
(0.69)\end{array}$ & $\begin{array}{c}0.554 \\
(0.69)\end{array}$ & $\begin{array}{l}0.600 \\
(0.73)\end{array}$ & $\begin{array}{r}0.615 \\
(0.71)\end{array}$ & $\begin{array}{r}0.640 \\
(0.70)\end{array}$ & $\begin{array}{c}0.650 \\
(0.96)\end{array}$ \\
\hline
\end{tabular}

Fig. 2.12b. Pin-power distribution for NITAWL benchmark PNL-35. Value in parentheses is percent standard deviation. 
Table 2.55. PNL-35 CENTRM reaction rates and fluxes

\begin{tabular}{ccccccc}
\hline Pin & Region & $\begin{array}{c}\mathrm{G}_{\mathrm{a}} \mathrm{M} \\
\left(\mathrm{cm}^{-3}-\mathrm{s}^{-1}\right)\end{array}$ & $\begin{array}{c}\mathrm{G}_{\mathrm{f}} \mathrm{M} \\
\left(\mathrm{cm}^{-3}-\mathrm{s}^{-1}\right)\end{array}$ & $\begin{array}{c}\mathrm{G}_{\mathrm{f}} \mathrm{M} \\
\left(\mathrm{cm}^{-3}-\mathrm{s}^{-1}\right)\end{array}$ & $\begin{array}{c}\mathrm{M} \\
\left(\mathrm{cm}^{-1}\right)\end{array}$ & $\mathrm{M}_{\mathrm{t}}$ \\
\hline \multirow{2}{*}{1} & Fuel & $3.409 \mathrm{E}-06$ & $5.555 \mathrm{E}-06$ & $1.980 \mathrm{E}-06$ & $3.018 \mathrm{E}-3$ & 0.205 \\
& Clad & $6.266 \mathrm{E}-08$ & 0.0 & 0.0 & $5.795 \mathrm{E}-4$ & 0.039 \\
& Mod. & $3.753 \mathrm{E}-07$ & 0.0 & 0.0 & $1.113 \mathrm{E}-2$ & 0.756 \\
\hline \multirow{2}{*}{99} & Fuel & $1.478 \mathrm{E}-05$ & $2.314 \mathrm{E}-05$ & $8.243 \mathrm{E}-06$ & $1.578 \mathrm{E}-2$ & 0.206 \\
& Clad & $2.862 \mathrm{E}-07$ & 0.0 & 0.0 & $3.049 \mathrm{E}-3$ & 0.039 \\
& Mod. & $1.467 \mathrm{E}-06$ & 0.0 & 0.0 & $5.794 \mathrm{E}-2$ & 0.755 \\
\hline
\end{tabular}

Table 2.56. PNL-35 NITAWL reaction rates and fluxes

\begin{tabular}{|c|c|c|c|c|c|c|}
\hline Pin & Region & $\begin{array}{c}\mathrm{G}_{\mathrm{a}} \mathrm{M} \\
\left(\mathrm{cm}^{-3}-\mathrm{s}^{-1}\right)\end{array}$ & $\begin{array}{c}\mathcal{G}_{\mathrm{f}} \mathrm{M} \\
\left(\mathrm{cm}^{-3}-\mathrm{s}^{-1}\right)\end{array}$ & $\begin{array}{c}\mathrm{G}_{\mathrm{f}} \mathrm{M} \\
\left(\mathrm{cm}^{-3}-\mathrm{s}^{-1}\right)\end{array}$ & $\begin{array}{c}\mathrm{M} \\
\left(\mathrm{cm}-\mathrm{s}^{-1}\right)\end{array}$ & $\mathrm{M} / \mathrm{M}_{\mathrm{t}}$ \\
\hline \multirow{3}{*}{1} & Fuel & $3.418 \mathrm{E}-06$ & $5.589 \mathrm{E}-06$ & 1.992E-06 & $3.030 \mathrm{E}-3$ & 0.206 \\
\hline & Clad & $6.288 \mathrm{E}-08$ & 0.0 & 0.0 & $5.820 \mathrm{E}-4$ & 0.040 \\
\hline & Mod. & 3.743E-07 & 0.0 & 0.0 & $1.107 \mathrm{E}-2$ & 0.754 \\
\hline \multirow{3}{*}{99} & Fuel & $1.486 \mathrm{E}-05$ & $2.341 \mathrm{E}-05$ & 8.338E-06 & $1.603 \mathrm{E}-2$ & 0.207 \\
\hline & Clad & $2.817 \mathrm{E}-07$ & 0.0 & 0.0 & $3.073 \mathrm{E}-3$ & 0.040 \\
\hline & Mod. & $1.487 \mathrm{E}-06$ & 0.0 & 0.0 & $5.834 \mathrm{E}-2$ & 0.753 \\
\hline
\end{tabular}


Table 2.57. PNL-35, Pin-1 four-group fluxes

\begin{tabular}{ccc}
\hline Group & $\begin{array}{c}\text { CENTRM M } \\
\left(\mathrm{cm}^{-2}-\mathrm{s}^{-1}\right)\end{array}$ & $\begin{array}{c}\text { NITAWL M } \\
\left(\mathrm{cm}^{-2}-\mathrm{s}^{-1}\right)\end{array}$ \\
\hline 1 & $1.534 \mathrm{E}-05$ & $1.545 \mathrm{E}-05$ \\
2 & $4.935 \mathrm{E}-06$ & $4.897 \mathrm{E}-06$ \\
3 & $1.254 \mathrm{E}-06$ & $1.264 \mathrm{E}-06$ \\
4 & $5.413 \mathrm{E}-06$ & $5.443 \mathrm{E}-06$ \\
\hline
\end{tabular}

Table 2.58. PNL-35, Pin-1 four-group U-235 cross sections

\begin{tabular}{cccccccc}
\hline Group & \multicolumn{3}{c}{ CENTRM cross sections $\left(\mathrm{cm}^{-1}\right)$} & \multicolumn{3}{c}{ NITAWL cross sections $\left(\mathrm{cm}^{-1}\right)$} \\
\hline & $\frac{\mathrm{G}_{\mathrm{a}}}{4.29059 \mathrm{E}-04}$ & $\frac{\varangle_{\mathrm{f}}}{5.17093 \mathrm{E}-04}$ & $\frac{\mathrm{G}_{\mathrm{f}}}{1.99589 \mathrm{E}-04}$ & $\frac{2.29390 \mathrm{E}-04}{\mathrm{G}}$ & $\frac{\mathrm{G}_{\mathrm{f}}}{5.17691 \mathrm{E}-04}$ & $\frac{\mathrm{G}_{\mathrm{f}}}{1.99788 \mathrm{E}-04}$ \\
1 & $5.45929 \mathrm{E}-03$ & $8.37664 \mathrm{E}-03$ & $3.43769 \mathrm{E}-03$ & $5.46541 \mathrm{E}-03$ & $8.36718 \mathrm{E}-03$ & $3.43382 \mathrm{E}-03$ \\
2 & $8.30534 \mathrm{E}-03$ & $1.69238 \mathrm{E}-02$ & $6.94538 \mathrm{E}-03$ & $8.28758 \mathrm{E}-03$ & $1.68623 \mathrm{E}-02$ & $6.92012 \mathrm{E}-03$ \\
3 & $7.30078 \mathrm{E}-02$ & $1.51919 \mathrm{E}-01$ & $6.23460 \mathrm{E}-02$ & $7.27680 \mathrm{E}-02$ & $1.51408 \mathrm{E}-01$ & $6.21367 \mathrm{E}-02$ \\
\hline
\end{tabular}

Table 2.59. PNL-35, Pin-1 four-group U-238 cross sections

\begin{tabular}{|c|c|c|c|c|c|c|}
\hline \multirow[t]{2}{*}{ Group } & \multicolumn{3}{|c|}{ CENTRM cross sections $\left(\mathrm{cm}^{-1}\right)$} & \multicolumn{3}{|c|}{ NITAWL cross sections $\left(\mathrm{cm}^{-1}\right)$} \\
\hline & G & $\ll G_{.}$ & $\mathrm{G}_{\mathrm{f}}$ & G & $\ll G_{f}$ & $\mathrm{G}_{\mathrm{f}}$ \\
\hline 1 & $6.96200 \mathrm{E}-03$ & $\overline{1.25983 \mathrm{E}-02}$ & $\overline{4.46724 \mathrm{E}-03}$ & $6.97774 \bar{E}-03$ & $1.26269 \mathrm{E}-02$ & $\overline{4.47453 \mathrm{E}-03}$ \\
\hline 2 & $5.05345 \mathrm{E}-02$ & $5.59431 \mathrm{E}-06$ & $2.31769 \mathrm{E}-06$ & $4.85328 \mathrm{E}-02$ & $5.78528 \mathrm{E}-06$ & $2.39681 \mathrm{E}-06$ \\
\hline 3 & $1.11140 \mathrm{E}-02$ & $3.88695 \mathrm{E}-08$ & $1.61043 \mathrm{E}-08$ & $1.10876 \mathrm{E}-02$ & $3.87287 \mathrm{E}-08$ & $1.60460 \mathrm{E}-08$ \\
\hline 4 & 4.19366E-02 & $1.96955 \mathrm{E}-07$ & $8.16021 \mathrm{E}-08$ & $4.18109 \mathrm{E}-02$ & $1.96348 \mathrm{E}-07$ & $8.13505 \mathrm{E}-08$ \\
\hline
\end{tabular}

Table 2.60. PNL-35, Pin-1 four-group Pu-239 cross sections

\begin{tabular}{cccccccc}
\hline Group & \multicolumn{3}{c}{ CENTRM cross sections $\left(\mathrm{cm}^{-1}\right)$} & \multicolumn{3}{c}{ NITAWL cross sections $\left(\mathrm{cm}^{-1}\right)$} \\
\hline & $\frac{\mathrm{G}_{\mathrm{a}}}{}$ & $7.31004 \mathrm{E}-04$ & $\frac{\mathrm{G}_{\mathrm{f}}}{2.12002 \mathrm{E}-03}$ & $\frac{\mathrm{G}_{\mathrm{f}}}{6.83898 \mathrm{E}-04}$ & $\frac{\mathrm{G}_{\mathrm{a}}}{7.31003 \mathrm{E}-04}$ & $\frac{\mathrm{G}_{\mathrm{f}}}{2.11949 \mathrm{E}-03}$ & $\frac{\mathrm{G}_{\mathrm{f}}}{6.83599 \mathrm{E}-04}$ \\
2 & $1.57027 \mathrm{E}-02$ & $2.57978 \mathrm{E}-02$ & $8.95814 \mathrm{E}-03$ & $1.48272 \mathrm{E}-02$ & $2.46432 \mathrm{E}-02$ & $8.55722 \mathrm{E}-03$ \\
3 & $4.68010 \mathrm{E}-02$ & $9.21946 \mathrm{E}-02$ & $3.20143 \mathrm{E}-02$ & $4.68134 \mathrm{E}-02$ & $9.21519 \mathrm{E}-02$ & $3.19994 \mathrm{E}-02$ \\
4 & $3.84903 \mathrm{E}-01$ & $7.69989 \mathrm{E}-01$ & $2.66394 \mathrm{E}-01$ & $3.86636 \mathrm{E}-01$ & $7.72458 \mathrm{E}-01$ & $2.67253 \mathrm{E}-01$ \\
\hline
\end{tabular}


Table 2.61. PNL-35, Pin-99 four-group fluxes

\begin{tabular}{ccc}
\hline Group & $\begin{array}{c}\text { CENTRM M } \\
\left(\mathrm{cm}^{-2}-\mathrm{s}^{-1}\right)\end{array}$ & $\begin{array}{c}\text { NITAWL M } \\
\left(\mathrm{cm}^{-2}-\mathrm{s}^{-1}\right)\end{array}$ \\
\hline 1 & $8.383 \mathrm{E}-05$ & $8.501 \mathrm{E}-05$ \\
2 & $2.858 \mathrm{E}-05$ & $2.911 \mathrm{E}-05$ \\
3 & $6.805 \mathrm{E}-06$ & $7.098 \mathrm{E}-06$ \\
4 & $2.163 \mathrm{E}-05$ & $2.187 \mathrm{E}-05$ \\
\hline
\end{tabular}

Table 2.62. PNL-35, Pin-99 four-group U-235 cross sections

\begin{tabular}{cccccccc}
\hline Group & \multicolumn{3}{c}{ CENTRM cross sections $\left(\mathrm{cm}^{-1}\right)$} & \multicolumn{3}{c}{ NITAWL cross sections $\left(\mathrm{cm}^{-1}\right)$} \\
\hline & $\frac{\mathrm{G}_{\mathrm{a}}}{2.33860 \mathrm{E}-04}$ & $\frac{<\mathrm{G}_{\mathrm{f}}}{5.21028 \mathrm{E}-04}$ & $\frac{\mathrm{G}_{\mathrm{f}}}{2.02116 \mathrm{E}-04}$ & $\frac{\mathrm{G}_{\mathrm{a}}}{2.31754 \mathrm{E}-04}$ & $\frac{\mathrm{G}_{\mathrm{f}}}{5.19152 \mathrm{E}-04}$ & $\frac{\mathrm{G}_{\mathrm{f}}}{2.00942 \mathrm{E}-04}$ \\
1 & $5.36679 \mathrm{E}-03$ & $8.26977 \mathrm{E}-03$ & $3.39384 \mathrm{E}-03$ & $5.36675 \mathrm{E}-03$ & $8.27252 \mathrm{E}-03$ & $3.39496 \mathrm{E}-03$ \\
2 & $8.17348 \mathrm{E}-03$ & $1.67070 \mathrm{E}-02$ & $6.85640 \mathrm{E}-03$ & $8.15523 \mathrm{E}-03$ & $1.66066 \mathrm{E}-02$ & $6.81524 \mathrm{E}-03$ \\
3 & $7.08756 \mathrm{E}-02$ & $1.47418 \mathrm{E}-01$ & $6.04988 \mathrm{E}-02$ & $7.06159 \mathrm{E}-02$ & $1.46859 \mathrm{E}-01$ & $6.02693 \mathrm{E}-02$ \\
\hline
\end{tabular}

Table 2.63. PNL-35, Pin-99 four-group U-238 cross sections

\begin{tabular}{cccccccc}
\hline Group & \multicolumn{3}{c}{ CENTRM cross sections $\left(\mathrm{cm}^{-1}\right)$} & \multicolumn{3}{c}{ NITAWL cross sections $\left(\mathrm{cm}^{-1}\right)$} \\
\hline & $\frac{\mathrm{G}_{\mathrm{a}}}{4.85097 \mathrm{E}-03}$ & $\frac{\mathrm{G}_{\mathrm{f}}}{1.17780 \mathrm{E}-02}$ & $\frac{\mathrm{G}_{\mathrm{f}}}{4.19479 \mathrm{E}-03}$ & $\frac{6.87710 \mathrm{E}-03}{1.20714 \mathrm{E}-02}$ & $\frac{\mathrm{G}_{\mathrm{a}}}{4.28595 \mathrm{E}-03}$ \\
1 & $5.03681 \mathrm{E}-02$ & $5.57278 \mathrm{E}-06$ & $2.30878 \mathrm{E}-06$ & $4.60701 \mathrm{E}-02$ & $5.75479 \mathrm{E}-06$ & $2.38419 \mathrm{E}-06$ \\
2 & $1.10871 \mathrm{E}-02$ & $3.86935 \mathrm{E}-08$ & $1.60313 \mathrm{E}-08$ & $1.10723 \mathrm{E}-02$ & $3.85789 \mathrm{E}-08$ & $1.59839 \mathrm{E}-08$ \\
3 & $4.08300 \mathrm{E}-02$ & $1.91640 \mathrm{E}-07$ & $7.94004 \mathrm{E}-08$ & $4.06994 \mathrm{E}-02$ & $1.91015 \mathrm{E}-07$ & $7.91408 \mathrm{E}-08$ \\
\hline
\end{tabular}

Table 2.64. PNL-35, Pin-99 four-group Pu-239 cross sections

\begin{tabular}{cccccccc}
\hline Group & \multicolumn{3}{c}{ CENTRM cross sections $\left(\mathrm{cm}^{-1}\right)$} & \multicolumn{3}{c}{ NITAWL cross sections $\left(\mathrm{cm}^{-1}\right)$} \\
\hline & $\frac{\mathrm{G}_{\mathrm{a}}}{7.33474 \mathrm{E}-04}$ & $\frac{\mathrm{G}_{\mathrm{f}}}{2.10363 \mathrm{E}-03}$ & $\frac{\mathrm{G}_{\mathrm{f}}}{6.81678 \mathrm{E}-04}$ & $\overline{7.31953 \mathrm{E}-04}$ & $\frac{\mathrm{G}_{\mathrm{f}}}{2.10893 \mathrm{E}-03}$ & $\frac{\mathrm{G}_{\mathrm{f}}}{6.82076 \mathrm{E}-04}$ \\
1 & $1.48751 \mathrm{E}-02$ & $2.48097 \mathrm{E}-02$ & $8.61505 \mathrm{E}-03$ & $1.48705 \mathrm{E}-02$ & $2.44772 \mathrm{E}-02$ & $8.49959 \mathrm{E}-03$ \\
2 & $4.59681 \mathrm{E}-02$ & $9.06342 \mathrm{E}-02$ & $3.14723 \mathrm{E}-02$ & $4.52842 \mathrm{E}-02$ & $8.93873 \mathrm{E}-02$ & $3.10394 \mathrm{E}-02$ \\
3 & $3.95694 \mathrm{E}-01$ & $7.84138 \mathrm{E}-01$ & $2.71343 \mathrm{E}-01$ & $3.95679 \mathrm{E}-01$ & $7.83429 \mathrm{E}-01$ & $2.71097 \mathrm{E}-01$ \\
\hline
\end{tabular}





\section{MIX-COMP-THERM-3 (SAXTON-1 TO SAXTON-6)}

\subsection{DESCRIPTION}

This section describes a set of six critical experiments, each consisting of a square-pitched array of mixed plutonium-uranium fuel rods submerged in water surrounded by a water reflector. The fuel rods are identical in all cases. Criticality is controlled by varying the number of rods, rod pitch, boron in water, and water level. This set of experiments is contained in the International Handbook of Evaluated Criticality Safety Benchmark Experiments. ${ }^{3}$

This set of experiments was performed in 1965 at the in the Critical Reactor Experiment Facility at the Westinghouse Reactor Evaluation Center. The benchmark experiments are light-watermoderated critical assemblies consisting of a core array supported by upper, middle, and lower lattice plates. The lower and middle lattice plates are $0.635 \mathrm{~cm}$ thick. The upper lattice plate is $1.27 \mathrm{~cm}$ thick. The middle lattice plate was not included in the models to facilitate the accumulation of pinpower distributions. The reactor is brought to critical by raising the water level in the tank, thus avoiding the use of control rods. The fuel rods sit on a support plate above the bottom of the tank. The tank is wide enough to assume an infinite moderator on the sides and bottom $(\sim 30 \mathrm{~cm}$ of water).

All fuel rods have the same physical dimensions. A schematic diagram of the fuel rods and bottom reflector is given in Fig. 3.1. Each fuel rod has an active fuel length of $92.964 \mathrm{~cm}$, a 1.905$\mathrm{cm}$-long cladding plug on the bottom and a 4.320-cm-long cladding plug on the top. The fuel has a diameter of $0.856996 \mathrm{~cm}$. The cladding outside diameter is $0.99314 \mathrm{~cm}$ with a $0.059055-\mathrm{cm}$-thick wall. This leaves a $0.009017-\mathrm{cm}$ gap between the fuel and cladding.

The bottom of the fuel rod and lower lattice plate rest on a $2.54-\mathrm{cm}$ aluminum support plate. Between the bottom of the aluminum support plate and the top of a 5.08-cm aluminum slab is 6.35 $\mathrm{cm}$ of water. The space between the top of the lower lattice plate and bottom of the middle lattice plate is $46.99 \mathrm{~cm}$. The space between the top of the middle lattice plate and bottom of the top lattice plate is $47.625 \mathrm{~cm}$. To simplify the problem the middle lattice plate was removed and replaced with water. The water level varies with each problem but is always between the middle and upper lattice plates. The tank is large enough to assume an infinite water reflector on the sides and bottom. An infinite water reflector can be effectively modeled using $30 \mathrm{~cm}$ of water, which is used to model the reflector for this set of benchmarks.

The primary differences between the six benchmarks are lattice pitch, number of rods in the lattice, water level, and for problem 3, boron density in the water. The physical characteristics of each benchmark case, including moderator temperature, are given in Table 3.1. The atom densities for all the materials in the problem except B-10, B-11 H, and $\mathrm{O}$ in the moderator are contained in Table 3.2. The atom densities in Table 3.2 are constant for all benchmarks. Table 3.3 contain the atom densities of B-10, B-11, $\mathrm{H}$, and $\mathrm{O}$ in the moderator for each benchmark.

The lattice is filled from the $1 / 8$ th or $1 / 4$ th section by inserting additional pins in a mirror image. Figures 3.2 through 3.6 show the lattice map for each problem. Figure 3.2 shows a 1/4th lattice mapping while the other figures contain only $1 / 8$ th lattices. This difference exists because SAXTON1 contains a different number of pins in the $\mathrm{X}$ and $\mathrm{Y}$ directions. 
Table 3.1. Lattice description for SAXTON benchmark cases

\begin{tabular}{cccccc}
\hline $\begin{array}{c}\text { Benchmark } \\
\text { No. }\end{array}$ & $\begin{array}{c}\text { Boron con. } \\
(\mathrm{ppm})\end{array}$ & Lattice & $\begin{array}{c}\text { Lattice } \\
\text { pitch } \\
(\mathrm{cm})\end{array}$ & $\begin{array}{c}\text { Water level } \\
\text { from bottom } \\
\text { of fuel } \\
(\mathrm{cm})\end{array}$ & $\begin{array}{c}\text { Water } \\
\text { temp. } \\
\left({ }^{\circ} \mathrm{C}\right)\end{array}$ \\
\hline 1 & 0.0 & $22 \times 23$ & 1.3208 & 82.90 & 25.8 \\
2 & 0.0 & $19 \times 19$ & 1.4224 & 80.80 & 17.0 \\
3 & 337 & $21 \times 21$ & 1.4224 & 88.06 & 18.0 \\
4 & 0.0 & $13 \times 13$ & 1.86789 & 68.41 & 24.1 \\
5 & 0.0 & $12 \times 12$ & 2.01158 & 76.76 & 16.1 \\
6 & 0.0 & $11 \times 11$ & 2.6416 & 79.50 & 19.9 \\
\hline
\end{tabular}

Table 3.2. Constant benchmark atom densities

\begin{tabular}{|c|c|c|c|c|c|}
\hline Material & Isotope & $\begin{array}{c}\text { Atom density } \\
\text { (atoms/barn-cm) }\end{array}$ & Material & Isotope & $\begin{array}{c}\text { Atom density } \\
\text { (atoms/barn-cm) }\end{array}$ \\
\hline $\begin{array}{c}\text { Fuel } \\
\mathrm{UO}_{2}-\mathrm{PuO}_{2}\end{array}$ & $\begin{array}{c}\mathrm{U}-234 \\
\mathrm{U}-235 \\
\mathrm{U}-238 \\
\mathrm{Pu}-239 \\
\mathrm{Pu}-240 \\
\mathrm{Pu}-241 \\
\mathrm{Pu}-242 \\
\mathrm{Am}-241 \\
\mathrm{O}-16\end{array}$ & $\begin{array}{l}4.6590 \times 10^{-6} \\
1.5301 \times 10^{-4} \\
2.1097 \times 10^{-2} \\
1.3526 \times 10^{-3} \\
1.2759 \times 10^{-4} \\
1.1407 \times 10^{-5} \\
6.0318 \times 10^{-7} \\
1.7783 \times 10^{-6} \\
4.3779 \times 10^{-2}\end{array}$ & $\begin{array}{c}\text { Al lattice plate, } \\
\text { Al support } \\
\text { plate, Al slab } \\
(2.69 \mathrm{gm} / \mathrm{cc})\end{array}$ & $\begin{array}{l}\mathrm{Sn} \\
\mathrm{Fe} \\
\mathrm{Cr} \\
\mathrm{O} \\
\mathrm{Zr} \\
\mathrm{Al}\end{array}$ & $\begin{array}{l}4.6590 \times 10^{-4} \\
1.4148 \times 10^{-4} \\
7.5977 \times 10^{-5} \\
2.9630 \times 10^{-4} \\
4.2517 \times 10^{-2} \\
6.0039 \times 10^{-2}\end{array}$ \\
\hline
\end{tabular}




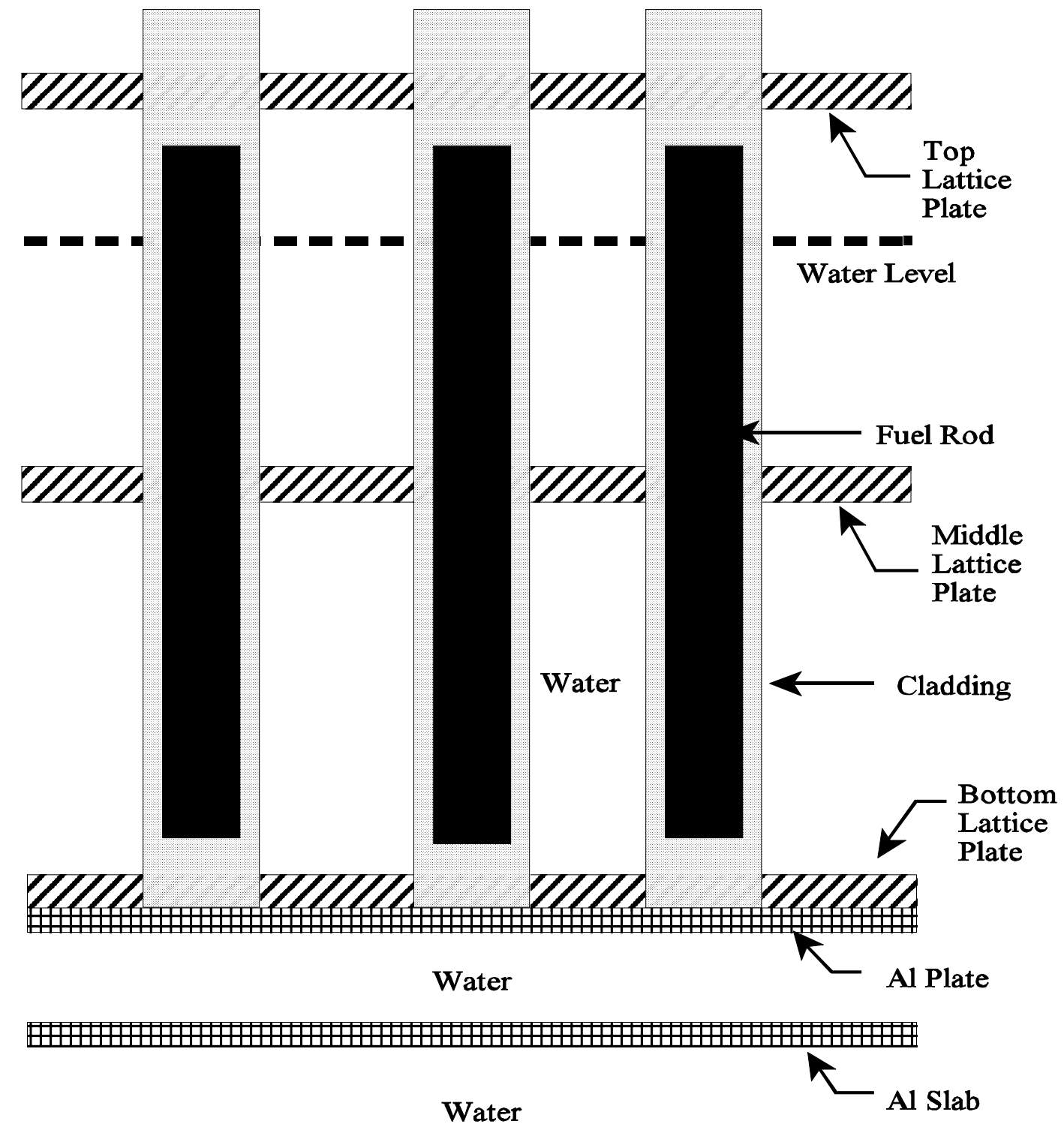

Fig. 3.1. Schematic of the SAXTON fuel rods with reflector. 
Table 3.3. Moderator atom densities

\begin{tabular}{ccccc}
\hline \multirow{2}{*}{$\begin{array}{c}\text { Benchmark } \\
\text { No. }\end{array}$} & $\mathrm{4}$ & $\mathrm{O}$ & $\mathrm{B}-10$ & $\mathrm{~B}-11$ \\
\cline { 2 - 5 } & $\mathrm{H}$ & 0.0 & 0.0 \\
\hline SAXTON-1 & $6.6643 \times 10^{-2}$ & $3.3322 \times 10^{-2}$ & 0.0 & 0.0 \\
SAXTON-2 & $6.6781 \times 10^{-2}$ & $3.3390 \times 10^{-2}$ & 0.070 \\
SAXTON-3 & $6.6751 \times 10^{-2}$ & $3.3404 \times 10^{-2}$ & $3.7338 \times 10^{-6}$ & $1.5029 \times 10^{-5}$ \\
SAXTON-4 & $6.6673 \times 10^{-2}$ & $3.3336 \times 10^{-2}$ & 0.0 & 0.0 \\
SAXTON-5 & $6.6783 \times 10^{-2}$ & $3.3392 \times 10^{-2}$ & 0.0 & 0.0 \\
SAXTON-6 & $6.6737 \times 10^{-2}$ & $3.3368 \times 10^{-2}$ & 0.0 & 0.0 \\
\hline
\end{tabular}

\subsection{ANALYSIS}

All six computational benchmarks in this section were processed twice using SCALE 5.0. The set labeled NITAWL uses the NITAWL resonance processor to self-shield the resolved resonance region for all nuclides in the unit cell. An identical set, labeled CENTRM, replaces NITAWL with the CENTRM/PMC code sequence. The NITAWL and CENTRM results are then compared.

Table 3.4 contains the $k_{e f f}$ and Energy of the Average Lethargy Causing Fission (EALCF). The $k_{\text {eff }}$ values for all the benchmark cases are very close to 1.0, the worst NITAWL benchmark being $0.68 \%$ high and the worst CENTRM case being $0.59 \%$ high. There appears to be a small negative bias between NITAWL and CENTRM, the CENTRM cases being about $0.3 \%$ lower on average than the NITAWL cases. The EALCF values listed are from the CENTRM cases. For all cases, the difference between this value for CENTRM and NITAWL was less than $0.1 \%$. Both CENTRM and NITAWL produce excellent results for the $k_{\text {eff }}$ and EALCF for this set of problems.

Also, calculated for each problem are the pin-power distributions; absorption, $\varangle_{\mathrm{f}}$, and fission reaction rates and fluxes in the pin fuel, clad, and moderator; and four group cross sections and fluxes for a corner outside pin and the center pin. Each set of data is calculated using both CENTRM and NITAWL. All the data for each case are contained in the following Figs. 3.7a through 3.12b and Tables 3.5 through 3.64 .

The pir-power distributions for the first problem assume 1/4th core symmetry, the pin-power distributions for the remaining problems assume 1/8th core symmetry, with a surrounding reflector in all problems. The pin-power data consist of a value and a standard deviation for each pin. The values for the pin power are actually in units of fissions per $\mathrm{cm}^{3}$-source particle $\times 10^{-5}$. The value in parentheses is the percent standard deviation of the pin-power value. The pin-power distributions in the 1/8th or full-core representations are the portion of the pin covered by water. The value off to the side in the pin-power distributions represents all the fuel not covered by water. Separating the fuel rod was necessary to model this in KENO-V.a.

In most cases the CENTRM and NITAWL results for each benchmark case agree within 2 standard deviations. The peak-to-low power changes with respect to pitch. The peak-to-low power ratio initially decreased with increasing pitch, starting with a high of 2.6 for SAXTON-1 and decreasing to a low of 1.8 for SAXTON-5. A further increase in pitch, as shown in SAXTON-6, 
increases the ratio to 2.5. The small pitch in SAXTON-1 and -2 cause the array to be under moderated. This under moderation results in the highest power pin being on the edge of the array and the lowest power pin being several pins in from the edge where the thermal flux is low due to lack of moderator and the fast flux is also low because it is not deep within the array.

The total fluxes, and flux ratios for the CENTRM and NITAWL cases of each benchmark case, are also in good agreement, seldom varying by more than $1 \%$. These values are included for a corner pin and the center pin for each case. The reaction rates in the fuel and cladding are also in good agreement. However, the reaction rates for the moderator appear to be significantly different, with the CENTRM value being almost a factor of 2 higher for all cases.

Finally, four-group fluxes and cross sections were calculated for selected nuclides in the fuel region of the same corner and center pins. The macroscopic cross sections listed include the radiative capture, fission, and nu*fission cross sections for U-235, U-238, and Pu-239. The four groups are collapsed from the 238-group multigroup cross-section set using the flux profile calculated in KENOV.a as follows: group 1 is from $20 \mathrm{Mev}$ to $9.5 \mathrm{keV}$, group 2 is from $9.5 \mathrm{keV}$ to $3.0 \mathrm{eV}$, group 3 is from $3 \mathrm{eV}$ to $0.4 \mathrm{eV}$, and group 4 is from $0.4 \mathrm{eV}$ to $10^{-5} \mathrm{eV}$. The $0.4 \mathrm{eV}$ was chosen as a boundary because it is the cadmium cutoff energy. Groups 2 and 3 contain the resolved resonance regions for most of the nuclides used in these cases. Most of the cross-section data for the CENTRM and NITAWL cases of a given benchmark case agree within $1 \%$.

\subsection{CONCLUSIONS}

For this set of benchmark cases using either NITAWL and CENTRM as the resonance region processor produces acceptable results of the $k_{\text {eff }}$ values. All other values produced using CENTRM and NITAWL are also consistent with each. For all cases, the $k_{\text {eff }} s$ produced using CENTRM are slightly lower $(\sim 0.3 \%)$ than those produced using NITAWL to do the resonance self-shielding. As a result the average $k_{\text {eff }}$ of all the CENTRM cases is closer to 1.0 than the average $k_{\text {eff }}$ of all the NITAWL cases for this set of benchmarks. The pin-power distributions, fluxes, most reactions rates, and macroscopic cross sections generally agree to approximately $1 \%$ between CENTRM and NITAWL. The only significant discrepancy is the absorption reaction rate for the moderator. CENTRM calculates a value that is about a factor of 2 higher than the same value using NITAWL. 
Table 3.4 Comparison of $k_{\text {eff }}$ and EALCF from CENTRM and NITAWL ${ }^{a}$

\begin{tabular}{ccccc}
\hline $\begin{array}{c}\text { CASE } \\
\text { MIX-COMP-THERM-3 }\end{array}$ & $\begin{array}{c}\text { NITAWL } \\
k_{\text {eff }}( \pm \mathrm{F})\end{array}$ & $\begin{array}{c}\text { CENTRM } \\
k_{\text {eff }}( \pm \mathrm{F})\end{array}$ & \% DIFF & $\begin{array}{c}\text { EALCF } \\
(\mathrm{eV})\end{array}$ \\
\hline SAXTON-1 & $1.0046(0.0005)$ & $1.0007(0.0004)$ & -0.39 & 0.881 \\
SAXTON-2 & $1.0058(0.0004)$ & $1.0012(0.0004)$ & -0.46 & 0.537 \\
SAXTON-3 & $1.0054(0.0004)$ & $1.0017(0.0004)$ & -0.37 & 0.633 \\
SAXTON-4 & $1.0056(0.0004)$ & $1.0029(0.0004)$ & -0.27 & 0.186 \\
SAXTON-5 & $1.0062(0.0004)$ & $1.0035(0.0004)$ & -0.27 & 0.154 \\
SAXTON-6 & $1.0068(0.0005)$ & $1.0059(0.0004)$ & -0.09 & 0.100 \\
\hline
\end{tabular}

${ }^{a}$ Value in parentheses is percent standard deviation. 


\begin{tabular}{|l|c|c|c|c|c|c|c|c|c|c|c|}
\hline 121 & 122 & 123 & 124 & 125 & 126 & 127 & 128 & 129 & 130 & 131 & 132 \\
\hline 109 & 110 & 111 & 112 & 113 & 114 & 115 & 116 & 117 & 118 & 119 & 120 \\
\hline 97 & 98 & 99 & 100 & 101 & 102 & 103 & 104 & 105 & 106 & 107 & 108 \\
\hline 85 & 86 & 87 & 88 & 89 & 90 & 91 & 92 & 93 & 94 & 95 & 96 \\
\hline 73 & 74 & 75 & 76 & 77 & 78 & 79 & 80 & 81 & 82 & 83 & 84 \\
\hline 61 & 62 & 63 & 64 & 65 & 66 & 67 & 68 & 69 & 70 & 71 & 72 \\
\hline 49 & 50 & 51 & 52 & 53 & 54 & 55 & 56 & 57 & 58 & 59 & 60 \\
\hline 37 & 38 & 39 & 40 & 41 & 42 & 43 & 44 & 45 & 46 & 47 & 48 \\
\hline 25 & 26 & 27 & 28 & 29 & 30 & 31 & 32 & 33 & 34 & 35 & 36 \\
\hline 13 & 14 & 15 & 16 & 17 & 18 & 19 & 20 & 21 & 22 & 23 & 24 \\
\hline 1 & 2 & 3 & 4 & 5 & 6 & 7 & 8 & 9 & 10 & 11 & 12 \\
\hline
\end{tabular}

Fig.3.2. Pin layout of benchmark case SAXTON-1. 


\begin{tabular}{|c|c|c|c|c|c|c|c|c|c|}
\hline & 55 \\
\hline & & & & & & & \multirow[b]{2}{*}{50} & 53 & 54 \\
\hline & & & & & & & & 51 & 52 \\
\hline & & & & & & 46 & 47 & 48 & 49 \\
\hline & & & & & 41 & 42 & 43 & 44 & 45 \\
\hline & & & & 35 & 36 & 37 & 38 & 39 & 40 \\
\hline & & & 28 & 29 & 30 & 31 & 32 & 33 & 34 \\
\hline & & 20 & 21 & 22 & 23 & 24 & 25 & 26 & 27 \\
\hline & 11 & 12 & 13 & 14 & 15 & 16 & 17 & 18 & 19 \\
\hline 1 & 2 & 3 & 4 & 5 & 6 & 7 & 8 & 9 & 10 \\
\hline
\end{tabular}

Fig. 3.3. Pin layout of benchmark case SAXTON-2.

\begin{tabular}{|c|c|c|c|c|c|c|c|c|c|c|}
\hline & & & & & & & & & & 66 \\
\hline & & & & & & & & & 64 & 65 \\
\hline & & & & & & & & 61 & 62 & 63 \\
\hline & & & & & & & 57 & 58 & 59 & 60 \\
\hline & & & & & & 52 & 53 & 54 & 55 & 56 \\
\hline & & & & & 46 & 47 & 48 & 49 & 50 & 51 \\
\hline & & & & 39 & 40 & 41 & 42 & 43 & 44 & 45 \\
\hline & & & 31 & 32 & 33 & 34 & 35 & 36 & 37 & 38 \\
\hline & & 22 & 23 & 24 & 25 & 26 & 27 & 28 & 29 & 30 \\
\hline & 12 & 13 & 14 & 15 & 16 & 17 & 18 & 19 & 20 & 21 \\
\hline 1 & 2 & 3 & 4 & 5 & 6 & 7 & 8 & 9 & 10 & 11 \\
\hline
\end{tabular}

Fig. 3.4. Pin layout of benchmark case SAXTON-3. 


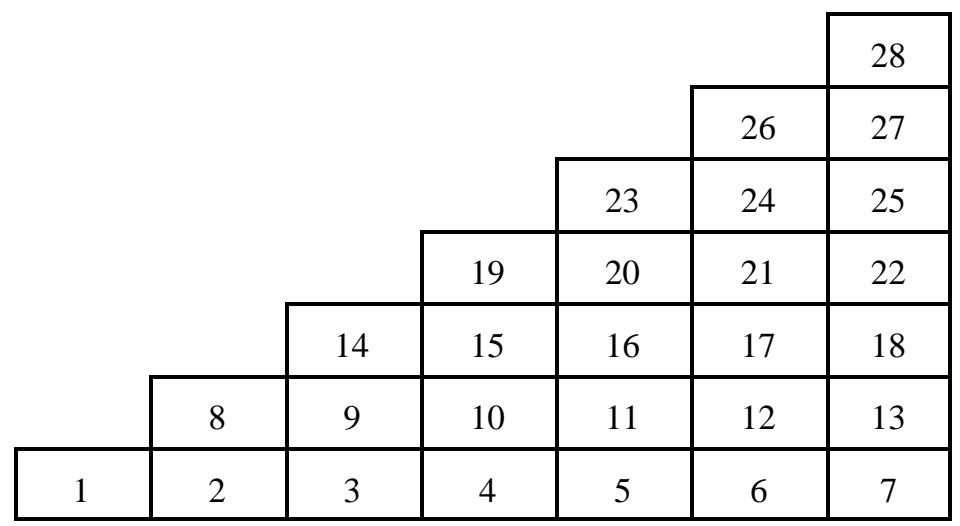

Fig. 3.5. Pin layout of benchmark case SAXTON-4.

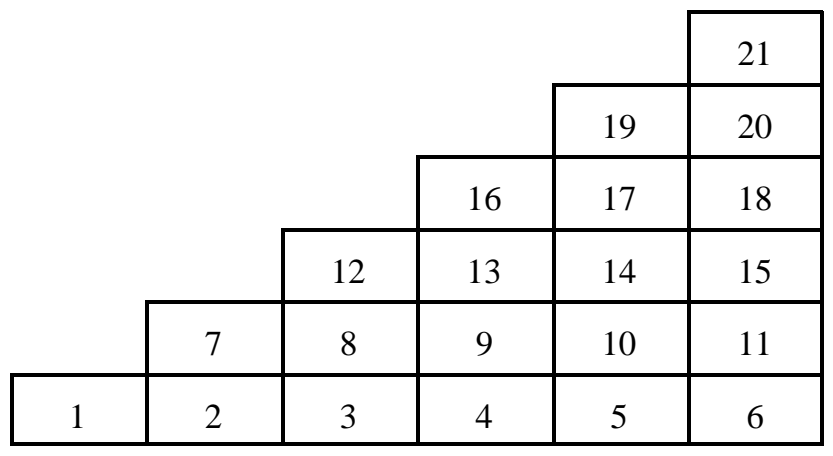

Fig. 3.6. Pin layout of benchmark cases SAXTON-5 and 6. 


\begin{tabular}{|c|c|c|c|c|c|c|c|c|c|c|c|}
\hline & & & & & & $\begin{array}{c}0.150 \\
(0.73)\end{array}$ & & & & & \\
\hline $\begin{array}{l}6.485 \\
(0.53)\end{array}$ & $\begin{array}{l}3.658 \\
(0.59)\end{array}$ & $\begin{array}{l}3.513 \\
(0.64)\end{array}$ & $\begin{array}{l}3.704 \\
(0.60)\end{array}$ & $\begin{array}{l}3.968 \\
(0.55)\end{array}$ & $\begin{array}{l}4.199 \\
(0.57)\end{array}$ & $\begin{array}{l}4.486 \\
(0.55)\end{array}$ & $\begin{array}{l}4.633 \\
(0.49)\end{array}$ & $\begin{array}{l}4.770 \\
(0.54)\end{array}$ & $\begin{array}{l}4.894 \\
(0.55)\end{array}$ & $\begin{array}{l}4.914 \\
(0.50)\end{array}$ & $\begin{array}{l}4.971 \\
(0.73)\end{array}$ \\
\hline $\begin{array}{l}6.446 \\
(0.50)\end{array}$ & $\begin{array}{l}3.716 \\
(0.58)\end{array}$ & $\begin{array}{l}3.461 \\
(0.61)\end{array}$ & $\begin{array}{l}3.693 \\
(0.61)\end{array}$ & $\begin{array}{l}3.957 \\
(0.59)\end{array}$ & $\begin{array}{l}4.222 \\
(0.55)\end{array}$ & $\begin{array}{l}4.421 \\
(0.57)\end{array}$ & $\begin{array}{l}4.570 \\
(0.57)\end{array}$ & $\begin{array}{l}4.687 \\
(0.51)\end{array}$ & $\begin{array}{l}4.871 \\
(0.51)\end{array}$ & $\begin{array}{l}4.858 \\
(0.53)\end{array}$ & $\begin{array}{l}4.962 \\
(0.66)\end{array}$ \\
\hline $\begin{array}{l}6.319 \\
(0.48)\end{array}$ & $\begin{array}{l}3.630 \\
(0.63)\end{array}$ & $\begin{array}{l}3.434 \\
(0.62)\end{array}$ & $\begin{array}{l}3.601 \\
(0.61)\end{array}$ & $\begin{array}{l}3.837 \\
(0.54)\end{array}$ & $\begin{array}{l}4.133 \\
(0.57)\end{array}$ & $\begin{array}{l}4.381 \\
(0.56)\end{array}$ & $\begin{array}{l}4.493 \\
(0.49)\end{array}$ & $\begin{array}{l}4.664 \\
(0.56)\end{array}$ & $\begin{array}{l}4.782 \\
(0.56)\end{array}$ & $\begin{array}{l}4.846 \\
(0.52)\end{array}$ & $\begin{array}{l}4.847 \\
(0.71)\end{array}$ \\
\hline $\begin{array}{l}6.148 \\
(0.50)\end{array}$ & $\begin{array}{l}3.531 \\
(0.62)\end{array}$ & $\begin{array}{l}3.368 \\
(0.59)\end{array}$ & $\begin{array}{l}3.524 \\
(0.66)\end{array}$ & $\begin{array}{l}3.729 \\
(0.56)\end{array}$ & $\begin{array}{l}3.986 \\
(0.58)\end{array}$ & $\begin{array}{l}4.175 \\
(0.52)\end{array}$ & $\begin{array}{l}4.397 \\
(0.55)\end{array}$ & $\begin{array}{l}4.503 \\
(0.50)\end{array}$ & $\begin{array}{l}4.649 \\
(0.51)\end{array}$ & $\begin{array}{l}4.680 \\
(0.52)\end{array}$ & $\begin{array}{l}4.705 \\
(0.75)\end{array}$ \\
\hline $\begin{array}{l}5.884 \\
(0.49)\end{array}$ & $\begin{array}{l}3.410 \\
(0.63)\end{array}$ & $\begin{array}{c}3.176 \\
(0.67)\end{array}$ & $\begin{array}{l}3.433 \\
(0.59)\end{array}$ & $\begin{array}{l}3.686 \\
(0.59)\end{array}$ & $\begin{array}{l}3.876 \\
(0.58)\end{array}$ & $\begin{array}{l}4.087 \\
(0.61)\end{array}$ & $\begin{array}{l}4.247 \\
(0.55)\end{array}$ & $\begin{array}{l}4.338 \\
(0.54)\end{array}$ & $\begin{array}{l}4.491 \\
(0.54)\end{array}$ & $\begin{array}{l}4.514 \\
(0.53)\end{array}$ & $\begin{array}{l}4.556 \\
(0.75)\end{array}$ \\
\hline $\begin{array}{l}5.635 \\
(0.50)\end{array}$ & $\begin{array}{l}3.234 \\
(0.67)\end{array}$ & $\begin{array}{l}3.058 \\
(0.64)\end{array}$ & $\begin{array}{l}3.231 \\
(0.66)\end{array}$ & $\begin{array}{l}3.496 \\
(0.60)\end{array}$ & $\begin{array}{l}3.668 \\
(0.54)\end{array}$ & $\begin{array}{l}3.879 \\
(0.60)\end{array}$ & $\begin{array}{l}4.029 \\
(0.57)\end{array}$ & $\begin{array}{l}4.171 \\
(0.52)\end{array}$ & $\begin{array}{l}4.254 \\
(0.57)\end{array}$ & $\begin{array}{l}4.283 \\
(0.51)\end{array}$ & $\begin{array}{l}4.344 \\
(0.75)\end{array}$ \\
\hline $\begin{array}{l}5.340 \\
(0.53)\end{array}$ & $\begin{array}{l}3.066 \\
(0.66)\end{array}$ & $\begin{array}{l}2.898 \\
(0.63)\end{array}$ & $\begin{array}{l}3.037 \\
(0.71)\end{array}$ & $\begin{array}{l}3.280 \\
(0.62)\end{array}$ & $\begin{array}{l}3.477 \\
(0.56)\end{array}$ & $\begin{array}{l}3.662 \\
(0.61)\end{array}$ & $\begin{array}{l}3.794 \\
(0.59)\end{array}$ & $\begin{array}{l}3.908 \\
(0.61)\end{array}$ & $\begin{array}{l}4.018 \\
(0.57)\end{array}$ & $\begin{array}{l}4.067 \\
(0.56)\end{array}$ & $\begin{array}{l}4.085 \\
(0.75)\end{array}$ \\
\hline $\begin{array}{l}4.988 \\
(0.55)\end{array}$ & $\begin{array}{l}2.822 \\
(0.70)\end{array}$ & $\begin{array}{l}2.693 \\
(0.72)\end{array}$ & $\begin{array}{l}2.864 \\
(0.66)\end{array}$ & $\begin{array}{l}3.054 \\
(0.70)\end{array}$ & $\begin{array}{l}3.264 \\
(0.61)\end{array}$ & $\begin{array}{l}3.412 \\
(0.61)\end{array}$ & $\begin{array}{l}3.552 \\
(0.61)\end{array}$ & $\begin{array}{l}3.652 \\
(0.59)\end{array}$ & $\begin{array}{l}3.747 \\
(0.59)\end{array}$ & $\begin{array}{l}3.772 \\
(0.58)\end{array}$ & $\begin{array}{l}3.830 \\
(0.84)\end{array}$ \\
\hline $\begin{array}{l}4.609 \\
(0.58)\end{array}$ & $\begin{array}{l}4.609 \\
(0.67)\end{array}$ & $\begin{array}{l}2.520 \\
(0.72)\end{array}$ & $\begin{array}{l}2.716 \\
(0.71)\end{array}$ & $\begin{array}{l}2.878 \\
(0.67)\end{array}$ & $\begin{array}{l}3.057 \\
(0.67)\end{array}$ & $\begin{array}{l}3.187 \\
(0.62)\end{array}$ & $\begin{array}{l}3.360 \\
(0.58)\end{array}$ & $\begin{array}{l}3.473 \\
(0.62)\end{array}$ & $\begin{array}{l}3.531 \\
(0.59)\end{array}$ & $\begin{array}{l}3.574 \\
(0.70)\end{array}$ & $\begin{array}{l}3.571 \\
(0.88)\end{array}$ \\
\hline $\begin{array}{l}4.611 \\
(0.62)\end{array}$ & $\begin{array}{l}2.773 \\
(0.75)\end{array}$ & $\begin{array}{l}2.701 \\
(0.67)\end{array}$ & $\begin{array}{l}2.827 \\
(0.68)\end{array}$ & $\begin{array}{l}3.079 \\
(0.65)\end{array}$ & $\begin{array}{l}3.255 \\
(0.69)\end{array}$ & $\begin{array}{l}3.427 \\
(0.66)\end{array}$ & $\begin{array}{l}3.557 \\
(0.63)\end{array}$ & $\begin{array}{l}3.717 \\
(0.60)\end{array}$ & $\begin{array}{l}3.757 \\
(0.54)\end{array}$ & $\begin{array}{c}3.81 \\
(0.62)\end{array}$ & $\begin{array}{l}3.815 \\
(0.88)\end{array}$ \\
\hline $\begin{array}{l}6.090 \\
(0.50)\end{array}$ & $\begin{array}{l}4.599 \\
(0.56)\end{array}$ & $\begin{array}{l}4.683 \\
(0.52)\end{array}$ & $\begin{array}{l}5.010 \\
(0.56)\end{array}$ & $\begin{array}{l}5.313 \\
(0.51)\end{array}$ & $\begin{array}{l}5.712 \\
(0.53)\end{array}$ & $\begin{array}{l}5.943 \\
(0.52)\end{array}$ & $\begin{array}{c}6.227 \\
(0.49)\end{array}$ & $\begin{array}{l}6.425 \\
(0.51)\end{array}$ & $\begin{array}{l}6.539 \\
(0.50)\end{array}$ & $\begin{array}{l}6.636 \\
(0.48)\end{array}$ & $\begin{array}{l}6.635 \\
(0.67)\end{array}$ \\
\hline
\end{tabular}

Fig. 3.7a. Pin-power distribution for CENTRM benchmark SAXTON-1. Value in parentheses is the percent standard deviation. 


\begin{tabular}{|c|c|c|c|c|c|c|c|c|c|c|c|}
\hline & & & & & & $\begin{array}{l}4.967 \\
(0.78)\end{array}$ & & & & & \\
\hline $\begin{array}{l}6.502 \\
(0.50)\end{array}$ & $\begin{array}{l}3.746 \\
(0.64)\end{array}$ & $\begin{array}{l}3.555 \\
(0.60)\end{array}$ & $\begin{array}{l}3.711 \\
(0.62)\end{array}$ & $\begin{array}{l}4.013 \\
(0.54)\end{array}$ & $\begin{array}{l}4.274 \\
(0.56)\end{array}$ & $\begin{array}{l}4.457 \\
(0.58)\end{array}$ & $\begin{array}{l}4.689 \\
(0.54)\end{array}$ & $\begin{array}{l}4.801 \\
(0.55)\end{array}$ & $\begin{array}{l}4.913 \\
(0.55)\end{array}$ & $\begin{array}{l}4.997 \\
(0.49)\end{array}$ & $\begin{array}{l}4.967 \\
(0.78)\end{array}$ \\
\hline $\begin{array}{l}6.441 \\
(0.49)\end{array}$ & $\begin{array}{c}3.706 \\
(0.59)\end{array}$ & $\begin{array}{c}3.493 \\
(0.67)\end{array}$ & $\begin{array}{c}3.699 \\
(0.65)\end{array}$ & $\begin{array}{l}3.939 \\
(0.63)\end{array}$ & $\begin{array}{l}4.244 \\
(0.56)\end{array}$ & $\begin{array}{l}4.422 \\
(0.53)\end{array}$ & $\begin{array}{l}4.641 \\
(0.55)\end{array}$ & $\begin{array}{l}4.780 \\
(0.55)\end{array}$ & $\begin{array}{l}4.840 \\
(0.51)\end{array}$ & $\begin{array}{l}4.917 \\
(0.49)\end{array}$ & $\begin{array}{l}4.912 \\
(0.74)\end{array}$ \\
\hline $\begin{array}{l}6.347 \\
(0.45)\end{array}$ & $\begin{array}{l}3.634 \\
(0.54)\end{array}$ & $\begin{array}{l}3.368 \\
(0.65)\end{array}$ & $\begin{array}{l}3.599 \\
(0.59)\end{array}$ & $\begin{array}{l}3.884 \\
(0.61)\end{array}$ & $\begin{array}{l}4.115 \\
(0.57)\end{array}$ & $\begin{array}{l}4.390 \\
(0.51)\end{array}$ & $\begin{array}{l}4.502 \\
(0.52)\end{array}$ & $\begin{array}{l}4.684 \\
(0.54)\end{array}$ & $\begin{array}{l}4.757 \\
(0.56)\end{array}$ & $\begin{array}{l}4.854 \\
(0.51)\end{array}$ & $\begin{array}{l}4.879 \\
(0.78)\end{array}$ \\
\hline $\begin{array}{l}6.197 \\
(0.52)\end{array}$ & $\begin{array}{l}3.522 \\
(0.63)\end{array}$ & $\begin{array}{l}3.356 \\
(0.64)\end{array}$ & $\begin{array}{c}3.356 \\
(0.59)\end{array}$ & $\begin{array}{l}3.836 \\
(0.60)\end{array}$ & $\begin{array}{l}4.052 \\
(0.56)\end{array}$ & $\begin{array}{l}4.279 \\
(0.57)\end{array}$ & $\begin{array}{l}4.446 \\
(0.54)\end{array}$ & $\begin{array}{l}4.547 \\
(0.54)\end{array}$ & $\begin{array}{l}4.614 \\
(0.55)\end{array}$ & $\begin{array}{l}4.711 \\
(0.49)\end{array}$ & $\begin{array}{l}4.737 \\
(0.76)\end{array}$ \\
\hline $\begin{array}{l}5.882 \\
(0.52)\end{array}$ & $\begin{array}{l}3.391 \\
(0.62)\end{array}$ & $\begin{array}{l}3.212 \\
(0.63)\end{array}$ & $\begin{array}{l}3.408 \\
(0.64)\end{array}$ & $\begin{array}{l}3.685 \\
(0.61)\end{array}$ & $\begin{array}{l}3.868 \\
(0.56)\end{array}$ & $\begin{array}{l}4.112 \\
(0.57)\end{array}$ & $\begin{array}{l}4.229 \\
(0.55)\end{array}$ & $\begin{array}{l}4.343 \\
(049 .)\end{array}$ & $\begin{array}{l}4.500 \\
(0.54)\end{array}$ & $\begin{array}{l}4.497 \\
(0.57)\end{array}$ & $\begin{array}{l}4.520 \\
(0.76)\end{array}$ \\
\hline $\begin{array}{l}5.619 \\
(0.54)\end{array}$ & $\begin{array}{l}3.243 \\
(0.70)\end{array}$ & $\begin{array}{l}3.060 \\
(0.66)\end{array}$ & $\begin{array}{l}3.260 \\
(0.61)\end{array}$ & $\begin{array}{l}3.477 \\
(0.64)\end{array}$ & $\begin{array}{c}3.708 \\
(0.59)\end{array}$ & $\begin{array}{l}3.880 \\
(0.57)\end{array}$ & $\begin{array}{l}4.050 \\
(0.60)\end{array}$ & $\begin{array}{l}4.189 \\
(0.60)\end{array}$ & $\begin{array}{l}4.265 \\
(0.55)\end{array}$ & $\begin{array}{l}4.321 \\
(0.58)\end{array}$ & $\begin{array}{l}4.305 \\
(0.71)\end{array}$ \\
\hline $\begin{array}{l}5.353 \\
(0.51)\end{array}$ & $\begin{array}{l}3.065 \\
(0.67)\end{array}$ & $\begin{array}{l}2.880 \\
(0.64)\end{array}$ & $\begin{array}{l}3.041 \\
(0.64)\end{array}$ & $\begin{array}{l}3.296 \\
(0.66)\end{array}$ & $\begin{array}{l}3.479 \\
(0.62)\end{array}$ & $\begin{array}{l}3.650 \\
(0.59)\end{array}$ & $\begin{array}{l}3.879 \\
(0.60)\end{array}$ & $\begin{array}{l}3.922 \\
(0.67)\end{array}$ & $\begin{array}{l}3.990 \\
(0.52)\end{array}$ & $\begin{array}{l}4.087 \\
(0.54)\end{array}$ & $\begin{array}{l}4.126 \\
(0.78)\end{array}$ \\
\hline $\begin{array}{l}4.982 \\
(0.54)\end{array}$ & $\begin{array}{l}2.844 \\
(0.73)\end{array}$ & $\begin{array}{l}2.673 \\
(0.69)\end{array}$ & $\begin{array}{l}2.857 \\
(0.73)\end{array}$ & $\begin{array}{l}3.063 \\
(0.66)\end{array}$ & $\begin{array}{l}3.264 \\
(0.62)\end{array}$ & $\begin{array}{l}3.422 \\
(0.68)\end{array}$ & $\begin{array}{l}3.577 \\
(0.62)\end{array}$ & $\begin{array}{l}3.698 \\
(0.59)\end{array}$ & $\begin{array}{l}3.787 \\
(0.62)\end{array}$ & $\begin{array}{l}3.847 \\
(0.59)\end{array}$ & $\begin{array}{l}3.823 \\
(0.83)\end{array}$ \\
\hline $\begin{array}{l}4.689 \\
(0.60)\end{array}$ & $\begin{array}{l}2.684 \\
(0.76)\end{array}$ & $\begin{array}{l}2.526 \\
(0.71)\end{array}$ & $\begin{array}{l}2.697 \\
(0.68)\end{array}$ & $\begin{array}{l}2.904 \\
(0.69)\end{array}$ & $\begin{array}{l}3.064 \\
(0.68)\end{array}$ & $\begin{array}{l}3.243 \\
(0.65)\end{array}$ & $\begin{array}{l}3.361 \\
(0.64)\end{array}$ & $\begin{array}{l}3.478 \\
(0.62)\end{array}$ & $\begin{array}{l}3.538 \\
(0.61)\end{array}$ & $\begin{array}{l}3.578 \\
(0.63)\end{array}$ & $\begin{array}{l}3.626 \\
(0.89)\end{array}$ \\
\hline $\begin{array}{l}4.621 \\
(0.58)\end{array}$ & $\begin{array}{l}2.801 \\
(0.72)\end{array}$ & $\begin{array}{l}2.677 \\
(0.75)\end{array}$ & $\begin{array}{l}2.839 \\
(0.78)\end{array}$ & $\begin{array}{l}3.079 \\
(0.61)\end{array}$ & $\begin{array}{l}3.258 \\
(0.64)\end{array}$ & $\begin{array}{l}3.417 \\
(0.65)\end{array}$ & $\begin{array}{l}3.558 \\
(0.61)\end{array}$ & $\begin{array}{l}3.735 \\
(0.53)\end{array}$ & $\begin{array}{l}3.801 \\
(0.60)\end{array}$ & $\begin{array}{l}3.882 \\
(0.63)\end{array}$ & $\begin{array}{l}3.872 \\
(0.86)\end{array}$ \\
\hline $\begin{array}{l}6.047 \\
(0.48)\end{array}$ & $\begin{array}{l}4.602 \\
(0.56)\end{array}$ & $\begin{array}{l}4.618 \\
(0.58)\end{array}$ & $\begin{array}{l}4.932 \\
(0.58)\end{array}$ & $\begin{array}{l}5.360 \\
(0.51)\end{array}$ & $\begin{array}{l}5.681 \\
(0.53)\end{array}$ & $\begin{array}{l}6.037 \\
(0.46)\end{array}$ & $\begin{array}{l}6.282 \\
(0.44)\end{array}$ & $\begin{array}{l}6.440 \\
(0.48)\end{array}$ & $\begin{array}{l}6.562 \\
(0.47)\end{array}$ & $\begin{array}{c}6.694 \\
(0.48)\end{array}$ & $\begin{array}{c}6.750 \\
(0.68)\end{array}$ \\
\hline
\end{tabular}

Fig. 3.7b. Pin-power distribution for NITAWL benchmark SAXTON-1. Value in parentheses is the percent standard deviation. 
Table 3.5. SAXTON-1 CENTRM reaction rates and fluxes

\begin{tabular}{|c|c|c|c|c|c|c|}
\hline Pin & Region & $\begin{array}{c}\mathrm{G}_{\mathrm{a}} \mathrm{M} \\
\left(\mathrm{cm}^{-3}-\mathrm{s}^{-1}\right)\end{array}$ & $\begin{array}{c}\mathcal{G}_{\mathrm{f}} \mathrm{M} \\
\left(\mathrm{cm}^{-3}-\mathrm{s}^{-1}\right)\end{array}$ & $\begin{array}{c}\mathrm{G}_{\mathrm{f}} \mathrm{M} \\
\left(\mathrm{cm}^{-3}-\mathrm{s}^{-1}\right)\end{array}$ & $\begin{array}{c}\mathrm{M} \\
\left(\mathrm{cm}-\mathrm{s}^{-1}\right)\end{array}$ & $\mathrm{MVM}_{\mathrm{t}}$ \\
\hline \multirow{3}{*}{1} & Fuel & $3.400 \mathrm{E}-05$ & $6.075 \mathrm{E}-05$ & $2.123 \mathrm{E}-05$ & 7.209E-03 & 0.344 \\
\hline & Clad & 3.137E-07 & 0.0 & 0.0 & $2.114 \mathrm{E}-03$ & 0.101 \\
\hline & Mod. & $1.303 \mathrm{E}-06$ & 0.0 & 0.0 & $1.162 \mathrm{E}-02$ & 0.555 \\
\hline \multirow{3}{*}{132} & Fuel & $3.446 \mathrm{E}-05$ & 5.001E-05 & $1.747 \mathrm{E}-05$ & $1.905 \mathrm{E}-02$ & 0.339 \\
\hline & Clad & 5.304E-07 & 0.0 & 0.0 & 5.690E-03 & 0.101 \\
\hline & Mod. & $9.171 \mathrm{E}-07$ & 0.0 & 0.0 & 3.141E-02 & 0.559 \\
\hline
\end{tabular}

Table 3.6. SAXTON-1 NITAWL reaction rates and fluxes

\begin{tabular}{ccccccc}
\hline \multirow{2}{*}{ Pin } & Region & $\begin{array}{c}\mathrm{G}_{\mathrm{a}} \mathrm{M} \\
\left(\mathrm{cm}^{-3}-\mathrm{s}^{-1}\right)\end{array}$ & $\begin{array}{c}\mathrm{G}_{\mathrm{f}} \mathrm{M} \\
\left(\mathrm{cm}^{-3}-\mathrm{s}^{-1}\right)\end{array}$ & $\begin{array}{c}\mathrm{G}_{\mathrm{f}} \mathrm{M} \\
\left(\mathrm{cm}^{-3}-\mathrm{s}^{-1}\right)\end{array}$ & $\begin{array}{c}\mathrm{M} \\
\left(\mathrm{cm}-\mathrm{s}^{-1}\right)\end{array}$ & $\mathrm{M}_{\mathrm{t}}$ \\
\hline \multirow{2}{*}{1} & Fuel & $3.407 \mathrm{E}-05$ & $6.097 \mathrm{E}-05$ & $2.131 \mathrm{E}-05$ & $7.160 \mathrm{E}-03$ & 0.344 \\
& Clad & $3.038 \mathrm{E}-07$ & 0.0 & 0.0 & $2.102 \mathrm{E}-03$ & 0.101 \\
& Mod. & $6.505 \mathrm{E}-07$ & 0.0 & 0.0 & $1.157 \mathrm{E}-02$ & 0.556 \\
\hline \multirow{2}{*}{132} & Fuel & $3.445 \mathrm{E}-05$ & $4.998 \mathrm{E}-05$ & $1.746 \mathrm{E}-05$ & $1.895 \mathrm{E}-02$ & 0.338 \\
& Clad & $5.131 \mathrm{E}-07$ & 0.0 & 0.0 & $5.682 \mathrm{E}-03$ & 0.101 \\
& Mod. & $4.648 \mathrm{E}-07$ & 0.0 & 0.0 & $3.143 \mathrm{E}-02$ & 0.561 \\
\hline
\end{tabular}


Table 3.7. SAXTON-1, Pin-1 four-group fluxes

\begin{tabular}{ccc}
\hline Group & $\begin{array}{c}\text { CENTRM M } \\
\left(\mathrm{cm}^{-2}-\mathrm{s}^{-1}\right)\end{array}$ & $\begin{array}{c}\text { NITAWL M } \\
\left(\mathrm{cm}^{-2}-\mathrm{s}^{-1}\right)\end{array}$ \\
\hline 1 & $9.920 \mathrm{E}-05$ & $9.850 \mathrm{E}-05$ \\
2 & $2.598 \mathrm{E}-05$ & $2.555 \mathrm{E}-05$ \\
3 & $5.448 \mathrm{E}-06$ & $5.416 \mathrm{E}-06$ \\
4 & $2.012 \mathrm{E}-05$ & $2.026 \mathrm{E}-05$ \\
\hline
\end{tabular}

Table 3.8. SAXTON-1, Pin-1 four-group U-235 cross sections

\begin{tabular}{ccccccc}
\hline Group & \multicolumn{3}{c}{ CENTRM cross sections $\left(\mathrm{cm}^{-1}\right)$} & \multicolumn{2}{c}{ NITAWL cross sections $\left(\mathrm{cm}^{-1}\right)$} \\
\hline & $\frac{\mathrm{G}_{\mathrm{a}}}{2.32297 \mathrm{E}-04}$ & $\frac{\mathrm{G}_{\mathrm{f}}}{5.28702 \mathrm{E}-04}$ & $\frac{\mathrm{G}_{\mathrm{f}}}{2.03588 \mathrm{E}-04}$ & $\frac{\mathrm{G}_{\mathrm{a}}}{2.31560 \mathrm{E}-04}$ & $\frac{\mathrm{G}_{\mathrm{f}}}{5.28129 \mathrm{E}-04}$ & $\frac{\mathrm{G}_{\mathrm{f}}}{2.03214 \mathrm{E}-04}$ \\
1 & $5.36940 \mathrm{E}-03$ & $8.29315 \mathrm{E}-03$ & $3.40344 \mathrm{E}-03$ & $5.43045 \mathrm{E}-03$ & $8.32423 \mathrm{E}-03$ & $3.41618 \mathrm{E}-03$ \\
2 & $7.84363 \mathrm{E}-03$ & $1.58948 \mathrm{E}-02$ & $6.52311 \mathrm{E}-03$ & $7.88612 \mathrm{E}-03$ & $1.60166 \mathrm{E}-02$ & $6.57304 \mathrm{E}-03$ \\
3 & $7.43305 \mathrm{E}-02$ & $1.54727 \mathrm{E}-01$ & $6.34985 \mathrm{E}-02$ & $7.41841 \mathrm{E}-02$ & $1.54426 \mathrm{E}-01$ & $6.33751 \mathrm{E}-02$ \\
\hline
\end{tabular}

Table 3.9. SAXTON-1, Pin-1 four-group U-238 cross sections

\begin{tabular}{cccccccc}
\hline Group & \multicolumn{3}{c}{ CENTRM cross sections $\left(\mathrm{cm}^{-1}\right)$} & \multicolumn{3}{c}{ NITAWL cross sections $\left(\mathrm{cm}^{-1}\right)$} \\
\hline & $\frac{\mathrm{G}_{\mathrm{a}}}{7.21435 \mathrm{E}-03}$ & $\frac{\mathrm{G}_{\mathrm{f}}}{1.34429 \mathrm{E}-02}$ & $\frac{\mathrm{G}_{\mathrm{f}}}{4.77316 \mathrm{E}-03}$ & $\frac{\mathrm{G}_{\mathrm{a}}}{7.22446 \mathrm{E}-03}$ & $\frac{\mathrm{G}_{\mathrm{f}}}{1.35539 \mathrm{E}-02}$ & $\frac{\mathrm{G}_{\mathrm{f}}}{4.80883 \mathrm{E}-03}$ \\
1 & $5.31239 \mathrm{E}-02$ & $5.82914 \mathrm{E}-06$ & $2.41498 \mathrm{E}-06$ & $5.09276 \mathrm{E}-02$ & $5.71143 \mathrm{E}-06$ & $2.36622 \mathrm{E}-06$ \\
2 & $1.12361 \mathrm{E}-02$ & $3.85156 \mathrm{E}-08$ & $1.59577 \mathrm{E}-08$ & $1.12440 \mathrm{E}-02$ & $3.86270 \mathrm{E}-08$ & $1.60039 \mathrm{E}-08$ \\
3 & $4.26642 \mathrm{E}-02$ & $2.00382 \mathrm{E}-07$ & $8.30224 \mathrm{E}-08$ & $4.25934 \mathrm{E}-02$ & $2.00045 \mathrm{E}-07$ & $8.28825 \mathrm{E}-08$ \\
\hline
\end{tabular}

Table 3.10. SAXTON-1, Pin-1 four-group Pu-239 cross sections

\begin{tabular}{ccccccc}
\hline Group & \multicolumn{2}{c}{ CENTRM cross sections $\left(\mathrm{cm}^{-1}\right)$} & \multicolumn{2}{c}{ NITAWL cross sections $\left(\mathrm{cm}^{-1}\right)$} \\
\hline & $\frac{\mathrm{G}_{\mathrm{a}}}{2.51521 \mathrm{E}-03}$ & $\frac{\mathrm{G}_{\mathrm{f}}}{7.34395 \mathrm{E}-03}$ & $\frac{\mathrm{G}_{\mathrm{f}}}{2.36412 \mathrm{E}-03}$ & $\frac{\mathrm{G}_{\mathrm{a}}}{2.51413 \mathrm{E}-03}$ & $\frac{\mathrm{G}_{\mathrm{f}}}{7.35233 \mathrm{E}-03}$ & $\frac{\mathrm{G}_{\mathrm{f}}}{2.36542 \mathrm{E}-03}$ \\
1 & $4.31528 \mathrm{E}-02$ & $7.26582 \mathrm{E}-02$ & $2.52301 \mathrm{E}-02$ & $4.23284 \mathrm{E}-02$ & $7.17029 \mathrm{E}-02$ & $2.48984 \mathrm{E}-02$ \\
2 & $1.42157 \mathrm{E}-01$ & $2.82164 \mathrm{E}-01$ & $9.79800 \mathrm{E}-02$ & $1.43048 \mathrm{E}-01$ & $2.83783 \mathrm{E}-01$ & $9.85430 \mathrm{E}-02$ \\
3 & $1.26177 \mathrm{E}+00$ & $2.54082 \mathrm{E}+00$ & $8.78966 \mathrm{E}-01$ & $1.25963 \mathrm{E}+00$ & $2.53651 \mathrm{E}+00$ & $8.77483 \mathrm{E}-01$ \\
\hline
\end{tabular}


Table 3.11. SAXTON-1, Pin-132 four-group fluxes

\begin{tabular}{ccc}
\hline Group & $\begin{array}{c}\text { CENTRM M } \\
\left(\mathrm{cm}^{-2}-\mathrm{s}^{-1}\right)\end{array}$ & $\begin{array}{c}\text { NITAWL M } \\
\left(\mathrm{cm}^{-2}-\mathrm{s}^{-1}\right)\end{array}$ \\
\hline 1 & $2.750 \mathrm{E}-04$ & $2.726 \mathrm{E}-04$ \\
2 & $9.570 \mathrm{E}-05$ & $9.640 \mathrm{E}-05$ \\
3 & $1.651 \mathrm{E}-05$ & $1.607 \mathrm{E}-05$ \\
4 & $1.110 \mathrm{E}-05$ & $1.112 \mathrm{E}-05$ \\
\hline
\end{tabular}

Table 3.12. SAXTON-1, Pin-132 four-group U-235 cross sections

\begin{tabular}{ccccccc}
\hline Group & \multicolumn{2}{c}{ CENTRM cross sections $\left(\mathrm{cm}^{-1}\right)$} & \multicolumn{3}{c}{ NITAWL cross sections $\left(\mathrm{cm}^{-1}\right)$} \\
\hline & $\frac{\mathrm{G}_{\mathrm{a}}}{2.45122 \mathrm{E}-04}$ & $\frac{\varangle_{\mathrm{f}}}{5.39109 \mathrm{E}-04}$ & $\frac{\mathrm{G}_{\mathrm{f}}}{2.10038 \mathrm{E}-04}$ & $\frac{\mathrm{G}_{\mathrm{a}}}{2.44993 \mathrm{E}-04}$ & $\frac{\mathrm{G}_{\mathrm{f}}}{5.38844 \mathrm{E}-04}$ & $\frac{\mathrm{G}_{\mathrm{f}}}{2.09938 \mathrm{E}-04}$ \\
1 & $5.15441 \mathrm{E}-03$ & $7.97880 \mathrm{E}-03$ & $3.27443 \mathrm{E}-03$ & $5.09111 \mathrm{E}-03$ & $7.85028 \mathrm{E}-03$ & $3.22168 \mathrm{E}-03$ \\
2 & $7.58571 \mathrm{E}-03$ & $1.52693 \mathrm{E}-02$ & $6.26638 \mathrm{E}-03$ & $7.63367 \mathrm{E}-03$ & $1.54170 \mathrm{E}-02$ & $6.32701 \mathrm{E}-03$ \\
3 & $6.21247 \mathrm{E}-02$ & $1.28918 \mathrm{E}-01$ & $5.29069 \mathrm{E}-02$ & $6.23526 \mathrm{E}-02$ & $1.29385 \mathrm{E}-01$ & $5.30985 \mathrm{E}-02$ \\
\hline
\end{tabular}

Table 3.13. SAXTON-1, Pin-132 four-group U-238 cross sections

\begin{tabular}{|c|c|c|c|c|c|c|}
\hline \multirow[t]{2}{*}{ Group } & \multicolumn{3}{|c|}{ CENTRM cross sections $\left(\mathrm{cm}^{-1}\right)$} & \multicolumn{3}{|c|}{ NITAWL cross sections $\left(\mathrm{cm}^{-1}\right)$} \\
\hline & $\mathrm{G}_{\mathrm{a}}$ & $<G_{f}$ & $\mathrm{G}_{\mathrm{f}}$ & $\mathrm{G}_{\mathrm{a}}$ & $<\mathrm{G}_{\mathrm{f}}$ & $\mathrm{G}_{\mathrm{f}}$ \\
\hline 1 & $6.80382 \mathrm{E}-03$ & $1.10027 \mathrm{E}-02$ & $3.91622 \mathrm{E}-03$ & $6.78188 \mathrm{E}-03$ & $1.09576 \mathrm{E}-02$ & $3.89839 \mathrm{E}-03$ \\
\hline 2 & 4.89435E-02 & $6.26429 \mathrm{E}-06$ & $2.59527 \mathrm{E}-06$ & 4.75272E-02 & $6.04762 \mathrm{E}-06$ & $2.50550 \mathrm{E}-06$ \\
\hline 3 & $1.11343 \mathrm{E}-02$ & 3.75412E-08 & $1.55540 \mathrm{E}-08$ & 1.11699E-02 & $3.77752 \mathrm{E}-08$ & 1.56510E-08 \\
\hline 4 & $3.63750 \mathrm{E}-02$ & $1.70185 \mathrm{E}-07$ & 7.05107E-08 & 3.64831E-02 & $1.70704 \mathrm{E}-07$ & $7.07262 \mathrm{E}-08$ \\
\hline
\end{tabular}

Table 3.14. SAXTON-1, Pin-132 four-group Pu-239 cross sections

\begin{tabular}{cccccccc}
\hline Group & \multicolumn{3}{c}{ CENTRM cross sections $\left(\mathrm{cm}^{-1}\right)$} & \multicolumn{3}{c}{ NITAWL cross sections $\left(\mathrm{cm}^{-1}\right)$} \\
\hline & $\frac{\mathrm{G}_{\mathrm{a}}}{2.52991 \mathrm{E}-03}$ & $\frac{\mathrm{G}_{\mathrm{f}}}{7.17411 \mathrm{E}-03}$ & $\frac{\mathrm{G}_{\mathrm{f}}}{2.33467 \mathrm{E}-03}$ & $\frac{\mathrm{G}_{\mathrm{a}}}{2.52913 \mathrm{E}-03}$ & $\frac{\mathrm{G}_{\mathrm{f}}}{7.17146 \mathrm{E}-03}$ & $\frac{\mathrm{G}_{\mathrm{f}}}{2.33393 \mathrm{E}-03}$ \\
1 & $4.10734 \mathrm{E}-02$ & $6.89915 \mathrm{E}-02$ & $2.39569 \mathrm{E}-02$ & $3.95781 \mathrm{E}-02$ & $6.68722 \mathrm{E}-02$ & $2.32210 \mathrm{E}-02$ \\
2 & $1.30973 \mathrm{E}-01$ & $2.61059 \mathrm{E}-01$ & $9.06517 \mathrm{E}-02$ & $1.35790 \mathrm{E}-01$ & $2.70087 \mathrm{E}-01$ & $9.37866 \mathrm{E}-02$ \\
3 & $1.45288 \mathrm{E}+00$ & $2.78304 \mathrm{E}+00$ & $9.63703 \mathrm{E}-01$ & $1.45769 \mathrm{E}+00$ & $2.79201 \mathrm{E}+00$ & $9.66808 \mathrm{E}-01$ \\
\hline
\end{tabular}




\begin{tabular}{|c|c|c|c|c|c|c|c|c|c|}
\hline & & & & & & & & & $\begin{array}{l}7.032 \\
(0.91)\end{array}$ \\
\hline & $\begin{array}{l}0.171 \\
(0.75)\end{array}$ & & & & & & & $\begin{array}{l}7.088 \\
(0.42)\end{array}$ & $\begin{array}{l}7.085 \\
(0.46)\end{array}$ \\
\hline & & & & & & & $\begin{array}{l}6.809 \\
(0.46)\end{array}$ & $\begin{array}{l}6.904 \\
(0.32)\end{array}$ & $\begin{array}{l}6.960 \\
(0.49)\end{array}$ \\
\hline & & & & & & $\begin{array}{l}6.408 \\
(0.49)\end{array}$ & $\begin{array}{l}6.587 \\
(0.33)\end{array}$ & $\begin{array}{l}6.701 \\
(0.33)\end{array}$ & $\begin{array}{l}6.721 \\
(0.48)\end{array}$ \\
\hline & & & & & $\begin{array}{l}5.874 \\
(0.49)\end{array}$ & $\begin{array}{l}6.137 \\
(0.35)\end{array}$ & $\begin{array}{l}6.268 \\
(0.37)\end{array}$ & $\begin{array}{l}6.459 \\
(0.35)\end{array}$ & $\begin{array}{l}6.431 \\
(0.48)\end{array}$ \\
\hline & & & & $\begin{array}{l}5.267 \\
(0.53)\end{array}$ & $\begin{array}{l}5.498 \\
(0.34)\end{array}$ & $\begin{array}{l}5.807 \\
(0.36)\end{array}$ & $\begin{array}{l}5.922 \\
(0.34)\end{array}$ & $\begin{array}{l}6.082 \\
(0.36)\end{array}$ & $\begin{array}{l}6.127 \\
(0.49)\end{array}$ \\
\hline & & & $\begin{array}{l}4.501 \\
(0.61)\end{array}$ & $\begin{array}{l}4.877 \\
(0.40)\end{array}$ & $\begin{array}{l}5.169 \\
(0.39)\end{array}$ & $\begin{array}{l}5.362 \\
(0.37)\end{array}$ & $\begin{array}{l}5.580 \\
(0.37)\end{array}$ & $\begin{array}{l}5.629 \\
(0.38)\end{array}$ & $\begin{array}{l}5.695 \\
(0.52)\end{array}$ \\
\hline & & $\begin{array}{l}3.937 \\
(0.61)\end{array}$ & $\begin{array}{l}4.239 \\
(0.41)\end{array}$ & $\begin{array}{l}4.533 \\
(0.40)\end{array}$ & $\begin{array}{l}4.826 \\
(0.42)\end{array}$ & $\begin{array}{l}5.017 \\
(0.39)\end{array}$ & $\begin{array}{l}5.191 \\
(0.39)\end{array}$ & $\begin{array}{l}5.256 \\
(0.37)\end{array}$ & $\begin{array}{l}5.308 \\
(0.56)\end{array}$ \\
\hline & $\begin{array}{l}4.056 \\
(0.65)\end{array}$ & $\begin{array}{l}4.036 \\
(0.42)\end{array}$ & $\begin{array}{l}4.308 \\
(0.40)\end{array}$ & $\begin{array}{l}4.628 \\
(0.40)\end{array}$ & $\begin{array}{l}4.898 \\
(0.37)\end{array}$ & $\begin{array}{l}5.101 \\
(0.39)\end{array}$ & $\begin{array}{l}5.261 \\
(0.38)\end{array}$ & $\begin{array}{l}5.374 \\
(0.35)\end{array}$ & $\begin{array}{l}5.422 \\
(0.51)\end{array}$ \\
\hline $\begin{array}{c}7.779 \\
(0.47)\end{array}$ & $\begin{array}{l}6.169 \\
(0.37)\end{array}$ & $\begin{array}{l}6.394 \\
(0.34)\end{array}$ & $\begin{array}{l}6.876 \\
(0.36)\end{array}$ & $\begin{array}{c}7.378 \\
(0.34)\end{array}$ & $\begin{array}{c}7.815 \\
(0.30)\end{array}$ & $\begin{array}{l}8.167 \\
(0.33)\end{array}$ & $\begin{array}{l}8.417 \\
(0.30)\end{array}$ & $\begin{array}{l}8.574 \\
(0.31)\end{array}$ & $\begin{array}{l}8.669 \\
(0.41)\end{array}$ \\
\hline
\end{tabular}

Fig. 3.8a. Pin-power distribution for CENTRM benchmark SAXTON-2. Value in parentheses is percent standard deviation. 


\begin{tabular}{|c|c|c|c|c|c|c|c|c|c|}
\hline \multirow[t]{9}{*}{$\begin{array}{l}0.172 \\
(0.72)\end{array}$} & & & & & & & & & $\begin{array}{c}7.111 \\
(0.85)\end{array}$ \\
\hline & & & & & & & & $\begin{array}{l}7.073 \\
(0.45)\end{array}$ & $\begin{array}{r}7.107 \\
(0.46)\end{array}$ \\
\hline & & & & & & & $\begin{array}{l}6.803 \\
(0.46)\end{array}$ & $\begin{array}{l}6.929 \\
(0.32)\end{array}$ & $\begin{array}{c}7.015 \\
(0.47)\end{array}$ \\
\hline & & & & & & $\begin{array}{l}6.476 \\
(0.49)\end{array}$ & $\begin{array}{l}6.655 \\
(0.35)\end{array}$ & $\begin{array}{l}6.786 \\
(0.34)\end{array}$ & $\begin{array}{l}6.773 \\
(0.44)\end{array}$ \\
\hline & & & & & $\begin{array}{l}5.890 \\
(0.52)\end{array}$ & $\begin{array}{l}6.194 \\
(0.36)\end{array}$ & $\begin{array}{l}6.362 \\
(0.34)\end{array}$ & $\begin{array}{l}6.449 \\
(0.34)\end{array}$ & $\begin{array}{l}6.504 \\
(0.46)\end{array}$ \\
\hline & & & & $\begin{array}{l}5.255 \\
(0.53)\end{array}$ & $\begin{array}{l}5.527 \\
(0.39)\end{array}$ & $\begin{array}{l}5.826 \\
(0.36)\end{array}$ & $\begin{array}{l}5.985 \\
(0.35)\end{array}$ & $\begin{array}{l}6.159 \\
(0.37)\end{array}$ & $\begin{array}{c}6.139 \\
(0.50)\end{array}$ \\
\hline & & & $\begin{array}{l}4.556 \\
(0.57)\end{array}$ & $\begin{array}{l}4.871 \\
(0.39)\end{array}$ & $\begin{array}{l}5.198 \\
(0.36)\end{array}$ & $\begin{array}{l}5.449 \\
(0.38)\end{array}$ & $\begin{array}{l}5.562 \\
(0.37)\end{array}$ & $\begin{array}{l}5.690 \\
(0.38)\end{array}$ & $\begin{array}{l}5.706 \\
(0.48)\end{array}$ \\
\hline & & $\begin{array}{l}3.988 \\
(0.57)\end{array}$ & $\begin{array}{l}4.229 \\
(0.40)\end{array}$ & $\begin{array}{l}4.534 \\
(0.40)\end{array}$ & $\begin{array}{l}4.839 \\
(040 .)\end{array}$ & $\begin{array}{l}5.022 \\
(0.42)\end{array}$ & $\begin{array}{l}5.209 \\
(0.39)\end{array}$ & $\begin{array}{l}5.298 \\
(0.39)\end{array}$ & $\begin{array}{l}5.338 \\
(054 .)\end{array}$ \\
\hline & $\begin{array}{l}4.073 \\
(0.62)\end{array}$ & $\begin{array}{l}3.989 \\
(0.46)\end{array}$ & $\begin{array}{l}4.314 \\
(0.38)\end{array}$ & $\begin{array}{l}4.651 \\
(0.40)\end{array}$ & $\begin{array}{l}4.909 \\
(0.36)\end{array}$ & $\begin{array}{l}5.172 \\
(0.39)\end{array}$ & $\begin{array}{l}5.260 \\
(040 .)\end{array}$ & $\begin{array}{l}5.401 \\
(0.36)\end{array}$ & $\begin{array}{l}5.401 \\
(048 .)\end{array}$ \\
\hline $\begin{array}{c}7.878 \\
(0.40)\end{array}$ & $\begin{array}{c}6.193 \\
(0.34)\end{array}$ & $\begin{array}{l}6.355 \\
(0.33)\end{array}$ & $\begin{array}{c}6.870 \\
(0.34)\end{array}$ & $\begin{array}{c}7.353 \\
(0.32)\end{array}$ & $\begin{array}{c}7.839 \\
(0.32)\end{array}$ & $\begin{array}{l}8.201 \\
(0.30)\end{array}$ & $\begin{array}{l}8.457 \\
(0.29)\end{array}$ & $\begin{array}{l}8.657 \\
(0.29)\end{array}$ & $\begin{array}{l}8.684 \\
(0.44)\end{array}$ \\
\hline
\end{tabular}

Fig. 3.8b. Pin-power distribution for NITAWL benchmark SAXTON-2. Value in parentheses is percent standard deviation. 
Table 3.15. SAXTON-2 CENTRM reaction rates and fluxes

\begin{tabular}{|c|c|c|c|c|c|c|}
\hline Pin & Region & $\begin{array}{c}\mathrm{G}_{\mathrm{a}} \mathrm{M} \\
\left(\mathrm{cm}^{-3}-\mathrm{s}^{-1}\right)\end{array}$ & $\begin{array}{c}\mathcal{G}_{\mathrm{f}} \mathrm{M} \\
\left(\mathrm{cm}^{-3}-\mathrm{s}^{-1}\right)\end{array}$ & $\begin{array}{c}\mathrm{G}_{\mathrm{f}} \mathrm{M} \\
\left(\mathrm{cm}^{-3}-\mathrm{s}^{-1}\right)\end{array}$ & $\begin{array}{c}\mathrm{M} \\
\left(\mathrm{cm}-\mathrm{s}^{-1}\right)\end{array}$ & $\mathrm{MM}_{\mathrm{t}}$ \\
\hline \multirow{3}{*}{1} & Fuel & $4.361 \mathrm{E}-05$ & $7.811 \mathrm{E}-05$ & $2.729 \mathrm{E}-05$ & $8.590 \mathrm{E}-03$ & 0.296 \\
\hline & Clad & $3.882 \mathrm{E}-07$ & 0.0 & 0.0 & $2.531 \mathrm{E}-03$ & 0.087 \\
\hline & Mod. & $1.731 \mathrm{E}-06$ & 0.0 & 0.0 & $1.793 \mathrm{E}-02$ & 0.617 \\
\hline \multirow{3}{*}{55} & Fuel & $4.675 \mathrm{E}-05$ & $7.033 \mathrm{E}-05$ & $2.457 \mathrm{E}-05$ & $2.148 \mathrm{E}-02$ & 0.293 \\
\hline & Clad & $6.310 \mathrm{E}-07$ & 0.0 & 0.0 & $6.403 \mathrm{E}-03$ & 0.087 \\
\hline & Mod. & $1.351 \mathrm{E}-06$ & 0.0 & 0.0 & 4.552E- 02 & 0.620 \\
\hline
\end{tabular}

Table 3.16. SAXTON-2 NITAWL reaction rates and fluxes

\begin{tabular}{ccccccc}
\hline \multirow{2}{*}{ Pin } & Region & $\begin{array}{c}\mathrm{G}_{\mathrm{a}} \mathrm{M} \\
\left(\mathrm{cm}^{-3}-\mathrm{s}^{-1}\right)\end{array}$ & $\begin{array}{c}\mathrm{G}_{\mathrm{f}} \mathrm{M} \\
\left(\mathrm{cm}^{-3}-\mathrm{s}^{-1}\right)\end{array}$ & $\begin{array}{c}\mathrm{G}_{\mathrm{f}} \mathrm{M} \\
\left(\mathrm{cm}^{-3}-\mathrm{s}^{-1}\right)\end{array}$ & $\begin{array}{c}\mathrm{M} \\
\left(\mathrm{cm}^{-1}\right)\end{array}$ & $\mathrm{M}_{\mathrm{t}}$ \\
\hline \multirow{2}{*}{1} & Fuel & $4.393 \mathrm{E}-05$ & $7.895 \mathrm{E}-05$ & $2.759 \mathrm{E}-05$ & $8.596 \mathrm{E}-03$ & 0.296 \\
& Clad & $3.874 \mathrm{E}-07$ & 0.0 & 0.0 & $2.523 \mathrm{E}-03$ & 0.087 \\
& Mod. & $8.732 \mathrm{E}-07$ & 0.0 & 0.0 & $1.790 \mathrm{E}-02$ & 0.617 \\
\hline \multirow{2}{*}{55} & Fuel & $4.723 \mathrm{E}-05$ & $7.130 \mathrm{E}-05$ & $2.491 \mathrm{E}-05$ & $2.145 \mathrm{E}-02$ & 0.292 \\
& Clad & $6.079 \mathrm{E}-07$ & 0.0 & 0.0 & $6.411 \mathrm{E}-03$ & 0.087 \\
& Mod. & $6.803 \mathrm{E}-07$ & 0.0 & 0.0 & $4.565 \mathrm{E}-02$ & 0.621 \\
\hline
\end{tabular}


Table 3.17. SAXTON-2, Pin-1 four-group fluxes

\begin{tabular}{ccc}
\hline Group & $\begin{array}{c}\text { CENTRM M } \\
\left(\mathrm{cm}^{-2}-\mathrm{s}^{-1}\right)\end{array}$ & $\begin{array}{c}\text { NITAWL M } \\
\left(\mathrm{cm}^{-2}-\mathrm{s}^{-1}\right)\end{array}$ \\
\hline 1 & $1.210 \mathrm{E}-04$ & $1.209 \mathrm{E}-04$ \\
2 & $3.068 \mathrm{E}-05$ & $3.075 \mathrm{E}-05$ \\
3 & $6.650 \mathrm{E}-06$ & $6.426 \mathrm{E}-06$ \\
4 & $2.598 \mathrm{E}-05$ & $2.635 \mathrm{E}-05$ \\
\hline
\end{tabular}

Table 3.18. SAXTON-2, Pin-1 four-group U-235 cross sections

\begin{tabular}{cccccccc}
\hline Group & \multicolumn{3}{c}{ CENTRM cross sections $\left(\mathrm{cm}^{-1}\right)$} & \multicolumn{3}{c}{ NITAWL cross sections $\left(\mathrm{cm}^{-1}\right)$} \\
\hline & $\frac{\mathrm{G}_{\mathrm{a}}}{2.31295 \mathrm{E}-04}$ & $\frac{\mathrm{G}_{\mathrm{f}}}{5.28168 \mathrm{E}-04}$ & $\frac{\mathrm{G}_{\mathrm{f}}}{2.03137 \mathrm{E}-04}$ & $\frac{\mathrm{G}_{\mathrm{a}}}{2.31046 \mathrm{E}-04}$ & $\frac{\mathrm{G}_{\mathrm{f}}}{5.27611 \mathrm{E}-04}$ & $\frac{\mathrm{G}_{\mathrm{f}}}{2.02942 \mathrm{E}-04}$ \\
1 & $5.50818 \mathrm{E}-03$ & $8.46995 \mathrm{E}-03$ & $3.47598 \mathrm{E}-03$ & $5.34062 \mathrm{E}-03$ & $8.20683 \mathrm{E}-03$ & $3.36800 \mathrm{E}-03$ \\
2 & $7.88307 \mathrm{E}-03$ & $1.60481 \mathrm{E}-02$ & $6.58600 \mathrm{E}-03$ & $7.88335 \mathrm{E}-03$ & $1.59830 \mathrm{E}-02$ & $6.55932 \mathrm{E}-03$ \\
3 & $7.48945 \mathrm{E}-02$ & $1.55911 \mathrm{E}-01$ & $6.39846 \mathrm{E}-02$ & $7.47985 \mathrm{E}-02$ & $1.55708 \mathrm{E}-01$ & $6.39009 \mathrm{E}-02$ \\
\hline
\end{tabular}

Table 3.19. SAXTON-2, Pin-1 four-group U-238 cross sections

\begin{tabular}{cccccccc}
\hline Group & \multicolumn{3}{c}{ CENTRM cross sections $\left(\mathrm{cm}^{-1}\right)$} & \multicolumn{3}{c}{ NITAWL cross sections $\left(\mathrm{cm}^{-1}\right)$} \\
\hline & $\frac{\mathrm{G}_{\mathrm{a}}}{7.27299 \mathrm{E}-03}$ & $\frac{\mathrm{G}_{\mathrm{f}}}{1.37200 \mathrm{E}-02}$ & $\frac{\mathrm{G}_{\mathrm{f}}}{4.87017 \mathrm{E}-03}$ & $\frac{\mathrm{G}_{\mathrm{a}}}{7.25772 \mathrm{E}-03}$ & $\frac{\mathrm{G}_{\mathrm{f}}}{1.36913 \mathrm{E}-02}$ & $\frac{\mathrm{G}_{\mathrm{f}}}{4.86098 \mathrm{E}-03}$ \\
1 & $5.45685 \mathrm{E}-02$ & $6.04055 \mathrm{E}-06$ & $2.50257 \mathrm{E}-06$ & $5.15366 \mathrm{E}-02$ & $5.85011 \mathrm{E}-06$ & $2.42368 \mathrm{E}-06$ \\
2 & $1.12498 \mathrm{E}-02$ & $3.86744 \mathrm{E}-08$ & $1.60235 \mathrm{E}-08$ & $1.12126 \mathrm{E}-02$ & $3.84197 \mathrm{E}-08$ & $1.59180 \mathrm{E}-08$ \\
3 & $4.29376 \mathrm{E}-02$ & $2.01691 \mathrm{E}-07$ & $8.35643 \mathrm{E}-08$ & $4.28896 \mathrm{E}-02$ & $2.01461 \mathrm{E}-07$ & $8.34691 \mathrm{E}-08$ \\
\hline
\end{tabular}

Table 3.20. SAXTON-2, Pin-1 four-group Pu-239 cross sections

\begin{tabular}{ccccccc}
\hline Group & \multicolumn{3}{c}{ CENTRM cross sections $\left(\mathrm{cm}^{-1}\right)$} & \multicolumn{3}{c}{ NITAWL cross sections $\left(\mathrm{cm}^{-1}\right)$} \\
\hline & $\frac{\mathrm{G}_{\mathrm{a}}}{2.51538 \mathrm{E}-03}$ & $\frac{\mathrm{G}_{\mathrm{f}}}{7.36394 \mathrm{E}-03}$ & $\frac{\mathrm{G}_{\mathrm{f}}}{2.36798 \mathrm{E}-03}$ & $\frac{\mathrm{G}_{\mathrm{a}}}{2.51302 \mathrm{E}-03}$ & $\frac{\mathrm{G}_{\mathrm{f}}}{7.35725 \mathrm{E}-03}$ & $\frac{\mathrm{G}_{\mathrm{f}}}{2.36609 \mathrm{E}-03}$ \\
1 & $4.36441 \mathrm{E}-02$ & $7.27138 \mathrm{E}-02$ & $2.52495 \mathrm{E}-02$ & $4.25694 \mathrm{E}-02$ & $7.20315 \mathrm{E}-02$ & $2.50126 \mathrm{E}-02$ \\
2 & $1.44153 \mathrm{E}-01$ & $2.85821 \mathrm{E}-01$ & $9.92499 \mathrm{E}-02$ & $1.40219 \mathrm{E}-01$ & $2.78403 \mathrm{E}-01$ & $9.66744 \mathrm{E}-02$ \\
3 & $1.26117 \mathrm{E}+00$ & $2.54337 \mathrm{E}+00$ & $8.79827 \mathrm{E}-01$ & $1.26115 \mathrm{E}+00$ & $2.54272 \mathrm{E}+00$ & $8.79601 \mathrm{E}-01$ \\
\hline
\end{tabular}


Table 3.21. SAXTON-2, Pin-55 four-group fluxes

\begin{tabular}{ccc}
\hline Group & $\begin{array}{c}\text { CENTRM M } \\
\left(\mathrm{cm}^{-2}-\mathrm{s}^{-1}\right)\end{array}$ & $\begin{array}{c}\text { NITAWL M } \\
\left(\mathrm{cm}^{-2}-\mathrm{s}^{-1}\right)\end{array}$ \\
\hline 1 & $3.168 \mathrm{E}-04$ & $3.145 \mathrm{E}-04$ \\
2 & $1.074 \mathrm{E}-04$ & $1.086 \mathrm{E}-04$ \\
3 & $1.945 \mathrm{E}-05$ & $1.953 \mathrm{E}-05$ \\
4 & $1.722 \mathrm{E}-05$ & $1.751 \mathrm{E}-05$ \\
\hline
\end{tabular}

Table 3.22 SAXTON-2, Pin-55 four-group U-235 cross sections

\begin{tabular}{cccccccc}
\hline Group & \multicolumn{2}{c}{ CENTRM cross sections $\left(\mathrm{cm}^{-1}\right)$} & \multicolumn{2}{c}{ NITAWL cross sections $\left(\mathrm{cm}^{-1}\right)$} \\
\hline & $\frac{\mathrm{G}_{\mathrm{a}}}{2.42818 \mathrm{E}-04}$ & $\frac{\mathrm{G}_{\mathrm{f}}}{5.37235 \mathrm{E}-04}$ & $\frac{\mathrm{G}_{\mathrm{f}}}{2.08871 \mathrm{E}-04}$ & $\frac{\mathrm{G}_{\mathrm{a}}}{2.42331 \mathrm{E}-04}$ & $\frac{\mathrm{G}_{\mathrm{f}}}{5.36480 \mathrm{E}-04}$ & $\frac{\mathrm{G}_{\mathrm{f}}}{2.08578 \mathrm{E}-04}$ \\
1 & $5.28301 \mathrm{E}-03$ & $8.16913 \mathrm{E}-03$ & $3.35254 \mathrm{E}-03$ & $5.21227 \mathrm{E}-03$ & $8.02944 \mathrm{E}-03$ & $3.29521 \mathrm{E}-03$ \\
3 & $7.65558 \mathrm{E}-03$ & $1.53980 \mathrm{E}-02$ & $6.31918 \mathrm{E}-03$ & $7.58530 \mathrm{E}-03$ & $1.53401 \mathrm{E}-02$ & $6.29544 \mathrm{E}-03$ \\
4 & $6.42364 \mathrm{E}-02$ & $1.33386 \mathrm{E}-01$ & $5.47403 \mathrm{E}-02$ & $6.47836 \mathrm{E}-02$ & $1.34511 \mathrm{E}-01$ & $5.52022 \mathrm{E}-02$ \\
\hline
\end{tabular}

Table 3.23. SAXTON-2, Pin-55 four-group U-238 cross sections

\begin{tabular}{cccccccc}
\hline Group & \multicolumn{3}{c}{ CENTRM cross sections $\left(\mathrm{cm}^{-1}\right)$} & \multicolumn{3}{c}{ NITAWL cross sections $\left(\mathrm{cm}^{-1}\right)$} \\
\hline & $\frac{\mathrm{G}_{\mathrm{a}}}{4}$ & $\frac{\mathrm{G}_{\mathrm{f}}}{1.14866 \mathrm{E}-02}$ & $\frac{\mathrm{G}_{\mathrm{f}}}{4.08747 \mathrm{E}-03}$ & $\frac{\mathrm{G}_{\mathrm{a}}}{6.87320 \mathrm{E}-03}$ & $\frac{\mathrm{G}_{\mathrm{f}}}{1.14586 \mathrm{E}-02}$ & $\frac{\mathrm{G}_{\mathrm{f}}}{4.07844 \mathrm{E}-03}$ \\
2 & $5.89564 \mathrm{E}-03$ & $1.14845 \mathrm{E}-02$ & $5.98081 \mathrm{E}-06$ & $2.47782 \mathrm{E}-06$ & $5.07872 \mathrm{E}-02$ & $5.96084 \mathrm{E}-06$ & $2.46955 \mathrm{E}-06$ \\
3 & $1.11261 \mathrm{E}-02$ & $3.76231 \mathrm{E}-08$ & $1.55879 \mathrm{E}-08$ & $1.11419 \mathrm{E}-02$ & $3.76606 \mathrm{E}-08$ & $1.56035 \mathrm{E}-08$ \\
4 & $3.74682 \mathrm{E}-02$ & $1.75440 \mathrm{E}-07$ & $7.26882 \mathrm{E}-08$ & $3.77212 \mathrm{E}-02$ & $1.76632 \mathrm{E}-07$ & $7.31816 \mathrm{E}-08$ \\
\hline
\end{tabular}

Table 3.24. SAXTON-2, Pin-55 four-group Pu-239 cross sections

\begin{tabular}{cccccccc}
\hline Group & \multicolumn{3}{c}{ CENTRM cross sections $\left(\mathrm{cm}^{-1}\right)$} & \multicolumn{2}{c}{ NITAWL cross sections $\left(\mathrm{cm}^{-1}\right)$} \\
\hline & $\frac{\mathrm{G}_{\mathrm{a}}}{2.52700 \mathrm{E}-03}$ & $\frac{\mathrm{G}_{\mathrm{f}}}{7.20417 \mathrm{E}-03}$ & $\frac{\mathrm{G}_{\mathrm{f}}}{2.33954 \mathrm{E}-03}$ & $\frac{\mathrm{G}_{\mathrm{a}}}{2.52549 \mathrm{E}-03}$ & $\frac{\mathrm{G}_{\mathrm{f}}}{7.20372 \mathrm{E}-03}$ & $\frac{\mathrm{G}_{\mathrm{f}}}{2.33961 \mathrm{E}-03}$ \\
1 & $4.23034 \mathrm{E}-02$ & $7.11892 \mathrm{E}-02$ & $2.47201 \mathrm{E}-02$ & $4.01978 \mathrm{E}-02$ & $6.76656 \mathrm{E}-02$ & $2.34965 \mathrm{E}-02$ \\
2 & $1.33769 \mathrm{E}-01$ & $2.66117 \mathrm{E}-01$ & $9.24077 \mathrm{E}-02$ & $1.31144 \mathrm{E}-01$ & $2.61407 \mathrm{E}-01$ & $9.07727 \mathrm{E}-02$ \\
3 & $1.41690 \mathrm{E}+00$ & $2.73612 \mathrm{E}+00$ & $9.47311 \mathrm{E}-01$ & $1.43265 \mathrm{E}+00$ & $2.76589 \mathrm{E}+00$ & $9.57596 \mathrm{E}-01$ \\
\hline
\end{tabular}




\begin{tabular}{|c|c|c|c|c|c|c|c|c|c|c|}
\hline \multirow[t]{10}{*}{$\begin{array}{l}0.186 \\
(0.99)\end{array}$} & & & & & & & & & & $\begin{array}{l}5.900 \\
(0.99)\end{array}$ \\
\hline & & & & & & & & & $\begin{array}{l}5.929 \\
(0.48)\end{array}$ & $\begin{array}{l}5.958 \\
(0.50)\end{array}$ \\
\hline & & & & & & & & $\begin{array}{l}5.672 \\
(0.51)\end{array}$ & $\begin{array}{l}5.819 \\
(0.31)\end{array}$ & $\begin{array}{l}5.884 \\
(0.49)\end{array}$ \\
\hline & & & & & & & $\begin{array}{l}5.436 \\
(0.48)\end{array}$ & $\begin{array}{l}5.585 \\
(0.33)\end{array}$ & $\begin{array}{l}5.666 \\
(0.33)\end{array}$ & $\begin{array}{l}5.630 \\
(0.49)\end{array}$ \\
\hline & & & & & & $\begin{array}{l}5.000 \\
(0.54)\end{array}$ & $\begin{array}{l}5.206 \\
(0.35)\end{array}$ & $\begin{array}{l}5.373 \\
(0.33)\end{array}$ & $\begin{array}{l}5.425 \\
(0.33)\end{array}$ & $\begin{array}{l}5.486 \\
(0.55)\end{array}$ \\
\hline & & & & & $\begin{array}{l}4.473 \\
(0.49)\end{array}$ & $\begin{array}{l}4.718 \\
(0.35)\end{array}$ & $\begin{array}{l}4.935 \\
(0.37)\end{array}$ & $\begin{array}{l}5.060 \\
(0.34)\end{array}$ & $\begin{array}{l}5.148 \\
(0.34)\end{array}$ & $\begin{array}{l}5.191 \\
(0 .) 55\end{array}$ \\
\hline & & & & $\begin{array}{l}3.921 \\
(0.58)\end{array}$ & $\begin{array}{l}4.189 \\
(0.42)\end{array}$ & $\begin{array}{l}4.440 \\
(0.38)\end{array}$ & $\begin{array}{l}4.590 \\
(0.38)\end{array}$ & $\begin{array}{l}4.750 \\
(0.36)\end{array}$ & $\begin{array}{l}4.833 \\
(0.34)\end{array}$ & $\begin{array}{l}4.836 \\
(0.52)\end{array}$ \\
\hline & & & $\begin{array}{l}3.331 \\
(0.66)\end{array}$ & $\begin{array}{l}3.599 \\
(0.45)\end{array}$ & $\begin{array}{l}3.842 \\
(0.38)\end{array}$ & $\begin{array}{l}4.096 \\
(0.43)\end{array}$ & $\begin{array}{l}4.271 \\
(0.41)\end{array}$ & $\begin{array}{l}4.357 \\
(0.41)\end{array}$ & $\begin{array}{l}4.399 \\
(0.42)\end{array}$ & $\begin{array}{l}4.447 \\
(0.52)\end{array}$ \\
\hline & & $\begin{array}{l}2.821 \\
(0.75)\end{array}$ & $\begin{array}{l}3.029 \\
(0.47)\end{array}$ & $\begin{array}{l}3.314 \\
(0.50)\end{array}$ & $\begin{array}{l}3.555 \\
(0.43)\end{array}$ & $\begin{array}{l}3.722 \\
(0.39)\end{array}$ & $\begin{array}{l}3.898 \\
(0.40)\end{array}$ & $\begin{array}{r}3.976 \\
(0.39)\end{array}$ & $\begin{array}{l}4.078 \\
(0.39)\end{array}$ & $\begin{array}{l}4.096 \\
(0.57)\end{array}$ \\
\hline & $\begin{array}{l}2.693 \\
(0.80)\end{array}$ & $\begin{array}{c}2.750 \\
(0.51)\end{array}$ & $\begin{array}{l}2.987 \\
(0.44)\end{array}$ & $\begin{array}{r}3.235 \\
(0.47)\end{array}$ & $\begin{array}{c}3.464 \\
(0.40)\end{array}$ & $\begin{array}{c}3.672 \\
(0.41)\end{array}$ & $\begin{array}{l}3.860 \\
(0.41)\end{array}$ & $\begin{array}{l}3.906 \\
(0.40)\end{array}$ & $\begin{array}{l}3.977 \\
(0.41)\end{array}$ & $\begin{array}{c}3.972 \\
(0.56)\end{array}$ \\
\hline $\begin{array}{l}4.495 \\
(0.57)\end{array}$ & $\begin{array}{l}3.726 \\
(0.48)\end{array}$ & $\begin{array}{c}3.956 \\
(0.41)\end{array}$ & $\begin{array}{l}4.320 \\
(0.37)\end{array}$ & $\begin{array}{l}4.667 \\
(0.37)\end{array}$ & $\begin{array}{l}5.028 \\
(0.37)\end{array}$ & $\begin{array}{l}5.272 \\
(0.37)\end{array}$ & $\begin{array}{l}5.506 \\
(0.38)\end{array}$ & $\begin{array}{l}5.679 \\
(0.38)\end{array}$ & $\begin{array}{l}5.763 \\
(0.36)\end{array}$ & $\begin{array}{l}5.781 \\
(0.51)\end{array}$ \\
\hline
\end{tabular}

Fig. 3.9a. Pin-power distribution for CENTRM benchmark SAXTON-3. Value in parentheses is percent standard deviation. 


\begin{tabular}{|c|c|c|c|c|c|c|c|c|c|c|}
\hline \multirow[t]{10}{*}{$\begin{array}{l}0.187 \\
(1.06)\end{array}$} & & & & & & & & & & $\begin{array}{l}5.920 \\
(1.07)\end{array}$ \\
\hline & & & & & & & & & $\begin{array}{l}5.932 \\
(0.47)\end{array}$ & $\begin{array}{l}5.983 \\
(0.45)\end{array}$ \\
\hline & & & & & & & & $\begin{array}{l}5.779 \\
(0.48)\end{array}$ & $\begin{array}{l}5.826 \\
(0.33)\end{array}$ & $\begin{array}{l}5.906 \\
(0.48)\end{array}$ \\
\hline & & & & & & & $\begin{array}{l}5.446 \\
(0.46)\end{array}$ & $\begin{array}{l}5.598 \\
(0.33)\end{array}$ & $\begin{array}{l}5.704 \\
(0.33)\end{array}$ & $\begin{array}{l}5.705 \\
(0.45)\end{array}$ \\
\hline & & & & & & $\begin{array}{l}5.019 \\
(0.56)\end{array}$ & $\begin{array}{l}5.257 \\
(0.36)\end{array}$ & $\begin{array}{l}5.406 \\
(0.36)\end{array}$ & $\begin{array}{l}5.478 \\
(0.36)\end{array}$ & $\begin{array}{l}5.496 \\
(0.47)\end{array}$ \\
\hline & & & & & $\begin{array}{c}4.482 \\
(0.54)\end{array}$ & $\begin{array}{l}4.743 \\
(0.40)\end{array}$ & $\begin{array}{l}4.946 \\
(0.34)\end{array}$ & $\begin{array}{l}5.088 \\
(0.35)\end{array}$ & $\begin{array}{l}5.205 \\
(0.34)\end{array}$ & $\begin{array}{l}5.195 \\
(0.51)\end{array}$ \\
\hline & & & & $\begin{array}{c}3.988 \\
(0.58)\end{array}$ & $\begin{array}{c}4.209 \\
(0.38)\end{array}$ & $\begin{array}{l}4.447 \\
(0.36)\end{array}$ & $\begin{array}{l}4.582 \\
(0.37)\end{array}$ & $\begin{array}{l}4.738 \\
(0.36)\end{array}$ & $\begin{array}{l}4.822 \\
(0.36)\end{array}$ & $\begin{array}{l}4.866 \\
(0.54)\end{array}$ \\
\hline & & & $\begin{array}{l}3.317 \\
(0.60)\end{array}$ & $\begin{array}{l}3.625 \\
(0.44)\end{array}$ & $\begin{array}{c}3.856 \\
(0.39)\end{array}$ & $\begin{array}{l}4.081 \\
(0.38)\end{array}$ & $\begin{array}{l}4.245 \\
(0.37)\end{array}$ & $\begin{array}{r}4.400 \\
(0.36)\end{array}$ & $\begin{array}{l}4.439 \\
(0.34)\end{array}$ & $\begin{array}{l}4.515 \\
(0.51)\end{array}$ \\
\hline & & $\begin{array}{l}2.787 \\
(0.67)\end{array}$ & $\begin{array}{c}3.058 \\
(0.48)\end{array}$ & $\begin{array}{l}3.340 \\
(0.44)\end{array}$ & $\begin{array}{l}3.557 \\
(0.45)\end{array}$ & $\begin{array}{l}3.734 \\
(0.42)\end{array}$ & $\begin{array}{l}3.912 \\
(0.43)\end{array}$ & $\begin{array}{l}4.022 \\
(0.39)\end{array}$ & $\begin{array}{l}4.049 \\
(0.41)\end{array}$ & $\begin{array}{l}4.134 \\
(0.59)\end{array}$ \\
\hline & $\begin{array}{l}2.676 \\
(0.74)\end{array}$ & $\begin{array}{r}2.740 \\
(0.51)\end{array}$ & $\begin{array}{l}3.007 \\
(0.46)\end{array}$ & $\begin{array}{c}3.254 \\
(0.49)\end{array}$ & $\begin{array}{c}3.491 \\
(0.42)\end{array}$ & $\begin{array}{l}3.663 \\
(0.47)\end{array}$ & $\begin{array}{l}3.821 \\
(0.42)\end{array}$ & $\begin{array}{l}3.954 \\
(0.42)\end{array}$ & $\begin{array}{l}4.004 \\
(0.43)\end{array}$ & $\begin{array}{r}4.050 \\
(0.61)\end{array}$ \\
\hline $\begin{array}{l}4.515 \\
(0.53)\end{array}$ & $\begin{array}{l}3.758 \\
(0.45)\end{array}$ & $\begin{array}{l}3.949 \\
(0.41)\end{array}$ & $\begin{array}{l}4.335 \\
(0.40)\end{array}$ & $\begin{array}{l}4.695 \\
(0.39)\end{array}$ & $\begin{array}{c}5.019 \\
(0.36)\end{array}$ & $\begin{array}{l}5.313 \\
(0.36)\end{array}$ & $\begin{array}{l}5.493 \\
(0.38)\end{array}$ & $\begin{array}{l}5.685 \\
(0.38)\end{array}$ & $\begin{array}{l}5.746 \\
(0.34)\end{array}$ & $\begin{array}{l}5.823 \\
(0.46)\end{array}$ \\
\hline
\end{tabular}

Fig. 3.9b. Pin-power distribution for NITAWL benchmark SAXTON-3. Value in parentheses is percent standard deviation. 
Table 3.25. SAXTON-3 CENTRM reaction rates and fluxes

\begin{tabular}{ccccccc}
\hline \multirow{2}{*}{ Pin } & Region & $\begin{array}{c}\mathrm{G}_{\mathrm{a}} \mathrm{M} \\
\left(\mathrm{cm}^{-3}-\mathrm{s}^{-1}\right)\end{array}$ & $\begin{array}{c}\mathrm{G}_{\mathrm{f}} \mathrm{M} \\
\left(\mathrm{cm}^{-3}-\mathrm{s}^{-1}\right)\end{array}$ & $\begin{array}{c}\mathrm{G}_{\mathrm{f}} \mathrm{M} \\
\left(\mathrm{cm}^{-3}-\mathrm{s}^{-1}\right)\end{array}$ & $\begin{array}{c}\mathrm{M} \\
\left(\mathrm{cm}^{-1}\right)\end{array}$ & $\mathrm{MM}_{\mathrm{t}}$ \\
\hline \multirow{2}{*}{1} & Fuel & $2.543 \mathrm{E}-05$ & $4.469 \mathrm{E}-05$ & $1.562 \mathrm{E}-05$ & $6.051 \mathrm{E}-03$ & 0.295 \\
& Clad & $2.340 \mathrm{E}-07$ & 0.0 & 0.0 & $1.781 \mathrm{E}-03$ & 0.087 \\
& Mod. & $1.588 \mathrm{E}-06$ & 0.0 & 0.0 & $1.266 \mathrm{E}-02$ & 0.618 \\
\hline \multirow{2}{*}{66} & Fuel & $3.944 \mathrm{E}-05$ & $5.901 \mathrm{E}-05$ & $2.061 \mathrm{E}-05$ & $1.985 \mathrm{E}-02$ & 0.292 \\
& Clad & $5.323 \mathrm{E}-07$ & 0.0 & 0.0 & $5.950 \mathrm{E}-03$ & 0.087 \\
& Mod. & $1.782 \mathrm{E}-06$ & 0.0 & 0.0 & $4.227 \mathrm{E}-02$ & 0.621 \\
\hline
\end{tabular}

Table 3.26. SAXTON-3 NITAWL reaction rates and fluxes

\begin{tabular}{|c|c|c|c|c|c|c|}
\hline Pin & Region & $\begin{array}{c}\mathrm{G}_{\mathrm{a}} \mathrm{M} \\
\left(\mathrm{cm}^{-3}-\mathrm{s}^{-1}\right)\end{array}$ & $\begin{array}{c}\mathcal{G}_{\mathrm{f}} \mathrm{M} \\
\left(\mathrm{cm}^{-3}-\mathrm{s}^{-1}\right)\end{array}$ & $\begin{array}{c}\mathrm{G}_{\mathrm{f}} \mathrm{M} \\
\left(\mathrm{cm}^{-3}-\mathrm{s}^{-1}\right)\end{array}$ & $\begin{array}{c}\mathrm{M} \\
\left(\mathrm{cm}-\mathrm{s}^{-1}\right)\end{array}$ & $M M_{t}$ \\
\hline \multirow{3}{*}{1} & Fuel & $2.574 \mathrm{E}-05$ & $4.529 \mathrm{E}-05$ & $1.589 \mathrm{E}-05$ & $6.060 \mathrm{E}-03$ & 0.295 \\
\hline & Clad & $2.313 \mathrm{E}-07$ & 0.0 & 0.0 & $1.785 \mathrm{E}-03$ & 0.087 \\
\hline & Mod. & 7.923E-07 & 0.0 & 0.0 & $1.270 \mathrm{E}-02$ & 0.618 \\
\hline \multirow{3}{*}{66} & Fuel & $3.956 \mathrm{E}-05$ & $5.952 \mathrm{E}-05$ & $2.079 \mathrm{E}-05$ & $1.999 \mathrm{E}-02$ & 0.293 \\
\hline & Clad & $5.281 \mathrm{E}-07$ & 0.0 & 0.0 & $5.968 \mathrm{E}-03$ & 0.087 \\
\hline & Mod. & $8.857 \mathrm{E}-07$ & 0.0 & 0.0 & 4.235E-02 & 0.620 \\
\hline
\end{tabular}


Table 3.27. SAXTON-3, Pin-1 four-group fluxes

\begin{tabular}{ccc}
\hline Group & $\begin{array}{c}\text { CENTRM M } \\
\left(\mathrm{cm}^{-2}-\mathrm{s}^{-1}\right)\end{array}$ & $\begin{array}{c}\text { NITAWL M } \\
\left(\mathrm{cm}^{-2}-\mathrm{s}^{-1}\right)\end{array}$ \\
\hline 1 & $7.796 \mathrm{E}-05$ & $7.800 \mathrm{E}-05$ \\
2 & $2.181 \mathrm{E}-05$ & $2.192 \mathrm{E}-05$ \\
3 & $4.829 \mathrm{E}-06$ & $4.689 \mathrm{E}-06$ \\
4 & $1.453 \mathrm{E}-05$ & $1.470 \mathrm{E}-05$ \\
\hline
\end{tabular}

Table 3.28. SAXTON-3, Pin-1 four-group U-235 cross sections

\begin{tabular}{cccccccc}
\hline Group & \multicolumn{3}{c}{ CENTRM cross sections $\left(\mathrm{cm}^{-1}\right)$} & \multicolumn{3}{c}{ NITAWL cross sections $\left(\mathrm{cm}^{-1}\right)$} \\
\hline & $\frac{\mathrm{G}_{\mathrm{a}}}{4.32521 \mathrm{E}-04}$ & $\frac{\mathrm{G}_{\mathrm{f}}}{5.28612 \mathrm{E}-04}$ & $\frac{\mathrm{G}_{\mathrm{f}}}{2.03658 \mathrm{E}-04}$ & $\frac{\mathrm{G}_{\mathrm{a}}}{2.32862 \mathrm{E}-04}$ & $\frac{\mathrm{G}_{\mathrm{f}}}{5.29454 \mathrm{E}-04}$ & $\frac{\mathrm{G}_{\mathrm{f}}}{2.03931 \mathrm{E}-04}$ \\
1 & $5.50005 \mathrm{E}-03$ & $8.43228 \mathrm{E}-03$ & $3.46053 \mathrm{E}-03$ & $5.39193 \mathrm{E}-03$ & $8.28878 \mathrm{E}-03$ & $3.40165 \mathrm{E}-03$ \\
2 & $7.92452 \mathrm{E}-03$ & $1.60789 \mathrm{E}-02$ & $6.59865 \mathrm{E}-03$ & $7.90441 \mathrm{E}-03$ & $1.60146 \mathrm{E}-02$ & $6.57223 \mathrm{E}-03$ \\
3 & $7.31686 \mathrm{E}-02$ & $1.52279 \mathrm{E}-01$ & $6.24939 \mathrm{E}-02$ & $7.33599 \mathrm{E}-02$ & $1.52663 \mathrm{E}-01$ & $6.26517 \mathrm{E}-02$ \\
\hline
\end{tabular}

Table 3.29. SAXTON-3, Pin-1 four-group U-238 cross sections

\begin{tabular}{cccccccc}
\hline Group & \multicolumn{3}{c}{ CENTRM cross sections $\left(\mathrm{cm}^{-1}\right)$} & \multicolumn{3}{c}{ NITAWL cross sections $\left(\mathrm{cm}^{-1}\right)$} \\
\hline & $\frac{\mathrm{G}_{\mathrm{a}}}{4.20136 \mathrm{E}-03}$ & $\frac{\mathrm{G}_{\mathrm{f}}}{1.33762 \mathrm{E}-02}$ & $\frac{\mathrm{G}_{\mathrm{f}}}{4.75174 \mathrm{E}-03}$ & $\frac{\mathrm{G}_{\mathrm{a}}}{7.23290 \mathrm{E}-03}$ & $\frac{\mathrm{G}_{\mathrm{f}}}{1.34483 \mathrm{E}-02}$ & $\frac{\mathrm{G}_{\mathrm{f}}}{4.77628 \mathrm{E}-03}$ \\
1 & $5.41784 \mathrm{E}-02$ & $5.69922 \mathrm{E}-06$ & $2.36116 \mathrm{E}-06$ & $5.29886 \mathrm{E}-02$ & $5.86670 \mathrm{E}-06$ & $2.43054 \mathrm{E}-06$ \\
2 & $1.12448 \mathrm{E}-02$ & $3.86080 \mathrm{E}-08$ & $1.59959 \mathrm{E}-08$ & $1.12161 \mathrm{E}-02$ & $3.84319 \mathrm{E}-08$ & $1.59231 \mathrm{E}-08$ \\
3 & $4.20736 \mathrm{E}-02$ & $1.97550 \mathrm{E}-07$ & $8.18486 \mathrm{E}-08$ & $4.21529 \mathrm{E}-02$ & $1.97922 \mathrm{E}-07$ & $8.20034 \mathrm{E}-08$ \\
\hline
\end{tabular}

Table 3.30. SAXTON-3, Pin-1 four-group Pu-239 cross sections

\begin{tabular}{cccccccc}
\hline Group & \multicolumn{3}{c}{ CENTRM cross sections $\left(\mathrm{cm}^{-1}\right)$} & \multicolumn{3}{c}{ NITAWL cross sections $\left(\mathrm{cm}^{-1}\right)$} \\
\hline & $\frac{\mathrm{G}_{\mathrm{a}}}{4.51444 \mathrm{E}-03}$ & $\frac{\mathrm{G}_{\mathrm{f}}}{7.33436 \mathrm{E}-03}$ & $\frac{\mathrm{G}_{\mathrm{f}}}{2.36219 \mathrm{E}-03}$ & $\frac{\mathrm{G}_{\mathrm{a}}}{2.51691 \mathrm{E}-03}$ & $\frac{\mathrm{G}_{\mathrm{f}}}{7.34278 \mathrm{E}-03}$ & $\frac{\mathrm{G}_{\mathrm{f}}}{2.36421 \mathrm{E}-03}$ \\
1 & $4.43213 \mathrm{E}-02$ & $7.47680 \mathrm{E}-02$ & $2.59628 \mathrm{E}-02$ & $4.17771 \mathrm{E}-02$ & $7.10830 \mathrm{E}-02$ & $2.46832 \mathrm{E}-02$ \\
2 & $1.44573 \mathrm{E}-01$ & $2.86496 \mathrm{E}-01$ & $9.94842 \mathrm{E}-02$ & $1.40383 \mathrm{E}-01$ & $2.78776 \mathrm{E}-01$ & $9.68036 \mathrm{E}-02$ \\
3 & $1.27177 \mathrm{E}+00$ & $2.55010 \mathrm{E}+00$ & $8.82257 \mathrm{E}-01$ & $1.28214 \mathrm{E}+00$ & $2.56855 \mathrm{E}+00$ & $8.88646 \mathrm{E}-01$ \\
\hline
\end{tabular}


Table 3.31. SAXTON-3, Pin-66 four-group fluxes

\begin{tabular}{ccc}
\hline Group & $\begin{array}{c}\text { CENTRM M } \\
\left(\mathrm{cm}^{-2}-\mathrm{s}^{-1}\right)\end{array}$ & $\begin{array}{c}\text { NITAWL M } \\
\left(\mathrm{cm}^{-2}-\mathrm{s}^{-1}\right)\end{array}$ \\
\hline 1 & $2.666 \mathrm{E}-04$ & $2.684 \mathrm{E}-04$ \\
2 & $9.313 \mathrm{E}-05$ & $9.364 \mathrm{E}-05$ \\
3 & $1.707 \mathrm{E}-05$ & $1.685 \mathrm{E}-05$ \\
4 & $1.407 \mathrm{E}-05$ & $1.456 \mathrm{E}-05$ \\
\hline
\end{tabular}

Table 3.32. SAXTON-3, Pin-66 four-group U-235 cross sections

\begin{tabular}{cccccccc}
\hline Group & \multicolumn{3}{c}{ CENTRM cross sections $\left(\mathrm{cm}^{-1}\right)$} & \multicolumn{3}{c}{ NITAWL cross sections $\left(\mathrm{cm}^{-1}\right)$} \\
\hline & $\frac{\mathrm{G}_{\mathrm{a}}}{2.42733 \mathrm{E}-04}$ & $\frac{\mathrm{G}_{\mathrm{f}}}{5.37472 \mathrm{E}-04}$ & $\frac{\mathrm{G}_{\mathrm{f}}}{2.08870 \mathrm{E}-04}$ & $\frac{\mathrm{G}_{\mathrm{a}}}{2.43387 \mathrm{E}-04}$ & $\frac{\mathrm{G}_{\mathrm{f}}}{5.37876 \mathrm{E}-04}$ & $\frac{\mathrm{G}_{\mathrm{f}}}{2.09178 \mathrm{E}-04}$ \\
1 & $5.30011 \mathrm{E}-03$ & $8.16540 \mathrm{E}-03$ & $3.35100 \mathrm{E}-03$ & $5.27422 \mathrm{E}-03$ & $8.12879 \mathrm{E}-03$ & $3.33598 \mathrm{E}-03$ \\
2 & $7.70943 \mathrm{E}-03$ & $1.55532 \mathrm{E}-02$ & $6.38289 \mathrm{E}-03$ & $7.67703 \mathrm{E}-03$ & $1.54383 \mathrm{E}-02$ & $6.33579 \mathrm{E}-03$ \\
3 & $6.52259 \mathrm{E}-02$ & $1.35439 \mathrm{E}-01$ & $5.55832 \mathrm{E}-02$ & $6.49576 \mathrm{E}-02$ & $1.34906 \mathrm{E}-01$ & $5.53641 \mathrm{E}-02$ \\
\hline
\end{tabular}

Table 3.33. SAXTON-3, Pin-66 four-group U-238 cross sections

\begin{tabular}{cccccccc}
\hline Group & \multicolumn{3}{c}{ CENTRM cross sections $\left(\mathrm{cm}^{-1}\right)$} & \multicolumn{3}{c}{ NITAWL cross sections $\left(\mathrm{cm}^{-1}\right)$} \\
\hline & $\frac{\mathrm{G}_{\mathrm{a}}}{6.92119 \mathrm{E}-03}$ & $\frac{\mathrm{G}_{\mathrm{f}}}{1.15793 \mathrm{E}-02}$ & $\frac{\mathrm{G}_{\mathrm{f}}}{4.11808 \mathrm{E}-03}$ & $\frac{\mathrm{G}_{\mathrm{a}}}{6.86967 \mathrm{E}-03}$ & $\frac{\mathrm{G}_{\mathrm{f}}}{1.13727 \mathrm{E}-02}$ & $\frac{\mathrm{G}_{\mathrm{f}}}{4.04314 \mathrm{E}-03}$ \\
1 & $5.09692 \mathrm{E}-02$ & $6.02439 \mathrm{E}-06$ & $2.49588 \mathrm{E}-06$ & $4.92231 \mathrm{E}-02$ & $6.08021 \mathrm{E}-06$ & $2.51901 \mathrm{E}-06$ \\
2 & $1.11677 \mathrm{E}-02$ & $3.78609 \mathrm{E}-08$ & $1.56865 \mathrm{E}-08$ & $1.11602 \mathrm{E}-02$ & $3.77671 \mathrm{E}-08$ & $1.56476 \mathrm{E}-08$ \\
3 & $3.79241 \mathrm{E}-02$ & $1.77598 \mathrm{E}-07$ & $7.35821 \mathrm{E}-08$ & $3.78323 \mathrm{E}-02$ & $1.77181 \mathrm{E}-07$ & $7.34093 \mathrm{E}-08$ \\
\hline
\end{tabular}

Table 3.34. SAXTON-3, Pin-66 four-group Pu-239 cross sections

\begin{tabular}{cccccccc}
\hline Group & \multicolumn{3}{c}{ CENTRM cross sections $\left(\mathrm{cm}^{-1}\right)$} & \multicolumn{3}{c}{ NITAWL cross sections $\left(\mathrm{cm}^{-1}\right)$} \\
\hline & $\frac{\mathrm{G}_{\mathrm{a}}}{2.52720 \mathrm{E}-03}$ & $\frac{\mathrm{G}_{\mathrm{f}}}{7.21032 \mathrm{E}-03}$ & $\frac{\mathrm{G}_{\mathrm{f}}}{2.34053 \mathrm{E}-03}$ & $\frac{\mathrm{G}_{\mathrm{a}}}{2.52750 \mathrm{E}-03}$ & $\frac{\mathrm{G}_{\mathrm{f}}}{7.19788 \mathrm{E}-03}$ & $\frac{\mathrm{G}_{\mathrm{f}}}{2.33835 \mathrm{E}-03}$ \\
1 & $4.12518 \mathrm{E}-02$ & $6.95573 \mathrm{E}-02$ & $2.41534 \mathrm{E}-02$ & $4.03271 \mathrm{E}-02$ & $6.74581 \mathrm{E}-02$ & $2.34245 \mathrm{E}-02$ \\
2 & $1.37500 \mathrm{E}-01$ & $2.73101 \mathrm{E}-01$ & $9.48330 \mathrm{E}-02$ & $1.37757 \mathrm{E}-01$ & $2.73564 \mathrm{E}-01$ & $9.49941 \mathrm{E}-02$ \\
3 & $1.43800 \mathrm{E}+00$ & $2.77757 \mathrm{E}+00$ & $9.61635 \mathrm{E}-01$ & $1.41120 \mathrm{E}+00$ & $2.73126 \mathrm{E}+00$ & $9.45577 \mathrm{E}-01$ \\
\hline
\end{tabular}




\begin{tabular}{|c|c|c|c|c|c|c|}
\hline \multirow[t]{6}{*}{$\begin{array}{l}0.218 \\
(0.73)\end{array}$} & & & & & & $\begin{array}{c}18.89 \\
(0.69)\end{array}$ \\
\hline & & & & & $\begin{array}{l}18.60 \\
(0.33)\end{array}$ & $\begin{array}{l}18.82 \\
(0.36)\end{array}$ \\
\hline & & & & $\begin{array}{l}17.23 \\
(0.35)\end{array}$ & $\begin{array}{l}17.81 \\
(0.24)\end{array}$ & $\begin{array}{l}18.18 \\
(0.33)\end{array}$ \\
\hline & & & $\begin{array}{l}14.90 \\
(0.35)\end{array}$ & $\begin{array}{l}16.09 \\
(0.26)\end{array}$ & $\begin{array}{c}1.6 .9 \\
(0.26)\end{array}$ & $\begin{array}{l}16.95 \\
(0.35)\end{array}$ \\
\hline & & $\begin{array}{r}12.39 \\
(0.38)\end{array}$ & $\begin{array}{l}13.62 \\
(0.29)\end{array}$ & $\begin{array}{l}14.58 \\
(0.24)\end{array}$ & $\begin{array}{l}15.18 \\
(0.25)\end{array}$ & $\begin{array}{l}15.37 \\
(0.38)\end{array}$ \\
\hline & $\begin{array}{c}10.54 \\
(0.30)\end{array}$ & $\begin{array}{c}11.44 \\
(0.28)\end{array}$ & $\begin{array}{c}12.55 \\
(0.28)\end{array}$ & $\begin{array}{c}13.40 \\
(0.26)\end{array}$ & $\begin{array}{l}13.91 \\
(0.27)\end{array}$ & $\begin{array}{c}14.11 \\
(0.40)\end{array}$ \\
\hline $\begin{array}{c}13.71 \\
(0.41)\end{array}$ & $\begin{array}{l}12.57 \\
(0.29)\end{array}$ & $\begin{array}{l}13.86 \\
(0.29)\end{array}$ & $\begin{array}{c}15.06 \\
(0.26)\end{array}$ & $\begin{array}{l}16.18 \\
(0.25)\end{array}$ & $\begin{array}{c}16.83 \\
(0.25)\end{array}$ & $\begin{array}{c}17.04 \\
(0.30)\end{array}$ \\
\hline
\end{tabular}

Fig. 3.10a. Pin-power distribution for CENTRM benchmark SAXTON-4. Value in parentheses is percent standard deviation.

\begin{tabular}{|c|c|c|c|c|c|c|}
\hline \multirow[t]{6}{*}{$\begin{array}{c}0.216 \\
(0.71)\end{array}$} & & & & & & $\begin{array}{l}19.22 \\
(0.63)\end{array}$ \\
\hline & & & & & $\begin{array}{l}18.69 \\
(0.34)\end{array}$ & $\begin{array}{l}18.74 \\
(0.33)\end{array}$ \\
\hline & & & & $\begin{array}{c}17.22 \\
(0.35)\end{array}$ & $\begin{array}{l}17.97 \\
(0.23)\end{array}$ & $\begin{array}{l}18.11 \\
(0.32)\end{array}$ \\
\hline & & & $\begin{array}{l}15.01 \\
(0.37)\end{array}$ & $\begin{array}{l}16.06 \\
(0.26)\end{array}$ & $\begin{array}{l}16.70 \\
(0.24)\end{array}$ & $\begin{array}{l}16.94 \\
(0.35)\end{array}$ \\
\hline & & $\begin{array}{l}12.38 \\
(0.42)\end{array}$ & $\begin{array}{c}13.63 \\
(0.29)\end{array}$ & $\begin{array}{l}14.56 \\
(0.25)\end{array}$ & $\begin{array}{c}15.19 \\
(0.26)\end{array}$ & $\begin{array}{l}15.46 \\
(0.37)\end{array}$ \\
\hline & $\begin{array}{c}10.58 \\
(0.45)\end{array}$ & $\begin{array}{c}11.45 \\
(0.28)\end{array}$ & $\begin{array}{l}12.55 \\
(0.30)\end{array}$ & $\begin{array}{l}13.47 \\
(0.29)\end{array}$ & $\begin{array}{l}14.02 \\
(0.27)\end{array}$ & $\begin{array}{l}14.19 \\
(0.39)\end{array}$ \\
\hline $\begin{array}{c}13.72 \\
(0.38)\end{array}$ & $\begin{array}{c}12.61 \\
(0.27)\end{array}$ & $\begin{array}{r}13.89 \\
(0.27)\end{array}$ & $\begin{array}{l}15.18 \\
(0.25)\end{array}$ & $\begin{array}{l}16.16 \\
(0.28)\end{array}$ & $\begin{array}{c}16.86 \\
(0.24)\end{array}$ & $\begin{array}{l}17.12 \\
(0.33)\end{array}$ \\
\hline
\end{tabular}

Fig. 3.10b. Pin-power distribution for NITAWL benchmark SAXTON-4. Value in parentheses is percent standard deviation. 
Table 3.35. SAXTON-4 CENTRM reaction rates and fluxes

\begin{tabular}{ccccccc}
\hline \multirow{2}{*}{ Pin } & Region & $\begin{array}{c}\mathrm{G}_{\mathrm{a}} \mathrm{M} \\
\left(\mathrm{cm}^{-3}-\mathrm{s}^{-1}\right)\end{array}$ & $\begin{array}{c}\mathrm{G}_{\mathrm{f}} \mathrm{M} \\
\left(\mathrm{cm}^{-3}-\mathrm{s}^{-1}\right)\end{array}$ & $\begin{array}{c}\mathrm{G}_{\mathrm{f}} \mathrm{M} \\
\left(\mathrm{cm}^{-3}-\mathrm{s}^{-1}\right)\end{array}$ & $\begin{array}{c}\mathrm{M} \\
\left(\mathrm{cm}^{-1}\right)\end{array}$ & $\mathrm{MM}_{\mathrm{t}}$ \\
\hline \multirow{2}{*}{1} & Fuel & $7.531 \mathrm{E}-05$ & $1.363 \mathrm{E}-04$ & $4.765 \mathrm{E}-05$ & $1.085 \mathrm{E}-02$ & 0.172 \\
& Clad & $6.208 \mathrm{E}-07$ & 0.0 & 0.0 & $3.159 \mathrm{E}-03$ & 0.050 \\
& Mod. & $3.325 \mathrm{E}-06$ & 0.0 & 0.0 & $4.911 \mathrm{E}-02$ & 0.778 \\
\hline \multirow{2}{*}{28} & Fuel & $1.133 \mathrm{E}-04$ & $1.888 \mathrm{E}-04$ & $6.597 \mathrm{E}-05$ & $2.644 \mathrm{E}-02$ & 0.170 \\
& Clad & $1.093 \mathrm{E}-06$ & 0.0 & 0.0 & $7.857 \mathrm{E}-03$ & 0.051 \\
& Mod. & $4.152 \mathrm{E}-06$ & 0.0 & 0.0 & $1.212 \mathrm{E}-01$ & 0.779 \\
\hline
\end{tabular}

Table 3.36. SAXTON-4 NITAWL reaction rates and fluxes

\begin{tabular}{ccccccc}
\hline Pin & Region & $\begin{array}{c}\mathrm{G}_{\mathrm{a}} \mathrm{M} \\
\left(\mathrm{cm}^{-3}-\mathrm{s}^{-1}\right)\end{array}$ & $\begin{array}{c}\mathrm{G}_{\mathrm{f}} \mathrm{M} \\
\left(\mathrm{cm}^{-3}-\mathrm{s}^{-1}\right)\end{array}$ & $\begin{array}{c}\mathrm{G}_{\mathrm{f}} \mathrm{M} \\
\left(\mathrm{cm}^{-3}-\mathrm{s}^{-1}\right)\end{array}$ & $\begin{array}{c}\mathrm{M} \\
\left(\mathrm{cm} \mathrm{-} \mathrm{s}^{-1}\right)\end{array}$ & $\mathrm{MM}_{\mathrm{t}}$ \\
\hline \multirow{2}{*}{1} & Fuel & $7.555 \mathrm{E}-05$ & $1.372 \mathrm{E}-04$ & $4.796 \mathrm{E}-05$ & $1.091 \mathrm{E}-02$ & 0.172 \\
& Clad & $6.298 \mathrm{E}-07$ & 0.0 & 0.0 & $3.186 \mathrm{E}-03$ & 0.050 \\
& Mod. & $1.677 \mathrm{E}-06$ & 0.0 & 0.0 & $4.926 \mathrm{E}-02$ & 0.778 \\
\hline \multirow{2}{*}{28} & Fuel & $1.153 \mathrm{E}-04$ & $1.923 \mathrm{E}-04$ & $6.721 \mathrm{E}-05$ & $2.661 \mathrm{E}-02$ & 0.171 \\
& Clad & $1.090 \mathrm{E}-06$ & 0.0 & 0.0 & $7.874 \mathrm{E}-03$ & 0.050 \\
& Mod. & $2.076 \mathrm{E}-06$ & 0.0 & 0.0 & $1.214 \mathrm{E}-01$ & 0.779 \\
\hline
\end{tabular}


Table 3.37. SAXTON-4, Pin-1 four-group fluxes

\begin{tabular}{ccc}
\hline Group & $\begin{array}{c}\text { CENTRM M } \\
\left(\mathrm{cm}^{-2}-\mathrm{s}^{-1}\right)\end{array}$ & $\begin{array}{c}\text { NITAWL M } \\
\left(\mathrm{cm}^{-2}-\mathrm{s}^{-1}\right)\end{array}$ \\
\hline 1 & $1.767 \mathrm{E}-04$ & $1.772 \mathrm{E}-04$ \\
2 & $4.238 \mathrm{E}-05$ & $4.314 \mathrm{E}-05$ \\
3 & $9.788 \mathrm{E}-06$ & $9.606 \mathrm{E}-06$ \\
4 & $4.606 \mathrm{E}-05$ & $4.644 \mathrm{E}-05$ \\
\hline
\end{tabular}

Table 3.38. SAXTON-4, Pin-1 four-group U-235 cross sections

\begin{tabular}{cccccccc}
\hline Group & \multicolumn{3}{c}{ CENTRM cross sections $\left(\mathrm{cm}^{-1}\right)$} & \multicolumn{3}{c}{ NITAWL cross sections $\left(\mathrm{cm}^{-1}\right)$} \\
\hline & $\frac{\mathrm{G}_{\mathrm{a}}}{2.28768 \mathrm{E}-04}$ & $\frac{\mathrm{G}_{\mathrm{f}}}{5.25936 \mathrm{E}-04}$ & $\frac{\mathrm{G}_{\mathrm{f}}}{2.01824 \mathrm{E}-04}$ & $\frac{\mathrm{G}_{\mathrm{a}}}{2.28888 \mathrm{E}-04}$ & $\frac{\mathrm{G}_{\mathrm{f}}}{5.26218 \mathrm{E}-04}$ & $\frac{\mathrm{G}_{\mathrm{f}}}{2.01905 \mathrm{E}-04}$ \\
1 & $5.58388 \mathrm{E}-03$ & $8.56052 \mathrm{E}-03$ & $3.51317 \mathrm{E}-03$ & $5.50211 \mathrm{E}-03$ & $8.45190 \mathrm{E}-03$ & $3.46859 \mathrm{E}-03$ \\
2 & $8.12596 \mathrm{E}-03$ & $1.65337 \mathrm{E}-02$ & $6.78529 \mathrm{E}-03$ & $8.00644 \mathrm{E}-03$ & $1.62619 \mathrm{E}-02$ & $6.67374 \mathrm{E}-03$ \\
3 & $7.52875 \mathrm{E}-02$ & $1.56735 \mathrm{E}-01$ & $6.43226 \mathrm{E}-02$ & $7.54139 \mathrm{E}-02$ & $1.57003 \mathrm{E}-01$ & $6.44324 \mathrm{E}-02$ \\
\hline
\end{tabular}

Table 3.39. SAXTON-4, Pin-1 four-group U-238 cross sections

\begin{tabular}{cccccccc}
\hline Group & \multicolumn{3}{c}{ CENTRM cross sections $\left(\mathrm{cm}^{-1}\right)$} & \multicolumn{3}{c}{ NITAWL cross sections $\left(\mathrm{cm}^{-1}\right)$} \\
\hline & $\frac{\mathrm{G}_{\mathrm{a}}}{4.34533 \mathrm{E}-03}$ & $\frac{\mathrm{G}_{\mathrm{f}}}{1.41658 \mathrm{E}-02}$ & $\frac{\mathrm{G}_{\mathrm{f}}}{5.02602 \mathrm{E}-03}$ & $\frac{7.35536 \mathrm{E}-03}{1.41852 \mathrm{E}-02}$ & $\frac{\mathrm{G}_{\mathrm{f}}}{5.03150 \mathrm{E}-03}$ \\
1 & $5.77836 \mathrm{E}-02$ & $6.06902 \mathrm{E}-06$ & $2.51437 \mathrm{E}-06$ & $5.53505 \mathrm{E}-02$ & $5.94471 \mathrm{E}-06$ & $2.46287 \mathrm{E}-06$ \\
2 & $1.12800 \mathrm{E}-02$ & $3.90072 \mathrm{E}-08$ & $1.61613 \mathrm{E}-08$ & $1.12638 \mathrm{E}-02$ & $3.87636 \mathrm{E}-08$ & $1.60605 \mathrm{E}-08$ \\
3 & $4.31348 \mathrm{E}-02$ & $2.02633 \mathrm{E}-07$ & $8.39546 \mathrm{E}-08$ & $4.31960 \mathrm{E}-02$ & $2.02927 \mathrm{E}-07$ & $8.40764 \mathrm{E}-08$ \\
\hline
\end{tabular}

Table 3.40. SAXTON-4, Pin-1 four-group Pu-239 cross sections

\begin{tabular}{cccccccc}
\hline Group & \multicolumn{3}{c}{ CENTRM cross sections $\left(\mathrm{cm}^{-1}\right)$} & \multicolumn{3}{c}{ NITAWL cross sections $\left(\mathrm{cm}^{-1}\right)$} \\
\hline & $\frac{\mathrm{G}_{\mathrm{a}}}{2.51088 \mathrm{E}-03}$ & $\frac{\mathrm{G}_{\mathrm{f}}}{7.39032 \mathrm{E}-03}$ & $\frac{\mathrm{G}_{\mathrm{f}}}{2.37191 \mathrm{E}-03}$ & $\frac{\mathrm{G}_{\mathrm{a}}}{2.51148 \mathrm{E}-03}$ & $\frac{\mathrm{G}_{\mathrm{f}}}{7.39283 \mathrm{E}-03}$ & $\frac{\mathrm{G}_{\mathrm{f}}}{2.37221 \mathrm{E}-03}$ \\
1 & $4.48570 \mathrm{E}-02$ & $7.50776 \mathrm{E}-02$ & $2.60703 \mathrm{E}-02$ & $4.28458 \mathrm{E}-02$ & $7.19822 \mathrm{E}-02$ & $2.49954 \mathrm{E}-02$ \\
2 & $1.48740 \mathrm{E}-01$ & $2.94317 \mathrm{E}-01$ & $1.02200 \mathrm{E}-01$ & $1.46544 \mathrm{E}-01$ & $2.90227 \mathrm{E}-01$ & $1.00780 \mathrm{E}-01$ \\
3 & $1.25958 \mathrm{E}+00$ & $2.54316 \mathrm{E}+00$ & $8.79726 \mathrm{E}-01$ & $1.25886 \mathrm{E}+00$ & $2.54285 \mathrm{E}+00$ & $8.79615 \mathrm{E}-01$ \\
\hline
\end{tabular}


Table 3.41. SAXTON-4, Pin-28 four-group fluxes

\begin{tabular}{ccc}
\hline Group & $\begin{array}{c}\text { CENTRM M } \\
\left(\mathrm{cm}^{-2}-\mathrm{s}^{-1}\right)\end{array}$ & $\begin{array}{c}\text { NITAWL M } \\
\left(\mathrm{cm}^{-2}-\mathrm{s}^{-1}\right)\end{array}$ \\
\hline 1 & $4.435 \mathrm{E}-04$ & $4.456 \mathrm{E}-04$ \\
2 & $1.401 \mathrm{E}-04$ & $1.409 \mathrm{E}-04$ \\
3 & $2.995 \mathrm{E}-05$ & $2.997 \mathrm{E}-05$ \\
4 & $5.644 \mathrm{E}-05$ & $5.789 \mathrm{E}-05$ \\
\hline
\end{tabular}

Table 3.42. SAXTON-4, Pin-28 four-group U-235 cross sections

\begin{tabular}{cccccccc}
\hline Group & \multicolumn{3}{c}{ CENTRM cross sections $\left(\mathrm{cm}^{-1}\right)$} & \multicolumn{3}{c}{ NITAWL cross sections $\left(\mathrm{cm}^{-1}\right)$} \\
\hline & $\frac{\mathrm{G}_{\mathrm{a}}}{4.37576 \mathrm{E}-04}$ & $\frac{\mathrm{G}_{\mathrm{f}}}{5.33610 \mathrm{E}-04}$ & $\frac{\mathrm{G}_{\mathrm{f}}}{2.06345 \mathrm{E}-04}$ & $\frac{\mathrm{G}_{\mathrm{a}}}{2.36654 \mathrm{E}-04}$ & $\frac{\mathrm{G}_{\mathrm{f}}}{5.32933 \mathrm{E}-04}$ & $\frac{\mathrm{G}_{\mathrm{f}}}{2.05880 \mathrm{E}-04}$ \\
1 & $5.42624 \mathrm{E}-03$ & $8.34946 \mathrm{E}-03$ & $3.42656 \mathrm{E}-03$ & $5.49204 \mathrm{E}-03$ & $8.44613 \mathrm{E}-03$ & $3.46621 \mathrm{E}-03$ \\
2 & $8.00180 \mathrm{E}-03$ & $1.62249 \mathrm{E}-02$ & $6.65856 \mathrm{E}-03$ & $7.91521 \mathrm{E}-03$ & $1.60134 \mathrm{E}-02$ & $6.57174 \mathrm{E}-03$ \\
3 & $7.09472 \mathrm{E}-02$ & $1.47553 \mathrm{E}-01$ & $6.05542 \mathrm{E}-02$ & $7.02795 \mathrm{E}-02$ & $1.46150 \mathrm{E}-01$ & $5.99789 \mathrm{E}-02$ \\
\hline
\end{tabular}

Table 3.43. SAXTON-4, Pin-28 four-group U-238 cross sections

\begin{tabular}{cccccccc}
\hline Group & \multicolumn{3}{c}{ CENTRM cross sections $\left(\mathrm{cm}^{-1}\right)$} & \multicolumn{3}{c}{ NITAWL cross sections $\left(\mathrm{cm}^{-1}\right)$} \\
\hline & $\frac{\mathrm{G}_{\mathrm{a}}}{7.11109 \mathrm{E}-03}$ & $\frac{\mathrm{G}_{\mathrm{f}}}{1.26244 \mathrm{E}-02}$ & $\frac{\mathrm{G}_{\mathrm{f}}}{4.48453 \mathrm{E}-03}$ & $\frac{7.14856 \mathrm{E}-03}{\mathrm{G}}$ & $\frac{\mathrm{G}_{\mathrm{f}}}{1.28317 \mathrm{E}-02}$ & $\frac{\mathrm{G}_{\mathrm{f}}}{4.55624 \mathrm{E}-03}$ \\
1 & $5.52425 \mathrm{E}-02$ & $5.99818 \mathrm{E}-06$ & $2.48502 \mathrm{E}-06$ & $5.40680 \mathrm{E}-02$ & $5.89807 \mathrm{E}-06$ & $2.44355 \mathrm{E}-06$ \\
2 & $1.12522 \mathrm{E}-02$ & $3.86845 \mathrm{E}-08$ & $1.60276 \mathrm{E}-08$ & $1.12054 \mathrm{E}-02$ & $3.82593 \mathrm{E}-08$ & $1.58516 \mathrm{E}-08$ \\
3 & $4.09068 \mathrm{E}-02$ & $1.91933 \mathrm{E}-07$ & $7.95213 \mathrm{E}-08$ & $4.05719 \mathrm{E}-02$ & $1.90337 \mathrm{E}-07$ & $7.88606 \mathrm{E}-08$ \\
\hline
\end{tabular}

Table 3.44. SAXTON-4, Pin-28 four-group Pu-239 cross sections

\begin{tabular}{|c|c|c|c|c|c|c|}
\hline \multirow[t]{2}{*}{ Group } & \multicolumn{3}{|c|}{ CENTRM cross sections $\left(\mathrm{cm}^{-1}\right)$} & \multicolumn{3}{|c|}{ NITAWL cross sections $\left(\mathrm{cm}^{-1}\right)$} \\
\hline & $\mathrm{G}_{\mathrm{a}}$ & $\mathrm{G}_{\mathrm{f}}$ & $\mathrm{G}_{\mathrm{f}}$ & $\mathrm{G}_{\mathrm{a}}$ & $<\mathrm{G}_{\mathrm{f}}$ & $\mathrm{G}_{\mathrm{f}}$ \\
\hline 1 & $2 . \overline{52286 \mathrm{E}-03}$ & $\overline{7.28582 \mathrm{E}-03}$ & $\overline{2.35416 \mathrm{E}-03}$ & $2 . \overline{52139 \mathrm{E}-03}$ & $\overline{7.29800 \mathrm{E}-03}$ & $\overline{2.35595 \mathrm{E}-03}$ \\
\hline 2 & 4.55287E-02 & 7.65767E-02 & 2.65909E-02 & 4.36759E-02 & 7.33527E-02 & 2.54713E-02 \\
\hline 3 & $1.45672 \mathrm{E}-01$ & $2.88541 \mathrm{E}-01$ & $1.00195 \mathrm{E}-01$ & $1.41609 \mathrm{E}-01$ & $2.80817 \mathrm{E}-01$ & $9.75128 \mathrm{E}-02$ \\
\hline 4 & $1.32655 \mathrm{E}+00$ & $2.62741 \mathrm{E}+00$ & $9.09199 \mathrm{E}-01$ & $1.32947 \mathrm{E}+00$ & $2.62807 \mathrm{E}+00$ & $9.09471 \mathrm{E}-01$ \\
\hline
\end{tabular}




\begin{tabular}{|c|c|c|c|c|c|}
\hline \multirow[t]{5}{*}{$\begin{array}{l}0.274 \\
(0.94)\end{array}$} & & & & & $\begin{array}{l}20.09 \\
(0.35)\end{array}$ \\
\hline & & & & $\begin{array}{c}18.84 \\
(0.33)\end{array}$ & $\begin{array}{c}19.55 \\
(0.23)\end{array}$ \\
\hline & & & $\begin{array}{l}16.65 \\
(0.35)\end{array}$ & $\begin{array}{l}17.71 \\
(0.21)\end{array}$ & $\begin{array}{l}18.36 \\
(0.23)\end{array}$ \\
\hline & & $\begin{array}{c}13.79 \\
(0.34)\end{array}$ & $\begin{array}{l}15.16 \\
(0.22)\end{array}$ & $\begin{array}{l}16.11 \\
(0.24)\end{array}$ & $\begin{array}{l}16.66 \\
(0.24)\end{array}$ \\
\hline & $\begin{array}{l}11.35 \\
(0.40)\end{array}$ & $\begin{array}{c}12.45 \\
(0.27)\end{array}$ & $\begin{array}{l}13.69 \\
(0.26)\end{array}$ & $\begin{array}{l}14.59 \\
(0.26)\end{array}$ & $\begin{array}{l}15.00 \\
(0.23)\end{array}$ \\
\hline $\begin{array}{c}13.36 \\
(0.35)\end{array}$ & $\begin{array}{l}12.75 \\
(0.28)\end{array}$ & $\begin{array}{c}14.14 \\
(0.23)\end{array}$ & $\begin{array}{l}15.59 \\
(0.23)\end{array}$ & $\begin{array}{l}16.47 \\
(0.23)\end{array}$ & $\begin{array}{c}17.04 \\
(0.24)\end{array}$ \\
\hline
\end{tabular}

Fig. 3.11a. Pin-power distribution for CENTRM benchmark SAXTON-5. Value in parentheses is percent standard deviation.

\begin{tabular}{|c|c|c|c|c|c|}
\hline \multirow[t]{5}{*}{$\begin{array}{l}0.273 \\
(0.90)\end{array}$} & & & & & $\begin{array}{l}20.26 \\
(0.30)\end{array}$ \\
\hline & & & & $\begin{array}{l}19.04 \\
(0.33)\end{array}$ & $\begin{array}{l}19.51 \\
(0.21)\end{array}$ \\
\hline & & & $\begin{array}{l}16.69 \\
(0.35)\end{array}$ & $\begin{array}{c}17.88 \\
(0.23)\end{array}$ & $\begin{array}{l}18.32 \\
(0.22)\end{array}$ \\
\hline & & $\begin{array}{l}13.84 \\
(0.38)\end{array}$ & $\begin{array}{l}15.25 \\
(0.24)\end{array}$ & $\begin{array}{l}16.23 \\
(0.23)\end{array}$ & $\begin{array}{l}16.66 \\
(0.23)\end{array}$ \\
\hline & $\begin{array}{l}11.26 \\
(0.44)\end{array}$ & $\begin{array}{c}12.43 \\
(0.29)\end{array}$ & $\begin{array}{c}13.78 \\
(0.25)\end{array}$ & $\begin{array}{l}14.67 \\
(0.26)\end{array}$ & $\begin{array}{l}15.07 \\
(0.25)\end{array}$ \\
\hline $\begin{array}{l}13.29 \\
(0.34)\end{array}$ & $\begin{array}{l}12.74 \\
(0.26)\end{array}$ & $\begin{array}{l}14.20 \\
(0.24)\end{array}$ & $\begin{array}{c}15.58 \\
(0.22)\end{array}$ & $\begin{array}{l}16.46 \\
(0.22)\end{array}$ & $\begin{array}{l}17.07 \\
(0.24)\end{array}$ \\
\hline
\end{tabular}

Fig. 3.11b. Pin-power distribution for NITAWL Benchmark SAXTON-5. Value in parentheses is percent standard deviation. 
Table 3.45. SAXTON-5 CENTRM reaction rates and fluxes

\begin{tabular}{|c|c|c|c|c|c|c|}
\hline Pin & Region & $\begin{array}{c}\mathrm{G}_{\mathrm{a}} \mathrm{M} \\
\left(\mathrm{cm}^{-3}-\mathrm{s}^{-1}\right)\end{array}$ & $\begin{array}{c}\mathcal{G}_{\mathrm{f}} \mathrm{M} \\
\left(\mathrm{cm}^{-3}-\mathrm{s}^{-1}\right)\end{array}$ & $\begin{array}{c}\mathrm{G}_{\mathrm{f}} \mathrm{M} \\
\left(\mathrm{cm}^{-3}-\mathrm{s}^{-1}\right)\end{array}$ & $\begin{array}{c}M \\
\left(\mathrm{~cm}^{\left.-\mathrm{s}^{-1}\right)}\right.\end{array}$ & $\mathrm{MM}_{\mathrm{t}}$ \\
\hline \multirow{3}{*}{1} & Fuel & $7.340 \mathrm{E}-05$ & $1.334 \mathrm{E}-04$ & 4.665E-05 & $1.142 \mathrm{E}-02$ & 0.148 \\
\hline & Clad & $6.001 \mathrm{E}-07$ & 0.0 & 0.0 & $3.327 \mathrm{E}-03$ & 0.043 \\
\hline & Mod. & 3.369E-06 & 0.0 & 0.0 & $6.246 \mathrm{E}-02$ & 0.809 \\
\hline \multirow{3}{*}{21} & Fuel & $1.175 \mathrm{E}-04$ & $2.005 \mathrm{E}-04$ & $7.008 \mathrm{E}-05$ & $2.753 \mathrm{E}-02$ & 0.147 \\
\hline & Clad & $1.081 \mathrm{E}-06$ & 0.0 & 0.0 & 8.169E-03 & 0.043 \\
\hline & Mod. & $4.571 \mathrm{E}-06$ & 0.0 & 0.0 & $1.519 \mathrm{E}-01$ & 0.810 \\
\hline
\end{tabular}

Table 3.46. SAXTON-5 NITAWL reaction rates and fluxes

\begin{tabular}{ccccccc}
\hline Pin & Region & $\begin{array}{c}\mathrm{G}_{\mathrm{a}} \mathrm{M} \\
\left(\mathrm{cm}^{-3}-\mathrm{s}^{-1}\right)\end{array}$ & $\begin{array}{c}\mathrm{G}_{\mathrm{f}} \mathrm{M} \\
\left(\mathrm{cm}^{-3}-\mathrm{s}^{-1}\right)\end{array}$ & $\begin{array}{c}\mathrm{G}_{\mathrm{f}} \mathrm{M} \\
\left(\mathrm{cm}^{-3}-\mathrm{s}^{-1}\right)\end{array}$ & $\begin{array}{c}\mathrm{M} \\
\left({\left.\mathrm{cm}-\mathrm{s}^{-1}\right)}^{2}\right.\end{array}$ & $\mathrm{MM}_{\mathrm{t}}$ \\
\hline \multirow{2}{*}{1} & Fuel & $7.276 \mathrm{E}-05$ & $1.326 \mathrm{E}-04$ & $4.636 \mathrm{E}-05$ & $1.134 \mathrm{E}-02$ & 0.147 \\
& Clad & $5.939 \mathrm{E}-07$ & 0.0 & 0.0 & $3.314 \mathrm{E}-03$ & 0.043 \\
& Mod. & $1.683 \mathrm{E}-06$ & 0.0 & 0.0 & $6.233 \mathrm{E}-02$ & 0.810 \\
\hline \multirow{2}{*}{21} & Fuel & $1.185 \mathrm{E}-04$ & $2.024 \mathrm{E}-04$ & $7.072 \mathrm{E}-05$ & $2.776 \mathrm{E}-02$ & 0.147 \\
& Clad & $1.081 \mathrm{E}-06$ & 0.0 & 0.0 & $8.220 \mathrm{E}-03$ & 0.044 \\
& Mod. & $2.306 \mathrm{E}-06$ & 0.0 & 0.0 & $1.528 \mathrm{E}-01$ & 0.809 \\
\hline
\end{tabular}


Table 3.47. SAXTON-5, Pin-1 four-group fluxes

\begin{tabular}{ccc}
\hline Group & $\begin{array}{c}\text { CENTRM M } \\
\left(\mathrm{cm}^{-2}-\mathrm{s}^{-1}\right)\end{array}$ & $\begin{array}{c}\text { NITAWL M } \\
\left(\mathrm{cm}^{-2}-\mathrm{s}^{-1}\right)\end{array}$ \\
\hline 1 & $1.642 \mathrm{E}-04$ & $1.632 \mathrm{E}-04$ \\
2 & $3.938 \mathrm{E}-05$ & $3.897 \mathrm{E}-05$ \\
3 & $9.086 \mathrm{E}-06$ & $8.830 \mathrm{E}-06$ \\
4 & $4.517 \mathrm{E}-05$ & $4.505 \mathrm{E}-05$ \\
\hline
\end{tabular}

Table 3.48. SAXTON-5, Pin-1 four-group U-235 cross sections

\begin{tabular}{cccccccc}
\hline Group & \multicolumn{3}{c}{ CENTRM cross sections $\left(\mathrm{cm}^{-1}\right)$} & \multicolumn{3}{c}{ NITAWL cross sections $\left(\mathrm{cm}^{-1}\right)$} \\
\hline & $\frac{\mathrm{G}_{\mathrm{a}}}{4.28516 \mathrm{E}-04}$ & $\frac{\mathrm{G}_{\mathrm{f}}}{5.26334 \mathrm{E}-04}$ & $\frac{\mathrm{G}_{\mathrm{f}}}{2.01776 \mathrm{E}-04}$ & $\frac{\mathrm{G}_{\mathrm{a}}}{2.28104 \mathrm{E}-04}$ & $\frac{\mathrm{G}_{\mathrm{f}}}{5.25678 \mathrm{E}-04}$ & $\frac{\mathrm{G}_{\mathrm{f}}}{2.01507 \mathrm{E}-04}$ \\
1 & $5.57692 \mathrm{E}-03$ & $8.53438 \mathrm{E}-03$ & $3.50244 \mathrm{E}-03$ & $5.57593 \mathrm{E}-03$ & $8.52166 \mathrm{E}-03$ & $3.49720 \mathrm{E}-03$ \\
2 & $8.00912 \mathrm{E}-03$ & $1.62733 \mathrm{E}-02$ & $6.67842 \mathrm{E}-03$ & $7.98496 \mathrm{E}-03$ & $1.61621 \mathrm{E}-02$ & $6.63276 \mathrm{E}-03$ \\
3 & $7.58687 \mathrm{E}-02$ & $1.57957 \mathrm{E}-01$ & $6.48244 \mathrm{E}-02$ & $7.56584 \mathrm{E}-02$ & $1.57516 \mathrm{E}-01$ & $6.46433 \mathrm{E}-02$ \\
\hline
\end{tabular}

Table 3.49. SAXTON-5, Pin-1 four-group U-238 cross sections

\begin{tabular}{cccccccc}
\hline Group & \multicolumn{3}{c}{ CENTRM cross sections $\left(\mathrm{cm}^{-1}\right)$} & \multicolumn{3}{c}{ NITAWL cross sections $\left(\mathrm{cm}^{-1}\right)$} \\
\hline & $\frac{\mathrm{G}_{\mathrm{a}}}{7.40499 \mathrm{E}-03}$ & $\frac{\mathrm{G}_{\mathrm{f}}}{1.43721 \mathrm{E}-02}$ & $\frac{\mathrm{G}_{\mathrm{f}}}{5.09562 \mathrm{E}-03}$ & $\frac{7.36961 \mathrm{E}-03}{\mathrm{G}}$ & $\frac{\mathrm{G}_{\mathrm{f}}}{1.43170 \mathrm{E}-02}$ & $\frac{\mathrm{G}_{\mathrm{f}}}{5.07374 \mathrm{E}-03}$ \\
1 & $6.01938 \mathrm{E}-02$ & $6.08276 \mathrm{E}-06$ & $2.52006 \mathrm{E}-06$ & $5.60690 \mathrm{E}-02$ & $5.89946 \mathrm{E}-06$ & $2.44412 \mathrm{E}-06$ \\
2 & $1.12744 \mathrm{E}-02$ & $3.88600 \mathrm{E}-08$ & $1.61004 \mathrm{E}-08$ & $1.12557 \mathrm{E}-02$ & $3.87165 \mathrm{E}-08$ & $1.60410 \mathrm{E}-08$ \\
3 & $4.34215 \mathrm{E}-02$ & $2.04004 \mathrm{E}-07$ & $8.45229 \mathrm{E}-08$ & $4.33185 \mathrm{E}-02$ & $2.03512 \mathrm{E}-07$ & $8.43190 \mathrm{E}-08$ \\
\hline
\end{tabular}

Table 3.50. SAXTON-5, Pin-1 four-group Pu-239 cross sections

\begin{tabular}{|c|c|c|c|c|c|c|}
\hline \multirow[t]{2}{*}{ Group } & \multicolumn{3}{|c|}{ CENTRM cross sections $\left(\mathrm{cm}^{-1}\right)$} & \multicolumn{3}{|c|}{ NITAWL cross sections $\left(\mathrm{cm}^{-1}\right)$} \\
\hline & $\mathrm{G}_{\mathrm{a}}$ & $\mathrm{G}_{\mathrm{f}}$ & $\mathrm{G}_{\mathrm{f}}$ & $\mathrm{G}_{\mathrm{a}}$ & $<\mathrm{G}_{\mathrm{f}}$ & $\mathrm{G}_{\mathrm{f}}$ \\
\hline 1 & $2 . \overline{51193 \mathrm{E}-03}$ & $\overline{7.40536 \mathrm{E}-03}$ & $\overline{2.37431 \mathrm{E}-03}$ & $2 . \overline{51011 \mathrm{E}-03}$ & $\overline{7.40294 \mathrm{E}-03}$ & $\overline{2.37356 \mathrm{E}-03}$ \\
\hline 2 & 4.59985E-02 & 7.75008E-02 & $2.69116 \mathrm{E}-02$ & 4.40523E-02 & 7.41950E-02 & 2.57639E-02 \\
\hline 3 & $1.50511 \mathrm{E}-01$ & $2.97520 \mathrm{E}-01$ & $1.03313 \mathrm{E}-01$ & $1.47223 \mathrm{E}-01$ & $2.91456 \mathrm{E}-01$ & $1.01207 \mathrm{E}-01$ \\
\hline 4 & $1.25860 \mathrm{E}+00$ & $2.54527 \mathrm{E}+00$ & 8.80429E-01 & $1.25753 \mathrm{E}+00$ & $2.54209 \mathrm{E}+00$ & 8.79338E-01 \\
\hline
\end{tabular}


Table 3.51. SAXTON-5, Pin-21 four-group fluxes

\begin{tabular}{ccc}
\hline Group & $\begin{array}{c}\text { CENTRM M } \\
\left(\mathrm{cm}^{-2}-\mathrm{s}^{-1}\right)\end{array}$ & $\begin{array}{c}\text { NITAWL M } \\
\left(\mathrm{cm}^{-2}-\mathrm{s}^{-1}\right)\end{array}$ \\
\hline 1 & $4.092 \mathrm{E}-04$ & $4.112 \mathrm{E}-04$ \\
2 & $1.239 \mathrm{E}-04$ & $1.261 \mathrm{E}-04$ \\
3 & $2.677 \mathrm{E}-05$ & $2.696 \mathrm{E}-05$ \\
4 & $6.193 \mathrm{E}-05$ & $6.260 \mathrm{E}-05$ \\
\hline
\end{tabular}

Table 3.52. SAXTON-5, Pin-21 four-group U-235 cross sections

\begin{tabular}{cccccccc}
\hline Group & \multicolumn{3}{c}{ CENTRM cross sections $\left(\mathrm{cm}^{-1}\right)$} & \multicolumn{2}{c}{ NITAWL cross sections $\left(\mathrm{cm}^{-1}\right)$} \\
\hline & $\frac{\mathrm{G}_{\mathrm{a}}}{2.36162 \mathrm{E}-04}$ & $\frac{\mathrm{G}_{\mathrm{f}}}{5.32867 \mathrm{E}-04}$ & $\frac{\mathrm{G}_{\mathrm{f}}}{2.05677 \mathrm{E}-04}$ & $\frac{\mathrm{G}_{\mathrm{a}}}{2.35623 \mathrm{E}-04}$ & $\frac{\mathrm{G}_{\mathrm{f}}}{5.32179 \mathrm{E}-04}$ & $\frac{\mathrm{G}_{\mathrm{f}}}{2.05354 \mathrm{E}-04}$ \\
1 & $5.48935 \mathrm{E}-03$ & $8.40097 \mathrm{E}-03$ & $3.44768 \mathrm{E}-03$ & $5.44674 \mathrm{E}-03$ & $8.35559 \mathrm{E}-03$ & $3.42905 \mathrm{E}-03$ \\
2 & $7.96989 \mathrm{E}-03$ & $1.61823 \mathrm{E}-02$ & $6.64109 \mathrm{E}-03$ & $7.98750 \mathrm{E}-03$ & $1.62231 \mathrm{E}-02$ & $6.65779 \mathrm{E}-03$ \\
3 & $7.21016 \mathrm{E}-02$ & $1.49992 \mathrm{E}-01$ & $6.15555 \mathrm{E}-02$ & $7.19091 \mathrm{E}-02$ & $1.49587 \mathrm{E}-01$ & $6.13891 \mathrm{E}-02$ \\
\hline
\end{tabular}

Table 3.53. SAXTON-5, Pin-21 four-group U-238 cross sections

\begin{tabular}{cccccccc}
\hline Group & \multicolumn{3}{c}{ CENTRM cross sections $\left(\mathrm{cm}^{-1}\right)$} & \multicolumn{3}{c}{ NITAWL cross sections $\left(\mathrm{cm}^{-1}\right)$} \\
\hline & $\frac{\mathrm{G}_{\mathrm{a}}}{4.19130 \mathrm{E}-03}$ & $\frac{\varangle \mathrm{G}_{\mathrm{f}}}{1.30115 \mathrm{E}-02}$ & $\frac{\mathrm{G}_{\mathrm{f}}}{4.61767 \mathrm{E}-03}$ & $\frac{\mathrm{G}_{\mathrm{a}}}{7.19283 \mathrm{E}-03}$ & $\frac{\mathrm{G}_{\mathrm{f}}}{1.30642 \mathrm{E}-02}$ & $\frac{\mathrm{G}_{\mathrm{f}}}{4.63670 \mathrm{E}-03}$ \\
1 & $5.75995 \mathrm{E}-02$ & $6.14358 \mathrm{E}-06$ & $2.54525 \mathrm{E}-06$ & $5.55646 \mathrm{E}-02$ & $6.04202 \mathrm{E}-06$ & $2.50319 \mathrm{E}-06$ \\
2 & $1.12575 \mathrm{E}-02$ & $3.87725 \mathrm{E}-08$ & $1.60642 \mathrm{E}-08$ & $1.12617 \mathrm{E}-02$ & $3.87641 \mathrm{E}-08$ & $1.60607 \mathrm{E}-08$ \\
3 & $4.14883 \mathrm{E}-02$ & $1.94726 \mathrm{E}-07$ & $8.06790 \mathrm{E}-08$ & $4.13946 \mathrm{E}-02$ & $1.94278 \mathrm{E}-07$ & $8.04931 \mathrm{E}-08$ \\
\hline
\end{tabular}

Table 3.54. SAXTON-5, Pin-21 four-group Pu-239 cross sections

\begin{tabular}{cccccccc}
\hline Group & \multicolumn{3}{c}{ CENTRM cross sections $\left(\mathrm{cm}^{-1}\right)$} & \multicolumn{3}{c}{ NITAWL cross sections $\left(\mathrm{cm}^{-1}\right)$} \\
\hline & $\frac{\mathrm{G}_{\mathrm{a}}}{2.52182 \mathrm{E}-03}$ & $\frac{\mathrm{G}_{\mathrm{f}}}{7.31114 \mathrm{E}-03}$ & $\frac{\mathrm{G}_{\mathrm{f}}}{2.35818 \mathrm{E}-03}$ & $\frac{\mathrm{G}_{\mathrm{a}}}{2.52033 \mathrm{E}-03}$ & $\frac{\mathrm{G}_{\mathrm{f}}}{7.31286 \mathrm{E}-03}$ & $\frac{\mathrm{G}_{\mathrm{f}}}{2.35814 \mathrm{E}-03}$ \\
1 & $4.59107 \mathrm{E}-02$ & $7.65671 \mathrm{E}-02$ & $2.65875 \mathrm{E}-02$ & $4.38622 \mathrm{E}-02$ & $7.38489 \mathrm{E}-02$ & $2.56436 \mathrm{E}-02$ \\
2 & $1.46728 \mathrm{E}-01$ & $2.90546 \mathrm{E}-01$ & $1.00891 \mathrm{E}-01$ & $1.47469 \mathrm{E}-01$ & $2.91907 \mathrm{E}-01$ & $1.01364 \mathrm{E}-01$ \\
3 & $1.31306 \mathrm{E}+00$ & $2.61230 \mathrm{E}+00$ & $9.03898 \mathrm{E}-01$ & $1.31380 \mathrm{E}+00$ & $2.61230 \mathrm{E}+00$ & $9.03907 \mathrm{E}-01$ \\
\hline
\end{tabular}




\begin{tabular}{|c|c|c|c|c|c|}
\hline \multirow[t]{5}{*}{$\begin{array}{l}0.349 \\
(1.00)\end{array}$} & & & & & $\begin{array}{l}26.71 \\
(0.54)\end{array}$ \\
\hline & & & & $\begin{array}{l}25.24 \\
(0.26)\end{array}$ & $\begin{array}{l}26.06 \\
(0.27)\end{array}$ \\
\hline & & & $\begin{array}{l}22.18 \\
(0.30)\end{array}$ & $\begin{array}{l}23.68 \\
(0.19)\end{array}$ & $\begin{array}{l}24.15 \\
(0.28)\end{array}$ \\
\hline & & $\begin{array}{l}17.62 \\
(0.35)\end{array}$ & $\begin{array}{l}19.75 \\
(0.21)\end{array}$ & $\begin{array}{l}21.10 \\
(0.19)\end{array}$ & $\begin{array}{l}21.46 \\
(0.29)\end{array}$ \\
\hline & $\begin{array}{l}12.89 \\
(0.35)\end{array}$ & $\begin{array}{l}15.03 \\
(0.22)\end{array}$ & $\begin{array}{l}16.85 \\
(0.22)\end{array}$ & $\begin{array}{l}17.95 \\
(0.20)\end{array}$ & $\begin{array}{r}18.30 \\
(0.31)\end{array}$ \\
\hline $\begin{array}{c}10.69 \\
(0.39)\end{array}$ & $\begin{array}{c}11.87 \\
(0.28)\end{array}$ & $\begin{array}{c}13.89 \\
(0.26)\end{array}$ & $\begin{array}{l}15.51 \\
(0.25)\end{array}$ & $\begin{array}{c}16.58 \\
(0.23)\end{array}$ & $\begin{array}{c}16.92 \\
(0.35)\end{array}$ \\
\hline
\end{tabular}

Fig. 3.12a. Pin-power distribution for CENTRM benchmark SAXTON-6. Value in parentheses is percent standard deviation.

\begin{tabular}{|c|c|c|c|c|c|}
\hline \multirow[t]{5}{*}{$\begin{array}{l}0.341 \\
(1.01)\end{array}$} & & & & & $\begin{array}{l}26.34 \\
(0.53)\end{array}$ \\
\hline & & & & $\begin{array}{l}25.27 \\
(0.28)\end{array}$ & $\begin{array}{l}25.86 \\
(0.27)\end{array}$ \\
\hline & & & $\begin{array}{c}22.14 \\
(0.31)\end{array}$ & $\begin{array}{l}23.68 \\
(0.20)\end{array}$ & $\begin{array}{l}24.16 \\
(0.27)\end{array}$ \\
\hline & & $\begin{array}{l}17.65 \\
(0.32)\end{array}$ & $\begin{array}{l}19.77 \\
(0.21)\end{array}$ & $\begin{array}{l}21.15 \\
(0.19)\end{array}$ & $\begin{array}{c}21.58 \\
(0.30)\end{array}$ \\
\hline & $\begin{array}{c}12.84 \\
(0.40)\end{array}$ & $\begin{array}{l}15.03 \\
(0.24)\end{array}$ & $\begin{array}{l}16.86 \\
(0.23)\end{array}$ & $\begin{array}{l}17.98 \\
(0.20)\end{array}$ & $\begin{array}{l}18.46 \\
(0.31)\end{array}$ \\
\hline $\begin{array}{l}10.75 \\
(0.42)\end{array}$ & $\begin{array}{c}11.84 \\
(0.27)\end{array}$ & $\begin{array}{c}13.94 \\
(0.26)\end{array}$ & $\begin{array}{c}15.58 \\
(0.24)\end{array}$ & $\begin{array}{l}16.62 \\
(0.23)\end{array}$ & $\begin{array}{c}16.94 \\
(0.30)\end{array}$ \\
\hline
\end{tabular}

Fig. 3.12b. Pin-power distribution for NITAWL benchmark SAXTON-6. Value in parentheses is percent standard deviation. 
Table 3.55. SAXTON-6 CENTRM reaction rates and fluxes

\begin{tabular}{|c|c|c|c|c|c|c|}
\hline Pin & Region & $\begin{array}{c}\mathrm{G}_{\mathrm{a}} \mathrm{M} \\
\left(\mathrm{cm}^{-3}-\mathrm{s}^{-1}\right)\end{array}$ & $\begin{array}{c}\mathcal{G}_{\mathrm{f}} \mathrm{M} \\
\left(\mathrm{cm}^{-3}-\mathrm{s}^{-1}\right)\end{array}$ & $\begin{array}{c}\mathrm{G}_{\mathrm{f}} \mathrm{M} \\
\left(\mathrm{cm}^{-3}-\mathrm{s}^{-1}\right)\end{array}$ & $\begin{array}{c}\mathrm{M} \\
\left(\mathrm{cm}-\mathrm{s}^{-1}\right)\end{array}$ & $\mathrm{MM}_{\mathrm{t}}$ \\
\hline \multirow{3}{*}{1} & Fuel & $5.801 \mathrm{E}-05$ & $1.068 \mathrm{E}-04$ & $3.734 \mathrm{E}-05$ & $8.227 \mathrm{E}-03$ & 0.085 \\
\hline & Clad & $4.571 \mathrm{E}-07$ & 0.0 & 0.0 & $2.381 \mathrm{E}-03$ & 0.024 \\
\hline & Mod. & $2.974 \mathrm{E}-06$ & 0.0 & 0.0 & 8.646E-02 & 0.891 \\
\hline \multirow{3}{*}{21} & Fuel & $1.483 \mathrm{E}-04$ & $2.649 \mathrm{E}-04$ & $9.259 \mathrm{E}-05$ & $2.605 \mathrm{E}-02$ & 0.085 \\
\hline & Clad & $1.224 \mathrm{E}-06$ & 0.0 & 0.0 & $7.665 \mathrm{E}-03$ & 0.025 \\
\hline & Mod. & $6.914 \mathrm{E}-06$ & 0.0 & 0.0 & $2.718 \mathrm{E}-01$ & 0.890 \\
\hline
\end{tabular}

Table 3.56. SAXTON-6 NITAWL reaction rates and fluxes

\begin{tabular}{|c|c|c|c|c|c|c|}
\hline Pin & Region & $\begin{array}{c}\mathrm{G}_{\mathrm{a}} \mathrm{M} \\
\left(\mathrm{cm}^{-3}-\mathrm{s}^{-1}\right)\end{array}$ & $\begin{array}{c}\mathcal{G}_{\mathrm{f}} \mathrm{M} \\
\left(\mathrm{cm}^{-3}-\mathrm{s}^{-1}\right)\end{array}$ & $\begin{array}{c}\mathrm{G}_{\mathrm{f}} \mathrm{M} \\
\left(\mathrm{cm}^{-3}-\mathrm{s}^{-1}\right)\end{array}$ & $\begin{array}{c}M \\
\left(\mathrm{~cm}-\mathrm{s}^{-1}\right)\end{array}$ & $M / M_{t}$ \\
\hline \multirow{3}{*}{1} & Fuel & $5.849 \mathrm{E}-05$ & $1.078 \mathrm{E}-04$ & $3.768 \mathrm{E}-05$ & $8.318 \mathrm{E}-03$ & 0.085 \\
\hline & Clad & $4.597 \mathrm{E}-07$ & 0.0 & 0.0 & $2.405 \mathrm{E}-03$ & 0.025 \\
\hline & Mod. & $1.489 \mathrm{E}-06$ & 0.0 & 0.0 & 8.696E-02 & 0.890 \\
\hline \multirow{3}{*}{21} & Fuel & $1.484 \mathrm{E}-04$ & $2.656 \mathrm{E}-04$ & $9.284 \mathrm{E}-05$ & $2.592 \mathrm{E}-02$ & 0.085 \\
\hline & Clad & $1.212 \mathrm{E}-06$ & 0.0 & 0.0 & $7.589 \mathrm{E}-03$ & 0.025 \\
\hline & Mod. & $3.439 \mathrm{E}-06$ & 0.0 & 0.0 & $2.708 \mathrm{E}-01$ & 0.890 \\
\hline
\end{tabular}


Table 3.57. SAXTON-6, Pin-1 four-group fluxes

\begin{tabular}{ccc}
\hline Group & $\begin{array}{c}\text { CENTRM M } \\
\left(\mathrm{cm}^{-2}-\mathrm{s}^{-1}\right)\end{array}$ & $\begin{array}{c}\text { NITAWL M } \\
\left(\mathrm{cm}^{-2}-\mathrm{s}^{-1}\right)\end{array}$ \\
\hline 1 & $1.127 \mathrm{E}-04$ & $1.136 \mathrm{E}-04$ \\
2 & $2.431 \mathrm{E}-05$ & $2.487 \mathrm{E}-05$ \\
3 & $5.596 \mathrm{E}-06$ & $5.708 \mathrm{E}-06$ \\
4 & $3.680 \mathrm{E}-05$ & $3.720 \mathrm{E}-05$ \\
\hline
\end{tabular}

Table 3.58. SAXTON-6, Pin-1 four-group U-235 cross sections

\begin{tabular}{cccccccc}
\hline Group & \multicolumn{2}{c}{ CENTRM cross sections $\left(\mathrm{cm}^{-1}\right)$} & \multicolumn{3}{c}{ NITAWL cross sections $\left(\mathrm{cm}^{-1}\right)$} \\
\hline & $\frac{\mathrm{G}_{\mathrm{a}}}{4.25419 \mathrm{E}-04}$ & $\frac{\leftarrow_{\mathrm{f}}}{5.23524 \mathrm{E}-04}$ & $\frac{\mathrm{G}_{\mathrm{f}}}{2.00192 \mathrm{E}-04}$ & $\frac{\mathrm{G}_{\mathrm{a}}}{2.25627 \mathrm{E}-04}$ & $\frac{\mathrm{G}_{\mathrm{f}}}{5.23460 \mathrm{E}-04}$ & $\frac{\mathrm{G}_{\mathrm{f}}}{2.00250 \mathrm{E}-04}$ \\
1 & $5.64554 \mathrm{E}-03$ & $8.59223 \mathrm{E}-03$ & $3.52618 \mathrm{E}-03$ & $5.66043 \mathrm{E}-03$ & $8.68922 \mathrm{E}-03$ & $3.56598 \mathrm{E}-03$ \\
2 & $8.10920 \mathrm{E}-03$ & $1.64809 \mathrm{E}-02$ & $6.76363 \mathrm{E}-03$ & $8.14676 \mathrm{E}-03$ & $1.65761 \mathrm{E}-02$ & $6.80268 \mathrm{E}-03$ \\
3 & $7.66356 \mathrm{E}-02$ & $1.59575 \mathrm{E}-01$ & $6.54883 \mathrm{E}-02$ & $7.61884 \mathrm{E}-02$ & $1.58640 \mathrm{E}-01$ & $6.51043 \mathrm{E}-02$ \\
\hline
\end{tabular}

Table 3.59. SAXTON-6, Pin-1 four-group U-238 cross sections

\begin{tabular}{cccccccc}
\hline Group & \multicolumn{3}{c}{ CENTRM cross sections $\left(\mathrm{cm}^{-1}\right)$} & \multicolumn{3}{c}{ NITAWL cross sections $\left(\mathrm{cm}^{-1}\right)$} \\
\hline & $\frac{\mathrm{G}_{\mathrm{a}}}{7.46961 \mathrm{E}-03}$ & $\frac{\mathrm{G}_{\mathrm{f}}}{1.48484 \mathrm{E}-02}$ & $\frac{\mathrm{G}_{\mathrm{f}}}{5.26453 \mathrm{E}-03}$ & $\frac{\mathrm{G}_{\mathrm{a}}}{7.45908 \mathrm{E}-03}$ & $\frac{\mathrm{G}_{\mathrm{f}}}{1.47830 \mathrm{E}-02}$ & $\frac{\mathrm{G}_{\mathrm{f}}}{5.24259 \mathrm{E}-03}$ \\
1 & $6.30267 \mathrm{E}-02$ & $6.27162 \mathrm{E}-06$ & $2.59830 \mathrm{E}-06$ & $5.76920 \mathrm{E}-02$ & $5.74105 \mathrm{E}-06$ & $2.37850 \mathrm{E}-06$ \\
3 & $1.12849 \mathrm{E}-02$ & $3.89762 \mathrm{E}-08$ & $1.61485 \mathrm{E}-08$ & $1.13126 \mathrm{E}-02$ & $3.92234 \mathrm{E}-08$ & $1.62510 \mathrm{E}-08$ \\
4 & $4.38120 \mathrm{E}-02$ & $2.05877 \mathrm{E}-07$ & $8.52986 \mathrm{E}-08$ & $4.35935 \mathrm{E}-02$ & $2.04834 \mathrm{E}-07$ & $8.48667 \mathrm{E}-08$ \\
\hline
\end{tabular}

Table 3.60. SAXTON-6, Pin-1 four-group Pu-239 cross sections

\begin{tabular}{cccccccc}
\hline Group & \multicolumn{3}{c}{ CENTRM cross sections $\left(\mathrm{cm}^{-1}\right)$} & \multicolumn{3}{c}{ NITAWL cross sections $\left(\mathrm{cm}^{-1}\right)$} \\
\hline & $\frac{\mathrm{G}_{\mathrm{a}}}{2.50829 \mathrm{E}-03}$ & $\frac{\mathrm{G}_{\mathrm{f}}}{7.44201 \mathrm{E}-03}$ & $\frac{\mathrm{G}_{\mathrm{f}}}{2.38103 \mathrm{E}-03}$ & $\frac{\mathrm{G}_{\mathrm{a}}}{2.50741 \mathrm{E}-03}$ & $\frac{\mathrm{G}_{\mathrm{f}}}{7.43347 \mathrm{E}-03}$ & $\frac{\mathrm{G}_{\mathrm{f}}}{2.37922 \mathrm{E}-03}$ \\
1 & $4.78379 \mathrm{E}-02$ & $8.01259 \mathrm{E}-02$ & $2.78233 \mathrm{E}-02$ & $4.63764 \mathrm{E}-02$ & $7.75412 \mathrm{E}-02$ & $2.69258 \mathrm{E}-02$ \\
2 & $1.52214 \mathrm{E}-01$ & $3.00611 \mathrm{E}-01$ & $1.04386 \mathrm{E}-01$ & $1.54150 \mathrm{E}-01$ & $3.04373 \mathrm{E}-01$ & $1.05692 \mathrm{E}-01$ \\
3 & $1.24949 \mathrm{E}+00$ & $2.53488 \mathrm{E}+00$ & $8.76780 \mathrm{E}-01$ & $1.24891 \mathrm{E}+00$ & $2.53120 \mathrm{E}+00$ & $8.75527 \mathrm{E}-01$ \\
\hline
\end{tabular}


Table 3.61. SAXTON-6, Pin-21 four-group fluxes

\begin{tabular}{ccc}
\hline Group & $\begin{array}{c}\text { CENTRM M } \\
\left(\mathrm{cm}^{-2}-\mathrm{s}^{-1}\right)\end{array}$ & $\begin{array}{c}\text { NITAWL M } \\
\left(\mathrm{cm}^{-2}-\mathrm{s}^{-1}\right)\end{array}$ \\
\hline 1 & $3.626 \mathrm{E}-04$ & $3.592 \mathrm{E}-04$ \\
2 & $9.528 \mathrm{E}-05$ & $9.575 \mathrm{E}-05$ \\
3 & $2.230 \mathrm{E}-05$ & $2.206 \mathrm{E}-05$ \\
4 & $8.798 \mathrm{E}-05$ & $8.816 \mathrm{E}-05$ \\
\hline
\end{tabular}

Table 3.62. SAXTON-6, Pin-21 four-group U-235 cross sections

\begin{tabular}{cccccccc}
\hline Group & \multicolumn{3}{c}{ CENTRM cross sections $\left(\mathrm{cm}^{-1}\right)$} & \multicolumn{3}{c}{ NITAWL cross sections $\left(\mathrm{cm}^{-1}\right)$} \\
\hline & $\frac{\mathrm{G}_{\mathrm{a}}}{4.30565 \mathrm{E}-04}$ & $\frac{\mathrm{G}_{\mathrm{f}}}{5.27350 \mathrm{E}-04}$ & $\frac{\mathrm{G}_{\mathrm{f}}}{2.02688 \mathrm{E}-04}$ & $\frac{\mathrm{G}_{\mathrm{a}}}{2.30477 \mathrm{E}-04}$ & $\frac{\mathrm{G}_{\mathrm{f}}}{5.28068 \mathrm{E}-04}$ & $\frac{\mathrm{G}_{\mathrm{f}}}{2.02763 \mathrm{E}-04}$ \\
1 & $5.63375 \mathrm{E}-03$ & $8.59935 \mathrm{E}-03$ & $3.52909 \mathrm{E}-03$ & $5.56879 \mathrm{E}-03$ & $8.53534 \mathrm{E}-03$ & $3.50283 \mathrm{E}-03$ \\
2 & $8.06789 \mathrm{E}-03$ & $1.64302 \mathrm{E}-02$ & $6.74278 \mathrm{E}-03$ & $8.03178 \mathrm{E}-03$ & $1.64159 \mathrm{E}-02$ & $6.73694 \mathrm{E}-03$ \\
3 & $7.49118 \mathrm{E}-02$ & $1.55931 \mathrm{E}-01$ & $6.39925 \mathrm{E}-02$ & $7.47374 \mathrm{E}-02$ & $1.55564 \mathrm{E}-01$ & $6.38420 \mathrm{E}-02$ \\
\hline
\end{tabular}

Table 3.63. SAXTON-6, Pin-21 four-group U-238 cross sections

\begin{tabular}{cccccccc}
\hline Group & \multicolumn{2}{c}{ CENTRM cross sections $\left(\mathrm{cm}^{-1}\right)$} & \multicolumn{2}{c}{ NITAWL cross sections $\left(\mathrm{cm}^{-1}\right)$} \\
& $\frac{\mathrm{G}_{\mathrm{a}}}{7.31139 \mathrm{E}-03}$ & $\frac{\mathrm{G}_{\mathrm{f}}}{1.38701 \mathrm{E}-02}$ & $\frac{\mathrm{G}_{\mathrm{f}}}{4.92308 \mathrm{E}-03}$ & $\overline{7.34112 \mathrm{E}-03}$ & $\frac{\mathrm{G}_{\mathrm{f}}}{1.40133 \mathrm{E}-02}$ & $\frac{\mathrm{G}_{\mathrm{f}}}{4.96478 \mathrm{E}-03}$ \\
1 & $6.04901 \mathrm{E}-02$ & $6.16474 \mathrm{E}-06$ & $2.55402 \mathrm{E}-06$ & $5.89630 \mathrm{E}-02$ & $6.22799 \mathrm{E}-06$ & $2.58023 \mathrm{E}-06$ \\
2 & $1.12898 \mathrm{E}-02$ & $3.90791 \mathrm{E}-08$ & $1.61912 \mathrm{E}-08$ & $1.12885 \mathrm{E}-02$ & $3.90972 \mathrm{E}-08$ & $1.61987 \mathrm{E}-08$ \\
3 & $4.29311 \mathrm{E}-02$ & $2.01652 \mathrm{E}-07$ & $8.35480 \mathrm{E}-08$ & $4.28370 \mathrm{E}-02$ & $2.01200 \mathrm{E}-07$ & $8.33613 \mathrm{E}-08$ \\
\hline
\end{tabular}

Table 3.64. SAXTON-6, Pin-21 four-group Pu-239 cross sections

\begin{tabular}{cccccccc}
\hline Group & \multicolumn{3}{c}{ CENTRM cross sections $\left(\mathrm{cm}^{-1}\right)$} & \multicolumn{3}{c}{ NITAWL cross sections $\left(\mathrm{cm}^{-1}\right)$} \\
\hline & $\frac{\mathrm{G}_{\mathrm{a}}}{2.51210 \mathrm{E}-03}$ & $\frac{\mathrm{G}_{\mathrm{f}}}{7.36437 \mathrm{E}-03}$ & $\frac{\mathrm{G}_{\mathrm{f}}}{2.36645 \mathrm{E}-03}$ & $\frac{\mathrm{G}_{\mathrm{a}}}{2.51355 \mathrm{E}-03}$ & $\frac{\mathrm{G}_{\mathrm{f}}}{7.37904 \mathrm{E}-03}$ & $\frac{\mathrm{G}_{\mathrm{f}}}{2.36928 \mathrm{E}-03}$ \\
1 & $4.63848 \mathrm{E}-02$ & $7.74581 \mathrm{E}-02$ & $2.68969 \mathrm{E}-02$ & $4.49666 \mathrm{E}-02$ & $7.52761 \mathrm{E}-02$ & $2.61392 \mathrm{E}-02$ \\
2 & $1.47063 \mathrm{E}-01$ & $2.91411 \mathrm{E}-01$ & $1.01191 \mathrm{E}-01$ & $1.47665 \mathrm{E}-01$ & $2.92500 \mathrm{E}-01$ & $1.01570 \mathrm{E}-01$ \\
3 & $1.27191 \mathrm{E}+00$ & $2.56133 \mathrm{E}+00$ & $8.86051 \mathrm{E}-01$ & $1.27598 \mathrm{E}+00$ & $2.56709 \mathrm{E}+00$ & $8.88067 \mathrm{E}-01$ \\
\hline
\end{tabular}




\section{MIX-COMP-THERM-4 (TCA-1 TO TCA-11)}

\subsection{DESCRIPTION}

This section describes a set of 11 critical experiments, each consisting of a square-pitched array of mixed Plutonium-Uranium fuel rods partially submerged in water surrounded by a water reflector. The water-to-fuel ratios for the arrays range from 2.4 to 5.6. This set of experiments is contained in the International Handbook of Evaluated Criticality Safety Benchmark Experiment. 3

This set of experiments was performed between 1972 and 1975 at the Tokai Research Establishment of JAERI. The Tank-Type Critical Assembly (TCA) benchmark cases are light-water moderated critical assemblies, consisting of a core array supported by upper, middle, and lower grid plates. The grid plates do not pass through the fuel region. The fuel is sufficiently long such that the water level is below the top of the fuel region in all cases. The reactor is brought to critical by raising the water level in the tank, thus avoiding the use of control rods. The fuel rods sit on a support plate above the bottom of the tank. The tank is wide enough to assume an infinite moderator on the sides ( $30 \mathrm{~cm}$ of water).

All fuel rods have the same physical dimensions. A schematic diagram of the fuel rods and bottom reflector is given in Fig. 4.1. Each fuel rod has an active fuel length of $70.6 \mathrm{~cm}$ and a $16.83-$ $\mathrm{cm}$-long bottom aluminum end plug that sits on a $1.27-\mathrm{cm}$-thick aluminum support plate. The fuel has a radius of $0.5325 \mathrm{~cm}$. The cladding has an outside radius of $0.6115 \mathrm{~cm}$. For the calculation, the cladding is extended $9.97 \mathrm{~cm}$ above the active fuel to the bottom of the middle grid plate. The middle grid plate and everything above are assumed to be insignificant and thus excluded from the model. The fuel lattice is surrounded by $30 \mathrm{~cm}$ of water on the four sides from the bottom of the tank to the top of the critical water level.

From the top of the aluminum support plate is a $4.445-\mathrm{cm}$ water gap and $0.601-\mathrm{cm}$ lower aluminum grid plate. Below the aluminum support plate is a 2.2-cm-thick stainless steel (SS) support plate, a 13.8-cm water gap, 0.5-cm-thick SS tank liner and $37.0 \mathrm{~cm}$ of concrete.

The primary differences between the 11 benchmarks are lattice pitch, number of rods in the lattice, water level, and Pu-241 and Am-241 number densities. All other benchmark characteristics are constant. The 11 benchmarks are divided into four different lattice pitches: $1.825 \mathrm{~cm}, 1.956 \mathrm{~cm}$, $2.225 \mathrm{~cm}$, and $2.474 \mathrm{~cm}$. For a given pitch, the number of pins in the lattice is given. The fuel is arranged in a square-pitched square lattice. The characteristics of each of the four lattices are given in Table 4.1. The critical fuel height variations are due to the changes in $\mathrm{Pu}-241$ and $\mathrm{Am}-241$ atom densities. Table 4.2 contains the atom densities for all the materials in the problem except $\mathrm{Pu}-241$ and Am-241. The atom densities in Table 4.2 are constant for all benchmarks. Table 4.3 contains the atom densities for Pu-241 and Am-241 for each benchmark. All material temperatures are assumed to be $20^{\circ} \mathrm{C}$. 

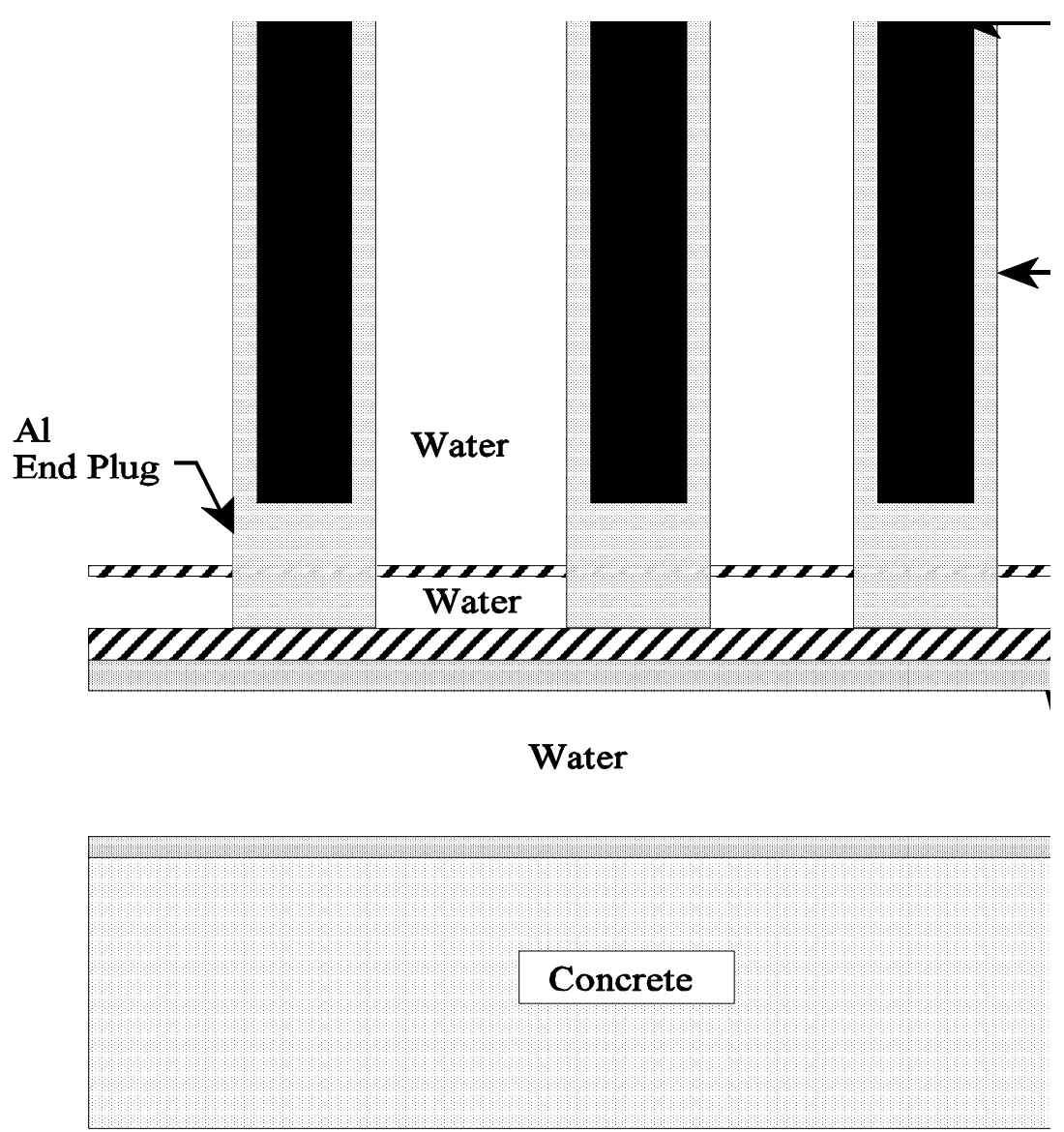

Fig. 4.1. Schematic of the TCA fuel rods and bottom reflector. 
Table 4.1. Lattice description for benchmarks

\begin{tabular}{cccccc}
\hline $\begin{array}{c}\text { Benchmark } \\
\text { No. }\end{array}$ & $\begin{array}{c}\text { Water/Fuel } \\
\text { volume ratio } \\
(\text { H/Pu ratio })\end{array}$ & $\begin{array}{c}\text { Lattice } \\
\text { pitch } \\
(\mathrm{cm})\end{array}$ & $\begin{array}{c}\text { Number } \\
\text { of rods } \\
\text { on a Side }\end{array}$ & $\begin{array}{c}\text { Lattice side } \\
\text { dimension } \\
(\mathrm{cm})\end{array}$ & $\begin{array}{c}\text { Critical } \\
\text { water level } \\
(\mathrm{cm})\end{array}$ \\
\hline 1 & & & & & 59.5 \\
2 & $\begin{array}{c}2.42 \\
(402)\end{array}$ & 1.825 & 23 & 41.975 & 91.90 \\
3 & & & & & 64.06 \\
\hline 4 & 2.98 & 1.956 & 21 & 41.076 & 61.50 \\
5 & $(494)$ & & & & 64.40 \\
6 & & & & & 69.40 \\
\hline 7 & 4.24 & $\mathrm{p} 2.225$ & 20 & 44.500 & 60.32 \\
8 & $(703)$ & & & & 62.99 \\
9 & & & & & 65.63 \\
\hline 10 & 5.55 & 2.474 & 21 & 51.954 & 62.05 \\
11 & $(921)$ & & & & 64.53 \\
\hline
\end{tabular}

The first three benchmarks; TCA-1, TCA-2, and TCA-3 have a pitch of $1.825 \mathrm{~cm}$ and $23 \times 23$ pin lattice. This pitch produces a water-to-fuel volume ratio of $2.42 \mathrm{~cm}$ and a $\mathrm{H} / \mathrm{Pu}$ ratio of 402 . To simplify the production of the lattice pin-power distribution and improve the statistics, the lattice advantage is taken of the lattice symmetry. Only $1 / 8$ th of the lattice needs to be modeled precisely to produce the pin-power distribution. The lattice is then filled from the $1 / 8$ th section by inserting additional pins in a mirror image. Figure 4.2 is a lattice map of the pin locations.

The same approach is used for the next three benchmarks: TCA-4, TCA-5, and TCA-6. These benchmarks have a pitch of $1.956 \mathrm{~cm}$ and $21 \times 21$ pin lattice. This pitch produces a water-to-fuel volume ratio of $2.98 \mathrm{~cm}$ and a $\mathrm{H} / \mathrm{Pu}$ ratio of 494 . The symmetry of the lattice is used in the same way it was used in the first three benchmarks. One-eighth of the lattice is filled, and the remaining lattice is filled assuming a mirror image. Figure 4.3 is a lattice map of the pin locations.

The next three benchmark problemsCTCA-7, TCA-8, and TCA-9Cagain use the same approach. These benchmarks have a pitch of $2.225 \mathrm{~cm}$ and a $20 \times 20$ pin lattice. This pitch produces a water-to-fuel volume ratio of $4.24 \mathrm{~cm}$ and a $\mathrm{H} / \mathrm{Pu}$ ratio of 703 . Figure 4.4 is a lattice map of the pin locations.

The last benchmark problemsCTCA-10 and TCA-11Chave the same lattice arrangement as the second set of benchmarks (see Fig. 4.3). However, these benchmarks have of pitch of $2.474 \mathrm{~cm}$ which produces a water-to-fuel volume ratio of $5.55 \mathrm{~cm}$ and a $\mathrm{H} / \mathrm{Pu}$ ratio of 921 . The input files for each of the benchmark problems can be found in Appendix C. 


\subsection{ANALYSIS}

All 11 computational benchmarks in this section were processed twice using SCALE 5.0. The set labeled NITAWL uses the NITAWL resonance processor to self-shield the resolved resonance region for all nuclides in the fuel and clad. An identical set, labeled CENTRM, replaces NITAWL with the CENTRM/PMC code sequence. The NITAWL and CENTRM results are then compared.

Table 4.4 contains the $k_{\text {eff }}$ and Energy of the Average Lethargy Causing Fission (EALCF). The $k_{\text {eff }}$ values for all the benchmark cases are very close to 1.0: the worst NITAWL benchmark is $0.26 \%$ high, and the worst CENTRM case is $0.37 \%$ low. There does appear to be a small negative bias between NITAWL and CENTRM, the CENTRM cases being about $0.2 \%$ lower on average than the NITAWL cases. The EALCF values listed are from the CENTRM cases. For all cases the difference between this value for CENTRM and NITAWL was less than $0.1 \%$. Both CENTRM and NITAWL produce excellent results for the $k_{\text {eff }}$ and fission energy for this set of problems.

Also, calculated for each problem is the: pin-power distributions; absorption, $\mathbb{G}_{\mathrm{f}}$, and fission reaction rates and fluxes in the pin fuel, clad, and moderator; and four group cross sections and fluxes for a corner outside pin and the innermost pin. Each of these sets of data are calculated using both CENTRM and NITAWL. The data for each case are contained in Figs. 4.5a through 4.15b and Tables 4.5 through 4.114.

The pin-power distributions for this problem assumed 1/8th core symmetry with a surrounding reflector. The pin-power data consist of a value and a standard deviation for each pin. The values for the pin-powers are actually in units of fissions per $\mathrm{cm}^{3}$-s-source particle $\times 10^{-5}$. The value in parentheses is the percent standard deviation of the pin-power value. In most cases, the CENTRM and NITAWL results for each benchmark case agree within 2 standard deviations. The pin-power distributions represent that portion of the pin covered by water. The value off to the side in the pinpower distributions represents all the fuel not covered by water. The peak-to-low power changed with respect to pitch. The peak-to-low-power ratio changed from a low of 2.7 for the smallest pitch to a high of 4.9 at the largest pitch.

The reaction rates, total fluxes, and flux ratios for the CENTRM and NITAWL cases of each benchmark case are also in good agreement, seldom varying by more than $1 \%$. These values are included for a corner pin and the center pin for each case. The flux ratios over the fuel, clad, and moderator stay relatively constant for a given pitch. However, as the pitch increases the proportion of the total pin flux in the moderator increases.

Finally, four-group fluxes and cross sections were calculated for selected nuclides in the fuel region of the same corner and center pins. The macroscopic cross sections include the radiative capture, fission, and nu*fission cross sections for $\mathrm{U}-235, \mathrm{U}-238$, and $\mathrm{Pu}-239$. The four groups are collapsed from the 238-group multigroup cross-section set using the flux profile calculated in KENOV.a as follows: group 1 is from $20 \mathrm{Mev}$ to $9.5 \mathrm{keV}$, group 2 is from $9.5 \mathrm{keV}$ to $3.0 \mathrm{eV}$, Group 3 is from $3 \mathrm{eV}$ to $0.4 \mathrm{eV}$, and group 4 is from $0.4 \mathrm{eV}$ to $10^{-5} \mathrm{eV}$. The $0.4 \mathrm{eV}$ was chosen as a boundary because it is the cadmium cutoff energy. Groups 2 and 3 contain the resolved resonance regions for most of the nuclides used in these cases. Most of the cross-section data for the CENTRM and NITAWL cases of a given benchmark case agree within $1 \%$. 


\subsection{CONCLUSIONS}

For this set of benchmark cases, using either NITAWL and CENTRM as the resonance region processor produces acceptable results of the $k_{\text {eff }}$ values. All other values produced using CENTRM and NITAWL are also consistent with each. The $k_{\text {eff }}$ values are slightly lower, $\sim 0.2 \%$, for all CENTRM cases. The pin-power distributions, fluxes, reaction rates, and macroscopic cross sections generally agree within approximately $1 \%$ between CENTRM and NITAWL. No significant differences were identified between the results produced by NITAWL and CENTRM for these benchmark cases. 
Table 4.2. Benchmark atom densities

\begin{tabular}{|c|c|c|c|c|c|}
\hline Material & Isotope & $\begin{array}{c}\text { Atom density } \\
\text { (atoms/barn-cm) }\end{array}$ & Material & Isotope & $\begin{array}{c}\text { Atom density } \\
\text { (atoms/barn-cm) }\end{array}$ \\
\hline Fuel & $\begin{array}{c}\mathrm{U}-234 \\
\mathrm{U}-235 \\
\mathrm{U}-238 \\
\mathrm{Pu}-238 \\
\mathrm{Pu}-239 \\
\mathrm{Pu}-240 \\
\mathrm{Pu}-241 \\
\mathrm{Pu}-242 \\
\mathrm{Am}-241 \\
\mathrm{O}-16 \\
\mathrm{~B}-10 \\
\mathrm{~B}-11\end{array}$ & $\begin{array}{c}7.1749 \times 10^{-7} \\
9.3926 \times 10^{-5} \\
1.2951 \times 10^{-2} \\
2.0003 \times 10^{-6} \\
2.7491 \times 10^{-4} \\
8.8417 \times 10^{-5} \\
(\text { See Table } 3.3) \\
8.1234 \times 10^{-6} \\
(\text { See Table } 3.3) \\
2.7837 \times 10^{-2} \\
6.0418 \times 10^{-8} \\
2.4319 \times 10^{-7}\end{array}$ & $\begin{array}{l}\text { Ordinary } \\
\text { concrete }\end{array}$ & $\begin{array}{c}\mathrm{H} \\
\mathrm{O} \\
\mathrm{C} \\
\mathrm{Na} \\
\mathrm{Mg} \\
\mathrm{Al} \\
\mathrm{Si} \\
\mathrm{K} \\
\mathrm{Ca} \\
\mathrm{Fe}\end{array}$ & $\begin{array}{l}1.3742 \times 10^{-2} \\
4.5919 \times 10^{-2} \\
1.1532 \times 10^{-4} \\
9.6395 \times 10^{-4} \\
1.2388 \times 10^{-4} \\
1.7409 \times 10^{-3} \\
1.6617 \times 10^{-2} \\
4.6052 \times 10^{-4} \\
1.5025 \times 10^{-3} \\
3.4492 \times 10^{-4}\end{array}$ \\
\hline $\begin{array}{l}\text { Cladding } \\
+ \text { Air gap }\end{array}$ & $\begin{array}{l}\mathrm{Zr} \\
\mathrm{Sn} \\
\mathrm{Fe} \\
\mathrm{Cr} \\
\mathrm{Ni}\end{array}$ & $\begin{array}{l}3.7772 \times 10^{-2} \\
4.3737 \times 10^{-4} \\
8.8570 \times 10^{-5} \\
6.6119 \times 10^{-5} \\
3.5864 \times 10^{-5}\end{array}$ & $\begin{array}{c}\text { Stainless } \\
\text { steel } \\
(\mathrm{SS}-304 \mathrm{~L})\end{array}$ & $\begin{array}{c}\mathrm{C} \\
\mathrm{Si} \\
\mathrm{Mn} \\
\mathrm{P} \\
\mathrm{S} \\
\mathrm{Ni} \\
\mathrm{Cr} \\
\mathrm{Fe}\end{array}$ & $\begin{array}{l}1.1928 \times 10^{-4} \\
1.7003 \times 10^{-3} \\
1.7385 \times 10^{-3} \\
6.9381 \times 10^{-5} \\
4.4673 \times 10^{-5} \\
8.9506 \times 10^{-3} \\
1.7450 \times 10^{-2} \\
5.7202 \times 10^{-2}\end{array}$ \\
\hline $\begin{array}{l}\text { Water }\left(20^{\circ} \mathrm{C}\right) \\
0.9982 \mathrm{~g} / \mathrm{cc}\end{array}$ & $\begin{array}{l}\mathrm{H} \\
\mathrm{O}\end{array}$ & $\begin{array}{l}6.6735 \times 10^{-2} \\
3.3368 \times 10^{-2}\end{array}$ & Aluminum & $\mathrm{Al}$ & $6.0224 \times 10^{-2}$ \\
\hline
\end{tabular}


Table 4.3. Pu-241 and Am-241 atom densities

\begin{tabular}{ccc}
\hline & \multicolumn{2}{c}{ Atom densities (atoms/barn-cm $)$} \\
\cline { 2 - 3 } $\begin{array}{c}\text { Benchmark } \\
\text { No. }\end{array}$ & Pu-241 & Am-241 \\
\hline 1 & $2.7923 \times 10^{-5}$ & $1.3531 \times 10^{-6}$ \\
2 & $2.6701 \times 10^{-5}$ & $2.5812 \times 10^{-6}$ \\
3 & $2.5447 \times 10^{-5}$ & $3.8361 \times 10^{-6}$ \\
4 & $2.8003 \times 10^{-5}$ & $1.2793 \times 10^{-6}$ \\
5 & $2.6670 \times 10^{-5}$ & $2.6129 \times 10^{-6}$ \\
6 & $2.4228 \times 10^{-5}$ & $5.0543 \times 10^{-6}$ \\
7 & $2.8133 \times 10^{-5}$ & $1.1498 \times 10^{-6}$ \\
8 & $2.6649 \times 10^{-5}$ & $2.6340 \times 10^{-6}$ \\
9 & $2.5373 \times 10^{-5}$ & $3.9098 \times 10^{-6}$ \\
10 & $2.8077 \times 10^{-5}$ & $1.2053 \times 10^{-6}$ \\
11 & $2.6617 \times 10^{-5}$ & $2.6656 \times 10^{-6}$ \\
\hline
\end{tabular}

Table 4.4. Comparison of $k_{\text {eff }}$ and EALCF from CENTRM and NITAWL ${ }^{a}$

\begin{tabular}{ccccc}
\hline $\begin{array}{c}\text { Benchmark } \\
\text { identification }\end{array}$ & $\begin{array}{c}\text { CENTRM } \\
k_{\text {eff }}( \pm \mathrm{F})\end{array}$ & $\begin{array}{c}\text { NITAWL } \\
k_{\text {eff }}( \pm \mathrm{F})\end{array}$ & $\begin{array}{c}\text { EALF } \\
(\mathrm{eV})\end{array}$ & $\begin{array}{c}k_{\text {eff }} \\
\% \text { DIFF }\end{array}$ \\
\hline TCA-1 & $0.9963(0.0005)$ & $0.9993(0.0004)$ & 0.145 & -0.3 \\
TCA-2 & $0.9969(0.0004)$ & $1.0001(0.0004)$ & 0.144 & -0.32 \\
TCA-3 & $0.9985(0.0005)$ & $1.0002(0.0004)$ & 0.143 & -0.17 \\
TCA-4 & $0.9973(0.0004)$ & $0.9995(0.0004)$ & 0.119 & -0.22 \\
TCA-5 & $0.9982(0.0004)$ & $1.0008(0.0004)$ & 0.118 & -0.26 \\
TCA-6 & $0.9988(0.0004)$ & $1.0003(0.0003)$ & 0.117 & -0.15 \\
TCA-7 & $0.9994(0.0004)$ & $1.0016(0.0004)$ & 0.0927 & -0.22 \\
TCA-8 & $1.0002(0.0004)$ & $1.0017(0.0003)$ & 0.0923 & -0.15 \\
TCA-9 & $1.0009(0.0004)$ & $1.0024(0.0004)$ & 0.0916 & -0.15 \\
TCA-10 & $1.0003(0.0003)$ & $1.0020(0.0004)$ & 0.0797 & -0.17 \\
TCA-11 & $1.0010(0.0004)$ & $1.0026(0.0004)$ & 0.0792 & -0.16 \\
\hline
\end{tabular}

${ }^{a}$ Value in parentheses is percent standard deviation. 


\begin{tabular}{|c|c|c|c|c|c|c|c|c|c|c|c|}
\hline & 78 \\
\hline & & & & & & & & & & 76 & 77 \\
\hline & & & & & & & & & 73 & 74 & 75 \\
\hline & & & & & & & & 69 & 70 & 71 & 72 \\
\hline & & & & & & & 64 & 65 & 66 & 67 & 68 \\
\hline & & & & & & 58 & 59 & 60 & 61 & 62 & 63 \\
\hline & & & & & 51 & 52 & 53 & 54 & 55 & 56 & 57 \\
\hline & & & & 43 & 44 & 45 & 46 & 47 & 48 & 49 & 50 \\
\hline & & & 34 & 35 & 36 & 37 & 38 & 39 & 40 & 41 & 42 \\
\hline & & 24 & 25 & 26 & 27 & 28 & 29 & 30 & 31 & 32 & 33 \\
\hline & 13 & 14 & 15 & 16 & 17 & 18 & 19 & 20 & 21 & 22 & 23 \\
\hline 1 & 2 & 3 & 4 & 5 & 6 & 7 & 8 & 9 & 10 & 11 & 12 \\
\hline
\end{tabular}

Fig. 4.2. Pin layout of benchmark cases TCA-1, TCA-2, and TCA-3.

\begin{tabular}{|c|c|c|c|c|c|c|c|c|c|c|}
\hline & & & & & & & & & & 66 \\
\hline & & & & & & & & & 64 & 65 \\
\hline & & & & & & & & 61 & 62 & 63 \\
\hline & & & & & & & 57 & 58 & 59 & 60 \\
\hline & & & & & & 52 & 53 & 54 & 55 & 56 \\
\hline & & & & & 46 & 47 & 48 & 49 & 50 & 51 \\
\hline & & & & 39 & 40 & 41 & 42 & 43 & 44 & 45 \\
\hline & & & 31 & 32 & 33 & 34 & 35 & 36 & 37 & 38 \\
\hline & & 22 & 23 & 24 & 25 & 26 & 27 & 28 & 29 & 30 \\
\hline & 12 & 13 & 14 & 15 & 16 & 17 & 18 & 19 & 20 & 21 \\
\hline 1 & 2 & 3 & 4 & 5 & 6 & 7 & 8 & 9 & 10 & 11 \\
\hline
\end{tabular}

Fig. 4.3. Pin layout of benchmark cases TCA-4, TCA-5 ,TCA-6, TCA-10, and TCA-11. 


\begin{tabular}{|c|c|c|c|c|c|c|c|c|c|}
\hline & & & & & & & & & 55 \\
\hline & & & & & & & & 53 & 54 \\
\hline & & & & & & & 50 & 51 & 52 \\
\hline & & & & & & 46 & 47 & 48 & 49 \\
\hline & & & & & 41 & 42 & 43 & 44 & 45 \\
\hline & & & & 35 & 36 & 37 & 38 & 39 & 40 \\
\hline & & & 28 & 29 & 30 & 31 & 32 & 33 & 34 \\
\hline & & 20 & 21 & 22 & 23 & 24 & 25 & 26 & 27 \\
\hline & 11 & 12 & 13 & 14 & 15 & 16 & 17 & 18 & 19 \\
\hline 1 & 2 & 3 & 4 & 5 & 6 & 7 & 8 & 9 & 10 \\
\hline
\end{tabular}

Fig. 4.4. Pin layout of benchmark cases TCA-7, TCA-8, and TCA-9. 


\begin{tabular}{|c|c|c|c|c|c|c|c|c|c|c|c|}
\hline & & & & & & & & & & & $\begin{array}{l}5.299 \\
(0.91)\end{array}$ \\
\hline & $\begin{array}{l}0.131 \\
(0.59)\end{array}$ & & & & & & & & & $\begin{array}{c}5.191 \\
(0.46)\end{array}$ & $\begin{array}{l}5.175 \\
(0.48)\end{array}$ \\
\hline & & & & & & & & & $\begin{array}{l}4.954 \\
(0.48)\end{array}$ & $\begin{array}{c}5104 \\
(0.36)\end{array}$ & $\begin{array}{l}5.242 \\
(0.48)\end{array}$ \\
\hline & & & & & & & & $\begin{array}{l}4.722 \\
(0.51)\end{array}$ & $\begin{array}{l}4.871 \\
(0.33)\end{array}$ & $\begin{array}{l}4.971 \\
(0.34)\end{array}$ & $\begin{array}{l}4.937 \\
(0.48)\end{array}$ \\
\hline & & & & & & & $\begin{array}{l}4.426 \\
(0.51)\end{array}$ & $\begin{array}{l}4.601 \\
(0.33)\end{array}$ & $\begin{array}{l}4.697 \\
(0.36)\end{array}$ & $\begin{array}{l}4.759 \\
(0.36)\end{array}$ & $\begin{array}{l}4.815 \\
(0.54)\end{array}$ \\
\hline & & & & & & $\begin{array}{l}3.982 \\
(0.50)\end{array}$ & $\begin{array}{l}4.212 \\
(0.39)\end{array}$ & $\begin{array}{l}4.340 \\
(0.37)\end{array}$ & $\begin{array}{l}4.479 \\
0.36)\end{array}$ & $\begin{array}{l}4.554 \\
(0.33)\end{array}$ & $\begin{array}{l}4.551 \\
(0.44)\end{array}$ \\
\hline & & & & & $\begin{array}{c}3.535 \\
(0.54)\end{array}$ & $\begin{array}{l}3.766 \\
(0.44)\end{array}$ & $\begin{array}{l}3.934 \\
(0.40)\end{array}$ & $\begin{array}{l}4.068 \\
(0.40)\end{array}$ & $\begin{array}{l}4.184 \\
(0.38)\end{array}$ & $\begin{array}{l}4.252 \\
(0.38)\end{array}$ & $\begin{array}{r}4.286 \\
(0.53)\end{array}$ \\
\hline & & & & $\begin{array}{l}3.058 \\
(060)\end{array}$ & $\begin{array}{l}3.295 \\
(0.43)\end{array}$ & $\begin{array}{l}3.456 \\
(0.45)\end{array}$ & $\begin{array}{l}3.650 \\
(0.35)\end{array}$ & $\begin{array}{l}3.781 \\
(0.36)\end{array}$ & $\begin{array}{l}3.895 \\
(0.37)\end{array}$ & $\begin{array}{l}3.942 \\
(0.39)\end{array}$ & $\begin{array}{l}3.989 \\
(0.53)\end{array}$ \\
\hline & & & $\begin{array}{l}2.518 \\
(0.75)\end{array}$ & $\begin{array}{l}2.769 \\
(0.48)\end{array}$ & $\begin{array}{l}2.963 \\
(0.45)\end{array}$ & $\begin{array}{l}3.180 \\
(0.44)\end{array}$ & $\begin{array}{l}3.354 \\
(0.46)\end{array}$ & $\begin{array}{r}3.457 \\
(0.41)\end{array}$ & $\begin{array}{l}3.555 \\
(0.38)\end{array}$ & $\begin{array}{l}3.617 \\
(0.40)\end{array}$ & $\begin{array}{c}3.640 \\
(0.56)\end{array}$ \\
\hline & & $\begin{array}{l}2.104 \\
(0.83)\end{array}$ & $\begin{array}{l}2.321 \\
(0.51)\end{array}$ & $\begin{array}{l}2.533 \\
(0.48)\end{array}$ & $\begin{array}{l}2.736 \\
(0.45)\end{array}$ & $\begin{array}{l}2.893 \\
(0.47)\end{array}$ & $\begin{array}{l}3.058 \\
(0.39)\end{array}$ & $\begin{array}{r}3.159 \\
(0.41)\end{array}$ & $\begin{array}{l}3.257 \\
(0.42)\end{array}$ & $\begin{array}{l}3.271 \\
(0.41)\end{array}$ & $\begin{array}{c}3.331 \\
(0.58)\end{array}$ \\
\hline & $\begin{array}{c}1.889 \\
(0.78)\end{array}$ & $\begin{array}{c}1.980 \\
(0.57)\end{array}$ & $\begin{array}{l}2.223 \\
(0.55)\end{array}$ & $\begin{array}{l}2.402 \\
(0.51)\end{array}$ & $\begin{array}{l}2.624 \\
(0.50)\end{array}$ & $\begin{array}{l}2.789 \\
(0.41)\end{array}$ & $\begin{array}{l}2.917 \\
(0.41)\end{array}$ & $\begin{array}{l}3.037 \\
(0.47)\end{array}$ & $\begin{array}{c}3.092 \\
(0.45)\end{array}$ & $\begin{array}{c}3.150 \\
(0.42)\end{array}$ & $\begin{array}{c}3.184 \\
(0.65)\end{array}$ \\
\hline $\begin{array}{l}2.329 \\
(0.66)\end{array}$ & $\begin{array}{c}2.178 \\
(0.54)\end{array}$ & $\begin{array}{l}2.356 \\
(0.50)\end{array}$ & $\begin{array}{l}2.589 \\
(0.47)\end{array}$ & $\begin{array}{l}2.844 \\
(0.47)\end{array}$ & $\begin{array}{l}3.066 \\
(0.41)\end{array}$ & $\begin{array}{l}3.258 \\
(0.42)\end{array}$ & $\begin{array}{l}3.398 \\
(0.43)\end{array}$ & $\begin{array}{l}3.576 \\
(0.39)\end{array}$ & $\begin{array}{l}3.644 \\
(0.44)\end{array}$ & $\begin{array}{c}3.710 \\
(0.40)\end{array}$ & $\begin{array}{r}3.729 \\
(0.57)\end{array}$ \\
\hline
\end{tabular}

Fig. 4.5a. Pin-power distribution for CENTRM benchmark TCA-1. Value in parentheses is percent standard deviation. 


\begin{tabular}{|c|c|c|c|c|c|c|c|c|c|c|c|}
\hline & & & & & & & & & & & $\begin{array}{l}5.219 \\
(0.86)\end{array}$ \\
\hline & $\begin{array}{l}0.131 \\
(0.53)\end{array}$ & & & & & & & & & $\begin{array}{l}5.153 \\
(0.50)\end{array}$ & $\begin{array}{l}5.235 \\
(0.47)\end{array}$ \\
\hline & & & & & & & & & $\begin{array}{l}5.022 \\
(0.49)\end{array}$ & $\begin{array}{l}5.083 \\
(0.36)\end{array}$ & $\begin{array}{l}5.140 \\
(0.46)\end{array}$ \\
\hline & & & & & & & & $\begin{array}{l}4.775 \\
(0.48)\end{array}$ & $\begin{array}{l}4.893 \\
(0.35)\end{array}$ & $\begin{array}{l}4.968 \\
(0.38)\end{array}$ & $\begin{array}{l}5.010 \\
(0.49)\end{array}$ \\
\hline & & & & & & & $\begin{array}{c}4.453 \\
(0.54\end{array}$ & $\begin{array}{l}4.607 \\
(0.35)\end{array}$ & $\begin{array}{l}4.712 \\
(0.36)\end{array}$ & $\begin{array}{l}4.779 \\
(0.33)\end{array}$ & $\begin{array}{l}4.840 \\
(0.50)\end{array}$ \\
\hline & & & & & & $\begin{array}{l}4.006 \\
(0.55)\end{array}$ & $\begin{array}{l}4.225 \\
(0.41)\end{array}$ & $\begin{array}{l}4.384 \\
(0.36)\end{array}$ & $\begin{array}{l}4.474 \\
(0.36)\end{array}$ & $\begin{array}{l}4.594 \\
(0.35)\end{array}$ & $\begin{array}{l}4.547 \\
(0.53)\end{array}$ \\
\hline & & & & & $\begin{array}{l}3.541 \\
(0.55)\end{array}$ & $\begin{array}{l}3.807 \\
(0.39)\end{array}$ & $\begin{array}{l}3.978 \\
(0.41)\end{array}$ & $\begin{array}{l}4.086 \\
(0.36)\end{array}$ & $\begin{array}{l}4.200 \\
(0.39)\end{array}$ & $\begin{array}{l}4.270 \\
(0.37)\end{array}$ & $\begin{array}{l}4.341 \\
(0.47)\end{array}$ \\
\hline & & & & $\begin{array}{l}3.013 \\
(0.60)\end{array}$ & $\begin{array}{l}3.298 \\
(0.40)\end{array}$ & $\begin{array}{l}3.496 \\
(0.39)\end{array}$ & $\begin{array}{l}3.645 \\
(0.42)\end{array}$ & $\begin{array}{l}3.821 \\
(0.38)\end{array}$ & $\begin{array}{l}3.921 \\
(0.43)\end{array}$ & $\begin{array}{l}3.981 \\
(0.37)\end{array}$ & $\begin{array}{l}3.948 \\
(0.55)\end{array}$ \\
\hline & & & $\begin{array}{l}2.552 \\
(0.65)\end{array}$ & $\begin{array}{l}2.767 \\
(0.46)\end{array}$ & $\begin{array}{l}2.999 \\
(0.44)\end{array}$ & $\begin{array}{l}3.200 \\
(0.40)\end{array}$ & $\begin{array}{l}3.362 \\
(0.36)\end{array}$ & $\begin{array}{l}3.471 \\
(0.38)\end{array}$ & $\begin{array}{l}3.581 \\
(0.41)\end{array}$ & $\begin{array}{l}3.631 \\
(0.41)\end{array}$ & $\begin{array}{l}3.624 \\
(0.59)\end{array}$ \\
\hline & & $\begin{array}{l}2.113 \\
(0.73)\end{array}$ & $\begin{array}{l}2.284 \\
(0.48)\end{array}$ & $\begin{array}{l}2.509 \\
0.50)\end{array}$ & $\begin{array}{l}2.749 \\
(0.46)\end{array}$ & $\begin{array}{l}2.906 \\
(0.47)\end{array}$ & $\begin{array}{l}3.036 \\
(0.42)\end{array}$ & $\begin{array}{l}3.153 \\
(0.42)\end{array}$ & $\begin{array}{l}3.238 \\
(0.41)\end{array}$ & $\begin{array}{l}3.276 \\
(0.44)\end{array}$ & $\begin{array}{l}3.329 \\
(0.53)\end{array}$ \\
\hline & $\begin{array}{l}1.911 \\
(0.77)\end{array}$ & $\begin{array}{l}1.997 \\
(0.47)\end{array}$ & $\begin{array}{l}2.202 \\
(0.57)\end{array}$ & $\begin{array}{l}2.417 \\
(0.51)\end{array}$ & $\begin{array}{l}2.623 \\
(0.44)\end{array}$ & $\begin{array}{l}2.768 \\
(0.46)\end{array}$ & $\begin{array}{l}2.927 \\
(0.46)\end{array}$ & $\begin{array}{l}3.029 \\
(0.40)\end{array}$ & $\begin{array}{l}3.071 \\
(0.44)\end{array}$ & $\begin{array}{l}3.176 \\
(0.41)\end{array}$ & $\begin{array}{l}3.180 \\
(0.65)\end{array}$ \\
\hline $\begin{array}{l}2.279 \\
(0.69)\end{array}$ & $\begin{array}{l}2.171 \\
(0.53)\end{array}$ & $\begin{array}{l}2.347 \\
(0.50)\end{array}$ & $\begin{array}{l}2.571 \\
(0.48)\end{array}$ & $\begin{array}{l}2.845 \\
(0.43)\end{array}$ & $\begin{array}{l}3.066 \\
(0.47)\end{array}$ & $\begin{array}{l}3.274 \\
(0.39)\end{array}$ & $\begin{array}{l}3.446 \\
(0.41)\end{array}$ & $\begin{array}{l}3.601 \\
(0.38)\end{array}$ & $\begin{array}{c}3.673 \\
(0.38)\end{array}$ & $\begin{array}{l}3.729 \\
(0.41)\end{array}$ & $\begin{array}{c}3.776 \\
(0.55)\end{array}$ \\
\hline
\end{tabular}

Fig. 4.5b. Pin-power distribution for NITAWL benchmark TCA-1. Value in parentheses is percent standard deviation. 
Table 4.5. Selected reaction rates for CENTRM case TCA-1

\begin{tabular}{ccccccc}
\hline Pin & Region & $\begin{array}{c}\mathrm{G}_{\mathrm{a}} \mathrm{M} \\
\left(\mathrm{cm}^{-3}-\mathrm{s}^{-1}\right)\end{array}$ & $\begin{array}{c}\mathrm{G}_{\mathrm{f}} \mathrm{M} \\
\left(\mathrm{cm}^{-3}-\mathrm{s}^{-1}\right)\end{array}$ & $\begin{array}{c}\mathrm{G}_{\mathrm{f}} \mathrm{M} \\
\left(\mathrm{cm}^{-3}-\mathrm{s}^{-1}\right)\end{array}$ & $\begin{array}{c}\mathrm{M} \\
\left(\mathrm{cm}-\mathrm{s}^{-1}\right)\end{array}$ & $\mathrm{M}_{\mathrm{t}}$ \\
\hline \multirow{2}{*}{1} & Fuel & $1.400 \mathrm{E}-05$ & $2.320 \mathrm{E}-05$ & $8.219 \mathrm{E}-06$ & $5.406 \mathrm{E}-03$ & 0.271 \\
& Clad & $2.686 \mathrm{E}-07$ & 0.0 & 0.0 & $1.687 \mathrm{E}-03$ & 0.085 \\
& Mod. & $7.396 \mathrm{E}-07$ & 0.0 & 0.0 & $1.285 \mathrm{E}-02$ & 0.644 \\
\hline \multirow{2}{*}{78} & Fuel & $3.547 \mathrm{E}-05$ & $5.287 \mathrm{E}-05$ & $1.869 \mathrm{E}-05$ & $2.162 \mathrm{E}-02$ & 0.270 \\
& Clad & $7.404 \mathrm{E}-07$ & 0.0 & 0.0 & $6.840 \mathrm{E}-03$ & 0.085 \\
& Mod. & $1.542 \mathrm{E}-06$ & 0.0 & 0.0 & $5.169 \mathrm{E}-02$ & 0.645 \\
\hline
\end{tabular}

Table 4.6. Selected reaction rates for NITAWL case TCA-1

\begin{tabular}{ccccccc}
\hline \multirow{2}{*}{ Pin } & Region & $\begin{array}{c}\mathrm{G}_{\mathrm{a}} \mathrm{M} \\
\left(\mathrm{cm}^{-3}-\mathrm{s}^{-1}\right)\end{array}$ & $\begin{array}{c}\mathrm{G}_{\mathrm{f}} \mathrm{M} \\
\left(\mathrm{cm}^{-3}-\mathrm{s}^{-1}\right)\end{array}$ & $\begin{array}{c}\mathrm{G}_{\mathrm{f}} \mathrm{M} \\
\left(\mathrm{cm}^{-3}-\mathrm{s}^{-1}\right)\end{array}$ & $\begin{array}{c}\mathrm{M} \\
\left(\mathrm{cm}-\mathrm{s}^{-1}\right)\end{array}$ & $\mathrm{M}_{\mathrm{t}}$ \\
\hline \multirow{2}{*}{1} & Fuel & $1.386 \mathrm{E}-05$ & $2.292 \mathrm{E}-05$ & $8.121 \mathrm{E}-06$ & $5.354 \mathrm{E}-03$ & 0.271 \\
& Clad & $2.641 \mathrm{E}-07$ & 0.0 & 0.0 & $1.665 \mathrm{E}-03$ & 0.084 \\
& Mod. & $7.348 \mathrm{E}-07$ & 0.0 & 0.0 & $1.277 \mathrm{E}-02$ & 0.645 \\
\hline \multirow{2}{*}{78} & Fuel & $3.457 \mathrm{E}-05$ & $5.164 \mathrm{E}-05$ & $1.827 \mathrm{E}-05$ & $2.134 \mathrm{E}-02$ & 0.268 \\
& Clad & $7.151 \mathrm{E}-07$ & 0.0 & 0.0 & $6.771 \mathrm{E}-03$ & 0.085 \\
& Mod. & $1.529 \mathrm{E}-06$ & 0.0 & 0.0 & $5.146 \mathrm{E}-02$ & 0.647 \\
\hline
\end{tabular}


Table 4.7. TCA-1, Pin-1 four-group fluxes

\begin{tabular}{ccc}
\hline Group & $\begin{array}{c}\text { CENTRM M } \\
\left(\mathrm{cm}^{-2}-\mathrm{s}^{-1}\right)\end{array}$ & $\begin{array}{c}\text { NITAWL M } \\
\left(\mathrm{cm}^{-2}-\mathrm{s}^{-1}\right)\end{array}$ \\
\hline 1 & $5.205 \mathrm{E}-05$ & $5.150 \mathrm{E}-05$ \\
2 & $1.550 \mathrm{E}-05$ & $1.533 \mathrm{E}-05$ \\
3 & $3.677 \mathrm{E}-06$ & $3.705 \mathrm{E}-06$ \\
4 & $3.068 \mathrm{E}-05$ & $3.040 \mathrm{E}-05$ \\
\hline
\end{tabular}

Table 4.8. TCA-1, Pin-1 four-group U-235 cross sections

\begin{tabular}{ccccccc}
\hline Group & \multicolumn{2}{c}{ CENTRM cross sections $\left(\mathrm{cm}^{-1}\right)$} & \multicolumn{3}{c}{ NITAWL cross sections $\left(\mathrm{cm}^{-1}\right)$} \\
\hline & $\frac{\mathrm{G}_{\mathrm{a}}}{1.43276 \mathrm{E}-04}$ & $\frac{\mathrm{G}_{\mathrm{f}}}{3.25948 \mathrm{E}-04}$ & $\frac{\mathrm{G}_{\mathrm{f}}}{1.25443 \mathrm{E}-04}$ & $\frac{\mathrm{G}_{\mathrm{a}}}{1.43133 \mathrm{E}-04}$ & $\frac{\mathrm{G}_{\mathrm{f}}}{3.25491 \mathrm{E}-04}$ & $\frac{\mathrm{G}_{\mathrm{f}}}{1.25308 \mathrm{E}-04}$ \\
1 & $3.47383 \mathrm{E}-03$ & $5.32334 \mathrm{E}-03$ & $2.18465 \mathrm{E}-03$ & $3.41501 \mathrm{E}-03$ & $5.21704 \mathrm{E}-03$ & $2.14103 \mathrm{E}-03$ \\
2 & $5.07797 \mathrm{E}-03$ & $1.03603 \mathrm{E}-02$ & $4.25178 \mathrm{E}-03$ & $5.13482 \mathrm{E}-03$ & $1.05164 \mathrm{E}-02$ & $4.31584 \mathrm{E}-03$ \\
3 & $4.86542 \mathrm{E}-02$ & $1.01291 \mathrm{E}-01$ & $4.15691 \mathrm{E}-02$ & $4.83612 \mathrm{E}-02$ & $1.00680 \mathrm{E}-01$ & $4.13181 \mathrm{E}-02$ \\
\hline
\end{tabular}

Table 4.9. TCA-1, Pin-1 four-group U-238 cross sections

\begin{tabular}{ccccccc}
\hline Group & \multicolumn{3}{c}{ CENTRM cross sections $\left(\mathrm{cm}^{-1}\right)$} & \multicolumn{2}{c}{ NITAWL cross sections $\left(\mathrm{cm}^{-1}\right)$} \\
\hline & $\frac{\mathrm{G}_{\mathrm{a}}}{4.48868 \mathrm{E}-03}$ & $\frac{\mathrm{G}_{\mathrm{f}}}{8.37908 \mathrm{E}-03}$ & $\frac{\mathrm{G}_{\mathrm{f}}}{2.97182 \mathrm{E}-03}$ & $\frac{\mathrm{G}_{\mathrm{a}}}{4.45235 \mathrm{E}-03}$ & $\frac{\mathrm{G}_{\mathrm{f}}}{8.27583 \mathrm{E}-03}$ & $\frac{\mathrm{G}_{\mathrm{f}}}{2.93388 \mathrm{E}-03}$ \\
1 & $3.79279 \mathrm{E}-02$ & $3.70803 \mathrm{E}-06$ & $1.53622 \mathrm{E}-06$ & $3.78200 \mathrm{E}-02$ & $3.60508 \mathrm{E}-06$ & $1.49357 \mathrm{E}-06$ \\
2 & $6.96274 \mathrm{E}-03$ & $2.42790 \mathrm{E}-08$ & $1.00592 \mathrm{E}-08$ & $6.99725 \mathrm{E}-03$ & $2.45979 \mathrm{E}-08$ & $1.01914 \mathrm{E}-08$ \\
3 & $2.76422 \mathrm{E}-02$ & $1.29929 \mathrm{E}-07$ & $5.38321 \mathrm{E}-08$ & $2.75005 \mathrm{E}-02$ & $1.29251 \mathrm{E}-07$ & $5.35513 \mathrm{E}-08$ \\
\hline
\end{tabular}

Table 4.10. TCA-1, Pin-1 four-group Pu-239 cross sections

\begin{tabular}{cccccccc}
\hline Group & \multicolumn{3}{c}{ CENTRM cross sections $\left(\mathrm{cm}^{-1}\right)$} & \multicolumn{3}{c}{ NITAWL cross sections $\left(\mathrm{cm}^{-1}\right)$} \\
\hline & $\frac{\mathrm{G}_{\mathrm{a}}}{}$ & $\frac{\mathrm{G}_{\mathrm{f}}}{12119 \mathrm{E}-04}$ & $\frac{\mathrm{G}_{\mathrm{f}}}{1.49477 \mathrm{E}-03}$ & $\frac{4.80859 \mathrm{E}-04}{5.11680 \mathrm{E}-04}$ & $\frac{\mathrm{G}_{\mathrm{f}}}{1.49305 \mathrm{E}-03}$ & $\frac{\mathrm{G}_{\mathrm{f}}}{4.80499 \mathrm{E}-04}$ \\
2 & $1.13759 \mathrm{E}-02$ & $1.87090 \mathrm{E}-02$ & $6.49658 \mathrm{E}-03$ & $1.06255 \mathrm{E}-02$ & $1.76083 \mathrm{E}-02$ & $6.11439 \mathrm{E}-03$ \\
3 & $3.27337 \mathrm{E}-02$ & $6.44523 \mathrm{E}-02$ & $2.23808 \mathrm{E}-02$ & $3.35760 \mathrm{E}-02$ & $6.60534 \mathrm{E}-02$ & $2.29368 \mathrm{E}-02$ \\
4 & $2.66846 \mathrm{E}-01$ & $5.40163 \mathrm{E}-01$ & $1.86831 \mathrm{E}-01$ & $2.66855 \mathrm{E}-01$ & $5.39595 \mathrm{E}-01$ & $1.86638 \mathrm{E}-01$ \\
\hline
\end{tabular}


Table 4.11. TCA-1, Pin-78 four-group fluxes

\begin{tabular}{ccc}
\hline Group & $\begin{array}{c}\text { CENTRM M } \\
\left(\mathrm{cm}^{-2}-\mathrm{s}^{-1}\right)\end{array}$ & $\begin{array}{c}\text { NITAWL M } \\
\left(\mathrm{cm}^{-2}-\mathrm{s}^{-1}\right)\end{array}$ \\
\hline 1 & $2.399 \mathrm{E}-04$ & $2.360 \mathrm{E}-04$ \\
2 & $8.524 \mathrm{E}-05$ & $8.507 \mathrm{E}-05$ \\
3 & $1.867 \mathrm{E}-05$ & $1.851 \mathrm{E}-05$ \\
4 & $6.380 \mathrm{E}-05$ & $6.276 \mathrm{E}-05$ \\
\hline
\end{tabular}

Table 4.12. TCA-1, Pin-78 four-group U-235 cross sections

\begin{tabular}{cccccccc}
\hline Group & \multicolumn{3}{c}{ CENTRM cross sections $\left(\mathrm{cm}^{-1}\right)$} & \multicolumn{3}{c}{ NITAWL cross sections $\left(\mathrm{cm}^{-1}\right)$} \\
\hline & $\frac{\mathrm{G}_{\mathrm{a}}}{1.48131 \mathrm{E}-04}$ & $\frac{\mathrm{G}_{\mathrm{f}}}{3.30102 \mathrm{E}-04}$ & $\frac{\mathrm{G}_{\mathrm{f}}}{1.27921 \mathrm{E}-04}$ & $\frac{\mathrm{G}_{\mathrm{a}}}{1.47652 \mathrm{E}-04}$ & $\frac{\mathrm{G}_{\mathrm{f}}}{3.28787 \mathrm{E}-04}$ & $\frac{\mathrm{G}_{\mathrm{f}}}{1.27540 \mathrm{E}-04}$ \\
1 & $3.33968 \mathrm{E}-03$ & $5.12318 \mathrm{E}-03$ & $2.10251 \mathrm{E}-03$ & $3.40930 \mathrm{E}-03$ & $5.23865 \mathrm{E}-03$ & $2.14990 \mathrm{E}-03$ \\
3 & $5.10391 \mathrm{E}-03$ & $1.04015 \mathrm{E}-02$ & $4.26869 \mathrm{E}-03$ & $5.13030 \mathrm{E}-03$ & $1.04663 \mathrm{E}-02$ & $4.29526 \mathrm{E}-03$ \\
4 & $4.54602 \mathrm{E}-02$ & $9.45345 \mathrm{E}-02$ & $3.87961 \mathrm{E}-02$ & $4.53139 \mathrm{E}-02$ & $9.42388 \mathrm{E}-02$ & $3.86748 \mathrm{E}-02$ \\
\hline
\end{tabular}

Table 4.13. TCA-1, Pin-78 four-group U-238 cross sections

\begin{tabular}{cccccccc}
\hline Group & \multicolumn{3}{c}{ CENTRM cross sections $\left(\mathrm{cm}^{-1}\right)$} & \multicolumn{3}{c}{ NITAWL cross sections $\left(\mathrm{cm}^{-1}\right)$} \\
\hline & $\frac{\mathrm{G}_{\mathrm{a}}}{4.34327 \mathrm{E}-03}$ & $\frac{\mathrm{G}_{\mathrm{f}}}{7.48462 \mathrm{E}-03}$ & $\frac{\mathrm{G}_{\mathrm{f}}}{2.65627 \mathrm{E}-03}$ & $\frac{\mathrm{G}_{\mathrm{a}}}{4.30530 \mathrm{E}-03}$ & $\frac{\mathrm{G}_{\mathrm{f}}}{7.37008 \mathrm{E}-03}$ & $\frac{\mathrm{G}_{\mathrm{f}}}{2.62250 \mathrm{E}-03}$ \\
1 & $3.98175 \mathrm{E}-02$ & $3.68536 \mathrm{E}-06$ & $1.52683 \mathrm{E}-06$ & $3.74190 \mathrm{E}-02$ & $3.60909 \mathrm{E}-06$ & $1.49523 \mathrm{E}-06$ \\
2 & $6.98275 \mathrm{E}-03$ & $2.43235 \mathrm{E}-08$ & $1.00777 \mathrm{E}-08$ & $6.98957 \mathrm{E}-03$ & $2.44139 \mathrm{E}-08$ & $1.01151 \mathrm{E}-08$ \\
3 & $2.59889 \mathrm{E}-02$ & $1.21997 \mathrm{E}-07$ & $5.05458 \mathrm{E}-08$ & $2.59301 \mathrm{E}-02$ & $1.21719 \mathrm{E}-07$ & $5.04304 \mathrm{E}-08$ \\
\hline
\end{tabular}

Table 4.14. TCA-1, Pin-78 four-group Pu-239 cross sections

\begin{tabular}{|c|c|c|c|c|c|c|}
\hline \multirow[t]{2}{*}{ Group } & \multicolumn{3}{|c|}{ CENTRM cross sections $\left(\mathrm{cm}^{-1}\right)$} & \multicolumn{3}{|c|}{ NITAWL cross sections $\left(\mathrm{cm}^{-1}\right)$} \\
\hline & $\mathrm{G}_{\mathrm{a}}$ & $<\mathrm{G}_{\mathrm{f}}$ & $\mathrm{G}_{\mathrm{f}}$ & $\mathrm{G}_{\mathrm{a}}$ & $\mathrm{G}_{\mathrm{f}}$ & $\mathrm{G}_{\mathrm{f}}$ \\
\hline 1 & $5.14181 \mathrm{E}-04$ & $\overline{1.47485 \mathrm{E}-03}$ & $\overline{4.77461 \mathrm{E}-04}$ & $5.13292 \mathrm{E}-04$ & $\overline{1.47159 \mathrm{E}-03}$ & $\overline{4.76847 \mathrm{E}-04}$ \\
\hline 2 & $1.06372 \mathrm{E}-02$ & $1.75772 \mathrm{E}-02$ & $6.10359 \mathrm{E}-03$ & $1.08006 \mathrm{E}-02$ & 1.79107E-02 & $6.21938 \mathrm{E}-03$ \\
\hline 3 & $3.42975 \mathrm{E}-02$ & $6.73203 \mathrm{E}-02$ & $2.33768 \mathrm{E}-02$ & $3.44553 \mathrm{E}-02$ & $6.76072 \mathrm{E}-02$ & $2.34764 \mathrm{E}-02$ \\
\hline 4 & $2.85886 \mathrm{E}-01$ & $5.65284 \mathrm{E}-01$ & $1.95610 \mathrm{E}-01$ & 2.82398E-01 & 5.59148E-01 & $1.93483 \mathrm{E}-01$ \\
\hline
\end{tabular}




\begin{tabular}{|c|c|c|c|c|c|c|c|c|c|c|c|}
\hline & & & & & & & & & & & $\begin{array}{l}4.965 \\
(0.99)\end{array}$ \\
\hline & $\begin{array}{r}0.139 \\
(0.64) \\
\end{array}$ & & & & & & & & & $\begin{array}{l}4.937 \\
(0.46)\end{array}$ & $\begin{array}{l}4.994 \\
(0.49)\end{array}$ \\
\hline & & & & & & & & & $\begin{array}{l}4.776 \\
(0.50)\end{array}$ & $\begin{array}{l}4.866 \\
(0.34)\end{array}$ & $\begin{array}{l}4.932 \\
(0.47)\end{array}$ \\
\hline & & & & & & & & $\begin{array}{l}4.569 \\
(0.53)\end{array}$ & $\begin{array}{l}4.688 \\
(0.32)\end{array}$ & $\begin{array}{l}4.750 \\
(0.35)\end{array}$ & $\begin{array}{l}4.781 \\
(0.47)\end{array}$ \\
\hline & & & & & & & $\begin{array}{l}4.283 \\
(0.57)\end{array}$ & $\begin{array}{l}4.402 \\
(0.38)\end{array}$ & $\begin{array}{l}4.527 \\
(0.36)\end{array}$ & $\begin{array}{l}4.600 \\
(0.35)\end{array}$ & $\begin{array}{l}4.677 \\
(0.48)\end{array}$ \\
\hline & & & & & & $\begin{array}{l}3.846 \\
(0.55)\end{array}$ & $\begin{array}{l}4.048 \\
(0.38)\end{array}$ & $\begin{array}{l}4.205 \\
(0.37)\end{array}$ & $\begin{array}{l}4.302 \\
(0.36)\end{array}$ & $\begin{array}{l}4.359 \\
(0.34)\end{array}$ & $\begin{array}{l}4.376 \\
(0.50)\end{array}$ \\
\hline & & & & & $\begin{array}{l}3.405 \\
(0.57)\end{array}$ & $\begin{array}{l}3.636 \\
(0.43)\end{array}$ & $\begin{array}{l}3.795 \\
(0.37)\end{array}$ & $\begin{array}{l}3.946 \\
(0.36)\end{array}$ & $\begin{array}{l}4.058 \\
(0.34)\end{array}$ & $\begin{array}{l}4.111 \\
(0.39)\end{array}$ & $\begin{array}{l}4.125 \\
(0.49)\end{array}$ \\
\hline & & & & $\begin{array}{l}2.910 \\
(0.64)\end{array}$ & $\begin{array}{l}3.171 \\
(0.47)\end{array}$ & $\begin{array}{l}3.388 \\
(0.42)\end{array}$ & $\begin{array}{l}3.532 \\
(0.36)\end{array}$ & $\begin{array}{l}3.649 \\
(0.41)\end{array}$ & $\begin{array}{l}3.739 \\
(0.41)\end{array}$ & $\begin{array}{l}3.822 \\
(0.40)\end{array}$ & $\begin{array}{l}3.849 \\
(0.55)\end{array}$ \\
\hline & & & $\begin{array}{l}2.455 \\
(0.64)\end{array}$ & $\begin{array}{l}2.666 \\
(0.48)\end{array}$ & $\begin{array}{l}2.882 \\
(0.43)\end{array}$ & $\begin{array}{l}3.063 \\
(0.42)\end{array}$ & $\begin{array}{l}3.207 \\
(0.41)\end{array}$ & $\begin{array}{l}3.324 \\
(0.42)\end{array}$ & $\begin{array}{l}3.449 \\
(0.41)\end{array}$ & $\begin{array}{l}3.489 \\
(0.42)\end{array}$ & $\begin{array}{l}3.486 \\
(0.55)\end{array}$ \\
\hline & & $\begin{array}{l}2.021 \\
(0.74)\end{array}$ & $\begin{array}{l}2.222 \\
(0.54)\end{array}$ & $\begin{array}{l}2.433 \\
0.46)\end{array}$ & $\begin{array}{l}2.634 \\
(0.46)\end{array}$ & $\begin{array}{l}2.808 \\
(0.47)\end{array}$ & $\begin{array}{l}2.906 \\
(0.42)\end{array}$ & $\begin{array}{l}3.043 \\
(0.45)\end{array}$ & $\begin{array}{l}3.141 \\
(0.44)\end{array}$ & $\begin{array}{l}3.166 \\
(0.45)\end{array}$ & $\begin{array}{l}3.189 \\
(0.61)\end{array}$ \\
\hline & $\begin{array}{l}1.841 \\
(0.80)\end{array}$ & $\begin{array}{l}1.935 \\
(0.54)\end{array}$ & $\begin{array}{c}2.120 \\
(0.48)\end{array}$ & $\begin{array}{l}2.324 \\
(0.52)\end{array}$ & $\begin{array}{l}2.503 \\
(0.44)\end{array}$ & $\begin{array}{l}2.645 \\
(0.46)\end{array}$ & $\begin{array}{l}2.825 \\
(0.44)\end{array}$ & $\begin{array}{l}2.902 \\
(0.43)\end{array}$ & $\begin{array}{l}2.982 \\
(0.43)\end{array}$ & $\begin{array}{c}3.040 \\
(0.46)\end{array}$ & $\begin{array}{l}3.036 \\
(0.61)\end{array}$ \\
\hline $\begin{array}{l}2.226 \\
(0.69)\end{array}$ & $\begin{array}{l}2.099 \\
(0.51)\end{array}$ & $\begin{array}{l}2.276 \\
(0.50)\end{array}$ & $\begin{array}{l}2.506 \\
(0.50)\end{array}$ & $\begin{array}{l}2.739 \\
(0.44)\end{array}$ & $\begin{array}{l}2.952 \\
(0.41)\end{array}$ & $\begin{array}{l}3.138 \\
(0.43)\end{array}$ & $\begin{array}{l}3.341 \\
(0.35)\end{array}$ & $\begin{array}{l}3.425 \\
(0.39)\end{array}$ & $\begin{array}{c}3.533 \\
(0.37)\end{array}$ & $\begin{array}{l}3.606 \\
(0.40)\end{array}$ & $\begin{array}{l}3.590 \\
(0.59)\end{array}$ \\
\hline
\end{tabular}

Fig. 4.6a. Pin-power distribution for CENTRM benchmark TCA-2. Value in parentheses is percent standard deviation. 


\begin{tabular}{|c|c|c|c|c|c|c|c|c|c|c|c|}
\hline & & & & & & & & & & & $\begin{array}{l}5.023 \\
(0.99)\end{array}$ \\
\hline & $\begin{array}{l}0.139 \\
(0.63)\end{array}$ & & & & & & & & & $\begin{array}{l}4.960 \\
(0.46)\end{array}$ & $\begin{array}{l}5.042 \\
(0.47)\end{array}$ \\
\hline & & & & & & & & & $\begin{array}{l}4.835 \\
(0.48)\end{array}$ & $\begin{array}{l}4.922 \\
(0.33)\end{array}$ & $\begin{array}{l}4.929 \\
(0.47)\end{array}$ \\
\hline & & & & & & & & $\begin{array}{l}4.569 \\
(0.52)\end{array}$ & $\begin{array}{l}4.721 \\
(0.32)\end{array}$ & $\begin{array}{l}4.780 \\
(0.36)\end{array}$ & $\begin{array}{l}4.776 \\
(0.48)\end{array}$ \\
\hline & & & & & & & $\begin{array}{l}4.250 \\
(0.50) \\
\end{array}$ & $\begin{array}{r}4.420 \\
(0.35) \\
\end{array}$ & $\begin{array}{l}4.521 \\
(0.36) \\
\end{array}$ & $\begin{array}{l}4.608 \\
(0.35) \\
\end{array}$ & $\begin{array}{l}4.612 \\
(0.50) \\
\end{array}$ \\
\hline & & & & & & $\begin{array}{l}3.803 \\
(0.58)\end{array}$ & $\begin{array}{l}4.057 \\
(0.34)\end{array}$ & $\begin{array}{l}4.179 \\
(0.39)\end{array}$ & $\begin{array}{l}4.303 \\
(0.35)\end{array}$ & $\begin{array}{l}4.406 \\
(0.35)\end{array}$ & $\begin{array}{r}4.409 \\
(0.51)\end{array}$ \\
\hline & & & & & $\begin{array}{c}3.430 \\
(0.58)\end{array}$ & $\begin{array}{l}3.641 \\
(0.41)\end{array}$ & $\begin{array}{l}3.836 \\
(0.39)\end{array}$ & $\begin{array}{l}3.972 \\
(0.37)\end{array}$ & $\begin{array}{l}4.049 \\
(0.39)\end{array}$ & $\begin{array}{l}4.121 \\
(0.37)\end{array}$ & $\begin{array}{l}4.127 \\
(0.54)\end{array}$ \\
\hline & & & & $\begin{array}{l}2.949 \\
(0.66)\end{array}$ & $\begin{array}{l}3.164 \\
(0.42)\end{array}$ & $\begin{array}{l}3.374 \\
(0.41)\end{array}$ & $\begin{array}{l}3.549 \\
(0.43)\end{array}$ & $\begin{array}{l}3.649 \\
(0.40)\end{array}$ & $\begin{array}{l}3.775 \\
(0.38)\end{array}$ & $\begin{array}{l}3.806 \\
(0.42)\end{array}$ & $\begin{array}{l}3.858 \\
(0.53)\end{array}$ \\
\hline & & & $\begin{array}{l}2.447 \\
(0.66)\end{array}$ & $\begin{array}{l}2.659 \\
(0.43)\end{array}$ & $\begin{array}{l}2.876 \\
(0.44)\end{array}$ & $\begin{array}{l}3.101 \\
(0.45)\end{array}$ & $\begin{array}{l}3.229 \\
(0.44)\end{array}$ & $\begin{array}{l}3.359 \\
(0.40)\end{array}$ & $\begin{array}{l}3.459 \\
(0.38)\end{array}$ & $\begin{array}{l}3.487 \\
(0.43)\end{array}$ & $\begin{array}{c}3.504 \\
(0.59)\end{array}$ \\
\hline & & $\begin{array}{l}2.029 \\
(0.71)\end{array}$ & $\begin{array}{l}2.232 \\
(0.51)\end{array}$ & $\begin{array}{l}2.431 \\
0.48)\end{array}$ & $\begin{array}{l}2.644 \\
(0.45)\end{array}$ & $\begin{array}{l}2.793 \\
(0.49)\end{array}$ & $\begin{array}{l}2.940 \\
(0.44)\end{array}$ & $\begin{array}{l}3.042 \\
(0.45)\end{array}$ & $\begin{array}{l}3.132 \\
(0.39)\end{array}$ & $\begin{array}{l}3.194 \\
(0.43)\end{array}$ & $\begin{array}{l}3.205 \\
(0.58)\end{array}$ \\
\hline & $\begin{array}{l}1.837 \\
(0.75)\end{array}$ & $\begin{array}{l}1.930 \\
(0.56)\end{array}$ & $\begin{array}{l}2.140 \\
(0.51)\end{array}$ & $\begin{array}{l}2.325 \\
(0.53)\end{array}$ & $\begin{array}{l}2.522 \\
(0.47)\end{array}$ & $\begin{array}{l}2.677 \\
(0.44)\end{array}$ & $\begin{array}{l}2.816 \\
(0.44)\end{array}$ & $\begin{array}{l}2.936 \\
(0.40)\end{array}$ & $\begin{array}{l}3.008 \\
(0.44)\end{array}$ & $\begin{array}{l}3.035 \\
(0.40)\end{array}$ & $\begin{array}{l}3.064 \\
(0.61)\end{array}$ \\
\hline $\begin{array}{l}2.222 \\
(0.68)\end{array}$ & $\begin{array}{c}2.106 \\
(0.51)\end{array}$ & $\begin{array}{l}2.274 \\
(0.52)\end{array}$ & $\begin{array}{l}2.527 \\
(0.47)\end{array}$ & $\begin{array}{l}2.756 \\
(0.43)\end{array}$ & $\begin{array}{c}2.976 \\
(0.41)\end{array}$ & $\begin{array}{l}3.149 \\
(0.44)\end{array}$ & $\begin{array}{l}3.323 \\
(0.39)\end{array}$ & $\begin{array}{l}3.477 \\
(0.38)\end{array}$ & $\begin{array}{l}3.562 \\
(0.38)\end{array}$ & $\begin{array}{c}3.583 \\
(0.41)\end{array}$ & $\begin{array}{l}3.598 \\
(0.59)\end{array}$ \\
\hline
\end{tabular}

Fig. 4.6b. Pin-power distribution for NITAWL benchmark TCA-2. Value in parentheses is percent standard deviation. 
Table 4.15. Selected reaction rates for CENTRM case TCA-2

\begin{tabular}{ccccccc}
\hline Pin & Region & $\begin{array}{c}\mathrm{G}_{\mathrm{a}} \mathrm{M} \\
\left(\mathrm{cm}^{-3}-\mathrm{s}^{-1}\right)\end{array}$ & $\begin{array}{c}\mathrm{G}_{\mathrm{f}} \mathrm{M} \\
\left(\mathrm{cm}^{-3}-\mathrm{s}^{-1}\right)\end{array}$ & $\begin{array}{c}\mathrm{G}_{\mathrm{f}} \mathrm{M} \\
\left(\mathrm{cm}^{-3}-\mathrm{s}^{-1}\right)\end{array}$ & $\begin{array}{c}\mathrm{M} \\
\left(\mathrm{cm}^{-1}\right)\end{array}$ & $\mathrm{M}_{\mathrm{t}}$ \\
\hline \multirow{2}{*}{1} & Fuel & $1.356 \mathrm{E}-05$ & $2.236 \mathrm{E}-05$ & $7.923 \mathrm{E}-06$ & $5.453 \mathrm{E}-03$ & 0.270 \\
& Clad & $2.613 \mathrm{E}-07$ & 0.0 & 0.0 & $1.705 \mathrm{E}-03$ & 0.085 \\
& Mod. & $7.206 \mathrm{E}-07$ & 0.0 & 0.0 & $1.301 \mathrm{E}-02$ & 0.645 \\
\hline \multirow{2}{*}{78} & Fuel & $3.394 \mathrm{E}-05$ & $5.051 \mathrm{E}-05$ & $1.786 \mathrm{E}-05$ & $2.155 \mathrm{E}-02$ & 0.269 \\
& Clad & $7.023 \mathrm{E}-07$ & 0.0 & 0.0 & $6.812 \mathrm{E}-03$ & 0.085 \\
& Mod. & $1.495 \mathrm{E}-06$ & 0.0 & 0.0 & $5.179 \mathrm{E}-02$ & 0.646 \\
\hline
\end{tabular}

Table 4.16. Selected reaction rates for NITAWL case TCA-2

\begin{tabular}{|c|c|c|c|c|c|c|}
\hline Pin & Region & $\begin{array}{c}\mathrm{G}_{\mathrm{a}} \mathrm{M} \\
\left(\mathrm{cm}^{-3}-\mathrm{s}^{-1}\right)\end{array}$ & $\begin{array}{c}\mathcal{G}_{\mathrm{f}} \mathrm{M} \\
\left(\mathrm{cm}^{-3}-\mathrm{s}^{-1}\right)\end{array}$ & $\begin{array}{c}\mathrm{G}_{\mathrm{f}} \mathrm{M} \\
\left(\mathrm{cm}^{-3}-\mathrm{s}^{-1}\right)\end{array}$ & $\begin{array}{c}\mathrm{M} \\
\left(\mathrm{cm}-\mathrm{s}^{-1}\right)\end{array}$ & $\mathrm{M} / \mathrm{M}_{\mathrm{t}}$ \\
\hline \multirow{3}{*}{1} & Fuel & $1.356 \mathrm{E}-05$ & $2.241 \mathrm{E}-05$ & 7.942E-06 & $5.429 \mathrm{E}-03$ & 0.271 \\
\hline & Clad & 2.591E-07 & 0.0 & 0.0 & $1.693 \mathrm{E}-03$ & 0.084 \\
\hline & Mod. & 7.211E-07 & 0.0 & 0.0 & $1.293 \mathrm{E}-02$ & 0.645 \\
\hline \multirow{3}{*}{78} & Fuel & 3.354E-05 & $5.009 \mathrm{E}-05$ & 1.771E-05 & $2.137 \mathrm{E}-02$ & 0.268 \\
\hline & Clad & $6.772 \mathrm{E}-07$ & 0.0 & 0.0 & $6.756 \mathrm{E}-03$ & 0.084 \\
\hline & Mod. & $1.478 \mathrm{E}-06$ & 0.0 & 0.0 & $5.172 \mathrm{E}-02$ & 0.648 \\
\hline
\end{tabular}


Table 4.17. TCA-2, Pin-1 four-group fluxes

\begin{tabular}{ccc}
\hline Group & $\begin{array}{c}\text { CENTRM M } \\
\left(\mathrm{cm}^{-2}-\mathrm{s}^{-1}\right)\end{array}$ & $\begin{array}{c}\text { NITAWL M } \\
\left(\mathrm{cm}^{-2}-\mathrm{s}^{-1}\right)\end{array}$ \\
\hline 1 & $5.046 \mathrm{E}-05$ & $5.017 \mathrm{E}-05$ \\
2 & $1.503 \mathrm{E}-05$ & $1.485 \mathrm{E}-05$ \\
3 & $3.705 \mathrm{E}-06$ & $3.620 \mathrm{E}-06$ \\
4 & $2.970 \mathrm{E}-05$ & $2.981 \mathrm{E}-05$ \\
\hline
\end{tabular}

Table 4.18. TCA-2, Pin-1 four-group U-235 cross sections

\begin{tabular}{cccccccc}
\hline Group & \multicolumn{2}{c}{ CENTRM cross sections $\left(\mathrm{cm}^{-1}\right)$} & \multicolumn{3}{c}{ NITAWL cross sections $\left(\mathrm{cm}^{-1}\right)$} \\
\hline & $\frac{\mathrm{G}_{\mathrm{a}}}{1.43119 \mathrm{E}-04}$ & $\frac{\mathrm{G}_{\mathrm{f}}}{3.25379 \mathrm{E}-04}$ & $\frac{\mathrm{G}_{\mathrm{f}}}{1.25277 \mathrm{E}-04}$ & $\frac{\mathrm{G}_{\mathrm{a}}}{1.42796 \mathrm{E}-04}$ & $\frac{\mathrm{G}_{\mathrm{f}}}{3.25595 \mathrm{E}-04}$ & $\frac{\mathrm{G}_{\mathrm{f}}}{1.25157 \mathrm{E}-04}$ \\
1 & $3.53013 \mathrm{E}-03$ & $5.42307 \mathrm{E}-03$ & $2.22558 \mathrm{E}-03$ & $3.53101 \mathrm{E}-03$ & $5.37976 \mathrm{E}-03$ & $2.20780 \mathrm{E}-03$ \\
2 & $5.06839 \mathrm{E}-03$ & $1.03472 \mathrm{E}-02$ & $4.24642 \mathrm{E}-03$ & $5.21793 \mathrm{E}-03$ & $1.06593 \mathrm{E}-02$ & $4.37445 \mathrm{E}-03$ \\
3 & $4.85643 \mathrm{E}-02$ & $1.01103 \mathrm{E}-01$ & $4.14919 \mathrm{E}-02$ & $4.86105 \mathrm{E}-02$ & $1.01201 \mathrm{E}-01$ & $4.15317 \mathrm{E}-02$ \\
\hline
\end{tabular}

Table 4.19. TCA-2, Pin-1 four-group U-238 cross sections

\begin{tabular}{cccccccc}
\hline Group & \multicolumn{3}{c}{ CENTRM cross sections $\left(\mathrm{cm}^{-1}\right)$} & \multicolumn{3}{c}{ NITAWL cross sections $\left(\mathrm{cm}^{-1}\right)$} \\
\hline & $\frac{\mathrm{G}_{\mathrm{a}}}{4.44405 \mathrm{E}-03}$ & $\frac{\mathrm{G}_{\mathrm{f}}}{8.24665 \mathrm{E}-03}$ & $\frac{\mathrm{G}_{\mathrm{f}}}{2.92378 \mathrm{E}-03}$ & $\frac{\mathrm{G}_{\mathrm{a}}}{4.49711 \mathrm{E}-03}$ & $\frac{\mathrm{G}_{\mathrm{f}}}{8.46895 \mathrm{E}-03}$ & $\frac{\mathrm{G}_{\mathrm{f}}}{2.99705 \mathrm{E}-03}$ \\
1 & $3.85408 \mathrm{E}-02$ & $3.81629 \mathrm{E}-06$ & $1.58108 \mathrm{E}-06$ & $3.63117 \mathrm{E}-02$ & $3.48554 \mathrm{E}-06$ & $1.44405 \mathrm{E}-06$ \\
2 & $6.97263 \mathrm{E}-03$ & $2.42518 \mathrm{E}-08$ & $1.00480 \mathrm{E}-08$ & $6.99494 \mathrm{E}-03$ & $2.45706 \mathrm{E}-08$ & $1.01801 \mathrm{E}-08$ \\
3 & $2.75966 \mathrm{E}-02$ & $1.29711 \mathrm{E}-07$ & $5.37418 \mathrm{E}-08$ & $2.76225 \mathrm{E}-02$ & $1.29835 \mathrm{E}-07$ & $5.37930 \mathrm{E}-08$ \\
\hline
\end{tabular}

Table 4.20. TCA-2, Pin-1 four-group $\mathrm{Pu}-239$ cross sections

\begin{tabular}{|c|c|c|c|c|c|c|}
\hline \multirow[t]{2}{*}{ Group } & \multicolumn{3}{|c|}{ CENTRM cross sections $\left(\mathrm{cm}^{-1}\right)$} & \multicolumn{3}{|c|}{ NITAWL cross sections $\left(\mathrm{cm}^{-1}\right)$} \\
\hline & $\mathrm{G}_{\mathrm{a}}$ & $<\mathrm{G}_{\mathrm{f}}$ & $\mathrm{G}_{\mathrm{f}}$ & $\mathrm{G}_{\mathrm{a}}$ & $<\mathrm{G}_{\mathrm{f}}$ & $\mathrm{G}_{\mathrm{f}}$ \\
\hline 1 & $5.11583 \mathrm{E}-04$ & $\overline{1.49239 \mathrm{E}-03}$ & $\overline{4.80303 \mathrm{E}-04}$ & $\overline{5.11437 \mathrm{E}-04}$ & $1.49567 \mathrm{E}-03$ & $4.80666 \mathrm{E}-04$ \\
\hline 2 & 1.10651E-02 & $1.80525 \mathrm{E}-02$ & $6.26864 \mathrm{E}-03$ & 1.07872E-02 & $1.76378 \mathrm{E}-02$ & $6.12465 \mathrm{E}-03$ \\
\hline 3 & $3.33360 \mathrm{E}-02$ & $6.55390 \mathrm{E}-02$ & $2.27581 \mathrm{E}-02$ & $3.37318 \mathrm{E}-02$ & 6.63232E-02 & $2.30304 \mathrm{E}-02$ \\
\hline 4 & 2.67093E-01 & 5.40384E-01 & $1.86909 \mathrm{E}-01$ & $2.66605 \mathrm{E}-01$ & 5.39661E-01 & $1.86656 \mathrm{E}-01$ \\
\hline
\end{tabular}


Table 4.21. TCA-2, Pin-78 four-group fluxes

\begin{tabular}{ccc}
\hline Group & $\begin{array}{c}\text { CENTRM M } \\
\left(\mathrm{cm}^{-2}-\mathrm{s}^{-1}\right)\end{array}$ & $\begin{array}{c}\text { NITAWL M } \\
\left(\mathrm{cm}^{-2}-\mathrm{s}^{-1}\right)\end{array}$ \\
\hline 1 & $2.302 \mathrm{E}-04$ & $2.272 \mathrm{E}-04$ \\
2 & $8.173 \mathrm{E}-05$ & $8.176 \mathrm{E}-05$ \\
3 & $1.759 \mathrm{E}-05$ & $1.791 \mathrm{E}-05$ \\
4 & $6.122 \mathrm{E}-05$ & $6.071 \mathrm{E}-05$ \\
\hline
\end{tabular}

Table 4.22. TCA-2, Pin-78 four-group U-235 cross sections

\begin{tabular}{cccccccc}
\hline Group & \multicolumn{3}{c}{ CENTRM cross sections $\left(\mathrm{cm}^{-1}\right)$} & \multicolumn{3}{c}{ NITAWL cross sections $\left(\mathrm{cm}^{-1}\right)$} \\
\hline & $\frac{\mathrm{G}_{\mathrm{a}}}{1.47775 \mathrm{E}-04}$ & $\frac{\mathrm{G}_{\mathrm{f}}}{3.29361 \mathrm{E}-04}$ & $\frac{\mathrm{G}_{\mathrm{f}}}{1.27654 \mathrm{E}-04}$ & $\frac{\mathrm{G}_{\mathrm{a}}}{1.47727 \mathrm{E}-04}$ & $\frac{\mathrm{G}_{\mathrm{f}}}{3.29384 \mathrm{E}-04}$ & $\frac{\mathrm{G}_{\mathrm{f}}}{1.27666 \mathrm{E}-04}$ \\
1 & $3.40270 \mathrm{E}-03$ & $5.23267 \mathrm{E}-03$ & $2.14744 \mathrm{E}-03$ & $3.44284 \mathrm{E}-03$ & $5.28022 \mathrm{E}-03$ & $2.16695 \mathrm{E}-03$ \\
2 & $5.19904 \mathrm{E}-03$ & $1.06173 \mathrm{E}-02$ & $4.35723 \mathrm{E}-03$ & $5.12518 \mathrm{E}-03$ & $1.04631 \mathrm{E}-02$ & $4.29398 \mathrm{E}-03$ \\
3 & $4.56001 \mathrm{E}-02$ & $9.48282 \mathrm{E}-02$ & $3.89167 \mathrm{E}-02$ & $4.54184 \mathrm{E}-02$ & $9.44484 \mathrm{E}-02$ & $3.87607 \mathrm{E}-02$ \\
\hline
\end{tabular}

Table 4.23. TCA-2, Pin-78 four-group U-238 cross sections

\begin{tabular}{|c|c|c|c|c|c|c|}
\hline \multirow[t]{2}{*}{ Group } & \multicolumn{3}{|c|}{ CENTRM cross sections $\left(\mathrm{cm}^{-1}\right)$} & \multicolumn{3}{|c|}{ NITAWL cross sections $\left(\mathrm{cm}^{-1}\right)$} \\
\hline & $\mathrm{G}_{\mathrm{a}}$ & $<G_{f}$ & $\mathrm{G}_{\mathrm{f}}$ & $\mathrm{G}_{\mathrm{a}}$ & $<\mathrm{G}_{\mathrm{f}}$ & $\mathrm{G}_{\mathrm{f}}$ \\
\hline 1 & $4.32970 \mathrm{E}-03$ & $7.46321 \mathrm{E}-03$ & $2.65044 \mathrm{E}-03$ & $4.32945 \mathrm{E}-03$ & $7.46893 \mathrm{E}-03$ & $2.65359 \mathrm{E}-03$ \\
\hline 2 & 3.93173E-02 & $3.71651 \mathrm{E}-06$ & $1.53973 \mathrm{E}-06$ & $3.66084 \mathrm{E}-02$ & 3.70903E-06 & $1.53663 \mathrm{E}-06$ \\
\hline 3 & 7.00819E-03 & $2.46566 \mathrm{E}-08$ & $1.02156 \mathrm{E}-08$ & 6.99481E-03 & 2.44808E-08 & 1.01429E-08 \\
\hline 4 & $2.60606 \mathrm{E}-02$ & $1.22341 \mathrm{E}-07$ & $5.06882 \mathrm{E}-08$ & 2.59687E-02 & $1.21901 \mathrm{E}-07$ & $5.05058 \mathrm{E}-08$ \\
\hline
\end{tabular}

Table 4.24. TCA-2, Pin-78 four-group Pu-239 cross sections

\begin{tabular}{|c|c|c|c|c|c|c|}
\hline \multirow[t]{2}{*}{ Group } & \multicolumn{3}{|c|}{ CENTRM cross sections $\left(\mathrm{cm}^{-1}\right)$} & \multicolumn{3}{|c|}{ NITAWL cross sections $\left(\mathrm{cm}^{-1}\right)$} \\
\hline & $\mathrm{G}_{\mathrm{a}}$ & $\mathrm{G}_{\mathrm{f}}$ & $\mathrm{G}_{\mathrm{f}}$ & $\mathrm{G}_{\mathrm{a}}$ & $\mathrm{G}_{\mathrm{f}}$ & $\mathrm{G}_{\mathrm{f}}$ \\
\hline 1 & $5.13614 \mathrm{E}-04$ & $\overline{1.47354 \mathrm{E}-03}$ & $\overline{4.77076 \mathrm{E}-04}$ & $5.13794 \mathrm{E}-04$ & $\overline{1.47461 \mathrm{E}-03}$ & $\overline{4.77474 \mathrm{E}-04}$ \\
\hline 2 & $1.10415 \mathrm{E}-02$ & $1.82425 \mathrm{E}-02$ & $6.33460 \mathrm{E}-03$ & $1.06940 \mathrm{E}-02$ & 1.76497E-02 & $6.12879 \mathrm{E}-03$ \\
\hline 3 & $3.48073 \mathrm{E}-02$ & 6.83161E-02 & 2.37224E-02 & 3.39004E-02 & $6.66510 \mathrm{E}-02$ & $2.31443 \mathrm{E}-02$ \\
\hline 4 & $2.84748 \mathrm{E}-01$ & 5.63652E-01 & $1.95041 \mathrm{E}-01$ & $2.85625 \mathrm{E}-01$ & 5.64792E-01 & $1.95441 \mathrm{E}-01$ \\
\hline
\end{tabular}




\begin{tabular}{|c|c|c|c|c|c|c|c|c|c|c|c|}
\hline & & & & & & & & & & & $\begin{array}{l}4.889 \\
(1.00)\end{array}$ \\
\hline & $\begin{array}{l}0.143 \\
(0.72)\end{array}$ & & & & & & & & & $\begin{array}{l}4.823 \\
(0.45)\end{array}$ & $\begin{array}{l}4.841 \\
(0.48)\end{array}$ \\
\hline & & & & & & & & & $\begin{array}{l}4.680 \\
(0.49)\end{array}$ & $\begin{array}{l}4.775 \\
(0.35)\end{array}$ & $\begin{array}{l}4.801 \\
(0.51)\end{array}$ \\
\hline & & & & & & & & $\begin{array}{l}4.418 \\
(0.49)\end{array}$ & $\begin{array}{l}4.556 \\
(0.35)\end{array}$ & $\begin{array}{l}4.662 \\
(0.34)\end{array}$ & $\begin{array}{l}4.671 \\
(0.44)\end{array}$ \\
\hline & & & & & & & $\begin{array}{l}4.131 \\
(0.53)\end{array}$ & $\begin{array}{l}4.290 \\
(0.37)\end{array}$ & $\begin{array}{l}4.381 \\
(0.40)\end{array}$ & $\begin{array}{l}4.473 \\
(0.37)\end{array}$ & $\begin{array}{l}4.486 \\
(0.50)\end{array}$ \\
\hline & & & & & & $\begin{array}{l}3.736 \\
(0.54)\end{array}$ & $\begin{array}{l}3.929 \\
(0.37)\end{array}$ & $\begin{array}{l}4.067 \\
(0.37)\end{array}$ & $\begin{array}{l}4.181 \\
(0.37)\end{array}$ & $\begin{array}{l}4.232 \\
(0.35)\end{array}$ & $\begin{array}{l}4.253 \\
(0.47)\end{array}$ \\
\hline & & & & & $\begin{array}{l}3.282 \\
(0.51)\end{array}$ & $\begin{array}{c}3.485 \\
(0.38)\end{array}$ & $\begin{array}{l}3.672 \\
(0.38)\end{array}$ & $\begin{array}{l}3.812 \\
(0.35)\end{array}$ & $\begin{array}{l}3.911 \\
(0.36)\end{array}$ & $\begin{array}{l}3.997 \\
(0.36)\end{array}$ & $\begin{array}{l}4.014 \\
(0.57)\end{array}$ \\
\hline & & & & $\begin{array}{c}2.803 \\
(0.63)\end{array}$ & $\begin{array}{l}3.040 \\
(0.42)\end{array}$ & $\begin{array}{l}3.236 \\
(0.40)\end{array}$ & $\begin{array}{l}3.429 \\
(0.41)\end{array}$ & $\begin{array}{l}3.556 \\
(0.41)\end{array}$ & $\begin{array}{c}3.634 \\
(0.40)\end{array}$ & $\begin{array}{l}3.682 \\
(0.39)\end{array}$ & $\begin{array}{l}3.716 \\
(0.53)\end{array}$ \\
\hline & & & $\begin{array}{l}2.356 \\
(0.60)\end{array}$ & $\begin{array}{c}2.576 \\
(0.50)\end{array}$ & $\begin{array}{l}2.763 \\
(0.42)\end{array}$ & $\begin{array}{l}2.948 \\
(0.40)\end{array}$ & $\begin{array}{l}3.121 \\
(0.39)\end{array}$ & $\begin{array}{l}3.244 \\
(0.43)\end{array}$ & $\begin{array}{c}3.319 \\
(0.40)\end{array}$ & $\begin{array}{l}3.364 \\
(0.43)\end{array}$ & $\begin{array}{c}3.384 \\
(0.59)\end{array}$ \\
\hline & & $\begin{array}{l}1.962 \\
(0.81)\end{array}$ & $\begin{array}{l}2.133 \\
(0.49)\end{array}$ & $\begin{array}{l}2.338 \\
0.47)\end{array}$ & $\begin{array}{l}2.571 \\
(0.43)\end{array}$ & $\begin{array}{l}2.695 \\
(0.42)\end{array}$ & $\begin{array}{l}2.838 \\
(0.44)\end{array}$ & $\begin{array}{l}2.947 \\
(0.47)\end{array}$ & $\begin{array}{l}3.038 \\
(0.46)\end{array}$ & $\begin{array}{l}3.085 \\
(0.43)\end{array}$ & $\begin{array}{l}3.097 \\
(0.55)\end{array}$ \\
\hline & $\begin{array}{l}1.782 \\
(0.78)\end{array}$ & $\begin{array}{c}1.874 \\
(0.52)\end{array}$ & $\begin{array}{l}2.046 \\
(0.52)\end{array}$ & $\begin{array}{l}2.263 \\
(0.47)\end{array}$ & $\begin{array}{l}2.423 \\
(0.44)\end{array}$ & $\begin{array}{l}2.573 \\
(0.48)\end{array}$ & $\begin{array}{l}2.708 \\
(0.46)\end{array}$ & $\begin{array}{l}2.819 \\
(0.40)\end{array}$ & $\begin{array}{l}2.891 \\
(0.44)\end{array}$ & $\begin{array}{l}2.907 \\
(0.42)\end{array}$ & $\begin{array}{l}2.936 \\
(0.54)\end{array}$ \\
\hline $\begin{array}{l}2.156 \\
(0.74)\end{array}$ & $\begin{array}{l}2.021 \\
(0.52)\end{array}$ & $\begin{array}{l}2.204 \\
(0.50)\end{array}$ & $\begin{array}{l}2.429 \\
(0.49)\end{array}$ & $\begin{array}{c}2.663 \\
(0.44)\end{array}$ & $\begin{array}{l}2.876 \\
(0.42)\end{array}$ & $\begin{array}{l}3.083 \\
(0.41)\end{array}$ & $\begin{array}{l}3.215 \\
(0.40)\end{array}$ & $\begin{array}{l}3.338 \\
(0.42)\end{array}$ & $\begin{array}{l}3.414 \\
(0.40)\end{array}$ & $\begin{array}{l}3.474 \\
(0.33)\end{array}$ & $\begin{array}{l}3.480 \\
(0.59)\end{array}$ \\
\hline
\end{tabular}

Fig. 4.7a. Pin-power distribution for CENTRM benchmark TCA-3. Value in parentheses is percent standard deviation. 


\begin{tabular}{|c|c|c|c|c|c|c|c|c|c|c|c|}
\hline & & & & & & & & & & & $\begin{array}{l}4.857 \\
(1.00)\end{array}$ \\
\hline & $\begin{array}{l}0.143 \\
(0.73)\end{array}$ & & & & & & & & & $\begin{array}{l}4.845 \\
(0.46)\end{array}$ & $\begin{array}{l}4.826 \\
(0.50)\end{array}$ \\
\hline & & & & & & & & & $\begin{array}{l}4.692 \\
(0.49)\end{array}$ & $\begin{array}{l}4.713 \\
(0.37)\end{array}$ & $\begin{array}{l}4.814 \\
(0.46)\end{array}$ \\
\hline & & & & & & & & $\begin{array}{l}4.430 \\
(0.53)\end{array}$ & $\begin{array}{l}4.565 \\
(0.34)\end{array}$ & $\begin{array}{l}4.635 \\
(0.34)\end{array}$ & $\begin{array}{l}4.646 \\
(0.48)\end{array}$ \\
\hline & & & & & & & $\begin{array}{l}4.145 \\
(0.54) \\
\end{array}$ & $\begin{array}{r}4.284 \\
(0.39) \\
\end{array}$ & $\begin{array}{l}4.400 \\
(0.36) \\
\end{array}$ & $\begin{array}{r}4.464 \\
(0.36) \\
\end{array}$ & $\begin{array}{l}4.512 \\
(0.50) \\
\end{array}$ \\
\hline & & & & & & $\begin{array}{l}3.718 \\
(0.51)\end{array}$ & $\begin{array}{l}3.929 \\
(0.35)\end{array}$ & $\begin{array}{l}4.066 \\
(0.38)\end{array}$ & $\begin{array}{l}4.195 \\
(0.37)\end{array}$ & $\begin{array}{l}4.262 \\
(0.33)\end{array}$ & $\begin{array}{l}4.242 \\
(0.50)\end{array}$ \\
\hline & & & & & $\begin{array}{c}3.318 \\
(0.57)\end{array}$ & $\begin{array}{l}3.525 \\
(0.36)\end{array}$ & $\begin{array}{l}3.701 \\
(0.40)\end{array}$ & $\begin{array}{l}3.836 \\
(0.37)\end{array}$ & $\begin{array}{l}3.934 \\
(0.37)\end{array}$ & $\begin{array}{l}3.997 \\
(0.35)\end{array}$ & $\begin{array}{r}4.006 \\
(0.51)\end{array}$ \\
\hline & & & & $\begin{array}{l}2.807 \\
(0.61)\end{array}$ & $\begin{array}{l}3.095 \\
(0.41)\end{array}$ & $\begin{array}{l}3.287 \\
(0.39)\end{array}$ & $\begin{array}{l}3.406 \\
(0.41)\end{array}$ & $\begin{array}{l}3.538 \\
(0.40)\end{array}$ & $\begin{array}{l}3.646 \\
(0.36)\end{array}$ & $\begin{array}{l}3.714 \\
(0.36)\end{array}$ & $\begin{array}{l}3.704 \\
(0.49)\end{array}$ \\
\hline & & & $\begin{array}{l}2.365 \\
(0.67)\end{array}$ & $\begin{array}{l}2.589 \\
(0.48)\end{array}$ & $\begin{array}{l}2.799 \\
(0.44)\end{array}$ & $\begin{array}{l}2.975 \\
(0.41)\end{array}$ & $\begin{array}{l}3.132 \\
(0.41)\end{array}$ & $\begin{array}{l}3.230 \\
(0.40)\end{array}$ & $\begin{array}{l}3.314 \\
(0.41)\end{array}$ & $\begin{array}{l}3.391 \\
(0.39)\end{array}$ & $\begin{array}{l}3.396 \\
(0.59)\end{array}$ \\
\hline & & $\begin{array}{l}1.949 \\
(0.76)\end{array}$ & $\begin{array}{l}2.150 \\
(0.51)\end{array}$ & $\begin{array}{l}2.363 \\
0.47)\end{array}$ & $\begin{array}{l}2.541 \\
(0.48)\end{array}$ & $\begin{array}{l}2.706 \\
(0.45)\end{array}$ & $\begin{array}{l}2.838 \\
(0.44)\end{array}$ & $\begin{array}{l}2.977 \\
(0.42)\end{array}$ & $\begin{array}{l}3.035 \\
(0.43)\end{array}$ & $\begin{array}{l}3.082 \\
(0.43)\end{array}$ & $\begin{array}{l}3.092 \\
(0.55)\end{array}$ \\
\hline & $\begin{array}{l}1.762 \\
(0.77)\end{array}$ & $\begin{array}{l}1.861 \\
(0.58)\end{array}$ & $\begin{array}{l}2.063 \\
(0.49)\end{array}$ & $\begin{array}{l}2.238 \\
(0.53)\end{array}$ & $\begin{array}{l}2.433 \\
(0.47)\end{array}$ & $\begin{array}{l}2.572 \\
(0.46)\end{array}$ & $\begin{array}{l}2.729 \\
(0.48)\end{array}$ & $\begin{array}{l}2.840 \\
(0.40)\end{array}$ & $\begin{array}{l}2.894 \\
(0.44)\end{array}$ & $\begin{array}{l}2.944 \\
(0.42)\end{array}$ & $\begin{array}{c}2.962 \\
(0.59)\end{array}$ \\
\hline $\begin{array}{l}2.155 \\
(0.76)\end{array}$ & $\begin{array}{l}2.037 \\
(0.53)\end{array}$ & $\begin{array}{l}2.194 \\
(0.47\end{array}$ & $\begin{array}{l}2.420 \\
(0.50)\end{array}$ & $\begin{array}{l}2.654 \\
(0.46)\end{array}$ & $\begin{array}{l}2.866 \\
(0.42)\end{array}$ & $\begin{array}{l}3.058 \\
(0.41)\end{array}$ & $\begin{array}{l}3.225 \\
(0.40)\end{array}$ & $\begin{array}{l}3.315 \\
(0.41)\end{array}$ & $\begin{array}{l}3.430 \\
(0.39)\end{array}$ & $\begin{array}{l}3.480 \\
(0.37)\end{array}$ & $\begin{array}{l}3.483 \\
(0.58)\end{array}$ \\
\hline
\end{tabular}

Fig. 4.7b. Pin-power distribution for NITAWL benchmark TCA-3. Value in parentheses is percent standard deviation. 
Table 4.25. Selected reaction rates for CENTRM case TCA-3

\begin{tabular}{|c|c|c|c|c|c|c|}
\hline Pin & Region & $\begin{array}{c}\mathrm{G}_{\mathrm{a}} \mathrm{M} \\
\left(\mathrm{cm}^{-3}-\mathrm{s}^{-1}\right)\end{array}$ & $\begin{array}{c}\mathcal{G}_{\mathrm{f}} \mathrm{M} \\
\left(\mathrm{cm}^{-3}-\mathrm{s}^{-1}\right)\end{array}$ & $\begin{array}{c}\mathrm{G}_{\mathrm{f}} \mathrm{M} \\
\left(\mathrm{cm}^{-3}-\mathrm{s}^{-1}\right)\end{array}$ & $\begin{array}{c}\mathrm{M} \\
\left(\mathrm{cm}-\mathrm{s}^{-1}\right)\end{array}$ & $\mathrm{M} / \mathrm{M}_{\mathrm{t}}$ \\
\hline \multirow{3}{*}{1} & Fuel & $1.307 \mathrm{E}-05$ & $2.156 \mathrm{E}-05$ & 7.643E-06 & $5.476 \mathrm{E}-03$ & 0.271 \\
\hline & Clad & 2.496E-07 & 0.0 & 0.0 & $1.701 \mathrm{E}-03$ & 0.085 \\
\hline & Mod. & $6.938 \mathrm{E}-07$ & 0.0 & 0.0 & $1.300 \mathrm{E}-02$ & 0.644 \\
\hline \multirow{3}{*}{78} & Fuel & 3.318E-05 & 4.938E-05 & $1.747 \mathrm{E}-05$ & $2.182 \mathrm{E}-02$ & 0.269 \\
\hline & Clad & $6.794 \mathrm{E}-07$ & 0.0 & 0.0 & $6.875 \mathrm{E}-03$ & 0.085 \\
\hline & Mod. & $1.442 \mathrm{E}-06$ & 0.0 & 0.0 & $5.233 \mathrm{E}-02$ & 0.646 \\
\hline
\end{tabular}

Table 4.26. Selected reaction rates for NITAWL case TCA-3

\begin{tabular}{|c|c|c|c|c|c|c|}
\hline Pin & Region & $\begin{array}{c}\mathrm{G}_{\mathrm{a}} \mathrm{M} \\
\left(\mathrm{cm}^{-3}-\mathrm{s}^{-1}\right)\end{array}$ & $\begin{array}{c}\mathcal{G}_{\mathrm{f}} \mathrm{M} \\
\left(\mathrm{cm}^{-3}-\mathrm{s}^{-1}\right)\end{array}$ & $\begin{array}{c}\mathrm{G}_{\mathrm{f}} \mathrm{M} \\
\left(\mathrm{cm}^{-3}-\mathrm{s}^{-1}\right)\end{array}$ & $\begin{array}{c}\mathrm{M} \\
\left(\mathrm{cm}-\mathrm{s}^{-1}\right)\end{array}$ & $\mathrm{M} / \mathrm{M}_{\mathrm{t}}$ \\
\hline \multirow{3}{*}{1} & Fuel & $1.296 \mathrm{E}-05$ & $2.142 \mathrm{E}-05$ & 7.591E-06 & $5.418 \mathrm{E}-03$ & 0.272 \\
\hline & Clad & 2.463E-07 & 0.0 & 0.0 & $1.676 \mathrm{E}-03$ & 0.084 \\
\hline & Mod. & $6.886 \mathrm{E}-07$ & 0.0 & 0.0 & $1.285 \mathrm{E}-02$ & 0.644 \\
\hline \multirow{3}{*}{78} & Fuel & $3.290 \mathrm{E}-05$ & 4.896E-05 & 1.732E-05 & $2.172 \mathrm{E}-02$ & 0.269 \\
\hline & Clad & $6.746 \mathrm{E}-07$ & 0.0 & 0.0 & $6.861 \mathrm{E}-03$ & 0.085 \\
\hline & Mod. & $1.449 \mathrm{E}-06$ & 0.0 & 0.0 & $5.222 \mathrm{E}-02$ & 0.646 \\
\hline
\end{tabular}


Table 4.27. TCA-3, Pin-1 four-group fluxes

\begin{tabular}{ccc}
\hline Group & $\begin{array}{c}\text { CENTRM M } \\
\left(\mathrm{cm}^{-2}-\mathrm{s}^{-1}\right)\end{array}$ & $\begin{array}{c}\text { NITAWL M } \\
\left(\mathrm{cm}^{-2}-\mathrm{s}^{-1}\right)\end{array}$ \\
\hline 1 & $4.915 \mathrm{E}-05$ & $4.860 \mathrm{E}-05$ \\
2 & $1.453 \mathrm{E}-05$ & $1.440 \mathrm{E}-05$ \\
3 & $3.519 \mathrm{E}-06$ & $3.300 \mathrm{E}-06$ \\
4 & $2.876 \mathrm{E}-05$ & $2.864 \mathrm{E}-05$ \\
\hline
\end{tabular}

Table 4.28. TCA-3, Pin-1 four-group U-235 cross sections

\begin{tabular}{cccccccc}
\hline Group & \multicolumn{2}{c}{ CENTRM cross sections $\left(\mathrm{cm}^{-1}\right)$} & \multicolumn{3}{c}{ NITAWL cross sections $\left(\mathrm{cm}^{-1}\right)$} \\
\hline & $\frac{\mathrm{G}_{\mathrm{a}}}{1.42818 \mathrm{E}-04}$ & $\frac{\mathrm{G}_{\mathrm{f}}}{3.25054 \mathrm{E}-04}$ & $\frac{\mathrm{G}_{\mathrm{f}}}{1.25124 \mathrm{E}-04}$ & $\frac{\mathrm{G}_{\mathrm{a}}}{1.43107 \mathrm{E}-04}$ & $\frac{\mathrm{G}_{\mathrm{f}}}{3.25739 \mathrm{E}-04}$ & $\frac{\mathrm{G}_{\mathrm{f}}}{1.25325 \mathrm{E}-04}$ \\
1 & $3.36929 \mathrm{E}-03$ & $5.19745 \mathrm{E}-03$ & $2.13299 \mathrm{E}-03$ & $3.46178 \mathrm{E}-03$ & $5.31513 \mathrm{E}-03$ & $2.18128 \mathrm{E}-03$ \\
2 & $5.17928 \mathrm{E}-03$ & $1.05930 \mathrm{E}-02$ & $4.34726 \mathrm{E}-03$ & $5.19818 \mathrm{E}-03$ & $1.06224 \mathrm{E}-02$ & $4.35933 \mathrm{E}-03$ \\
3 & $4.86050 \mathrm{E}-02$ & $1.01190 \mathrm{E}-01$ & $4.15275 \mathrm{E}-02$ & $4.84951 \mathrm{E}-02$ & $1.00958 \mathrm{E}-01$ & $4.14323 \mathrm{E}-02$ \\
\hline
\end{tabular}

Table 4.29. TCA-3, Pin-1 four-group U-238 cross sections

\begin{tabular}{cccccccc}
\hline Group & \multicolumn{3}{c}{ CENTRM cross sections $\left(\mathrm{cm}^{-1}\right)$} & \multicolumn{2}{c}{ NITAWL cross sections $\left(\mathrm{cm}^{-1}\right)$} \\
\hline & $\frac{\mathrm{G}_{\mathrm{a}}}{4.44993 \mathrm{E}-03}$ & $\frac{\mathrm{G}_{\mathrm{f}}}{8.27601 \mathrm{E}-03}$ & $\frac{\mathrm{G}_{\mathrm{f}}}{2.93516 \mathrm{E}-03}$ & $\frac{\mathrm{G}_{\mathrm{a}}}{4.47772 \mathrm{E}-03}$ & $\frac{\mathrm{G}_{\mathrm{f}}}{8.36366 \mathrm{E}-03}$ & $\frac{\mathrm{G}_{\mathrm{f}}}{2.96352 \mathrm{E}-03}$ \\
1 & $3.65872 \mathrm{E}-02$ & $3.54715 \mathrm{E}-06$ & $1.46957 \mathrm{E}-06$ & $3.65101 \mathrm{E}-02$ & $3.54449 \mathrm{E}-06$ & $1.46847 \mathrm{E}-06$ \\
2 & $7.01523 \mathrm{E}-03$ & $2.46279 \mathrm{E}-08$ & $1.02038 \mathrm{E}-08$ & $7.00700 \mathrm{E}-03$ & $2.46391 \mathrm{E}-08$ & $1.02084 \mathrm{E}-08$ \\
3 & $2.76173 \mathrm{E}-02$ & $1.29810 \mathrm{E}-07$ & $5.37830 \mathrm{E}-08$ & $2.75642 \mathrm{E}-02$ & $1.29555 \mathrm{E}-07$ & $5.36770 \mathrm{E}-08$ \\
\hline
\end{tabular}

Table 4.30. TCA-3, Pin-1 four-group Pu-239 cross sections

\begin{tabular}{ccccccc}
\hline Group & \multicolumn{3}{c}{ CENTRM cross sections $\left(\mathrm{cm}^{-1}\right)$} & \multicolumn{3}{c}{ NITAWL cross sections $\left(\mathrm{cm}^{-1}\right)$} \\
\hline & $\frac{\mathrm{G}_{\mathrm{a}}}{1}$ & $\frac{\mathrm{G}_{\mathrm{f}}}{1.11491 \mathrm{E}-04}$ & $\frac{\mathrm{G}_{\mathrm{f}}}{1.49361 \mathrm{E}-03}$ & $\frac{4.80637 \mathrm{E}-04}{4.11798 \mathrm{E}-04}$ & $\frac{\mathrm{G}_{\mathrm{f}}}{1.49459 \mathrm{E}-03}$ & $\frac{\mathrm{G}_{\mathrm{f}}}{4.80689 \mathrm{E}-04}$ \\
2 & $1.09264 \mathrm{E}-02$ & $1.82048 \mathrm{E}-02$ & $6.32153 \mathrm{E}-03$ & $1.06379 \mathrm{E}-02$ & $1.74194 \mathrm{E}-02$ & $6.04882 \mathrm{E}-03$ \\
3 & $3.56962 \mathrm{E}-02$ & $6.99270 \mathrm{E}-02$ & $2.42818 \mathrm{E}-02$ & $3.44527 \mathrm{E}-02$ & $6.76673 \mathrm{E}-02$ & $2.34972 \mathrm{E}-02$ \\
4 & $2.66921 \mathrm{E}-01$ & $5.40191 \mathrm{E}-01$ & $1.86841 \mathrm{E}-01$ & $2.66795 \mathrm{E}-01$ & $5.39735 \mathrm{E}-01$ & $1.86684 \mathrm{E}-01$ \\
\hline
\end{tabular}


Table 4.31. TCA-3, Pin-78 four-group fluxes

\begin{tabular}{ccc}
\hline Group & $\begin{array}{c}\text { CENTRM M } \\
\left(\mathrm{cm}^{-2}-\mathrm{s}^{-1}\right)\end{array}$ & $\begin{array}{c}\text { NITAWL M } \\
\left(\mathrm{cm}^{-2}-\mathrm{s}^{-1}\right)\end{array}$ \\
\hline 1 & $2.243 \mathrm{E}-04$ & $2.215 \mathrm{E}-04$ \\
2 & $8.038 \mathrm{E}-05$ & $8.190 \mathrm{E}-05$ \\
3 & $1.810 \mathrm{E}-05$ & $1.765 \mathrm{E}-05$ \\
4 & $5.962 \mathrm{E}-05$ & $5.956 \mathrm{E}-05$ \\
\hline
\end{tabular}

Table 4.32. TCA-3, Pin-78 four-group U-235 cross sections

\begin{tabular}{cccccccc}
\hline Group & \multicolumn{3}{c}{ CENTRM cross sections $\left(\mathrm{cm}^{-1}\right)$} & \multicolumn{3}{c}{ NITAWL cross sections $\left(\mathrm{cm}^{-1}\right)$} \\
\hline & $\frac{\mathrm{G}_{\mathrm{a}}}{1.47728 \mathrm{E}-04}$ & $\frac{\mathrm{G}_{\mathrm{f}}}{3.29228 \mathrm{E}-04}$ & $\frac{\mathrm{G}_{\mathrm{f}}}{1.27610 \mathrm{E}-04}$ & $\frac{\mathrm{G}_{\mathrm{a}}}{1.47738 \mathrm{E}-04}$ & $\frac{\mathrm{G}_{\mathrm{f}}}{3.29429 \mathrm{E}-04}$ & $\frac{\mathrm{G}_{\mathrm{f}}}{1.27667 \mathrm{E}-04}$ \\
1 & $3.36825 \mathrm{E}-03$ & $5.14716 \mathrm{E}-03$ & $2.11235 \mathrm{E}-03$ & $3.40828 \mathrm{E}-03$ & $5.21512 \mathrm{E}-03$ & $2.14023 \mathrm{E}-03$ \\
2 & $5.12983 \mathrm{E}-03$ & $1.04660 \mathrm{E}-02$ & $4.29517 \mathrm{E}-03$ & $5.13172 \mathrm{E}-03$ & $1.04717 \mathrm{E}-02$ & $4.29747 \mathrm{E}-03$ \\
3 & $4.59777 \mathrm{E}-02$ & $9.56112 \mathrm{E}-02$ & $3.92380 \mathrm{E}-02$ & $4.54550 \mathrm{E}-02$ & $9.45238 \mathrm{E}-02$ & $3.87918 \mathrm{E}-02$ \\
\hline
\end{tabular}

Table 4.33. TCA-3, Pin-78 four-group U-238 cross sections

\begin{tabular}{|c|c|c|c|c|c|c|}
\hline \multirow[t]{2}{*}{ Group } & \multicolumn{3}{|c|}{ CENTRM cross sections $\left(\mathrm{cm}^{-1}\right)$} & \multicolumn{3}{|c|}{ NITAWL cross sections $\left(\mathrm{cm}^{-1}\right)$} \\
\hline & $\mathrm{G}_{\mathrm{a}}$ & $<G_{f}$ & $\mathrm{G}_{\mathrm{f}}$ & $\mathrm{G}_{\mathrm{a}}$ & $<\mathrm{G}_{\mathrm{f}}$ & $\mathrm{G}_{\mathrm{f}}$ \\
\hline 1 & $4.31758 \mathrm{E}-03$ & $7.42746 \mathrm{E}-03$ & $2.63695 \mathrm{E}-03$ & $4.34475 \mathrm{E}-03$ & $7.50781 \mathrm{E}-03$ & $2.66748 \mathrm{E}-03$ \\
\hline 2 & 3.69603E-02 & 3.91179E-06 & $1.62064 \mathrm{E}-06$ & $3.60504 \mathrm{E}-02$ & 3.75949E-06 & $1.55755 \mathrm{E}-06$ \\
\hline 3 & 6.98884E-03 & $2.44361 \mathrm{E}-08$ & $1.01243 \mathrm{E}-08$ & $6.98652 \mathrm{E}-03$ & 2.44809E-08 & 1.01429E-08 \\
\hline 4 & $2.62422 \mathrm{E}-02$ & $1.23201 \mathrm{E}-07$ & 5.10444E-08 & $2.59876 \mathrm{E}-02$ & $1.21988 \mathrm{E}-07$ & $5.05418 \mathrm{E}-08$ \\
\hline
\end{tabular}

Table 4.34. TCA-3, Pin-78 four-group Pu-239 cross sections

\begin{tabular}{ccccccc}
\hline Group & \multicolumn{3}{c}{ CENTRM cross sections $\left(\mathrm{cm}^{-1}\right)$} & \multicolumn{3}{c}{ NITAWL cross sections $\left(\mathrm{cm}^{-1}\right)$} \\
\hline & $\frac{\mathrm{G}_{\mathrm{a}}}{4}$ & $\frac{\mathrm{G}_{\mathrm{f}}}{1.43304 \mathrm{E}-04}$ & $\frac{\mathrm{G}_{\mathrm{f}}}{1.47258 \mathrm{E}-03}$ & $\frac{4.76835 \mathrm{E}-04}{5.13774 \mathrm{E}-04}$ & $\frac{\mathrm{G}_{\mathrm{f}}}{1.47465 \mathrm{E}-03}$ & $\frac{\mathrm{G}_{\mathrm{f}}}{4.77404 \mathrm{E}-04}$ \\
2 & $1.08802 \mathrm{E}-02$ & $1.78741 \mathrm{E}-02$ & $6.20669 \mathrm{E}-03$ & $1.05497 \mathrm{E}-02$ & $1.73471 \mathrm{E}-02$ & $6.02371 \mathrm{E}-03$ \\
3 & $3.36764 \mathrm{E}-02$ & $6.62210 \mathrm{E}-02$ & $2.29950 \mathrm{E}-02$ & $3.26723 \mathrm{E}-02$ & $6.44142 \mathrm{E}-02$ & $2.23675 \mathrm{E}-02$ \\
4 & $2.87561 \mathrm{E}-01$ & $5.69182 \mathrm{E}-01$ & $1.96949 \mathrm{E}-01$ & $2.86127 \mathrm{E}-01$ & $5.65714 \mathrm{E}-01$ & $1.95759 \mathrm{E}-01$ \\
\hline
\end{tabular}




\begin{tabular}{|c|c|c|c|c|c|c|c|c|c|c|}
\hline & & & & & & & & & & $\begin{array}{l}6.107 \\
(0.90)\end{array}$ \\
\hline & $\begin{array}{l}0.155 \\
(0.61)\end{array}$ & & & & & & & & $\begin{array}{l}6.067 \\
(0.43)\end{array}$ & $\begin{array}{l}6.093 \\
(0.43)\end{array}$ \\
\hline & & & & & & & & $\begin{array}{l}5.865 \\
(0.43)\end{array}$ & $\begin{array}{l}5.933 \\
(0.35)\end{array}$ & $\begin{array}{l}5.977 \\
(0.46)\end{array}$ \\
\hline & & & & & & & $\begin{array}{l}5.480 \\
(0.46)\end{array}$ & $\begin{array}{l}5.673 \\
(0.35)\end{array}$ & $\begin{array}{l}5.759 \\
(0.31)\end{array}$ & $\begin{array}{l}5.797 \\
(0.43)\end{array}$ \\
\hline & & & & & & $\begin{array}{r}4.989 \\
(0.43) \\
\end{array}$ & $\begin{array}{l}5.244 \\
(0.34) \\
\end{array}$ & $\begin{array}{l}5.447 \\
(0.34) \\
\end{array}$ & $\begin{array}{l}5.513 \\
(0.35) \\
\end{array}$ & $\begin{array}{l}5.571 \\
(0.47) \\
\end{array}$ \\
\hline & & & & & $\begin{array}{l}4.464 \\
(0.56)\end{array}$ & $\begin{array}{l}4.722 \\
(0.35)\end{array}$ & $\begin{array}{l}4.957 \\
(0.33)\end{array}$ & $\begin{array}{l}5.109 \\
(0.36)\end{array}$ & $\begin{array}{l}5.207 \\
(0.35)\end{array}$ & $\begin{array}{l}5.226 \\
(0.47)\end{array}$ \\
\hline & & & & $\begin{array}{c}3.839 \\
(0.55)\end{array}$ & $\begin{array}{l}4.125 \\
(0.38)\end{array}$ & $\begin{array}{l}4.412 \\
(0.33)\end{array}$ & $\begin{array}{l}4.611 \\
(0.35)\end{array}$ & $\begin{array}{l}4.762 \\
(0.32)\end{array}$ & $\begin{array}{l}4.805 \\
(0.34)\end{array}$ & $\begin{array}{l}4.824 \\
(0.46)\end{array}$ \\
\hline & & & $\begin{array}{c}3.152 \\
(0.60)\end{array}$ & $\begin{array}{l}3.489 \\
(0.40)\end{array}$ & $\begin{array}{c}3.765 \\
(0.38)\end{array}$ & $\begin{array}{l}3.994 \\
(0.35)\end{array}$ & $\begin{array}{l}4.201 \\
(0.34)\end{array}$ & $\begin{array}{l}4.308 \\
(0.38)\end{array}$ & $\begin{array}{l}4.384 \\
(0.35)\end{array}$ & $\begin{array}{l}4.402 \\
(0.52)\end{array}$ \\
\hline & & $\begin{array}{l}2.601 \\
(0.64)\end{array}$ & $\begin{array}{l}2.827 \\
(0.45)\end{array}$ & $\begin{array}{c}3.120 \\
(0.42)\end{array}$ & $\begin{array}{c}3.374 \\
(0.40)\end{array}$ & $\begin{array}{l}3.577 \\
(0.41)\end{array}$ & $\begin{array}{l}3.735 \\
(0.37)\end{array}$ & $\begin{array}{l}3.861 \\
(0.39)\end{array}$ & $\begin{array}{l}3.933 \\
(0.37)\end{array}$ & $\begin{array}{l}3.954 \\
(0.56)\end{array}$ \\
\hline & $\begin{array}{l}2.180 \\
(0.76)\end{array}$ & $\begin{array}{l}2.373 \\
(0.47)\end{array}$ & $\begin{array}{l}2.634 \\
(0.45)\end{array}$ & $\begin{array}{l}2.897 \\
(0.44)\end{array}$ & $\begin{array}{l}3.116 \\
(0.42)\end{array}$ & $\begin{array}{l}3.317 \\
(0.44)\end{array}$ & $\begin{array}{l}3.488 \\
(0.39)\end{array}$ & $\begin{array}{l}3.597 \\
(0.37)\end{array}$ & $\begin{array}{c}3.641 \\
(0.41)\end{array}$ & $\begin{array}{c}3.642 \\
(0.57)\end{array}$ \\
\hline $\begin{array}{l}2.423 \\
(0.70)\end{array}$ & $\begin{array}{l}2.365 \\
(0.50)\end{array}$ & $\begin{array}{l}2.614 \\
(0.49)\end{array}$ & $\begin{array}{l}2.928 \\
(0.42)\end{array}$ & $\begin{array}{c}3.239 \\
(0.43)\end{array}$ & $\begin{array}{c}3.492 \\
(0.39)\end{array}$ & $\begin{array}{l}3.697 \\
(0.36)\end{array}$ & $\begin{array}{c}3.883 \\
(0.36)\end{array}$ & $\begin{array}{l}3.998 \\
(0.39)\end{array}$ & $\begin{array}{l}4.081 \\
(0.36)\end{array}$ & $\begin{array}{l}4.134 \\
(0.53)\end{array}$ \\
\hline
\end{tabular}

Fig. 4.8a. Pin-power distribution for CENTRM benchmark TCA-4. Value in parentheses is percent standard deviation. 


\begin{tabular}{|c|c|c|c|c|c|c|c|c|c|c|}
\hline & & & & & & & & & & $\begin{array}{l}6.212 \\
(0.86)\end{array}$ \\
\hline & $\begin{array}{c}1.54 \\
(0.66)\end{array}$ & & & & & & & & $\begin{array}{l}6.114 \\
(0.44)\end{array}$ & $\begin{array}{l}6.159 \\
(0.45)\end{array}$ \\
\hline & & & & & & & & $\begin{array}{l}5.831 \\
(0.44)\end{array}$ & $\begin{array}{l}5.974 \\
(0.31)\end{array}$ & $\begin{array}{l}5.984 \\
(0.46)\end{array}$ \\
\hline & & & & & & & $\begin{array}{l}5.529 \\
(0.46)\end{array}$ & $\begin{array}{l}5.708 \\
(0.33)\end{array}$ & $\begin{array}{l}5.770 \\
(0.31)\end{array}$ & $\begin{array}{l}5.819 \\
(0.41)\end{array}$ \\
\hline & & & & & & $\begin{array}{l}5.102 \\
(0.47) \\
\end{array}$ & $\begin{array}{l}5.229 \\
(0.35) \\
\end{array}$ & $\begin{array}{l}5.445 \\
(0.32) \\
\end{array}$ & $\begin{array}{l}5.522 \\
(0.33) \\
\end{array}$ & $\begin{array}{l}5.533 \\
(0.42) \\
\end{array}$ \\
\hline & & & & & $\begin{array}{l}4.470 \\
(0.51)\end{array}$ & $\begin{array}{l}4.777 \\
(0.34)\end{array}$ & $\begin{array}{l}4.968 \\
(0.33)\end{array}$ & $\begin{array}{l}5.140 \\
(0.35)\end{array}$ & $\begin{array}{l}5.202 \\
(0.33)\end{array}$ & $\begin{array}{l}5.243 \\
(0.45)\end{array}$ \\
\hline & & & & $\begin{array}{c}3.832 \\
(0.54)\end{array}$ & $\begin{array}{l}4.116 \\
(0.40)\end{array}$ & $\begin{array}{l}4.387 \\
(0.37)\end{array}$ & $\begin{array}{l}4.630 \\
(0.35)\end{array}$ & $\begin{array}{l}4.727 \\
(0.32)\end{array}$ & $\begin{array}{l}4.837 \\
(0.33)\end{array}$ & $\begin{array}{l}4.850 \\
(0.46)\end{array}$ \\
\hline & & & $\begin{array}{l}3.129 \\
(0.62)\end{array}$ & $\begin{array}{l}3.495 \\
(0.38)\end{array}$ & $\begin{array}{c}3.735 \\
(0.41)\end{array}$ & $\begin{array}{l}4.030 \\
(0.39)\end{array}$ & $\begin{array}{l}4.197 \\
(0.38)\end{array}$ & $\begin{array}{l}4.309 \\
(0.34)\end{array}$ & $\begin{array}{l}4.374 \\
(0.35)\end{array}$ & $\begin{array}{l}4.458 \\
(0.50)\end{array}$ \\
\hline & & $\begin{array}{l}2.582 \\
(0.69)\end{array}$ & $\begin{array}{l}2.840 \\
(0.45)\end{array}$ & $\begin{array}{c}3.132 \\
(0.46)\end{array}$ & $\begin{array}{c}3.388 \\
(0.42)\end{array}$ & $\begin{array}{l}3.581 \\
(0.39)\end{array}$ & $\begin{array}{c}3.754 \\
(0.40)\end{array}$ & $\begin{array}{l}3.870 \\
(0.38)\end{array}$ & $\begin{array}{l}3.961 \\
(0.38)\end{array}$ & $\begin{array}{l}3.971 \\
(0.51)\end{array}$ \\
\hline & $\begin{array}{l}2.185 \\
(0.71)\end{array}$ & $\begin{array}{l}2.350 \\
(0.49)\end{array}$ & $\begin{array}{l}2.643 \\
(0.44)\end{array}$ & $\begin{array}{l}2.904 \\
(0.44)\end{array}$ & $\begin{array}{l}3.128 \\
(0.43)\end{array}$ & $\begin{array}{l}3.330 \\
(0.42)\end{array}$ & $\begin{array}{l}3.500 \\
(0.40)\end{array}$ & $\begin{array}{l}3.601 \\
(0.40)\end{array}$ & $\begin{array}{c}3.672 \\
(0.39)\end{array}$ & $\begin{array}{l}3.710 \\
(0.48)\end{array}$ \\
\hline $\begin{array}{l}2.436 \\
(0.71)\end{array}$ & $\begin{array}{c}2.386 \\
(0.49)\end{array}$ & $\begin{array}{l}2.626 \\
(0.48)\end{array}$ & $\begin{array}{l}2.916 \\
(0.44)\end{array}$ & $\begin{array}{c}3.228 \\
(0.44)\end{array}$ & $\begin{array}{l}3.505 \\
(0.41)\end{array}$ & $\begin{array}{l}3.706 \\
(0.43)\end{array}$ & $\begin{array}{l}3.883 \\
(0.39)\end{array}$ & $\begin{array}{l}3.981 \\
(0.41)\end{array}$ & $\begin{array}{l}4.062 \\
(0.38)\end{array}$ & $\begin{array}{l}4.100 \\
(0.55)\end{array}$ \\
\hline
\end{tabular}

Fig. 4.8b. Pin-power distribution for NITAWL benchmark TCA-4. Value in parentheses is percent standard deviation. 
Table 4.35. Selected reaction rates for CENTRM case TCA-4

\begin{tabular}{|c|c|c|c|c|c|c|}
\hline Pin & Region & $\begin{array}{c}\mathrm{G}_{\mathrm{a}} \mathrm{M} \\
\left(\mathrm{cm}^{-3}-\mathrm{s}^{-1}\right)\end{array}$ & $\begin{array}{c}\mathcal{G}_{\mathrm{f}} \mathrm{M} \\
\left(\mathrm{cm}^{-3}-\mathrm{s}^{-1}\right)\end{array}$ & $\begin{array}{c}\mathrm{G}_{\mathrm{f}} \mathrm{M} \\
\left(\mathrm{cm}^{-3}-\mathrm{s}^{-1}\right)\end{array}$ & $\begin{array}{c}\mathrm{M} \\
\left(\mathrm{cm}-\mathrm{s}^{-1}\right)\end{array}$ & $M / M_{t}$ \\
\hline \multirow{3}{*}{1} & Fuel & $1.440 \mathrm{E}-05$ & $2.393 \mathrm{E}-05$ & $8.480 \mathrm{E}-06$ & $5.581 \mathrm{E}-03$ & 0.235 \\
\hline & Clad & $2.746 \mathrm{E}-07$ & 0.0 & 0.0 & $1.739 \mathrm{E}-03$ & 0.073 \\
\hline & Mod. & 7.889E-07 & 0.0 & 0.0 & $1.642 \mathrm{E}-02$ & 0.692 \\
\hline \multirow{3}{*}{66} & Fuel & $4.055 \mathrm{E}-05$ & $6.181 \mathrm{E}-05$ & $2.186 \mathrm{E}-05$ & $2.281 \mathrm{E}-02$ & 0.235 \\
\hline & Clad & $8.066 \mathrm{E}-07$ & 0.0 & 0.0 & 7.166E-03 & 0.074 \\
\hline & Mod. & $1.867 \mathrm{E}-06$ & 0.0 & 0.0 & 6.712E-02 & 0.691 \\
\hline
\end{tabular}

Table 4.36. Selected reaction rates for NITAWL case TCA-4

\begin{tabular}{|c|c|c|c|c|c|c|}
\hline Pin & Region & $\begin{array}{c}\mathrm{G}_{\mathrm{a}} \mathrm{M} \\
\left(\mathrm{cm}^{-3}-\mathrm{s}^{-1}\right)\end{array}$ & $\begin{array}{c}\mathcal{G}_{\mathrm{f}} \mathrm{M} \\
\left(\mathrm{cm}^{-3}-\mathrm{s}^{-1}\right)\end{array}$ & $\begin{array}{c}\mathrm{G}_{\mathrm{f}} \mathrm{M} \\
\left(\mathrm{cm}^{-3}-\mathrm{s}^{-1}\right) \\
\end{array}$ & $\begin{array}{c}\mathrm{M} \\
\left(\mathrm{cm}-\mathrm{s}^{-1}\right)\end{array}$ & $M / M_{t}$ \\
\hline \multirow{3}{*}{1} & Fuel & $1.464 \mathrm{E}-05$ & $2.446 \mathrm{E}-05$ & 8.665E-06 & 5.642E-03 & 0.236 \\
\hline & Clad & $2.772 \mathrm{E}-07$ & 0.0 & 0.0 & 1.754E-03 & 0.073 \\
\hline & Mod. & 7.942E-07 & 0.0 & 0.0 & $1.652 \mathrm{E}-02$ & 0.691 \\
\hline \multirow{3}{*}{66} & Fuel & $4.061 \mathrm{E}-05$ & $6.263 \mathrm{E}-05$ & $2.216 \mathrm{E}-05$ & $2.295 \mathrm{E}-02$ & 0.234 \\
\hline & Clad & $8.106 \mathrm{E}-07$ & 0.0 & 0.0 & 7.227E-03 & 0.074 \\
\hline & Mod. & $1.885 \mathrm{E}-06$ & 0.0 & 0.0 & $6.790 \mathrm{E}-02$ & 0.692 \\
\hline
\end{tabular}


Table 4.37. TCA-4, Pin-1 four-group fluxes

\begin{tabular}{ccc}
\hline Group & $\begin{array}{c}\text { CENTRM M } \\
\left(\mathrm{cm}^{-2}-\mathrm{s}^{-1}\right)\end{array}$ & $\begin{array}{c}\text { NITAWL M } \\
\left(\mathrm{cm}^{-2}-\mathrm{s}^{-1}\right)\end{array}$ \\
\hline 1 & $5.137 \mathrm{E}-05$ & $5.198 \mathrm{E}-05$ \\
2 & $1.508 \mathrm{E}-05$ & $1.488 \mathrm{E}-05$ \\
3 & $3.637 \mathrm{E}-06$ & $3.684 \mathrm{E}-06$ \\
4 & $3.178 \mathrm{E}-05$ & $3.244 \mathrm{E}-05$ \\
\hline
\end{tabular}

Table 4.38. TCA-4, Pin-1 four-group U-235 cross sections

\begin{tabular}{cccccccc}
\hline Group & \multicolumn{3}{c}{ CENTRM cross sections $\left(\mathrm{cm}^{-1}\right)$} & \multicolumn{3}{c}{ NITAWL cross sections $\left(\mathrm{cm}^{-1}\right)$} \\
\hline & $\frac{\mathrm{G}_{\mathrm{a}}}{1.42972 \mathrm{E}-04}$ & $\frac{\mathrm{G}_{\mathrm{f}}}{3.25729 \mathrm{E}-04}$ & $\frac{\mathrm{G}_{\mathrm{f}}}{1.25284 \mathrm{E}-04}$ & $\frac{\mathrm{G}_{\mathrm{a}}}{1.42717 \mathrm{E}-04}$ & $\frac{\mathrm{G}_{\mathrm{f}}}{3.25108 \mathrm{E}-04}$ & $\frac{\mathrm{G}_{\mathrm{f}}}{1.25072 \mathrm{E}-04}$ \\
1 & $3.54777 \mathrm{E}-03$ & $5.40060 \mathrm{E}-03$ & $2.21636 \mathrm{E}-03$ & $3.49262 \mathrm{E}-03$ & $5.32638 \mathrm{E}-03$ & $2.18590 \mathrm{E}-03$ \\
2 & $5.19612 \mathrm{E}-03$ & $1.06384 \mathrm{E}-02$ & $4.36591 \mathrm{E}-03$ & $5.19563 \mathrm{E}-03$ & $1.06220 \mathrm{E}-02$ & $4.35917 \mathrm{E}-03$ \\
3 & $4.87823 \mathrm{E}-02$ & $1.01563 \mathrm{E}-01$ & $4.16806 \mathrm{E}-02$ & $4.89171 \mathrm{E}-02$ & $1.01845 \mathrm{E}-01$ & $4.17962 \mathrm{E}-02$ \\
\hline
\end{tabular}

Table 4.39. TCA-4, Pin-1 four-group U-238 cross sections

\begin{tabular}{cccccccc}
\hline Group & \multicolumn{3}{c}{ CENTRM cross sections $\left(\mathrm{cm}^{-1}\right)$} & \multicolumn{2}{c}{ NITAWL cross sections $\left(\mathrm{cm}^{-1}\right)$} \\
\hline & $\frac{\mathrm{G}_{\mathrm{a}}}{4.51308 \mathrm{E}-03}$ & $\frac{\mathrm{G}_{\mathrm{f}}}{8.46385 \mathrm{E}-03}$ & $\frac{\mathrm{G}_{\mathrm{f}}}{3.00241 \mathrm{E}-03}$ & $\overline{4.47482 \mathrm{E}-03}$ & $\overline{8.38222 \mathrm{E}-03}$ & $\overline{2.97205 \mathrm{E}-03}$ \\
1 & $3.86113 \mathrm{E}-02$ & $3.72395 \mathrm{E}-06$ & $1.54282 \mathrm{E}-06$ & $3.54513 \mathrm{E}-02$ & $3.52350 \mathrm{E}-06$ & $1.45977 \mathrm{E}-06$ \\
3 & $7.00737 \mathrm{E}-03$ & $2.46436 \mathrm{E}-08$ & $1.02103 \mathrm{E}-08$ & $7.01355 \mathrm{E}-03$ & $2.47048 \mathrm{E}-08$ & $1.02357 \mathrm{E}-08$ \\
4 & $2.77151 \mathrm{E}-02$ & $1.30278 \mathrm{E}-07$ & $5.39767 \mathrm{E}-08$ & $2.77735 \mathrm{E}-02$ & $1.30557 \mathrm{E}-07$ & $5.40922 \mathrm{E}-08$ \\
\hline
\end{tabular}

Table 4.40. TCA-4, Pin-1 four-group Pu-239 cross sections

\begin{tabular}{|c|c|c|c|c|c|c|}
\hline \multirow[t]{2}{*}{ Group } & \multicolumn{3}{|c|}{ CENTRM cross sections $\left(\mathrm{cm}^{-1}\right)$} & \multicolumn{3}{|c|}{ NITAWL cross sections $\left(\mathrm{cm}^{-1}\right)$} \\
\hline & $\mathrm{G}_{\mathrm{a}}$ & $<\mathrm{G}_{\mathrm{f}}$ & $\mathrm{G}_{\mathrm{f}}$ & $\mathrm{G}_{\mathrm{a}}$ & $<\mathrm{G}_{\mathrm{f}}$ & $\mathrm{G}_{\mathrm{f}}$ \\
\hline 1 & $5.12178 \mathrm{E}-04$ & $1.49692 \mathrm{E}-03$ & $4.81232 \mathrm{E}-04$ & $5.11274 \mathrm{E}-04$ & $1.49406 \mathrm{E}-03$ & $4.80446 \mathrm{E}-04$ \\
\hline 2 & $1.11540 \mathrm{E}-02$ & $1.83841 \mathrm{E}-02$ & 6.38377E-03 & 1.08239E-02 & 1.78689E-02 & $6.20489 \mathrm{E}-03$ \\
\hline 3 & $3.46250 \mathrm{E}-02$ & 6.79736E-02 & $2.36035 \mathrm{E}-02$ & $3.48586 \mathrm{E}-02$ & 6.84167E-02 & $2.37574 \mathrm{E}-02$ \\
\hline 4 & $2.65578 \mathrm{E}-01$ & 5.38310E-01 & $1.86183 \mathrm{E}-01$ & $2.66229 \mathrm{E}-01$ & $5.39667 \mathrm{E}-01$ & $1.86654 \mathrm{E}-01$ \\
\hline
\end{tabular}


Table 4.41. TCA-4, Pin-66 four-group fluxes

\begin{tabular}{ccc}
\hline Group & $\begin{array}{c}\text { CENTRM M } \\
\left(\mathrm{cm}^{-2}-\mathrm{s}^{-1}\right)\end{array}$ & $\begin{array}{c}\text { NITAWL M } \\
\left(\mathrm{cm}^{-2}-\mathrm{s}^{-1}\right)\end{array}$ \\
\hline 1 & $2.378 \mathrm{E}-04$ & $2.383 \mathrm{E}-04$ \\
2 & $8.358 \mathrm{E}-05$ & $8.422 \mathrm{E}-05$ \\
3 & $1.882 \mathrm{E}-05$ & $1.898 \mathrm{E}-05$ \\
4 & $7.623 \mathrm{E}-05$ & $7.746 \mathrm{E}-05$ \\
\hline
\end{tabular}

Table 4.42. TCA-4, Pin-66 four-group U-235 cross sections

\begin{tabular}{ccccccc}
\hline Group & \multicolumn{2}{c}{ CENTRM cross sections $\left(\mathrm{cm}^{-1}\right)$} & \multicolumn{3}{c}{ NITAWL cross sections $\left(\mathrm{cm}^{-1}\right)$} \\
\hline & $\frac{\mathrm{G}_{\mathrm{a}}}{}$ & $\frac{\mathrm{G}_{\mathrm{f}}}{1.47181 \mathrm{E}-04}$ & $\frac{3.29323 \mathrm{E}-04}{1.27404 \mathrm{E}-04}$ & $\frac{\mathrm{G}_{\mathrm{f}}}{1.47030 \mathrm{E}-04}$ & $\frac{\mathrm{G}_{\mathrm{f}}}{3.28757 \mathrm{E}-04}$ & $\frac{\mathrm{G}_{\mathrm{f}}}{1.27281 \mathrm{E}-04}$ \\
1 & $3.46397 \mathrm{E}-03$ & $5.28525 \mathrm{E}-03$ & $2.16902 \mathrm{E}-03$ & $3.45056 \mathrm{E}-03$ & $5.27803 \mathrm{E}-03$ & $2.16605 \mathrm{E}-03$ \\
3 & $5.05350 \mathrm{E}-03$ & $1.03193 \mathrm{E}-02$ & $4.23494 \mathrm{E}-03$ & $5.07799 \mathrm{E}-03$ & $1.03695 \mathrm{E}-02$ & $4.25556 \mathrm{E}-03$ \\
4 & $4.64067 \mathrm{E}-02$ & $9.65304 \mathrm{E}-02$ & $3.96152 \mathrm{E}-02$ & $4.62458 \mathrm{E}-02$ & $9.61957 \mathrm{E}-02$ & $3.94779 \mathrm{E}-02$ \\
\hline
\end{tabular}

Table 4.43. TCA-4, Pin-66 four-group U-238 cross sections

\begin{tabular}{cccccccc}
\hline Group & \multicolumn{3}{c}{ CENTRM cross sections $\left(\mathrm{cm}^{-1}\right)$} & \multicolumn{3}{c}{ NITAWL cross sections $\left(\mathrm{cm}^{-1}\right)$} \\
\hline & $\frac{\mathrm{G}_{\mathrm{a}}}{1}$ & $4.36716 \mathrm{E}-03$ & $\frac{\mathrm{G}_{\mathrm{f}}}{7.64672 \mathrm{E}-03}$ & $\frac{\mathrm{G}_{\mathrm{f}}}{2.70927 \mathrm{E}-03}$ & $\frac{\mathrm{G}_{\mathrm{a}}}{4.35812 \mathrm{E}-03}$ & $\frac{\mathrm{G}_{\mathrm{f}}}{7.61288 \mathrm{E}-03}$ & $\frac{\mathrm{G}_{\mathrm{f}}}{2.70342 \mathrm{E}-03}$ \\
2 & $4.02182 \mathrm{E}-02$ & $3.82265 \mathrm{E}-06$ & $1.58372 \mathrm{E}-06$ & $3.67393 \mathrm{E}-02$ & $3.69650 \mathrm{E}-06$ & $1.53144 \mathrm{E}-06$ \\
3 & $6.96068 \mathrm{E}-03$ & $2.41878 \mathrm{E}-08$ & $1.00214 \mathrm{E}-08$ & $6.97476 \mathrm{E}-03$ & $2.43271 \mathrm{E}-08$ & $1.00792 \mathrm{E}-08$ \\
4 & $2.64749 \mathrm{E}-02$ & $1.24324 \mathrm{E}-07$ & $5.15096 \mathrm{E}-08$ & $2.63939 \mathrm{E}-02$ & $1.23940 \mathrm{E}-07$ & $5.13506 \mathrm{E}-08$ \\
\hline
\end{tabular}

Table 4.44. TCA-4, Pin-66 four-group Pu-239 cross sections

\begin{tabular}{|c|c|c|c|c|c|c|}
\hline \multirow[t]{2}{*}{ Group } & \multicolumn{3}{|c|}{ CENTRM cross sections $\left(\mathrm{cm}^{-1}\right)$} & \multicolumn{3}{|c|}{ NITAWL cross sections $\left(\mathrm{cm}^{-1}\right)$} \\
\hline & $\mathrm{G}_{\mathrm{a}}$ & $<\mathrm{G}_{\mathrm{f}}$ & $\mathrm{G}_{\mathrm{f}}$ & $\mathrm{G}_{\mathrm{a}}$ & $<\mathrm{G}_{\mathrm{f}}$ & $\mathrm{G}_{\mathrm{f}}$ \\
\hline 1 & $5.13616 \mathrm{E}-04$ & $\overline{1.47840 \mathrm{E}-03}$ & $\overline{4.77839 \mathrm{E}-04}$ & $5.13256 \mathrm{E}-04$ & $\overline{1.47677 \mathrm{E}-03}$ & $\overline{4.77633 \mathrm{E}-04}$ \\
\hline 2 & $1.11268 \mathrm{E}-02$ & 1.82952E-02 & $6.35292 \mathrm{E}-03$ & $1.07160 \mathrm{E}-02$ & $1.75431 \mathrm{E}-02$ & $6.09175 \mathrm{E}-03$ \\
\hline 3 & $3.23976 \mathrm{E}-02$ & $6.38268 \mathrm{E}-02$ & $2.21636 \mathrm{E}-02$ & $3.30910 \mathrm{E}-02$ & $6.51217 \mathrm{E}-02$ & $2.26133 \mathrm{E}-02$ \\
\hline 4 & $2.81830 \mathrm{E}-01$ & $5.60508 \mathrm{E}-01$ & $1.93933 \mathrm{E}-01$ & 2.81951E-01 & 5.60363E-01 & $1.93887 \mathrm{E}-01$ \\
\hline
\end{tabular}




\begin{tabular}{|c|c|c|c|c|c|c|c|c|c|c|}
\hline & & & & & & & & & & $\begin{array}{l}5.973 \\
(0.85)\end{array}$ \\
\hline & \multirow[t]{8}{*}{$\begin{array}{l}0.164 \\
(0.72)\end{array}$} & & & & & & & & $\begin{array}{l}5.841 \\
(0.44)\end{array}$ & $\begin{array}{l}5.875 \\
(0.49)\end{array}$ \\
\hline & & & & & & & & $\begin{array}{l}5.617 \\
(0.45)\end{array}$ & $\begin{array}{l}5.723 \\
(0.34)\end{array}$ & $\begin{array}{l}5.821 \\
(0.42)\end{array}$ \\
\hline & & & & & & & $\begin{array}{l}5.245 \\
(0.44)\end{array}$ & $\begin{array}{l}5.464 \\
(0.33)\end{array}$ & $\begin{array}{l}5.537 \\
(0.33)\end{array}$ & $\begin{array}{l}5.623 \\
(0.44)\end{array}$ \\
\hline & & & & & & $\begin{array}{l}4.824 \\
(0.47) \\
\end{array}$ & $\begin{array}{l}5.071 \\
(0.31) \\
\end{array}$ & $\begin{array}{l}5.205 \\
(0.30) \\
\end{array}$ & $\begin{array}{l}5.288 \\
(0.29) \\
\end{array}$ & $\begin{array}{l}5.352 \\
(0.47) \\
\end{array}$ \\
\hline & & & & & $\begin{array}{l}4.271 \\
(0.53)\end{array}$ & $\begin{array}{l}4.523 \\
(0.33)\end{array}$ & $\begin{array}{l}4.753 \\
(0.30)\end{array}$ & $\begin{array}{l}4.916 \\
(0.31)\end{array}$ & $\begin{array}{l}4.981 \\
(0.34)\end{array}$ & $\begin{array}{l}5.050 \\
(0.44)\end{array}$ \\
\hline & & & & $\begin{array}{l}3.643 \\
(0.55)\end{array}$ & $\begin{array}{l}3.966 \\
(0.37)\end{array}$ & $\begin{array}{l}4.187 \\
(0.38)\end{array}$ & $\begin{array}{l}4.389 \\
(0.35)\end{array}$ & $\begin{array}{l}4.507 \\
(0.34)\end{array}$ & $\begin{array}{l}4.630 \\
(0.34)\end{array}$ & $\begin{array}{l}4.648 \\
(0.50)\end{array}$ \\
\hline & & & $\begin{array}{c}3.029 \\
(0.59)\end{array}$ & $\begin{array}{l}3.330 \\
(0.42)\end{array}$ & $\begin{array}{l}3.604 \\
(0.41)\end{array}$ & $\begin{array}{l}3.798 \\
(0.40)\end{array}$ & $\begin{array}{l}3.987 \\
(0.38)\end{array}$ & $\begin{array}{l}4.110 \\
(0.36)\end{array}$ & $\begin{array}{l}4.165 \\
(0.36)\end{array}$ & $\begin{array}{l}4.228 \\
(0.48)\end{array}$ \\
\hline & & $\begin{array}{l}2.450 \\
(0.65)\end{array}$ & $\begin{array}{l}2.713 \\
(0.44)\end{array}$ & $\begin{array}{l}2.973 \\
(0.41)\end{array}$ & $\begin{array}{l}3.230 \\
(0.39)\end{array}$ & $\begin{array}{l}3.385 \\
(0.41)\end{array}$ & $\begin{array}{l}3.574 \\
(0.39)\end{array}$ & $\begin{array}{l}3.709 \\
(0.42)\end{array}$ & $\begin{array}{l}3.777 \\
(0.37)\end{array}$ & $\begin{array}{l}3.781 \\
(0.56)\end{array}$ \\
\hline & $\begin{array}{l}2.050 \\
(0.75)\end{array}$ & $\begin{array}{l}2.254 \\
(0.48)\end{array}$ & $\begin{array}{c}2.522 \\
(0.47)\end{array}$ & $\begin{array}{l}2.750 \\
(0.45)\end{array}$ & $\begin{array}{l}2.981 \\
(0.46)\end{array}$ & $\begin{array}{l}3.176 \\
(0.38)\end{array}$ & $\begin{array}{l}3.331 \\
(0.38)\end{array}$ & $\begin{array}{l}3.429 \\
(0.35)\end{array}$ & $\begin{array}{c}3.508 \\
(0.41)\end{array}$ & $\begin{array}{l}3.534 \\
(0.55)\end{array}$ \\
\hline $\begin{array}{l}2.283 \\
(0.66)\end{array}$ & $\begin{array}{c}2.260 \\
(0.50)\end{array}$ & $\begin{array}{l}2.500 \\
(0.47)\end{array}$ & $\begin{array}{l}2.828 \\
(0.43)\end{array}$ & $\begin{array}{c}3.060 \\
(0.41)\end{array}$ & $\begin{array}{l}3.331 \\
(0.40)\end{array}$ & $\begin{array}{l}3.528 \\
(0.37)\end{array}$ & $\begin{array}{l}3.725 \\
(0.37)\end{array}$ & $\begin{array}{l}3.815 \\
(0.39)\end{array}$ & $\begin{array}{c}3.867 \\
(0.37)\end{array}$ & $\begin{array}{l}3.921 \\
(0.50)\end{array}$ \\
\hline
\end{tabular}

Fig. 4.9a. Pin-power distribution for CENTRM benchmark TCA-5. Value in parentheses is percent standard deviation. 


\begin{tabular}{|c|c|c|c|c|c|c|c|c|c|c|}
\hline & & & & & & & & & & $\begin{array}{l}5.872 \\
(0.90)\end{array}$ \\
\hline & \multirow[t]{8}{*}{$\begin{array}{l}0.161 \\
(0.77)\end{array}$} & & & & & & & & $\begin{array}{l}5.824 \\
(0.44)\end{array}$ & $\begin{array}{l}5.930 \\
(0.43)\end{array}$ \\
\hline & & & & & & & & $\begin{array}{l}5.663 \\
(0.47)\end{array}$ & $\begin{array}{l}5.775 \\
(0.33)\end{array}$ & $\begin{array}{l}5.815 \\
(0.46)\end{array}$ \\
\hline & & & & & & & $\begin{array}{l}5.278 \\
(0.44)\end{array}$ & $\begin{array}{l}5.523 \\
(0.31)\end{array}$ & $\begin{array}{l}5.575 \\
(0.32)\end{array}$ & $\begin{array}{l}5.572 \\
(0.46)\end{array}$ \\
\hline & & & & & & $\begin{array}{l}4.844 \\
(0.50) \\
\end{array}$ & $\begin{array}{l}5.069 \\
(0.31) \\
\end{array}$ & $\begin{array}{l}5.219 \\
(0.32) \\
\end{array}$ & $\begin{array}{l}5.311 \\
(0.31) \\
\end{array}$ & $\begin{array}{l}5.323 \\
(0.47) \\
\end{array}$ \\
\hline & & & & & $\begin{array}{l}4.326 \\
(0.49)\end{array}$ & $\begin{array}{l}4.538 \\
(0.35)\end{array}$ & $\begin{array}{l}4.742 \\
(0.33)\end{array}$ & $\begin{array}{l}4.945 \\
(0.33)\end{array}$ & $\begin{array}{l}5.011 \\
(0.32)\end{array}$ & $\begin{array}{l}5.054 \\
(0.46)\end{array}$ \\
\hline & & & & $\begin{array}{l}3.679 \\
(0.54)\end{array}$ & $\begin{array}{l}3.942 \\
(0.34)\end{array}$ & $\begin{array}{l}4.189 \\
(0.36)\end{array}$ & $\begin{array}{l}4.400 \\
(0.36)\end{array}$ & $\begin{array}{l}4.534 \\
(0.35)\end{array}$ & $\begin{array}{l}4.638 \\
(0.33)\end{array}$ & $\begin{array}{l}4.691 \\
(0.53)\end{array}$ \\
\hline & & & $\begin{array}{c}3.002 \\
(0.59)\end{array}$ & $\begin{array}{l}3.342 \\
(0.40)\end{array}$ & $\begin{array}{l}3.606 \\
(0.38)\end{array}$ & $\begin{array}{l}3.840 \\
(0.35)\end{array}$ & $\begin{array}{l}4.021 \\
(0.35)\end{array}$ & $\begin{array}{l}4.138 \\
(0.38)\end{array}$ & $\begin{array}{l}4.209 \\
(0.38)\end{array}$ & $\begin{array}{l}4.213 \\
(0.54)\end{array}$ \\
\hline & & $\begin{array}{l}2.468 \\
(0.64)\end{array}$ & $\begin{array}{l}2.720 \\
(0.49)\end{array}$ & $\begin{array}{l}2.999 \\
(0.42)\end{array}$ & $\begin{array}{l}3.225 \\
(0.45)\end{array}$ & $\begin{array}{l}3.440 \\
(0.39)\end{array}$ & $\begin{array}{l}3.582 \\
(0.41)\end{array}$ & $\begin{array}{l}3.702 \\
(0.40)\end{array}$ & $\begin{array}{l}3.771 \\
(0.38)\end{array}$ & $\begin{array}{l}3.783 \\
(0.53)\end{array}$ \\
\hline & $\begin{array}{c}2.075 \\
(0.66)\end{array}$ & $\begin{array}{l}2.259 \\
(0.54)\end{array}$ & $\begin{array}{l}2.518 \\
(0.45)\end{array}$ & $\begin{array}{l}2.768 \\
(0.45)\end{array}$ & $\begin{array}{l}3.015 \\
(0.41)\end{array}$ & $\begin{array}{l}3.168 \\
(0.43)\end{array}$ & $\begin{array}{l}3.345 \\
(0.39)\end{array}$ & $\begin{array}{l}3.455 \\
(0.40)\end{array}$ & $\begin{array}{c}3.474 \\
(0.39)\end{array}$ & $\begin{array}{l}3.504 \\
(0.54)\end{array}$ \\
\hline $\begin{array}{l}2.297 \\
(0.69)\end{array}$ & $\begin{array}{c}2.263 \\
(0.45)\end{array}$ & $\begin{array}{l}2.480 \\
(0.49)\end{array}$ & $\begin{array}{c}2.792 \\
(0.42)\end{array}$ & $\begin{array}{c}3.046 \\
(0.38)\end{array}$ & $\begin{array}{l}3.316 \\
(0.41)\end{array}$ & $\begin{array}{l}3.535 \\
(0.39)\end{array}$ & $\begin{array}{l}3.708 \\
(0.38)\end{array}$ & $\begin{array}{l}3.801 \\
(0.38)\end{array}$ & $\begin{array}{c}3.876 \\
(0.36)\end{array}$ & $\begin{array}{l}3.904 \\
(0.55)\end{array}$ \\
\hline
\end{tabular}

Fig. 4.9b. Pin-power distribution for NITAWL benchmark TCA-5. Value in parentheses is percent standard deviation. 
Table 4.45. Selected reaction rates for CENTRM case TCA-5

\begin{tabular}{|c|c|c|c|c|c|c|}
\hline Pin & Region & $\begin{array}{c}\mathrm{G}_{\mathrm{a}} \mathrm{M} \\
\left(\mathrm{cm}^{-3}-\mathrm{s}^{-1}\right)\end{array}$ & $\begin{array}{c}\mathcal{G}_{\mathrm{f}} \mathrm{M} \\
\left(\mathrm{cm}^{-3}-\mathrm{s}^{-1}\right)\end{array}$ & $\begin{array}{c}\mathrm{G}_{\mathrm{f}} \mathrm{M} \\
\left(\mathrm{cm}^{-3}-\mathrm{s}^{-1}\right)\end{array}$ & $\begin{array}{c}\mathrm{M} \\
\left(\mathrm{cm}-\mathrm{s}^{-1}\right)\end{array}$ & $\mathrm{M} / \mathrm{M}_{\mathrm{t}}$ \\
\hline \multirow{3}{*}{1} & Fuel & $1.374 \mathrm{E}-05$ & $2.280 \mathrm{E}-05$ & $8.078 \mathrm{E}-06$ & $5.603 \mathrm{E}-03$ & 0.235 \\
\hline & Clad & $2.618 \mathrm{E}-07$ & 0.0 & 0.0 & $1.747 \mathrm{E}-03$ & 0.074 \\
\hline & Mod. & 7.503E-07 & 0.0 & 0.0 & $1.646 \mathrm{E}-02$ & 0.691 \\
\hline \multirow{3}{*}{66} & Fuel & 3.907E-05 & $5.970 \mathrm{E}-05$ & $2.112 \mathrm{E}-05$ & $2.311 \mathrm{E}-02$ & 0.235 \\
\hline & Clad & 7.783E-07 & 0.0 & 0.0 & $7.263 \mathrm{E}-03$ & 0.074 \\
\hline & Mod. & $1.793 \mathrm{E}-06$ & 0.0 & 0.0 & $6.806 \mathrm{E}-02$ & 0.691 \\
\hline
\end{tabular}

Table 4.46. Selected reaction rates for NITAWL case TCA-5

\begin{tabular}{ccccccc}
\hline Pin & Region & $\begin{array}{c}\mathrm{G}_{\mathrm{a}} \mathrm{M} \\
\left(\mathrm{cm}^{-3}-\mathrm{s}^{-1}\right)\end{array}$ & $\begin{array}{c}\mathrm{G}_{\mathrm{f}} \mathrm{M} \\
\left(\mathrm{cm}^{-3}-\mathrm{s}^{-1}\right)\end{array}$ & $\begin{array}{c}\mathrm{G}_{\mathrm{f}} \mathrm{M} \\
\left(\mathrm{cm}^{-3}-\mathrm{s}^{-1}\right)\end{array}$ & $\begin{array}{c}\mathrm{M} \\
\left(\mathrm{cm}^{-1}\right)\end{array}$ & $\mathrm{sM}_{\mathrm{t}}$ \\
\hline \multirow{2}{*}{1} & Fuel & $1.378 \mathrm{E}-05$ & $2.293 \mathrm{E}-05$ & $8.127 \mathrm{E}-06$ & $5.603 \mathrm{E}-03$ & 0.235 \\
& Clad & $2.619 \mathrm{E}-07$ & 0.0 & 0.0 & $1.745 \mathrm{E}-03$ & 0.074 \\
& Mod. & $7.543 \mathrm{E}-07$ & 0.0 & 0.0 & $1.646 \mathrm{E}-02$ & 0.691 \\
\hline \multirow{2}{*}{66} & Fuel & $3.868 \mathrm{E}-05$ & $5.889 \mathrm{E}-05$ & $2.084 \mathrm{E}-05$ & $2.304 \mathrm{E}-02$ & 0.234 \\
& Clad & $7.727 \mathrm{E}-07$ & 0.0 & 0.0 & $7.281 \mathrm{E}-03$ & 0.074 \\
& Mod. & $1.803 \mathrm{E}-06$ & 0.0 & 0.0 & $6.824 \mathrm{E}-02$ & 0.692 \\
\hline
\end{tabular}


Table 4.47. TCA-5, Pin-1 four-group fluxes

\begin{tabular}{ccc}
\hline Group & $\begin{array}{c}\text { CENTRM M } \\
\left(\mathrm{cm}^{-2}-\mathrm{s}^{-1}\right)\end{array}$ & $\begin{array}{c}\text { NITAWL M } \\
\left(\mathrm{cm}^{-2}-\mathrm{s}^{-1}\right)\end{array}$ \\
\hline 1 & $4.939 \mathrm{E}-05$ & $4.938 \mathrm{E}-05$ \\
2 & $1.446 \mathrm{E}-05$ & $1.412 \mathrm{E}-05$ \\
3 & $3.466 \mathrm{E}-06$ & $3.579 \mathrm{E}-06$ \\
4 & $3.035 \mathrm{E}-05$ & $3.059 \mathrm{E}-05$ \\
\hline
\end{tabular}

Table 4.48. TCA-5, Pin-1 four-group U-235 cross sections

\begin{tabular}{cccccccc}
\hline Group & \multicolumn{2}{c}{ CENTRM cross sections $\left(\mathrm{cm}^{-1}\right)$} & \multicolumn{3}{c}{ NITAWL cross sections $\left(\mathrm{cm}^{-1}\right)$} \\
\hline & $\frac{\mathrm{G}_{\mathrm{a}}}{1.42994 \mathrm{E}-04}$ & $\frac{\mathrm{G}_{\mathrm{f}}}{3.26070 \mathrm{E}-04}$ & $\frac{\mathrm{G}_{\mathrm{f}}}{1.25328 \mathrm{E}-04}$ & $\frac{\mathrm{G}_{\mathrm{a}}}{1.43122 \mathrm{E}-04}$ & $\frac{4 \mathrm{G}_{\mathrm{f}}}{3.25555 \mathrm{E}-04}$ & $\frac{\mathrm{G}_{\mathrm{f}}}{1.25325 \mathrm{E}-04}$ \\
1 & $3.42309 \mathrm{E}-03$ & $5.26214 \mathrm{E}-03$ & $2.15954 \mathrm{E}-03$ & $3.47849 \mathrm{E}-03$ & $5.29943 \mathrm{E}-03$ & $2.17484 \mathrm{E}-03$ \\
2 & $5.14146 \mathrm{E}-03$ & $1.04884 \mathrm{E}-02$ & $4.30436 \mathrm{E}-03$ & $5.22434 \mathrm{E}-03$ & $1.06706 \mathrm{E}-02$ & $4.37913 \mathrm{E}-03$ \\
3 & $4.87407 \mathrm{E}-02$ & $1.01475 \mathrm{E}-01$ & $4.16445 \mathrm{E}-02$ & $4.86829 \mathrm{E}-02$ & $1.01355 \mathrm{E}-01$ & $4.15953 \mathrm{E}-02$ \\
\hline
\end{tabular}

Table 4.49. TCA-5, Pin-1 four-group U-238 cross sections

\begin{tabular}{cccccccc}
\hline Group & \multicolumn{3}{c}{ CENTRM cross sections $\left(\mathrm{cm}^{-1}\right)$} & \multicolumn{3}{c}{ NITAWL cross sections $\left(\mathrm{cm}^{-1}\right)$} \\
\hline & $\frac{\mathrm{G}_{\mathrm{a}}}{4.50583 \mathrm{E}-03}$ & $\frac{\mathrm{G}_{\mathrm{f}}}{8.47688 \mathrm{E}-03}$ & $\frac{\mathrm{G}_{\mathrm{f}}}{2.99982 \mathrm{E}-03}$ & $\frac{\mathrm{G}_{\mathrm{a}}}{4.48281 \mathrm{E}-03}$ & $\frac{\mathrm{G}_{\mathrm{f}}}{8.35527 \mathrm{E}-03}$ & $\frac{\mathrm{G}_{\mathrm{f}}}{2.96632 \mathrm{E}-03}$ \\
1 & $3.83667 \mathrm{E}-02$ & $3.84273 \mathrm{E}-06$ & $1.59203 \mathrm{E}-06$ & $3.62671 \mathrm{E}-02$ & $3.53982 \mathrm{E}-06$ & $1.46653 \mathrm{E}-06$ \\
3 & $7.00150 \mathrm{E}-03$ & $2.45134 \mathrm{E}-08$ & $1.01563 \mathrm{E}-08$ & $7.02110 \mathrm{E}-03$ & $2.47448 \mathrm{E}-08$ & $1.02522 \mathrm{E}-08$ \\
4 & $2.76871 \mathrm{E}-02$ & $1.30144 \mathrm{E}-07$ & $5.39213 \mathrm{E}-08$ & $2.76564 \mathrm{E}-02$ & $1.30000 \mathrm{E}-07$ & $5.38614 \mathrm{E}-08$ \\
\hline
\end{tabular}

Table 4.50. TCA-5, Pin-1 four-group Pu-239 cross sections

\begin{tabular}{|c|c|c|c|c|c|c|}
\hline \multirow[t]{2}{*}{ Group } & \multicolumn{3}{|c|}{ CENTRM cross sections $\left(\mathrm{cm}^{-1}\right)$} & \multicolumn{3}{|c|}{ NITAWL cross sections $\left(\mathrm{cm}^{-1}\right)$} \\
\hline & $\mathrm{G}_{\mathrm{a}}$ & $<\mathrm{G}_{\mathrm{f}}$ & $\mathrm{G}_{\mathrm{f}}$ & $\mathrm{G}_{\mathrm{a}}$ & $\mathcal{G}_{f}$ & $\mathrm{G}_{\mathrm{f}}$ \\
\hline 1 & $5.12069 \mathrm{E}-04$ & $1.49754 \mathrm{E}-03$ & $4.81215 \mathrm{E}-04$ & $5.11841 \mathrm{E}-04$ & $1.49393 \mathrm{E}-03$ & 4.80664E-04 \\
\hline 2 & $1.09615 \mathrm{E}-02$ & $1.82420 \mathrm{E}-02$ & $6.33444 \mathrm{E}-03$ & $1.10261 \mathrm{E}-02$ & $1.81861 \mathrm{E}-02$ & 6.31503E-03 \\
\hline 3 & 3.50990E-02 & 6.88148E-02 & $2.38956 \mathrm{E}-02$ & $3.48620 \mathrm{E}-02$ & 6.84733E-02 & 2.37771E-02 \\
\hline 4 & $2.66585 \mathrm{E}-01$ & 5.39901E-01 & $1.86737 \mathrm{E}-01$ & $2.66263 \mathrm{E}-01$ & 5.39232E-01 & $1.86508 \mathrm{E}-01$ \\
\hline
\end{tabular}


Table 4.51. TCA-5, Pin-66 four-group fluxes

\begin{tabular}{ccc}
\hline Group & $\begin{array}{c}\text { CENTRM M } \\
\left(\mathrm{cm}^{-2}-\mathrm{s}^{-1}\right)\end{array}$ & $\begin{array}{c}\text { NITAWL M } \\
\left(\mathrm{cm}^{-2}-\mathrm{s}^{-1}\right)\end{array}$ \\
\hline 1 & $2.303 \mathrm{E}-04$ & $2.296 \mathrm{E}-04$ \\
2 & $8.041 \mathrm{E}-05$ & $8.059 \mathrm{E}-05$ \\
3 & $1.804 \mathrm{E}-05$ & $1.799 \mathrm{E}-05$ \\
4 & $7.411 \mathrm{E}-05$ & $7.336 \mathrm{E}-05$ \\
\hline
\end{tabular}

Table 4.52. TCA-5, Pin-66 four-group U-235 cross sections

\begin{tabular}{cccccccc}
\hline Group & \multicolumn{2}{c}{ CENTRM cross sections $\left(\mathrm{cm}^{-1}\right)$} & \multicolumn{3}{c}{ NITAWL cross sections $\left(\mathrm{cm}^{-1}\right)$} \\
\hline & $\frac{\mathrm{G}_{\mathrm{a}}}{}$ & $\frac{\mathrm{G}_{\mathrm{f}}}{1.47516 \mathrm{E}-04}$ & $\frac{\mathrm{G}_{\mathrm{f}}}{3.29683 \mathrm{E}-04}$ & $\frac{1.27617 \mathrm{E}-04}{1.46906 \mathrm{E}-04}$ & $\frac{\mathrm{G}_{\mathrm{f}}}{3.28595 \mathrm{E}-04}$ & $\frac{\mathrm{G}_{\mathrm{f}}}{1.27226 \mathrm{E}-04}$ \\
1 & $3.45804 \mathrm{E}-03$ & $5.28540 \mathrm{E}-03$ & $2.16908 \mathrm{E}-03$ & $3.42799 \mathrm{E}-03$ & $5.19278 \mathrm{E}-03$ & $2.13107 \mathrm{E}-03$ \\
3 & $5.21778 \mathrm{E}-03$ & $1.06625 \mathrm{E}-02$ & $4.37578 \mathrm{E}-03$ & $5.17950 \mathrm{E}-03$ & $1.05856 \mathrm{E}-02$ & $4.34425 \mathrm{E}-03$ \\
4 & $4.63091 \mathrm{E}-02$ & $9.63284 \mathrm{E}-02$ & $3.95323 \mathrm{E}-02$ & $4.61333 \mathrm{E}-02$ & $9.59635 \mathrm{E}-02$ & $3.93825 \mathrm{E}-02$ \\
\hline
\end{tabular}

Table 4.53. TCA-5, Pin-66 four-group U-238 cross sections

\begin{tabular}{cccccccc}
\hline Group & \multicolumn{3}{c}{ CENTRM cross sections $\left(\mathrm{cm}^{-1}\right)$} & \multicolumn{3}{c}{ NITAWL cross sections $\left(\mathrm{cm}^{-1}\right)$} \\
\hline & $\frac{\mathrm{G}_{\mathrm{a}}}{1}$ & $4.38259 \mathrm{E}-03$ & $\frac{\mathrm{G}_{\mathrm{f}}}{7.65041 \mathrm{E}-03}$ & $\frac{\mathrm{G}_{\mathrm{f}}}{2.71517 \mathrm{E}-03}$ & $\frac{\mathrm{G}_{\mathrm{a}}}{4.35318 \mathrm{E}-03}$ & $\frac{\mathrm{G}_{\mathrm{f}}}{7.60571 \mathrm{E}-03}$ & $\frac{\mathrm{G}_{\mathrm{f}}}{2.70175 \mathrm{E}-03}$ \\
2 & $3.76966 \mathrm{E}-02$ & $3.76282 \mathrm{E}-06$ & $1.55892 \mathrm{E}-06$ & $3.76657 \mathrm{E}-02$ & $3.47384 \mathrm{E}-06$ & $1.43920 \mathrm{E}-06$ \\
3 & $7.01397 \mathrm{E}-03$ & $2.46319 \mathrm{E}-08$ & $1.02054 \mathrm{E}-08$ & $6.99839 \mathrm{E}-03$ & $2.45177 \mathrm{E}-08$ & $1.01581 \mathrm{E}-08$ \\
4 & $2.64310 \mathrm{E}-02$ & $1.24116 \mathrm{E}-07$ & $5.14236 \mathrm{E}-08$ & $2.63412 \mathrm{E}-02$ & $1.23688 \mathrm{E}-07$ & $5.12462 \mathrm{E}-08$ \\
\hline
\end{tabular}

Table 4.54. TCA-5, Pin-66 four-group Pu-239 cross sections

\begin{tabular}{|c|c|c|c|c|c|c|}
\hline \multirow[t]{2}{*}{ Group } & \multicolumn{3}{|c|}{ CENTRM cross sections $\left(\mathrm{cm}^{-1}\right)$} & \multicolumn{3}{|c|}{ NITAWL cross sections $\left(\mathrm{cm}^{-1}\right)$} \\
\hline & $\mathrm{G}_{\mathrm{a}}$ & $\mathrm{G}_{\mathrm{f}}$ & $\mathrm{G}_{\mathrm{f}}$ & $\mathrm{G}_{\mathrm{a}}$ & $\mathrm{G}_{\mathrm{f}}$ & $\mathrm{G}_{\mathrm{f}}$ \\
\hline 1 & $5.14155 \mathrm{E}-04$ & $1.47850 \mathrm{E}-03$ & $\overline{4.78094 \mathrm{E}-04}$ & $5.13349 \mathrm{E}-04$ & $1.47737 \mathrm{E}-03$ & $4.77888 \mathrm{E}-04$ \\
\hline 2 & $1.09920 \mathrm{E}-02$ & $1.80785 \mathrm{E}-02$ & $6.27768 \mathrm{E}-03$ & $1.06624 \mathrm{E}-02$ & 1.75079E-02 & $6.07953 \mathrm{E}-03$ \\
\hline 3 & $3.48251 \mathrm{E}-02$ & 6.83614E-02 & 2.37382E-02 & $3.45711 \mathrm{E}-02$ & 6.78516E-02 & $2.35613 \mathrm{E}-02$ \\
\hline 4 & 2.81194E-01 & $5.59228 \mathrm{E}-01$ & $1.93491 \mathrm{E}-01$ & $2.80259 \mathrm{E}-01$ & 5.57291E-01 & $1.92823 \mathrm{E}-01$ \\
\hline
\end{tabular}




\begin{tabular}{|c|c|c|c|c|c|c|c|c|c|c|}
\hline & & & & & & & & & & $\begin{array}{l}5.446 \\
(0.87)\end{array}$ \\
\hline & \multirow[t]{8}{*}{$\begin{array}{l}0.191 \\
(1.58)\end{array}$} & & & & & & & & $\begin{array}{l}5.462 \\
(0.45)\end{array}$ & $\begin{array}{l}5.433 \\
(0.42)\end{array}$ \\
\hline & & & & & & & & $\begin{array}{l}5.225 \\
(0.40)\end{array}$ & $\begin{array}{l}5.327 \\
(0.30)\end{array}$ & $\begin{array}{l}5.357 \\
(0.42)\end{array}$ \\
\hline & & & & & & & $\begin{array}{l}4.915 \\
(0.44)\end{array}$ & $\begin{array}{l}5.049 \\
(0.30)\end{array}$ & $\begin{array}{l}5.160 \\
(0.34)\end{array}$ & $\begin{array}{l}5.185 \\
(0.43)\end{array}$ \\
\hline & & & & & & $\begin{array}{l}4.477 \\
(0.48) \\
\end{array}$ & $\begin{array}{l}4.691 \\
(0.37) \\
\end{array}$ & $\begin{array}{r}4.860 \\
(0.33) \\
\end{array}$ & $\begin{array}{l}4.954 \\
(0.36) \\
\end{array}$ & $\begin{array}{l}4.957 \\
(0.47) \\
\end{array}$ \\
\hline & & & & & $\begin{array}{l}3.976 \\
(0.52)\end{array}$ & $\begin{array}{l}4.231 \\
(0.37)\end{array}$ & $\begin{array}{l}4.430 \\
(0.37)\end{array}$ & $\begin{array}{l}4.569 \\
(0.33)\end{array}$ & $\begin{array}{l}4.651 \\
(0.31)\end{array}$ & $\begin{array}{l}4.688 \\
(0.46)\end{array}$ \\
\hline & & & & $\begin{array}{l}3.440 \\
(0.56)\end{array}$ & $\begin{array}{l}3.685 \\
(0.42)\end{array}$ & $\begin{array}{l}3.916 \\
(0.36)\end{array}$ & $\begin{array}{l}4.084 \\
(0.36)\end{array}$ & $\begin{array}{l}4.239 \\
(0.34)\end{array}$ & $\begin{array}{l}4.317 \\
(0.35)\end{array}$ & $\begin{array}{l}4.338 \\
(0.50)\end{array}$ \\
\hline & & & $\begin{array}{l}2.811 \\
(0.62)\end{array}$ & $\begin{array}{l}3.097 \\
(0.39)\end{array}$ & $\begin{array}{l}3.347 \\
(0.37)\end{array}$ & $\begin{array}{l}3.552 \\
(0.34)\end{array}$ & $\begin{array}{l}3.704 \\
(0.36)\end{array}$ & $\begin{array}{l}3.855 \\
(0.39)\end{array}$ & $\begin{array}{l}3.900 \\
(0.33)\end{array}$ & $\begin{array}{l}3.925 \\
(0.48)\end{array}$ \\
\hline & & $\begin{array}{l}2.272 \\
(0.65)\end{array}$ & $\begin{array}{l}2.533 \\
(0.42)\end{array}$ & $\begin{array}{c}2797 \\
(0.37)\end{array}$ & $\begin{array}{l}3.010 \\
(0.43)\end{array}$ & $\begin{array}{l}3.216 \\
(0.43)\end{array}$ & $\begin{array}{l}3.339 \\
(0.40)\end{array}$ & $\begin{array}{l}3.445 \\
(0.33)\end{array}$ & $\begin{array}{l}3.528 \\
(0.38)\end{array}$ & $\begin{array}{l}3.528 \\
(0.59)\end{array}$ \\
\hline & $\begin{array}{c}1.928 \\
(0.76)\end{array}$ & $\begin{array}{l}2.090 \\
(0.51)\end{array}$ & $\begin{array}{l}2.327 \\
(0.47)\end{array}$ & $\begin{array}{c}2.570 \\
(0.45)\end{array}$ & $\begin{array}{l}2.789 \\
(0.40)\end{array}$ & $\begin{array}{l}2.962 \\
(0.40)\end{array}$ & $\begin{array}{c}3.081 \\
(0.42)\end{array}$ & $\begin{array}{l}3.182 \\
(0.38)\end{array}$ & $\begin{array}{c}3.274 \\
(0.42)\end{array}$ & $\begin{array}{l}3.260 \\
(0.56)\end{array}$ \\
\hline $\begin{array}{l}2.123 \\
(0.72)\end{array}$ & $\begin{array}{c}2.094 \\
(0.47)\end{array}$ & $\begin{array}{l}2.324 \\
(0.47)\end{array}$ & $\begin{array}{c}2.602 \\
(0.41)\end{array}$ & $\begin{array}{l}2.847 \\
(0.39)\end{array}$ & $\begin{array}{l}3.072 \\
(0.40)\end{array}$ & $\begin{array}{l}3.294 \\
(0.40)\end{array}$ & $\begin{array}{c}3.461 \\
(0.41)\end{array}$ & $\begin{array}{l}3.543 \\
(0.36)\end{array}$ & $\begin{array}{c}3.638 \\
(0.36)\end{array}$ & $\begin{array}{l}3.680 \\
(0.54)\end{array}$ \\
\hline
\end{tabular}

Fig. 4.10a. Pin-power distribution for CENTRM benchmark TCA-6. Value in parentheses is percent standard deviation. 


\begin{tabular}{|c|c|c|c|c|c|c|c|c|c|c|}
\hline & & & & & & & & & & $\begin{array}{l}5.535 \\
(0.82)\end{array}$ \\
\hline & $\begin{array}{l}0.192 \\
(1.50)\end{array}$ & & & & & & & & $\begin{array}{l}5.433 \\
(0.45)\end{array}$ & $\begin{array}{l}5.485 \\
(0.41)\end{array}$ \\
\hline & & & & & & & & $\begin{array}{l}5.208 \\
(0.43)\end{array}$ & $\begin{array}{l}5.336 \\
(0.30)\end{array}$ & $\begin{array}{l}5.380 \\
(0.44)\end{array}$ \\
\hline & & & & & & & $\begin{array}{l}4.903 \\
(0.45)\end{array}$ & $\begin{array}{l}5.056 \\
(0.32)\end{array}$ & $\begin{array}{l}5.155 \\
(0.31)\end{array}$ & $\begin{array}{l}5.224 \\
(0.42)\end{array}$ \\
\hline & & & & & & $\begin{array}{l}4.478 \\
(0.50)\end{array}$ & $\begin{array}{l}4.745 \\
(0.32)\end{array}$ & $\begin{array}{l}4.840 \\
(0.32)\end{array}$ & $\begin{array}{l}4.949 \\
(0.31)\end{array}$ & $\begin{array}{l}5.009 \\
(0.47)\end{array}$ \\
\hline & & & & & $\begin{array}{c}3.945 \\
(0.51)\end{array}$ & $\begin{array}{l}4.219 \\
(0.35)\end{array}$ & $\begin{array}{l}4.437 \\
(0.34)\end{array}$ & $\begin{array}{l}4.592 \\
(0.34)\end{array}$ & $\begin{array}{l}4.656 \\
(0.35)\end{array}$ & $\begin{array}{l}4.662 \\
(0.46)\end{array}$ \\
\hline & & & & $\begin{array}{c}3.402 \\
(0.55)\end{array}$ & $\begin{array}{l}3.677 \\
(0.37)\end{array}$ & $\begin{array}{l}3.935 \\
(0.36)\end{array}$ & $\begin{array}{l}4.103 \\
(0.36)\end{array}$ & $\begin{array}{l}4.259 \\
(0.35)\end{array}$ & $\begin{array}{l}4.279 \\
(0.33)\end{array}$ & $\begin{array}{l}4.347 \\
(0.51)\end{array}$ \\
\hline & & & $\begin{array}{c}2.808 \\
(0.56)\end{array}$ & $\begin{array}{l}3.096 \\
(0.43)\end{array}$ & $\begin{array}{l}3.349 \\
(0.38)\end{array}$ & $\begin{array}{l}3.556 \\
(0.37)\end{array}$ & $\begin{array}{l}3.711 \\
(0.36)\end{array}$ & $\begin{array}{l}3.858 \\
(0.35)\end{array}$ & $\begin{array}{c}3.908 \\
(0.35)\end{array}$ & $\begin{array}{l}3.932 \\
(0.47)\end{array}$ \\
\hline & & $\begin{array}{l}2.286 \\
(0.62)\end{array}$ & $\begin{array}{l}2.545 \\
(0.44)\end{array}$ & $\begin{array}{c}2795 \\
(0.38)\end{array}$ & $\begin{array}{l}3.014 \\
(0.42)\end{array}$ & $\begin{array}{l}3.211 \\
(0.41)\end{array}$ & $\begin{array}{l}3.340 \\
(0.38)\end{array}$ & $\begin{array}{l}3.434 \\
(0.35)\end{array}$ & $\begin{array}{l}3.501 \\
(0.39)\end{array}$ & $\begin{array}{l}3.547 \\
(0.56)\end{array}$ \\
\hline & $\begin{array}{l}1.930 \\
(0.79)\end{array}$ & $\begin{array}{l}2.114 \\
(0.47)\end{array}$ & $\begin{array}{l}2.328 \\
(0.48)\end{array}$ & $\begin{array}{l}2.571 \\
(0.47)\end{array}$ & $\begin{array}{l}2.784 \\
(0.43)\end{array}$ & $\begin{array}{l}2.977 \\
(0.38)\end{array}$ & $\begin{array}{l}3.106 \\
(0.36)\end{array}$ & $\begin{array}{l}3.198 \\
(0.41)\end{array}$ & $\begin{array}{l}3.269 \\
(0.38)\end{array}$ & $\begin{array}{l}3.308 \\
(0.52)\end{array}$ \\
\hline $\begin{array}{l}2.129 \\
(0.65)\end{array}$ & $\begin{array}{l}2.105 \\
(0.45)\end{array}$ & $\begin{array}{l}2.328 \\
(0.49)\end{array}$ & $\begin{array}{c}2.622 \\
(0.41)\end{array}$ & $\begin{array}{c}2.861 \\
(0.43)\end{array}$ & $\begin{array}{c}3.096 \\
(0.38)\end{array}$ & $\begin{array}{l}3.292 \\
(0.40)\end{array}$ & $\begin{array}{c}3.450 \\
(0.38)\end{array}$ & $\begin{array}{l}3.563 \\
(0.40)\end{array}$ & $\begin{array}{c}3.618 \\
(0.35)\end{array}$ & $\begin{array}{l}3.656 \\
(0.57)\end{array}$ \\
\hline
\end{tabular}

Fig. 4.10b. Pin-power distribution for NITAWL benchmark TCA-6. Value in parentheses is percent standard deviation. 
Table 4.55. Selected reaction rates for CENTRM case TCA-6

\begin{tabular}{|c|c|c|c|c|c|c|}
\hline Pin & Region & $\begin{array}{c}\mathrm{G}_{\mathrm{a}} \mathrm{M} \\
\left(\mathrm{cm}^{-3}-\mathrm{s}^{-1}\right)\end{array}$ & $\begin{array}{c}\mathcal{G}_{\mathrm{f}} \mathrm{M} \\
\left(\mathrm{cm}^{-3}-\mathrm{s}^{-1}\right)\end{array}$ & $\begin{array}{c}\mathrm{G}_{\mathrm{f}} \mathrm{M} \\
\left(\mathrm{cm}^{-3}-\mathrm{s}^{-1}\right)\end{array}$ & $\begin{array}{c}\mathrm{M} \\
\left(\mathrm{cm}-\mathrm{s}^{-1}\right)\end{array}$ & $\mathrm{M} / \mathrm{M}_{\mathrm{t}}$ \\
\hline \multirow{3}{*}{1} & Fuel & $1.298 \mathrm{E}-05$ & $2.136 \mathrm{E}-05$ & 7.574E-06 & $5.637 \mathrm{E}-03$ & 0.235 \\
\hline & Clad & $2.474 \mathrm{E}-07$ & 0.0 & 0.0 & $1.759 \mathrm{E}-03$ & 0.073 \\
\hline & Mod. & 7.049E-07 & 0.0 & 0.0 & $1.659 \mathrm{E}-02$ & 0.692 \\
\hline \multirow{3}{*}{66} & Fuel & 3.599E-05 & $5.470 \mathrm{E}-05$ & $1.936 \mathrm{E}-05$ & $2.295 \mathrm{E}-02$ & 0.234 \\
\hline & Clad & $7.226 \mathrm{E}-07$ & 0.0 & 0.0 & $7.268 \mathrm{E}-03$ & 0.074 \\
\hline & Mod. & $1.669 \mathrm{E}-06$ & 0.0 & 0.0 & $6.776 \mathrm{E}-02$ & 0.692 \\
\hline
\end{tabular}

Table 4.56. Selected reaction rates for NITAWL case TCA-6

\begin{tabular}{ccccccc}
\hline Pin & Region & $\begin{array}{c}\mathrm{G}_{\mathrm{a}} \mathrm{M} \\
\left(\mathrm{cm}^{-3}-\mathrm{s}^{-1}\right)\end{array}$ & $\begin{array}{c}\mathrm{G}_{\mathrm{f}} \mathrm{M} \\
\left(\mathrm{cm}^{-3}-\mathrm{s}^{-1}\right)\end{array}$ & $\begin{array}{c}\mathrm{G}_{\mathrm{f}} \mathrm{M} \\
\left(\mathrm{cm}^{-3}-\mathrm{s}^{-1}\right)\end{array}$ & $\begin{array}{c}\mathrm{M} \\
\left(\mathrm{cm}^{-1}\right)\end{array}$ & $\mathrm{sM}_{\mathrm{t}}$ \\
\hline \multirow{2}{*}{1} & Fuel & $1.301 \mathrm{E}-05$ & $2.152 \mathrm{E}-05$ & $7.630 \mathrm{E}-06$ & $5.677 \mathrm{E}-03$ & 0.236 \\
& Clad & $2.482 \mathrm{E}-07$ & 0.0 & 0.0 & $1.771 \mathrm{E}-03$ & 0.073 \\
& Mod. & $7.104 \mathrm{E}-07$ & 0.0 & 0.0 & $1.663 \mathrm{E}-02$ & 0.691 \\
\hline \multirow{2}{*}{66} & Fuel & $3.641 \mathrm{E}-05$ & $5.549 \mathrm{E}-05$ & $1.964 \mathrm{E}-05$ & $2.328 \mathrm{E}-02$ & 0.235 \\
& Clad & $7.250 \mathrm{E}-07$ & 0.0 & 0.0 & $7.322 \mathrm{E}-03$ & 0.074 \\
& Mod. & $1.692 \mathrm{E}-06$ & 0.0 & 0.0 & $6.857 \mathrm{E}-02$ & 0.691 \\
\hline
\end{tabular}


Table 4.57. TCA-6, Pin-1 four-group fluxes

\begin{tabular}{ccc}
\hline Group & $\begin{array}{c}\text { CENTRM M } \\
\left(\mathrm{cm}^{-2}-\mathrm{s}^{-1}\right)\end{array}$ & $\begin{array}{c}\text { NITAWL M } \\
\left(\mathrm{cm}^{-2}-\mathrm{s}^{-1}\right)\end{array}$ \\
\hline 1 & $4.562 \mathrm{E}-05$ & $4.614 \mathrm{E}-05$ \\
2 & $1.353 \mathrm{E}-05$ & $1.361 \mathrm{E}-05$ \\
3 & $3.260 \mathrm{E}-06$ & $3.167 \mathrm{E}-06$ \\
4 & $2.877 \mathrm{E}-05$ & $2.891 \mathrm{E}-05$ \\
\hline
\end{tabular}

Table 4.58. TCA-6, Pin-1 four-group U-235 cross sections

\begin{tabular}{cccccccc}
\hline Group & \multicolumn{3}{c}{ CENTRM cross sections $\left(\mathrm{cm}^{-1}\right)$} & \multicolumn{3}{c}{ NITAWL cross sections $\left(\mathrm{cm}^{-1}\right)$} \\
\hline & $\frac{\mathrm{G}_{\mathrm{a}}}{1.43180 \mathrm{E}-04}$ & $\frac{\mathrm{G}_{\mathrm{f}}}{3.25454 \mathrm{E}-04}$ & $\frac{\mathrm{G}_{\mathrm{f}}}{1.25334 \mathrm{E}-04}$ & $\frac{\mathrm{G}_{\mathrm{a}}}{1.42890 \mathrm{E}-04}$ & $\frac{\mathrm{G}_{\mathrm{f}}}{3.25301 \mathrm{E}-04}$ & $\frac{\mathrm{G}_{\mathrm{f}}}{1.25170 \mathrm{E}-04}$ \\
1 & $3.45574 \mathrm{E}-03$ & $5.26068 \mathrm{E}-03$ & $2.15894 \mathrm{E}-03$ & $3.46874 \mathrm{E}-03$ & $5.30446 \mathrm{E}-03$ & $2.17691 \mathrm{E}-03$ \\
2 & $5.12187 \mathrm{E}-03$ & $1.04432 \mathrm{E}-02$ & $4.28580 \mathrm{E}-03$ & $5.18636 \mathrm{E}-03$ & $1.05942 \mathrm{E}-02$ & $4.34774 \mathrm{E}-03$ \\
3 & $4.87091 \mathrm{E}-02$ & $1.01408 \mathrm{E}-01$ & $4.16173 \mathrm{E}-02$ & $4.87392 \mathrm{E}-02$ & $1.01468 \mathrm{E}-01$ & $4.16413 \mathrm{E}-02$ \\
\hline
\end{tabular}

Table 4.59. TCA-6, Pin-1 four-group U-238 cross sections

\begin{tabular}{ccccccc}
\hline Group & \multicolumn{2}{c}{ CENTRM cross sections $\left(\mathrm{cm}^{-1}\right)$} & \multicolumn{3}{c}{ NITAWL cross sections $\left(\mathrm{cm}^{-1}\right)$} \\
\hline & $\frac{\mathrm{G}_{\mathrm{a}}}{4.44759 \mathrm{E}-03}$ & $\frac{\mathrm{G}_{\mathrm{f}}}{8.24022 \mathrm{E}-03}$ & $\frac{\mathrm{G}_{\mathrm{f}}}{2.92337 \mathrm{E}-03}$ & $\frac{\mathrm{G}_{\mathrm{a}}}{4.46693 \mathrm{E}-03}$ & $\overline{8.35598 \mathrm{E}-03}$ & $\overline{2.96179 \mathrm{E}-03}$ \\
1 & $3.90256 \mathrm{E}-02$ & $3.50820 \mathrm{E}-06$ & $1.45343 \mathrm{E}-06$ & $3.57765 \mathrm{E}-02$ & $3.56985 \mathrm{E}-06$ & $1.47897 \mathrm{E}-06$ \\
2 & $6.98181 \mathrm{E}-03$ & $2.44734 \mathrm{E}-08$ & $1.01398 \mathrm{E}-08$ & $7.00395 \mathrm{E}-03$ & $2.46168 \mathrm{E}-08$ & $1.01992 \mathrm{E}-08$ \\
3 & $2.76742 \mathrm{E}-02$ & $1.30082 \mathrm{E}-07$ & $5.38954 \mathrm{E}-08$ & $2.76818 \mathrm{E}-02$ & $1.30118 \mathrm{E}-07$ & $5.39104 \mathrm{E}-08$ \\
\hline
\end{tabular}

Table 4.60. TCA-6, Pin-1 four-group Pu-239 cross sections

\begin{tabular}{cccccccc}
\hline Group & \multicolumn{3}{c}{ CENTRM cross sections $\left(\mathrm{cm}^{-1}\right)$} & \multicolumn{3}{c}{ NITAWL cross sections $\left(\mathrm{cm}^{-1}\right)$} \\
\hline & $\frac{\mathrm{G}_{\mathrm{a}}}{1}$ & $5.11662 \mathrm{E}-04$ & $\frac{\leftarrow_{\mathrm{f}}}{1.49257 \mathrm{E}-03}$ & $\frac{\mathrm{G}_{\mathrm{f}}}{4.80489 \mathrm{E}-04}$ & $\frac{\mathrm{G}_{\mathrm{a}}}{5.11324 \mathrm{E}-04}$ & $\frac{\mathrm{G}_{\mathrm{f}}}{1.49329 \mathrm{E}-03}$ & $\frac{\mathrm{G}_{\mathrm{f}}}{4.80338 \mathrm{E}-04}$ \\
2 & $1.13135 \mathrm{E}-02$ & $1.86601 \mathrm{E}-02$ & $6.47963 \mathrm{E}-03$ & $1.07085 \mathrm{E}-02$ & $1.75213 \mathrm{E}-02$ & $6.08419 \mathrm{E}-03$ \\
3 & $3.39736 \mathrm{E}-02$ & $6.67666 \mathrm{E}-02$ & $2.31844 \mathrm{E}-02$ & $3.36473 \mathrm{E}-02$ & $6.62052 \mathrm{E}-02$ & $2.29895 \mathrm{E}-02$ \\
4 & $2.66162 \mathrm{E}-01$ & $5.39117 \mathrm{E}-01$ & $1.86465 \mathrm{E}-01$ & $2.67553 \mathrm{E}-01$ & $5.41501 \mathrm{E}-01$ & $1.87294 \mathrm{E}-01$ \\
\hline
\end{tabular}


Table 4.61. TCA-6, Pin-66 four-group fluxes

\begin{tabular}{ccc}
\hline Group & $\begin{array}{c}\text { CENTRM M } \\
\left(\mathrm{cm}^{-2}-\mathrm{s}^{-1}\right)\end{array}$ & $\begin{array}{c}\text { NITAWL M } \\
\left(\mathrm{cm}^{-2}-\mathrm{s}^{-1}\right)\end{array}$ \\
\hline 1 & $2.129 \mathrm{E}-04$ & $2.141 \mathrm{E}-04$ \\
2 & $7.322 \mathrm{E}-05$ & $7.593 \mathrm{E}-05$ \\
3 & $1.668 \mathrm{E}-05$ & $1.698 \mathrm{E}-05$ \\
4 & $6.844 \mathrm{E}-05$ & $6.955 \mathrm{E}-05$ \\
\hline
\end{tabular}

Table 4.62. TCA-6, Pin-66 four-group U-235 cross sections

\begin{tabular}{cccccccc}
\hline Group & \multicolumn{3}{c}{ CENTRM cross sections $\left(\mathrm{cm}^{-1}\right)$} & \multicolumn{3}{c}{ NITAWL cross sections $\left(\mathrm{cm}^{-1}\right)$} \\
\hline & $\frac{\mathrm{G}_{\mathrm{a}}}{1.46734 \mathrm{E}-04}$ & $\frac{\mathrm{G}_{\mathrm{f}}}{3.28401 \mathrm{E}-04}$ & $\frac{\mathrm{G}_{\mathrm{f}}}{1.27150 \mathrm{E}-04}$ & $\frac{\mathrm{G}_{\mathrm{a}}}{1.47522 \mathrm{E}-04}$ & $\frac{\mathrm{G}_{\mathrm{f}}}{3.29450 \mathrm{E}-04}$ & $\frac{\mathrm{G}_{\mathrm{f}}}{1.27598 \mathrm{E}-04}$ \\
1 & $3.38204 \mathrm{E}-03$ & $5.17330 \mathrm{E}-03$ & $2.12307 \mathrm{E}-03$ & $3.41355 \mathrm{E}-03$ & $5.23610 \mathrm{E}-03$ & $2.14885 \mathrm{E}-03$ \\
2 & $5.13990 \mathrm{E}-03$ & $1.04899 \mathrm{E}-02$ & $4.30497 \mathrm{E}-03$ & $5.04984 \mathrm{E}-03$ & $1.02714 \mathrm{E}-02$ & $4.21529 \mathrm{E}-03$ \\
3 & $4.64337 \mathrm{E}-02$ & $9.65915 \mathrm{E}-02$ & $3.96403 \mathrm{E}-02$ & $4.61970 \mathrm{E}-02$ & $9.60939 \mathrm{E}-02$ & $3.94361 \mathrm{E}-02$ \\
\hline
\end{tabular}

Table 4.63. TCA-6, Pin-66 four-group U-238 cross sections

\begin{tabular}{cccccccc}
\hline Group & \multicolumn{3}{c}{ CENTRM cross sections $\left(\mathrm{cm}^{-1}\right)$} & \multicolumn{3}{c}{ NITAWL cross sections $\left(\mathrm{cm}^{-1}\right)$} \\
\hline & $\frac{\mathrm{G}_{\mathrm{a}}}{4.34898 \mathrm{E}-03}$ & $\frac{\mathrm{G}_{\mathrm{f}}}{7.60907 \mathrm{E}-03}$ & $\frac{\mathrm{G}_{\mathrm{f}}}{2.70457 \mathrm{E}-03}$ & $\frac{\mathrm{G}_{\mathrm{a}}}{4.36295 \mathrm{E}-03}$ & $\frac{\mathrm{G}_{\mathrm{f}}}{7.58351 \mathrm{E}-03}$ & $\frac{\mathrm{G}_{\mathrm{f}}}{2.69381 \mathrm{E}-03}$ \\
1 & $3.85262 \mathrm{E}-02$ & $3.68752 \mathrm{E}-06$ & $1.52772 \mathrm{E}-06$ & $3.74174 \mathrm{E}-02$ & $3.59244 \mathrm{E}-06$ & $1.48833 \mathrm{E}-06$ \\
3 & $6.99508 \mathrm{E}-03$ & $2.44889 \mathrm{E}-08$ & $1.01462 \mathrm{E}-08$ & $6.96908 \mathrm{E}-03$ & $2.42193 \mathrm{E}-08$ & $1.00345 \mathrm{E}-08$ \\
4 & $2.64864 \mathrm{E}-02$ & $1.24380 \mathrm{E}-07$ & $5.15332 \mathrm{E}-08$ & $2.63675 \mathrm{E}-02$ & $1.23812 \mathrm{E}-07$ & $5.12979 \mathrm{E}-08$ \\
\hline
\end{tabular}

Table 4.64. TCA-6, Pin-66 four-group Pu-239 cross sections

\begin{tabular}{cccccccc}
\hline Group & \multicolumn{2}{c}{ CENTRM cross sections $\left(\mathrm{cm}^{-1}\right)$} & \multicolumn{3}{c}{ NITAWL cross sections $\left(\mathrm{cm}^{-1}\right)$} \\
\hline & $\frac{\mathrm{G}_{\mathrm{a}}}{5.13250 \mathrm{E}-04}$ & $\frac{\mathrm{G}_{\mathrm{f}}}{1.47784 \mathrm{E}-03}$ & $\frac{\mathrm{G}_{\mathrm{f}}}{4.78051 \mathrm{E}-04}$ & $\frac{\mathrm{G}_{\mathrm{a}}}{5.13989 \mathrm{E}-04}$ & $\frac{\mathrm{G}_{\mathrm{f}}}{1.47716 \mathrm{E}-03}$ & $\frac{\mathrm{G}_{\mathrm{f}}}{4.77940 \mathrm{E}-04}$ \\
1 & $1.13093 \mathrm{E}-02$ & $1.84779 \mathrm{E}-02$ & $6.41636 \mathrm{E}-03$ & $1.07147 \mathrm{E}-02$ & $1.76559 \mathrm{E}-02$ & $6.13092 \mathrm{E}-03$ \\
3 & $3.39521 \mathrm{E}-02$ & $6.67206 \mathrm{E}-02$ & $2.31683 \mathrm{E}-02$ & $3.32187 \mathrm{E}-02$ & $6.53557 \mathrm{E}-02$ & $2.26946 \mathrm{E}-02$ \\
4 & $2.81522 \mathrm{E}-01$ & $5.60045 \mathrm{E}-01$ & $1.93774 \mathrm{E}-01$ & $2.81843 \mathrm{E}-01$ & $5.60105 \mathrm{E}-01$ & $1.93799 \mathrm{E}-01$ \\
\hline
\end{tabular}




\begin{tabular}{|c|c|c|c|c|c|c|c|c|c|}
\hline & & & & & & & & & $\begin{array}{l}7.308 \\
(0.44)\end{array}$ \\
\hline & $\begin{array}{l}0.155 \\
(0.61)\end{array}$ & & & & & & & $\begin{array}{c}7.112 \\
(0.39)\end{array}$ & $\begin{array}{l}7.241 \\
(0.35)\end{array}$ \\
\hline & & & & & & & $\begin{array}{l}6.694 \\
(0.43)\end{array}$ & $\begin{array}{c}6.907 \\
(0.26)\end{array}$ & $\begin{array}{r}7.038 \\
(0.31)\end{array}$ \\
\hline & & & & & & $\begin{array}{l}6.068 \\
(0.46)\end{array}$ & $\begin{array}{l}6.408 \\
(0.30)\end{array}$ & $\begin{array}{c}6.595 \\
(0.30)\end{array}$ & $\begin{array}{l}6.694 \\
(0.30)\end{array}$ \\
\hline & & & & & $\begin{array}{l}5.368 \\
(0.55)\end{array}$ & $\begin{array}{l}5.743 \\
(0.35)\end{array}$ & $\begin{array}{l}6.028 \\
(0.33)\end{array}$ & $\begin{array}{c}6.153 \\
(0.34)\end{array}$ & $\begin{array}{l}6.308 \\
(0.29)\end{array}$ \\
\hline & & & & $\begin{array}{l}4.563 \\
(0.47)\end{array}$ & $\begin{array}{l}4.998 \\
(0.34)\end{array}$ & $\begin{array}{l}5.297 \\
(0.35)\end{array}$ & $\begin{array}{l}5.515 \\
(0.33)\end{array}$ & $\begin{array}{l}5.710 \\
(0.30)\end{array}$ & $\begin{array}{l}5.792 \\
(0.32)\end{array}$ \\
\hline & & & $\begin{array}{c}3.708 \\
(0.54)\end{array}$ & $\begin{array}{l}4.107 \\
(0.38)\end{array}$ & $\begin{array}{l}4.467 \\
(0.38)\end{array}$ & $\begin{array}{l}4.726 \\
(0.34)\end{array}$ & $\begin{array}{l}4.996 \\
(0.34)\end{array}$ & $\begin{array}{l}5.135 \\
(0.34)\end{array}$ & $\begin{array}{l}5.182 \\
(0.36)\end{array}$ \\
\hline & & $\begin{array}{l}2.884 \\
(0.72)\end{array}$ & $\begin{array}{l}3.250 \\
(0.41)\end{array}$ & $\begin{array}{c}3.615 \\
(0.38)\end{array}$ & $\begin{array}{l}3.950 \\
(0.39)\end{array}$ & $\begin{array}{l}4.203 \\
(0.34)\end{array}$ & $\begin{array}{l}4.373 \\
(0.37)\end{array}$ & $\begin{array}{l}4.511 \\
(0.36)\end{array}$ & $\begin{array}{r}4.579 \\
(0.39)\end{array}$ \\
\hline & $\begin{array}{l}2.218 \\
(0.76)\end{array}$ & $\begin{array}{l}2.524 \\
(0.48)\end{array}$ & $\begin{array}{l}2.866 \\
(0.44)\end{array}$ & $\begin{array}{c}3.179 \\
(0.45)\end{array}$ & $\begin{array}{l}3.463 \\
(0.41)\end{array}$ & $\begin{array}{l}3.694 \\
(0.41)\end{array}$ & $\begin{array}{l}3.852 \\
(0.37)\end{array}$ & $\begin{array}{l}3.966 \\
(0.40)\end{array}$ & $\begin{array}{l}4.027 \\
(0.40)\end{array}$ \\
\hline $\begin{array}{l}2.112 \\
(0.73)\end{array}$ & $\begin{array}{l}2.218 \\
(0.54)\end{array}$ & $\begin{array}{l}2.550 \\
(0.48)\end{array}$ & $\begin{array}{l}2.879 \\
(0.44)\end{array}$ & $\begin{array}{c}3.211 \\
(0.40)\end{array}$ & $\begin{array}{l}3.481 \\
(0.45)\end{array}$ & $\begin{array}{l}3.706 \\
(0.38)\end{array}$ & $\begin{array}{l}3.893 \\
(0.38)\end{array}$ & $\begin{array}{l}4.026 \\
(0.39)\end{array}$ & $\begin{array}{l}4.085 \\
(0.37)\end{array}$ \\
\hline
\end{tabular}

Fig. 4.11a. Pin-power distribution for CENTRM benchmark TCA-7. Value in parentheses is percent standard deviation. 


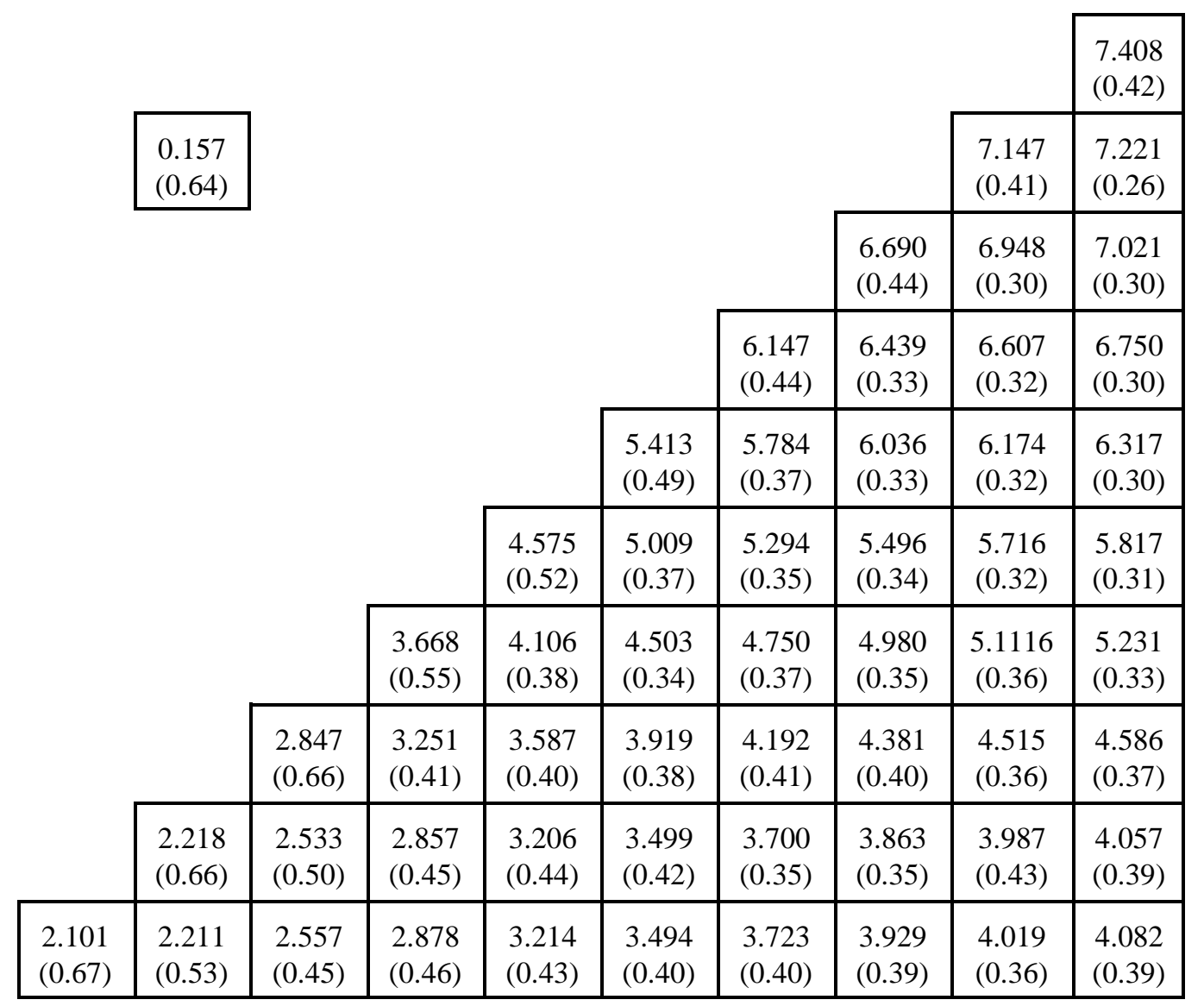

Fig. 4.11b. Pin-power distribution for NITAWL benchmark TCA-7. Value in parentheses is percent standard deviation. 
Table 4.65. Selected reaction rates for CENTRM case TCA-7

\begin{tabular}{|c|c|c|c|c|c|c|}
\hline Pin & Region & $\begin{array}{c}\mathrm{G}_{\mathrm{a}} \mathrm{M} \\
\left(\mathrm{cm}^{-3}-\mathrm{s}^{-1}\right)\end{array}$ & $\begin{array}{c}\mathcal{G}_{\mathrm{f}} \mathrm{M} \\
\left(\mathrm{cm}^{-3}-\mathrm{s}^{-1}\right)\end{array}$ & $\begin{array}{c}\mathrm{G}_{\mathrm{f}} \mathrm{M} \\
\left(\mathrm{cm}^{-3}-\mathrm{s}^{-1}\right)\end{array}$ & $\begin{array}{c}\mathrm{M} \\
\left(\mathrm{cm}-\mathrm{s}^{-1}\right)\end{array}$ & $\mathrm{M} / \mathrm{M}_{\mathrm{t}}$ \\
\hline \multirow{3}{*}{1} & Fuel & $1.250 \mathrm{E}-05$ & $2.098 \mathrm{E}-05$ & 7.436E-06 & $4.475 \mathrm{E}-03$ & 0.181 \\
\hline & Clad & $2.338 \mathrm{E}-07$ & 0.0 & 0.0 & $1.390 \mathrm{E}-03$ & 0.056 \\
\hline & Mod. & 7.159E-07 & 0.0 & 0.0 & $1.884 \mathrm{E}-02$ & 0.763 \\
\hline \multirow{3}{*}{55} & Fuel & 4.638E-05 & 7.357E-05 & 2.604E-05 & $2.167 \mathrm{E}-02$ & 0.182 \\
\hline & Clad & 8.926E-07 & 0.0 & 0.0 & $6.796 \mathrm{E}-03$ & 0.057 \\
\hline & Mod. & 2.331E-06 & 0.0 & 0.0 & $9.081 \mathrm{E}-02$ & 0.761 \\
\hline
\end{tabular}

Table 4.66. Selected reaction rates for NITAWL case TCA-7

\begin{tabular}{ccccccc}
\hline Pin & Region & $\begin{array}{c}\mathrm{G}_{\mathrm{a}} \mathrm{M} \\
\left(\mathrm{cm}^{-3}-\mathrm{s}^{-1}\right)\end{array}$ & $\begin{array}{c}\mathrm{G}_{\mathrm{f}} \mathrm{M} \\
\left(\mathrm{cm}^{-3}-\mathrm{s}^{-1}\right)\end{array}$ & $\begin{array}{c}\mathrm{G}_{\mathrm{f}} \mathrm{M} \\
\left(\mathrm{cm}^{-3}-\mathrm{s}^{-1}\right)\end{array}$ & $\begin{array}{c}\mathrm{M} \\
\left(\mathrm{cm}^{-1}\right)\end{array}$ & $\mathrm{MM}_{\mathrm{t}}$ \\
\hline \multirow{2}{*}{1} & Fuel & $1.239 \mathrm{E}-05$ & $2.081 \mathrm{E}-05$ & $7.375 \mathrm{E}-06$ & $4.405 \mathrm{E}-03$ & 0.181 \\
& Clad & $2.328 \mathrm{E}-07$ & 0.0 & 0.0 & $1.370 \mathrm{E}-03$ & 0.056 \\
& Mod. & $7.088 \mathrm{E}-07$ & 0.0 & 0.0 & $1.861 \mathrm{E}-02$ & 0.763 \\
\hline \multirow{2}{*}{55} & Fuel & $4.646 \mathrm{E}-05$ & $7.384 \mathrm{E}-05$ & $2.619 \mathrm{E}-05$ & $2.180 \mathrm{E}-02$ & 0.182 \\
& Clad & $8.849 \mathrm{E}-07$ & 0.0 & 0.0 & $6.807 \mathrm{E}-03$ & 0.057 \\
& Mod. & $2.324 \mathrm{E}-06$ & 0.0 & 0.0 & $9.112 \mathrm{E}-02$ & 0.761 \\
\hline
\end{tabular}


Table 4.67. TCA-7, Pin-1 four-group fluxes

\begin{tabular}{ccc}
\hline Group & $\begin{array}{c}\text { CENTRM M } \\
\left(\mathrm{cm}^{-2}-\mathrm{s}^{-1}\right)\end{array}$ & $\begin{array}{c}\text { NITAWL M } \\
\left(\mathrm{cm}^{-2}-\mathrm{s}^{-1}\right)\end{array}$ \\
\hline 1 & $4.076 \mathrm{E}-05$ & $4.002 \mathrm{E}-05$ \\
2 & $1.156 \mathrm{E}-05$ & $1.122 \mathrm{E}-05$ \\
3 & $2.916 \mathrm{E}-06$ & $2.849 \mathrm{E}-06$ \\
4 & $2.805 \mathrm{E}-05$ & $2.789 \mathrm{E}-05$ \\
\hline
\end{tabular}

Table 4.68. TCA-7, Pin-1 four-group U-235 cross sections

\begin{tabular}{cccccccc}
\hline Group & \multicolumn{3}{c}{ CENTRM cross sections $\left(\mathrm{cm}^{-1}\right)$} & \multicolumn{3}{c}{ NITAWL cross sections $\left(\mathrm{cm}^{-1}\right)$} \\
\hline & $\frac{\mathrm{G}_{\mathrm{a}}}{}$ & $\frac{\varangle_{\mathrm{f}}}{1.42491 \mathrm{E}-04}$ & $\frac{\mathrm{G}_{\mathrm{f}}}{3.25106 \mathrm{E}-04}$ & $\frac{\mathrm{G}_{\mathrm{f}}}{1.25005 \mathrm{E}-04}$ & $\frac{\mathrm{G}_{\mathrm{a}}}{1.41299 \mathrm{E}-04}$ & $\frac{\varangle_{\mathrm{f}}}{3.24300 \mathrm{E}-04}$ & $\frac{\mathrm{G}_{\mathrm{f}}}{1.24414 \mathrm{E}-04}$ \\
2 & $3.44975 \mathrm{E}-03$ & $5.22773 \mathrm{E}-03$ & $2.14542 \mathrm{E}-03$ & $3.47152 \mathrm{E}-03$ & $5.26026 \mathrm{E}-03$ & $2.15876 \mathrm{E}-03$ \\
3 & $5.21159 \mathrm{E}-03$ & $1.06607 \mathrm{E}-02$ & $4.37503 \mathrm{E}-03$ & $5.27529 \mathrm{E}-03$ & $1.08126 \mathrm{E}-02$ & $4.43738 \mathrm{E}-03$ \\
4 & $4.89248 \mathrm{E}-02$ & $1.01867 \mathrm{E}-01$ & $4.18053 \mathrm{E}-02$ & $4.88655 \mathrm{E}-02$ & $1.01743 \mathrm{E}-01$ & $4.17544 \mathrm{E}-02$ \\
\hline
\end{tabular}

Table 4.69. TCA-7, Pin-1 four-group U-238 cross sections

\begin{tabular}{cccccccc}
\hline Group & \multicolumn{3}{c}{ CENTRM cross sections $\left(\mathrm{cm}^{-1}\right)$} & \multicolumn{3}{c}{ NITAWL cross sections $\left(\mathrm{cm}^{-1}\right)$} \\
\hline & $\frac{\mathrm{G}_{\mathrm{a}}}{4.5114 \mathrm{E}-03}$ & $\frac{\mathrm{G}_{\mathrm{f}}}{8.48982 \mathrm{E}-03}$ & $\frac{\mathrm{G}_{\mathrm{f}}}{3.01170 \mathrm{E}-03}$ & $\frac{4.53551 \mathrm{E}-03}{\mathrm{G}_{\mathrm{a}}}$ & $\frac{{ }_{8}}{8.70929 \mathrm{E}-03}$ & $\frac{\mathrm{G}_{\mathrm{f}}}{3.08361 \mathrm{E}-03}$ \\
1 & $3.80608 \mathrm{E}-02$ & $3.86401 \mathrm{E}-06$ & $1.60085 \mathrm{E}-06$ & $3.81145 \mathrm{E}-02$ & $3.57483 \mathrm{E}-06$ & $1.48104 \mathrm{E}-06$ \\
3 & $7.01523 \mathrm{E}-03$ & $2.46902 \mathrm{E}-08$ & $1.02296 \mathrm{E}-08$ & $7.02506 \mathrm{E}-03$ & $2.48652 \mathrm{E}-08$ & $1.03021 \mathrm{E}-08$ \\
4 & $2.77876 \mathrm{E}-02$ & $1.30626 \mathrm{E}-07$ & $5.41212 \mathrm{E}-08$ & $2.77567 \mathrm{E}-02$ & $1.30480 \mathrm{E}-07$ & $5.40602 \mathrm{E}-08$ \\
\hline
\end{tabular}

Table 4.70. TCA-7, Pin-1 four-group Pu-239 cross sections

\begin{tabular}{cccccccc}
\hline Group & \multicolumn{3}{c}{ CENTRM cross sections $\left(\mathrm{cm}^{-1}\right)$} & \multicolumn{3}{c}{ NITAWL cross sections $\left(\mathrm{cm}^{-1}\right)$} \\
\hline & $\frac{\mathrm{G}_{\mathrm{a}}}{}$ & $\frac{\mathrm{G}_{\mathrm{f}}}{1.11889 \mathrm{E}-04}$ & $\frac{\mathrm{G}_{\mathrm{f}}}{4.49757 \mathrm{E}-03}$ & $4.81327 \mathrm{E}-04$ & $\frac{\mathrm{G}_{\mathrm{a}}}{5.11022 \mathrm{E}-04}$ & $\frac{\mathrm{G}_{\mathrm{f}}}{1.50238 \mathrm{E}-03}$ & $\frac{\mathrm{G}_{\mathrm{f}}}{4.81994 \mathrm{E}-04}$ \\
1 & $1.09768 \mathrm{E}-02$ & $1.79177 \mathrm{E}-02$ & $6.22185 \mathrm{E}-03$ & $1.09889 \mathrm{E}-02$ & $1.81187 \mathrm{E}-02$ & $6.29163 \mathrm{E}-03$ \\
3 & $3.46245 \mathrm{E}-02$ & $6.80175 \mathrm{E}-02$ & $2.36188 \mathrm{E}-02$ & $3.47959 \mathrm{E}-02$ & $6.83577 \mathrm{E}-02$ & $2.37369 \mathrm{E}-02$ \\
4 & $2.64817 \mathrm{E}-01$ & $5.37348 \mathrm{E}-01$ & $1.85848 \mathrm{E}-01$ & $2.63994 \mathrm{E}-01$ & $5.35812 \mathrm{E}-01$ & $1.85317 \mathrm{E}-01$ \\
\hline
\end{tabular}


Table 4.71. TCA-7, Pin-55 four-group fluxes

\begin{tabular}{ccc}
\hline Group & $\begin{array}{c}\text { CENTRM M } \\
\left(\mathrm{cm}^{-2}-\mathrm{s}^{-1}\right)\end{array}$ & $\begin{array}{c}\text { NITAWL M } \\
\left(\mathrm{cm}^{-2}-\mathrm{s}^{-1}\right)\end{array}$ \\
\hline 1 & $2.186 \mathrm{E}-04$ & $2.205 \mathrm{E}-04$ \\
2 & $7.342 \mathrm{E}-05$ & $7.405 \mathrm{E}-05$ \\
3 & $1.743 \mathrm{E}-05$ & $1.715 \mathrm{E}-05$ \\
4 & $9.388 \mathrm{E}-05$ & $9.404 \mathrm{E}-05$ \\
\hline
\end{tabular}

Table 4.72. TCA-7, Pin-55 four-group U-235 cross sections

\begin{tabular}{cccccccc}
\hline Group & \multicolumn{2}{c}{ CENTRM cross sections $\left(\mathrm{cm}^{-1}\right)$} & \multicolumn{3}{c}{ NITAWL cross sections $\left(\mathrm{cm}^{-1}\right)$} \\
\hline & $\frac{\mathrm{G}_{\mathrm{a}}}{}$ & $\frac{\mathrm{G}_{\mathrm{f}}}{1.46008 \mathrm{E}-04}$ & $\frac{3.28325 \mathrm{E}-04}{1.26835 \mathrm{E}-04}$ & $\frac{\mathrm{G}_{\mathrm{f}}}{1.45867 \mathrm{E}-04}$ & $\frac{\mathrm{G}_{\mathrm{f}}}{3.28061 \mathrm{E}-04}$ & $\frac{\mathrm{G}_{\mathrm{f}}}{1.26745 \mathrm{E}-04}$ \\
1 & $3.45407 \mathrm{E}-03$ & $5.28814 \mathrm{E}-03$ & $2.17020 \mathrm{E}-03$ & $3.44685 \mathrm{E}-03$ & $5.26736 \mathrm{E}-03$ & $2.16167 \mathrm{E}-03$ \\
3 & $5.22363 \mathrm{E}-03$ & $1.06689 \mathrm{E}-02$ & $4.37843 \mathrm{E}-03$ & $5.15115 \mathrm{E}-03$ & $1.05143 \mathrm{E}-02$ & $4.31500 \mathrm{E}-03$ \\
4 & $4.73673 \mathrm{E}-02$ & $9.85703 \mathrm{E}-02$ & $4.04524 \mathrm{E}-02$ & $4.74047 \mathrm{E}-02$ & $9.86425 \mathrm{E}-02$ & $4.04820 \mathrm{E}-02$ \\
\hline
\end{tabular}

Table 4.73. TCA-7, Pin-55 four-group U-238 cross sections

\begin{tabular}{cccccccc}
\hline Group & \multicolumn{3}{c}{ CENTRM cross sections $\left(\mathrm{cm}^{-1}\right)$} & \multicolumn{3}{c}{ NITAWL cross sections $\left(\mathrm{cm}^{-1}\right)$} \\
\hline & $\frac{\mathrm{G}_{\mathrm{a}}}{1}$ & $\frac{\mathrm{G}_{\mathrm{f}}}{7.42752 \mathrm{E}-03}$ & $\frac{\mathrm{G}_{\mathrm{f}}}{2.91693 \mathrm{E}-03}$ & $\frac{2.81011 \mathrm{E}-03}{4.41279 \mathrm{E}-03}$ & $\frac{\mathrm{G}_{\mathrm{f}}}{7.88612 \mathrm{E}-03}$ & $\frac{\mathrm{G}_{\mathrm{f}}}{2.79909 \mathrm{E}-03}$ \\
2 & $3.99843 \mathrm{E}-02$ & $3.82517 \mathrm{E}-06$ & $1.58476 \mathrm{E}-06$ & $3.88724 \mathrm{E}-02$ & $3.70637 \mathrm{E}-06$ & $1.53553 \mathrm{E}-06$ \\
3 & $7.01291 \mathrm{E}-03$ & $2.46832 \mathrm{E}-08$ & $1.02267 \mathrm{E}-08$ & $6.99207 \mathrm{E}-03$ & $2.45220 \mathrm{E}-08$ & $1.01599 \mathrm{E}-08$ \\
4 & $2.69759 \mathrm{E}-02$ & $1.26733 \mathrm{E}-07$ & $5.25080 \mathrm{E}-08$ & $2.69906 \mathrm{E}-02$ & $1.26799 \mathrm{E}-07$ & $5.25354 \mathrm{E}-08$ \\
\hline
\end{tabular}

Table 4.74. TCA-7, Pin-55 four-group Pu-239 cross sections

\begin{tabular}{cccccccc}
\hline Group & \multicolumn{3}{c}{ CENTRM cross sections $\left(\mathrm{cm}^{-1}\right)$} & \multicolumn{3}{c}{ NITAWL cross sections $\left(\mathrm{cm}^{-1}\right)$} \\
\hline & $\frac{\mathrm{G}_{\mathrm{a}}}{4}$ & $5.13362 \mathrm{E}-04$ & $\frac{\mathrm{G}_{\mathrm{f}}}{1.48414 \mathrm{E}-03}$ & $\frac{\mathrm{G}_{\mathrm{f}}}{4.78998 \mathrm{E}-04}$ & $\overline{5.13238 \mathrm{E}-04}$ & $\frac{\mathrm{G}_{\mathrm{f}}}{1.48384 \mathrm{E}-03}$ & $\frac{\mathrm{G}_{\mathrm{f}}}{4.78975 \mathrm{E}-04}$ \\
2 & $1.13505 \mathrm{E}-02$ & $1.86607 \mathrm{E}-02$ & $6.47983 \mathrm{E}-03$ & $1.09736 \mathrm{E}-02$ & $1.80388 \mathrm{E}-02$ & $6.26385 \mathrm{E}-03$ \\
3 & $3.48495 \mathrm{E}-02$ & $6.84159 \mathrm{E}-02$ & $2.37571 \mathrm{E}-02$ & $3.38470 \mathrm{E}-02$ & $6.65440 \mathrm{E}-02$ & $2.31071 \mathrm{E}-02$ \\
4 & $2.74694 \mathrm{E}-01$ & $5.50591 \mathrm{E}-01$ & $1.90475 \mathrm{E}-01$ & $2.75966 \mathrm{E}-01$ & $5.52786 \mathrm{E}-01$ & $1.91235 \mathrm{E}-01$ \\
\hline
\end{tabular}




\begin{tabular}{|c|c|c|c|c|c|c|c|c|c|}
\hline & & & & & & & & & $\begin{array}{l}7.058 \\
(0.42)\end{array}$ \\
\hline & \multirow[t]{7}{*}{$\begin{array}{l}0.163 \\
(0.75) \\
\end{array}$} & & & & & & & $\begin{array}{c}6.778 \\
(0.45)\end{array}$ & $\begin{array}{r}6.930 \\
(0.30)\end{array}$ \\
\hline & & & & & & & $\begin{array}{l}6.434 \\
(0.40)\end{array}$ & $\begin{array}{l}6.596 \\
(0.28)\end{array}$ & $\begin{array}{r}6.745 \\
(0.31) \\
\end{array}$ \\
\hline & & & & & & $\begin{array}{l}5.871 \\
(0.47)\end{array}$ & $\begin{array}{l}6.133 \\
(0.33)\end{array}$ & $\begin{array}{l}6.327 \\
(0.30)\end{array}$ & $\begin{array}{l}6.451 \\
(0.29)\end{array}$ \\
\hline & & & & & $\begin{array}{l}5.168 \\
(0.48)\end{array}$ & $\begin{array}{l}5.489 \\
(0.32)\end{array}$ & $\begin{array}{l}5.742 \\
(0.35)\end{array}$ & $\begin{array}{l}5.939 \\
(0.31)\end{array}$ & $\begin{array}{l}6.053 \\
(0.31)\end{array}$ \\
\hline & & & & $\begin{array}{l}4.377 \\
(0.49)\end{array}$ & $\begin{array}{l}4.767 \\
(0.33)\end{array}$ & $\begin{array}{l}5.039 \\
(0.34)\end{array}$ & $\begin{array}{l}5.336 \\
(0.33)\end{array}$ & $\begin{array}{l}5.465 \\
(0.33)\end{array}$ & $\begin{array}{l}5.519 \\
(0.32)\end{array}$ \\
\hline & & & $\begin{array}{l}3.520 \\
(0.56)\end{array}$ & $\begin{array}{l}3.936 \\
(0.40)\end{array}$ & $\begin{array}{l}4.294 \\
(0.38)\end{array}$ & $\begin{array}{l}4.552 \\
(0.35)\end{array}$ & $\begin{array}{l}4.754 \\
(0.36)\end{array}$ & $\begin{array}{l}4.920 \\
(0.32)\end{array}$ & $\begin{array}{l}4.979 \\
(0.34)\end{array}$ \\
\hline & & $\begin{array}{l}2.777 \\
(0.66) \\
\end{array}$ & $\begin{array}{l}3.119 \\
(0.45) \\
\end{array}$ & $\begin{array}{l}3.500 \\
(0.39) \\
\end{array}$ & $\begin{array}{l}3.787 \\
(0.45) \\
\end{array}$ & $\begin{array}{l}4.040 \\
(0.37) \\
\end{array}$ & $\begin{array}{l}4.186 \\
(0.35) \\
\end{array}$ & $\begin{array}{l}4.325 \\
(0.33) \\
\end{array}$ & $\begin{array}{r}4.397 \\
(0.36) \\
\end{array}$ \\
\hline & $\begin{array}{l}2.148 \\
(0.70)\end{array}$ & $\begin{array}{l}2.439 \\
(0.51)\end{array}$ & $\begin{array}{l}2.772 \\
(0.46)\end{array}$ & $\begin{array}{l}3.062 \\
(0.39)\end{array}$ & $\begin{array}{l}3.330 \\
(0.39)\end{array}$ & $\begin{array}{l}3.540 \\
(0.40)\end{array}$ & $\begin{array}{l}3.714 \\
(0.42)\end{array}$ & $\begin{array}{l}3.803 \\
(0.37)\end{array}$ & $\begin{array}{l}3.857 \\
(0.39)\end{array}$ \\
\hline $\begin{array}{l}2.037 \\
(0.75)\end{array}$ & $\begin{array}{c}2.140 \\
(0.50)\end{array}$ & $\begin{array}{l}2.445 \\
(0.49)\end{array}$ & $\begin{array}{l}2.777 \\
(0.46)\end{array}$ & $\begin{array}{c}3.109 \\
(0.42)\end{array}$ & $\begin{array}{l}3.342 \\
(0.42)\end{array}$ & $\begin{array}{l}3.553 \\
(0.38)\end{array}$ & $\begin{array}{c}3.728 \\
(0.41)\end{array}$ & $\begin{array}{c}3.851 \\
(0.40)\end{array}$ & $\begin{array}{l}3.923 \\
(0.39)\end{array}$ \\
\hline
\end{tabular}

Fig. 4.12a. Pin-power distribution for CENTRM benchmark TCA-8. Value in parentheses is percent standard deviation. 


\begin{tabular}{|c|c|c|c|c|c|c|c|c|c|}
\hline & & & & & & & & & $\begin{array}{l}7.087 \\
(0.46)\end{array}$ \\
\hline & \multirow[t]{7}{*}{$\begin{array}{l}0.162 \\
(0.72) \\
\end{array}$} & & & & & & & $\begin{array}{c}6.790 \\
(0.41)\end{array}$ & $\begin{array}{l}6.919 \\
(0.28)\end{array}$ \\
\hline & & & & & & & $\begin{array}{l}6.419 \\
(0.42)\end{array}$ & $\begin{array}{l}6.669 \\
(0.27)\end{array}$ & $\begin{array}{r}6.737 \\
(0.32) \\
\end{array}$ \\
\hline & & & & & & $\begin{array}{l}5.900 \\
(0.43)\end{array}$ & $\begin{array}{l}6.151 \\
(0.30)\end{array}$ & $\begin{array}{c}6.328 \\
(0.27)\end{array}$ & $\begin{array}{l}6.378 \\
(0.33)\end{array}$ \\
\hline & & & & & $\begin{array}{l}5.179 \\
(0.50)\end{array}$ & $\begin{array}{l}5.512 \\
(0.31)\end{array}$ & $\begin{array}{l}5.799 \\
(0.30)\end{array}$ & $\begin{array}{l}5.942 \\
(0.31)\end{array}$ & $\begin{array}{l}6.021 \\
(0.31)\end{array}$ \\
\hline & & & & $\begin{array}{l}4.388 \\
(0.53)\end{array}$ & $\begin{array}{l}4.751 \\
(0.35)\end{array}$ & $\begin{array}{l}5.082 \\
(0.35)\end{array}$ & $\begin{array}{l}5.308 \\
(0.31)\end{array}$ & $\begin{array}{l}5.501 \\
(0.31)\end{array}$ & $\begin{array}{l}5.566 \\
(0.31)\end{array}$ \\
\hline & & & $\begin{array}{l}3.547 \\
(0.55)\end{array}$ & $\begin{array}{l}3.954 \\
(0.39)\end{array}$ & $\begin{array}{l}4.305 \\
(0.40)\end{array}$ & $\begin{array}{l}4.595 \\
(0.33)\end{array}$ & $\begin{array}{l}4.764 \\
(0.36)\end{array}$ & $\begin{array}{l}4.941 \\
(0.34)\end{array}$ & $\begin{array}{l}4.987 \\
(0.34)\end{array}$ \\
\hline & & $\begin{array}{r}2.717 \\
(0.67) \\
\end{array}$ & $\begin{array}{l}3.141 \\
(0.42) \\
\end{array}$ & $\begin{array}{l}3.469 \\
(0.43) \\
\end{array}$ & $\begin{array}{l}3.797 \\
(0.38) \\
\end{array}$ & $\begin{array}{l}4.026 \\
(0.36) \\
\end{array}$ & $\begin{array}{l}4.223 \\
(0.36) \\
\end{array}$ & $\begin{array}{l}4.326 \\
(0.36) \\
\end{array}$ & $\begin{array}{l}4.387 \\
(0.37) \\
\end{array}$ \\
\hline & $\begin{array}{l}2.140 \\
(0.71)\end{array}$ & $\begin{array}{l}2.434 \\
(0.47)\end{array}$ & $\begin{array}{l}2.753 \\
(0.43)\end{array}$ & $\begin{array}{l}3.061 \\
(0.44)\end{array}$ & $\begin{array}{l}3.313 \\
(0.40)\end{array}$ & $\begin{array}{l}3.540 \\
(0.40)\end{array}$ & $\begin{array}{l}3.695 \\
(0.40)\end{array}$ & $\begin{array}{l}3.832 \\
(0.42)\end{array}$ & $\begin{array}{l}3.886 \\
(0.38)\end{array}$ \\
\hline $\begin{array}{l}2.055 \\
(0.75)\end{array}$ & $\begin{array}{l}2.128 \\
(0.49)\end{array}$ & $\begin{array}{c}2.452 \\
(0.45)\end{array}$ & $\begin{array}{l}2.780 \\
(0.44)\end{array}$ & $\begin{array}{c}3.082 \\
(0.44)\end{array}$ & $\begin{array}{l}3.377 \\
(0.39)\end{array}$ & $\begin{array}{l}3.615 \\
(0.40)\end{array}$ & $\begin{array}{l}3.740 \\
(0.39)\end{array}$ & $\begin{array}{c}3.859 \\
(0.40)\end{array}$ & $\begin{array}{l}3.928 \\
(0.38)\end{array}$ \\
\hline
\end{tabular}

Fig. 4.12b. Pin-power distribution for NITAWL benchmark TCA-8. Value in parentheses is percent standard deviation. 
Table 4.75. Selected reaction rates for CENTRM case TCA-8

\begin{tabular}{|c|c|c|c|c|c|c|}
\hline Pin & Region & $\begin{array}{c}\mathrm{G}_{\mathrm{a}} \mathrm{M} \\
\left(\mathrm{cm}^{-3}-\mathrm{s}^{-1}\right)\end{array}$ & $\begin{array}{c}\mathcal{G}_{\mathrm{f}} \mathrm{M} \\
\left(\mathrm{cm}^{-3}-\mathrm{s}^{-1}\right)\end{array}$ & $\begin{array}{c}\mathrm{G}_{\mathrm{f}} \mathrm{M} \\
\left(\mathrm{cm}^{-3}-\mathrm{s}^{-1}\right)\end{array}$ & $\begin{array}{c}\mathrm{M} \\
\left(\mathrm{cm}-\mathrm{s}^{-1}\right)\end{array}$ & $\mathrm{M} / \mathrm{M}_{\mathrm{t}}$ \\
\hline \multirow{3}{*}{1} & Fuel & $1.219 \mathrm{E}-05$ & $2.033 \mathrm{E}-05$ & 7.204E-06 & $4.521 \mathrm{E}-03$ & 0.182 \\
\hline & Clad & $2.267 \mathrm{E}-07$ & 0.0 & 0.0 & $1.397 \mathrm{E}-03$ & 0.056 \\
\hline & Mod. & 6.904E-07 & 0.0 & 0.0 & $1.890 \mathrm{E}-02$ & 0.762 \\
\hline \multirow{3}{*}{55} & Fuel & 4.454E-05 & 7.082E-05 & $2.507 \mathrm{E}-05$ & 2.182E-02 & 0.182 \\
\hline & Clad & 8.569E-07 & 0.0 & 0.0 & $6.833 \mathrm{E}-03$ & 0.057 \\
\hline & Mod. & 2.247E-06 & 0.0 & 0.0 & $9.140 \mathrm{E}-02$ & 0.761 \\
\hline
\end{tabular}

Table 4.76. Selected reaction rates for NITAWL case TCA-8

\begin{tabular}{ccccccc}
\hline \multirow{2}{*}{ Pin } & Region & $\begin{array}{c}\mathrm{G}_{\mathrm{a}} \mathrm{M} \\
\left(\mathrm{cm}^{-3}-\mathrm{s}^{-1}\right)\end{array}$ & $\begin{array}{c}\mathrm{G}_{\mathrm{f}} \mathrm{M} \\
\left(\mathrm{cm}^{-3}-\mathrm{s}^{-1}\right)\end{array}$ & $\begin{array}{c}\mathrm{G}_{\mathrm{f}} \mathrm{M} \\
\left(\mathrm{cm}^{-3}-\mathrm{s}^{-1}\right)\end{array}$ & $\begin{array}{c}\mathrm{M} \\
\left({\left.\mathrm{cm}-\mathrm{s}^{-1}\right)}^{2}\right.\end{array}$ & $\mathrm{MM}_{\mathrm{t}}$ \\
\hline \multirow{2}{*}{1} & Fuel & $1.228 \mathrm{E}-05$ & $2.056 \mathrm{E}-05$ & $7.288 \mathrm{E}-06$ & $4.508 \mathrm{E}-03$ & 0.182 \\
& Clad & $2.273 \mathrm{E}-07$ & 0.0 & 0.0 & $1.396 \mathrm{E}-03$ & 0.056 \\
& Mod. & $6.917 \mathrm{E}-07$ & 0.0 & 0.0 & $1.890 \mathrm{E}-02$ & 0.762 \\
\hline \multirow{2}{*}{55} & Fuel & $4.442 \mathrm{E}-05$ & $7.058 \mathrm{E}-05$ & $2.499 \mathrm{E}-05$ & $2.172 \mathrm{E}-02$ & 0.182 \\
& Clad & $8.512 \mathrm{E}-07$ & 0.0 & 0.0 & $6.810 \mathrm{E}-03$ & 0.057 \\
& Mod. & $2.244 \mathrm{E}-06$ & 0.0 & 0.0 & $9.104 \mathrm{E}-02$ & 0.761 \\
\hline
\end{tabular}


Table 4.77. TCA-8, Pin-1 four-group fluxes

\begin{tabular}{ccc}
\hline Group & $\begin{array}{c}\text { CENTRM M } \\
\left(\mathrm{cm}^{-2}-\mathrm{s}^{-1}\right)\end{array}$ & $\begin{array}{c}\text { NITAWL M } \\
\left(\mathrm{cm}^{-2}-\mathrm{s}^{-1}\right)\end{array}$ \\
\hline 1 & $3.944 \mathrm{E}-05$ & $3.890 \mathrm{E}-05$ \\
2 & $1.103 \mathrm{E}-05$ & $1.116 \mathrm{E}-05$ \\
3 & $2.823 \mathrm{E}-06$ & $2.632 \mathrm{E}-06$ \\
4 & $2.727 \mathrm{E}-05$ & $2.765 \mathrm{E}-05$ \\
\hline
\end{tabular}

Table 4.78. TCA-8, Pin-1 four-group U-235 cross sections

\begin{tabular}{cccccccc}
\hline Group & \multicolumn{2}{c}{ CENTRM cross sections $\left(\mathrm{cm}^{-1}\right)$} & \multicolumn{3}{c}{ NITAWL cross sections $\left(\mathrm{cm}^{-1}\right)$} \\
\hline & $\frac{\mathrm{G}_{\mathrm{a}}}{1.42334 \mathrm{E}-04}$ & $\frac{\mathrm{G}_{\mathrm{f}}}{3.25312 \mathrm{E}-04}$ & $\frac{\mathrm{G}_{\mathrm{f}}}{1.24980 \mathrm{E}-04}$ & $\frac{\mathrm{G}_{\mathrm{a}}}{1.42088 \mathrm{E}-04}$ & $\frac{\mathrm{G}_{\mathrm{f}}}{3.25219 \mathrm{E}-04}$ & $\frac{\mathrm{G}_{\mathrm{f}}}{1.24828 \mathrm{E}-04}$ \\
1 & $3.53127 \mathrm{E}-03$ & $5.35172 \mathrm{E}-03$ & $2.19630 \mathrm{E}-03$ & $3.46204 \mathrm{E}-03$ & $5.26653 \mathrm{E}-03$ & $2.16133 \mathrm{E}-03$ \\
2 & $5.12848 \mathrm{E}-03$ & $1.04583 \mathrm{E}-02$ & $4.29199 \mathrm{E}-03$ & $5.21250 \mathrm{E}-03$ & $1.06698 \mathrm{E}-02$ & $4.37876 \mathrm{E}-03$ \\
3 & $4.88629 \mathrm{E}-02$ & $1.01736 \mathrm{E}-01$ & $4.17514 \mathrm{E}-02$ & $4.88872 \mathrm{E}-02$ & $1.01784 \mathrm{E}-01$ & $4.17714 \mathrm{E}-02$ \\
\hline
\end{tabular}

Table 4.79. TCA-8, Pin-1 four-group U-238 cross sections

\begin{tabular}{cccccccc}
\hline Group & \multicolumn{3}{c}{ CENTRM cross sections $\left(\mathrm{cm}^{-1}\right)$} & \multicolumn{3}{c}{ NITAWL cross sections $\left(\mathrm{cm}^{-1}\right)$} \\
\hline & $\frac{\mathrm{G}_{\mathrm{a}}}{4.53489 \mathrm{E}-03}$ & $\frac{\mathrm{G}_{\mathrm{f}}}{8.60396 \mathrm{E}-03}$ & $\frac{\mathrm{G}_{\mathrm{f}}}{3.05003 \mathrm{E}-03}$ & $\frac{\mathrm{G}_{\mathrm{a}}}{4.52758 \mathrm{E}-03}$ & $\frac{\mathrm{G}_{\mathrm{f}}}{8.62663 \mathrm{E}-03}$ & $\frac{\mathrm{G}_{\mathrm{f}}}{3.05064 \mathrm{E}-03}$ \\
1 & $4.11256 \mathrm{E}-02$ & $3.90524 \mathrm{E}-06$ & $1.61793 \mathrm{E}-06$ & $3.90452 \mathrm{E}-02$ & $3.64034 \mathrm{E}-06$ & $1.50818 \mathrm{E}-06$ \\
3 & $6.97793 \mathrm{E}-03$ & $2.43563 \mathrm{E}-08$ & $1.00913 \mathrm{E}-08$ & $7.01079 \mathrm{E}-03$ & $2.46993 \mathrm{E}-08$ & $1.02334 \mathrm{E}-08$ \\
4 & $2.77529 \mathrm{E}-02$ & $1.30460 \mathrm{E}-07$ & $5.40521 \mathrm{E}-08$ & $2.77661 \mathrm{E}-02$ & $1.30523 \mathrm{E}-07$ & $5.40785 \mathrm{E}-08$ \\
\hline
\end{tabular}

Table 4.80. TCA-8, Pin-1 four-group Pu-239 cross sections

\begin{tabular}{|c|c|c|c|c|c|c|}
\hline \multirow[t]{2}{*}{ Group } & \multicolumn{3}{|c|}{ CENTRM cross sections $\left(\mathrm{cm}^{-1}\right)$} & \multicolumn{3}{|c|}{ NITAWL cross sections $\left(\mathrm{cm}^{-1}\right)$} \\
\hline & $\mathrm{G}_{\mathrm{a}}$ & $<\mathrm{G}_{\mathrm{f}}$ & $\mathrm{G}_{\mathrm{f}}$ & $\mathrm{G}_{\mathrm{a}}$ & $\mathcal{G}_{f}$ & $\mathrm{G}_{\mathrm{f}}$ \\
\hline 1 & $5.11790 \mathrm{E}-04$ & $1.49952 \mathrm{E}-03$ & $4.81643 \mathrm{E}-04$ & $5.11429 \mathrm{E}-04$ & $1.50018 \mathrm{E}-03$ & 4.81447E-04 \\
\hline 2 & $1.15051 \mathrm{E}-02$ & $1.87178 \mathrm{E}-02$ & $6.49966 \mathrm{E}-03$ & $1.06573 \mathrm{E}-02$ & $1.75647 \mathrm{E}-02$ & 6.09924E-03 \\
\hline 3 & 3.42439E-02 & $6.71877 \mathrm{E}-02$ & $2.33306 \mathrm{E}-02$ & $3.42088 \mathrm{E}-02$ & $6.72576 \mathrm{E}-02$ & $2.33550 \mathrm{E}-02$ \\
\hline 4 & $2.65649 \mathrm{E}-01$ & $5.38621 \mathrm{E}-01$ & $1.86291 \mathrm{E}-01$ & $2.65522 \mathrm{E}-01$ & 5.38441E-01 & $1.86228 \mathrm{E}-01$ \\
\hline
\end{tabular}


Table 4.81. TCA-8, Pin-55 four-group fluxes

\begin{tabular}{ccc}
\hline Group & $\begin{array}{c}\text { CENTRM M } \\
\left(\mathrm{cm}^{-2}-\mathrm{s}^{-1}\right)\end{array}$ & $\begin{array}{c}\text { NITAWL M } \\
\left(\mathrm{cm}^{-2}-\mathrm{s}^{-1}\right)\end{array}$ \\
\hline 1 & $2.110 \mathrm{E}-04$ & $2.107 \mathrm{E}-04$ \\
2 & $7.037 \mathrm{E}-05$ & $6.962 \mathrm{E}-05$ \\
3 & $1.673 \mathrm{E}-05$ & $1.667 \mathrm{E}-05$ \\
4 & $9.070 \mathrm{E}-05$ & $9.010 \mathrm{E}-05$ \\
\hline
\end{tabular}

Table 4.82. TCA-8, Pin-55 four-group U-235 cross sections

\begin{tabular}{cccccccc}
\hline Group & \multicolumn{2}{c}{ CENTRM cross sections $\left(\mathrm{cm}^{-1}\right)$} & \multicolumn{3}{c}{ NITAWL cross sections $\left(\mathrm{cm}^{-1}\right)$} \\
\hline & $\frac{\mathrm{G}_{\mathrm{a}}}{1.45480 \mathrm{E}-04}$ & $\frac{\mathrm{G}_{\mathrm{f}}}{3.27674 \mathrm{E}-04}$ & $\frac{\mathrm{G}_{\mathrm{f}}}{1.26526 \mathrm{E}-04}$ & $\frac{\mathrm{G}_{\mathrm{a}}}{1.45435 \mathrm{E}-04}$ & $\frac{\mathrm{G}_{\mathrm{f}}}{3.27709 \mathrm{E}-04}$ & $\frac{\mathrm{G}_{\mathrm{f}}}{1.26510 \mathrm{E}-04}$ \\
1 & $3.39048 \mathrm{E}-03$ & $5.20276 \mathrm{E}-03$ & $2.13517 \mathrm{E}-03$ & $3.47111 \mathrm{E}-03$ & $5.30325 \mathrm{E}-03$ & $2.17640 \mathrm{E}-03$ \\
2 & $5.13229 \mathrm{E}-03$ & $1.04562 \mathrm{E}-02$ & $4.29112 \mathrm{E}-03$ & $5.19965 \mathrm{E}-03$ & $1.06013 \mathrm{E}-02$ & $4.35069 \mathrm{E}-03$ \\
3 & $4.74630 \mathrm{E}-02$ & $9.87671 \mathrm{E}-02$ & $4.05332 \mathrm{E}-02$ & $4.77188 \mathrm{E}-02$ & $9.92999 \mathrm{E}-02$ & $4.07518 \mathrm{E}-02$ \\
\hline
\end{tabular}

Table 4.83. TCA-8, Pin-55 four-group U-238 cross sections

\begin{tabular}{cccccccc}
\hline Group & \multicolumn{3}{c}{ CENTRM cross sections $\left(\mathrm{cm}^{-1}\right)$} & \multicolumn{3}{c}{ NITAWL cross sections $\left(\mathrm{cm}^{-1}\right)$} \\
\hline & $\frac{\mathrm{G}_{\mathrm{a}}}{1}$ & $\frac{\mathrm{G}_{\mathrm{f}}}{7.41327 \mathrm{E}-03}$ & $\frac{\mathrm{G}_{\mathrm{f}}}{7.93014 \mathrm{E}-03}$ & $\frac{2.81274 \mathrm{E}-03}{4.42132 \mathrm{E}-03}$ & $\frac{\mathrm{G}_{\mathrm{f}}}{7.95819 \mathrm{E}-03}$ & $\frac{\mathrm{G}_{\mathrm{f}}}{2.82199 \mathrm{E}-03}$ \\
2 & $3.87344 \mathrm{E}-02$ & $3.80772 \mathrm{E}-06$ & $1.57752 \mathrm{E}-06$ & $3.76439 \mathrm{E}-02$ & $3.63710 \mathrm{E}-06$ & $1.50684 \mathrm{E}-06$ \\
3 & $6.98380 \mathrm{E}-03$ & $2.43899 \mathrm{E}-08$ & $1.01051 \mathrm{E}-08$ & $7.00174 \mathrm{E}-03$ & $2.45717 \mathrm{E}-08$ & $1.01805 \mathrm{E}-08$ \\
4 & $2.70204 \mathrm{E}-02$ & $1.26942 \mathrm{E}-07$ & $5.25946 \mathrm{E}-08$ & $2.71446 \mathrm{E}-02$ & $1.27535 \mathrm{E}-07$ & $5.28402 \mathrm{E}-08$ \\
\hline
\end{tabular}

Table 4.84. TCA-8, Pin-55 four-group Pu-239 cross sections

\begin{tabular}{|c|c|c|c|c|c|c|}
\hline \multirow[t]{2}{*}{ Group } & \multicolumn{3}{|c|}{ CENTRM cross sections $\left(\mathrm{cm}^{-1}\right)$} & \multicolumn{3}{|c|}{ NITAWL cross sections $\left(\mathrm{cm}^{-1}\right)$} \\
\hline & $\mathrm{G}_{\mathrm{a}}$ & $<\mathrm{G}_{\mathrm{f}}$ & $\mathrm{G}_{\mathrm{f}}$ & $\mathrm{G}_{\mathrm{a}}$ & $<\mathrm{G}_{\mathrm{f}}$ & $\mathrm{G}_{\mathrm{f}}$ \\
\hline 1 & $5 . \overline{12787 \mathrm{E}-04}$ & $\overline{1.48453 \mathrm{E}-03}$ & $\overline{4.78981 \mathrm{E}-04}$ & $5 . \overline{12848 \mathrm{E}-04}$ & $\overline{1.48514 \mathrm{E}-03}$ & $\overline{4.79084 \mathrm{E}-04}$ \\
\hline 2 & 1.11398E-02 & 1.83762E-02 & 6.38104E-03 & $1.10696 \mathrm{E}-02$ & 1.81030E-02 & $6.28615 \mathrm{E}-03$ \\
\hline 3 & $3.39692 \mathrm{E}-02$ & $6.67376 \mathrm{E}-02$ & 2.31743E-02 & 3.43528E-02 & $6.74775 \mathrm{E}-02$ & 2.34313E-02 \\
\hline 4 & $2.75637 \mathrm{E}-01$ & $5.52375 \mathrm{E}-01$ & $1.91090 \mathrm{E}-01$ & $2.76264 \mathrm{E}-01$ & $5.53948 \mathrm{E}-01$ & 1.91632E-01 \\
\hline
\end{tabular}




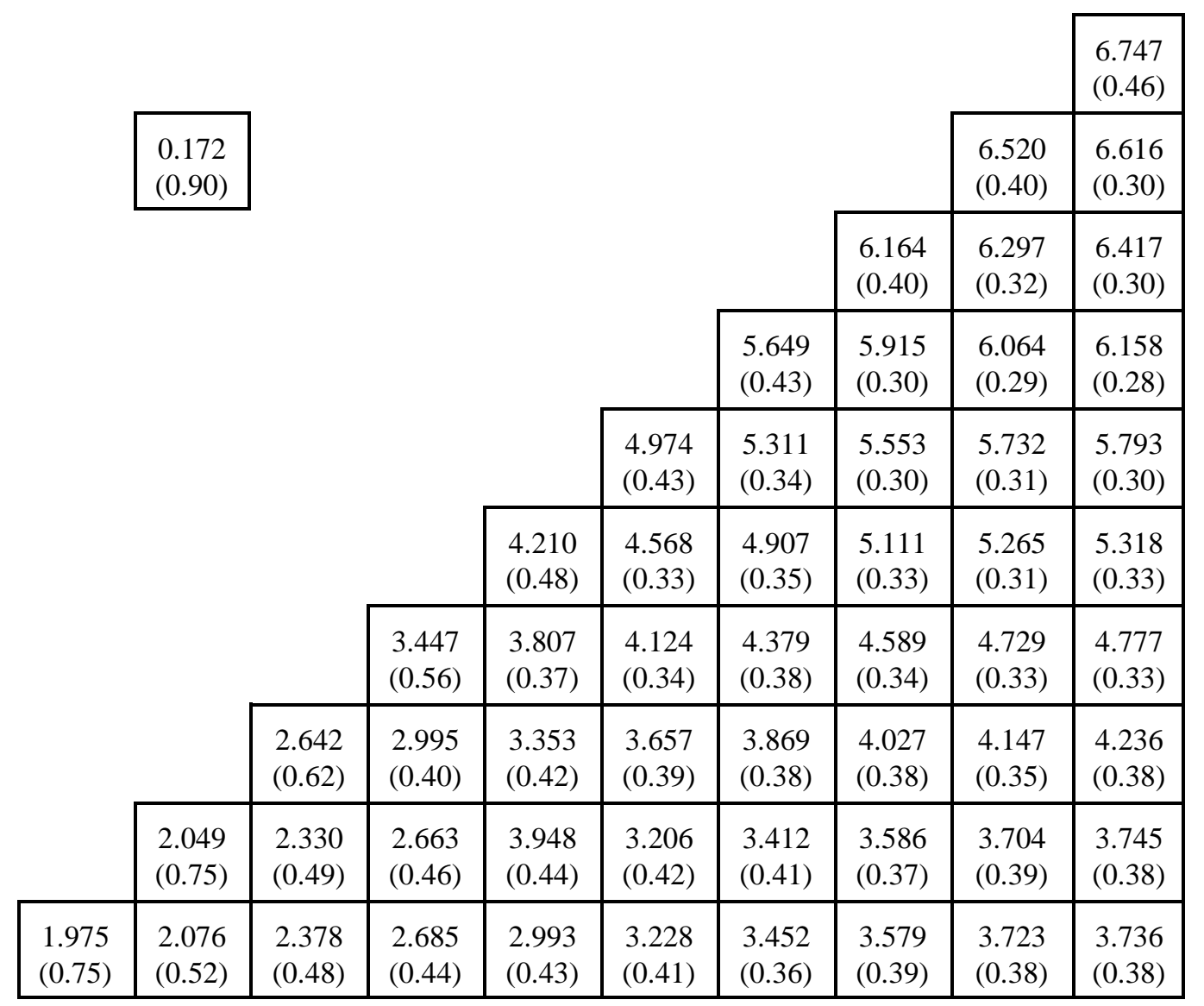

Fig. 4.13a. Pin-power distribution for CENTRM benchmark TCA-9. Value in parentheses is percent standard deviation. 


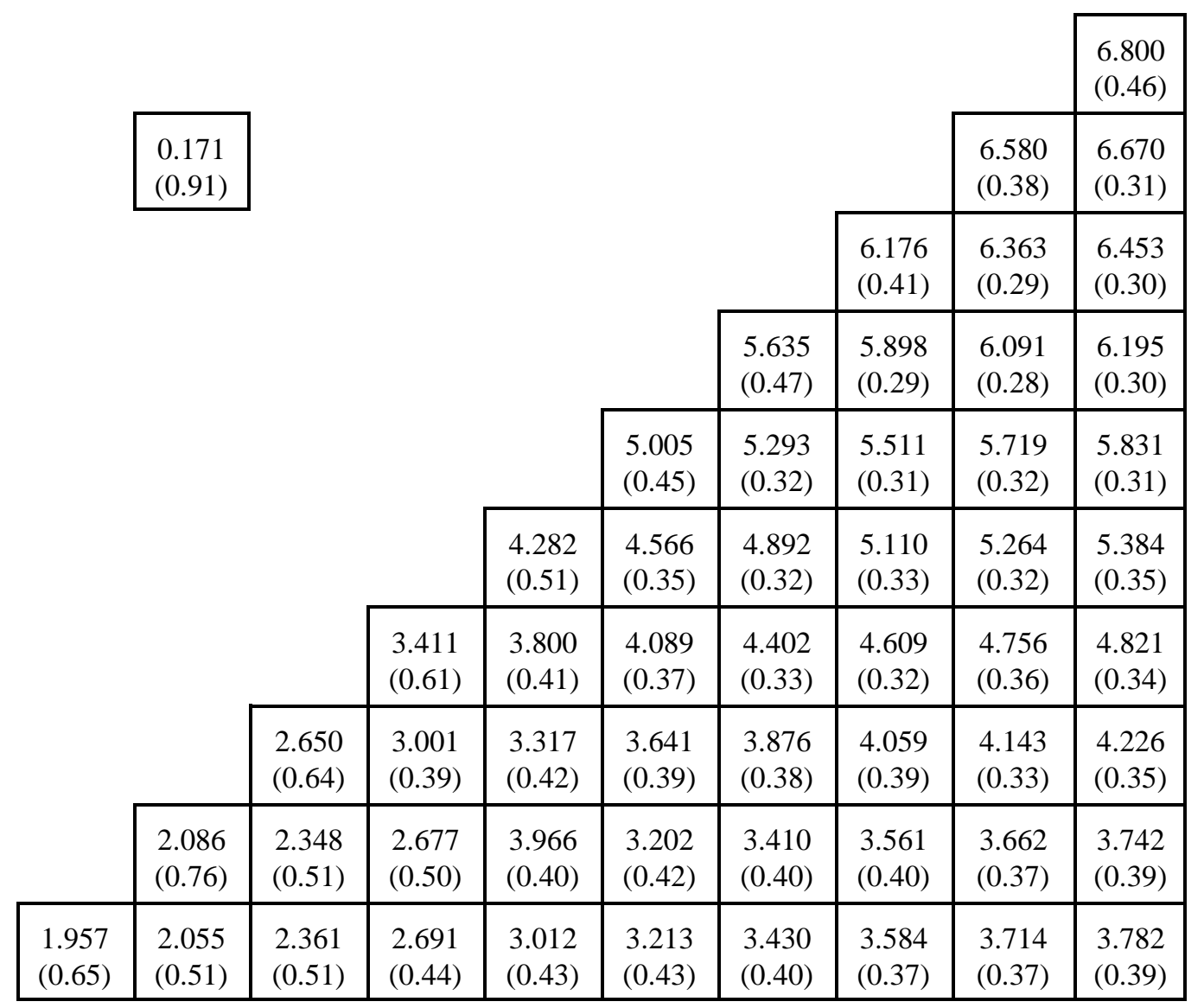

Fig. 4.13b. Pin-power distribution for NITAWL benchmark TCA-9. Value in parentheses is percent standard deviation. 
Table 4.85. Selected reaction rates for CENTRM case TCA-9

\begin{tabular}{|c|c|c|c|c|c|c|}
\hline Pin & Region & $\begin{array}{c}\mathrm{G}_{\mathrm{a}} \mathrm{M} \\
\left(\mathrm{cm}^{-3}-\mathrm{s}^{-1}\right)\end{array}$ & $\begin{array}{c}\mathcal{G}_{\mathrm{f}} \mathrm{M} \\
\left(\mathrm{cm}^{-3}-\mathrm{s}^{-1}\right)\end{array}$ & $\begin{array}{c}\mathrm{G}_{\mathrm{f}} \mathrm{M} \\
\left(\mathrm{cm}^{-3}-\mathrm{s}^{-1}\right)\end{array}$ & $\begin{array}{c}\mathrm{M} \\
\left(\mathrm{cm}-\mathrm{s}^{-1}\right)\end{array}$ & $\mathrm{M} / \mathrm{M}_{\mathrm{t}}$ \\
\hline \multirow{3}{*}{1} & Fuel & 1.197E-05 & $1.995 \mathrm{E}-05$ & 7.072E-06 & 4.613E-03 & 0.183 \\
\hline & Clad & $2.247 \mathrm{E}-07$ & 0.0 & 0.0 & $1.425 \mathrm{E}-03$ & 0.056 \\
\hline & Mod. & 6.729E-07 & 0.0 & 0.0 & 1.919E-02 & 0.761 \\
\hline \multirow{3}{*}{55} & Fuel & $4.255 \mathrm{E}-05$ & $6.730 \mathrm{E}-05$ & $2.383 \mathrm{E}-05$ & $2.168 \mathrm{E}-02$ & 0.181 \\
\hline & Clad & 8.192E-07 & 0.0 & 0.0 & $6.794 \mathrm{E}-03$ & 0.057 \\
\hline & Mod. & $2.155 \mathrm{E}-06$ & 0.0 & 0.0 & $9.111 \mathrm{E}-02$ & 0.762 \\
\hline
\end{tabular}

Table 4.86. Selected reaction rates for NITAWL case TCA-9

\begin{tabular}{ccccccc}
\hline Pin & Region & $\begin{array}{c}\mathrm{G}_{\mathrm{a}} \mathrm{M} \\
\left(\mathrm{cm}^{-3}-\mathrm{s}^{-1}\right)\end{array}$ & $\begin{array}{c}\mathrm{G}_{\mathrm{f}} \mathrm{M} \\
\left(\mathrm{cm}^{-3}-\mathrm{s}^{-1}\right)\end{array}$ & $\begin{array}{c}\mathrm{G}_{\mathrm{f}} \mathrm{M} \\
\left(\mathrm{cm}^{-3}-\mathrm{s}^{-1}\right)\end{array}$ & $\begin{array}{c}\mathrm{M} \\
\left(\mathrm{cm} \mathrm{-} \mathrm{s}^{-1}\right)\end{array}$ & $\mathrm{MM}_{\mathrm{t}}$ \\
\hline \multirow{2}{*}{1} & Fuel & $1.173 \mathrm{E}-05$ & $1.955 \mathrm{E}-05$ & $6.931 \mathrm{E}-06$ & $4.554 \mathrm{E}-03$ & 0.182 \\
& Clad & $2.206 \mathrm{E}-07$ & 0.0 & 0.0 & $1.420 \mathrm{E}-03$ & 0.056 \\
& Mod. & $6.680 \mathrm{E}-07$ & 0.0 & 0.0 & $1.910 \mathrm{E}-02$ & 0.762 \\
\hline \multirow{2}{*}{55} & Fuel & $4.279 \mathrm{E}-05$ & $6.771 \mathrm{E}-05$ & $2.398 \mathrm{E}-05$ & $2.188 \mathrm{E}-02$ & 0.182 \\
& Clad & $8.213 \mathrm{E}-07$ & 0.0 & 0.0 & $6.844 \mathrm{E}-03$ & 0.057 \\
& Mod. & $2.172 \mathrm{E}-06$ & 0.0 & 0.0 & $9.168 \mathrm{E}-02$ & 0.761 \\
\hline
\end{tabular}


Table 4.87. TCA-9, Pin-1 four-group fluxes

\begin{tabular}{ccc}
\hline Group & $\begin{array}{c}\text { CENTRM M } \\
\left(\mathrm{cm}^{-2}-\mathrm{s}^{-1}\right)\end{array}$ & $\begin{array}{c}\text { NITAWL M } \\
\left(\mathrm{cm}^{-2}-\mathrm{s}^{-1}\right)\end{array}$ \\
\hline 1 & $3.851 \mathrm{E}-05$ & $3.808 \mathrm{E}-05$ \\
2 & $1.088 \mathrm{E}-05$ & $1.068 \mathrm{E}-05$ \\
3 & $2.665 \mathrm{E}-06$ & $2.690 \mathrm{E}-06$ \\
4 & $2.684 \mathrm{E}-05$ & $2.644 \mathrm{E}-05$ \\
\hline
\end{tabular}

Table 4.88. TCA-9, Pin-1 four-group U-235 cross sections

\begin{tabular}{cccccccc}
\hline Group & \multicolumn{2}{c}{ CENTRM cross sections $\left(\mathrm{cm}^{-1}\right)$} & \multicolumn{3}{c}{ NITAWL cross sections $\left(\mathrm{cm}^{-1}\right)$} \\
\hline & $\frac{\mathrm{G}_{\mathrm{a}}}{1.42657 \mathrm{E}-04}$ & $\frac{\mathrm{G}_{\mathrm{f}}}{3.25414 \mathrm{E}-04}$ & $\frac{\mathrm{G}_{\mathrm{f}}}{1.25101 \mathrm{E}-04}$ & $\frac{\mathrm{G}_{\mathrm{a}}}{1.41875 \mathrm{E}-04}$ & $\frac{\mathrm{G}_{\mathrm{f}}}{3.24320 \mathrm{E}-04}$ & $\frac{\mathrm{G}_{\mathrm{f}}}{1.24670 \mathrm{E}-04}$ \\
1 & $3.42835 \mathrm{E}-03$ & $5.22619 \mathrm{E}-03$ & $2.14479 \mathrm{E}-03$ & $3.51588 \mathrm{E}-03$ & $5.33745 \mathrm{E}-03$ & $2.19045 \mathrm{E}-03$ \\
2 & $5.02206 \mathrm{E}-03$ & $1.01901 \mathrm{E}-02$ & $4.18195 \mathrm{E}-03$ & $5.22963 \mathrm{E}-03$ & $1.07319 \mathrm{E}-02$ & $4.40428 \mathrm{E}-03$ \\
4 & $4.91554 \mathrm{E}-02$ & $1.02344 \mathrm{E}-01$ & $4.20007 \mathrm{E}-02$ & $4.86914 \mathrm{E}-02$ & $1.01377 \mathrm{E}-01$ & $4.16040 \mathrm{E}-02$ \\
\hline
\end{tabular}

Table 4.89. TCA-9, Pin-1 four-group U-238 cross sections

\begin{tabular}{cccccccc}
\hline Group & \multicolumn{2}{c}{ CENTRM cross sections $\left(\mathrm{cm}^{-1}\right)$} & \multicolumn{3}{c}{ NITAWL cross sections $\left(\mathrm{cm}^{-1}\right)$} \\
\hline & $\frac{\mathrm{G}_{\mathrm{a}}}{4.50192 \mathrm{E}-03}$ & $\frac{\mathrm{G}_{\mathrm{f}}}{8.46604 \mathrm{E}-03}$ & $\frac{\mathrm{G}_{\mathrm{f}}}{2.99920 \mathrm{E}-03}$ & $\frac{\mathrm{G}_{\mathrm{a}}}{4.50218 \mathrm{E}-03}$ & $\frac{\mathrm{G}_{\mathrm{f}}}{8.53582 \mathrm{E}-03}$ & $\frac{\mathrm{G}_{\mathrm{f}}}{3.03046 \mathrm{E}-03}$ \\
1 & $4.14411 \mathrm{E}-02$ & $3.76574 \mathrm{E}-06$ & $1.56013 \mathrm{E}-06$ & $3.83075 \mathrm{E}-02$ & $3.58055 \mathrm{E}-06$ & $1.48341 \mathrm{E}-06$ \\
2 & $6.94867 \mathrm{E}-03$ & $2.40724 \mathrm{E}-08$ & $9.97362 \mathrm{E}-09$ & $7.02602 \mathrm{E}-03$ & $2.48603 \mathrm{E}-08$ & $1.03001 \mathrm{E}-08$ \\
3 & $2.78966 \mathrm{E}-02$ & $1.31143 \mathrm{E}-07$ & $5.43353 \mathrm{E}-08$ & $2.76690 \mathrm{E}-02$ & $1.30061 \mathrm{E}-07$ & $5.38869 \mathrm{E}-08$ \\
\hline
\end{tabular}

Table 4.90 TCA-9, Pin-1 four-group Pu-239 cross sections

\begin{tabular}{|c|c|c|c|c|c|c|}
\hline \multirow[t]{2}{*}{ Group } & \multicolumn{3}{|c|}{ CENTRM cross sections $\left(\mathrm{cm}^{-1}\right)$} & \multicolumn{3}{|c|}{ NITAWL cross sections $\left(\mathrm{cm}^{-1}\right)$} \\
\hline & $\mathrm{G}_{\mathrm{a}}$ & $<\mathrm{G}_{\mathrm{f}}$ & $\mathrm{G}_{\mathrm{f}}$ & $\mathrm{G}_{\mathrm{a}}$ & $<\mathrm{G}_{\mathrm{f}}$ & $\mathrm{G}_{\mathrm{f}}$ \\
\hline 1 & $5 . \overline{11763 \mathrm{E}-04}$ & $1.49708 \mathrm{E}-03$ & $4.81127 \mathrm{E}-04$ & $\overline{5.10995 \mathrm{E}-04}$ & $\overline{1.49750 \mathrm{E}-03}$ & $4.81258 \mathrm{E}-04$ \\
\hline 2 & 1.10924E-02 & $1.84326 \mathrm{E}-02$ & $6.40062 \mathrm{E}-03$ & 1.10329E-02 & 1.80993E-02 & $6.28488 \mathrm{E}-03$ \\
\hline 3 & $3.20870 \mathrm{E}-02$ & $6.32248 \mathrm{E}-02$ & $2.19544 \mathrm{E}-02$ & $3.47840 \mathrm{E}-02$ & $6.83283 \mathrm{E}-02$ & $2.37267 \mathrm{E}-02$ \\
\hline 4 & $2.66228 \mathrm{E}-01$ & $5.40183 \mathrm{E}-01$ & $1.86826 \mathrm{E}-01$ & $2.64971 E-01$ & 5.37118E-01 & $1.85773 \mathrm{E}-01$ \\
\hline
\end{tabular}


Table 4.91. TCA-9, Pin-55 four-group fluxes

\begin{tabular}{ccc}
\hline Group & $\begin{array}{c}\text { CENTRM M } \\
\left(\mathrm{cm}^{-2}-\mathrm{s}^{-1}\right)\end{array}$ & $\begin{array}{c}\text { NITAWL M } \\
\left(\mathrm{cm}^{-2}-\mathrm{s}^{-1}\right)\end{array}$ \\
\hline 1 & $2.010 \mathrm{E}-04$ & $2.034 \mathrm{E}-04$ \\
2 & $6.739 \mathrm{E}-05$ & $6.769 \mathrm{E}-05$ \\
3 & $1.576 \mathrm{E}-05$ & $1.591 \mathrm{E}-05$ \\
4 & $8.667 \mathrm{E}-05$ & $8.728 \mathrm{E}-05$ \\
\hline
\end{tabular}

Table 4.92. TCA-9, Pin-55 four-group U-235 cross sections

\begin{tabular}{cccccccc}
\hline Group & \multicolumn{3}{c}{ CENTRM cross sections $\left(\mathrm{cm}^{-1}\right)$} & \multicolumn{3}{c}{ NITAWL cross sections $\left(\mathrm{cm}^{-1}\right)$} \\
\hline & $\frac{\mathrm{G}_{\mathrm{a}}}{1.45454 \mathrm{E}-04}$ & $\frac{\mathrm{G}_{\mathrm{f}}}{3.27423 \mathrm{E}-04}$ & $\frac{\mathrm{G}_{\mathrm{f}}}{1.26486 \mathrm{E}-04}$ & $\frac{\mathrm{G}_{\mathrm{a}}}{1.45607 \mathrm{E}-04}$ & $\frac{\mathrm{G}_{\mathrm{f}}}{3.27771 \mathrm{E}-04}$ & $\frac{\mathrm{G}_{\mathrm{f}}}{1.26597 \mathrm{E}-04}$ \\
1 & $3.41193 \mathrm{E}-03$ & $5.23067 \mathrm{E}-03$ & $2.14662 \mathrm{E}-03$ & $3.48574 \mathrm{E}-03$ & $5.30232 \mathrm{E}-03$ & $2.17602 \mathrm{E}-03$ \\
2 & $5.17154 \mathrm{E}-03$ & $1.05678 \mathrm{E}-02$ & $4.33692 \mathrm{E}-03$ & $5.13163 \mathrm{E}-03$ & $1.04658 \mathrm{E}-02$ & $4.29508 \mathrm{E}-03$ \\
3 & $4.74074 \mathrm{E}-02$ & $9.86494 \mathrm{E}-02$ & $4.04848 \mathrm{E}-02$ & $4.74735 \mathrm{E}-02$ & $9.87892 \mathrm{E}-02$ & $4.05423 \mathrm{E}-02$ \\
\hline
\end{tabular}

Table 4.93. TCA-9, Pin-55 four-group U-238 cross sections

\begin{tabular}{|c|c|c|c|c|c|c|}
\hline \multirow[t]{2}{*}{ Group } & \multicolumn{3}{|c|}{ CENTRM cross sections $\left(\mathrm{cm}^{-1}\right)$} & \multicolumn{3}{|c|}{ NITAWL cross sections $\left(\mathrm{cm}^{-1}\right)$} \\
\hline & $\mathrm{G}_{\mathrm{a}}$ & $\mathrm{G}_{\mathrm{f}}$ & $\mathrm{G}_{\mathrm{f}}$ & $\mathrm{G}_{\mathrm{a}}$ & $<\mathrm{G}_{\mathrm{f}}$ & $\mathrm{G}_{\mathrm{f}}$ \\
\hline 1 & $4.40327 \mathrm{E}-03$ & $7.89249 \mathrm{E}-03$ & $2.80210 \mathrm{E}-03$ & $4.40840 \mathrm{E}-03$ & $7.90103 \mathrm{E}-03$ & $2.80352 \mathrm{E}-03$ \\
\hline 2 & 3.82984E-02 & $3.87376 \mathrm{E}-06$ & $1.60488 \mathrm{E}-06$ & 3.80297E-02 & 3.68119E-06 & $1.52510 \mathrm{E}-06$ \\
\hline 3 & 6.99689E-03 & $2.45553 \mathrm{E}-08$ & $1.01737 \mathrm{E}-08$ & $6.98259 \mathrm{E}-03$ & $2.44124 \mathrm{E}-08$ & $1.01145 \mathrm{E}-08$ \\
\hline 4 & 2.69919E-02 & $1.26807 \mathrm{E}-07$ & $5.25386 \mathrm{E}-08$ & 2.70262E-02 & $1.26972 \mathrm{E}-07$ & 5.26067E-08 \\
\hline
\end{tabular}

Table 4.94. TCA-9, Pin-55 four-group Pu-239 cross sections

\begin{tabular}{cccccccc}
\hline Group & \multicolumn{2}{c}{ CENTRM cross sections $\left(\mathrm{cm}^{-1}\right)$} & \multicolumn{3}{c}{ NITAWL cross sections $\left(\mathrm{cm}^{-1}\right)$} \\
\hline & $\frac{\mathrm{G}_{\mathrm{a}}}{5.12642 \mathrm{E}-04}$ & $\frac{\mathrm{G}_{\mathrm{f}}}{1.48335 \mathrm{E}-03}$ & $\frac{\mathrm{G}_{\mathrm{f}}}{4.78804 \mathrm{E}-04}$ & $\frac{\mathrm{G}_{\mathrm{a}}}{5.12945 \mathrm{E}-04}$ & $\frac{\mathrm{G}_{\mathrm{f}}}{1.48419 \mathrm{E}-03}$ & $\frac{\mathrm{G}_{\mathrm{f}}}{4.78995 \mathrm{E}-04}$ \\
1 & $1.10458 \mathrm{E}-02$ & $1.82405 \mathrm{E}-02$ & $6.33394 \mathrm{E}-03$ & $1.08837 \mathrm{E}-02$ & $1.78922 \mathrm{E}-02$ & $6.21299 \mathrm{E}-03$ \\
3 & $3.38276 \mathrm{E}-02$ & $6.65209 \mathrm{E}-02$ & $2.30991 \mathrm{E}-02$ & $3.35685 \mathrm{E}-02$ & $6.60145 \mathrm{E}-02$ & $2.29233 \mathrm{E}-02$ \\
4 & $2.75620 \mathrm{E}-01$ & $5.52218 \mathrm{E}-01$ & $1.91037 \mathrm{E}-01$ & $2.75080 \mathrm{E}-01$ & $5.51449 \mathrm{E}-01$ & $1.90770 \mathrm{E}-01$ \\
\hline
\end{tabular}




\begin{tabular}{|c|c|c|c|c|c|c|c|c|c|c|}
\hline & & & & & & & & & & $\begin{array}{l}6.856 \\
(0.85)\end{array}$ \\
\hline & \multirow[t]{8}{*}{$\begin{array}{l}0.141 \\
(0.76)\end{array}$} & & & & & & & & $\begin{array}{l}6.768 \\
(0.41)\end{array}$ & $\begin{array}{l}6.823 \\
(0.42)\end{array}$ \\
\hline & & & & & & & & $\begin{array}{l}6.535 \\
(0.43)\end{array}$ & $\begin{array}{l}6.644 \\
(0.30)\end{array}$ & $\begin{array}{l}6.692 \\
(0.44)\end{array}$ \\
\hline & & & & & & & $\begin{array}{l}6.073 \\
(0.44)\end{array}$ & $\begin{array}{l}6.278 \\
(0.31)\end{array}$ & $\begin{array}{l}6.452 \\
(0.32)\end{array}$ & $\begin{array}{l}6.462 \\
(0.48)\end{array}$ \\
\hline & & & & & & $\begin{array}{l}5.443 \\
(0.46) \\
\end{array}$ & $\begin{array}{l}5.760 \\
(0.35) \\
\end{array}$ & $\begin{array}{l}5.984 \\
(0.30) \\
\end{array}$ & $\begin{array}{l}6.074 \\
(0.35) \\
\end{array}$ & $\begin{array}{l}6.161 \\
(0.44) \\
\end{array}$ \\
\hline & & & & & $\begin{array}{l}4.729 \\
(0.48)\end{array}$ & $\begin{array}{l}5.080 \\
(0.34)\end{array}$ & $\begin{array}{l}5.339 \\
(0.33)\end{array}$ & $\begin{array}{l}5.561 \\
(0.32)\end{array}$ & $\begin{array}{l}5.648 \\
(0.33)\end{array}$ & $\begin{array}{l}5.659 \\
(0.48)\end{array}$ \\
\hline & & & & $\begin{array}{c}3.888 \\
(0.57)\end{array}$ & $\begin{array}{l}4.322 \\
(0.37)\end{array}$ & $\begin{array}{l}4.641 \\
(0.36)\end{array}$ & $\begin{array}{l}4.858 \\
(0.32)\end{array}$ & $\begin{array}{l}5.062 \\
(0.33)\end{array}$ & $\begin{array}{l}5.192 \\
(0.34)\end{array}$ & $\begin{array}{l}5.200 \\
(0.49)\end{array}$ \\
\hline & & & $\begin{array}{c}3.115 \\
(0.64)\end{array}$ & $\begin{array}{l}3.500 \\
(0.43)\end{array}$ & $\begin{array}{l}3.840 \\
(0.39)\end{array}$ & $\begin{array}{l}4.093 \\
(0.37)\end{array}$ & $\begin{array}{l}4.339 \\
(0.38)\end{array}$ & $\begin{array}{l}4.483 \\
(0.39)\end{array}$ & $\begin{array}{l}4.605 \\
(0.39)\end{array}$ & $\begin{array}{l}4.604 \\
(0.53)\end{array}$ \\
\hline & & $\begin{array}{l}2.358 \\
(0.72)\end{array}$ & $\begin{array}{l}2.681 \\
(0.47)\end{array}$ & $\begin{array}{l}3.033 \\
(0.43)\end{array}$ & $\begin{array}{l}3.323 \\
(0.38)\end{array}$ & $\begin{array}{l}3.532 \\
(0.43)\end{array}$ & $\begin{array}{l}3.760 \\
(0.40)\end{array}$ & $\begin{array}{l}3.875 \\
(0.42)\end{array}$ & $\begin{array}{l}3.955 \\
(0.37)\end{array}$ & $\begin{array}{l}3.936 \\
(0.55)\end{array}$ \\
\hline & $\begin{array}{l}1.684 \\
(0.85)\end{array}$ & $\begin{array}{l}1.983 \\
(0.56)\end{array}$ & $\begin{array}{l}2.291 \\
(0.51)\end{array}$ & $\begin{array}{l}2.553 \\
(0.46)\end{array}$ & $\begin{array}{l}2.823 \\
(0.46)\end{array}$ & $\begin{array}{l}3.029 \\
(0.41)\end{array}$ & $\begin{array}{l}3.208 \\
(0.45)\end{array}$ & $\begin{array}{c}3.286 \\
(0.41)\end{array}$ & $\begin{array}{l}3.384 \\
(0.39)\end{array}$ & $\begin{array}{l}3.418 \\
(0.59)\end{array}$ \\
\hline $\begin{array}{l}1.428 \\
(0.77)\end{array}$ & $\begin{array}{c}1.569 \\
(0.61)\end{array}$ & $\begin{array}{r}1.859 \\
(0.57)\end{array}$ & $\begin{array}{l}2.158 \\
(0.49)\end{array}$ & $\begin{array}{c}2.398 \\
(0.51)\end{array}$ & $\begin{array}{l}2.643 \\
(0.49)\end{array}$ & $\begin{array}{l}2.831 \\
(0.45)\end{array}$ & $\begin{array}{l}2.995 \\
(0.47)\end{array}$ & $\begin{array}{l}3.099 \\
(0.42)\end{array}$ & $\begin{array}{l}3.141 \\
(0.43)\end{array}$ & $\begin{array}{l}3.185 \\
(0.58)\end{array}$ \\
\hline
\end{tabular}

Fig. 4.14a. Pin-power distribution for CENTRM benchmark TCA-10. Value in parentheses is percent standard deviation. 


\begin{tabular}{|c|c|c|c|c|c|c|c|c|c|c|}
\hline & & & & & & & & & & $\begin{array}{l}6.908 \\
(0.85)\end{array}$ \\
\hline & \multirow[t]{8}{*}{$\begin{array}{l}0.144 \\
(0.76)\end{array}$} & & & & & & & & $\begin{array}{l}6.810 \\
(0.41)\end{array}$ & $\begin{array}{c}6.858 \\
(0.43)\end{array}$ \\
\hline & & & & & & & & $\begin{array}{l}6.535 \\
(0.42)\end{array}$ & $\begin{array}{l}6.640 \\
(0.31)\end{array}$ & $\begin{array}{l}6.685 \\
(0.43)\end{array}$ \\
\hline & & & & & & & $\begin{array}{l}6.057 \\
(0.47)\end{array}$ & $\begin{array}{l}6.279 \\
(0.33)\end{array}$ & $\begin{array}{c}6.387 \\
(0.31)\end{array}$ & $\begin{array}{r}6.470 \\
(0.43)\end{array}$ \\
\hline & & & & & & $\begin{array}{l}5.486 \\
(0.45)\end{array}$ & $\begin{array}{l}5.776 \\
(0.31)\end{array}$ & $\begin{array}{c}6.008 \\
(0.31)\end{array}$ & $\begin{array}{c}6.085 \\
(0.33)\end{array}$ & $\begin{array}{l}6.152 \\
(0.45)\end{array}$ \\
\hline & & & & & $\begin{array}{l}4.750 \\
(0.51)\end{array}$ & $\begin{array}{l}5.094 \\
(0.33)\end{array}$ & $\begin{array}{l}5.371 \\
(0.31)\end{array}$ & $\begin{array}{l}5.545 \\
(0.30)\end{array}$ & $\begin{array}{l}5.669 \\
(0.34)\end{array}$ & $\begin{array}{l}5.702 \\
(0.44)\end{array}$ \\
\hline & & & & $\begin{array}{l}3.961 \\
(0.55)\end{array}$ & $\begin{array}{l}4.306 \\
(0.33)\end{array}$ & $\begin{array}{l}4.671 \\
(0.38)\end{array}$ & $\begin{array}{l}4.906 \\
(0.32)\end{array}$ & $\begin{array}{l}5.079 \\
(0.34)\end{array}$ & $\begin{array}{l}5.159 \\
(0.36)\end{array}$ & $\begin{array}{l}5.240 \\
(0.48)\end{array}$ \\
\hline & & & $\begin{array}{c}3.100 \\
(0.59)\end{array}$ & $\begin{array}{l}3.517 \\
(0.38)\end{array}$ & $\begin{array}{l}3.850 \\
(0.40)\end{array}$ & $\begin{array}{l}4.088 \\
(0.40)\end{array}$ & $\begin{array}{l}4.342 \\
(0.36)\end{array}$ & $\begin{array}{l}4.512 \\
(0.35)\end{array}$ & $\begin{array}{l}4.599 \\
(0.34)\end{array}$ & $\begin{array}{l}4.621 \\
(0.50)\end{array}$ \\
\hline & & $\begin{array}{l}2.323 \\
(0.70)\end{array}$ & $\begin{array}{l}2.712 \\
(0.44)\end{array}$ & $\begin{array}{l}3.010 \\
(0.46)\end{array}$ & $\begin{array}{l}3.318 \\
(0.45)\end{array}$ & $\begin{array}{l}3.576 \\
(0.42)\end{array}$ & $\begin{array}{l}3.745 \\
(0.40)\end{array}$ & $\begin{array}{l}3.898 \\
(0.41)\end{array}$ & $\begin{array}{l}3.962 \\
(0.39)\end{array}$ & $\begin{array}{l}4.025 \\
(0.59)\end{array}$ \\
\hline & $\begin{array}{c}1.667 \\
(0.84)\end{array}$ & $\begin{array}{l}2.010 \\
(0.56)\end{array}$ & $\begin{array}{l}2.295 \\
(0.51)\end{array}$ & $\begin{array}{l}2.584 \\
(0.45)\end{array}$ & $\begin{array}{l}2.808 \\
(0.48)\end{array}$ & $\begin{array}{l}3.022 \\
(0.46)\end{array}$ & $\begin{array}{c}3.184 \\
(0.45)\end{array}$ & $\begin{array}{c}3.312 \\
(0.43)\end{array}$ & $\begin{array}{c}3.374 \\
(0.41)\end{array}$ & $\begin{array}{c}3.412 \\
(0.57)\end{array}$ \\
\hline $\begin{array}{l}1.422 \\
(0.85)\end{array}$ & $\begin{array}{c}1.576 \\
(0.65)\end{array}$ & $\begin{array}{c}1.865 \\
(0.58)\end{array}$ & $\begin{array}{c}2.122 \\
(0.51)\end{array}$ & $\begin{array}{l}2.401 \\
(0.50)\end{array}$ & $\begin{array}{l}2.644 \\
(0.46)\end{array}$ & $\begin{array}{l}2.824 \\
(0.47)\end{array}$ & $\begin{array}{c}2.996 \\
(0.45)\end{array}$ & $\begin{array}{c}3.102 \\
(0.39)\end{array}$ & $\begin{array}{c}3.155 \\
(0.44)\end{array}$ & $\begin{array}{l}3.217 \\
(0.56)\end{array}$ \\
\hline
\end{tabular}

Fig. 4.14b. Pin-power distribution for NITAWL benchmark TCA-10. Value in parentheses is percent standard deviation. 
Table 4.95. Selected reaction rates for CENTRM case TCA-10

\begin{tabular}{|c|c|c|c|c|c|c|}
\hline Pin & Region & $\begin{array}{c}\mathrm{G}_{\mathrm{a}} \mathrm{M} \\
\left(\mathrm{cm}^{-3}-\mathrm{s}^{-1}\right)\end{array}$ & $\begin{array}{c}\mathcal{G}_{\mathrm{f}} \mathrm{M} \\
\left(\mathrm{cm}^{-3}-\mathrm{s}^{-1}\right)\end{array}$ & $\begin{array}{c}\mathrm{G}_{\mathrm{f}} \mathrm{M} \\
\left(\mathrm{cm}^{-3}-\mathrm{s}^{-1}\right)\end{array}$ & $\begin{array}{c}\mathrm{M} \\
\left(\mathrm{cm}-\mathrm{s}^{-1}\right)\end{array}$ & $\mathrm{MM}_{\mathrm{t}}$ \\
\hline \multirow{3}{*}{1} & Fuel & $8.487 \mathrm{E}-06$ & $1.430 \mathrm{E}-05$ & $5.068 \mathrm{E}-06$ & $2.952 \mathrm{E}-03$ & 0.146 \\
\hline & Clad & $1.587 \mathrm{E}-07$ & 0.0 & 0.0 & $9.168 \mathrm{E}-04$ & 0.046 \\
\hline & Mod. & 4.949E-07 & 0.0 & 0.0 & $1.630 \mathrm{E}-02$ & 0.808 \\
\hline \multirow{3}{*}{66} & Fuel & $4.238 \mathrm{E}-05$ & $6.861 \mathrm{E}-05$ & $2.429 \mathrm{E}-05$ & $1.833 \mathrm{E}-02$ & 0.147 \\
\hline & Clad & $8.072 \mathrm{E}-07$ & 0.0 & 0.0 & $5.748 \mathrm{E}-03$ & 0.046 \\
\hline & Mod. & $2.263 \mathrm{E}-06$ & 0.0 & 0.0 & $1.007 \mathrm{E}-01$ & 0.807 \\
\hline
\end{tabular}

Table 4.96. Selected reaction rates for NITAWL case TCA-10

\begin{tabular}{ccccccc}
\hline Pin & Region & $\begin{array}{c}\mathrm{G}_{\mathrm{a}} \mathrm{M} \\
\left(\mathrm{cm}^{-3}-\mathrm{s}^{-1}\right)\end{array}$ & $\begin{array}{c}\mathrm{G}_{\mathrm{f}} \mathrm{M} \\
\left(\mathrm{cm}^{-3}-\mathrm{s}^{-1}\right)\end{array}$ & $\begin{array}{c}\mathrm{G}_{\mathrm{f}} \mathrm{M} \\
\left(\mathrm{cm}^{-3}-\mathrm{s}^{-1}\right)\end{array}$ & $\begin{array}{c}\mathrm{M} \\
\left(\mathrm{cm} \mathrm{-} \mathrm{s}^{-1}\right)\end{array}$ & $\mathrm{MM}_{\mathrm{t}}$ \\
\hline \multirow{2}{*}{1} & Fuel & $8.415 \mathrm{E}-06$ & $1.413 \mathrm{E}-05$ & $5.006 \mathrm{E}-06$ & $2.931 \mathrm{E}-03$ & 0.146 \\
& Clad & $1.562 \mathrm{E}-07$ & 0.0 & 0.0 & $9.141 \mathrm{E}-04$ & 0.046 \\
& Mod. & $4.895 \mathrm{E}-07$ & 0.0 & 0.0 & $1.618 \mathrm{E}-02$ & 0.808 \\
\hline \multirow{2}{*}{66} & Fuel & $4.266 \mathrm{E}-05$ & $6.930 \mathrm{E}-05$ & $2.454 \mathrm{E}-05$ & $1.832 \mathrm{E}-02$ & 0.147 \\
& Clad & $8.014 \mathrm{E}-07$ & 0.0 & 0.0 & $5.729 \mathrm{E}-03$ & 0.045 \\
& Mod. & $2.297 \mathrm{E}-06$ & 0.0 & 0.0 & $1.009 \mathrm{E}-01$ & 0.808 \\
\hline
\end{tabular}


Table 4.97. TCA-10, Pin-1 four-group fluxes

\begin{tabular}{ccc}
\hline Group & $\begin{array}{c}\text { CENTRM M } \\
\left(\mathrm{cm}^{-2}-\mathrm{s}^{-1}\right)\end{array}$ & $\begin{array}{c}\text { NITAWL M } \\
\left(\mathrm{cm}^{-2}-\mathrm{s}^{-1}\right)\end{array}$ \\
\hline 1 & $2.547 \mathrm{E}-05$ & $2.512 \mathrm{E}-05$ \\
2 & $7.003 \mathrm{E}-06$ & $7.231 \mathrm{E}-06$ \\
3 & $1.709 \mathrm{E}-06$ & $1.737 \mathrm{E}-06$ \\
4 & $1.922 \mathrm{E}-05$ & $1.894 \mathrm{E}-05$ \\
\hline
\end{tabular}

Table 4.98. TCA-10, Pin-1 four-group U-235 cross sections

\begin{tabular}{cccccccc}
\hline Group & \multicolumn{2}{c}{ CENTRM cross sections $\left(\mathrm{cm}^{-1}\right)$} & \multicolumn{3}{c}{ NITAWL cross sections $\left(\mathrm{cm}^{-1}\right)$} \\
\hline & $\frac{\mathrm{G}_{\mathrm{a}}}{1.40788 \mathrm{E}-04}$ & $\frac{\mathrm{G}_{\mathrm{f}}}{3.23363 \mathrm{E}-04}$ & $\frac{\mathrm{G}_{\mathrm{f}}}{1.24110 \mathrm{E}-04}$ & $\frac{\mathrm{G}_{\mathrm{a}}}{1.41106 \mathrm{E}-04}$ & $\frac{4 \mathrm{G}_{\mathrm{f}}}{3.24338 \mathrm{E}-04}$ & $\frac{\mathrm{G}_{\mathrm{f}}}{1.24311 \mathrm{E}-04}$ \\
1 & $3.64170 \mathrm{E}-03$ & $5.55391 \mathrm{E}-03$ & $2.27928 \mathrm{E}-03$ & $3.51527 \mathrm{E}-03$ & $5.32391 \mathrm{E}-03$ & $2.18488 \mathrm{E}-03$ \\
2 & $5.30350 \mathrm{E}-03$ & $1.08803 \mathrm{E}-02$ & $4.46517 \mathrm{E}-03$ & $5.18458 \mathrm{E}-03$ & $1.06225 \mathrm{E}-02$ & $4.35935 \mathrm{E}-03$ \\
4 & $4.91298 \mathrm{E}-02$ & $1.02298 \mathrm{E}-01$ & $4.19822 \mathrm{E}-02$ & $4.92076 \mathrm{E}-02$ & $1.02458 \mathrm{E}-01$ & $4.20476 \mathrm{E}-02$ \\
\hline
\end{tabular}

Table 4.99. TCA-10, Pin-1 four-group U-238 cross sections

\begin{tabular}{cccccccc}
\hline Group & \multicolumn{3}{c}{ CENTRM cross sections $\left(\mathrm{cm}^{-1}\right)$} & \multicolumn{3}{c}{ NITAWL cross sections $\left(\mathrm{cm}^{-1}\right)$} \\
\hline & $\frac{\mathrm{G}_{\mathrm{a}}}{4.53168 \mathrm{E}-03}$ & $\frac{\mathrm{G}_{\mathrm{f}}}{8.72356 \mathrm{E}-03}$ & $\frac{\mathrm{G}_{\mathrm{f}}}{3.09660 \mathrm{E}-03}$ & $\frac{\mathrm{G}_{\mathrm{a}}}{4.53467 \mathrm{E}-03}$ & $\frac{\mathrm{G}_{\mathrm{f}}}{8.75482 \mathrm{E}-03}$ & $\frac{\mathrm{G}_{\mathrm{f}}}{3.09219 \mathrm{E}-03}$ \\
1 & $4.29217 \mathrm{E}-02$ & $3.81724 \mathrm{E}-06$ & $1.58147 \mathrm{E}-06$ & $4.01630 \mathrm{E}-02$ & $3.94182 \mathrm{E}-06$ & $1.63309 \mathrm{E}-06$ \\
3 & $7.03335 \mathrm{E}-03$ & $2.49018 \mathrm{E}-08$ & $1.03173 \mathrm{E}-08$ & $7.00443 \mathrm{E}-03$ & $2.45869 \mathrm{E}-08$ & $1.01868 \mathrm{E}-08$ \\
4 & $2.78924 \mathrm{E}-02$ & $1.31130 \mathrm{E}-07$ & $5.43293 \mathrm{E}-08$ & $2.79251 \mathrm{E}-02$ & $1.31284 \mathrm{E}-07$ & $5.43933 \mathrm{E}-08$ \\
\hline
\end{tabular}

Table 4.100. TCA-10, Pin-1 four-group Pu-239 cross sections

\begin{tabular}{|c|c|c|c|c|c|c|}
\hline \multirow[t]{2}{*}{ Group } & \multicolumn{3}{|c|}{ CENTRM cross sections $\left(\mathrm{cm}^{-1}\right)$} & \multicolumn{3}{|c|}{ NITAWL cross sections $\left(\mathrm{cm}^{-1}\right)$} \\
\hline & $\mathrm{G}_{\mathrm{a}}$ & $<\mathrm{G}_{\mathrm{f}}$ & $\mathrm{G}_{\mathrm{f}}$ & $\mathrm{G}_{\mathrm{a}}$ & $\mathcal{G}_{\mathrm{f}}$ & $\mathrm{G}_{\mathrm{f}}$ \\
\hline 1 & $5 . \overline{10742 \mathrm{E}-04}$ & $\overline{1.50232 \mathrm{E}-03}$ & $\overline{4.82148 \mathrm{E}-04}$ & $\overline{5.10768 \mathrm{E}-04}$ & $\overline{1.50322 \mathrm{E}-03}$ & $\overline{4.81907 \mathrm{E}-04}$ \\
\hline 2 & $1.13157 \mathrm{E}-02$ & $1.86831 \mathrm{E}-02$ & $6.48763 \mathrm{E}-03$ & $1.14534 \mathrm{E}-02$ & 1.87359E-02 & $6.50593 \mathrm{E}-03$ \\
\hline 3 & $3.56099 \mathrm{E}-02$ & $6.98250 \mathrm{E}-02$ & $2.42465 \mathrm{E}-02$ & 3.42803E-02 & $6.73431 \mathrm{E}-02$ & 2.33847E-02 \\
\hline 4 & $2.63607 \mathrm{E}-01$ & 5.35739E-01 & $1.85285 \mathrm{E}-01$ & $2.64216 \mathrm{E}-01$ & $5.36885 \mathrm{E}-01$ & 1.85682E-01 \\
\hline
\end{tabular}


Table 4.101. TCA-10, Pin-66 four-group fluxes

\begin{tabular}{ccc}
\hline Group & $\begin{array}{c}\text { CENTRM M } \\
\left(\mathrm{cm}^{-2}-\mathrm{s}^{-1}\right)\end{array}$ & $\begin{array}{c}\text { NITAWL M } \\
\left(\mathrm{cm}^{-2}-\mathrm{s}^{-1}\right)\end{array}$ \\
\hline 1 & $1.721 \mathrm{E}-04$ & $1.716 \mathrm{E}-04$ \\
2 & $5.659 \mathrm{E}-05$ & $5.611 \mathrm{E}-05$ \\
3 & $1.360 \mathrm{E}-05$ & $1.336 \mathrm{E}-05$ \\
4 & $8.928 \mathrm{E}-05$ & $9.041 \mathrm{E}-05$ \\
\hline
\end{tabular}

Table 4.102. TCA-10, Pin-66 four-group U-235 cross sections

\begin{tabular}{cccccccc}
\hline Group & \multicolumn{2}{c}{ CENTRM cross sections $\left(\mathrm{cm}^{-1}\right)$} & \multicolumn{3}{c}{ NITAWL cross sections $\left(\mathrm{cm}^{-1}\right)$} \\
\hline & $\frac{\mathrm{G}_{\mathrm{a}}}{1.44765 \mathrm{E}-04}$ & $\frac{\mathrm{G}_{\mathrm{f}}}{3.27146 \mathrm{E}-04}$ & $\frac{\mathrm{G}_{\mathrm{f}}}{1.26179 \mathrm{E}-04}$ & $\frac{\mathrm{G}_{\mathrm{a}}}{1.44207 \mathrm{E}-04}$ & $\frac{\mathrm{G}_{\mathrm{f}}}{3.26568 \mathrm{E}-04}$ & $\frac{\mathrm{G}_{\mathrm{f}}}{1.25881 \mathrm{E}-04}$ \\
1 & $3.42458 \mathrm{E}-03$ & $5.21627 \mathrm{E}-03$ & $2.14071 \mathrm{E}-03$ & $3.45678 \mathrm{E}-03$ & $5.26794 \mathrm{E}-03$ & $2.16191 \mathrm{E}-03$ \\
2 & $5.10952 \mathrm{E}-03$ & $1.04097 \mathrm{E}-02$ & $4.27204 \mathrm{E}-03$ & $5.28578 \mathrm{E}-03$ & $1.08415 \mathrm{E}-02$ & $4.44925 \mathrm{E}-03$ \\
3 & $4.80156 \mathrm{E}-02$ & $9.99418 \mathrm{E}-02$ & $4.10152 \mathrm{E}-02$ & $4.77411 \mathrm{E}-02$ & $9.93647 \mathrm{E}-02$ & $4.07783 \mathrm{E}-02$ \\
\hline
\end{tabular}

Table 4.103. TCA-10, Pin-66 four-group U-238 cross sections

\begin{tabular}{cccccccc}
\hline Group & \multicolumn{2}{c}{ CENTRM cross sections $\left(\mathrm{cm}^{-1}\right)$} & \multicolumn{3}{c}{ NITAWL cross sections $\left(\mathrm{cm}^{-1}\right)$} \\
\hline & $\frac{\mathrm{G}_{\mathrm{a}}}{4.43964 \mathrm{E}-03}$ & $\frac{\mathrm{G}_{\mathrm{f}}}{8.07600 \mathrm{E}-03}$ & $\frac{\mathrm{G}_{\mathrm{f}}}{2.86439 \mathrm{E}-03}$ & $\frac{\mathrm{G}_{\mathrm{a}}}{4.43787 \mathrm{E}-03}$ & $\frac{\mathrm{G}_{\mathrm{f}}}{8.12821 \mathrm{E}-03}$ & $\frac{\mathrm{G}_{\mathrm{f}}}{2.88138 \mathrm{E}-03}$ \\
1 & $3.89892 \mathrm{E}-02$ & $3.80924 \mathrm{E}-06$ & $1.57815 \mathrm{E}-06$ & $3.85192 \mathrm{E}-02$ & $3.58239 \mathrm{E}-06$ & $1.48417 \mathrm{E}-06$ \\
2 & $6.97667 \mathrm{E}-03$ & $2.43379 \mathrm{E}-08$ & $1.00837 \mathrm{E}-08$ & $7.03071 \mathrm{E}-03$ & $2.48865 \mathrm{E}-08$ & $1.03109 \mathrm{E}-08$ \\
3 & $2.73091 \mathrm{E}-02$ & $1.28331 \mathrm{E}-07$ & $5.31703 \mathrm{E}-08$ & $2.71792 \mathrm{E}-02$ & $1.27709 \mathrm{E}-07$ & $5.29124 \mathrm{E}-08$ \\
\hline
\end{tabular}

Table 4.104. TCA-10, Pin-66 four-group Pu-239 cross sections

\begin{tabular}{|c|c|c|c|c|c|c|}
\hline \multirow[t]{2}{*}{ Group } & \multicolumn{3}{|c|}{ CENTRM cross sections $\left(\mathrm{cm}^{-1}\right)$} & \multicolumn{3}{|c|}{ NITAWL cross sections $\left(\mathrm{cm}^{-1}\right)$} \\
\hline & $\mathrm{G}_{\mathrm{a}}$ & $\mathcal{G}_{\mathrm{f}}$ & $\mathrm{G}_{f_{-}}$ & $\mathrm{G}_{\mathrm{a}}$ & $<\mathrm{G}_{\mathrm{f}}$ & $\mathrm{G}_{\mathrm{f}}$ \\
\hline 1 & $5.12740 \mathrm{E}-04$ & $\overline{1.48833 \mathrm{E}-03}$ & $\overline{4.79719 \mathrm{E}-04}$ & $5.12294 \mathrm{E}-04$ & $\overline{1.48952 \mathrm{E}-03}$ & $4.79891 \mathrm{E}-04$ \\
\hline 2 & 1.11449E-02 & 1.83349E-02 & $6.36671 \mathrm{E}-03$ & $1.12214 \mathrm{E}-02$ & 1.84762E-02 & $6.41579 \mathrm{E}-03$ \\
\hline 3 & 3.30546E-02 & $6.50683 \mathrm{E}-02$ & $2.25947 \mathrm{E}-02$ & $3.57615 \mathrm{E}-02$ & 7.00931E-02 & $2.43396 \mathrm{E}-02$ \\
\hline 4 & 2.70972E-01 & $5.45716 \mathrm{E}-01$ & $1.88771 \mathrm{E}-01$ & $2.70533 \mathrm{E}-01$ & 5.44404E-01 & 1.88319E-01 \\
\hline
\end{tabular}




\begin{tabular}{|c|c|c|c|c|c|c|c|c|c|c|}
\hline & & & & & & & & & & $\begin{array}{l}6.654 \\
(0.79)\end{array}$ \\
\hline & $\begin{array}{l}0.147 \\
(0.81)\end{array}$ & & & & & & & & $\begin{array}{c}6.562 \\
(0.44)\end{array}$ & $\begin{array}{l}6.611 \\
(0.46)\end{array}$ \\
\hline & & & & & & & & $\begin{array}{r}6.263 \\
(0.44)\end{array}$ & $\begin{array}{c}6.409 \\
(0.32)\end{array}$ & $\begin{array}{l}6.449 \\
(0.43)\end{array}$ \\
\hline & & & & & & & $\begin{array}{l}5.818 \\
(0.46)\end{array}$ & $\begin{array}{l}6.026 \\
(0.31)\end{array}$ & $\begin{array}{l}6.170 \\
(0.31)\end{array}$ & $\begin{array}{l}6.207 \\
(0.44)\end{array}$ \\
\hline & & & & & & $\begin{array}{l}5.216 \\
(0.46)\end{array}$ & $\begin{array}{l}5.552 \\
(0.35)\end{array}$ & $\begin{array}{l}5.746 \\
(0.30)\end{array}$ & $\begin{array}{l}5.878 \\
(0.30)\end{array}$ & $\begin{array}{l}5.943 \\
(0.39)\end{array}$ \\
\hline & & & & & $\begin{array}{l}4.527 \\
(0.58)\end{array}$ & $\begin{array}{l}4.898 \\
(0.35)\end{array}$ & $\begin{array}{l}5.157 \\
(0.31)\end{array}$ & $\begin{array}{l}5.351 \\
(0.31)\end{array}$ & $\begin{array}{l}5.483 \\
(0.32)\end{array}$ & $\begin{array}{l}5.471 \\
(0.45)\end{array}$ \\
\hline & & & & $\begin{array}{c}3.745 \\
(0.51)\end{array}$ & $\begin{array}{l}4.139 \\
(0.38)\end{array}$ & $\begin{array}{l}4.467 \\
(0.35)\end{array}$ & $\begin{array}{l}4.724 \\
(0.35)\end{array}$ & $\begin{array}{l}4.888 \\
(0.33)\end{array}$ & $\begin{array}{l}5.001 \\
(0.33)\end{array}$ & $\begin{array}{l}4.991 \\
(0.49)\end{array}$ \\
\hline & & & $\begin{array}{l}3.030 \\
(0.65)\end{array}$ & $\begin{array}{l}3.377 \\
(0.41)\end{array}$ & $\begin{array}{l}3.691 \\
(0.40)\end{array}$ & $\begin{array}{l}3.991 \\
(0.38)\end{array}$ & $\begin{array}{l}4.178 \\
(0.39)\end{array}$ & $\begin{array}{l}4.331 \\
(0.34)\end{array}$ & $\begin{array}{l}4.457 \\
(0.36)\end{array}$ & $\begin{array}{l}4.431 \\
(0.50)\end{array}$ \\
\hline & & $\begin{array}{l}2.212 \\
(0.70)\end{array}$ & $\begin{array}{l}2.576 \\
(0.52)\end{array}$ & $\begin{array}{l}2.912 \\
(0.43)\end{array}$ & $\begin{array}{l}3.174 \\
(0.42)\end{array}$ & $\begin{array}{l}3.439 \\
(0.38)\end{array}$ & $\begin{array}{l}3.611 \\
(0.39)\end{array}$ & $\begin{array}{l}3.748 \\
(0.36)\end{array}$ & $\begin{array}{l}3.825 \\
(0.40)\end{array}$ & $\begin{array}{l}3.884 \\
(0.50)\end{array}$ \\
\hline & $\begin{array}{l}1.604 \\
(0.82)\end{array}$ & $\begin{array}{l}1.905 \\
(0.50)\end{array}$ & $\begin{array}{l}2.200 \\
(0.49)\end{array}$ & $\begin{array}{l}2.472 \\
(0.45)\end{array}$ & $\begin{array}{l}2.710 \\
(0.48)\end{array}$ & $\begin{array}{l}2.905 \\
(0.41)\end{array}$ & $\begin{array}{c}3.084 \\
(0.47)\end{array}$ & $\begin{array}{l}3.215 \\
(0.41)\end{array}$ & $\begin{array}{c}3.268 \\
(0.43)\end{array}$ & $\begin{array}{l}3.263 \\
(0.55)\end{array}$ \\
\hline $\begin{array}{l}1.350 \\
(0.84)\end{array}$ & $\begin{array}{c}1.492 \\
(0.56)\end{array}$ & $\begin{array}{l}1.764 \\
(0.57)\end{array}$ & $\begin{array}{l}2.050 \\
(0.50)\end{array}$ & $\begin{array}{l}2.302 \\
(0.49)\end{array}$ & $\begin{array}{l}2.553 \\
(0.45)\end{array}$ & $\begin{array}{l}2.722 \\
(0.44)\end{array}$ & $\begin{array}{l}2.886 \\
(0.45)\end{array}$ & $\begin{array}{r}2.980 \\
(0.46)\end{array}$ & $\begin{array}{l}3.036 \\
(0.44)\end{array}$ & $\begin{array}{l}3.092 \\
(0.56)\end{array}$ \\
\hline
\end{tabular}

Fig. 4.15a. Pin-power distribution for CENTRM benchmark TCA-11. Value in parentheses is percent standard deviation. 


\begin{tabular}{|c|c|c|c|c|c|c|c|c|c|c|}
\hline & & & & & & & & & & $\begin{array}{l}6.756 \\
(0.82)\end{array}$ \\
\hline & \multirow[t]{8}{*}{$\begin{array}{l}0.148 \\
(0.84)\end{array}$} & & & & & & & & $\begin{array}{l}6.609 \\
(0.41)\end{array}$ & $\begin{array}{l}6.637 \\
(0.39)\end{array}$ \\
\hline & & & & & & & & $\begin{array}{l}6.358 \\
(0.42)\end{array}$ & $\begin{array}{l}6.453 \\
(0.31)\end{array}$ & $\begin{array}{r}6.510 \\
(0.44)\end{array}$ \\
\hline & & & & & & & $\begin{array}{l}5.866 \\
(0.43)\end{array}$ & $\begin{array}{r}6.087 \\
(0.31)\end{array}$ & $\begin{array}{l}6.184 \\
(0.31)\end{array}$ & $\begin{array}{l}6.208 \\
(0.44)\end{array}$ \\
\hline & & & & & & $\begin{array}{l}5.248 \\
(0.45) \\
\end{array}$ & $\begin{array}{l}5.518 \\
(0.31) \\
\end{array}$ & $\begin{array}{l}5.748 \\
(0.29) \\
\end{array}$ & $\begin{array}{l}5.874 \\
(0.31) \\
\end{array}$ & $\begin{array}{l}5.922 \\
(0.43) \\
\end{array}$ \\
\hline & & & & & $\begin{array}{l}4.565 \\
(0.51)\end{array}$ & $\begin{array}{l}4.901 \\
(0.35)\end{array}$ & $\begin{array}{l}5.149 \\
(0.31)\end{array}$ & $\begin{array}{l}5.364 \\
(0.31)\end{array}$ & $\begin{array}{l}5.480 \\
(0.32)\end{array}$ & $\begin{array}{l}5.535 \\
(0.47)\end{array}$ \\
\hline & & & & $\begin{array}{c}3.782 \\
(0.57)\end{array}$ & $\begin{array}{l}4.171 \\
(0.39)\end{array}$ & $\begin{array}{l}4.444 \\
(0.38)\end{array}$ & $\begin{array}{l}4.672 \\
(0.37)\end{array}$ & $\begin{array}{l}4.911 \\
(0.33)\end{array}$ & $\begin{array}{l}4.984 \\
(0.30)\end{array}$ & $\begin{array}{l}5.039 \\
(0.47)\end{array}$ \\
\hline & & & $\begin{array}{c}2.969 \\
(0.61)\end{array}$ & $\begin{array}{l}3.380 \\
(0.38)\end{array}$ & $\begin{array}{l}3.683 \\
(0.38)\end{array}$ & $\begin{array}{l}3.961 \\
(0.37)\end{array}$ & $\begin{array}{l}4.175 \\
(0.40)\end{array}$ & $\begin{array}{l}4.337 \\
(0.35)\end{array}$ & $\begin{array}{l}4.431 \\
(0.36)\end{array}$ & $\begin{array}{l}4.489 \\
(0.53)\end{array}$ \\
\hline & & $\begin{array}{l}2.266 \\
(0.73)\end{array}$ & $\begin{array}{l}2.599 \\
(0.49)\end{array}$ & $\begin{array}{l}2.912 \\
(0.47)\end{array}$ & $\begin{array}{l}3.209 \\
(0.40)\end{array}$ & $\begin{array}{l}3.419 \\
(0.44)\end{array}$ & $\begin{array}{l}3.625 \\
(0.39)\end{array}$ & $\begin{array}{l}3.746 \\
(0.38)\end{array}$ & $\begin{array}{l}3.823 \\
(0.39)\end{array}$ & $\begin{array}{l}3.855 \\
(0.60)\end{array}$ \\
\hline & $\begin{array}{c}1.628 \\
(0.86)\end{array}$ & $\begin{array}{c}1.918 \\
(0.54)\end{array}$ & $\begin{array}{l}2.205 \\
(0.51)\end{array}$ & $\begin{array}{c}2.470 \\
(0.48)\end{array}$ & $\begin{array}{l}2.727 \\
(0.45)\end{array}$ & $\begin{array}{l}2.946 \\
(0.52)\end{array}$ & $\begin{array}{c}3.062 \\
(0.42)\end{array}$ & $\begin{array}{c}3.184 \\
(0.42)\end{array}$ & $\begin{array}{c}3.282 \\
(0.42)\end{array}$ & $\begin{array}{l}3.293 \\
(0.59)\end{array}$ \\
\hline $\begin{array}{l}1.376 \\
(0.81)\end{array}$ & $\begin{array}{c}1.498 \\
(0.61)\end{array}$ & $\begin{array}{l}1.770 \\
(0.54)\end{array}$ & $\begin{array}{c}2.049 \\
(0.51)\end{array}$ & $\begin{array}{l}2.320 \\
(0.50)\end{array}$ & $\begin{array}{l}2.536 \\
(0.45)\end{array}$ & $\begin{array}{l}2.723 \\
(0.45)\end{array}$ & $\begin{array}{l}2.861 \\
(0.49)\end{array}$ & $\begin{array}{c}2.983 \\
(0.44)\end{array}$ & $\begin{array}{c}3.037 \\
(0.42)\end{array}$ & $\begin{array}{l}3.032 \\
(0.61)\end{array}$ \\
\hline
\end{tabular}

Fig. 4.15b. Pin-power distribution for NITAWL benchmark TCA-11. Value in parentheses is percent standard deviation. 
Table 4.105. Selected reaction rates for CENTRM case TCA-11

\begin{tabular}{ccccccc}
\hline Pin & Region & $\begin{array}{c}\mathrm{G}_{\mathrm{a}} \mathrm{M} \\
\left(\mathrm{cm}^{-3}-\mathrm{s}^{-1}\right)\end{array}$ & $\begin{array}{c}\mathrm{G}_{\mathrm{f}} \mathrm{M} \\
\left(\mathrm{cm}^{-3}-\mathrm{s}^{-1}\right)\end{array}$ & $\begin{array}{c}\mathrm{G}_{\mathrm{f}} \mathrm{M} \\
\left(\mathrm{cm}^{-3}-\mathrm{s}^{-1}\right)\end{array}$ & $\begin{array}{c}\mathrm{M} \\
\left(\mathrm{cm}^{-1}\right)\end{array}$ & $\mathrm{MM}_{\mathrm{t}}$ \\
\hline \multirow{2}{*}{1} & Fuel & $7.995 \mathrm{E}-06$ & $1.342 \mathrm{E}-05$ & $4.759 \mathrm{E}-06$ & $2.922 \mathrm{E}-03$ & 0.146 \\
& Clad & $1.494 \mathrm{E}-07$ & 0.0 & 0.0 & $8.994 \mathrm{E}-04$ & 0.046 \\
& Mod. & $4.727 \mathrm{E}-07$ & 0.0 & 0.0 & $1.613 \mathrm{E}-02$ & 0.808 \\
\hline \multirow{2}{*}{66} & Fuel & $4.112 \mathrm{E}-05$ & $6.652 \mathrm{E}-05$ & $2.356 \mathrm{E}-05$ & $1.831 \mathrm{E}-02$ & 0.147 \\
& Clad & $7.801 \mathrm{E}-07$ & 0.0 & 0.0 & $5.719 \mathrm{E}-03$ & 0.045 \\
& Mod. & $2.193 \mathrm{E}-06$ & 0.0 & 0.0 & $1.009 \mathrm{E}-01$ & 0.808 \\
\hline
\end{tabular}

Table 4.106. Selected reaction rates for NITAWL case TCA-11

\begin{tabular}{ccccccc}
\hline Pin & Region & $\begin{array}{c}\mathrm{G}_{\mathrm{a}} \mathrm{M} \\
\left(\mathrm{cm}^{-3}-\mathrm{s}^{-1}\right)\end{array}$ & $\begin{array}{c}\mathrm{G}_{\mathrm{f}} \mathrm{M} \\
\left(\mathrm{cm}^{-3}-\mathrm{s}^{-1}\right)\end{array}$ & $\begin{array}{c}\mathrm{G}_{\mathrm{f}} \mathrm{M} \\
\left(\mathrm{cm}^{-3}-\mathrm{s}^{-1}\right)\end{array}$ & $\begin{array}{c}\mathrm{M} \\
\left(\mathrm{cm}^{-1}\right)\end{array}$ & $\mathrm{M}_{\mathrm{t}}$ \\
\hline \multirow{2}{*}{1} & Fuel & $8.237 \mathrm{E}-06$ & $1.382 \mathrm{E}-05$ & $4.898 \mathrm{E}-06$ & $2.946 \mathrm{E}-03$ & 0.146 \\
& Clad & $1.536 \mathrm{E}-07$ & 0.0 & 0.0 & $9.201 \mathrm{E}-04$ & 0.046 \\
& Mod. & $4.780 \mathrm{E}-07$ & 0.0 & 0.0 & $1.632 \mathrm{E}-02$ & 0.808 \\
\hline \multirow{2}{*}{66} & Fuel & $4.210 \mathrm{E}-05$ & $6.785 \mathrm{E}-05$ & $2.403 \mathrm{E}-05$ & $1.881 \mathrm{E}-02$ & 0.149 \\
& Clad & $7.944 \mathrm{E}-07$ & 0.0 & 0.0 & $5.881 \mathrm{E}-03$ & 0.045 \\
& Mod. & $2.226 \mathrm{E}-06$ & 0.0 & 0.0 & $1.019 \mathrm{E}-01$ & 0.805 \\
\hline
\end{tabular}


Table 4.107. TCA-11, Pin-1 four-group fluxes

\begin{tabular}{ccc}
\hline Group & $\begin{array}{c}\text { CENTRM M } \\
\left(\mathrm{cm}^{-2}-\mathrm{s}^{-1}\right)\end{array}$ & $\begin{array}{c}\text { NITAWL M } \\
\left(\mathrm{cm}^{-2}-\mathrm{s}^{-1}\right)\end{array}$ \\
\hline 1 & $2.428 \mathrm{E}-05$ & $2.418 \mathrm{E}-05$ \\
2 & $6.754 \mathrm{E}-06$ & $6.771 \mathrm{E}-06$ \\
3 & $1.616 \mathrm{E}-06$ & $1.716 \mathrm{E}-06$ \\
4 & $1.818 \mathrm{E}-05$ & $1.859 \mathrm{E}-05$ \\
\hline
\end{tabular}

Table 4.108. TCA-11, Pin-1 four-group U-235 cross sections

\begin{tabular}{cccccccc}
\hline Group & \multicolumn{2}{c}{ CENTRM cross sections $\left(\mathrm{cm}^{-1}\right)$} & \multicolumn{3}{c}{ NITAWL cross sections $\left(\mathrm{cm}^{-1}\right)$} \\
\hline & $\frac{\mathrm{G}_{\mathrm{a}}}{1.41872 \mathrm{E}-04}$ & $\frac{\mathrm{G}_{\mathrm{f}}}{3.24906 \mathrm{E}-04}$ & $\frac{\mathrm{G}_{\mathrm{f}}}{1.24701 \mathrm{E}-04}$ & $\frac{\mathrm{G}_{\mathrm{a}}}{1.41020 \mathrm{E}-04}$ & $\frac{4 \mathrm{G}_{\mathrm{f}}}{3.23700 \mathrm{E}-04}$ & $\frac{\mathrm{G}_{\mathrm{f}}}{1.24222 \mathrm{E}-04}$ \\
1 & $3.59017 \mathrm{E}-03$ & $5.46494 \mathrm{E}-03$ & $2.24276 \mathrm{E}-03$ & $3.58779 \mathrm{E}-03$ & $5.48985 \mathrm{E}-03$ & $2.25299 \mathrm{E}-03$ \\
2 & $5.18994 \mathrm{E}-03$ & $1.06233 \mathrm{E}-02$ & $4.35970 \mathrm{E}-03$ & $5.10914 \mathrm{E}-03$ & $1.04368 \mathrm{E}-02$ & $4.28316 \mathrm{E}-03$ \\
4 & $4.91039 \mathrm{E}-02$ & $1.02248 \mathrm{E}-01$ & $4.19618 \mathrm{E}-02$ & $4.92082 \mathrm{E}-02$ & $1.02458 \mathrm{E}-01$ & $4.20477 \mathrm{E}-02$ \\
\hline
\end{tabular}

Table 4.109. TCA-11, Pin-1 four-group U-238 cross sections

\begin{tabular}{cccccccc}
\hline Group & \multicolumn{3}{c}{ CENTRM cross sections $\left(\mathrm{cm}^{-1}\right)$} & \multicolumn{3}{c}{ NITAWL cross sections $\left(\mathrm{cm}^{-1}\right)$} \\
\hline & $\frac{\mathrm{G}_{\mathrm{a}}}{4.53707 \mathrm{E}-03}$ & $\frac{\leftarrow_{\mathrm{f}}}{8.65445 \mathrm{E}-03}$ & $\frac{\mathrm{G}_{\mathrm{f}}}{3.06352 \mathrm{E}-03}$ & $\frac{\mathrm{G}_{\mathrm{a}}}{4.50757 \mathrm{E}-03}$ & $\frac{\mathrm{G}_{\mathrm{f}}}{8.64413 \mathrm{E}-03}$ & $\frac{\mathrm{G}_{\mathrm{f}}}{3.06142 \mathrm{E}-03}$ \\
1 & $3.77442 \mathrm{E}-02$ & $3.89227 \mathrm{E}-06$ & $1.61256 \mathrm{E}-06$ & $4.14119 \mathrm{E}-02$ & $3.44468 \mathrm{E}-06$ & $1.42711 \mathrm{E}-06$ \\
3 & $6.97847 \mathrm{E}-03$ & $2.44523 \mathrm{E}-08$ & $1.01310 \mathrm{E}-08$ & $6.97541 \mathrm{E}-03$ & $2.44034 \mathrm{E}-08$ & $1.01108 \mathrm{E}-08$ \\
4 & $2.78818 \mathrm{E}-02$ & $1.31080 \mathrm{E}-07$ & $5.43088 \mathrm{E}-08$ & $2.79222 \mathrm{E}-02$ & $1.31269 \mathrm{E}-07$ & $5.43873 \mathrm{E}-08$ \\
\hline
\end{tabular}

Table 4.110. TCA-11, Pin-1 four-group Pu-239 cross sections

\begin{tabular}{|c|c|c|c|c|c|c|}
\hline \multirow[t]{2}{*}{ Group } & \multicolumn{3}{|c|}{ CENTRM cross sections $\left(\mathrm{cm}^{-1}\right)$} & \multicolumn{3}{|c|}{ NITAWL cross sections $\left(\mathrm{cm}^{-1}\right)$} \\
\hline & $\mathrm{G}_{\mathrm{a}}$ & $\leftarrow_{\mathrm{f}}$ & $\mathrm{G}_{\mathrm{f}}$ & $\mathrm{G}_{\mathrm{a}}$ & $<\mathrm{G}_{\mathrm{f}}$ & $\mathrm{G}_{\mathrm{f}}$ \\
\hline 1 & $5 . \overline{11446 \mathrm{E}-04}$ & $\overline{1.50099 \mathrm{E}-03}$ & $\overline{4.81647 \mathrm{E}-04}$ & $\overline{5.10571 \mathrm{E}-04}$ & $\overline{1.50108 \mathrm{E}-03}$ & $\overline{4.81762 \mathrm{E}-04}$ \\
\hline 2 & 1.15859E-02 & $1.88543 \mathrm{E}-02$ & $6.54708 \mathrm{E}-03$ & $1.15115 \mathrm{E}-02$ & $1.90902 \mathrm{E}-02$ & 6.62899E-03 \\
\hline 3 & $3.43188 \mathrm{E}-02$ & $6.73416 \mathrm{E}-02$ & $2.33841 \mathrm{E}-02$ & $3.42125 \mathrm{E}-02$ & 6.71562E-02 & $2.33196 \mathrm{E}-02$ \\
\hline 4 & $2.62911 \mathrm{E}-01$ & $5.34534 \mathrm{E}-01$ & $1.84867 \mathrm{E}-01$ & 2.65492E-01 & 5.39053E-01 & $1.86434 \mathrm{E}-01$ \\
\hline
\end{tabular}


Table 4.111. TCA-11, Pin-66 four-group fluxes

\begin{tabular}{ccc}
\hline Group & $\begin{array}{c}\text { CENTRM M } \\
\left(\mathrm{cm}^{-2}-\mathrm{s}^{-1}\right)\end{array}$ & $\begin{array}{c}\text { NITAWL M } \\
\left(\mathrm{cm}^{-2}-\mathrm{s}^{-1}\right)\end{array}$ \\
\hline 1 & $1.657 \mathrm{E}-04$ & $1.701 \mathrm{E}-04$ \\
2 & $5.293 \mathrm{E}-05$ & $5.558 \mathrm{E}-05$ \\
3 & $1.316 \mathrm{E}-05$ & $1.301 \mathrm{E}-05$ \\
4 & $8.674 \mathrm{E}-05$ & $8.849 \mathrm{E}-05$ \\
\hline
\end{tabular}

Table 4.112. TCA-11, Pin-66 four-group U-235 cross sections

\begin{tabular}{cccccccc}
\hline Group & \multicolumn{2}{c}{ CENTRM cross sections $\left(\mathrm{cm}^{-1}\right)$} & \multicolumn{3}{c}{ NITAWL cross sections $\left(\mathrm{cm}^{-1}\right)$} \\
\hline & $\frac{\mathrm{G}_{\mathrm{a}}}{1.44711 \mathrm{E}-04}$ & $\frac{\mathrm{G}_{\mathrm{f}}}{3.27220 \mathrm{E}-04}$ & $\frac{\mathrm{G}_{\mathrm{f}}}{1.26172 \mathrm{E}-04}$ & $\frac{\mathrm{G}_{\mathrm{a}}}{1.44755 \mathrm{E}-04}$ & $\frac{\mathrm{G}_{\mathrm{f}}}{3.27436 \mathrm{E}-04}$ & $\frac{\mathrm{G}_{\mathrm{f}}}{1.26225 \mathrm{E}-04}$ \\
1 & $3.46220 \mathrm{E}-03$ & $5.28876 \mathrm{E}-03$ & $2.17045 \mathrm{E}-03$ & $3.45516 \mathrm{E}-03$ & $5.25847 \mathrm{E}-03$ & $2.15802 \mathrm{E}-03$ \\
2 & $5.16589 \mathrm{E}-03$ & $1.05595 \mathrm{E}-02$ & $4.33352 \mathrm{E}-03$ & $5.18064 \mathrm{E}-03$ & $1.05839 \mathrm{E}-02$ & $4.34357 \mathrm{E}-03$ \\
4 & $4.81825 \mathrm{E}-02$ & $1.00288 \mathrm{E}-01$ & $4.11571 \mathrm{E}-02$ & $4.80322 \mathrm{E}-02$ & $9.99713 \mathrm{E}-02$ & $4.10273 \mathrm{E}-02$ \\
\hline
\end{tabular}

Table 4.113. TCA-11, Pin-66 four-group U-238 cross sections

\begin{tabular}{cccccccc}
\hline Group & \multicolumn{3}{c}{ CENTRM cross sections $\left(\mathrm{cm}^{-1}\right)$} & \multicolumn{3}{c}{ NITAWL cross sections $\left(\mathrm{cm}^{-1}\right)$} \\
\hline & $\frac{\mathrm{G}_{\mathrm{a}}}{4.47467 \mathrm{E}-03}$ & $\frac{\mathrm{G}_{\mathrm{f}}}{8.17996 \mathrm{E}-03}$ & $\frac{\mathrm{G}_{\mathrm{f}}}{2.90346 \mathrm{E}-03}$ & $\frac{\mathrm{G}_{\mathrm{a}}}{4.47739 \mathrm{E}-03}$ & $\frac{\mathrm{G}_{\mathrm{f}}}{8.19447 \mathrm{E}-03}$ & $\frac{\mathrm{G}_{\mathrm{f}}}{2.90690 \mathrm{E}-03}$ \\
1 & $3.91615 \mathrm{E}-02$ & $3.93752 \mathrm{E}-06$ & $1.63130 \mathrm{E}-06$ & $4.02064 \mathrm{E}-02$ & $3.76053 \mathrm{E}-06$ & $1.55797 \mathrm{E}-06$ \\
3 & $6.99303 \mathrm{E}-03$ & $2.44910 \mathrm{E}-08$ & $1.01470 \mathrm{E}-08$ & $6.99198 \mathrm{E}-03$ & $2.45410 \mathrm{E}-08$ & $1.01678 \mathrm{E}-08$ \\
4 & $2.73917 \mathrm{E}-02$ & $1.28724 \mathrm{E}-07$ & $5.33326 \mathrm{E}-08$ & $2.73197 \mathrm{E}-02$ & $1.28377 \mathrm{E}-07$ & $5.31893 \mathrm{E}-08$ \\
\hline
\end{tabular}

Table 4.114. TCA-11, Pin-66 four-group Pu-239 cross sections

\begin{tabular}{cccccccc}
\hline Group & \multicolumn{3}{c}{ CENTRM cross sections $\left(\mathrm{cm}^{-1}\right)$} & \multicolumn{3}{c}{ NITAWL cross sections $\left(\mathrm{cm}^{-1}\right)$} \\
\hline & $\frac{\mathrm{G}_{\mathrm{a}}}{4.12696 \mathrm{E}-04}$ & $\frac{\mathrm{G}_{\mathrm{f}}}{1.48906 \mathrm{E}-03}$ & $\frac{\mathrm{G}_{\mathrm{f}}}{4.79759 \mathrm{E}-04}$ & $\frac{5.13031 \mathrm{E}-04}{1.49057 \mathrm{E}-03}$ & $\frac{\mathrm{G}_{\mathrm{a}}}{4.80148 \mathrm{E}-04}$ \\
1 & $1.14152 \mathrm{E}-02$ & $1.88409 \mathrm{E}-02$ & $6.54241 \mathrm{E}-03$ & $1.11940 \mathrm{E}-02$ & $1.81913 \mathrm{E}-02$ & $6.31686 \mathrm{E}-03$ \\
2 & $3.48957 \mathrm{E}-02$ & $6.84264 \mathrm{E}-02$ & $2.37608 \mathrm{E}-02$ & $3.45817 \mathrm{E}-02$ & $6.78782 \mathrm{E}-02$ & $2.35705 \mathrm{E}-02$ \\
3 & $2.71706 \mathrm{E}-01$ & $5.47283 \mathrm{E}-01$ & $1.89309 \mathrm{E}-01$ & $2.72174 \mathrm{E}-01$ & $5.47792 \mathrm{E}-01$ & $1.89488 \mathrm{E}-01$ \\
\hline
\end{tabular}




\section{CALCULATIONAL BENCHMARKS}

\subsection{DESCRIPTION}

The calculational benchmarks examined in this report are divided into two distinct geometric sets of cases. The first set of cases consists of an infinitely long pin in an infinite triangular-pitched lattice. The second set of cases consists of a buckled pin in an infinite triangular-pitched lattice. The radii of the fuel, clad, and moderator are all the same for all variants and states. Also, the buckling height is the same in all buckled problems. All variants and states are calculated in both geometric configurations. The state parameters are given in Table 5.1. ${ }^{4}$

The calculational benchmarks in each geometric set are divided into variants, with five states in each of the variants. The variants differ only by the isotopic composition of the fuel. Within each variant, the fuel composition is the same for each state, with the exception of the presence or absence of ${ }^{135} \mathrm{Xe}$ and ${ }^{149} \mathrm{Sm}$. The differences in the states are due to the presence or absence of ${ }^{135} \mathrm{Xe}$ and ${ }^{149} \mathrm{Sm}$ in the fuel, the fuel temperature, and the type of moderator.

There are four basic types of fuel: fresh uranium fuel, fresh MOX fuel, spent uranium fuel and fresh MOX fuel containing Pu recycled from a reactor. The spent uranium fuel is used in two variants: variant V3 uses spent uranium fuel with only the actinides, whereas variant V4 uses spent uranium fuel with actinides and fission products. There are also four variations of the fresh MOX fuel: fresh MOX fuel, MOX fuel with only ${ }^{239} \mathrm{Pu}$, MOX fuel with only ${ }^{240} \mathrm{Pu}$, and MOX fuel with only ${ }^{241} \mathrm{Pu}$. The basic fuel material isotopic composition for each variant is given in Table 5.2.

There are four nonfuel material compositions in the set of calculational benchmarks: 1 clad composition, which is common to all problems, and 3 moderator material compositions. The variations in the moderator compositions are due to the presence or absence of ${ }^{10} \mathrm{~B}$ and ${ }^{11} \mathrm{~B}$, the clad and moderator temperature, and the moderator density. The material isotopic compositions for the clad and each moderator are given in Table 5.3.

Table 5.1. State parameters for the calculational benchmark variants

\begin{tabular}{cccccc}
\hline State & $\begin{array}{c}\text { Fuel zone } \\
\text { temp }(\mathrm{K})\end{array}$ & $\begin{array}{c}\text { Nonfuel zone } \\
\text { temp }(\mathrm{K})\end{array}$ & $\begin{array}{c}{ }^{135} \mathrm{Xe},{ }^{149} \mathrm{Sm} \\
\text { in fuel }\end{array}$ & $\begin{array}{c}{ }^{10} \mathrm{~B},{ }^{11} \mathrm{~B} \text { in } \\
\text { moderator }\end{array}$ & $\begin{array}{c}\text { Moderator } \\
\text { material }\end{array}$ \\
\hline S1 & 1027 & 579 & Yes & Yes & MOD1 \\
S3 & 1027 & 579 & Yes & No & MOD2 \\
S4 & 1027 & 579 & No & Yes & MOD1 \\
S5 & 579 & 579 & No & Yes & MOD1 \\
S6 & 300 & 300 & No & Yes & MOD3 \\
\hline
\end{tabular}


Table 5.2. Basic fuel isotopic composition for each computational benchmark variant

\begin{tabular}{|c|c|c|c|c|c|}
\hline Variant & Comment & Isotope & $\begin{array}{l}\text { Atom density } \\
\left(10^{-24} \mathrm{~cm}^{-3}\right)\end{array}$ & $\begin{array}{c}\text { Isotop } \\
\mathrm{e}\end{array}$ & $\begin{array}{l}\text { Atom density } \\
\left(10^{-24} \mathrm{~cm}^{-3}\right)\end{array}$ \\
\hline V1 & Fresh uranium fuel & ${ }^{235} \mathrm{U}$ & $\begin{array}{l}8.7370 \times 10^{-4} \\
1.8744 \times 10^{-2}\end{array}$ & ${ }^{16} \mathrm{O}$ & $3.9235 \times 10^{-2}$ \\
\hline $\mathrm{V} 2$ & Fresh MOX fuel & ${ }^{235} \mathrm{U}$ & $\begin{array}{l}3.8393 \times 10^{-5} \\
1.8917 \times 10^{-2} \\
4.1707 \times 10^{-2}\end{array}$ & $\begin{array}{l}{ }^{239} \mathrm{Pu} \\
{ }^{240} \mathrm{Pu} \\
{ }^{41} \mathrm{Pu}\end{array}$ & $\begin{array}{l}6.5875 \times 10^{-4} \\
4.2323 \times 10^{-5} \\
7.0246 \times 10^{-4}\end{array}$ \\
\hline V3 & $\begin{array}{l}\text { Spent uranium fuel with } \\
\text { no fission products } \\
\text { ( actinides only ) }\end{array}$ & $\begin{array}{l}{ }^{235} \mathrm{U} \\
{ }^{236} \mathrm{U} \\
{ }^{238} \mathrm{U} \\
{ }^{237} \mathrm{~Np} \\
{ }^{238} \mathrm{Pu} \\
{ }^{239} \mathrm{~Np} \\
{ }^{239} \mathrm{Pu} \\
{ }^{240} \mathrm{Pu} \\
{ }^{241} \mathrm{Pu}\end{array}$ & $\begin{array}{l}3.7843 \times 10^{-4} \\
8.6365 \times 10^{-5} \\
1.8327 \times 10^{-2} \\
2.4823 \times 10^{-5} \\
6.7254 \times 10^{-6} \\
1.8332 \times 10^{-6} \\
1.3111 \times 10^{-4} \\
3.6233 \times 10^{-5} \\
2.1701 \times 10^{-5}\end{array}$ & $\begin{array}{l}{ }^{242} \mathrm{Pu} \\
{ }^{241} \mathrm{Am} \\
{ }^{242 \mathrm{~m}} \mathrm{Am} \\
{ }^{243} \mathrm{Am} \\
{ }^{242} \mathrm{Cm} \\
{ }^{243} \mathrm{Cm} \\
{ }^{244} \mathrm{Cm} \\
{ }^{16} \mathrm{O}\end{array}$ & $\begin{array}{l}4.7576 \times 10^{-6} \\
4.9491 \times 10^{-7} \\
7.9194 \times 10^{-9} \\
6.6925 \times 10^{-7} \\
1.2582 \times 10^{-7} \\
2.0629 \times 10^{-9} \\
1.2387 \times 10^{-7} \\
3.9235 \times 10^{-2}\end{array}$ \\
\hline V4 & $\begin{array}{l}\text { Spent uranium fuel } \\
\text { ( actinides }+ \\
\text { fission products ) } \\
\text { ( V3 + listed isotopes ) }\end{array}$ & $\begin{array}{l}{ }^{103} \mathrm{Rh} \\
{ }^{131} \mathrm{Xe} \\
{ }^{143} \mathrm{Nd} \\
{ }^{147} \mathrm{Pm} \\
{ }^{133} \mathrm{Cs} \\
{ }^{99} \mathrm{Tc} \\
{ }^{152} \mathrm{Sm} \\
{ }^{151} \mathrm{Sm}\end{array}$ & $\begin{array}{l}1.8890 \times 10^{-4} \\
1.4255 \times 10^{-5} \\
2.6692 \times 10^{-2} \\
6.1574 \times 10^{-5} \\
3.5974 \times 10^{-6} \\
3.3320 \times 10^{-6} \\
2.6842 \times 10^{-4} \\
3.0757 \times 10^{-5}\end{array}$ & $\begin{array}{l}{ }^{145} \mathrm{Nd} \\
{ }^{153} \mathrm{Eu} \\
{ }^{109} \mathrm{Ag} \\
{ }^{155} \mathrm{Eu} \\
{ }^{95} \mathrm{Mo} \\
{ }^{154} \mathrm{Eu} \\
{ }^{101} \mathrm{Ru}\end{array}$ & $\begin{array}{l}1.9975 \times 10^{-6} \\
2.4801 \times 10^{-7} \\
2.2037 \times 10^{-9} \\
9.6857 \times 10^{-7} \\
3.3720 \times 10^{-7} \\
5.1189 \times 10^{-9} \\
3.1134 \times 10^{-7}\end{array}$ \\
\hline V7 & $\begin{array}{l}\text { Fresh MOX fuel with } \\
{ }^{239} \mathrm{Pu} \text { as only } \mathrm{Pu}\end{array}$ & ${ }^{235} \mathrm{U}$ & $\begin{array}{l}3.8393 \times 10^{-5} \\
1.8917 \times 10^{-2}\end{array}$ & ${ }^{{ }^{239} \mathrm{Pu}}$ & $\begin{array}{l}6.5875 \times 10^{-4} \\
4.1707 \times 10^{-2}\end{array}$ \\
\hline V8 & $\begin{array}{l}\text { Fresh MOX fuel with } \\
{ }^{240} \mathrm{Pu} \text { as only } \mathrm{Pu}\end{array}$ & ${ }^{235} \mathrm{U}$ & $\begin{array}{l}6.9714 \times 10^{-4} \\
1.8917 \times 10^{-2}\end{array}$ & ${ }^{240} \mathrm{Pu}$ & $\begin{array}{l}4.2323 \times 10^{-5} \\
4.1707 \times 10^{-2}\end{array}$ \\
\hline V9 & $\begin{array}{l}\text { Fresh MOX fuel with } \\
{ }^{241} \mathrm{Pu} \text { as only } \mathrm{Pu}\end{array}$ & ${ }^{235} \mathrm{U}$ & $\begin{array}{l}3.8393 \times 10^{-5} \\
1.8917 \times 10^{-2}\end{array}$ & $\begin{array}{l}{ }^{41} \mathrm{Pu} \\
{ }^{16} \mathrm{O}\end{array}$ & $\begin{array}{l}6.6577 \times 10^{-4} \\
4.1707 \times 10^{-2}\end{array}$ \\
\hline V10 & $\begin{array}{l}\text { Fresh MOX fuel with } \\
\text { recycled reactor } \mathrm{Pu}\end{array}$ & $\begin{array}{c}{ }^{235} \mathrm{U} \\
{ }^{238} \mathrm{U} \\
{ }^{16} \mathrm{O} \\
{ }^{238} \mathrm{Pu} \\
{ }^{239} \mathrm{Pu}\end{array}$ & $\begin{array}{l}5.0000 \times 10^{-5} \\
2.2100 \times 10^{-2} \\
4.6300 \times 10^{-2} \\
3.0000 \times 10^{-5} \\
1.1600 \times 10^{-3}\end{array}$ & $\begin{array}{l}{ }^{240} \mathrm{Pu} \\
{ }^{241} \mathrm{Pu} \\
{ }^{242} \mathrm{Pu} \\
{ }^{241} \mathrm{Am}\end{array}$ & $\begin{array}{l}4.9000 \times 10^{-4} \\
1.9000 \times 10^{-4} \\
1.0500 \times 10^{-4} \\
2.5000 \times 10^{-5}\end{array}$ \\
\hline
\end{tabular}


Table 5.3. Nonfuel material isotopic composition

\begin{tabular}{|c|c|c|c|c|c|}
\hline Material & Comment & Isotope & $\begin{array}{l}\text { Atom density } \\
\left(10^{-24} \mathrm{~cm}^{-3}\right)\end{array}$ & Isotope & $\begin{array}{l}\text { Atom density } \\
\left(10^{-24} \mathrm{~cm}^{-3}\right)\end{array}$ \\
\hline Clad & Cladding & $\mathrm{Zr}$ & $4.2300 \times 10^{-2}$ & & \\
\hline MOD1 & $\begin{array}{l}\text { Hot moderator } \\
\text { with boric acid }\end{array}$ & $\begin{array}{c}\mathrm{H} \\
{ }^{16} \mathrm{O}\end{array}$ & $\begin{array}{l}4.7830 \times 10^{-2} \\
2.3910 \times 10^{-2}\end{array}$ & $\begin{array}{l}{ }^{10} \mathrm{~B} \\
{ }^{11} \mathrm{~B}\end{array}$ & $\begin{array}{l}4.7344 \times 10^{-6} \\
1.9177 \times 10^{-5}\end{array}$ \\
\hline MOD2 & $\begin{array}{l}\text { Hot moderator } \\
\text { without boric acid }\end{array}$ & $\begin{array}{c}\mathrm{H} \\
{ }^{16} \mathrm{O}\end{array}$ & $\begin{array}{l}4.7830 \times 10^{-2} \\
2.3910 \times 10^{-2}\end{array}$ & & \\
\hline MOD3 & $\begin{array}{l}\text { Cold moderator } \\
\text { with boric acid }\end{array}$ & $\begin{array}{c}\mathrm{H} \\
{ }^{16} \mathrm{O}\end{array}$ & $\begin{array}{l}6.6940 \times 10^{-2} \\
3.3470 \times 10^{-2}\end{array}$ & $\begin{array}{l}{ }^{10} \mathrm{~B} \\
{ }^{11} \mathrm{~B}\end{array}$ & $\begin{array}{l}6.6262 \times 10^{-6} \\
2.6839 \times 10^{-5}\end{array}$ \\
\hline
\end{tabular}

\subsection{ANALYSIS}

All computational benchmarks were processed using SCALE 5.0. The cases labeled NITAWL utilize the following sequence: CSAS, BONAMI, NITAWL, XSDRNPM. The cases labeled CENTRM utilize the following sequence: CSAS, BONAMI, NITAWL, CENTRM, PMC, WORKER, XSDRNPM. In the NITAWL cases the resolved resonance region is self-shielded using NITAWL. In the CENTRM cases the resolved resonance region is self-shielded using CENTRM and PMC, which removes the limitations of the Nordheim Integral Treatment. Table 5.4 contains the results for the infinite arrays, and Table 5.5 contains the results for the buckled arrays.

For each variant and state, the differences between the NITAWL and CENTRM multiplication factors $\left(k_{4}\right.$ and buckled $\left.k_{4}\right)$ and the energy of the average lethargy causing fission (EALCF) are almost identical for the infinite and buckled geometries as shown in Tables 5.4 and 5.5. The differences in the multiplication between the CENTRM and NITAWL cases range from $-0.09 \%$ to $-0.55 \%$. Trends are apparent based on both fuel material and temperature. The smallest difference occurs for variant V4, state S6, which is spent uranium fuel with actinides and fission products at room temperature. The largest difference occurs for variant V9, state S1, which is fresh MOX fuel with all the $\mathrm{Pu}$ as ${ }^{241} \mathrm{Pu}$ at reactor temperatures.

The selection and number of nuclides in the fuel has little effect on the difference between NITAWL and CENTRM, at most $\sim 0.25 \%$, which occurs when the amount of ${ }^{241} \mathrm{Pu}$ is at its maximum. The effects of resonance overlap do not seem to be significant. The lowest difference at any given temperature occurs for spent uranium fuel with actinides and fission products. The amount of absorbers in the fuel $\left({ }^{135} \mathrm{Xe}\right.$ and $\left.{ }^{149} \mathrm{Sm}\right)$ and in the moderator $\left({ }^{10} \mathrm{~B}\right)$ also does not affect the differences.

Fuel and moderator temperature have the most obvious, albeit still small, effect on the difference between NITAWL and CENTRM results. For a given variant, the largest difference always occurs with the fuel at $1027 \mathrm{~K}$ and the moderator at $579 \mathrm{~K}$. Decreasing the fuel temperature to $579 \mathrm{~K}$ always decreases the difference, as much as $0.1 \%$. Further decreasing the fuel and moderator temperature to $300 \mathrm{~K}$ always further decreases the difference, as much as another $0.1 \%$. Both NITAWL and 
CENTRM interpolate data between temperatures.

The EALCF is also calculated for each variant and state. The differences in the average-energy between the NITAWL and CENTRM cases range from a low of $-0.41 \%$ to a high of $1.66 \%$. Trends are apparent based only on temperature. The smallest differences with each variant occur in state S6, and the largest differences occurs for states 1,2, and 3. As the pin temperature drops, the differences between the CSAS and CENTRM results lessen.

\subsection{CONCLUSIONS}

For this set of cases using either NITAWL or CENTRM as the resonance region processor produces consistent results. The $k_{\text {eff }}$ values are slightly lower, $\sim 0.3 \%$, for all CENTRM cases. The nuclides selected do not appear to have an effect on the difference, but the temperature of the system does have a slight effect: the point library has temperature data well above that available in the multigroup library. The approximations used to process the resolved resonance data in NITAWL do not significantly affect the results, as shown by the good agreement with the CENTRM results. 
Table 5.4a. $k_{4}$ and EALCF values and differences for the variant cases

\begin{tabular}{|c|c|c|c|c|c|c|}
\hline $\begin{array}{c}\text { VARIANTS } \\
\text { V1 - V4 }\end{array}$ & $\begin{array}{c}\text { NITAWL } \\
k_{4}\end{array}$ & $\begin{array}{c}\text { CENTRM } \\
k_{4}\end{array}$ & $\begin{array}{c}k_{4} \\
\% \text { DIFF }\end{array}$ & $\begin{array}{c}\text { NITAWL } \\
\text { EALCF } \\
(\mathrm{eV})\end{array}$ & $\begin{array}{c}\text { CENTRM } \\
\text { EALCF } \\
(\mathrm{eV})\end{array}$ & $\begin{array}{l}\text { EALCF } \\
\% \text { DIFF }\end{array}$ \\
\hline V1S1 & 1.2593 & 1.2541 & -0.41 & 0.7766 & 0.7806 & 0.52 \\
\hline V1S3 & 1.3144 & 1.3090 & -0.41 & 0.6984 & 0.7019 & 0.5 \\
\hline V1S4 & 1.3069 & 1.3015 & -0.41 & 0.7052 & 0.7087 & 0.5 \\
\hline V1S5 & 1.3249 & 1.3205 & -0.33 & 0.6846 & 0.6877 & 0.45 \\
\hline V1S6 & 1.3669 & 1.3634 & -0.26 & 0.2808 & 0.2819 & 0.39 \\
\hline V2S1 & 1.2074 & 1.2017 & -0.47 & 1.5678 & 1.5873 & 1.24 \\
\hline V2S3 & 1.2360 & 1.2300 & -0.49 & 1.4690 & 1.4871 & 1.23 \\
\hline V2S4 & 1.2270 & 1.2212 & -0.47 & 1.4907 & 1.5090 & 1.23 \\
\hline V2S5 & 1.2474 & 1.2428 & -0.37 & 1.4305 & 1.4460 & 1.08 \\
\hline V2S6 & 1.3256 & 1.3210 & -0.35 & 0.5514 & 0.5568 & 0.98 \\
\hline V3S1 & 1.0821 & 1.0767 & -0.5 & 0.9976 & 1.0064 & 0.88 \\
\hline V3S3 & 1.1267 & 1.1210 & -0.51 & 0.8982 & 0.9058 & 0.85 \\
\hline V3S4 & 1.1218 & 1.1162 & -0.5 & 0.9027 & 0.9103 & 0.84 \\
\hline V3S5 & 1.1412 & 1.1357 & -0.48 & 0.8694 & 0.877 & 0.87 \\
\hline V3S6 & 1.1902 & 1.1862 & -0.34 & 0.3271 & 0.3289 & 0.55 \\
\hline V4S1 & 1.0158 & 1.0140 & -0.18 & 1.1496 & 1.1532 & 0.31 \\
\hline V4S3 & 1.0558 & 1.0536 & -0.21 & 1.0346 & 1.0385 & 0.38 \\
\hline V4S4 & 1.0510 & 1.0490 & -0.19 & 1.0411 & 1.0442 & 0.3 \\
\hline V4S5 & 1.0688 & 1.0669 & -0.18 & 1.0081 & 1.0055 & -0.26 \\
\hline V4S6 & 1.1197 & 1.1187 & -0.09 & 0.3678 & 0.368 & 0.05 \\
\hline
\end{tabular}


Table 5.4b. $k_{4}$ and EALCF values and differences for the variant cases (cont.)

\begin{tabular}{|c|c|c|c|c|c|c|}
\hline $\begin{array}{c}\text { VARIANTS } \\
\text { V7 - V10 } \\
\end{array}$ & $\begin{array}{c}\text { CSAS } \\
k_{4} \\
\end{array}$ & $\begin{array}{c}\text { CENTRM } \\
k_{4} \\
\end{array}$ & $\begin{array}{c}k_{4} \\
\% \text { DIFF } \\
\end{array}$ & $\begin{array}{c}\text { CSAS } \\
\text { EALCF } \\
(\mathrm{eV}) \\
\end{array}$ & $\begin{array}{c}\text { CENTRM } \\
\text { EALCF } \\
(\mathrm{eV}) \\
\end{array}$ & $\begin{array}{l}\text { EALCF } \\
\% \text { DIFF }\end{array}$ \\
\hline V7S1 & 1.3047 & 1.2984 & -0.48 & 1.2704 & 1.2851 & 1.16 \\
\hline V7S3 & 1.3372 & 1.3307 & -0.49 & 1.1922 & 1.2058 & 1.14 \\
\hline V7S4 & 1.3270 & 1.3206 & -0.48 & 1.2090 & 1.2228 & 1.14 \\
\hline V7S5 & 1.3459 & 1.3404 & -0.41 & 1.1687 & 1.1816 & 1.1 \\
\hline V7S6 & 1.4112 & 1.4067 & -0.32 & 0.4793 & 0.4835 & 0.88 \\
\hline V8S1 & 1.0904 & 1.0859 & -0.41 & 0.8038 & 0.8083 & 0.56 \\
\hline V8S3 & 1.1442 & 1.1395 & -0.41 & 0.7108 & 0.7146 & 0.53 \\
\hline V8S4 & 1.1399 & 1.1351 & -0.42 & 0.7147 & 0.7186 & 0.55 \\
\hline V8S5 & 1.1596 & 1.1557 & -0.34 & 0.6881 & 0.6912 & 0.45 \\
\hline V8S6 & 1.2115 & 1.2084 & -0.26 & 0.2694 & 0.2704 & 0.37 \\
\hline V9S1 & 1.5290 & 1.5207 & -0.54 & 1.4193 & 1.4140 & -0.37 \\
\hline V9S3 & 1.5644 & 1.5561 & -0.53 & 1.3346 & 1.3293 & -0.4 \\
\hline V9S4 & 1.5515 & 1.5432 & -0.53 & 1.3585 & 1.3529 & -0.41 \\
\hline V9S5 & 1.5736 & 1.5668 & -0.43 & 1.3191 & 1.3181 & -0.08 \\
\hline V9S6 & 1.6195 & 1.6140 & -0.34 & 0.5601 & 0.5602 & 0.02 \\
\hline V10S1 & 1.0952 & 1.0902 & -0.46 & 9.5809 & 9.7345 & 1.6 \\
\hline V10S3 & 1.1078 & 1.1027 & -0.46 & 9.118 & 9.2636 & 1.6 \\
\hline V10S4 & 1.1010 & 1.0958 & -0.47 & 9.3374 & 9.4863 & 1.59 \\
\hline V10S5 & 1.1196 & 1.1160 & -0.32 & 8.7717 & 8.8831 & 1.27 \\
\hline V10S6 & 1.1937 & 1.1917 & -0.17 & 2.614 & 2.6373 & 0.89 \\
\hline
\end{tabular}


Table 5.5a. Buckled $k_{4}$ and EALCF values and differences for the variant cases

\begin{tabular}{|c|c|c|c|c|c|c|}
\hline $\begin{array}{l}\text { VARIANTS } \\
\text { V1 - V4 }\end{array}$ & $\begin{array}{c}\text { CSAS } \\
\text { buckled } \\
k_{4} \\
\end{array}$ & $\begin{array}{c}\text { CENTRM } \\
\text { buckled } \\
k_{4} \\
\end{array}$ & $\begin{array}{c}k_{4} \\
\% \mathrm{DIFF}\end{array}$ & $\begin{array}{c}\text { CSAS } \\
\text { EALCF } \\
(\mathrm{eV})\end{array}$ & $\begin{array}{c}\text { CENTRM } \\
\text { EALCF } \\
(\mathrm{eV})\end{array}$ & $\begin{array}{l}\text { EALCF } \\
\% \text { DIFF }\end{array}$ \\
\hline V1S1 & 1.0802 & 1.0757 & -0.42 & 0.8408 & 0.8455 & 0.56 \\
\hline V1S3 & 1.1266 & 1.1218 & -0.43 & 0.755 & 0.7592 & 0.56 \\
\hline V1S4 & 1.1201 & 1.1154 & -0.42 & 0.7628 & 0.7670 & 0.55 \\
\hline V1S5 & 1.1353 & 1.1314 & -0.34 & 0.7402 & 0.7438 & 0.49 \\
\hline V1S6 & 1.2286 & 1.2253 & -0.27 & 0.2932 & 0.2944 & 0.41 \\
\hline V2S1 & 1.0396 & 1.0346 & -0.48 & 1.6927 & 1.7146 & 1.29 \\
\hline V2S3 & 1.0638 & 1.0586 & -0.49 & 1.5847 & 1.605 & 1.28 \\
\hline $\mathrm{V} 2 \mathrm{~S} 4$ & 1.0562 & 1.0511 & -0.48 & 1.6086 & 1.6293 & 1.29 \\
\hline V2S5 & 1.1050 & 1.1007 & -0.39 & 1.5477 & 1.5659 & 1.18 \\
\hline V2S6 & 1.2167 & 1.2125 & -0.35 & 0.5755 & 0.5814 & 1.03 \\
\hline V3S1 & 0.9547 & 0.9499 & -0.5 & 1.0872 & 1.0973 & 0.93 \\
\hline V3S3 & 0.9932 & 0.9882 & -0.5 & 0.9775 & 0.9863 & 0.9 \\
\hline V3S4 & 0.9889 & 0.9839 & -0.51 & 0.9827 & 0.9917 & 0.92 \\
\hline V3S5 & 1.0059 & 1.0011 & -0.48 & 0.9457 & 0.9542 & 0.9 \\
\hline V3S6 & 1.0887 & 1.0850 & -0.34 & 0.3425 & 0.3445 & 0.58 \\
\hline V4S1 & 0.8975 & 0.8958 & -0.19 & 1.2555 & 1.2600 & 0.36 \\
\hline V4S3 & 0.9322 & 0.9301 & -0.23 & 1.1285 & 1.1334 & 0.43 \\
\hline V4S4 & 0.9279 & 0.9261 & -0.19 & 1.1361 & 1.1400 & 0.34 \\
\hline V4S5 & 0.9435 & 0.9418 & -0.18 & 1.0923 & 1.0963 & 0.37 \\
\hline V4S6 & 1.0253 & 1.0242 & -0.11 & 0.3847 & 0.3858 & 0.29 \\
\hline
\end{tabular}


Table 5.5b. Buckled $k_{4}$ and EALCF values and differences for the variant cases (cont.)

\begin{tabular}{|c|c|c|c|c|c|c|}
\hline $\begin{array}{c}\text { VARIANTS } \\
\text { V7 - V10 }\end{array}$ & $\begin{array}{c}\text { CSAS } \\
\text { buckled } \\
k_{4} \\
\end{array}$ & $\begin{array}{c}\text { CENTRM } \\
\text { buckled } \\
k_{4} \\
\end{array}$ & $\begin{array}{c}k_{4} \\
\% \mathrm{DIFF} \\
\end{array}$ & $\begin{array}{c}\text { CSAS } \\
\text { EALCF } \\
(\mathrm{eV})\end{array}$ & $\begin{array}{c}\text { CENTRM } \\
\text { EALCF } \\
(\mathrm{eV}) \\
\end{array}$ & $\begin{array}{l}\text { EALCF } \\
\% \text { DIFF }\end{array}$ \\
\hline V7S 1 & 1.1560 & 1.1504 & -0.48 & 1.3698 & 1.3865 & 1.22 \\
\hline V7S3 & 1.1844 & 1.1786 & -0.49 & 1.2846 & 1.300 & 1.2 \\
\hline V7S4 & 1.1755 & 1.1697 & -0.49 & 1.3031 & 1.3187 & 1.2 \\
\hline V7S5 & 1.1920 & 1.1870 & -0.42 & 1.2589 & 1.2734 & 1.15 \\
\hline V7S6 & 1.2955 & 1.2913 & -0.32 & 0.4991 & 0.5037 & 0.92 \\
\hline V8S1 & 0.9628 & 0.9588 & -0.42 & 0.8777 & 0.8831 & 0.62 \\
\hline V8S3 & 1.0093 & 1.0050 & -0.43 & 0.7748 & 0.7794 & 0.59 \\
\hline V8S4 & 1.0055 & 1.0012 & -0.43 & 0.7794 & 0.7841 & 0.6 \\
\hline V8S5 & 1.0227 & 1.0192 & -0.34 & 0.7496 & 0.7534 & 0.51 \\
\hline V8S6 & 1.1095 & 1.1066 & -0.26 & 0.2819 & 0.2829 & 0.35 \\
\hline V9S1 & 1.3591 & 1.3515 & -0.56 & 1.5135 & 1.5087 & -0.32 \\
\hline V9S3 & 1.3901 & 1.3825 & -0.55 & 1.4225 & 1.4177 & -0.34 \\
\hline V9S4 & 1.3788 & 1.3712 & -0.55 & 1.4481 & 1.4431 & -0.35 \\
\hline V9S5 & 1.3982 & 1.3920 & -0.44 & 1.4055 & 1.4052 & -0.02 \\
\hline V9S6 & 1.4906 & 1.4855 & -0.34 & 0.5802 & 0.5803 & 0.02 \\
\hline V10S1 & 0.9876 & 0.9831 & -0.46 & 10.483 & 10.657 & 1.66 \\
\hline V10S3 & 0.9988 & 0.9941 & -0.47 & 9.9769 & 10.142 & 1.65 \\
\hline V10S4 & 0.9927 & 0.9881 & -0.46 & 10.216 & 10.385 & 1.65 \\
\hline V10S5 & 1.0094 & 1.0061 & -0.33 & 9.5901 & 9.7165 & 1.32 \\
\hline V10S6 & 1.1087 & 1.1069 & -0.16 & 2.7455 & 2.7705 & 0.91 \\
\hline
\end{tabular}




\section{REFERENCES}

1. SCALE: A Modular Code System for Performing Standardized Analyses for Licensing Evaluations, NUREG/CR-0200, Rev. 4 (ORNL/NUREG/CSD-2/R4), Vols. I, II, and III, April 1995. Available from Radiation Safety Information Computational Center at Oak Ridge National Laboratory as CCC-545.

2. M. Williams and M. Asgari, "Computation of Continuous-Energy Neutron Spectra with Discrete Ordinates Transport Theory," Nucl. Sci. Eng. 121, 173B201 (1995).

3. International Handbook of Evaluated Criticality Safety Benchmark Experiments, HEU-METFAST-035 Benchmark, The Uranium/Iron Benchmark Assembly: A 235U(93\%)/Iron Cylinder Reflected by Stainless Steel, NEA/NSC/DOC(95)03 (September 1998).

4. Neutronics Benchmarks for the Utilization of Mixed-oxide Fuel: Joint U.S./Russian Progress Report for Fiscal Year 1997, Volume 3 - Calculations Performed in the Russian Federation, ORNL/TM-13603/V3, June 1998 



\section{APPENDIX A \\ SCALE INPUT OF MIX-COMP-THERM-2 PROBLEM SET}





\section{APPENDIX A \\ SCALE INPUT OF MIX-COMP-THERM-2 PROBLEM SET}

This appendix contains the CENTRM inputs for the six MIX-COMP-THERM-02 cases, PNL30, PNL31, PNL32, PNL33, PNL34, and PNL35. The inputs can be changed to standard CSAS cases by replacing the $=$ CSASC 5 record with $=$ CSAS25. The input for the cases are listed below.

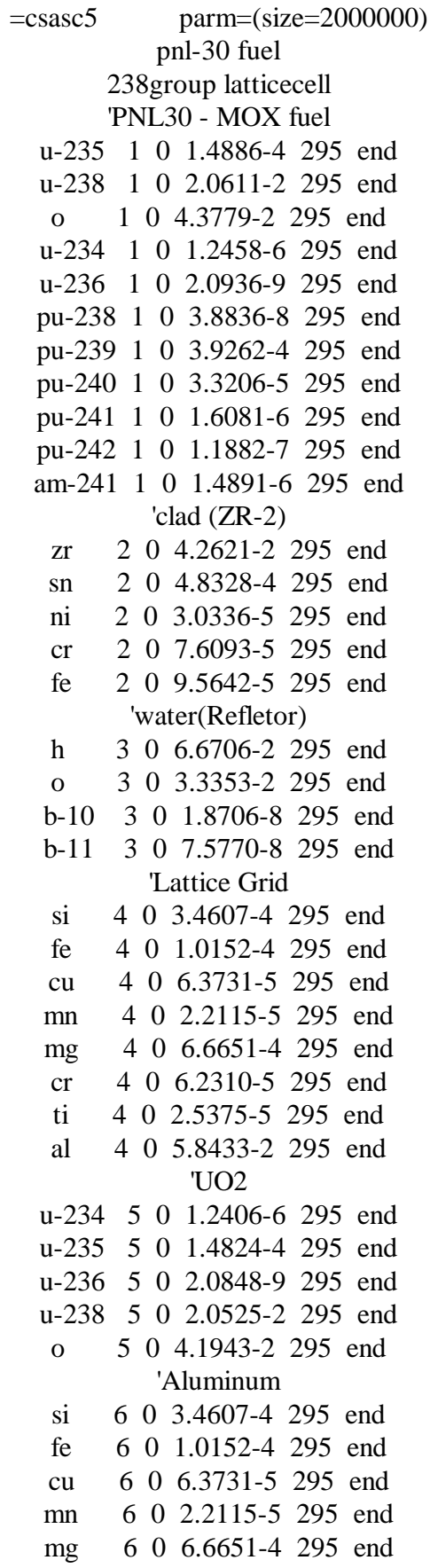

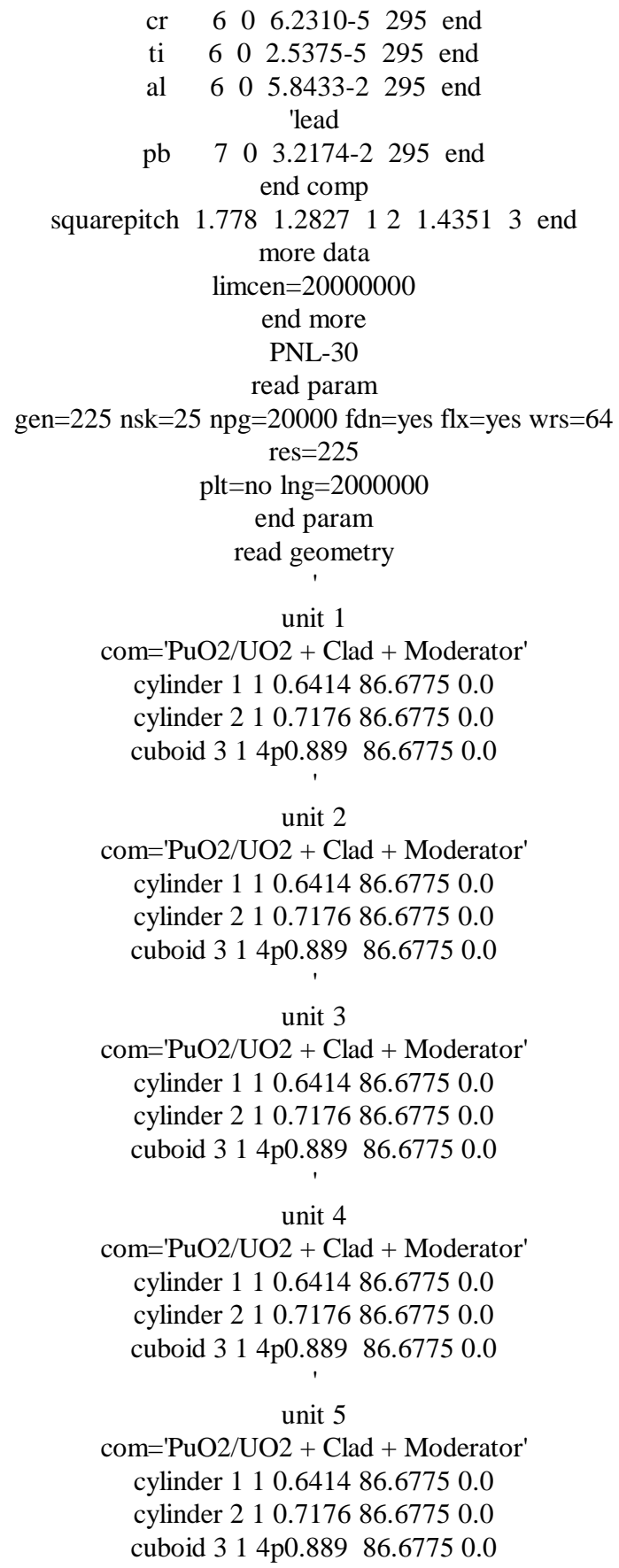




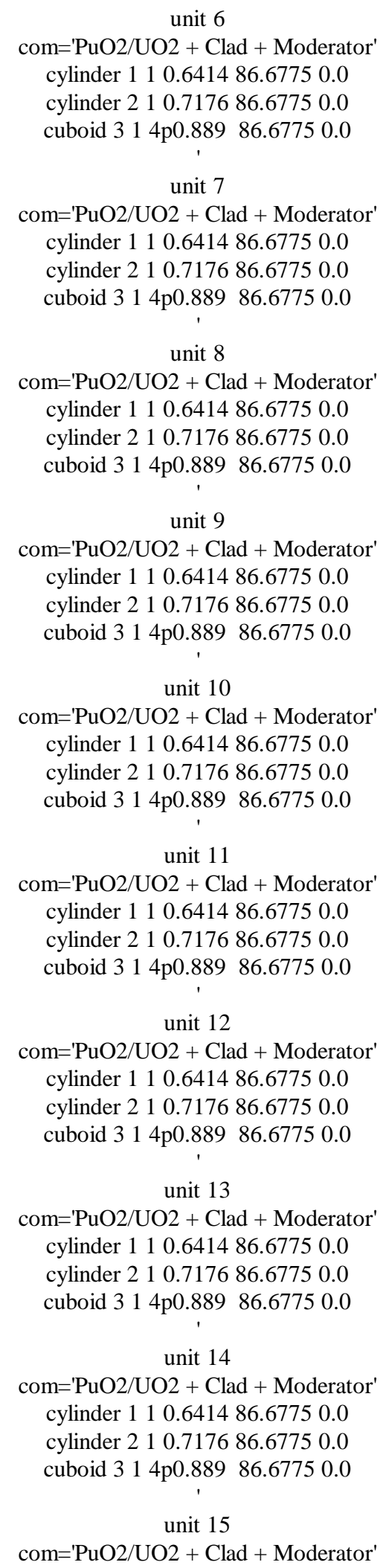

cylinder 110.641486 .67750 .0 cylinder 210.717686 .67750 .0 cuboid $314 \mathrm{p} 0.88986 .67750 .0$

$$
\text { unit } 16
$$

com='PuO2/UO2 + Clad + Moderator' cylinder 110.641486 .67750 .0 cylinder 210.717686 .67750 .0 cuboid $314 \mathrm{p} 0.88986 .67750 .0$

$$
\text { unit } 17
$$

com='PuO2/UO2 + Clad + Moderator' cylinder 110.641486 .67750 .0 cylinder 210.717686 .67750 .0 cuboid 314 p0.889 86.67750 .0

$$
\begin{gathered}
\text { unit } 18 \\
\text { com='PuO2/UO2 + Clad + Moderator' } \\
\text { cylinder } 110.641486 .67750 .0 \\
\text { cylinder } 210.717686 .67750 .0 \\
\text { cuboid } 314 \mathrm{p} 0.88986 .67750 .0 \\
\text { unit } 19 \\
\text { com='PuO2/UO2 + Clad + Moderator' } \\
\text { cylinder } 110.641486 .67750 .0 \\
\text { cylinder } 210.717686 .67750 .0 \\
\text { cuboid } 314 \text { 4 } 0.88986 .67750 .0 \\
\text { ' } \\
\text { unit } 20 \\
\text { com='PuO2/UO2 + Clad + Moderator' }
\end{gathered}
$$
cylinder 110.641486 .67750 .0 cylinder 210.717686 .67750 .0 cuboid $314 \mathrm{p} 0.88986 .67750 .0$

$$
\text { unit } 21
$$

com='PuO2/UO2 + Clad + Moderator' cylinder 110.641486 .67750 .0 cylinder 210.717686 .67750 .0 cuboid 314 p0.889 86.67750 .0

$$
\text { unit } 22
$$

com='PuO2/UO2 + Clad + Moderator' cylinder 110.641486 .67750 .0 cylinder 210.717686 .67750 .0 cuboid $314 \mathrm{p} 0.88986 .67750 .0$

$$
\begin{gathered}
\text { unit } 23 \\
\text { com='PuO2/UO2 + Clad + Moderator' } \\
\text { cylinder } 110.641486 .67750 .0 \\
\text { cylinder } 210.717686 .67750 .0 \\
\text { cuboid } 314 \text { p0.889 } 86.67750 .0 \\
\text { unit } 24 \\
\text { com='PuO2/UO2 + Clad + Moderator' } \\
\text { cylinder 1 } 110.641486 .67750 .0 \\
\text { cylinder 2 } 110.717686 .67750 .0 \\
\text { cuboid 3 } 14 \text { 4p0.889 } 86.67750 .0
\end{gathered}
$$




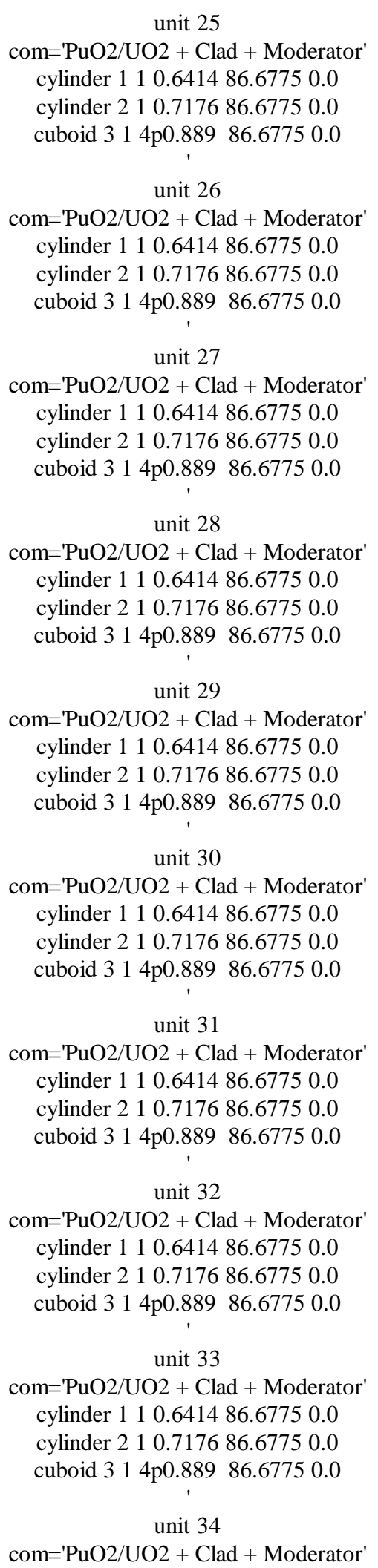

cylinder 110.641486 .67750 .0 cylinder 210.717686 .67750 .0 cuboid $314 \mathrm{p} 0.88986 .67750 .0$

$$
\text { unit } 35
$$

com='PuO2/UO2 + Clad + Moderator' cylinder 110.641486 .67750 .0 cylinder 210.717686 .67750 .0 cuboid $314 \mathrm{p} 0.88986 .67750 .0$

$$
\text { unit } 36
$$

com='PuO2/UO2 + Clad + Moderator' cylinder 110.641486 .67750 .0 cylinder 210.717686 .67750 .0 cuboid 31 4p0.889 86.67750 .0

$$
\text { unit } 37
$$

com='PuO2/UO2 + Clad + Moderator' cylinder 110.641486 .67750 .0 cylinder 210.717686 .67750 .0 cuboid 314 p0.889 86.67750 .0

$$
\text { unit } 38
$$

com='PuO2/UO2 + Clad + Moderator' cylinder 110.641486 .67750 .0 cylinder 210.717686 .67750 .0 cuboid $314 \mathrm{p} 0.88986 .67750 .0$

$$
\text { unit } 39
$$

com='PuO2/UO2 + Clad + Moderator' cylinder 110.641486 .67750 .0 cylinder 210.717686 .67750 .0 cuboid $314 \mathrm{p} 0.88986 .67750 .0$

$$
\text { unit } 40
$$

com='PuO2/UO2 + Clad + Moderator' cylinder 110.641486 .67750 .0 cylinder 210.717686 .67750 .0 cuboid 314 p0.889 86.67750 .0

$$
\text { unit } 41
$$

com='PuO2/UO2 + Clad + Moderator' cylinder 110.641486 .67750 .0 cylinder 210.717686 .67750 .0 cuboid $314 \mathrm{p} 0.88986 .67750 .0$

$$
\begin{gathered}
\text { unit } 42 \\
\text { com='PuO2/UO2 + Clad + Moderator' } \\
\text { cylinder } 110.641486 .67750 .0 \\
\text { cylinder } 210.717686 .67750 .0 \\
\text { cuboid } 314 \text { p0.889 } 86.67750 .0 \\
\text { unit } 43 \\
\text { com='PuO2/UO2 + Clad + Moderator' } \\
\text { cylinder 1 } 110.641486 .67750 .0 \\
\text { cylinder 2 } 110.717686 .67750 .0 \\
\text { cuboid 3 } 14 \text { 4p0.889 } 86.67750 .0
\end{gathered}
$$




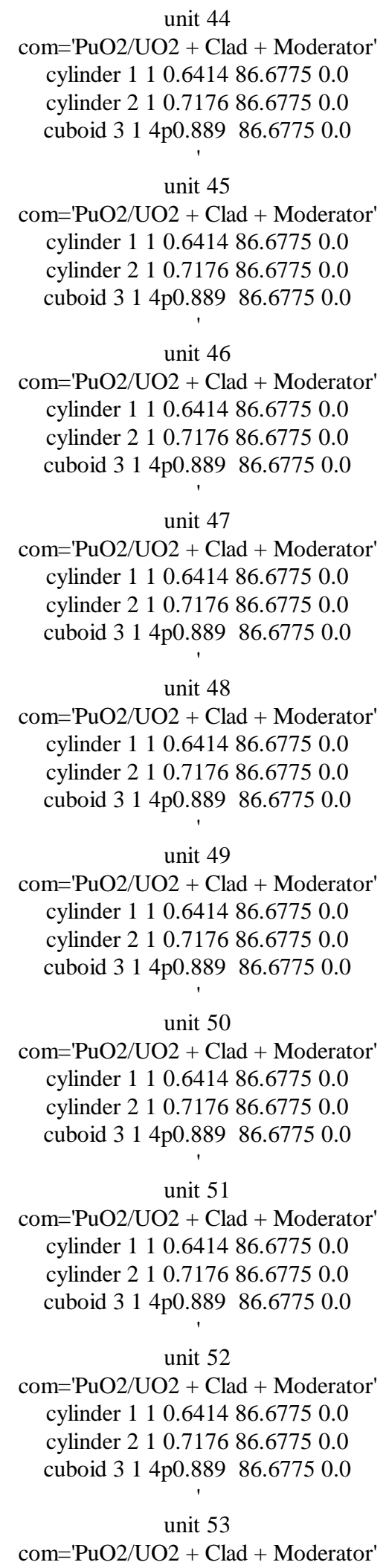

cylinder 110.641486 .67750 .0 cylinder 210.717686 .67750 .0 cuboid $314 \mathrm{p} 0.88986 .67750 .0$

$$
\text { unit } 54
$$

com='PuO2/UO2 + Clad + Moderator' cylinder 110.641486 .67750 .0 cylinder 210.717686 .67750 .0 cuboid $314 \mathrm{p} 0.88986 .67750 .0$

$$
\text { unit } 55
$$

com='PuO2/UO2 + Clad + Moderator' cylinder 110.641486 .67750 .0 cylinder 210.717686 .67750 .0 cuboid 314 p0.889 86.67750 .0 unit 56
com='PuO2/UO2 + Clad + Moderator' cylinder 110.641486 .67750 .0 cylinder 210.717686 .67750 .0 cuboid $314 \mathrm{p} 0.88986 .67750 .0$

$$
\text { unit } 57
$$

com='PuO2/UO2 + Clad + Moderator' cylinder 110.641486 .67750 .0 cylinder 210.717686 .67750 .0 cuboid $314 \mathrm{p} 0.88986 .67750 .0$

$$
\text { unit } 58
$$

com='PuO2/UO2 + Clad + Moderator' cylinder 110.641486 .67750 .0 cylinder 210.717686 .67750 .0 cuboid $314 \mathrm{p} 0.88986 .67750 .0$

$$
\text { unit } 59
$$

com='PuO2/UO2 + Clad + Moderator' cylinder 110.641486 .67750 .0 cylinder 210.717686 .67750 .0 cuboid 314 p0.889 86.67750 .0

$$
\text { unit } 60
$$

com='PuO2/UO2 + Clad + Moderator' cylinder 110.641486 .67750 .0 cylinder 210.717686 .67750 .0 cuboid $314 \mathrm{p} 0.88986 .67750 .0$

$$
\begin{gathered}
\text { unit } 61 \\
\text { com='PuO2/UO2 + Clad + Moderator' } \\
\text { cylinder } 110.641486 .67750 .0 \\
\text { cylinder } 210.717686 .67750 .0 \\
\text { cuboid } 314 \text { p0.889 } 86.67750 .0 \\
\text { unit } 62 \\
\text { com='PuO2/UO2 + Clad + Moderator' } \\
\text { cylinder 1 } 110.641486 .67750 .0 \\
\text { cylinder 2 } 110.717686 .67750 .0 \\
\text { cuboid 3 } 14 \text { 4p0.889 } 86.67750 .0
\end{gathered}
$$




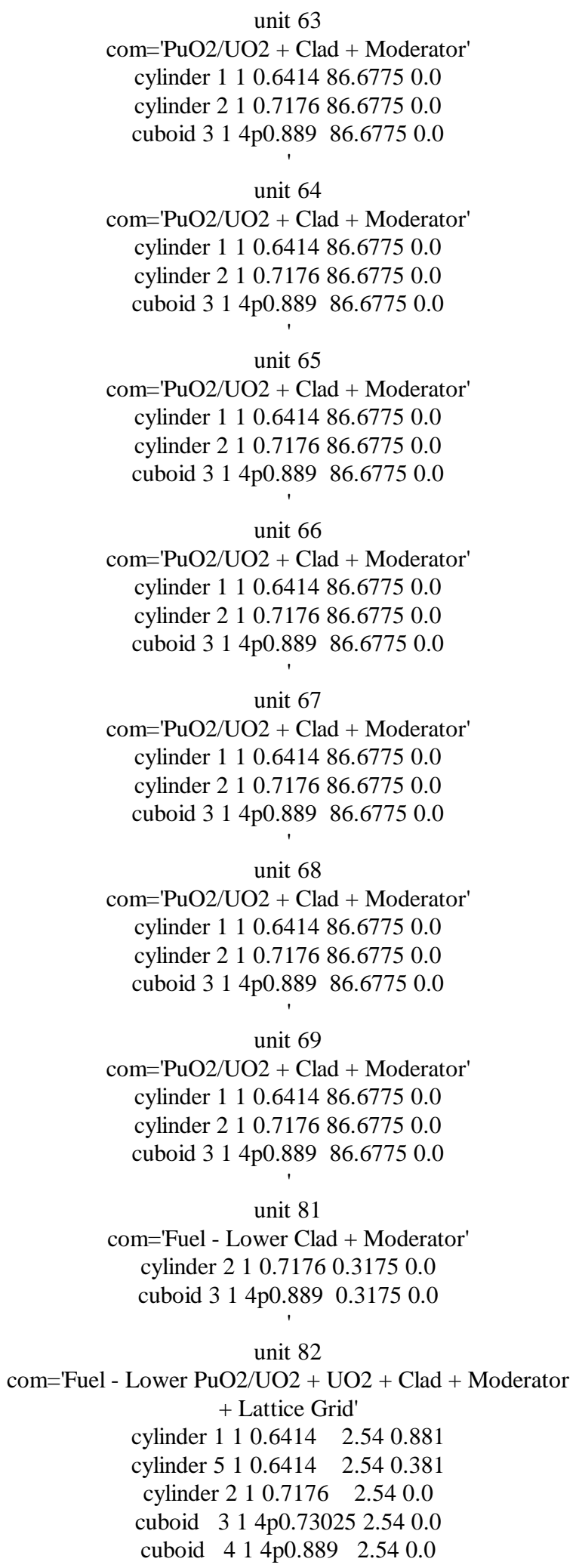

unit 83

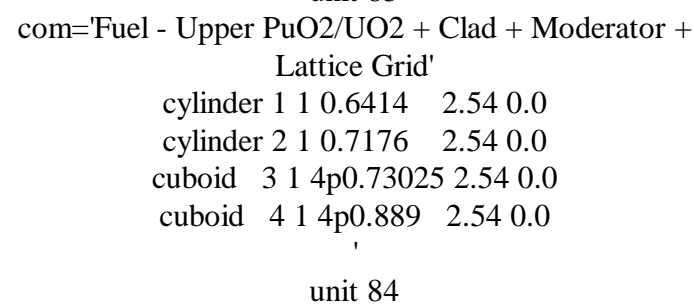

'Lower Cladding and Moderator 858585858581818181818181 1b11 858585858181818181818181 lb11 858585818181818181818181 lb11 858581818181818181818181 lb11 858181818181818181818181 lb11 818181818181818181818181 1b11 818181818181818181818181 lb11 818181818181818181818181 lb11 
818181818181818181818181 1b11 818181818181818181818181 lb11 818181818181818181818181 1b11 818181818181818181818181 1b264 'Lower Lattice Grid

$868686868682828282828282 \quad 1 b 11$ $868686868282828282828282 \quad 1 b 11$ $\begin{array}{lll}868686828282828282828282 & 1 b 11\end{array}$ $\begin{array}{llllllll}868682828282828282828282 & 1 b 11\end{array}$

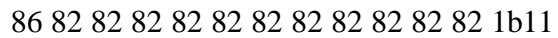
828282828282828282828282 1b11 $\begin{array}{ll}828282828282828282828282 \quad 1 b 11 & 0\end{array}$

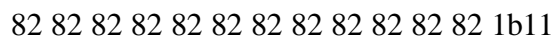
$828282828282828282828282 \quad 1 b 11$

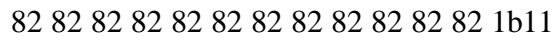
828282828282828282828282 1b11 828282828282828282828282 1b264

'Fuel and Reflector

909090909012334567 1b11 $90909090 \quad 8 \quad 9101112131415 \quad 1 b 11$ $909090161718192021222324 \quad 1 b 11$ 909016252627282930313233 1b11 $90 \quad 817263435363738394041 \quad 1 b 11$

$1918273542434445464748 \quad 1 b 11$ $210192836434950515253541 b 11$ $311202937445055565758591 \mathrm{~b} 11$ 412213038455156606162631 b11 $513223139465257616465661 b 11$ 614233240475358626567681 b11 $715243341485459636668691 \mathrm{~b} 264$ 'Upper Lattice Grid

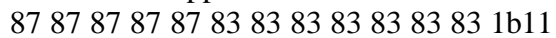
$878787878383838383838383 \quad 1 b 11$

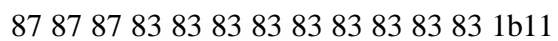
$\begin{array}{lllllllll}878783838383838383838383 & 1 b 11\end{array}$ $\begin{array}{lllllllll}878383838383838383838383 & 1 b 11\end{array}$

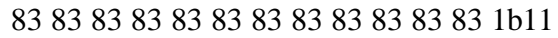
$\begin{array}{llllllllll}838383838383838383838383 & 1 b 11\end{array}$

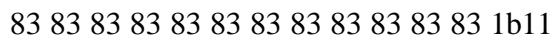
$\begin{array}{llllllllll}838383838383838383838383 & 1 b 11\end{array}$

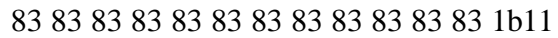
838383838383838383838383 1b11 838383838383838383838383 1b264 'Upper Cladding and Moderator 888888888884848484848484 1b11 888888888484848484848484 1b11 888888848484848484848484 1b11 888884848484848484848484 1b11 888484848484848484848484 1b11 848484848484848484848484 1b11 848484848484848484848484 1b11 848484848484848484848484 1b11 848484848484848484848484 1b11 848484848484848484848484 1b11 848484848484848484848484 1b11 848484848484848484848484 1b264 end fill

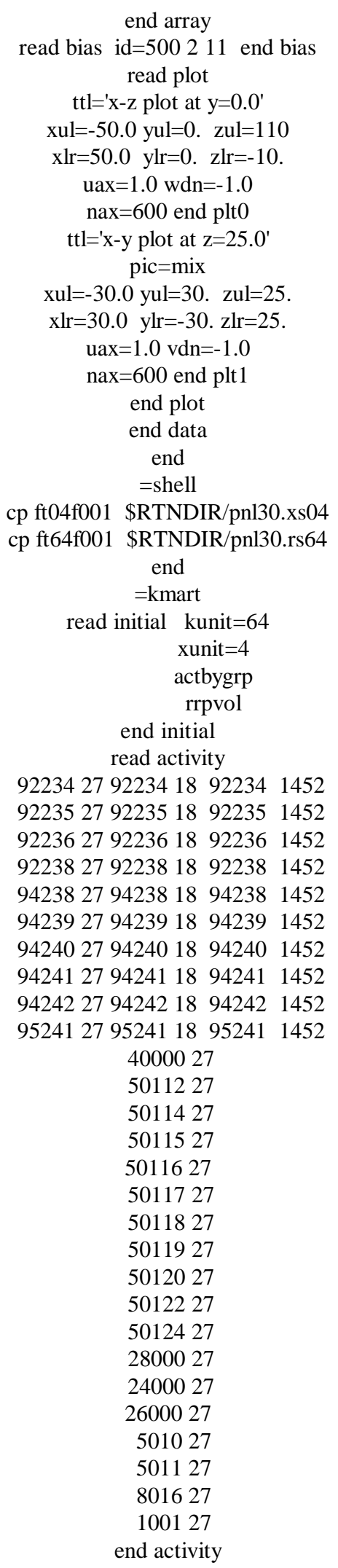




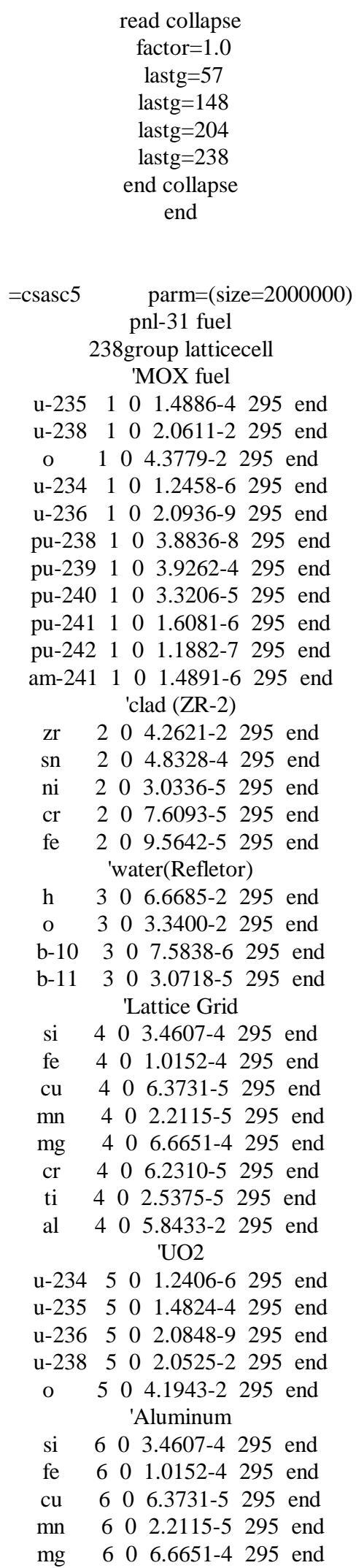

read collapse

lastg $=148$

lastg $=204$

lastg $=238$

collapse

end

pnl-31 fuel

'MOX fuel

u-235 $11001.4886-4295$ end

10 2.0611-2 295 end

u-234 10 1.2458-6 295 end u-236 $11002.0936-9295$ end pu-238 $110 \quad 3.8836-8 \quad 295$ end pu-239 $1 \quad 0 \quad 3.9262-4295$ end pu-240 110 3.3206-5 295 end pu-241 1 0 $1.6081-6295$ end pu-242 100 1.1882-7 295 end 'clad (ZR-2)

20 4.2621-2 295 end

ni $2003.0336-5295$ end

cr $2007.6093-5295$ end 'water(Refletor)

b-10 $3007.5838-6295$ end 'Lattice Grid

si $\quad \begin{array}{lllll}4 & 0 & 3.4607-4 & 295 & \text { end }\end{array}$

cu $\quad \begin{array}{lllll}4 & 0 & 6.3731-5 & 295 & \text { end }\end{array}$

mn $4002.2115-5295$ end

6.6651-4 295 end

ti $4 \quad \begin{array}{llll}4 & 2.5375-5 & 295 & \text { end }\end{array}$

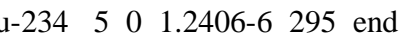
u-235 $5 \begin{array}{llll}5 & 0 & 1.4824-4 & 295 \\ \text { end }\end{array}$

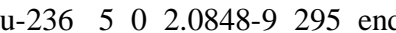

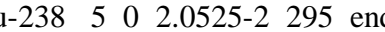
'Aluminum

si $\quad 6 \quad \begin{array}{lllll}6 & 0 & 3.4607-4 & 295 & \text { end }\end{array}$

mg $\quad \begin{array}{lllll}6 & 0 & 6.6651-4 & 295 & \text { end }\end{array}$ cr $\quad \begin{array}{llll}6 & 0 & 6.2310-5 & 295 \\ & \text { end }\end{array}$

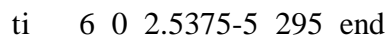

al $\quad \begin{array}{lllll}6 & 0 & 5.8433-2 & 295 & \text { end }\end{array}$ 'lead

pb $\quad \begin{array}{llll}7 & 0 & 3.2174-2 & 295\end{array}$ end end comp

squarepitch $1.778 \quad 1.2827 \quad 121.43513$ end more data

limcen $=20000000$

end more

PNL-31

read param

gen=225 nsk=25 npg=20000 fdn=yes flx=yes wrs $=64$ res $=225$

plt=no lng $=2000000$

end param

read geometry

unit 1

com $=' \mathrm{PuO} 2 / \mathrm{UO} 2+$ Clad + Moderator' cylinder 110.641486 .67750 .0

cylinder 210.717686 .67750 .0

cuboid $314 \mathrm{p} 0.88986 .67750 .0$

unit 2

com='PuO2/UO2 + Clad + Moderator' cylinder 110.641486 .67750 .0

cylinder 210.717686 .67750 .0

cuboid $314 \mathrm{p} 0.88986 .67750 .0$

unit 3

com='PuO2/UO2 + Clad + Moderator' cylinder 110.641486 .67750 .0

cylinder 210.717686 .67750 .0

cuboid $314 \mathrm{p} 0.88986 .67750 .0$

unit 4

com='PuO2/UO2 + Clad + Moderator' cylinder 110.641486 .67750 .0

cylinder 210.717686 .67750 .0

cuboid 314 p0.889 86.67750 .0

unit 5

com='PuO2/UO2 + Clad + Moderator' cylinder 110.641486 .67750 .0

cylinder 210.717686 .67750 .0

cuboid $314 \mathrm{p} 0.88986 .67750 .0$

unit 6

com='PuO2/UO2 + Clad + Moderator' cylinder 110.641486 .67750 .0

cylinder 210.717686 .67750 .0

cuboid $314 \mathrm{p} 0.88986 .67750 .0$

unit 7

com $=$ 'PuO2/UO2 + Clad + Moderator' cylinder 110.641486 .67750 .0 


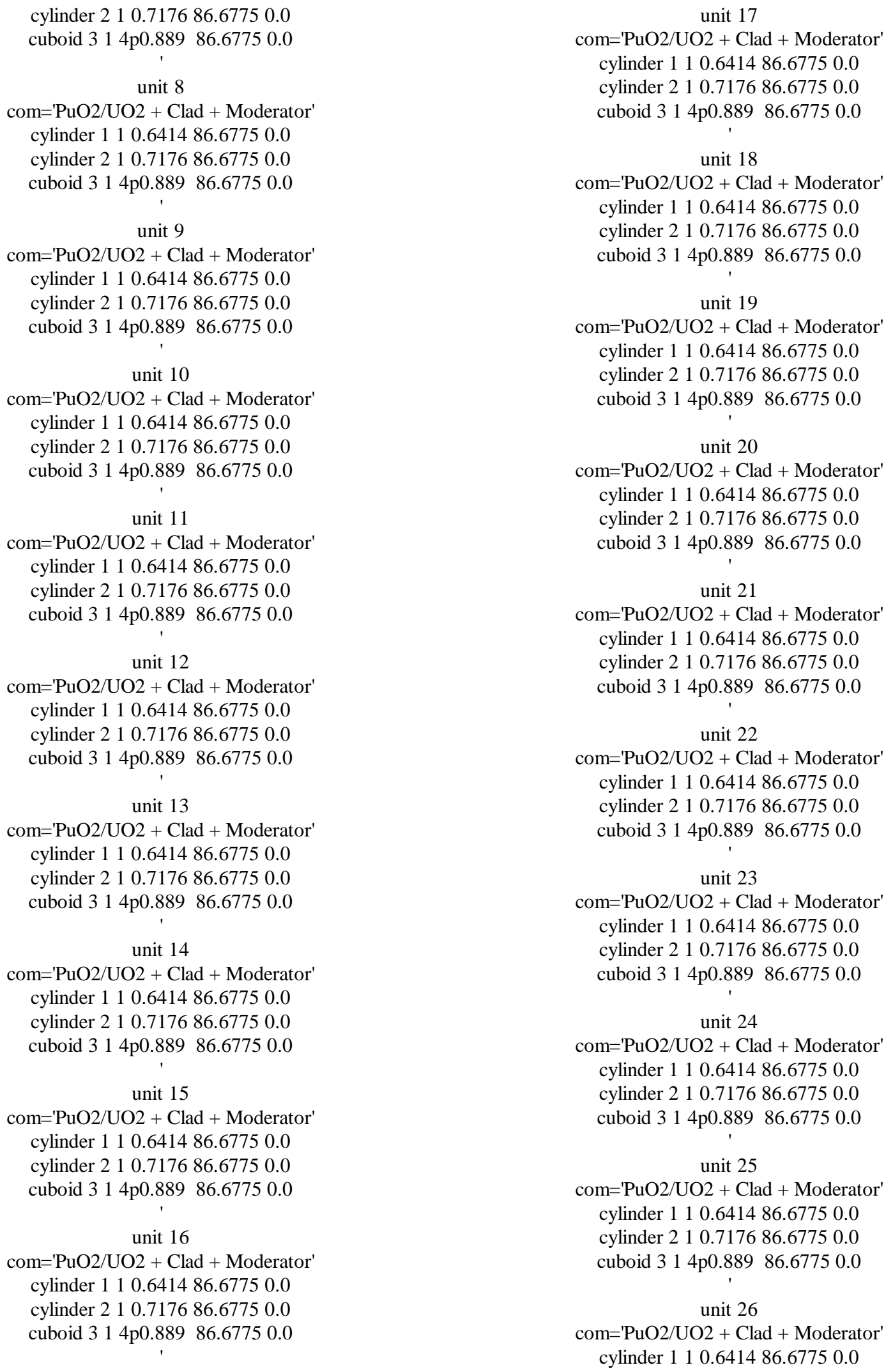

unit 17 
cylinder 210.717686 .67750 .0 cuboid 314 p0.889 86.67750 .0

unit 27

com='PuO2/UO2 + Clad + Moderator' cylinder 110.641486 .67750 .0 cylinder 210.717686 .67750 .0 cuboid $314 \mathrm{p} 0.88986 .67750 .0$

$$
\text { unit } 28
$$

com='PuO2/UO2 + Clad + Moderator' cylinder 110.641486 .67750 .0 cylinder 210.717686 .67750 .0 cuboid $314 \mathrm{p} 0.88986 .67750 .0$

unit 29

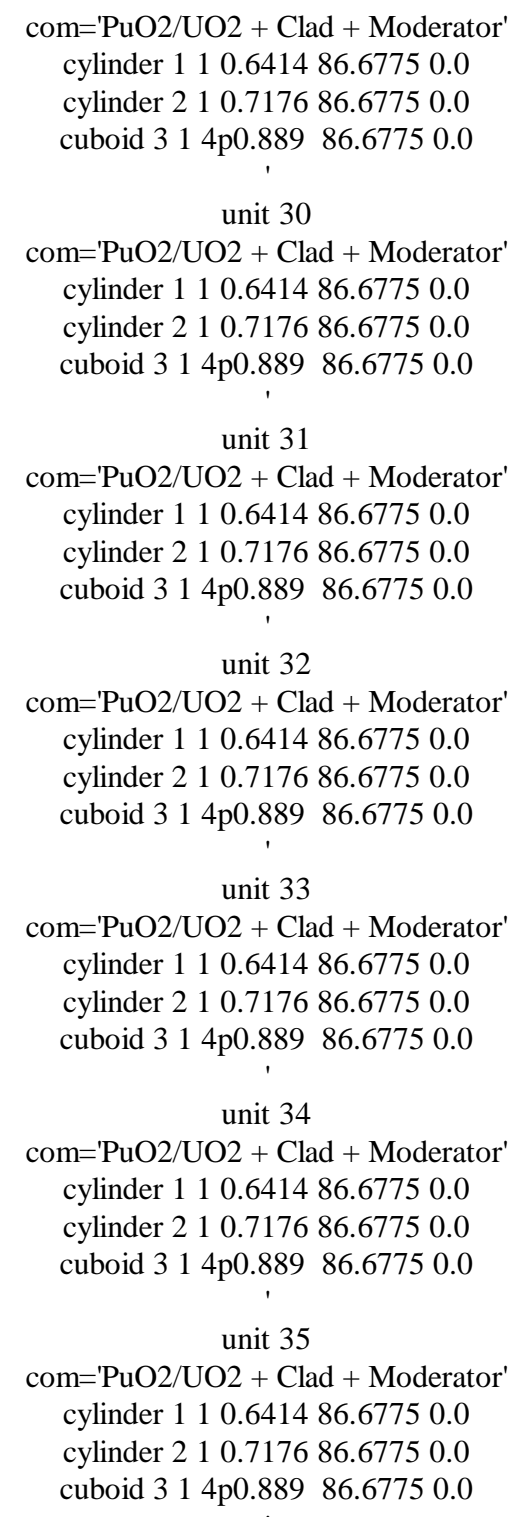

unit 36

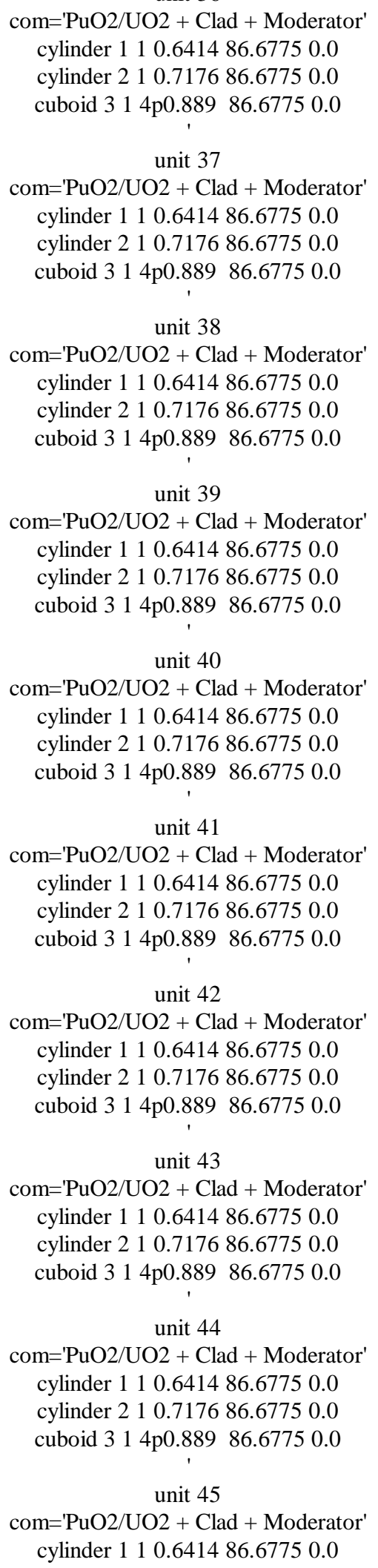




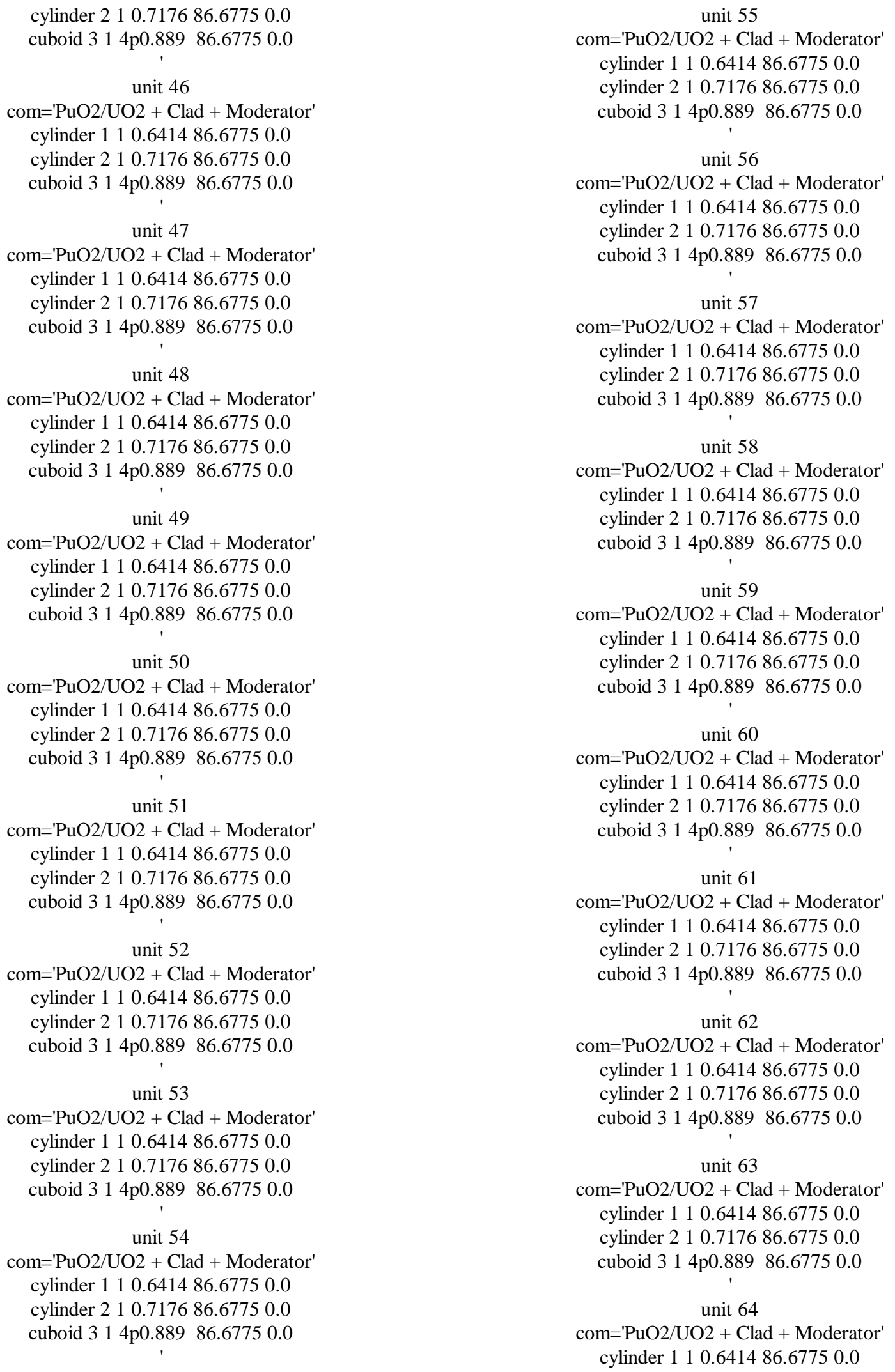




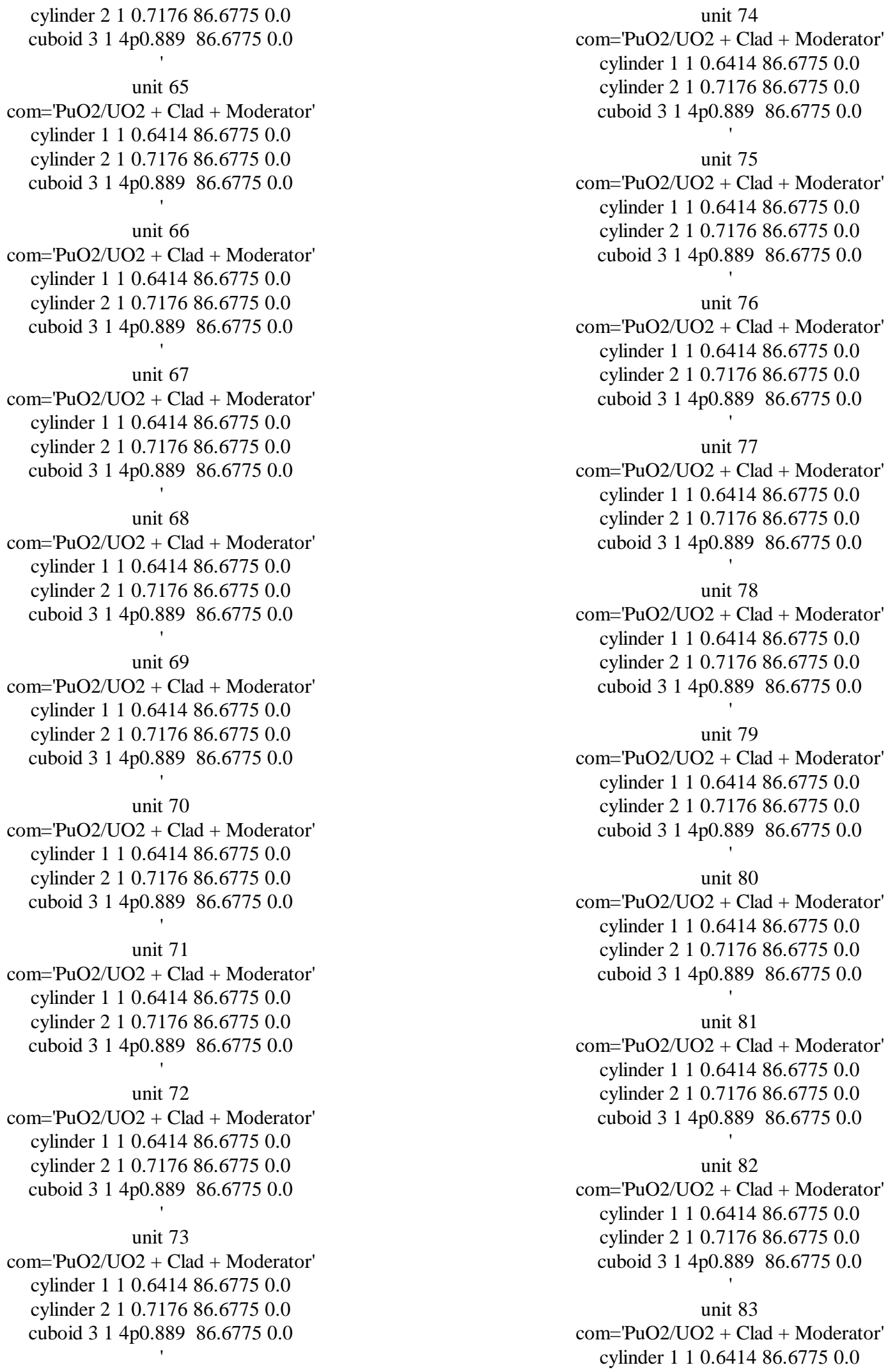




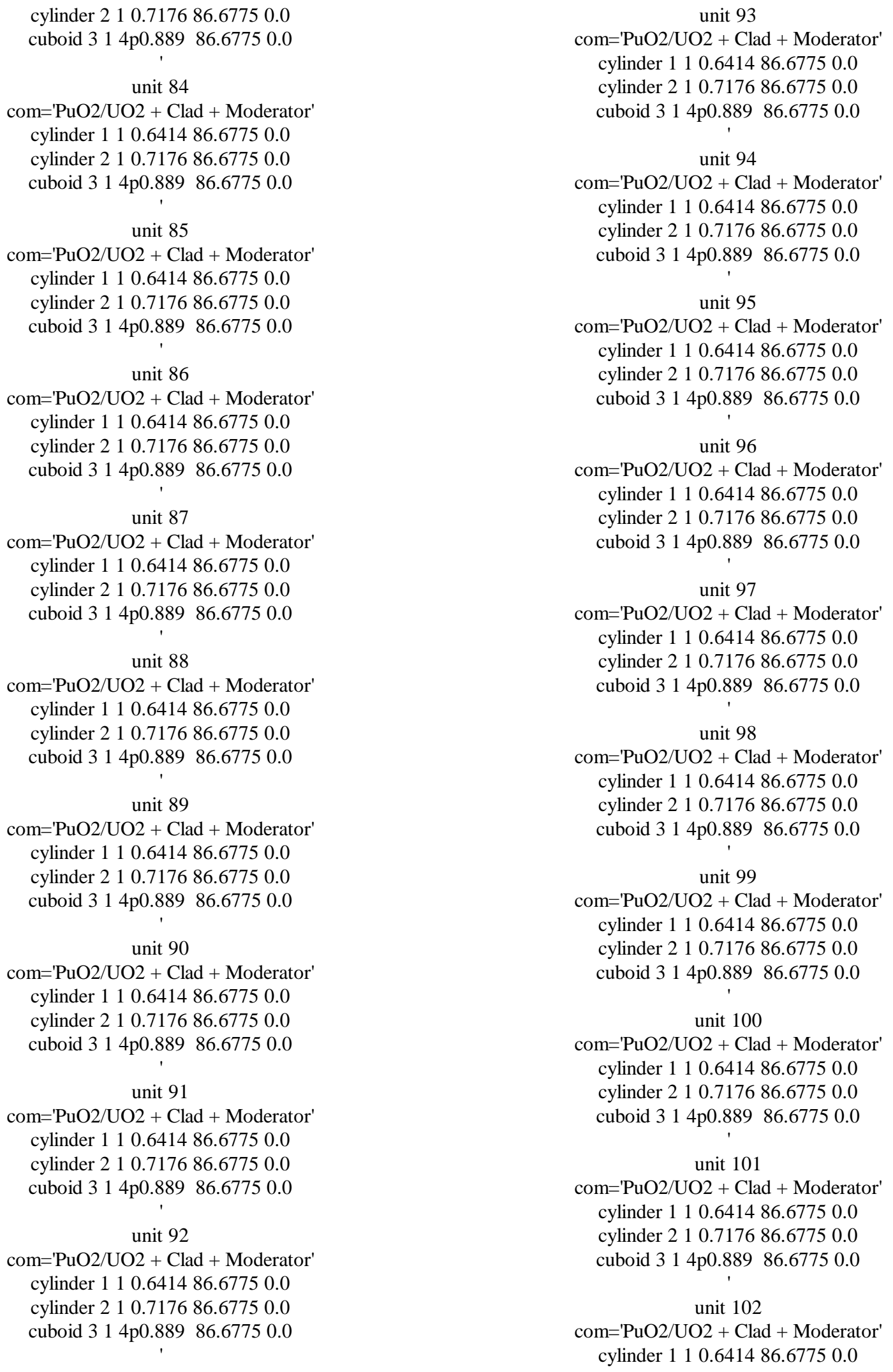


cylinder 210.717686 .67750 .0

cuboid $314 \mathrm{p} 0.88986 .67750 .0$

unit 103

com='PuO2/UO2 + Clad + Moderator' cylinder 110.641486 .67750 .0 cylinder 210.717686 .67750 .0 cuboid $314 \mathrm{p} 0.88986 .67750 .0$

$$
\text { unit } 104
$$

com='PuO2/UO2 + Clad + Moderator' cylinder 110.641486 .67750 .0 cylinder 210.717686 .67750 .0 cuboid 31 4p0.889 86.67750 .0

unit 105

com='PuO2/UO2 + Clad + Moderator' cylinder 110.641486 .67750 .0 cylinder 210.717686 .67750 .0 cuboid $314 \mathrm{p} 0.88986 .67750 .0$

unit 106

com='PuO2/UO2 + Clad + Moderator' cylinder 110.641486 .67750 .0 cylinder 210.717686 .67750 .0 cuboid $314 \mathrm{p} 0.88986 .67750 .0$

unit 107

com='PuO2/UO2 + Clad + Moderator' cylinder 110.641486 .67750 .0 cylinder 210.717686 .67750 .0 cuboid $314 \mathrm{p} 0.88986 .67750 .0$

unit 108

com='PuO2/UO2 + Clad + Moderator' cylinder 110.641486 .67750 .0 cylinder 210.717686 .67750 .0 cuboid $314 \mathrm{p} 0.88986 .67750 .0$

unit 109

com='PuO2/UO2 + Clad + Moderator' cylinder 110.641486 .67750 .0 cylinder 210.717686 .67750 .0 cuboid $314 \mathrm{p} 0.88986 .67750 .0$

unit 111

com='Fuel - Lower Clad + Moderator' cylinder 210.71760 .31750 .0 cuboid $314 \mathrm{p} 0.8890 .31750 .0$

unit 112

com='Fuel - Lower PuO2/UO2 + UO2 + Clad + Moderator + Lattice Grid'

cylinder $110.6414 \quad 2.540 .881$

cylinder $510.6414 \quad 2.540 .381$

cylinder $210.7176 \quad 2.540 .0$

cuboid $314 \mathrm{p} 0.730252 .540 .0$ cuboid $414 \mathrm{p} 0.889 \quad 2.540 .0$

unit 113

com='Fuel - Upper PuO2/UO2 + Clad + Moderator + Lattice Grid'

cylinder $110.6414 \quad 2.540 .0$

cylinder $210.7176 \quad 2.540 .0$

cuboid $314 \mathrm{p} 0.730252 .540 .0$

cuboid $414 \mathrm{p} 0.8892 .540 .0$

unit 114

com='Fuel - Upper PuO2/UO2 + Clad + Moderator' cylinder 110.64140 .06350 .0 cylinder 210.71760 .06350 .0 cuboid $314 \mathrm{p} 0.8890 .06350 .0$

unit 115

com='Reflector - Lower Moderator' cuboid $314 \mathrm{p} 0.8890 .31750 .0$

unit 116

com='Reflector - Lower Moderator + Lattice Grid' cuboid $314 \mathrm{p} 0.730252 .540 .0$

cuboid $414 \mathrm{p} 0.8892 .540 .0$

unit 117

com='Reflector - Upper Moderator + Lattice Grid' cuboid $314 \mathrm{p} 0.730252 .540 .0$ cuboid $414 \mathrm{p} 0.8892 .540 .0$

unit 118

com='Reflector - Upper Moderator' cuboid $314 \mathrm{p} 0.8890 .06350 .0$

unit 120

com='Moderator'

cuboid 314 p0.889 86.67750.0

global unit 130

com='Cells in assembly' array $1-27.559-27.5590$

cuboid $614 \mathrm{p} 27.559 \quad 92.9640 \quad-2.8575$

cuboid $714 \mathrm{p} 27.559 \quad 93.9165-2.8575$

replicate $326 * 3.04$

replicate $364 * 3.01 .4623 .01$

replicate $374 * 3.00 .0 \quad 3.05$

end geometry

read array

ara $=1$ nux $=31$ nuy=31 nuz=5

fill

'Lower Cladding and Moderator

115115115115115115115115115115115115111 $1111111111 \mathrm{~b} 15$

115115115115115115115115115111111111111 $1111111111 \mathrm{~b} 15$

115115115115115115115111111111111111111 $1111111111 \mathrm{~b} 15$ 
115115115115115111111111111111111111111 $1111111111 \mathrm{~b} 15$

115115115115111111111111111111111111111 $1111111111 \mathrm{~b} 15$

115115115111111111111111111111111111111 $1111111111 \mathrm{~b} 15$

115115115111111111111111111111111111111 $1111111111 \mathrm{~b} 15$

115115111111111111111111111111111111111 $1111111111 \mathrm{~b} 15$

115115111111111111111111111111111111111 $1111111111 \mathrm{~b} 15$

115111111111111111111111111111111111111 $1111111111 \mathrm{~b} 15$

115111111111111111111111111111111111111 $1111111111 \mathrm{~b} 15$

115111111111111111111111111111111111111 $1111111111 \mathrm{~b} 15$

111111111111111111111111111111111111111 $1111111111 \mathrm{~b} 15$

111111111111111111111111111111111111111 $1111111111 \mathrm{~b} 15$

111111111111111111111111111111111111111 $1111111111 \mathrm{~b} 15$

111111111111111111111111111111111111111 $1111111111 \mathrm{~b} 480$ 'Lower Lattice Grid

116116116116116116116116116116116116112 $1121121121 \mathrm{~b} 15$

116116116116116116116116116112112112112 $1121121121 \mathrm{~b} 15$

116116116116116116116112112112112112112 $1121121121 \mathrm{~b} 15$

116116116116116112112112112112112112112 $1121121121 \mathrm{~b} 15$

116116116116112112112112112112112112112 $1121121121 \mathrm{~b} 15$

116116116112112112112112112112112112112 $1121121121 \mathrm{~b} 15$

116116116112112112112112112112112112112 $1121121121 \mathrm{~b} 15$

116116112112112112112112112112112112112 $1121121121 \mathrm{~b} 15$

116116112112112112112112112112112112112 $1121121121 \mathrm{~b} 15$

116112112112112112112112112112112112112 $1121121121 \mathrm{~b} 15$

116112112112112112112112112112112112112 $1121121121 \mathrm{~b} 15$

116112112112112112112112112112112112112 $1121121121 \mathrm{~b} 15$

112112112112112112112112112112112112112 $1121121121 \mathrm{~b} 15$

112112112112112112112112112112112112112 $1121121121 \mathrm{~b} 15$

112112112112112112112112112112112112112 $1121121121 \mathrm{~b} 15$
112112112112112112112112112112112112112 $1121121121 \mathrm{~b} 480$

'Fuel and Reflector

$120120120120120120120120120120120120 \quad 12$ $3 \quad 4 \quad 1 b 15$

$120120120120120120120120120 \quad 5 \quad 6 \quad 7 \quad 8 \quad 9 \quad 10$ $111 \mathrm{~b} 15$

$12012012012012012012012 \quad 13 \quad 14 \quad 15 \quad 16 \quad 17 \quad 18$ $19201 \mathrm{~b} 15$

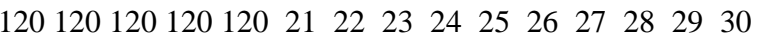
$311 \mathrm{~b} 15$

$120120120120 \quad 32 \quad 33 \quad 34 \quad 35 \quad 36 \quad 37 \quad 38 \quad 3940 \quad 41 \quad 42$ $431 \mathrm{~b} 15$

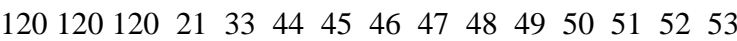
$541 \mathrm{~b} 15$

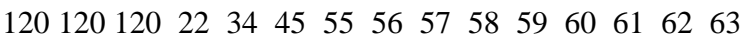
$641 \mathrm{~b} 15$

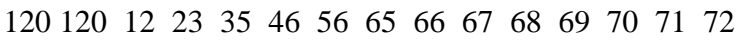
$731 \mathrm{~b} 15$

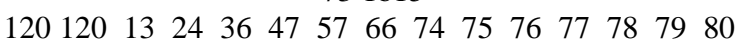
$811 \mathrm{~b} 15$

$\begin{array}{llllllllllllllll}120 & 5 & 14 & 25 & 37 & 48 & 58 & 67 & 75 & 82 & 83 & 84 & 85 & 86 & 87 & 88\end{array}$ $1 \mathrm{~b} 15$

$\begin{array}{llllllllllllllll}120 & 6 & 15 & 26 & 38 & 49 & 59 & 68 & 76 & 83 & 89 & 90 & 91 & 92 & 93 & 94\end{array}$ $1 \mathrm{~b} 15$

$\begin{array}{llllllllllllllll}120 & 7 & 16 & 27 & 39 & 50 & 60 & 69 & 77 & 84 & 90 & 95 & 96 & 97 & 98 & 99\end{array}$ $1 \mathrm{~b} 15$

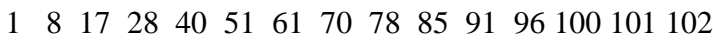
$1031 \mathrm{~b} 15$

$\begin{array}{lllllllllllllll}2 & 9 & 18 & 29 & 41 & 52 & 62 & 71 & 79 & 86 & 92 & 97 & 101 & 104 & 105\end{array}$ $1061 \mathrm{~b} 15$

$\begin{array}{lllllllllllllll}3 & 10 & 19 & 30 & 42 & 53 & 63 & 72 & 80 & 87 & 93 & 98 & 102 & 105 & 107\end{array}$ $1081 \mathrm{~b} 15$

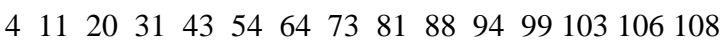
$1091 \mathrm{~b} 480$

'Upper Lattice Grid

117117117117117117117117117117117117113 $1131131131 \mathrm{~b} 15$

117117117117117117117117117113113113113 $1131131131 b 15$

117117117117117117117113113113113113113 $1131131131 \mathrm{~b} 15$

117117117117117113113113113113113113113 $1131131131 \mathrm{~b} 15$

117117117117113113113113113113113113113 $1131131131 \mathrm{~b} 15$

117117117113113113113113113113113113113 $1131131131 \mathrm{~b} 15$

117117117113113113113113113113113113113 $1131131131 \mathrm{~b} 15$

117117113113113113113113113113113113113 $1131131131 \mathrm{~b} 15$

117117113113113113113113113113113113113 $1131131131 \mathrm{~b} 15$

117113113113113113113113113113113113113 $1131131131 \mathrm{~b} 15$

117113113113113113113113113113113113113 
$1131131131 \mathrm{~b} 15$

117113113113113113113113113113113113113 $1131131131 \mathrm{~b} 15$

113113113113113113113113113113113113113 $1131131131 \mathrm{~b} 15$

113113113113113113113113113113113113113 $1131131131 \mathrm{~b} 15$

113113113113113113113113113113113113113 $1131131131 \mathrm{~b} 15$

113113113113113113113113113113113113113 $1131131131 \mathrm{~b} 480$

'Upper Cladding and Moderator

114114114114114114114114114114114114118 $1181181181 \mathrm{~b} 15$

114114114114114114114114114118118118118 $1181181181 \mathrm{~b} 15$

114114114114114114114118118118118118118 $1181181181 \mathrm{~b} 15$

114114114114114118118118118118118118118 $1181181181 \mathrm{~b} 15$

114114114114118118118118118118118118118 $1181181181 \mathrm{~b} 15$

114114114118118118118118118118118118118 $1181181181 \mathrm{~b} 15$

114114114118118118118118118118118118118 $1181181181 \mathrm{~b} 15$

114114118118118118118118118118118118118 $1181181181 \mathrm{~b} 15$

114114118118118118118118118118118118118 $1181181181 \mathrm{~b} 15$

114118118118118118118118118118118118118 $1181181181 \mathrm{~b} 15$

114118118118118118118118118118118118118 $1181181181 \mathrm{~b} 15$

114118118118118118118118118118118118118 $1181181181 \mathrm{~b} 15$

118118118118118118118118118118118118118 $1181181181 \mathrm{~b} 15$

118118118118118118118118118118118118118 $1181181181 \mathrm{~b} 15$

118118118118118118118118118118118118118 $1181181181 \mathrm{~b} 15$

118118118118118118118118118118118118118 $1181181181 \mathrm{~b} 480$ end fill end array

read bias id=500 211 end bias read plot

$\mathrm{ttl}={ }^{\prime} \mathrm{x}-\mathrm{z}$ plot at $\mathrm{y}=0.0^{\prime}$

$\mathrm{xul}=-50.0 \mathrm{yul}=0 . \mathrm{zul}=110$

$\mathrm{xlr}=50.0$ ylr $=0 . \quad \mathrm{zlr}=-10$.

$\mathrm{uax}=1.0 \mathrm{wdn}=-1.0$

nax $=600$ end plt0

$\mathrm{ttl}=$ 'x-y plot at $\mathrm{z}=25.0^{\prime}$ pic $=$ mix

xul=-30.0 yul $=30 . \quad \mathrm{zul}=25$.

$\mathrm{xlr}=30.0$ ylr=-30. $\mathrm{zlr}=25$.

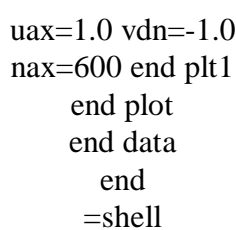

cp ft04f001 \$RTNDIR/pnl31.xs04 cp ft64f001 \$RTNDIR/pnl31.rs64 end $=\mathrm{kmart}$ read initial kunit $=64$ xunit $=4$ actbygrp rrpvol

end initial read activity 92234279223418922341452 $9223527922351892235 \quad 1452$ 92236279223618922361452 $9223827922381892238 \quad 1452$ 94238279423818942381452 $9423927942391894239 \quad 1452$ $9424027942401894240 \quad 1452$ $9424127942411894241 \quad 1452$ $9424227942421894242 \quad 1452$ 95241279524118952411452

4000027

5011227

5011427

5011527

5011627

5011727

5011827

5011927

5012027

5012227

5012427

2800027

2400027

2600027

501027

501127

801627

100127

end activity read collapse factor $=1.0$ lastg $=57$ lastg $=148$ lastg $=204$ last $\mathrm{g}=238$ end collapse end

$=\operatorname{csasc} 5 \quad$ parm $=($ size $=1000000)$ pnl-32 fuel 


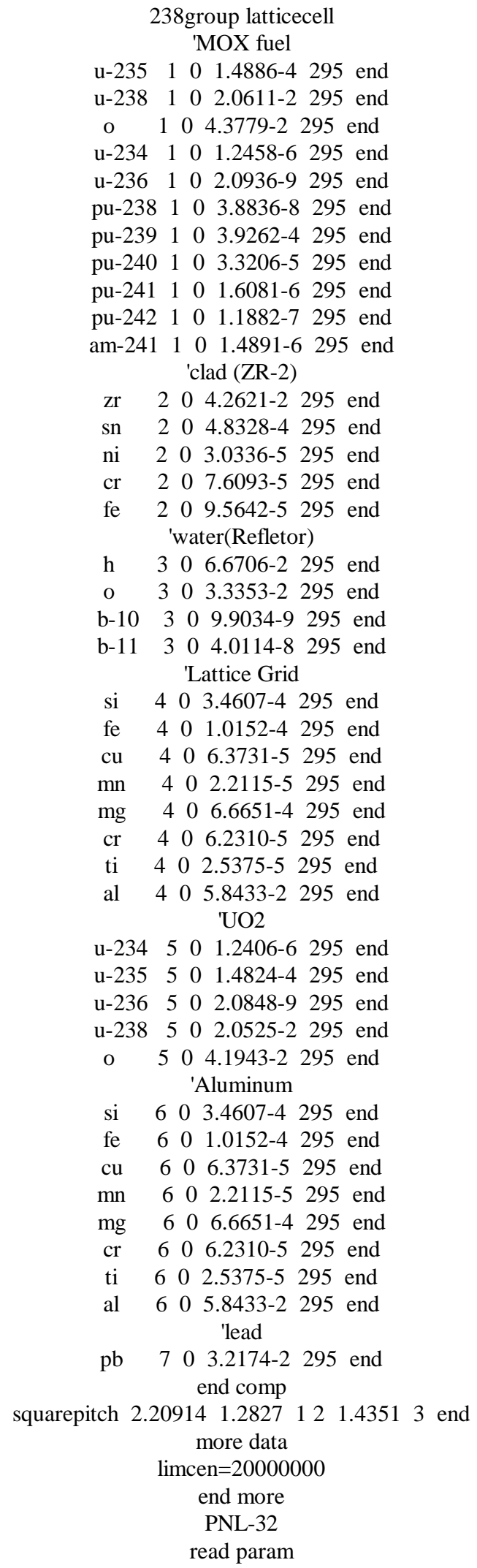

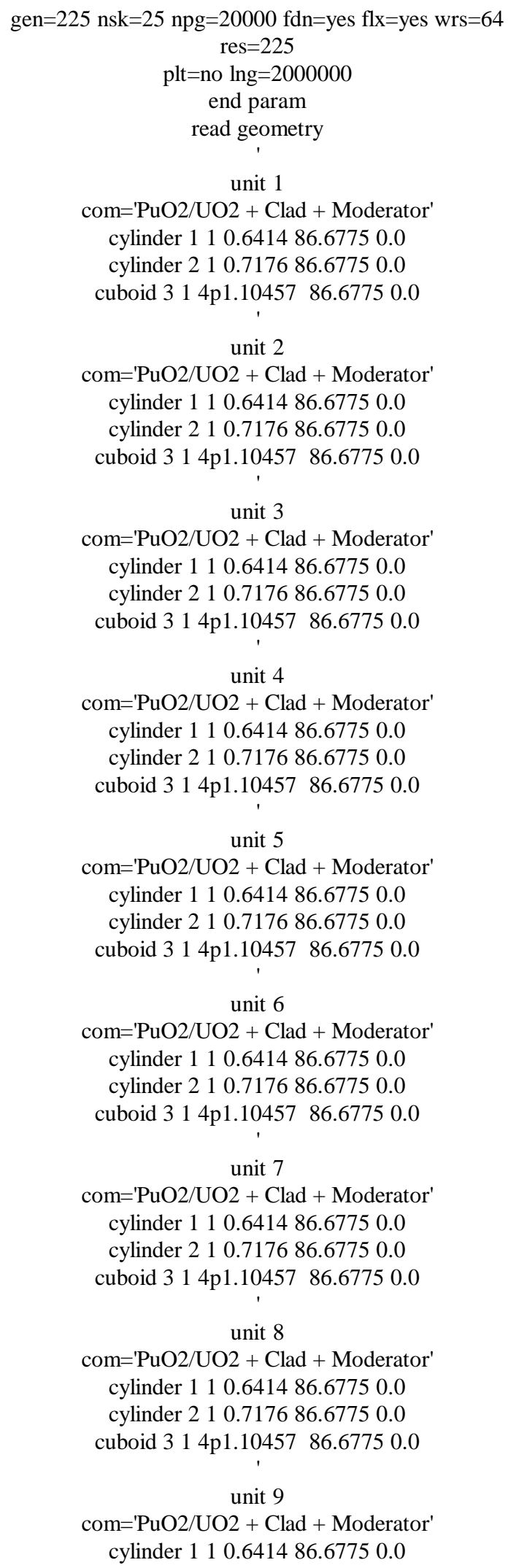


cylinder 210.717686 .67750 .0
cuboid $314 \mathrm{p} 1.1045786 .67750 .0$

$$
\begin{aligned}
& \text { unit } 10 \\
& \text { com='PuO2/UO2 + Clad + Moderator' } \\
& \text { cylinder } 110.641486 .67750 .0 \\
& \text { cylinder } 210.717686 .67750 .0 \\
& \text { cuboid } 314 p 1.1045786 .67750 .0 \\
& \text { unit } 11 \\
& \text { com='PuO2/UO2 + Clad + Moderator' } \\
& \text { cylinder } 110.641486 .67750 .0 \\
& \text { cylinder } 210.717686 .67750 .0 \\
& \text { cuboid } 314 \mathrm{p} 1.1045786 .67750 .0 \\
& \text { unit } 12 \\
& \text { com='PuO2/UO2 + Clad + Moderator' } \\
& \text { cylinder } 110.641486 .67750 .0 \\
& \text { cylinder } 210.717686 .67750 .0 \\
& \text { cuboid } 314 p 1.1045786 .67750 .0 \\
& \text { unit } 13 \\
& \text { com='PuO2/UO2 + Clad + Moderator' } \\
& \text { cylinder } 110.641486 .67750 .0 \\
& \text { cylinder } 210.717686 .67750 .0 \\
& \text { cuboid } 314 \mathrm{p} 1.1045786 .67750 .0 \\
& \text { unit } 14 \\
& \text { com='PuO2/UO2 + Clad + Moderator' } \\
& \text { cylinder } 110.641486 .67750 .0 \\
& \text { cylinder } 210.717686 .67750 .0 \\
& \text { cuboid } 314 p 1.1045786 .67750 .0 \\
& \text { unit } 15 \\
& \text { com='PuO2/UO2 + Clad + Moderator' } \\
& \text { cylinder } 110.641486 .67750 .0 \\
& \text { cylinder } 210.717686 .67750 .0 \\
& \text { cuboid } 314 p 1.1045786 .67750 .0 \\
& \text { unit } 16 \\
& \text { com='PuO2/UO2 + Clad + Moderator' } \\
& \text { cylinder } 110.641486 .67750 .0 \\
& \text { cylinder } 210.717686 .67750 .0 \\
& \text { cuboid } 314 \mathrm{p} 1.1045786 .67750 .0 \\
& \text { unit } 17 \\
& \text { com='PuO2/UO2 + Clad + Moderator' } \\
& \text { cylinder } 110.641486 .67750 .0 \\
& \text { cylinder } 210.717686 .67750 .0 \\
& \text { cuboid } 314 \mathrm{p} 1.1045786 .67750 .0 \\
& \text { unit } 18 \\
& \text { com='PuO2/UO2 + Clad + Moderator' } \\
& \text { cylinder } 110.641486 .67750 .0 \\
& \text { cylinder } 210.717686 .67750 .0 \\
& \text { cuboid } 314 \mathrm{p} 1.1045786 .67750 .0
\end{aligned}
$$

unit 19

$$
\begin{aligned}
& \text { com='PuO2/UO2 + Clad + Moderator' } \\
& \text { cylinder } 110.641486 .67750 .0 \\
& \text { cylinder } 210.717686 .67750 .0 \\
& \text { cuboid } 314 p 1.1045786 .67750 .0 \\
& \text { unit } 20 \\
& \text { com='PuO2/UO2 + Clad + Moderator' } \\
& \text { cylinder } 110.641486 .67750 .0 \\
& \text { cylinder } 210.717686 .67750 .0 \\
& \text { cuboid } 314 p 1.1045786 .67750 .0 \\
& \text { unit } 21 \\
& \text { com='PuO2/UO2 + Clad + Moderator' } \\
& \text { cylinder } 110.641486 .67750 .0 \\
& \text { cylinder } 210.717686 .67750 .0 \\
& \text { cuboid } 314 \mathrm{p} 1.1045786 .67750 .0 \\
& \text { unit } 22 \\
& \text { com='PuO2/UO2 + Clad + Moderator' } \\
& \text { cylinder } 110.641486 .67750 .0 \\
& \text { cylinder } 210.717686 .67750 .0 \\
& \text { cuboid } 314 \mathrm{p} 1.1045786 .67750 .0 \\
& \text { unit } 23 \\
& \text { com='PuO2/UO2 + Clad + Moderator' } \\
& \text { cylinder } 110.641486 .67750 .0 \\
& \text { cylinder } 210.717686 .67750 .0 \\
& \text { cuboid } 314 p 1.1045786 .67750 .0 \\
& \text { unit } 24 \\
& \text { com='PuO2/UO2 + Clad + Moderator' } \\
& \text { cylinder } 110.641486 .67750 .0 \\
& \text { cylinder } 210.717686 .67750 .0 \\
& \text { cuboid } 314 p 1.1045786 .67750 .0 \\
& \text { unit } 25 \\
& \text { com='PuO2/UO2 + Clad + Moderator' } \\
& \text { cylinder } 110.641486 .67750 .0 \\
& \text { cylinder } 210.717686 .67750 .0 \\
& \text { cuboid } 31 \text { 4p1.10457 } 86.67750 .0 \\
& \text { unit } 26 \\
& \text { com='PuO2/UO2 + Clad + Moderator' } \\
& \text { cylinder } 110.641486 .67750 .0 \\
& \text { cylinder } 210.717686 .67750 .0 \\
& \text { cuboid } 31 \text { 4p1.10457 } 86.67750 .0 \\
& \text { unit } 27 \\
& \text { com='PuO2/UO2 + Clad + Moderator' } \\
& \text { cylinder } 110.641486 .67750 .0 \\
& \text { cylinder } 210.717686 .67750 .0 \\
& \text { cuboid } 314 \mathrm{p} 1.1045786 .67750 .0 \\
& \text { unit } 28 \\
& \text { com='PuO2/UO2 + Clad + Moderator' } \\
& \text { cylinder } 110.641486 .67750 .0
\end{aligned}
$$


cylinder 210.717686 .67750 .0
cuboid $314 \mathrm{p} 1.1045786 .67750 .0$

unit 29

com='PuO2/UO2 + Clad + Moderator' cylinder 110.641486 .67750 .0 cylinder 210.717686 .67750 .0 cuboid $314 p 1.1045786 .67750 .0$

$$
\text { unit } 30
$$

com $=$ 'PuO2/UO2 + Clad + Moderator' cylinder 110.641486 .67750 .0 cylinder 210.717686 .67750 .0 cuboid 31 4p1.10457 86.67750 .0

unit 31

com='PuO2/UO2 + Clad + Moderator' cylinder 110.641486 .67750 .0 cylinder 210.717686 .67750 .0 cuboid $314 p 1.1045786 .67750 .0$

unit 32

com='PuO2/UO2 + Clad + Moderator' cylinder 110.641486 .67750 .0 cylinder 210.717686 .67750 .0 cuboid 31 4p1.10457 86.67750 .0

unit 33

com='PuO2/UO2 + Clad + Moderator' cylinder 110.641486 .67750 .0 cylinder 210.717686 .67750 .0 cuboid $314 \mathrm{p} 1.1045786 .67750 .0$

unit 34

com='PuO2/UO2 + Clad + Moderator' cylinder 110.641486 .67750 .0 cylinder 210.717686 .67750 .0 cuboid $314 p 1.1045786 .67750 .0$

unit 35

com='PuO2/UO2 + Clad + Moderator' cylinder 110.641486 .67750 .0 cylinder 210.717686 .67750 .0 cuboid $314 p 1.1045786 .67750 .0$

unit 36

com='PuO2/UO2 + Clad + Moderator' cylinder 110.641486 .67750 .0 cylinder 210.717686 .67750 .0 cuboid $314 \mathrm{p} 1.1045786 .67750 .0$

unit 37

com='PuO2/UO2 + Clad + Moderator' cylinder 110.641486 .67750 .0 cylinder 210.717686 .67750 .0 cuboid 314 p1.10457 86.67750 .0 unit 38

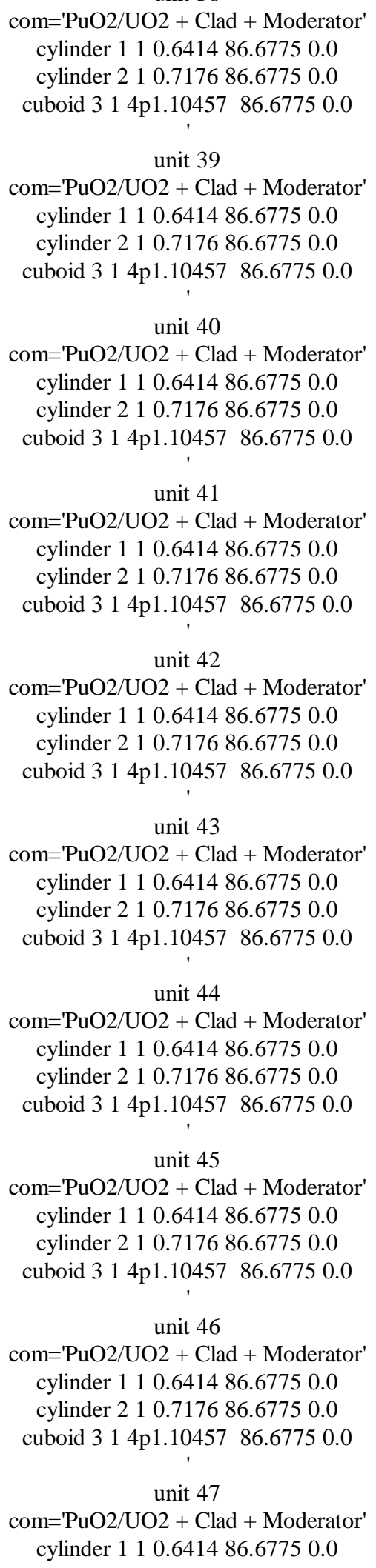




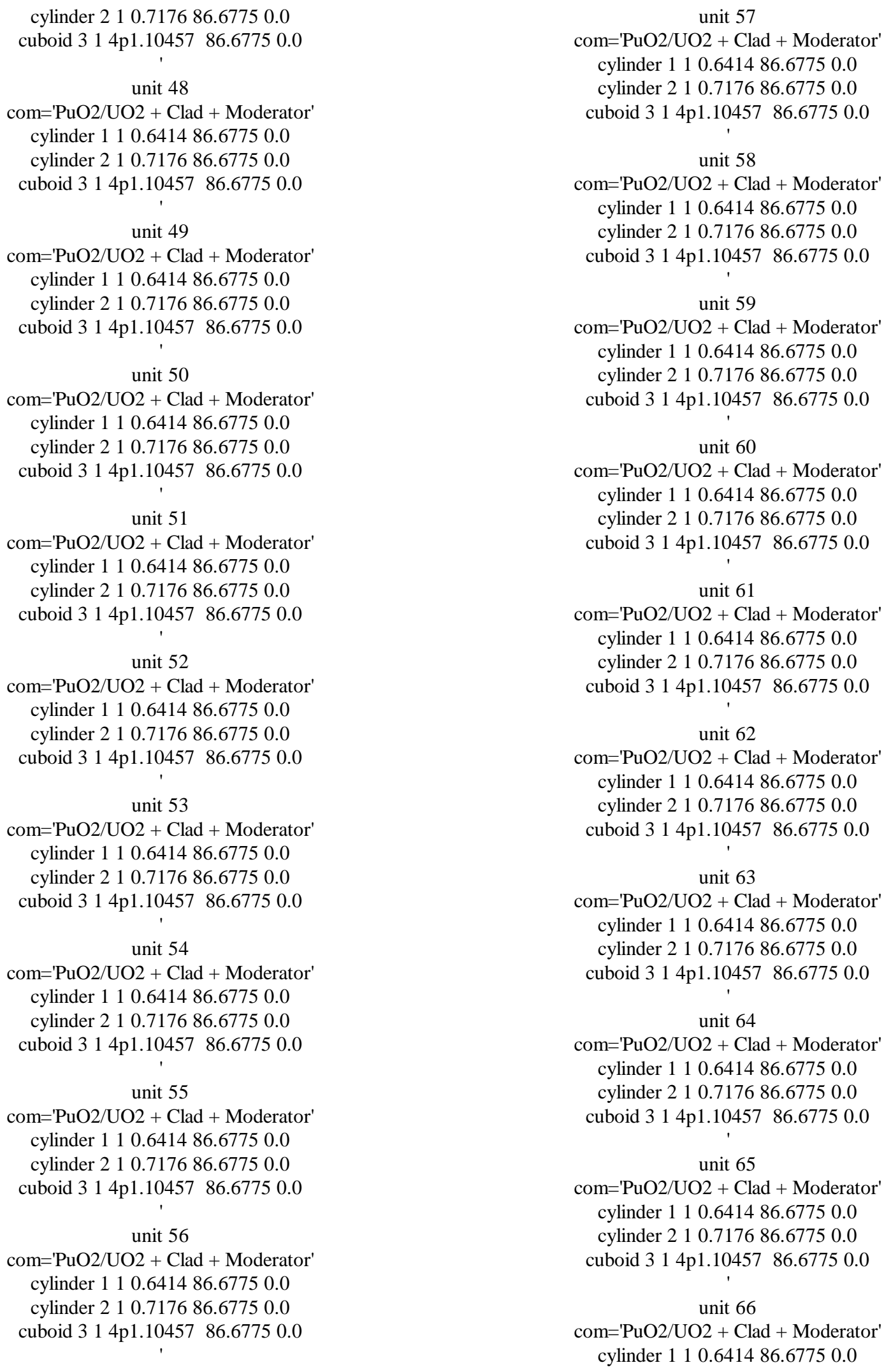




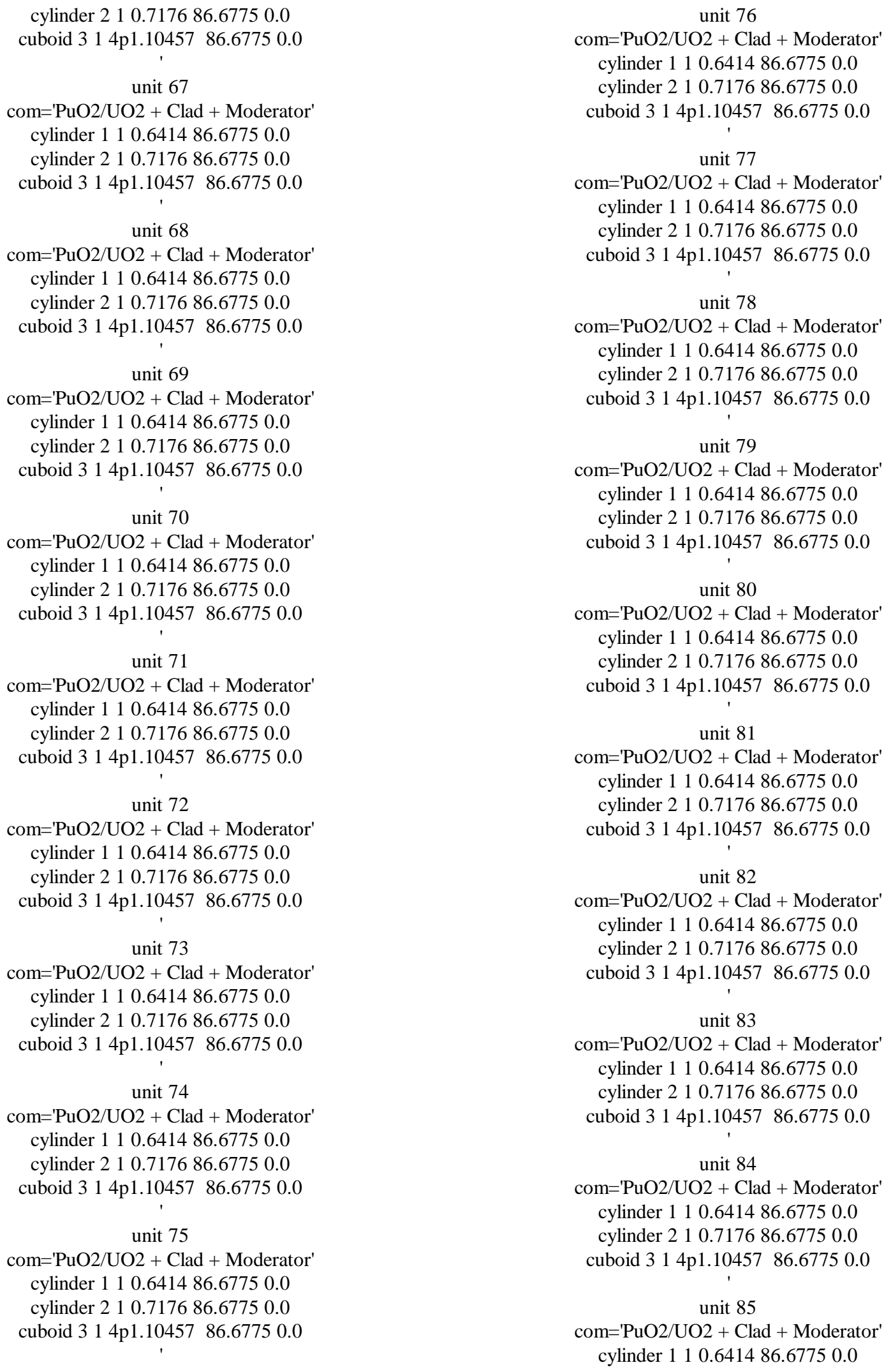




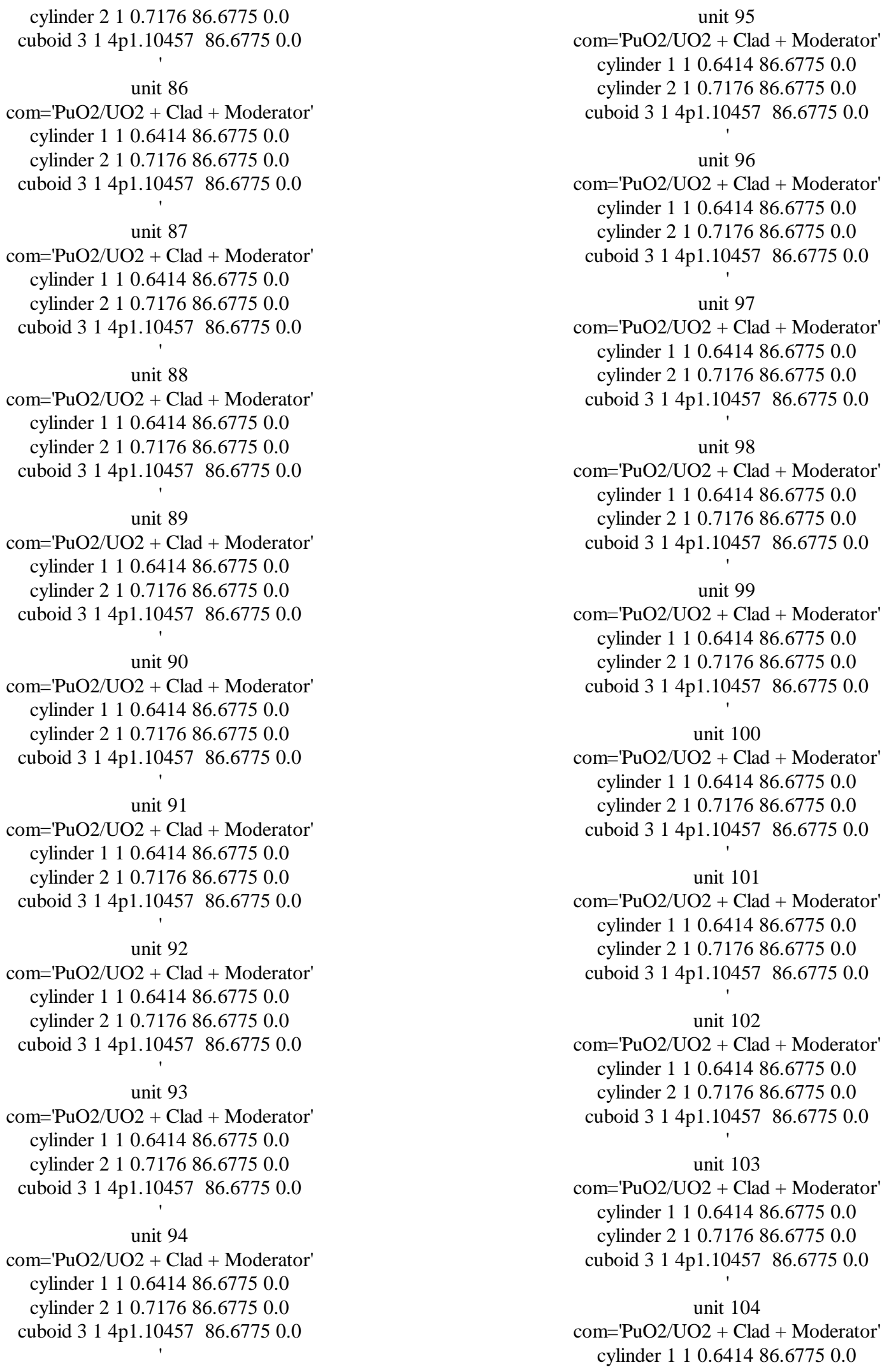




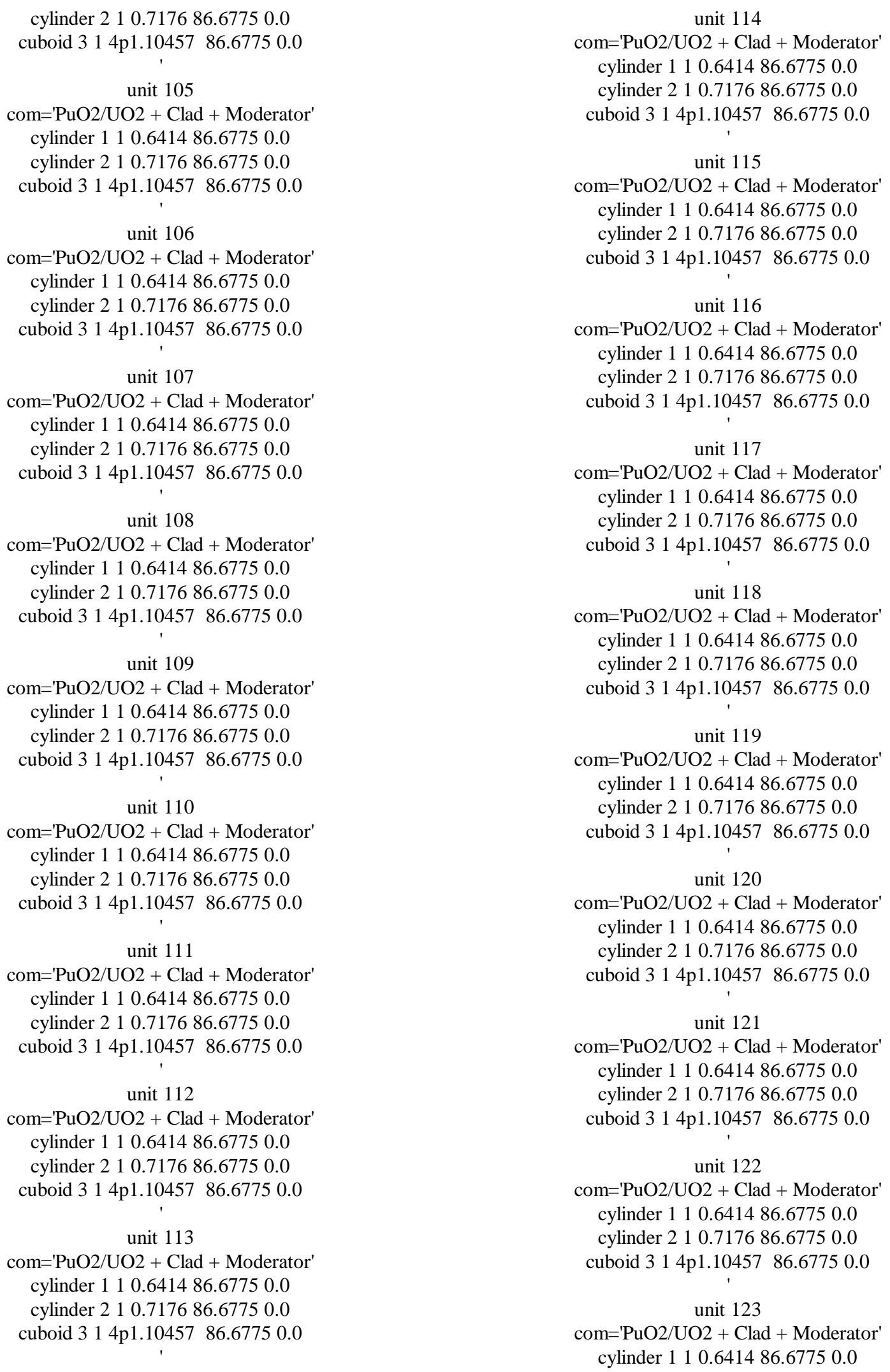

cylinder 210.717686 .67750 .0 cuboid $314 \mathrm{p} 1.1045786 .67750 .0$

unit 105

com='PuO2/UO2 + Clad + Moderator' cylinder 110.641486 .67750 .0 cylinder 210.717686 .67750 .0 cuboid $314 \mathrm{p} 1.1045786 .67750 .0$

unit 106

com='PuO2/UO2 + Clad + Moderator' cylinder 110.641486 .67750 .0 cylinder 210.717686 .67750 .0 cuboid $314 \mathrm{p} 1.1045786 .67750 .0$

unit 107

com='PuO2/UO2 + Clad + Moderator' cylinder 110.641486 .67750 .0 cylinder 210.717686 .67750 .0 cuboid $314 p 1.1045786 .67750 .0$ unit 108

com='PuO2/UO2 + Clad + Moderator' cylinder 110.641486 .67750 .0 cylinder 210.717686 .67750 .0 cuboid 31 4p1.10457 86.67750 .0

unit 109

com='PuO2/UO2 + Clad + Moderator' cylinder 110.641486 .67750 .0 cylinder 210.717686 .67750 .0 cuboid $314 \mathrm{p} 1.1045786 .67750 .0$

unit 110

com='PuO2/UO2 + Clad + Moderator' cylinder 110.641486 .67750 .0 cylinder 210.717686 .67750 .0 cuboid $314 \mathrm{p} 1.1045786 .67750 .0$

unit 111

com='PuO2/UO2 + Clad + Moderator' cylinder 110.641486 .67750 .0 cylinder 210.717686 .67750 .0 cuboid 31 4p1.10457 86.67750 .0

unit 112

com='PuO2/UO2 + Clad + Moderator' cylinder 110.641486 .67750 .0 cylinder 210.717686 .67750 .0 cuboid $314 \mathrm{p} 1.1045786 .67750 .0$

unit 113

com='PuO2/UO2 + Clad + Moderator' cylinder 110.641486 .67750 .0 cylinder 210.717686 .67750 .0 cuboid $314 \mathrm{p} 1.1045786 .67750 .0$

unit 114 


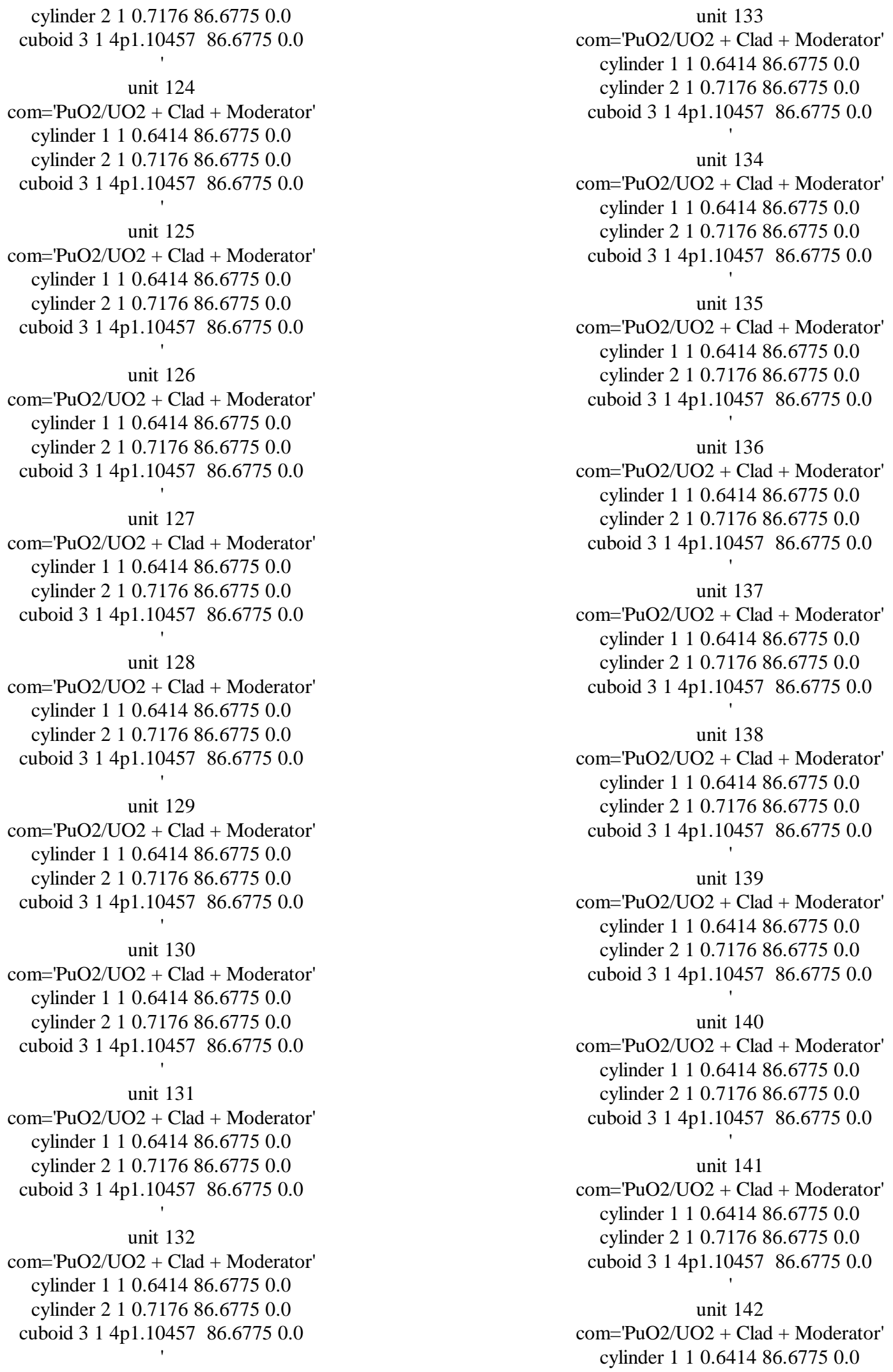

unit 133 


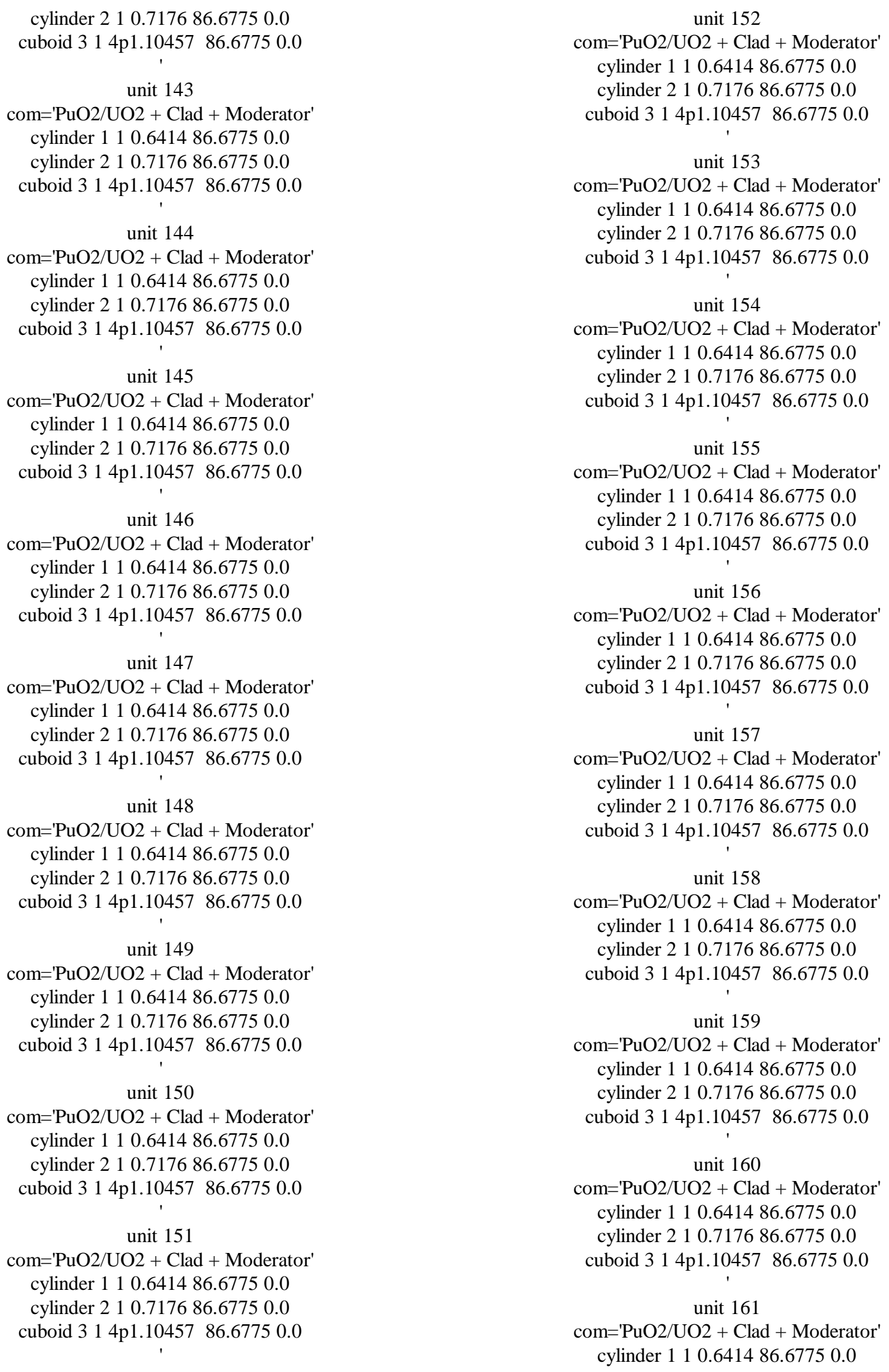

cylinder 210.717686 .67750 .0 cuboid $314 \mathrm{p} 1.1045786 .67750 .0$

unit 143

com='PuO2/UO2 + Clad + Moderator' cylinder 110.641486 .67750 .0 cylinder 210.717686 .67750 .0 cuboid $314 \mathrm{p} 1.1045786 .67750 .0$

unit 144

com='PuO2/UO2 + Clad + Moderator' cylinder 110.641486 .67750 .0 cylinder 210.717686 .67750 .0 cuboid 31 4p1.10457 86.67750 .0

unit 145

com='PuO2/UO2 + Clad + Moderator' cylinder 110.641486 .67750 .0 cylinder 210.717686 .67750 .0 cuboid $314 \mathrm{p} 1.1045786 .67750 .0$

unit 146

com='PuO2/UO2 + Clad + Moderator' cylinder 110.641486 .67750 .0 cylinder 210.717686 .67750 .0 cuboid 31 4p1.10457 86.67750 .0

unit 147

com='PuO2/UO2 + Clad + Moderator' cylinder 110.641486 .67750 .0 cylinder 210.717686 .67750 .0 cuboid $314 p 1.1045786 .67750 .0$

unit 148

com='PuO2/UO2 + Clad + Moderator' cylinder 110.641486 .67750 .0 cylinder 210.717686 .67750 .0 cuboid $314 \mathrm{p} 1.1045786 .67750 .0$

unit 149

com $=$ 'PuO2/UO2 + Clad + Moderator' cylinder 110.641486 .67750 .0 cylinder 210.717686 .67750 .0 cuboid 31 4p1.10457 86.67750 .0

unit 150

com='PuO2/UO2 + Clad + Moderator' cylinder 110.641486 .67750 .0 cylinder 210.717686 .67750 .0 cuboid $314 p 1.1045786 .67750 .0$

unit 151

com='PuO2/UO2 + Clad + Moderator' cylinder 110.641486 .67750 .0 cylinder 210.717686 .67750 .0 cuboid $314 \mathrm{p} 1.1045786 .67750 .0$

unit 152 


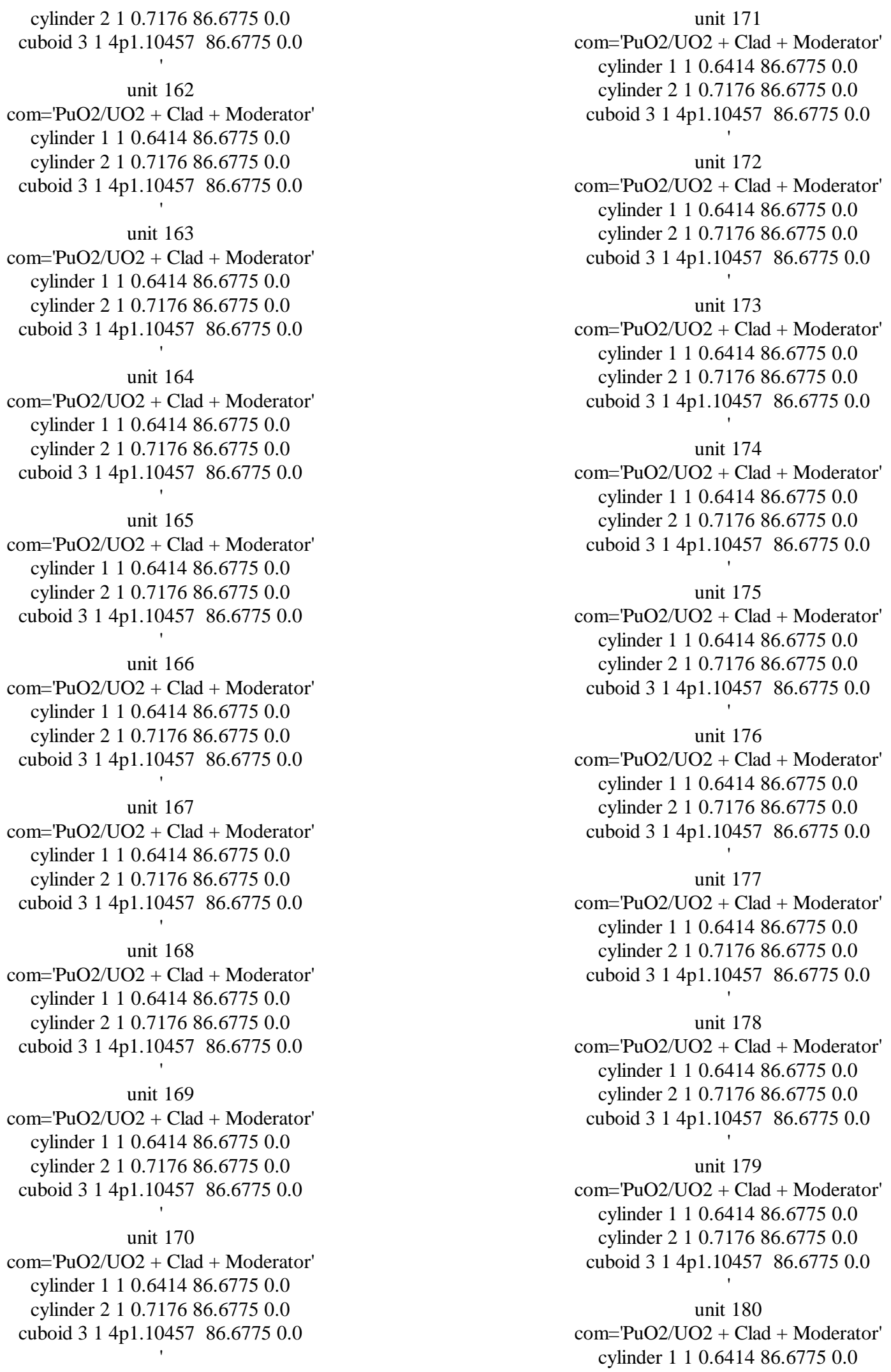

unit 171 
cylinder 210.717686 .67750 .0 cuboid $314 \mathrm{p} 1.1045786 .67750 .0$

unit 181

com='PuO2/UO2 + Clad + Moderator' cylinder 110.641486 .67750 .0 cylinder 210.717686 .67750 .0 cuboid $314 p 1.1045786 .67750 .0$

unit 182

com $=$ 'PuO2/UO2 + Clad + Moderator' cylinder 110.641486 .67750 .0 cylinder 210.717686 .67750 .0 cuboid $314 \mathrm{p} 1.1045786 .67750 .0$

unit 183

com='PuO2/UO2 + Clad + Moderator' cylinder 110.641486 .67750 .0 cylinder 210.717686 .67750 .0 cuboid $314 p 1.1045786 .67750 .0$

unit 184

com='PuO2/UO2 + Clad + Moderator' cylinder 110.641486 .67750 .0 cylinder 210.717686 .67750 .0 cuboid $314 \mathrm{p} 1.1045786 .67750 .0$

unit 185

com='PuO2/UO2 + Clad + Moderator' cylinder 110.641486 .67750 .0 cylinder 210.717686 .67750 .0 cuboid $314 p 1.1045786 .67750 .0$

unit 186

com='PuO2/UO2 + Clad + Moderator' cylinder 110.641486 .67750 .0 cylinder 210.717686 .67750 .0 cuboid $314 p 1.1045786 .67750 .0$

unit 187

com='PuO2/UO2 + Clad + Moderator' cylinder 110.641486 .67750 .0 cylinder 210.717686 .67750 .0 cuboid 314 p1.10457 86.67750 .0

unit 188

com='PuO2/UO2 + Clad + Moderator' cylinder 110.641486 .67750 .0 cylinder 210.717686 .67750 .0 cuboid $314 \mathrm{p} 1.1045786 .67750 .0$

unit 189

com='PuO2/UO2 + Clad + Moderator' cylinder 110.641486 .67750 .0 cylinder 210.717686 .67750 .0 cuboid 314 p1.10457 86.67750 .0 unit 190

com $=$ 'PuO2/UO2 + Clad + Moderator' cylinder 110.641486 .67750 .0 cylinder 210.717686 .67750 .0 cuboid $314 p 1.1045786 .67750 .0$

unit 191

com='PuO2/UO2 + Clad + Moderator' cylinder 110.641486 .67750 .0 cylinder 210.717686 .67750 .0 cuboid $314 p 1.1045786 .67750 .0$

unit 192

com $=$ 'PuO2/UO2 + Clad + Moderator' cylinder 110.641486 .67750 .0 cylinder 210.717686 .67750 .0 cuboid $314 \mathrm{p} 1.1045786 .67750 .0$

unit 193

com='PuO2/UO2 + Clad + Moderator' cylinder 110.641486 .67750 .0 cylinder 210.717686 .67750 .0 cuboid $314 \mathrm{p} 1.1045786 .67750 .0$

unit 194

com='1PuO2/UO2 + Clad + Moderator' cylinder 110.641486 .67750 .0 cylinder 210.717686 .67750 .0 cuboid $314 \mathrm{p} 1.1045786 .67750 .0$

unit 195

com='PuO2/UO2 + Clad + Moderator' cylinder 110.641486 .67750 .0 cylinder 210.717686 .67750 .0 cuboid $314 p 1.1045786 .67750 .0$

unit 201

com='Fuel - Lower Clad + Moderator' cylinder 210.71760 .31750 .0 cuboid $314 \mathrm{p} 1.104570 .31750 .0$

unit 202

com='Fuel - Lower PuO2/UO2 + UO2 + Clad + Moderator + Lattice Grid'

cylinder $110.6414 \quad 2.540 .881$

cylinder $510.6414 \quad 2.540 .381$

cylinder $210.7176 \quad 2.540 .0$

cuboid $314 \mathrm{p} 1.023292 .540 .0$

cuboid $414 \mathrm{p} 1.10457 \quad 2.540 .0$

unit 203

com='Fuel - Upper PuO2/UO2 + Clad + Moderator + Lattice Grid' cylinder $110.6414 \quad 2.540 .0$ cylinder $210.7176 \quad 2.540 .0$ cuboid $314 \mathrm{p} 1.023292 .540 .0$ cuboid $414 \mathrm{p} 1.10457 \quad 2.540 .0$ 


$$
\text { unit } 204
$$

com='Fuel - Upper PuO2/UO2 + Clad + Moderator' cylinder 110.64140 .06350 .0 cylinder 210.71760 .06350 .0 cuboid $314 \mathrm{p} 1.104570 .06350 .0$

$$
\text { unit } 205
$$

com='Reflector - Lower Moderator' cuboid $314 \mathrm{p} 1.104570 .31750 .0$

$$
\text { unit } 206
$$

com='Reflector - Lower Moderator + Lattice Grid' cuboid $314 \mathrm{p} 1.023292 .540 .0$ cuboid $414 \mathrm{p} 1.10457 \quad 2.540 .0$ unit 207

com='Reflector - Upper Moderator + Lattice Grid' cuboid $314 \mathrm{p} 1.023292 .540 .0$ cuboid $414 \mathrm{p} 1.10457 \quad 2.540 .0$ unit 208 com='Reflector - Upper Moderator' cuboid $314 \mathrm{p} 1.104570 .06350 .0$ unit 210 com='Moderator' cuboid $314 \mathrm{p} 1.1045786 .67750 .0$ global unit 220 com='Cells in assembly' array $1-18.77769-18.777690$ cuboid 61 4p18.77769 $92.9640 \quad-2.8575$ cuboid 71 4p18.77769 93.9165 -2.8575 replicate $324 * 3.03 .9373 .01$ replicate $334 * 3.00 .0 \quad 3.09$ cuboid 31 4p48.77769 97.8535 -32.8575 end geometry read array ara=1 nux=17 nuy=17 nuz=5 fill

'Lower Cladding and Moderator

205205205205205205205201201201205205205 205205205205

205205205205205205201201201201201201205 205205205205

205205205205201201201201201201201201201 205205205205

205205205201201201201201201201201201201 201205205205

205205201201201201201201201201201201201 201201205205

205201201201201201201201201201201201201 201201201205

205201201201201201201201201201201201201 201201201205

201201201201201201201201201201201201201
201201201201

201201201201201201201201201201201201201 201201201201

201201201201201201201201201201201201201 201201201201

205201201201201201201201201201201201201 201201201205

205201201201201201201201201201201201201 201201201205

205205201201201201201201201201201201201 201201205205

205205205201201201201201201201201201201 201205205205

205205205205201201201201201201201201201 205205205205

205205205205205201201201201201201205205 205205205205

205205205205205205205201201201205205205 205205205205

'Lower Lattice Grid

206206206206206206206202202202206206206 206206206206

206206206206206206202202202202202202206 206206206206

206206206206202202202202202202202202202 206206206206

206206206202202202202202202202202202202 202206206206

206206202202202202202202202202202202202 202202206206

206202202202202202202202202202202202202 202202202206

206202202202202202202202202202202202202 202202202206

202202202202202202202202202202202202202 202202202202

202202202202202202202202202202202202202 202202202202

202202202202202202202202202202202202202 202202202202

206202202202202202202202202202202202202 202202202206

206202202202202202202202202202202202202 202202202206

206206202202202202202202202202202202202 202202206206

206206206202202202202202202202202202202 202206206206

206206206206202202202202202202202202202 206206206206

206206206206206202202202202202202206206 206206206206

206206206206206206206202202202206206206 206206206206

'Fuel and Reflector

210210210210210210210193194195210210210 210210210210 
210210210210210210187188189190191192210 210210210210

210210210210178179180181182183184185186 210210210210

210210210167168169170171172173174175176 177210210210

210210154155156157158159160161162163164 165166210210

210139140141142143144145146147148149150 151152153210

210124125126127128129130131132133134135 136137138210

107108109110111112113114115116117118119 120121122123

90919293949596979899100101102103104 105106

$\begin{array}{llllllllllllllll}73 & 74 & 75 & 76 & 77 & 78 & 79 & 80 & 81 & 82 & 83 & 84 & 85 & 86 & 87 & 88\end{array}$ 89

$\begin{array}{lllllllllllllll}210 & 58 & 59 & 60 & 61 & 62 & 63 & 64 & 65 & 66 & 67 & 68 & 69 & 70 & 71\end{array}$ 72210

$\begin{array}{lllllllllllllll}210 & 43 & 44 & 45 & 46 & 47 & 48 & 49 & 50 & 51 & 52 & 53 & 54 & 55 & 56\end{array}$ 57210

$\begin{array}{lllllllllllllll}210 & 210 & 30 & 31 & 32 & 33 & 34 & 35 & 36 & 37 & 38 & 39 & 40 & 41 & 42\end{array}$ 210210

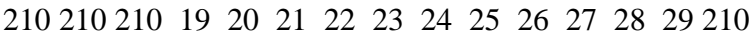
210210

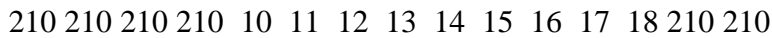
210210

$210210210210210 \quad 4 \quad 5 \quad 6 \quad 7 \quad 8 \quad 9210210210210$ 210210

$210210210210210210210 \quad 1 \quad 2 \quad 3210210210210$ 210210210

'Upper Lattice Grid

207207207207207207207203203203207207207 207207207207

207207207207207207203203203203203203207 207207207207

207207207207203203203203203203203203203 207207207207

207207207203203203203203203203203203203 203207207207

207207203203203203203203203203203203203 203203207207

207203203203203203203203203203203203203 203203203207

207203203203203203203203203203203203203 203203203207

203203203203203203203203203203203203203 203203203203

203203203203203203203203203203203203203 203203203203

203203203203203203203203203203203203203 203203203203

207203203203203203203203203203203203203 203203203207

207203203203203203203203203203203203203 203203203207
207207203203203203203203203203203203203 203203207207

207207207203203203203203203203203203203 203207207207

207207207207203203203203203203203203203 207207207207

207207207207207203203203203203203207207 207207207207

207207207207207207207203203203207207207 207207207207

'Upper Cladding and Moderator

208208208208208208208204204204208208208 208208208208

208208208208208208204204204204204204208 208208208208

208208208208204204204204204204204204204 208208208208

208208208204204204204204204204204204204 204208208208

208208204204204204204204204204204204204 204204208208

208204204204204204204204204204204204204 204204204208

208204204204204204204204204204204204204 204204204208

204204204204204204204204204204204204204 204204204204

204204204204204204204204204204204204204 204204204204

204204204204204204204204204204204204204 204204204204

208204204204204204204204204204204204204 204204204208

208204204204204204204204204204204204204 204204204208

208208204204204204204204204204204204204 204204208208

208208208204204204204204204204204204204 204208208208

208208208208204204204204204204204204204 208208208208

208208208208208204204204204204204208208 208208208208

208208208208208208208204204204208208208 208208208208

end fill

end array

read bias id=500 211 end bias read plot

$\mathrm{ttl}=$ 'x-z plot at $\mathrm{y}=0.0^{\prime}$

$\mathrm{xul}=-30.0$ yul $=0 . \quad \mathrm{zul}=110$

$x l r=30.0$ ylr $=0 . \quad z l r=-10$.

$\mathrm{uax}=1.0 \mathrm{wdn}=-1.0$

nax $=600$ end plt0

$\mathrm{tt} l=' \mathrm{x}-\mathrm{y}$ plot at $\mathrm{z}=25.0^{\prime}$ pic $=$ mix

$\mathrm{xul}=-30.0 \mathrm{yul}=30 . \mathrm{zul}=25$. 


$$
\begin{gathered}
\mathrm{xlr}=30.0 \text { ylr }=-30 . \mathrm{zlr}=25 . \\
\mathrm{uax}=1.0 \text { vdn=-1.0 } \\
\text { nax }=600 \text { end plt } 1 \\
\text { end plot } \\
\text { end data } \\
\text { end } \\
=\text { shell }
\end{gathered}
$$

cp ft04f001 \$RTNDIR/pnl32.xs04 cp ft64f001 \$RTNDIR/pnl32.rs64

end $=\mathrm{kmart}$

read initial kunit $=64$

xunit=4

actbygrp

rrpvol

end initial

read activity

92234279223418922341452

92235279223518922351452

92236279223618922361452

$9223827922381892238 \quad 1452$

94238279423818942381452

94239279423918942391452

94240279424018942401452

94241279424118942411452

$9424227942421894242 \quad 1452$

95241279524118952411452

4000027

5011227

5011427

5011527

5011627

5011727

5011827

5011927

5012027

5012227

5012427

2800027

2400027

2600027

501027

501127

801627

100127

end activity

read collapse

factor $=1.0$

lastg $=57$

lastg $=148$

lastg $=204$

lastg $=238$

end collapse

end

$=\operatorname{csasc} 5 \quad$ parm $=($ size $=1000000)$ pnl-33 fuel

238group latticecell

'MOX fuel

u-235 100 1.4886-4 295 end

u-238 110 2.0611-2 295 end

$\begin{array}{lllll}\text { o } & 1 & 0 & 4.3779-2 & 295\end{array}$ end

u-234 $100 \quad 1.2458-6 \quad 295$ end u-236 $11002.0936-9295$ end pu-238 $1003.8836-8 \quad 295$ end pu-239 $11003.9262-4295$ end pu-240 $1103.3206-5295$ end pu-241 100 1.6081-6 295 end pu-242 $11001.1882-7 \quad 295$ end am-241 10 1.4891-6 295 end 'clad (ZR-2)

zr $\quad 2 \quad 0 \quad 4.2621-2 \quad 295$ end

sn $\quad 2 \quad 0 \quad 4.8328-4295$ end

ni $2003.0336-5295$ end

cr $2007.6093-5295$ end

fe $\quad 2 \quad 0 \quad 9.5642-5295$ end 'water(Refletor)

h $3 \quad 0 \quad 6.6672-2 \quad 295$ end

o $3003.3427-2 \quad 295$ end

b-10 $3 \quad 0 \quad 1.2034-5295$ end

b-11 30 4.8746-5 295 end 'Lattice Grid

si $4 \quad \begin{array}{llll} & 3.4607-4 & 295 & \text { end }\end{array}$

fe $\quad \begin{array}{lllll}4 & 0 & 1.0152-4 & 295 & \text { end }\end{array}$

cu $\quad \begin{array}{llll}4 & 0 & 6.3731-5 & 295 \\ & \text { end }\end{array}$

mn $4002.2115-5295$ end

mg $4 \begin{array}{llll}4 & 0 & 6.6651-4 & 295\end{array}$ end

cr $4 \begin{array}{llll}4 & 0 & 6.2310-5 & 295 \\ \text { end }\end{array}$

ti $4002.5375-5 \quad 295$ end

al $4 \quad \begin{array}{llll}4 & 5.8433-2 & 295 & \text { end }\end{array}$ 'UO2

u-234 50 1.2406-6 295 end

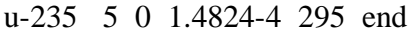

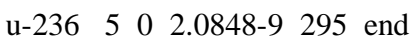

u-238 500 2.0525-2 295 end

o $\quad 5 \quad 0 \quad 4.1943-2 \quad 295$ end 'Aluminum

si $\quad \begin{array}{llllll}6 & 0 & 3.4607-4 & 295 & \text { end }\end{array}$

fe $\quad \begin{array}{lllll}6 & 0 & 1.0152-4 & 295 & \text { end }\end{array}$

cu $\quad \begin{array}{lllll}6 & 0 & 6.3731-5 & 295 & \text { end }\end{array}$

mn $\quad \begin{array}{llll}6 & 0 & 2.2115-5 & 295 \\ & \text { end }\end{array}$

mg $\quad \begin{array}{llll}6 & 0 & 6.6651-4 & 295 \\ \text { end }\end{array}$

cr $\quad \begin{array}{lllll}6 & 0 & 6.2310-5 & 295 & \text { end }\end{array}$

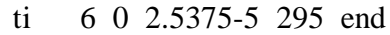

al $\quad \begin{array}{lllll}6 & 0 & 5.8433-2 & 295 & \text { end }\end{array}$ 'lead

pb $\quad \begin{array}{llll}7 & 0 & 3.2174-2 & 295 \\ \text { end }\end{array}$ end comp

squarepitch $2.20914 \quad 1.2827 \quad 121.43513$ end more data

limcen $=20000000$ end more PNL-33 
read param

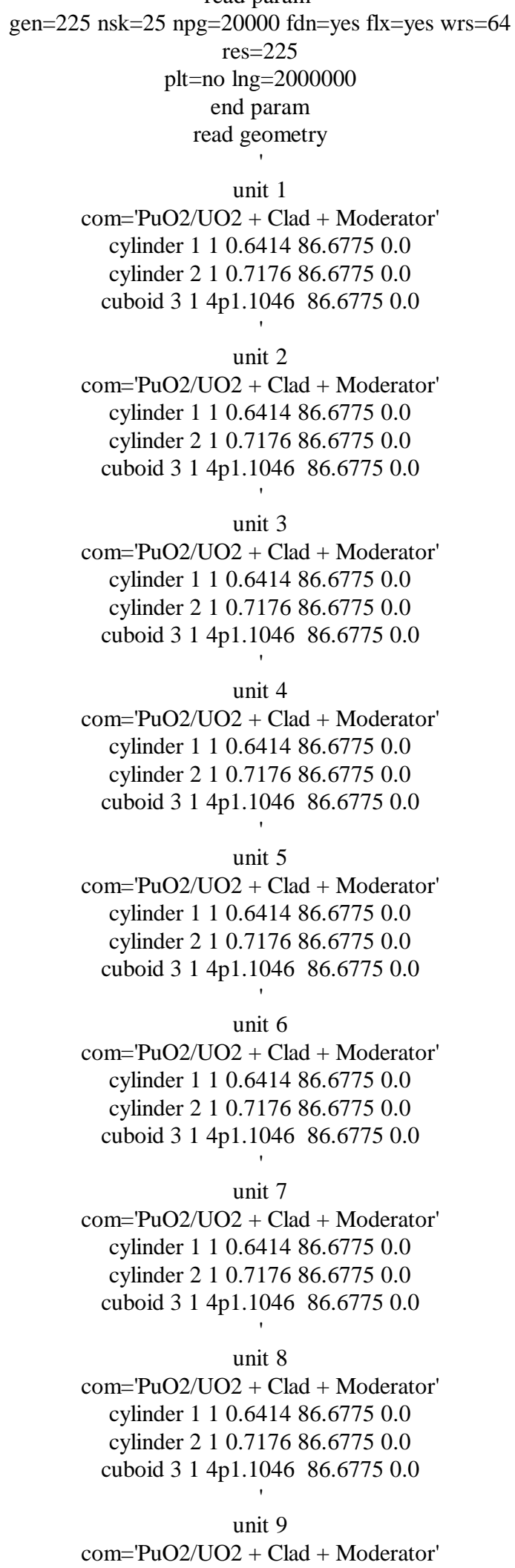

cylinder 110.641486 .67750 .0 cylinder 210.717686 .67750 .0 cuboid $314 \mathrm{p} 1.104686 .67750 .0$ unit 10

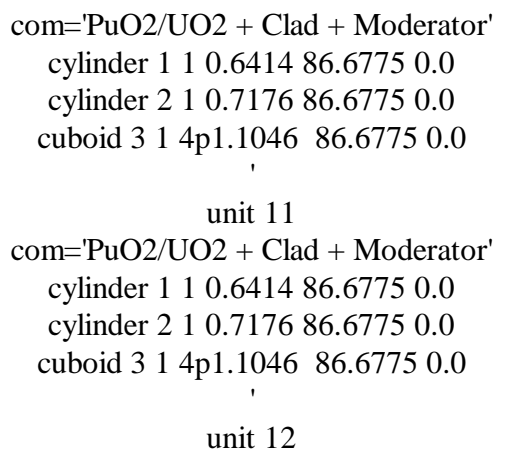




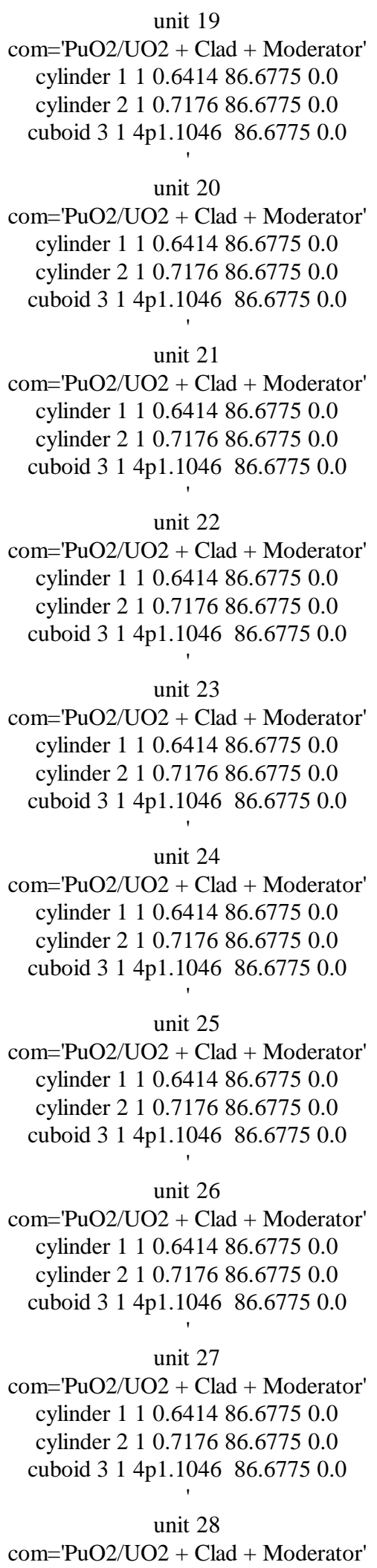

cylinder 110.641486 .67750 .0 cylinder 210.717686 .67750 .0 cuboid $314 \mathrm{p} 1.104686 .67750 .0$ unit 29 


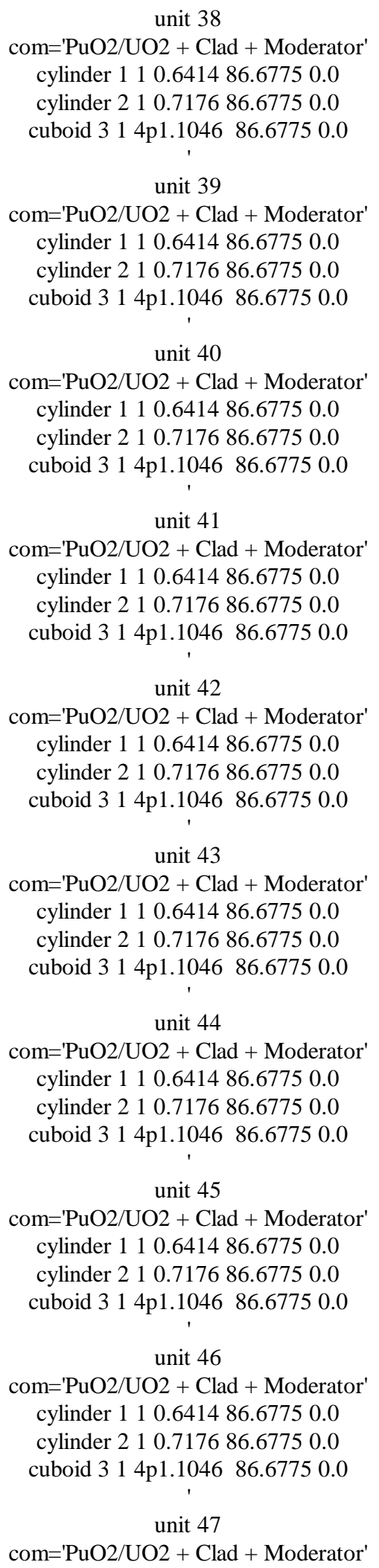

cylinder 110.641486 .67750 .0 cylinder 210.717686 .67750 .0 cuboid $314 \mathrm{p} 1.104686 .67750 .0$ unit 48

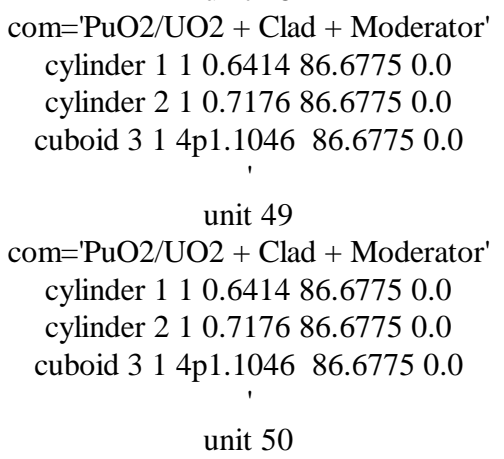




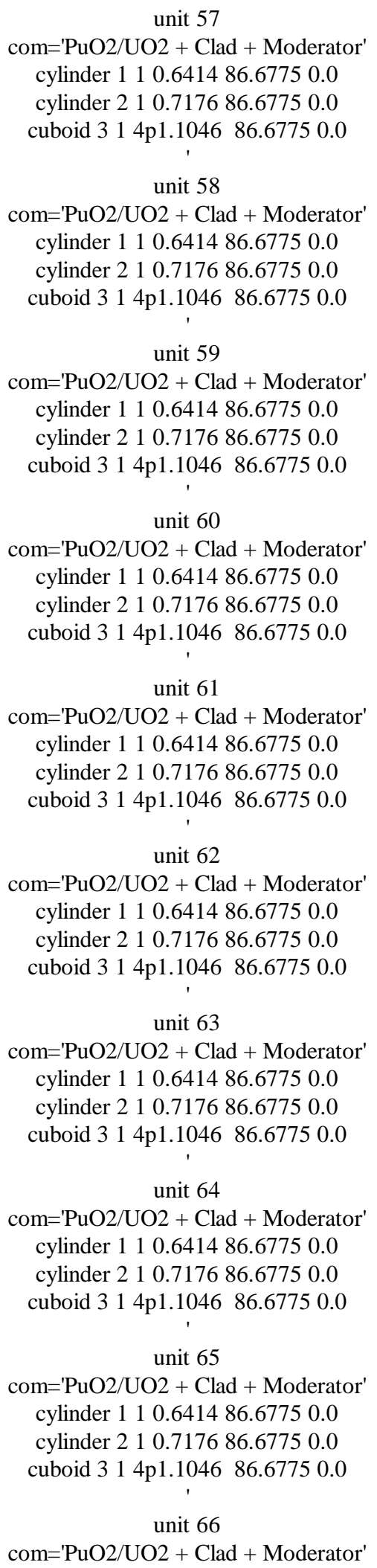

cylinder 110.641486 .67750 .0 cylinder 210.717686 .67750 .0 cuboid $314 \mathrm{p} 1.104686 .67750 .0$ unit 67

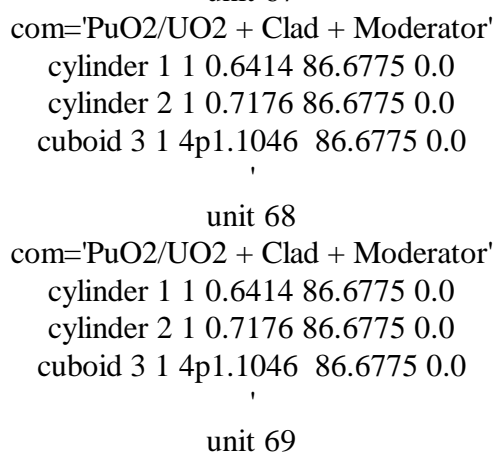




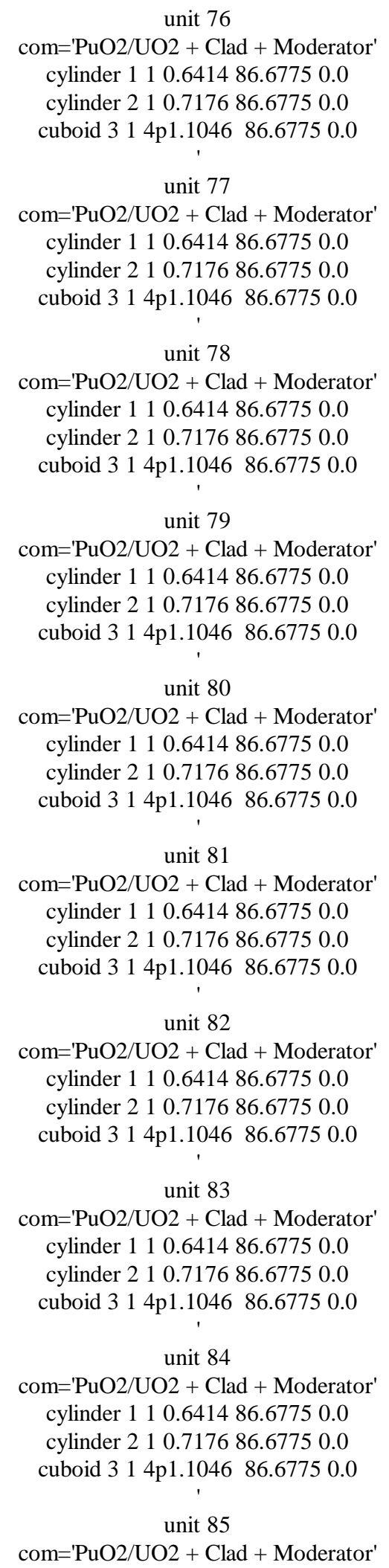

cylinder 110.641486 .67750 .0 cylinder 210.717686 .67750 .0 cuboid 31 4p1.1046 86.67750 .0 unit 86 


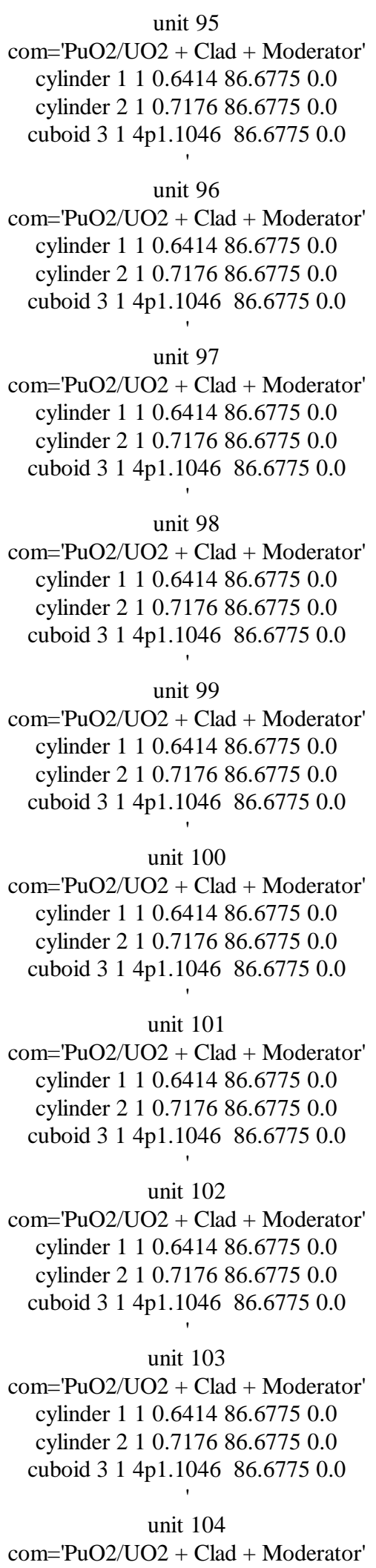

cylinder 110.641486 .67750 .0 cylinder 210.717686 .67750 .0 cuboid $314 \mathrm{p} 1.104686 .67750 .0$

unit 105

com='PuO2/UO2 + Clad + Moderator' cylinder 110.641486 .67750 .0 cylinder 210.717686 .67750 .0 cuboid $314 \mathrm{p} 1.104686 .67750 .0$

unit 106

com='PuO2/UO2 + Clad + Moderator' cylinder 110.641486 .67750 .0 cylinder 210.717686 .67750 .0 cuboid 31 4p1.1046 86.67750 .0

unit 107

com='PuO2/UO2 + Clad + Moderator' cylinder 110.641486 .67750 .0 cylinder 210.717686 .67750 .0 cuboid $314 \mathrm{p} 1.104686 .67750 .0$

unit 108

com='PuO2/UO2 + Clad + Moderator' cylinder 110.641486 .67750 .0 cylinder 210.717686 .67750 .0 cuboid $314 \mathrm{p} 1.104686 .67750 .0$

unit 109

com='PuO2/UO2 + Clad + Moderator' cylinder 110.641486 .67750 .0 cylinder 210.717686 .67750 .0 cuboid $314 \mathrm{p} 1.104686 .67750 .0$ unit 111

com='Fuel - Lower Clad + Moderator' cylinder 210.71760 .31750 .0 cuboid $314 \mathrm{p} 1.10460 .31750 .0$

unit 112

com='Fuel - Lower PuO2/UO2 + UO2 + Clad + Moderator + Lattice Grid' cylinder $110.6414 \quad 2.540 .881$ cylinder $510.6414 \quad 2.540 .381$ cylinder $210.7176 \quad 2.540 .0$ cuboid $314 \mathrm{p} 1.023292 .540 .0$ cuboid $414 \mathrm{p} 1.10462 .540 .0$

unit 113 com='Fuel - Upper PuO2/UO2 + Clad + Moderator + Lattice Grid'

cylinder $110.6414 \quad 2.540 .0$ cylinder $210.7176 \quad 2.540 .0$ cuboid $314 \mathrm{p} 1.023292 .540 .0$ cuboid $414 \mathrm{p} 1.10462 .540 .0$

unit 114 


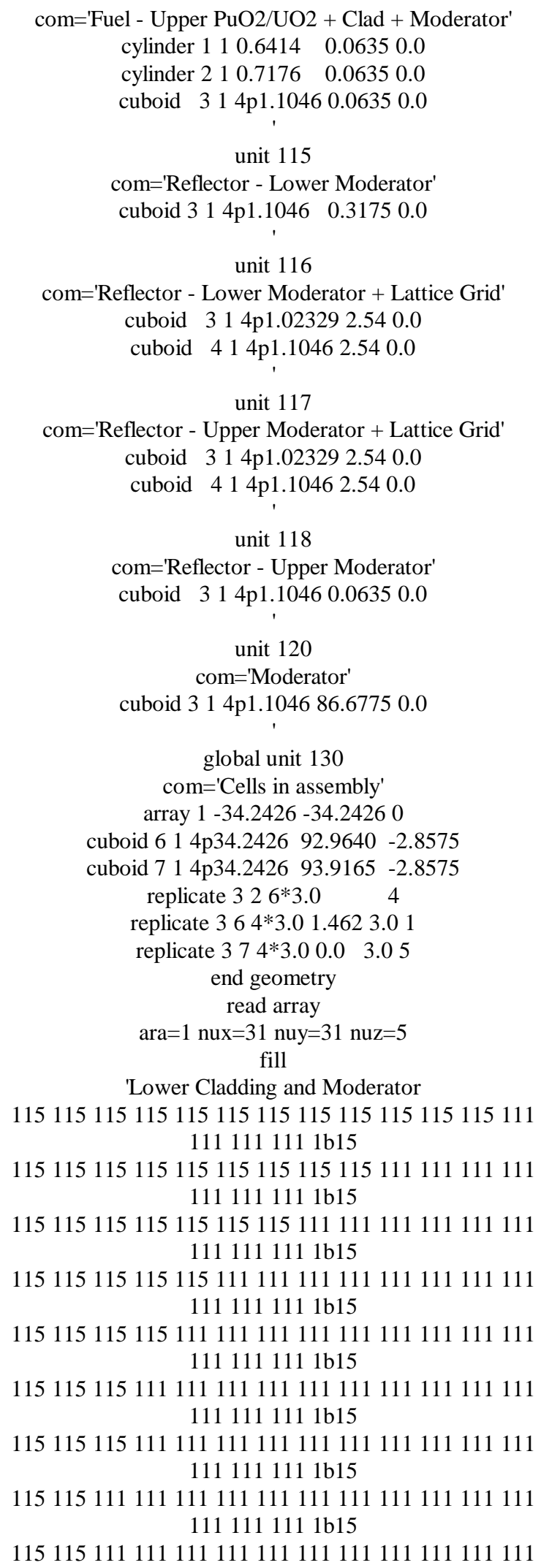

$1111111111 \mathrm{~b} 15$

115111111111111111111111111111111111111 $1111111111 \mathrm{~b} 15$

115111111111111111111111111111111111111 $1111111111 \mathrm{~b} 15$

115111111111111111111111111111111111111 $1111111111 \mathrm{~b} 15$

111111111111111111111111111111111111111 $1111111111 \mathrm{~b} 15$

111111111111111111111111111111111111111 $1111111111 \mathrm{~b} 15$

111111111111111111111111111111111111111 $1111111111 \mathrm{~b} 15$

111111111111111111111111111111111111111 $1111111111 \mathrm{~b} 480$ 'Lower Lattice Grid

116116116116116116116116116116116116112 $1121121121 \mathrm{~b} 15$

116116116116116116116116116112112112112 $1121121121 \mathrm{~b} 15$

116116116116116116116112112112112112112 $1121121121 \mathrm{~b} 15$

116116116116116112112112112112112112112 $1121121121 \mathrm{~b} 15$

116116116116112112112112112112112112112 $1121121121 \mathrm{~b} 15$

116116116112112112112112112112112112112 $1121121121 \mathrm{~b} 15$

116116116112112112112112112112112112112 $1121121121 \mathrm{~b} 15$

116116112112112112112112112112112112112 $1121121121 \mathrm{~b} 15$

116116112112112112112112112112112112112 $1121121121 \mathrm{~b} 15$

116112112112112112112112112112112112112 $1121121121 \mathrm{~b} 15$

116112112112112112112112112112112112112 $1121121121 \mathrm{~b} 15$

116112112112112112112112112112112112112 $1121121121 \mathrm{~b} 15$

112112112112112112112112112112112112112 $1121121121 \mathrm{~b} 15$

112112112112112112112112112112112112112 $1121121121 \mathrm{~b} 15$

112112112112112112112112112112112112112 $1121121121 \mathrm{~b} 15$

112112112112112112112112112112112112112 $1121121121 \mathrm{~b} 480$

'Fuel and Reflector

$120120120120120120120120120120120120 \quad 1 \quad 2$ $3 \quad 4 \quad 1 b 15$

$120120120120120120120120120 \quad 5 \quad 6 \quad 7 \quad 8 \quad 9 \quad 10$ $111 \mathrm{~b} 15$

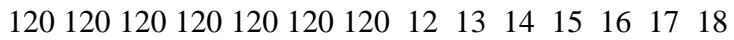
$19201 \mathrm{~b} 15$

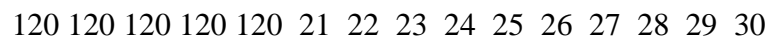
$311 \mathrm{~b} 15$ 


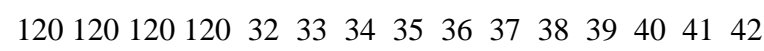
$431 \mathrm{~b} 15$

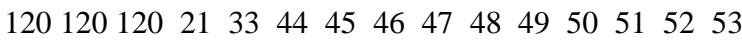
$541 \mathrm{~b} 15$

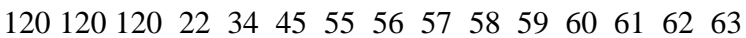
$641 \mathrm{~b} 15$

$\begin{array}{lllllllllllllll}120 & 120 & 12 & 23 & 35 & 46 & 56 & 65 & 66 & 67 & 68 & 69 & 70 & 71 & 72\end{array}$ $731 \mathrm{~b} 15$

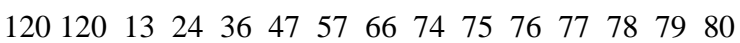
$811 \mathrm{~b} 15$

$\begin{array}{llllllllllllllll}120 & 5 & 14 & 25 & 37 & 48 & 58 & 67 & 75 & 82 & 83 & 84 & 85 & 86 & 87 & 88\end{array}$ $1 \mathrm{~b} 15$

$\begin{array}{llllllllllllllll}120 & 6 & 15 & 26 & 38 & 49 & 59 & 68 & 76 & 83 & 89 & 90 & 91 & 92 & 93 & 94\end{array}$ $1 \mathrm{~b} 15$

$\begin{array}{llllllllllllllll}120 & 7 & 16 & 27 & 39 & 50 & 60 & 69 & 77 & 84 & 90 & 95 & 96 & 97 & 98 & 99\end{array}$ $1 \mathrm{~b} 15$

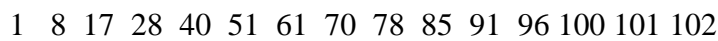
$1031 \mathrm{~b} 15$

$\begin{array}{lllllllllllllll}2 & 9 & 18 & 29 & 41 & 52 & 62 & 71 & 79 & 86 & 92 & 97 & 101 & 104 & 105\end{array}$ $1061 \mathrm{~b} 15$

$\begin{array}{lllllllllllllll}3 & 10 & 19 & 30 & 42 & 53 & 63 & 72 & 80 & 87 & 93 & 98 & 102 & 105 & 107\end{array}$ $1081 \mathrm{~b} 15$

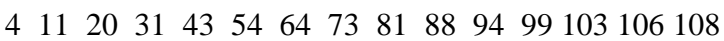
$1091 \mathrm{~b} 480$

'Upper Lattice Grid

117117117117117117117117117117117117113 $1131131131 \mathrm{~b} 15$

117117117117117117117117117113113113113 $1131131131 b 15$

117117117117117117117113113113113113113 $1131131131 \mathrm{~b} 15$

117117117117117113113113113113113113113 $1131131131 \mathrm{~b} 15$

117117117117113113113113113113113113113 $1131131131 \mathrm{~b} 15$

117117117113113113113113113113113113113 $1131131131 \mathrm{~b} 15$

117117117113113113113113113113113113113 $1131131131 \mathrm{~b} 15$

117117113113113113113113113113113113113 $1131131131 \mathrm{~b} 15$

117117113113113113113113113113113113113 $1131131131 \mathrm{~b} 15$

117113113113113113113113113113113113113 $1131131131 \mathrm{~b} 15$

117113113113113113113113113113113113113 $1131131131 \mathrm{~b} 15$

117113113113113113113113113113113113113 $1131131131 \mathrm{~b} 15$

113113113113113113113113113113113113113 $1131131131 \mathrm{~b} 15$

113113113113113113113113113113113113113 $1131131131 \mathrm{~b} 15$

113113113113113113113113113113113113113 $1131131131 \mathrm{~b} 15$

113113113113113113113113113113113113113 $1131131131 \mathrm{~b} 480$
'Upper Cladding and Moderator

114114114114114114114114114114114114118 $1181181181 \mathrm{~b} 15$

114114114114114114114114114118118118118 $1181181181 \mathrm{~b} 15$

114114114114114114114118118118118118118 $1181181181 \mathrm{~b} 15$

114114114114114118118118118118118118118 $1181181181 \mathrm{~b} 15$

114114114114118118118118118118118118118 $1181181181 \mathrm{~b} 15$

114114114118118118118118118118118118118 $1181181181 \mathrm{~b} 15$

114114114118118118118118118118118118118 $1181181181 \mathrm{~b} 15$

114114118118118118118118118118118118118 $1181181181 \mathrm{~b} 15$

114114118118118118118118118118118118118 $1181181181 \mathrm{~b} 15$

114118118118118118118118118118118118118 $1181181181 \mathrm{~b} 15$

114118118118118118118118118118118118118 $1181181181 \mathrm{~b} 15$

114118118118118118118118118118118118118 $1181181181 \mathrm{~b} 15$

118118118118118118118118118118118118118 $1181181181 \mathrm{~b} 15$

118118118118118118118118118118118118118 $1181181181 \mathrm{~b} 15$

118118118118118118118118118118118118118 $1181181181 \mathrm{~b} 15$

118118118118118118118118118118118118118 $1181181181 \mathrm{~b} 480$ end fill end array

read bias id=500 211 end bias read plot $\mathrm{ttl}=\mathrm{x} \mathrm{x}-\mathrm{z}$ plot at $\mathrm{y}=0.0^{\prime}$ $\mathrm{xul}=-50.0$ yul $=0 . \mathrm{zul}=110$ $\mathrm{xlr}=50.0 \quad \mathrm{ylr}=0 . \quad \mathrm{zlr}=-10$. uax $=1.0 \mathrm{wdn}=-1.0$ nax $=600$ end plt0 $\mathrm{ttl}={ }^{\prime} \mathrm{x}-\mathrm{y}$ plot at $\mathrm{z}=25.0^{\prime}$ pic $=$ mix

xul= $=30.0$ yul $=30 . \quad z u l=25$.

$\mathrm{xlr}=30.0$ ylr $=-30 . \mathrm{zlr}=25$.

uax $=1.0 \mathrm{vdn}=-1.0$

nax $=600$ end plt 1 end plot

end data end $=$ shell

cp ft04f001 \$RTNDIR/pnl33.xs04 cp ft64f001 \$RTNDIR/pnl33.rs64 end $=\mathrm{kmart}$

read initial kunit $=64$ 


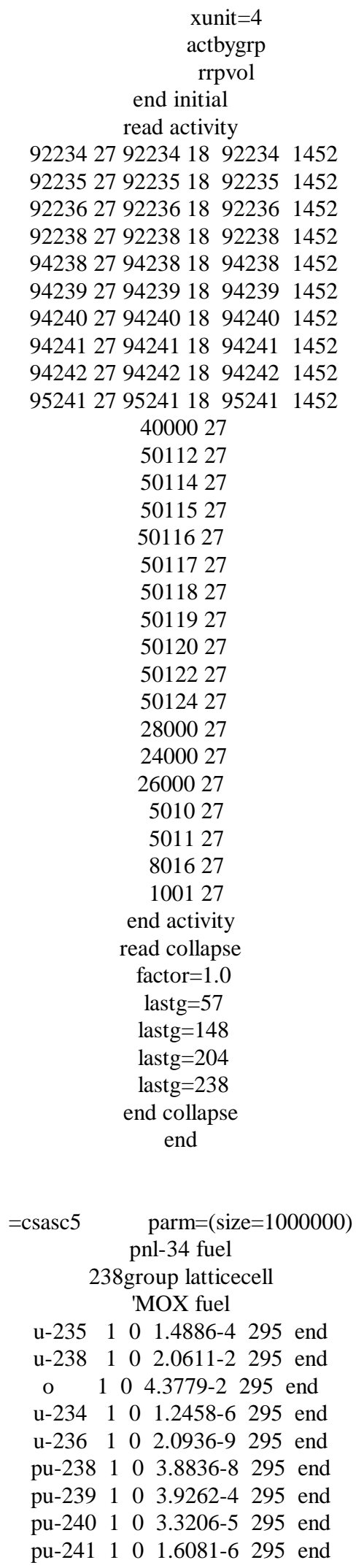

pu-242 $11001.1882-7 \quad 295$ end am-241 10 1.4891-6 295 end 'clad (ZR-2)

zr $\quad 2 \quad 0 \quad 4.2621-2 \quad 295$ end

sn $\quad 2 \quad 0 \quad 4.8328-4295$ end

ni $20 \begin{array}{llll}2 & 0 & 3.0336-5 & 295 \\ \text { end }\end{array}$

cr 20 7.6093-5 295 end

fe $\quad \begin{array}{llll}2 & 0 & 9.5642-5 & 295\end{array}$ end 'water(Refletor)

h $3 \quad 0 \quad 6.6706-2 \quad 295$ end

o $303.3353-2295$ end

b-10 $3001.7606-8 \quad 295$ end

b-11 $3007.1313-8295$ end 'Lattice Grid

si $\quad \begin{array}{lllll}4 & 0 & 3.4607-4 & 295 & \text { end }\end{array}$

fe $\quad \begin{array}{lllll}4 & 0 & 1.0152-4 & 295 & \text { end }\end{array}$

cu $\quad \begin{array}{llll}4 & 0 & 6.3731-5 & 295 \\ \text { end }\end{array}$

mn $4 \quad 0 \quad 2.2115-5295$ end

mg $400.6651-4295$ end

cr $4 \begin{array}{llll}4 & 0 & 6.2310-5 & 295\end{array}$ end

ti $4 \quad 0 \quad 2.5375-5 \quad 295$ end

al $4 \quad 0 \quad 5.8433-2 \quad 295$ end 'UO2

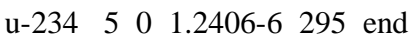

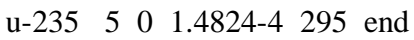

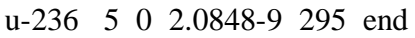
u-238 500 2.0525-2 295 end o $50 \quad 4.1943-2295$ end 'Aluminum

si $\quad \begin{array}{lllll}6 & 0 & 3.4607-4 & 295 & \text { end }\end{array}$

fe $\quad \begin{array}{llll}6 & 0 & 1.0152-4 & 295 \\ \text { end }\end{array}$

cu $\quad \begin{array}{llll}6 & 0 & 6.3731-5 & 295 \\ & \text { end }\end{array}$

mn $\quad \begin{array}{llll}6 & 0 & 2.2115-5 & 295 \\ \text { end }\end{array}$

mg $\quad \begin{array}{lllll}6 & 0 & 6.6651-4 & 295 & \text { end }\end{array}$

cr $\quad \begin{array}{llll}6 & 0 & 6.2310-5 & 295\end{array}$ end

ti $\quad \begin{array}{lllll}6 & 0 & 2.5375-5 & 295 & \text { end }\end{array}$

al $\quad \begin{array}{llll}6 & 0 & 5.8433-2 & 295 \\ \text { end }\end{array}$ 'lead

pb $\quad \begin{array}{llll}7 & 0 & 3.2174-2 & 295 \\ & \text { end }\end{array}$ end comp

squarepitch $2.51447 \quad 1.2827 \quad 121.43513$ end more data

limcen $=20000000$ end more PNL-34 read param gen=225 nsk=25 npg=20000 fdn=yes flx=yes wrs=64 res $=225$

plt $=$ no lng $=2000000$ end param read geometry

unit 1 com='PuO2/UO2 + Clad + Moderator' cylinder 110.641486 .67750 .0 cylinder 210.717686 .67750 .0 cuboid $314 \mathrm{p} 1.25723586 .67750 .0$ 


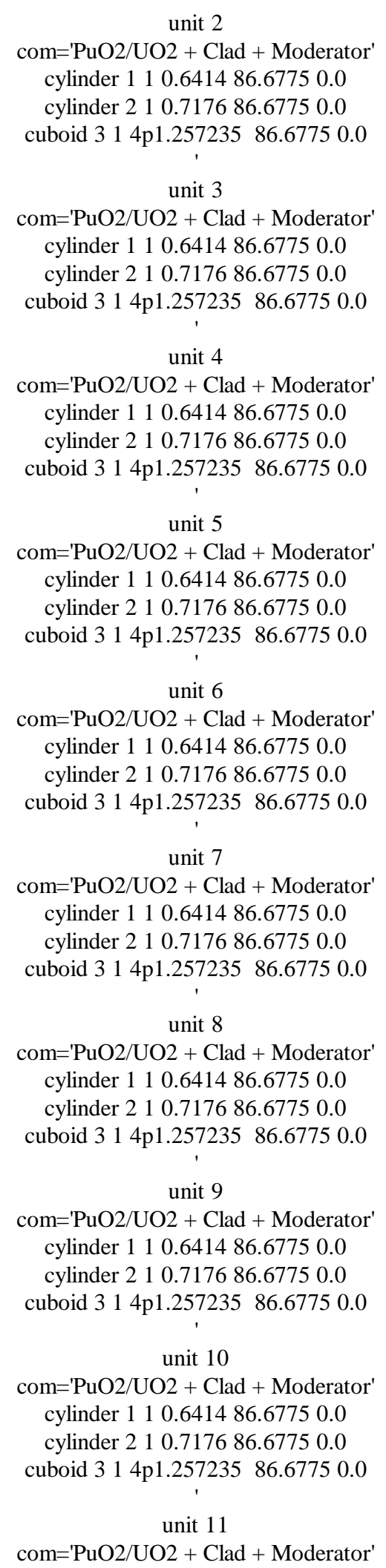

cylinder 110.641486 .67750 .0

cylinder 210.717686 .67750 .0

cuboid 31 4p1.25723586.67750.0

unit 12

com='PuO2/UO2 + Clad + Moderator'
cylinder 110.641486 .67750 .0
cylinder 210.717686 .67750 .0
cuboid $314 \mathrm{p} 1.25723586 .67750 .0$

unit 13

com='PuO2/UO2 + Clad + Moderator' cylinder 110.641486 .67750 .0 cylinder 210.717686 .67750 .0 cuboid $314 \mathrm{p} 1.25723586 .67750 .0$

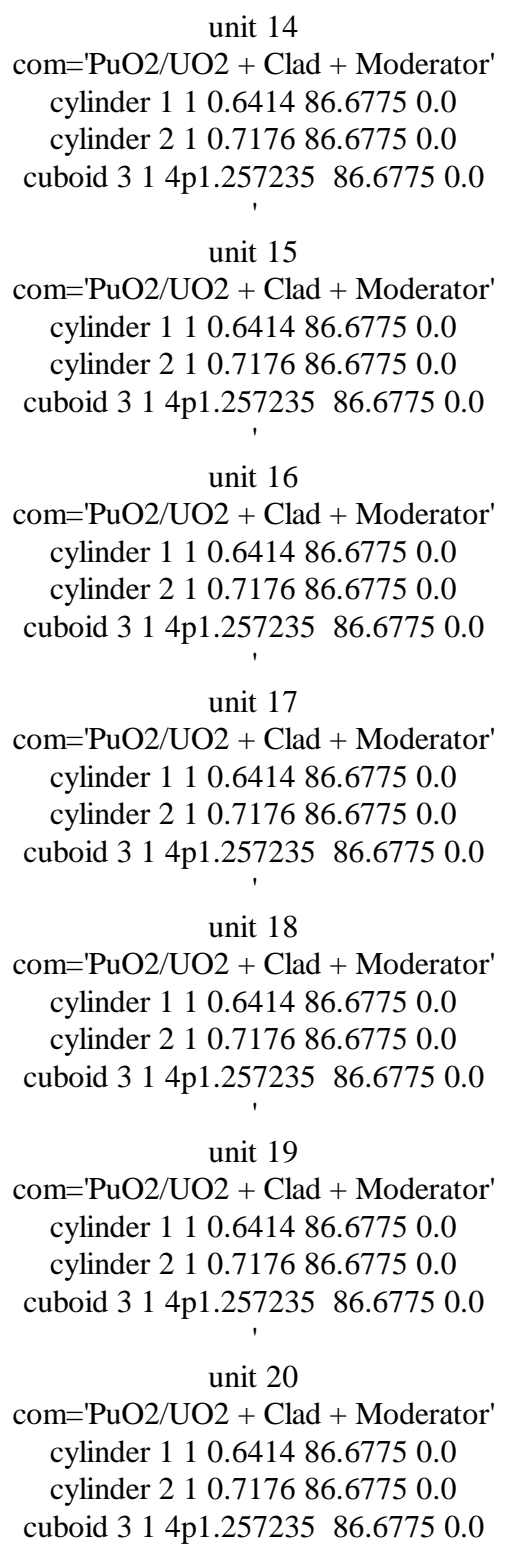




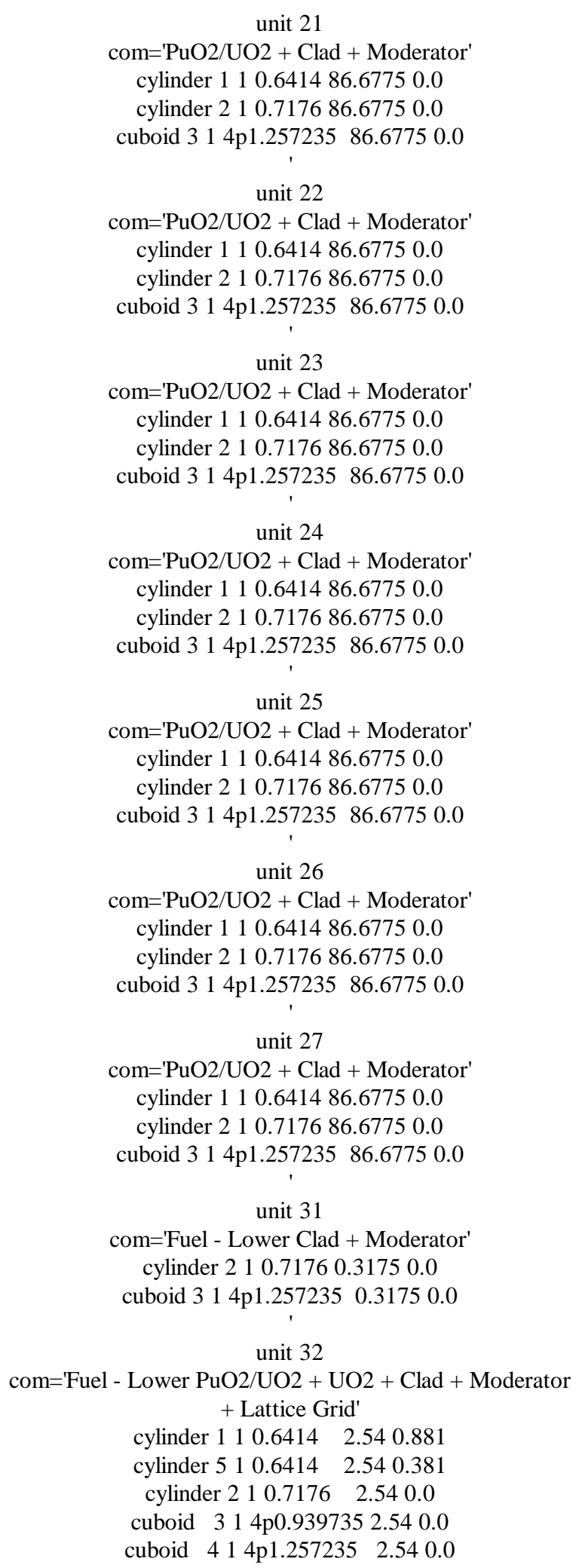

unit 33

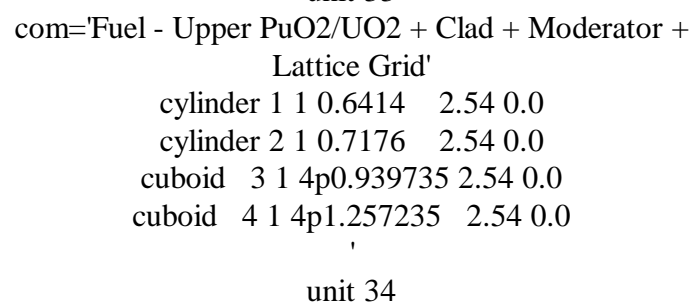

'Lower Cladding and Moderator $3535353535353535311 \mathrm{~b} 8$ $3535353535353531311 \mathrm{~b} 8$ $3535353535313131311 \mathrm{~b} 8$ $3535353531313131311 b 8$ $3535353131313131311 \mathrm{~b} 8$ $3535313131313131311 \mathrm{~b} 8$ $3535313131313131311 \mathrm{~b} 8$ $3531313131313131311 \mathrm{~b} 8$ $3131313131313131311 b 144$ 
'Lower Lattice Grid 363636363636363632 1b8 $363636363636363232 \quad 1 b 8$ $363636363632323232 \quad 1 b 8$ $363636363232323232 \quad 1 b 8$ $363636323232323232 \quad 1 b 8$ $363632323232323232 \quad 1 b 8$ $363632323232323232 \quad 1 b 8$

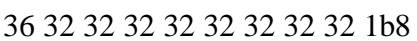
323232323232323232 1b144

'Fuel and Reflector $4040404040404040 \quad 1 \quad 1 b 8$ $40404040404040231 \mathrm{~b} 8$ $4040404040 \quad 45671 \mathrm{~b} 8$ $40404040 \quad 8 \quad 91011121 \mathrm{~b} 8$ $404040 \quad 813141516171 \mathrm{~b} 8$ $4040 \quad 4 \quad 91418192021 \quad 1 b 8$ $404051015192223241 b 8$ $40261116202325261 \mathrm{~b} 8$

$\begin{array}{llll}13 & 71217212426271 \mathrm{~b} 144\end{array}$ 'Upper Lattice Grid 373737373737373733 1b8 373737373737373333 1b8 373737373733333333 lb8 $3737373733333333331 b 8$ $3737373333333333331 b 8$ $3737333333333333331 b 8$ $373733333333333333 \quad 1 b 8$ $3733333333333333331 \mathrm{~b} 8$ 333333333333333333 1b144 'Upper Cladding and Moderator 383838383838383834 1b8 383838383838383434 1b8 383838383834343434 1b8 383838383434343434 1b8 383838343434343434 1b8 383834343434343434 1b8 383834343434343434 1b8 383434343434343434 1b8 343434343434343434 1b144 end fill end array

read bias id=500 211 end bias read plot

$\mathrm{ttl}=\mathrm{x}^{\mathrm{x}} \mathrm{x} \mathrm{z}$ plot at $\mathrm{y}=0.0^{\prime}$

$\mathrm{xul}=-30.0 \mathrm{yul}=0 . \mathrm{zul}=110$

$x l r=30.0 \quad$ ylr $=0 . \quad z l r=-10$.

uax $=1.0 \mathrm{wdn}=-1.0$

nax $=600$ end plt0

$\mathrm{tt} l=' \mathrm{x}-\mathrm{y}$ plot at $\mathrm{z}=25.0^{\prime}$ $\mathrm{pic}=\mathrm{mix}$

xul=-30.0 yul $=30 . \quad \mathrm{zul}=25$.

$\mathrm{xlr}=30.0 \quad \mathrm{ylr}=-30 . \mathrm{zlr}=25$.

uax $=1.0 \mathrm{vdn}=-1.0$

nax $=600$ end plt 1

end plot

end data

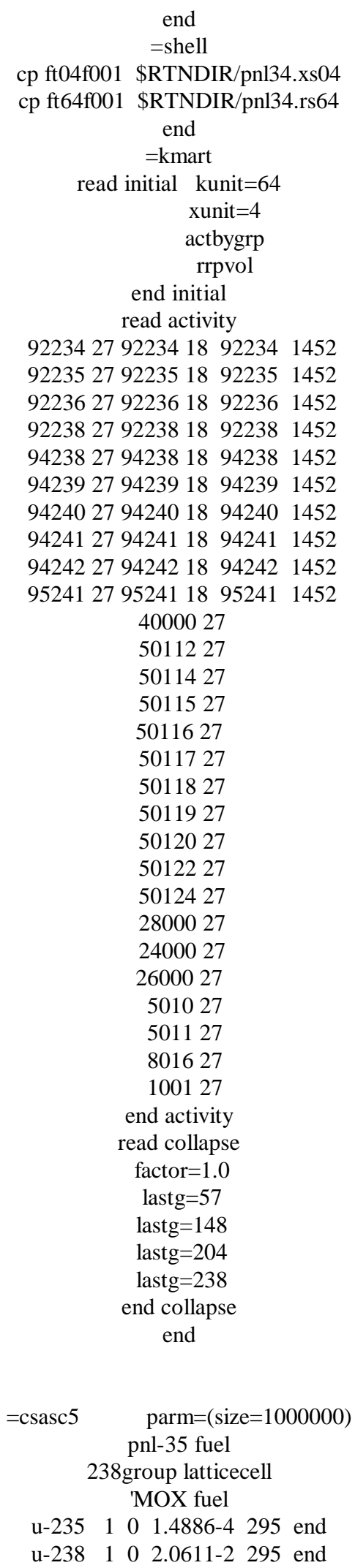




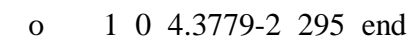
u-234 $100 \quad 1.2458-6 \quad 295$ end u-236 $11002.0936-9 \quad 295$ end pu-238 10 3.8836-8 295 end pu-239 10 3.9262-4 295 end pu-240 10 3.3206-5 295 end pu-241 10 1.6081-6 295 end pu-242 $100 \quad 1.1882-7 \quad 295$ end am-241 10 1.4891-6 295 end 'clad (ZR-2)

zr $\quad 2 \quad 0 \quad 4.2621-2295$ end

sn $2004.8328-4295$ end

ni $2003.0336-5295$ end

cr $2007.6093-5295$ end

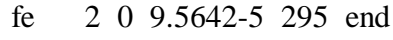
'water(Refletor)

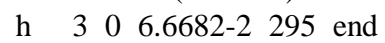

o 30 3.3405-2 295 end

b-10 $3008.4597-6295$ end

b-11 30 3.4266-5 295 end 'Lattice Grid

si $\quad \begin{array}{lllll}4 & 0 & 3.4607-4 & 295 & \text { end }\end{array}$

fe $4001.0152-4295$ end

cu $\quad \begin{array}{llll}4 & 0 & 6.3731-5 & 295\end{array}$ end

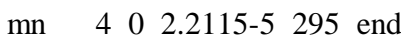

mg $4 \begin{array}{lllll}4 & 6.6651-4 & 295 & \text { end }\end{array}$

cr $4 \quad \begin{array}{llll}4 & 6.2310-5 & 295 & \text { end }\end{array}$

ti $4002.5375-5 \quad 295$ end

al $4 \quad 0 \quad 5.8433-2 \quad 295$ end 'UO2

u-234 $5 \quad 0 \quad 1.2406-6 \quad 295$ end u-235 $500 \quad 1.4824-4 \quad 295$ end

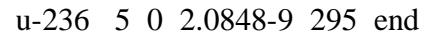
u-238 $500 \quad 2.0525-2 \quad 295$ end o $50 \quad 4.1943-2295$ end 'Aluminum

si $\quad \begin{array}{llllll}6 & 0 & 3.4607-4 & 295 & \text { end }\end{array}$

fe $\quad \begin{array}{llll}6 & 0 & 1.0152-4 & 295 \\ \text { end }\end{array}$

cu $\quad \begin{array}{llll}6 & 0 & 6.3731-5 & 295 \\ \text { end }\end{array}$

mn $\quad \begin{array}{llll}6 & 0 & 2.2115-5 & 295 \\ \text { end }\end{array}$

mg $\quad \begin{array}{llll}6 & 0 & 6.6651-4 & 295 \\ \text { end }\end{array}$

cr $\quad \begin{array}{lllll}6 & 0 & 6.2310-5 & 295 & \text { end }\end{array}$

ti $\quad \begin{array}{lllll}6 & 0 & 2.5375-5 & 295 & \text { end }\end{array}$

al $\begin{array}{llll}6 & 0 & 5.8433-2 & 295\end{array}$ end 'lead

pb $\quad \begin{array}{llll}7 & 0 & 3.2174-2 & 295\end{array}$ end end comp

squarepitch $2.51447 \quad 1.2827 \quad 121.43513$ end more data

limcen $=20000000$

end more

PNL-35

read param

gen=225 nsk=25 npg=20000 $\mathrm{fdn}=$ yes $f l x=$ yes wrs $=64$ res $=225$

$\mathrm{plt}=$ no $\operatorname{lng}=2000000$

end param

\author{
read geometry \\ unit 1 \\ com='PuO2/UO2 + Clad + Moderator' \\ cylinder 110.641486 .67750 .0 \\ cylinder 210.717686 .67750 .0 \\ cuboid $314 p 1.25723586 .67750 .0$ \\ unit 2 \\ com='PuO2/UO2 + Clad + Moderator' \\ cylinder 110.641486 .67750 .0 \\ cylinder 210.717686 .67750 .0 \\ cuboid $314 \mathrm{p} 1.25723586 .67750 .0$ \\ unit 3 \\ com='PuO2/UO2 + Clad + Moderator' \\ cylinder 110.641486 .67750 .0 \\ cylinder 210.717686 .67750 .0 \\ cuboid $314 p 1.25723586 .67750 .0$ \\ unit 4 \\ com='PuO2/UO2 + Clad + Moderator' \\ cylinder 110.641486 .67750 .0 \\ cylinder 210.717686 .67750 .0 \\ cuboid 31 4p1.25723586.67750.0 \\ unit 5 \\ com='PuO2/UO2 + Clad + Moderator' \\ cylinder 110.641486 .67750 .0 \\ cylinder 210.717686 .67750 .0 \\ cuboid 31 4p1.257235 86.67750 .0 \\ unit 6 \\ com='PuO2/UO2 + Clad + Moderator' \\ cylinder 110.641486 .67750 .0 \\ cylinder 210.717686 .67750 .0 \\ cuboid 31 4p1.257235 86.67750 .0 \\ unit 7 \\ com='PuO2/UO2 + Clad + Moderator' \\ cylinder 110.641486 .67750 .0 \\ cylinder 210.717686 .67750 .0 \\ cuboid $314 p 1.25723586 .67750 .0$ \\ unit 8 \\ com='PuO2/UO2 + Clad + Moderator' \\ cylinder 110.641486 .67750 .0 \\ cylinder 210.717686 .67750 .0 \\ cuboid 31 4p1.257235 86.67750 .0 \\ unit 9 \\ com='PuO2/UO2 + Clad + Moderator' \\ cylinder 110.641486 .67750 .0 \\ cylinder 210.717686 .67750 .0 \\ cuboid 31 4p1.25723586.67750.0 \\ unit 10
}


com='PuO2/UO2 + Clad + Moderator' cylinder 110.641486 .67750 .0 cylinder 210.717686 .67750 .0 cuboid $314 p 1.25723586 .67750 .0$

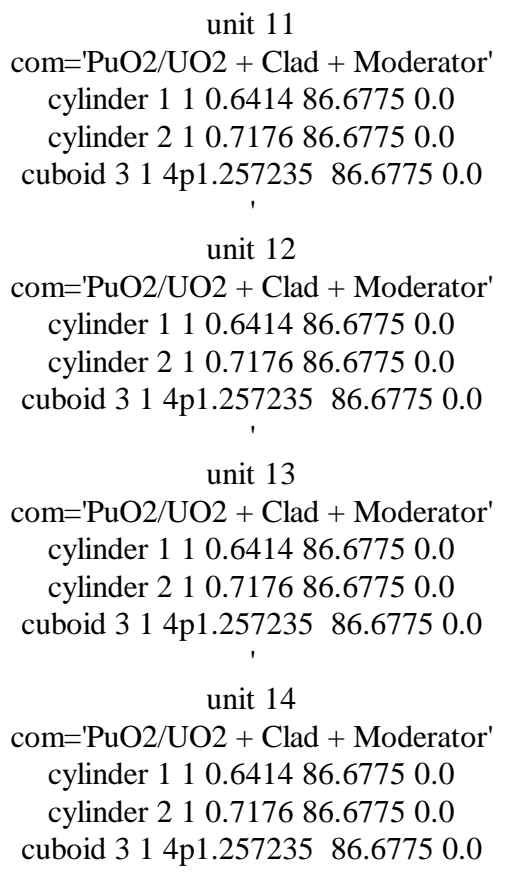

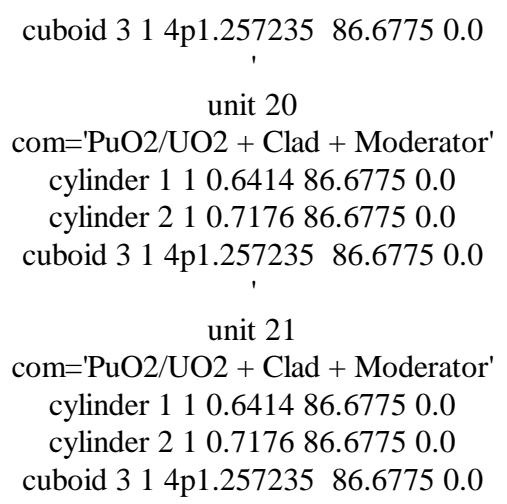

unit 29 
com='PuO2/UO2 + Clad + Moderator' cylinder 110.641486 .67750 .0 cylinder 210.717686 .67750 .0 cuboid 31 4p1.257235 86.67750 .0

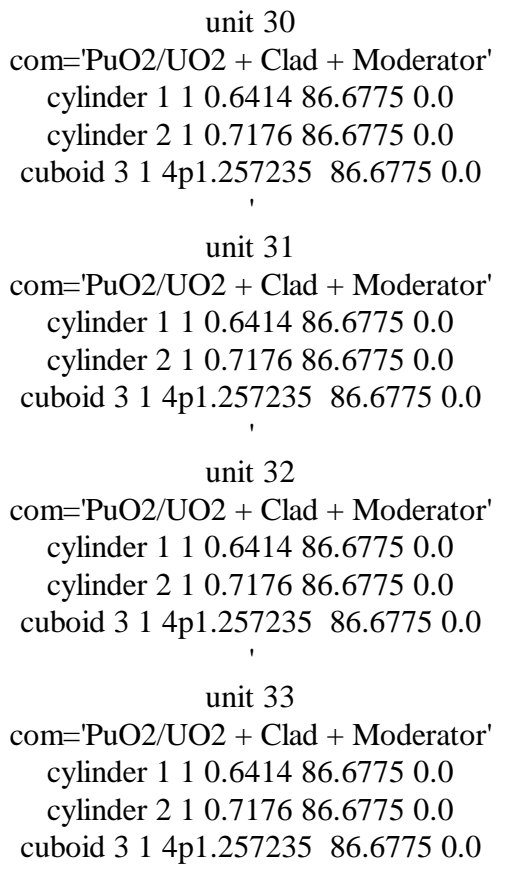

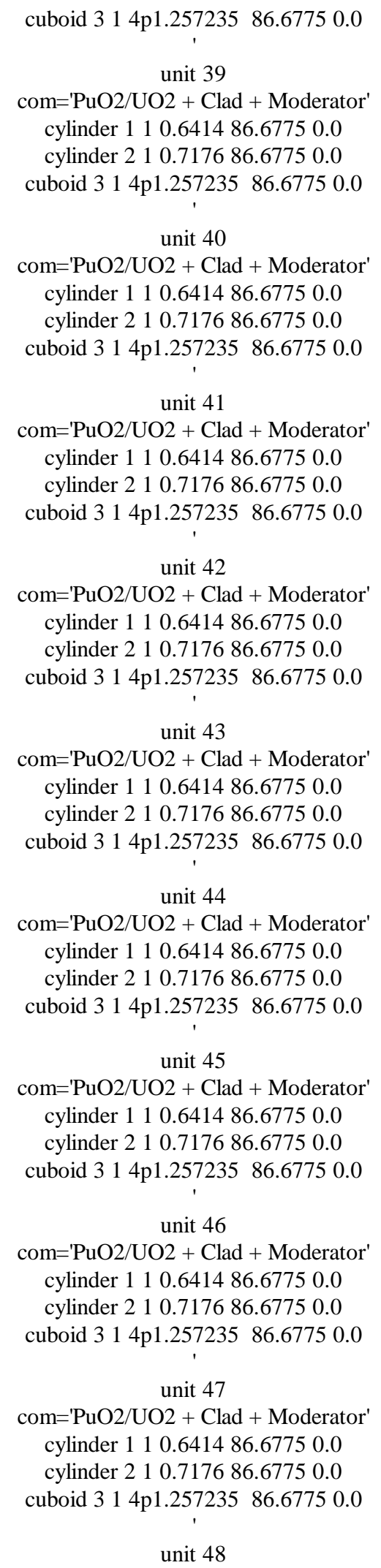


com='PuO2/UO2 + Clad + Moderator' cylinder 110.641486 .67750 .0 cylinder 210.717686 .67750 .0 cuboid 31 4p1.257235 86.67750 .0

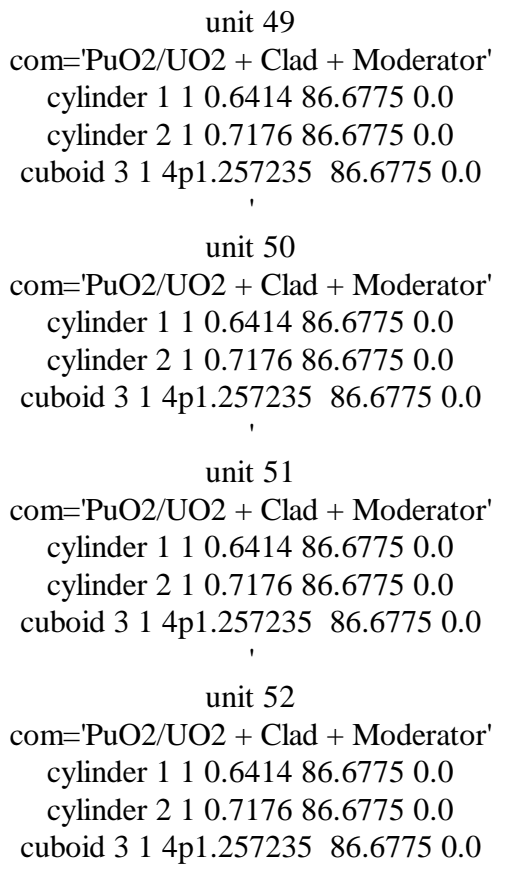

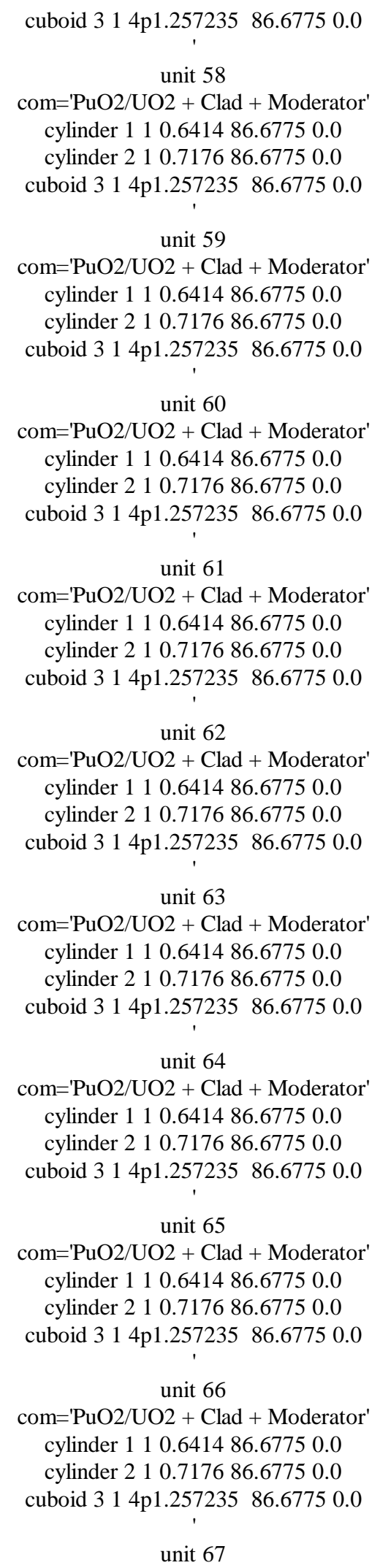


com='PuO2/UO2 + Clad + Moderator' cylinder 110.641486 .67750 .0 cylinder 210.717686 .67750 .0 cuboid $314 p 1.25723586 .67750 .0$

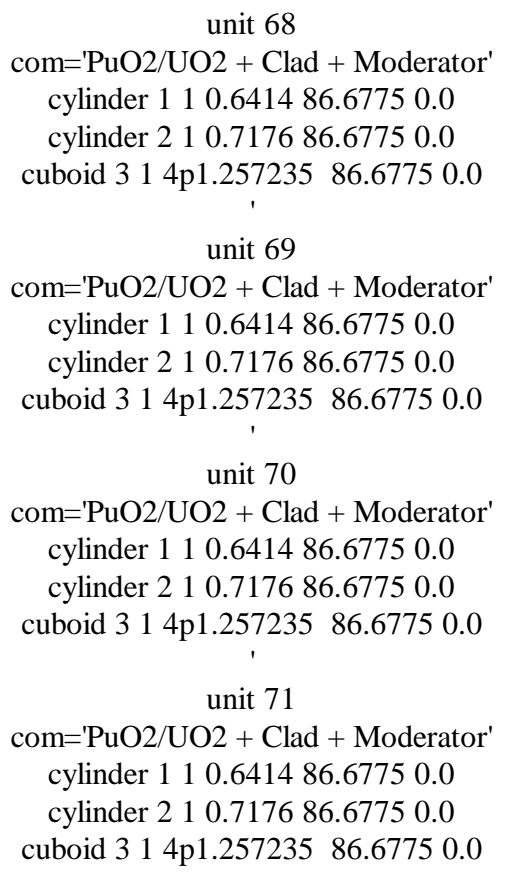

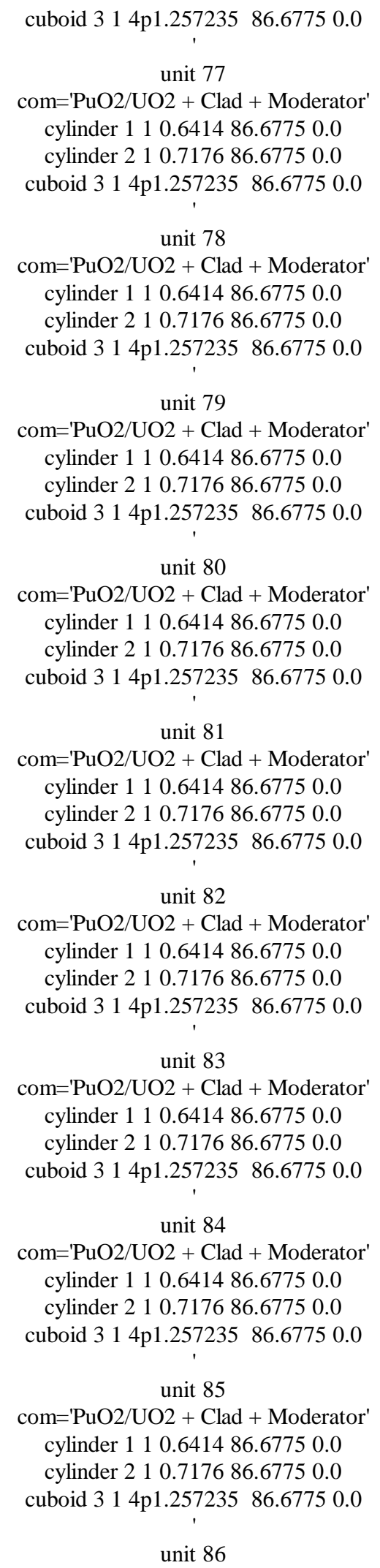


com='PuO2/UO2 + Clad + Moderator' cylinder 110.641486 .67750 .0 cylinder 210.717686 .67750 .0 cuboid 31 4p1.257235 86.67750 .0

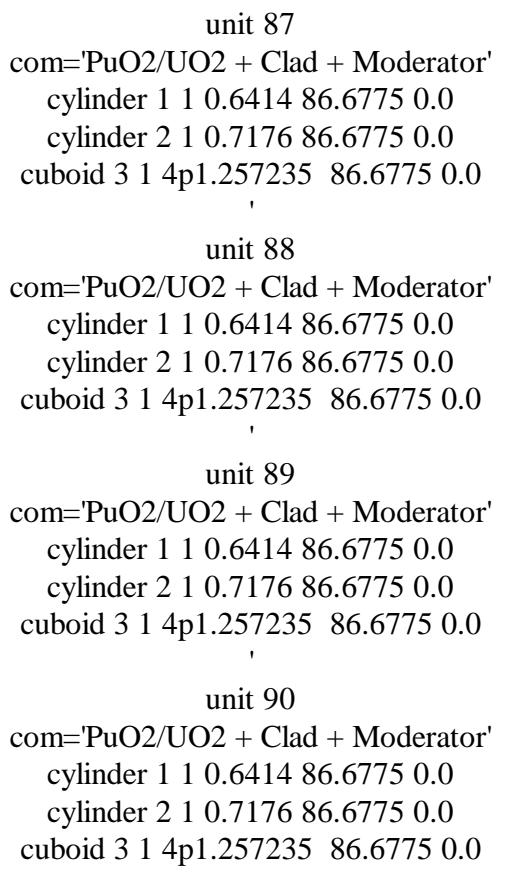

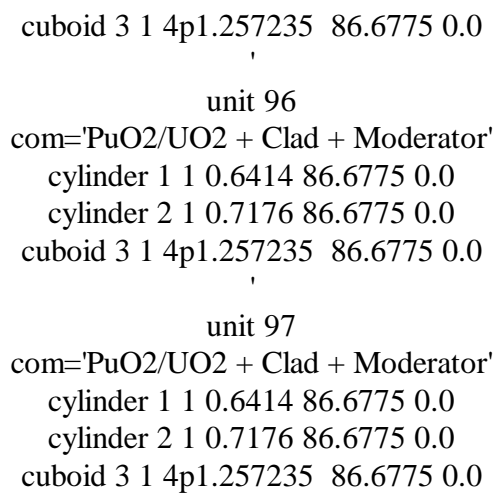




$$
\text { unit } 106
$$

com='Reflector - Lower Moderator + Lattice Grid' cuboid $314 \mathrm{p} 0.9397352 .540 .0$

cuboid $414 \mathrm{p} 1.2572352 .540 .0$

unit 107

com='Reflector - Upper Moderator + Lattice Grid' cuboid $314 \mathrm{p} 0.9397352 .540 .0$

cuboid $414 \mathrm{p} 1.2572352 .540 .0$

unit 108

com='Reflector - Upper Moderator'

cuboid $314 \mathrm{p} 1.2572350 .06350 .0$

unit 110

com='Moderator'

cuboid 314 p1.25723586.67750.0

global unit 120

com='Cells in assembly'

array $1-36.459815-36.4598150$

cuboid $614 \mathrm{p} 36.45981592 .9640 \quad-2.8575$

cuboid $714 \mathrm{p} 36.45981593 .9165-2.8575$

replicate $326 * 3.0 \quad 4$

replicate $364 * 3.01 .4623 .01$

replicate $374 * 3.00 .0 \quad 3.05$

end geometry

read array

ara $=1$ nux=29 nuy=29 nuz=5

fill

'Lower Cladding and Moderator

105105105105105105105105105101101101101 $1011011 \mathrm{~b} 14$

105105105105105105105101101101101101101 $1011011 \mathrm{~b} 14$

105105105105105105101101101101101101101 $1011011 \mathrm{~b} 14$

105105105105105101101101101101101101101 $1011011 \mathrm{~b} 14$

105105105105101101101101101101101101101 $1011011 \mathrm{~b} 14$

105105105101101101101101101101101101101 $1011011 \mathrm{~b} 14$

105105101101101101101101101101101101101 $1011011 \mathrm{~b} 14$

105101101101101101101101101101101101101 $1011011 \mathrm{~b} 14$

105101101101101101101101101101101101101 $1011011 \mathrm{~b} 14$

101101101101101101101101101101101101101 $1011011 \mathrm{~b} 14$

101101101101101101101101101101101101101 $1011011 \mathrm{~b} 14$

101101101101101101101101101101101101101 $1011011 \mathrm{~b} 14$

101101101101101101101101101101101101101
$1011011 \mathrm{~b} 14$

101101101101101101101101101101101101101 $1011011 \mathrm{~b} 14$

101101101101101101101101101101101101101 $1011011 \mathrm{~b} 420$

'Lower Lattice Grid

106106106106106106106106106102102102102 $1021021 \mathrm{~b} 14$

106106106106106106106102102102102102102 $1021021 \mathrm{~b} 14$

106106106106106106102102102102102102102 $1021021 \mathrm{~b} 14$

106106106106106102102102102102102102102 $1021021 \mathrm{~b} 14$

106106106106102102102102102102102102102 $1021021 \mathrm{~b} 14$

106106106102102102102102102102102102102 $1021021 \mathrm{~b} 14$

106106102102102102102102102102102102102 $1021021 \mathrm{~b} 14$

106102102102102102102102102102102102102 $1021021 \mathrm{~b} 14$

106102102102102102102102102102102102102 $1021021 \mathrm{~b} 14$

102102102102102102102102102102102102102 $1021021 \mathrm{~b} 14$

102102102102102102102102102102102102102 $1021021 \mathrm{~b} 14$

102102102102102102102102102102102102102 $1021021 \mathrm{~b} 14$

102102102102102102102102102102102102102 $1021021 \mathrm{~b} 14$

102102102102102102102102102102102102102 $1021021 \mathrm{~b} 14$

102102102102102102102102102102102102102 $1021021 \mathrm{~b} 420$

'Fuel and Reflector

$110110110110110110110110110 \quad 1 \quad 2 \quad 3 \quad 4 \quad 556$ $1 \mathrm{~b} 14$

$110110110110110110110 \quad 7 \quad 8 \quad 9 \quad 1011 \quad 12 \quad 13 \quad 14$ $1 \mathrm{~b} 14$

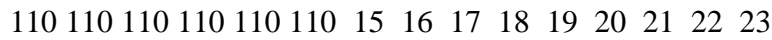
$1 \mathrm{~b} 14$

$11011011011011024252627 \quad 2829 \quad 30 \quad 31 \quad 32 \quad 33$ $1 \mathrm{~b} 14$

$110110110110 \quad 34 \quad 35 \quad 36 \quad 37 \quad 38 \quad 39 \quad 40 \quad 41 \quad 42 \quad 43 \quad 44$ $1 \mathrm{~b} 14$

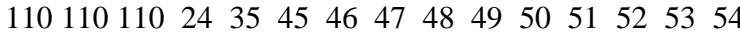
$1 \mathrm{~b} 14$

$\begin{array}{lllllllllllllll}110 & 110 & 15 & 25 & 36 & 46 & 55 & 56 & 57 & 58 & 59 & 60 & 61 & 62 & 63\end{array}$ $1 \mathrm{~b} 14$

$\begin{array}{lllllllllllllll}110 & 7 & 16 & 26 & 37 & 47 & 56 & 64 & 65 & 66 & 67 & 68 & 69 & 70 & 71\end{array}$ $1 \mathrm{~b} 14$

$\begin{array}{lllllllllllllll}110 & 8 & 17 & 27 & 38 & 48 & 57 & 65 & 72 & 73 & 74 & 75 & 76 & 77 & 78\end{array}$ $1 \mathrm{~b} 14$

$\begin{array}{lllllllllllllll}1 & 9 & 18 & 28 & 39 & 49 & 58 & 66 & 73 & 79 & 80 & 81 & 82 & 83 & 84\end{array}$ $1 \mathrm{~b} 14$ 
$\begin{array}{lllllllllllllll}2 & 10 & 19 & 29 & 40 & 50 & 59 & 67 & 74 & 80 & 85 & 86 & 87 & 88 & 89\end{array}$ $1 \mathrm{~b} 14$

$\begin{array}{lllllllllllllll}3 & 11 & 20 & 30 & 41 & 51 & 60 & 68 & 75 & 81 & 86 & 90 & 91 & 92 & 93\end{array}$ $1 \mathrm{~b} 14$

$\begin{array}{lllllllllllllll}4 & 12 & 21 & 31 & 42 & 52 & 61 & 69 & 76 & 82 & 87 & 91 & 94 & 95 & 96\end{array}$ $1 \mathrm{~b} 14$

$\begin{array}{lllllllllllllll}5 & 13 & 22 & 32 & 43 & 53 & 62 & 70 & 77 & 83 & 88 & 92 & 95 & 97 & 98\end{array}$ $1 \mathrm{~b} 14$

$\begin{array}{lllllllllllllll}6 & 14 & 23 & 33 & 44 & 54 & 63 & 71 & 78 & 84 & 89 & 93 & 96 & 98 & 99\end{array}$ $1 \mathrm{~b} 420$

'Upper Lattice Grid

107107107107107107107107107103103103103 $1031031 \mathrm{~b} 14$

107107107107107107107103103103103103103 $1031031 \mathrm{~b} 14$

107107107107107107103103103103103103103 $1031031 \mathrm{~b} 14$

107107107107107103103103103103103103103 $1031031 \mathrm{~b} 14$

107107107107103103103103103103103103103 $1031031 \mathrm{~b} 14$

107107107103103103103103103103103103103 $1031031 \mathrm{~b} 14$

107107103103103103103103103103103103103 $1031031 \mathrm{~b} 14$

107103103103103103103103103103103103103 $1031031 \mathrm{~b} 14$

107103103103103103103103103103103103103 $1031031 \mathrm{~b} 14$

103103103103103103103103103103103103103 $1031031 \mathrm{~b} 14$

103103103103103103103103103103103103103 $1031031 \mathrm{~b} 14$

103103103103103103103103103103103103103 $1031031 \mathrm{~b} 14$

103103103103103103103103103103103103103 $1031031 \mathrm{~b} 14$

103103103103103103103103103103103103103 $1031031 \mathrm{~b} 14$

103103103103103103103103103103103103103 $1031031 \mathrm{~b} 420$

'Upper Cladding and Moderator

108108108108108108108108108104104104104 $1041041 \mathrm{~b} 14$

108108108108108108108104104104104104104 $1041041 \mathrm{~b} 14$

108108108108108108104104104104104104104 $1041041 \mathrm{~b} 14$

108108108108108104104104104104104104104 $1041041 \mathrm{~b} 14$

108108108108104104104104104104104104104 $1041041 \mathrm{~b} 14$

108108108104104104104104104104104104104 $1041041 \mathrm{~b} 14$

108108104104104104104104104104104104104 $1041041 \mathrm{~b} 14$

108104104104104104104104104104104104104
$1041041 \mathrm{~b} 14$

108104104104104104104104104104104104104 $1041041 \mathrm{~b} 14$

104104104104104104104104104104104104104 $1041041 \mathrm{~b} 14$

104104104104104104104104104104104104104 $1041041 \mathrm{~b} 14$

104104104104104104104104104104104104104 $1041041 \mathrm{~b} 14$

104104104104104104104104104104104104104 $1041041 \mathrm{~b} 14$

104104104104104104104104104104104104104 $1041041 \mathrm{~b} 14$

104104104104104104104104104104104104104 104104 1b420 end fill end array

read bias id=500 211 end bias read plot

$\mathrm{tt} l={ }^{\prime} \mathrm{x}-\mathrm{z}$ plot at $\mathrm{y}=0.0^{\prime}$

$\mathrm{xul}=-50.0$ yul $=0 . \quad \mathrm{zul}=110$

$\mathrm{xlr}=50.0 \quad \mathrm{ylr}=0 . \quad \mathrm{zlr}=-10$.

uax $=1.0 \mathrm{wdn}=-1.0$

nax $=600$ end plt0

$\mathrm{ttl}={ }^{\prime} \mathrm{x}-\mathrm{y}$ plot at $\mathrm{z}=25.0^{\prime}$ pic $=$ mix

$\mathrm{xul}=-30.0 \mathrm{yul}=30 . \mathrm{zul}=25$.

$\mathrm{xlr}=30.0$ ylr $=-30 . \mathrm{zlr}=25$.

$\mathrm{uax}=1.0 \mathrm{vdn}=-1.0$

nax $=600$ end plt 1

end plot

end data

end

$=$ shell

cp ft04f001 \$RTNDIR/pnl35.xs04

cp ft64f001 \$RTNDIR/pnl35.rs64

end

$=\mathrm{kmart}$

read initial kunit $=64$

xunit $=4$

actbygrp

rrpvol

end initial

read activity

92234279223418922341452

92235279223518922351452

92236279223618922361452

$9223827922381892238 \quad 1452$

$9423827942381894238 \quad 1452$

94239279423918942391452

94240279424018942401452

$9424127942411894241 \quad 1452$

$9424227942421894242 \quad 1452$

95241279524118952411452

4000027

5011227

5011427 
5011527

5011627

5011727

5011827

5011927

5012027

5012227

5012427

2800027

2400027

2600027

501027

501127

801627

100127

end activity

read collapse

factor $=1.0$

lastg $=57$

lastg $=148$

lastg $=204$

lastg $=238$

end collapse end 
APPENDIX B

SCALE INPUT OF MIX-COMP-THERM-3 PROBLEM SET 



\section{APPENDIX B \\ SCALE INPUT OF MIX-COMP-THERM-3 PROBLEM SET}

This appendix contains the CENTRM inputs for the six MIX-COMP-THERM-03 cases, SAXTON01, SAXTON02, SAXTON03, SAXTON04, SAXTON05, and SAXTON06. The inputs can be changed to standard CSAS cases by replacing the $=$ CSASC5 record with $=$ CSAS25.

The input for the cases are listed below.

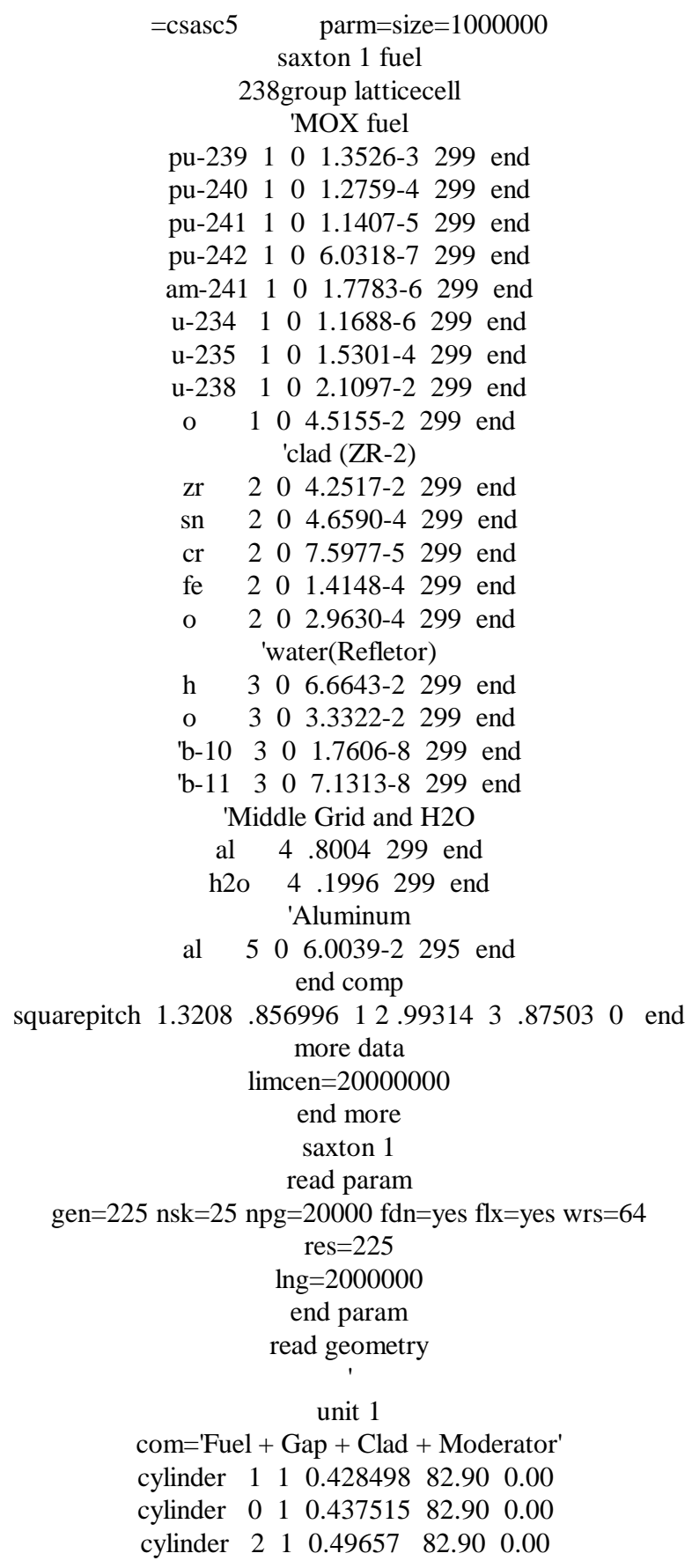

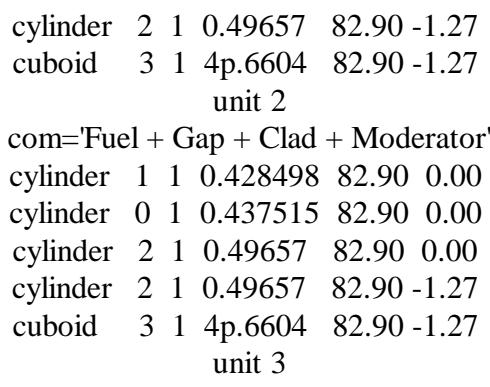

com='Fuel + Gap + Clad + Moderator $\begin{array}{lllllll}\text { cylinder } & 1 & 1 & 0.428498 & 82.90 & 0.00\end{array}$ $\begin{array}{lllllll}\text { cylinder } & 0 & 1 & 0.437515 & 82.90 & 0.00\end{array}$ $\begin{array}{lllllll}\text { cylinder } & 2 & 1 & 0.49657 & 82.90 & 0.00\end{array}$ $\begin{array}{llllll}\text { cylinder } & 2 & 1 & 0.49657 & 82.90 & -1.27\end{array}$ cuboid 314 p.6604 $82.90-1.27$ unit 4

com='Fuel + Gap + Clad + Moderator' $\begin{array}{llllll}\text { cylinder } & 1 & 1 & 0.428498 & 82.90 & 0.00\end{array}$ $\begin{array}{llllllll}\text { cylinder } & 0 & 1 & 0.437515 & 82.90 & 0.00\end{array}$ $\begin{array}{lllllll}\text { cylinder } & 2 & 1 & 0.49657 & 82.90 & 0.00\end{array}$ $\begin{array}{llllll}\text { cylinder } & 2 & 1 & 0.49657 & 82.90 & -1.27\end{array}$ cuboid $314 \mathrm{p} .6604 \quad 82.90-1.27$ unit 5

com='Fuel + Gap + Clad + Moderator' $\begin{array}{lllllll}\text { cylinder } & 1 & 1 & 0.428498 & 82.90 & 0.00\end{array}$ $\begin{array}{lllllll}\text { cylinder } & 0 & 1 & 0.437515 & 82.90 & 0.00\end{array}$ $\begin{array}{lllllll}\text { cylinder } & 2 & 1 & 0.49657 & 82.90 & 0.00\end{array}$ $\begin{array}{llllll}\text { cylinder } & 2 & 1 & 0.49657 & 82.90 & -1.27\end{array}$ cuboid $3 \quad 1 \quad 4 p .6604 \quad 82.90-1.27$ unit 6

com='Fuel + Gap + Clad + Moderator' $\begin{array}{lllllll}\text { cylinder } & 1 & 1 & 0.428498 & 82.90 & 0.00\end{array}$ $\begin{array}{lllllll}\text { cylinder } & 0 & 1 & 0.437515 & 82.90 & 0.00\end{array}$ $\begin{array}{lllllll}\text { cylinder } & 2 & 1 & 0.49657 & 82.90 & 0.00\end{array}$ $\begin{array}{llllll}\text { cylinder } & 2 & 1 & 0.49657 & 82.90 & -1.27\end{array}$ cuboid $3 \quad 1 \quad 4 p .6604 \quad 82.90-1.27$ unit 7

com='Fuel + Gap + Clad + Moderator' cylinder $\begin{array}{llllll}1 & 1 & 0.428498 & 82.90 & 0.00\end{array}$ $\begin{array}{lllllll}\text { cylinder } & 0 & 1 & 0.437515 & 82.90 & 0.00\end{array}$ $\begin{array}{lllllll}\text { cylinder } & 2 & 1 & 0.49657 & 82.90 & 0.00\end{array}$ $\begin{array}{llllll}\text { cylinder } & 2 & 1 & 0.49657 & 82.90 & -1.27\end{array}$ cuboid $3 \quad 1 \quad 4 p .6604 \quad 82.90-1.27$ unit 8

com='Fuel + Gap + Clad + Moderator' cylinder $\begin{array}{llllll}1 & 1 & 0.428498 & 82.90 & 0.00\end{array}$ 
$\begin{array}{llllll}\text { cylinder } & 0 & 1 & 0.437515 & 82.90 & 0.00\end{array}$ $\begin{array}{lllllll}\text { cylinder } & 2 & 1 & 0.49657 & 82.90 & 0.00\end{array}$ $\begin{array}{llllll}\text { cylinder } & 2 & 1 & 0.49657 & 82.90 & -1.27\end{array}$ cuboid $3 \quad 1 \quad 4 p .6604 \quad 82.90-1.27$ unit 9

com='Fuel + Gap + Clad + Moderator' $\begin{array}{lllllll}\text { cylinder } & 1 & 1 & 0.428498 & 82.90 & 0.00\end{array}$ $\begin{array}{lllllll}\text { cylinder } & 0 & 1 & 0.437515 & 82.90 & 0.00\end{array}$ $\begin{array}{lllllll}\text { cylinder } & 2 & 1 & 0.49657 & 82.90 & 0.00\end{array}$ $\begin{array}{llllll}\text { cylinder } & 2 & 1 & 0.49657 & 82.90 & -1.27\end{array}$ cuboid $3 \quad 1 \quad 4 p .6604 \quad 82.90-1.27$ unit 10

com='Fuel + Gap + Clad + Moderator' $\begin{array}{lllllll}\text { cylinder } & 1 & 1 & 0.428498 & 82.90 & 0.00\end{array}$ $\begin{array}{lllllllll}\text { cylinder } & 0 & 1 & 0.437515 & 82.90 & 0.00\end{array}$ $\begin{array}{lllllll}\text { cylinder } & 2 & 1 & 0.49657 & 82.90 & 0.00\end{array}$ $\begin{array}{llllll}\text { cylinder } & 2 & 1 & 0.49657 & 82.90 & -1.27\end{array}$ cuboid 3 1 4 p.6604 $82.90-1.27$ unit 11

com='Fuel + Gap + Clad + Moderator' $\begin{array}{llllll}\text { cylinder } & 1 & 1 & 0.428498 & 82.90 & 0.00\end{array}$ $\begin{array}{lllllll}\text { cylinder } & 0 & 1 & 0.437515 & 82.90 & 0.00\end{array}$ $\begin{array}{lllllll}\text { cylinder } & 2 & 1 & 0.49657 & 82.90 & 0.00\end{array}$ $\begin{array}{llllll}\text { cylinder } & 2 & 1 & 0.49657 & 82.90 & -1.27\end{array}$ cuboid $3 \quad 1 \quad 4 p .6604 \quad 82.90-1.27$ unit 12

com='Fuel + Gap + Clad + Moderator' $\begin{array}{llllll}\text { cylinder } & 1 & 1 & 0.428498 & 82.90 & 0.00\end{array}$ $\begin{array}{lllllll}\text { cylinder } & 0 & 1 & 0.437515 & 82.90 & 0.00\end{array}$ $\begin{array}{lllllll}\text { cylinder } & 2 & 1 & 0.49657 & 82.90 & 0.00\end{array}$ $\begin{array}{llllll}\text { cylinder } & 2 & 1 & 0.49657 & 82.90 & -1.27\end{array}$ cuboid $3 \quad 1 \quad 4 p .6604 \quad 82.90-1.27$ unit 13

com='Fuel + Gap + Clad + Moderator' $\begin{array}{llllll}\text { cylinder } & 1 & 1 & 0.428498 & 82.90 & 0.00\end{array}$ $\begin{array}{lllllll}\text { cylinder } & 0 & 1 & 0.437515 & 82.90 & 0.00\end{array}$ $\begin{array}{lllllll}\text { cylinder } & 2 & 1 & 0.49657 & 82.90 & 0.00\end{array}$ $\begin{array}{llllll}\text { cylinder } & 2 & 1 & 0.49657 & 82.90 & -1.27\end{array}$ cuboid 3 1 4 p.6604 $82.90-1.27$ unit 14

com='Fuel + Gap + Clad + Moderator' $\begin{array}{lllllll}\text { cylinder } & 1 & 1 & 0.428498 & 82.90 & 0.00\end{array}$ $\begin{array}{lllllll}\text { cylinder } & 0 & 1 & 0.437515 & 82.90 & 0.00\end{array}$ $\begin{array}{lllllll}\text { cylinder } & 2 & 1 & 0.49657 & 82.90 & 0.00\end{array}$ $\begin{array}{llllll}\text { cylinder } & 2 & 1 & 0.49657 & 82.90 & -1.27\end{array}$ cuboid 3 1 4 p.6604 $82.90-1.27$ unit 15

com='Fuel + Gap + Clad + Moderator' $\begin{array}{lllllll}\text { cylinder } & 1 & 1 & 0.428498 & 82.90 & 0.00\end{array}$ $\begin{array}{lllllll}\text { cylinder } & 0 & 1 & 0.437515 & 82.90 & 0.00\end{array}$ $\begin{array}{lllllll}\text { cylinder } & 2 & 1 & 0.49657 & 82.90 & 0.00\end{array}$ $\begin{array}{llllll}\text { cylinder } & 2 & 1 & 0.49657 & 82.90 & -1.27\end{array}$ cuboid 3 1 4 p.6604 $82.90-1.27$ unit 16

com='Fuel + Gap + Clad + Moderator' $\begin{array}{lllllll}\text { cylinder } & 1 & 1 & 0.428498 & 82.90 & 0.00\end{array}$ $\begin{array}{lllllll}\text { cylinder } & 0 & 1 & 0.437515 & 82.90 & 0.00\end{array}$ $\begin{array}{llllll}\text { cylinder } & 2 & 1 & 0.49657 & 82.90 & 0.00\end{array}$ $\begin{array}{llllll}\text { cylinder } & 2 & 1 & 0.49657 & 82.90 & -1.27\end{array}$ cuboid $314 \mathrm{p} .6604 \quad 82.90-1.27$ unit 17

com='Fuel + Gap + Clad + Moderator $\begin{array}{lllllll}\text { cylinder } & 1 & 1 & 0.428498 & 82.90 & 0.00\end{array}$ $\begin{array}{lllllll}\text { cylinder } & 0 & 1 & 0.437515 & 82.90 & 0.00\end{array}$ $\begin{array}{lllllll}\text { cylinder } & 2 & 1 & 0.49657 & 82.90 & 0.00\end{array}$ $\begin{array}{llllll}\text { cylinder } & 2 & 1 & 0.49657 & 82.90 & -1.27\end{array}$ cuboid $3 \quad 1 \quad 4 p .6604 \quad 82.90-1.27$ unit 18

com='Fuel + Gap + Clad + Moderator $\begin{array}{llllll}\text { cylinder } & 1 & 1 & 0.428498 & 82.90 & 0.00\end{array}$ $\begin{array}{llllllll}\text { cylinder } & 0 & 1 & 0.437515 & 82.90 & 0.00\end{array}$ $\begin{array}{llllllll}\text { cylinder } & 2 & 1 & 0.49657 & 82.90 & 0.00\end{array}$ $\begin{array}{llllll}\text { cylinder } & 2 & 1 & 0.49657 & 82.90 & -1.27\end{array}$ cuboid $3 \quad 1 \quad 4 p .6604 \quad 82.90-1.27$ unit 19

com='Fuel + Gap + Clad + Moderator' $\begin{array}{llllll}\text { cylinder } & 1 & 1 & 0.428498 & 82.90 & 0.00\end{array}$ $\begin{array}{lllllll}\text { cylinder } & 0 & 1 & 0.437515 & 82.90 & 0.00\end{array}$ $\begin{array}{lllllll}\text { cylinder } & 2 & 1 & 0.49657 & 82.90 & 0.00\end{array}$ $\begin{array}{llllll}\text { cylinder } & 2 & 1 & 0.49657 & 82.90 & -1.27\end{array}$ cuboid 31 4p.6604 $82.90-1.27$ unit 20

com='Fuel + Gap + Clad + Moderator' $\begin{array}{lllllll}\text { cylinder } & 1 & 1 & 0.428498 & 82.90 & 0.00\end{array}$ $\begin{array}{lllllll}\text { cylinder } & 0 & 1 & 0.437515 & 82.90 & 0.00\end{array}$ $\begin{array}{lllllll}\text { cylinder } & 2 & 1 & 0.49657 & 82.90 & 0.00\end{array}$ $\begin{array}{llllll}\text { cylinder } & 2 & 1 & 0.49657 & 82.90 & -1.27\end{array}$ cuboid $314 \mathrm{p} .6604 \quad 82.90-1.27$ unit 21

com='Fuel + Gap + Clad + Moderator $\begin{array}{lllllll}\text { cylinder } & 1 & 1 & 0.428498 & 82.90 & 0.00\end{array}$ $\begin{array}{lllllll}\text { cylinder } & 0 & 1 & 0.437515 & 82.90 & 0.00\end{array}$ $\begin{array}{llllllll}\text { cylinder } & 2 & 1 & 0.49657 & 82.90 & 0.00\end{array}$ $\begin{array}{llllll}\text { cylinder } & 2 & 1 & 0.49657 & 82.90 & -1.27\end{array}$ cuboid $3 \quad 1 \quad 4 p .6604 \quad 82.90-1.27$ unit 22

com='Fuel + Gap + Clad + Moderator' $\begin{array}{lllllll}\text { cylinder } & 1 & 1 & 0.428498 & 82.90 & 0.00\end{array}$ $\begin{array}{lllllll}\text { cylinder } & 0 & 1 & 0.437515 & 82.90 & 0.00\end{array}$ $\begin{array}{lllllll}\text { cylinder } & 2 & 1 & 0.49657 & 82.90 & 0.00\end{array}$ $\begin{array}{llllll}\text { cylinder } & 2 & 1 & 0.49657 & 82.90 & -1.27\end{array}$ cuboid $3 \quad 1$ 4p.6604 $82.90-1.27$ unit 23

com='Fuel + Gap + Clad + Moderator' $\begin{array}{lllllll}\text { cylinder } & 1 & 1 & 0.428498 & 82.90 & 0.00\end{array}$ $\begin{array}{lllllll}\text { cylinder } & 0 & 1 & 0.437515 & 82.90 & 0.00\end{array}$ $\begin{array}{lllllll}\text { cylinder } & 2 & 1 & 0.49657 & 82.90 & 0.00\end{array}$ $\begin{array}{llllll}\text { cylinder } & 2 & 1 & 0.49657 & 82.90 & -1.27\end{array}$ cuboid $3 \quad 1$ 4p.6604 82.90-1.27 unit 24

com='Fuel + Gap + Clad + Moderator' $\begin{array}{llllll}\text { cylinder } & 1 & 1 & 0.428498 & 82.90 & 0.00\end{array}$ $\begin{array}{lllllll}\text { cylinder } & 0 & 1 & 0.437515 & 82.90 & 0.00\end{array}$ $\begin{array}{lllllll}\text { cylinder } & 2 & 1 & 0.49657 & 82.90 & 0.00\end{array}$ 
$\begin{array}{llllll}\text { cylinder } & 2 & 1 & 0.49657 & 82.90 & -1.27\end{array}$

cuboid 314 p.6604 $82.90-1.27$ unit 25

com='Fuel + Gap + Clad + Moderator' $\begin{array}{lllllll}\text { cylinder } & 1 & 1 & 0.428498 & 82.90 & 0.00\end{array}$ $\begin{array}{llllllll}\text { cylinder } & 0 & 1 & 0.437515 & 82.90 & 0.00\end{array}$ $\begin{array}{lllllll}\text { cylinder } & 2 & 1 & 0.49657 & 82.90 & 0.00\end{array}$ $\begin{array}{llllll}\text { cylinder } & 2 & 1 & 0.49657 & 82.90 & -1.27\end{array}$ cuboid $3 \quad 1$ 4p.6604 $82.90-1.27$ unit 26

com='Fuel + Gap + Clad + Moderator' $\begin{array}{lllllll}\text { cylinder } & 1 & 1 & 0.428498 & 82.90 & 0.00\end{array}$ $\begin{array}{lllllll}\text { cylinder } & 0 & 1 & 0.437515 & 82.90 & 0.00\end{array}$ $\begin{array}{llllllll}\text { cylinder } & 2 & 1 & 0.49657 & 82.90 & 0.00\end{array}$ $\begin{array}{llllll}\text { cylinder } & 2 & 1 & 0.49657 & 82.90 & -1.27\end{array}$ cuboid 3 1 4 p.6604 $82.90-1.27$ unit 27

com='Fuel + Gap + Clad + Moderator' $\begin{array}{lllllll}\text { cylinder } & 1 & 1 & 0.428498 & 82.90 & 0.00\end{array}$ $\begin{array}{lllllll}\text { cylinder } & 0 & 1 & 0.437515 & 82.90 & 0.00\end{array}$ $\begin{array}{lllllll}\text { cylinder } & 2 & 1 & 0.49657 & 82.90 & 0.00\end{array}$ $\begin{array}{llllll}\text { cylinder } & 2 & 1 & 0.49657 & 82.90 & -1.27\end{array}$ cuboid $3 \begin{array}{llll}4 & 4 p .6604 & 82.90 & -1.27\end{array}$ unit 28

com='Fuel + Gap + Clad + Moderator' $\begin{array}{lllllll}\text { cylinder } & 1 & 1 & 0.428498 & 82.90 & 0.00\end{array}$ $\begin{array}{lllllll}\text { cylinder } & 0 & 1 & 0.437515 & 82.90 & 0.00\end{array}$ $\begin{array}{lllllll}\text { cylinder } & 2 & 1 & 0.49657 & 82.90 & 0.00\end{array}$ $\begin{array}{llllll}\text { cylinder } & 2 & 1 & 0.49657 & 82.90 & -1.27\end{array}$ cuboid $3 \begin{array}{lllll} & 4 & 4 p .6604 & 82.90 & -1.27\end{array}$ unit 29

com='Fuel + Gap + Clad + Moderator' $\begin{array}{lllllll}\text { cylinder } & 1 & 1 & 0.428498 & 82.90 & 0.00\end{array}$ $\begin{array}{llllllll}\text { cylinder } & 0 & 1 & 0.437515 & 82.90 & 0.00\end{array}$ $\begin{array}{lllllll}\text { cylinder } & 2 & 1 & 0.49657 & 82.90 & 0.00\end{array}$ $\begin{array}{llllll}\text { cylinder } & 2 & 1 & 0.49657 & 82.90 & -1.27\end{array}$ cuboid $3 \begin{array}{lllll} & 4 & 4 p .6604 & 82.90 & -1.27\end{array}$ unit 30

com='Fuel + Gap + Clad + Moderator' $\begin{array}{llllll}\text { cylinder } & 1 & 1 & 0.428498 & 82.90 & 0.00\end{array}$ $\begin{array}{llllllll}\text { cylinder } & 0 & 1 & 0.437515 & 82.90 & 0.00\end{array}$ $\begin{array}{lllllll}\text { cylinder } & 2 & 1 & 0.49657 & 82.90 & 0.00\end{array}$ $\begin{array}{llllll}\text { cylinder } & 2 & 1 & 0.49657 & 82.90 & -1.27\end{array}$ cuboid 31 4p.6604 $82.90-1.27$ unit 31

com='Fuel + Gap + Clad + Moderator' $\begin{array}{lllllll}\text { cylinder } & 1 & 1 & 0.428498 & 82.90 & 0.00\end{array}$ $\begin{array}{lllllll}\text { cylinder } & 0 & 1 & 0.437515 & 82.90 & 0.00\end{array}$ $\begin{array}{lllllll}\text { cylinder } & 2 & 1 & 0.49657 & 82.90 & 0.00\end{array}$ $\begin{array}{llllll}\text { cylinder } & 2 & 1 & 0.49657 & 82.90 & -1.27\end{array}$ cuboid $3 \quad 1 \quad 4 p .6604 \quad 82.90-1.27$ unit 32

com='Fuel + Gap + Clad + Moderator' $\begin{array}{llllll}\text { cylinder } & 1 & 1 & 0.428498 & 82.90 & 0.00\end{array}$ $\begin{array}{lllllll}\text { cylinder } & 0 & 1 & 0.437515 & 82.90 & 0.00\end{array}$ $\begin{array}{lllllll}\text { cylinder } & 2 & 1 & 0.49657 & 82.90 & 0.00\end{array}$ $\begin{array}{llllll}\text { cylinder } & 2 & 1 & 0.49657 & 82.90 & -1.27\end{array}$ cuboid $3 \begin{array}{lllll} & 1 & 4 p .6604 & 82.90 & -1.27\end{array}$ unit 33

com='Fuel + Gap + Clad + Moderator' $\begin{array}{lllllll}\text { cylinder } & 1 & 1 & 0.428498 & 82.90 & 0.00\end{array}$ $\begin{array}{lllllll}\text { cylinder } & 0 & 1 & 0.437515 & 82.90 & 0.00\end{array}$ $\begin{array}{lllllll}\text { cylinder } & 2 & 1 & 0.49657 & 82.90 & 0.00\end{array}$ $\begin{array}{llllll}\text { cylinder } & 2 & 1 & 0.49657 & 82.90 & -1.27\end{array}$ cuboid 31 4p.6604 82.90-1.27 unit 34

com='Fuel + Gap + Clad + Moderator' $\begin{array}{lllllll}\text { cylinder } & 1 & 1 & 0.428498 & 82.90 & 0.00\end{array}$ $\begin{array}{lllllll}\text { cylinder } & 0 & 1 & 0.437515 & 82.90 & 0.00\end{array}$ $\begin{array}{lllllll}\text { cylinder } & 2 & 1 & 0.49657 & 82.90 & 0.00\end{array}$ $\begin{array}{llllll}\text { cylinder } & 2 & 1 & 0.49657 & 82.90 & -1.27\end{array}$ cuboid $3 \quad 1 \quad 4 p .6604 \quad 82.90-1.27$ unit 35

com='Fuel + Gap + Clad + Moderator' $\begin{array}{lllllll}\text { cylinder } & 1 & 1 & 0.428498 & 82.90 & 0.00\end{array}$ $\begin{array}{lllllll}\text { cylinder } & 0 & 1 & 0.437515 & 82.90 & 0.00\end{array}$ $\begin{array}{lllllll}\text { cylinder } & 2 & 1 & 0.49657 & 82.90 & 0.00\end{array}$ $\begin{array}{llllll}\text { cylinder } & 2 & 1 & 0.49657 & 82.90 & -1.27\end{array}$ cuboid $3 \quad 1 \quad 4 p .6604 \quad 82.90-1.27$ unit 36

com='Fuel + Gap + Clad + Moderator' $\begin{array}{lllllll}\text { cylinder } & 1 & 1 & 0.428498 & 82.90 & 0.00\end{array}$ $\begin{array}{llllllll}\text { cylinder } & 0 & 1 & 0.437515 & 82.90 & 0.00\end{array}$ $\begin{array}{lllllll}\text { cylinder } & 2 & 1 & 0.49657 & 82.90 & 0.00\end{array}$ $\begin{array}{llllll}\text { cylinder } & 2 & 1 & 0.49657 & 82.90 & -1.27\end{array}$ cuboid $3 \quad 1 \quad 4 p .6604 \quad 82.90-1.27$ unit 37

com='Fuel + Gap + Clad + Moderator' $\begin{array}{llllll}\text { cylinder } & 1 & 1 & 0.428498 & 82.90 & 0.00\end{array}$ $\begin{array}{lllllll}\text { cylinder } & 0 & 1 & 0.437515 & 82.90 & 0.00\end{array}$ $\begin{array}{lllllll}\text { cylinder } & 2 & 1 & 0.49657 & 82.90 & 0.00\end{array}$ $\begin{array}{llllll}\text { cylinder } & 2 & 1 & 0.49657 & 82.90 & -1.27\end{array}$ cuboid $3 \quad 1 \quad 4 p .6604 \quad 82.90-1.27$ unit 38

com='Fuel + Gap + Clad + Moderator' $\begin{array}{lllllll}\text { cylinder } & 1 & 1 & 0.428498 & 82.90 & 0.00\end{array}$ $\begin{array}{lllllll}\text { cylinder } & 0 & 1 & 0.437515 & 82.90 & 0.00\end{array}$ $\begin{array}{lllllll}\text { cylinder } & 2 & 1 & 0.49657 & 82.90 & 0.00\end{array}$ $\begin{array}{llllll}\text { cylinder } & 2 & 1 & 0.49657 & 82.90 & -1.27\end{array}$ cuboid $3 \quad 1 \quad 4 p .6604 \quad 82.90-1.27$ unit 39

com='Fuel + Gap + Clad + Moderator' $\begin{array}{lllllll}\text { cylinder } & 1 & 1 & 0.428498 & 82.90 & 0.00\end{array}$ $\begin{array}{lllllll}\text { cylinder } & 0 & 1 & 0.437515 & 82.90 & 0.00\end{array}$ $\begin{array}{lllllll}\text { cylinder } & 2 & 1 & 0.49657 & 82.90 & 0.00\end{array}$ $\begin{array}{llllll}\text { cylinder } & 2 & 1 & 0.49657 & 82.90 & -1.27\end{array}$ cuboid 3 1 4p.6604 82.90-1.27 unit 40

com='Fuel + Gap + Clad + Moderator' $\begin{array}{lllllll}\text { cylinder } & 1 & 1 & 0.428498 & 82.90 & 0.00\end{array}$ $\begin{array}{lllllll}\text { cylinder } & 0 & 1 & 0.437515 & 82.90 & 0.00\end{array}$ $\begin{array}{lllllll}\text { cylinder } & 2 & 1 & 0.49657 & 82.90 & 0.00\end{array}$ $\begin{array}{llllll}\text { cylinder } & 2 & 1 & 0.49657 & 82.90 & -1.27\end{array}$ cuboid $3 \quad 1 \quad 4 p .6604 \quad 82.90-1.27$ 
unit 41

com='Fuel + Gap + Clad + Moderator' $\begin{array}{lllllll}\text { cylinder } & 1 & 1 & 0.428498 & 82.90 & 0.00\end{array}$ $\begin{array}{lllllll}\text { cylinder } & 0 & 1 & 0.437515 & 82.90 & 0.00\end{array}$ $\begin{array}{lllllll}\text { cylinder } & 2 & 1 & 0.49657 & 82.90 & 0.00\end{array}$ $\begin{array}{llllll}\text { cylinder } & 2 & 1 & 0.49657 & 82.90 & -1.27\end{array}$ cuboid $3 \quad 1 \quad 4 p .6604 \quad 82.90-1.27$ unit 42

com='Fuel + Gap + Clad + Moderator' $\begin{array}{lllllll}\text { cylinder } & 1 & 1 & 0.428498 & 82.90 & 0.00\end{array}$ $\begin{array}{lllllll}\text { cylinder } & 0 & 1 & 0.437515 & 82.90 & 0.00\end{array}$ $\begin{array}{lllllll}\text { cylinder } & 2 & 1 & 0.49657 & 82.90 & 0.00\end{array}$ $\begin{array}{llllll}\text { cylinder } & 2 & 1 & 0.49657 & 82.90 & -1.27\end{array}$ cuboid 314 p.6604 $82.90-1.27$ unit 43

com='Fuel + Gap + Clad + Moderator' $\begin{array}{llllll}\text { cylinder } & 1 & 1 & 0.428498 & 82.90 & 0.00\end{array}$ $\begin{array}{lllllll}\text { cylinder } & 0 & 1 & 0.437515 & 82.90 & 0.00\end{array}$ $\begin{array}{lllllll}\text { cylinder } & 2 & 1 & 0.49657 & 82.90 & 0.00\end{array}$ $\begin{array}{llllll}\text { cylinder } & 2 & 1 & 0.49657 & 82.90 & -1.27\end{array}$ cuboid $3 \quad 1 \quad 4 p .6604 \quad 82.90-1.27$ unit 44

com='Fuel + Gap + Clad + Moderator' $\begin{array}{llllll}\text { cylinder } & 1 & 1 & 0.428498 & 82.90 & 0.00\end{array}$ $\begin{array}{lllllll}\text { cylinder } & 0 & 1 & 0.437515 & 82.90 & 0.00\end{array}$ $\begin{array}{llllllll}\text { cylinder } & 2 & 1 & 0.49657 & 82.90 & 0.00\end{array}$ $\begin{array}{llllll}\text { cylinder } & 2 & 1 & 0.49657 & 82.90 & -1.27\end{array}$ cuboid 3 1 4 p.6604 $82.90-1.27$ unit 45

com='Fuel + Gap + Clad + Moderator' $\begin{array}{lllllll}\text { cylinder } & 1 & 1 & 0.428498 & 82.90 & 0.00\end{array}$ $\begin{array}{lllllll}\text { cylinder } & 0 & 1 & 0.437515 & 82.90 & 0.00\end{array}$ $\begin{array}{lllllll}\text { cylinder } & 2 & 1 & 0.49657 & 82.90 & 0.00\end{array}$ $\begin{array}{llllll}\text { cylinder } & 2 & 1 & 0.49657 & 82.90 & -1.27\end{array}$ cuboid 31 4p.6604 82.90-1.27 unit 46

com='Fuel + Gap + Clad + Moderator' $\begin{array}{lllllll}\text { cylinder } & 1 & 1 & 0.428498 & 82.90 & 0.00\end{array}$ $\begin{array}{lllllll}\text { cylinder } & 0 & 1 & 0.437515 & 82.90 & 0.00\end{array}$ $\begin{array}{lllllll}\text { cylinder } & 2 & 1 & 0.49657 & 82.90 & 0.00\end{array}$ $\begin{array}{llllll}\text { cylinder } & 2 & 1 & 0.49657 & 82.90 & -1.27\end{array}$ cuboid 31 4p.6604 $82.90-1.27$ unit 47

com='Fuel + Gap + Clad + Moderator' $\begin{array}{llllll}\text { cylinder } & 1 & 1 & 0.428498 & 82.90 & 0.00\end{array}$ $\begin{array}{lllllll}\text { cylinder } & 0 & 1 & 0.437515 & 82.90 & 0.00\end{array}$ $\begin{array}{lllllll}\text { cylinder } & 2 & 1 & 0.49657 & 82.90 & 0.00\end{array}$ $\begin{array}{llllll}\text { cylinder } & 2 & 1 & 0.49657 & 82.90 & -1.27\end{array}$ cuboid 3 1 4 p.6604 $82.90-1.27$ unit 48

com='Fuel + Gap + Clad + Moderator' $\begin{array}{lllllll}\text { cylinder } & 1 & 1 & 0.428498 & 82.90 & 0.00\end{array}$ $\begin{array}{lllllll}\text { cylinder } & 0 & 1 & 0.437515 & 82.90 & 0.00\end{array}$ $\begin{array}{lllllll}\text { cylinder } & 2 & 1 & 0.49657 & 82.90 & 0.00\end{array}$ $\begin{array}{llllll}\text { cylinder } & 2 & 1 & 0.49657 & 82.90 & -1.27\end{array}$ cuboid $3 \quad 1 \quad 4 p .6604 \quad 82.90-1.27$ unit 49
com='Fuel + Gap + Clad + Moderator' $\begin{array}{lllllll}\text { cylinder } & 1 & 1 & 0.428498 & 82.90 & 0.00\end{array}$ $\begin{array}{lllllll}\text { cylinder } & 0 & 1 & 0.437515 & 82.90 & 0.00\end{array}$ $\begin{array}{llllllll}\text { cylinder } & 2 & 1 & 0.49657 & 82.90 & 0.00\end{array}$ $\begin{array}{llllll}\text { cylinder } & 2 & 1 & 0.49657 & 82.90 & -1.27\end{array}$ cuboid $314 \mathrm{p} .6604 \quad 82.90-1.27$ unit 50

com='Fuel + Gap + Clad + Moderator' $\begin{array}{llllll}\text { cylinder } & 1 & 1 & 0.428498 & 82.90 & 0.00\end{array}$ $\begin{array}{llllllll}\text { cylinder } & 0 & 1 & 0.437515 & 82.90 & 0.00\end{array}$ $\begin{array}{lllllll}\text { cylinder } & 2 & 1 & 0.49657 & 82.90 & 0.00\end{array}$ $\begin{array}{llllll}\text { cylinder } & 2 & 1 & 0.49657 & 82.90 & -1.27\end{array}$ cuboid 31 4p.6604 $82.90-1.27$ unit 51

com='Fuel + Gap + Clad + Moderator' $\begin{array}{llllll}\text { cylinder } & 1 & 1 & 0.428498 & 82.90 & 0.00\end{array}$ $\begin{array}{lllllll}\text { cylinder } & 0 & 1 & 0.437515 & 82.90 & 0.00\end{array}$ $\begin{array}{lllllll}\text { cylinder } & 2 & 1 & 0.49657 & 82.90 & 0.00\end{array}$ $\begin{array}{llllll}\text { cylinder } & 2 & 1 & 0.49657 & 82.90 & -1.27\end{array}$ cuboid 3 1 4 p.6604 $82.90-1.27$ unit 52

com='Fuel + Gap + Clad + Moderator $\begin{array}{lllllll}\text { cylinder } & 1 & 1 & 0.428498 & 82.90 & 0.00\end{array}$ $\begin{array}{lllllll}\text { cylinder } & 0 & 1 & 0.437515 & 82.90 & 0.00\end{array}$ $\begin{array}{llllllll}\text { cylinder } & 2 & 1 & 0.49657 & 82.90 & 0.00\end{array}$ $\begin{array}{llllll}\text { cylinder } & 2 & 1 & 0.49657 & 82.90 & -1.27\end{array}$ cuboid $314 \mathrm{p} .6604 \quad 82.90-1.27$ unit 53

com='Fuel + Gap + Clad + Moderator' $\begin{array}{lllllll}\text { cylinder } & 1 & 1 & 0.428498 & 82.90 & 0.00\end{array}$ $\begin{array}{lllllll}\text { cylinder } & 0 & 1 & 0.437515 & 82.90 & 0.00\end{array}$ $\begin{array}{llllll}\text { cylinder } & 2 & 1 & 0.49657 & 82.90 & 0.00\end{array}$ $\begin{array}{llllll}\text { cylinder } & 2 & 1 & 0.49657 & 82.90 & -1.27\end{array}$ cuboid $3 \quad 1$ 4p.6604 $82.90-1.27$ unit 54

com='Fuel + Gap + Clad + Moderator' $\begin{array}{lllllll}\text { cylinder } & 1 & 1 & 0.428498 & 82.90 & 0.00\end{array}$ $\begin{array}{lllllll}\text { cylinder } & 0 & 1 & 0.437515 & 82.90 & 0.00\end{array}$ $\begin{array}{lllllll}\text { cylinder } & 2 & 1 & 0.49657 & 82.90 & 0.00\end{array}$ $\begin{array}{llllll}\text { cylinder } & 2 & 1 & 0.49657 & 82.90 & -1.27\end{array}$ cuboid $314 \mathrm{p} .6604 \quad 82.90-1.27$ unit 55

com='Fuel + Gap + Clad + Moderator $\begin{array}{llllll}\text { cylinder } & 1 & 1 & 0.428498 & 82.90 & 0.00\end{array}$ $\begin{array}{llllllll}\text { cylinder } & 0 & 1 & 0.437515 & 82.90 & 0.00\end{array}$ $\begin{array}{lllllll}\text { cylinder } & 2 & 1 & 0.49657 & 82.90 & 0.00\end{array}$ $\begin{array}{llllll}\text { cylinder } & 2 & 1 & 0.49657 & 82.90 & -1.27\end{array}$ cuboid $3 \quad 1 \quad 4 p .6604 \quad 82.90-1.27$ unit 56

com='Fuel + Gap + Clad + Moderator' $\begin{array}{lllllll}\text { cylinder } & 1 & 1 & 0.428498 & 82.90 & 0.00\end{array}$ $\begin{array}{llllllll}\text { cylinder } & 0 & 1 & 0.437515 & 82.90 & 0.00\end{array}$ $\begin{array}{llllll}\text { cylinder } & 2 & 1 & 0.49657 & 82.90 & 0.00\end{array}$ $\begin{array}{llllll}\text { cylinder } & 2 & 1 & 0.49657 & 82.90 & -1.27\end{array}$ cuboid $3 \quad 1 \quad 4 p .6604 \quad 82.90-1.27$ unit 57

com='Fuel + Gap + Clad + Moderator' 
$\begin{array}{llllll}\text { cylinder } & 1 & 1 & 0.428498 & 82.90 & 0.00\end{array}$ $\begin{array}{lllllll}\text { cylinder } & 0 & 1 & 0.437515 & 82.90 & 0.00\end{array}$ $\begin{array}{lllllll}\text { cylinder } & 2 & 1 & 0.49657 & 82.90 & 0.00\end{array}$ $\begin{array}{llllll}\text { cylinder } & 2 & 1 & 0.49657 & 82.90 & -1.27\end{array}$ cuboid 3 1 4 p.6604 $82.90-1.27$ unit 58

com='Fuel + Gap + Clad + Moderator' $\begin{array}{lllllll}\text { cylinder } & 1 & 1 & 0.428498 & 82.90 & 0.00\end{array}$ $\begin{array}{llllllll}\text { cylinder } & 0 & 1 & 0.437515 & 82.90 & 0.00\end{array}$ $\begin{array}{lllllll}\text { cylinder } & 2 & 1 & 0.49657 & 82.90 & 0.00\end{array}$ $\begin{array}{llllll}\text { cylinder } & 2 & 1 & 0.49657 & 82.90 & -1.27\end{array}$ cuboid $3 \begin{array}{lllll} & 4 & 4 p .6604 & 82.90 & -1.27\end{array}$ unit 59

com='Fuel + Gap + Clad + Moderator' $\begin{array}{lllllll}\text { cylinder } & 1 & 1 & 0.428498 & 82.90 & 0.00\end{array}$ $\begin{array}{lllllll}\text { cylinder } & 0 & 1 & 0.437515 & 82.90 & 0.00\end{array}$ $\begin{array}{lllllll}\text { cylinder } & 2 & 1 & 0.49657 & 82.90 & 0.00\end{array}$ $\begin{array}{llllll}\text { cylinder } & 2 & 1 & 0.49657 & 82.90 & -1.27\end{array}$ cuboid $3 \quad 1$ 4p.6604 $82.90-1.27$ unit 60

com='Fuel + Gap + Clad + Moderator' $\begin{array}{lllllll}\text { cylinder } & 1 & 1 & 0.428498 & 82.90 & 0.00\end{array}$ $\begin{array}{lllllll}\text { cylinder } & 0 & 1 & 0.437515 & 82.90 & 0.00\end{array}$ $\begin{array}{lllllll}\text { cylinder } & 2 & 1 & 0.49657 & 82.90 & 0.00\end{array}$ $\begin{array}{llllll}\text { cylinder } & 2 & 1 & 0.49657 & 82.90 & -1.27\end{array}$ cuboid $3 \quad 1 \quad 4 p .6604 \quad 82.90-1.27$ unit 61

com='Fuel + Gap + Clad + Moderator' $\begin{array}{llllll}\text { cylinder } & 1 & 1 & 0.428498 & 82.90 & 0.00\end{array}$ $\begin{array}{lllllll}\text { cylinder } & 0 & 1 & 0.437515 & 82.90 & 0.00\end{array}$ $\begin{array}{lllllll}\text { cylinder } & 2 & 1 & 0.49657 & 82.90 & 0.00\end{array}$ $\begin{array}{llllll}\text { cylinder } & 2 & 1 & 0.49657 & 82.90 & -1.27\end{array}$ cuboid $3 \quad 1 \quad 4 p .6604 \quad 82.90-1.27$ unit 62

com='Fuel + Gap + Clad + Moderator' $\begin{array}{llllll}\text { cylinder } & 1 & 1 & 0.428498 & 82.90 & 0.00\end{array}$ $\begin{array}{llllllll}\text { cylinder } & 0 & 1 & 0.437515 & 82.90 & 0.00\end{array}$ $\begin{array}{lllllll}\text { cylinder } & 2 & 1 & 0.49657 & 82.90 & 0.00\end{array}$ $\begin{array}{llllll}\text { cylinder } & 2 & 1 & 0.49657 & 82.90 & -1.27\end{array}$ cuboid 3 1 4 p.6604 $82.90-1.27$ unit 63

com='Fuel + Gap + Clad + Moderator' $\begin{array}{llllll}\text { cylinder } & 1 & 1 & 0.428498 & 82.90 & 0.00\end{array}$ $\begin{array}{lllllll}\text { cylinder } & 0 & 1 & 0.437515 & 82.90 & 0.00\end{array}$ $\begin{array}{lllllll}\text { cylinder } & 2 & 1 & 0.49657 & 82.90 & 0.00\end{array}$ $\begin{array}{llllll}\text { cylinder } & 2 & 1 & 0.49657 & 82.90 & -1.27\end{array}$ cuboid 31 4p.6604 $82.90-1.27$ unit 64

com='Fuel + Gap + Clad + Moderator' $\begin{array}{lllllll}\text { cylinder } & 1 & 1 & 0.428498 & 82.90 & 0.00\end{array}$ $\begin{array}{lllllll}\text { cylinder } & 0 & 1 & 0.437515 & 82.90 & 0.00\end{array}$ $\begin{array}{lllllll}\text { cylinder } & 2 & 1 & 0.49657 & 82.90 & 0.00\end{array}$ $\begin{array}{llllll}\text { cylinder } & 2 & 1 & 0.49657 & 82.90 & -1.27\end{array}$ cuboid $3 \quad 1 \quad 4 p .6604 \quad 82.90-1.27$ unit 65

com='Fuel + Gap + Clad + Moderator' $\begin{array}{llllll}\text { cylinder } & 1 & 1 & 0.428498 & 82.90 & 0.00\end{array}$ $\begin{array}{lllllll}\text { cylinder } & 0 & 1 & 0.437515 & 82.90 & 0.00\end{array}$ $\begin{array}{lllllll}\text { cylinder } & 2 & 1 & 0.49657 & 82.90 & 0.00\end{array}$ $\begin{array}{llllll}\text { cylinder } & 2 & 1 & 0.49657 & 82.90 & -1.27\end{array}$ cuboid 31 4p.6604 $82.90-1.27$ unit 66

com='Fuel + Gap + Clad + Moderator' $\begin{array}{lllllll}\text { cylinder } & 1 & 1 & 0.428498 & 82.90 & 0.00\end{array}$ $\begin{array}{llllllll}\text { cylinder } & 0 & 1 & 0.437515 & 82.90 & 0.00\end{array}$ $\begin{array}{llllll}\text { cylinder } & 2 & 1 & 0.49657 & 82.90 & 0.00\end{array}$ $\begin{array}{llllll}\text { cylinder } & 2 & 1 & 0.49657 & 82.90 & -1.27\end{array}$ cuboid $3 \quad 1$ 4p.6604 $82.90-1.27$ unit 67

com='Fuel + Gap + Clad + Moderator $\begin{array}{llllll}\text { cylinder } & 1 & 1 & 0.428498 & 82.90 & 0.00\end{array}$ $\begin{array}{llllllll}\text { cylinder } & 0 & 1 & 0.437515 & 82.90 & 0.00\end{array}$ $\begin{array}{lllllll}\text { cylinder } & 2 & 1 & 0.49657 & 82.90 & 0.00\end{array}$ $\begin{array}{llllll}\text { cylinder } & 2 & 1 & 0.49657 & 82.90 & -1.27\end{array}$ cuboid 3 1 4 p.6604 $82.90-1.27$ unit 68

com='Fuel + Gap + Clad + Moderator' $\begin{array}{llllll}\text { cylinder } & 1 & 1 & 0.428498 & 82.90 & 0.00\end{array}$ $\begin{array}{llllllll}\text { cylinder } & 0 & 1 & 0.437515 & 82.90 & 0.00\end{array}$ $\begin{array}{llllllll}\text { cylinder } & 2 & 1 & 0.49657 & 82.90 & 0.00\end{array}$ $\begin{array}{llllll}\text { cylinder } & 2 & 1 & 0.49657 & 82.90 & -1.27\end{array}$ cuboid $314 \mathrm{p} .6604 \quad 82.90-1.27$ unit 69

com='Fuel + Gap + Clad + Moderator' $\begin{array}{lllllll}\text { cylinder } & 1 & 1 & 0.428498 & 82.90 & 0.00\end{array}$ $\begin{array}{lllllll}\text { cylinder } & 0 & 1 & 0.437515 & 82.90 & 0.00\end{array}$ $\begin{array}{lllllll}\text { cylinder } & 2 & 1 & 0.49657 & 82.90 & 0.00\end{array}$ $\begin{array}{llllll}\text { cylinder } & 2 & 1 & 0.49657 & 82.90 & -1.27\end{array}$ cuboid 3 1 4 4p.6604 82.90-1.27 unit 70

com='Fuel + Gap + Clad + Moderator $\begin{array}{llllll}\text { cylinder } & 1 & 1 & 0.428498 & 82.90 & 0.00\end{array}$ $\begin{array}{llllllll}\text { cylinder } & 0 & 1 & 0.437515 & 82.90 & 0.00\end{array}$ $\begin{array}{lllllll}\text { cylinder } & 2 & 1 & 0.49657 & 82.90 & 0.00\end{array}$ $\begin{array}{llllll}\text { cylinder } & 2 & 1 & 0.49657 & 82.90 & -1.27\end{array}$ cuboid $3 \quad 1 \quad 4 p .6604 \quad 82.90-1.27$ unit 71

com='Fuel + Gap + Clad + Moderator' $\begin{array}{llllll}\text { cylinder } & 1 & 1 & 0.428498 & 82.90 & 0.00\end{array}$ $\begin{array}{lllllll}\text { cylinder } & 0 & 1 & 0.437515 & 82.90 & 0.00\end{array}$ $\begin{array}{lllllll}\text { cylinder } & 2 & 1 & 0.49657 & 82.90 & 0.00\end{array}$ $\begin{array}{llllll}\text { cylinder } & 2 & 1 & 0.49657 & 82.90 & -1.27\end{array}$ cuboid $3 \quad 1 \quad 4 p .6604 \quad 82.90-1.27$ unit 72

com='Fuel + Gap + Clad + Moderator' $\begin{array}{lllllll}\text { cylinder } & 1 & 1 & 0.428498 & 82.90 & 0.00\end{array}$ $\begin{array}{lllllll}\text { cylinder } & 0 & 1 & 0.437515 & 82.90 & 0.00\end{array}$ $\begin{array}{lllllll}\text { cylinder } & 2 & 1 & 0.49657 & 82.90 & 0.00\end{array}$ $\begin{array}{llllll}\text { cylinder } & 2 & 1 & 0.49657 & 82.90 & -1.27\end{array}$ cuboid $3 \quad 1 \quad 4 p .6604 \quad 82.90-1.27$ unit 73

com='Fuel + Gap + Clad + Moderator' $\begin{array}{lllllll}\text { cylinder } & 1 & 1 & 0.428498 & 82.90 & 0.00\end{array}$ $\begin{array}{lllllll}\text { cylinder } & 0 & 1 & 0.437515 & 82.90 & 0.00\end{array}$ 
$\begin{array}{llllll}\text { cylinder } & 2 & 1 & 0.49657 & 82.90 & 0.00\end{array}$ $\begin{array}{llllll}\text { cylinder } & 2 & 1 & 0.49657 & 82.90 & -1.27\end{array}$ cuboid 3 1 4 p.6604 $82.90-1.27$ unit 74

com='Fuel + Gap + Clad + Moderator' $\begin{array}{llllll}\text { cylinder } & 1 & 1 & 0.428498 & 82.90 & 0.00\end{array}$ $\begin{array}{lllllll}\text { cylinder } & 0 & 1 & 0.437515 & 82.90 & 0.00\end{array}$ $\begin{array}{lllllll}\text { cylinder } & 2 & 1 & 0.49657 & 82.90 & 0.00\end{array}$ $\begin{array}{llllll}\text { cylinder } & 2 & 1 & 0.49657 & 82.90 & -1.27\end{array}$ cuboid 31 4p.6604 $82.90-1.27$ unit 75

com='Fuel + Gap + Clad + Moderator' $\begin{array}{lllllll}\text { cylinder } & 1 & 1 & 0.428498 & 82.90 & 0.00\end{array}$ $\begin{array}{lllllll}\text { cylinder } & 0 & 1 & 0.437515 & 82.90 & 0.00\end{array}$ $\begin{array}{lllllll}\text { cylinder } & 2 & 1 & 0.49657 & 82.90 & 0.00\end{array}$ $\begin{array}{llllll}\text { cylinder } & 2 & 1 & 0.49657 & 82.90 & -1.27\end{array}$ cuboid 31 4p.6604 $82.90-1.27$ unit 76

com='Fuel + Gap + Clad + Moderator' $\begin{array}{llllll}\text { cylinder } & 1 & 1 & 0.428498 & 82.90 & 0.00\end{array}$ $\begin{array}{lllllll}\text { cylinder } & 0 & 1 & 0.437515 & 82.90 & 0.00\end{array}$ $\begin{array}{lllllll}\text { cylinder } & 2 & 1 & 0.49657 & 82.90 & 0.00\end{array}$ $\begin{array}{llllll}\text { cylinder } & 2 & 1 & 0.49657 & 82.90 & -1.27\end{array}$ cuboid 31 4p.6604 $82.90-1.27$ unit 77

com='Fuel + Gap + Clad + Moderator' $\begin{array}{llllll}\text { cylinder } & 1 & 1 & 0.428498 & 82.90 & 0.00\end{array}$ $\begin{array}{lllllll}\text { cylinder } & 0 & 1 & 0.437515 & 82.90 & 0.00\end{array}$ $\begin{array}{lllllll}\text { cylinder } & 2 & 1 & 0.49657 & 82.90 & 0.00\end{array}$ $\begin{array}{llllll}\text { cylinder } & 2 & 1 & 0.49657 & 82.90 & -1.27\end{array}$ cuboid $3 \quad 1 \quad 4 p .6604 \quad 82.90-1.27$ unit 78

com='Fuel + Gap + Clad + Moderator' $\begin{array}{lllllll}\text { cylinder } & 1 & 1 & 0.428498 & 82.90 & 0.00\end{array}$ $\begin{array}{lllllll}\text { cylinder } & 0 & 1 & 0.437515 & 82.90 & 0.00\end{array}$ $\begin{array}{lllllll}\text { cylinder } & 2 & 1 & 0.49657 & 82.90 & 0.00\end{array}$ $\begin{array}{llllll}\text { cylinder } & 2 & 1 & 0.49657 & 82.90 & -1.27\end{array}$ cuboid 314 p.6604 $82.90-1.27$ unit 79

com='Fuel + Gap + Clad + Moderator' $\begin{array}{lllllll}\text { cylinder } & 1 & 1 & 0.428498 & 82.90 & 0.00\end{array}$ $\begin{array}{lllllll}\text { cylinder } & 0 & 1 & 0.437515 & 82.90 & 0.00\end{array}$ $\begin{array}{llllll}\text { cylinder } & 2 & 1 & 0.49657 & 82.90 & 0.00\end{array}$ $\begin{array}{llllll}\text { cylinder } & 2 & 1 & 0.49657 & 82.90 & -1.27\end{array}$ cuboid $3 \quad 1 \quad 4 p .6604 \quad 82.90-1.27$ unit 80

com='Fuel + Gap + Clad + Moderator' $\begin{array}{lllllll}\text { cylinder } & 1 & 1 & 0.428498 & 82.90 & 0.00\end{array}$ $\begin{array}{lllllll}\text { cylinder } & 0 & 1 & 0.437515 & 82.90 & 0.00\end{array}$ $\begin{array}{lllllll}\text { cylinder } & 2 & 1 & 0.49657 & 82.90 & 0.00\end{array}$ $\begin{array}{llllll}\text { cylinder } & 2 & 1 & 0.49657 & 82.90 & -1.27\end{array}$ cuboid $3 \quad 1 \quad 4 p .6604 \quad 82.90-1.27$ unit 81

com='Fuel + Gap + Clad + Moderator' $\begin{array}{lllllll}\text { cylinder } & 1 & 1 & 0.428498 & 82.90 & 0.00\end{array}$ $\begin{array}{lllllll}\text { cylinder } & 0 & 1 & 0.437515 & 82.90 & 0.00\end{array}$ $\begin{array}{llllll}\text { cylinder } & 2 & 1 & 0.49657 & 82.90 & 0.00\end{array}$ $\begin{array}{llllll}\text { cylinder } & 2 & 1 & 0.49657 & 82.90 & -1.27\end{array}$ cuboid 314 p.6604 $82.90-1.27$ unit 82

com='Fuel + Gap + Clad + Moderator' $\begin{array}{llllll}\text { cylinder } & 1 & 1 & 0.428498 & 82.90 & 0.00\end{array}$ $\begin{array}{lllllll}\text { cylinder } & 0 & 1 & 0.437515 & 82.90 & 0.00\end{array}$ $\begin{array}{lllllll}\text { cylinder } & 2 & 1 & 0.49657 & 82.90 & 0.00\end{array}$ $\begin{array}{llllll}\text { cylinder } & 2 & 1 & 0.49657 & 82.90 & -1.27\end{array}$ cuboid $3 \quad 1 \quad 4 p .6604 \quad 82.90-1.27$ unit 83

com='Fuel + Gap + Clad + Moderator' $\begin{array}{llllll}\text { cylinder } & 1 & 1 & 0.428498 & 82.90 & 0.00\end{array}$ $\begin{array}{lllllll}\text { cylinder } & 0 & 1 & 0.437515 & 82.90 & 0.00\end{array}$ $\begin{array}{lllllll}\text { cylinder } & 2 & 1 & 0.49657 & 82.90 & 0.00\end{array}$ $\begin{array}{llllll}\text { cylinder } & 2 & 1 & 0.49657 & 82.90 & -1.27\end{array}$ cuboid $314 \mathrm{p} .6604 \quad 82.90-1.27$ unit 84

com='Fuel + Gap + Clad + Moderator' $\begin{array}{lllllll}\text { cylinder } & 1 & 1 & 0.428498 & 82.90 & 0.00\end{array}$ $\begin{array}{lllllll}\text { cylinder } & 0 & 1 & 0.437515 & 82.90 & 0.00\end{array}$ $\begin{array}{llllll}\text { cylinder } & 2 & 1 & 0.49657 & 82.90 & 0.00\end{array}$ $\begin{array}{llllll}\text { cylinder } & 2 & 1 & 0.49657 & 82.90 & -1.27\end{array}$ cuboid $3 \quad 1$ 4p.6604 $82.90-1.27$ unit 85

com='Fuel + Gap + Clad + Moderator $\begin{array}{lllllll}\text { cylinder } & 1 & 1 & 0.428498 & 82.90 & 0.00\end{array}$ $\begin{array}{llllllll}\text { cylinder } & 0 & 1 & 0.437515 & 82.90 & 0.00\end{array}$ $\begin{array}{llllll}\text { cylinder } & 2 & 1 & 0.49657 & 82.90 & 0.00\end{array}$ $\begin{array}{llllll}\text { cylinder } & 2 & 1 & 0.49657 & 82.90 & -1.27\end{array}$ cuboid $3 \quad 1 \quad 4 p .6604 \quad 82.90-1.27$ unit 86

com='Fuel + Gap + Clad + Moderator $\begin{array}{lllllll}\text { cylinder } & 1 & 1 & 0.428498 & 82.90 & 0.00\end{array}$ $\begin{array}{lllllll}\text { cylinder } & 0 & 1 & 0.437515 & 82.90 & 0.00\end{array}$ $\begin{array}{llllll}\text { cylinder } & 2 & 1 & 0.49657 & 82.90 & 0.00\end{array}$ $\begin{array}{llllll}\text { cylinder } & 2 & 1 & 0.49657 & 82.90 & -1.27\end{array}$ cuboid $3 \quad 1$ 4p.6604 $82.90-1.27$ unit 87

com='Fuel + Gap + Clad + Moderator' $\begin{array}{lllllll}\text { cylinder } & 1 & 1 & 0.428498 & 82.90 & 0.00\end{array}$ $\begin{array}{lllllll}\text { cylinder } & 0 & 1 & 0.437515 & 82.90 & 0.00\end{array}$ $\begin{array}{lllllll}\text { cylinder } & 2 & 1 & 0.49657 & 82.90 & 0.00\end{array}$ $\begin{array}{llllll}\text { cylinder } & 2 & 1 & 0.49657 & 82.90 & -1.27\end{array}$ cuboid $3 \quad 1 \quad 4 p .6604 \quad 82.90-1.27$ unit 88

com='Fuel + Gap + Clad + Moderator' $\begin{array}{lllllll}\text { cylinder } & 1 & 1 & 0.428498 & 82.90 & 0.00\end{array}$ $\begin{array}{llllllll}\text { cylinder } & 0 & 1 & 0.437515 & 82.90 & 0.00\end{array}$ $\begin{array}{lllllll}\text { cylinder } & 2 & 1 & 0.49657 & 82.90 & 0.00\end{array}$ $\begin{array}{llllll}\text { cylinder } & 2 & 1 & 0.49657 & 82.90 & -1.27\end{array}$ cuboid $314 \mathrm{p} .6604 \quad 82.90-1.27$ unit 89

com='Fuel + Gap + Clad + Moderator' $\begin{array}{llllll}\text { cylinder } & 1 & 1 & 0.428498 & 82.90 & 0.00\end{array}$ $\begin{array}{llllllll}\text { cylinder } & 0 & 1 & 0.437515 & 82.90 & 0.00\end{array}$ $\begin{array}{lllllll}\text { cylinder } & 2 & 1 & 0.49657 & 82.90 & 0.00\end{array}$ $\begin{array}{llllll}\text { cylinder } & 2 & 1 & 0.49657 & 82.90 & -1.27\end{array}$ 
$\begin{array}{cccc}\text { cuboid } \quad 3 & 1 & 4 p .6604 & 82.90-1.27 \\ & \text { unit } 90 & \end{array}$

com='Fuel + Gap + Clad + Moderator' $\begin{array}{lllllll}\text { cylinder } & 1 & 1 & 0.428498 & 82.90 & 0.00\end{array}$ $\begin{array}{llllllll}\text { cylinder } & 0 & 1 & 0.437515 & 82.90 & 0.00\end{array}$ $\begin{array}{lllllll}\text { cylinder } & 2 & 1 & 0.49657 & 82.90 & 0.00\end{array}$ $\begin{array}{llllll}\text { cylinder } & 2 & 1 & 0.49657 & 82.90 & -1.27\end{array}$ cuboid $3 \quad 1 \quad 4 p .6604 \quad 82.90-1.27$ unit 91

com='Fuel + Gap + Clad + Moderator' $\begin{array}{lllllll}\text { cylinder } & 1 & 1 & 0.428498 & 82.90 & 0.00\end{array}$ $\begin{array}{llllllll}\text { cylinder } & 0 & 1 & 0.437515 & 82.90 & 0.00\end{array}$ $\begin{array}{lllllll}\text { cylinder } & 2 & 1 & 0.49657 & 82.90 & 0.00\end{array}$ $\begin{array}{llllll}\text { cylinder } & 2 & 1 & 0.49657 & 82.90 & -1.27\end{array}$ cuboid $3 \quad 1 \quad 4 p .6604 \quad 82.90-1.27$ unit 92

com='Fuel + Gap + Clad + Moderator' $\begin{array}{llllll}\text { cylinder } & 1 & 1 & 0.428498 & 82.90 & 0.00\end{array}$ $\begin{array}{lllllll}\text { cylinder } & 0 & 1 & 0.437515 & 82.90 & 0.00\end{array}$ $\begin{array}{lllllll}\text { cylinder } & 2 & 1 & 0.49657 & 82.90 & 0.00\end{array}$ $\begin{array}{llllll}\text { cylinder } & 2 & 1 & 0.49657 & 82.90 & -1.27\end{array}$ cuboid $3 \quad 1 \quad 4 p .6604 \quad 82.90-1.27$ unit 93

com='Fuel + Gap + Clad + Moderator' $\begin{array}{lllllll}\text { cylinder } & 1 & 1 & 0.428498 & 82.90 & 0.00\end{array}$ $\begin{array}{llllllll}\text { cylinder } & 0 & 1 & 0.437515 & 82.90 & 0.00\end{array}$ $\begin{array}{lllllll}\text { cylinder } & 2 & 1 & 0.49657 & 82.90 & 0.00\end{array}$ $\begin{array}{llllll}\text { cylinder } & 2 & 1 & 0.49657 & 82.90 & -1.27\end{array}$ cuboid $3 \begin{array}{llll}3 & 4 p .6604 & 82.90 & -1.27\end{array}$ unit 94

com='Fuel + Gap + Clad + Moderator' $\begin{array}{lllllll}\text { cylinder } & 1 & 1 & 0.428498 & 82.90 & 0.00\end{array}$ $\begin{array}{lllllll}\text { cylinder } & 0 & 1 & 0.437515 & 82.90 & 0.00\end{array}$ $\begin{array}{lllllll}\text { cylinder } & 2 & 1 & 0.49657 & 82.90 & 0.00\end{array}$ $\begin{array}{llllll}\text { cylinder } & 2 & 1 & 0.49657 & 82.90 & -1.27\end{array}$ cuboid 3 1 4 p.6604 $82.90-1.27$ unit 95

com='Fuel + Gap + Clad + Moderator' $\begin{array}{lllllll}\text { cylinder } & 1 & 1 & 0.428498 & 82.90 & 0.00\end{array}$ $\begin{array}{lllllll}\text { cylinder } & 0 & 1 & 0.437515 & 82.90 & 0.00\end{array}$ $\begin{array}{lllllll}\text { cylinder } & 2 & 1 & 0.49657 & 82.90 & 0.00\end{array}$ $\begin{array}{llllll}\text { cylinder } & 2 & 1 & 0.49657 & 82.90 & -1.27\end{array}$ cuboid $314 \mathrm{p} .6604 \quad 82.90-1.27$ unit 96

com='Fuel + Gap + Clad + Moderator' $\begin{array}{llllll}\text { cylinder } & 1 & 1 & 0.428498 & 82.90 & 0.00\end{array}$ $\begin{array}{lllllll}\text { cylinder } & 0 & 1 & 0.437515 & 82.90 & 0.00\end{array}$ $\begin{array}{lllllll}\text { cylinder } & 2 & 1 & 0.49657 & 82.90 & 0.00\end{array}$ $\begin{array}{llllll}\text { cylinder } & 2 & 1 & 0.49657 & 82.90 & -1.27\end{array}$ cuboid 31 4p.6604 82.90-1.27 unit 97

com='Fuel + Gap + Clad + Moderator' $\begin{array}{llllll}\text { cylinder } & 1 & 1 & 0.428498 & 82.90 & 0.00\end{array}$ $\begin{array}{lllllll}\text { cylinder } & 0 & 1 & 0.437515 & 82.90 & 0.00\end{array}$ $\begin{array}{lllllll}\text { cylinder } & 2 & 1 & 0.49657 & 82.90 & 0.00\end{array}$ $\begin{array}{llllll}\text { cylinder } & 2 & 1 & 0.49657 & 82.90 & -1.27\end{array}$

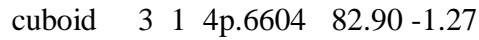

unit 98

com='Fuel + Gap + Clad + Moderator' $\begin{array}{llllll}\text { cylinder } & 1 & 1 & 0.428498 & 82.90 & 0.00\end{array}$ $\begin{array}{lllllll}\text { cylinder } & 0 & 1 & 0.437515 & 82.90 & 0.00\end{array}$ $\begin{array}{llllll}\text { cylinder } & 2 & 1 & 0.49657 & 82.90 & 0.00\end{array}$ $\begin{array}{llllll}\text { cylinder } & 2 & 1 & 0.49657 & 82.90 & -1.27\end{array}$ cuboid 3 1 4 p.6604 $82.90-1.27$ unit 99

com='Fuel + Gap + Clad + Moderator' $\begin{array}{lllllll}\text { cylinder } & 1 & 1 & 0.428498 & 82.90 & 0.00\end{array}$ $\begin{array}{lllllll}\text { cylinder } & 0 & 1 & 0.437515 & 82.90 & 0.00\end{array}$ $\begin{array}{llllllll}\text { cylinder } & 2 & 1 & 0.49657 & 82.90 & 0.00\end{array}$ $\begin{array}{llllll}\text { cylinder } & 2 & 1 & 0.49657 & 82.90 & -1.27\end{array}$ cuboid $3 \quad 1 \quad 4 p .6604 \quad 82.90-1.27$ unit 100

com='Fuel + Gap + Clad + Moderator' $\begin{array}{lllllll}\text { cylinder } & 1 & 1 & 0.428498 & 82.90 & 0.00\end{array}$ $\begin{array}{lllllll}\text { cylinder } & 0 & 1 & 0.437515 & 82.90 & 0.00\end{array}$ $\begin{array}{lllllll}\text { cylinder } & 2 & 1 & 0.49657 & 82.90 & 0.00\end{array}$ $\begin{array}{llllll}\text { cylinder } & 2 & 1 & 0.49657 & 82.90 & -1.27\end{array}$ cuboid $3 \quad 1 \quad 4 p .6604 \quad 82.90-1.27$ unit 101

com='Fuel + Gap + Clad + Moderator' $\begin{array}{llllll}\text { cylinder } & 1 & 1 & 0.428498 & 82.90 & 0.00\end{array}$ $\begin{array}{lllllll}\text { cylinder } & 0 & 1 & 0.437515 & 82.90 & 0.00\end{array}$ $\begin{array}{llllllll}\text { cylinder } & 2 & 1 & 0.49657 & 82.90 & 0.00\end{array}$ $\begin{array}{llllll}\text { cylinder } & 2 & 1 & 0.49657 & 82.90 & -1.27\end{array}$ cuboid 3 1 4p.6604 82.90-1.27 unit 102

com='Fuel + Gap + Clad + Moderator' $\begin{array}{lllllll}\text { cylinder } & 1 & 1 & 0.428498 & 82.90 & 0.00\end{array}$ $\begin{array}{lllllll}\text { cylinder } & 0 & 1 & 0.437515 & 82.90 & 0.00\end{array}$ $\begin{array}{lllllll}\text { cylinder } & 2 & 1 & 0.49657 & 82.90 & 0.00\end{array}$ $\begin{array}{llllll}\text { cylinder } & 2 & 1 & 0.49657 & 82.90 & -1.27\end{array}$ cuboid $3 \quad 1 \quad 4 p .6604 \quad 82.90-1.27$ unit 103

com='Fuel + Gap + Clad + Moderator' $\begin{array}{lllllll}\text { cylinder } & 1 & 1 & 0.428498 & 82.90 & 0.00\end{array}$ $\begin{array}{lllllll}\text { cylinder } & 0 & 1 & 0.437515 & 82.90 & 0.00\end{array}$ $\begin{array}{lllllll}\text { cylinder } & 2 & 1 & 0.49657 & 82.90 & 0.00\end{array}$ $\begin{array}{llllll}\text { cylinder } & 2 & 1 & 0.49657 & 82.90 & -1.27\end{array}$ cuboid 3 1 4 p.6604 $82.90-1.27$ unit 104

com='Fuel + Gap + Clad + Moderator' $\begin{array}{lllllll}\text { cylinder } & 1 & 1 & 0.428498 & 82.90 & 0.00\end{array}$ $\begin{array}{lllllll}\text { cylinder } & 0 & 1 & 0.437515 & 82.90 & 0.00\end{array}$ $\begin{array}{llllllll}\text { cylinder } & 2 & 1 & 0.49657 & 82.90 & 0.00\end{array}$ $\begin{array}{llllll}\text { cylinder } & 2 & 1 & 0.49657 & 82.90 & -1.27\end{array}$ cuboid $3 \quad 1 \quad 4 p .6604 \quad 82.90-1.27$ unit 105

com='Fuel + Gap + Clad + Moderator' $\begin{array}{lllllll}\text { cylinder } & 1 & 1 & 0.428498 & 82.90 & 0.00\end{array}$ $\begin{array}{lllllll}\text { cylinder } & 0 & 1 & 0.437515 & 82.90 & 0.00\end{array}$ $\begin{array}{lllllll}\text { cylinder } & 2 & 1 & 0.49657 & 82.90 & 0.00\end{array}$

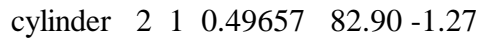
cuboid $3 \quad 1 \quad 4 p .6604 \quad 82.90-1.27$ unit 106 
com='Fuel + Gap + Clad + Moderator' $\begin{array}{lllllll}\text { cylinder } & 1 & 1 & 0.428498 & 82.90 & 0.00\end{array}$ $\begin{array}{lllllll}\text { cylinder } & 0 & 1 & 0.437515 & 82.90 & 0.00\end{array}$ $\begin{array}{llllllll}\text { cylinder } & 2 & 1 & 0.49657 & 82.90 & 0.00\end{array}$ $\begin{array}{llllll}\text { cylinder } & 2 & 1 & 0.49657 & 82.90 & -1.27\end{array}$ cuboid $3 \quad 1 \quad 4 p .6604 \quad 82.90-1.27$ unit 107

com='Fuel + Gap + Clad + Moderator' $\begin{array}{lllllll}\text { cylinder } & 1 & 1 & 0.428498 & 82.90 & 0.00\end{array}$ $\begin{array}{lllllll}\text { cylinder } & 0 & 1 & 0.437515 & 82.90 & 0.00\end{array}$ $\begin{array}{lllllll}\text { cylinder } & 2 & 1 & 0.49657 & 82.90 & 0.00\end{array}$ $\begin{array}{llllll}\text { cylinder } & 2 & 1 & 0.49657 & 82.90 & -1.27\end{array}$ cuboid 314 p.6604 $82.90-1.27$ unit 108

com='Fuel + Gap + Clad + Moderator' $\begin{array}{lllllll}\text { cylinder } & 1 & 1 & 0.428498 & 82.90 & 0.00\end{array}$ $\begin{array}{lllllll}\text { cylinder } & 0 & 1 & 0.437515 & 82.90 & 0.00\end{array}$ $\begin{array}{lllllll}\text { cylinder } & 2 & 1 & 0.49657 & 82.90 & 0.00\end{array}$ $\begin{array}{llllll}\text { cylinder } & 2 & 1 & 0.49657 & 82.90 & -1.27\end{array}$ cuboid 3 1 4 p.6604 $82.90-1.27$ unit 109

com='Fuel + Gap + Clad + Moderator' $\begin{array}{lllllll}\text { cylinder } & 1 & 1 & 0.428498 & 82.90 & 0.00\end{array}$ $\begin{array}{lllllll}\text { cylinder } & 0 & 1 & 0.437515 & 82.90 & 0.00\end{array}$ $\begin{array}{llllllll}\text { cylinder } & 2 & 1 & 0.49657 & 82.90 & 0.00\end{array}$ $\begin{array}{lllllll}\text { cylinder } & 2 & 1 & 0.49657 & 82.90 & -1.27\end{array}$ cuboid 3 1 4 p.6604 $82.90-1.27$ unit 110

com='Fuel + Gap + Clad + Moderator' $\begin{array}{lllllll}\text { cylinder } & 1 & 1 & 0.428498 & 82.90 & 0.00\end{array}$ $\begin{array}{lllllll}\text { cylinder } & 0 & 1 & 0.437515 & 82.90 & 0.00\end{array}$ $\begin{array}{lllllll}\text { cylinder } & 2 & 1 & 0.49657 & 82.90 & 0.00\end{array}$ $\begin{array}{llllll}\text { cylinder } & 2 & 1 & 0.49657 & 82.90 & -1.27\end{array}$ cuboid $3 \quad 1 \quad 4 p .6604 \quad 82.90-1.27$ unit 111

com='Fuel + Gap + Clad + Moderator' $\begin{array}{lllllll}\text { cylinder } & 1 & 1 & 0.428498 & 82.90 & 0.00\end{array}$ $\begin{array}{lllllll}\text { cylinder } & 0 & 1 & 0.437515 & 82.90 & 0.00\end{array}$ $\begin{array}{lllllll}\text { cylinder } & 2 & 1 & 0.49657 & 82.90 & 0.00\end{array}$ $\begin{array}{llllll}\text { cylinder } & 2 & 1 & 0.49657 & 82.90 & -1.27\end{array}$ cuboid $3 \quad 1$ 4p.6604 $82.90-1.27$ unit 112

com='Fuel + Gap + Clad + Moderator' $\begin{array}{lllllll}\text { cylinder } & 1 & 1 & 0.428498 & 82.90 & 0.00\end{array}$ $\begin{array}{llllllll}\text { cylinder } & 0 & 1 & 0.437515 & 82.90 & 0.00\end{array}$ $\begin{array}{lllllll}\text { cylinder } & 2 & 1 & 0.49657 & 82.90 & 0.00\end{array}$ $\begin{array}{llllll}\text { cylinder } & 2 & 1 & 0.49657 & 82.90 & -1.27\end{array}$ cuboid $3 \quad 1$ 4p.6604 $82.90-1.27$ unit 113

com='Fuel + Gap + Clad + Moderator $\begin{array}{llllll}\text { cylinder } & 1 & 1 & 0.428498 & 82.90 & 0.00\end{array}$ $\begin{array}{lllllll}\text { cylinder } & 0 & 1 & 0.437515 & 82.90 & 0.00\end{array}$ $\begin{array}{lllllll}\text { cylinder } & 2 & 1 & 0.49657 & 82.90 & 0.00\end{array}$ $\begin{array}{llllll}\text { cylinder } & 2 & 1 & 0.49657 & 82.90 & -1.27\end{array}$ cuboid $3 \quad 1 \quad 4 p .6604 \quad 82.90-1.27$ unit 114

com='Fuel + Gap + Clad + Moderator' $\begin{array}{llllll}\text { cylinder } & 1 & 1 & 0.428498 & 82.90 & 0.00\end{array}$ $\begin{array}{lllllll}\text { cylinder } & 0 & 1 & 0.437515 & 82.90 & 0.00\end{array}$ $\begin{array}{lllllll}\text { cylinder } & 2 & 1 & 0.49657 & 82.90 & 0.00\end{array}$ $\begin{array}{llllll}\text { cylinder } & 2 & 1 & 0.49657 & 82.90 & -1.27\end{array}$ cuboid $3 \quad 1 \quad 4 p .6604 \quad 82.90-1.27$ unit 115

com='Fuel + Gap + Clad + Moderator' $\begin{array}{lllllll}\text { cylinder } & 1 & 1 & 0.428498 & 82.90 & 0.00\end{array}$ $\begin{array}{lllllll}\text { cylinder } & 0 & 1 & 0.437515 & 82.90 & 0.00\end{array}$ $\begin{array}{lllllll}\text { cylinder } & 2 & 1 & 0.49657 & 82.90 & 0.00\end{array}$ $\begin{array}{llllll}\text { cylinder } & 2 & 1 & 0.49657 & 82.90 & -1.27\end{array}$ cuboid $3 \quad 1 \quad 4 p .6604 \quad 82.90-1.27$ unit 116

com='Fuel + Gap + Clad + Moderator' $\begin{array}{lllllll}\text { cylinder } & 1 & 1 & 0.428498 & 82.90 & 0.00\end{array}$ $\begin{array}{llllllll}\text { cylinder } & 0 & 1 & 0.437515 & 82.90 & 0.00\end{array}$ $\begin{array}{lllllll}\text { cylinder } & 2 & 1 & 0.49657 & 82.90 & 0.00\end{array}$ $\begin{array}{llllll}\text { cylinder } & 2 & 1 & 0.49657 & 82.90 & -1.27\end{array}$ cuboid $3 \quad 1$ 4p.6604 82.90-1.27 unit 117

com='Fuel + Gap + Clad + Moderator' $\begin{array}{lllllll}\text { cylinder } & 1 & 1 & 0.428498 & 82.90 & 0.00\end{array}$ $\begin{array}{llllllll}\text { cylinder } & 0 & 1 & 0.437515 & 82.90 & 0.00\end{array}$ $\begin{array}{lllllll}\text { cylinder } & 2 & 1 & 0.49657 & 82.90 & 0.00\end{array}$ $\begin{array}{llllll}\text { cylinder } & 2 & 1 & 0.49657 & 82.90 & -1.27\end{array}$ cuboid $3 \quad 1 \quad 4 p .6604 \quad 82.90-1.27$ unit 118

com='Fuel + Gap + Clad + Moderator' $\begin{array}{lllllll}\text { cylinder } & 1 & 1 & 0.428498 & 82.90 & 0.00\end{array}$ $\begin{array}{lllllll}\text { cylinder } & 0 & 1 & 0.437515 & 82.90 & 0.00\end{array}$ $\begin{array}{lllllll}\text { cylinder } & 2 & 1 & 0.49657 & 82.90 & 0.00\end{array}$

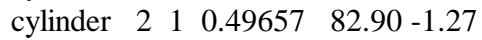
cuboid $3 \quad 1 \quad 4 p .6604 \quad 82.90-1.27$ unit 119

com='Fuel + Gap + Clad + Moderator' $\begin{array}{lllllll}\text { cylinder } & 1 & 1 & 0.428498 & 82.90 & 0.00\end{array}$ $\begin{array}{lllllll}\text { cylinder } & 0 & 1 & 0.437515 & 82.90 & 0.00\end{array}$ $\begin{array}{lllllll}\text { cylinder } & 2 & 1 & 0.49657 & 82.90 & 0.00\end{array}$ $\begin{array}{llllll}\text { cylinder } & 2 & 1 & 0.49657 & 82.90 & -1.27\end{array}$ cuboid 3 1 4 p.6604 $82.90-1.27$ unit 120

com='Fuel + Gap + Clad + Moderator' $\begin{array}{llllll}\text { cylinder } & 1 & 1 & 0.428498 & 82.90 & 0.00\end{array}$ $\begin{array}{lllllll}\text { cylinder } & 0 & 1 & 0.437515 & 82.90 & 0.00\end{array}$ $\begin{array}{lllllll}\text { cylinder } & 2 & 1 & 0.49657 & 82.90 & 0.00\end{array}$ $\begin{array}{llllll}\text { cylinder } & 2 & 1 & 0.49657 & 82.90 & -1.27\end{array}$ cuboid $3 \quad 1 \quad 4 p .6604 \quad 82.90-1.27$ unit 121

com='Fuel + Gap + Clad + Moderator' $\begin{array}{llllll}\text { cylinder } & 1 & 1 & 0.428498 & 82.90 & 0.00\end{array}$ $\begin{array}{lllllll}\text { cylinder } & 0 & 1 & 0.437515 & 82.90 & 0.00\end{array}$ $\begin{array}{lllllll}\text { cylinder } & 2 & 1 & 0.49657 & 82.90 & 0.00\end{array}$ $\begin{array}{llllll}\text { cylinder } & 2 & 1 & 0.49657 & 82.90 & -1.27\end{array}$ cuboid $3 \quad 1 \quad 4 p .6604 \quad 82.90-1.27$ unit 122

com='Fuel + Gap + Clad + Moderator' $\begin{array}{llllll}\text { cylinder } & 1 & 1 & 0.428498 & 82.90 & 0.00\end{array}$ 
$\begin{array}{llllll}\text { cylinder } & 0 & 1 & 0.437515 & 82.90 & 0.00\end{array}$ $\begin{array}{lllllll}\text { cylinder } & 2 & 1 & 0.49657 & 82.90 & 0.00\end{array}$ $\begin{array}{llllll}\text { cylinder } & 2 & 1 & 0.49657 & 82.90 & -1.27\end{array}$ cuboid $3 \quad 1 \quad 4 p .6604 \quad 82.90-1.27$ unit 123

com='Fuel + Gap + Clad + Moderator' $\begin{array}{lllllll}\text { cylinder } & 1 & 1 & 0.428498 & 82.90 & 0.00\end{array}$ $\begin{array}{lllllll}\text { cylinder } & 0 & 1 & 0.437515 & 82.90 & 0.00\end{array}$ $\begin{array}{lllllll}\text { cylinder } & 2 & 1 & 0.49657 & 82.90 & 0.00\end{array}$ $\begin{array}{llllll}\text { cylinder } & 2 & 1 & 0.49657 & 82.90 & -1.27\end{array}$ cuboid $3 \begin{array}{lllll} & 1 & 4 p .6604 & 82.90 & -1.27\end{array}$ unit 124

com='Fuel + Gap + Clad + Moderator' $\begin{array}{lllllll}\text { cylinder } & 1 & 1 & 0.428498 & 82.90 & 0.00\end{array}$ $\begin{array}{lllllllll}\text { cylinder } & 0 & 1 & 0.437515 & 82.90 & 0.00\end{array}$ $\begin{array}{lllllll}\text { cylinder } & 2 & 1 & 0.49657 & 82.90 & 0.00\end{array}$ $\begin{array}{llllll}\text { cylinder } & 2 & 1 & 0.49657 & 82.90 & -1.27\end{array}$ cuboid 3 1 4 p.6604 $82.90-1.27$ unit 125

com='Fuel + Gap + Clad + Moderator' $\begin{array}{llllll}\text { cylinder } & 1 & 1 & 0.428498 & 82.90 & 0.00\end{array}$ $\begin{array}{lllllll}\text { cylinder } & 0 & 1 & 0.437515 & 82.90 & 0.00\end{array}$ $\begin{array}{lllllll}\text { cylinder } & 2 & 1 & 0.49657 & 82.90 & 0.00\end{array}$ $\begin{array}{llllll}\text { cylinder } & 2 & 1 & 0.49657 & 82.90 & -1.27\end{array}$ cuboid 314 p.6604 $82.90-1.27$ unit 126

com='Fuel + Gap + Clad + Moderator' $\begin{array}{lllllll}\text { cylinder } & 1 & 1 & 0.428498 & 82.90 & 0.00\end{array}$ $\begin{array}{lllllll}\text { cylinder } & 0 & 1 & 0.437515 & 82.90 & 0.00\end{array}$ $\begin{array}{lllllll}\text { cylinder } & 2 & 1 & 0.49657 & 82.90 & 0.00\end{array}$ $\begin{array}{llllll}\text { cylinder } & 2 & 1 & 0.49657 & 82.90 & -1.27\end{array}$ cuboid $3 \quad 1 \quad 4 p .6604 \quad 82.90-1.27$ unit 127

com='Fuel + Gap + Clad + Moderator' $\begin{array}{llllll}\text { cylinder } & 1 & 1 & 0.428498 & 82.90 & 0.00\end{array}$ $\begin{array}{llllllll}\text { cylinder } & 0 & 1 & 0.437515 & 82.90 & 0.00\end{array}$ $\begin{array}{llllllll}\text { cylinder } & 2 & 1 & 0.49657 & 82.90 & 0.00\end{array}$ $\begin{array}{llllll}\text { cylinder } & 2 & 1 & 0.49657 & 82.90 & -1.27\end{array}$ cuboid 3 1 4 p.6604 $82.90-1.27$ unit 128

com='Fuel + Gap + Clad + Moderator' $\begin{array}{lllllll}\text { cylinder } & 1 & 1 & 0.428498 & 82.90 & 0.00\end{array}$ $\begin{array}{lllllll}\text { cylinder } & 0 & 1 & 0.437515 & 82.90 & 0.00\end{array}$ $\begin{array}{lllllll}\text { cylinder } & 2 & 1 & 0.49657 & 82.90 & 0.00\end{array}$ $\begin{array}{llllll}\text { cylinder } & 2 & 1 & 0.49657 & 82.90 & -1.27\end{array}$ cuboid 3 1 4 p.6604 $82.90-1.27$ unit 129

com='Fuel + Gap + Clad + Moderator' $\begin{array}{llllll}\text { cylinder } & 1 & 1 & 0.428498 & 82.90 & 0.00\end{array}$ $\begin{array}{lllllll}\text { cylinder } & 0 & 1 & 0.437515 & 82.90 & 0.00\end{array}$ $\begin{array}{lllllll}\text { cylinder } & 2 & 1 & 0.49657 & 82.90 & 0.00\end{array}$ $\begin{array}{llllll}\text { cylinder } & 2 & 1 & 0.49657 & 82.90 & -1.27\end{array}$ cuboid 3 1 4 p.6604 $82.90-1.27$ unit 130

com='Fuel + Gap + Clad + Moderator' $\begin{array}{llllll}\text { cylinder } & 1 & 1 & 0.428498 & 82.90 & 0.00\end{array}$ $\begin{array}{lllllll}\text { cylinder } & 0 & 1 & 0.437515 & 82.90 & 0.00\end{array}$ $\begin{array}{llllll}\text { cylinder } & 2 & 1 & 0.49657 & 82.90 & 0.00\end{array}$ $\begin{array}{llllll}\text { cylinder } & 2 & 1 & 0.49657 & 82.90 & -1.27\end{array}$ cuboid $314 \mathrm{p} .6604 \quad 82.90-1.27$ unit 131

com='Fuel + Gap + Clad + Moderator $\begin{array}{llllll}\text { cylinder } & 1 & 1 & 0.428498 & 82.90 & 0.00\end{array}$ $\begin{array}{llllllll}\text { cylinder } & 0 & 1 & 0.437515 & 82.90 & 0.00\end{array}$ $\begin{array}{lllllll}\text { cylinder } & 2 & 1 & 0.49657 & 82.90 & 0.00\end{array}$ $\begin{array}{llllll}\text { cylinder } & 2 & 1 & 0.49657 & 82.90 & -1.27\end{array}$ cuboid $3 \quad 1 \quad 4 p .6604 \quad 82.90-1.27$ unit 132

com='Fuel + Gap + Clad + Moderator $\begin{array}{llllll}\text { cylinder } & 1 & 1 & 0.428498 & 82.90 & 0.00\end{array}$ $\begin{array}{lllllll}\text { cylinder } & 0 & 1 & 0.437515 & 82.90 & 0.00\end{array}$ $\begin{array}{lllllll}\text { cylinder } & 2 & 1 & 0.49657 & 82.90 & 0.00\end{array}$ $\begin{array}{llllll}\text { cylinder } & 2 & 1 & 0.49657 & 82.90 & -1.27\end{array}$ cuboid 3 1 4 p.6604 $82.90-1.27$

unit 141

com='Clad + Mod + AL Grid' $\begin{array}{lllll}\text { cylinder } 2 & 1 & 0.49657 & 0.635 & 0.0\end{array}$ $\begin{array}{llllll}\text { cylinder } 3 & 1 & 0.50419 & 0.635 & 0.0\end{array}$ $\begin{array}{lllllll}\text { cuboid } \quad 5 & 1 & 4 \mathrm{p} .6604 & 0.635 & 0.0\end{array}$

unit 143

com='Fuel + Gap + Clad + Air' $\begin{array}{llllll}\text { cylinder } & 1 & 1 & 0.428498 & 10.064 & 0.0\end{array}$ $\begin{array}{lllllll}\text { cylinder } & 0 & 1 & 0.437515 & 10.064 & 0.0\end{array}$ $\begin{array}{llllll}\text { cylinder } 2 & 1 & 0.49657 & 10.064 & 0.0\end{array}$ $\begin{array}{lllllll}\text { cuboid } & 0 & 1 & 4 \mathrm{p} .6604 & 10.064 & 0.0\end{array}$

unit 144 com='Top Clad + Void' $\begin{array}{llllll}\text { cylinder } 2 & 1 & 0.49657 & 1.016 & 0.0\end{array}$ $\begin{array}{lllllll}\text { cuboid } \quad 0 & 1 & 4 p .6604 & 1.016 & 0.0\end{array}$

unit 145

com='Top Clad + Void + AL grid' $\begin{array}{lllll}\text { cylinder } 2 & 1 & 0.49657 & 1.27 & 0.0\end{array}$ cylinder $0 \begin{array}{llll}0 & 0.50419 & 1.27 & 0.0\end{array}$ cuboid $514 \mathrm{p} .6604 \quad 1.27 \quad 0.0$

unit 151

com='array of Clad + Mod + AL Grid + Reflector' array $1-15.1892-14.52880 .0$

cuboid $412 \mathrm{p} 45.1892$ 2p44.5288 0.6350 .0

unit 152

com='array of Fuel + Gap + Clad + Moderator + Reflector' array $2-15.1892-14.5288-1.27$

cuboid 31 2p45.1892 2p44.5288 $82.90 \quad-1.27$

unit 153

com='array of Fuel + Gap + Clad + Air' array $3-15.1892-14.52880 .0$

cuboid $012 \mathrm{p} 45.1892$ 2p44.5288 10.0640 .0 
unit 154

com='array of Top Clad + Void'

array $4-15.1892-14.52880 .0$

cuboid 01 2p45.1892 2p44.5288 1.0160 .0

unit 155

com='array of Top Clad + Void + AL grid' array $5-15.1892-14.52880 .0$

cuboid $412 \mathrm{p} 45.1892$ 2p44.5288 $1.27 \quad 0.0$

global unit 160

com='array of arrays of fuel element sections + reflector' array $6-45.1892 \quad-44.5288 \quad 0.0$

cuboid $\begin{array}{llllll}5 & 1 & 2 \mathrm{p} 45.1892 & 2 \mathrm{p} 44.5288 & 97.155 & -2.54\end{array}$

cuboid $3 \quad 1 \quad 2 p 45.1892 \quad 2 p 44.5288 \quad 97.155 \quad-8.89$

cuboid $5 \quad 1 \quad 2 p 45.1892$ 2p44.5288 $97.155 \quad-13.97$

cuboid $3 \quad 1$ 2p45.1892 2p44.5288 97.155 -43.97

end geom

read array

$\operatorname{ara}=1$ nux $=23$ nuy=22 nuz=1

fill $\mathrm{f} 141$ end fill

$\operatorname{ara}=2$ nux $=23$ nuy $=22$ nuz $=1$

fill

$\begin{array}{llllllllllllll}1 & 2 & 3 & 4 & 5 & 6 & 7 & 8 & 9 & 10 & 11 & 12 & 1 \mathrm{~b} 11\end{array}$

$\begin{array}{lllllllllllll}13 & 14 & 15 & 16 & 17 & 18 & 19 & 20 & 21 & 22 & 23 & 24 & 1 \mathrm{~b} 11\end{array}$

$\begin{array}{lllllllllllll}25 & 26 & 27 & 28 & 29 & 30 & 31 & 32 & 33 & 34 & 35 & 36 & 1 \mathrm{~b} 11\end{array}$

$\begin{array}{lllllllllllll}37 & 38 & 39 & 40 & 41 & 42 & 43 & 44 & 45 & 46 & 47 & 48 & 1 \mathrm{~b} 11\end{array}$

$\begin{array}{lllllllllllll}49 & 50 & 51 & 52 & 53 & 54 & 55 & 56 & 57 & 58 & 59 & 60 & 1 \mathrm{~b} 11\end{array}$

$\begin{array}{lllllllllllll}61 & 62 & 63 & 64 & 65 & 66 & 67 & 68 & 69 & 70 & 71 & 72 & 1 \mathrm{~b} 11\end{array}$

$\begin{array}{lllllllllllll}73 & 74 & 75 & 76 & 77 & 78 & 79 & 80 & 81 & 82 & 83 & 84 & 1 b 11\end{array}$

$\begin{array}{lllllllllllll}85 & 86 & 87 & 88 & 89 & 90 & 91 & 92 & 93 & 94 & 95 & 96 & 1 \mathrm{~b} 11\end{array}$

979899100101102103104105106107108 1b11

$1091101111121131141151161171181191201 \mathrm{~b} 11$

$1211221231241251261271281291301311321 b 11$ $\ln 253$

end fill

ara $=3$ nux $=23$ nuy=22 nuz=1 fill $\mathrm{f} 143$ end fill

ara $=4$ nux $=23$ nuy=22 nuz $=1$ fill $\mathrm{f} 144$ end fill

ara $=5$ nux $=23$ nuy=22 nuz=1 fill $\mathrm{f} 145$ end fill

ara $=6$ nux $=1$ nuy=1 nuz=5

fill 151152153154155 end fill

end array

read plot

$\mathrm{ttl}=$ 'simple plot 1 '

$\mathrm{lpi}=10 \mathrm{scr}=$ yes $\mathrm{pic}=\operatorname{mix}$ nax $=400$

$\mathrm{xul}=-20.0 \mathrm{yul}=0.6 \mathrm{zul}=100$

$\mathrm{xlr}=20.0 \quad \mathrm{ylr}=0.6 \quad \mathrm{zlr}=-10$

uax $=1.0 \mathrm{wdn}=-1.0$ end plt 0

$\mathrm{ttl}=$ 'simple plot $2^{\prime}$

pic $=$ mix nax $=400$

$\mathrm{xul}=-20.0$ yul $=20 . \quad \mathrm{zul}=25$.

$\mathrm{xlr}=20.0 \quad \mathrm{ylr}=-20 . \quad \mathrm{zlr}=25$.

$\mathrm{uax}=1.0 \mathrm{vdn}=-1.0$ end plt 1 end plot

end data

end

$=$ shell

cp ft04f001 \$RTNDIR/sax01.xs04

cp ft64f001 \$RTNDIR/sax01.rs64

end

$=\mathrm{kmart}$

read initial kunit $=64$

xunit $=4$

actbygrp

end initial

rrpvol

read activity

92234279223418922341452

$9223527922351892235 \quad 1452$

92238279223818922381452

94239279423918942391452

94240279424018942401452

$9424127942411894241 \quad 1452$

$9424227942421894242 \quad 1452$

$9524127952411895241 \quad 1452$

501027

501127

801627

4000027

2400027

2600027

100127

5011227

5011427

5011527

5011627

5011727

5011827

5011927

5012027

5012227

5012427

end activity

read collapse

factor $=1.0$

lastg $=57$

lastg $=148$

last $\mathrm{g}=204$

lastg $=238$

end collapse

end

$=\operatorname{csasc} 5 \quad$ parm $=$ size $=1000000$ saxton 2 fuel

238group latticecell

'MOX fuel

pu-239 $1001.3526-3290$ end

pu-240 $120 \quad 1.2759-4290$ end

pu-241 100 1.1407-5 290 end 
pu-242 $1106.0318-7290$ end am-241 $1001.7783-6290$ end u-234 $100 \quad 1.1688-6290$ end u-235 $11001.5301-4290$ end u-238 $11002.1097-2 \quad 290$ end

o $\quad 1 \quad 0 \quad 4.5155-2290$ end 'clad (ZR-2)

zr $\quad 2 \quad 0 \quad 4.2517-2 \quad 290$ end

sn $\quad 2 \quad 0 \quad 4.6590-4290$ end

cr $\quad 2 \quad 0 \quad 7.5977-5290$ end

fe $\quad \begin{array}{llll}2 & 0 & 1.4148-4 & 290 \\ & \text { end }\end{array}$

o $\quad 2002.9630-4290$ end 'water(Refletor)

h $3 \quad 3 \quad 0 \quad 6.6781-2 \quad 290$ end

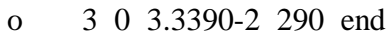

'b-10 $3 \begin{array}{llll}3 & 0 & 1.7606-8 & 290 \\ \text { end }\end{array}$

'b-11 $3007.1313-8290$ end

'Middle Grid and $\mathrm{H} 2 \mathrm{O}$

$$
\begin{array}{cccc}
\text { al } & 4 & .8458 & 290 \text { end } \\
\text { h2o } & 4 & .1542 & 290 \text { end } \\
& \multicolumn{4}{c}{\text { 'Aluminum }}
\end{array}
$$

al $\quad \begin{array}{llll}5 & 0 & 6.0039-2 & 290\end{array}$ end end comp

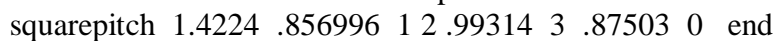
more data

limcen $=20000000$

end more

saxton 2

read param

gen=225 nsk=25 npg=20000 fdn=yes flx=yes wrs=64 res $=225$

$\operatorname{lng}=2000000$

end param

read geometry

unit 1

com='Fuel + Gap + Clad + Moderator'

$\begin{array}{lllllll}\text { cylinder } & 1 & 1 & 0.428498 & 80.80 & 0.00\end{array}$

$\begin{array}{lllllll}\text { cylinder } & 0 & 1 & 0.437515 & 80.80 & 0.00\end{array}$

$\begin{array}{lllllll}\text { cylinder } & 2 & 1 & 0.49657 & 80.80 & 0.00\end{array}$

$\begin{array}{llllll}\text { cylinder } & 2 & 1 & 0.49657 & 80.80 & -1.27\end{array}$

cuboid $3 \quad 1 \quad 4 p .7112 \quad 80.80-1.27$

unit 2

com='Fuel + Gap + Clad + Moderator'

$\begin{array}{lllllll}\text { cylinder } & 1 & 1 & 0.428498 & 80.80 & 0.00\end{array}$

$\begin{array}{lllllll}\text { cylinder } & 0 & 1 & 0.437515 & 80.80 & 0.00\end{array}$

$\begin{array}{llllllll}\text { cylinder } & 2 & 1 & 0.49657 & 80.80 & 0.00\end{array}$

$\begin{array}{llllll}\text { cylinder } & 2 & 1 & 0.49657 & 80.80 & -1.27\end{array}$

cuboid $3 \quad 1 \quad 4 p .7112 \quad 80.80-1.27$

unit 3

com='Fuel + Gap + Clad + Moderator'

$\begin{array}{lllllll}\text { cylinder } & 1 & 1 & 0.428498 & 80.80 & 0.00\end{array}$

$\begin{array}{lllllll}\text { cylinder } & 0 & 1 & 0.437515 & 80.80 & 0.00\end{array}$

$\begin{array}{lllllll}\text { cylinder } & 2 & 1 & 0.49657 & 80.80 & 0.00\end{array}$

$\begin{array}{llllll}\text { cylinder } & 2 & 1 & 0.49657 & 80.80 & -1.27\end{array}$

cuboid $3 \quad 1 \quad 4 p .7112 \quad 80.80-1.27$

unit 4
com='Fuel + Gap + Clad + Moderator' $\begin{array}{lllllll}\text { cylinder } & 1 & 1 & 0.428498 & 80.80 & 0.00\end{array}$ $\begin{array}{lllllll}\text { cylinder } & 0 & 1 & 0.437515 & 80.80 & 0.00\end{array}$ $\begin{array}{llllllll}\text { cylinder } & 2 & 1 & 0.49657 & 80.80 & 0.00\end{array}$ $\begin{array}{llllll}\text { cylinder } & 2 & 1 & 0.49657 & 80.80 & -1.27\end{array}$ cuboid $3 \quad 1 \quad 4 p .7112 \quad 80.80-1.27$ unit 5

com='Fuel + Gap + Clad + Moderator' $\begin{array}{llllll}\text { cylinder } & 1 & 1 & 0.428498 & 80.80 & 0.00\end{array}$ $\begin{array}{lllllll}\text { cylinder } & 0 & 1 & 0.437515 & 80.80 & 0.00\end{array}$ $\begin{array}{lllllll}\text { cylinder } & 2 & 1 & 0.49657 & 80.80 & 0.00\end{array}$ $\begin{array}{llllll}\text { cylinder } & 2 & 1 & 0.49657 & 80.80 & -1.27\end{array}$ cuboid 31 4p.7112 $80.80-1.27$ unit 6

com='Fuel + Gap + Clad + Moderator' $\begin{array}{llllll}\text { cylinder } & 1 & 1 & 0.428498 & 80.80 & 0.00\end{array}$ $\begin{array}{lllllll}\text { cylinder } & 0 & 1 & 0.437515 & 80.80 & 0.00\end{array}$ $\begin{array}{lllllll}\text { cylinder } & 2 & 1 & 0.49657 & 80.80 & 0.00\end{array}$ $\begin{array}{llllll}\text { cylinder } & 2 & 1 & 0.49657 & 80.80 & -1.27\end{array}$ cuboid $3 \quad 1 \quad 4 p .7112 \quad 80.80-1.27$ unit 7

com='Fuel + Gap + Clad + Moderator' $\begin{array}{lllllll}\text { cylinder } & 1 & 1 & 0.428498 & 80.80 & 0.00\end{array}$ $\begin{array}{lllllll}\text { cylinder } & 0 & 1 & 0.437515 & 80.80 & 0.00\end{array}$ $\begin{array}{llllllll}\text { cylinder } & 2 & 1 & 0.49657 & 80.80 & 0.00\end{array}$ $\begin{array}{llllll}\text { cylinder } & 2 & 1 & 0.49657 & 80.80 & -1.27\end{array}$ cuboid 3 1 4 p.7112 $80.80-1.27$ unit 8

com='Fuel + Gap + Clad + Moderator' $\begin{array}{lllllll}\text { cylinder } & 1 & 1 & 0.428498 & 80.80 & 0.00\end{array}$ $\begin{array}{lllllll}\text { cylinder } & 0 & 1 & 0.437515 & 80.80 & 0.00\end{array}$ $\begin{array}{lllllll}\text { cylinder } & 2 & 1 & 0.49657 & 80.80 & 0.00\end{array}$ $\begin{array}{llllll}\text { cylinder } & 2 & 1 & 0.49657 & 80.80 & -1.27\end{array}$ cuboid $3 \quad 1 \quad 4 p .7112 \quad 80.80-1.27$ unit 9

com='Fuel + Gap + Clad + Moderator' $\begin{array}{lllllll}\text { cylinder } & 1 & 1 & 0.428498 & 80.80 & 0.00\end{array}$ $\begin{array}{lllllll}\text { cylinder } & 0 & 1 & 0.437515 & 80.80 & 0.00\end{array}$ $\begin{array}{lllllll}\text { cylinder } & 2 & 1 & 0.49657 & 80.80 & 0.00\end{array}$ $\begin{array}{llllll}\text { cylinder } & 2 & 1 & 0.49657 & 80.80 & -1.27\end{array}$ cuboid $3 \quad 1 \quad 4 p .7112 \quad 80.80-1.27$ unit 10

com='Fuel + Gap + Clad + Moderator' $\begin{array}{lllllll}\text { cylinder } & 1 & 1 & 0.428498 & 80.80 & 0.00\end{array}$ $\begin{array}{llllllll}\text { cylinder } & 0 & 1 & 0.437515 & 80.80 & 0.00\end{array}$ $\begin{array}{lllllll}\text { cylinder } & 2 & 1 & 0.49657 & 80.80 & 0.00\end{array}$ $\begin{array}{llllll}\text { cylinder } & 2 & 1 & 0.49657 & 80.80 & -1.27\end{array}$ cuboid 31 4p.7112 $80.80-1.27$ unit 11

com='Fuel + Gap + Clad + Moderator $\begin{array}{lllllll}\text { cylinder } & 1 & 1 & 0.428498 & 80.80 & 0.00\end{array}$ $\begin{array}{lllllll}\text { cylinder } & 0 & 1 & 0.437515 & 80.80 & 0.00\end{array}$ $\begin{array}{lllllll}\text { cylinder } & 2 & 1 & 0.49657 & 80.80 & 0.00\end{array}$ $\begin{array}{llllll}\text { cylinder } & 2 & 1 & 0.49657 & 80.80 & -1.27\end{array}$ cuboid $3 \quad 1 \quad 4$ p.7112 $80.80-1.27$ unit 12

com='Fuel + Gap + Clad + Moderator' 
$\begin{array}{llllll}\text { cylinder } & 1 & 1 & 0.428498 & 80.80 & 0.00\end{array}$ $\begin{array}{lllllll}\text { cylinder } & 0 & 1 & 0.437515 & 80.80 & 0.00\end{array}$ $\begin{array}{lllllll}\text { cylinder } & 2 & 1 & 0.49657 & 80.80 & 0.00\end{array}$ $\begin{array}{lllllll}\text { cylinder } & 2 & 1 & 0.49657 & 80.80 & -1.27\end{array}$ cuboid 3 1 4 p.7112 $80.80-1.27$ unit 13

com='Fuel + Gap + Clad + Moderator' $\begin{array}{lllllll}\text { cylinder } & 1 & 1 & 0.428498 & 80.80 & 0.00\end{array}$ $\begin{array}{llllllll}\text { cylinder } & 0 & 1 & 0.437515 & 80.80 & 0.00\end{array}$ $\begin{array}{lllllll}\text { cylinder } & 2 & 1 & 0.49657 & 80.80 & 0.00\end{array}$ $\begin{array}{llllll}\text { cylinder } & 2 & 1 & 0.49657 & 80.80 & -1.27\end{array}$ cuboid $\quad 3 \quad 1 \quad 4$ p.7112 $80.80-1.27$ unit 14

com='Fuel + Gap + Clad + Moderator' $\begin{array}{lllllll}\text { cylinder } & 1 & 1 & 0.428498 & 80.80 & 0.00\end{array}$ $\begin{array}{lllllll}\text { cylinder } & 0 & 1 & 0.437515 & 80.80 & 0.00\end{array}$ $\begin{array}{lllllll}\text { cylinder } & 2 & 1 & 0.49657 & 80.80 & 0.00\end{array}$ $\begin{array}{llllll}\text { cylinder } & 2 & 1 & 0.49657 & 80.80 & -1.27\end{array}$ cuboid $3 \quad 1$ 4p.7112 $80.80-1.27$ unit 15

com='Fuel + Gap + Clad + Moderator' $\begin{array}{lllllll}\text { cylinder } & 1 & 1 & 0.428498 & 80.80 & 0.00\end{array}$ $\begin{array}{lllllll}\text { cylinder } & 0 & 1 & 0.437515 & 80.80 & 0.00\end{array}$ $\begin{array}{lllllll}\text { cylinder } & 2 & 1 & 0.49657 & 80.80 & 0.00\end{array}$ $\begin{array}{llllll}\text { cylinder } & 2 & 1 & 0.49657 & 80.80 & -1.27\end{array}$ $\begin{array}{llllll}\text { cuboid } \quad 3 & 1 & 4 p .7112 & 80.80 & -1.27\end{array}$ unit 16

com='Fuel + Gap + Clad + Moderator' $\begin{array}{llllll}\text { cylinder } & 1 & 1 & 0.428498 & 80.80 & 0.00\end{array}$ $\begin{array}{lllllll}\text { cylinder } & 0 & 1 & 0.437515 & 80.80 & 0.00\end{array}$ $\begin{array}{lllllll}\text { cylinder } & 2 & 1 & 0.49657 & 80.80 & 0.00\end{array}$ $\begin{array}{llllll}\text { cylinder } & 2 & 1 & 0.49657 & 80.80 & -1.27\end{array}$ cuboid $3 \quad 1 \quad 4$ p.7112 $80.80-1.27$ unit 17

com='Fuel + Gap + Clad + Moderator' $\begin{array}{lllllll}\text { cylinder } & 1 & 1 & 0.428498 & 80.80 & 0.00\end{array}$ $\begin{array}{llllllll}\text { cylinder } & 0 & 1 & 0.437515 & 80.80 & 0.00\end{array}$ $\begin{array}{lllllll}\text { cylinder } & 2 & 1 & 0.49657 & 80.80 & 0.00\end{array}$ $\begin{array}{llllll}\text { cylinder } & 2 & 1 & 0.49657 & 80.80 & -1.27\end{array}$ cuboid 3 1 4 p.7112 $80.80-1.27$ unit 18

com='Fuel + Gap + Clad + Moderator' $\begin{array}{llllll}\text { cylinder } & 1 & 1 & 0.428498 & 80.80 & 0.00\end{array}$ $\begin{array}{lllllll}\text { cylinder } & 0 & 1 & 0.437515 & 80.80 & 0.00\end{array}$ $\begin{array}{lllllll}\text { cylinder } & 2 & 1 & 0.49657 & 80.80 & 0.00\end{array}$ $\begin{array}{llllll}\text { cylinder } & 2 & 1 & 0.49657 & 80.80 & -1.27\end{array}$ cuboid $3 \quad 1$ 4p.7112 $80.80-1.27$ unit 19

com='Fuel + Gap + Clad + Moderator' $\begin{array}{lllllll}\text { cylinder } & 1 & 1 & 0.428498 & 80.80 & 0.00\end{array}$ $\begin{array}{lllllll}\text { cylinder } & 0 & 1 & 0.437515 & 80.80 & 0.00\end{array}$ $\begin{array}{lllllll}\text { cylinder } & 2 & 1 & 0.49657 & 80.80 & 0.00\end{array}$ $\begin{array}{llllll}\text { cylinder } & 2 & 1 & 0.49657 & 80.80 & -1.27\end{array}$ cuboid 3 1 4 p.7112 $80.80-1.27$ unit 20

com='Fuel + Gap + Clad + Moderator' $\begin{array}{llllll}\text { cylinder } & 1 & 1 & 0.428498 & 80.80 & 0.00\end{array}$ $\begin{array}{lllllll}\text { cylinder } & 0 & 1 & 0.437515 & 80.80 & 0.00\end{array}$ $\begin{array}{lllllll}\text { cylinder } & 2 & 1 & 0.49657 & 80.80 & 0.00\end{array}$ $\begin{array}{llllll}\text { cylinder } & 2 & 1 & 0.49657 & 80.80 & -1.27\end{array}$ cuboid $3 \quad 1 \quad 4 p .7112 \quad 80.80-1.27$ unit 21

com='Fuel + Gap + Clad + Moderator' $\begin{array}{lllllll}\text { cylinder } & 1 & 1 & 0.428498 & 80.80 & 0.00\end{array}$ $\begin{array}{llllllll}\text { cylinder } & 0 & 1 & 0.437515 & 80.80 & 0.00\end{array}$ $\begin{array}{lllllll}\text { cylinder } & 2 & 1 & 0.49657 & 80.80 & 0.00\end{array}$ $\begin{array}{llllll}\text { cylinder } & 2 & 1 & 0.49657 & 80.80 & -1.27\end{array}$ cuboid $3 \quad 1$ 4p.7112 $80.80-1.27$ unit 22

com='Fuel + Gap + Clad + Moderator' $\begin{array}{lllllll}\text { cylinder } & 1 & 1 & 0.428498 & 80.80 & 0.00\end{array}$ $\begin{array}{llllllll}\text { cylinder } & 0 & 1 & 0.437515 & 80.80 & 0.00\end{array}$ $\begin{array}{lllllll}\text { cylinder } & 2 & 1 & 0.49657 & 80.80 & 0.00\end{array}$ $\begin{array}{llllll}\text { cylinder } & 2 & 1 & 0.49657 & 80.80 & -1.27\end{array}$ cuboid 314 p.7112 $80.80-1.27$ unit 23

com='Fuel + Gap + Clad + Moderator' $\begin{array}{lllllll}\text { cylinder } & 1 & 1 & 0.428498 & 80.80 & 0.00\end{array}$ $\begin{array}{llllllll}\text { cylinder } & 0 & 1 & 0.437515 & 80.80 & 0.00\end{array}$ $\begin{array}{lllllll}\text { cylinder } & 2 & 1 & 0.49657 & 80.80 & 0.00\end{array}$ $\begin{array}{llllll}\text { cylinder } & 2 & 1 & 0.49657 & 80.80 & -1.27\end{array}$ cuboid 31 4p.7112 $80.80-1.27$ unit 24

com='Fuel + Gap + Clad + Moderator' $\begin{array}{lllllll}\text { cylinder } & 1 & 1 & 0.428498 & 80.80 & 0.00\end{array}$ $\begin{array}{lllllll}\text { cylinder } & 0 & 1 & 0.437515 & 80.80 & 0.00\end{array}$ $\begin{array}{lllllll}\text { cylinder } & 2 & 1 & 0.49657 & 80.80 & 0.00\end{array}$ $\begin{array}{llllll}\text { cylinder } & 2 & 1 & 0.49657 & 80.80 & -1.27\end{array}$ cuboid $3 \quad 1 \quad 4 p .7112 \quad 80.80-1.27$ unit 25

com='Fuel + Gap + Clad + Moderator' $\begin{array}{llllll}\text { cylinder } & 1 & 1 & 0.428498 & 80.80 & 0.00\end{array}$ $\begin{array}{llllllll}\text { cylinder } & 0 & 1 & 0.437515 & 80.80 & 0.00\end{array}$ $\begin{array}{lllllll}\text { cylinder } & 2 & 1 & 0.49657 & 80.80 & 0.00\end{array}$ $\begin{array}{llllll}\text { cylinder } & 2 & 1 & 0.49657 & 80.80 & -1.27\end{array}$ cuboid $3 \quad 1 \quad 4 p .7112 \quad 80.80-1.27$ unit 26

com='Fuel + Gap + Clad + Moderator' $\begin{array}{llllll}\text { cylinder } & 1 & 1 & 0.428498 & 80.80 & 0.00\end{array}$ $\begin{array}{lllllll}\text { cylinder } & 0 & 1 & 0.437515 & 80.80 & 0.00\end{array}$ $\begin{array}{lllllll}\text { cylinder } & 2 & 1 & 0.49657 & 80.80 & 0.00\end{array}$ $\begin{array}{llllll}\text { cylinder } & 2 & 1 & 0.49657 & 80.80 & -1.27\end{array}$ cuboid $3 \quad 1 \quad 4 p .7112 \quad 80.80-1.27$ unit 27

com='Fuel + Gap + Clad + Moderator' $\begin{array}{lllllll}\text { cylinder } & 1 & 1 & 0.428498 & 80.80 & 0.00\end{array}$ $\begin{array}{lllllll}\text { cylinder } & 0 & 1 & 0.437515 & 80.80 & 0.00\end{array}$ $\begin{array}{lllllll}\text { cylinder } & 2 & 1 & 0.49657 & 80.80 & 0.00\end{array}$ $\begin{array}{llllll}\text { cylinder } & 2 & 1 & 0.49657 & 80.80 & -1.27\end{array}$ cuboid 314 p.7112 $80.80-1.27$ unit 28

com='Fuel + Gap + Clad + Moderator' $\begin{array}{llllll}\text { cylinder } & 1 & 1 & 0.428498 & 80.80 & 0.00\end{array}$ $\begin{array}{lllllll}\text { cylinder } & 0 & 1 & 0.437515 & 80.80 & 0.00\end{array}$ 
$\begin{array}{llllll}\text { cylinder } & 2 & 1 & 0.49657 & 80.80 & 0.00\end{array}$ $\begin{array}{llllll}\text { cylinder } & 2 & 1 & 0.49657 & 80.80 & -1.27\end{array}$ cuboid 3 1 4 p.7112 $80.80-1.27$ unit 29

com='Fuel + Gap + Clad + Moderator' $\begin{array}{llllll}\text { cylinder } & 1 & 1 & 0.428498 & 80.80 & 0.00\end{array}$ $\begin{array}{lllllll}\text { cylinder } & 0 & 1 & 0.437515 & 80.80 & 0.00\end{array}$ $\begin{array}{lllllll}\text { cylinder } & 2 & 1 & 0.49657 & 80.80 & 0.00\end{array}$ $\begin{array}{llllll}\text { cylinder } & 2 & 1 & 0.49657 & 80.80 & -1.27\end{array}$ cuboid $3 \quad 1 \quad 4$ p.7112 $80.80-1.27$ unit 30

com='Fuel + Gap + Clad + Moderator' $\begin{array}{llllll}\text { cylinder } & 1 & 1 & 0.428498 & 80.80 & 0.00\end{array}$ $\begin{array}{lllllll}\text { cylinder } & 0 & 1 & 0.437515 & 80.80 & 0.00\end{array}$ $\begin{array}{llllllll}\text { cylinder } & 2 & 1 & 0.49657 & 80.80 & 0.00\end{array}$ $\begin{array}{llllll}\text { cylinder } & 2 & 1 & 0.49657 & 80.80 & -1.27\end{array}$ cuboid 3 1 4 p.7112 $80.80-1.27$ unit 31

com='Fuel + Gap + Clad + Moderator' $\begin{array}{llllll}\text { cylinder } & 1 & 1 & 0.428498 & 80.80 & 0.00\end{array}$ $\begin{array}{lllllll}\text { cylinder } & 0 & 1 & 0.437515 & 80.80 & 0.00\end{array}$ $\begin{array}{lllllll}\text { cylinder } & 2 & 1 & 0.49657 & 80.80 & 0.00\end{array}$ $\begin{array}{llllll}\text { cylinder } & 2 & 1 & 0.49657 & 80.80 & -1.27\end{array}$ cuboid 31 4p.7112 $80.80-1.27$ unit 32

com='Fuel + Gap + Clad + Moderator' $\begin{array}{llllll}\text { cylinder } & 1 & 1 & 0.428498 & 80.80 & 0.00\end{array}$ $\begin{array}{lllllll}\text { cylinder } & 0 & 1 & 0.437515 & 80.80 & 0.00\end{array}$ $\begin{array}{lllllll}\text { cylinder } & 2 & 1 & 0.49657 & 80.80 & 0.00\end{array}$ $\begin{array}{llllll}\text { cylinder } & 2 & 1 & 0.49657 & 80.80 & -1.27\end{array}$ cuboid $3 \quad 1 \quad 4 p .7112 \quad 80.80-1.27$ unit 33

com='Fuel + Gap + Clad + Moderator' $\begin{array}{lllllll}\text { cylinder } & 1 & 1 & 0.428498 & 80.80 & 0.00\end{array}$ $\begin{array}{lllllll}\text { cylinder } & 0 & 1 & 0.437515 & 80.80 & 0.00\end{array}$ $\begin{array}{lllllll}\text { cylinder } & 2 & 1 & 0.49657 & 80.80 & 0.00\end{array}$ $\begin{array}{lllllll}\text { cylinder } & 2 & 1 & 0.49657 & 80.80 & -1.27\end{array}$ cuboid 31 4p.7112 $80.80-1.27$ unit 34

com='Fuel + Gap + Clad + Moderator' $\begin{array}{lllllll}\text { cylinder } & 1 & 1 & 0.428498 & 80.80 & 0.00\end{array}$ $\begin{array}{lllllll}\text { cylinder } & 0 & 1 & 0.437515 & 80.80 & 0.00\end{array}$ $\begin{array}{lllllll}\text { cylinder } & 2 & 1 & 0.49657 & 80.80 & 0.00\end{array}$ $\begin{array}{llllll}\text { cylinder } & 2 & 1 & 0.49657 & 80.80 & -1.27\end{array}$ cuboid $3 \quad 1 \quad 4$ p.7112 $80.80-1.27$ unit 35

com='Fuel + Gap + Clad + Moderator' $\begin{array}{lllllll}\text { cylinder } & 1 & 1 & 0.428498 & 80.80 & 0.00\end{array}$ $\begin{array}{lllllll}\text { cylinder } & 0 & 1 & 0.437515 & 80.80 & 0.00\end{array}$ $\begin{array}{lllllll}\text { cylinder } & 2 & 1 & 0.49657 & 80.80 & 0.00\end{array}$ $\begin{array}{llllll}\text { cylinder } & 2 & 1 & 0.49657 & 80.80 & -1.27\end{array}$ cuboid $3 \quad 1$ 4p.7112 $80.80-1.27$ unit 36

com='Fuel + Gap + Clad + Moderator' $\begin{array}{lllllll}\text { cylinder } & 1 & 1 & 0.428498 & 80.80 & 0.00\end{array}$ $\begin{array}{llllllll}\text { cylinder } & 0 & 1 & 0.437515 & 80.80 & 0.00\end{array}$ $\begin{array}{lllllll}\text { cylinder } & 2 & 1 & 0.49657 & 80.80 & 0.00\end{array}$ $\begin{array}{llllll}\text { cylinder } & 2 & 1 & 0.49657 & 80.80 & -1.27\end{array}$ cuboid 31 4p.7112 $80.80-1.27$ unit 37

com='Fuel + Gap + Clad + Moderator' $\begin{array}{lllllll}\text { cylinder } & 1 & 1 & 0.428498 & 80.80 & 0.00\end{array}$ $\begin{array}{lllllll}\text { cylinder } & 0 & 1 & 0.437515 & 80.80 & 0.00\end{array}$ $\begin{array}{lllllll}\text { cylinder } & 2 & 1 & 0.49657 & 80.80 & 0.00\end{array}$ $\begin{array}{llllll}\text { cylinder } & 2 & 1 & 0.49657 & 80.80 & -1.27\end{array}$ cuboid $3 \quad 1 \quad 4 p .7112 \quad 80.80-1.27$ unit 38

com='Fuel + Gap + Clad + Moderator' $\begin{array}{llllll}\text { cylinder } & 1 & 1 & 0.428498 & 80.80 & 0.00\end{array}$ $\begin{array}{lllllll}\text { cylinder } & 0 & 1 & 0.437515 & 80.80 & 0.00\end{array}$ $\begin{array}{lllllll}\text { cylinder } & 2 & 1 & 0.49657 & 80.80 & 0.00\end{array}$ $\begin{array}{lllllll}\text { cylinder } & 2 & 1 & 0.49657 & 80.80 & -1.27\end{array}$ cuboid $3 \quad 1 \quad 4 p .7112 \quad 80.80-1.27$ unit 39

com='Fuel + Gap + Clad + Moderator' $\begin{array}{lllllll}\text { cylinder } & 1 & 1 & 0.428498 & 80.80 & 0.00\end{array}$ $\begin{array}{lllllll}\text { cylinder } & 0 & 1 & 0.437515 & 80.80 & 0.00\end{array}$ $\begin{array}{llllll}\text { cylinder } & 2 & 1 & 0.49657 & 80.80 & 0.00\end{array}$ $\begin{array}{llllll}\text { cylinder } & 2 & 1 & 0.49657 & 80.80 & -1.27\end{array}$ cuboid 31 4p.7112 $80.80-1.27$ unit 40

com='Fuel + Gap + Clad + Moderator $\begin{array}{lllllll}\text { cylinder } & 1 & 1 & 0.428498 & 80.80 & 0.00\end{array}$ $\begin{array}{llllllll}\text { cylinder } & 0 & 1 & 0.437515 & 80.80 & 0.00\end{array}$ $\begin{array}{lllllll}\text { cylinder } & 2 & 1 & 0.49657 & 80.80 & 0.00\end{array}$ $\begin{array}{llllll}\text { cylinder } & 2 & 1 & 0.49657 & 80.80 & -1.27\end{array}$ cuboid $3 \quad 1$ 4p.7112 $80.80-1.27$ unit 41

com='Fuel + Gap + Clad + Moderator $\begin{array}{lllllll}\text { cylinder } & 1 & 1 & 0.428498 & 80.80 & 0.00\end{array}$ $\begin{array}{llllllll}\text { cylinder } & 0 & 1 & 0.437515 & 80.80 & 0.00\end{array}$ $\begin{array}{llllll}\text { cylinder } & 2 & 1 & 0.49657 & 80.80 & 0.00\end{array}$ $\begin{array}{llllll}\text { cylinder } & 2 & 1 & 0.49657 & 80.80 & -1.27\end{array}$ cuboid $3 \quad 1 \quad 4 p .7112 \quad 80.80-1.27$ unit 42

com='Fuel + Gap + Clad + Moderator' $\begin{array}{lllllll}\text { cylinder } & 1 & 1 & 0.428498 & 80.80 & 0.00\end{array}$ $\begin{array}{lllllll}\text { cylinder } & 0 & 1 & 0.437515 & 80.80 & 0.00\end{array}$ $\begin{array}{lllllll}\text { cylinder } & 2 & 1 & 0.49657 & 80.80 & 0.00\end{array}$ $\begin{array}{llllll}\text { cylinder } & 2 & 1 & 0.49657 & 80.80 & -1.27\end{array}$ cuboid $3 \quad 1 \quad 4 p .7112 \quad 80.80-1.27$ unit 43

com='Fuel + Gap + Clad + Moderator' $\begin{array}{llllll}\text { cylinder } & 1 & 1 & 0.428498 & 80.80 & 0.00\end{array}$ $\begin{array}{llllllll}\text { cylinder } & 0 & 1 & 0.437515 & 80.80 & 0.00\end{array}$ $\begin{array}{lllllll}\text { cylinder } & 2 & 1 & 0.49657 & 80.80 & 0.00\end{array}$ $\begin{array}{llllll}\text { cylinder } & 2 & 1 & 0.49657 & 80.80 & -1.27\end{array}$ cuboid 314 p.7112 $80.80-1.27$ unit 44

com='Fuel + Gap + Clad + Moderator' $\begin{array}{llllll}\text { cylinder } & 1 & 1 & 0.428498 & 80.80 & 0.00\end{array}$ $\begin{array}{llllllll}\text { cylinder } & 0 & 1 & 0.437515 & 80.80 & 0.00\end{array}$ $\begin{array}{lllllll}\text { cylinder } & 2 & 1 & 0.49657 & 80.80 & 0.00\end{array}$ $\begin{array}{llllll}\text { cylinder } & 2 & 1 & 0.49657 & 80.80 & -1.27\end{array}$ 


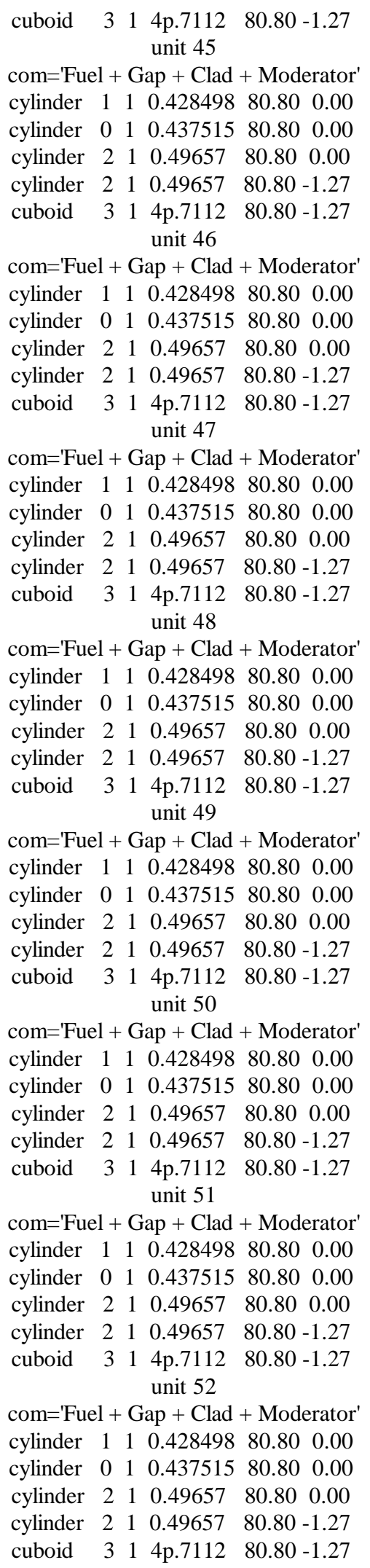

com='Fuel + Gap + Clad + Moderator' $\begin{array}{lllllll}\text { cylinder } & 1 & 1 & 0.428498 & 80.80 & 0.00\end{array}$ $\begin{array}{lllllll}\text { cylinder } & 0 & 1 & 0.437515 & 80.80 & 0.00\end{array}$ $\begin{array}{lllllll}\text { cylinder } & 2 & 1 & 0.49657 & 80.80 & 0.00\end{array}$ $\begin{array}{llllll}\text { cylinder } & 2 & 1 & 0.49657 & 80.80 & -1.27\end{array}$ cuboid $3 \quad 1 \quad 4 p .7112 \quad 80.80-1.27$

unit 53

com='Fuel + Gap + Clad + Moderator' $\begin{array}{lllllll}\text { cylinder } & 1 & 1 & 0.428498 & 80.80 & 0.00\end{array}$ $\begin{array}{lllllll}\text { cylinder } & 0 & 1 & 0.437515 & 80.80 & 0.00\end{array}$ $\begin{array}{lllllll}\text { cylinder } & 2 & 1 & 0.49657 & 80.80 & 0.00\end{array}$ $\begin{array}{llllll}\text { cylinder } & 2 & 1 & 0.49657 & 80.80 & -1.27\end{array}$ cuboid $3 \quad 1$ 4p.7112 $80.80-1.27$ unit 54

com='Fuel + Gap + Clad + Moderator' $\begin{array}{lllllll}\text { cylinder } & 1 & 1 & 0.428498 & 80.80 & 0.00\end{array}$ $\begin{array}{llllllll}\text { cylinder } & 0 & 1 & 0.437515 & 80.80 & 0.00\end{array}$ $\begin{array}{llllllll}\text { cylinder } & 2 & 1 & 0.49657 & 80.80 & 0.00\end{array}$ $\begin{array}{llllll}\text { cylinder } & 2 & 1 & 0.49657 & 80.80 & -1.27\end{array}$ cuboid 3 1 4 p.7112 $80.80-1.27$ unit 55

com='Fuel + Gap + Clad + Moderator' $\begin{array}{lllllll}\text { cylinder } & 1 & 1 & 0.428498 & 80.80 & 0.00\end{array}$ $\begin{array}{lllllll}\text { cylinder } & 0 & 1 & 0.437515 & 80.80 & 0.00\end{array}$ $\begin{array}{lllllll}\text { cylinder } & 2 & 1 & 0.49657 & 80.80 & 0.00\end{array}$ $\begin{array}{llllll}\text { cylinder } & 2 & 1 & 0.49657 & 80.80 & -1.27\end{array}$ cuboid $3 \quad 1 \quad 4 p .7112 \quad 80.80-1.27$

unit 71

com='Clad + Mod + AL Grid' $\begin{array}{lllll}\text { cylinder } 2 & 1 & 0.49657 & 0.635 & 0.0\end{array}$ $\begin{array}{llllll}\text { cylinder } 3 & 1 & 0.50419 & 0.635 & 0.0\end{array}$ $\begin{array}{lllllll}\text { cuboid } \quad 5 & 1 & 4 \mathrm{p} .7112 & 0.635 & 0.0\end{array}$

unit 73

com='Fuel + Gap + Clad + Air' $\begin{array}{llllll}\text { cylinder } & 1 & 1 & 0.428498 & 12.164 & 0.0\end{array}$ $\begin{array}{lllllll}\text { cylinder } & 0 & 1 & 0.437515 & 12.164 & 0.0\end{array}$ $\begin{array}{llllll}\text { cylinder } 2 & 1 & 0.49657 & 12.164 & 0.0\end{array}$ $\begin{array}{lllllll}\text { cuboid } & 0 & 1 & 4 p .7112 & 12.164 & 0.0\end{array}$

unit 74 com='Top Clad + Void' $\begin{array}{llllll}\text { cylinder } 2 & 1 & 0.49657 & 1.016 & 0.0\end{array}$ $\begin{array}{llllll}\text { cuboid } 0 & 0 & 1 & 4 p .7112 & 1.016 & 0.0\end{array}$

unit 75

com='Top Clad + Void + AL grid' cylinder $210.49657 \quad 1.27 \quad 0.0$ cylinder $\begin{array}{lllll}0 & 1 & 0.50419 & 1.27 & 0.0\end{array}$ cuboid 514 p.7112 $1.27 \quad 0.0$

unit 81

com='array of Clad + Mod + AL Grid + Reflector' array $1-13.5128-13.51280 .0$ cuboid 41 4p43.5128 0.6350 .0

unit 82

com='array of Fuel + Gap + Clad + Moderator + Reflector' array $2-13.5128-13.5128-1.27$ cuboid $314 \mathrm{p} 43.5128 \quad 80.80 \quad-1.27$

unit 83 
com='array of Fuel + Gap + Clad + Air'

array $3-13.5128-13.51280 .0$

cuboid $014 \mathrm{p} 43.512812 .164 \quad 0.0$

unit 84

com='array of Top Clad + Void'

array $4-13.5128-13.51280 .0$

cuboid $014 \mathrm{p} 43.51281 .0160 .0$

unit 85

com='array of Top Clad + Void + AL grid' array $5-13.5128-13.51280 .0$

cuboid 41 4p43.5128 $1.27 \quad 0.0$

global unit 100

com='array of arrays of fuel element sections + reflector' array $6-43.5128-43.5128 \quad 0.0$

cuboid 5 1 $14 \mathrm{p} 43.5128 \quad 97.155 \quad-2.54$

$\begin{array}{llllll}\text { cuboid } 3 & 1 & 4 \mathrm{p} 43.5128 & 97.155 & -8.89\end{array}$

cuboid $5 \quad 1 \quad 4 p 43.5128 \quad 97.155 \quad-13.97$

cuboid $3 \quad 1 \quad 4 p 43.5128 \quad 97.155 \quad-43.97$

end geom

read array

ara=1 nux=19 nuy=19 nuz=1

fill $\mathrm{f} 71$ end fill

ara=2 nux=19 nuy=19 nuz=1

fill

$\begin{array}{llllllllllll}1 & 2 & 3 & 4 & 5 & 6 & 7 & 8 & 9 & 10 & 1 \mathrm{~b} 9\end{array}$

$2111213141516171819 \quad 1 b 9$

$3122021222324252627 \quad 1$ b9

4132128293031323334 1b9

5142229353637383940 1b9

$6152330364142434445 \quad 1 b 9$

7162431374246474849 1b9

8172532384347505152 1b9

9182633394448515354 1b9

10192734404549525455 1b180 end fill

ara=3 nux=19 nuy=19 nuz=1 fill $\mathrm{f} 73$ end fill

ara=4 nux=19 nuy=19 nuz=1 fill f74 end fill

ara $=5$ nux $=19$ nuy=19 nuz=1 fill $\mathrm{f} 75$ end fill

ara $=6$ nux $=1$ nuy=1 nuz=5

fill 8182838485 end fill

end array

read plot

$\mathrm{ttl}=$ 'simple plot 1 '

$\mathrm{lpi}=10 \mathrm{scr}=$ yes $\mathrm{pic}=\operatorname{mix} \operatorname{nax}=400$

$\mathrm{xul}=-20.0$ yul $=0.0 \mathrm{zul}=100$

$\mathrm{xlr}=20.0 \quad \mathrm{ylr}=0.0 \quad \mathrm{zlr}=-10$

uax $=1.0 \mathrm{wdn}=-1.0$ end plt0

$\mathrm{ttl}=$ 'simple plot 2'

pic $=\operatorname{mix}$ nax $=400$

$\mathrm{xul}=-20.0 \mathrm{yul}=20 . \mathrm{zul}=25$.

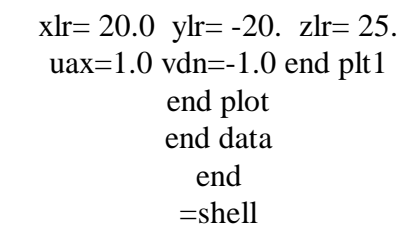

cp ft04f001 \$RTNDIR/sax02.xs04 cp ft64f001 \$RTNDIR/sax02.rs64 end $=\mathrm{kmart}$

read initial kunit $=64$ xunit $=4$ actbygrp rrpvol

end initial read activity

92234279223418922341452 92235279223518922351452 92238279223818922381452

94239279423918942391452 94240279424018942401452 $9424127942411894241 \quad 1452$ $9424227942421894242 \quad 1452$ 95241279524118952411452 501027

501127

801627

4000027

2400027

2600027

100127

5011227

5011427

5011527

5011627

5011727

5011827

5011927

5012027

5012227

5012427

end activity read collapse

factor $=1.0$ lastg $=57$

lastg $=148$

lastg $=204$

lastg $=238$

end collapse

end
$=\operatorname{csasc} 5 \quad$ parm $=$ size $=1000000$ saxton 3 fuel
238group latticecell
'MOX fuel
pu-239 10 1.3526-3 291 end


pu-240 $11001.2759-4291$ end pu-241 110 1.1407-5 291 end pu-242 $1106.0318-7291$ end am-241 10 1.7783-6 291 end u-234 $100 \quad 1.1688-6291$ end u-235 $11001.5301-4 \quad 291$ end u-238 $11002.1097-2 \quad 291$ end

o $\quad \begin{array}{llll}1 & 0 & 4.5155-2 & 291 \\ \text { end }\end{array}$ 'clad (ZR-2)

zr $\quad 2 \quad 0 \quad 4.2517-2 \quad 291$ end

sn $\quad 2 \quad 0 \quad 4.6590-4291$ end

cr $\quad 2007.5977-5291$ end

fe $\quad \begin{array}{llll}2 & 0 & 1.4148-4 & 291 \\ \text { end }\end{array}$

o $20 \quad 2.9630-4291$ end 'water(Refletor)

h $\quad 3 \quad 0 \quad 6.6751-2 \quad 291$ end

o 30 3.3404-2 291 end

b-10 $3003.7338-6291$ end

b-11 $3001.5029-5291$ end

'Middle Grid and $\mathrm{H} 2 \mathrm{O}$

al $\quad 4.8458 \quad 291$ end

h2o $4.1542 \quad 291$ end 'Aluminum

al $\quad \begin{array}{llll}5 & 0 & 6.0039-2 & 291 \\ \text { end }\end{array}$ end comp

$\begin{array}{llllllll}\text { squarepitch } & 1.4224 & 856996 & 1 & 2.99314 & 3 & 87503 & 0\end{array}$ end more data

limcen $=20000000$

end more

saxton 3

read param

gen=225 nsk=25 npg=20000 $\mathrm{fdn}=$ yes $\mathrm{flx}=$ yes wrs $=64$ res $=225$

$\operatorname{lng}=2000000$

end param

read geometry unit 1

com='Fuel + Gap + Clad + Moderator' $\begin{array}{llllll}\text { cylinder } & 1 & 1 & 0.428498 & 88.06 & 0.00\end{array}$ $\begin{array}{lllllll}\text { cylinder } & 0 & 1 & 0.437515 & 88.06 & 0.00\end{array}$ $\begin{array}{lllllll}\text { cylinder } & 2 & 1 & 0.49657 & 88.06 & 0.00\end{array}$ $\begin{array}{llllll}\text { cylinder } & 2 & 1 & 0.49657 & 88.06 & -1.27\end{array}$ cuboid $3 \quad 1 \quad 4 p .7112 \quad 88.06-1.27$ unit 2

com='Fuel + Gap + Clad + Moderator' $\begin{array}{lllllll}\text { cylinder } & 1 & 1 & 0.428498 & 88.06 & 0.00\end{array}$ $\begin{array}{lllllll}\text { cylinder } & 0 & 1 & 0.437515 & 88.06 & 0.00\end{array}$ $\begin{array}{lllllll}\text { cylinder } & 2 & 1 & 0.49657 & 88.06 & 0.00\end{array}$ $\begin{array}{llllll}\text { cylinder } & 2 & 1 & 0.49657 & 88.06 & -1.27\end{array}$ cuboid 31 4p.7112 $88.06-1.27$ unit 3

com='Fuel + Gap + Clad + Moderator' $\begin{array}{lllllll}\text { cylinder } & 1 & 1 & 0.428498 & 88.06 & 0.00\end{array}$ $\begin{array}{lllllll}\text { cylinder } & 0 & 1 & 0.437515 & 88.06 & 0.00\end{array}$ $\begin{array}{lllllll}\text { cylinder } & 2 & 1 & 0.49657 & 88.06 & 0.00\end{array}$ $\begin{array}{llllll}\text { cylinder } & 2 & 1 & 0.49657 & 88.06 & -1.27\end{array}$ cuboid $3 \begin{array}{llll}3 & 4 p .7112 & 88.06 & -1.27\end{array}$ unit 4

com='Fuel + Gap + Clad + Moderator' $\begin{array}{lllllll}\text { cylinder } & 1 & 1 & 0.428498 & 88.06 & 0.00\end{array}$ $\begin{array}{lllllll}\text { cylinder } & 0 & 1 & 0.437515 & 88.06 & 0.00\end{array}$ $\begin{array}{lllllll}\text { cylinder } & 2 & 1 & 0.49657 & 88.06 & 0.00\end{array}$ $\begin{array}{llllll}\text { cylinder } & 2 & 1 & 0.49657 & 88.06 & -1.27\end{array}$ cuboid $3 \quad 1$ 4p.7112 88.06-1.27 unit 5

com='Fuel + Gap + Clad + Moderator' $\begin{array}{lllllll}\text { cylinder } & 1 & 1 & 0.428498 & 88.06 & 0.00\end{array}$ $\begin{array}{llllllll}\text { cylinder } & 0 & 1 & 0.437515 & 88.06 & 0.00\end{array}$ $\begin{array}{llllllll}\text { cylinder } & 2 & 1 & 0.49657 & 88.06 & 0.00\end{array}$ $\begin{array}{llllll}\text { cylinder } & 2 & 1 & 0.49657 & 88.06 & -1.27\end{array}$ cuboid $3 \quad 1 \quad 4 p .7112 \quad 88.06-1.27$ unit 6

com='Fuel + Gap + Clad + Moderator' $\begin{array}{lllllll}\text { cylinder } & 1 & 1 & 0.428498 & 88.06 & 0.00\end{array}$ $\begin{array}{lllllll}\text { cylinder } & 0 & 1 & 0.437515 & 88.06 & 0.00\end{array}$ $\begin{array}{lllllll}\text { cylinder } & 2 & 1 & 0.49657 & 88.06 & 0.00\end{array}$ $\begin{array}{llllll}\text { cylinder } & 2 & 1 & 0.49657 & 88.06 & -1.27\end{array}$ cuboid $3 \quad 1 \quad 4$ p.7112 88.06-1.27 unit 7

com='Fuel + Gap + Clad + Moderator' $\begin{array}{llllll}\text { cylinder } & 1 & 1 & 0.428498 & 88.06 & 0.00\end{array}$ $\begin{array}{lllllll}\text { cylinder } & 0 & 1 & 0.437515 & 88.06 & 0.00\end{array}$ $\begin{array}{llllllll}\text { cylinder } & 2 & 1 & 0.49657 & 88.06 & 0.00\end{array}$ $\begin{array}{llllll}\text { cylinder } & 2 & 1 & 0.49657 & 88.06 & -1.27\end{array}$ cuboid 3 1 4 p.7112 88.06-1.27 unit 8

com='Fuel + Gap + Clad + Moderator' $\begin{array}{lllllll}\text { cylinder } & 1 & 1 & 0.428498 & 88.06 & 0.00\end{array}$ $\begin{array}{lllllll}\text { cylinder } & 0 & 1 & 0.437515 & 88.06 & 0.00\end{array}$ $\begin{array}{lllllll}\text { cylinder } & 2 & 1 & 0.49657 & 88.06 & 0.00\end{array}$ $\begin{array}{llllll}\text { cylinder } & 2 & 1 & 0.49657 & 88.06 & -1.27\end{array}$ cuboid 3 1 4 4p.7112 88.06 -1.27 unit 9

com='Fuel + Gap + Clad + Moderator' $\begin{array}{lllllll}\text { cylinder } & 1 & 1 & 0.428498 & 88.06 & 0.00\end{array}$ $\begin{array}{lllllll}\text { cylinder } & 0 & 1 & 0.437515 & 88.06 & 0.00\end{array}$ $\begin{array}{lllllll}\text { cylinder } & 2 & 1 & 0.49657 & 88.06 & 0.00\end{array}$ $\begin{array}{llllll}\text { cylinder } & 2 & 1 & 0.49657 & 88.06 & -1.27\end{array}$ cuboid 3 1 4 p.7112 88.06 - 1.27 unit 10

com='Fuel + Gap + Clad + Moderator' $\begin{array}{lllllll}\text { cylinder } & 1 & 1 & 0.428498 & 88.06 & 0.00\end{array}$ $\begin{array}{lllllll}\text { cylinder } & 0 & 1 & 0.437515 & 88.06 & 0.00\end{array}$ $\begin{array}{llllllll}\text { cylinder } & 2 & 1 & 0.49657 & 88.06 & 0.00\end{array}$ $\begin{array}{llllll}\text { cylinder } & 2 & 1 & 0.49657 & 88.06 & -1.27\end{array}$ cuboid $3 \quad 1 \quad 4 p .7112 \quad 88.06-1.27$ unit 11

com='Fuel + Gap + Clad + Moderator' $\begin{array}{lllllll}\text { cylinder } & 1 & 1 & 0.428498 & 88.06 & 0.00\end{array}$ $\begin{array}{lllllll}\text { cylinder } & 0 & 1 & 0.437515 & 88.06 & 0.00\end{array}$ $\begin{array}{lllllll}\text { cylinder } & 2 & 1 & 0.49657 & 88.06 & 0.00\end{array}$ $\begin{array}{llllll}\text { cylinder } & 2 & 1 & 0.49657 & 88.06 & -1.27\end{array}$ cuboid $3 \quad 1$ 4p.7112 88.06-1.27 unit 12 
com='Fuel + Gap + Clad + Moderator' $\begin{array}{lllllll}\text { cylinder } & 1 & 1 & 0.428498 & 88.06 & 0.00\end{array}$ $\begin{array}{lllllll}\text { cylinder } & 0 & 1 & 0.437515 & 88.06 & 0.00\end{array}$ $\begin{array}{llllllll}\text { cylinder } & 2 & 1 & 0.49657 & 88.06 & 0.00\end{array}$ $\begin{array}{llllll}\text { cylinder } & 2 & 1 & 0.49657 & 88.06 & -1.27\end{array}$ cuboid $3 \quad 1 \quad 4 p .7112 \quad 88.06-1.27$ unit 13

com='Fuel + Gap + Clad + Moderator' $\begin{array}{llllll}\text { cylinder } & 1 & 1 & 0.428498 & 88.06 & 0.00\end{array}$ $\begin{array}{llllllll}\text { cylinder } & 0 & 1 & 0.437515 & 88.06 & 0.00\end{array}$ $\begin{array}{lllllll}\text { cylinder } & 2 & 1 & 0.49657 & 88.06 & 0.00\end{array}$ $\begin{array}{llllll}\text { cylinder } & 2 & 1 & 0.49657 & 88.06 & -1.27\end{array}$ cuboid 31 4p.7112 $88.06-1.27$ unit 14

com='Fuel + Gap + Clad + Moderator' $\begin{array}{lllllll}\text { cylinder } & 1 & 1 & 0.428498 & 88.06 & 0.00\end{array}$ $\begin{array}{lllllll}\text { cylinder } & 0 & 1 & 0.437515 & 88.06 & 0.00\end{array}$ $\begin{array}{lllllll}\text { cylinder } & 2 & 1 & 0.49657 & 88.06 & 0.00\end{array}$ $\begin{array}{llllll}\text { cylinder } & 2 & 1 & 0.49657 & 88.06 & -1.27\end{array}$ cuboid $3 \quad 1 \quad 4 p .7112 \quad 88.06-1.27$ unit 15

com='Fuel + Gap + Clad + Moderator' $\begin{array}{lllllll}\text { cylinder } & 1 & 1 & 0.428498 & 88.06 & 0.00\end{array}$ $\begin{array}{lllllll}\text { cylinder } & 0 & 1 & 0.437515 & 88.06 & 0.00\end{array}$ $\begin{array}{lllllll}\text { cylinder } & 2 & 1 & 0.49657 & 88.06 & 0.00\end{array}$ $\begin{array}{lllllll}\text { cylinder } & 2 & 1 & 0.49657 & 88.06 & -1.27\end{array}$ cuboid 314 p.7112 $88.06-1.27$ unit 16

com='Fuel + Gap + Clad + Moderator' $\begin{array}{lllllll}\text { cylinder } & 1 & 1 & 0.428498 & 88.06 & 0.00\end{array}$ $\begin{array}{lllllll}\text { cylinder } & 0 & 1 & 0.437515 & 88.06 & 0.00\end{array}$ $\begin{array}{lllllll}\text { cylinder } & 2 & 1 & 0.49657 & 88.06 & 0.00\end{array}$ $\begin{array}{llllll}\text { cylinder } & 2 & 1 & 0.49657 & 88.06 & -1.27\end{array}$ cuboid $3 \quad 1 \quad 4$ p.7112 $88.06-1.27$ unit 17

com='Fuel + Gap + Clad + Moderator' $\begin{array}{lllllll}\text { cylinder } & 1 & 1 & 0.428498 & 88.06 & 0.00\end{array}$ $\begin{array}{lllllll}\text { cylinder } & 0 & 1 & 0.437515 & 88.06 & 0.00\end{array}$ $\begin{array}{lllllll}\text { cylinder } & 2 & 1 & 0.49657 & 88.06 & 0.00\end{array}$ $\begin{array}{llllll}\text { cylinder } & 2 & 1 & 0.49657 & 88.06 & -1.27\end{array}$ cuboid $3 \quad 1 \quad 4 p .7112 \quad 88.06-1.27$ unit 18

com='Fuel + Gap + Clad + Moderator $\begin{array}{lllllll}\text { cylinder } & 1 & 1 & 0.428498 & 88.06 & 0.00\end{array}$ $\begin{array}{llllllll}\text { cylinder } & 0 & 1 & 0.437515 & 88.06 & 0.00\end{array}$ $\begin{array}{lllllll}\text { cylinder } & 2 & 1 & 0.49657 & 88.06 & 0.00\end{array}$ $\begin{array}{llllll}\text { cylinder } & 2 & 1 & 0.49657 & 88.06 & -1.27\end{array}$ cuboid $3 \quad 1$ 4p.7112 $88.06-1.27$ unit 19

com='Fuel + Gap + Clad + Moderator' $\begin{array}{lllllll}\text { cylinder } & 1 & 1 & 0.428498 & 88.06 & 0.00\end{array}$ $\begin{array}{llllllll}\text { cylinder } & 0 & 1 & 0.437515 & 88.06 & 0.00\end{array}$ $\begin{array}{lllllll}\text { cylinder } & 2 & 1 & 0.49657 & 88.06 & 0.00\end{array}$ $\begin{array}{llllll}\text { cylinder } & 2 & 1 & 0.49657 & 88.06 & -1.27\end{array}$ cuboid 31 4p.7112 $88.06-1.27$ unit 20

com='Fuel + Gap + Clad + Moderator' $\begin{array}{lllllll}\text { cylinder } & 1 & 1 & 0.428498 & 88.06 & 0.00\end{array}$ $\begin{array}{lllllll}\text { cylinder } & 0 & 1 & 0.437515 & 88.06 & 0.00\end{array}$ $\begin{array}{lllllll}\text { cylinder } & 2 & 1 & 0.49657 & 88.06 & 0.00\end{array}$ $\begin{array}{lllllll}\text { cylinder } & 2 & 1 & 0.49657 & 88.06 & -1.27\end{array}$ cuboid $3 \quad 1$ 4p.7112 88.06-1.27 unit 21

com='Fuel + Gap + Clad + Moderator' $\begin{array}{lllllll}\text { cylinder } & 1 & 1 & 0.428498 & 88.06 & 0.00\end{array}$ $\begin{array}{lllllll}\text { cylinder } & 0 & 1 & 0.437515 & 88.06 & 0.00\end{array}$ $\begin{array}{lllllll}\text { cylinder } & 2 & 1 & 0.49657 & 88.06 & 0.00\end{array}$ $\begin{array}{llllll}\text { cylinder } & 2 & 1 & 0.49657 & 88.06 & -1.27\end{array}$ cuboid 3 1 4 p.7112 88.06-1.27 unit 22

com='Fuel + Gap + Clad + Moderator $\begin{array}{lllllll}\text { cylinder } & 1 & 1 & 0.428498 & 88.06 & 0.00\end{array}$ $\begin{array}{lllllll}\text { cylinder } & 0 & 1 & 0.437515 & 88.06 & 0.00\end{array}$ $\begin{array}{lllllll}\text { cylinder } & 2 & 1 & 0.49657 & 88.06 & 0.00\end{array}$ $\begin{array}{llllll}\text { cylinder } & 2 & 1 & 0.49657 & 88.06 & -1.27\end{array}$ cuboid 31 4p.7112 $88.06-1.27$ unit 23

com='Fuel + Gap + Clad + Moderator' $\begin{array}{lllllll}\text { cylinder } & 1 & 1 & 0.428498 & 88.06 & 0.00\end{array}$ $\begin{array}{llllllll}\text { cylinder } & 0 & 1 & 0.437515 & 88.06 & 0.00\end{array}$ $\begin{array}{lllllll}\text { cylinder } & 2 & 1 & 0.49657 & 88.06 & 0.00\end{array}$ $\begin{array}{llllll}\text { cylinder } & 2 & 1 & 0.49657 & 88.06 & -1.27\end{array}$ cuboid $3 \quad 1$ 4p.7112 $88.06-1.27$ unit 24

com='Fuel + Gap + Clad + Moderator' $\begin{array}{lllllll}\text { cylinder } & 1 & 1 & 0.428498 & 88.06 & 0.00\end{array}$ $\begin{array}{lllllll}\text { cylinder } & 0 & 1 & 0.437515 & 88.06 & 0.00\end{array}$ $\begin{array}{lllllll}\text { cylinder } & 2 & 1 & 0.49657 & 88.06 & 0.00\end{array}$ $\begin{array}{llllll}\text { cylinder } & 2 & 1 & 0.49657 & 88.06 & -1.27\end{array}$ cuboid $3 \quad 1 \quad 4 p .7112 \quad 88.06-1.27$ unit 25

com='Fuel + Gap + Clad + Moderator' $\begin{array}{lllllll}\text { cylinder } & 1 & 1 & 0.428498 & 88.06 & 0.00\end{array}$ $\begin{array}{llllllll}\text { cylinder } & 0 & 1 & 0.437515 & 88.06 & 0.00\end{array}$ $\begin{array}{lllllll}\text { cylinder } & 2 & 1 & 0.49657 & 88.06 & 0.00\end{array}$ $\begin{array}{llllll}\text { cylinder } & 2 & 1 & 0.49657 & 88.06 & -1.27\end{array}$ cuboid 314 p.7112 $88.06-1.27$ unit 26

com='Fuel + Gap + Clad + Moderator' $\begin{array}{lllllll}\text { cylinder } & 1 & 1 & 0.428498 & 88.06 & 0.00\end{array}$ $\begin{array}{lllllll}\text { cylinder } & 0 & 1 & 0.437515 & 88.06 & 0.00\end{array}$ $\begin{array}{lllllll}\text { cylinder } & 2 & 1 & 0.49657 & 88.06 & 0.00\end{array}$ $\begin{array}{llllll}\text { cylinder } & 2 & 1 & 0.49657 & 88.06 & -1.27\end{array}$ cuboid $3 \quad 1$ 4p.7112 $88.06-1.27$ unit 27

com='Fuel + Gap + Clad + Moderator $\begin{array}{lllllll}\text { cylinder } & 1 & 1 & 0.428498 & 88.06 & 0.00\end{array}$ $\begin{array}{lllllll}\text { cylinder } & 0 & 1 & 0.437515 & 88.06 & 0.00\end{array}$ $\begin{array}{lllllll}\text { cylinder } & 2 & 1 & 0.49657 & 88.06 & 0.00\end{array}$ $\begin{array}{llllll}\text { cylinder } & 2 & 1 & 0.49657 & 88.06 & -1.27\end{array}$ cuboid 3 1 4 p.7112 88.06 - 1.27 unit 28

com='Fuel + Gap + Clad + Moderator' $\begin{array}{llllll}\text { cylinder } & 1 & 1 & 0.428498 & 88.06 & 0.00\end{array}$ 
$\begin{array}{lllllll}\text { cylinder } & 0 & 1 & 0.437515 & 88.06 & 0.00\end{array}$ $\begin{array}{lllllll}\text { cylinder } & 2 & 1 & 0.49657 & 88.06 & 0.00\end{array}$ $\begin{array}{llllll}\text { cylinder } & 2 & 1 & 0.49657 & 88.06 & -1.27\end{array}$ cuboid $3 \quad 1 \quad 4 p .7112 \quad 88.06-1.27$ unit 29

com='Fuel + Gap + Clad + Moderator' $\begin{array}{lllllll}\text { cylinder } & 1 & 1 & 0.428498 & 88.06 & 0.00\end{array}$ $\begin{array}{lllllll}\text { cylinder } & 0 & 1 & 0.437515 & 88.06 & 0.00\end{array}$ $\begin{array}{lllllll}\text { cylinder } & 2 & 1 & 0.49657 & 88.06 & 0.00\end{array}$ $\begin{array}{llllll}\text { cylinder } & 2 & 1 & 0.49657 & 88.06 & -1.27\end{array}$ cuboid $3 \quad 1 \quad 4 p .7112 \quad 88.06-1.27$ unit 30

com='Fuel + Gap + Clad + Moderator' $\begin{array}{lllllll}\text { cylinder } & 1 & 1 & 0.428498 & 88.06 & 0.00\end{array}$ $\begin{array}{llllllll}\text { cylinder } & 0 & 1 & 0.437515 & 88.06 & 0.00\end{array}$ $\begin{array}{lllllll}\text { cylinder } & 2 & 1 & 0.49657 & 88.06 & 0.00\end{array}$ $\begin{array}{llllll}\text { cylinder } & 2 & 1 & 0.49657 & 88.06 & -1.27\end{array}$ cuboid $3 \quad 1 \quad 4 p .7112 \quad 88.06-1.27$ unit 31

com='Fuel + Gap + Clad + Moderator' $\begin{array}{llllll}\text { cylinder } & 1 & 1 & 0.428498 & 88.06 & 0.00\end{array}$ $\begin{array}{llllllll}\text { cylinder } & 0 & 1 & 0.437515 & 88.06 & 0.00\end{array}$ $\begin{array}{lllllll}\text { cylinder } & 2 & 1 & 0.49657 & 88.06 & 0.00\end{array}$ $\begin{array}{llllll}\text { cylinder } & 2 & 1 & 0.49657 & 88.06 & -1.27\end{array}$ cuboid $3 \quad 1 \quad 4 p .7112 \quad 88.06-1.27$ unit 32

com='Fuel + Gap + Clad + Moderator' $\begin{array}{llllll}\text { cylinder } & 1 & 1 & 0.428498 & 88.06 & 0.00\end{array}$ $\begin{array}{lllllll}\text { cylinder } & 0 & 1 & 0.437515 & 88.06 & 0.00\end{array}$ $\begin{array}{lllllll}\text { cylinder } & 2 & 1 & 0.49657 & 88.06 & 0.00\end{array}$ $\begin{array}{llllll}\text { cylinder } & 2 & 1 & 0.49657 & 88.06 & -1.27\end{array}$ cuboid 31 4p.7112 $88.06-1.27$ unit 33

com='Fuel + Gap + Clad + Moderator' $\begin{array}{llllll}\text { cylinder } & 1 & 1 & 0.428498 & 88.06 & 0.00\end{array}$ $\begin{array}{llllllll}\text { cylinder } & 0 & 1 & 0.437515 & 88.06 & 0.00\end{array}$ $\begin{array}{llllllll}\text { cylinder } & 2 & 1 & 0.49657 & 88.06 & 0.00\end{array}$ $\begin{array}{llllll}\text { cylinder } & 2 & 1 & 0.49657 & 88.06 & -1.27\end{array}$ cuboid 3 1 4 p.7112 $88.06-1.27$ unit 34

com='Fuel + Gap + Clad + Moderator' $\begin{array}{lllllll}\text { cylinder } & 1 & 1 & 0.428498 & 88.06 & 0.00\end{array}$ $\begin{array}{llllllll}\text { cylinder } & 0 & 1 & 0.437515 & 88.06 & 0.00\end{array}$ $\begin{array}{lllllll}\text { cylinder } & 2 & 1 & 0.49657 & 88.06 & 0.00\end{array}$ $\begin{array}{llllll}\text { cylinder } & 2 & 1 & 0.49657 & 88.06 & -1.27\end{array}$ cuboid 314 p.7112 $88.06-1.27$ unit 35

com='Fuel + Gap + Clad + Moderator' $\begin{array}{lllllll}\text { cylinder } & 1 & 1 & 0.428498 & 88.06 & 0.00\end{array}$ $\begin{array}{lllllll}\text { cylinder } & 0 & 1 & 0.437515 & 88.06 & 0.00\end{array}$ $\begin{array}{lllllll}\text { cylinder } & 2 & 1 & 0.49657 & 88.06 & 0.00\end{array}$ $\begin{array}{llllll}\text { cylinder } & 2 & 1 & 0.49657 & 88.06 & -1.27\end{array}$ cuboid 3 1 4 p.7112 $88.06-1.27$ unit 36

com='Fuel + Gap + Clad + Moderator' $\begin{array}{lllllll}\text { cylinder } & 1 & 1 & 0.428498 & 88.06 & 0.00\end{array}$ $\begin{array}{lllllll}\text { cylinder } & 0 & 1 & 0.437515 & 88.06 & 0.00\end{array}$ $\begin{array}{llllll}\text { cylinder } & 2 & 1 & 0.49657 & 88.06 & 0.00\end{array}$ $\begin{array}{llllll}\text { cylinder } & 2 & 1 & 0.49657 & 88.06 & -1.27\end{array}$ cuboid 314 p.7112 $88.06-1.27$ unit 37

com='Fuel + Gap + Clad + Moderator' $\begin{array}{llllll}\text { cylinder } & 1 & 1 & 0.428498 & 88.06 & 0.00\end{array}$ $\begin{array}{llllllll}\text { cylinder } & 0 & 1 & 0.437515 & 88.06 & 0.00\end{array}$ $\begin{array}{lllllll}\text { cylinder } & 2 & 1 & 0.49657 & 88.06 & 0.00\end{array}$ $\begin{array}{llllll}\text { cylinder } & 2 & 1 & 0.49657 & 88.06 & -1.27\end{array}$ cuboid $3 \quad 1 \quad 4 p .7112 \quad 88.06-1.27$ unit 38

com='Fuel + Gap + Clad + Moderator $\begin{array}{lllllll}\text { cylinder } & 1 & 1 & 0.428498 & 88.06 & 0.00\end{array}$ $\begin{array}{lllllll}\text { cylinder } & 0 & 1 & 0.437515 & 88.06 & 0.00\end{array}$ $\begin{array}{llllllll}\text { cylinder } & 2 & 1 & 0.49657 & 88.06 & 0.00\end{array}$ $\begin{array}{llllll}\text { cylinder } & 2 & 1 & 0.49657 & 88.06 & -1.27\end{array}$ cuboid 314 p.7112 $88.06-1.27$ unit 39

com='Fuel + Gap + Clad + Moderator' $\begin{array}{llllll}\text { cylinder } & 1 & 1 & 0.428498 & 88.06 & 0.00\end{array}$ $\begin{array}{llllllll}\text { cylinder } & 0 & 1 & 0.437515 & 88.06 & 0.00\end{array}$ $\begin{array}{lllllll}\text { cylinder } & 2 & 1 & 0.49657 & 88.06 & 0.00\end{array}$ $\begin{array}{llllll}\text { cylinder } & 2 & 1 & 0.49657 & 88.06 & -1.27\end{array}$ cuboid 31 4p.7112 $88.06-1.27$ unit 40

com='Fuel + Gap + Clad + Moderator' $\begin{array}{lllllll}\text { cylinder } & 1 & 1 & 0.428498 & 88.06 & 0.00\end{array}$ $\begin{array}{lllllll}\text { cylinder } & 0 & 1 & 0.437515 & 88.06 & 0.00\end{array}$ $\begin{array}{lllllll}\text { cylinder } & 2 & 1 & 0.49657 & 88.06 & 0.00\end{array}$ $\begin{array}{llllll}\text { cylinder } & 2 & 1 & 0.49657 & 88.06 & -1.27\end{array}$ cuboid 314 p.7112 $88.06-1.27$ unit 41

com='Fuel + Gap + Clad + Moderator' $\begin{array}{lllllll}\text { cylinder } & 1 & 1 & 0.428498 & 88.06 & 0.00\end{array}$ $\begin{array}{lllllll}\text { cylinder } & 0 & 1 & 0.437515 & 88.06 & 0.00\end{array}$ $\begin{array}{lllllll}\text { cylinder } & 2 & 1 & 0.49657 & 88.06 & 0.00\end{array}$ $\begin{array}{llllll}\text { cylinder } & 2 & 1 & 0.49657 & 88.06 & -1.27\end{array}$ cuboid 31 4p.7112 $88.06-1.27$ unit 42

com='Fuel + Gap + Clad + Moderator' $\begin{array}{lllllll}\text { cylinder } & 1 & 1 & 0.428498 & 88.06 & 0.00\end{array}$ $\begin{array}{lllllll}\text { cylinder } & 0 & 1 & 0.437515 & 88.06 & 0.00\end{array}$ $\begin{array}{lllllll}\text { cylinder } & 2 & 1 & 0.49657 & 88.06 & 0.00\end{array}$ $\begin{array}{llllll}\text { cylinder } & 2 & 1 & 0.49657 & 88.06 & -1.27\end{array}$ cuboid $3 \quad 1 \quad 4$ p.7112 $88.06-1.27$ unit 43

com='Fuel + Gap + Clad + Moderator' $\begin{array}{lllllll}\text { cylinder } & 1 & 1 & 0.428498 & 88.06 & 0.00\end{array}$ $\begin{array}{lllllll}\text { cylinder } & 0 & 1 & 0.437515 & 88.06 & 0.00\end{array}$ $\begin{array}{lllllll}\text { cylinder } & 2 & 1 & 0.49657 & 88.06 & 0.00\end{array}$ $\begin{array}{llllll}\text { cylinder } & 2 & 1 & 0.49657 & 88.06 & -1.27\end{array}$ cuboid 314 p.7112 $88.06-1.27$ unit 44

com='Fuel + Gap + Clad + Moderator' $\begin{array}{lllllll}\text { cylinder } & 1 & 1 & 0.428498 & 88.06 & 0.00\end{array}$ $\begin{array}{lllllll}\text { cylinder } & 0 & 1 & 0.437515 & 88.06 & 0.00\end{array}$ $\begin{array}{lllllll}\text { cylinder } & 2 & 1 & 0.49657 & 88.06 & 0.00\end{array}$ 
$\begin{array}{llllll}\text { cylinder } & 2 & 1 & 0.49657 & 88.06 & -1.27\end{array}$

cuboid 31 4p.7112 $88.06-1.27$ unit 45

com='Fuel + Gap + Clad + Moderator' $\begin{array}{lllllll}\text { cylinder } & 1 & 1 & 0.428498 & 88.06 & 0.00\end{array}$ $\begin{array}{llllllll}\text { cylinder } & 0 & 1 & 0.437515 & 88.06 & 0.00\end{array}$ $\begin{array}{lllllll}\text { cylinder } & 2 & 1 & 0.49657 & 88.06 & 0.00\end{array}$ $\begin{array}{llllll}\text { cylinder } & 2 & 1 & 0.49657 & 88.06 & -1.27\end{array}$ cuboid $3 \quad 1$ 4p.7112 $88.06-1.27$ unit 46

com='Fuel + Gap + Clad + Moderator' $\begin{array}{lllllll}\text { cylinder } & 1 & 1 & 0.428498 & 88.06 & 0.00\end{array}$ $\begin{array}{lllllll}\text { cylinder } & 0 & 1 & 0.437515 & 88.06 & 0.00\end{array}$ $\begin{array}{lllllll}\text { cylinder } & 2 & 1 & 0.49657 & 88.06 & 0.00\end{array}$ $\begin{array}{llllll}\text { cylinder } & 2 & 1 & 0.49657 & 88.06 & -1.27\end{array}$ cuboid 314 p.7112 $88.06-1.27$ unit 47

com='Fuel + Gap + Clad + Moderator' $\begin{array}{lllllll}\text { cylinder } & 1 & 1 & 0.428498 & 88.06 & 0.00\end{array}$ $\begin{array}{lllllll}\text { cylinder } & 0 & 1 & 0.437515 & 88.06 & 0.00\end{array}$ $\begin{array}{lllllll}\text { cylinder } & 2 & 1 & 0.49657 & 88.06 & 0.00\end{array}$ $\begin{array}{llllll}\text { cylinder } & 2 & 1 & 0.49657 & 88.06 & -1.27\end{array}$ cuboid 31 4p.7112 $88.06-1.27$ unit 48

com='Fuel + Gap + Clad + Moderator' $\begin{array}{lllllll}\text { cylinder } & 1 & 1 & 0.428498 & 88.06 & 0.00\end{array}$ $\begin{array}{lllllll}\text { cylinder } & 0 & 1 & 0.437515 & 88.06 & 0.00\end{array}$ $\begin{array}{lllllll}\text { cylinder } & 2 & 1 & 0.49657 & 88.06 & 0.00\end{array}$ $\begin{array}{llllll}\text { cylinder } & 2 & 1 & 0.49657 & 88.06 & -1.27\end{array}$ cuboid $3 \begin{array}{llll}3 & 4 p .7112 \quad 88.06 & -1.27\end{array}$ unit 49

com='Fuel + Gap + Clad + Moderator $\begin{array}{lllllll}\text { cylinder } & 1 & 1 & 0.428498 & 88.06 & 0.00\end{array}$ $\begin{array}{llllllll}\text { cylinder } & 0 & 1 & 0.437515 & 88.06 & 0.00\end{array}$ $\begin{array}{lllllll}\text { cylinder } & 2 & 1 & 0.49657 & 88.06 & 0.00\end{array}$ $\begin{array}{llllll}\text { cylinder } & 2 & 1 & 0.49657 & 88.06 & -1.27\end{array}$ cuboid $3 \quad 1 \quad 4 p .7112 \quad 88.06-1.27$ unit 50

com='Fuel + Gap + Clad + Moderator' $\begin{array}{lllllll}\text { cylinder } & 1 & 1 & 0.428498 & 88.06 & 0.00\end{array}$ $\begin{array}{lllllll}\text { cylinder } & 0 & 1 & 0.437515 & 88.06 & 0.00\end{array}$ $\begin{array}{lllllll}\text { cylinder } & 2 & 1 & 0.49657 & 88.06 & 0.00\end{array}$ $\begin{array}{llllll}\text { cylinder } & 2 & 1 & 0.49657 & 88.06 & -1.27\end{array}$ cuboid 31 4p.7112 $88.06-1.27$ unit 51

com='Fuel + Gap + Clad + Moderator' $\begin{array}{llllll}\text { cylinder } & 1 & 1 & 0.428498 & 88.06 & 0.00\end{array}$ $\begin{array}{llllllll}\text { cylinder } & 0 & 1 & 0.437515 & 88.06 & 0.00\end{array}$ $\begin{array}{lllllll}\text { cylinder } & 2 & 1 & 0.49657 & 88.06 & 0.00\end{array}$ $\begin{array}{llllll}\text { cylinder } & 2 & 1 & 0.49657 & 88.06 & -1.27\end{array}$ cuboid 314 p.7112 $88.06-1.27$ unit 52

com='Fuel + Gap + Clad + Moderator' $\begin{array}{lllllll}\text { cylinder } & 1 & 1 & 0.428498 & 88.06 & 0.00\end{array}$ $\begin{array}{lllllll}\text { cylinder } & 0 & 1 & 0.437515 & 88.06 & 0.00\end{array}$ $\begin{array}{lllllll}\text { cylinder } & 2 & 1 & 0.49657 & 88.06 & 0.00\end{array}$ $\begin{array}{llllll}\text { cylinder } & 2 & 1 & 0.49657 & 88.06 & -1.27\end{array}$ cuboid $3 \quad 1 \quad 4 p .7112 \quad 88.06-1.27$ unit 53

com='Fuel + Gap + Clad + Moderator' $\begin{array}{lllllll}\text { cylinder } & 1 & 1 & 0.428498 & 88.06 & 0.00\end{array}$ $\begin{array}{lllllll}\text { cylinder } & 0 & 1 & 0.437515 & 88.06 & 0.00\end{array}$ $\begin{array}{lllllll}\text { cylinder } & 2 & 1 & 0.49657 & 88.06 & 0.00\end{array}$ $\begin{array}{llllll}\text { cylinder } & 2 & 1 & 0.49657 & 88.06 & -1.27\end{array}$ cuboid $3 \quad 1$ 4p.7112 88.06-1.27 unit 54

com='Fuel + Gap + Clad + Moderator' $\begin{array}{lllllll}\text { cylinder } & 1 & 1 & 0.428498 & 88.06 & 0.00\end{array}$ $\begin{array}{llllllll}\text { cylinder } & 0 & 1 & 0.437515 & 88.06 & 0.00\end{array}$ $\begin{array}{lllllll}\text { cylinder } & 2 & 1 & 0.49657 & 88.06 & 0.00\end{array}$ $\begin{array}{lllllll}\text { cylinder } & 2 & 1 & 0.49657 & 88.06 & -1.27\end{array}$ cuboid $3 \quad 1$ 4p.7112 88.06 -1.27 unit 55

com='Fuel + Gap + Clad + Moderator' $\begin{array}{lllllll}\text { cylinder } & 1 & 1 & 0.428498 & 88.06 & 0.00\end{array}$ $\begin{array}{llllllll}\text { cylinder } & 0 & 1 & 0.437515 & 88.06 & 0.00\end{array}$ $\begin{array}{lllllll}\text { cylinder } & 2 & 1 & 0.49657 & 88.06 & 0.00\end{array}$ $\begin{array}{llllll}\text { cylinder } & 2 & 1 & 0.49657 & 88.06 & -1.27\end{array}$ cuboid $3 \quad 1 \quad 4 p .7112 \quad 88.06-1.27$ unit 56

com='Fuel + Gap + Clad + Moderator $\begin{array}{lllllll}\text { cylinder } & 1 & 1 & 0.428498 & 88.06 & 0.00\end{array}$ $\begin{array}{llllllll}\text { cylinder } & 0 & 1 & 0.437515 & 88.06 & 0.00\end{array}$ $\begin{array}{lllllll}\text { cylinder } & 2 & 1 & 0.49657 & 88.06 & 0.00\end{array}$ $\begin{array}{llllll}\text { cylinder } & 2 & 1 & 0.49657 & 88.06 & -1.27\end{array}$ cuboid 314 p.7112 $88.06-1.27$ unit 57

com='Fuel + Gap + Clad + Moderator' $\begin{array}{lllllll}\text { cylinder } & 1 & 1 & 0.428498 & 88.06 & 0.00\end{array}$ $\begin{array}{llllllll}\text { cylinder } & 0 & 1 & 0.437515 & 88.06 & 0.00\end{array}$ $\begin{array}{lllllll}\text { cylinder } & 2 & 1 & 0.49657 & 88.06 & 0.00\end{array}$ $\begin{array}{llllll}\text { cylinder } & 2 & 1 & 0.49657 & 88.06 & -1.27\end{array}$ cuboid $3 \quad 1$ 4p.7112 $88.06-1.27$ unit 58

com='Fuel + Gap + Clad + Moderator $\begin{array}{lllllll}\text { cylinder } & 1 & 1 & 0.428498 & 88.06 & 0.00\end{array}$ $\begin{array}{lllllll}\text { cylinder } & 0 & 1 & 0.437515 & 88.06 & 0.00\end{array}$ $\begin{array}{lllllll}\text { cylinder } & 2 & 1 & 0.49657 & 88.06 & 0.00\end{array}$ $\begin{array}{llllll}\text { cylinder } & 2 & 1 & 0.49657 & 88.06 & -1.27\end{array}$ cuboid 3 1 4 4p.7112 88.06-1.27 unit 59

com='Fuel + Gap + Clad + Moderator' $\begin{array}{llllll}\text { cylinder } & 1 & 1 & 0.428498 & 88.06 & 0.00\end{array}$ $\begin{array}{llllllll}\text { cylinder } & 0 & 1 & 0.437515 & 88.06 & 0.00\end{array}$ $\begin{array}{llllllll}\text { cylinder } & 2 & 1 & 0.49657 & 88.06 & 0.00\end{array}$ $\begin{array}{llllll}\text { cylinder } & 2 & 1 & 0.49657 & 88.06 & -1.27\end{array}$ cuboid 314 p.7112 $88.06-1.27$ unit 60

com='Fuel + Gap + Clad + Moderator' $\begin{array}{lllllll}\text { cylinder } & 1 & 1 & 0.428498 & 88.06 & 0.00\end{array}$ $\begin{array}{lllllll}\text { cylinder } & 0 & 1 & 0.437515 & 88.06 & 0.00\end{array}$ $\begin{array}{lllllll}\text { cylinder } & 2 & 1 & 0.49657 & 88.06 & 0.00\end{array}$ $\begin{array}{llllll}\text { cylinder } & 2 & 1 & 0.49657 & 88.06 & -1.27\end{array}$ cuboid 31 4p.7112 $88.06-1.27$ 
unit 61

com='Fuel + Gap + Clad + Moderator' $\begin{array}{lllllll}\text { cylinder } & 1 & 1 & 0.428498 & 88.06 & 0.00\end{array}$ $\begin{array}{llllllll}\text { cylinder } & 0 & 1 & 0.437515 & 88.06 & 0.00\end{array}$ $\begin{array}{lllllll}\text { cylinder } & 2 & 1 & 0.49657 & 88.06 & 0.00\end{array}$ $\begin{array}{llllll}\text { cylinder } & 2 & 1 & 0.49657 & 88.06 & -1.27\end{array}$ cuboid $3 \quad 1 \quad 4$ p.7112 $88.06-1.27$ unit 62

com='Fuel + Gap + Clad + Moderator' $\begin{array}{lllllll}\text { cylinder } & 1 & 1 & 0.428498 & 88.06 & 0.00\end{array}$ $\begin{array}{lllllll}\text { cylinder } & 0 & 1 & 0.437515 & 88.06 & 0.00\end{array}$ $\begin{array}{lllllll}\text { cylinder } & 2 & 1 & 0.49657 & 88.06 & 0.00\end{array}$ $\begin{array}{llllll}\text { cylinder } & 2 & 1 & 0.49657 & 88.06 & -1.27\end{array}$ cuboid $3 \quad 1 \quad 4 p .7112 \quad 88.06-1.27$ unit 63

com='Fuel + Gap + Clad + Moderator' $\begin{array}{llllll}\text { cylinder } & 1 & 1 & 0.428498 & 88.06 & 0.00\end{array}$ $\begin{array}{llllllll}\text { cylinder } & 0 & 1 & 0.437515 & 88.06 & 0.00\end{array}$ $\begin{array}{lllllll}\text { cylinder } & 2 & 1 & 0.49657 & 88.06 & 0.00\end{array}$ $\begin{array}{llllll}\text { cylinder } & 2 & 1 & 0.49657 & 88.06 & -1.27\end{array}$ cuboid $3 \quad 1 \quad 4$ p.7112 $88.06-1.27$ unit 64

com='Fuel + Gap + Clad + Moderator' $\begin{array}{llllll}\text { cylinder } & 1 & 1 & 0.428498 & 88.06 & 0.00\end{array}$ $\begin{array}{lllllll}\text { cylinder } & 0 & 1 & 0.437515 & 88.06 & 0.00\end{array}$ $\begin{array}{lllllll}\text { cylinder } & 2 & 1 & 0.49657 & 88.06 & 0.00\end{array}$ $\begin{array}{llllll}\text { cylinder } & 2 & 1 & 0.49657 & 88.06 & -1.27\end{array}$ cuboid 3 1 4 p.7112 $88.06-1.27$ unit 65

com='Fuel + Gap + Clad + Moderator' $\begin{array}{llllll}\text { cylinder } & 1 & 1 & 0.428498 & 88.06 & 0.00\end{array}$ $\begin{array}{lllllll}\text { cylinder } & 0 & 1 & 0.437515 & 88.06 & 0.00\end{array}$ $\begin{array}{lllllll}\text { cylinder } & 2 & 1 & 0.49657 & 88.06 & 0.00\end{array}$ $\begin{array}{llllll}\text { cylinder } & 2 & 1 & 0.49657 & 88.06 & -1.27\end{array}$ cuboid 31 4p.7112 $88.06-1.27$ unit 66

com='Fuel + Gap + Clad + Moderator' $\begin{array}{lllllll}\text { cylinder } & 1 & 1 & 0.428498 & 88.06 & 0.00\end{array}$ $\begin{array}{lllllll}\text { cylinder } & 0 & 1 & 0.437515 & 88.06 & 0.00\end{array}$ $\begin{array}{lllllll}\text { cylinder } & 2 & 1 & 0.49657 & 88.06 & 0.00\end{array}$ $\begin{array}{llllll}\text { cylinder } & 2 & 1 & 0.49657 & 88.06 & -1.27\end{array}$ cuboid $3 \quad 1 \quad 4 p .7112 \quad 88.06-1.27$

unit 71

com='Clad + Mod + AL Grid'

$\begin{array}{lllll}\text { cylinder } 2 & 1 & 0.49657 & 0.635 & 0.0\end{array}$

$\begin{array}{llllll}\text { cylinder } & 3 & 1 & 0.50419 & 0.635 & 0.0\end{array}$

$\begin{array}{lllllll}\text { cuboid } 5 & 5 & 1 & 4 \text { p.7112 } & 0.635 & 0.0\end{array}$

unit 73

com='Fuel + Gap + Clad + Air'

$\begin{array}{llllll}\text { cylinder } & 1 & 1 & 0.428498 & 4.904 & 0.0\end{array}$

cylinder $\begin{array}{llllll}0 & 1 & 0.437515 & 4.904 & 0.0\end{array}$

$\begin{array}{llllll}\text { cylinder } 2 & 1 & 0.49657 & 4.904 & 0.0\end{array}$

$\begin{array}{lllllll}\text { cuboid } & 0 & 1 & 4 p .7112 & 4.904 & 0.0\end{array}$

unit 74
com='Top Clad + Void'

$\begin{array}{llllll}\text { cylinder } & 2 & 1 & 0.49657 & 1.016 & 0.0\end{array}$

cuboid $\begin{array}{lllllll}0 & 1 & 4 p .7112 & 1.016 & 0.0\end{array}$

unit 75

com='Top Clad + Void + AL grid'

cylinder $210.49657 \quad 1.27 \quad 0.0$

cylinder $010.50419 \quad 1.27 \quad 0.0$

cuboid 51 4p.7112 $1.27 \quad 0.0$

unit 81

com='array of Clad + Mod + AL Grid + Reflector'

array $1-14.9499-14.94990 .0$

cuboid $414 \mathrm{p} 44.94990 .6350 .0$

unit 82

com='array of Fuel + Gap + Clad + Moderator + Reflector' array $2-14.9499-14.9499-1.27$

cuboid $314 p 44.949988 .06-1.27$

unit 83

com='array of Fuel + Gap + Clad + Air'

array $3-14.9499-14.94990 .0$

cuboid $014 \mathrm{p} 44.94994 .9040 .0$

unit 84

com='array of Top Clad + Void'

array $4-14.9499-14.94990 .0$

cuboid $014 \mathrm{p} 44.94991 .0160 .0$

unit 85

com='array of Top Clad + Void + AL grid'

array $5-14.9499-14.94990 .0$

cuboid 41 4p44.9499 $1.27 \quad 0.0$

global unit 100

com='array of arrays of fuel element sections + reflector' array $6-44.9499-44.94990 .0$

$\begin{array}{llllll}\text { cuboid } 5 & 1 & 4 \mathrm{p} 44.9499 & 97.155 & -2.54\end{array}$

$\begin{array}{llllll}\text { cuboid } 3 & 1 & 4 p 44.9499 & 97.155 & -8.89\end{array}$

cuboid $5 \quad 1 \quad 4 p 44.9499 \quad 97.155 \quad-13.97$

cuboid $3 \quad 1 \quad 4 p 44.9499 \quad 97.155 \quad-43.97$

end geom

read array

ara=1 nux=21 nuy=21 nuz=1

fill $\mathrm{f} 7 \mathrm{1}$ end fill

ara $=2$ nux $=21$ nuy=21 nuz=1

fill

$\begin{array}{llllllllll}1 & 2 & 3 & 4 & 5 & 6 & 8 & 9 & 1011 & 1 b \\ 10\end{array}$

$212131415161718192021 \quad 1 b 10$

313222324252627282930 1b 10

414233132333435363738 1b 10

515243239404142434445 1b 10

616253340464748495051 1b 10

717263441475253545556 1b 10

818273542485357585960 1b 10 
919283643495458616263 1b 10 $1020293744505559626465 \quad 1 b 10$ 1121303845515660636566 1b 220 end fill

ara $=3$ nux $=21$ nuy=21 nuz=1 fill $\mathrm{f} 73$ end fill

ara $=4$ nux $=21$ nuy $=21$ nuz $=1$ fill $\mathrm{f} 74$ end fill

ara=5 nux=21 nuy=21 nuz=1 fill f75 end fill

ara $=6$ nux $=1$ nuy $=1$ nuz $=5$

fill 8182838485 end fill

end array

read plot

$\mathrm{ttl}=$ 'simple plot 1

lpi $=10 \mathrm{scr}=$ yes pic $=\operatorname{mix}$ nax $=400$

$\mathrm{xul}=-20.0$ yul $=0.0 \mathrm{zul}=100$

$\mathrm{xlr}=20.0 \quad \mathrm{ylr}=0.0 \quad \mathrm{zlr}=-10$

uax $=1.0 \mathrm{wdn}=-1.0$ end plt0

$\mathrm{ttl}=$ 'simple plot $2^{\prime}$

pic $=$ mix nax $=400$

$\mathrm{xul}=-20.0$ yul $=20 . \quad \mathrm{zul}=25$.

$\mathrm{xlr}=20.0 \quad \mathrm{ylr}=-20 . \quad \mathrm{zlr}=25$.

uax $=1.0 \mathrm{vdn}=-1.0$ end plt 1

end plot

end data

end

$=$ shell

cp ft04f001 \$RTNDIR/sax03.xs04

cp ft64f001 \$RTNDIR/sax03.rs64

end

$=\mathrm{kmart}$

read initial kunit $=64$

xunit $=4$

actbygrp

rrpvol

end initial

read activity

92234279223418922341452

92235279223518922351452

$9223827922381892238 \quad 1452$

94239279423918942391452

94240279424018942401452

94241279424118942411452

$9424227942421894242 \quad 1452$

95241279524118952411452

501027

501127

801627

4000027

2400027

2600027

100127

5011227

5011427

5011527

5011627
5011727

5011827

5011927

5012027

5012227

5012427

end activity

read collapse

factor $=1.0$

lastg $=57$

lastg $=148$

last $\mathrm{g}=204$

lastg $=238$

end collapse

end

$=\operatorname{csasc} 5 \quad$ parm $=$ size $=1000000$

saxton 4 fuel

238group latticecell

'MOX fuel

pu-239 10 1.3526-3 297 end

pu-240 $11001.2759-4297$ end

pu-241 110 1.1407-5 297 end

pu-242 $11006.0318-7297$ end

am-241 $11001.7783-6297$ end

u-234 $1001.1688-6297$ end

u-235 $11001.5301-4297$ end

u-238 110 2.1097-2 297 end

o $104.5155-2297$ end 'clad (ZR-2)

zr $\quad 2 \quad 0 \quad 4.2517-2297$ end

sn $\quad 2 \quad 0 \quad 4.6590-4297$ end

cr $\quad 2 \quad 0 \quad 7.5977-5297$ end

fe $\quad \begin{array}{llll}2 & 0 & 1.4148-4 & 297 \\ \text { end }\end{array}$

o $202.9630-4297$ end 'water(Refletor)

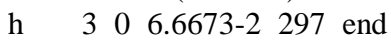

o 30 3.3336-2 297 end

'b-10 $3003.7338-6297$ end

'b-11 $3001.5029-5297$ end

'Middle Grid and H2O

\begin{tabular}{cccc} 
al & 4.93 & 297 & end \\
h2o & 4.07 & 297 & end \\
\multicolumn{4}{c}{ 'Aluminum }
\end{tabular}

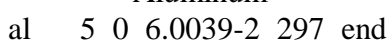
end comp

$\begin{array}{lllllllll}\text { squarepitch } & 1.8679 & 856996 & 1 & 2.99314 & 3 & 87503 & 0 & \text { end }\end{array}$ more data

limcen $=20000000$

end more

saxton 4

read param

gen=225 nsk=25 npg=20000 fdn=yes flx=yes wrs=64

$$
\text { res }=225
$$

lng $=2000000$

end param 
read geometry

unit 1

com='Fuel + Gap + Clad + Moderator' $\begin{array}{lllllll}\text { cylinder } & 1 & 1 & 0.428498 & 68.41 & 0.00\end{array}$ $\begin{array}{llllllll}\text { cylinder } & 0 & 1 & 0.437515 & 68.41 & 0.00\end{array}$ $\begin{array}{lllllll}\text { cylinder } & 2 & 1 & 0.49657 & 68.41 & 0.00\end{array}$ $\begin{array}{llllll}\text { cylinder } & 2 & 1 & 0.49657 & 68.41 & -1.27\end{array}$ cuboid $\quad 3 \quad 1 \quad 4 p .93395 \quad 68.41-1.27$ unit 2

com='Fuel + Gap + Clad + Moderator' $\begin{array}{lllllll}\text { cylinder } & 1 & 1 & 0.428498 & 68.41 & 0.00\end{array}$ $\begin{array}{lllllll}\text { cylinder } & 0 & 1 & 0.437515 & 68.41 & 0.00\end{array}$ $\begin{array}{llllllll}\text { cylinder } & 2 & 1 & 0.49657 & 68.41 & 0.00\end{array}$ $\begin{array}{lllllll}\text { cylinder } & 2 & 1 & 0.49657 & 68.41 & -1.27\end{array}$ cuboid $3114 p .93395 \quad 68.41-1.27$ unit 3

com='Fuel + Gap + Clad + Moderator' $\begin{array}{lllllll}\text { cylinder } & 1 & 1 & 0.428498 & 68.41 & 0.00\end{array}$ $\begin{array}{lllllll}\text { cylinder } & 0 & 1 & 0.437515 & 68.41 & 0.00\end{array}$ $\begin{array}{lllllll}\text { cylinder } & 2 & 1 & 0.49657 & 68.41 & 0.00\end{array}$ $\begin{array}{llllll}\text { cylinder } & 2 & 1 & 0.49657 & 68.41 & -1.27\end{array}$ cuboid $3 \quad 1 \quad 4 p .93395 \quad 68.41-1.27$ unit 4

com='Fuel + Gap + Clad + Moderator' $\begin{array}{lllllll}\text { cylinder } & 1 & 1 & 0.428498 & 68.41 & 0.00\end{array}$ $\begin{array}{lllllll}\text { cylinder } & 0 & 1 & 0.437515 & 68.41 & 0.00\end{array}$ $\begin{array}{lllllll}\text { cylinder } & 2 & 1 & 0.49657 & 68.41 & 0.00\end{array}$ $\begin{array}{llllll}\text { cylinder } & 2 & 1 & 0.49657 & 68.41 & -1.27\end{array}$ cuboid $\quad 3 \quad 1 \quad 4 p .93395 \quad 68.41-1.27$ unit 5

com='Fuel + Gap + Clad + Moderator' $\begin{array}{lllllll}\text { cylinder } & 1 & 1 & 0.428498 & 68.41 & 0.00\end{array}$ $\begin{array}{llllllll}\text { cylinder } & 0 & 1 & 0.437515 & 68.41 & 0.00\end{array}$ $\begin{array}{lllllll}\text { cylinder } & 2 & 1 & 0.49657 & 68.41 & 0.00\end{array}$ $\begin{array}{llllll}\text { cylinder } & 2 & 1 & 0.49657 & 68.41 & -1.27\end{array}$ cuboid $3 \quad 11$ 4p.93395 $68.41-1.27$ unit 6

com='Fuel + Gap + Clad + Moderator' $\begin{array}{llllll}\text { cylinder } & 1 & 1 & 0.428498 & 68.41 & 0.00\end{array}$ $\begin{array}{llllllll}\text { cylinder } & 0 & 1 & 0.437515 & 68.41 & 0.00\end{array}$ $\begin{array}{lllllll}\text { cylinder } & 2 & 1 & 0.49657 & 68.41 & 0.00\end{array}$ $\begin{array}{llllll}\text { cylinder } & 2 & 1 & 0.49657 & 68.41 & -1.27\end{array}$ cuboid $3 \quad 1 \quad 4 p .93395 \quad 68.41-1.27$ unit 7

com='Fuel + Gap + Clad + Moderator' $\begin{array}{lllllll}\text { cylinder } & 1 & 1 & 0.428498 & 68.41 & 0.00\end{array}$ $\begin{array}{lllllll}\text { cylinder } & 0 & 1 & 0.437515 & 68.41 & 0.00\end{array}$ $\begin{array}{lllllll}\text { cylinder } & 2 & 1 & 0.49657 & 68.41 & 0.00\end{array}$ $\begin{array}{llllll}\text { cylinder } & 2 & 1 & 0.49657 & 68.41 & -1.27\end{array}$ cuboid $3 \quad 1 \quad 4 p .93395 \quad 68.41-1.27$ unit 8

com='Fuel + Gap + Clad + Moderator' $\begin{array}{llllll}\text { cylinder } & 1 & 1 & 0.428498 & 68.41 & 0.00\end{array}$ $\begin{array}{lllllll}\text { cylinder } & 0 & 1 & 0.437515 & 68.41 & 0.00\end{array}$ $\begin{array}{lllllll}\text { cylinder } & 2 & 1 & 0.49657 & 68.41 & 0.00\end{array}$ $\begin{array}{llllll}\text { cylinder } & 2 & 1 & 0.49657 & 68.41 & -1.27\end{array}$ cuboid $\quad 3 \quad 1 \quad 4 p .93395 \quad 68.41-1.27$ unit 9

com='Fuel + Gap + Clad + Moderator' $\begin{array}{lllllll}\text { cylinder } & 1 & 1 & 0.428498 & 68.41 & 0.00\end{array}$ $\begin{array}{lllllll}\text { cylinder } & 0 & 1 & 0.437515 & 68.41 & 0.00\end{array}$ $\begin{array}{lllllll}\text { cylinder } & 2 & 1 & 0.49657 & 68.41 & 0.00\end{array}$ $\begin{array}{llllll}\text { cylinder } & 2 & 1 & 0.49657 & 68.41 & -1.27\end{array}$ cuboid $\quad 3 \quad 1 \quad 4 p .93395 \quad 68.41-1.27$ unit 10

com='Fuel + Gap + Clad + Moderator' $\begin{array}{lllllll}\text { cylinder } & 1 & 1 & 0.428498 & 68.41 & 0.00\end{array}$ $\begin{array}{lllllll}\text { cylinder } & 0 & 1 & 0.437515 & 68.41 & 0.00\end{array}$ $\begin{array}{lllllll}\text { cylinder } & 2 & 1 & 0.49657 & 68.41 & 0.00\end{array}$ $\begin{array}{lllllll}\text { cylinder } & 2 & 1 & 0.49657 & 68.41 & -1.27\end{array}$ $\begin{array}{lllllll}\text { cuboid } \quad 3 & 1 & 4 p .93395 & 68.41 & -1.27\end{array}$ unit 11

com='Fuel + Gap + Clad + Moderator' $\begin{array}{lllllll}\text { cylinder } & 1 & 1 & 0.428498 & 68.41 & 0.00\end{array}$ $\begin{array}{lllllll}\text { cylinder } & 0 & 1 & 0.437515 & 68.41 & 0.00\end{array}$ $\begin{array}{lllllll}\text { cylinder } & 2 & 1 & 0.49657 & 68.41 & 0.00\end{array}$ $\begin{array}{llllll}\text { cylinder } & 2 & 1 & 0.49657 & 68.41 & -1.27\end{array}$ cuboid $\quad \begin{array}{llllll}3 & 1 & 4 p .93395 & 68.41 & -1.27\end{array}$ unit 12

com='Fuel + Gap + Clad + Moderator' $\begin{array}{lllllll}\text { cylinder } & 1 & 1 & 0.428498 & 68.41 & 0.00\end{array}$ $\begin{array}{llllllll}\text { cylinder } & 0 & 1 & 0.437515 & 68.41 & 0.00\end{array}$ $\begin{array}{lllllll}\text { cylinder } & 2 & 1 & 0.49657 & 68.41 & 0.00\end{array}$ $\begin{array}{llllll}\text { cylinder } & 2 & 1 & 0.49657 & 68.41 & -1.27\end{array}$ cuboid $\quad \begin{array}{llllll}3 & 1 & 4 p .93395 & 68.41 & -1.27\end{array}$ unit 13

com='Fuel + Gap + Clad + Moderator' $\begin{array}{llllll}\text { cylinder } & 1 & 1 & 0.428498 & 68.41 & 0.00\end{array}$ $\begin{array}{lllllll}\text { cylinder } & 0 & 1 & 0.437515 & 68.41 & 0.00\end{array}$ $\begin{array}{lllllll}\text { cylinder } & 2 & 1 & 0.49657 & 68.41 & 0.00\end{array}$ $\begin{array}{llllll}\text { cylinder } & 2 & 1 & 0.49657 & 68.41 & -1.27\end{array}$ cuboid $3 \quad 1 \quad 4 p .93395 \quad 68.41-1.27$ unit 14

com='Fuel + Gap + Clad + Moderator' $\begin{array}{lllllll}\text { cylinder } & 1 & 1 & 0.428498 & 68.41 & 0.00\end{array}$ $\begin{array}{lllllll}\text { cylinder } & 0 & 1 & 0.437515 & 68.41 & 0.00\end{array}$ $\begin{array}{lllllll}\text { cylinder } & 2 & 1 & 0.49657 & 68.41 & 0.00\end{array}$ $\begin{array}{llllll}\text { cylinder } & 2 & 1 & 0.49657 & 68.41 & -1.27\end{array}$ cuboid $3 \quad 1 \quad 4 p .93395 \quad 68.41-1.27$ unit 15

com='Fuel + Gap + Clad + Moderator' $\begin{array}{lllllll}\text { cylinder } & 1 & 1 & 0.428498 & 68.41 & 0.00\end{array}$ $\begin{array}{lllllll}\text { cylinder } & 0 & 1 & 0.437515 & 68.41 & 0.00\end{array}$ $\begin{array}{llllllll}\text { cylinder } & 2 & 1 & 0.49657 & 68.41 & 0.00\end{array}$ $\begin{array}{llllll}\text { cylinder } & 2 & 1 & 0.49657 & 68.41 & -1.27\end{array}$ cuboid $3 \quad 1 \quad 4 p .93395 \quad 68.41-1.27$ unit 16

com='Fuel + Gap + Clad + Moderator' $\begin{array}{lllllll}\text { cylinder } & 1 & 1 & 0.428498 & 68.41 & 0.00\end{array}$ $\begin{array}{lllllll}\text { cylinder } & 0 & 1 & 0.437515 & 68.41 & 0.00\end{array}$ $\begin{array}{lllllll}\text { cylinder } & 2 & 1 & 0.49657 & 68.41 & 0.00\end{array}$ $\begin{array}{lllllll}\text { cylinder } & 2 & 1 & 0.49657 & 68.41 & -1.27\end{array}$ cuboid $3 \quad 1 \quad 4 p .93395 \quad 68.41-1.27$ 
unit 17

com='Fuel + Gap + Clad + Moderator' $\begin{array}{lllllll}\text { cylinder } & 1 & 1 & 0.428498 & 68.41 & 0.00\end{array}$ $\begin{array}{llllllll}\text { cylinder } & 0 & 1 & 0.437515 & 68.41 & 0.00\end{array}$ $\begin{array}{lllllll}\text { cylinder } & 2 & 1 & 0.49657 & 68.41 & 0.00\end{array}$ $\begin{array}{llllll}\text { cylinder } & 2 & 1 & 0.49657 & 68.41 & -1.27\end{array}$ cuboid $\quad 3 \quad 1 \quad 4 p .93395 \quad 68.41-1.27$ unit 18

com='Fuel + Gap + Clad + Moderator' $\begin{array}{lllllll}\text { cylinder } & 1 & 1 & 0.428498 & 68.41 & 0.00\end{array}$ $\begin{array}{lllllll}\text { cylinder } & 0 & 1 & 0.437515 & 68.41 & 0.00\end{array}$ $\begin{array}{lllllll}\text { cylinder } & 2 & 1 & 0.49657 & 68.41 & 0.00\end{array}$ $\begin{array}{llllll}\text { cylinder } & 2 & 1 & 0.49657 & 68.41 & -1.27\end{array}$ cuboid $3 \quad 1 \quad 4 p .93395 \quad 68.41-1.27$ unit 19

com='Fuel + Gap + Clad + Moderator' $\begin{array}{lllllll}\text { cylinder } & 1 & 1 & 0.428498 & 68.41 & 0.00\end{array}$ $\begin{array}{lllllll}\text { cylinder } & 0 & 1 & 0.437515 & 68.41 & 0.00\end{array}$ $\begin{array}{lllllll}\text { cylinder } & 2 & 1 & 0.49657 & 68.41 & 0.00\end{array}$ $\begin{array}{lllllll}\text { cylinder } & 2 & 1 & 0.49657 & 68.41 & -1.27\end{array}$ cuboid $3 \quad 1 \quad 4 p .93395 \quad 68.41-1.27$ unit 20

com='Fuel + Gap + Clad + Moderator' $\begin{array}{llllll}\text { cylinder } & 1 & 1 & 0.428498 & 68.41 & 0.00\end{array}$ $\begin{array}{lllllll}\text { cylinder } & 0 & 1 & 0.437515 & 68.41 & 0.00\end{array}$ $\begin{array}{lllllll}\text { cylinder } & 2 & 1 & 0.49657 & 68.41 & 0.00\end{array}$ $\begin{array}{llllll}\text { cylinder } & 2 & 1 & 0.49657 & 68.41 & -1.27\end{array}$ cuboid $\quad 3 \quad 1 \quad 4 p .93395 \quad 68.41-1.27$ unit 21

com='Fuel + Gap + Clad + Moderator' $\begin{array}{lllllll}\text { cylinder } & 1 & 1 & 0.428498 & 68.41 & 0.00\end{array}$ $\begin{array}{llllllll}\text { cylinder } & 0 & 1 & 0.437515 & 68.41 & 0.00\end{array}$ $\begin{array}{lllllll}\text { cylinder } & 2 & 1 & 0.49657 & 68.41 & 0.00\end{array}$ $\begin{array}{llllll}\text { cylinder } & 2 & 1 & 0.49657 & 68.41 & -1.27\end{array}$ cuboid $\quad 3 \quad 1 \quad 4 p .93395 \quad 68.41-1.27$ unit 22

com='Fuel + Gap + Clad + Moderator' $\begin{array}{lllllll}\text { cylinder } & 1 & 1 & 0.428498 & 68.41 & 0.00\end{array}$ $\begin{array}{lllllll}\text { cylinder } & 0 & 1 & 0.437515 & 68.41 & 0.00\end{array}$ $\begin{array}{lllllll}\text { cylinder } & 2 & 1 & 0.49657 & 68.41 & 0.00\end{array}$ $\begin{array}{llllll}\text { cylinder } & 2 & 1 & 0.49657 & 68.41 & -1.27\end{array}$ cuboid 3 1 4 p.93395 $68.41-1.27$ unit 23

com='Fuel + Gap + Clad + Moderator' $\begin{array}{lllllll}\text { cylinder } & 1 & 1 & 0.428498 & 68.41 & 0.00\end{array}$ $\begin{array}{lllllll}\text { cylinder } & 0 & 1 & 0.437515 & 68.41 & 0.00\end{array}$ $\begin{array}{lllllll}\text { cylinder } & 2 & 1 & 0.49657 & 68.41 & 0.00\end{array}$ $\begin{array}{lllllll}\text { cylinder } & 2 & 1 & 0.49657 & 68.41 & -1.27\end{array}$ cuboid $3 \quad 1 \quad 4 p .93395 \quad 68.41-1.27$ unit 24

com='Fuel + Gap + Clad + Moderator' $\begin{array}{lllllll}\text { cylinder } & 1 & 1 & 0.428498 & 68.41 & 0.00\end{array}$ $\begin{array}{lllllll}\text { cylinder } & 0 & 1 & 0.437515 & 68.41 & 0.00\end{array}$ $\begin{array}{lllllll}\text { cylinder } & 2 & 1 & 0.49657 & 68.41 & 0.00\end{array}$ $\begin{array}{llllll}\text { cylinder } & 2 & 1 & 0.49657 & 68.41 & -1.27\end{array}$ cuboid $3 \quad 1 \quad 4 p .93395 \quad 68.41-1.27$ unit 25
com='Fuel + Gap + Clad + Moderator' $\begin{array}{lllllll}\text { cylinder } & 1 & 1 & 0.428498 & 68.41 & 0.00\end{array}$ $\begin{array}{lllllll}\text { cylinder } & 0 & 1 & 0.437515 & 68.41 & 0.00\end{array}$ $\begin{array}{lllllll}\text { cylinder } & 2 & 1 & 0.49657 & 68.41 & 0.00\end{array}$ $\begin{array}{llllll}\text { cylinder } & 2 & 1 & 0.49657 & 68.41 & -1.27\end{array}$ cuboid $3 \quad 1 \quad 4 p .93395 \quad 68.41-1.27$ unit 26

com='Fuel + Gap + Clad + Moderator' $\begin{array}{llllll}\text { cylinder } & 1 & 1 & 0.428498 & 68.41 & 0.00\end{array}$ $\begin{array}{lllllll}\text { cylinder } & 0 & 1 & 0.437515 & 68.41 & 0.00\end{array}$ $\begin{array}{lllllll}\text { cylinder } & 2 & 1 & 0.49657 & 68.41 & 0.00\end{array}$ $\begin{array}{llllll}\text { cylinder } & 2 & 1 & 0.49657 & 68.41 & -1.27\end{array}$ cuboid $\quad 3 \quad 1 \quad 4 p .93395 \quad 68.41-1.27$ unit 27

com='Fuel + Gap + Clad + Moderator' $\begin{array}{lllllll}\text { cylinder } & 1 & 1 & 0.428498 & 68.41 & 0.00\end{array}$ $\begin{array}{lllllll}\text { cylinder } & 0 & 1 & 0.437515 & 68.41 & 0.00\end{array}$ $\begin{array}{lllllll}\text { cylinder } & 2 & 1 & 0.49657 & 68.41 & 0.00\end{array}$ $\begin{array}{llllll}\text { cylinder } & 2 & 1 & 0.49657 & 68.41 & -1.27\end{array}$ cuboid $\quad 3 \quad 1 \quad 4 p .93395 \quad 68.41-1.27$ unit 28

com='Fuel + Gap + Clad + Moderator' $\begin{array}{lllllll}\text { cylinder } & 1 & 1 & 0.428498 & 68.41 & 0.00\end{array}$ $\begin{array}{lllllll}\text { cylinder } & 0 & 1 & 0.437515 & 68.41 & 0.00\end{array}$ $\begin{array}{lllllll}\text { cylinder } & 2 & 1 & 0.49657 & 68.41 & 0.00\end{array}$ $\begin{array}{lllllll}\text { cylinder } & 2 & 1 & 0.49657 & 68.41 & -1.27\end{array}$ cuboid $\quad 3 \quad 1 \quad 4 p .93395 \quad 68.41-1.27$

unit 71

com='Clad + Mod + AL Grid'

$\begin{array}{lllll}\text { cylinder } 2 & 1 & 0.49657 & 0.635 & 0.0\end{array}$

$\begin{array}{llllll}\text { cylinder } 3 & 1 & 0.50419 & 0.635 & 0.0\end{array}$

$\begin{array}{lllllll}\text { cuboid } 5 & 5 & 4 p .93395 & 0.635 & 0.0\end{array}$

unit 73

com='Fuel + Gap + Clad + Air' $\begin{array}{llllll}\text { cylinder } & 1 & 1 & 0.428498 & 24.554 & 0.0\end{array}$ $\begin{array}{lllllll}\text { cylinder } & 0 & 1 & 0.437515 & 24.554 & 0.0\end{array}$ $\begin{array}{llllll}\text { cylinder } 2 & 1 & 0.49657 & 24.554 & 0.0\end{array}$ cuboid $\begin{array}{lllllll}0 & 1 & 4 p .93395 & 24.554 & 0.0\end{array}$

unit 74 com='Top Clad + Void' $\begin{array}{llllll}\text { cylinder } 2 & 1 & 0.49657 & 1.016 & 0.0\end{array}$ cuboid $\quad \begin{array}{lllllll}0 & 1 & 4 p .93395 & 1.016 & 0.0\end{array}$

unit 75

com='Top Clad + Void + AL grid' cylinder $210.49657 \quad 1.27 \quad 0.0$ cylinder $\begin{array}{lllll}0 & 1 & 0.50419 & 1.27 & 0.0\end{array}$ cuboid 51 4p.93395 1.270 .0

unit 81

com='array of Clad + Mod + AL Grid + Reflector' array $1-12.14135-12.141350 .0$ cuboid 41 4p42.14135 $0.635 \quad 0.0$ 
unit 82

com='array of Fuel + Gap + Clad + Moderator + Reflector' array $2-12.14135-12.14135-1.27$

cuboid $314 \mathrm{p} 42.14135 \quad 68.41 \quad-1.27$

unit 83

com='array of Fuel + Gap + Clad + Air'

array $3-12.14135-12.141350 .0$

cuboid $014 \mathrm{p} 42.1413524 .554 \quad 0.0$

unit 84

com='array of Top Clad + Void'

array $4-12.14135-12.141350 .0$

cuboid $014 \mathrm{p} 42.141351 .0160 .0$

unit 85

com='array of Top Clad + Void + AL grid' array $5-12.14135-12.141350 .0$

cuboid 41 4p42.14135 $1.27 \quad 0.0$

global unit 100

com='array of arrays of fuel element sections + reflector' array $6-42.14135-42.141350 .0$

$\begin{array}{llllll}\text { cuboid } 0 & 1 & 4 p 42.14135 & 97.15501 & 0.00\end{array}$

cuboid $5 \quad 1 \quad 4 p 42.1413597 .15501 \quad-2.54$

cuboid $3 \quad 1 \quad 4 p 42.1413597 .15501 \quad-8.89$

cuboid $5 \begin{array}{llllll} & 1 & 4 p 42.14135 & 97.15501 & -13.97\end{array}$

cuboid $3 \quad 1 \quad 4 p 42.14135 \quad 97.15501 \quad-43.97$

end geom

read array

ara $=1$ nux $=13$ nuy=13 nuz=1

fill $\mathrm{f} 71$ end fill

ara $=2$ nux $=13$ nuy=13 nuz=1 fill

$\begin{array}{llllllll}1 & 2 & 3 & 4 & 5 & 6 & 7 & 1 \mathrm{~b} 6\end{array}$

$\begin{array}{llllllll}2 & 8 & 9 & 10 & 11 & 12 & 13 & 1 \mathrm{~b} 6\end{array}$

$391415161718 \quad 1 \mathrm{~b} 6$

$4101519202122 \quad 1 b 6$

51116202324251 b6

$6121721242627 \quad 1 b 6$

$7131822252728 \quad 1 b 84$ end fill

ara $=3$ nux $=13$ nuy=13 nuz=1 fill $\mathrm{f} 73$ end fill

ara=4 nux=13 nuy=13 nuz=1 fill $\mathrm{f} 74$ end fill

ara $=5$ nux $=13$ nuy $=13$ nuz=1 fill $\mathrm{f} 75$ end fill

ara $=6$ nux=1 nuy=1 nuz=5

fill 8182838485 end fill

end array

read plot

$\mathrm{ttl}=$ 'simple plot 1 '

lpi $=10 \mathrm{scr}=$ yes pic $=\operatorname{mix}$ nax $=400$

$\mathrm{xul}=-20.0 \mathrm{yul}=0.0 \mathrm{zul}=100$

$\mathrm{xlr}=20.0 \mathrm{ylr}=0.0 \mathrm{zlr}=-10$

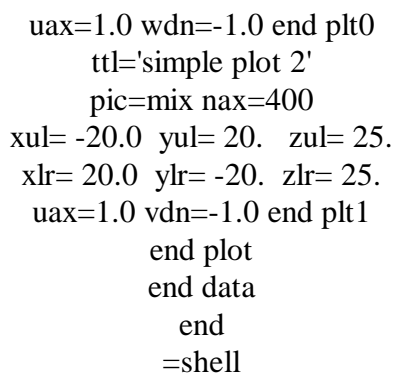

cp ft04f001 \$RTNDIR/sax04.xs04 cp ft64f001 \$RTNDIR/sax04.rs64 end $=\mathrm{kmart}$

read initial kunit $=64$ xunit $=4$ actbygrp rrpvol

end initial read activity

92234279223418922341452 92235279223518922351452 92238279223818922381452 94239279423918942391452 94240279424018942401452 $9424127942411894241 \quad 1452$ 94242279424218942421452 95241279524118952411452 501027

501127

801627

4000027

2400027

2600027

100127

5011227

5011427

5011527

5011627

5011727

5011827

5011927

5012027

5012227

5012427

end activity

read collapse

factor $=1.0$

lastg $=57$

lastg $=148$

lastg $=204$

lastg $=238$

end collapse

end

$=\operatorname{csasc} 5 \quad$ parm $=$ size $=1000000$ 
saxton 5 fuel

238group latticecell

'MOX fuel

pu-239 100 1.3526-3 289 end pu-240 110 1.2759-4 289 end pu-241 $11001.1407-5289$ end pu-242 $1106.0318-7289$ end am-241 $11001.7783-6289$ end u-234 $100 \quad 1.1688-6 \quad 289$ end u-235 $11001.5301-4 \quad 289$ end u-238 $11002.1097-2 \quad 289$ end

o $1 \quad 0 \quad 4.5155-2289$ end 'clad (ZR-2)

zr $\quad 2 \quad 0 \quad 4.2517-2 \quad 289$ end

sn $\quad 2 \quad 0 \quad 4.6590-4298$ end

cr $\quad 2 \quad 0 \quad 7.5977-5289$ end

fe $\quad \begin{array}{llll}2 & 0 & 1.4148-4 & 289 \\ & \text { end }\end{array}$

o $\quad 2 \quad 0 \quad 2.9630-4289$ end 'water(Refletor)

h $\quad 3 \quad 0 \quad 6.6783-2 \quad 289$ end

o 30 3.3392-2 289 end

'Middle Grid and $\mathrm{H} 2 \mathrm{O}$

al $\quad 4.942 \quad 289$ end

h2o $\quad 4 \quad .058 \quad 289$ end

'Aluminum

al $\quad \begin{array}{llll}5 & 0 & 6.0039-2 & 289\end{array}$ end end comp

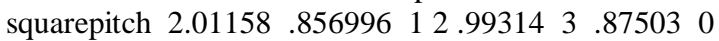
end

more data

limcen $=20000000$

end more

saxton 5

read param

gen=225 nsk=25 npg=20000 fdn=yes flx=yes wrs=64 res $=225$

$\operatorname{lng}=2000000$

end param

read geometry

unit 1

com='Fuel + Gap + Clad + Moderator' $\begin{array}{lllllll}\text { cylinder } & 1 & 1 & 0.428498 & 76.76 & 0.00\end{array}$

$\begin{array}{lllllll}\text { cylinder } & 0 & 1 & 0.437515 & 76.76 & 0.00\end{array}$

$\begin{array}{lllllll}\text { cylinder } & 2 & 1 & 0.49657 & 76.76 & 0.00\end{array}$

$\begin{array}{llllll}\text { cylinder } & 2 & 1 & 0.49657 & 76.76 & -1.27\end{array}$

cuboid $314 \mathrm{p} 1.00579 \quad 76.76-1.27$ unit 2

com='Fuel + Gap + Clad + Moderator' $\begin{array}{lllllll}\text { cylinder } & 1 & 1 & 0.428498 & 76.76 & 0.00\end{array}$ $\begin{array}{lllllll}\text { cylinder } & 0 & 1 & 0.437515 & 76.76 & 0.00\end{array}$ $\begin{array}{lllllll}\text { cylinder } & 2 & 1 & 0.49657 & 76.76 & 0.00\end{array}$ $\begin{array}{lllll}\text { cylinder } & 2 & 1 & 0.49657 & 76.76-1.27\end{array}$ cuboid 314 p1.00579 $76.76-1.27$ unit 3

com='Fuel + Gap + Clad + Moderator' $\begin{array}{lllllll}\text { cylinder } & 1 & 1 & 0.428498 & 76.76 & 0.00\end{array}$ $\begin{array}{lllllll}\text { cylinder } & 0 & 1 & 0.437515 & 76.76 & 0.00\end{array}$ $\begin{array}{lllllll}\text { cylinder } & 2 & 1 & 0.49657 & 76.76 & 0.00\end{array}$ $\begin{array}{llllll}\text { cylinder } & 2 & 1 & 0.49657 & 76.76 & -1.27\end{array}$ cuboid $314 \mathrm{p} 1.0057976 .76-1.27$ unit 4

com='Fuel + Gap + Clad + Moderator' $\begin{array}{lllllll}\text { cylinder } & 1 & 1 & 0.428498 & 76.76 & 0.00\end{array}$ $\begin{array}{lllllll}\text { cylinder } & 0 & 1 & 0.437515 & 76.76 & 0.00\end{array}$ $\begin{array}{lllllll}\text { cylinder } & 2 & 1 & 0.49657 & 76.76 & 0.00\end{array}$ $\begin{array}{llllll}\text { cylinder } & 2 & 1 & 0.49657 & 76.76 & -1.27\end{array}$ cuboid $3 \quad 1 \quad 4 p 1.0057976 .76-1.27$ unit 5

com='Fuel + Gap + Clad + Moderator $\begin{array}{lllllll}\text { cylinder } & 1 & 1 & 0.428498 & 76.76 & 0.00\end{array}$ $\begin{array}{lllllllll}\text { cylinder } & 0 & 1 & 0.437515 & 76.76 & 0.00\end{array}$ $\begin{array}{lllllll}\text { cylinder } & 2 & 1 & 0.49657 & 76.76 & 0.00\end{array}$ $\begin{array}{llllll}\text { cylinder } & 2 & 1 & 0.49657 & 76.76 & -1.27\end{array}$ cuboid $3 \quad 1 \quad 4 p 1.00579 \quad 76.76-1.27$ unit 6

com='Fuel + Gap + Clad + Moderator' $\begin{array}{lllllll}\text { cylinder } & 1 & 1 & 0.428498 & 76.76 & 0.00\end{array}$ $\begin{array}{llllllll}\text { cylinder } & 0 & 1 & 0.437515 & 76.76 & 0.00\end{array}$ $\begin{array}{llllll}\text { cylinder } & 2 & 1 & 0.49657 & 76.76 & 0.00\end{array}$ $\begin{array}{llllll}\text { cylinder } & 2 & 1 & 0.49657 & 76.76-1.27\end{array}$ cuboid $314 \mathrm{p} 1.0057976 .76-1.27$ unit 7

com='Fuel + Gap + Clad + Moderator' $\begin{array}{lllllll}\text { cylinder } & 1 & 1 & 0.428498 & 76.76 & 0.00\end{array}$ $\begin{array}{lllllll}\text { cylinder } & 0 & 1 & 0.437515 & 76.76 & 0.00\end{array}$ $\begin{array}{lllllll}\text { cylinder } & 2 & 1 & 0.49657 & 76.76 & 0.00\end{array}$

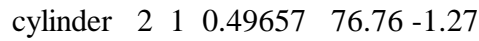
cuboid $3 \quad 1 \quad 4 p 1.0057976 .76-1.27$ unit 8

com='Fuel + Gap + Clad + Moderator' $\begin{array}{lllllll}\text { cylinder } & 1 & 1 & 0.428498 & 76.76 & 0.00\end{array}$ $\begin{array}{llllllll}\text { cylinder } & 0 & 1 & 0.437515 & 76.76 & 0.00\end{array}$ $\begin{array}{lllllll}\text { cylinder } & 2 & 1 & 0.49657 & 76.76 & 0.00\end{array}$ $\begin{array}{llllll}\text { cylinder } & 2 & 1 & 0.49657 & 76.76 & -1.27\end{array}$ cuboid $314 \mathrm{p} 1.00579 \quad 76.76-1.27$ unit 9

com='Fuel + Gap + Clad + Moderator' $\begin{array}{lllllll}\text { cylinder } & 1 & 1 & 0.428498 & 76.76 & 0.00\end{array}$ $\begin{array}{lllllll}\text { cylinder } & 0 & 1 & 0.437515 & 76.76 & 0.00\end{array}$ $\begin{array}{lllllll}\text { cylinder } & 2 & 1 & 0.49657 & 76.76 & 0.00\end{array}$ $\begin{array}{llllll}\text { cylinder } & 2 & 1 & 0.49657 & 76.76 & -1.27\end{array}$ cuboid $314 \mathrm{p} 1.0057976 .76-1.27$ unit 10

com='Fuel + Gap + Clad + Moderator' $\begin{array}{lllllll}\text { cylinder } & 1 & 1 & 0.428498 & 76.76 & 0.00\end{array}$ $\begin{array}{lllllll}\text { cylinder } & 0 & 1 & 0.437515 & 76.76 & 0.00\end{array}$ $\begin{array}{lllllll}\text { cylinder } & 2 & 1 & 0.49657 & 76.76 & 0.00\end{array}$ $\begin{array}{lllllll}\text { cylinder } & 2 & 1 & 0.49657 & 76.76 & -1.27\end{array}$ cuboid $\quad 3 \quad 1 \quad 4 p 1.00579 \quad 76.76-1.27$ unit 11

com='Fuel + Gap + Clad + Moderator' $\begin{array}{lllllll}\text { cylinder } & 1 & 1 & 0.428498 & 76.76 & 0.00\end{array}$ $\begin{array}{llllllll}\text { cylinder } & 0 & 1 & 0.437515 & 76.76 & 0.00\end{array}$ 
$\begin{array}{llllll}\text { cylinder } & 2 & 1 & 0.49657 & 76.76 & 0.00\end{array}$ $\begin{array}{llllll}\text { cylinder } & 2 & 1 & 0.49657 & 76.76-1.27\end{array}$

cuboid $3 \quad 14$ p1.00579 $76.76-1.27$ unit 12

com='Fuel + Gap + Clad + Moderator' $\begin{array}{lllllll}\text { cylinder } & 1 & 1 & 0.428498 & 76.76 & 0.00\end{array}$ $\begin{array}{llllllll}\text { cylinder } & 0 & 1 & 0.437515 & 76.76 & 0.00\end{array}$ $\begin{array}{lllllll}\text { cylinder } & 2 & 1 & 0.49657 & 76.76 & 0.00\end{array}$ $\begin{array}{llllll}\text { cylinder } & 2 & 1 & 0.49657 & 76.76 & -1.27\end{array}$ cuboid $3 \quad 1 \quad 4 p 1.0057976 .76-1.27$ unit 13

com='Fuel + Gap + Clad + Moderator' $\begin{array}{lllllll}\text { cylinder } & 1 & 1 & 0.428498 & 76.76 & 0.00\end{array}$ $\begin{array}{lllllll}\text { cylinder } & 0 & 1 & 0.437515 & 76.76 & 0.00\end{array}$ $\begin{array}{llllllll}\text { cylinder } & 2 & 1 & 0.49657 & 76.76 & 0.00\end{array}$ $\begin{array}{llllll}\text { cylinder } & 2 & 1 & 0.49657 & 76.76 & -1.27\end{array}$ cuboid $314 \mathrm{p} 1.00579$ 76.76 -1.27 unit 14

com='Fuel + Gap + Clad + Moderator' $\begin{array}{lllllll}\text { cylinder } & 1 & 1 & 0.428498 & 76.76 & 0.00\end{array}$ $\begin{array}{llllllll}\text { cylinder } & 0 & 1 & 0.437515 & 76.76 & 0.00\end{array}$ $\begin{array}{llllllll}\text { cylinder } & 2 & 1 & 0.49657 & 76.76 & 0.00\end{array}$ $\begin{array}{llllll}\text { cylinder } & 2 & 1 & 0.49657 & 76.76 & -1.27\end{array}$ cuboid $314 \mathrm{p} 1.0057976 .76-1.27$ unit 15

com='Fuel + Gap + Clad + Moderator' $\begin{array}{lllllll}\text { cylinder } & 1 & 1 & 0.428498 & 76.76 & 0.00\end{array}$ $\begin{array}{llllllll}\text { cylinder } & 0 & 1 & 0.437515 & 76.76 & 0.00\end{array}$ $\begin{array}{lllllll}\text { cylinder } & 2 & 1 & 0.49657 & 76.76 & 0.00\end{array}$ $\begin{array}{llllll}\text { cylinder } & 2 & 1 & 0.49657 & 76.76 & -1.27\end{array}$ cuboid $314 \mathrm{p} 1.0057976 .76-1.27$ unit 16

com='Fuel + Gap + Clad + Moderator' $\begin{array}{lllllll}\text { cylinder } & 1 & 1 & 0.428498 & 76.76 & 0.00\end{array}$ $\begin{array}{lllllll}\text { cylinder } & 0 & 1 & 0.437515 & 76.76 & 0.00\end{array}$ $\begin{array}{llllllll}\text { cylinder } & 2 & 1 & 0.49657 & 76.76 & 0.00\end{array}$ $\begin{array}{llllll}\text { cylinder } & 2 & 1 & 0.49657 & 76.76-1.27\end{array}$ cuboid $314 \mathrm{p} 1.0057976 .76-1.27$ unit 17

com='Fuel + Gap + Clad + Moderator' $\begin{array}{lllllll}\text { cylinder } & 1 & 1 & 0.428498 & 76.76 & 0.00\end{array}$ $\begin{array}{llllllll}\text { cylinder } & 0 & 1 & 0.437515 & 76.76 & 0.00\end{array}$ $\begin{array}{lllllll}\text { cylinder } & 2 & 1 & 0.49657 & 76.76 & 0.00\end{array}$ $\begin{array}{lllll}\text { cylinder } & 2 & 1 & 0.49657 & 76.76-1.27\end{array}$ cuboid $314 \mathrm{p} 1.0057976 .76-1.27$ unit 18

com='Fuel + Gap + Clad + Moderator' $\begin{array}{lllllll}\text { cylinder } & 1 & 1 & 0.428498 & 76.76 & 0.00\end{array}$ $\begin{array}{lllllll}\text { cylinder } & 0 & 1 & 0.437515 & 76.76 & 0.00\end{array}$ $\begin{array}{lllllll}\text { cylinder } & 2 & 1 & 0.49657 & 76.76 & 0.00\end{array}$ $\begin{array}{llllll}\text { cylinder } & 2 & 1 & 0.49657 & 76.76 & -1.27\end{array}$ cuboid $3 \quad 1 \quad 4 p 1.0057976 .76-1.27$ unit 19

com='Fuel + Gap + Clad + Moderator' $\begin{array}{lllllll}\text { cylinder } & 1 & 1 & 0.428498 & 76.76 & 0.00\end{array}$ $\begin{array}{lllllll}\text { cylinder } & 0 & 1 & 0.437515 & 76.76 & 0.00\end{array}$ $\begin{array}{llllll}\text { cylinder } & 2 & 1 & 0.49657 & 76.76 & 0.00\end{array}$ $\begin{array}{lllll}\text { cylinder } & 2 & 1 & 0.49657 & 76.76-1.27\end{array}$

cuboid $314 \mathrm{p} 1.0057976 .76-1.27$ unit 20

com='Fuel + Gap + Clad + Moderator' $\begin{array}{lllllll}\text { cylinder } & 1 & 1 & 0.428498 & 76.76 & 0.00\end{array}$ $\begin{array}{lllllll}\text { cylinder } & 0 & 1 & 0.437515 & 76.76 & 0.00\end{array}$ $\begin{array}{lllllll}\text { cylinder } & 2 & 1 & 0.49657 & 76.76 & 0.00\end{array}$ $\begin{array}{llllll}\text { cylinder } & 2 & 1 & 0.49657 & 76.76-1.27\end{array}$ cuboid $314 \mathrm{p} 1.0057976 .76-1.27$ unit 21

com='Fuel + Gap + Clad + Moderator' $\begin{array}{lllllll}\text { cylinder } & 1 & 1 & 0.428498 & 76.76 & 0.00\end{array}$ $\begin{array}{lllllll}\text { cylinder } & 0 & 1 & 0.437515 & 76.76 & 0.00\end{array}$ $\begin{array}{lllllll}\text { cylinder } & 2 & 1 & 0.49657 & 76.76 & 0.00\end{array}$ $\begin{array}{lllllll}\text { cylinder } & 2 & 1 & 0.49657 & 76.76 & -1.27\end{array}$ cuboid $314 \mathrm{p} 1.0057976 .76-1.27$

unit 71

com='Clad + Mod + AL Grid'

$\begin{array}{lllll}\text { cylinder } 2 & 1 & 0.49657 & 0.635 & 0.0\end{array}$ $\begin{array}{llllll}\text { cylinder } 3 & 1 & 0.50419 & 0.635 & 0.0\end{array}$ cuboid $5 \quad 1 \quad 4 p 1.005790 .6350 .0$

unit 73

com='Fuel + Gap + Clad + Air' $\begin{array}{lllllll}\text { cylinder } & 1 & 1 & 0.428498 & 16.204 & 0.0\end{array}$ $\begin{array}{llllll}\text { cylinder } & 0 & 1 & 0.437515 & 16.204 & 0.0\end{array}$ $\begin{array}{llllll}\text { cylinder } 2 & 1 & 0.49657 & 16.204 & 0.0\end{array}$ cuboid $\quad \begin{array}{lllllll}0 & 1 & 4 p 1.00579 & 16.204 & 0.0\end{array}$

unit 74 com='Top Clad + Void' cylinder $2 \quad 1 \quad 0.49657 \quad 1.016 \quad 0.0$ cuboid $\begin{array}{lllllll}0 & 1 & 4 \mathrm{p} 1.00579 & 1.016 & 0.0\end{array}$

unit 75

com='Top Clad + Void + AL grid' $\begin{array}{lllll}\text { cylinder } 2 & 1 & 0.49657 & 1.27 & 0.0\end{array}$ cylinder $\begin{array}{lllll}0 & 1 & 0.50419 & 1.27 & 0.0\end{array}$ cuboid $514 \mathrm{p} 1.005791 .27 \quad 0.0$

unit 81

com='array of Clad + Mod + AL Grid + Reflector' array $1-12.06948-12.069480 .0$ cuboid 41 4p42.06948 $0.635 \quad 0.0$

unit 82

com='array of Fuel + Gap + Clad + Moderator + Reflector' array $2-12.06948-12.06948-1.27$ cuboid $314 \mathrm{p} 42.0694876 .76-1.27$

unit 83 com='array of Fuel + Gap + Clad + Air' array $3-12.06948-12.069480 .0$ cuboid 014 p42.06948 $16.204 \quad 0.0$

unit 84 
com='array of Top Clad + Void'

array $4-12.06948-12.069480 .0$

cuboid $014 \mathrm{p} 42.069481 .0160 .0$

unit 85

com='array of Top Clad + Void + AL grid' array $5-12.06948-12.069480 .0$

cuboid 41 4p42.06948 $1.27 \quad 0.0$

global unit 100

com='array of arrays of fuel element sections + reflector' array $6-42.06948-42.06948 \quad 0.0$

cuboid $5 \quad 1 \quad 4 p 42.06948 \quad 97.15500 \quad-2.54$

cuboid $3 \quad 14 \mathrm{p} 42.06948 \quad 97.15500 \quad-8.89$

cuboid $5 \quad 1 \quad 4 p 42.06948 \quad 97.15500 \quad-13.97$

cuboid $3 \begin{array}{llllll} & 1 & 4 p 42.06948 & 97.15500 & -43.97\end{array}$

end geom

read array

ara $=1$ nux=12 nuy=12 nuz=1

fill $\mathrm{f} 71$ end fill

ara=2 nux=12 nuy=12 nuz=1

fill

$\begin{array}{lllllll}1 & 2 & 3 & 4 & 5 & 6 & \mathrm{n} 6\end{array}$

$278891011 \mathrm{n} 6$

$\begin{array}{lllllll}3 & 8 & 12 & 13 & 14 & 15 & \text { n6 }\end{array}$

4913161718 n6

51014171920 n6

61115182021 n6

n72

end fill

ara=3 nux=12 nuy=12 nuz=1 fill $\mathrm{f} 73$ end fill

ara=4 nux=12 nuy=12 nuz=1 fill f74 end fill

ara $=5$ nux $=12$ nuy=12 nuz=1 fill f75 end fill

ara $=6$ nux $=1$ nuy=1 nuz $=5$

fill 8182838485 end fill

end array

read plot

$\mathrm{ttl}=$ 'simple plot $1^{\prime}$

lpi $=10 \mathrm{scr}=$ yes pic $=\operatorname{mix}$ nax $=400$

$\mathrm{xul}=-20.0 \mathrm{yul}=1.006 \mathrm{zul}=100$

$\mathrm{xlr}=20.0 \mathrm{ylr}=1.006 \mathrm{zlr}=-10$

$\mathrm{uax}=1.0 \mathrm{wdn}=-1.0$ end plt 0

ttl='simple plot 2'

pic $=$ mix nax $=400$

$\mathrm{xul}=-20.0$ yul $=20 . \quad \mathrm{zul}=25$.

$\mathrm{xlr}=20.0 \quad \mathrm{ylr}=-20 . \quad \mathrm{zlr}=25$.

$\mathrm{uax}=1.0 \mathrm{vdn}=-1.0$ end plt 1

end plot

end data

end

$=$ shell

cp ft04f001 \$RTNDIR/sax05.xs04

cp ft64f001 \$RTNDIR/sax05.rs64

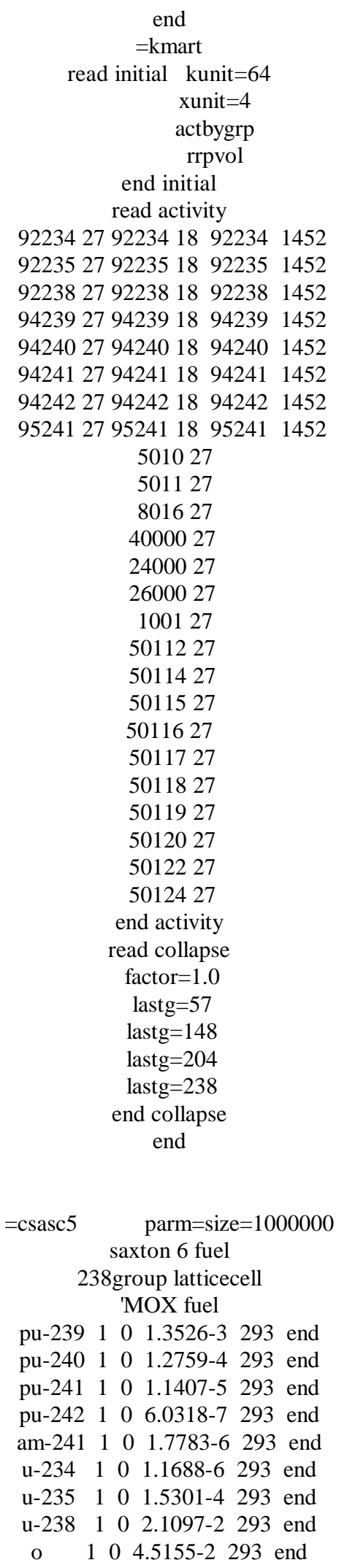


'clad (ZR-2)

zr $2004.2517-2 \quad 293$ end

sn $2004.6590-4293$ end

cr $2 \quad 0 \quad 7.5977-5293$ end

fe $\quad 2 \quad 0 \quad 1.4148-4293$ end

o $\quad 202.9630-4293$ end 'water(Refletor)

h $\quad 3 \quad 0 \quad 6.6737-2 \quad 293$ end

o $\quad 30$ 3.3368-2 293 end

'Middle Grid and $\mathrm{H} 2 \mathrm{O}$

\begin{tabular}{cccc} 
al & 4 & .969 & 293 end \\
h2o & 4 & .031 & 293 end \\
\multicolumn{4}{c}{ 'Aluminum }
\end{tabular}

al $\quad \begin{array}{llll}5 & 0 & 6.0039-2 & 293 \\ \text { end }\end{array}$ end comp

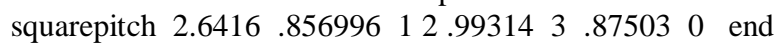
more data

limcen $=20000000$

end more

saxton 6

read param

gen=225 nsk=25 npg=20000 $\mathrm{fdn}=$ yes $\mathrm{flx}=$ yes wrs $=64$ res $=225$

$\operatorname{lng}=2000000$

end param

read geometry

unit 1

com='Fuel + Gap + Clad + Moderator'

$\begin{array}{lllllll}\text { cylinder } & 1 & 1 & 0.428498 & 79.50 & 0.00\end{array}$

$\begin{array}{lllllll}\text { cylinder } & 0 & 1 & 0.437515 & 79.50 & 0.00\end{array}$

$\begin{array}{llllllll}\text { cylinder } & 2 & 1 & 0.49657 & 79.50 & 0.00\end{array}$

$\begin{array}{lllllll}\text { cylinder } & 2 & 1 & 0.49657 & 79.50 & -1.27\end{array}$

cuboid $3 \quad 1 \quad 4 p 1.320879 .50-1.27$ unit 2

com='Fuel + Gap + Clad + Moderator' $\begin{array}{lllllll}\text { cylinder } & 1 & 1 & 0.428498 & 79.50 & 0.00\end{array}$

$\begin{array}{lllllll}\text { cylinder } & 0 & 1 & 0.43751579 .50 & 0.00\end{array}$

$\begin{array}{llllllll}\text { cylinder } & 2 & 1 & 0.49657 & 79.50 & 0.00\end{array}$

$\begin{array}{lllllll}\text { cylinder } & 2 & 1 & 0.49657 & 79.50 & -1.27\end{array}$

cuboid $3 \quad 14 p 1.320879 .50-1.27$ unit 3

com='Fuel + Gap + Clad + Moderator'

$\begin{array}{lllllll}\text { cylinder } & 1 & 1 & 0.428498 & 79.50 & 0.00\end{array}$

$\begin{array}{llllllll}\text { cylinder } & 0 & 1 & 0.437515 & 79.50 & 0.00\end{array}$

$\begin{array}{llllllll}\text { cylinder } & 2 & 1 & 0.49657 & 79.50 & 0.00\end{array}$

$\begin{array}{llllll}\text { cylinder } & 2 & 1 & 0.49657 & 79.50 & -1.27\end{array}$

cuboid $3 \quad 14$ p1.3208 $79.50-1.27$ unit 4

com='Fuel + Gap + Clad + Moderator'

$\begin{array}{lllllll}\text { cylinder } & 1 & 1 & 0.428498 & 79.50 & 0.00\end{array}$

$\begin{array}{llllllll}\text { cylinder } & 0 & 1 & 0.437515 & 79.50 & 0.00\end{array}$

$\begin{array}{lllllll}\text { cylinder } & 2 & 1 & 0.49657 & 79.50 & 0.00\end{array}$

$\begin{array}{lllllll}\text { cylinder } & 2 & 1 & 0.49657 & 79.50 & -1.27\end{array}$

cuboid $\quad 3 \quad 14 p 1.320879 .50-1.27$

unit 5

com='Fuel + Gap + Clad + Moderator' $\begin{array}{lllllll}\text { cylinder } & 1 & 1 & 0.428498 & 79.50 & 0.00\end{array}$

$\begin{array}{llllllll}\text { cylinder } & 0 & 1 & 0.437515 & 79.50 & 0.00\end{array}$

$\begin{array}{lllllll}\text { cylinder } & 2 & 1 & 0.49657 & 79.50 & 0.00\end{array}$

$\begin{array}{llllll}\text { cylinder } & 2 & 1 & 0.49657 & 79.50 & -1.27\end{array}$

cuboid $314 \mathrm{p} 1.320879 .50-1.27$ unit 6

com='Fuel + Gap + Clad + Moderator' $\begin{array}{lllllll}\text { cylinder } & 1 & 1 & 0.428498 & 79.50 & 0.00\end{array}$ $\begin{array}{llllllll}\text { cylinder } & 0 & 1 & 0.437515 & 79.50 & 0.00\end{array}$ $\begin{array}{llllllllllll}\text { cylinder } & 2 & 1 & 0.49657 & 79.50 & 0.00\end{array}$ $\begin{array}{lllllll}\text { cylinder } & 2 & 1 & 0.49657 & 79.50 & -1.27\end{array}$ cuboid $314 \mathrm{p} 1.320879 .50-1.27$ unit 7

com='Fuel + Gap + Clad + Moderator' $\begin{array}{lllllll}\text { cylinder } & 1 & 1 & 0.428498 & 79.50 & 0.00\end{array}$ $\begin{array}{lllllll}\text { cylinder } & 0 & 1 & 0.437515 & 79.50 & 0.00\end{array}$ $\begin{array}{llllllll}\text { cylinder } & 2 & 1 & 0.49657 & 79.50 & 0.00\end{array}$ $\begin{array}{lllllll}\text { cylinder } & 2 & 1 & 0.49657 & 79.50 & -1.27\end{array}$ cuboid $3 \quad 14 p 1.320879 .50-1.27$ unit 8

com='Fuel + Gap + Clad + Moderator' $\begin{array}{lllllllll}\text { cylinder } & 1 & 1 & 0.428498 & 79.50 & 0.00\end{array}$ $\begin{array}{lllllllll}\text { cylinder } & 0 & 1 & 0.437515 & 79.50 & 0.00\end{array}$ $\begin{array}{lllllll}\text { cylinder } & 2 & 1 & 0.49657 & 79.50 & 0.00\end{array}$ $\begin{array}{lllllll}\text { cylinder } & 2 & 1 & 0.49657 & 79.50 & -1.27\end{array}$ cuboid $314 \mathrm{p} 1.320879 .50-1.27$ unit 9

com='Fuel + Gap + Clad + Moderator' $\begin{array}{lllllll}\text { cylinder } & 1 & 1 & 0.428498 & 79.50 & 0.00\end{array}$ $\begin{array}{lllllllll}\text { cylinder } & 0 & 1 & 0.437515 & 79.50 & 0.00\end{array}$ $\begin{array}{lllllll}\text { cylinder } & 2 & 1 & 0.49657 & 79.50 & 0.00\end{array}$ $\begin{array}{lllllll}\text { cylinder } & 2 & 1 & 0.49657 & 79.50 & -1.27\end{array}$ cuboid $314 \mathrm{p} 1.320879 .50-1.27$ unit 10

com='Fuel + Gap + Clad + Moderator' $\begin{array}{lllllllll}\text { cylinder } & 1 & 1 & 0.428498 & 79.50 & 0.00\end{array}$ $\begin{array}{llllllll}\text { cylinder } & 0 & 1 & 0.437515 & 79.50 & 0.00\end{array}$ $\begin{array}{lllllll}\text { cylinder } & 2 & 1 & 0.49657 & 79.50 & 0.00\end{array}$ $\begin{array}{lllllll}\text { cylinder } & 2 & 1 & 0.49657 & 79.50 & -1.27\end{array}$ cuboid $314 \mathrm{p} 1.320879 .50-1.27$ unit 11

com='Fuel + Gap + Clad + Moderator' $\begin{array}{lllllll}\text { cylinder } & 1 & 1 & 0.428498 & 79.50 & 0.00\end{array}$ $\begin{array}{llllllll}\text { cylinder } & 0 & 1 & 0.437515 & 79.50 & 0.00\end{array}$ $\begin{array}{lllllll}\text { cylinder } & 2 & 1 & 0.49657 & 79.50 & 0.00\end{array}$ $\begin{array}{llllll}\text { cylinder } & 2 & 1 & 0.49657 & 79.50 & -1.27\end{array}$ cuboid $314 \mathrm{p} 1.320879 .50-1.27$ unit 12

com='Fuel + Gap + Clad + Moderator' $\begin{array}{lllllll}\text { cylinder } & 1 & 1 & 0.428498 & 79.50 & 0.00\end{array}$ $\begin{array}{lllllll}\text { cylinder } & 0 & 1 & 0.437515 & 79.50 & 0.00\end{array}$ $\begin{array}{lllllll}\text { cylinder } & 2 & 1 & 0.49657 & 79.50 & 0.00\end{array}$ $\begin{array}{lllllll}\text { cylinder } & 2 & 1 & 0.49657 & 79.50 & -1.27\end{array}$ cuboid $\quad 3 \quad 1 \quad 4 p 1.320879 .50-1.27$ unit 13

com='Fuel + Gap + Clad + Moderator' $\begin{array}{lllllll}\text { cylinder } & 1 & 1 & 0.428498 & 79.50 & 0.00\end{array}$ 
$\begin{array}{lllllll}\text { cylinder } & 0 & 1 & 0.437515 & 79.50 & 0.00\end{array}$ $\begin{array}{lllllll}\text { cylinder } & 2 & 1 & 0.49657 & 79.50 & 0.00\end{array}$ $\begin{array}{llllll}\text { cylinder } & 2 & 1 & 0.49657 & 79.50 & -1.27\end{array}$ cuboid $\quad 3 \quad 14$ p1.3208 79.50 -1.27 unit 14

com='Fuel + Gap + Clad + Moderator' $\begin{array}{lllllll}\text { cylinder } & 1 & 1 & 0.428498 & 79.50 & 0.00\end{array}$ $\begin{array}{lllllll}\text { cylinder } & 0 & 1 & 0.437515 & 79.50 & 0.00\end{array}$ $\begin{array}{llllllll}\text { cylinder } & 2 & 1 & 0.49657 & 79.50 & 0.00\end{array}$ $\begin{array}{llllll}\text { cylinder } & 2 & 1 & 0.49657 & 79.50 & -1.27\end{array}$ cuboid 3 1 4 p1.3208 $79.50-1.27$ unit 15

com='Fuel + Gap + Clad + Moderator' $\begin{array}{llllllll}\text { cylinder } & 1 & 1 & 0.428498 & 79.50 & 0.00\end{array}$ $\begin{array}{lllllllllll}\text { cylinder } & 0 & 1 & 0.437515 & 79.50 & 0.00\end{array}$ $\begin{array}{llllllllllllll}\text { cylinder } & 2 & 1 & 0.49657 & 79.50 & 0.00\end{array}$ $\begin{array}{lllllll}\text { cylinder } & 2 & 1 & 0.49657 & 79.50 & -1.27\end{array}$ cuboid 3 1 4 p1.3208 $79.50-1.27$ unit 16

com='Fuel + Gap + Clad + Moderator' $\begin{array}{lllllll}\text { cylinder } & 1 & 1 & 0.428498 & 79.50 & 0.00\end{array}$ $\begin{array}{lllllll}\text { cylinder } & 0 & 1 & 0.437515 & 79.50 & 0.00\end{array}$ $\begin{array}{llllllllll}\text { cylinder } & 2 & 1 & 0.49657 & 79.50 & 0.00\end{array}$ $\begin{array}{lllllll}\text { cylinder } & 2 & 1 & 0.49657 & 79.50 & -1.27\end{array}$ cuboid 3 1 4 p1.3208 $79.50-1.27$ unit 17

com='Fuel + Gap + Clad + Moderator' $\begin{array}{lllllll}\text { cylinder } & 1 & 1 & 0.428498 & 79.50 & 0.00\end{array}$ $\begin{array}{lllllll}\text { cylinder } & 0 & 1 & 0.437515 & 79.50 & 0.00\end{array}$ $\begin{array}{llllllll}\text { cylinder } & 2 & 1 & 0.49657 & 79.50 & 0.00\end{array}$ $\begin{array}{lllllll}\text { cylinder } & 2 & 1 & 0.49657 & 79.50 & -1.27\end{array}$ cuboid $3 \quad 14 p 1.320879 .50-1.27$ unit 18

com='Fuel + Gap + Clad + Moderator' $\begin{array}{lllllll}\text { cylinder } & 1 & 1 & 0.428498 & 79.50 & 0.00\end{array}$ $\begin{array}{lllllllllllllll}\text { cylinder } & 0 & 1 & 0.437515 & 79.50 & 0.00\end{array}$ $\begin{array}{llllllllllllll}\text { cylinder } & 2 & 1 & 0.49657 & 79.50 & 0.00\end{array}$ $\begin{array}{lllllll}\text { cylinder } & 2 & 1 & 0.49657 & 79.50 & -1.27\end{array}$ cuboid $\quad 3 \quad 1 \quad 4 p 1.320879 .50-1.27$ unit 19

com='Fuel + Gap + Clad + Moderator' $\begin{array}{lllllll}\text { cylinder } & 1 & 1 & 0.428498 & 79.50 & 0.00\end{array}$ $\begin{array}{lllllllllllll}\text { cylinder } & 0 & 1 & 0.437515 & 79.50 & 0.00\end{array}$ $\begin{array}{lllllll}\text { cylinder } & 2 & 1 & 0.49657 & 79.50 & 0.00\end{array}$ $\begin{array}{lllllll}\text { cylinder } & 2 & 1 & 0.49657 & 79.50 & -1.27\end{array}$ cuboid $314 \mathrm{p} 1.320879 .50-1.27$ unit 20

com='Fuel + Gap + Clad + Moderator' $\begin{array}{lllllll}\text { cylinder } & 1 & 1 & 0.428498 & 79.50 & 0.00\end{array}$ $\begin{array}{lllllll}\text { cylinder } & 0 & 1 & 0.437515 & 79.50 & 0.00\end{array}$ $\begin{array}{llllllll}\text { cylinder } & 2 & 1 & 0.49657 & 79.50 & 0.00\end{array}$ $\begin{array}{lllllll}\text { cylinder } & 2 & 1 & 0.49657 & 79.50 & -1.27\end{array}$ cuboid $314 \mathrm{p} 1.320879 .50-1.27$ unit 21

com='Fuel + Gap + Clad + Moderator' $\begin{array}{lllllll}\text { cylinder } & 1 & 1 & 0.428498 & 79.50 & 0.00\end{array}$ $\begin{array}{lllllll}\text { cylinder } & 0 & 1 & 0.437515 & 79.50 & 0.00\end{array}$ $\begin{array}{llllll}\text { cylinder } & 2 & 1 & 0.49657 & 79.50 & 0.00\end{array}$ $\begin{array}{lllllll}\text { cylinder } & 2 & 1 & 0.49657 & 79.50 & -1.27\end{array}$ cuboid $314 p 1.320879 .50-1.27$ unit 22

com='Fuel + Gap + Clad + Moderator' $\begin{array}{llllllll}\text { cylinder } & 1 & 1 & 0.428498 & 79.50 & 0.00\end{array}$ $\begin{array}{llllllll}\text { cylinder } & 0 & 1 & 0.437515 & 79.50 & 0.00\end{array}$ $\begin{array}{llllllllllllll}\text { cylinder } & 2 & 1 & 0.49657 & 79.50 & 0.00\end{array}$ $\begin{array}{lllllll}\text { cylinder } & 2 & 1 & 0.49657 & 79.50 & -1.27\end{array}$ cuboid $314 p 1.320879 .50-1.27$ unit 23

com='Fuel + Gap + Clad + Moderator' $\begin{array}{lllllll}\text { cylinder } & 1 & 1 & 0.428498 & 79.50 & 0.00\end{array}$ $\begin{array}{llllllll}\text { cylinder } & 0 & 1 & 0.437515 & 79.50 & 0.00\end{array}$ $\begin{array}{lllllllllllllll}\text { cylinder } & 2 & 1 & 0.49657 & 79.50 & 0.00\end{array}$ $\begin{array}{lllllll}\text { cylinder } & 2 & 1 & 0.49657 & 79.50 & -1.27\end{array}$ cuboid $3 \begin{array}{llll}4 & 4 p 1.320879 .50-1.27\end{array}$ unit 24

com='Fuel + Gap + Clad + Moderator' $\begin{array}{lllllll}\text { cylinder } & 1 & 1 & 0.428498 & 79.50 & 0.00\end{array}$ $\begin{array}{llllllll}\text { cylinder } & 0 & 1 & 0.437515 & 79.50 & 0.00\end{array}$ $\begin{array}{llllllllllll}\text { cylinder } & 2 & 1 & 0.49657 & 79.50 & 0.00\end{array}$ $\begin{array}{llllll}\text { cylinder } & 2 & 1 & 0.49657 & 79.50 & -1.27\end{array}$ cuboid $314 p 1.320879 .50-1.27$ unit 25

com='Fuel + Gap + Clad + Moderator' $\begin{array}{lllllll}\text { cylinder } & 1 & 1 & 0.428498 & 79.50 & 0.00\end{array}$ $\begin{array}{lllllll}\text { cylinder } & 0 & 1 & 0.437515 & 79.50 & 0.00\end{array}$ $\begin{array}{lllllll}\text { cylinder } & 2 & 1 & 0.49657 & 79.50 & 0.00\end{array}$ $\begin{array}{lllllll}\text { cylinder } & 2 & 1 & 0.49657 & 79.50 & -1.27\end{array}$ cuboid $3 \quad 14$ p1.3208 $79.50-1.27$ unit 26

com='Fuel + Gap + Clad + Moderator' $\begin{array}{llllllll}\text { cylinder } & 1 & 1 & 0.428498 & 79.50 & 0.00\end{array}$ $\begin{array}{lllllll}\text { cylinder } & 0 & 1 & 0.437515 & 79.50 & 0.00\end{array}$ $\begin{array}{llllllllllll}\text { cylinder } & 2 & 1 & 0.49657 & 79.50 & 0.00\end{array}$ $\begin{array}{llllll}\text { cylinder } & 2 & 1 & 0.49657 & 79.50 & -1.27\end{array}$ cuboid $314 p 1.320879 .50-1.27$ unit 27

com='Fuel + Gap + Clad + Moderator' cylinder $\begin{array}{llllll}1 & 1 & 0.428498 & 79.50 & 0.00\end{array}$ $\begin{array}{lllllll}\text { cylinder } & 0 & 1 & 0.437515 & 79.50 & 0.00\end{array}$ $\begin{array}{llllllllllll}\text { cylinder } & 2 & 1 & 0.49657 & 79.50 & 0.00\end{array}$ $\begin{array}{llllll}\text { cylinder } & 2 & 1 & 0.49657 & 79.50 & -1.27\end{array}$ cuboid $3 \quad 14$ p1.3208 $79.50-1.27$ unit 28

com='Fuel + Gap + Clad + Moderator' $\begin{array}{llllllll}\text { cylinder } & 1 & 1 & 0.428498 & 79.50 & 0.00\end{array}$ $\begin{array}{lllllll}\text { cylinder } & 0 & 1 & 0.437515 & 79.50 & 0.00\end{array}$ $\begin{array}{llllllll}\text { cylinder } & 2 & 1 & 0.49657 & 79.50 & 0.00\end{array}$ $\begin{array}{llllll}\text { cylinder } & 2 & 1 & 0.49657 & 79.50 & -1.27\end{array}$ cuboid 314 p1.3208 79.50-1.27

unit 71

com='Clad + Mod + AL Grid' $\begin{array}{lllll}\text { cylinder } 2 & 1 & 0.49657 & 0.635 & 0.0\end{array}$ $\begin{array}{llllll}\text { cylinder } 3 & 1 & 0.50419 & 0.635 & 0.0\end{array}$ 
$\begin{array}{lllllll}\text { cuboid } & 5 & 1 & 4 \mathrm{p} 1.3208 & 0.635 & 0.0\end{array}$

unit 73

com='Fuel + Gap + Clad + Air'

cylinder $\begin{array}{llllll}1 & 1 & 0.428498 & 13.464 & 0.0\end{array}$

$\begin{array}{lllllll}\text { cylinder } & 0 & 1 & 0.437515 & 13.464 & 0.0\end{array}$

$\begin{array}{llllll}\text { cylinder } 2 & 1 & 0.49657 & 13.464 & 0.0\end{array}$

$\begin{array}{llllllll}\text { cuboid } & 0 & 1 & 4 p 1.3208 & 13.464 & 0.0\end{array}$

unit 74

com='Top Clad + Void'

$\begin{array}{lllll}\text { cylinder } 2 & 1 & 0.49657 & 1.016 & 0.0\end{array}$

cuboid $\quad 0 \quad 1 \quad 4 p 1.32081 .016 \quad 0.0$

unit 75

com='Top Clad + Void + AL grid'

cylinder $210.49657 \quad 1.27 \quad 0.0$

cylinder $\begin{array}{llllll}0 & 1 & 0.50419 & 1.27 & 0.0\end{array}$

cuboid $514 \mathrm{p} 1.32081 .27 \quad 0.0$

unit 81

com='array of Clad + Mod + AL Grid + Reflector'

array $1-14.5288-14.52880 .0$

cuboid 41 4p44.5288 0.6350 .0

unit 82

com='array of Fuel + Gap + Clad + Moderator + Reflector'

array $2-14.5288-14.5288-1.27$

cuboid $314 \mathrm{p} 44.528879 .50-1.27$

unit 83

com='array of Fuel + Gap + Clad + Air'

array $3-14.5288-14.52880 .0$

cuboid $014 \mathrm{p} 44.528813 .4640 .0$

unit 84

com='array of Top Clad + Void'

array $4-14.5288-14.52880 .0$

cuboid $014 \mathrm{p} 44.52881 .0160 .0$

unit 85

com='array of Top Clad + Void + AL grid'

array $5-14.5288-14.52880 .0$

cuboid $414 \mathrm{p} 44.52881 .27 \quad 0.0$

global unit 100

com='array of arrays of fuel element sections + reflector' array $6-44.5288-44.5288 \quad 0.0$

cuboid $5 \begin{array}{lllll}5 & 4 \mathrm{p} 44.5288 & 97.15500 & -2.54\end{array}$

cuboid $3 \quad 1 \quad 4 p 44.5288 \quad 97.15500 \quad-8.89$

cuboid $5 \quad 1 \quad 4 p 44.528897 .15500$-13.97

cuboid $3 \quad 14$ p44.5288 97.15500 -43.97

end geom

read array

ara=1 nux=11 nuy=11 nuz=1

fill $\mathrm{f} 7 \mathrm{l}$ end fill

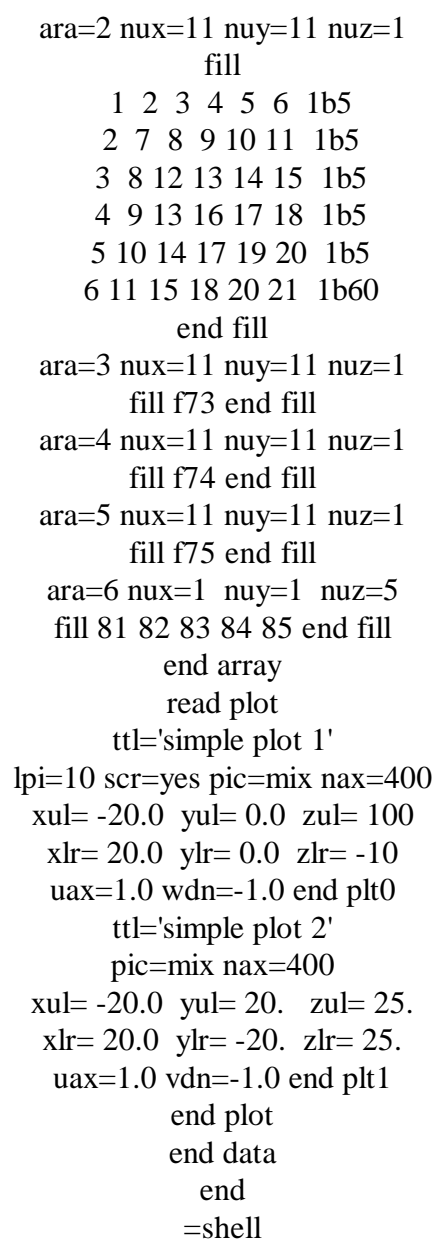

cp ft04f001 \$RTNDIR/sax06.xs04 cp ft64f001 \$RTNDIR/sax06.rs64 end $=\mathrm{kmart}$

read initial kunit $=64$ xunit $=4$ actbygrp rrpvol

end initial read activity

$9223427922341892234 \quad 1452$ 92235279223518922351452

92238279223818922381452

94239279423918942391452

94240279424018942401452

$9424127942411894241 \quad 1452$

94242279424218942421452

95241279524118952411452

501027

501127

801627

4000027

2400027

2600027 
100127

5011227

5011427

5011527

5011627

5011727

5011827

5011927

5012027

5012227

5012427

end activity

read collapse

factor $=1.0$

lastg $=57$

lastg $=148$

lastg $=204$

lastg $=238$

end collapse end 

APPENDIX C

SCALE INPUT OF MIX-COMP-THERM-4 PROBLEM SET 



\section{APPENDIX C \\ SCALE INPUT OF MIX-COMP-THERM-4 PROBLEM SET}

This appendix contains the CENTRM inputs for the eleven MIX-COMP-THERM-04 cases, TCA01, TCA02, TCA03, TCA04, TCA05, TCA06, TCA07, TCA08, TCA09, TCA10, and TCA11. The inputs can be changed to standard CSAS cases by replacing the $=$ CSASC 5 record with $=$ CSAS25.

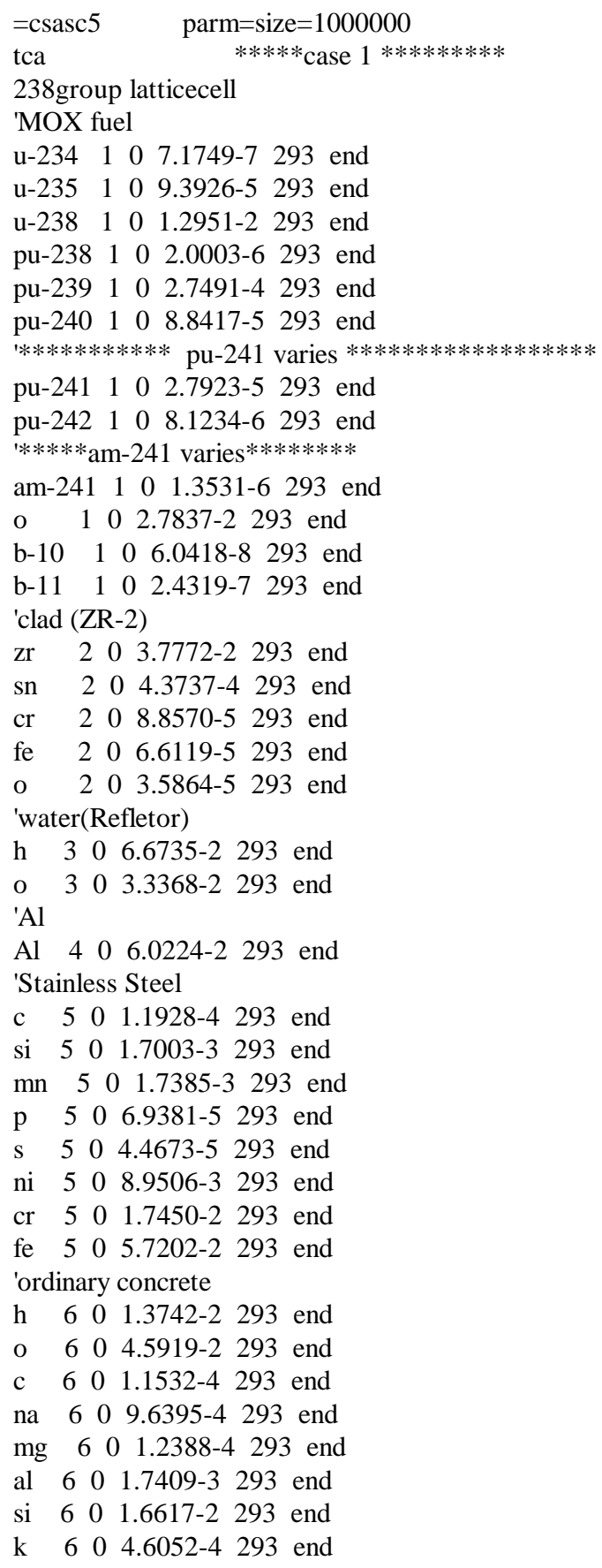

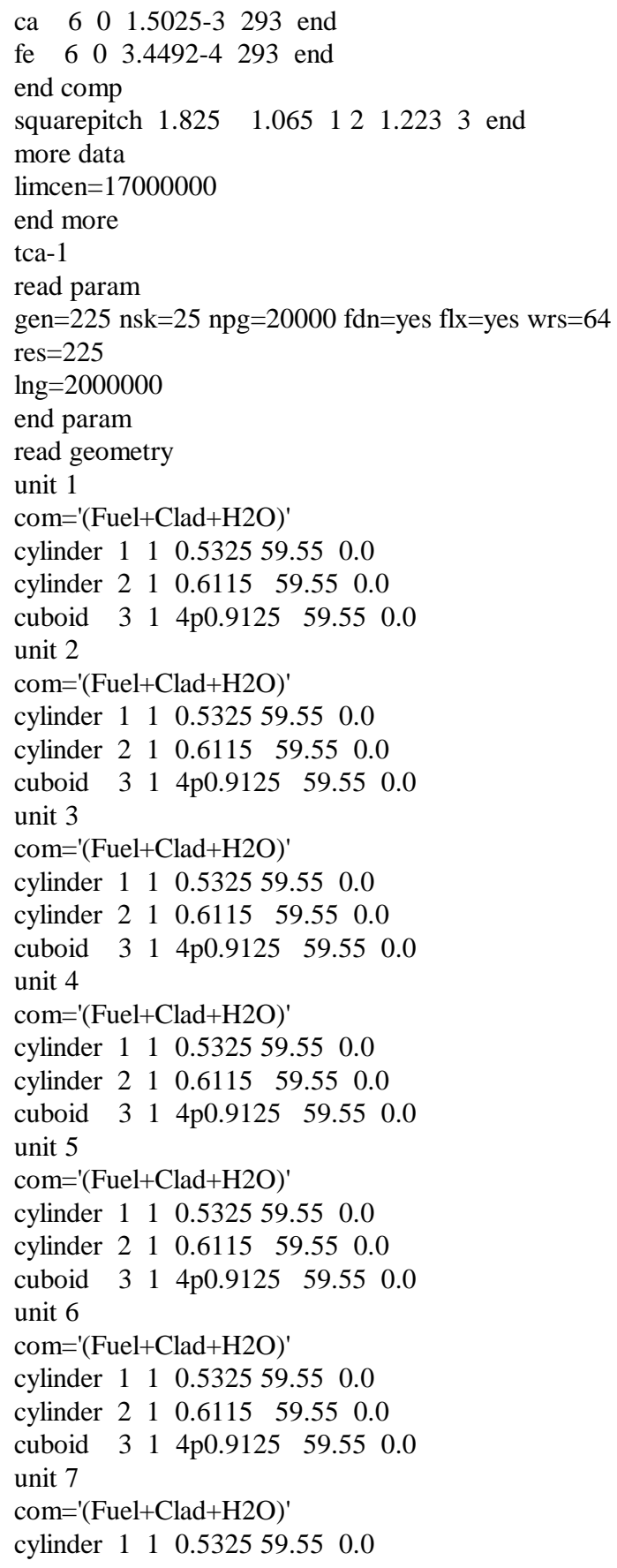


$\begin{array}{llllll}\text { cylinder } & 2 & 1 & 0.6115 & 59.55 & 0.0\end{array}$ $\begin{array}{llllll}\text { cuboid } & 3 & 1 & 4 \mathrm{p} 0.9125 & 59.55 & 0.0\end{array}$ unit 8 com='(Fuel+Clad $+\mathrm{H} 2 \mathrm{O})^{\prime}$

cylinder $1110.532559 .55 \quad 0.0$

$\begin{array}{lllll}\text { cylinder } 2 & 1 & 0.6115 & 59.55 & 0.0\end{array}$

$\begin{array}{llllll}\text { cuboid } & 3 & 1 & 4 \mathrm{p} 0.9125 & 59.55 & 0.0\end{array}$

unit 9

com='(Fuel+Clad $+\mathrm{H} 2 \mathrm{O})^{\prime}$

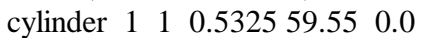

$\begin{array}{llllll}\text { cylinder } & 2 & 1 & 0.6115 & 59.55 & 0.0\end{array}$

$\begin{array}{lllllll}\text { cuboid } & 3 & 1 & 4 \mathrm{p} 0.9125 & 59.55 & 0.0\end{array}$

unit 10

com $=$ '(Fuel+Clad $+\mathrm{H} 2 \mathrm{O})^{\prime}$

$\begin{array}{llllll}\text { cylinder } & 1 & 1 & 0.5325 & 59.55 & 0.0\end{array}$

$\begin{array}{llllll}\text { cylinder } 2 & 1 & 0.6115 & 59.55 & 0.0\end{array}$

$\begin{array}{llllll}\text { cuboid } & 3 & 1 & 4 \mathrm{p} 0.9125 & 59.55 & 0.0\end{array}$

unit 11

com='(Fuel+Clad $+\mathrm{H} 2 \mathrm{O})^{\prime}$

cylinder 1110.532559 .550 .0

$\begin{array}{llllll}\text { cylinder } 2 & 1 & 0.6115 & 59.55 & 0.0\end{array}$

$\begin{array}{llllll}\text { cuboid } & 3 & 1 & 4 \mathrm{p} 0.9125 & 59.55 & 0.0\end{array}$

unit 12

com='(Fuel+Clad $+\mathrm{H} 2 \mathrm{O})^{\prime}$

$\begin{array}{llllll}\text { cylinder } & 1 & 1 & 0.5325 & 59.55 & 0.0\end{array}$

$\begin{array}{llllll}\text { cylinder } & 2 & 1 & 0.6115 & 59.55 & 0.0\end{array}$

$\begin{array}{lllllll}\text { cuboid } & 3 & 1 & 4 \mathrm{p} 0.9125 & 59.55 & 0.0\end{array}$

unit 13

com='(Fuel+Clad $+\mathrm{H} 2 \mathrm{O})^{\prime}$

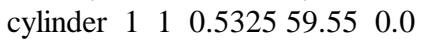

$\begin{array}{lllll}\text { cylinder } 2 & 1 & 0.6115 & 59.55 & 0.0\end{array}$

$\begin{array}{lllllll}\text { cuboid } & 3 & 1 & 4 \mathrm{p} 0.9125 & 59.55 & 0.0\end{array}$

unit 14

com='(Fuel+Clad $+\mathrm{H} 2 \mathrm{O})^{\prime}$

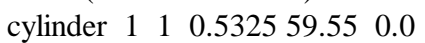

$\begin{array}{llllll}\text { cylinder } 2 & 1 & 0.6115 & 59.55 & 0.0\end{array}$

$\begin{array}{lllllll}\text { cuboid } & 3 & 1 & 4 \mathrm{p} 0.9125 & 59.55 & 0.0\end{array}$

unit 15

com='(Fuel+Clad $+\mathrm{H} 2 \mathrm{O})^{\prime}$

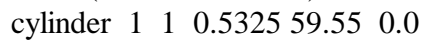

$\begin{array}{llllll}\text { cylinder } & 2 & 1 & 0.6115 & 59.55 & 0.0\end{array}$

$\begin{array}{lllllll}\text { cuboid } & 3 & 1 & 4 \mathrm{p} 0.9125 & 59.55 & 0.0\end{array}$

unit 16

com $=$ '(Fuel+Clad $+\mathrm{H} 2 \mathrm{O})^{\prime}$

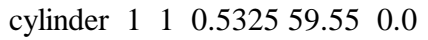

$\begin{array}{lllll}\text { cylinder } 2 & 1 & 0.6115 & 59.55 & 0.0\end{array}$

$\begin{array}{lllllll}\text { cuboid } & 3 & 1 & 4 \mathrm{p} 0.9125 & 59.55 & 0.0\end{array}$

unit 17

com='(Fuel+Clad $+\mathrm{H} 2 \mathrm{O})^{\prime}$

$\begin{array}{llllll}\text { cylinder } & 1 & 1 & 0.5325 & 59.55 & 0.0\end{array}$

$\begin{array}{llllll}\text { cylinder } & 2 & 1 & 0.6115 & 59.55 & 0.0\end{array}$

$\begin{array}{lllllll}\text { cuboid } & 3 & 1 & 4 \mathrm{p} 0.9125 & 59.55 & 0.0\end{array}$

unit 18

com $=$ '(Fuel + Clad $+\mathrm{H} 2 \mathrm{O})^{\prime}$

cylinder 1110.532559 .550 .0

$\begin{array}{llllll}\text { cylinder } 2 & 1 & 0.6115 & 59.55 & 0.0\end{array}$

$\begin{array}{llllll}\text { cuboid } & 3 & 1 & 4 \mathrm{p} 0.9125 & 59.55 & 0.0\end{array}$ unit 19

com $=$ '(Fuel + Clad $+\mathrm{H} 2 \mathrm{O})^{\prime}$

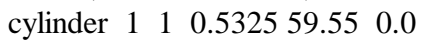

$\begin{array}{llllll}\text { cylinder } & 2 & 1 & 0.6115 & 59.55 & 0.0\end{array}$

$\begin{array}{llllll}\text { cuboid } & 3 & 1 & 4 \mathrm{p} 0.9125 & 59.55 & 0.0\end{array}$

unit 20

com='(Fuel+Clad $+\mathrm{H} 2 \mathrm{O})^{\prime}$

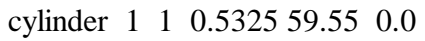

$\begin{array}{llllll}\text { cylinder } 2 & 1 & 0.6115 & 59.55 & 0.0\end{array}$

$\begin{array}{lllllll}\text { cuboid } & 3 & 1 & 4 \mathrm{p} 0.9125 & 59.55 & 0.0\end{array}$

unit 21

com='(Fuel+Clad $+\mathrm{H} 2 \mathrm{O})^{\prime}$

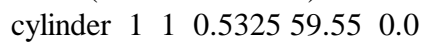

$\begin{array}{llllll}\text { cylinder } & 2 & 1 & 0.6115 & 59.55 & 0.0\end{array}$

$\begin{array}{llllll}\text { cuboid } & 3 & 1 & 4 \mathrm{p} 0.9125 & 59.55 & 0.0\end{array}$

unit 22

com $=$ '(Fuel+Clad $+\mathrm{H} 2 \mathrm{O})^{\prime}$

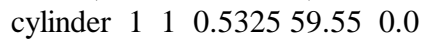

$\begin{array}{llllll}\text { cylinder } & 2 & 1 & 0.6115 & 59.55 & 0.0\end{array}$

$\begin{array}{lllllll}\text { cuboid } & 3 & 1 & 4 \mathrm{p} 0.9125 & 59.55 & 0.0\end{array}$

unit 23

com $=$ '(Fuel + Clad $+\mathrm{H} 2 \mathrm{O})^{\prime}$

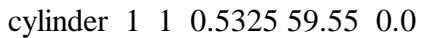

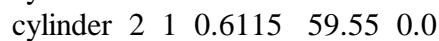

$\begin{array}{llllll}\text { cuboid } & 3 & 1 & 4 \mathrm{p} 0.9125 & 59.55 & 0.0\end{array}$

unit 24

com $=$ '(Fuel + Clad $+\mathrm{H} 2 \mathrm{O})^{\prime}$

cylinder $\begin{array}{lllll}1 & 1 & 0.5325 & 59.55 & 0.0\end{array}$

$\begin{array}{llllll}\text { cylinder } & 2 & 1 & 0.6115 & 59.55 & 0.0\end{array}$

$\begin{array}{llllll}\text { cuboid } & 3 & 1 & 4 \mathrm{p} 0.9125 & 59.55 & 0.0\end{array}$

unit 25

com='(Fuel+Clad $+\mathrm{H} 2 \mathrm{O})^{\prime}$

$\begin{array}{lllll}\text { cylinder } 1 & 1 & 0.5325 & 59.55 & 0.0\end{array}$

$\begin{array}{llllll}\text { cylinder } & 2 & 1 & 0.6115 & 59.55 & 0.0\end{array}$

$\begin{array}{llllll}\text { cuboid } & 3 & 1 & 4 \mathrm{p} 0.9125 & 59.55 & 0.0\end{array}$

unit 26

com='(Fuel+Clad $+\mathrm{H} 2 \mathrm{O})^{\prime}$

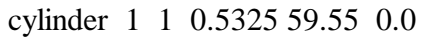

$\begin{array}{llllll}\text { cylinder } 2 & 1 & 0.6115 & 59.55 & 0.0\end{array}$

$\begin{array}{llllll}\text { cuboid } 3 & 1 & 4 \mathrm{p} 0.9125 & 59.55 & 0.0\end{array}$

unit 27

com $=$ '(Fuel $+\mathrm{Clad}+\mathrm{H} 2 \mathrm{O})^{\prime}$

cylinder $\begin{array}{lllll}1 & 1 & 0.5325 & 59.55 & 0.0\end{array}$

$\begin{array}{llllll}\text { cylinder } 2 & 1 & 0.6115 & 59.55 & 0.0\end{array}$

$\begin{array}{lllllll}\text { cuboid } & 3 & 1 & 4 \mathrm{p} 0.9125 & 59.55 & 0.0\end{array}$

unit 28

com $=$ '(Fuel+Clad $+\mathrm{H} 2 \mathrm{O})^{\prime}$

cylinder $\begin{array}{lllll}1 & 1 & 0.5325 & 59.55 & 0.0\end{array}$

$\begin{array}{llllll}\text { cylinder } 2 & 1 & 0.6115 & 59.55 & 0.0\end{array}$

$\begin{array}{lllllll}\text { cuboid } & 3 & 1 & 4 \mathrm{p} 0.9125 & 59.55 & 0.0\end{array}$

unit 29

com $=$ '(Fuel + Clad $+\mathrm{H} 2 \mathrm{O})^{\prime}$

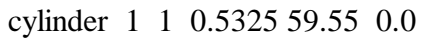

$\begin{array}{llllll}\text { cylinder } 2 & 1 & 0.6115 & 59.55 & 0.0\end{array}$

$\begin{array}{lllllll}\text { cuboid } & 3 & 1 & 4 \mathrm{p} 0.9125 & 59.55 & 0.0\end{array}$

unit 30

com='(Fuel+Clad $+\mathrm{H} 2 \mathrm{O})^{\prime}$ 
$\begin{array}{llllll}\text { cylinder } & 1 & 1 & 0.5325 & 59.55 & 0.0\end{array}$

$\begin{array}{llllll}\text { cylinder } 2 & 1 & 0.6115 & 59.55 & 0.0\end{array}$

$\begin{array}{lllllll}\text { cuboid } & 3 & 1 & 4 \mathrm{p} 0.9125 & 59.55 & 0.0\end{array}$

unit 31

com='(Fuel+Clad $+\mathrm{H} 2 \mathrm{O})^{\prime}$

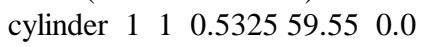

$\begin{array}{llllll}\text { cylinder } & 2 & 1 & 0.6115 & 59.55 & 0.0\end{array}$

$\begin{array}{llllll}\text { cuboid } & 3 & 1 & 4 \mathrm{p} 0.9125 & 59.55 & 0.0\end{array}$

unit 32

com $=$ '(Fuel+Clad $+\mathrm{H} 2 \mathrm{O})^{\prime}$

cylinder $\begin{array}{lllll}1 & 1 & 0.5325 & 59.55 & 0.0\end{array}$

$\begin{array}{llllll}\text { cylinder } & 2 & 1 & 0.6115 & 59.55 & 0.0\end{array}$

$\begin{array}{llllll}\text { cuboid } & 3 & 1 & 4 \mathrm{p} 0.9125 & 59.55 & 0.0\end{array}$

unit 33

com='(Fuel+Clad $+\mathrm{H} 2 \mathrm{O})^{\prime}$

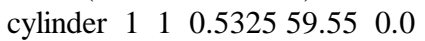

$\begin{array}{llllll}\text { cylinder } & 2 & 1 & 0.6115 & 59.55 & 0.0\end{array}$

$\begin{array}{lllllll}\text { cuboid } & 3 & 1 & 4 \mathrm{p} 0.9125 & 59.55 & 0.0\end{array}$

unit 34

com='(Fuel+Clad $+\mathrm{H} 2 \mathrm{O})^{\prime}$

$\begin{array}{llllll}\text { cylinder } & 1 & 1 & 0.5325 & 59.55 & 0.0\end{array}$

$\begin{array}{llllll}\text { cylinder } & 2 & 1 & 0.6115 & 59.55 & 0.0\end{array}$

$\begin{array}{llllll}\text { cuboid } & 3 & 1 & 4 \mathrm{p} 0.9125 & 59.55 & 0.0\end{array}$

unit 35

com $=$ '(Fuel+Clad $+\mathrm{H} 2 \mathrm{O})^{\prime}$

cylinder $\begin{array}{lllll}1 & 1 & 0.5325 & 59.55 & 0.0\end{array}$

$\begin{array}{llllll}\text { cylinder } 2 & 1 & 0.6115 & 59.55 & 0.0\end{array}$

$\begin{array}{lllllll}\text { cuboid } & 3 & 1 & 4 \mathrm{p} 0.9125 & 59.55 & 0.0\end{array}$

unit 36

com='(Fuel+Clad $+\mathrm{H} 2 \mathrm{O})^{\prime}$

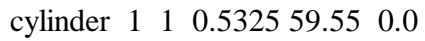

$\begin{array}{llllll}\text { cylinder } 2 & 1 & 0.6115 & 59.55 & 0.0\end{array}$

$\begin{array}{lllllll}\text { cuboid } & 3 & 1 & 4 \mathrm{p} 0.9125 & 59.55 & 0.0\end{array}$

unit 37

com='(Fuel+Clad $+\mathrm{H} 2 \mathrm{O})^{\prime}$

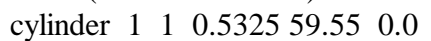

$\begin{array}{llllll}\text { cylinder } & 2 & 1 & 0.6115 & 59.55 & 0.0\end{array}$

$\begin{array}{lllllll}\text { cuboid } & 3 & 1 & 4 \mathrm{p} 0.9125 & 59.55 & 0.0\end{array}$

unit 38

com='(Fuel+Clad $+\mathrm{H} 2 \mathrm{O})^{\prime}$

cylinder $\begin{array}{lllll}1 & 1 & 0.5325 & 59.55 & 0.0\end{array}$

$\begin{array}{llllll}\text { cylinder } 2 & 1 & 0.6115 & 59.55 & 0.0\end{array}$

$\begin{array}{lllllll}\text { cuboid } & 3 & 1 & 4 \mathrm{p} 0.9125 & 59.55 & 0.0\end{array}$

unit 39

com='(Fuel+Clad $+\mathrm{H} 2 \mathrm{O})^{\prime}$

cylinder $\begin{array}{lllll}1 & 1 & 0.5325 & 59.55 & 0.0\end{array}$

$\begin{array}{llllll}\text { cylinder } 2 & 1 & 0.6115 & 59.55 & 0.0\end{array}$

$\begin{array}{lllllll}\text { cuboid } & 3 & 1 & 4 \mathrm{p} 0.9125 & 59.55 & 0.0\end{array}$

unit 40

com='(Fuel+Clad $+\mathrm{H} 2 \mathrm{O})^{\prime}$

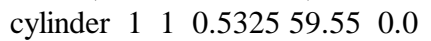

$\begin{array}{llllll}\text { cylinder } & 2 & 1 & 0.6115 & 59.55 & 0.0\end{array}$

$\begin{array}{lllllll}\text { cuboid } & 3 & 1 & 4 \mathrm{p} 0.9125 & 59.55 & 0.0\end{array}$

unit 41

com $=$ '(Fuel+Clad $+\mathrm{H} 2 \mathrm{O})^{\prime}$

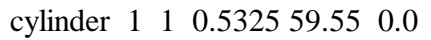

$\begin{array}{llllll}\text { cylinder } 2 & 1 & 0.6115 & 59.55 & 0.0\end{array}$ $\begin{array}{lllllll}\text { cuboid } & 3 & 1 & 4 \mathrm{p} 0.9125 & 59.55 & 0.0\end{array}$

unit 42

com $=$ '(Fuel + Clad $+\mathrm{H} 2 \mathrm{O})^{\prime}$

$\begin{array}{llllll}\text { cylinder } & 1 & 1 & 0.5325 & 59.55 & 0.0\end{array}$

$\begin{array}{llllll}\text { cylinder } 2 & 1 & 0.6115 & 59.55 & 0.0\end{array}$

$\begin{array}{lllllll}\text { cuboid } & 3 & 1 & 4 \mathrm{p} 0.9125 & 59.55 & 0.0\end{array}$

unit 43

com='(Fuel $+\mathrm{Clad}+\mathrm{H} 2 \mathrm{O})^{\prime}$

cylinder $\begin{array}{lllll}1 & 1 & 0.5325 & 59.55 & 0.0\end{array}$

$\begin{array}{llllll}\text { cylinder } 2 & 1 & 0.6115 & 59.55 & 0.0\end{array}$

$\begin{array}{lllllll}\text { cuboid } & 3 & 1 & 4 \mathrm{p} 0.9125 & 59.55 & 0.0\end{array}$

unit 44

com $=$ '(Fuel $+\mathrm{Clad}+\mathrm{H} 2 \mathrm{O})^{\prime}$

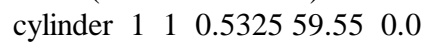

$\begin{array}{lllllll}\text { cylinder } & 2 & 1 & 0.6115 & 59.55 & 0.0\end{array}$

$\begin{array}{lllllll}\text { cuboid } & 3 & 1 & 4 \mathrm{p} 0.9125 & 59.55 & 0.0\end{array}$

unit 45

com='(Fuel+Clad $+\mathrm{H} 2 \mathrm{O})^{\prime}$

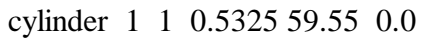

$\begin{array}{lllll}\text { cylinder } 2 & 1 & 0.6115 & 59.55 & 0.0\end{array}$

$\begin{array}{llllll}\text { cuboid } 3 & 1 & 4 \mathrm{p} 0.9125 & 59.55 & 0.0\end{array}$

unit 46

com='(Fuel+Clad+H2O)'

cylinder $\begin{array}{lllll}1 & 1 & 0.5325 & 59.55 & 0.0\end{array}$

$\begin{array}{llllll}\text { cylinder } 2 & 1 & 0.6115 & 59.55 & 0.0\end{array}$

$\begin{array}{lllllll}\text { cuboid } & 3 & 1 & 4 \mathrm{p} 0.9125 & 59.55 & 0.0\end{array}$

unit 47

com $=$ '(Fuel + Clad $+\mathrm{H} 2 \mathrm{O})^{\prime}$

cylinder $\begin{array}{lllll}1 & 1 & 0.5325 & 59.55 & 0.0\end{array}$

$\begin{array}{llllll}\text { cylinder } & 2 & 1 & 0.6115 & 59.55 & 0.0\end{array}$

$\begin{array}{lllllll}\text { cuboid } & 3 & 1 & 4 \mathrm{p} 0.9125 & 59.55 & 0.0\end{array}$

unit 48

com $=$ '(Fuel+Clad $+\mathrm{H} 2 \mathrm{O})^{\prime}$

cylinder 1110.532559 .550 .0

$\begin{array}{llllll}\text { cylinder } & 2 & 1 & 0.6115 & 59.55 & 0.0\end{array}$

$\begin{array}{lllllll}\text { cuboid } & 3 & 1 & 4 \mathrm{p} 0.9125 & 59.55 & 0.0\end{array}$

unit 49

com $=$ '(Fuel $+\mathrm{Clad}+\mathrm{H} 2 \mathrm{O})^{\prime}$

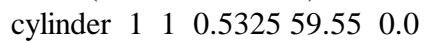

$\begin{array}{llllll}\text { cylinder } 2 & 1 & 0.6115 & 59.55 & 0.0\end{array}$

$\begin{array}{lllllll}\text { cuboid } & 3 & 1 & 4 \mathrm{p} 0.9125 & 59.55 & 0.0\end{array}$

unit 50

com $=$ '(Fuel+Clad $+\mathrm{H} 2 \mathrm{O})^{\prime}$

$\begin{array}{lllll}\text { cylinder } 1 & 1 & 0.5325 & 59.55 & 0.0\end{array}$

$\begin{array}{llllll}\text { cylinder } 2 & 1 & 0.6115 & 59.55 & 0.0\end{array}$

$\begin{array}{llllll}\text { cuboid } & 3 & 1 & 4 \mathrm{p} 0.9125 & 59.55 & 0.0\end{array}$

unit 51

com $=$ '(Fuel + Clad $+\mathrm{H} 2 \mathrm{O})^{\prime}$

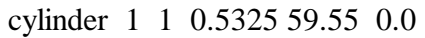

$\begin{array}{llllll}\text { cylinder } & 2 & 1 & 0.6115 & 59.55 & 0.0\end{array}$

$\begin{array}{llllll}\text { cuboid } & 3 & 1 & 4 \mathrm{p} 0.9125 & 59.55 & 0.0\end{array}$

unit 52

com='(Fuel+Clad $+\mathrm{H} 2 \mathrm{O})^{\prime}$

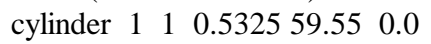

$\begin{array}{llllll}\text { cylinder } 2 & 1 & 0.6115 & 59.55 & 0.0\end{array}$

$\begin{array}{lllllll}\text { cuboid } 3 & 1 & 4 \mathrm{p} 0.9125 & 59.55 & 0.0\end{array}$

unit 53 
com='(Fuel+Clad+H2O)'

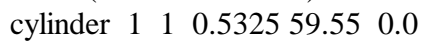

$\begin{array}{llllll}\text { cylinder } & 2 & 1 & 0.6115 & 59.55 & 0.0\end{array}$

$\begin{array}{lllllll}\text { cuboid } & 3 & 1 & 4 \mathrm{p} 0.9125 & 59.55 & 0.0\end{array}$

unit 54

com $=$ '(Fuel + Clad $+\mathrm{H} 2 \mathrm{O})^{\prime}$

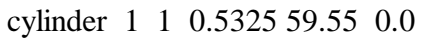

$\begin{array}{llllll}\text { cylinder } & 2 & 1 & 0.6115 & 59.55 & 0.0\end{array}$

$\begin{array}{llllll}\text { cuboid } & 3 & 1 & 4 \mathrm{p} 0.9125 & 59.55 & 0.0\end{array}$

unit 55

com $=$ '(Fuel + Clad $+\mathrm{H} 2 \mathrm{O})^{\prime}$

cylinder 1110.532559 .550 .0

$\begin{array}{llllll}\text { cylinder } 2 & 1 & 0.6115 & 59.55 & 0.0\end{array}$

$\begin{array}{lllllll}\text { cuboid } & 3 & 1 & 4 \mathrm{p} 0.9125 & 59.55 & 0.0\end{array}$

unit 56

com $=$ '(Fuel + Clad $+\mathrm{H} 2 \mathrm{O})^{\prime}$

cylinder $\begin{array}{lllll}1 & 1 & 0.5325 & 59.55 & 0.0\end{array}$

$\begin{array}{llllll}\text { cylinder } & 2 & 1 & 0.6115 & 59.55 & 0.0\end{array}$

$\begin{array}{llllll}\text { cuboid } & 3 & 1 & 4 \mathrm{p} 0.9125 & 59.55 & 0.0\end{array}$

unit 57

com $=$ '(Fuel + Clad $+\mathrm{H} 2 \mathrm{O})^{\prime}$

$\begin{array}{llllll}\text { cylinder } & 1 & 1 & 0.5325 & 59.55 & 0.0\end{array}$

$\begin{array}{llllll}\text { cylinder } & 2 & 1 & 0.6115 & 59.55 & 0.0\end{array}$

$\begin{array}{llllll}\text { cuboid } & 3 & 1 & 4 \mathrm{p} 0.9125 & 59.55 & 0.0\end{array}$

unit 58

com $=$ '(Fuel + Clad $+\mathrm{H} 2 \mathrm{O})^{\prime}$

cylinder $1110.532559 .55 \quad 0.0$

$\begin{array}{llllll}\text { cylinder } & 2 & 1 & 0.6115 & 59.55 & 0.0\end{array}$

$\begin{array}{llllll}\text { cuboid } & 3 & 1 & 4 \mathrm{p} 0.9125 & 59.55 & 0.0\end{array}$

unit 59

com='(Fuel+Clad $+\mathrm{H} 2 \mathrm{O})^{\prime}$

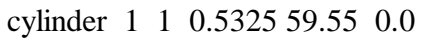

$\begin{array}{llllll}\text { cylinder } 2 & 1 & 0.6115 & 59.55 & 0.0\end{array}$

$\begin{array}{lllllll}\text { cuboid } & 3 & 1 & 4 \mathrm{p} 0.9125 & 59.55 & 0.0\end{array}$

unit 60

com $=$ '(Fuel + Clad $+\mathrm{H} 2 \mathrm{O})^{\prime}$

$\begin{array}{llllll}\text { cylinder } & 1 & 1 & 0.5325 & 59.55 & 0.0\end{array}$

$\begin{array}{llllll}\text { cylinder } 2 & 1 & 0.6115 & 59.55 & 0.0\end{array}$

$\begin{array}{lllllll}\text { cuboid } & 3 & 1 & 4 \mathrm{p} 0.9125 & 59.55 & 0.0\end{array}$

unit 61

com='(Fuel+Clad $+\mathrm{H} 2 \mathrm{O})^{\prime}$

cylinder $\begin{array}{lllll}1 & 1 & 0.5325 & 59.55 & 0.0\end{array}$

$\begin{array}{lllll}\text { cylinder } 2 & 1 & 0.6115 & 59.55 & 0.0\end{array}$

$\begin{array}{lllllll}\text { cuboid } & 3 & 1 & 4 \mathrm{p} 0.9125 & 59.55 & 0.0\end{array}$

unit 62

com='(Fuel+Clad $+\mathrm{H} 2 \mathrm{O})^{\prime}$

cylinder $1110.532559 .55 \quad 0.0$

$\begin{array}{llllll}\text { cylinder } 2 & 1 & 0.6115 & 59.55 & 0.0\end{array}$

$\begin{array}{lllllll}\text { cuboid } & 3 & 1 & 4 \mathrm{p} 0.9125 & 59.55 & 0.0\end{array}$

unit 63

com $=$ '(Fuel $+\mathrm{Clad}+\mathrm{H} 2 \mathrm{O})^{\prime}$

$\begin{array}{llllll}\text { cylinder } & 1 & 1 & 0.5325 & 59.55 & 0.0\end{array}$

$\begin{array}{llllll}\text { cylinder } 2 & 1 & 0.6115 & 59.55 & 0.0\end{array}$

$\begin{array}{lllllll}\text { cuboid } & 3 & 1 & 4 \mathrm{p} 0.9125 & 59.55 & 0.0\end{array}$

unit 64

com $=$ '(Fuel+Clad $+\mathrm{H} 2 \mathrm{O})^{\prime}$

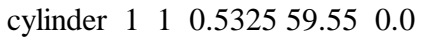

$\begin{array}{llllll}\text { cylinder } & 2 & 1 & 0.6115 & 59.55 & 0.0\end{array}$

$\begin{array}{lllllll}\text { cuboid } & 3 & 1 & 4 \mathrm{p} 0.9125 & 59.55 & 0.0\end{array}$

unit 65

com $=$ '(Fuel $+\mathrm{Clad}+\mathrm{H} 2 \mathrm{O})^{\prime}$

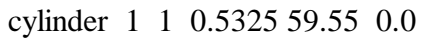

$\begin{array}{llllll}\text { cylinder } & 2 & 1 & 0.6115 & 59.55 & 0.0\end{array}$

$\begin{array}{lllllll}\text { cuboid } & 3 & 1 & 4 \mathrm{p} 0.9125 & 59.55 & 0.0\end{array}$

unit 66

com='(Fuel+Clad $+\mathrm{H} 2 \mathrm{O})^{\prime}$

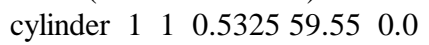

$\begin{array}{llllll}\text { cylinder } 2 & 1 & 0.6115 & 59.55 & 0.0\end{array}$

$\begin{array}{lllllll}\text { cuboid } & 3 & 1 & 4 \mathrm{p} 0.9125 & 59.55 & 0.0\end{array}$

unit 67

com $='(\text { Fuel }+\mathrm{Clad}+\mathrm{H} 2 \mathrm{O})^{\prime}$

cylinder $\begin{array}{lllll}1 & 1 & 0.5325 & 59.55 & 0.0\end{array}$

$\begin{array}{llllll}\text { cylinder } 2 & 1 & 0.6115 & 59.55 & 0.0\end{array}$

$\begin{array}{lllllll}\text { cuboid } & 3 & 1 & 4 \mathrm{p} 0.9125 & 59.55 & 0.0\end{array}$

unit 68

com='(Fuel $+\mathrm{Clad}+\mathrm{H} 2 \mathrm{O})^{\prime}$

$\begin{array}{llllll}\text { cylinder } & 1 & 1 & 0.5325 & 59.55 & 0.0\end{array}$

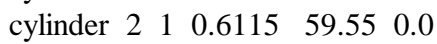

$\begin{array}{lllllll}\text { cuboid } & 3 & 1 & 4 \mathrm{p} 0.9125 & 59.55 & 0.0\end{array}$

unit 69

com $=$ '(Fuel+Clad $+\mathrm{H} 2 \mathrm{O})^{\prime}$

$\begin{array}{llllll}\text { cylinder } & 1 & 1 & 0.5325 & 59.55 & 0.0\end{array}$

$\begin{array}{llllll}\text { cylinder } & 2 & 1 & 0.6115 & 59.55 & 0.0\end{array}$

$\begin{array}{lllllll}\text { cuboid } & 3 & 1 & 4 \mathrm{p} 0.9125 & 59.55 & 0.0\end{array}$

unit 70

com='(Fuel+Clad $+\mathrm{H} 2 \mathrm{O})^{\prime}$

$\begin{array}{llllll}\text { cylinder } & 1 & 1 & 0.5325 & 59.55 & 0.0\end{array}$

$\begin{array}{lllll}\text { cylinder } 2 & 1 & 0.6115 & 59.55 & 0.0\end{array}$

$\begin{array}{lllllll}\text { cuboid } & 3 & 1 & 4 \mathrm{p} 0.9125 & 59.55 & 0.0\end{array}$

unit 71

com $=$ '(Fuel+Clad $+\mathrm{H} 2 \mathrm{O})^{\prime}$

cylinder $\begin{array}{lllll}1 & 1 & 0.5325 & 59.55 & 0.0\end{array}$

$\begin{array}{llllll}\text { cylinder } 2 & 1 & 0.6115 & 59.55 & 0.0\end{array}$

$\begin{array}{lllllll}\text { cuboid } & 3 & 1 & 4 \mathrm{p} 0.9125 & 59.55 & 0.0\end{array}$

unit 72

com $=$ '(Fuel $+\mathrm{Clad}+\mathrm{H} 2 \mathrm{O})^{\prime}$

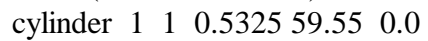

$\begin{array}{llllll}\text { cylinder } 2 & 1 & 0.6115 & 59.55 & 0.0\end{array}$

$\begin{array}{lllllll}\text { cuboid } & 3 & 1 & 4 \mathrm{p} 0.9125 & 59.55 & 0.0\end{array}$

unit 73

com='(Fuel+Clad $+\mathrm{H} 2 \mathrm{O})^{\prime}$

$\begin{array}{llllll}\text { cylinder } & 1 & 1 & 0.5325 & 59.55 & 0.0\end{array}$

$\begin{array}{lllll}\text { cylinder } 2 & 1 & 0.6115 & 59.55 & 0.0\end{array}$

$\begin{array}{lllllll}\text { cuboid } & 3 & 1 & 4 \mathrm{p} 0.9125 & 59.55 & 0.0\end{array}$

unit 74

com $=$ '(Fuel + Clad $+\mathrm{H} 2 \mathrm{O})^{\prime}$

cylinder $\begin{array}{lllll}1 & 1 & 0.5325 & 59.55 & 0.0\end{array}$

$\begin{array}{llllll}\text { cylinder } & 2 & 1 & 0.6115 & 59.55 & 0.0\end{array}$

$\begin{array}{lllllll}\text { cuboid } & 3 & 1 & 4 \mathrm{p} 0.9125 & 59.55 & 0.0\end{array}$

unit 75

com $=$ '(Fuel + Clad $+\mathrm{H} 2 \mathrm{O})^{\prime}$

cylinder $\begin{array}{lllll}1 & 1 & 0.5325 & 59.55 & 0.0\end{array}$

$\begin{array}{llllll}\text { cylinder } & 2 & 1 & 0.6115 & 59.55 & 0.0\end{array}$

$\begin{array}{lllllll}\text { cuboid } & 3 & 1 & 4 \mathrm{p} 0.9125 & 59.55 & 0.0\end{array}$ 
unit 76

com $=$ '(Fuel + Clad $+\mathrm{H} 2 \mathrm{O})^{\prime}$

cylinder $\begin{array}{lllll}1 & 1 & 0.5325 & 59.55 & 0.0\end{array}$

$\begin{array}{llllll}\text { cylinder } 2 & 1 & 0.6115 & 59.55 & 0.0\end{array}$

$\begin{array}{lllllll}\text { cuboid } & 3 & 1 & 4 \mathrm{p} 0.9125 & 59.55 & 0.0\end{array}$

unit 77

com $='(\text { Fuel }+ \text { Clad }+\mathrm{H} 2 \mathrm{O})^{\prime}$

cylinder $1 \quad 1 \quad 0.532559 .55 \quad 0.0$

$\begin{array}{lllll}\text { cylinder } 2 & 1 & 0.6115 & 59.55 & 0.0\end{array}$

$\begin{array}{lllllll}\text { cuboid } & 3 & 1 & 4 \mathrm{p} 0.9125 & 59.55 & 0.0\end{array}$

unit 78

com='(Fuel+Clad $+\mathrm{H} 2 \mathrm{O})^{\prime}$

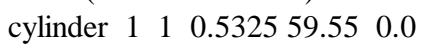

$\begin{array}{llllll}\text { cylinder } & 2 & 1 & 0.6115 & 59.55 & 0.0\end{array}$

$\begin{array}{lllllll}\text { cuboid } & 3 & 1 & 4 \mathrm{p} 0.9125 & 59.55 & 0.0\end{array}$

unit 81

com='h2o and end plug below Al grid plate - fill array 1'

$\begin{array}{lllll}\text { cylinder } 4 & 1 & 0.6115 & 4.445 & 0.0\end{array}$

$\begin{array}{lllllll}\text { cuboid } 3 & 1 & 4 \mathrm{p} .9125 & 4.445 & 0.0\end{array}$

unit 82

com='h2o and end plug above $\mathrm{Al}$ grid plate - fill array 2'

$\begin{array}{lllll}\text { cylinder } 4 & 1 & 0.6115 & 11.784 & 0.0\end{array}$

$\begin{array}{lllllll}\text { cuboid } 3 & 1 & 4 p .9125 & 11.784 & 0.0\end{array}$

unit 83

com='(Fuel+Clad+Void) - fill array 3'

$\begin{array}{llllll}\text { cylinder } & 1 & 1 & .5325 & 11.05 & 0.0\end{array}$

$\begin{array}{lllll}\text { cylinder } 2 & 1 & 6115 & 11.05 & 0.0\end{array}$

cuboid 014 p.9125 $11.05 \quad 0.0$

unit 84

com='(void+Clad+void $)^{\prime}$

cylinder $\begin{array}{lllll}0 & 1 & 0.5325 & 9.97 & 0.0\end{array}$

$\begin{array}{lllll}\text { cylinder } 2 & 1 & 0.6115 & 9.97 & 0.0\end{array}$

$\begin{array}{lllllll}\text { cuboid } & 0 & 1 & 4 \mathrm{p} 0.9125 & 9.97 & 0.0\end{array}$

unit 100

com='Lower Al grid plate'

cuboid $414 \mathrm{p} 50.9875 .601 \quad 0.0$

unit 101

com='array of h2o and end plugs below Al grid plate'

array $1-20.9875-20.98750 .0$

cuboid $314 \mathrm{p} 50.9875 \quad 4.445 \quad 0.0$

unit 102

com='array of h2o and end plugs above Al grid plate '

array $2-20.9875-20.98750 .0$

$\begin{array}{lllll}\text { cuboid } 3 & 1 & 4 p 50.9875 & 11.784 & 0.0\end{array}$

unit 103

com='array of Fuel+Clad+Void'

array $3-20.9875-20.98750 .0$

cuboid 01 4p50.9875 $11.05 \quad 0.0$

unit 104

com='array of void+Clad+void'

array $4-20.9875-20.98750 .0$

cuboid 014 p50.9875 $9.97 \quad 0.0$

unit 105

com='array of Fuel+Clad+H2O'

array $5-20.9875-20.98750 .0$

cuboid 31 4p50.9875 59.550 .0

global unit 110 com='array of arrays of fuel element sections + reflector'

array $6-50.9875-50.9875 \quad 0$

cuboid $4 \begin{array}{lllll}4 & 4 p 50.9875 & 97.40 & -1.27\end{array}$

cuboid $5 \begin{array}{lllll}5 & 4 p 50.9875 & 97.40 & -3.47\end{array}$

cuboid $3 \quad 1 \quad 4 p 50.9875 \quad 97.40 \quad-17.27$

$\begin{array}{lllllll}\text { cuboid } 5 & 1 & 4 p 50.9875 & 97.40 & -17.77\end{array}$

cuboid $6 \begin{array}{lllll}6 & 4 p 50.9875 & 97.40 & -54.77\end{array}$

end geometry

read array

ara $=1$ nux=23 nuy=23 nuz=1

fill f81 end fill

ara $=2$ nux $=23$ nuy=23 nuz $=1$

fill $\mathrm{f} 82$ end fill

ara $=3$ nux=23 nuy=23 nuz $=1$

fill $\mathrm{f} 83$ end fill

ara $=4$ nux $=23$ nuy=23 nuz=1

fill $\mathrm{f} 84$ end fill

ara $=5$ nux $=23$ nuy=23 nuz $=1$

fill

$\begin{array}{llllllllllllll}1 & 2 & 3 & 4 & 5 & 6 & 7 & 8 & 9 & 10 & 11 & 12 & 1 \mathrm{~b} & 11\end{array}$

$21314151617181920212223 \quad 1 b 11$

$31424252627282930313233 \quad 1 b 11$

$41525343536373839404142 \quad 1 b 11$

51626354344454647484950 1b 11

61727364451525354555657 1b 11

71828374552585960616263 1b 11

81929384653596465666768 1b 11

92030394754606569707172 1b 11

102131404855616670737475 1b 11

112232414956626771747677 1b 11

122333425057636872757778 1b 264 end fill

ara $=6$ nux $=1$ nuy=1 nuz=6

fill 101100102105103104 end fill

end array

end data

end

$=$ shell

cp ft04f001 \$RTNDIR/tca01.xs04 cp ft64f001 \$RTNDIR/tca01.rs64

end

$=\mathrm{kmart}$

read initial kunit $=64$

xunit $=4$

actbygrp

end initial

rrpvol

read activity

92234279223418922341452

92235279223518922351452

$92238279223818 \quad 92238 \quad 1452$

94238279423818942381452

94239279423918942391452

94240279424018942401452

$9424127942411894241 \quad 1452$

$9424227942421894242 \quad 1452$

$9524127952411895241 \quad 1452$ 


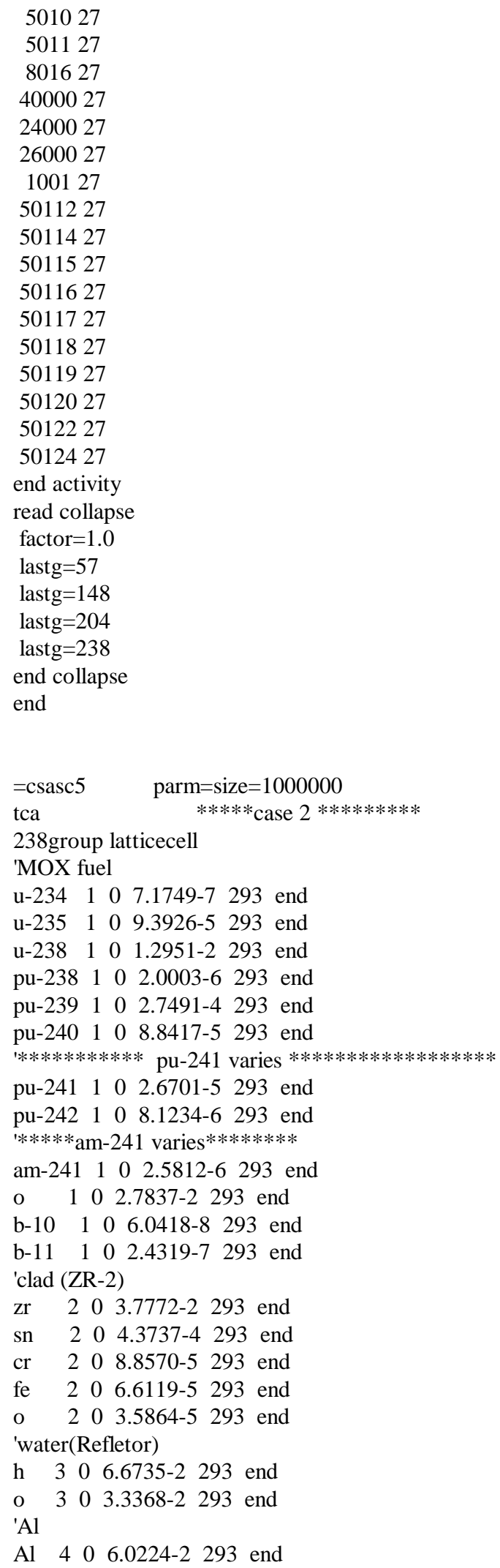


unit 6

com $=$ '(Fuel $+\mathrm{Clad}+\mathrm{H} 2 \mathrm{O})^{\prime}$

$\begin{array}{llllll}\text { cylinder } & 1 & 1 & 0.5325 & 61.90 & 0.0\end{array}$

$\begin{array}{llllll}\text { cylinder } 2 & 1 & 0.6115 & 61.90 & 0.0\end{array}$

$\begin{array}{llllll}\text { cuboid } & 3 & 1 & 4 \mathrm{p} 0.9125 & 61.90 & 0.0\end{array}$

unit 7

com $=$ '(Fuel $+\mathrm{Clad}+\mathrm{H} 2 \mathrm{O})^{\prime}$

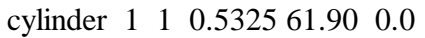

$\begin{array}{llllll}\text { cylinder } 2 & 1 & 0.6115 & 61.90 & 0.0\end{array}$

$\begin{array}{lllllll}\text { cuboid } & 3 & 1 & 4 \mathrm{p} 0.9125 & 61.90 & 0.0\end{array}$

unit 8

com='(Fuel+Clad $+\mathrm{H} 2 \mathrm{O})^{\prime}$

$\begin{array}{llllll}\text { cylinder } & 1 & 1 & 0.5325 & 61.90 & 0.0\end{array}$

$\begin{array}{llllll}\text { cylinder } & 2 & 1 & 0.6115 & 61.90 & 0.0\end{array}$

$\begin{array}{lllllll}\text { cuboid } & 3 & 1 & 4 \mathrm{p} 0.9125 & 61.90 & 0.0\end{array}$

unit 9

com='(Fuel+Clad $+\mathrm{H} 2 \mathrm{O})^{\prime}$

cylinder $\begin{array}{lllll}1 & 1 & 0.5325 & 61.90 & 0.0\end{array}$

$\begin{array}{llllll}\text { cylinder } 2 & 1 & 0.6115 & 61.90 & 0.0\end{array}$

$\begin{array}{llllll}\text { cuboid } & 3 & 1 & 4 \mathrm{p} 0.9125 & 61.90 & 0.0\end{array}$

unit 10

com $=$ '(Fuel $+\mathrm{Clad}+\mathrm{H} 2 \mathrm{O})^{\prime}$

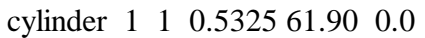

$\begin{array}{llllll}\text { cylinder } 2 & 1 & 0.6115 & 61.90 & 0.0\end{array}$

$\begin{array}{lllllll}\text { cuboid } & 3 & 1 & 4 \mathrm{p} 0.9125 & 61.90 & 0.0\end{array}$

unit 11

com='(Fuel+Clad $+\mathrm{H} 2 \mathrm{O})^{\prime}$

$\begin{array}{llllll}\text { cylinder } & 1 & 1 & 0.5325 & 61.90 & 0.0\end{array}$

$\begin{array}{llllll}\text { cylinder } & 2 & 1 & 0.6115 & 61.90 & 0.0\end{array}$

$\begin{array}{llllll}\text { cuboid } & 3 & 1 & 4 \mathrm{p} 0.9125 & 61.90 & 0.0\end{array}$

unit 12

com='(Fuel+Clad $+\mathrm{H} 2 \mathrm{O})^{\prime}$

$\begin{array}{llllll}\text { cylinder } & 1 & 1 & 0.5325 & 61.90 & 0.0\end{array}$

$\begin{array}{llllll}\text { cylinder } & 2 & 1 & 0.6115 & 61.90 & 0.0\end{array}$

$\begin{array}{lllllll}\text { cuboid } & 3 & 1 & 4 \mathrm{p} 0.9125 & 61.90 & 0.0\end{array}$

unit 13

com='(Fuel+Clad $+\mathrm{H} 2 \mathrm{O})^{\prime}$

$\begin{array}{llllll}\text { cylinder } & 1 & 1 & 0.5325 & 61.90 & 0.0\end{array}$

$\begin{array}{llllll}\text { cylinder } 2 & 1 & 0.6115 & 61.90 & 0.0\end{array}$

$\begin{array}{lllllll}\text { cuboid } & 3 & 1 & 4 \mathrm{p} 0.9125 & 61.90 & 0.0\end{array}$

unit 14

com='(Fuel+Clad $+\mathrm{H} 2 \mathrm{O})^{\prime}$

$\begin{array}{llllll}\text { cylinder } & 1 & 1 & 0.5325 & 61.90 & 0.0\end{array}$

$\begin{array}{llllll}\text { cylinder } 2 & 1 & 0.6115 & 61.90 & 0.0\end{array}$

$\begin{array}{lllllll}\text { cuboid } & 3 & 1 & 4 \mathrm{p} 0.9125 & 61.90 & 0.0\end{array}$

unit 15

com $=$ '(Fuel+Clad $+\mathrm{H} 2 \mathrm{O})^{\prime}$

cylinder $\begin{array}{lllll}1 & 1 & 0.5325 & 61.90 & 0.0\end{array}$

$\begin{array}{llllll}\text { cylinder } 2 & 1 & 0.6115 & 61.90 & 0.0\end{array}$

$\begin{array}{lllllll}\text { cuboid } & 3 & 1 & 4 \mathrm{p} 0.9125 & 61.90 & 0.0\end{array}$

unit 16

com $=$ '(Fuel $+\mathrm{Clad}+\mathrm{H} 2 \mathrm{O})^{\prime}$

cylinder $1110.532561 .90 \quad 0.0$

$\begin{array}{llllll}\text { cylinder } 2 & 1 & 0.6115 & 61.90 & 0.0\end{array}$

$\begin{array}{lllllll}\text { cuboid } & 3 & 1 & 4 \mathrm{p} 0.9125 & 61.90 & 0.0\end{array}$

unit 17

com='(Fuel+Clad $+\mathrm{H} 2 \mathrm{O})^{\prime}$ $\begin{array}{llllll}\text { cylinder } & 1 & 1 & 0.5325 & 61.90 & 0.0\end{array}$

$\begin{array}{llllll}\text { cylinder } & 2 & 1 & 0.6115 & 61.90 & 0.0\end{array}$

$\begin{array}{lllllll}\text { cuboid } & 3 & 1 & 4 \mathrm{p} 0.9125 & 61.90 & 0.0\end{array}$

unit 18

com='(Fuel+Clad $+\mathrm{H} 2 \mathrm{O})^{\prime}$

$\begin{array}{llllll}\text { cylinder } & 1 & 1 & 0.5325 & 61.90 & 0.0\end{array}$

$\begin{array}{llllll}\text { cylinder } & 2 & 1 & 0.6115 & 61.90 & 0.0\end{array}$

$\begin{array}{llllll}\text { cuboid } & 3 & 1 & 4 \mathrm{p} 0.9125 & 61.90 & 0.0\end{array}$

unit 19

com='(Fuel+Clad $+\mathrm{H} 2 \mathrm{O})^{\prime}$

cylinder $\begin{array}{lllll}1 & 1 & 0.5325 & 61.90 & 0.0\end{array}$

$\begin{array}{llllll}\text { cylinder } 2 & 1 & 0.6115 & 61.90 & 0.0\end{array}$

$\begin{array}{lllllll}\text { cuboid } \quad 3 & 1 & 4 \mathrm{p} 0.9125 & 61.90 & 0.0\end{array}$

unit 20

com='(Fuel+Clad $+\mathrm{H} 2 \mathrm{O})^{\prime}$

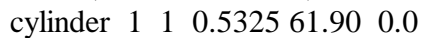

$\begin{array}{llllll}\text { cylinder } 2 & 1 & 0.6115 & 61.90 & 0.0\end{array}$

$\begin{array}{llllll}\text { cuboid } & 3 & 1 & 4 \mathrm{p} 0.9125 & 61.90 & 0.0\end{array}$

unit 21

com='(Fuel+Clad $+\mathrm{H} 2 \mathrm{O})^{\prime}$

cylinder $1110.532561 .90 \quad 0.0$

$\begin{array}{llllll}\text { cylinder } 2 & 1 & 0.6115 & 61.90 & 0.0\end{array}$

$\begin{array}{lllllll}\text { cuboid } & 3 & 1 & 4 \mathrm{p} 0.9125 & 61.90 & 0.0\end{array}$

unit 22

com='(Fuel+Clad $+\mathrm{H} 2 \mathrm{O})^{\prime}$

cylinder $\begin{array}{lllll}1 & 1 & 0.5325 & 61.90 & 0.0\end{array}$

$\begin{array}{llllll}\text { cylinder } 2 & 1 & 0.6115 & 61.90 & 0.0\end{array}$

$\begin{array}{lllllll}\text { cuboid } & 3 & 1 & 4 \mathrm{p} 0.9125 & 61.90 & 0.0\end{array}$

unit 23

com $=(\text { Fuel }+ \text { Clad }+\mathrm{H} 2 \mathrm{O})^{\prime}$

cylinder $\begin{array}{lllll}1 & 1 & 0.5325 & 61.90 & 0.0\end{array}$

$\begin{array}{llllll}\text { cylinder } 2 & 1 & 0.6115 & 61.90 & 0.0\end{array}$

$\begin{array}{llllll}\text { cuboid } & 3 & 1 & 4 \mathrm{p} 0.9125 & 61.90 & 0.0\end{array}$

unit 24

com $=$ '(Fuel + Clad $+\mathrm{H} 2 \mathrm{O})^{\prime}$

$\begin{array}{llllll}\text { cylinder } & 1 & 1 & 0.5325 & 61.90 & 0.0\end{array}$

$\begin{array}{llllll}\text { cylinder } & 2 & 1 & 0.6115 & 61.90 & 0.0\end{array}$

$\begin{array}{lllllll}\text { cuboid } & 3 & 1 & 4 \mathrm{p} 0.9125 & 61.90 & 0.0\end{array}$

unit 25

com='(Fuel+Clad $+\mathrm{H} 2 \mathrm{O})^{\prime}$

cylinder $1110.532561 .90 \quad 0.0$

$\begin{array}{lllll}\text { cylinder } 2 & 1 & 0.6115 & 61.90 & 0.0\end{array}$

$\begin{array}{lllllll}\text { cuboid } & 3 & 1 & 4 \mathrm{p} 0.9125 & 61.90 & 0.0\end{array}$

unit 26

com $=$ '(Fuel+Clad $+\mathrm{H} 2 \mathrm{O})^{\prime}$

cylinder $\begin{array}{lllll}1 & 1 & 0.5325 & 61.90 & 0.0\end{array}$

$\begin{array}{llllll}\text { cylinder } & 2 & 1 & 0.6115 & 61.90 & 0.0\end{array}$

$\begin{array}{lllllll}\text { cuboid } & 3 & 1 & 4 \mathrm{p} 0.9125 & 61.90 & 0.0\end{array}$

unit 27

com $=$ '(Fuel + Clad $+\mathrm{H} 2 \mathrm{O})^{\prime}$

cylinder $\begin{array}{lllll}1 & 1 & 0.5325 & 61.90 & 0.0\end{array}$

$\begin{array}{lllllll}\text { cylinder } & 2 & 1 & 0.6115 & 61.90 & 0.0\end{array}$

$\begin{array}{lllllll}\text { cuboid } & 3 & 1 & 4 \mathrm{p} 0.9125 & 61.90 & 0.0\end{array}$

unit 28

com $='(\text { Fuel }+\mathrm{Clad}+\mathrm{H} 2 \mathrm{O})^{\prime}$

$\begin{array}{llllll}\text { cylinder } & 1 & 1 & 0.5325 & 61.90 & 0.0\end{array}$

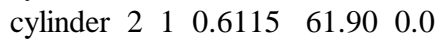


$\begin{array}{lllllll}\text { cuboid } \quad 3 & 1 & 4 p 0.9125 & 61.90 & 0.0\end{array}$

unit 29

com $='(\text { Fuel }+ \text { Clad }+\mathrm{H} 2 \mathrm{O})^{\prime}$

$\begin{array}{llllll}\text { cylinder } & 1 & 1 & 0.5325 & 61.90 & 0.0\end{array}$

$\begin{array}{llllll}\text { cylinder } 2 & 1 & 0.6115 & 61.90 & 0.0\end{array}$

$\begin{array}{lllllll}\text { cuboid } & 3 & 1 & 4 \mathrm{p} 0.9125 & 61.90 & 0.0\end{array}$

unit 30

com='(Fuel+Clad $+\mathrm{H} 2 \mathrm{O})^{\prime}$

cylinder $\begin{array}{lllll}1 & 1 & 0.5325 & 61.90 & 0.0\end{array}$

$\begin{array}{llllll}\text { cylinder } 2 & 1 & 0.6115 & 61.90 & 0.0\end{array}$

$\begin{array}{lllllll}\text { cuboid } & 3 & 1 & 4 \mathrm{p} 0.9125 & 61.90 & 0.0\end{array}$

unit 31

com $=$ '(Fuel + Clad $+\mathrm{H} 2 \mathrm{O})^{\prime}$

cylinder $\begin{array}{llllll}1 & 1 & 0.5325 & 61.90 & 0.0\end{array}$

$\begin{array}{llllll}\text { cylinder } & 2 & 1 & 0.6115 & 61.90 & 0.0\end{array}$

$\begin{array}{lllllll}\text { cuboid } & 3 & 1 & 4 \mathrm{p} 0.9125 & 61.90 & 0.0\end{array}$

unit 32

com='(Fuel+Clad $+\mathrm{H} 2 \mathrm{O})^{\prime}$

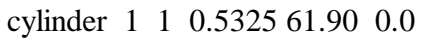

$\begin{array}{llllll}\text { cylinder } 2 & 1 & 0.6115 & 61.90 & 0.0\end{array}$

$\begin{array}{llllll}\text { cuboid } & 3 & 1 & 4 \mathrm{p} 0.9125 & 61.90 & 0.0\end{array}$

unit 33

com $='(\text { Fuel+Clad }+\mathrm{H} 2 \mathrm{O})^{\prime}$

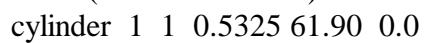

$\begin{array}{llllll}\text { cylinder } & 2 & 1 & 0.6115 & 61.90 & 0.0\end{array}$

$\begin{array}{lllllll}\text { cuboid } & 3 & 1 & 4 \mathrm{p} 0.9125 & 61.90 & 0.0\end{array}$

unit 34

com='(Fuel+Clad $+\mathrm{H} 2 \mathrm{O})^{\prime}$

cylinder $\begin{array}{lllll}1 & 1 & 0.5325 & 61.90 & 0.0\end{array}$

$\begin{array}{lllllll}\text { cylinder } & 2 & 1 & 0.6115 & 61.90 & 0.0\end{array}$

$\begin{array}{lllllll}\text { cuboid } & 3 & 1 & 4 \mathrm{p} 0.9125 & 61.90 & 0.0\end{array}$

unit 35

com $=$ '(Fuel $+\mathrm{Clad}+\mathrm{H} 2 \mathrm{O})^{\prime}$

$\begin{array}{llllll}\text { cylinder } & 1 & 1 & 0.5325 & 61.90 & 0.0\end{array}$

$\begin{array}{llllll}\text { cylinder } 2 & 1 & 0.6115 & 61.90 & 0.0\end{array}$

$\begin{array}{lllllll}\text { cuboid } & 3 & 1 & 4 \mathrm{p} 0.9125 & 61.90 & 0.0\end{array}$

unit 36

com='(Fuel+Clad $+\mathrm{H} 2 \mathrm{O})^{\prime}$

cylinder $\begin{array}{lllll}1 & 1 & 0.5325 & 61.90 & 0.0\end{array}$

$\begin{array}{llllll}\text { cylinder } & 2 & 1 & 0.6115 & 61.90 & 0.0\end{array}$

$\begin{array}{lllllll}\text { cuboid } & 3 & 1 & 4 \mathrm{p} 0.9125 & 61.90 & 0.0\end{array}$

unit 37

com $='(\text { Fuel }+ \text { Clad }+\mathrm{H} 2 \mathrm{O})^{\prime}$

$\begin{array}{llllll}\text { cylinder } & 1 & 1 & 0.5325 & 61.90 & 0.0\end{array}$

$\begin{array}{llllll}\text { cylinder } 2 & 1 & 0.6115 & 61.90 & 0.0\end{array}$

$\begin{array}{lllllll}\text { cuboid } & 3 & 1 & 4 \mathrm{p} 0.9125 & 61.90 & 0.0\end{array}$

unit 38

com $=$ '(Fuel $+\mathrm{Clad}+\mathrm{H} 2 \mathrm{O})^{\prime}$

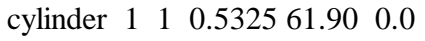

$\begin{array}{llllll}\text { cylinder } 2 & 1 & 0.6115 & 61.90 & 0.0\end{array}$

$\begin{array}{lllllll}\text { cuboid } 3 & 1 & 4 \mathrm{p} 0.9125 & 61.90 & 0.0\end{array}$

unit 39

com='(Fuel+Clad $+\mathrm{H} 2 \mathrm{O})^{\prime}$

cylinder $\begin{array}{lllll}1 & 1 & 0.5325 & 61.90 & 0.0\end{array}$

$\begin{array}{llllll}\text { cylinder } 2 & 1 & 0.6115 & 61.90 & 0.0\end{array}$

$\begin{array}{llllll}\text { cuboid } & 3 & 1 & 4 \mathrm{p} 0.9125 & 61.90 & 0.0\end{array}$

unit 40

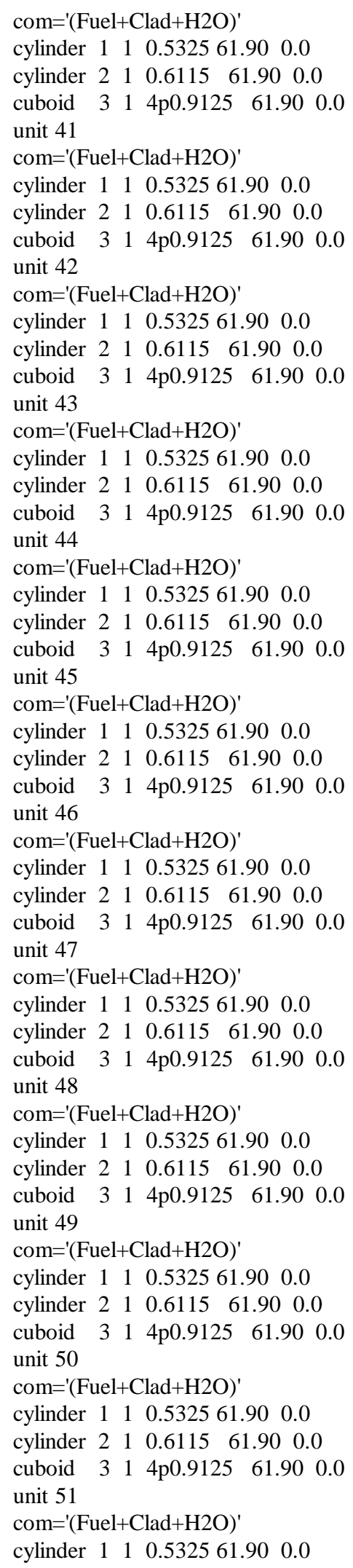


$\begin{array}{llllll}\text { cylinder } 2 & 1 & 0.6115 & 61.90 & 0.0\end{array}$ $\begin{array}{lllllll}\text { cuboid } & 3 & 1 & 4 \mathrm{p} 0.9125 & 61.90 & 0.0\end{array}$ unit 52

com='(Fuel+Clad $+\mathrm{H} 2 \mathrm{O})^{\prime}$

cylinder $\begin{array}{lllll}1 & 1 & 0.5325 & 61.90 & 0.0\end{array}$

$\begin{array}{llllll}\text { cylinder } 2 & 1 & 0.6115 & 61.90 & 0.0\end{array}$

$\begin{array}{lllllll}\text { cuboid } & 3 & 1 & 4 \mathrm{p} 0.9125 & 61.90 & 0.0\end{array}$

unit 53

com='(Fuel+Clad $+\mathrm{H} 2 \mathrm{O})^{\prime}$

$\begin{array}{llllll}\text { cylinder } & 1 & 1 & 0.5325 & 61.90 & 0.0\end{array}$

$\begin{array}{llllll}\text { cylinder } 2 & 1 & 0.6115 & 61.90 & 0.0\end{array}$

$\begin{array}{llllll}\text { cuboid } & 3 & 1 & 4 \mathrm{p} 0.9125 & 61.90 & 0.0\end{array}$

unit 54

com $=$ '(Fuel+Clad $+\mathrm{H} 2 \mathrm{O})^{\prime}$

cylinder $\begin{array}{lllll}1 & 1 & 0.5325 & 61.90 & 0.0\end{array}$

$\begin{array}{lllll}\text { cylinder } 2 & 1 & 0.6115 \quad 61.90 & 0.0\end{array}$

$\begin{array}{lllllll}\text { cuboid } & 3 & 1 & 4 \mathrm{p} 0.9125 & 61.90 & 0.0\end{array}$

unit 55

com='(Fuel+Clad $+\mathrm{H} 2 \mathrm{O})^{\prime}$

cylinder $\begin{array}{lllll}1 & 1 & 0.5325 & 61.90 & 0.0\end{array}$

$\begin{array}{llllll}\text { cylinder } 2 & 1 & 0.6115 & 61.90 & 0.0\end{array}$

$\begin{array}{lllllll}\text { cuboid } & 3 & 1 & 4 \mathrm{p} 0.9125 & 61.90 & 0.0\end{array}$

unit 56

com='(Fuel+Clad $+\mathrm{H} 2 \mathrm{O})^{\prime}$

$\begin{array}{llllll}\text { cylinder } & 1 & 1 & 0.5325 & 61.90 & 0.0\end{array}$

$\begin{array}{llllll}\text { cylinder } 2 & 1 & 0.6115 & 61.90 & 0.0\end{array}$

$\begin{array}{lllllll}\text { cuboid } & 3 & 1 & 4 \mathrm{p} 0.9125 & 61.90 & 0.0\end{array}$

unit 57

com='(Fuel+Clad $+\mathrm{H} 2 \mathrm{O})^{\prime}$

$\begin{array}{llllll}\text { cylinder } & 1 & 1 & 0.5325 & 61.90 & 0.0\end{array}$

$\begin{array}{llllll}\text { cylinder } 2 & 1 & 0.6115 & 61.90 & 0.0\end{array}$

$\begin{array}{llllll}\text { cuboid } & 3 & 1 & 4 \mathrm{p} 0.9125 & 61.90 & 0.0\end{array}$

unit 58

com='(Fuel+Clad $+\mathrm{H} 2 \mathrm{O})^{\prime}$

cylinder $\begin{array}{lllll}1 & 1 & 0.5325 & 61.90 & 0.0\end{array}$

$\begin{array}{llllll}\text { cylinder } 2 & 1 & 0.6115 & 61.90 & 0.0\end{array}$

$\begin{array}{lllllll}\text { cuboid } & 3 & 1 & 4 \mathrm{p} 0.9125 & 61.90 & 0.0\end{array}$

unit 59

com $=$ '(Fuel + Clad $+\mathrm{H} 2 \mathrm{O})^{\prime}$

cylinder $\begin{array}{lllll}1 & 1 & 0.5325 & 61.90 & 0.0\end{array}$

$\begin{array}{llllll}\text { cylinder } 2 & 1 & 0.6115 & 61.90 & 0.0\end{array}$

$\begin{array}{llllll}\text { cuboid } & 3 & 1 & 4 \mathrm{p} 0.9125 & 61.90 & 0.0\end{array}$

unit 60

com $='(\text { Fuel }+ \text { Clad }+\mathrm{H} 2 \mathrm{O})^{\prime}$

$\begin{array}{llllll}\text { cylinder } & 1 & 1 & 0.5325 & 61.90 & 0.0\end{array}$

$\begin{array}{llllll}\text { cylinder } 2 & 1 & 0.6115 & 61.90 & 0.0\end{array}$

$\begin{array}{lllllll}\text { cuboid } & 3 & 1 & 4 \mathrm{p} 0.9125 & 61.90 & 0.0\end{array}$

unit 61

com='(Fuel+Clad $+\mathrm{H} 2 \mathrm{O})^{\prime}$

$\begin{array}{llllll}\text { cylinder } & 1 & 1 & 0.5325 & 61.90 & 0.0\end{array}$

$\begin{array}{llllll}\text { cylinder } & 2 & 1 & 0.6115 & 61.90 & 0.0\end{array}$

$\begin{array}{lllllll}\text { cuboid } & 3 & 1 & 4 p 0.9125 & 61.90 & 0.0\end{array}$

unit 62

com='(Fuel+Clad $+\mathrm{H} 2 \mathrm{O})^{\prime}$

$\begin{array}{llllll}\text { cylinder } & 1 & 1 & 0.5325 & 61.90 & 0.0\end{array}$

$\begin{array}{llllll}\text { cylinder } 2 & 1 & 0.6115 & 61.90 & 0.0\end{array}$

$\begin{array}{llllll}\text { cuboid } 3 & 1 & 4 \mathrm{p} 0.9125 & 61.90 & 0.0\end{array}$ unit 63

com $=$ '(Fuel + Clad $+\mathrm{H} 2 \mathrm{O})^{\prime}$

$\begin{array}{llllll}\text { cylinder } & 1 & 1 & 0.5325 & 61.90 & 0.0\end{array}$

$\begin{array}{llllll}\text { cylinder } & 2 & 1 & 0.6115 & 61.90 & 0.0\end{array}$

$\begin{array}{llllll}\text { cuboid } & 3 & 1 & 4 \mathrm{p} 0.9125 & 61.90 & 0.0\end{array}$

unit 64

com $=$ '(Fuel+Clad $+\mathrm{H} 2 \mathrm{O})^{\prime}$

$\begin{array}{llllll}\text { cylinder } & 1 & 1 & 0.5325 & 61.90 & 0.0\end{array}$

$\begin{array}{llllll}\text { cylinder } 2 & 1 & 0.6115 & 61.90 & 0.0\end{array}$

$\begin{array}{lllllll}\text { cuboid } & 3 & 1 & 4 \mathrm{p} 0.9125 & 61.90 & 0.0\end{array}$

unit 65

com='(Fuel+Clad $+\mathrm{H} 2 \mathrm{O})^{\prime}$

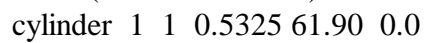

$\begin{array}{llllll}\text { cylinder } 2 & 1 & 0.6115 & 61.90 & 0.0\end{array}$

$\begin{array}{lllllll}\text { cuboid } & 3 & 1 & 4 \mathrm{p} 0.9125 & 61.90 & 0.0\end{array}$

unit 66

com $=$ '(Fuel+Clad $+\mathrm{H} 2 \mathrm{O})^{\prime}$

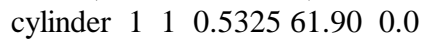

$\begin{array}{lllll}\text { cylinder } 2 & 1 & 0.6115 & 61.90 & 0.0\end{array}$

$\begin{array}{lllllll}\text { cuboid } \quad 3 & 1 & 4 \mathrm{p} 0.9125 & 61.90 & 0.0\end{array}$

unit 67

com $=$ '(Fuel + Clad $+\mathrm{H} 2 \mathrm{O})^{\prime}$

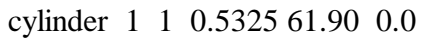

$\begin{array}{llllll}\text { cylinder } & 2 & 1 & 0.6115 & 61.90 & 0.0\end{array}$

$\begin{array}{llllll}\text { cuboid } 3 & 1 & 4 \mathrm{p} 0.9125 & 61.90 & 0.0\end{array}$

unit 68

com $=$ '(Fuel + Clad $+\mathrm{H} 2 \mathrm{O})^{\prime}$

$\begin{array}{llllll}\text { cylinder } & 1 & 1 & 0.5325 & 61.90 & 0.0\end{array}$

$\begin{array}{llllll}\text { cylinder } & 2 & 1 & 0.6115 & 61.90 & 0.0\end{array}$

$\begin{array}{lllllll}\text { cuboid } & 3 & 1 & 4 \mathrm{p} 0.9125 & 61.90 & 0.0\end{array}$

unit 69

com='(Fuel+Clad $+\mathrm{H} 2 \mathrm{O})^{\prime}$

$\begin{array}{llllll}\text { cylinder } & 1 & 1 & 0.5325 & 61.90 & 0.0\end{array}$

$\begin{array}{llllll}\text { cylinder } 2 & 1 & 0.6115 & 61.90 & 0.0\end{array}$

$\begin{array}{llllll}\text { cuboid } & 3 & 1 & 4 \mathrm{p} 0.9125 & 61.90 & 0.0\end{array}$

unit 70

com $=$ '(Fuel + Clad $+\mathrm{H} 2 \mathrm{O})^{\prime}$

$\begin{array}{llllll}\text { cylinder } & 1 & 1 & 0.5325 & 61.90 & 0.0\end{array}$

$\begin{array}{llllll}\text { cylinder } & 2 & 1 & 0.6115 & 61.90 & 0.0\end{array}$

$\begin{array}{llllll}\text { cuboid } 3 & 1 & 4 p 0.9125 & 61.90 & 0.0\end{array}$

unit 71

com $=$ '(Fuel $+\mathrm{Clad}+\mathrm{H} 2 \mathrm{O})^{\prime}$

cylinder $\begin{array}{lllll}1 & 1 & 0.5325 & 61.90 & 0.0\end{array}$

$\begin{array}{llllll}\text { cylinder } 2 & 1 & 0.6115 & 61.90 & 0.0\end{array}$

$\begin{array}{lllllll}\text { cuboid } & 3 & 1 & 4 \mathrm{p} 0.9125 & 61.90 & 0.0\end{array}$

unit 72

com='(Fuel+Clad $+\mathrm{H} 2 \mathrm{O})^{\prime}$

cylinder $\begin{array}{lllll}1 & 1 & 0.5325 & 61.90 & 0.0\end{array}$

$\begin{array}{llllll}\text { cylinder } 2 & 1 & 0.6115 & 61.90 & 0.0\end{array}$

$\begin{array}{llllll}\text { cuboid } 3 & 1 & 4 \mathrm{p} 0.9125 & 61.90 & 0.0\end{array}$

unit 73

com $=$ '(Fuel + Clad $+\mathrm{H} 2 \mathrm{O})^{\prime}$

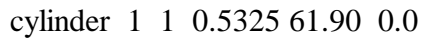

$\begin{array}{llllll}\text { cylinder } 2 & 1 & 0.6115 & 61.90 & 0.0\end{array}$

$\begin{array}{llllll}\text { cuboid } & 3 & 1 & 4 \mathrm{p} 0.9125 & 61.90 & 0.0\end{array}$

unit 74

com='(Fuel+Clad $+\mathrm{H} 2 \mathrm{O})^{\prime}$ 
$\begin{array}{llllll}\text { cylinder } & 1 & 1 & 0.5325 & 61.90 & 0.0\end{array}$

$\begin{array}{llllll}\text { cylinder } 2 & 1 & 0.6115 & 61.90 & 0.0\end{array}$

$\begin{array}{lllllll}\text { cuboid } & 3 & 1 & 4 \mathrm{p} 0.9125 & 61.90 & 0.0\end{array}$

unit 75

com='(Fuel+Clad $+\mathrm{H} 2 \mathrm{O})^{\prime}$

$\begin{array}{llllll}\text { cylinder } & 1 & 1 & 0.5325 & 61.90 & 0.0\end{array}$

$\begin{array}{llllll}\text { cylinder } & 2 & 1 & 0.6115 & 61.90 & 0.0\end{array}$

$\begin{array}{lllllll}\text { cuboid } & 3 & 1 & 4 \mathrm{p} 0.9125 & 61.90 & 0.0\end{array}$

unit 76

com='(Fuel+Clad $+\mathrm{H} 2 \mathrm{O})^{\prime}$

$\begin{array}{llllll}\text { cylinder } & 1 & 1 & 0.5325 & 61.90 & 0.0\end{array}$

$\begin{array}{llllll}\text { cylinder } & 2 & 1 & 0.6115 & 61.90 & 0.0\end{array}$

$\begin{array}{lllllll}\text { cuboid } & 3 & 1 & 4 \mathrm{p} 0.9125 & 61.90 & 0.0\end{array}$

unit 77

com $=$ '(Fuel $+\mathrm{Clad}+\mathrm{H} 2 \mathrm{O})^{\prime}$

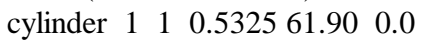

$\begin{array}{llllll}\text { cylinder } & 2 & 1 & 0.6115 & 61.90 & 0.0\end{array}$

$\begin{array}{lllllll}\text { cuboid } & 3 & 1 & 4 \mathrm{p} 0.9125 & 61.90 & 0.0\end{array}$

unit 78

com='(Fuel+Clad $+\mathrm{H} 2 \mathrm{O})^{\prime}$

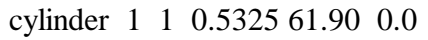

$\begin{array}{llllll}\text { cylinder } 2 & 1 & 0.6115 & 61.90 & 0.0\end{array}$

$\begin{array}{lllllll}\text { cuboid } & 3 & 1 & 4 \mathrm{p} 0.9125 & 61.90 & 0.0\end{array}$

unit 81

com='h2o and end plug below Al grid plate - fill array 1'

$\begin{array}{llllll}\text { cylinder } 4 & 1 & 0.6115 & 4.445 & 0.0\end{array}$

$\begin{array}{lllllll}\text { cuboid } 3 & 1 & 4 \mathrm{p} .9125 & 4.445 & 0.0\end{array}$

unit 82

com='h2o and end plug above Al grid plate - fill array 2'

$\begin{array}{lllll}\text { cylinder } 4 & 1 & 0.6115 & 11.784 & 0.0\end{array}$

$\begin{array}{lllllll}\text { cuboid } 3 & 1 & 4 p .9125 & 11.784 & 0.0\end{array}$

unit 83

com='(Fuel+Clad+Void) - fill array 3'

$\begin{array}{llllll}\text { cylinder } & 1 & 1 & .5325 & 8.7 & 0.0\end{array}$

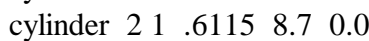

cuboid $\quad 014$ p $.91258 .7 \quad 0.0$

unit 84

com='(void+Clad+void $)^{\prime}$

cylinder $0 \begin{array}{lllll}0 & 1 & 0.5325 & 9.97 & 0.0\end{array}$

$\begin{array}{lllll}\text { cylinder } 2 & 1 & 0.6115 & 9.97 & 0.0\end{array}$

$\begin{array}{lllllll}\text { cuboid } & 0 & 1 & 4 \mathrm{p} 0.9125 & 9.97 & 0.0\end{array}$

unit 100

com='Lower Al grid plate'

cuboid $414 \mathrm{p} 50.9875 .601 \quad 0.0$

unit 101

com='array of h2o and end plugs below Al grid plate'

array $1-20.9875-20.98750 .0$

cuboid 31 4p50.9875 $4.445 \quad 0.0$

unit 102

com='array of h2o and end plugs above Al grid plate '

array $2-20.9875-20.98750 .0$

cuboid 314 p50.9875 $11.784 \quad 0.0$

unit 103

com='array of Fuel+Clad+Void'

array $3-20.9875-20.98750 .0$

cuboid $014 \mathrm{p} 50.98758 .7 \quad 0.0$

unit 104 com='array of void+Clad+void'

array $4-20.9875-20.98750 .0$

cuboid 014 p50.9875 $9.97 \quad 0.0$

unit 105

com='array of Fuel+Clad+H2O'

array $5-20.9875-20.98750 .0$

cuboid 31 4p50.9875 $61.90 \quad 0.0$

global unit 110

com='array of arrays of fuel element sections + reflector'

array $6-50.9875 \quad-50.9875 \quad 0.0$

$\begin{array}{llllll}\text { cuboid } & 4 & 1 & 4 p 50.9875 & 97.40 & -1.27\end{array}$

$\begin{array}{llllll}\text { cuboid } & 5 & 1 & 4 \mathrm{p} 50.9875 & 97.40 & -3.47\end{array}$

cuboid $3 \quad 1 \quad 4 p 50.9875 \quad 97.40 \quad-17.27$

$\begin{array}{lllllll}\text { cuboid } 5 & 1 & 4 p 50.9875 & 97.40 & -17.77\end{array}$

cuboid $\begin{array}{llllll}6 & 1 & 4 p 50.9875 & 97.40 & -54.77\end{array}$

end geometry

read array

ara $=1$ nux=23 nuy=23 nuz=1

fill $\mathrm{f} 81$ end fill

ara $=2$ nux $=23$ nuy=23 nuz=1

fill $\mathrm{f} 82$ end fill

ara $=3$ nux $=23$ nuy $=23$ nuz $=1$

fill $\mathrm{f} 83$ end fill

ara $=4$ nux $=23$ nuy=23 nuz $=1$

fill $\mathrm{f} 84$ end fill

ara $=5$ nux $=23$ nuy=23 nuz $=1$

fill

$\begin{array}{lllllllllllll}1 & 2 & 3 & 4 & 5 & 6 & 7 & 8 & 9 & 10 & 11 & 12 & 1 b \\ 11\end{array}$

$213141516171819202122231 b 11$

$31424252627282930313233 \quad 1 b 11$

41525343536373839404142 1b 11

51626354344454647484950 1b 11

61727364451525354555657 1b 11

71828374552585960616263 1b 11

81929384653596465666768 1b 11

$92030394754606569707172 \quad 1 b 11$

102131404855616670737475 1b 11

112232414956626771747677 1b 11

122333425057636872757778 1b 264 end fill

ara $=6$ nux $=1$ nuy $=1$ nuz $=6$

fill 101100102105103104 end fill

end array

end data

end

$=$ shell

cp ft04f001 \$RTNDIR/tca02.xs04

cp ft64f001 \$RTNDIR/tca02.rs64

end

$=\mathrm{kmart}$

read initial kunit $=64$

xunit $=4$

actbygrp

end initial

rrpvol

read activity

92234279223418922341452 


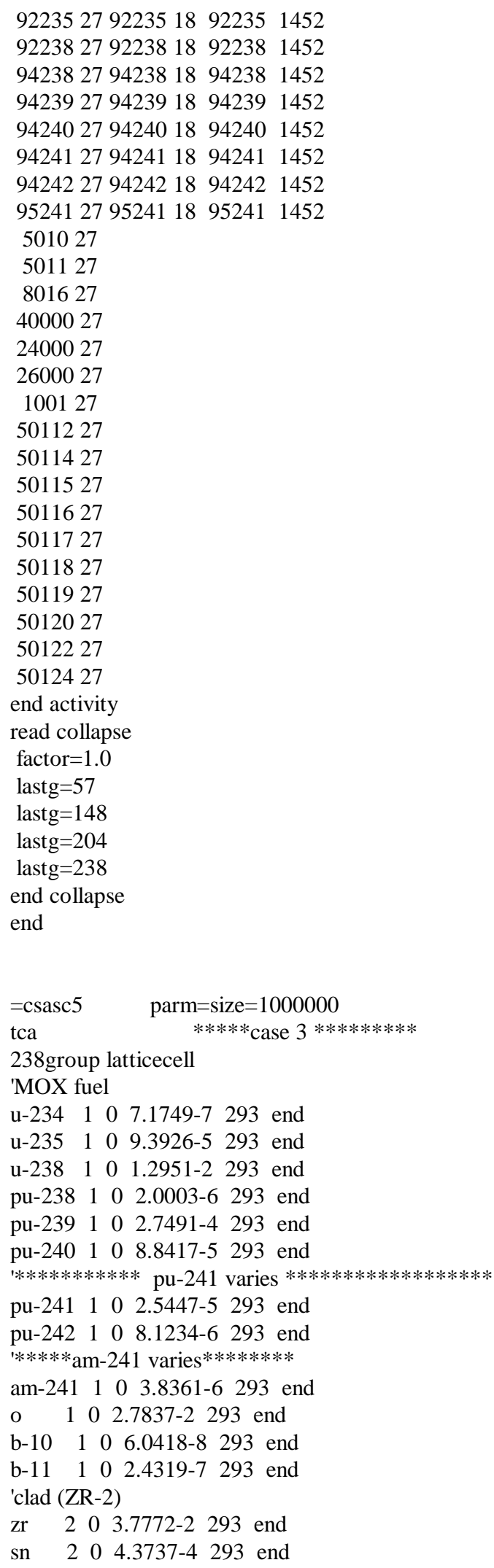

cr $\quad 2 \quad 0 \quad 8.8570-5293$ end

fe $\quad \begin{array}{llll}2 & 0 & 6.6119-5 & 293\end{array}$ end

o 20 3.5864-5 293 end 'water(Refletor)

h $3 \quad 0 \quad 6.6735-2 \quad 293$ end o 30 3.3368-2 293 end 'Al

Al $406.0224-2293$ end 'Stainless Steel

c 50 1.1928-4 293 end si 5 O $1.7003-3293$ end mn $5 \quad 0 \quad 1.7385-3293$ end p $\quad \begin{array}{llll}5 & 0 & 6.9381-5 & 293 \\ & \text { end }\end{array}$ s $504.4673-5293$ end ni $\quad \begin{array}{llll}5 & 0 & 8.9506-3 & 293 \\ & \text { end }\end{array}$ cr $5 \quad 0 \quad 1.7450-2293$ end fe 50 5.7202-2 293 end 'ordinary concrete

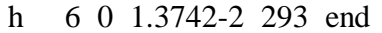

o $604.5919-2293$ end

c 60 1.1532-4 293 end

na $\begin{array}{llll}6 & 0 & 9.6395-4 & 293 \\ \text { end }\end{array}$

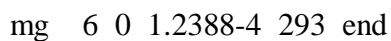

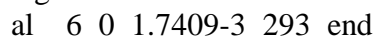
si $6 \begin{array}{llll}6 & 0 & 1.6617-2 & 293 \\ \text { end }\end{array}$ k $\quad \begin{array}{lllll}6 & 0 & 4.6052-4 & 293 & \text { end }\end{array}$ ca $6 \begin{array}{llll}6 & 0 & 1.5025-3 & 293 \\ \text { end }\end{array}$

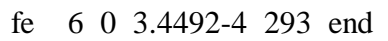
end comp squarepitch $1.825 \quad 1.065 \quad 121.223 \quad 3$ end more data limcen $=17000000$ end more tca-3 read param gen=225 nsk=25 npg=20000 $\mathrm{fdn}=$ yes $\mathrm{flx}=$ yes wrs $=64$ res $=225$

$\operatorname{lng}=2000000$

end param

read geometry

unit 1

com $=$ '(Fuel $+\mathrm{Clad}+\mathrm{H} 2 \mathrm{O})^{\prime}$

cylinder $\begin{array}{lllll}1 & 1 & 0.5325 & 64.06 & 0.0\end{array}$

$\begin{array}{llllll}\text { cylinder } 2 & 1 & 0.6115 & 64.06 & 0.0\end{array}$

$\begin{array}{lllllll}\text { cuboid } & 3 & 1 & 4 \mathrm{p} 0.9125 & 64.06 & 0.0\end{array}$

unit 2

com $=$ '(Fuel+Clad $+\mathrm{H} 2 \mathrm{O})^{\prime}$

$\begin{array}{llllll}\text { cylinder } & 1 & 1 & 0.5325 & 64.06 & 0.0\end{array}$

$\begin{array}{llllll}\text { cylinder } 2 & 1 & 0.6115 & 64.06 & 0.0\end{array}$

$\begin{array}{lllllll}\text { cuboid } 3 & 1 & 4 \mathrm{p} 0.9125 & 64.06 & 0.0\end{array}$

unit 3

com $=$ '(Fuel + Clad $+\mathrm{H} 2 \mathrm{O})^{\prime}$

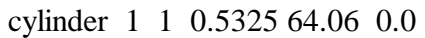

$\begin{array}{llllll}\text { cylinder } 2 & 1 & 0.6115 & 64.06 & 0.0\end{array}$

$\begin{array}{lllllll}\text { cuboid } & 3 & 1 & 4 \mathrm{p} 0.9125 & 64.06 & 0.0\end{array}$

unit 4

com='(Fuel+Clad $+\mathrm{H} 2 \mathrm{O})^{\prime}$ 
$\begin{array}{llllll}\text { cylinder } & 1 & 1 & 0.5325 & 64.06 & 0.0\end{array}$

$\begin{array}{llllll}\text { cylinder } & 2 & 1 & 0.6115 & 64.06 & 0.0\end{array}$

$\begin{array}{lllllll}\text { cuboid } & 3 & 1 & 4 \mathrm{p} 0.9125 & 64.06 & 0.0\end{array}$

unit 5

com='(Fuel+Clad $+\mathrm{H} 2 \mathrm{O})^{\prime}$

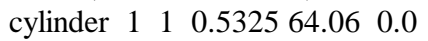

$\begin{array}{llllll}\text { cylinder } & 2 & 1 & 0.6115 & 64.06 & 0.0\end{array}$

$\begin{array}{lllllll}\text { cuboid } & 3 & 1 & 4 \mathrm{p} 0.9125 & 64.06 & 0.0\end{array}$

unit 6

com='(Fuel+Clad $+\mathrm{H} 2 \mathrm{O})^{\prime}$

$\begin{array}{llllll}\text { cylinder } & 1 & 1 & 0.5325 & 64.06 & 0.0\end{array}$

$\begin{array}{llllll}\text { cylinder } & 2 & 1 & 0.6115 & 64.06 & 0.0\end{array}$

$\begin{array}{lllllll}\text { cuboid } & 3 & 1 & 4 \mathrm{p} 0.9125 & 64.06 & 0.0\end{array}$

unit 7

com $=$ '(Fuel $+\mathrm{Clad}+\mathrm{H} 2 \mathrm{O})^{\prime}$

$\begin{array}{llllll}\text { cylinder } & 1 & 1 & 0.5325 & 64.06 & 0.0\end{array}$

$\begin{array}{llllll}\text { cylinder } & 2 & 1 & 0.6115 & 64.06 & 0.0\end{array}$

$\begin{array}{lllllll}\text { cuboid } & 3 & 1 & 4 \mathrm{p} 0.9125 & 64.06 & 0.0\end{array}$

unit 8

com $=$ '(Fuel+Clad $+\mathrm{H} 2 \mathrm{O})^{\prime}$

$\begin{array}{llllll}\text { cylinder } & 1 & 1 & 0.5325 & 64.06 & 0.0\end{array}$

$\begin{array}{llllll}\text { cylinder } 2 & 1 & 0.6115 & 64.06 & 0.0\end{array}$

$\begin{array}{lllllll}\text { cuboid } & 3 & 1 & 4 \mathrm{p} 0.9125 & 64.06 & 0.0\end{array}$

unit 9

com $=$ '(Fuel+Clad $+\mathrm{H} 2 \mathrm{O})^{\prime}$

$\begin{array}{lllllll}\text { cylinder } & 1 & 1 & 0.5325 & 64.06 & 0.0\end{array}$

$\begin{array}{llllll}\text { cylinder } 2 & 1 & 0.6115 & 64.06 & 0.0\end{array}$

$\begin{array}{lllllll}\text { cuboid } 3 & 1 & 4 \mathrm{p} 0.9125 & 64.06 & 0.0\end{array}$

unit 10

com='(Fuel+Clad $+\mathrm{H} 2 \mathrm{O})^{\prime}$

$\begin{array}{llllll}\text { cylinder } & 1 & 1 & 0.5325 & 64.06 & 0.0\end{array}$

$\begin{array}{llllll}\text { cylinder } 2 & 1 & 0.6115 & 64.06 & 0.0\end{array}$

$\begin{array}{lllllll}\text { cuboid } & 3 & 1 & 4 \mathrm{p} 0.9125 & 64.06 & 0.0\end{array}$

unit 11

com='(Fuel+Clad $+\mathrm{H} 2 \mathrm{O})^{\prime}$

$\begin{array}{llllll}\text { cylinder } & 1 & 1 & 0.5325 & 64.06 & 0.0\end{array}$

$\begin{array}{lllllll}\text { cylinder } & 2 & 1 & 0.6115 & 64.06 & 0.0\end{array}$

$\begin{array}{lllllll}\text { cuboid } & 3 & 1 & 4 \mathrm{p} 0.9125 & 64.06 & 0.0\end{array}$

unit 12

com='(Fuel + Clad $+\mathrm{H} 2 \mathrm{O})^{\prime}$

cylinder $1110.532564 .06 \quad 0.0$

$\begin{array}{llllll}\text { cylinder } 2 & 1 & 0.6115 & 64.06 & 0.0\end{array}$

$\begin{array}{lllllll}\text { cuboid } & 3 & 1 & 4 \mathrm{p} 0.9125 & 64.06 & 0.0\end{array}$

unit 13

com $='(\text { Fuel }+ \text { Clad }+\mathrm{H} 2 \mathrm{O})^{\prime}$

cylinder $\begin{array}{lllll}1 & 1 & 0.5325 & 64.06 & 0.0\end{array}$

$\begin{array}{llllll}\text { cylinder } 2 & 1 & 0.6115 & 64.06 & 0.0\end{array}$

$\begin{array}{lllllll}\text { cuboid } & 3 & 1 & 4 \mathrm{p} 0.9125 & 64.06 & 0.0\end{array}$

unit 14

com='(Fuel+Clad $+\mathrm{H} 2 \mathrm{O})^{\prime}$

cylinder $\begin{array}{lllll}1 & 1 & 0.5325 & 64.06 & 0.0\end{array}$

$\begin{array}{llllll}\text { cylinder } 2 & 1 & 0.6115 & 64.06 & 0.0\end{array}$

$\begin{array}{lllllll}\text { cuboid } & 3 & 1 & 4 \mathrm{p} 0.9125 & 64.06 & 0.0\end{array}$

unit 15

com $=$ '(Fuel+Clad $+\mathrm{H} 2 \mathrm{O})^{\prime}$

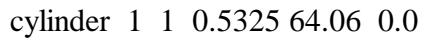

$\begin{array}{llllll}\text { cylinder } 2 & 1 & 0.6115 & 64.06 & 0.0\end{array}$ $\begin{array}{lllllll}\text { cuboid } & 3 & 1 & 4 \mathrm{p} 0.9125 & 64.06 & 0.0\end{array}$

unit 16

com $='(\text { Fuel }+\mathrm{Clad}+\mathrm{H} 2 \mathrm{O})^{\prime}$

$\begin{array}{lllllll}\text { cylinder } & 1 & 1 & 0.5325 & 64.06 & 0.0\end{array}$

$\begin{array}{llllll}\text { cylinder } 2 & 1 & 0.6115 & 64.06 & 0.0\end{array}$

$\begin{array}{lllllll}\text { cuboid } & 3 & 1 & 4 \mathrm{p} 0.9125 & 64.06 & 0.0\end{array}$

unit 17

com $=(\text { Fuel }+\mathrm{Clad}+\mathrm{H} 2 \mathrm{O})^{\prime}$

cylinder $\begin{array}{lllll}1 & 1 & 0.5325 & 64.06 & 0.0\end{array}$

$\begin{array}{llllll}\text { cylinder } 2 & 1 & 0.6115 & 64.06 & 0.0\end{array}$

$\begin{array}{lllllll}\text { cuboid } & 3 & 1 & 4 \mathrm{p} 0.9125 & 64.06 & 0.0\end{array}$

unit 18

com $=$ '(Fuel + Clad $+\mathrm{H} 2 \mathrm{O})^{\prime}$

$\begin{array}{llllll}\text { cylinder } & 1 & 1 & 0.5325 & 64.06 & 0.0\end{array}$

$\begin{array}{llllllll}\text { cylinder } & 2 & 1 & 0.6115 & 64.06 & 0.0\end{array}$

$\begin{array}{lllllll}\text { cuboid } & 3 & 1 & 4 \mathrm{p} 0.9125 & 64.06 & 0.0\end{array}$

unit 19

com='(Fuel+Clad $+\mathrm{H} 2 \mathrm{O})^{\prime}$

$\begin{array}{llllll}\text { cylinder } & 1 & 1 & 0.5325 & 64.06 & 0.0\end{array}$

$\begin{array}{lllll}\text { cylinder } 2 & 1 & 0.6115 & 64.06 & 0.0\end{array}$

$\begin{array}{lllllll}\text { cuboid } & 3 & 1 & 4 \mathrm{p} 0.9125 & 64.06 & 0.0\end{array}$

unit 20

com $=$ '(Fuel+Clad $+\mathrm{H} 2 \mathrm{O})^{\prime}$

cylinder $\begin{array}{lllll}1 & 1 & 0.5325 & 64.06 & 0.0\end{array}$

$\begin{array}{llllll}\text { cylinder } & 2 & 1 & 0.6115 & 64.06 & 0.0\end{array}$

$\begin{array}{lllllll}\text { cuboid } & 3 & 1 & 4 \mathrm{p} 0.9125 & 64.06 & 0.0\end{array}$

unit 21

com $=$ '(Fuel + Clad $+\mathrm{H} 2 \mathrm{O})^{\prime}$

$\begin{array}{llllll}\text { cylinder } & 1 & 1 & 0.5325 & 64.06 & 0.0\end{array}$

$\begin{array}{llllll}\text { cylinder } & 2 & 1 & 0.6115 & 64.06 & 0.0\end{array}$

$\begin{array}{lllllll}\text { cuboid } & 3 & 1 & 4 \mathrm{p} 0.9125 & 64.06 & 0.0\end{array}$

unit 22

com $=$ '(Fuel + Clad $+\mathrm{H} 2 \mathrm{O})^{\prime}$

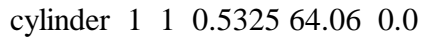

$\begin{array}{llllll}\text { cylinder } 2 & 1 & 0.6115 & 64.06 & 0.0\end{array}$

$\begin{array}{lllllll}\text { cuboid } & 3 & 1 & 4 \mathrm{p} 0.9125 & 64.06 & 0.0\end{array}$

unit 23

com $=$ '(Fuel $+\mathrm{Clad}+\mathrm{H} 2 \mathrm{O})^{\prime}$

$\begin{array}{llllll}\text { cylinder } & 1 & 1 & 0.5325 & 64.06 & 0.0\end{array}$

$\begin{array}{llllll}\text { cylinder } 2 & 1 & 0.6115 & 64.06 & 0.0\end{array}$

$\begin{array}{lllllll}\text { cuboid } & 3 & 1 & 4 \mathrm{p} 0.9125 & 64.06 & 0.0\end{array}$

unit 24

com $=$ '(Fuel + Clad $+\mathrm{H} 2 \mathrm{O})^{\prime}$

cylinder $\begin{array}{lllll}1 & 1 & 0.5325 & 64.06 & 0.0\end{array}$

$\begin{array}{lllll}\text { cylinder } 2 & 1 & 0.6115 & 64.06 & 0.0\end{array}$

$\begin{array}{lllllll}\text { cuboid } & 3 & 1 & 4 \mathrm{p} 0.9125 & 64.06 & 0.0\end{array}$

unit 25

com $=$ '(Fuel + Clad $+\mathrm{H} 2 \mathrm{O})^{\prime}$

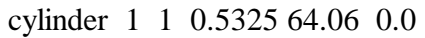

$\begin{array}{llllll}\text { cylinder } & 2 & 1 & 0.6115 & 64.06 & 0.0\end{array}$

$\begin{array}{lllllll}\text { cuboid } & 3 & 1 & 4 \mathrm{p} 0.9125 & 64.06 & 0.0\end{array}$

unit 26

com='(Fuel+Clad $+\mathrm{H} 2 \mathrm{O})^{\prime}$

$\begin{array}{lllll}\text { cylinder } & 1 & 1 & 0.532564 .06 & 0.0\end{array}$

$\begin{array}{llllll}\text { cylinder } 2 & 1 & 0.6115 & 64.06 & 0.0\end{array}$

$\begin{array}{lllllll}\text { cuboid } 3 & 1 & 4 \mathrm{p} 0.9125 & 64.06 & 0.0\end{array}$

unit 27 
com='(Fuel+Clad+H2O)'

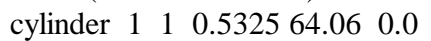

$\begin{array}{llllll}\text { cylinder } 2 & 1 & 0.6115 & 64.06 & 0.0\end{array}$

$\begin{array}{lllllll}\text { cuboid } & 3 & 1 & 4 \mathrm{p} 0.9125 & 64.06 & 0.0\end{array}$

unit 28

com='(Fuel+Clad $+\mathrm{H} 2 \mathrm{O})^{\prime}$

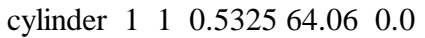

$\begin{array}{llllll}\text { cylinder } & 2 & 1 & 0.6115 & 64.06 & 0.0\end{array}$

$\begin{array}{lllllll}\text { cuboid } 3 & 1 & 4 \mathrm{p} 0.9125 & 64.06 & 0.0\end{array}$

unit 29

com $='(\text { Fuel }+\mathrm{Clad}+\mathrm{H} 2 \mathrm{O})^{\prime}$

cylinder $1110.532564 .06 \quad 0.0$

$\begin{array}{lllll}\text { cylinder } 2 & 1 & 0.6115 & 64.06 & 0.0\end{array}$

$\begin{array}{lllllll}\text { cuboid } 3 & 1 & 4 \mathrm{p} 0.9125 & 64.06 & 0.0\end{array}$

unit 30

com='(Fuel+Clad $+\mathrm{H} 2 \mathrm{O})^{\prime}$

$\begin{array}{llllll}\text { cylinder } & 1 & 1 & 0.5325 & 64.06 & 0.0\end{array}$

$\begin{array}{llllll}\text { cylinder } & 2 & 1 & 0.6115 & 64.06 & 0.0\end{array}$

$\begin{array}{lllllll}\text { cuboid } & 3 & 1 & 4 \mathrm{p} 0.9125 & 64.06 & 0.0\end{array}$

unit 31

com='(Fuel+Clad $+\mathrm{H} 2 \mathrm{O})^{\prime}$

$\begin{array}{llllll}\text { cylinder } & 1 & 1 & 0.5325 & 64.06 & 0.0\end{array}$

$\begin{array}{llllll}\text { cylinder } & 2 & 1 & 0.6115 & 64.06 & 0.0\end{array}$

$\begin{array}{lllllll}\text { cuboid } & 3 & 1 & 4 \mathrm{p} 0.9125 & 64.06 & 0.0\end{array}$

unit 32

com='(Fuel $+\mathrm{Clad}+\mathrm{H} 2 \mathrm{O})^{\prime}$

$\begin{array}{llllll}\text { cylinder } & 1 & 1 & 0.5325 & 64.06 & 0.0\end{array}$

$\begin{array}{llllll}\text { cylinder } & 2 & 1 & 0.6115 & 64.06 & 0.0\end{array}$

$\begin{array}{lllllll}\text { cuboid } & 3 & 1 & 4 \mathrm{p} 0.9125 & 64.06 & 0.0\end{array}$

unit 33

com='(Fuel+Clad $+\mathrm{H} 2 \mathrm{O})^{\prime}$

$\begin{array}{llllll}\text { cylinder } & 1 & 1 & 0.5325 & 64.06 & 0.0\end{array}$

$\begin{array}{llllll}\text { cylinder } 2 & 1 & 0.6115 & 64.06 & 0.0\end{array}$

$\begin{array}{lllllll}\text { cuboid } & 3 & 1 & 4 \mathrm{p} 0.9125 & 64.06 & 0.0\end{array}$

unit 34

com $=$ '(Fuel+Clad $+\mathrm{H} 2 \mathrm{O})^{\prime}$

$\begin{array}{lllllll}\text { cylinder } & 1 & 1 & 0.5325 & 64.06 & 0.0\end{array}$

$\begin{array}{llllll}\text { cylinder } & 2 & 1 & 0.6115 & 64.06 & 0.0\end{array}$

$\begin{array}{lllllll}\text { cuboid } 3 & 1 & 4 \mathrm{p} 0.9125 & 64.06 & 0.0\end{array}$

unit 35

com='(Fuel+Clad $+\mathrm{H} 2 \mathrm{O})^{\prime}$

cylinder $1110.532564 .06 \quad 0.0$

$\begin{array}{llllll}\text { cylinder } 2 & 1 & 0.6115 & 64.06 & 0.0\end{array}$

$\begin{array}{lllllll}\text { cuboid } & 3 & 1 & 4 \mathrm{p} 0.9125 & 64.06 & 0.0\end{array}$

unit 36

com='(Fuel+Clad $+\mathrm{H} 2 \mathrm{O})^{\prime}$

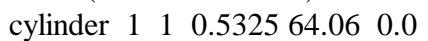

$\begin{array}{lllllll}\text { cylinder } & 2 & 1 & 0.6115 & 64.06 & 0.0\end{array}$

$\begin{array}{lllllll}\text { cuboid } & 3 & 1 & 4 \mathrm{p} 0.9125 & 64.06 & 0.0\end{array}$

unit 37

com $='(\text { Fuel }+\mathrm{Clad}+\mathrm{H} 2 \mathrm{O})^{\prime}$

$\begin{array}{llllll}\text { cylinder } & 1 & 1 & 0.5325 & 64.06 & 0.0\end{array}$

$\begin{array}{llllll}\text { cylinder } 2 & 1 & 0.6115 & 64.06 & 0.0\end{array}$

$\begin{array}{lllllll}\text { cuboid } & 3 & 1 & 4 \mathrm{p} 0.9125 & 64.06 & 0.0\end{array}$

unit 38

com='(Fuel+Clad $+\mathrm{H} 2 \mathrm{O})^{\prime}$

$\begin{array}{llllll}\text { cylinder } & 1 & 1 & 0.5325 & 64.06 & 0.0\end{array}$ $\begin{array}{llllll}\text { cylinder } & 2 & 1 & 0.6115 & 64.06 & 0.0\end{array}$ $\begin{array}{lllllll}\text { cuboid } & 3 & 1 & 4 \mathrm{p} 0.9125 & 64.06 & 0.0\end{array}$ unit 39

com $=$ '(Fuel $+\mathrm{Clad}+\mathrm{H} 2 \mathrm{O})^{\prime}$

$\begin{array}{llllll}\text { cylinder } & 1 & 1 & 0.5325 & 64.06 & 0.0\end{array}$

$\begin{array}{llllll}\text { cylinder } & 2 & 1 & 0.6115 & 64.06 & 0.0\end{array}$

$\begin{array}{lllllll}\text { cuboid } & 3 & 1 & 4 \mathrm{p} 0.9125 & 64.06 & 0.0\end{array}$

unit 40

com $=$ '(Fuel+Clad $+\mathrm{H} 2 \mathrm{O})^{\prime}$

cylinder $\begin{array}{lllll}1 & 1 & 0.5325 & 64.06 & 0.0\end{array}$

$\begin{array}{llllll}\text { cylinder } 2 & 1 & 0.6115 & 64.06 & 0.0\end{array}$

$\begin{array}{lllllll}\text { cuboid } & 3 & 1 & 4 \mathrm{p} 0.9125 & 64.06 & 0.0\end{array}$

unit 41

com $=$ '(Fuel + Clad $+\mathrm{H} 2 \mathrm{O})^{\prime}$

cylinder $1110.532564 .06 \quad 0.0$

$\begin{array}{llllll}\text { cylinder } 2 & 1 & 0.6115 & 64.06 & 0.0\end{array}$

$\begin{array}{lllllll}\text { cuboid } & 3 & 1 & 4 \mathrm{p} 0.9125 & 64.06 & 0.0\end{array}$

unit 42

com='(Fuel $+\mathrm{Clad}+\mathrm{H} 2 \mathrm{O})^{\prime}$

cylinder $\begin{array}{llll}1 & 1 & 0.532564 .06 & 0.0\end{array}$

$\begin{array}{llllll}\text { cylinder } 2 & 1 & 0.6115 & 64.06 & 0.0\end{array}$

$\begin{array}{lllllll}\text { cuboid } & 3 & 1 & 4 \mathrm{p} 0.9125 & 64.06 & 0.0\end{array}$

unit 43

com $=$ '(Fuel $+\mathrm{Clad}+\mathrm{H} 2 \mathrm{O})^{\prime}$

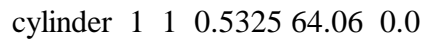

$\begin{array}{lllllll}\text { cylinder } & 2 & 1 & 0.6115 & 64.06 & 0.0\end{array}$

$\begin{array}{llllll}\text { cuboid } 3 & 1 & 4 \mathrm{p} 0.9125 & 64.06 & 0.0\end{array}$

unit 44

com $=$ '(Fuel + Clad $+\mathrm{H} 2 \mathrm{O})^{\prime}$

cylinder $1110.532564 .06 \quad 0.0$

$\begin{array}{llllll}\text { cylinder } & 2 & 1 & 0.6115 & 64.06 & 0.0\end{array}$

$\begin{array}{lllllll}\text { cuboid } & 3 & 1 & 4 \mathrm{p} 0.9125 & 64.06 & 0.0\end{array}$

unit 45

com $='(\text { Fuel }+\mathrm{Clad}+\mathrm{H} 2 \mathrm{O})^{\prime}$

cylinder $\begin{array}{lllll}1 & 1 & 0.5325 & 64.06 & 0.0\end{array}$

$\begin{array}{llllll}\text { cylinder } 2 & 1 & 0.6115 & 64.06 & 0.0\end{array}$

$\begin{array}{lllllll}\text { cuboid } & 3 & 1 & 4 \mathrm{p} 0.9125 & 64.06 & 0.0\end{array}$

unit 46

com $=$ '(Fuel + Clad $+\mathrm{H} 2 \mathrm{O})^{\prime}$

$\begin{array}{llllll}\text { cylinder } & 1 & 1 & 0.5325 & 64.06 & 0.0\end{array}$

$\begin{array}{llllll}\text { cylinder } 2 & 1 & 0.6115 & 64.06 & 0.0\end{array}$

$\begin{array}{lllllll}\text { cuboid } & 3 & 1 & 4 \mathrm{p} 0.9125 & 64.06 & 0.0\end{array}$

unit 47

com $='(\text { Fuel }+\mathrm{Clad}+\mathrm{H} 2 \mathrm{O})^{\prime}$

cylinder $1110.532564 .06 \quad 0.0$

$\begin{array}{llllll}\text { cylinder } 2 & 1 & 0.6115 & 64.06 & 0.0\end{array}$

$\begin{array}{lllllll}\text { cuboid } & 3 & 1 & 4 \mathrm{p} 0.9125 & 64.06 & 0.0\end{array}$

unit 48

com $=$ '(Fuel + Clad $+\mathrm{H} 2 \mathrm{O})^{\prime}$

$\begin{array}{llllll}\text { cylinder } & 1 & 1 & 0.5325 & 64.06 & 0.0\end{array}$

$\begin{array}{llllll}\text { cylinder } & 2 & 1 & 0.6115 & 64.06 & 0.0\end{array}$

$\begin{array}{lllllll}\text { cuboid } 3 & 1 & 4 \mathrm{p} 0.9125 & 64.06 & 0.0\end{array}$

unit 49

com $=$ '(Fuel + Clad $+\mathrm{H} 2 \mathrm{O})^{\prime}$

cylinder $\begin{array}{lllll}1 & 1 & 0.5325 & 64.06 & 0.0\end{array}$

$\begin{array}{llllll}\text { cylinder } & 2 & 1 & 0.6115 & 64.06 & 0.0\end{array}$

$\begin{array}{lllllll}\text { cuboid } & 3 & 1 & 4 \mathrm{p} 0.9125 & 64.06 & 0.0\end{array}$ 
unit 50

com='(Fuel+Clad $+\mathrm{H} 2 \mathrm{O})^{\prime}$

cylinder $\begin{array}{lllll}1 & 1 & 0.5325 & 64.06 & 0.0\end{array}$

cylinder $2 \quad 1 \quad 0.6115 \quad 64.06 \quad 0.0$

$\begin{array}{lllllll}\text { cuboid } & 3 & 1 & 4 p 0.9125 & 64.06 & 0.0\end{array}$

unit 51

com $=$ '(Fuel $+\mathrm{Clad}+\mathrm{H} 2 \mathrm{O})^{\prime}$

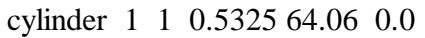

$\begin{array}{llllll}\text { cylinder } 2 & 1 & 0.6115 & 64.06 & 0.0\end{array}$

$\begin{array}{lllllll}\text { cuboid } & 3 & 1 & 4 \mathrm{p} 0.9125 & 64.06 & 0.0\end{array}$

unit 52

com='(Fuel+Clad $+\mathrm{H} 2 \mathrm{O})^{\prime}$

$\begin{array}{llllll}\text { cylinder } & 1 & 1 & 0.5325 & 64.06 & 0.0\end{array}$

$\begin{array}{llllll}\text { cylinder } & 2 & 1 & 0.6115 & 64.06 & 0.0\end{array}$

$\begin{array}{lllllll}\text { cuboid } & 3 & 1 & 4 \mathrm{p} 0.9125 & 64.06 & 0.0\end{array}$

unit 53

com='(Fuel+Clad $+\mathrm{H} 2 \mathrm{O})^{\prime}$

cylinder $\begin{array}{lllll}1 & 1 & 0.5325 & 64.06 & 0.0\end{array}$

$\begin{array}{llllll}\text { cylinder } & 2 & 1 & 0.6115 & 64.06 & 0.0\end{array}$

$\begin{array}{llllll}\text { cuboid } 3 & 1 & 4 \mathrm{p} 0.9125 & 64.06 & 0.0\end{array}$

unit 54

com $=$ '(Fuel $+\mathrm{Clad}+\mathrm{H} 2 \mathrm{O})^{\prime}$

cylinder $1110.532564 .06 \quad 0.0$

$\begin{array}{llllll}\text { cylinder } 2 & 1 & 0.6115 & 64.06 & 0.0\end{array}$

$\begin{array}{llllll}\text { cuboid } & 3 & 1 & 4 \mathrm{p} 0.9125 & 64.06 & 0.0\end{array}$

unit 55

com='(Fuel+Clad $+\mathrm{H} 2 \mathrm{O})^{\prime}$

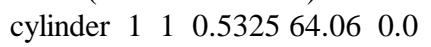

$\begin{array}{llllll}\text { cylinder } & 2 & 1 & 0.6115 & 64.06 & 0.0\end{array}$

$\begin{array}{lllllll}\text { cuboid } & 3 & 1 & 4 \mathrm{p} 0.9125 & 64.06 & 0.0\end{array}$

unit 56

com='(Fuel+Clad $+\mathrm{H} 2 \mathrm{O})^{\prime}$

$\begin{array}{lllllll}\text { cylinder } & 1 & 1 & 0.5325 & 64.06 & 0.0\end{array}$

$\begin{array}{llllll}\text { cylinder } & 2 & 1 & 0.6115 & 64.06 & 0.0\end{array}$

$\begin{array}{lllllll}\text { cuboid } & 3 & 1 & 4 \mathrm{p} 0.9125 & 64.06 & 0.0\end{array}$

unit 57

com $=$ '(Fuel $+\mathrm{Clad}+\mathrm{H} 2 \mathrm{O})^{\prime}$

$\begin{array}{llllll}\text { cylinder } & 1 & 1 & 0.5325 & 64.06 & 0.0\end{array}$

$\begin{array}{llllll}\text { cylinder } & 2 & 1 & 0.6115 & 64.06 & 0.0\end{array}$

$\begin{array}{lllllll}\text { cuboid } 3 & 1 & 4 \mathrm{p} 0.9125 & 64.06 & 0.0\end{array}$

unit 58

com='(Fuel+Clad $+\mathrm{H} 2 \mathrm{O})^{\prime}$

$\begin{array}{llllll}\text { cylinder } & 1 & 1 & 0.5325 & 64.06 & 0.0\end{array}$

$\begin{array}{llllll}\text { cylinder } 2 & 1 & 0.6115 & 64.06 & 0.0\end{array}$

$\begin{array}{lllllll}\text { cuboid } & 3 & 1 & 4 \mathrm{p} 0.9125 & 64.06 & 0.0\end{array}$

unit 59

com $=$ '(Fuel+Clad $+\mathrm{H} 2 \mathrm{O})^{\prime}$

cylinder $\begin{array}{llllll}1 & 1 & 0.5325 & 64.06 & 0.0\end{array}$

$\begin{array}{llllll}\text { cylinder } 2 & 1 & 0.6115 & 64.06 & 0.0\end{array}$

$\begin{array}{lllllll}\text { cuboid } & 3 & 1 & 4 \mathrm{p} 0.9125 & 64.06 & 0.0\end{array}$

unit 60

com $=$ '(Fuel $+\mathrm{Clad}+\mathrm{H} 2 \mathrm{O})^{\prime}$

cylinder $1110.532564 .06 \quad 0.0$

$\begin{array}{llllll}\text { cylinder } 2 & 1 & 0.6115 & 64.06 & 0.0\end{array}$

$\begin{array}{lllllll}\text { cuboid } & 3 & 1 & 4 \mathrm{p} 0.9125 & 64.06 & 0.0\end{array}$

unit 61

com='(Fuel+Clad $+\mathrm{H} 2 \mathrm{O})^{\prime}$ $\begin{array}{llllll}\text { cylinder } & 1 & 1 & 0.5325 & 64.06 & 0.0\end{array}$

$\begin{array}{llllll}\text { cylinder } & 2 & 1 & 0.6115 & 64.06 & 0.0\end{array}$

$\begin{array}{lllllll}\text { cuboid } & 3 & 1 & 4 \mathrm{p} 0.9125 & 64.06 & 0.0\end{array}$

unit 62

com='(Fuel+Clad $+\mathrm{H} 2 \mathrm{O})^{\prime}$

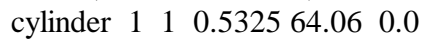

$\begin{array}{llllll}\text { cylinder } & 2 & 1 & 0.6115 & 64.06 & 0.0\end{array}$

$\begin{array}{lllllll}\text { cuboid } & 3 & 1 & 4 \mathrm{p} 0.9125 & 64.06 & 0.0\end{array}$

unit 63

com='(Fuel+Clad $+\mathrm{H} 2 \mathrm{O})^{\prime}$

cylinder $\begin{array}{lllll}1 & 1 & 0.5325 & 64.06 & 0.0\end{array}$

$\begin{array}{llllll}\text { cylinder } 2 & 1 & 0.6115 & 64.06 & 0.0\end{array}$

$\begin{array}{lllllll}\text { cuboid } & 3 & 1 & 4 \mathrm{p} 0.9125 & 64.06 & 0.0\end{array}$

unit 64

com $=$ '(Fuel + Clad $+\mathrm{H} 2 \mathrm{O})^{\prime}$

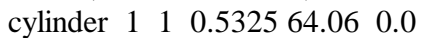

$\begin{array}{llllll}\text { cylinder } 2 & 1 & 0.6115 & 64.06 & 0.0\end{array}$

$\begin{array}{lllllll}\text { cuboid } & 3 & 1 & 4 \mathrm{p} 0.9125 & 64.06 & 0.0\end{array}$

unit 65

com='(Fuel+Clad $+\mathrm{H} 2 \mathrm{O})^{\prime}$

cylinder $1110.532564 .06 \quad 0.0$

$\begin{array}{lllllll}\text { cylinder } 2 & 1 & 0.6115 & 64.06 & 0.0\end{array}$

$\begin{array}{lllllll}\text { cuboid } & 3 & 1 & 4 \mathrm{p} 0.9125 & 64.06 & 0.0\end{array}$

unit 66

com $=$ '(Fuel + Clad $+\mathrm{H} 2 \mathrm{O})^{\prime}$

cylinder $\begin{array}{lllll}1 & 1 & 0.5325 & 64.06 & 0.0\end{array}$

$\begin{array}{llllll}\text { cylinder } 2 & 1 & 0.6115 & 64.06 & 0.0\end{array}$

$\begin{array}{lllllll}\text { cuboid } & 3 & 1 & 4 \mathrm{p} 0.9125 & 64.06 & 0.0\end{array}$

unit 67

com $=(\text { Fuel }+ \text { Clad }+\mathrm{H} 2 \mathrm{O})^{\prime}$

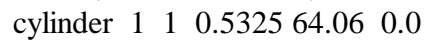

$\begin{array}{llllll}\text { cylinder } 2 & 1 & 0.6115 & 64.06 & 0.0\end{array}$

$\begin{array}{lllllll}\text { cuboid } & 3 & 1 & 4 \mathrm{p} 0.9125 & 64.06 & 0.0\end{array}$

unit 68

com $=$ '(Fuel + Clad $+\mathrm{H} 2 \mathrm{O})^{\prime}$

$\begin{array}{llllll}\text { cylinder } & 1 & 1 & 0.5325 & 64.06 & 0.0\end{array}$

$\begin{array}{lllllll}\text { cylinder } & 2 & 1 & 0.6115 & 64.06 & 0.0\end{array}$

$\begin{array}{lllllll}\text { cuboid } & 3 & 1 & 4 \mathrm{p} 0.9125 & 64.06 & 0.0\end{array}$

unit 69

com $=$ '(Fuel $+\mathrm{Clad}+\mathrm{H} 2 \mathrm{O})^{\prime}$

cylinder $\begin{array}{lllll}1 & 1 & 0.5325 & 64.06 & 0.0\end{array}$

$\begin{array}{llllll}\text { cylinder } 2 & 1 & 0.6115 & 64.06 & 0.0\end{array}$

$\begin{array}{lllllll}\text { cuboid } & 3 & 1 & 4 \mathrm{p} 0.9125 & 64.06 & 0.0\end{array}$

unit 70

com $=$ '(Fuel+Clad $+\mathrm{H} 2 \mathrm{O})^{\prime}$

cylinder $\begin{array}{lllll}1 & 1 & 0.5325 & 64.06 & 0.0\end{array}$

$\begin{array}{llllll}\text { cylinder } 2 & 1 & 0.6115 & 64.06 & 0.0\end{array}$

$\begin{array}{lllllll}\text { cuboid } & 3 & 1 & 4 \mathrm{p} 0.9125 & 64.06 & 0.0\end{array}$

unit 71

com $=$ '(Fuel + Clad $+\mathrm{H} 2 \mathrm{O})^{\prime}$

$\begin{array}{llllll}\text { cylinder } & 1 & 1 & 0.5325 & 64.06 & 0.0\end{array}$

$\begin{array}{llllllll}\text { cylinder } & 2 & 1 & 0.6115 & 64.06 & 0.0\end{array}$

$\begin{array}{lllllll}\text { cuboid } 3 & 1 & 4 \mathrm{p} 0.9125 & 64.06 & 0.0\end{array}$

unit 72

com $='(\text { Fuel }+\mathrm{Clad}+\mathrm{H} 2 \mathrm{O})^{\prime}$

$\begin{array}{llllll}\text { cylinder } & 1 & 1 & 0.5325 & 64.06 & 0.0\end{array}$

$\begin{array}{llllll}\text { cylinder } & 2 & 1 & 0.6115 & 64.06 & 0.0\end{array}$ 
$\begin{array}{lllllll}\text { cuboid } & 3 & 1 & 4 \mathrm{p} 0.9125 & 64.06 & 0.0\end{array}$

unit 73

com $='(\text { Fuel }+\mathrm{Clad}+\mathrm{H} 2 \mathrm{O})^{\prime}$

$\begin{array}{llllll}\text { cylinder } & 1 & 1 & 0.5325 & 64.06 & 0.0\end{array}$

$\begin{array}{lllll}\text { cylinder } 2 & 1 & 0.6115 & 64.06 & 0.0\end{array}$

$\begin{array}{lllllll}\text { cuboid } & 3 & 1 & 4 \mathrm{p} 0.9125 & 64.06 & 0.0\end{array}$

unit 74

com='(Fuel+Clad $+\mathrm{H} 2 \mathrm{O})^{\prime}$

$\begin{array}{llllll}\text { cylinder } & 1 & 1 & 0.5325 & 64.06 & 0.0\end{array}$

$\begin{array}{llllll}\text { cylinder } 2 & 1 & 0.6115 & 64.06 & 0.0\end{array}$

$\begin{array}{lllllll}\text { cuboid } & 3 & 1 & 4 \mathrm{p} 0.9125 & 64.06 & 0.0\end{array}$

unit 75

com='(Fuel+Clad $+\mathrm{H} 2 \mathrm{O})^{\prime}$

$\begin{array}{llllll}\text { cylinder } & 1 & 1 & 0.5325 & 64.06 & 0.0\end{array}$

$\begin{array}{lllllll}\text { cylinder } 2 & 1 & 0.6115 & 64.06 & 0.0\end{array}$

$\begin{array}{lllllll}\text { cuboid } & 3 & 1 & 4 \mathrm{p} 0.9125 & 64.06 & 0.0\end{array}$

unit 76

com='(Fuel+Clad $+\mathrm{H} 2 \mathrm{O})^{\prime}$

$\begin{array}{llllll}\text { cylinder } & 1 & 1 & 0.5325 & 64.06 & 0.0\end{array}$

$\begin{array}{llllll}\text { cylinder } 2 & 1 & 0.6115 & 64.06 & 0.0\end{array}$

$\begin{array}{lllllll}\text { cuboid } & 3 & 1 & 4 \mathrm{p} 0.9125 & 64.06 & 0.0\end{array}$

unit 77

com='(Fuel+Clad $+\mathrm{H} 2 \mathrm{O})^{\prime}$

$\begin{array}{llllll}\text { cylinder } & 1 & 1 & 0.5325 & 64.06 & 0.0\end{array}$

cylinder $2 \quad 1 \quad 0.6115 \quad 64.06 \quad 0.0$

$\begin{array}{lllllll}\text { cuboid } & 3 & 1 & 4 \mathrm{p} 0.9125 & 64.06 & 0.0\end{array}$

unit 78

com='(Fuel + Clad $+\mathrm{H} 2 \mathrm{O})^{\prime}$

cylinder $\begin{array}{lllll}1 & 1 & 0.5325 & 64.06 & 0.0\end{array}$

$\begin{array}{llllll}\text { cylinder } 2 & 1 & 0.6115 & 64.06 & 0.0\end{array}$

$\begin{array}{lllllll}\text { cuboid } & 3 & 1 & 4 \mathrm{p} 0.9125 & 64.06 & 0.0\end{array}$

unit 81

com='h2o and end plug below Al grid plate - fill array 1'

$\begin{array}{llllll}\text { cylinder } 4 & 1 & 0.6115 & 4.445 & 0.0\end{array}$

$\begin{array}{lllllll}\text { cuboid } 3 & 1 & 4 p .9125 & 4.445 & 0.0\end{array}$

unit 82

com='h2o and end plug above Al grid plate - fill array 2'

$\begin{array}{llllll}\text { cylinder } & 4 & 1 & 0.6115 & 11.784 & 0.0\end{array}$

$\begin{array}{lllllll}\text { cuboid } 3 & 1 & 4 p .9125 & 11.784 & 0.0\end{array}$

unit 83

com='(Fuel+Clad+Void) - fill array 3'

$\begin{array}{llllll}\text { cylinder } & 1 & 1 & .5325 & 6.54 & 0.0\end{array}$

$\begin{array}{llllll}\text { cylinder } 2 & 1 & .6115 & 6.54 & 0.0\end{array}$

cuboid $\quad 011$ 4p.9125 $6.54 \quad 0.0$

unit 84

com='(void+Clad+void $)^{\prime}$

cylinder $\begin{array}{lllll}0 & 1 & 0.5325 & 9.97 & 0.0\end{array}$

$\begin{array}{llllll}\text { cylinder } 2 & 1 & 0.6115 & 9.97 & 0.0\end{array}$

$\begin{array}{llllll}\text { cuboid } & 0 & 1 & 4 \mathrm{p} 0.9125 & 9.97 & 0.0\end{array}$

unit 100

com='Lower Al grid plate'

cuboid 414 p50.9875.601 0.0

unit 101

com='array of h2o and end plugs below Al grid plate'

array $1-20.9875-20.98750 .0$

cuboid 314 p50.9875 $4.445 \quad 0.0$

unit 102 com='array of h2o and end plugs above $\mathrm{Al}$ grid plate '

array $2-20.9875-20.98750 .0$

cuboid 31 4p50.9875 $11.784 \quad 0.0$

unit 103

com='array of Fuel+Clad+Void'

array $3-20.9875-20.98750 .0$

cuboid 014 p50.9875 $6.54 \quad 0.0$

unit 104

com='array of void+Clad+void'

array $4-20.9875-20.98750 .0$

cuboid 014 p50.9875 $9.97 \quad 0.0$

unit 105

com='array of Fuel+Clad+H2O'

array $5-20.9875-20.98750 .0$

cuboid 31 4p50.9875 64.060 .0

global unit 110

com='array of arrays of fuel element sections + reflector'

array $6-50.9875 \quad-50.9875 \quad 0.0$

$\begin{array}{llllll}\text { cuboid } \quad 4 & 1 & 4 p 50.9875 & 97.40 & -1.27\end{array}$

cuboid $5 \begin{array}{lllll}5 & 4 p 50.9875 & 97.40 & -3.47\end{array}$

cuboid $3 \quad 1 \quad 4 p 50.9875 \quad 97.40 \quad-17.27$

$\begin{array}{lllllll}\text { cuboid } 5 & 1 & 4 p 50.9875 & 97.40 & -17.77\end{array}$

cuboid $\begin{array}{llllll}6 & 1 & 4 \mathrm{p} 50.9875 & 97.40 & -54.77\end{array}$

end geometry

read array

ara $=1$ nux=23 nuy=23 nuz=1

fill $\mathrm{f} 81$ end fill

ara $=2$ nux $=23$ nuy=23 nuz $=1$

fill $\mathrm{f} 82$ end fill

ara=3 nux=23 nuy=23 nuz=1

fill $\mathrm{f} 83$ end fill

ara $=4$ nux $=23$ nuy=23 nuz=1

fill $\mathrm{f} 84$ end fill

ara $=5$ nux $=23$ nuy=23 nuz $=1$

fill

1223456789101112 lb 11

$21314151617181920212223 \quad 1 b 11$

$31424252627282930313233 \quad 1 b 11$

41525343536373839404142 1b 11

51626354344454647484950 1b 11

61727364451525354555657 1b 11

$71828374552585960616263 \quad 1 b 11$

81929384653596465666768 1b 11

92030394754606569707172 1b 11

102131404855616670737475 1b 11

$112232414956626771747677 \quad 1 b 11$

122333425057636872757778 1b 264

end fill

ara $=6$ nux=1 nuy=1 nuz=6

fill 101100102105103104 end fill

end array

end data

end

$=$ shell

cp ft04f001 \$RTNDIR/tca03.xs04

cp ft64f001 \$RTNDIR/tca03.rs64

end 
$=\mathrm{kmart}$

read initial kunit $=64$

xunit $=4$

actbygrp

end initial

rrpvol

read activity

92234279223418922341452

92235279223518922351452

92238279223818922381452

$9423827942381894238 \quad 1452$

94239279423918942391452

$9424027942401894240 \quad 1452$

$9424127942411894241 \quad 1452$

$9424227942421894242 \quad 1452$

95241279524118952411452

501027

501127

801627

4000027

2400027

2600027

100127

5011227

5011427

5011527

5011627

5011727

5011827

5011927

5012027

5012227

5012427

end activity

read collapse

factor $=1.0$

lastg $=57$

lastg $=148$

lastg $=204$

lastg $=238$

end collapse

end

$=\operatorname{csasc} 5 \quad$ parm $=$ size $=1000000$

tca $\quad * * * * *$ case $4 * * * * * * * * *$

238group latticecell

'MOX fuel

u-234 $1007.1749-7 \quad 293$ end

u-235 $1100.3926-5293$ end

u-238 $1001.2951-2 \quad 293$ end

pu-238 $1002.0003-6293$ end

pu-239 $1002.7491-4293$ end

pu-240 $108.8417-5293$ end

'*********** pu-241 varies $* * * * * * * * * * * * * * * * * *$

pu-241 102 2.8003-5 293 end

pu-242 10 8.1234-6 293 end

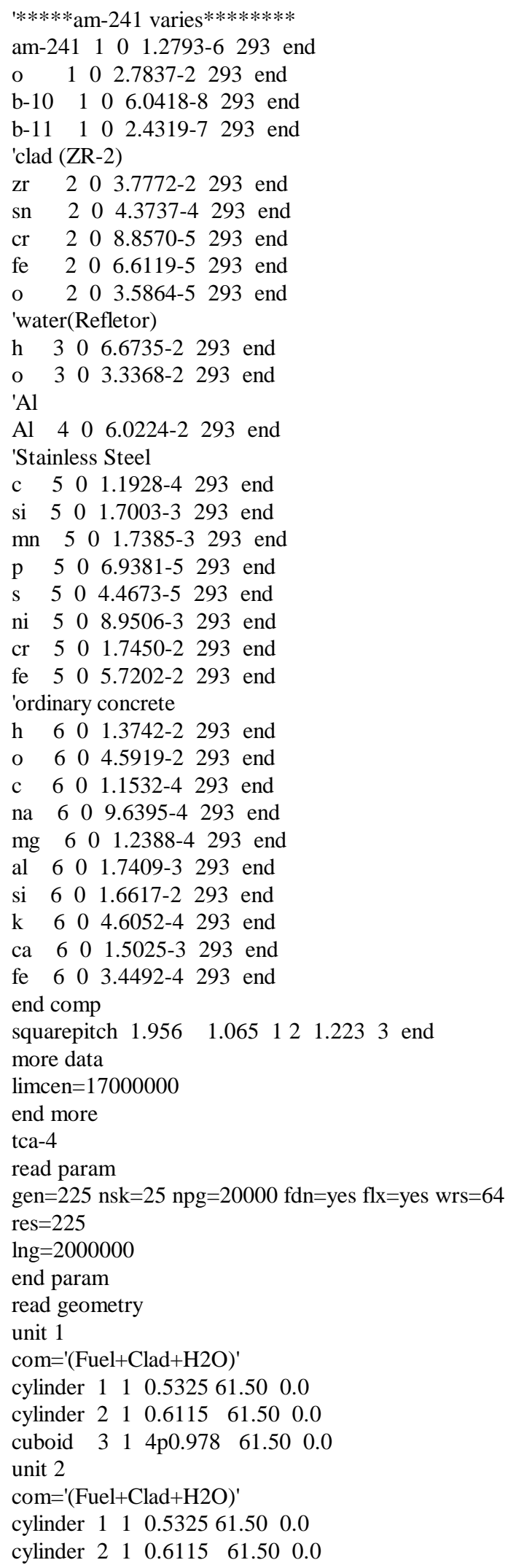


$\begin{array}{lllllll}\text { cuboid } \quad 3 & 1 & 4 \mathrm{p} 0.978 & 61.50 & 0.0\end{array}$

unit 3

com $='(\text { Fuel }+ \text { Clad }+\mathrm{H} 2 \mathrm{O})^{\prime}$

$\begin{array}{llllll}\text { cylinder } & 1 & 1 & 0.5325 & 61.50 & 0.0\end{array}$

$\begin{array}{llllll}\text { cylinder } & 2 & 1 & 0.6115 & 61.50 & 0.0\end{array}$

$\begin{array}{lllllll}\text { cuboid } & 3 & 1 & 4 \mathrm{p} 0.978 & 61.50 & 0.0\end{array}$

unit 4

com='(Fuel+Clad $+\mathrm{H} 2 \mathrm{O})^{\prime}$

$\begin{array}{llllll}\text { cylinder } & 1 & 1 & 0.5325 & 61.50 & 0.0\end{array}$

$\begin{array}{llllll}\text { cylinder } & 2 & 1 & 0.6115 & 61.50 & 0.0\end{array}$

$\begin{array}{lllllll}\text { cuboid } & 3 & 1 & 4 p 0.978 & 61.50 & 0.0\end{array}$

unit 5

com='(Fuel+Clad $+\mathrm{H} 2 \mathrm{O})^{\prime}$

$\begin{array}{llllll}\text { cylinder } & 1 & 1 & 0.5325 & 61.50 & 0.0\end{array}$

$\begin{array}{llllll}\text { cylinder } & 2 & 1 & 0.6115 & 61.50 & 0.0\end{array}$

$\begin{array}{llllll}\text { cuboid } & 3 & 1 & 4 \mathrm{p} 0.978 & 61.50 & 0.0\end{array}$

unit 6

com='(Fuel+Clad $+\mathrm{H} 2 \mathrm{O})^{\prime}$

$\begin{array}{llllll}\text { cylinder } & 1 & 1 & 0.5325 & 61.50 & 0.0\end{array}$

$\begin{array}{llllll}\text { cylinder } 2 & 1 & 0.6115 & 61.50 & 0.0\end{array}$

$\begin{array}{lllllll}\text { cuboid } 3 & 1 & 4 \mathrm{p} 0.978 & 61.50 & 0.0\end{array}$

unit 7

com='(Fuel+Clad $+\mathrm{H} 2 \mathrm{O})^{\prime}$

$\begin{array}{llllll}\text { cylinder } & 1 & 1 & 0.5325 & 61.50 & 0.0\end{array}$

$\begin{array}{llllll}\text { cylinder } 2 & 1 & 0.6115 & 61.50 & 0.0\end{array}$

$\begin{array}{lllllll}\text { cuboid } & 3 & 1 & 4 \mathrm{p} 0.978 & 61.50 & 0.0\end{array}$

unit 8

com='(Fuel+Clad $+\mathrm{H} 2 \mathrm{O})^{\prime}$

$\begin{array}{llllll}\text { cylinder } & 1 & 1 & 0.5325 & 61.50 & 0.0\end{array}$

$\begin{array}{llllll}\text { cylinder } 2 & 1 & 0.6115 & 61.50 & 0.0\end{array}$

$\begin{array}{lllllll}\text { cuboid } 3 & 3 & 4 \mathrm{p} 0.978 & 61.50 & 0.0\end{array}$

unit 9

com $=$ '(Fuel $+\mathrm{Clad}+\mathrm{H} 2 \mathrm{O})^{\prime}$

$\begin{array}{llllll}\text { cylinder } & 1 & 1 & 0.5325 & 61.50 & 0.0\end{array}$

$\begin{array}{llllll}\text { cylinder } 2 & 1 & 0.6115 & 61.50 & 0.0\end{array}$

$\begin{array}{lllllll}\text { cuboid } & 3 & 1 & 4 \mathrm{p} 0.978 & 61.50 & 0.0\end{array}$

unit 10

com='(Fuel+Clad $+\mathrm{H} 2 \mathrm{O})^{\prime}$

$\begin{array}{llllll}\text { cylinder } & 1 & 1 & 0.5325 & 61.50 & 0.0\end{array}$

$\begin{array}{llllll}\text { cylinder } 2 & 1 & 0.6115 & 61.50 & 0.0\end{array}$

$\begin{array}{lllllll}\text { cuboid } & 3 & 1 & 4 \mathrm{p} 0.978 & 61.50 & 0.0\end{array}$

unit 11

com='(Fuel+Clad $+\mathrm{H} 2 \mathrm{O})^{\prime}$

$\begin{array}{llllll}\text { cylinder } & 1 & 1 & 0.5325 & 61.50 & 0.0\end{array}$

$\begin{array}{llllll}\text { cylinder } & 2 & 1 & 0.6115 & 61.50 & 0.0\end{array}$

$\begin{array}{lllllll}\text { cuboid } 3 & 1 & 4 p 0.978 & 61.50 & 0.0\end{array}$

unit 12

com $=$ '(Fuel $+\mathrm{Clad}+\mathrm{H} 2 \mathrm{O})^{\prime}$

$\begin{array}{llllll}\text { cylinder } & 1 & 1 & 0.5325 & 61.50 & 0.0\end{array}$

$\begin{array}{llllll}\text { cylinder } 2 & 1 & 0.6115 & 61.50 & 0.0\end{array}$

$\begin{array}{lllllll}\text { cuboid } 3 & 3 & 4 \mathrm{p} 0.978 & 61.50 & 0.0\end{array}$

unit 13

com='(Fuel+Clad $+\mathrm{H} 2 \mathrm{O})^{\prime}$

$\begin{array}{llllll}\text { cylinder } & 1 & 1 & 0.5325 & 61.50 & 0.0\end{array}$

$\begin{array}{llllll}\text { cylinder } & 2 & 1 & 0.6115 & 61.50 & 0.0\end{array}$

$\begin{array}{lllllll}\text { cuboid } & 3 & 1 & 4 \mathrm{p} 0.978 & 61.50 & 0.0\end{array}$

unit 14

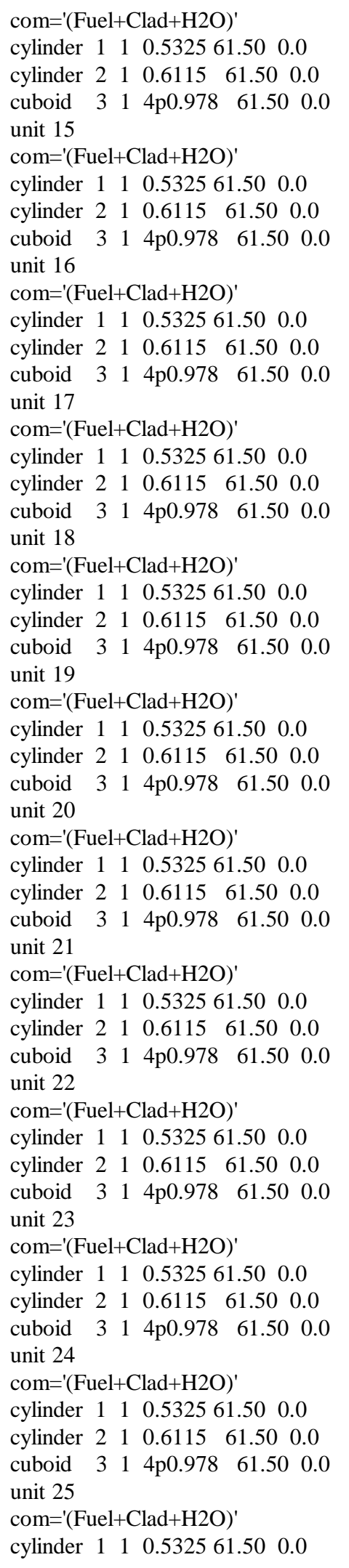


$\begin{array}{llllll}\text { cylinder } & 2 & 1 & 0.6115 & 61.50 & 0.0\end{array}$ $\begin{array}{lllllll}\text { cuboid } 3 & 1 & 4 p 0.978 & 61.50 & 0.0\end{array}$ unit 26

com='(Fuel+Clad $+\mathrm{H} 2 \mathrm{O})^{\prime}$

$\begin{array}{llllll}\text { cylinder } & 1 & 1 & 0.5325 & 61.50 & 0.0\end{array}$

$\begin{array}{llllll}\text { cylinder } 2 & 1 & 0.6115 & 61.50 & 0.0\end{array}$

$\begin{array}{lllllll}\text { cuboid } & 3 & 1 & 4 \mathrm{p} 0.978 & 61.50 & 0.0\end{array}$

unit 27

com='(Fuel+Clad $+\mathrm{H} 2 \mathrm{O})^{\prime}$

$\begin{array}{llllll}\text { cylinder } & 1 & 1 & 0.5325 & 61.50 & 0.0\end{array}$

$\begin{array}{lllllll}\text { cylinder } & 2 & 1 & 0.6115 & 61.50 & 0.0\end{array}$

$\begin{array}{lllllll}\text { cuboid } & 3 & 1 & 4 \mathrm{p} 0.978 & 61.50 & 0.0\end{array}$ unit 28

com $=$ '(Fuel+Clad $+\mathrm{H} 2 \mathrm{O})^{\prime}$

$\begin{array}{llllll}\text { cylinder } & 1 & 1 & 0.5325 & 61.50 & 0.0\end{array}$

$\begin{array}{llllll}\text { cylinder } 2 & 1 & 0.6115 & 61.50 & 0.0\end{array}$

$\begin{array}{lllllll}\text { cuboid } & 3 & 1 & 4 \mathrm{p} 0.978 & 61.50 & 0.0\end{array}$

unit 29

com='(Fuel+Clad $+\mathrm{H} 2 \mathrm{O})^{\prime}$

$\begin{array}{llllll}\text { cylinder } & 1 & 1 & 0.5325 & 61.50 & 0.0\end{array}$

$\begin{array}{llllll}\text { cylinder } 2 & 1 & 0.6115 & 61.50 & 0.0\end{array}$

$\begin{array}{lllllll}\text { cuboid } & 3 & 1 & 4 \mathrm{p} 0.978 & 61.50 & 0.0\end{array}$ unit 30

com='(Fuel+Clad $+\mathrm{H} 2 \mathrm{O})^{\prime}$

$\begin{array}{llllll}\text { cylinder } & 1 & 1 & 0.5325 & 61.50 & 0.0\end{array}$

$\begin{array}{llllll}\text { cylinder } 2 & 1 & 0.6115 & 61.50 & 0.0\end{array}$

$\begin{array}{lllllll}\text { cuboid } 3 & 1 & 4 \mathrm{p} 0.978 & 61.50 & 0.0\end{array}$

unit 31

com='(Fuel+Clad $+\mathrm{H} 2 \mathrm{O})^{\prime}$

$\begin{array}{llllll}\text { cylinder } & 1 & 1 & 0.5325 & 61.50 & 0.0\end{array}$

$\begin{array}{llllll}\text { cylinder } 2 & 1 & 0.6115 & 61.50 & 0.0\end{array}$

$\begin{array}{lllllll}\text { cuboid } & 3 & 1 & 4 \mathrm{p} 0.978 & 61.50 & 0.0\end{array}$

unit 32

com='(Fuel+Clad $+\mathrm{H} 2 \mathrm{O})^{\prime}$

$\begin{array}{llllll}\text { cylinder } & 1 & 1 & 0.5325 & 61.50 & 0.0\end{array}$

$\begin{array}{llllll}\text { cylinder } 2 & 1 & 0.6115 & 61.50 & 0.0\end{array}$

$\begin{array}{lllllll}\text { cuboid } & 3 & 1 & 4 \mathrm{p} 0.978 & 61.50 & 0.0\end{array}$ unit 33

com $=$ '(Fuel + Clad $+\mathrm{H} 2 \mathrm{O})^{\prime}$

$\begin{array}{llllll}\text { cylinder } & 1 & 1 & 0.5325 & 61.50 & 0.0\end{array}$

$\begin{array}{llllll}\text { cylinder } & 2 & 1 & 0.6115 & 61.50 & 0.0\end{array}$

$\begin{array}{lllllll}\text { cuboid } & 3 & 1 & 4 \mathrm{p} 0.978 & 61.50 & 0.0\end{array}$

unit 34

com $='(\text { Fuel }+ \text { Clad }+\mathrm{H} 2 \mathrm{O})^{\prime}$

$\begin{array}{llllll}\text { cylinder } & 1 & 1 & 0.5325 & 61.50 & 0.0\end{array}$

$\begin{array}{llllll}\text { cylinder } & 2 & 1 & 0.6115 & 61.50 & 0.0\end{array}$

$\begin{array}{lllllll}\text { cuboid } \quad 3 & 1 & 4 \mathrm{p} 0.978 & 61.50 & 0.0\end{array}$

unit 35

com='(Fuel+Clad $+\mathrm{H} 2 \mathrm{O})^{\prime}$

$\begin{array}{llllll}\text { cylinder } & 1 & 1 & 0.5325 & 61.50 & 0.0\end{array}$

$\begin{array}{llllll}\text { cylinder } & 2 & 1 & 0.6115 & 61.50 & 0.0\end{array}$

$\begin{array}{lllllll}\text { cuboid } & 3 & 1 & 4 \mathrm{p} 0.978 & 61.50 & 0.0\end{array}$

unit 36

com $=$ '(Fuel + Clad $+\mathrm{H} 2 \mathrm{O})^{\prime}$

$\begin{array}{llllll}\text { cylinder } & 1 & 1 & 0.5325 & 61.50 & 0.0\end{array}$

$\begin{array}{llllll}\text { cylinder } & 2 & 1 & 0.6115 & 61.50 & 0.0\end{array}$

$\begin{array}{lllllll}\text { cuboid } 3 & 1 & 4 \mathrm{p} 0.978 & 61.50 & 0.0\end{array}$ unit 37

com $=$ '(Fuel + Clad $+\mathrm{H} 2 \mathrm{O})^{\prime}$

$\begin{array}{llllll}\text { cylinder } & 1 & 1 & 0.5325 & 61.50 & 0.0\end{array}$

$\begin{array}{llllll}\text { cylinder } & 2 & 1 & 0.6115 & 61.50 & 0.0\end{array}$

$\begin{array}{lllllll}\text { cuboid } & 3 & 1 & 4 \mathrm{p} 0.978 & 61.50 & 0.0\end{array}$

unit 38

com $=$ '(Fuel + Clad $+\mathrm{H} 2 \mathrm{O})^{\prime}$

$\begin{array}{llllll}\text { cylinder } & 1 & 1 & 0.5325 & 61.50 & 0.0\end{array}$

$\begin{array}{lllll}\text { cylinder } 2 & 1 & 0.6115 & 61.50 & 0.0\end{array}$

$\begin{array}{lllllll}\text { cuboid } & 3 & 1 & 4 \mathrm{p} 0.978 & 61.50 & 0.0\end{array}$

unit 39

com='(Fuel+Clad $+\mathrm{H} 2 \mathrm{O})^{\prime}$

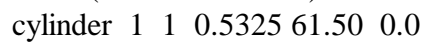

$\begin{array}{llllll}\text { cylinder } 2 & 1 & 0.6115 & 61.50 & 0.0\end{array}$

$\begin{array}{lllllll}\text { cuboid } & 3 & 1 & 4 \mathrm{p} 0.978 & 61.50 & 0.0\end{array}$

unit 40

com $=$ '(Fuel + Clad $+\mathrm{H} 2 \mathrm{O})^{\prime}$

$\begin{array}{llllll}\text { cylinder } & 1 & 1 & 0.5325 & 61.50 & 0.0\end{array}$

$\begin{array}{llllll}\text { cylinder } & 2 & 1 & 0.6115 & 61.50 & 0.0\end{array}$

$\begin{array}{lllllll}\text { cuboid } & 3 & 1 & 4 \mathrm{p} 0.978 & 61.50 & 0.0\end{array}$

unit 41

com $=$ '(Fuel + Clad $+\mathrm{H} 2 \mathrm{O})^{\prime}$

$\begin{array}{llllll}\text { cylinder } & 1 & 1 & 0.5325 & 61.50 & 0.0\end{array}$

$\begin{array}{llllll}\text { cylinder } & 2 & 1 & 0.6115 & 61.50 & 0.0\end{array}$

$\begin{array}{lllllll}\text { cuboid } & 3 & 1 & 4 \mathrm{p} 0.978 & 61.50 & 0.0\end{array}$

unit 42

com $=$ '(Fuel + Clad $+\mathrm{H} 2 \mathrm{O})^{\prime}$

$\begin{array}{llllll}\text { cylinder } & 1 & 1 & 0.5325 & 61.50 & 0.0\end{array}$

$\begin{array}{llllll}\text { cylinder } 2 & 1 & 0.6115 & 61.50 & 0.0\end{array}$

$\begin{array}{lllllll}\text { cuboid } 3 & 1 & 4 \mathrm{p} 0.978 & 61.50 & 0.0\end{array}$ unit 43

com='(Fuel+Clad $+\mathrm{H} 2 \mathrm{O})^{\prime}$

$\begin{array}{llllll}\text { cylinder } & 1 & 1 & 0.5325 & 61.50 & 0.0\end{array}$

$\begin{array}{llllll}\text { cylinder } & 2 & 1 & 0.6115 & 61.50 & 0.0\end{array}$

$\begin{array}{lllllll}\text { cuboid } & 3 & 1 & 4 p 0.978 & 61.50 & 0.0\end{array}$

unit 44

com $=$ '(Fuel+Clad $+\mathrm{H} 2 \mathrm{O})^{\prime}$

$\begin{array}{llllll}\text { cylinder } & 1 & 1 & 0.5325 & 61.50 & 0.0\end{array}$

$\begin{array}{llllll}\text { cylinder } 2 & 1 & 0.6115 & 61.50 & 0.0\end{array}$

$\begin{array}{lllllll}\text { cuboid } 3 & 1 & 4 p 0.978 & 61.50 & 0.0\end{array}$

unit 45

com $=$ '(Fuel + Clad $+\mathrm{H} 2 \mathrm{O})^{\prime}$

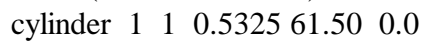

$\begin{array}{llllll}\text { cylinder } 2 & 1 & 0.6115 & 61.50 & 0.0\end{array}$

$\begin{array}{lllllll}\text { cuboid } & 3 & 1 & 4 \mathrm{p} 0.978 & 61.50 & 0.0\end{array}$

unit 46

com $=$ '(Fuel+Clad $+\mathrm{H} 2 \mathrm{O})^{\prime}$

cylinder $\begin{array}{llllll}1 & 1 & 0.5325 & 61.50 & 0.0\end{array}$

$\begin{array}{llllll}\text { cylinder } 2 & 1 & 0.6115 & 61.50 & 0.0\end{array}$

$\begin{array}{lllllll}\text { cuboid } & 3 & 1 & 4 p 0.978 & 61.50 & 0.0\end{array}$

unit 47

com $=$ '(Fuel + Clad $+\mathrm{H} 2 \mathrm{O})^{\prime}$

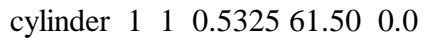

$\begin{array}{llllll}\text { cylinder } & 2 & 1 & 0.6115 & 61.50 & 0.0\end{array}$

$\begin{array}{lllllll}\text { cuboid } & 3 & 1 & 4 \mathrm{p} 0.978 & 61.50 & 0.0\end{array}$

unit 48

com='(Fuel+Clad $+\mathrm{H} 2 \mathrm{O})^{\prime}$ 
$\begin{array}{llllll}\text { cylinder } & 1 & 1 & 0.5325 & 61.50 & 0.0\end{array}$

$\begin{array}{llllll}\text { cylinder } & 2 & 1 & 0.6115 & 61.50 & 0.0\end{array}$

$\begin{array}{lllllll}\text { cuboid } 3 & 1 & 4 p 0.978 & 61.50 & 0.0\end{array}$

unit 49

com='(Fuel+Clad $+\mathrm{H} 2 \mathrm{O})^{\prime}$

$\begin{array}{llllll}\text { cylinder } & 1 & 1 & 0.5325 & 61.50 & 0.0\end{array}$

$\begin{array}{llllll}\text { cylinder } 2 & 1 & 0.6115 & 61.50 & 0.0\end{array}$

$\begin{array}{lllllll}\text { cuboid } & 3 & 1 & 4 \mathrm{p} 0.978 & 61.50 & 0.0\end{array}$

unit 50

com $=$ '(Fuel + Clad $+\mathrm{H} 2 \mathrm{O})^{\prime}$

$\begin{array}{llllll}\text { cylinder } & 1 & 1 & 0.5325 & 61.50 & 0.0\end{array}$

$\begin{array}{llllll}\text { cylinder } & 2 & 1 & 0.6115 & 61.50 & 0.0\end{array}$

$\begin{array}{llllll}\text { cuboid } 3 & 1 & 4 \mathrm{p} 0.978 & 61.50 & 0.0\end{array}$

unit 51

com $=$ '(Fuel $+\mathrm{Clad}+\mathrm{H} 2 \mathrm{O})^{\prime}$

$\begin{array}{llllll}\text { cylinder } & 1 & 1 & 0.5325 & 61.50 & 0.0\end{array}$

$\begin{array}{llllll}\text { cylinder } & 2 & 1 & 0.6115 & 61.50 & 0.0\end{array}$

$\begin{array}{lllllll}\text { cuboid } & 3 & 1 & 4 p 0.978 & 61.50 & 0.0\end{array}$

unit 52

com='(Fuel+Clad $+\mathrm{H} 2 \mathrm{O})^{\prime}$

$\begin{array}{llllll}\text { cylinder } & 1 & 1 & 0.5325 & 61.50 & 0.0\end{array}$

$\begin{array}{llllll}\text { cylinder } 2 & 1 & 0.6115 & 61.50 & 0.0\end{array}$

$\begin{array}{lllllll}\text { cuboid } & 3 & 1 & 4 \mathrm{p} 0.978 & 61.50 & 0.0\end{array}$ unit 53

com $=$ '(Fuel+Clad $+\mathrm{H} 2 \mathrm{O})^{\prime}$

$\begin{array}{lllllll}\text { cylinder } & 1 & 1 & 0.5325 & 61.50 & 0.0\end{array}$

$\begin{array}{llllll}\text { cylinder } 2 & 1 & 0.6115 & 61.50 & 0.0\end{array}$

$\begin{array}{lllllll}\text { cuboid } 3 & 1 & 4 \mathrm{p} 0.978 & 61.50 & 0.0\end{array}$

unit 54

com='(Fuel+Clad $+\mathrm{H} 2 \mathrm{O})^{\prime}$

$\begin{array}{llllll}\text { cylinder } & 1 & 1 & 0.5325 & 61.50 & 0.0\end{array}$

$\begin{array}{llllll}\text { cylinder } & 2 & 1 & 0.6115 & 61.50 & 0.0\end{array}$

$\begin{array}{lllllll}\text { cuboid } & 3 & 1 & 4 \mathrm{p} 0.978 & 61.50 & 0.0\end{array}$

unit 55

com='(Fuel+Clad $+\mathrm{H} 2 \mathrm{O})^{\prime}$

$\begin{array}{llllll}\text { cylinder } & 1 & 1 & 0.5325 & 61.50 & 0.0\end{array}$

$\begin{array}{llllll}\text { cylinder } & 2 & 1 & 0.6115 & 61.50 & 0.0\end{array}$

$\begin{array}{lllllll}\text { cuboid } & 3 & 1 & 4 \mathrm{p} 0.978 & 61.50 & 0.0\end{array}$

unit 56

com='(Fuel+Clad $+\mathrm{H} 2 \mathrm{O})^{\prime}$

$\begin{array}{llllll}\text { cylinder } & 1 & 1 & 0.5325 & 61.50 & 0.0\end{array}$

$\begin{array}{llllll}\text { cylinder } & 2 & 1 & 0.6115 & 61.50 & 0.0\end{array}$

$\begin{array}{llllll}\text { cuboid } 3 & 1 & 4 p 0.978 & 61.50 & 0.0\end{array}$

unit 57

com $='(\text { Fuel }+ \text { Clad }+\mathrm{H} 2 \mathrm{O})^{\prime}$

$\begin{array}{llllll}\text { cylinder } & 1 & 1 & 0.5325 & 61.50 & 0.0\end{array}$

$\begin{array}{llllll}\text { cylinder } & 2 & 1 & 0.6115 & 61.50 & 0.0\end{array}$

$\begin{array}{lllllll}\text { cuboid } & 3 & 1 & 4 p 0.978 & 61.50 & 0.0\end{array}$

unit 58

com $=$ '(Fuel + Clad $+\mathrm{H} 2 \mathrm{O})^{\prime}$

$\begin{array}{llllll}\text { cylinder } & 1 & 1 & 0.5325 & 61.50 & 0.0\end{array}$

$\begin{array}{llllll}\text { cylinder } & 2 & 1 & 0.6115 & 61.50 & 0.0\end{array}$

$\begin{array}{lllllll}\text { cuboid } & 3 & 1 & 4 \mathrm{p} 0.978 & 61.50 & 0.0\end{array}$

unit 59

com $=$ '(Fuel+Clad $+\mathrm{H} 2 \mathrm{O})^{\prime}$

$\begin{array}{llllll}\text { cylinder } & 1 & 1 & 0.5325 & 61.50 & 0.0\end{array}$

$\begin{array}{llllll}\text { cylinder } 2 & 1 & 0.6115 & 61.50 & 0.0\end{array}$

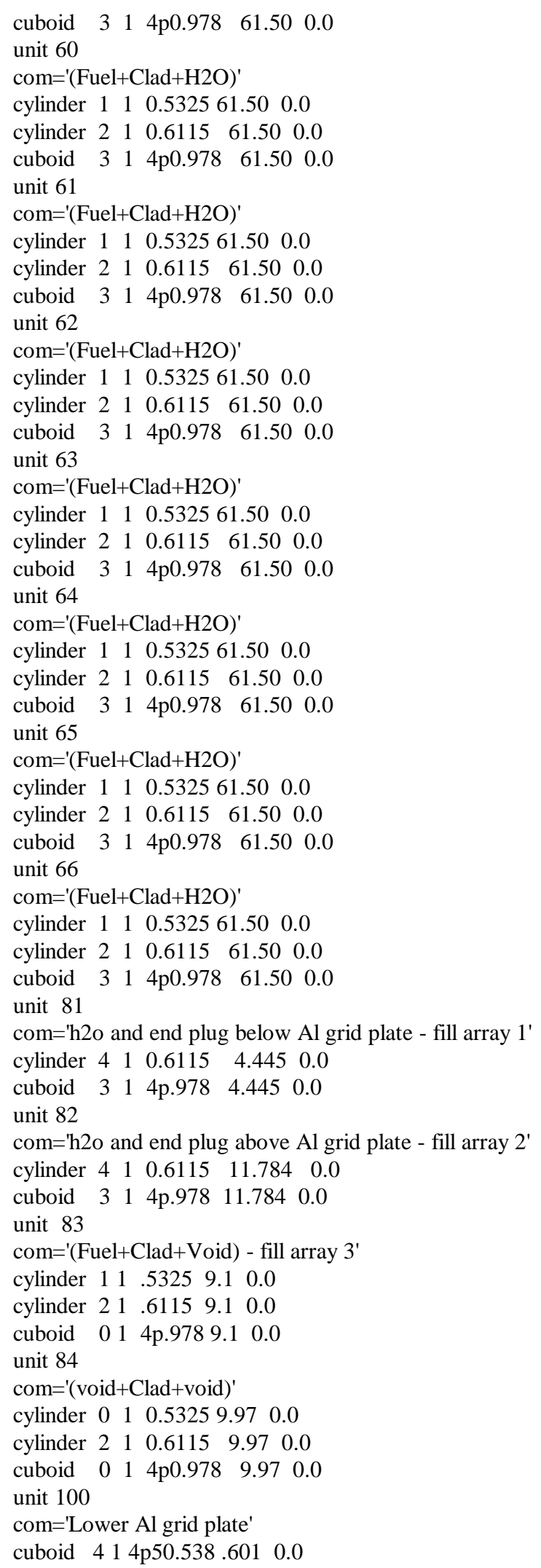


unit 101

com='array of h2o and end plugs below $\mathrm{Al}$ grid plate'

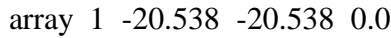

$\begin{array}{lllllll}\text { cuboid } & 3 & 1 & 4 p 50.538 & 4.445 & 0.0\end{array}$

unit 102

com='array of h2o and end plugs above $\mathrm{Al}$ grid plate '

array $2-20.538-20.538 \quad 0.0$

$\begin{array}{lllllll}\text { cuboid } & 3 & 1 & 4 p 50.538 & 11.784 & 0.0\end{array}$

unit 103

com='array of Fuel+Clad+Void'

$\begin{array}{llll}\text { array } 3 & -20.538 & -20.538 & 0.0\end{array}$

$\begin{array}{llllll}\text { cuboid } & 0 & 1 & 4 \mathrm{p} 50.5389 .1 & 0.0\end{array}$

unit 104

com='array of void+Clad+void'

$\begin{array}{llll}\text { array } 4 & -20.538 & -20.538 & 0.0\end{array}$

$\begin{array}{lllllll}\text { cuboid } & 0 & 1 & 4 \mathrm{p} 50.538 & 9.97 & 0.0\end{array}$

unit 105

com='array of Fuel+Clad+H2O'

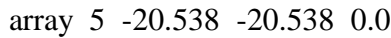

$\begin{array}{lllllll}\text { cuboid } & 3 & 1 & 4 p 50.538 & 61.50 & 0.0\end{array}$

global unit 110

com='array of arrays of fuel element sections + reflector'

$\begin{array}{llll}\text { array } 6 & -50.538 & -50.538 & 0.0\end{array}$

cuboid $4 \begin{array}{lllll}4 & 4 p 50.538 & 97.40 & -1.27\end{array}$

$\begin{array}{lllllll}\text { cuboid } & 5 & 1 & 4 p 50.538 & 97.40 & -3.47\end{array}$

$\begin{array}{lllllll}\text { cuboid } 3 & 1 & 4 p 50.538 & 97.40 & -17.27\end{array}$

$\begin{array}{lllllll}\text { cuboid } & 5 & 1 & 4 \mathrm{p} 50.538 & 97.40 & -17.77\end{array}$

$\begin{array}{lllllll}\text { cuboid } & 6 & 1 & 4 p 50.538 & 97.40 & -54.77\end{array}$

end geometry

read array

ara=1 nux=21 nuy=21 nuz=1

fill $\mathrm{f} 81$ end fill

ara $=2$ nux=21 nuy=21 nuz=1

fill $\mathrm{f} 82$ end fill

ara=3 nux=21 nuy=21 nuz=1

fill $\mathrm{f} 83$ end fill

ara $=4$ nux $=21$ nuy=21 nuz=1

fill $\mathrm{f} 84$ end fill

ara $=5$ nux=21 nuy=21 nuz=1

fill

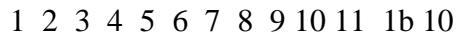

$212131415161718192021 \quad 1 b 10$

$313222324252627282930 \quad 1 b 10$

414233132333435363738 1b 10

515243239404142434445 1b 10

616253340464748495051 1b 10

717263441475253545556 1b 10

818273542485357585960 1b 10

919283643495458616263 1b 10

1020293744505559626465 1b 10

1121303845515660636566 1b 220

end fill

ara $=6$ nux=1 nuy=1 nuz=6

fill 101100102105103104 end fill

end array

end data

end

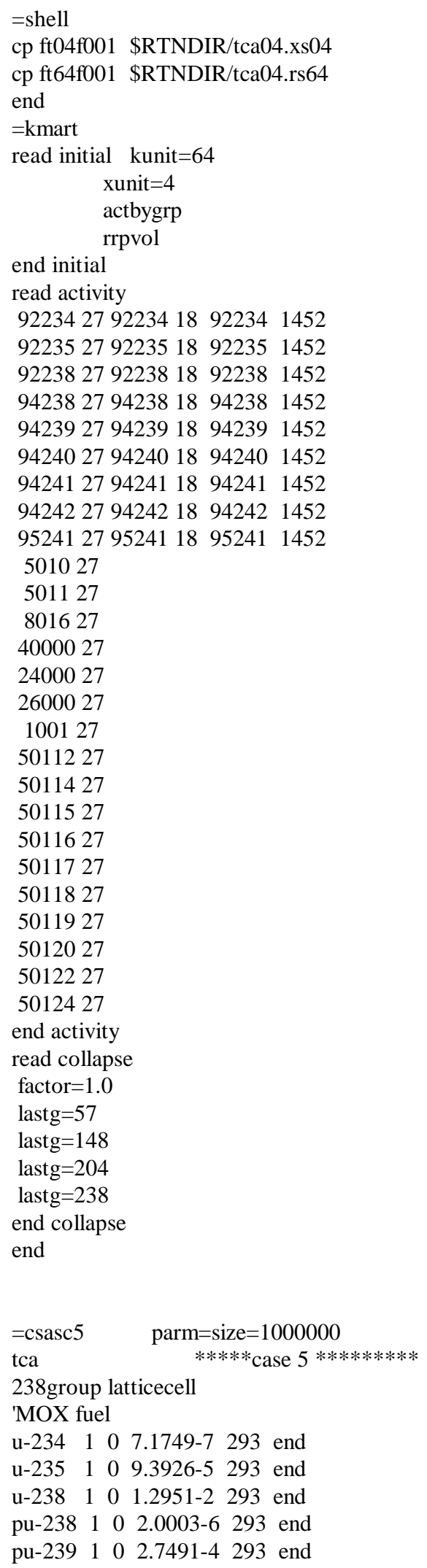




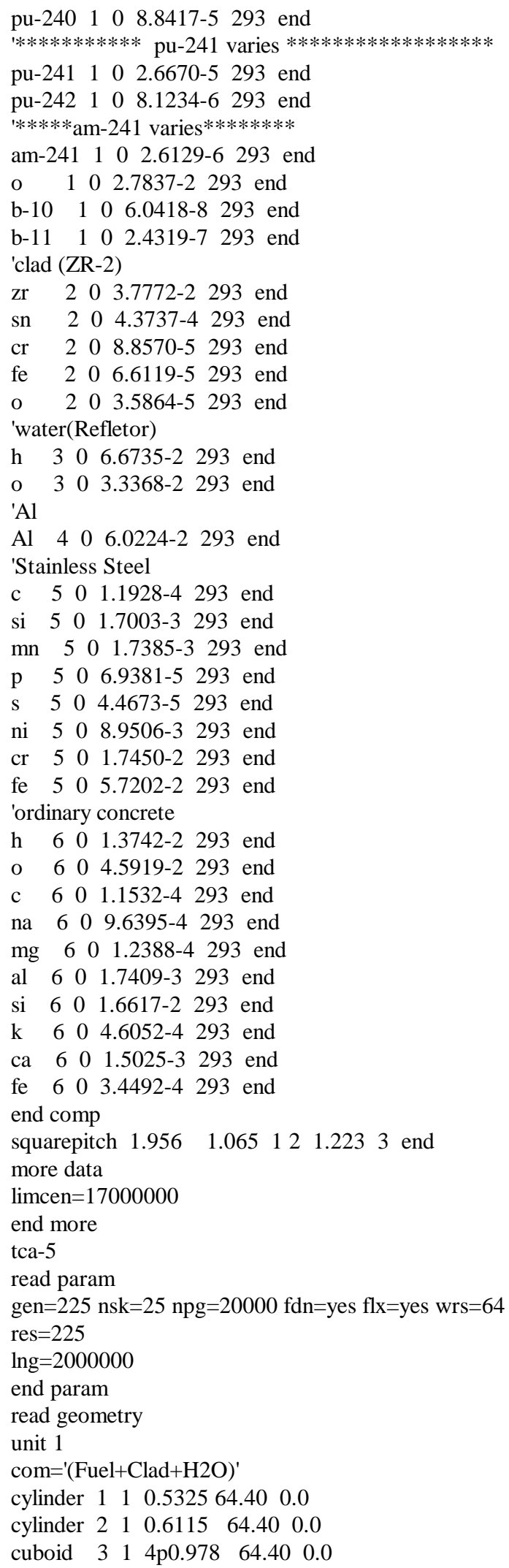

unit 2

com='(Fuel+Clad $+\mathrm{H} 2 \mathrm{O})^{\prime}$

cylinder $\begin{array}{lllll}1 & 1 & 0.5325 & 64.40 & 0.0\end{array}$

$\begin{array}{llllll}\text { cylinder } & 2 & 1 & 0.6115 & 64.40 & 0.0\end{array}$

$\begin{array}{lllllll}\text { cuboid } & 3 & 1 & 4 \mathrm{p} 0.978 & 64.40 & 0.0\end{array}$

unit 3

com $=$ '(Fuel + Clad $+\mathrm{H} 2 \mathrm{O})^{\prime}$

$\begin{array}{llllll}\text { cylinder } & 1 & 1 & 0.5325 & 64.40 & 0.0\end{array}$

$\begin{array}{llllll}\text { cylinder } & 2 & 1 & 0.6115 & 64.40 & 0.0\end{array}$

$\begin{array}{lllllll}\text { cuboid } & 3 & 1 & 4 \mathrm{p} 0.978 & 64.40 & 0.0\end{array}$

unit 4

com $=$ '(Fuel + Clad $+\mathrm{H} 2 \mathrm{O})^{\prime}$

cylinder $\begin{array}{lllll}1 & 1 & 0.5325 & 64.40 & 0.0\end{array}$

$\begin{array}{llllll}\text { cylinder } 2 & 1 & 0.6115 & 64.40 & 0.0\end{array}$

$\begin{array}{lllllll}\text { cuboid } & 3 & 1 & 4 \mathrm{p} 0.978 & 64.40 & 0.0\end{array}$

unit 5

com $=$ '(Fuel + Clad $+\mathrm{H} 2 \mathrm{O})^{\prime}$

$\begin{array}{llllll}\text { cylinder } & 1 & 1 & 0.5325 & 64.40 & 0.0\end{array}$

$\begin{array}{llllll}\text { cylinder } & 2 & 1 & 0.6115 & 64.40 & 0.0\end{array}$

$\begin{array}{lllllll}\text { cuboid } & 3 & 1 & 4 \mathrm{p} 0.978 & 64.40 & 0.0\end{array}$

unit 6

com $=$ '(Fuel + Clad $+\mathrm{H} 2 \mathrm{O})^{\prime}$

$\begin{array}{llllll}\text { cylinder } & 1 & 1 & 0.5325 & 64.40 & 0.0\end{array}$

$\begin{array}{llllll}\text { cylinder } 2 & 1 & 0.6115 & 64.40 & 0.0\end{array}$

$\begin{array}{lllllll}\text { cuboid } & 3 & 1 & 4 \mathrm{p} 0.978 & 64.40 & 0.0\end{array}$

unit 7

com $=$ '(Fuel + Clad $+\mathrm{H} 2 \mathrm{O})^{\prime}$

$\begin{array}{llllll}\text { cylinder } & 1 & 1 & 0.5325 & 64.40 & 0.0\end{array}$

$\begin{array}{llllll}\text { cylinder } 2 & 1 & 0.6115 & 64.40 & 0.0\end{array}$

$\begin{array}{lllllll}\text { cuboid } 3 & 1 & 4 p 0.978 & 64.40 & 0.0\end{array}$

unit 8

com $=$ '(Fuel + Clad $+\mathrm{H} 2 \mathrm{O})^{\prime}$

$\begin{array}{lllllll}\text { cylinder } & 1 & 1 & 0.5325 & 64.40 & 0.0\end{array}$

$\begin{array}{llllll}\text { cylinder } 2 & 1 & 0.6115 & 64.40 & 0.0\end{array}$

$\begin{array}{lllllll}\text { cuboid } & 3 & 1 & 4 p 0.978 & 64.40 & 0.0\end{array}$

unit 9

com $=(\text { Fuel }+ \text { Clad }+\mathrm{H} 2 \mathrm{O})^{\prime}$

$\begin{array}{lllllll}\text { cylinder } & 1 & 1 & 0.5325 & 64.40 & 0.0\end{array}$

$\begin{array}{llllll}\text { cylinder } & 2 & 1 & 0.6115 & 64.40 & 0.0\end{array}$

$\begin{array}{lllllll}\text { cuboid } 3 & 1 & 4 p 0.978 & 64.40 & 0.0\end{array}$

unit 10

com $=$ '(Fuel + Clad $+\mathrm{H} 2 \mathrm{O})^{\prime}$

$\begin{array}{llllll}\text { cylinder } & 1 & 1 & 0.5325 & 64.40 & 0.0\end{array}$

$\begin{array}{llllll}\text { cylinder } 2 & 1 & 0.6115 & 64.40 & 0.0\end{array}$

$\begin{array}{lllllll}\text { cuboid } \quad 3 & 1 & 4 \mathrm{p} 0.978 & 64.40 & 0.0\end{array}$ unit 11

com='(Fuel+Clad $+\mathrm{H} 2 \mathrm{O})^{\prime}$

$\begin{array}{lllllll}\text { cylinder } & 1 & 1 & 0.5325 & 64.40 & 0.0\end{array}$

$\begin{array}{llllll}\text { cylinder } 2 & 1 & 0.6115 & 64.40 & 0.0\end{array}$

$\begin{array}{lllllll}\text { cuboid } 3 & 1 & 4 p 0.978 & 64.40 & 0.0\end{array}$

unit 12

com $=$ '(Fuel + Clad $+\mathrm{H} 2 \mathrm{O})^{\prime}$

$\begin{array}{llllll}\text { cylinder } & 1 & 1 & 0.5325 & 64.40 & 0.0\end{array}$

$\begin{array}{llllll}\text { cylinder } & 2 & 1 & 0.6115 & 64.40 & 0.0\end{array}$

$\begin{array}{lllllll}\text { cuboid } & 3 & 1 & 4 \mathrm{p} 0.978 & 64.40 & 0.0\end{array}$

unit 13

com='(Fuel+Clad $+\mathrm{H} 2 \mathrm{O})^{\prime}$ 
$\begin{array}{llllll}\text { cylinder } & 1 & 1 & 0.5325 & 64.40 & 0.0\end{array}$

$\begin{array}{llllll}\text { cylinder } & 2 & 1 & 0.6115 & 64.40 & 0.0\end{array}$

$\begin{array}{llllll}\text { cuboad } & 3 & 1 & 4 \mathrm{p} 0.978 & 64.40 & 0.0\end{array}$

unit 14

com='(Fuel+Clad $+\mathrm{H} 2 \mathrm{O})^{\prime}$

$\begin{array}{llllll}\text { cylinder } & 1 & 1 & 0.5325 & 64.40 & 0.0\end{array}$

$\begin{array}{llllll}\text { cylinder } 2 & 1 & 0.6115 & 64.40 & 0.0\end{array}$

$\begin{array}{lllllll}\text { cuboid } & 3 & 1 & 4 \mathrm{p} 0.978 & 64.40 & 0.0\end{array}$

unit 15

com='(Fuel+Clad $+\mathrm{H} 2 \mathrm{O})^{\prime}$

$\begin{array}{lllllll}\text { cylinder } & 1 & 1 & 0.5325 & 64.40 & 0.0\end{array}$

$\begin{array}{llllll}\text { cylinder } & 2 & 1 & 0.6115 & 64.40 & 0.0\end{array}$

$\begin{array}{lllllll}\text { cuboid } 3 & 1 & 4 \mathrm{p} 0.978 & 64.40 & 0.0\end{array}$

unit 16

com $=$ '(Fuel $+\mathrm{Clad}+\mathrm{H} 2 \mathrm{O})^{\prime}$

$\begin{array}{llllll}\text { cylinder } & 1 & 1 & 0.5325 & 64.40 & 0.0\end{array}$

$\begin{array}{lllllll}\text { cylinder } 2 & 1 & 0.6115 & 64.40 & 0.0\end{array}$

$\begin{array}{lllllll}\text { cuboid } 3 & 1 & 4 \mathrm{p} 0.978 & 64.40 & 0.0\end{array}$

unit 17

com='(Fuel+Clad $+\mathrm{H} 2 \mathrm{O})^{\prime}$

$\begin{array}{llllll}\text { cylinder } & 1 & 1 & 0.5325 & 64.40 & 0.0\end{array}$

$\begin{array}{llllll}\text { cylinder } 2 & 1 & 0.6115 & 64.40 & 0.0\end{array}$

$\begin{array}{lllllll}\text { cuboid } & 3 & 1 & 4 \mathrm{p} 0.978 & 64.40 & 0.0\end{array}$

unit 18

com $=$ '(Fuel $+\mathrm{Clad}+\mathrm{H} 2 \mathrm{O})^{\prime}$

$\begin{array}{lllllll}\text { cylinder } & 1 & 1 & 0.5325 & 64.40 & 0.0\end{array}$

$\begin{array}{llllll}\text { cylinder } 2 & 1 & 0.6115 & 64.40 & 0.0\end{array}$

$\begin{array}{lllllll}\text { cuboid } 3 & 1 & 4 \mathrm{p} 0.978 & 64.40 & 0.0\end{array}$

unit 19

com='(Fuel+Clad $+\mathrm{H} 2 \mathrm{O})^{\prime}$

$\begin{array}{llllll}\text { cylinder } & 1 & 1 & 0.5325 & 64.40 & 0.0\end{array}$

$\begin{array}{llllll}\text { cylinder } 2 & 1 & 0.6115 & 64.40 & 0.0\end{array}$

$\begin{array}{lllllll}\text { cuboid } & 3 & 1 & 4 \mathrm{p} 0.978 & 64.40 & 0.0\end{array}$

unit 20

com='(Fuel+Clad $+\mathrm{H} 2 \mathrm{O})^{\prime}$

$\begin{array}{llllll}\text { cylinder } & 1 & 1 & 0.5325 & 64.40 & 0.0\end{array}$

$\begin{array}{lllllll}\text { cylinder } & 2 & 1 & 0.6115 & 64.40 & 0.0\end{array}$

$\begin{array}{lllllll}\text { cuboid } 3 & 1 & 4 \mathrm{p} 0.978 & 64.40 & 0.0\end{array}$

unit 21

com='(Fuel+Clad $+\mathrm{H} 2 \mathrm{O})^{\prime}$

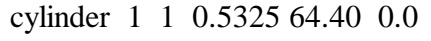

$\begin{array}{llllll}\text { cylinder } & 2 & 1 & 0.6115 & 64.40 & 0.0\end{array}$

$\begin{array}{lllllll}\text { cuboid } & 3 & 1 & 4 \mathrm{p} 0.978 & 64.40 & 0.0\end{array}$

unit 22

com $=$ '(Fuel + Clad $+\mathrm{H} 2 \mathrm{O})^{\prime}$

$\begin{array}{llllll}\text { cylinder } & 1 & 1 & 0.5325 & 64.40 & 0.0\end{array}$

$\begin{array}{llllll}\text { cylinder } & 2 & 1 & 0.6115 & 64.40 & 0.0\end{array}$

$\begin{array}{lllllll}\text { cuboid } & 3 & 1 & 4 p 0.978 & 64.40 & 0.0\end{array}$ unit 23

com='(Fuel+Clad $+\mathrm{H} 2 \mathrm{O})^{\prime}$

cylinder $\begin{array}{lllll}1 & 1 & 0.5325 & 64.40 & 0.0\end{array}$

$\begin{array}{llllll}\text { cylinder } & 2 & 1 & 0.6115 & 64.40 & 0.0\end{array}$

$\begin{array}{lllllll}\text { cuboid } 3 & 1 & 4 \mathrm{p} 0.978 & 64.40 & 0.0\end{array}$

unit 24

com $=$ '(Fuel+Clad $+\mathrm{H} 2 \mathrm{O})^{\prime}$

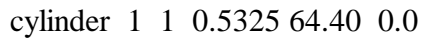

$\begin{array}{llllll}\text { cylinder } 2 & 1 & 0.6115 & 64.40 & 0.0\end{array}$ $\begin{array}{lllllll}\text { cuboid } \quad 3 & 1 & 4 \mathrm{p} 0.978 & 64.40 & 0.0\end{array}$

unit 25

com $=$ '(Fuel + Clad $+\mathrm{H} 2 \mathrm{O})^{\prime}$

$\begin{array}{llllll}\text { cylinder } & 1 & 1 & 0.5325 & 64.40 & 0.0\end{array}$

$\begin{array}{llllll}\text { cylinder } 2 & 1 & 0.6115 & 64.40 & 0.0\end{array}$

$\begin{array}{lllllll}\text { cuboid } 3 & 1 & 4 \mathrm{p} 0.978 & 64.40 & 0.0\end{array}$

unit 26

com='(Fuel+Clad $+\mathrm{H} 2 \mathrm{O})^{\prime}$

$\begin{array}{llllll}\text { cylinder } & 1 & 1 & 0.5325 & 64.40 & 0.0\end{array}$

$\begin{array}{llllll}\text { cylinder } 2 & 1 & 0.6115 & 64.40 & 0.0\end{array}$

$\begin{array}{lllllll}\text { cuboid } & 3 & 1 & 4 \mathrm{p} 0.978 & 64.40 & 0.0\end{array}$

unit 27

com $=$ '(Fuel + Clad $+\mathrm{H} 2 \mathrm{O})^{\prime}$

cylinder $\begin{array}{lllll}1 & 1 & 0.5325 & 64.40 & 0.0\end{array}$

$\begin{array}{llllllll}\text { cylinder } & 2 & 1 & 0.6115 & 64.40 & 0.0\end{array}$

$\begin{array}{lllllll}\text { cuboid } 3 & 1 & 4 \mathrm{p} 0.978 & 64.40 & 0.0\end{array}$

unit 28

com='(Fuel+Clad $+\mathrm{H} 2 \mathrm{O})^{\prime}$

$\begin{array}{llllll}\text { cylinder } & 1 & 1 & 0.5325 & 64.40 & 0.0\end{array}$

$\begin{array}{lllllll}\text { cylinder } 2 & 1 & 0.6115 & 64.40 & 0.0\end{array}$

$\begin{array}{lllllll}\text { cuboid } & 3 & 1 & 4 \mathrm{p} 0.978 & 64.40 & 0.0\end{array}$

unit 29

com $=$ '(Fuel + Clad $+\mathrm{H} 2 \mathrm{O})^{\prime}$

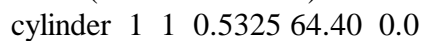

$\begin{array}{llllll}\text { cylinder } 2 & 1 & 0.6115 & 64.40 & 0.0\end{array}$

$\begin{array}{lllllll}\text { cuboid } & 3 & 1 & 4 \mathrm{p} 0.978 & 64.40 & 0.0\end{array}$

unit 30

com $=$ '(Fuel $+\mathrm{Clad}+\mathrm{H} 2 \mathrm{O})^{\prime}$

$\begin{array}{llllll}\text { cylinder } & 1 & 1 & 0.5325 & 64.40 & 0.0\end{array}$

$\begin{array}{llllll}\text { cylinder } 2 & 1 & 0.6115 & 64.40 & 0.0\end{array}$

$\begin{array}{lllllll}\text { cuboid } 3 & 1 & 4 p 0.978 & 64.40 & 0.0\end{array}$

unit 31

com $=$ '(Fuel + Clad $+\mathrm{H} 2 \mathrm{O})^{\prime}$

$\begin{array}{llllll}\text { cylinder } & 1 & 1 & 0.5325 & 64.40 & 0.0\end{array}$

$\begin{array}{llllll}\text { cylinder } 2 & 1 & 0.6115 & 64.40 & 0.0\end{array}$

$\begin{array}{lllllll}\text { cuboid } & 3 & 1 & 4 \mathrm{p} 0.978 & 64.40 & 0.0\end{array}$

unit 32

com $=$ '(Fuel + Clad $+\mathrm{H} 2 \mathrm{O})^{\prime}$

cylinder $\begin{array}{lllll}1 & 1 & 0.5325 & 64.40 & 0.0\end{array}$

$\begin{array}{llllll}\text { cylinder } 2 & 1 & 0.6115 & 64.40 & 0.0\end{array}$

$\begin{array}{lllllll}\text { cuboid } & 3 & 1 & 4 p 0.978 & 64.40 & 0.0\end{array}$

unit 33

com $=^{\prime}(\text { Fuel }+ \text { Clad }+\mathrm{H} 2 \mathrm{O})^{\prime}$

$\begin{array}{llllll}\text { cylinder } & 1 & 1 & 0.5325 & 64.40 & 0.0\end{array}$

$\begin{array}{llllll}\text { cylinder } 2 & 1 & 0.6115 & 64.40 & 0.0\end{array}$

$\begin{array}{lllllll}\text { cuboid } & 3 & 1 & 4 \mathrm{p} 0.978 & 64.40 & 0.0\end{array}$

unit 34

com $=$ '(Fuel + Clad $+\mathrm{H} 2 \mathrm{O})^{\prime}$

$\begin{array}{llllll}\text { cylinder } & 1 & 1 & 0.5325 & 64.40 & 0.0\end{array}$

$\begin{array}{llllll}\text { cylinder } 2 & 1 & 0.6115 & 64.40 & 0.0\end{array}$

$\begin{array}{lllllll}\text { cuboid } 3 & 1 & 4 p 0.978 & 64.40 & 0.0\end{array}$

unit 35

com $=$ '(Fuel + Clad $+\mathrm{H} 2 \mathrm{O})^{\prime}$

cylinder $\begin{array}{lllll}1 & 1 & 0.5325 & 64.40 & 0.0\end{array}$

$\begin{array}{llllll}\text { cylinder } 2 & 1 & 0.6115 & 64.40 & 0.0\end{array}$

$\begin{array}{lllllll}\text { cuboid } & 3 & 1 & 4 \mathrm{p} 0.978 & 64.40 & 0.0\end{array}$

unit 36 
com='(Fuel+Clad+H2O)'

$\begin{array}{llllll}\text { cylinder } & 1 & 1 & 0.5325 & 64.40 & 0.0\end{array}$

$\begin{array}{llllll}\text { cylinder } 2 & 1 & 0.6115 & 64.40 & 0.0\end{array}$

$\begin{array}{lllllll}\text { cuboid } & 3 & 1 & 4 \mathrm{p} 0.978 & 64.40 & 0.0\end{array}$

unit 37

com='(Fuel+Clad $+\mathrm{H} 2 \mathrm{O})^{\prime}$

$\begin{array}{llllll}\text { cylinder } & 1 & 1 & 0.5325 & 64.40 & 0.0\end{array}$

$\begin{array}{llllll}\text { cylinder } & 2 & 1 & 0.6115 & 64.40 & 0.0\end{array}$

$\begin{array}{lllllll}\text { cuboid } 3 & 1 & 4 \mathrm{p} 0.978 & 64.40 & 0.0\end{array}$

unit 38

com $=$ '(Fuel $+\mathrm{Clad}+\mathrm{H} 2 \mathrm{O})^{\prime}$

$\begin{array}{llllll}\text { cylinder } & 1 & 1 & 0.5325 & 64.40 & 0.0\end{array}$

$\begin{array}{llllll}\text { cylinder } & 2 & 1 & 0.6115 & 64.40 & 0.0\end{array}$

$\begin{array}{lllllll}\text { cuboid } & 3 & 1 & 4 \mathrm{p} 0.978 & 64.40 & 0.0\end{array}$

unit 39

com='(Fuel+Clad $+\mathrm{H} 2 \mathrm{O})^{\prime}$

$\begin{array}{llllll}\text { cylinder } & 1 & 1 & 0.5325 & 64.40 & 0.0\end{array}$

$\begin{array}{llllll}\text { cylinder } & 2 & 1 & 0.6115 & 64.40 & 0.0\end{array}$

$\begin{array}{lllllll}\text { cuboid } & 3 & 1 & 4 \mathrm{p} 0.978 & 64.40 & 0.0\end{array}$

unit 40

com $=$ '(Fuel+Clad $+\mathrm{H} 2 \mathrm{O})^{\prime}$

$\begin{array}{lllllll}\text { cylinder } & 1 & 1 & 0.5325 & 64.40 & 0.0\end{array}$

$\begin{array}{llllll}\text { cylinder } & 2 & 1 & 0.6115 & 64.40 & 0.0\end{array}$

$\begin{array}{lllllll}\text { cuboid } 3 & 1 & 4 p 0.978 & 64.40 & 0.0\end{array}$

unit 41

com $=$ '(Fuel + Clad $+\mathrm{H} 2 \mathrm{O})^{\prime}$

$\begin{array}{llllll}\text { cylinder } & 1 & 1 & 0.5325 & 64.40 & 0.0\end{array}$

$\begin{array}{llllll}\text { cylinder } & 2 & 1 & 0.6115 & 64.40 & 0.0\end{array}$

$\begin{array}{lllllll}\text { cuboid } 3 & 1 & 4 \mathrm{p} 0.978 & 64.40 & 0.0\end{array}$

unit 42

com='(Fuel+Clad $+\mathrm{H} 2 \mathrm{O})^{\prime}$

$\begin{array}{llllll}\text { cylinder } & 1 & 1 & 0.5325 & 64.40 & 0.0\end{array}$

$\begin{array}{llllll}\text { cylinder } 2 & 1 & 0.6115 & 64.40 & 0.0\end{array}$

$\begin{array}{lllllll}\text { cuboid } \quad 3 & 1 & 4 \mathrm{p} 0.978 & 64.40 & 0.0\end{array}$ unit 43

com $=$ '(Fuel+Clad $+\mathrm{H} 2 \mathrm{O})^{\prime}$

$\begin{array}{llllll}\text { cylinder } & 1 & 1 & 0.5325 & 64.40 & 0.0\end{array}$

$\begin{array}{llllll}\text { cylinder } & 2 & 1 & 0.6115 & 64.40 & 0.0\end{array}$

$\begin{array}{lllllll}\text { cuboid } 3 & 1 & 4 \mathrm{p} 0.978 & 64.40 & 0.0\end{array}$

unit 44

com $=$ '(Fuel+Clad $+\mathrm{H} 2 \mathrm{O})^{\prime}$

$\begin{array}{llllll}\text { cylinder } & 1 & 1 & 0.5325 & 64.40 & 0.0\end{array}$

$\begin{array}{llllll}\text { cylinder } & 2 & 1 & 0.6115 & 64.40 & 0.0\end{array}$

$\begin{array}{lllllll}\text { cuboid } & 3 & 1 & 4 \mathrm{p} 0.978 & 64.40 & 0.0\end{array}$

unit 45

com='(Fuel+Clad $+\mathrm{H} 2 \mathrm{O})^{\prime}$

cylinder $\begin{array}{lllll}1 & 1 & 0.5325 & 64.40 & 0.0\end{array}$

$\begin{array}{lllllll}\text { cylinder } & 2 & 1 & 0.6115 & 64.40 & 0.0\end{array}$

$\begin{array}{lllllll}\text { cuboid } 3 & 3 & 4 p 0.978 & 64.40 & 0.0\end{array}$

unit 46

com $=$ '(Fuel $+\mathrm{Clad}+\mathrm{H} 2 \mathrm{O})^{\prime}$

$\begin{array}{llllll}\text { cylinder } & 1 & 1 & 0.5325 & 64.40 & 0.0\end{array}$

$\begin{array}{llllll}\text { cylinder } & 2 & 1 & 0.6115 & 64.40 & 0.0\end{array}$

$\begin{array}{lllllll}\text { cuboid } 3 & 3 & 1 & 4 p 0.978 & 64.40 & 0.0\end{array}$

unit 47

com $='(\text { Fuel }+ \text { Clad }+\mathrm{H} 2 \mathrm{O})^{\prime}$

cylinder $\begin{array}{lllll}1 & 1 & 0.5325 & 64.40 & 0.0\end{array}$ $\begin{array}{llllll}\text { cylinder } & 2 & 1 & 0.6115 & 64.40 & 0.0\end{array}$ $\begin{array}{lllllll}\text { cuboid } 3 & 1 & 4 p 0.978 & 64.40 & 0.0\end{array}$ unit 48

com $=$ '(Fuel $+\mathrm{Clad}+\mathrm{H} 2 \mathrm{O})^{\prime}$

$\begin{array}{llllll}\text { cylinder } & 1 & 1 & 0.5325 & 64.40 & 0.0\end{array}$

$\begin{array}{llllll}\text { cylinder } 2 & 1 & 0.6115 & 64.40 & 0.0\end{array}$

$\begin{array}{lllllll}\text { cuboid } 3 & 1 & 4 \mathrm{p} 0.978 & 64.40 & 0.0\end{array}$

unit 49

com $=$ '(Fuel+Clad $+\mathrm{H} 2 \mathrm{O})^{\prime}$

$\begin{array}{llllll}\text { cylinder } & 1 & 1 & 0.5325 & 64.40 & 0.0\end{array}$

$\begin{array}{lllllll}\text { cylinder } 2 & 1 & 0.6115 & 64.40 & 0.0\end{array}$

$\begin{array}{lllllll}\text { cuboid } & 3 & 1 & 4 \mathrm{p} 0.978 & 64.40 & 0.0\end{array}$

unit 50

com $='(\text { Fuel }+\mathrm{Clad}+\mathrm{H} 2 \mathrm{O})^{\prime}$

$\begin{array}{lllllll}\text { cylinder } & 1 & 1 & 0.5325 & 64.40 & 0.0\end{array}$

$\begin{array}{llllll}\text { cylinder } 2 & 1 & 0.6115 & 64.40 & 0.0\end{array}$

$\begin{array}{lllllll}\text { cuboid } 3 & 1 & 4 \mathrm{p} 0.978 & 64.40 & 0.0\end{array}$

unit 51

com='(Fuel+Clad $+\mathrm{H} 2 \mathrm{O})^{\prime}$

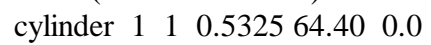

$\begin{array}{llllll}\text { cylinder } 2 & 1 & 0.6115 & 64.40 & 0.0\end{array}$

$\begin{array}{lllllll}\text { cuboid } & 3 & 1 & 4 \mathrm{p} 0.978 & 64.40 & 0.0\end{array}$

unit 52

com $=$ '(Fuel + Clad $+\mathrm{H} 2 \mathrm{O})^{\prime}$

$\begin{array}{llllll}\text { cylinder } & 1 & 1 & 0.5325 & 64.40 & 0.0\end{array}$

$\begin{array}{lllllll}\text { cylinder } & 2 & 1 & 0.6115 & 64.40 & 0.0\end{array}$

$\begin{array}{lllllll}\text { cuboid } 3 & 3 & 4 \mathrm{p} 0.978 & 64.40 & 0.0\end{array}$

unit 53

com $=$ '(Fuel + Clad $+\mathrm{H} 2 \mathrm{O})^{\prime}$

$\begin{array}{lllllll}\text { cylinder } & 1 & 1 & 0.5325 & 64.40 & 0.0\end{array}$

$\begin{array}{llllll}\text { cylinder } 2 & 1 & 0.6115 & 64.40 & 0.0\end{array}$

$\begin{array}{lllllll}\text { cuboid } & 3 & 1 & 4 \mathrm{p} 0.978 & 64.40 & 0.0\end{array}$

unit 54

com='(Fuel+Clad $+\mathrm{H} 2 \mathrm{O})^{\prime}$

$\begin{array}{llllll}\text { cylinder } & 1 & 1 & 0.5325 & 64.40 & 0.0\end{array}$

$\begin{array}{llllll}\text { cylinder } 2 & 1 & 0.6115 & 64.40 & 0.0\end{array}$

$\begin{array}{lllllll}\text { cuboid } & 3 & 1 & 4 \mathrm{p} 0.978 & 64.40 & 0.0\end{array}$

unit 55

com $=$ '(Fuel + Clad $+\mathrm{H} 2 \mathrm{O})^{\prime}$

$\begin{array}{llllll}\text { cylinder } & 1 & 1 & 0.5325 & 64.40 & 0.0\end{array}$

$\begin{array}{lllllll}\text { cylinder } & 2 & 1 & 0.6115 & 64.40 & 0.0\end{array}$

$\begin{array}{lllllll}\text { cuboid } & 3 & 1 & 4 \mathrm{p} 0.978 & 64.40 & 0.0\end{array}$

unit 56

com $=$ '(Fuel + Clad $+\mathrm{H} 2 \mathrm{O})^{\prime}$

$\begin{array}{lllllll}\text { cylinder } & 1 & 1 & 0.5325 & 64.40 & 0.0\end{array}$

$\begin{array}{llllll}\text { cylinder } 2 & 1 & 0.6115 & 64.40 & 0.0\end{array}$

$\begin{array}{lllllll}\text { cuboid } & 3 & 1 & 4 \mathrm{p} 0.978 & 64.40 & 0.0\end{array}$

unit 57

com $=$ '(Fuel + Clad $+\mathrm{H} 2 \mathrm{O})^{\prime}$

$\begin{array}{llllll}\text { cylinder } & 1 & 1 & 0.5325 & 64.40 & 0.0\end{array}$

$\begin{array}{llllll}\text { cylinder } 2 & 1 & 0.6115 & 64.40 & 0.0\end{array}$

$\begin{array}{lllllll}\text { cuboid } & 3 & 1 & 4 p 0.978 & 64.40 & 0.0\end{array}$

unit 58

com $=$ '(Fuel + Clad $+\mathrm{H} 2 \mathrm{O})^{\prime}$

cylinder $\begin{array}{lllll}1 & 1 & 0.5325 & 64.40 & 0.0\end{array}$

$\begin{array}{llllll}\text { cylinder } & 2 & 1 & 0.6115 & 64.40 & 0.0\end{array}$

$\begin{array}{lllllll}\text { cuboid } \quad 3 & 1 & 4 \mathrm{p} 0.978 & 64.40 & 0.0\end{array}$ 
unit 59

com='(Fuel+Clad $+\mathrm{H} 2 \mathrm{O})^{\prime}$

$\begin{array}{llllll}\text { cylinder } & 1 & 1 & 0.5325 & 64.40 & 0.0\end{array}$

$\begin{array}{llllll}\text { cylinder } 2 & 1 & 0.6115 & 64.40 & 0.0\end{array}$

$\begin{array}{lllllll}\text { cuboid } 3 & 1 & 4 \mathrm{p} 0.978 & 64.40 & 0.0\end{array}$

unit 60

com $=$ '(Fuel $+\mathrm{Clad}+\mathrm{H} 2 \mathrm{O})^{\prime}$

$\begin{array}{llllll}\text { cylinder } & 1 & 1 & 0.5325 & 64.40 & 0.0\end{array}$

$\begin{array}{llllll}\text { cylinder } 2 & 1 & 0.6115 & 64.40 & 0.0\end{array}$

$\begin{array}{lllllll}\text { cuboid } & 3 & 1 & 4 \mathrm{p} 0.978 & 64.40 & 0.0\end{array}$

unit 61

com='(Fuel+Clad $+\mathrm{H} 2 \mathrm{O})^{\prime}$

$\begin{array}{llllll}\text { cylinder } & 1 & 1 & 0.5325 & 64.40 & 0.0\end{array}$

$\begin{array}{llllll}\text { cylinder } & 2 & 1 & 0.6115 & 64.40 & 0.0\end{array}$

$\begin{array}{lllllll}\text { cuboid } & 3 & 1 & 4 \mathrm{p} 0.978 & 64.40 & 0.0\end{array}$

unit 62

com='(Fuel+Clad $+\mathrm{H} 2 \mathrm{O})^{\prime}$

$\begin{array}{llllll}\text { cylinder } & 1 & 1 & 0.5325 & 64.40 & 0.0\end{array}$

$\begin{array}{llllll}\text { cylinder } 2 & 1 & 0.6115 & 64.40 & 0.0\end{array}$

$\begin{array}{lllllll}\text { cuboid } & 3 & 1 & 4 \mathrm{p} 0.978 & 64.40 & 0.0\end{array}$

unit 63

com $='(\text { Fuel }+ \text { Clad }+\mathrm{H} 2 \mathrm{O})^{\prime}$

$\begin{array}{llllll}\text { cylinder } & 1 & 1 & 0.5325 & 64.40 & 0.0\end{array}$

$\begin{array}{llllll}\text { cylinder } 2 & 1 & 0.6115 & 64.40 & 0.0\end{array}$

$\begin{array}{lllllll}\text { cuboid } 3 & 1 & 4 \mathrm{p} 0.978 & 64.40 & 0.0\end{array}$

unit 64

com $=$ '(Fuel+Clad $+\mathrm{H} 2 \mathrm{O})^{\prime}$

$\begin{array}{llllll}\text { cylinder } & 1 & 1 & 0.5325 & 64.40 & 0.0\end{array}$

$\begin{array}{llllll}\text { cylinder } & 2 & 1 & 0.6115 & 64.40 & 0.0\end{array}$

$\begin{array}{lllllll}\text { cuboid } & 3 & 1 & 4 p 0.978 & 64.40 & 0.0\end{array}$

unit 65

com $=$ '(Fuel $+\mathrm{Clad}+\mathrm{H} 2 \mathrm{O})^{\prime}$

$\begin{array}{lllllll}\text { cylinder } & 1 & 1 & 0.5325 & 64.40 & 0.0\end{array}$

$\begin{array}{llllll}\text { cylinder } & 2 & 1 & 0.6115 & 64.40 & 0.0\end{array}$

$\begin{array}{lllllll}\text { cuboid } & 3 & 1 & 4 \mathrm{p} 0.978 & 64.40 & 0.0\end{array}$

unit 66

com $='(\text { Fuel }+ \text { Clad }+\mathrm{H} 2 \mathrm{O})^{\prime}$

$\begin{array}{llllll}\text { cylinder } & 1 & 1 & 0.5325 & 64.40 & 0.0\end{array}$

$\begin{array}{llllll}\text { cylinder } & 2 & 1 & 0.6115 & 64.40 & 0.0\end{array}$

$\begin{array}{lllllll}\text { cuboid } 3 & 1 & 4 \mathrm{p} 0.978 & 64.40 & 0.0\end{array}$

unit 81

com='h2o and end plug below Al grid plate - fill array 1'

$\begin{array}{lllll}\text { cylinder } 4 & 1 & 0.6115 & 4.445 & 0.0\end{array}$

$\begin{array}{lllllll}\text { cuboid } 3 & 1 & 4 p .978 & 4.445 & 0.0\end{array}$

unit 82

com='h2o and end plug above $\mathrm{Al}$ grid plate - fill array 2'

$\begin{array}{llllll}\text { cylinder } 4 & 1 & 0.6115 & 11.784 & 0.0\end{array}$

$\begin{array}{lllllll}\text { cuboid } & 3 & 1 & 4 p .978 & 11.784 & 0.0\end{array}$

unit 83

com='(Fuel+Clad+Void $)$ - fill array 3'

$\begin{array}{llllll}\text { cylinder } & 1 & 1 & .5325 & 6.2 & 0.0\end{array}$

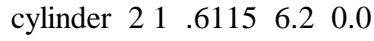

$\begin{array}{llllll}\text { cuboid } \quad 0 & 1 & 4 p .978 & 6.2 & 0.0\end{array}$

unit 84

com='(void+Clad+void)'

cylinder $0 \begin{array}{lllll}0 & 1 & 0.5325 & 9.97 & 0.0\end{array}$

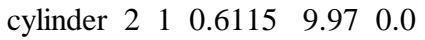

$\begin{array}{lllllll}\text { cuboid } & 0 & 1 & 4 p 0.978 & 9.97 & 0.0\end{array}$

unit 100

com='Lower $\mathrm{Al}$ grid plate'

cuboid $414 \mathrm{p} 50.538 .601 \quad 0.0$

unit 101

com='array of h2o and end plugs below $\mathrm{Al}$ grid plate'

array 1000

$\begin{array}{lllllll}\text { cuboid } & 3 & 1 & 4 \mathrm{p} 50.538 & 4.445 & 0.0\end{array}$

unit 102

com='array of $\mathrm{h} 2 \mathrm{o}$ and end plugs above $\mathrm{Al}$ grid plate '

array 2000

$\begin{array}{lllllll}\text { cuboid } & 3 & 1 & 4 \mathrm{p} 50.538 & 11.784 & 0.0\end{array}$

unit 103

com='array of Fuel+Clad+Void'

array 3000

$\begin{array}{lllllll}\text { cuboid } \quad 0 & 1 & 4 \mathrm{p} 50.538 & 6.2 & 0.0\end{array}$

unit 104

com='array of void+Clad+void'

array 4000

$\begin{array}{lllllll}\text { cuboid } & 0 & 1 & 4 \mathrm{p} 50.538 & 9.97 & 0.0\end{array}$

unit 105

com='array of Fuel+Clad+H2O'

array 5000

$\begin{array}{lllllll}\text { cuboid } & 3 & 1 & 4 \mathrm{p} 50.538 & 64.40 & 0.0\end{array}$

global unit 110

com='array of arrays of fuel element sections + reflector'

array $6-50.538 \quad-50.538 \quad 0$

cuboid $4 \begin{array}{lllll} & 1 & 4 \mathrm{p} 50.538 & 97.40 & -1.27\end{array}$

$\begin{array}{lllllll}\text { cuboid } & 5 & 1 & 4 p 50.538 & 97.40 & -3.47\end{array}$

cuboid $3 \quad 1 \quad 4 p 50.538 \quad 97.40 \quad-17.27$

$\begin{array}{lllllll}\text { cuboid } & 5 & 1 & 4 \mathrm{p} 50.538 & 97.40 & -17.77\end{array}$

$\begin{array}{lllllll}\text { cuboid } & 6 & 1 & 4 p 50.538 & 97.40 & -54.77\end{array}$

end geometry

read array

ara $=1$ nux $=21$ nuy=21 nuz=1

fill $\mathrm{f} 81$ end fill

ara=2 nux=21 nuy=21 nuz=1

fill $\mathrm{f} 82$ end fill

ara $=3$ nux $=21$ nuy=21 nuz=1

fill $\mathrm{f} 83$ end fill

ara $=4$ nux $=21$ nuy=21 nuz=1

fill $\mathrm{f} 84$ end fill

ara $=5$ nux=21 nuy=21 nuz=1

fill

1223455678991011 lb 10

$212131415161718192021 \quad 1 b 10$

313222324252627282930 1b 10

414233132333435363738 1b 10

$515243239404142434445 \quad 1 b 10$

616253340464748495051 bb 10

717263441475253545556 1b 10

818273542485357585960 1b 10

919283643495458616263 1b 10

1020293744505559626465 1b 10

1121303845515660636566 1b 220

end fill

ara $=6$ nux=1 nuy=1 nuz=6 
fill 101100102105103104 end fill end array

end data

end

=shell

cp ft04f001 \$RTNDIR/tca05.xs04 cp ft64f001 \$RTNDIR/tca05.rs64

end

$=\mathrm{kmart}$

read initial kunit $=64$

xunit $=4$

actbygrp

rrpvol

end initial

read activity

92234279223418922341452

92235279223518922351452

$9223827922381892238 \quad 1452$

$9423827942381894238 \quad 1452$

94239279423918942391452

94240279424018942401452

94241279424118942411452

$9424227942421894242 \quad 1452$

95241279524118952411452

501027

501027

501127

801627

4000027

2400027

2600027

100127

5011227

5011427

5011527

5011627

5011727

5011827

5011927

5012027

5012227

5012427

end activity

read collapse

factor $=1.0$

lastg $=57$

lastg $=148$

last $\mathrm{g}=204$

lastg $=238$

end collapse

end

$=\operatorname{csasc} 5 \quad$ parm $=$ size $=1000000$

tca $\quad * * * * *$ case $6 * * * * * * * * *$

238group latticecell

'MOX fuel u-234 $11007.1749-7 \quad 293$ end u-235 $1100.3926-5 \quad 293$ end u-238 $1001.2951-2293$ end pu-238 10 2.0003-6 293 end pu-239 100 2.7491-4 293 end pu-240 $10 \begin{array}{lll}0 & 8.8417-5 & 293 \\ \text { end }\end{array}$ ************ pu-241 varies $* * * * * * * * * * * * * * * * * *$ pu-241 $1002.4228-5293$ end pu-242 10 8.1234-6 293 end '*****am-241 varies $* * * * * * * *$ am-241 10 5.0543-6 293 end o $\quad \begin{array}{llll}1 & 0 & 2.7837-2 & 293\end{array}$ end b-10 $100.0418-8 \quad 293$ end b-11 $11002.4319-7293$ end 'clad (ZR-2)

zr $2 \quad \begin{array}{llll}0 & 3.7772-2 & 293 & \text { end }\end{array}$

sn $\quad 2 \quad 0 \quad 4.3737-4293$ end

cr $2008.8570-5293$ end

fe $200.6119-5293$ end

o $\quad 2 \quad 0 \quad 3.5864-5293$ end 'water(Refletor)

h $3 \quad 0 \quad 6.6735-2 \quad 293$ end

o 30 3.3368-2 293 end 'Al

Al 40 6.0224-2 293 end

'Stainless Steel

c 50 1.1928-4 293 end

si $5 \quad 0 \quad 1.7003-3293$ end

mn $5 \quad 0 \quad 1.7385-3293$ end

p $\quad 5 \quad 0 \quad 6.9381-5293$ end

s $5004.4673-5293$ end

ni $\begin{array}{lllll}5 & 0 & 8.9506-3 & 293 & \text { end }\end{array}$

cr $5 \quad 0 \quad 1.7450-2293$ end

fe $\begin{array}{llll}5 & 0 & 5.7202-2 & 293\end{array}$ end

'ordinary concrete

h $\quad \begin{array}{llll}6 & 0 & 1.3742-2 & 293 \\ & \text { end }\end{array}$

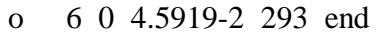

c 60 1.1532-4 293 end

na $6 \begin{array}{llll}6 & 0 & 9.6395-4 & 293 \\ \text { end }\end{array}$

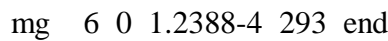

al $\begin{array}{lllll}6 & 0 & 1.7409-3 & 293 & \text { end }\end{array}$

si $6 \begin{array}{llll}6 & 0 & 1.6617-2 & 293 \\ & \text { end }\end{array}$

k $6 \begin{array}{llll}6 & 0 & 4.6052-4 & 293\end{array}$ end

ca $\begin{array}{lllll}6 & 0 & 1.5025-3 & 293 & \text { end }\end{array}$

fe $\begin{array}{llll}6 & 0 & 3.4492-4 & 293 \\ \text { end }\end{array}$

end comp

squarepitch $1.956 \quad 1.065 \quad 12 \quad 1.223 \quad 3$ end

more data

limcen $=17000000$

end more

tca-6

read param

gen=225 nsk=25 npg=20000 $\mathrm{fdn}=$ yes $f l x=$ yes wrs $=64$

res $=225$

$\operatorname{lng}=2000000$

end param

read geometry 
unit 1

com='(Fuel + Clad $+\mathrm{H} 2 \mathrm{O})^{\prime}$

$\begin{array}{llllll}\text { cylinder } & 1 & 1 & 0.5325 & 69.40 & 0.0\end{array}$

$\begin{array}{llllll}\text { cylinder } 2 & 1 & 0.6115 & 69.40 & 0.0\end{array}$

$\begin{array}{lllllll}\text { cuboid } 3 & 3 & 1 & 4 p 0.978 & 69.40 & 0.0\end{array}$

unit 2

com $=$ '(Fuel $+\mathrm{Clad}+\mathrm{H} 2 \mathrm{O})^{\prime}$

$\begin{array}{llllll}\text { cylinder } & 1 & 1 & 0.5325 & 69.40 & 0.0\end{array}$

$\begin{array}{llllll}\text { cylinder } 2 & 1 & 0.6115 & 69.40 & 0.0\end{array}$

$\begin{array}{lllllll}\text { cuboid } \quad 3 & 1 & 4 \mathrm{p} 0.978 & 69.40 & 0.0\end{array}$

unit 3

com='(Fuel+Clad $+\mathrm{H} 2 \mathrm{O})^{\prime}$

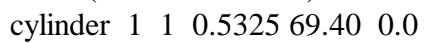

$\begin{array}{llllll}\text { cylinder } & 2 & 1 & 0.6115 & 69.40 & 0.0\end{array}$

$\begin{array}{lllllll}\text { cuboid } & 3 & 1 & 4 \mathrm{p} 0.978 & 69.40 & 0.0\end{array}$

unit 4

com='(Fuel+Clad $+\mathrm{H} 2 \mathrm{O})^{\prime}$

$\begin{array}{llllll}\text { cylinder } & 1 & 1 & 0.5325 & 69.40 & 0.0\end{array}$

$\begin{array}{llllll}\text { cylinder } & 2 & 1 & 0.6115 & 69.40 & 0.0\end{array}$

$\begin{array}{lllllll}\text { cuboid } 3 & 1 & 4 p 0.978 & 69.40 & 0.0\end{array}$

unit 5

com $=$ '(Fuel $+\mathrm{Clad}+\mathrm{H} 2 \mathrm{O})^{\prime}$

$\begin{array}{llllll}\text { cylinder } & 1 & 1 & 0.5325 & 69.40 & 0.0\end{array}$

$\begin{array}{llllll}\text { cylinder } & 2 & 1 & 0.6115 & 69.40 & 0.0\end{array}$

$\begin{array}{lllllll}\text { cuboid } 3 & 3 & 4 \mathrm{p} 0.978 & 69.40 & 0.0\end{array}$

unit 6

com $=$ '(Fuel + Clad $+\mathrm{H} 2 \mathrm{O})^{\prime}$

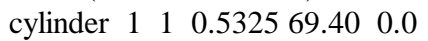

$\begin{array}{llllll}\text { cylinder } & 2 & 1 & 0.6115 & 69.40 & 0.0\end{array}$

$\begin{array}{lllllll}\text { cuboid } 3 & 3 & 4 \mathrm{p} 0.978 & 69.40 & 0.0\end{array}$

unit 7

com='(Fuel+Clad $+\mathrm{H} 2 \mathrm{O})^{\prime}$

$\begin{array}{lllllll}\text { cylinder } & 1 & 1 & 0.5325 & 69.40 & 0.0\end{array}$

$\begin{array}{llllll}\text { cylinder } & 2 & 1 & 0.6115 & 69.40 & 0.0\end{array}$

$\begin{array}{lllllll}\text { cuboid } & 3 & 1 & 4 \mathrm{p} 0.978 & 69.40 & 0.0\end{array}$

unit 8

com='(Fuel+Clad $+\mathrm{H} 2 \mathrm{O})^{\prime}$

$\begin{array}{llllll}\text { cylinder } & 1 & 1 & 0.5325 & 69.40 & 0.0\end{array}$

$\begin{array}{llllll}\text { cylinder } 2 & 1 & 0.6115 & 69.40 & 0.0\end{array}$

$\begin{array}{lllllll}\text { cuboid } 3 & 1 & 4 p 0.978 & 69.40 & 0.0\end{array}$

unit 9

com='(Fuel+Clad $+\mathrm{H} 2 \mathrm{O})^{\prime}$

$\begin{array}{llllll}\text { cylinder } & 1 & 1 & 0.5325 & 69.40 & 0.0\end{array}$

$\begin{array}{llllll}\text { cylinder } 2 & 1 & 0.6115 & 69.40 & 0.0\end{array}$

$\begin{array}{lllllll}\text { cuboid } \quad 3 & 1 & 4 \mathrm{p} 0.978 & 69.40 & 0.0\end{array}$

unit 10

com $=$ '(Fuel + Clad $+\mathrm{H} 2 \mathrm{O})^{\prime}$

cylinder $\begin{array}{llllll}1 & 1 & 0.5325 & 69.40 & 0.0\end{array}$

$\begin{array}{llllll}\text { cylinder } 2 & 1 & 0.6115 & 69.40 & 0.0\end{array}$

$\begin{array}{lllllll}\text { cuboid } 3 & 1 & 4 p 0.978 & 69.40 & 0.0\end{array}$

unit 11

com $=$ '(Fuel $+\mathrm{Clad}+\mathrm{H} 2 \mathrm{O})^{\prime}$

$\begin{array}{llllll}\text { cylinder } & 1 & 1 & 0.5325 & 69.40 & 0.0\end{array}$

$\begin{array}{llllll}\text { cylinder } 2 & 1 & 0.6115 & 69.40 & 0.0\end{array}$

$\begin{array}{lllllll}\text { cuboid } & 3 & 1 & 4 \mathrm{p} 0.978 & 69.40 & 0.0\end{array}$

unit 12

com='(Fuel+Clad $+\mathrm{H} 2 \mathrm{O})^{\prime}$ $\begin{array}{llllll}\text { cylinder } & 1 & 1 & 0.5325 & 69.40 & 0.0\end{array}$

$\begin{array}{llllll}\text { cylinder } & 2 & 1 & 0.6115 & 69.40 & 0.0\end{array}$

$\begin{array}{lllllll}\text { cuboid } 3 & 1 & 4 p 0.978 & 69.40 & 0.0\end{array}$

unit 13

com $=^{\prime}(\text { Fuel }+ \text { Clad }+\mathrm{H} 2 \mathrm{O})^{\prime}$

$\begin{array}{llllll}\text { cylinder } & 1 & 1 & 0.5325 & 69.40 & 0.0\end{array}$

$\begin{array}{lllllll}\text { cylinder } 2 & 1 & 0.6115 & 69.40 & 0.0\end{array}$

$\begin{array}{lllllll}\text { cuboid } 3 & 1 & 4 \mathrm{p} 0.978 & 69.40 & 0.0\end{array}$

unit 14

com='(Fuel+Clad $+\mathrm{H} 2 \mathrm{O})^{\prime}$

$\begin{array}{lllllll}\text { cylinder } & 1 & 1 & 0.5325 & 69.40 & 0.0\end{array}$

$\begin{array}{llllll}\text { cylinder } 2 & 1 & 0.6115 & 69.40 & 0.0\end{array}$

$\begin{array}{lllllll}\text { cuboid } 3 & 1 & 4 p 0.978 & 69.40 & 0.0\end{array}$

unit 15

com $=$ '(Fuel + Clad $+\mathrm{H} 2 \mathrm{O})^{\prime}$

$\begin{array}{llllll}\text { cylinder } & 1 & 1 & 0.5325 & 69.40 & 0.0\end{array}$

$\begin{array}{llllll}\text { cylinder } 2 & 1 & 0.6115 & 69.40 & 0.0\end{array}$

$\begin{array}{lllllll}\text { cuboid } 3 & 1 & 4 p 0.978 & 69.40 & 0.0\end{array}$

unit 16

com='(Fuel+Clad $+\mathrm{H} 2 \mathrm{O})^{\prime}$

$\begin{array}{llllll}\text { cylinder } & 1 & 1 & 0.5325 & 69.40 & 0.0\end{array}$

$\begin{array}{lllllll}\text { cylinder } 2 & 1 & 0.6115 & 69.40 & 0.0\end{array}$

$\begin{array}{lllllll}\text { cuboid } & 3 & 1 & 4 \mathrm{p} 0.978 & 69.40 & 0.0\end{array}$

unit 17

com $=$ '(Fuel+Clad $+\mathrm{H} 2 \mathrm{O})^{\prime}$

$\begin{array}{lllllll}\text { cylinder } & 1 & 1 & 0.5325 & 69.40 & 0.0\end{array}$

$\begin{array}{llllll}\text { cylinder } & 2 & 1 & 0.6115 & 69.40 & 0.0\end{array}$

$\begin{array}{lllllll}\text { cuboid } 3 & 1 & 4 \mathrm{p} 0.978 & 69.40 & 0.0\end{array}$

unit 18

com='(Fuel+Clad $+\mathrm{H} 2 \mathrm{O})^{\prime}$

$\begin{array}{llllll}\text { cylinder } & 1 & 1 & 0.5325 & 69.40 & 0.0\end{array}$

$\begin{array}{llllll}\text { cylinder } 2 & 1 & 0.6115 & 69.40 & 0.0\end{array}$

$\begin{array}{lllllll}\text { cuboid } & 3 & 1 & 4 \mathrm{p} 0.978 & 69.40 & 0.0\end{array}$

unit 19

com $=$ '(Fuel $+\mathrm{Clad}+\mathrm{H} 2 \mathrm{O})^{\prime}$

$\begin{array}{llllll}\text { cylinder } & 1 & 1 & 0.5325 & 69.40 & 0.0\end{array}$

$\begin{array}{lllllll}\text { cylinder } & 2 & 1 & 0.6115 & 69.40 & 0.0\end{array}$

$\begin{array}{lllllll}\text { cuboid } 3 & 1 & 4 p 0.978 & 69.40 & 0.0\end{array}$

unit 20

com='(Fuel+Clad $+\mathrm{H} 2 \mathrm{O})^{\prime}$

cylinder $\begin{array}{lllll}1 & 1 & 0.5325 & 69.40 & 0.0\end{array}$

$\begin{array}{llllll}\text { cylinder } 2 & 1 & 0.6115 & 69.40 & 0.0\end{array}$

$\begin{array}{lllllll}\text { cuboid } & 3 & 1 & 4 \mathrm{p} 0.978 & 69.40 & 0.0\end{array}$

unit 21

com='(Fuel+Clad $+\mathrm{H} 2 \mathrm{O})^{\prime}$

$\begin{array}{llllll}\text { cylinder } & 1 & 1 & 0.5325 & 69.40 & 0.0\end{array}$

$\begin{array}{llllll}\text { cylinder } 2 & 1 & 0.6115 & 69.40 & 0.0\end{array}$

$\begin{array}{lllllll}\text { cuboid } & 3 & 1 & 4 \mathrm{p} 0.978 & 69.40 & 0.0\end{array}$

unit 22

com $=$ '(Fuel + Clad $+\mathrm{H} 2 \mathrm{O})^{\prime}$

$\begin{array}{llllll}\text { cylinder } & 1 & 1 & 0.5325 & 69.40 & 0.0\end{array}$

$\begin{array}{llllllll}\text { cylinder } & 2 & 1 & 0.6115 & 69.40 & 0.0\end{array}$

$\begin{array}{lllllll}\text { cuboid } \quad 3 & 1 & 4 \mathrm{p} 0.978 & 69.40 & 0.0\end{array}$

unit 23

com $=$ '(Fuel+Clad $+\mathrm{H} 2 \mathrm{O})^{\prime}$

$\begin{array}{llllll}\text { cylinder } & 1 & 1 & 0.5325 & 69.40 & 0.0\end{array}$

$\begin{array}{lllll}\text { cylinder } 2 & 1 & 0.6115 & 69.40 & 0.0\end{array}$ 
$\begin{array}{lllllll}\text { cuboid } & 3 & 1 & 4 p 0.978 & 69.40 & 0.0\end{array}$ unit 24

com $='(\text { Fuel }+ \text { Clad }+\mathrm{H} 2 \mathrm{O})^{\prime}$

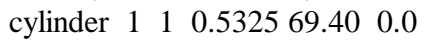

$\begin{array}{llllll}\text { cylinder } & 2 & 1 & 0.6115 & 69.40 & 0.0\end{array}$

$\begin{array}{lllllll}\text { cuboid } 3 & 1 & 4 \mathrm{p} 0.978 & 69.40 & 0.0\end{array}$

unit 25

com='(Fuel+Clad $+\mathrm{H} 2 \mathrm{O})^{\prime}$

$\begin{array}{llllll}\text { cylinder } & 1 & 1 & 0.5325 & 69.40 & 0.0\end{array}$

$\begin{array}{llllll}\text { cylinder } & 2 & 1 & 0.6115 & 69.40 & 0.0\end{array}$

$\begin{array}{lllllll}\text { cuboid } & 3 & 1 & 4 p 0.978 & 69.40 & 0.0\end{array}$

unit 26

com='(Fuel+Clad $+\mathrm{H} 2 \mathrm{O})^{\prime}$

$\begin{array}{llllll}\text { cylinder } & 1 & 1 & 0.5325 & 69.40 & 0.0\end{array}$

$\begin{array}{lllllll}\text { cylinder } 2 & 1 & 0.6115 & 69.40 & 0.0\end{array}$

$\begin{array}{lllllll}\text { cuboid } 3 & 3 & 1 & 4 p 0.978 & 69.40 & 0.0\end{array}$

unit 27

com='(Fuel+Clad $+\mathrm{H} 2 \mathrm{O})^{\prime}$

$\begin{array}{llllll}\text { cylinder } & 1 & 1 & 0.5325 & 69.40 & 0.0\end{array}$

$\begin{array}{llllll}\text { cylinder } 2 & 1 & 0.6115 & 69.40 & 0.0\end{array}$

$\begin{array}{lllllll}\text { cuboid } & 3 & 1 & 4 \mathrm{p} 0.978 & 69.40 & 0.0\end{array}$

unit 28

com='(Fuel+Clad $+\mathrm{H} 2 \mathrm{O})^{\prime}$

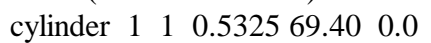

$\begin{array}{llllll}\text { cylinder } 2 & 1 & 0.6115 & 69.40 & 0.0\end{array}$

$\begin{array}{lllllll}\text { cuboid } & 3 & 1 & 4 \mathrm{p} 0.978 & 69.40 & 0.0\end{array}$

unit 29

com='(Fuel+Clad $+\mathrm{H} 2 \mathrm{O})^{\prime}$

cylinder $\begin{array}{lllll}1 & 1 & 0.5325 & 69.40 & 0.0\end{array}$

$\begin{array}{llllll}\text { cylinder } 2 & 1 & 0.6115 & 69.40 & 0.0\end{array}$

$\begin{array}{lllllll}\text { cuboid } 3 & 1 & 4 \mathrm{p} 0.978 & 69.40 & 0.0\end{array}$

unit 30

com $=$ '(Fuel $+\mathrm{Clad}+\mathrm{H} 2 \mathrm{O})^{\prime}$

$\begin{array}{llllll}\text { cylinder } & 1 & 1 & 0.5325 & 69.40 & 0.0\end{array}$

$\begin{array}{llllll}\text { cylinder } & 2 & 1 & 0.6115 & 69.40 & 0.0\end{array}$

$\begin{array}{lllllll}\text { cuboid } 3 & 1 & 4 \mathrm{p} 0.978 & 69.40 & 0.0\end{array}$

unit 31

com='(Fuel+Clad $+\mathrm{H} 2 \mathrm{O})^{\prime}$

$\begin{array}{llllll}\text { cylinder } & 1 & 1 & 0.5325 & 69.40 & 0.0\end{array}$

$\begin{array}{llllll}\text { cylinder } & 2 & 1 & 0.6115 & 69.40 & 0.0\end{array}$

$\begin{array}{lllllll}\text { cuboid } 3 & 1 & 4 \mathrm{p} 0.978 & 69.40 & 0.0\end{array}$

unit 32

com $='(\text { Fuel }+ \text { Clad }+\mathrm{H} 2 \mathrm{O})^{\prime}$

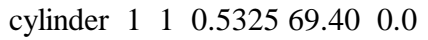

$\begin{array}{llllll}\text { cylinder } 2 & 1 & 0.6115 & 69.40 & 0.0\end{array}$

$\begin{array}{lllllll}\text { cuboid } & 3 & 1 & 4 \mathrm{p} 0.978 & 69.40 & 0.0\end{array}$

unit 33

com='(Fuel+Clad $+\mathrm{H} 2 \mathrm{O})^{\prime}$

$\begin{array}{llllll}\text { cylinder } & 1 & 1 & 0.5325 & 69.40 & 0.0\end{array}$

$\begin{array}{llllll}\text { cylinder } & 2 & 1 & 0.6115 & 69.40 & 0.0\end{array}$

$\begin{array}{lllllll}\text { cuboid } & 3 & 1 & 4 \mathrm{p} 0.978 & 69.40 & 0.0\end{array}$

unit 34

com $=$ '(Fuel + Clad $+\mathrm{H} 2 \mathrm{O})^{\prime}$

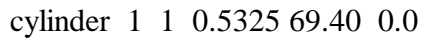

$\begin{array}{llllll}\text { cylinder } & 2 & 1 & 0.6115 & 69.40 & 0.0\end{array}$

$\begin{array}{lllllll}\text { cuboid } 3 & 1 & 4 \mathrm{p} 0.978 & 69.40 & 0.0\end{array}$ unit 35

com $=$ '(Fuel + Clad $+\mathrm{H} 2 \mathrm{O})^{\prime}$

$\begin{array}{llllll}\text { cylinder } & 1 & 1 & 0.5325 & 69.40 & 0.0\end{array}$

$\begin{array}{llllll}\text { cylinder } & 2 & 1 & 0.6115 & 69.40 & 0.0\end{array}$

$\begin{array}{lllllll}\text { cuboid } 3 & 1 & 4 \mathrm{p} 0.978 & 69.40 & 0.0\end{array}$

unit 36

com='(Fuel+Clad $+\mathrm{H} 2 \mathrm{O})^{\prime}$

$\begin{array}{llllll}\text { cylinder } & 1 & 1 & 0.5325 & 69.40 & 0.0\end{array}$

$\begin{array}{llllll}\text { cylinder } 2 & 1 & 0.6115 & 69.40 & 0.0\end{array}$

$\begin{array}{lllllll}\text { cuboid } & 3 & 1 & 4 \mathrm{p} 0.978 & 69.40 & 0.0\end{array}$

unit 37

com $=$ '(Fuel $+\mathrm{Clad}+\mathrm{H} 2 \mathrm{O})^{\prime}$

$\begin{array}{llllll}\text { cylinder } & 1 & 1 & 0.5325 & 69.40 & 0.0\end{array}$

$\begin{array}{llllll}\text { cylinder } 2 & 1 & 0.6115 & 69.40 & 0.0\end{array}$

$\begin{array}{lllllll}\text { cuboid } & 3 & 1 & 4 \mathrm{p} 0.978 & 69.40 & 0.0\end{array}$

unit 38

com $=^{\prime}(\text { Fuel }+ \text { Clad }+\mathrm{H} 2 \mathrm{O})^{\prime}$

$\begin{array}{llllll}\text { cylinder } & 1 & 1 & 0.5325 & 69.40 & 0.0\end{array}$

$\begin{array}{llllll}\text { cylinder } 2 & 1 & 0.6115 & 69.40 & 0.0\end{array}$

$\begin{array}{lllllll}\text { cuboid } 3 & 1 & 4 \mathrm{p} 0.978 & 69.40 & 0.0\end{array}$

unit 39

com $=$ '(Fuel + Clad $+\mathrm{H} 2 \mathrm{O})^{\prime}$

$\begin{array}{llllll}\text { cylinder } & 1 & 1 & 0.5325 & 69.40 & 0.0\end{array}$

$\begin{array}{llllll}\text { cylinder } & 2 & 1 & 0.6115 & 69.40 & 0.0\end{array}$

$\begin{array}{lllllll}\text { cuboid } & 3 & 1 & 4 \mathrm{p} 0.978 & 69.40 & 0.0\end{array}$

unit 40

com $=$ '(Fuel + Clad $+\mathrm{H} 2 \mathrm{O})^{\prime}$

$\begin{array}{llllll}\text { cylinder } & 1 & 1 & 0.5325 & 69.40 & 0.0\end{array}$

$\begin{array}{llllll}\text { cylinder } & 2 & 1 & 0.6115 & 69.40 & 0.0\end{array}$

$\begin{array}{lllllll}\text { cuboid } & 3 & 1 & 4 p 0.978 & 69.40 & 0.0\end{array}$

unit 41

com $=$ '(Fuel + Clad $+\mathrm{H} 2 \mathrm{O})^{\prime}$

$\begin{array}{llllll}\text { cylinder } & 1 & 1 & 0.5325 & 69.40 & 0.0\end{array}$

$\begin{array}{llllll}\text { cylinder } 2 & 1 & 0.6115 & 69.40 & 0.0\end{array}$

$\begin{array}{lllllll}\text { cuboid } & 3 & 1 & 4 p 0.978 & 69.40 & 0.0\end{array}$

unit 42

com $=$ '(Fuel + Clad $+\mathrm{H} 2 \mathrm{O})^{\prime}$

$\begin{array}{llllll}\text { cylinder } & 1 & 1 & 0.5325 & 69.40 & 0.0\end{array}$

$\begin{array}{llllll}\text { cylinder } 2 & 1 & 0.6115 & 69.40 & 0.0\end{array}$

$\begin{array}{lllllll}\text { cuboid } & 3 & 1 & 4 p 0.978 & 69.40 & 0.0\end{array}$

unit 43

com $=$ '(Fuel + Clad $+\mathrm{H} 2 \mathrm{O})^{\prime}$

$\begin{array}{llllll}\text { cylinder } & 1 & 1 & 0.5325 & 69.40 & 0.0\end{array}$

$\begin{array}{llllll}\text { cylinder } 2 & 1 & 0.6115 & 69.40 & 0.0\end{array}$

$\begin{array}{lllllll}\text { cuboid } & 3 & 1 & 4 \mathrm{p} 0.978 & 69.40 & 0.0\end{array}$

unit 44

com $=$ '(Fuel+Clad $+\mathrm{H} 2 \mathrm{O})^{\prime}$

cylinder $\begin{array}{llllll}1 & 1 & 0.5325 & 69.40 & 0.0\end{array}$

$\begin{array}{llllll}\text { cylinder } & 2 & 1 & 0.6115 & 69.40 & 0.0\end{array}$

$\begin{array}{lllllll}\text { cuboid } 3 & 1 & 4 p 0.978 & 69.40 & 0.0\end{array}$

unit 45

com='(Fuel+Clad $+\mathrm{H} 2 \mathrm{O})^{\prime}$

cylinder $\begin{array}{lllll}1 & 1 & 0.5325 & 69.40 & 0.0\end{array}$

$\begin{array}{llllll}\text { cylinder } & 2 & 1 & 0.6115 & 69.40 & 0.0\end{array}$

$\begin{array}{lllllll}\text { cuboid } & 3 & 1 & 4 \mathrm{p} 0.978 & 69.40 & 0.0\end{array}$

unit 46

com='(Fuel+Clad $+\mathrm{H} 2 \mathrm{O})^{\prime}$ 
$\begin{array}{llllll}\text { cylinder } & 1 & 1 & 0.5325 & 69.40 & 0.0\end{array}$

$\begin{array}{llllll}\text { cylinder } & 2 & 1 & 0.6115 & 69.40 & 0.0\end{array}$

$\begin{array}{lllllll}\text { cuboid } 3 & 1 & 4 p 0.978 & 69.40 & 0.0\end{array}$

unit 47

com='(Fuel+Clad $+\mathrm{H} 2 \mathrm{O})^{\prime}$

$\begin{array}{llllll}\text { cylinder } & 1 & 1 & 0.5325 & 69.40 & 0.0\end{array}$

$\begin{array}{llllll}\text { cylinder } 2 & 1 & 0.6115 & 69.40 & 0.0\end{array}$

$\begin{array}{lllllll}\text { cuboid } & 3 & 1 & 4 p 0.978 & 69.40 & 0.0\end{array}$ unit 48

com $=$ '(Fuel + Clad $+\mathrm{H} 2 \mathrm{O})^{\prime}$

$\begin{array}{llllll}\text { cylinder } & 1 & 1 & 0.5325 & 69.40 & 0.0\end{array}$

$\begin{array}{llllll}\text { cylinder } & 2 & 1 & 0.6115 & 69.40 & 0.0\end{array}$

$\begin{array}{lllllll}\text { cuboid } 3 & 1 & 4 \mathrm{p} 0.978 & 69.40 & 0.0\end{array}$

unit 49

com $=$ '(Fuel $+\mathrm{Clad}+\mathrm{H} 2 \mathrm{O})^{\prime}$

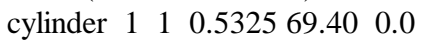

$\begin{array}{lllllll}\text { cylinder } 2 & 1 & 0.6115 & 69.40 & 0.0\end{array}$

$\begin{array}{lllllll}\text { cuboid } & 3 & 1 & 4 p 0.978 & 69.40 & 0.0\end{array}$

unit 50

com='(Fuel+Clad $+\mathrm{H} 2 \mathrm{O})^{\prime}$

$\begin{array}{llllll}\text { cylinder } & 1 & 1 & 0.5325 & 69.40 & 0.0\end{array}$

$\begin{array}{lllllll}\text { cylinder } 2 & 1 & 0.6115 & 69.40 & 0.0\end{array}$

$\begin{array}{lllllll}\text { cuboid } 3 & 1 & 4 \mathrm{p} 0.978 & 69.40 & 0.0\end{array}$ unit 51

com $=$ '(Fuel $+\mathrm{Clad}+\mathrm{H} 2 \mathrm{O})^{\prime}$

$\begin{array}{lllllll}\text { cylinder } & 1 & 1 & 0.5325 & 69.40 & 0.0\end{array}$

$\begin{array}{llllll}\text { cylinder } 2 & 1 & 0.6115 & 69.40 & 0.0\end{array}$

$\begin{array}{lllllll}\text { cuboid } 3 & 1 & 4 \mathrm{p} 0.978 & 69.40 & 0.0\end{array}$

unit 52

com='(Fuel+Clad $+\mathrm{H} 2 \mathrm{O})^{\prime}$

$\begin{array}{llllll}\text { cylinder } & 1 & 1 & 0.5325 & 69.40 & 0.0\end{array}$

$\begin{array}{llllll}\text { cylinder } 2 & 1 & 0.6115 & 69.40 & 0.0\end{array}$

$\begin{array}{lllllll}\text { cuboid } & 3 & 1 & 4 \mathrm{p} 0.978 & 69.40 & 0.0\end{array}$

unit 53

com='(Fuel+Clad $+\mathrm{H} 2 \mathrm{O})^{\prime}$

$\begin{array}{llllll}\text { cylinder } & 1 & 1 & 0.5325 & 69.40 & 0.0\end{array}$

$\begin{array}{lllllll}\text { cylinder } & 2 & 1 & 0.6115 & 69.40 & 0.0\end{array}$

$\begin{array}{lllllll}\text { cuboid } 3 & 1 & 4 \mathrm{p} 0.978 & 69.40 & 0.0\end{array}$

unit 54

com='(Fuel+Clad $+\mathrm{H} 2 \mathrm{O})^{\prime}$

$\begin{array}{llllll}\text { cylinder } & 1 & 1 & 0.5325 & 69.40 & 0.0\end{array}$

$\begin{array}{llllll}\text { cylinder } & 2 & 1 & 0.6115 & 69.40 & 0.0\end{array}$

$\begin{array}{lllllll}\text { cuboid } & 3 & 1 & 4 \mathrm{p} 0.978 & 69.40 & 0.0\end{array}$

unit 55

com $='(\text { Fuel }+ \text { Clad }+\mathrm{H} 2 \mathrm{O})^{\prime}$

cylinder $\begin{array}{lllll}1 & 1 & 0.5325 & 69.40 & 0.0\end{array}$

$\begin{array}{llllll}\text { cylinder } 2 & 1 & 0.6115 & 69.40 & 0.0\end{array}$

$\begin{array}{lllllll}\text { cuboid } & 3 & 1 & 4 p 0.978 & 69.40 & 0.0\end{array}$

unit 56

com $=^{\prime}(\text { Fuel }+\mathrm{Clad}+\mathrm{H} 2 \mathrm{O})^{\prime}$

$\begin{array}{llllll}\text { cylinder } & 1 & 1 & 0.5325 & 69.40 & 0.0\end{array}$

$\begin{array}{llllll}\text { cylinder } & 2 & 1 & 0.6115 & 69.40 & 0.0\end{array}$

$\begin{array}{lllllll}\text { cuboid } 3 & 1 & 4 \mathrm{p} 0.978 & 69.40 & 0.0\end{array}$

unit 57

com $=$ '(Fuel+Clad $+\mathrm{H} 2 \mathrm{O})^{\prime}$

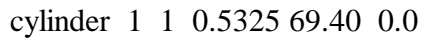

$\begin{array}{llllll}\text { cylinder } 2 & 1 & 0.6115 & 69.40 & 0.0\end{array}$

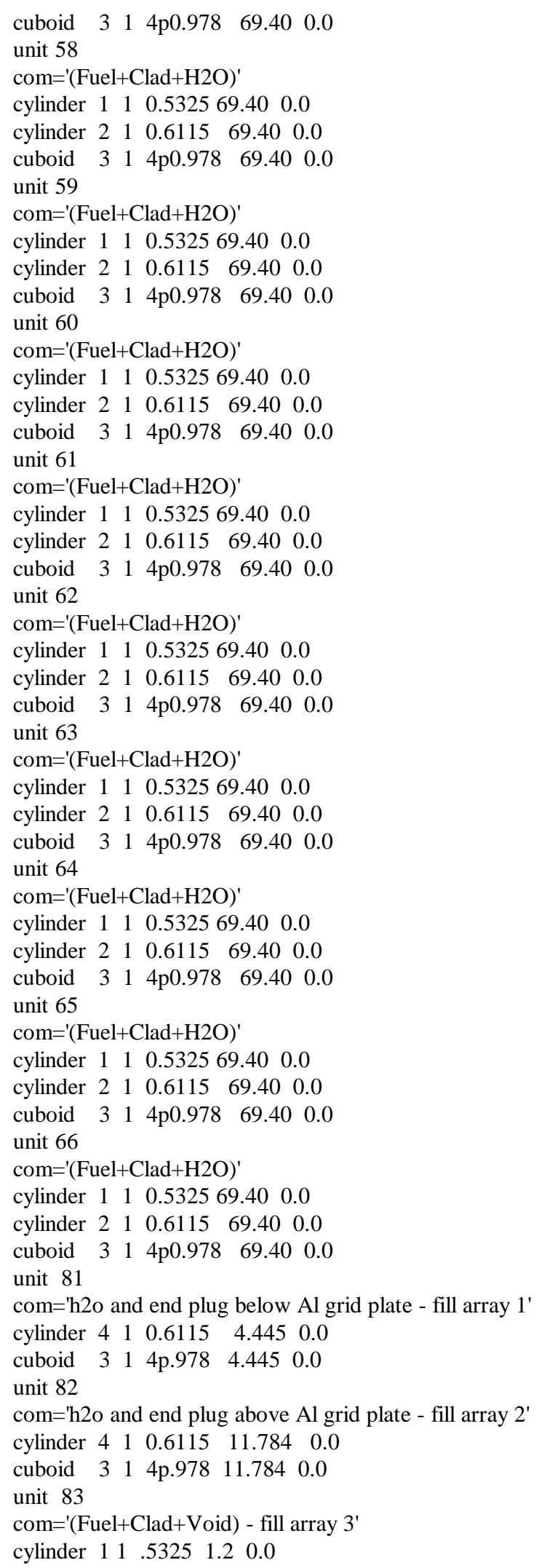




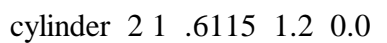

cuboid 014 p.978 1.20 .0

unit 84

com='(void+Clad+void)'

cylinder $\begin{array}{lllll}0 & 1 & 0.5325 & 9.97 & 0.0\end{array}$

$\begin{array}{lllll}\text { cylinder } 2 & 1 & 0.6115 & 9.97 & 0.0\end{array}$

$\begin{array}{lllllll}\text { cuboid } & 0 & 1 & 4 \mathrm{p} 0.978 & 9.97 & 0.0\end{array}$

unit 100

com='Lower Al grid plate'

cuboid $414 \mathrm{p} 50.538 .601 \quad 0.0$

unit 101

com='array of $\mathrm{h} 2 \mathrm{o}$ and end plugs below $\mathrm{Al}$ grid plate'

array 1000

$\begin{array}{lllllll}\text { cuboid } & 3 & 1 & 4 \mathrm{p} 50.538 & 4.445 & 0.0\end{array}$

unit 102

com='array of h2o and end plugs above Al grid plate '

array 20000

$\begin{array}{lllllll}\text { cuboid } & 3 & 1 & 4 p 50.538 & 11.784 & 0.0\end{array}$

unit 103

com='array of Fuel+Clad+Void'

array 3000

$\begin{array}{llllll}\text { cuboid } & 0 & 1 & 4 \mathrm{p} 50.538 & 1.2 & 0.0\end{array}$

unit 104

com='array of void+Clad+void'

array 4000

$\begin{array}{lllllll}\text { cuboid } & 0 & 1 & 4 \mathrm{p} 50.538 & 9.97 & 0.0\end{array}$

unit 105

com='array of Fuel+Clad+H2O'

array 50000

$\begin{array}{lllllll}\text { cuboid } & 3 & 1 & 4 p 50.538 & 69.40 & 0.0\end{array}$

global unit 110

com='array of arrays of fuel element sections + reflector'

array $6-50.538-50.5380$

$\begin{array}{lllllll}\text { cuboid } & 4 & 1 & 4 p 50.538 & 97.40 & -1.27\end{array}$

$\begin{array}{lllllll}\text { cuboid } & 5 & 1 & 4 p 50.538 & 97.40 & -3.47\end{array}$

$\begin{array}{lllllll}\text { cuboid } 3 & 1 & 4 p 50.538 & 97.40 & -17.27\end{array}$

$\begin{array}{lllllll}\text { cuboid } & 5 & 1 & 4 p 50.538 & 97.40 & -17.77\end{array}$

$\begin{array}{lllllll}\text { cuboid } & 6 & 1 & 4 p 50.538 & 97.40 & -54.77\end{array}$

end geometry

read array

ara=1 nux=21 nuy=21 nuz=1

fill $\mathrm{f} 81$ end fill

ara $=2$ nux $=21$ nuy=21 nuz=1

fill $\mathrm{f} 82$ end fill

ara=3 nux=21 nuy=21 nuz=1

fill $\mathrm{f} 83$ end fill

ara $=4$ nux=21 nuy=21 nuz=1

fill f84 end fill

ara $=5$ nux=21 nuy=21 nuz=1

fill

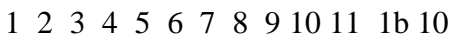

$212131415161718192021 \quad 1 b 10$

$313222324252627282930 \quad 1 b 10$

414233132333435363738 lb 10

515243239404142434445 1b 10

616253340464748495051 1b 10

717263441475253545556 1b 10
$818273542485357585960 \quad 1 b 10$

919283643495458616263 lb 10

1020293744505559626465 lb 10

1121303845515660636566 1b 220

end fill

ara $=6$ nux $=1$ nuy $=1$ nuz $=6$

fill 101100102105103104 end fill

end array

end data

end

$=$ shell

cp ft04f001 \$RTNDIR/tca06.xs04

cp ft64f001 \$RTNDIR/tca06.rs64

end

$=\mathrm{kmart}$

read initial kunit $=64$

xunit $=4$

actbygrp

rrpvol

end initial

read activity

92234279223418922341452

92235279223518922351452

92238279223818922381452

94238279423818942381452

94239279423918942391452

94240279424018942401452

$9424127942411894241 \quad 1452$

94242279424218942421452

95241279524118952411452

501027

501027

501127

801627

4000027

2400027

2600027

100127

5011227

5011427

5011527

5011627

5011727

5011827

5011927

5012027

5012227

5012427

end activity

read collapse

factor $=1.0$

lastg $=57$

last $\mathrm{g}=148$

last $\mathrm{g}=204$

last $\mathrm{g}=238$

end collapse

end 


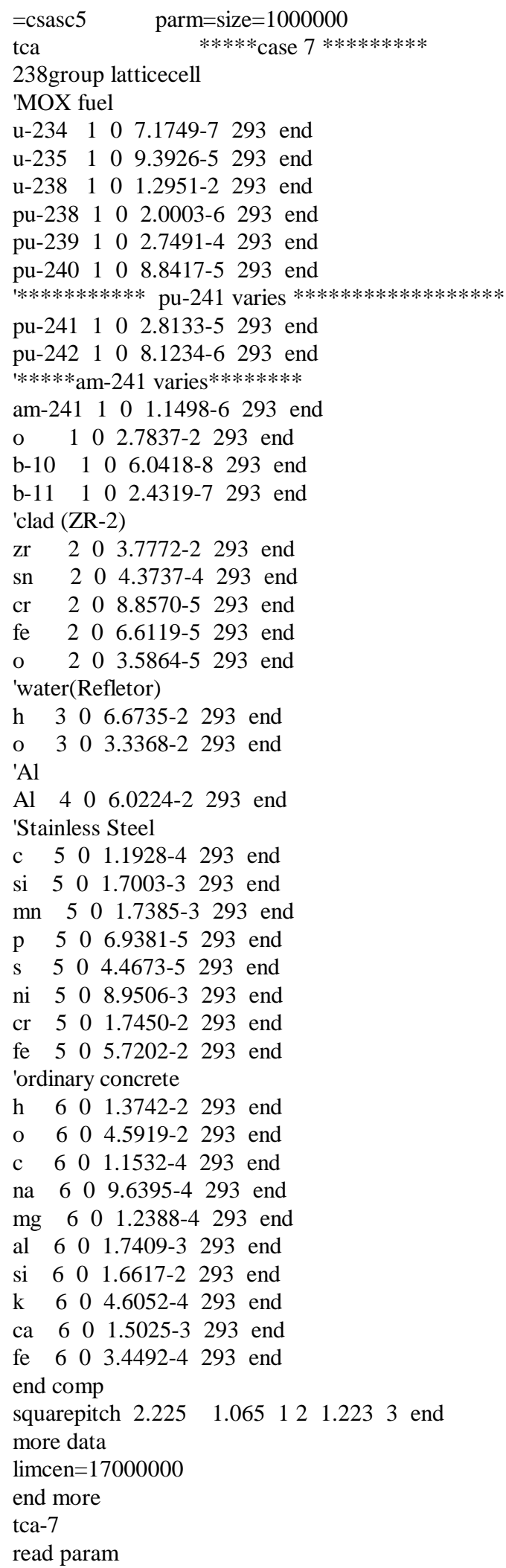

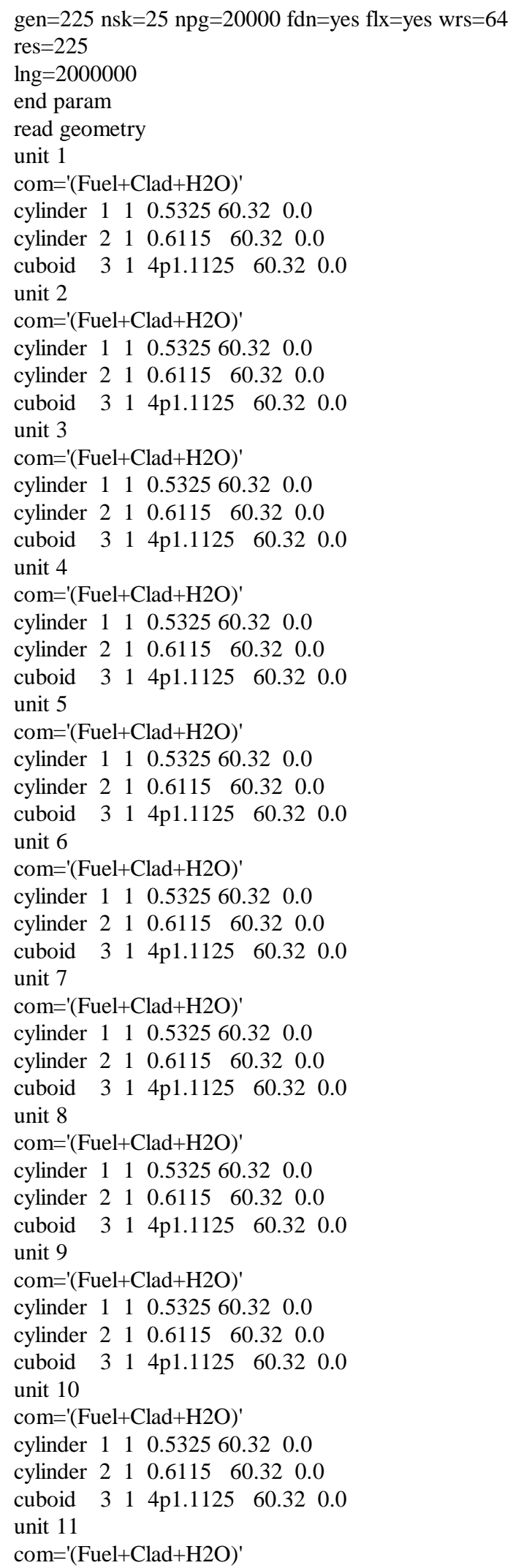


$\begin{array}{llllll}\text { cylinder } & 1 & 1 & 0.5325 & 60.32 & 0.0\end{array}$

$\begin{array}{llllll}\text { cylinder } & 2 & 1 & 0.6115 & 60.32 & 0.0\end{array}$

$\begin{array}{lllllll}\text { cuboid } & 3 & 1 & 4 \mathrm{p} 1.1125 & 60.32 & 0.0\end{array}$

unit 12

com='(Fuel+Clad $+\mathrm{H} 2 \mathrm{O})^{\prime}$

$\begin{array}{llllll}\text { cylinder } & 1 & 1 & 0.5325 & 60.32 & 0.0\end{array}$

$\begin{array}{llllll}\text { cylinder } 2 & 1 & 0.6115 & 60.32 & 0.0\end{array}$

$\begin{array}{lllllll}\text { cuboid } & 3 & 1 & 4 \mathrm{p} 1.1125 & 60.32 & 0.0\end{array}$

unit 13

com='(Fuel+Clad $+\mathrm{H} 2 \mathrm{O})^{\prime}$

$\begin{array}{lllllll}\text { cylinder } & 1 & 1 & 0.5325 & 60.32 & 0.0\end{array}$

$\begin{array}{llllll}\text { cylinder } 2 & 1 & 0.6115 & 60.32 & 0.0\end{array}$

$\begin{array}{lllllll}\text { cuboid } & 3 & 1 & 4 \mathrm{p} 1.1125 & 60.32 & 0.0\end{array}$

unit 14

com='(Fuel+Clad $+\mathrm{H} 2 \mathrm{O})^{\prime}$

$\begin{array}{llllll}\text { cylinder } & 1 & 1 & 0.5325 & 60.32 & 0.0\end{array}$

$\begin{array}{llllll}\text { cylinder } & 2 & 1 & 0.6115 & 60.32 & 0.0\end{array}$

$\begin{array}{lllllll}\text { cuboid } & 3 & 1 & 4 p 1.1125 & 60.32 & 0.0\end{array}$

unit 15

com='(Fuel+Clad $+\mathrm{H} 2 \mathrm{O})^{\prime}$

$\begin{array}{llllll}\text { cylinder } & 1 & 1 & 0.5325 & 60.32 & 0.0\end{array}$

$\begin{array}{llllll}\text { cylinder } 2 & 1 & 0.6115 & 60.32 & 0.0\end{array}$

$\begin{array}{lllllll}\text { cuboid } & 3 & 1 & 4 \mathrm{p} 1.1125 & 60.32 & 0.0\end{array}$

unit 16

com $=$ '(Fuel $+\mathrm{Clad}+\mathrm{H} 2 \mathrm{O})^{\prime}$

$\begin{array}{lllllll}\text { cylinder } & 1 & 1 & 0.5325 & 60.32 & 0.0\end{array}$

$\begin{array}{llllll}\text { cylinder } 2 & 1 & 0.6115 & 60.32 & 0.0\end{array}$

$\begin{array}{lllllll}\text { cuboid } 3 & 1 & 4 \mathrm{p} 1.1125 & 60.32 & 0.0\end{array}$

unit 17

com='(Fuel+Clad $+\mathrm{H} 2 \mathrm{O})^{\prime}$

$\begin{array}{llllll}\text { cylinder } & 1 & 1 & 0.5325 & 60.32 & 0.0\end{array}$

$\begin{array}{llllll}\text { cylinder } & 2 & 1 & 0.6115 & 60.32 & 0.0\end{array}$

$\begin{array}{lllllll}\text { cuboid } & 3 & 1 & 4 \mathrm{p} 1.1125 & 60.32 & 0.0\end{array}$

unit 18

com='(Fuel+Clad $+\mathrm{H} 2 \mathrm{O})^{\prime}$

$\begin{array}{llllll}\text { cylinder } & 1 & 1 & 0.5325 & 60.32 & 0.0\end{array}$

$\begin{array}{llllll}\text { cylinder } 2 & 1 & 0.6115 & 60.32 & 0.0\end{array}$

$\begin{array}{lllllll}\text { cuboid } & 3 & 1 & 4 \mathrm{p} 1.1125 & 60.32 & 0.0\end{array}$

unit 19

com='(Fuel+Clad $+\mathrm{H} 2 \mathrm{O})^{\prime}$

cylinder $1110.532560 .32 \quad 0.0$

$\begin{array}{llllll}\text { cylinder } 2 & 1 & 0.6115 & 60.32 & 0.0\end{array}$

$\begin{array}{lllllll}\text { cuboid } & 3 & 1 & 4 \mathrm{p} 1.1125 & 60.32 & 0.0\end{array}$

unit 20

com='(Fuel+Clad $+\mathrm{H} 2 \mathrm{O})^{\prime}$

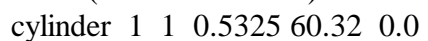

$\begin{array}{llllll}\text { cylinder } 2 & 1 & 0.6115 & 60.32 & 0.0\end{array}$

$\begin{array}{lllllll}\text { cuboid } & 3 & 1 & 4 \mathrm{p} 1.1125 & 60.32 & 0.0\end{array}$

unit 21

com $=^{\prime}(\text { Fuel }+\mathrm{Clad}+\mathrm{H} 2 \mathrm{O})^{\prime}$

$\begin{array}{llllll}\text { cylinder } & 1 & 1 & 0.5325 & 60.32 & 0.0\end{array}$

$\begin{array}{llllll}\text { cylinder } & 2 & 1 & 0.6115 & 60.32 & 0.0\end{array}$

$\begin{array}{lllllll}\text { cuboid } & 3 & 1 & 4 \mathrm{p} 1.1125 & 60.32 & 0.0\end{array}$

unit 22

com $=$ '(Fuel+Clad $+\mathrm{H} 2 \mathrm{O})^{\prime}$

$\begin{array}{llllll}\text { cylinder } & 1 & 1 & 0.5325 & 60.32 & 0.0\end{array}$

$\begin{array}{llllll}\text { cylinder } 2 & 1 & 0.6115 & 60.32 & 0.0\end{array}$

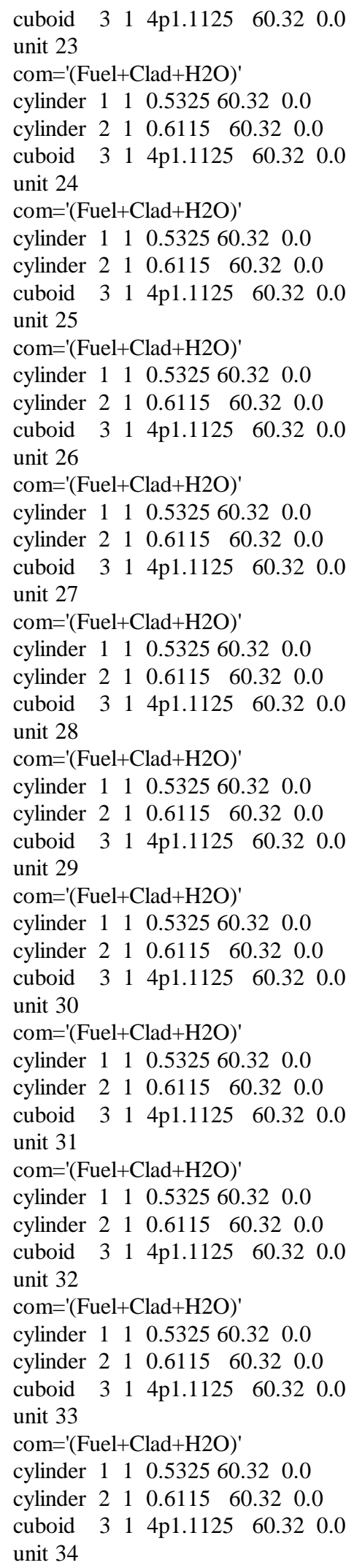


com='(Fuel+Clad+H2O $)^{\prime}$

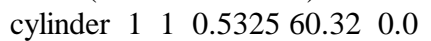

$\begin{array}{llllll}\text { cylinder } & 2 & 1 & 0.6115 & 60.32 & 0.0\end{array}$

$\begin{array}{lllllll}\text { cuboid } & 3 & 1 & 4 \mathrm{p} 1.1125 & 60.32 & 0.0\end{array}$

unit 35

com $=$ '(Fuel $+\mathrm{Clad}+\mathrm{H} 2 \mathrm{O})^{\prime}$

$\begin{array}{llllll}\text { cylinder } & 1 & 1 & 0.5325 & 60.32 & 0.0\end{array}$

$\begin{array}{llllll}\text { cylinder } & 2 & 1 & 0.6115 & 60.32 & 0.0\end{array}$

$\begin{array}{lllllll}\text { cuboid } & 3 & 1 & 4 \mathrm{p} 1.1125 & 60.32 & 0.0\end{array}$

unit 36

com $=$ '(Fuel $+\mathrm{Clad}+\mathrm{H} 2 \mathrm{O})^{\prime}$

$\begin{array}{llllll}\text { cylinder } & 1 & 1 & 0.5325 & 60.32 & 0.0\end{array}$

$\begin{array}{llllll}\text { cylinder } 2 & 1 & 0.6115 & 60.32 & 0.0\end{array}$

$\begin{array}{lllllll}\text { cuboid } & 3 & 1 & 4 \mathrm{p} 1.1125 & 60.32 & 0.0\end{array}$

unit 37

com $=$ '(Fuel + Clad $+\mathrm{H} 2 \mathrm{O})^{\prime}$

$\begin{array}{llllll}\text { cylinder } & 1 & 1 & 0.5325 & 60.32 & 0.0\end{array}$

$\begin{array}{llllll}\text { cylinder } & 2 & 1 & 0.6115 & 60.32 & 0.0\end{array}$

$\begin{array}{lllllll}\text { cuboid } & 3 & 1 & 4 \mathrm{p} 1.1125 & 60.32 & 0.0\end{array}$

unit 38

com $=$ '(Fuel + Clad $+\mathrm{H} 2 \mathrm{O})^{\prime}$

$\begin{array}{lllllll}\text { cylinder } & 1 & 1 & 0.5325 & 60.32 & 0.0\end{array}$

$\begin{array}{llllll}\text { cylinder } & 2 & 1 & 0.6115 & 60.32 & 0.0\end{array}$

$\begin{array}{lllllll}\text { cuboid } & 3 & 1 & 4 p 1.1125 & 60.32 & 0.0\end{array}$

unit 39

com $=$ '(Fuel + Clad $+\mathrm{H} 2 \mathrm{O})^{\prime}$

$\begin{array}{llllll}\text { cylinder } & 1 & 1 & 0.5325 & 60.32 & 0.0\end{array}$

$\begin{array}{llllll}\text { cylinder } 2 & 1 & 0.6115 & 60.32 & 0.0\end{array}$

$\begin{array}{lllllll}\text { cuboid } & 3 & 1 & 4 \mathrm{p} 1.1125 & 60.32 & 0.0\end{array}$

unit 40

com='(Fuel+Clad $+\mathrm{H} 2 \mathrm{O})^{\prime}$

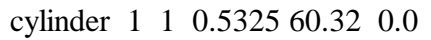

$\begin{array}{llllll}\text { cylinder } 2 & 1 & 0.6115 & 60.32 & 0.0\end{array}$

$\begin{array}{lllllll}\text { cuboid } & 3 & 1 & 4 \mathrm{p} 1.1125 & 60.32 & 0.0\end{array}$

unit 41

com $=$ '(Fuel + Clad $+\mathrm{H} 2 \mathrm{O})^{\prime}$

$\begin{array}{lllllll}\text { cylinder } & 1 & 1 & 0.5325 & 60.32 & 0.0\end{array}$

$\begin{array}{llllll}\text { cylinder } 2 & 1 & 0.6115 & 60.32 & 0.0\end{array}$

$\begin{array}{lllllll}\text { cuboid } & 3 & 1 & 4 \mathrm{p} 1.1125 & 60.32 & 0.0\end{array}$

unit 42

com='(Fuel+Clad $+\mathrm{H} 2 \mathrm{O})^{\prime}$

$\begin{array}{llllll}\text { cylinder } & 1 & 1 & 0.5325 & 60.32 & 0.0\end{array}$

$\begin{array}{llllll}\text { cylinder } 2 & 1 & 0.6115 & 60.32 & 0.0\end{array}$

$\begin{array}{lllllll}\text { cuboid } & 3 & 1 & 4 p 1.1125 & 60.32 & 0.0\end{array}$

unit 43

com='(Fuel+Clad $+\mathrm{H} 2 \mathrm{O})^{\prime}$

$\begin{array}{lllll}\text { cylinder } 1 & 1 & 0.5325 & 60.32 & 0.0\end{array}$

$\begin{array}{llllll}\text { cylinder } 2 & 1 & 0.6115 & 60.32 & 0.0\end{array}$

$\begin{array}{lllllll}\text { cuboid } & 3 & 1 & 4 \mathrm{p} 1.1125 & 60.32 & 0.0\end{array}$

unit 44

com='(Fuel+Clad $+\mathrm{H} 2 \mathrm{O})^{\prime}$

$\begin{array}{llllll}\text { cylinder } & 1 & 1 & 0.5325 & 60.32 & 0.0\end{array}$

$\begin{array}{llllll}\text { cylinder } 2 & 1 & 0.6115 & 60.32 & 0.0\end{array}$

$\begin{array}{lllllll}\text { cuboid } & 3 & 1 & 4 \mathrm{p} 1.1125 & 60.32 & 0.0\end{array}$

unit 45

com $=$ '(Fuel + Clad $+\mathrm{H} 2 \mathrm{O})^{\prime}$

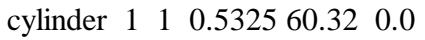

$\begin{array}{llllll}\text { cylinder } & 2 & 1 & 0.6115 & 60.32 & 0.0\end{array}$

$\begin{array}{lllllll}\text { cuboid } & 3 & 1 & 4 \mathrm{p} 1.1125 & 60.32 & 0.0\end{array}$

unit 46

com $=$ '(Fuel $+\mathrm{Clad}+\mathrm{H} 2 \mathrm{O})^{\prime}$

$\begin{array}{llllll}\text { cylinder } & 1 & 1 & 0.5325 & 60.32 & 0.0\end{array}$

$\begin{array}{llllll}\text { cylinder } 2 & 1 & 0.6115 & 60.32 & 0.0\end{array}$

$\begin{array}{lllllll}\text { cuboid } & 3 & 1 & 4 \mathrm{p} 1.1125 & 60.32 & 0.0\end{array}$

unit 47

com $=$ '(Fuel+Clad $+\mathrm{H} 2 \mathrm{O})^{\prime}$

cylinder $\begin{array}{lllll}1 & 1 & 0.5325 & 60.32 & 0.0\end{array}$

$\begin{array}{llllll}\text { cylinder } 2 & 1 & 0.6115 & 60.32 & 0.0\end{array}$

$\begin{array}{lllllll}\text { cuboid } & 3 & 1 & 4 \mathrm{p} 1.1125 & 60.32 & 0.0\end{array}$

unit 48

com $=$ '(Fuel + Clad $+\mathrm{H} 2 \mathrm{O})^{\prime}$

$\begin{array}{llllll}\text { cylinder } & 1 & 1 & 0.5325 & 60.32 & 0.0\end{array}$

$\begin{array}{llllll}\text { cylinder } 2 & 1 & 0.6115 & 60.32 & 0.0\end{array}$

$\begin{array}{lllllll}\text { cuboid } & 3 & 1 & 4 \mathrm{p} 1.1125 & 60.32 & 0.0\end{array}$

unit 49

com $=$ '(Fuel + Clad $+\mathrm{H} 2 \mathrm{O})^{\prime}$

$\begin{array}{llllll}\text { cylinder } & 1 & 1 & 0.5325 & 60.32 & 0.0\end{array}$

$\begin{array}{llllll}\text { cylinder } 2 & 1 & 0.6115 & 60.32 & 0.0\end{array}$

$\begin{array}{lllllll}\text { cuboid } & 3 & 1 & 4 \mathrm{p} 1.1125 & 60.32 & 0.0\end{array}$

unit 50

com $=$ '(Fuel + Clad $+\mathrm{H} 2 \mathrm{O})^{\prime}$

$\begin{array}{llllll}\text { cylinder } & 1 & 1 & 0.5325 & 60.32 & 0.0\end{array}$

$\begin{array}{lllllll}\text { cylinder } & 2 & 1 & 0.6115 & 60.32 & 0.0\end{array}$

$\begin{array}{lllllll}\text { cuboid } & 3 & 1 & 4 \mathrm{p} 1.1125 & 60.32 & 0.0\end{array}$

unit 51

com $=$ '(Fuel + Clad $+\mathrm{H} 2 \mathrm{O})^{\prime}$

$\begin{array}{llllll}\text { cylinder } & 1 & 1 & 0.5325 & 60.32 & 0.0\end{array}$

$\begin{array}{llllll}\text { cylinder } 2 & 1 & 0.6115 & 60.32 & 0.0\end{array}$

$\begin{array}{lllllll}\text { cuboid } & 3 & 1 & 4 \mathrm{p} 1.1125 & 60.32 & 0.0\end{array}$

unit 52

com $=$ '(Fuel + Clad $+\mathrm{H} 2 \mathrm{O})^{\prime}$

$\begin{array}{llllll}\text { cylinder } & 1 & 1 & 0.5325 & 60.32 & 0.0\end{array}$

$\begin{array}{llllll}\text { cylinder } & 2 & 1 & 0.6115 & 60.32 & 0.0\end{array}$

$\begin{array}{lllllll}\text { cuboid } & 3 & 1 & 4 \mathrm{p} 1.1125 & 60.32 & 0.0\end{array}$

unit 53

com $=$ '(Fuel + Clad $+\mathrm{H} 2 \mathrm{O})^{\prime}$

$\begin{array}{llllll}\text { cylinder } & 1 & 1 & 0.5325 & 60.32 & 0.0\end{array}$

$\begin{array}{llllll}\text { cylinder } 2 & 1 & 0.6115 & 60.32 & 0.0\end{array}$

$\begin{array}{lllllll}\text { cuboid } & 3 & 1 & 4 \mathrm{p} 1.1125 & 60.32 & 0.0\end{array}$

unit 54

com $=$ '(Fuel + Clad $+\mathrm{H} 2 \mathrm{O})^{\prime}$

$\begin{array}{llllll}\text { cylinder } & 1 & 1 & 0.5325 & 60.32 & 0.0\end{array}$

$\begin{array}{llllll}\text { cylinder } 2 & 1 & 0.6115 & 60.32 & 0.0\end{array}$

$\begin{array}{lllllll}\text { cuboid } & 3 & 1 & 4 \mathrm{p} 1.1125 & 60.32 & 0.0\end{array}$

unit 55

com $=$ '(Fuel + Clad $+\mathrm{H} 2 \mathrm{O})^{\prime}$

$\begin{array}{llllll}\text { cylinder } & 1 & 1 & 0.5325 & 60.32 & 0.0\end{array}$

$\begin{array}{llllll}\text { cylinder } 2 & 1 & 0.6115 & 60.32 & 0.0\end{array}$

$\begin{array}{lllllll}\text { cuboid } & 3 & 1 & 4 \mathrm{p} 1.1125 & 60.32 & 0.0\end{array}$

unit 81

com='h2o and end plug below Al grid plate - fill array 1'

$\begin{array}{lllll}\text { cylinder } 4 & 1 & 0.6115 & 4.445 & 0.0\end{array}$

$\begin{array}{lllllll}\text { cuboid } & 3 & 1 & 4 \mathrm{p} 1.1125 & 4.445 & 0.0\end{array}$

unit 82 
com='h2o and end plug above $\mathrm{Al}$ grid plate - fill array 2' $\begin{array}{lllll}\text { cylinder } 4 & 1 & 0.6115 & 11.784 & 0.0\end{array}$

$\begin{array}{llllllll}\text { cuboid } \quad 3 & 1 & 4 \mathrm{p} 1.1125 & 11.784 & 0.0\end{array}$

unit 83

com='(Fuel+Clad+Void $)$ - fill array 3'

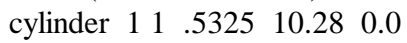

$\begin{array}{lllll}\text { cylinder } 2 & 1 & .6115 & 10.28 & 0.0\end{array}$

cuboid $0 \begin{array}{llllll}0 & 4 & 4 & 1.1125 & 10.28 & 0.0\end{array}$

unit 84

com='(void+Clad+void $)^{\prime}$

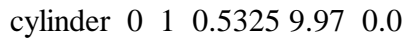

$\begin{array}{llllll}\text { cylinder } & 2 & 1 & 0.6115 & 9.97 & 0.0\end{array}$

cuboid $\begin{array}{llllll}0 & 1 & 4 \mathrm{p} 1.1125 & 9.97 & 0.0\end{array}$

unit 100

com='Lower $\mathrm{Al}$ grid plate'

cuboid $414 \mathrm{p} 52.25 .601 \quad 0.0$

unit 101

com='array of h2o and end plugs below Al grid plate'

$\begin{array}{lllll}\text { array } & 1 & -22.25 & -22.25 & 0.0\end{array}$

$\begin{array}{lllllll}\text { cuboid } & 3 & 1 & 4 \mathrm{p} 52.25 & 4.445 & 0.0\end{array}$

unit 102

com='array of h2o and end plugs above $\mathrm{Al}$ grid plate '

$\begin{array}{llll}\text { array } 2 & -22.25 & -22.25 & 0.0\end{array}$

$\begin{array}{lllllll}\text { cuboid } 3 & 1 & 4 \mathrm{p} 52.25 & 11.784 & 0.0\end{array}$

unit 103

com='array of Fuel+Clad+Void'

$\begin{array}{llll}\text { array } 3 & -22.25 & -22.25 & 0.0\end{array}$

$\begin{array}{lllllll}\text { cuboid } \quad 0 & 1 & 4 \mathrm{p} 52.25 & 10.28 & 0.0\end{array}$

unit 104

com='array of void+Clad+void'

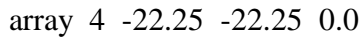

$\begin{array}{lllllll}\text { cuboid } & 0 & 1 & 4 \mathrm{p} 52.25 & 9.97 & 0.0\end{array}$

unit 105

com='array of Fuel+Clad+H2O'

$\begin{array}{llll}\text { array } 5 & -22.25 & -22.25 & 0.0\end{array}$

$\begin{array}{lllllll}\text { cuboid } 3 & 1 & 4 \mathrm{p} 52.25 & 60.32 & 0.0\end{array}$

global unit 110

com='array of arrays of fuel element sections + reflector'

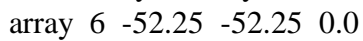

cuboid $\begin{array}{llllll}4 & 1 & 4 \mathrm{p} 52.25 & 97.40 & -1.27\end{array}$

cuboid $\begin{array}{llllll}5 & 1 & 4 p 52.25 & 97.40 & -3.47\end{array}$

$\begin{array}{lllllll}\text { cuboid } 3 & 1 & 4 p 52.25 & 97.40 & -17.27\end{array}$

$\begin{array}{llllll}\text { cuboid } 5 & 1 & 4 p 52.25 & 97.40 & -17.77\end{array}$

$\begin{array}{llllll}\text { cuboid } & 6 & 1 & 4 p 52.25 & 97.40 & -54.77\end{array}$

end geometry

read array

ara $=1$ nux $=20$ nuy=20 nuz=1

fill $\mathrm{f} 81$ end fill

ara=2 nux=20 nuy=20 nuz=1

fill $\mathrm{f} 82$ end fill

ara $=3$ nux $=20$ nuy=20 nuz $=1$

fill $\mathrm{f} 83$ end fill

ara $=4$ nux=20 nuy=20 nuz=1

fill $\mathrm{f} 84$ end fill

ara $=5$ nux=20 nuy=20 nuz=1

fill

$\begin{array}{llllllllllll}1 & 2 & 3 & 4 & 5 & 6 & 7 & 8 & 9 & 10 & \mathrm{n} 10\end{array}$
2111213141516171819 n 10 3122021222324252627 n 10 4132128293031323334 n 10 5142229353637383940 n 10 6152330364142434445 n 10 7162431374246474849 n 10 8172532384347505152 n 10

9182633394448515354 n 10 10192734404549525455 n 10 n200

end fill

ara $=6$ nux=1 nuy=1 nuz=6

fill 101100102105103104 end fill end array

end data

end

$=$ shell

cp ft04f001 \$RTNDIR/tca07.xs04 cp ft64f001 \$RTNDIR/tca07.rs64

end

$=\mathrm{kmart}$

read initial kunit $=64$

xunit $=4$

actbygrp

rrpvol

end initial

read activity

92234279223418922341452

92235279223518922351452

92238279223818922381452

94238279423818942381452

94239279423918942391452

94240279424018942401452

$9424127942411894241 \quad 1452$

$9424227942421894242 \quad 1452$

95241279524118952411452

501027

501127

801627

4000027

2400027

2600027

100127

5011227

5011427

5011527

5011627

5011727

5011827

5011927

5012027

5012227

5012427

end activity

read collapse

factor $=1.0$

lastg $=57$ 


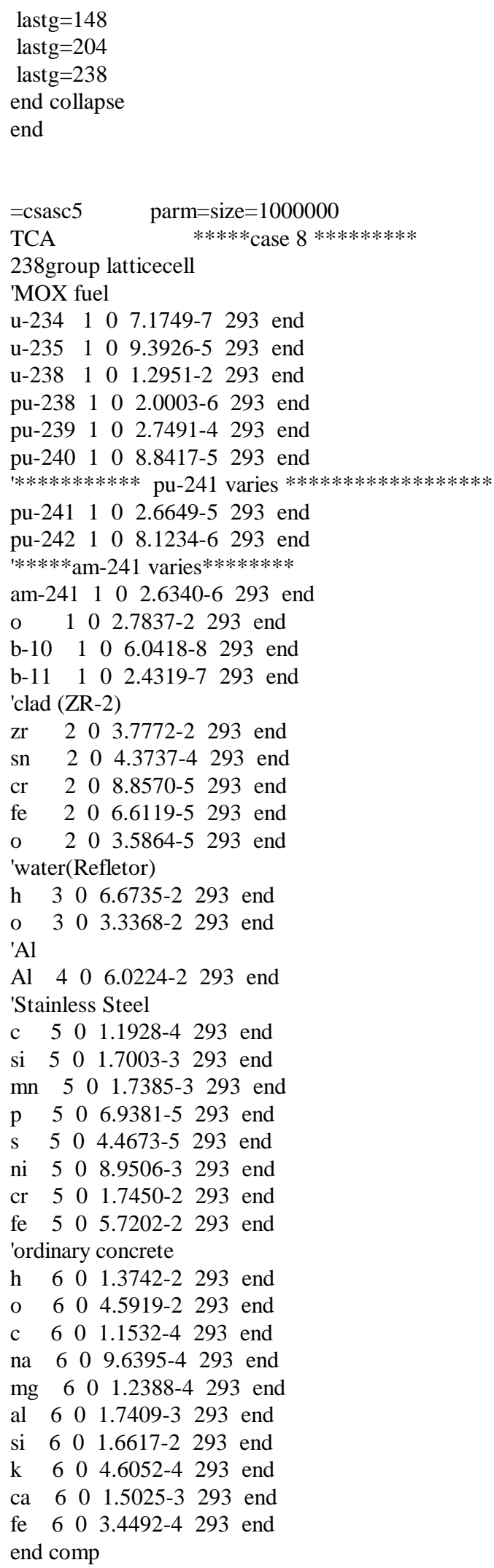

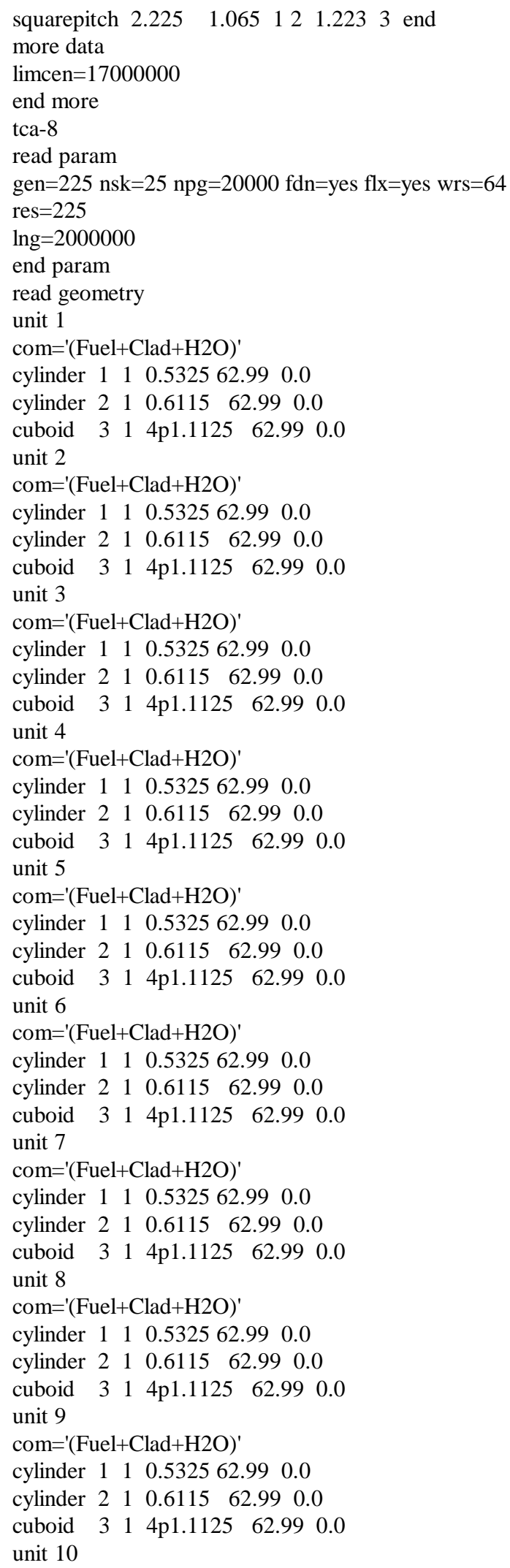


com='(Fuel+Clad+H2O)'

$\begin{array}{llllll}\text { cylinder } & 1 & 1 & 0.5325 & 62.99 & 0.0\end{array}$

$\begin{array}{llllll}\text { cylinder } & 2 & 1 & 0.6115 & 62.99 & 0.0\end{array}$

$\begin{array}{lllllll}\text { cuboid } & 3 & 1 & 4 \mathrm{p} 1.1125 & 62.99 & 0.0\end{array}$

unit 11

com $=$ '(Fuel $+\mathrm{Clad}+\mathrm{H} 2 \mathrm{O})^{\prime}$

$\begin{array}{llllll}\text { cylinder } & 1 & 1 & 0.5325 & 62.99 & 0.0\end{array}$

$\begin{array}{llllll}\text { cylinder } 2 & 1 & 0.6115 & 62.99 & 0.0\end{array}$

$\begin{array}{lllllll}\text { cuboid } & 3 & 1 & 4 \mathrm{p} 1.1125 & 62.99 & 0.0\end{array}$

unit 12

com $=$ '(Fuel $+\mathrm{Clad}+\mathrm{H} 2 \mathrm{O})^{\prime}$

cylinder $1110.532562 .99 \quad 0.0$

$\begin{array}{llllll}\text { cylinder } 2 & 1 & 0.6115 & 62.99 & 0.0\end{array}$

$\begin{array}{lllllll}\text { cuboid } & 3 & 1 & 4 \mathrm{p} 1.1125 & 62.99 & 0.0\end{array}$

unit 13

com='(Fuel+Clad $+\mathrm{H} 2 \mathrm{O})^{\prime}$

$\begin{array}{llllll}\text { cylinder } & 1 & 1 & 0.5325 & 62.99 & 0.0\end{array}$

$\begin{array}{llllll}\text { cylinder } & 2 & 1 & 0.6115 & 62.99 & 0.0\end{array}$

$\begin{array}{llllll}\text { cuboid } & 3 & 1 & 4 \mathrm{p} 1.1125 & 62.99 & 0.0\end{array}$

unit 14

com='(Fuel+Clad $+\mathrm{H} 2 \mathrm{O})^{\prime}$

$\begin{array}{llllll}\text { cylinder } & 1 & 1 & 0.5325 & 62.99 & 0.0\end{array}$

$\begin{array}{llllll}\text { cylinder } & 2 & 1 & 0.6115 & 62.99 & 0.0\end{array}$

$\begin{array}{llllll}\text { cuboid } & 3 & 1 & 4 p 1.1125 & 62.99 & 0.0\end{array}$

unit 15

com='(Fuel $+\mathrm{Clad}+\mathrm{H} 2 \mathrm{O})^{\prime}$

$\begin{array}{llllll}\text { cylinder } & 1 & 1 & 0.5325 & 62.99 & 0.0\end{array}$

$\begin{array}{llllll}\text { cylinder } & 2 & 1 & 0.6115 & 62.99 & 0.0\end{array}$

$\begin{array}{llllll}\text { cuboid } & 3 & 1 & 4 \mathrm{p} 1.1125 & 62.99 & 0.0\end{array}$

unit 16

com='(Fuel+Clad $+\mathrm{H} 2 \mathrm{O})^{\prime}$

$\begin{array}{llllll}\text { cylinder } & 1 & 1 & 0.5325 & 62.99 & 0.0\end{array}$

$\begin{array}{llllll}\text { cylinder } 2 & 1 & 0.6115 & 62.99 & 0.0\end{array}$

$\begin{array}{lllllll}\text { cuboid } & 3 & 1 & 4 \mathrm{p} 1.1125 & 62.99 & 0.0\end{array}$

unit 17

com $=$ '(Fuel+Clad $+\mathrm{H} 2 \mathrm{O})^{\prime}$

$\begin{array}{lllllll}\text { cylinder } & 1 & 1 & 0.5325 & 62.99 & 0.0\end{array}$

$\begin{array}{llllll}\text { cylinder } 2 & 1 & 0.6115 & 62.99 & 0.0\end{array}$

$\begin{array}{lllllll}\text { cuboid } & 3 & 1 & 4 \mathrm{p} 1.1125 & 62.99 & 0.0\end{array}$

unit 18

com='(Fuel+Clad $+\mathrm{H} 2 \mathrm{O})^{\prime}$

cylinder $\begin{array}{lllll}1 & 1 & 0.5325 & 62.99 & 0.0\end{array}$

$\begin{array}{llllll}\text { cylinder } 2 & 1 & 0.6115 & 62.99 & 0.0\end{array}$

$\begin{array}{lllllll}\text { cuboid } & 3 & 1 & 4 \mathrm{p} 1.1125 & 62.99 & 0.0\end{array}$

unit 19

com='(Fuel+Clad $+\mathrm{H} 2 \mathrm{O})^{\prime}$

$\begin{array}{llllll}\text { cylinder } & 1 & 1 & 0.5325 & 62.99 & 0.0\end{array}$

$\begin{array}{llllll}\text { cylinder } 2 & 1 & 0.6115 & 62.99 & 0.0\end{array}$

$\begin{array}{lllllll}\text { cuboid } & 3 & 1 & 4 \mathrm{p} 1.1125 & 62.99 & 0.0\end{array}$

unit 20

com='(Fuel+Clad $+\mathrm{H} 2 \mathrm{O})^{\prime}$

cylinder $\begin{array}{llllll}1 & 1 & 0.5325 & 62.99 & 0.0\end{array}$

$\begin{array}{llllll}\text { cylinder } & 2 & 1 & 0.6115 & 62.99 & 0.0\end{array}$

$\begin{array}{lllllll}\text { cuboid } & 3 & 1 & 4 \mathrm{p} 1.1125 & 62.99 & 0.0\end{array}$

unit 21

com='(Fuel+Clad $+\mathrm{H} 2 \mathrm{O})^{\prime}$

cylinder $\begin{array}{lllll}1 & 1 & 0.5325 & 62.99 & 0.0\end{array}$ $\begin{array}{llllll}\text { cylinder } & 2 & 1 & 0.6115 & 62.99 & 0.0\end{array}$

$\begin{array}{lllllll}\text { cuboid } & 3 & 1 & 4 \mathrm{p} 1.1125 & 62.99 & 0.0\end{array}$

unit 22

com $=$ '(Fuel $+\mathrm{Clad}+\mathrm{H} 2 \mathrm{O})^{\prime}$

$\begin{array}{llllll}\text { cylinder } & 1 & 1 & 0.5325 & 62.99 & 0.0\end{array}$

$\begin{array}{llllll}\text { cylinder } & 2 & 1 & 0.6115 & 62.99 & 0.0\end{array}$

$\begin{array}{lllllll}\text { cuboid } & 3 & 1 & 4 \mathrm{p} 1.1125 & 62.99 & 0.0\end{array}$

unit 23

com='(Fuel+Clad $+\mathrm{H} 2 \mathrm{O})^{\prime}$

cylinder $\begin{array}{lllll}1 & 1 & 0.5325 & 62.99 & 0.0\end{array}$

$\begin{array}{llllll}\text { cylinder } 2 & 1 & 0.6115 & 62.99 & 0.0\end{array}$

$\begin{array}{lllllll}\text { cuboid } & 3 & 1 & 4 \mathrm{p} 1.1125 & 62.99 & 0.0\end{array}$

unit 24

com $=$ '(Fuel+Clad $+\mathrm{H} 2 \mathrm{O})^{\prime}$

cylinder $\begin{array}{lllll}1 & 1 & 0.5325 & 62.99 & 0.0\end{array}$

$\begin{array}{llllll}\text { cylinder } 2 & 1 & 0.6115 & 62.99 & 0.0\end{array}$

$\begin{array}{lllllll}\text { cuboid } & 3 & 1 & 4 \mathrm{p} 1.1125 & 62.99 & 0.0\end{array}$

unit 25

com='(Fuel+Clad $+\mathrm{H} 2 \mathrm{O})^{\prime}$

$\begin{array}{llllll}\text { cylinder } & 1 & 1 & 0.5325 & 62.99 & 0.0\end{array}$

$\begin{array}{llllll}\text { cylinder } 2 & 1 & 0.6115 & 62.99 & 0.0\end{array}$

$\begin{array}{lllllll}\text { cuboid } & 3 & 1 & 4 \mathrm{p} 1.1125 & 62.99 & 0.0\end{array}$

unit 26

com $=$ '(Fuel + Clad $+\mathrm{H} 2 \mathrm{O})^{\prime}$

cylinder $\begin{array}{lllll}1 & 1 & 0.5325 & 62.99 & 0.0\end{array}$

$\begin{array}{llllll}\text { cylinder } & 2 & 1 & 0.6115 & 62.99 & 0.0\end{array}$

$\begin{array}{lllllll}\text { cuboid } & 3 & 1 & 4 \mathrm{p} 1.1125 & 62.99 & 0.0\end{array}$

unit 27

com $=$ '(Fuel + Clad $+\mathrm{H} 2 \mathrm{O})^{\prime}$

cylinder $\begin{array}{lllll}1 & 1 & 0.5325 & 62.99 & 0.0\end{array}$

$\begin{array}{llllll}\text { cylinder } 2 & 1 & 0.6115 & 62.99 & 0.0\end{array}$

$\begin{array}{lllllll}\text { cuboid } & 3 & 1 & 4 \mathrm{p} 1.1125 & 62.99 & 0.0\end{array}$

unit 28

com='(Fuel+Clad $+\mathrm{H} 2 \mathrm{O})^{\prime}$

$\begin{array}{llllll}\text { cylinder } & 1 & 1 & 0.5325 & 62.99 & 0.0\end{array}$

$\begin{array}{llllll}\text { cylinder } 2 & 1 & 0.6115 & 62.99 & 0.0\end{array}$

$\begin{array}{lllllll}\text { cuboid } & 3 & 1 & 4 \mathrm{p} 1.1125 & 62.99 & 0.0\end{array}$

unit 29

com $=$ '(Fuel + Clad $+\mathrm{H} 2 \mathrm{O})^{\prime}$

cylinder $\begin{array}{lllll}1 & 1 & 0.5325 & 62.99 & 0.0\end{array}$

$\begin{array}{llllll}\text { cylinder } & 2 & 1 & 0.6115 & 62.99 & 0.0\end{array}$

$\begin{array}{lllllll}\text { cuboid } & 3 & 1 & 4 \mathrm{p} 1.1125 & 62.99 & 0.0\end{array}$

unit 30

com $=$ '(Fuel + Clad $+\mathrm{H} 2 \mathrm{O})^{\prime}$

$\begin{array}{llllll}\text { cylinder } & 1 & 1 & 0.5325 & 62.99 & 0.0\end{array}$

$\begin{array}{llllll}\text { cylinder } & 2 & 1 & 0.6115 & 62.99 & 0.0\end{array}$

$\begin{array}{lllllll}\text { cuboid } & 3 & 1 & 4 \mathrm{p} 1.1125 & 62.99 & 0.0\end{array}$

unit 31

com='(Fuel+Clad $+\mathrm{H} 2 \mathrm{O})^{\prime}$

$\begin{array}{llllll}\text { cylinder } & 1 & 1 & 0.5325 & 62.99 & 0.0\end{array}$

$\begin{array}{llllll}\text { cylinder } & 2 & 1 & 0.6115 & 62.99 & 0.0\end{array}$

$\begin{array}{lllllll}\text { cuboid } & 3 & 1 & 4 \mathrm{p} 1.1125 & 62.99 & 0.0\end{array}$

unit 32

com $=$ '(Fuel + Clad $+\mathrm{H} 2 \mathrm{O})^{\prime}$

cylinder $\begin{array}{lllll}1 & 1 & 0.5325 & 62.99 & 0.0\end{array}$

$\begin{array}{llllll}\text { cylinder } & 2 & 1 & 0.6115 & 62.99 & 0.0\end{array}$

$\begin{array}{lllllll}\text { cuboid } & 3 & 1 & 4 \mathrm{p} 1.1125 & 62.99 & 0.0\end{array}$ 
unit 33

com='(Fuel+Clad $+\mathrm{H} 2 \mathrm{O})^{\prime}$

$\begin{array}{llllll}\text { cylinder } & 1 & 1 & 0.5325 & 62.99 & 0.0\end{array}$

$\begin{array}{llllll}\text { cylinder } & 2 & 1 & 0.6115 & 62.99 & 0.0\end{array}$

$\begin{array}{lllllll}\text { cuboid } & 3 & 1 & 4 \mathrm{p} 1.1125 & 62.99 & 0.0\end{array}$

unit 34

com $=$ '(Fuel + Clad $+\mathrm{H} 2 \mathrm{O})^{\prime}$

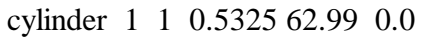

$\begin{array}{llllll}\text { cylinder } & 2 & 1 & 0.6115 & 62.99 & 0.0\end{array}$

$\begin{array}{lllllll}\text { cuboid } & 3 & 1 & 4 \mathrm{p} 1.1125 & 62.99 & 0.0\end{array}$

unit 35

com='(Fuel+Clad $+\mathrm{H} 2 \mathrm{O})^{\prime}$

$\begin{array}{llllll}\text { cylinder } & 1 & 1 & 0.5325 & 62.99 & 0.0\end{array}$

$\begin{array}{llllll}\text { cylinder } & 2 & 1 & 0.6115 & 62.99 & 0.0\end{array}$

$\begin{array}{lllllll}\text { cuboid } & 3 & 1 & 4 \mathrm{p} 1.1125 & 62.99 & 0.0\end{array}$

unit 36

com='(Fuel+Clad $+\mathrm{H} 2 \mathrm{O})^{\prime}$

cylinder $\begin{array}{lllll}1 & 1 & 0.5325 & 62.99 & 0.0\end{array}$

$\begin{array}{llllll}\text { cylinder } & 2 & 1 & 0.6115 & 62.99 & 0.0\end{array}$

$\begin{array}{lllllll}\text { cuboid } & 3 & 1 & 4 \mathrm{p} 1.1125 & 62.99 & 0.0\end{array}$

unit 37

com $=$ '(Fuel $+\mathrm{Clad}+\mathrm{H} 2 \mathrm{O})^{\prime}$

cylinder $1110.532562 .99 \quad 0.0$

$\begin{array}{llllll}\text { cylinder } 2 & 1 & 0.6115 & 62.99 & 0.0\end{array}$

$\begin{array}{llllll}\text { cuboid } & 3 & 1 & 4 \mathrm{p} 1.1125 & 62.99 & 0.0\end{array}$

unit 38

com $=$ '(Fuel + Clad $+\mathrm{H} 2 \mathrm{O})^{\prime}$

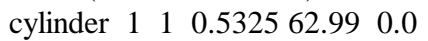

$\begin{array}{llllll}\text { cylinder } 2 & 1 & 0.6115 & 62.99 & 0.0\end{array}$

$\begin{array}{llllll}\text { cuboid } & 3 & 1 & 4 \mathrm{p} 1.1125 & 62.99 & 0.0\end{array}$

unit 39

com='(Fuel+Clad $+\mathrm{H} 2 \mathrm{O})^{\prime}$

$\begin{array}{lllllll}\text { cylinder } & 1 & 1 & 0.5325 & 62.99 & 0.0\end{array}$

$\begin{array}{llllll}\text { cylinder } & 2 & 1 & 0.6115 & 62.99 & 0.0\end{array}$

$\begin{array}{lllllll}\text { cuboid } & 3 & 1 & 4 \mathrm{p} 1.1125 & 62.99 & 0.0\end{array}$

unit 40

com $=$ '(Fuel $+\mathrm{Clad}+\mathrm{H} 2 \mathrm{O})^{\prime}$

$\begin{array}{llllll}\text { cylinder } & 1 & 1 & 0.5325 & 62.99 & 0.0\end{array}$

$\begin{array}{llllll}\text { cylinder } & 2 & 1 & 0.6115 & 62.99 & 0.0\end{array}$

$\begin{array}{lllllll}\text { cuboid } & 3 & 1 & 4 \mathrm{p} 1.1125 & 62.99 & 0.0\end{array}$

unit 41

com='(Fuel+Clad $+\mathrm{H} 2 \mathrm{O})^{\prime}$

$\begin{array}{llllll}\text { cylinder } & 1 & 1 & 0.5325 & 62.99 & 0.0\end{array}$

$\begin{array}{llllll}\text { cylinder } 2 & 1 & 0.6115 & 62.99 & 0.0\end{array}$

$\begin{array}{lllllll}\text { cuboid } & 3 & 1 & 4 \mathrm{p} 1.1125 & 62.99 & 0.0\end{array}$

unit 42

com $=$ '(Fuel+Clad $+\mathrm{H} 2 \mathrm{O})^{\prime}$

cylinder $\begin{array}{lllll}1 & 1 & 0.5325 & 62.99 & 0.0\end{array}$

$\begin{array}{llllll}\text { cylinder } 2 & 1 & 0.6115 & 62.99 & 0.0\end{array}$

$\begin{array}{lllllll}\text { cuboid } & 3 & 1 & 4 \mathrm{p} 1.1125 & 62.99 & 0.0\end{array}$

unit 43

com $=$ '(Fuel + Clad $+\mathrm{H} 2 \mathrm{O})^{\prime}$

cylinder $1110.532562 .99 \quad 0.0$

$\begin{array}{llllll}\text { cylinder } 2 & 1 & 0.6115 & 62.99 & 0.0\end{array}$

$\begin{array}{lllllll}\text { cuboid } & 3 & 1 & 4 \mathrm{p} 1.1125 & 62.99 & 0.0\end{array}$

unit 44

com='(Fuel+Clad $+\mathrm{H} 2 \mathrm{O})^{\prime}$ $\begin{array}{llllll}\text { cylinder } & 1 & 1 & 0.5325 & 62.99 & 0.0\end{array}$

$\begin{array}{llllll}\text { cylinder } & 2 & 1 & 0.6115 & 62.99 & 0.0\end{array}$

$\begin{array}{lllllll}\text { cuboid } & 3 & 1 & 4 \mathrm{p} 1.1125 & 62.99 & 0.0\end{array}$

unit 45

com $=$ '(Fuel + Clad $+\mathrm{H} 2 \mathrm{O})^{\prime}$

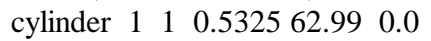

$\begin{array}{llllll}\text { cylinder } 2 & 1 & 0.6115 & 62.99 & 0.0\end{array}$

$\begin{array}{lllllll}\text { cuboid } & 3 & 1 & 4 p 1.1125 & 62.99 & 0.0\end{array}$

unit 46

com='(Fuel+Clad $+\mathrm{H} 2 \mathrm{O})^{\prime}$

$\begin{array}{llllll}\text { cylinder } & 1 & 1 & 0.5325 & 62.99 & 0.0\end{array}$

$\begin{array}{llllll}\text { cylinder } 2 & 1 & 0.6115 & 62.99 & 0.0\end{array}$

$\begin{array}{lllllll}\text { cuboid } & 3 & 1 & 4 \mathrm{p} 1.1125 & 62.99 & 0.0\end{array}$

unit 47

com $=$ '(Fuel + Clad $+\mathrm{H} 2 \mathrm{O})^{\prime}$

$\begin{array}{llllll}\text { cylinder } & 1 & 1 & 0.5325 & 62.99 & 0.0\end{array}$

$\begin{array}{llllll}\text { cylinder } & 2 & 1 & 0.6115 & 62.99 & 0.0\end{array}$

$\begin{array}{lllllll}\text { cuboid } & 3 & 1 & 4 \mathrm{p} 1.1125 & 62.99 & 0.0\end{array}$

unit 48

com='(Fuel+Clad $+\mathrm{H} 2 \mathrm{O})^{\prime}$

$\begin{array}{llllll}\text { cylinder } & 1 & 1 & 0.5325 & 62.99 & 0.0\end{array}$

$\begin{array}{llllll}\text { cylinder } 2 & 1 & 0.6115 & 62.99 & 0.0\end{array}$

$\begin{array}{lllllll}\text { cuboid } & 3 & 1 & 4 \mathrm{p} 1.1125 & 62.99 & 0.0\end{array}$

unit 49

com $=$ '(Fuel + Clad $+\mathrm{H} 2 \mathrm{O})^{\prime}$

cylinder $\begin{array}{llllll}1 & 1 & 0.5325 & 62.99 & 0.0\end{array}$

$\begin{array}{llllll}\text { cylinder } 2 & 1 & 0.6115 & 62.99 & 0.0\end{array}$

$\begin{array}{lllllll}\text { cuboid } & 3 & 1 & 4 \mathrm{p} 1.1125 & 62.99 & 0.0\end{array}$

unit 50

com $=$ '(Fuel + Clad $+\mathrm{H} 2 \mathrm{O})^{\prime}$

cylinder $\begin{array}{lllll}1 & 1 & 0.5325 & 62.99 & 0.0\end{array}$

$\begin{array}{llllll}\text { cylinder } 2 & 1 & 0.6115 & 62.99 & 0.0\end{array}$

$\begin{array}{lllllll}\text { cuboid } & 3 & 1 & 4 \mathrm{p} 1.1125 & 62.99 & 0.0\end{array}$

unit 51

com $=$ '(Fuel + Clad $+\mathrm{H} 2 \mathrm{O})^{\prime}$

cylinder $\begin{array}{lllll}1 & 1 & 0.5325 & 62.99 & 0.0\end{array}$

$\begin{array}{lllllll}\text { cylinder } & 2 & 1 & 0.6115 & 62.99 & 0.0\end{array}$

$\begin{array}{lllllll}\text { cuboid } & 3 & 1 & 4 \mathrm{p} 1.1125 & 62.99 & 0.0\end{array}$

unit 52

com='(Fuel+Clad $+\mathrm{H} 2 \mathrm{O})^{\prime}$

cylinder $\begin{array}{lllll}1 & 1 & 0.5325 & 62.99 & 0.0\end{array}$

$\begin{array}{llllll}\text { cylinder } 2 & 1 & 0.6115 & 62.99 & 0.0\end{array}$

$\begin{array}{lllllll}\text { cuboid } & 3 & 1 & 4 \mathrm{p} 1.1125 & 62.99 & 0.0\end{array}$

unit 53

com $=$ '(Fuel+Clad $+\mathrm{H} 2 \mathrm{O})^{\prime}$

$\begin{array}{llllll}\text { cylinder } & 1 & 1 & 0.5325 & 62.99 & 0.0\end{array}$

$\begin{array}{llllll}\text { cylinder } & 2 & 1 & 0.6115 & 62.99 & 0.0\end{array}$

$\begin{array}{lllllll}\text { cuboid } & 3 & 1 & 4 \mathrm{p} 1.1125 & 62.99 & 0.0\end{array}$

unit 54

com $=$ '(Fuel + Clad $+\mathrm{H} 2 \mathrm{O})^{\prime}$

$\begin{array}{llllll}\text { cylinder } & 1 & 1 & 0.5325 & 62.99 & 0.0\end{array}$

$\begin{array}{llllll}\text { cylinder } & 2 & 1 & 0.6115 & 62.99 & 0.0\end{array}$

$\begin{array}{lllllll}\text { cuboid } & 3 & 1 & 4 \mathrm{p} 1.1125 & 62.99 & 0.0\end{array}$

unit 55

com $=$ '(Fuel+Clad $+\mathrm{H} 2 \mathrm{O})^{\prime}$

$\begin{array}{lllllll}\text { cylinder } & 1 & 1 & 0.5325 & 62.99 & 0.0\end{array}$

$\begin{array}{llllll}\text { cylinder } & 2 & 1 & 0.6115 & 62.99 & 0.0\end{array}$ 
$\begin{array}{lllllll}\text { cuboid } & 3 & 1 & 4 \mathrm{p} 1.1125 & 62.99 & 0.0\end{array}$

unit 81

com='h2o and end plug below Al grid plate - fill array 1'

$\begin{array}{llllll}\text { cylinder } 4 & 1 & 0.6115 & 4.445 & 0.0\end{array}$

$\begin{array}{llllll}\text { cuboid } 3 & 1 & 4 \mathrm{p} 1.1125 & 4.445 & 0.0\end{array}$

unit 82

com='h2o and end plug above Al grid plate - fill array 2'

$\begin{array}{lllll}\text { cylinder } 4 & 1 & 0.6115 & 11.784 & 0.0\end{array}$

$\begin{array}{lllllll}\text { cuboid } \quad 3 & 1 & 4 \mathrm{p} 1.1125 & 11.784 & 0.0\end{array}$

unit 83

com='(Fuel+Clad+Void) - fill array 3'

$\begin{array}{lllllll}\text { cylinder } & 1 & 1 & .5325 & 7.61 & 0.0\end{array}$

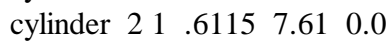

cuboid $\quad 014 \mathrm{p} 1.11257 .61 \quad 0.0$

unit 84

com='(void+Clad+void $)^{\prime}$

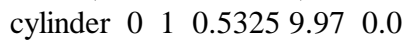

$\begin{array}{lllll}\text { cylinder } 2 & 1 & 0.6115 & 9.97 & 0.0\end{array}$

cuboid $\quad \begin{array}{llllll}0 & 1 & 4 \mathrm{p} 1.1125 & 9.97 & 0.0\end{array}$

unit 100

com='Lower Al grid plate'

cuboid 41 4p52.25.601 0.0

unit 101

com='array of h2o and end plugs below Al grid plate'

$\begin{array}{lllll}\text { array } & 1 & -22.25 & -22.25 & 0.0\end{array}$

$\begin{array}{lllllll}\text { cuboid } & 3 & 1 & 4 \mathrm{p} 52.25 & 4.445 & 0.0\end{array}$

unit 102

com='array of $\mathrm{h} 2 \mathrm{o}$ and end plugs above $\mathrm{Al}$ grid plate '

$\begin{array}{llll}\text { array } 2 & -22.25 & -22.25 & 0.0\end{array}$

cuboid $3 \quad 1 \quad 4$ p52.25 $11.784 \quad 0.0$

unit 103

com='array of Fuel+Clad+Void'

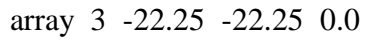

cuboid $\quad 011$ p $52.257 .61 \quad 0.0$

unit 104

com='array of void+Clad+void'

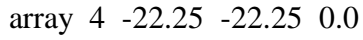

$\begin{array}{lllllll}\text { cuboid } & 0 & 1 & 4 \mathrm{p} 52.25 & 9.97 & 0.0\end{array}$

unit 105

com='array of Fuel+Clad+H2O'

$\begin{array}{llll}\text { array } 5 & -22.25 & -22.25 & 0.0\end{array}$

$\begin{array}{lllllll}\text { cuboid } 3 & 1 & 4 \mathrm{p} 52.25 & 62.99 & 0.0\end{array}$

global unit 110

com='array of arrays of fuel element sections + reflector'

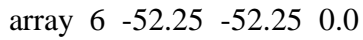

cuboid $4 \begin{array}{lllll} & 1 & 4 \mathrm{p} 52.25 & 97.40 & -1.27\end{array}$

cuboid $5 \begin{array}{lllll} & 1 & 4 \mathrm{p} 52.25 & 97.40 & -3.47\end{array}$

cuboid $3 \quad 1 \quad 4 p 52.25 \quad 97.40 \quad-17.27$

$\begin{array}{lllllll}\text { cuboid } 5 & 1 & 4 p 52.25 & 97.40 & -17.77\end{array}$

$\begin{array}{llllll}\text { cuboid } 6 & 1 & 4 p 52.25 & 97.40 & -54.77\end{array}$

end geometry

read array

ara=1 nux=20 nuy=20 nuz=1

fill $\mathrm{f} 81$ end fill

ara $=2$ nux=20 nuy=20 nuz=1

fill $\mathrm{f} 82$ end fill

ara=3 nux=20 nuy=20 nuz=1 fill $\mathrm{f} 83$ end fill

ara $=4$ nux $=20$ nuy=20 nuz=1

fill $\mathrm{f} 84$ end fill

ara=5 nux=20 nuy=20 nuz=1

fill

$\begin{array}{lllllllllll}1 & 2 & 3 & 4 & 5 & 6 & 7 & 8 & 9 & 10 & \mathrm{n} \\ 10\end{array}$

2111213141516171819 n 10

3122021222324252627 n 10

4132128293031323334 n 10

5142229353637383940 n 10

6152330364142434445 n 10

7162431374246474849 n 10

8172532384347505152 n 10

9182633394448515354 n 10

10192734404549525455 n 10

n200

end fill

ara $=6$ nux $=1$ nuy $=1$ nuz $=6$

fill 101100102105103104 end fill

end array

end data

end

$=$ shell

cp ft04f001 \$RTNDIR/tca08.xs04 cp ft64f001 \$RTNDIR/tca08.rs64

end

$=\mathrm{kmart}$

read initial kunit $=64$

xunit $=4$

actbygrp

end initial

rrpvol

read activity

92234279223418922341452

92235279223518922351452

$9223827922381892238 \quad 1452$

94238279423818942381452

94239279423918942391452

94240279424018942401452

$9424127942411894241 \quad 1452$

94242279424218942421452

$9524127952411895241 \quad 1452$

501027

501127

801627

4000027

2400027

2600027

100127

5011227

5011427

5011527

5011627

5011727

5011827

5011927

5012027 


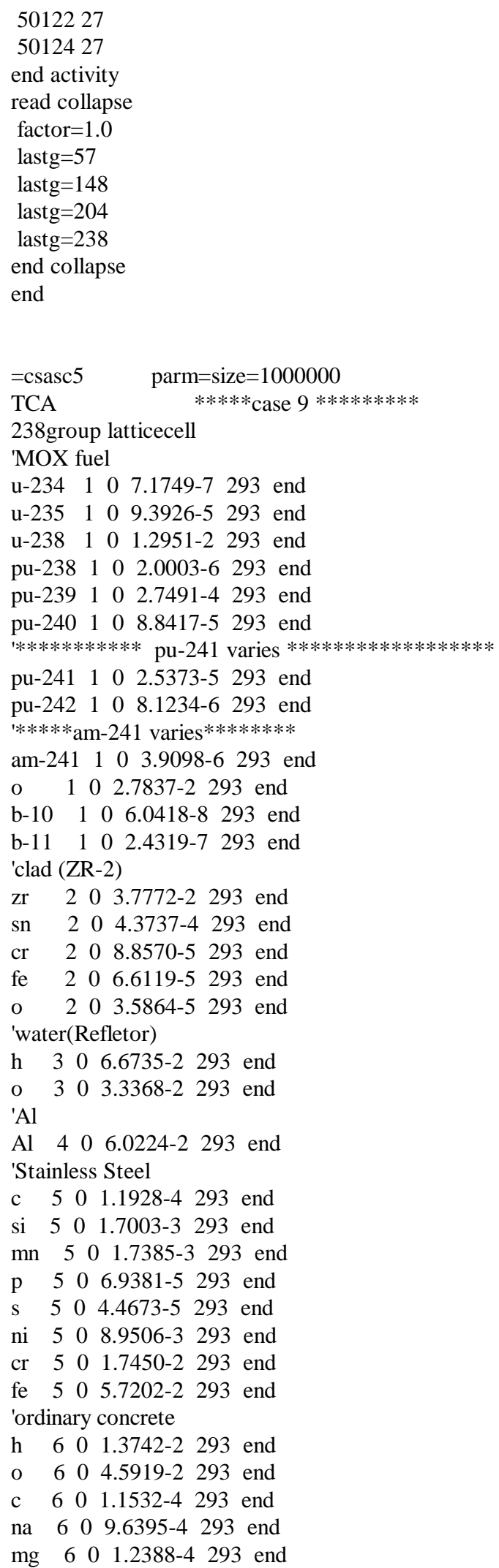

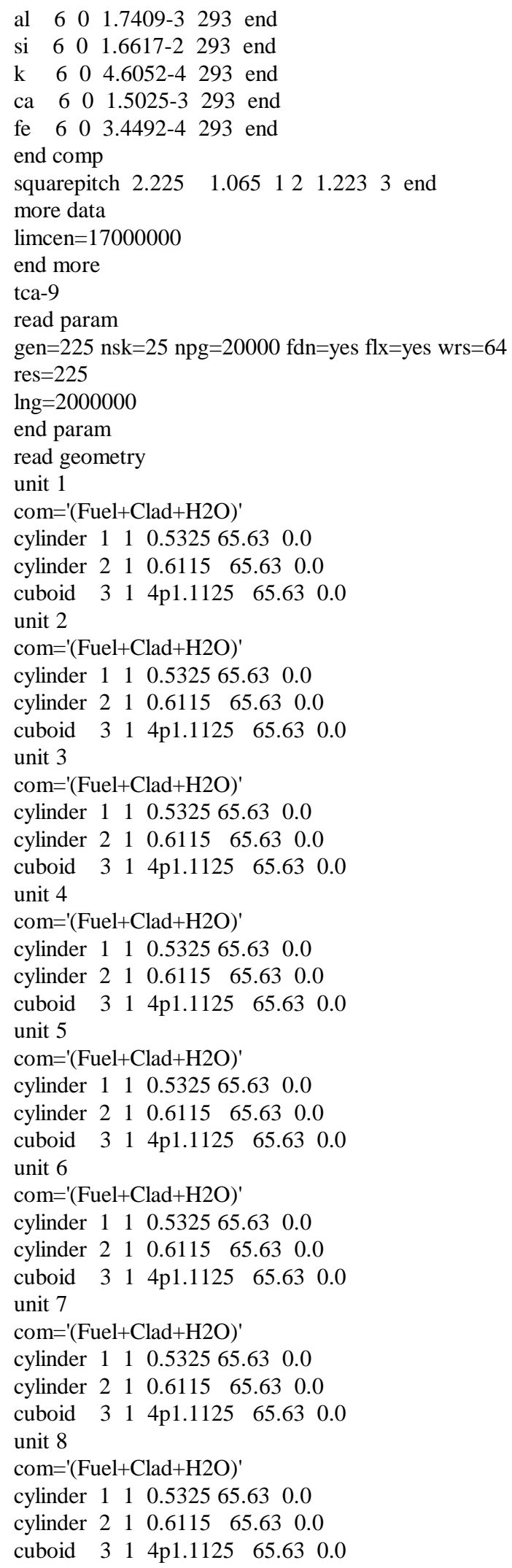


unit 9

com='(Fuel+Clad $+\mathrm{H} 2 \mathrm{O})^{\prime}$

$\begin{array}{llllll}\text { cylinder } & 1 & 1 & 0.5325 & 65.63 & 0.0\end{array}$

$\begin{array}{llllll}\text { cylinder } & 2 & 1 & 0.6115 & 65.63 & 0.0\end{array}$

$\begin{array}{lllllll}\text { cuboid } & 3 & 1 & 4 \mathrm{p} 1.1125 & 65.63 & 0.0\end{array}$

unit 10

com $=$ '(Fuel + Clad $+\mathrm{H} 2 \mathrm{O})^{\prime}$

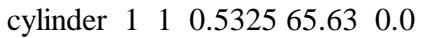

$\begin{array}{llllll}\text { cylinder } & 2 & 1 & 0.6115 & 65.63 & 0.0\end{array}$

$\begin{array}{lllllll}\text { cuboid } & 3 & 1 & 4 \mathrm{p} 1.1125 & 65.63 & 0.0\end{array}$

unit 11

com='(Fuel+Clad $+\mathrm{H} 2 \mathrm{O})^{\prime}$

$\begin{array}{llllll}\text { cylinder } & 1 & 1 & 0.5325 & 65.63 & 0.0\end{array}$

$\begin{array}{llllll}\text { cylinder } 2 & 1 & 0.6115 & 65.63 & 0.0\end{array}$

$\begin{array}{lllllll}\text { cuboid } & 3 & 1 & 4 \mathrm{p} 1.1125 & 65.63 & 0.0\end{array}$

unit 12

com='(Fuel+Clad $+\mathrm{H} 2 \mathrm{O})^{\prime}$

$\begin{array}{llllll}\text { cylinder } & 1 & 1 & 0.5325 & 65.63 & 0.0\end{array}$

$\begin{array}{llllll}\text { cylinder } & 2 & 1 & 0.6115 & 65.63 & 0.0\end{array}$

$\begin{array}{lllllll}\text { cuboid } & 3 & 1 & 4 \mathrm{p} 1.1125 & 65.63 & 0.0\end{array}$

unit 13

com $=$ '(Fuel $+\mathrm{Clad}+\mathrm{H} 2 \mathrm{O})^{\prime}$

cylinder $1110.532565 .63 \quad 0.0$

$\begin{array}{llllll}\text { cylinder } 2 & 1 & 0.6115 & 65.63 & 0.0\end{array}$

$\begin{array}{lllllll}\text { cuboid } & 3 & 1 & 4 \mathrm{p} 1.1125 & 65.63 & 0.0\end{array}$

unit 14

com='(Fuel+Clad $+\mathrm{H} 2 \mathrm{O})^{\prime}$

$\begin{array}{llllll}\text { cylinder } & 1 & 1 & 0.5325 & 65.63 & 0.0\end{array}$

$\begin{array}{llllll}\text { cylinder } 2 & 1 & 0.6115 & 65.63 & 0.0\end{array}$

$\begin{array}{lllllll}\text { cuboid } & 3 & 1 & 4 \mathrm{p} 1.1125 & 65.63 & 0.0\end{array}$

unit 15

com='(Fuel+Clad $+\mathrm{H} 2 \mathrm{O})^{\prime}$

$\begin{array}{lllllll}\text { cylinder } & 1 & 1 & 0.5325 & 65.63 & 0.0\end{array}$

$\begin{array}{llllll}\text { cylinder } & 2 & 1 & 0.6115 & 65.63 & 0.0\end{array}$

$\begin{array}{lllllll}\text { cuboid } & 3 & 1 & 4 \mathrm{p} 1.1125 & 65.63 & 0.0\end{array}$

unit 16

com='(Fuel $+\mathrm{Clad}+\mathrm{H} 2 \mathrm{O})^{\prime}$

$\begin{array}{llllll}\text { cylinder } & 1 & 1 & 0.5325 & 65.63 & 0.0\end{array}$

$\begin{array}{llllll}\text { cylinder } 2 & 1 & 0.6115 & 65.63 & 0.0\end{array}$

$\begin{array}{lllllll}\text { cuboid } & 3 & 1 & 4 \mathrm{p} 1.1125 & 65.63 & 0.0\end{array}$

unit 17

com='(Fuel+Clad $+\mathrm{H} 2 \mathrm{O})^{\prime}$

cylinder $1110.532565 .63 \quad 0.0$

$\begin{array}{llllll}\text { cylinder } 2 & 1 & 0.6115 & 65.63 & 0.0\end{array}$

$\begin{array}{lllllll}\text { cuboid } & 3 & 1 & 4 \mathrm{p} 1.1125 & 65.63 & 0.0\end{array}$

unit 18

com $=$ '(Fuel+Clad $+\mathrm{H} 2 \mathrm{O})^{\prime}$

cylinder $\begin{array}{lllll}1 & 1 & 0.5325 & 65.63 & 0.0\end{array}$

$\begin{array}{llllll}\text { cylinder } 2 & 1 & 0.6115 & 65.63 & 0.0\end{array}$

$\begin{array}{lllllll}\text { cuboid } & 3 & 1 & 4 \mathrm{p} 1.1125 & 65.63 & 0.0\end{array}$

unit 19

com $=$ '(Fuel $+\mathrm{Clad}+\mathrm{H} 2 \mathrm{O})^{\prime}$

cylinder $1110.532565 .63 \quad 0.0$

$\begin{array}{llllll}\text { cylinder } 2 & 1 & 0.6115 & 65.63 & 0.0\end{array}$

$\begin{array}{lllllll}\text { cuboid } & 3 & 1 & 4 \mathrm{p} 1.1125 & 65.63 & 0.0\end{array}$

unit 20

com='(Fuel+Clad $+\mathrm{H} 2 \mathrm{O})^{\prime}$ $\begin{array}{llllll}\text { cylinder } & 1 & 1 & 0.5325 & 65.63 & 0.0\end{array}$

$\begin{array}{llllll}\text { cylinder } & 2 & 1 & 0.6115 & 65.63 & 0.0\end{array}$

$\begin{array}{lllllll}\text { cuboid } & 3 & 1 & 4 \mathrm{p} 1.1125 & 65.63 & 0.0\end{array}$

unit 21

com $=$ '(Fuel + Clad $+\mathrm{H} 2 \mathrm{O})^{\prime}$

$\begin{array}{llllll}\text { cylinder } & 1 & 1 & 0.5325 & 65.63 & 0.0\end{array}$

$\begin{array}{llllll}\text { cylinder } 2 & 1 & 0.6115 & 65.63 & 0.0\end{array}$

$\begin{array}{lllllll}\text { cuboid } & 3 & 1 & 4 \mathrm{p} 1.1125 & 65.63 & 0.0\end{array}$

unit 22

com='(Fuel+Clad $+\mathrm{H} 2 \mathrm{O})^{\prime}$

cylinder $\begin{array}{lllll}1 & 1 & 0.5325 & 65.63 & 0.0\end{array}$

$\begin{array}{llllll}\text { cylinder } 2 & 1 & 0.6115 & 65.63 & 0.0\end{array}$

$\begin{array}{lllllll}\text { cuboid } & 3 & 1 & 4 \mathrm{p} 1.1125 & 65.63 & 0.0\end{array}$

unit 23

com='(Fuel $+\mathrm{Clad}+\mathrm{H} 2 \mathrm{O})^{\prime}$

$\begin{array}{llllll}\text { cylinder } & 1 & 1 & 0.5325 & 65.63 & 0.0\end{array}$

$\begin{array}{llllll}\text { cylinder } & 2 & 1 & 0.6115 & 65.63 & 0.0\end{array}$

$\begin{array}{lllllll}\text { cuboid } & 3 & 1 & 4 \mathrm{p} 1.1125 & 65.63 & 0.0\end{array}$

unit 24

com='(Fuel+Clad $+\mathrm{H} 2 \mathrm{O})^{\prime}$

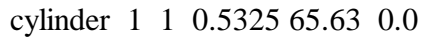

$\begin{array}{llllll}\text { cylinder } 2 & 1 & 0.6115 & 65.63 & 0.0\end{array}$

$\begin{array}{lllllll}\text { cuboid } & 3 & 1 & 4 \mathrm{p} 1.1125 & 65.63 & 0.0\end{array}$

unit 25

com='(Fuel+Clad $+\mathrm{H} 2 \mathrm{O})^{\prime}$

cylinder $\begin{array}{lllll}1 & 1 & 0.5325 & 65.63 & 0.0\end{array}$

$\begin{array}{llllll}\text { cylinder } 2 & 1 & 0.6115 & 65.63 & 0.0\end{array}$

$\begin{array}{lllllll}\text { cuboid } & 3 & 1 & 4 \mathrm{p} 1.1125 & 65.63 & 0.0\end{array}$

unit 26

com $=(\text { Fuel }+ \text { Clad }+\mathrm{H} 2 \mathrm{O})^{\prime}$

cylinder $\begin{array}{lllll}1 & 1 & 0.5325 & 65.63 & 0.0\end{array}$

$\begin{array}{llllll}\text { cylinder } 2 & 1 & 0.6115 & 65.63 & 0.0\end{array}$

$\begin{array}{lllllll}\text { cuboid } & 3 & 1 & 4 \mathrm{p} 1.1125 & 65.63 & 0.0\end{array}$

unit 27

com $=$ '(Fuel + Clad $+\mathrm{H} 2 \mathrm{O})^{\prime}$

$\begin{array}{llllll}\text { cylinder } & 1 & 1 & 0.5325 & 65.63 & 0.0\end{array}$

$\begin{array}{llllll}\text { cylinder } & 2 & 1 & 0.6115 & 65.63 & 0.0\end{array}$

$\begin{array}{lllllll}\text { cuboid } & 3 & 1 & 4 \mathrm{p} 1.1125 & 65.63 & 0.0\end{array}$

unit 28

com $=$ '(Fuel $+\mathrm{Clad}+\mathrm{H} 2 \mathrm{O})^{\prime}$

cylinder $\begin{array}{lllll}1 & 1 & 0.5325 & 65.63 & 0.0\end{array}$

$\begin{array}{llllll}\text { cylinder } 2 & 1 & 0.6115 & 65.63 & 0.0\end{array}$

$\begin{array}{lllllll}\text { cuboid } & 3 & 1 & 4 \mathrm{p} 1.1125 & 65.63 & 0.0\end{array}$

unit 29

com $=$ '(Fuel+Clad $+\mathrm{H} 2 \mathrm{O})^{\prime}$

$\begin{array}{llllll}\text { cylinder } & 1 & 1 & 0.5325 & 65.63 & 0.0\end{array}$

$\begin{array}{llllll}\text { cylinder } 2 & 1 & 0.6115 & 65.63 & 0.0\end{array}$

$\begin{array}{lllllll}\text { cuboid } & 3 & 1 & 4 \mathrm{p} 1.1125 & 65.63 & 0.0\end{array}$

unit 30

com $=$ '(Fuel + Clad $+\mathrm{H} 2 \mathrm{O})^{\prime}$

$\begin{array}{llllll}\text { cylinder } & 1 & 1 & 0.5325 & 65.63 & 0.0\end{array}$

$\begin{array}{lllllll}\text { cylinder } & 2 & 1 & 0.6115 & 65.63 & 0.0\end{array}$

$\begin{array}{lllllll}\text { cuboid } 3 & 1 & 4 p 1.1125 & 65.63 & 0.0\end{array}$

unit 31

com $=$ '(Fuel+Clad $+\mathrm{H} 2 \mathrm{O})^{\prime}$

$\begin{array}{llllll}\text { cylinder } & 1 & 1 & 0.5325 & 65.63 & 0.0\end{array}$

$\begin{array}{llllll}\text { cylinder } & 2 & 1 & 0.6115 & 65.63 & 0.0\end{array}$ 
$\begin{array}{lllllll}\text { cuboid } & 3 & 1 & 4 \mathrm{p} 1.1125 & 65.63 & 0.0\end{array}$

unit 32

com $='(\text { Fuel }+\mathrm{Clad}+\mathrm{H} 2 \mathrm{O})^{\prime}$

$\begin{array}{llllll}\text { cylinder } & 1 & 1 & 0.5325 & 65.63 & 0.0\end{array}$

$\begin{array}{llllll}\text { cylinder } 2 & 1 & 0.6115 & 65.63 & 0.0\end{array}$

$\begin{array}{lllllll}\text { cuboid } & 3 & 1 & 4 \mathrm{p} 1.1125 & 65.63 & 0.0\end{array}$

unit 33

com='(Fuel+Clad $+\mathrm{H} 2 \mathrm{O})^{\prime}$

$\begin{array}{llllll}\text { cylinder } & 1 & 1 & 0.5325 & 65.63 & 0.0\end{array}$

$\begin{array}{llllll}\text { cylinder } 2 & 1 & 0.6115 & 65.63 & 0.0\end{array}$

$\begin{array}{lllllll}\text { cuboid } & 3 & 1 & 4 \mathrm{p} 1.1125 & 65.63 & 0.0\end{array}$

unit 34

com='(Fuel+Clad $+\mathrm{H} 2 \mathrm{O})^{\prime}$

$\begin{array}{llllll}\text { cylinder } & 1 & 1 & 0.5325 & 65.63 & 0.0\end{array}$

$\begin{array}{llllll}\text { cylinder } 2 & 1 & 0.6115 & 65.63 & 0.0\end{array}$

$\begin{array}{lllllll}\text { cuboid } & 3 & 1 & 4 \mathrm{p} 1.1125 & 65.63 & 0.0\end{array}$

unit 35

com='(Fuel+Clad $+\mathrm{H} 2 \mathrm{O})^{\prime}$

$\begin{array}{llllll}\text { cylinder } & 1 & 1 & 0.5325 & 65.63 & 0.0\end{array}$

$\begin{array}{llllll}\text { cylinder } 2 & 1 & 0.6115 & 65.63 & 0.0\end{array}$

$\begin{array}{lllllll}\text { cuboid } & 3 & 1 & 4 \mathrm{p} 1.1125 & 65.63 & 0.0\end{array}$

unit 36

com $=$ '(Fuel+Clad $+\mathrm{H} 2 \mathrm{O})^{\prime}$

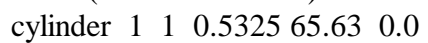

$\begin{array}{llllll}\text { cylinder } 2 & 1 & 0.6115 & 65.63 & 0.0\end{array}$

$\begin{array}{lllllll}\text { cuboid } & 3 & 1 & 4 p 1.1125 & 65.63 & 0.0\end{array}$

unit 37

com='(Fuel + Clad $+\mathrm{H} 2 \mathrm{O})^{\prime}$

cylinder $\begin{array}{lllll}1 & 1 & 0.5325 & 65.63 & 0.0\end{array}$

$\begin{array}{lllllll}\text { cylinder } & 2 & 1 & 0.6115 & 65.63 & 0.0\end{array}$

$\begin{array}{lllllll}\text { cuboid } & 3 & 1 & 4 \mathrm{p} 1.1125 & 65.63 & 0.0\end{array}$

unit 38

com $=$ '(Fuel $+\mathrm{Clad}+\mathrm{H} 2 \mathrm{O})^{\prime}$

$\begin{array}{llllll}\text { cylinder } & 1 & 1 & 0.5325 & 65.63 & 0.0\end{array}$

$\begin{array}{llllll}\text { cylinder } 2 & 1 & 0.6115 & 65.63 & 0.0\end{array}$

$\begin{array}{lllllll}\text { cuboid } & 3 & 1 & 4 \mathrm{p} 1.1125 & 65.63 & 0.0\end{array}$

unit 39

com='(Fuel+Clad $+\mathrm{H} 2 \mathrm{O})^{\prime}$

$\begin{array}{llllll}\text { cylinder } & 1 & 1 & 0.5325 & 65.63 & 0.0\end{array}$

$\begin{array}{llllll}\text { cylinder } 2 & 1 & 0.6115 & 65.63 & 0.0\end{array}$

$\begin{array}{lllllll}\text { cuboid } & 3 & 1 & 4 \mathrm{p} 1.1125 & 65.63 & 0.0\end{array}$

unit 40

com $=$ '(Fuel + Clad $+\mathrm{H} 2 \mathrm{O})^{\prime}$

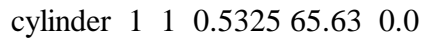

$\begin{array}{llllll}\text { cylinder } 2 & 1 & 0.6115 & 65.63 & 0.0\end{array}$

$\begin{array}{lllllll}\text { cuboid } & 3 & 1 & 4 \mathrm{p} 1.1125 & 65.63 & 0.0\end{array}$

unit 41

com='(Fuel $+\mathrm{Clad}+\mathrm{H} 2 \mathrm{O})^{\prime}$

$\begin{array}{lllllll}\text { cylinder } & 1 & 1 & 0.5325 & 65.63 & 0.0\end{array}$

$\begin{array}{llllll}\text { cylinder } 2 & 1 & 0.6115 & 65.63 & 0.0\end{array}$

$\begin{array}{lllllll}\text { cuboid } & 3 & 1 & 4 \mathrm{p} 1.1125 & 65.63 & 0.0\end{array}$

unit 42

com $=$ '(Fuel + Clad $+\mathrm{H} 2 \mathrm{O})^{\prime}$

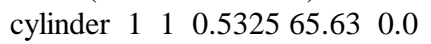

$\begin{array}{llllll}\text { cylinder } 2 & 1 & 0.6115 & 65.63 & 0.0\end{array}$

$\begin{array}{lllllll}\text { cuboid } & 3 & 1 & 4 \mathrm{p} 1.1125 & 65.63 & 0.0\end{array}$

unit 43

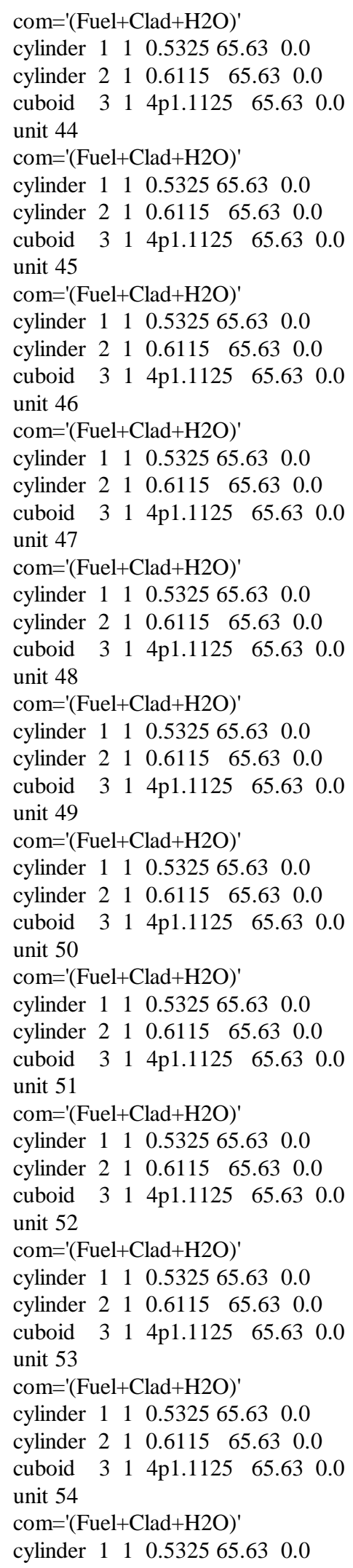


cylinder $2 \quad 1 \quad 0.6115 \quad 65.63 \quad 0.0$

$\begin{array}{lllllll}\text { cuboid } & 3 & 1 & 4 \mathrm{p} 1.1125 & 65.63 & 0.0\end{array}$

unit 55

com='(Fuel + Clad $+\mathrm{H} 2 \mathrm{O})^{\prime}$

cylinder $1 \quad 1 \quad 0.532565 .63 \quad 0.0$

cylinder $2 \quad 1 \quad 0.6115 \quad 65.63 \quad 0.0$

$\begin{array}{lllllll}\text { cuboid } & 3 & 1 & 4 \mathrm{p} 1.1125 & 65.63 & 0.0\end{array}$

unit 81

com='h2o and end plug below Al grid plate - fill array 1' $\begin{array}{lllll}\text { cylinder } 4 & 1 & 0.6115 & 4.445 & 0.0\end{array}$

$\begin{array}{lllllll}\text { cuboid } & 3 & 1 & 4 \mathrm{p} 1.1125 & 4.445 & 0.0\end{array}$

unit 82

com='h2o and end plug above $\mathrm{Al}$ grid plate - fill array 2' $\begin{array}{llllll}\text { cylinder } & 4 & 1 & 0.6115 & 11.784 & 0.0\end{array}$

$\begin{array}{llllllll}\text { cuboid } & 3 & 1 & 4 \mathrm{p} 1.1125 & 11.784 & 0.0\end{array}$

unit 83

com='(Fuel+Clad+Void $)$ - fill array 3'

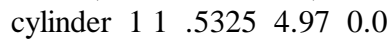

$\begin{array}{lllll}\text { cylinder } 2 & 1 & .6115 & 4.97 & 0.0\end{array}$

cuboid $014 \mathrm{p} 1.11254 .97 \quad 0.0$

unit 84

com='(void+Clad+void $)^{\prime}$

cylinder $\begin{array}{lllll}0 & 1 & 0.5325 & 9.97 & 0.0\end{array}$

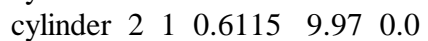

$\begin{array}{lllllll}\text { cuboid } & 0 & 1 & 4 \mathrm{p} 1.1125 & 9.97 & 0.0\end{array}$

unit 100

com='Lower Al grid plate'

cuboid $414 \mathrm{p} 52.25 .601 \quad 0.0$

unit 101

com='array of h2o and end plugs below Al grid plate'

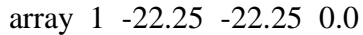

$\begin{array}{lllllll}\text { cuboid } 3 & 3 & 4 \mathrm{p} 52.25 & 4.445 & 0.0\end{array}$

unit 102

com='array of h2o and end plugs above $\mathrm{Al}$ grid plate '

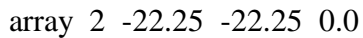

$\begin{array}{lllllll}\text { cuboid } 3 & 1 & 4 \mathrm{p} 52.25 & 11.784 & 0.0\end{array}$

unit 103

com='array of Fuel+Clad+Void'

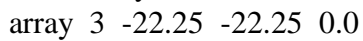

cuboid 014 p $52.254 .97 \quad 0.0$

unit 104

com='array of void+Clad+void'

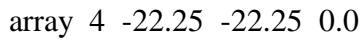

$\begin{array}{lllllll}\text { cuboid } & 0 & 1 & 4 \mathrm{p} 52.25 & 9.97 & 0.0\end{array}$

unit 105

com='array of Fuel+Clad+H2O'

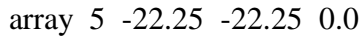

$\begin{array}{lllllll}\text { cuboid } & 3 & 1 & 4 \mathrm{p} 52.25 & 65.63 & 0.0\end{array}$

global unit 110

com='array of arrays of fuel element sections + reflector'

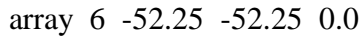

cuboid $4 \begin{array}{lllll}4 & 4 p 52.25 & 97.40 & -1.27\end{array}$

$\begin{array}{llllll}\text { cuboid } 5 & 1 & 4 p 52.25 & 97.40 & -3.47\end{array}$

$\begin{array}{lllllll}\text { cuboid } 3 & 1 & 4 p 52.25 & 97.40 & -17.27\end{array}$

cuboid $5 \begin{array}{llllll} & 1 & 4 p 52.25 & 97.40 & -17.77\end{array}$

$\begin{array}{lllllll}\text { cuboid } & 6 & 1 & 4 \mathrm{p} 52.25 & 97.40 & -54.77\end{array}$

end geometry read array

ara $=1$ nux=20 nuy=20 nuz=1

fill $\mathrm{f} 81$ end fill

ara=2 nux=20 nuy=20 nuz=1

fill $\mathrm{f} 82$ end fill

ara $=3$ nux $=20$ nuy=20 nuz=1

fill $\mathrm{f} 83$ end fill

ara $=4$ nux $=20$ nuy=20 nuz=1

fill $\mathrm{f} 84$ end fill

ara $=5$ nux $=20$ nuy $=20$ nuz $=1$

fill

$\begin{array}{lllllllllll}1 & 2 & 3 & 4 & 5 & 6 & 7 & 8 & 9 & 10 & \mathrm{n} \\ 10\end{array}$

2111213141516171819 n 10

3122021222324252627 n 10

4132128293031323334 n 10

5142229353637383940 n 10

6152330364142434445 n 10

7162431374246474849 n 10

8172532384347505152 n 10

9182633394448515354 n 10

10192734404549525455 n 10 n200

end fill

ara $=6$ nux= 1 nuy=1 nuz=6

fill 101100102105103104 end fill

end array

end data

end

$=$ shell

cp ft04f001 \$RTNDIR/tca09.xs04

cp ft64f001 \$RTNDIR/tca09.rs64

end

$=\mathrm{kmart}$

read initial kunit $=64$

xunit $=4$

actbygrp

rrpvol

end initial

read activity

$9223427922341892234 \quad 1452$

92235279223518922351452

92238279223818922381452

94238279423818942381452

94239279423918942391452

94240279424018942401452

$9424127942411894241 \quad 1452$

$9424227942421894242 \quad 1452$

95241279524118952411452

501027

501127

801627

4000027

2400027

2600027

100127

5011227

5011427 


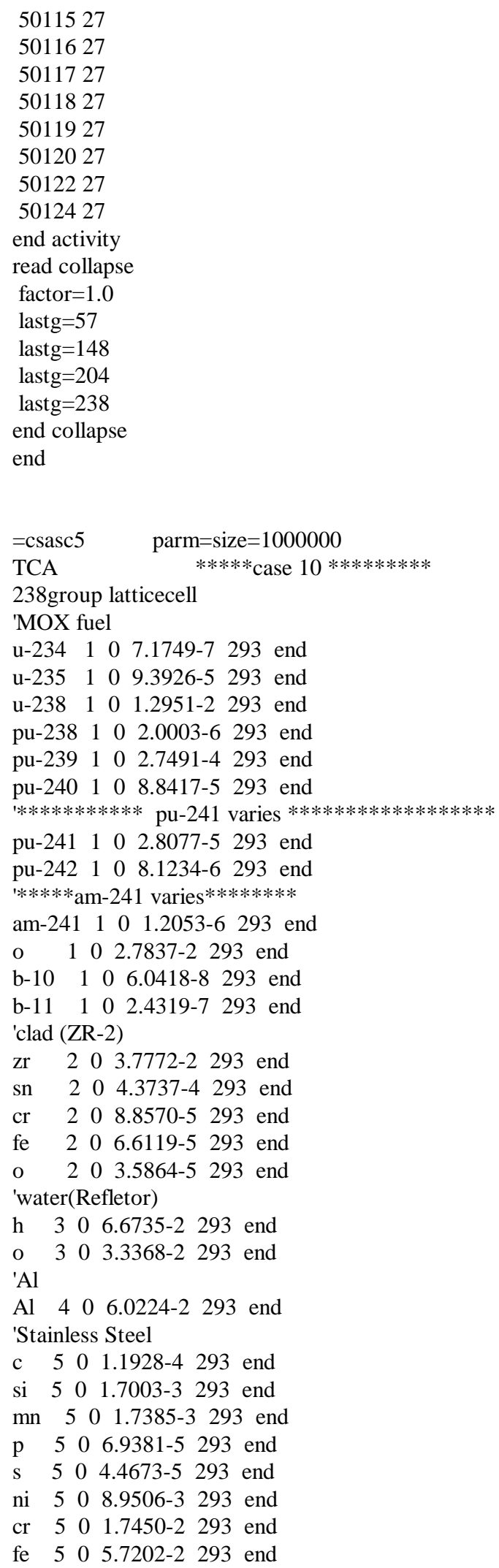

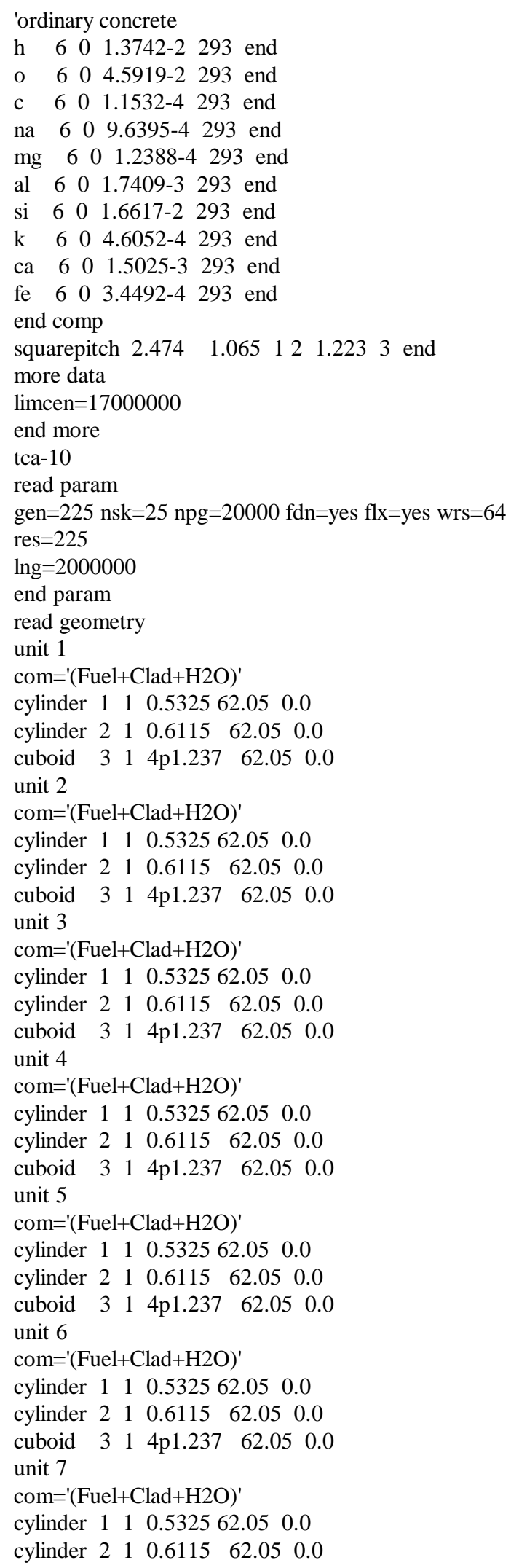


$\begin{array}{lllllll}\text { cuboid } & 3 & 1 & 4 \mathrm{p} 1.237 & 62.05 & 0.0\end{array}$ unit 8

com $='(\text { Fuel }+\mathrm{Clad}+\mathrm{H} 2 \mathrm{O})^{\prime}$

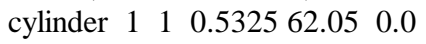

$\begin{array}{llllll}\text { cylinder } & 2 & 1 & 0.6115 & 62.05 & 0.0\end{array}$

$\begin{array}{lllllll}\text { cuboid } & 3 & 1 & 4 \mathrm{p} 1.237 & 62.05 & 0.0\end{array}$

unit 9

com='(Fuel+Clad $+\mathrm{H} 2 \mathrm{O})^{\prime}$

cylinder $\begin{array}{lllll}1 & 1 & 0.5325 & 62.05 & 0.0\end{array}$

$\begin{array}{llllll}\text { cylinder } 2 & 1 & 0.6115 & 62.05 & 0.0\end{array}$

$\begin{array}{lllllll}\text { cuboid } & 3 & 1 & 4 \mathrm{p} 1.237 & 62.05 & 0.0\end{array}$

unit 10

com='(Fuel+Clad $+\mathrm{H} 2 \mathrm{O})^{\prime}$

$\begin{array}{llllll}\text { cylinder } & 1 & 1 & 0.5325 & 62.05 & 0.0\end{array}$

$\begin{array}{llllll}\text { cylinder } & 2 & 1 & 0.6115 & 62.05 & 0.0\end{array}$

$\begin{array}{lllllll}\text { cuboid } & 3 & 1 & 4 \mathrm{p} 1.237 & 62.05 & 0.0\end{array}$

unit 11

com='(Fuel+Clad $+\mathrm{H} 2 \mathrm{O})^{\prime}$

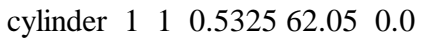

$\begin{array}{llllll}\text { cylinder } 2 & 1 & 0.6115 & 62.05 & 0.0\end{array}$

$\begin{array}{lllllll}\text { cuboid } & 3 & 1 & 4 \mathrm{p} 1.237 & 62.05 & 0.0\end{array}$

unit 12

com $=$ '(Fuel+Clad $+\mathrm{H} 2 \mathrm{O})^{\prime}$

$\begin{array}{llllll}\text { cylinder } & 1 & 1 & 0.5325 & 62.05 & 0.0\end{array}$

$\begin{array}{llllll}\text { cylinder } 2 & 1 & 0.6115 & 62.05 & 0.0\end{array}$

$\begin{array}{lllllll}\text { cuboid } & 3 & 1 & 4 \mathrm{p} 1.237 & 62.05 & 0.0\end{array}$

unit 13

com='(Fuel + Clad $+\mathrm{H} 2 \mathrm{O})^{\prime}$

$\begin{array}{llllll}\text { cylinder } & 1 & 1 & 0.5325 & 62.05 & 0.0\end{array}$

$\begin{array}{llllll}\text { cylinder } & 2 & 1 & 0.6115 & 62.05 & 0.0\end{array}$

$\begin{array}{lllllll}\text { cuboid } & 3 & 1 & 4 \mathrm{p} 1.237 & 62.05 & 0.0\end{array}$

unit 14

com $=$ '(Fuel $+\mathrm{Clad}+\mathrm{H} 2 \mathrm{O})^{\prime}$

$\begin{array}{llllll}\text { cylinder } & 1 & 1 & 0.5325 & 62.05 & 0.0\end{array}$

$\begin{array}{llllll}\text { cylinder } 2 & 1 & 0.6115 & 62.05 & 0.0\end{array}$

$\begin{array}{lllllll}\text { cuboid } & 3 & 1 & 4 \mathrm{p} 1.237 & 62.05 & 0.0\end{array}$

unit 15

com='(Fuel+Clad $+\mathrm{H} 2 \mathrm{O})^{\prime}$

$\begin{array}{llllll}\text { cylinder } & 1 & 1 & 0.5325 & 62.05 & 0.0\end{array}$

$\begin{array}{llllll}\text { cylinder } & 2 & 1 & 0.6115 & 62.05 & 0.0\end{array}$

$\begin{array}{lllllll}\text { cuboid } & 3 & 1 & 4 \mathrm{p} 1.237 & 62.05 & 0.0\end{array}$

unit 16

com='(Fuel+Clad $+\mathrm{H} 2 \mathrm{O})^{\prime}$

$\begin{array}{llllll}\text { cylinder } & 1 & 1 & 0.5325 & 62.05 & 0.0\end{array}$

$\begin{array}{llllll}\text { cylinder } & 2 & 1 & 0.6115 & 62.05 & 0.0\end{array}$

$\begin{array}{lllllll}\text { cuboid } & 3 & 1 & 4 \mathrm{p} 1.237 & 62.05 & 0.0\end{array}$

unit 17

com $=$ '(Fuel+Clad $+\mathrm{H} 2 \mathrm{O})^{\prime}$

$\begin{array}{llllll}\text { cylinder } & 1 & 1 & 0.5325 & 62.05 & 0.0\end{array}$

$\begin{array}{llllll}\text { cylinder } 2 & 1 & 0.6115 & 62.05 & 0.0\end{array}$

$\begin{array}{lllllll}\text { cuboid } & 3 & 1 & 4 \mathrm{p} 1.237 & 62.05 & 0.0\end{array}$

unit 18

com='(Fuel+Clad $+\mathrm{H} 2 \mathrm{O})^{\prime}$

$\begin{array}{llllll}\text { cylinder } & 1 & 1 & 0.5325 & 62.05 & 0.0\end{array}$

$\begin{array}{llllll}\text { cylinder } & 2 & 1 & 0.6115 & 62.05 & 0.0\end{array}$

$\begin{array}{lllllll}\text { cuboid } & 3 & 1 & 4 \mathrm{p} 1.237 & 62.05 & 0.0\end{array}$

unit 19
com='(Fuel+Clad+H2O)'

$\begin{array}{llllll}\text { cylinder } & 1 & 1 & 0.5325 & 62.05 & 0.0\end{array}$

$\begin{array}{llllll}\text { cylinder } & 2 & 1 & 0.6115 & 62.05 & 0.0\end{array}$

$\begin{array}{lllllll}\text { cuboid } & 3 & 1 & 4 \mathrm{p} 1.237 & 62.05 & 0.0\end{array}$

unit 20

com $=$ '(Fuel $+\mathrm{Clad}+\mathrm{H} 2 \mathrm{O})^{\prime}$

$\begin{array}{llllll}\text { cylinder } & 1 & 1 & 0.5325 & 62.05 & 0.0\end{array}$

$\begin{array}{llllll}\text { cylinder } 2 & 1 & 0.6115 & 62.05 & 0.0\end{array}$

$\begin{array}{lllllll}\text { cuboid } & 3 & 1 & 4 \mathrm{p} 1.237 & 62.05 & 0.0\end{array}$

unit 21

com $=$ '(Fuel + Clad $+\mathrm{H} 2 \mathrm{O})^{\prime}$

$\begin{array}{llllll}\text { cylinder } & 1 & 1 & 0.5325 & 62.05 & 0.0\end{array}$

$\begin{array}{llllll}\text { cylinder } 2 & 1 & 0.6115 & 62.05 & 0.0\end{array}$

$\begin{array}{lllllll}\text { cuboid } & 3 & 1 & 4 \mathrm{p} 1.237 & 62.05 & 0.0\end{array}$

unit 22

com $=$ '(Fuel + Clad $+\mathrm{H} 2 \mathrm{O})^{\prime}$

$\begin{array}{llllll}\text { cylinder } & 1 & 1 & 0.5325 & 62.05 & 0.0\end{array}$

$\begin{array}{llllll}\text { cylinder } & 2 & 1 & 0.6115 & 62.05 & 0.0\end{array}$

$\begin{array}{lllllll}\text { cuboid } & 3 & 1 & 4 \mathrm{p} 1.237 & 62.05 & 0.0\end{array}$

unit 23

com='(Fuel+Clad $+\mathrm{H} 2 \mathrm{O})^{\prime}$

$\begin{array}{llllll}\text { cylinder } & 1 & 1 & 0.5325 & 62.05 & 0.0\end{array}$

$\begin{array}{llllll}\text { cylinder } & 2 & 1 & 0.6115 & 62.05 & 0.0\end{array}$

$\begin{array}{lllllll}\text { cuboid } & 3 & 1 & 4 \mathrm{p} 1.237 & 62.05 & 0.0\end{array}$

unit 24

com='(Fuel $+\mathrm{Clad}+\mathrm{H} 2 \mathrm{O})^{\prime}$

$\begin{array}{llllll}\text { cylinder } & 1 & 1 & 0.5325 & 62.05 & 0.0\end{array}$

$\begin{array}{llllll}\text { cylinder } & 2 & 1 & 0.6115 & 62.05 & 0.0\end{array}$

$\begin{array}{lllllll}\text { cuboid } & 3 & 1 & 4 \mathrm{p} 1.237 & 62.05 & 0.0\end{array}$

unit 25

com='(Fuel+Clad $+\mathrm{H} 2 \mathrm{O})^{\prime}$

$\begin{array}{llllll}\text { cylinder } & 1 & 1 & 0.5325 & 62.05 & 0.0\end{array}$

$\begin{array}{llllll}\text { cylinder } 2 & 1 & 0.6115 & 62.05 & 0.0\end{array}$

$\begin{array}{lllllll}\text { cuboid } & 3 & 1 & 4 \mathrm{p} 1.237 & 62.05 & 0.0\end{array}$

unit 26

com='(Fuel+Clad $+\mathrm{H} 2 \mathrm{O})^{\prime}$

$\begin{array}{llllll}\text { cylinder } & 1 & 1 & 0.5325 & 62.05 & 0.0\end{array}$

$\begin{array}{llllll}\text { cylinder } & 2 & 1 & 0.6115 & 62.05 & 0.0\end{array}$

$\begin{array}{lllllll}\text { cuboid } & 3 & 1 & 4 \mathrm{p} 1.237 & 62.05 & 0.0\end{array}$

unit 27

com $=$ '(Fuel + Clad $+\mathrm{H} 2 \mathrm{O})^{\prime}$

cylinder $\begin{array}{lllll}1 & 1 & 0.5325 & 62.05 & 0.0\end{array}$

$\begin{array}{lllllll}\text { cylinder } 2 & 1 & 0.6115 & 62.05 & 0.0\end{array}$

$\begin{array}{lllllll}\text { cuboid } & 3 & 1 & 4 \mathrm{p} 1.237 & 62.05 & 0.0\end{array}$

unit 28

com='(Fuel+Clad $+\mathrm{H} 2 \mathrm{O})^{\prime}$

$\begin{array}{llllll}\text { cylinder } & 1 & 1 & 0.5325 & 62.05 & 0.0\end{array}$

$\begin{array}{llllll}\text { cylinder } & 2 & 1 & 0.6115 & 62.05 & 0.0\end{array}$

$\begin{array}{lllllll}\text { cuboid } 3 & 1 & 4 \mathrm{p} 1.237 & 62.05 & 0.0\end{array}$

unit 29

com $=$ '(Fuel+Clad $+\mathrm{H} 2 \mathrm{O})^{\prime}$

$\begin{array}{llllll}\text { cylinder } & 1 & 1 & 0.5325 & 62.05 & 0.0\end{array}$

$\begin{array}{llllll}\text { cylinder } 2 & 1 & 0.6115 & 62.05 & 0.0\end{array}$

$\begin{array}{lllllll}\text { cuboid } & 3 & 1 & 4 \mathrm{p} 1.237 & 62.05 & 0.0\end{array}$

unit 30

com $=(\text { Fuel }+\mathrm{Clad}+\mathrm{H} 2 \mathrm{O})^{\prime}$

cylinder $\begin{array}{lllll}1 & 1 & 0.5325 & 62.05 & 0.0\end{array}$ 
$\begin{array}{llllll}\text { cylinder } & 2 & 1 & 0.6115 & 62.05 & 0.0\end{array}$ $\begin{array}{lllllll}\text { cuboid } & 3 & 1 & 4 \mathrm{p} 1.237 & 62.05 & 0.0\end{array}$ unit 31

com='(Fuel+Clad $+\mathrm{H} 2 \mathrm{O})^{\prime}$

$\begin{array}{llllll}\text { cylinder } & 1 & 1 & 0.5325 & 62.05 & 0.0\end{array}$

$\begin{array}{llllll}\text { cylinder } 2 & 1 & 0.6115 & 62.05 & 0.0\end{array}$

$\begin{array}{lllllll}\text { cuboid } & 3 & 1 & 4 \mathrm{p} 1.237 & 62.05 & 0.0\end{array}$

unit 32

com='(Fuel+Clad $+\mathrm{H} 2 \mathrm{O})^{\prime}$

$\begin{array}{llllll}\text { cylinder } & 1 & 1 & 0.5325 & 62.05 & 0.0\end{array}$

$\begin{array}{llllll}\text { cylinder } & 2 & 1 & 0.6115 & 62.05 & 0.0\end{array}$

$\begin{array}{lllllll}\text { cuboid } & 3 & 1 & 4 \mathrm{p} 1.237 & 62.05 & 0.0\end{array}$

unit 33

com $=$ '(Fuel $+\mathrm{Clad}+\mathrm{H} 2 \mathrm{O})^{\prime}$

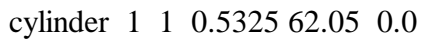

$\begin{array}{llllll}\text { cylinder } 2 & 1 & 0.6115 & 62.05 & 0.0\end{array}$

$\begin{array}{lllllll}\text { cuboid } & 3 & 1 & 4 \mathrm{p} 1.237 & 62.05 & 0.0\end{array}$

unit 34

com='(Fuel+Clad $+\mathrm{H} 2 \mathrm{O})^{\prime}$

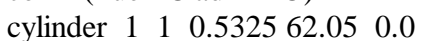

$\begin{array}{llllll}\text { cylinder } & 2 & 1 & 0.6115 & 62.05 & 0.0\end{array}$

$\begin{array}{lllllll}\text { cuboid } & 3 & 1 & 4 \mathrm{p} 1.237 & 62.05 & 0.0\end{array}$

unit 35

com $=$ '(Fuel+Clad $+\mathrm{H} 2 \mathrm{O})^{\prime}$

$\begin{array}{llllll}\text { cylinder } & 1 & 1 & 0.5325 & 62.05 & 0.0\end{array}$

$\begin{array}{lllllll}\text { cylinder } & 2 & 1 & 0.6115 & 62.05 & 0.0\end{array}$

$\begin{array}{lllllll}\text { cuboid } & 3 & 1 & 4 \mathrm{p} 1.237 & 62.05 & 0.0\end{array}$

unit 36

com='(Fuel+Clad $+\mathrm{H} 2 \mathrm{O})^{\prime}$

$\begin{array}{llllll}\text { cylinder } & 1 & 1 & 0.5325 & 62.05 & 0.0\end{array}$

$\begin{array}{llllll}\text { cylinder } 2 & 1 & 0.6115 & 62.05 & 0.0\end{array}$

$\begin{array}{lllllll}\text { cuboid } & 3 & 1 & 4 \mathrm{p} 1.237 & 62.05 & 0.0\end{array}$

unit 37

com='(Fuel+Clad $+\mathrm{H} 2 \mathrm{O})^{\prime}$

cylinder $\begin{array}{lllll}1 & 1 & 0.5325 & 62.05 & 0.0\end{array}$

$\begin{array}{llllll}\text { cylinder } & 2 & 1 & 0.6115 & 62.05 & 0.0\end{array}$

$\begin{array}{lllllll}\text { cuboid } & 3 & 1 & 4 \mathrm{p} 1.237 & 62.05 & 0.0\end{array}$

unit 38

com $=$ '(Fuel + Clad $+\mathrm{H} 2 \mathrm{O})^{\prime}$

$\begin{array}{llllll}\text { cylinder } & 1 & 1 & 0.5325 & 62.05 & 0.0\end{array}$

$\begin{array}{llllll}\text { cylinder } & 2 & 1 & 0.6115 & 62.05 & 0.0\end{array}$

$\begin{array}{lllllll}\text { cuboid } & 3 & 1 & 4 \mathrm{p} 1.237 & 62.05 & 0.0\end{array}$

unit 39

com $='(\text { Fuel }+ \text { Clad }+\mathrm{H} 2 \mathrm{O})^{\prime}$

$\begin{array}{llllll}\text { cylinder } & 1 & 1 & 0.5325 & 62.05 & 0.0\end{array}$

$\begin{array}{llllll}\text { cylinder } & 2 & 1 & 0.6115 & 62.05 & 0.0\end{array}$

$\begin{array}{lllllll}\text { cuboid } & 3 & 1 & 4 \mathrm{p} 1.237 & 62.05 & 0.0\end{array}$

unit 40

com='(Fuel+Clad $+\mathrm{H} 2 \mathrm{O})^{\prime}$

$\begin{array}{llllll}\text { cylinder } & 1 & 1 & 0.5325 & 62.05 & 0.0\end{array}$

$\begin{array}{llllll}\text { cylinder } & 2 & 1 & 0.6115 & 62.05 & 0.0\end{array}$

$\begin{array}{lllllll}\text { cuboid } & 3 & 1 & 4 \mathrm{p} 1.237 & 62.05 & 0.0\end{array}$

unit 41

com='(Fuel + Clad $+\mathrm{H} 2 \mathrm{O})^{\prime}$

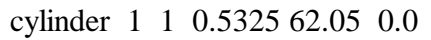

$\begin{array}{llllll}\text { cylinder } & 2 & 1 & 0.6115 & 62.05 & 0.0\end{array}$

$\begin{array}{lllllll}\text { cuboid } \quad 3 & 1 & 4 \mathrm{p} 1.237 & 62.05 & 0.0\end{array}$ unit 42

com $=$ '(Fuel + Clad $+\mathrm{H} 2 \mathrm{O})^{\prime}$

$\begin{array}{llllll}\text { cylinder } & 1 & 1 & 0.5325 & 62.05 & 0.0\end{array}$

$\begin{array}{llllll}\text { cylinder } & 2 & 1 & 0.6115 & 62.05 & 0.0\end{array}$

$\begin{array}{lllllll}\text { cuboid } & 3 & 1 & 4 \mathrm{p} 1.237 & 62.05 & 0.0\end{array}$

unit 43

com $=$ '(Fuel + Clad $+\mathrm{H} 2 \mathrm{O})^{\prime}$

$\begin{array}{llllll}\text { cylinder } & 1 & 1 & 0.5325 & 62.05 & 0.0\end{array}$

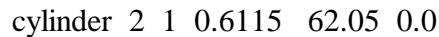

$\begin{array}{lllllll}\text { cuboid } & 3 & 1 & 4 \mathrm{p} 1.237 & 62.05 & 0.0\end{array}$

unit 44

com='(Fuel+Clad $+\mathrm{H} 2 \mathrm{O})^{\prime}$

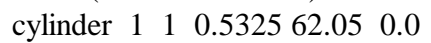

$\begin{array}{lllllll}\text { cylinder } 2 & 1 & 0.6115 & 62.05 & 0.0\end{array}$

$\begin{array}{lllllll}\text { cuboid } & 3 & 1 & 4 \mathrm{p} 1.237 & 62.05 & 0.0\end{array}$

unit 45

com $=$ '(Fuel + Clad $+\mathrm{H} 2 \mathrm{O})^{\prime}$

$\begin{array}{llllll}\text { cylinder } & 1 & 1 & 0.5325 & 62.05 & 0.0\end{array}$

$\begin{array}{llllll}\text { cylinder } 2 & 1 & 0.6115 & 62.05 & 0.0\end{array}$

$\begin{array}{lllllll}\text { cuboid } & 3 & 1 & 4 \mathrm{p} 1.237 & 62.05 & 0.0\end{array}$

unit 46

com $=$ '(Fuel + Clad $+\mathrm{H} 2 \mathrm{O})^{\prime}$

$\begin{array}{llllll}\text { cylinder } & 1 & 1 & 0.5325 & 62.05 & 0.0\end{array}$

$\begin{array}{llllll}\text { cylinder } 2 & 1 & 0.6115 & 62.05 & 0.0\end{array}$

$\begin{array}{lllllll}\text { cuboid } & 3 & 1 & 4 \mathrm{p} 1.237 & 62.05 & 0.0\end{array}$

unit 47

com $=$ '(Fuel + Clad $+\mathrm{H} 2 \mathrm{O})^{\prime}$

cylinder $\begin{array}{lllll}1 & 1 & 0.5325 & 62.05 & 0.0\end{array}$

$\begin{array}{llllll}\text { cylinder } & 2 & 1 & 0.6115 & 62.05 & 0.0\end{array}$

$\begin{array}{lllllll}\text { cuboid } 3 & 1 & 4 \mathrm{p} 1.237 & 62.05 & 0.0\end{array}$

unit 48

com $=$ '(Fuel + Clad $+\mathrm{H} 2 \mathrm{O})^{\prime}$

$\begin{array}{llllll}\text { cylinder } & 1 & 1 & 0.5325 & 62.05 & 0.0\end{array}$

$\begin{array}{llllll}\text { cylinder } & 2 & 1 & 0.6115 & 62.05 & 0.0\end{array}$

$\begin{array}{lllllll}\text { cuboid } & 3 & 1 & 4 \mathrm{p} 1.237 & 62.05 & 0.0\end{array}$

unit 49

com $=$ '(Fuel + Clad $+\mathrm{H} 2 \mathrm{O})^{\prime}$

$\begin{array}{llllll}\text { cylinder } & 1 & 1 & 0.5325 & 62.05 & 0.0\end{array}$

$\begin{array}{llllll}\text { cylinder } & 2 & 1 & 0.6115 & 62.05 & 0.0\end{array}$

$\begin{array}{lllllll}\text { cuboid } & 3 & 1 & 4 \mathrm{p} 1.237 & 62.05 & 0.0\end{array}$

unit 50

com $=$ '(Fuel + Clad $+\mathrm{H} 2 \mathrm{O})^{\prime}$

$\begin{array}{llllll}\text { cylinder } & 1 & 1 & 0.5325 & 62.05 & 0.0\end{array}$

$\begin{array}{llllll}\text { cylinder } 2 & 1 & 0.6115 & 62.05 & 0.0\end{array}$

$\begin{array}{lllllll}\text { cuboid } & 3 & 1 & 4 \mathrm{p} 1.237 & 62.05 & 0.0\end{array}$

unit 51

com $=$ '(Fuel+Clad $+\mathrm{H} 2 \mathrm{O})^{\prime}$

$\begin{array}{llllll}\text { cylinder } & 1 & 1 & 0.5325 & 62.05 & 0.0\end{array}$

$\begin{array}{llllll}\text { cylinder } 2 & 1 & 0.6115 & 62.05 & 0.0\end{array}$

$\begin{array}{lllllll}\text { cuboid } \quad 3 & 1 & 4 \mathrm{p} 1.237 & 62.05 & 0.0\end{array}$

unit 52

com $=$ '(Fuel + Clad $+\mathrm{H} 2 \mathrm{O})^{\prime}$

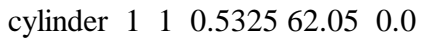

$\begin{array}{llllll}\text { cylinder } 2 & 1 & 0.6115 & 62.05 & 0.0\end{array}$

$\begin{array}{lllllll}\text { cuboid } & 3 & 1 & 4 \mathrm{p} 1.237 & 62.05 & 0.0\end{array}$

unit 53

com='(Fuel+Clad $+\mathrm{H} 2 \mathrm{O})^{\prime}$ 
$\begin{array}{llllll}\text { cylinder } & 1 & 1 & 0.5325 & 62.05 & 0.0\end{array}$

$\begin{array}{llllll}\text { cylinder } & 2 & 1 & 0.6115 & 62.05 & 0.0\end{array}$

$\begin{array}{lllllll}\text { cuboid } & 3 & 1 & 4 \mathrm{p} 1.237 & 62.05 & 0.0\end{array}$

unit 54

com='(Fuel+Clad $+\mathrm{H} 2 \mathrm{O})^{\prime}$

$\begin{array}{llllll}\text { cylinder } & 1 & 1 & 0.5325 & 62.05 & 0.0\end{array}$

$\begin{array}{llllll}\text { cylinder } & 2 & 1 & 0.6115 & 62.05 & 0.0\end{array}$

$\begin{array}{lllllll}\text { cuboid } & 3 & 1 & 4 \mathrm{p} 1.237 & 62.05 & 0.0\end{array}$

unit 55

com='(Fuel+Clad $+\mathrm{H} 2 \mathrm{O})^{\prime}$

$\begin{array}{llllll}\text { cylinder } & 1 & 1 & 0.5325 & 62.05 & 0.0\end{array}$

$\begin{array}{llllll}\text { cylinder } & 2 & 1 & 0.6115 & 62.05 & 0.0\end{array}$

$\begin{array}{lllllll}\text { cuboid } & 3 & 1 & 4 \mathrm{p} 1.237 & 62.05 & 0.0\end{array}$

unit 56

com='(Fuel + Clad $+\mathrm{H} 2 \mathrm{O})^{\prime}$

$\begin{array}{llllll}\text { cylinder } & 1 & 1 & 0.5325 & 62.05 & 0.0\end{array}$

$\begin{array}{llllll}\text { cylinder } & 2 & 1 & 0.6115 & 62.05 & 0.0\end{array}$

$\begin{array}{lllllll}\text { cuboid } & 3 & 1 & 4 \mathrm{p} 1.237 & 62.05 & 0.0\end{array}$

unit 57

com='(Fuel+Clad $+\mathrm{H} 2 \mathrm{O})^{\prime}$

$\begin{array}{llllll}\text { cylinder } & 1 & 1 & 0.5325 & 62.05 & 0.0\end{array}$

$\begin{array}{llllll}\text { cylinder } & 2 & 1 & 0.6115 & 62.05 & 0.0\end{array}$

$\begin{array}{lllllll}\text { cuboid } & 3 & 1 & 4 \mathrm{p} 1.237 & 62.05 & 0.0\end{array}$

unit 58

com $=$ '(Fuel+Clad $+\mathrm{H} 2 \mathrm{O})^{\prime}$

$\begin{array}{lllllll}\text { cylinder } & 1 & 1 & 0.5325 & 62.05 & 0.0\end{array}$

$\begin{array}{llllll}\text { cylinder } 2 & 1 & 0.6115 & 62.05 & 0.0\end{array}$

$\begin{array}{lllllll}\text { cuboid } & 3 & 1 & 4 \mathrm{p} 1.237 & 62.05 & 0.0\end{array}$

unit 59

com='(Fuel+Clad $+\mathrm{H} 2 \mathrm{O})^{\prime}$

$\begin{array}{llllll}\text { cylinder } & 1 & 1 & 0.5325 & 62.05 & 0.0\end{array}$

$\begin{array}{llllll}\text { cylinder } 2 & 1 & 0.6115 & 62.05 & 0.0\end{array}$

$\begin{array}{lllllll}\text { cuboid } & 3 & 1 & 4 \mathrm{p} 1.237 & 62.05 & 0.0\end{array}$

unit 60

com='(Fuel+Clad $+\mathrm{H} 2 \mathrm{O})^{\prime}$

$\begin{array}{llllll}\text { cylinder } & 1 & 1 & 0.5325 & 62.05 & 0.0\end{array}$

$\begin{array}{llllll}\text { cylinder } & 2 & 1 & 0.6115 & 62.05 & 0.0\end{array}$

$\begin{array}{lllllll}\text { cuboid } & 3 & 1 & 4 \mathrm{p} 1.237 & 62.05 & 0.0\end{array}$

unit 61

com='(Fuel+Clad $+\mathrm{H} 2 \mathrm{O})^{\prime}$

$\begin{array}{llllll}\text { cylinder } & 1 & 1 & 0.5325 & 62.05 & 0.0\end{array}$

$\begin{array}{llllll}\text { cylinder } & 2 & 1 & 0.6115 & 62.05 & 0.0\end{array}$

$\begin{array}{lllllll}\text { cuboid } & 3 & 1 & 4 \mathrm{p} 1.237 & 62.05 & 0.0\end{array}$

unit 62

com='(Fuel+Clad $+\mathrm{H} 2 \mathrm{O})^{\prime}$

cylinder $\begin{array}{lllll}1 & 1 & 0.5325 & 62.05 & 0.0\end{array}$

$\begin{array}{llllll}\text { cylinder } 2 & 1 & 0.6115 & 62.05 & 0.0\end{array}$

$\begin{array}{lllllll}\text { cuboid } & 3 & 1 & 4 \mathrm{p} 1.237 & 62.05 & 0.0\end{array}$

unit 63

com $=^{\prime}(\text { Fuel }+\mathrm{Clad}+\mathrm{H} 2 \mathrm{O})^{\prime}$

$\begin{array}{llllll}\text { cylinder } & 1 & 1 & 0.5325 & 62.05 & 0.0\end{array}$

$\begin{array}{llllll}\text { cylinder } & 2 & 1 & 0.6115 & 62.05 & 0.0\end{array}$

$\begin{array}{lllllll}\text { cuboid } & 3 & 1 & 4 \mathrm{p} 1.237 & 62.05 & 0.0\end{array}$

unit 64

com $='(\text { Fuel }+ \text { Clad }+\mathrm{H} 2 \mathrm{O})^{\prime}$

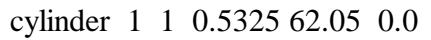

$\begin{array}{llllll}\text { cylinder } 2 & 1 & 0.6115 & 62.05 & 0.0\end{array}$ $\begin{array}{lllllll}\text { cuboid } \quad 3 & 1 & 4 \mathrm{p} 1.237 & 62.05 & 0.0\end{array}$

unit 65

com $=$ '(Fuel + Clad $+\mathrm{H} 2 \mathrm{O})^{\prime}$

$\begin{array}{lllllll}\text { cylinder } & 1 & 1 & 0.5325 & 62.05 & 0.0\end{array}$

$\begin{array}{llllll}\text { cylinder } 2 & 1 & 0.6115 & 62.05 & 0.0\end{array}$

$\begin{array}{lllllll}\text { cuboid } & 3 & 1 & 4 \mathrm{p} 1.237 & 62.05 & 0.0\end{array}$

unit 66

com='(Fuel+Clad $+\mathrm{H} 2 \mathrm{O})^{\prime}$

cylinder $\begin{array}{llllll}1 & 1 & 0.5325 & 62.05 & 0.0\end{array}$

$\begin{array}{llllll}\text { cylinder } 2 & 1 & 0.6115 & 62.05 & 0.0\end{array}$

$\begin{array}{lllllll}\text { cuboid } & 3 & 1 & 4 \mathrm{p} 1.237 & 62.05 & 0.0\end{array}$

unit 81

com='h2o and end plug below Al grid plate - fill array 1'

$\begin{array}{llllll}\text { cylinder } 4 & 1 & 0.6115 & 4.445 & 0.0\end{array}$

$\begin{array}{lllllll}\text { cuboid } & 3 & 1 & 4 \mathrm{p} 1.237 & 4.445 & 0.0\end{array}$

unit 82

com='h2o and end plug above $\mathrm{Al}$ grid plate - fill array 2'

$\begin{array}{lllll}\text { cylinder } 4 & 1 & 0.6115 & 11.784 & 0.0\end{array}$

$\begin{array}{lllllll}\text { cuboid } & 3 & 1 & 4 \mathrm{p} 1.237 & 11.784 & 0.0\end{array}$

unit 83

com='(Fuel+Clad+Void $)$ - fill array 3'

$\begin{array}{llllll}\text { cylinder } & 1 & 1 & .5325 & 8.55 & 0.0\end{array}$

$\begin{array}{lllll}\text { cylinder } 2 & 1 & 6115 & 8.55 & 0.0\end{array}$

cuboid $\quad 0114 \mathrm{p} 1.2378 .55 \quad 0.0$

unit 84

com='(void+Clad+void $)^{\prime}$

$\begin{array}{llllll}\text { cylinder } & 0 & 1 & 0.5325 & 9.97 & 0.0\end{array}$

$\begin{array}{lllll}\text { cylinder } 2 & 1 & 0.6115 & 9.97 & 0.0\end{array}$

$\begin{array}{llllllll}\text { cuboid } & 0 & 1 & 4 \mathrm{p} 1.237 & 9.97 & 0.0\end{array}$

unit 100

com='Lower $\mathrm{Al}$ grid plate'

cuboid 414 p55.977.601 0.0

unit 101

com='array of h2o and end plugs below Al grid plate'

$\begin{array}{lllll}\text { array } & 1 & -25.977 & -25.977 & 0.0\end{array}$

$\begin{array}{lllllll}\text { cuboid } & 3 & 1 & 4 \mathrm{p} 55.977 & 4.445 & 0.0\end{array}$

unit 102

com='array of h2o and end plugs above $\mathrm{Al}$ grid plate '

$\begin{array}{llll}\text { array } 2 & -25.977 & -25.977 & 0.0\end{array}$

$\begin{array}{lllllll}\text { cuboid } & 3 & 1 & 4 p 55.977 & 11.784 & 0.0\end{array}$

unit 103

com='array of Fuel+Clad+Void'

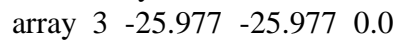

$\begin{array}{lllllll}\text { cuboid } & 0 & 1 & 4 \mathrm{p} 55.977 & 8.55 & 0.0\end{array}$

unit 104

com='array of void+Clad+void'

$\begin{array}{llll}\text { array } 4 & -25.977 & -25.977 & 0.0\end{array}$

$\begin{array}{lllllll}\text { cuboid } & 0 & 1 & 4 \mathrm{p} 55.977 & 9.97 & 0.0\end{array}$

unit 105

com='array of Fuel+Clad+H2O'

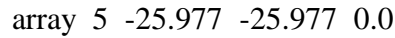

cuboid $\begin{array}{llllll}3 & 1 & 4 p 55.977 & 62.05 & 0.0\end{array}$

global unit 110

com='array of arrays of fuel element sections + reflector' $\begin{array}{lllll}\text { array } 6 & -55.977 & -55.977 & 0.0\end{array}$

$\begin{array}{lllllll}\text { cuboid } & 4 & 1 & 4 p 55.977 & 97.40 & -1.27\end{array}$

cuboid $\begin{array}{llllll}5 & 1 & 4 p 55.977 & 97.40 & -3.47\end{array}$ 
cuboid $3 \begin{array}{lllll} & 1 & 4 p 55.977 & 97.40 & -17.27\end{array}$

$\begin{array}{lllllll}\text { cuboid } & 5 & 1 & 4 p 55.977 & 97.40 & -17.77\end{array}$

$\begin{array}{lllllll}\text { cuboid } & 6 & 1 & 4 p 55.977 & 97.40 & -54.77\end{array}$

end geometry

read array

ara=1 nux=21 nuy=21 nuz=1

fill $\mathrm{f} 81$ end fill

ara=2 nux=21 nuy=21 nuz=1

fill $\mathrm{f} 82$ end fill

ara=3 nux=21 nuy=21 nuz=1

fill $\mathrm{f} 83$ end fill

ara $=4$ nux=21 nuy=21 nuz=1

fill $\mathrm{f} 84$ end fill

ara=5 nux=21 nuy=21 nuz=1

fill

$\begin{array}{lllllllllllll}1 & 2 & 3 & 4 & 5 & 6 & 7 & 8 & 9 & 10 & 11 & 1 \mathrm{~b} & 10\end{array}$

$212131415161718192021 \quad 1 b 10$

313222324252627282930 1b 10

414233132333435363738 1b 10

515243239404142434445 1b 10

616253340464748495051 lb 10

717263441475253545556 1b 10

818273542485357585960 1b 10

$919283643495458616263 \quad 1 b 10$

1020293744505559626465 1b 10

1121303845515660636566 1b 220

end fill

ara $=6$ nux $=1$ nuy $=1$ nuz $=6$

fill 101100102105103104 end fill

end array

end data

end

$=$ shell

cp ft04f001 \$RTNDIR/tca10.xs04

cp ft64f001 \$RTNDIR/tca10.rs64

end

$=\mathrm{kmart}$

read initial kunit $=64$

xunit=4

actbygrp

rrpvol

end initial

read activity

92234279223418922341452

$9223527922351892235 \quad 1452$

$9223827922381892238 \quad 1452$

$9423827942381894238 \quad 1452$

94239279423918942391452

$9424027942401894240 \quad 1452$

$9424127942411894241 \quad 1452$

$9424227942421894242 \quad 1452$

95241279524118952411452

501027

501127

801627

4000027

2400027
2600027

100127

5011227

5011427

5011527

5011627

5011727

5011827

5011927

5012027

5012227

5012427

end activity

read collapse

factor $=1.0$

lastg $=57$

lastg $=148$

lastg $=204$

lastg $=238$

end collapse

end

$=\operatorname{csasc} 5 \quad$ parm $=$ size $=1000000$

TCA $\quad * * * * *$ case $11 * * * * * * * * *$

238group latticecell

'MOX fuel

u-234 $1007.1749-7293$ end

u-235 $1100.3926-5293$ end

u-238 $11001.2951-2293$ end

pu-238 $1002.0003-6293$ end

pu-239 100 2.7491-4 293 end

pu-240 10 8.8417-5 293 end

'*********** pu-241 varies $* * * * * * * * * * * * * * * * * *$

pu-241 100 2.6617-5 293 end

pu-242 10 8.1234-6 293 end

'*****am-241 varies*********

am-241 $1002.6656-6293$ end

o $\quad \begin{array}{llll}1 & 0 & 2.7837-2 & 293\end{array}$ end

b-10 $100.0418-8293$ end

b-11 $1102.4319-7293$ end

'clad (ZR-2)

zr $\quad 2 \quad 0 \quad 3.7772-2 \quad 293$ end

sn $\quad 2 \quad 0 \quad 4.3737-4293$ end

cr $\quad 2 \quad 0 \quad 8.8570-5293$ end

fe $\quad \begin{array}{llll}2 & 0 & 6.6119-5 & 293\end{array}$ end

o 20 3.5864-5 293 end

'water(Refletor)

h $3 \quad 0 \quad 6.6735-2293$ end

o 30 3.3368-2 293 end

'Al

Al 40 6.0224-2 293 end

'Stainless Steel

c 50 1.1928-4 293 end

si $5001.7003-3293$ end

mn $\begin{array}{lllll}5 & 0 & 1.7385-3 & 293 & \text { end }\end{array}$

p $\quad \begin{array}{lllll}5 & 0 & 6.9381-5 & 293 & \text { end }\end{array}$ 


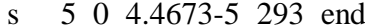

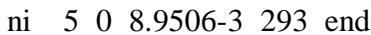

cr $5 \quad 0 \quad 1.7450-2293$ end

fe $500.7202-2293$ end 'ordinary concrete

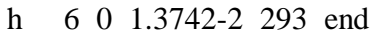

o $6 \begin{array}{llll}6 & 0 & 4.5919-2 & 293 \\ & \text { end }\end{array}$

c 60 1.1532-4 293 end

na $\begin{array}{llll}6 & 0 & 9.6395-4 & 293 \\ \text { end }\end{array}$

mg $6 \begin{array}{llll}6 & 1.2388-4 & 293 & \text { end }\end{array}$

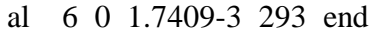

si $6 \begin{array}{llll}6 & 0 & 1.6617-2 & 293 \\ & \text { end }\end{array}$

k $6 \begin{array}{llll}6 & 0 & 4.6052-4 & 293\end{array}$ end

ca $\begin{array}{lllll}6 & 0 & 1.5025-3 & 293 & \text { end }\end{array}$

fe $\begin{array}{lllll}6 & 0 & 3.4492-4 & 293 & \text { end }\end{array}$

end comp

squarepitch $2.474 \quad 1.065121 .223 \quad 3$ end

more data

limcen $=17000000$

end more

tca-11

read param

gen=225 nsk=25 npg=20000 fdn=yes flx=yes wrs=64

res $=225$

lng $=2000000$

end param

read geometry

unit 1

com $=$ '(Fuel+Clad $+\mathrm{H} 2 \mathrm{O})^{\prime}$

cylinder $\begin{array}{lllll}1 & 1 & 0.5325 & 64.53 & 0.0\end{array}$

$\begin{array}{llllll}\text { cylinder } 2 & 1 & 0.6115 & 64.53 & 0.0\end{array}$

$\begin{array}{lllllll}\text { cuboid } \quad 3 & 1 & 4 \mathrm{p} 1.237 & 64.53 & 0.0\end{array}$

unit 2

com $=$ '(Fuel+Clad $+\mathrm{H} 2 \mathrm{O})^{\prime}$

cylinder $\begin{array}{lllll}1 & 1 & 0.5325 & 64.53 & 0.0\end{array}$

$\begin{array}{llllll}\text { cylinder } & 2 & 1 & 0.6115 & 64.53 & 0.0\end{array}$

$\begin{array}{lllllll}\text { cuboid } & 3 & 1 & 4 \mathrm{p} 1.237 & 64.53 & 0.0\end{array}$

unit 3

com='(Fuel+Clad $+\mathrm{H} 2 \mathrm{O})^{\prime}$

cylinder $\begin{array}{lllll}1 & 1 & 0.5325 & 64.53 & 0.0\end{array}$

$\begin{array}{lllllll}\text { cylinder } & 2 & 1 & 0.6115 & 64.53 & 0.0\end{array}$

$\begin{array}{llllllll}\text { cuboid } & 3 & 1 & 4 \mathrm{p} 1.237 & 64.53 & 0.0\end{array}$

unit 4

com $=$ '(Fuel $+\mathrm{Clad}+\mathrm{H} 2 \mathrm{O})^{\prime}$

$\begin{array}{llllll}\text { cylinder } & 1 & 1 & 0.5325 & 64.53 & 0.0\end{array}$

$\begin{array}{llllll}\text { cylinder } 2 & 1 & 0.6115 & 64.53 & 0.0\end{array}$

$\begin{array}{lllllll}\text { cuboid } \quad 3 & 1 & 4 \mathrm{p} 1.237 & 64.53 & 0.0\end{array}$

unit 5

com $=$ '(Fuel+Clad $+\mathrm{H} 2 \mathrm{O})^{\prime}$

cylinder $\begin{array}{lllll}1 & 1 & 0.5325 & 64.53 & 0.0\end{array}$

$\begin{array}{llllll}\text { cylinder } 2 & 1 & 0.6115 & 64.53 & 0.0\end{array}$

$\begin{array}{lllllll}\text { cuboid } & 3 & 1 & 4 \mathrm{p} 1.237 & 64.53 & 0.0\end{array}$

unit 6

com='(Fuel+Clad $+\mathrm{H} 2 \mathrm{O})^{\prime}$

cylinder $1110.532564 .53 \quad 0.0$

$\begin{array}{llllll}\text { cylinder } 2 & 1 & 0.6115 & 64.53 & 0.0\end{array}$

$\begin{array}{lllllll}\text { cuboid } \quad 3 & 1 & 4 \mathrm{p} 1.237 & 64.53 & 0.0\end{array}$ unit 7

com $=$ '(Fuel + Clad $+\mathrm{H} 2 \mathrm{O})^{\prime}$

cylinder $\begin{array}{lllll}1 & 1 & 0.5325 & 64.53 & 0.0\end{array}$

$\begin{array}{llllll}\text { cylinder } 2 & 1 & 0.6115 & 64.53 & 0.0\end{array}$

$\begin{array}{lllllll}\text { cuboid } & 3 & 1 & 4 \mathrm{p} 1.237 & 64.53 & 0.0\end{array}$

unit 8

com $=(\text { Fuel }+\mathrm{Clad}+\mathrm{H} 2 \mathrm{O})^{\prime}$

$\begin{array}{lllllll}\text { cylinder } & 1 & 1 & 0.5325 & 64.53 & 0.0\end{array}$

$\begin{array}{llllll}\text { cylinder } 2 & 1 & 0.6115 & 64.53 & 0.0\end{array}$

$\begin{array}{lllllll}\text { cuboid } & 3 & 1 & 4 \mathrm{p} 1.237 & 64.53 & 0.0\end{array}$

unit 9

com='(Fuel+Clad $+\mathrm{H} 2 \mathrm{O})^{\prime}$

$\begin{array}{llllll}\text { cylinder } & 1 & 1 & 0.5325 & 64.53 & 0.0\end{array}$

$\begin{array}{llllll}\text { cylinder } & 2 & 1 & 0.6115 & 64.53 & 0.0\end{array}$

$\begin{array}{lllllll}\text { cuboid } & 3 & 1 & 4 \mathrm{p} 1.237 & 64.53 & 0.0\end{array}$

unit 10

com='(Fuel+Clad $+\mathrm{H} 2 \mathrm{O})^{\prime}$

$\begin{array}{llllll}\text { cylinder } & 1 & 1 & 0.5325 & 64.53 & 0.0\end{array}$

$\begin{array}{llllll}\text { cylinder } & 2 & 1 & 0.6115 & 64.53 & 0.0\end{array}$

$\begin{array}{lllllll}\text { cuboid } & 3 & 1 & 4 \mathrm{p} 1.237 & 64.53 & 0.0\end{array}$

unit 11

com $=$ '(Fuel + Clad $+\mathrm{H} 2 \mathrm{O})^{\prime}$

$\begin{array}{llllll}\text { cylinder } & 1 & 1 & 0.5325 & 64.53 & 0.0\end{array}$

$\begin{array}{llllll}\text { cylinder } 2 & 1 & 0.6115 & 64.53 & 0.0\end{array}$

$\begin{array}{lllllll}\text { cuboid } & 3 & 1 & 4 \mathrm{p} 1.237 & 64.53 & 0.0\end{array}$

unit 12

com $=$ '(Fuel + Clad $+\mathrm{H} 2 \mathrm{O})^{\prime}$

cylinder $\begin{array}{lllll}1 & 1 & 0.5325 & 64.53 & 0.0\end{array}$

$\begin{array}{llllll}\text { cylinder } & 2 & 1 & 0.6115 & 64.53 & 0.0\end{array}$

$\begin{array}{lllllll}\text { cuboid } & 3 & 1 & 4 \mathrm{p} 1.237 & 64.53 & 0.0\end{array}$

unit 13

com='(Fuel+Clad $+\mathrm{H} 2 \mathrm{O})^{\prime}$

$\begin{array}{llllll}\text { cylinder } & 1 & 1 & 0.5325 & 64.53 & 0.0\end{array}$

$\begin{array}{llllll}\text { cylinder } 2 & 1 & 0.6115 & 64.53 & 0.0\end{array}$

$\begin{array}{lllllll}\text { cuboid } & 3 & 1 & 4 \mathrm{p} 1.237 & 64.53 & 0.0\end{array}$

unit 14

com $=(\text { Fuel }+ \text { Clad }+\mathrm{H} 2 \mathrm{O})^{\prime}$

$\begin{array}{llllll}\text { cylinder } & 1 & 1 & 0.5325 & 64.53 & 0.0\end{array}$

$\begin{array}{llllll}\text { cylinder } 2 & 1 & 0.6115 & 64.53 & 0.0\end{array}$

$\begin{array}{lllllll}\text { cuboid } 3 & 1 & 4 \mathrm{p} 1.237 & 64.53 & 0.0\end{array}$

unit 15

com $=$ '(Fuel $+\mathrm{Clad}+\mathrm{H} 2 \mathrm{O})^{\prime}$

cylinder $\begin{array}{lllll}1 & 1 & 0.5325 & 64.53 & 0.0\end{array}$

$\begin{array}{llllll}\text { cylinder } 2 & 1 & 0.6115 & 64.53 & 0.0\end{array}$

$\begin{array}{lllllll}\text { cuboid } & 3 & 1 & 4 \mathrm{p} 1.237 & 64.53 & 0.0\end{array}$

unit 16

com='(Fuel+Clad $+\mathrm{H} 2 \mathrm{O})^{\prime}$

cylinder $\begin{array}{lllll}1 & 1 & 0.5325 & 64.53 & 0.0\end{array}$

$\begin{array}{llllll}\text { cylinder } 2 & 1 & 0.6115 & 64.53 & 0.0\end{array}$

$\begin{array}{lllllll}\text { cuboid } 3 & 1 & 4 \mathrm{p} 1.237 & 64.53 & 0.0\end{array}$

unit 17

com='(Fuel+Clad $+\mathrm{H} 2 \mathrm{O})^{\prime}$

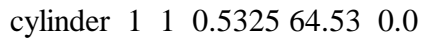

$\begin{array}{llllll}\text { cylinder } & 2 & 1 & 0.6115 & 64.53 & 0.0\end{array}$

$\begin{array}{lllllll}\text { cuboid } & 3 & 1 & 4 \mathrm{p} 1.237 & 64.53 & 0.0\end{array}$

unit 18

com='(Fuel+Clad $+\mathrm{H} 2 \mathrm{O})^{\prime}$ 
$\begin{array}{llllll}\text { cylinder } & 1 & 1 & 0.5325 & 64.53 & 0.0\end{array}$

$\begin{array}{llllll}\text { cylinder } & 2 & 1 & 0.6115 & 64.53 & 0.0\end{array}$

$\begin{array}{lllllll}\text { cuboid } 3 & 1 & 4 \mathrm{p} 1.237 & 64.53 & 0.0\end{array}$

unit 19

com='(Fuel+Clad $+\mathrm{H} 2 \mathrm{O})^{\prime}$

$\begin{array}{llllll}\text { cylinder } & 1 & 1 & 0.5325 & 64.53 & 0.0\end{array}$

$\begin{array}{llllll}\text { cylinder } 2 & 1 & 0.6115 & 64.53 & 0.0\end{array}$

$\begin{array}{lllllll}\text { cuboid } & 3 & 1 & 4 \mathrm{p} 1.237 & 64.53 & 0.0\end{array}$

unit 20

com $=$ '(Fuel + Clad $+\mathrm{H} 2 \mathrm{O})^{\prime}$

$\begin{array}{llllll}\text { cylinder } & 1 & 1 & 0.5325 & 64.53 & 0.0\end{array}$

$\begin{array}{llllll}\text { cylinder } & 2 & 1 & 0.6115 & 64.53 & 0.0\end{array}$

$\begin{array}{lllllll}\text { cuboid } & 3 & 1 & 4 \mathrm{p} 1.237 & 64.53 & 0.0\end{array}$

unit 21

com $=$ '(Fuel $+\mathrm{Clad}+\mathrm{H} 2 \mathrm{O})^{\prime}$

$\begin{array}{llllll}\text { cylinder } & 1 & 1 & 0.5325 & 64.53 & 0.0\end{array}$

$\begin{array}{lllllll}\text { cylinder } 2 & 1 & 0.6115 & 64.53 & 0.0\end{array}$

$\begin{array}{lllllll}\text { cuboid } 3 & 1 & 4 \mathrm{p} 1.237 & 64.53 & 0.0\end{array}$

unit 22

com='(Fuel+Clad $+\mathrm{H} 2 \mathrm{O})^{\prime}$

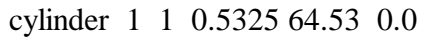

$\begin{array}{llllll}\text { cylinder } 2 & 1 & 0.6115 & 64.53 & 0.0\end{array}$

$\begin{array}{lllllll}\text { cuboid } & 3 & 1 & 4 \mathrm{p} 1.237 & 64.53 & 0.0\end{array}$

unit 23

com $=$ '(Fuel $+\mathrm{Clad}+\mathrm{H} 2 \mathrm{O})^{\prime}$

$\begin{array}{lllllll}\text { cylinder } & 1 & 1 & 0.5325 & 64.53 & 0.0\end{array}$

$\begin{array}{llllll}\text { cylinder } 2 & 1 & 0.6115 & 64.53 & 0.0\end{array}$

$\begin{array}{lllllll}\text { cuboid } & 3 & 1 & 4 \mathrm{p} 1.237 & 64.53 & 0.0\end{array}$

unit 24

com='(Fuel+Clad $+\mathrm{H} 2 \mathrm{O})^{\prime}$

$\begin{array}{llllll}\text { cylinder } & 1 & 1 & 0.5325 & 64.53 & 0.0\end{array}$

$\begin{array}{llllll}\text { cylinder } & 2 & 1 & 0.6115 & 64.53 & 0.0\end{array}$

$\begin{array}{lllllll}\text { cuboid } & 3 & 1 & 4 \mathrm{p} 1.237 & 64.53 & 0.0\end{array}$

unit 25

com='(Fuel+Clad $+\mathrm{H} 2 \mathrm{O})^{\prime}$

$\begin{array}{lllll}\text { cylinder } 1 & 1 & 0.5325 & 64.53 & 0.0\end{array}$

$\begin{array}{llllll}\text { cylinder } & 2 & 1 & 0.6115 & 64.53 & 0.0\end{array}$

$\begin{array}{lllllll}\text { cuboid } & 3 & 1 & 4 \mathrm{p} 1.237 & 64.53 & 0.0\end{array}$

unit 26

com='(Fuel + Clad $+\mathrm{H} 2 \mathrm{O})^{\prime}$

$\begin{array}{llllll}\text { cylinder } & 1 & 1 & 0.5325 & 64.53 & 0.0\end{array}$

$\begin{array}{llllll}\text { cylinder } & 2 & 1 & 0.6115 & 64.53 & 0.0\end{array}$

$\begin{array}{lllllll}\text { cuboid } & 3 & 1 & 4 \mathrm{p} 1.237 & 64.53 & 0.0\end{array}$

unit 27

com $=$ '(Fuel + Clad $+\mathrm{H} 2 \mathrm{O})^{\prime}$

cylinder $\begin{array}{lllll}1 & 1 & 0.5325 & 64.53 & 0.0\end{array}$

$\begin{array}{llllll}\text { cylinder } & 2 & 1 & 0.6115 & 64.53 & 0.0\end{array}$

$\begin{array}{lllllll}\text { cuboid } & 3 & 1 & 4 \mathrm{p} 1.237 & 64.53 & 0.0\end{array}$

unit 28

com $=^{\prime}(\text { Fuel }+\mathrm{Clad}+\mathrm{H} 2 \mathrm{O})^{\prime}$

$\begin{array}{llllll}\text { cylinder } & 1 & 1 & 0.5325 & 64.53 & 0.0\end{array}$

$\begin{array}{lllllll}\text { cylinder } & 2 & 1 & 0.6115 & 64.53 & 0.0\end{array}$

$\begin{array}{lllllll}\text { cuboid } & 3 & 1 & 4 \mathrm{p} 1.237 & 64.53 & 0.0\end{array}$

unit 29

com $=$ '(Fuel+Clad $+\mathrm{H} 2 \mathrm{O})^{\prime}$

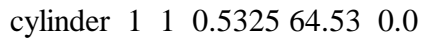

$\begin{array}{llllll}\text { cylinder } 2 & 1 & 0.6115 & 64.53 & 0.0\end{array}$ $\begin{array}{lllllll}\text { cuboid } \quad 3 & 1 & 4 \mathrm{p} 1.237 & 64.53 & 0.0\end{array}$

unit 30

com $=$ '(Fuel + Clad $+\mathrm{H} 2 \mathrm{O})^{\prime}$

$\begin{array}{llllll}\text { cylinder } & 1 & 1 & 0.5325 & 64.53 & 0.0\end{array}$

$\begin{array}{llllll}\text { cylinder } 2 & 1 & 0.6115 & 64.53 & 0.0\end{array}$

$\begin{array}{lllllll}\text { cuboid } & 3 & 1 & 4 \mathrm{p} 1.237 & 64.53 & 0.0\end{array}$

unit 31

com='(Fuel $+\mathrm{Clad}+\mathrm{H} 2 \mathrm{O})^{\prime}$

$\begin{array}{llllll}\text { cylinder } & 1 & 1 & 0.5325 & 64.53 & 0.0\end{array}$

$\begin{array}{llllll}\text { cylinder } 2 & 1 & 0.6115 & 64.53 & 0.0\end{array}$

$\begin{array}{lllllll}\text { cuboid } & 3 & 1 & 4 \mathrm{p} 1.237 & 64.53 & 0.0\end{array}$

unit 32

com $=$ '(Fuel + Clad $+\mathrm{H} 2 \mathrm{O})^{\prime}$

$\begin{array}{llllll}\text { cylinder } & 1 & 1 & 0.5325 & 64.53 & 0.0\end{array}$

$\begin{array}{lllllll}\text { cylinder } & 2 & 1 & 0.6115 & 64.53 & 0.0\end{array}$

$\begin{array}{lllllll}\text { cuboid } 3 & 1 & 4 \mathrm{p} 1.237 & 64.53 & 0.0\end{array}$

unit 33

com $=$ '(Fuel+Clad $+\mathrm{H} 2 \mathrm{O})^{\prime}$

$\begin{array}{llllll}\text { cylinder } & 1 & 1 & 0.5325 & 64.53 & 0.0\end{array}$

$\begin{array}{llllll}\text { cylinder } & 2 & 1 & 0.6115 & 64.53 & 0.0\end{array}$

$\begin{array}{lllllll}\text { cuboid } 3 & 1 & 4 \mathrm{p} 1.237 & 64.53 & 0.0\end{array}$

unit 34

com $=$ '(Fuel+Clad $+\mathrm{H} 2 \mathrm{O})^{\prime}$

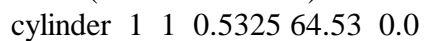

$\begin{array}{lllllll}\text { cylinder } 2 & 1 & 0.6115 & 64.53 & 0.0\end{array}$

$\begin{array}{lllllll}\text { cuboid } & 3 & 1 & 4 \mathrm{p} 1.237 & 64.53 & 0.0\end{array}$

unit 35

com $=$ '(Fuel + Clad $+\mathrm{H} 2 \mathrm{O})^{\prime}$

cylinder $\begin{array}{lllll}1 & 1 & 0.5325 & 64.53 & 0.0\end{array}$

$\begin{array}{llllll}\text { cylinder } & 2 & 1 & 0.6115 & 64.53 & 0.0\end{array}$

$\begin{array}{lllllll}\text { cuboid } & 3 & 1 & 4 \mathrm{p} 1.237 & 64.53 & 0.0\end{array}$

unit 36

com $=$ '(Fuel + Clad $+\mathrm{H} 2 \mathrm{O})^{\prime}$

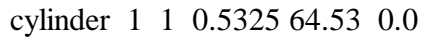

$\begin{array}{llllll}\text { cylinder } 2 & 1 & 0.6115 & 64.53 & 0.0\end{array}$

$\begin{array}{lllllll}\text { cuboid } 3 & 1 & 4 \mathrm{p} 1.237 & 64.53 & 0.0\end{array}$

unit 37

com $=$ '(Fuel $+\mathrm{Clad}+\mathrm{H} 2 \mathrm{O})^{\prime}$

cylinder $\begin{array}{lllll}1 & 1 & 0.5325 & 64.53 & 0.0\end{array}$

$\begin{array}{lllllll}\text { cylinder } 2 & 1 & 0.6115 & 64.53 & 0.0\end{array}$

$\begin{array}{lllllll}\text { cuboid } & 3 & 1 & 4 \mathrm{p} 1.237 & 64.53 & 0.0\end{array}$

unit 38

com $=^{\prime}(\text { Fuel }+ \text { Clad }+\mathrm{H} 2 \mathrm{O})^{\prime}$

cylinder $\begin{array}{lllll}1 & 1 & 0.5325 & 64.53 & 0.0\end{array}$

$\begin{array}{llllll}\text { cylinder } 2 & 1 & 0.6115 & 64.53 & 0.0\end{array}$

$\begin{array}{lllllll}\text { cuboid } & 3 & 1 & 4 \mathrm{p} 1.237 & 64.53 & 0.0\end{array}$

unit 39

com $=$ '(Fuel + Clad $+\mathrm{H} 2 \mathrm{O})^{\prime}$

$\begin{array}{llllll}\text { cylinder } & 1 & 1 & 0.5325 & 64.53 & 0.0\end{array}$

$\begin{array}{llllll}\text { cylinder } & 2 & 1 & 0.6115 & 64.53 & 0.0\end{array}$

$\begin{array}{lllllll}\text { cuboid } & 3 & 1 & 4 \mathrm{p} 1.237 & 64.53 & 0.0\end{array}$

unit 40

com $=$ '(Fuel + Clad $+\mathrm{H} 2 \mathrm{O})^{\prime}$

cylinder $\begin{array}{lllll}1 & 1 & 0.5325 & 64.53 & 0.0\end{array}$

$\begin{array}{llllll}\text { cylinder } 2 & 1 & 0.6115 & 64.53 & 0.0\end{array}$

$\begin{array}{lllllll}\text { cuboid } & 3 & 1 & 4 \mathrm{p} 1.237 & 64.53 & 0.0\end{array}$

unit 41 
com='(Fuel+Clad+H2O)'

$\begin{array}{lllll}\text { cylinder } 1 & 1 & 0.5325 & 64.53 & 0.0\end{array}$

$\begin{array}{llllll}\text { cylinder } 2 & 1 & 0.6115 & 64.53 & 0.0\end{array}$

$\begin{array}{lllllll}\text { cuboid } & 3 & 1 & 4 \mathrm{p} 1.237 & 64.53 & 0.0\end{array}$

unit 42

com='(Fuel+Clad $+\mathrm{H} 2 \mathrm{O})^{\prime}$

$\begin{array}{llllll}\text { cylinder } & 1 & 1 & 0.5325 & 64.53 & 0.0\end{array}$

$\begin{array}{llllll}\text { cylinder } & 2 & 1 & 0.6115 & 64.53 & 0.0\end{array}$

$\begin{array}{lllllll}\text { cuboid } 3 & 1 & 4 \mathrm{p} 1.237 & 64.53 & 0.0\end{array}$

unit 43

com $='(\text { Fuel }+\mathrm{Clad}+\mathrm{H} 2 \mathrm{O})^{\prime}$

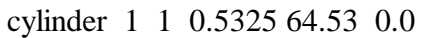

$\begin{array}{llllll}\text { cylinder } & 2 & 1 & 0.6115 & 64.53 & 0.0\end{array}$

$\begin{array}{lllllll}\text { cuboid } & 3 & 1 & 4 \mathrm{p} 1.237 & 64.53 & 0.0\end{array}$

unit 44

com $=$ '(Fuel + Clad $+\mathrm{H} 2 \mathrm{O})^{\prime}$

$\begin{array}{llllll}\text { cylinder } & 1 & 1 & 0.5325 & 64.53 & 0.0\end{array}$

$\begin{array}{llllll}\text { cylinder } & 2 & 1 & 0.6115 & 64.53 & 0.0\end{array}$

$\begin{array}{lllllll}\text { cuboid } & 3 & 1 & 4 \mathrm{p} 1.237 & 64.53 & 0.0\end{array}$

unit 45

com='(Fuel+Clad $+\mathrm{H} 2 \mathrm{O})^{\prime}$

$\begin{array}{llllll}\text { cylinder } & 1 & 1 & 0.5325 & 64.53 & 0.0\end{array}$

$\begin{array}{llllll}\text { cylinder } & 2 & 1 & 0.6115 & 64.53 & 0.0\end{array}$

$\begin{array}{lllllll}\text { cuboid } & 3 & 1 & 4 \mathrm{p} 1.237 & 64.53 & 0.0\end{array}$

unit 46

com='(Fuel $+\mathrm{Clad}+\mathrm{H} 2 \mathrm{O})^{\prime}$

cylinder $1110.532564 .53 \quad 0.0$

$\begin{array}{llllll}\text { cylinder } 2 & 1 & 0.6115 & 64.53 & 0.0\end{array}$

$\begin{array}{lllllll}\text { cuboid } & 3 & 1 & 4 \mathrm{p} 1.237 & 64.53 & 0.0\end{array}$

unit 47

com='(Fuel+Clad $+\mathrm{H} 2 \mathrm{O})^{\prime}$

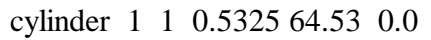

$\begin{array}{llllll}\text { cylinder } 2 & 1 & 0.6115 & 64.53 & 0.0\end{array}$

$\begin{array}{lllllll}\text { cuboid } & 3 & 1 & 4 \mathrm{p} 1.237 & 64.53 & 0.0\end{array}$

unit 48

com $=$ '(Fuel + Clad $+\mathrm{H} 2 \mathrm{O})^{\prime}$

$\begin{array}{lllllll}\text { cylinder } & 1 & 1 & 0.5325 & 64.53 & 0.0\end{array}$

$\begin{array}{llllll}\text { cylinder } & 2 & 1 & 0.6115 & 64.53 & 0.0\end{array}$

$\begin{array}{lllllll}\text { cuboid } & 3 & 1 & 4 \mathrm{p} 1.237 & 64.53 & 0.0\end{array}$

unit 49

com='(Fuel+Clad $+\mathrm{H} 2 \mathrm{O})^{\prime}$

cylinder $\begin{array}{lllll}1 & 1 & 0.5325 & 64.53 & 0.0\end{array}$

$\begin{array}{lllll}\text { cylinder } 2 & 1 & 0.6115 & 64.53 & 0.0\end{array}$

$\begin{array}{lllllll}\text { cuboid } & 3 & 1 & 4 \mathrm{p} 1.237 & 64.53 & 0.0\end{array}$

unit 50

com='(Fuel+Clad $+\mathrm{H} 2 \mathrm{O})^{\prime}$

$\begin{array}{lllll}\text { cylinder } 1 & 1 & 0.5325 & 64.53 & 0.0\end{array}$

$\begin{array}{lllllll}\text { cylinder } & 2 & 1 & 0.6115 & 64.53 & 0.0\end{array}$

$\begin{array}{lllllll}\text { cuboid } 3 & 1 & 4 \mathrm{p} 1.237 & 64.53 & 0.0\end{array}$

unit 51

com='(Fuel+Clad $+\mathrm{H} 2 \mathrm{O})^{\prime}$

$\begin{array}{llllll}\text { cylinder } & 1 & 1 & 0.5325 & 64.53 & 0.0\end{array}$

$\begin{array}{llllll}\text { cylinder } & 2 & 1 & 0.6115 & 64.53 & 0.0\end{array}$

$\begin{array}{lllllll}\text { cuboid } \quad 3 & 1 & 4 \mathrm{p} 1.237 & 64.53 & 0.0\end{array}$

unit 52

com $='(\text { Fuel }+ \text { Clad }+\mathrm{H} 2 \mathrm{O})^{\prime}$

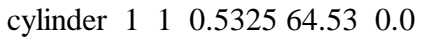

$\begin{array}{llllll}\text { cylinder } & 2 & 1 & 0.6115 & 64.53 & 0.0\end{array}$

$\begin{array}{lllllll}\text { cuboid } & 3 & 1 & 4 \mathrm{p} 1.237 & 64.53 & 0.0\end{array}$

unit 53

com $=$ '(Fuel + Clad $+\mathrm{H} 2 \mathrm{O})^{\prime}$

$\begin{array}{llllll}\text { cylinder } & 1 & 1 & 0.5325 & 64.53 & 0.0\end{array}$

$\begin{array}{lllllll}\text { cylinder } 2 & 1 & 0.6115 & 64.53 & 0.0\end{array}$

$\begin{array}{lllllll}\text { cuboid } & 3 & 1 & 4 \mathrm{p} 1.237 & 64.53 & 0.0\end{array}$

unit 54

com='(Fuel+Clad $+\mathrm{H} 2 \mathrm{O})^{\prime}$

cylinder $\begin{array}{lllll}1 & 1 & 0.5325 & 64.53 & 0.0\end{array}$

$\begin{array}{llllll}\text { cylinder } 2 & 1 & 0.6115 & 64.53 & 0.0\end{array}$

$\begin{array}{lllllll}\text { cuboid } & 3 & 1 & 4 \mathrm{p} 1.237 & 64.53 & 0.0\end{array}$

unit 55

com $='(\text { Fuel }+\mathrm{Clad}+\mathrm{H} 2 \mathrm{O})^{\prime}$

$\begin{array}{llllll}\text { cylinder } & 1 & 1 & 0.5325 & 64.53 & 0.0\end{array}$

$\begin{array}{llllll}\text { cylinder } 2 & 1 & 0.6115 & 64.53 & 0.0\end{array}$

$\begin{array}{lllllll}\text { cuboid } & 3 & 1 & 4 \mathrm{p} 1.237 & 64.53 & 0.0\end{array}$

unit 56

com='(Fuel+Clad $+\mathrm{H} 2 \mathrm{O})^{\prime}$

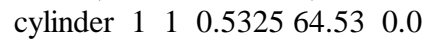

$\begin{array}{llllll}\text { cylinder } 2 & 1 & 0.6115 & 64.53 & 0.0\end{array}$

$\begin{array}{lllllll}\text { cuboid } & 3 & 1 & 4 \mathrm{p} 1.237 & 64.53 & 0.0\end{array}$

unit 57

com $=$ '(Fuel + Clad $+\mathrm{H} 2 \mathrm{O})^{\prime}$

$\begin{array}{llllll}\text { cylinder } & 1 & 1 & 0.5325 & 64.53 & 0.0\end{array}$

$\begin{array}{lllllll}\text { cylinder } & 2 & 1 & 0.6115 & 64.53 & 0.0\end{array}$

$\begin{array}{lllllll}\text { cuboid } & 3 & 1 & 4 \mathrm{p} 1.237 & 64.53 & 0.0\end{array}$

unit 58

com $=$ '(Fuel + Clad $+\mathrm{H} 2 \mathrm{O})^{\prime}$

$\begin{array}{llllll}\text { cylinder } & 1 & 1 & 0.5325 & 64.53 & 0.0\end{array}$

$\begin{array}{llllll}\text { cylinder } & 2 & 1 & 0.6115 & 64.53 & 0.0\end{array}$

$\begin{array}{lllllll}\text { cuboid } & 3 & 1 & 4 \mathrm{p} 1.237 & 64.53 & 0.0\end{array}$

unit 59

com $=$ '(Fuel+Clad $+\mathrm{H} 2 \mathrm{O})^{\prime}$

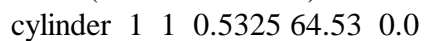

$\begin{array}{llllll}\text { cylinder } 2 & 1 & 0.6115 & 64.53 & 0.0\end{array}$

$\begin{array}{lllllll}\text { cuboid } & 3 & 1 & 4 \mathrm{p} 1.237 & 64.53 & 0.0\end{array}$

unit 60

com $=$ '(Fuel $+\mathrm{Clad}+\mathrm{H} 2 \mathrm{O})^{\prime}$

$\begin{array}{llllll}\text { cylinder } & 1 & 1 & 0.5325 & 64.53 & 0.0\end{array}$

$\begin{array}{lllllll}\text { cylinder } & 2 & 1 & 0.6115 & 64.53 & 0.0\end{array}$

$\begin{array}{lllllll}\text { cuboid } & 3 & 1 & 4 \mathrm{p} 1.237 & 64.53 & 0.0\end{array}$

unit 61

com $=$ '(Fuel + Clad $+\mathrm{H} 2 \mathrm{O})^{\prime}$

$\begin{array}{llllll}\text { cylinder } & 1 & 1 & 0.5325 & 64.53 & 0.0\end{array}$

$\begin{array}{llllll}\text { cylinder } 2 & 1 & 0.6115 & 64.53 & 0.0\end{array}$

$\begin{array}{lllllll}\text { cuboid } & 3 & 1 & 4 \mathrm{p} 1.237 & 64.53 & 0.0\end{array}$

unit 62

com $=$ '(Fuel + Clad $+\mathrm{H} 2 \mathrm{O})^{\prime}$

$\begin{array}{llllll}\text { cylinder } & 1 & 1 & 0.5325 & 64.53 & 0.0\end{array}$

$\begin{array}{llllll}\text { cylinder } & 2 & 1 & 0.6115 & 64.53 & 0.0\end{array}$

$\begin{array}{lllllll}\text { cuboid } & 3 & 1 & 4 \mathrm{p} 1.237 & 64.53 & 0.0\end{array}$

unit 63

com $=$ '(Fuel + Clad $+\mathrm{H} 2 \mathrm{O})^{\prime}$

cylinder $\begin{array}{lllll}1 & 1 & 0.5325 & 64.53 & 0.0\end{array}$

$\begin{array}{llllll}\text { cylinder } & 2 & 1 & 0.6115 & 64.53 & 0.0\end{array}$

$\begin{array}{lllllll}\text { cuboid } \quad 3 & 1 & 4 \mathrm{p} 1.237 & 64.53 & 0.0\end{array}$ 
unit 64

com $=$ '(Fuel + Clad $+\mathrm{H} 2 \mathrm{O})^{\prime}$

cylinder $\begin{array}{lllll}1 & 1 & 0.5325 & 64.53 & 0.0\end{array}$

$\begin{array}{llllll}\text { cylinder } 2 & 1 & 0.6115 & 64.53 & 0.0\end{array}$

$\begin{array}{lllllll}\text { cuboid } 3 & 1 & 4 \mathrm{p} 1.237 & 64.53 & 0.0\end{array}$

unit 65

com $='(\text { Fuel }+ \text { Clad }+\mathrm{H} 2 \mathrm{O})^{\prime}$

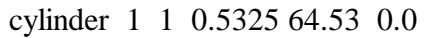

$\begin{array}{llllll}\text { cylinder } & 2 & 1 & 0.6115 & 64.53 & 0.0\end{array}$

$\begin{array}{llllllll}\text { cuboid } & 3 & 1 & 4 \mathrm{p} 1.237 & 64.53 & 0.0\end{array}$

unit 66

com='(Fuel+Clad $+\mathrm{H} 2 \mathrm{O})^{\prime}$

cylinder $\begin{array}{lllll}1 & 1 & 0.5325 & 64.53 & 0.0\end{array}$

$\begin{array}{llllll}\text { cylinder } & 2 & 1 & 0.6115 & 64.53 & 0.0\end{array}$

$\begin{array}{lllllll}\text { cuboid } & 3 & 1 & 4 \mathrm{p} 1.237 & 64.53 & 0.0\end{array}$

unit 81

com='h2o and end plug below Al grid plate - fill array 1'

$\begin{array}{lllll}\text { cylinder } 4 & 1 & 0.6115 & 4.445 & 0.0\end{array}$

$\begin{array}{lllllll}\text { cuboid } & 3 & 1 & 4 \mathrm{p} 1.237 & 4.445 & 0.0\end{array}$

unit 82

com='h2o and end plug above $\mathrm{Al}$ grid plate - fill array 2'

$\begin{array}{lllll}\text { cylinder } 4 & 1 & 0.6115 & 11.784 & 0.0\end{array}$

$\begin{array}{lllllll}\text { cuboid } 3 & 1 & 4 \mathrm{p} 1.237 & 11.784 & 0.0\end{array}$

unit 83

com='(Fuel+Clad+Void $)$ - fill array $3^{\prime}$

$\begin{array}{llllll}\text { cylinder } & 1 & 1 & .5325 & 6.07 & 0.0\end{array}$

$\begin{array}{lllll}\text { cylinder } 2 & 1 & .6115 & 6.07 & 0.0\end{array}$

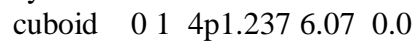

unit 84

com='(void+Clad+void $)^{\prime}$

cylinder $\begin{array}{lllll}0 & 1 & 0.5325 & 9.97 & 0.0\end{array}$

$\begin{array}{llllll}\text { cylinder } 2 & 1 & 0.6115 & 9.97 & 0.0\end{array}$

$\begin{array}{llllllll}\text { cuboid } & 0 & 1 & 4 \mathrm{p} 1.237 & 9.97 & 0.0\end{array}$

unit 100

com='Lower Al grid plate'

cuboid $414 \mathrm{p} 55.977 .601 \quad 0.0$

unit 101

com='array of h2o and end plugs below Al grid plate'

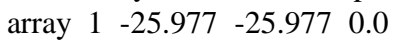

$\begin{array}{lllllll}\text { cuboid } & 3 & 1 & 4 \mathrm{p} 55.977 & 4.445 & 0.0\end{array}$

unit 102

com='array of h2o and end plugs above $\mathrm{Al}$ grid plate '

array $2-25.977-25.977 \quad 0.0$

$\begin{array}{lllllll}\text { cuboid } & 3 & 1 & 4 p 55.977 & 11.784 & 0.0\end{array}$

unit 103

com='array of Fuel+Clad+Void'

$\begin{array}{llll}\text { array } 3 & -25.977 & -25.977 & 0.0\end{array}$

$\begin{array}{lllllll}\text { cuboid } & 0 & 1 & 4 p 55.977 & 6.07 & 0.0\end{array}$

unit 104

com='array of void+Clad+void'

$\begin{array}{llll}\text { array } 4 & -25.977 & -25.977 & 0.0\end{array}$

$\begin{array}{lllllll}\text { cuboid } & 0 & 1 & 4 \mathrm{p} 55.977 & 9.97 & 0.0\end{array}$

unit 105

com='array of Fuel+Clad+H2O'

$\begin{array}{llll}\text { array } 5 & -25.977 & -25.977 & 0.0\end{array}$

$\begin{array}{lllllll}\text { cuboid } & 3 & 1 & 4 \mathrm{p} 55.977 & 64.53 & 0.0\end{array}$

global unit 110 com='array of arrays of fuel element sections + reflector'

$\begin{array}{llll}\text { array } 6 & -55.977 & -55.977 & 0.0\end{array}$

$\begin{array}{lllllll}\text { cuboid } 4 & 1 & 4 p 55.977 & 97.40 & -1.27\end{array}$

cuboid $5 \quad 1 \quad 4 p 55.977 \quad 97.40 \quad-3.47$

cuboid $3 \quad 1 \quad 4 p 55.977 \quad 97.40 \quad-17.27$

cuboid $5 \quad 1 \quad 4 p 55.977 \quad 97.40 \quad-17.77$

cuboid $\begin{array}{llllll}6 & 1 & 4 p 55.977 & 97.40 & -54.77\end{array}$

end geometry

read array

ara=1 nux=21 nuy=21 nuz=1

fill f81 end fill

ara $=2$ nux=21 nuy=21 nuz=1

fill $\mathrm{f} 82$ end fill

ara=3 nux=21 nuy=21 nuz=1

fill $\mathrm{f} 83$ end fill

ara $=4$ nux=21 nuy=21 nuz=1

fill $\mathrm{f} 84$ end fill

ara $=5$ nux=21 nuy=21 nuz=1

fill

12234456778991011 lb 10

$212131415161718192021 \quad 1 b 10$

$313222324252627282930 \quad 1 b 10$

414233132333435363738 1b 10

515243239404142434445 1b 10

$616253340464748495051 \quad 1 b 10$

717263441475253545556 1b 10

818273542485357585960 1b 10

919283643495458616263 1b 10

1020293744505559626465 1b 10

1121303845515660636566 1b 220

end fill

ara $=6$ nux $=1$ nuy=1 nuz=6

fill 101100102105103104 end fill

end array

end data

end

$=$ shell

cp ft04f001 \$RTNDIR/tca11.xs04

cp ft64f001 \$RTNDIR/tca11.rs64

end

$=\mathrm{kmart}$

read initial kunit=64

xunit $=4$

actbygrp

rrpvol

end initial

read activity

92234279223418922341452

92235279223518922351452

$9223827922381892238 \quad 1452$

94238279423818942381452

94239279423918942391452

$9424027942401894240 \quad 1452$

$9424127942411894241 \quad 1452$

$9424227942421894242 \quad 1452$

95241279524118952411452

501027 
501127

801627

4000027

2400027

2600027

100127

5011227

5011427

5011527

5011627

5011727

5011827

5011927

5012027

5012227

5012427

end activity

read collapse

factor $=1.0$

lastg $=57$

lastg $=148$

lastg $=204$

lastg $=238$

end collapse

end 

APPENDIX D

SCALE INPUT OF VARIANTS PROBLEM SET 



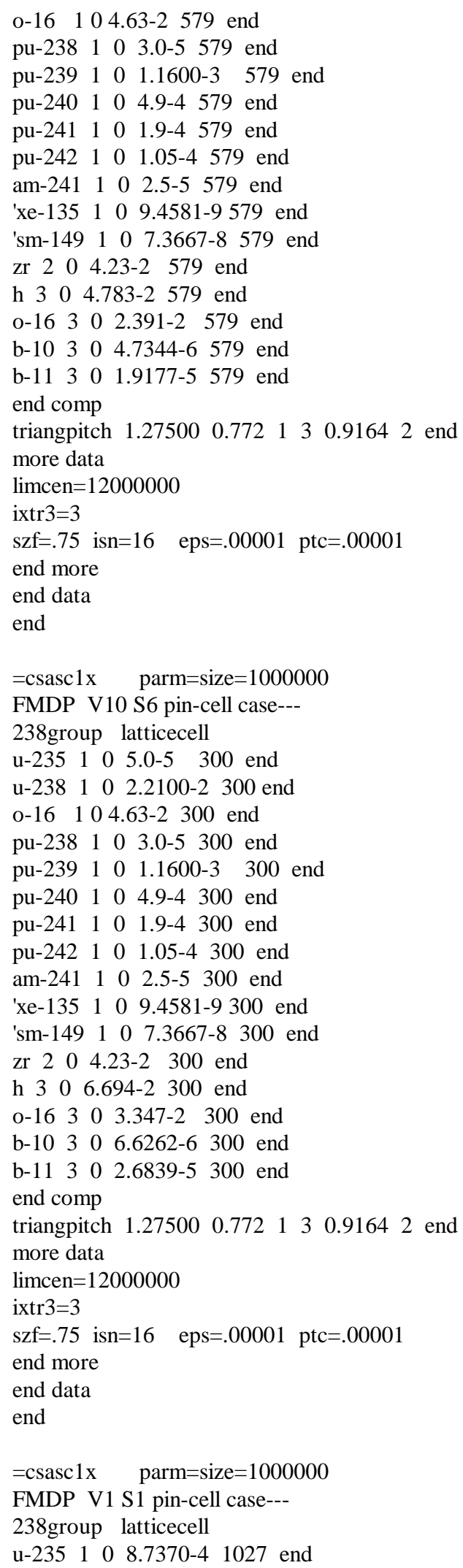

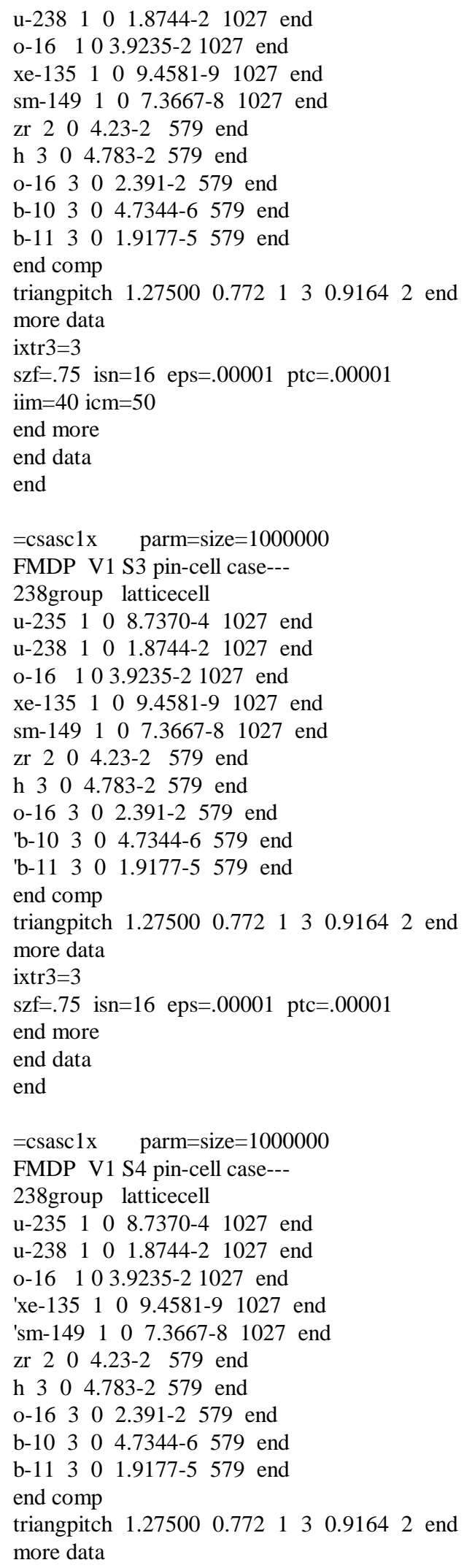


ixtr $3=3$

$\mathrm{szf}=.75$ isn $=16$ eps $=.00001 \mathrm{ptc}=.00001$

end more

end data

end

$=\operatorname{csasc} 1 \mathrm{x} \quad$ parm $=$ size $=1000000$

FMDP V1 S5 pin-cell case---

238group latticecell

u-235 $11008.7370-4579$ end

u-238 $11001.8744-2579$ end

o-16 $103.9235-2579$ end

'xe-135 $1100.4581-9579$ end

'sm-149 $1007.3667-8 \quad 579$ end

zr $2004.23-2 \quad 579$ end

h $3 \quad 0 \quad 4.783-2 \quad 579$ end

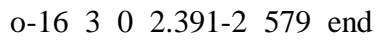

b-10 $3 \quad 0 \quad 4.7344-6579$ end

b-11 $3001.9177-5579$ end

end comp

triangpitch $1.27500 \quad 0.772 \quad 1 \quad 3 \quad 0.91642$ end more data

ixtr $3=3$

$\mathrm{szf}=.75$ isn $=16$ eps $=.00001 \mathrm{ptc}=.00001$

end more

end data

end

$=\operatorname{csasc} 1 \mathrm{x} \quad$ parm $=$ size $=1000000$

FMDP V1 S6 pin-cell case---

238group latticecell

u-235 $110 \begin{array}{llll}0 & 8.7370-4 & 300 & \text { end }\end{array}$

u-238 $1001.8744-2 \quad 300$ end

o-16 $103.9235-2300$ end

'xe-135 $1 \quad 0 \quad 9.4581-9 \quad 300$ end

'sm-149 $1 \quad 0 \quad 7.3667-8 \quad 300$ end

zr $20 \quad 4.23-2 \quad 300$ end

h $3 \quad 0 \quad 6.694-2 \quad 300$ end

o-16 $3003.347-2 \quad 300$ end

b-10 $3 \quad 0 \quad 6.6262-6 \quad 300$ end

b-11 $3 \quad 0 \quad 2.6839-5300$ end

end comp

triangpitch $1.27500 \quad 0.772 \quad 1 \quad 3 \quad 0.91642$ end

more data

limcen $=8000000$

ixtr $3=3$

$\mathrm{szf}=.75$ isn $=16$ eps $=.00001$ ptc $=.00001$

end more

end data

end

$=\operatorname{csasc} 1 \mathrm{x} \quad$ parm $=$ size $=1000000$

FMDP V2 S1 pin-cell case---

238group latticecell

u-235 $11003.8393-51027$ end

u-238 $100 \quad 1.8917-2 \quad 1027$ end

pu-239 $100.5875-41027$ end pu-240 $104.2323-51027$ end pu-241 $107.0246-61027$ end o-16 $103.9235-21027$ end xe-135 $1100.4581-91027$ end sm-149 $1107.3667-8 \quad 1027$ end

zr $2 \quad 0 \quad 4.23-2 \quad 579$ end

h $3 \quad 0 \quad 4.783-2579$ end o-16 $300 \quad 2.391-2579$ end

b-10 $3 \quad 0 \quad 4.7344-6579$ end b-11 $3 \quad 0 \quad 1.9177-5579$ end end comp

triangpitch $1.27500 \quad 0.772 \quad 1 \quad 3 \quad 0.91642$ end more data

limcen $=12000000$

ixtr $3=3$

$\mathrm{szf}=.75$ isn $=16$ eps $=.00001 \mathrm{ptc}=.00001$

end more

end data

end

$=\operatorname{csasc} 1 \mathrm{x} \quad$ parm $=$ size $=1000000$

FMDP V2 S3 pin-cell case---

238group latticecell

u-235 $11003.8393-5 \quad 1027$ end

u-238 $11001.8917-2 \quad 1027$ end

pu-239 $1006.5875-41027$ end

pu-240 $104.2323-51027$ end

pu-241 $107.0246-61027$ end

o-16 $103.9235-21027$ end

xe-135 $1100.4581-91027$ end

sm-149 $11007.3667-81027$ end

zr $2 \quad 0 \quad 4.23-2 \quad 579$ end

h $3 \quad 0 \quad 4.783-2 \quad 579$ end

o-16 $3 \quad 0 \quad 2.391-2579$ end

'b-10 $3 \begin{array}{llll}0 & 4.7344-6 & 579 & \text { end }\end{array}$

'b-11 $3001.9177-5579$ end

end comp

triangpitch $1.27500 \quad 0.772 \quad 1 \quad 3 \quad 0.91642$ end more data

limcen $=12000000$

ixtr $3=3$

$\mathrm{szf}=.75$ isn $=16$ eps $=.00001 \mathrm{ptc}=.00001$

end more

end data

end

$=\operatorname{csasc} 1 \mathrm{x} \quad$ parm $=$ size $=1000000$

FMDP V2 S4 pin-cell case---

238group latticecell

u-235 $11003.8393-51027$ end

u-238 $1001.8917-21027$ end

pu-239 1 0 6.5875-4 1027 end

pu-240 $104.2323-51027$ end

pu-241 $107.0246-61027$ end

o-16 $103.9235-21027$ end

'xe-135 $1 \quad 0 \quad 9.4581-91027$ end

'sm-149 10 7.3667-8 1027 end 
zr $2 \quad 0 \quad 4.23-2 \quad 579$ end

h $3 \quad 0 \quad 4.783-2 \quad 579$ end

o-16 $3 \begin{array}{lll}0 & 2.391-2 & 579\end{array}$ end

b-10 $3 \quad 0 \quad 4.7344-6579$ end

b-11 $3001.9177-5579$ end

end comp

triangpitch $1.27500 \quad 0.772 \quad 1 \quad 3 \quad 0.91642$ end

more data

limcen $=17000000$

ixtr3=3

$\mathrm{szf}=.75$ isn $=16$ eps $=.00001$ ptc $=.00001$

end more

end data

end

$=\operatorname{csasc} 1 \mathrm{x} \quad$ parm $=\operatorname{size}=1000000$

FMDP V2 S5 pin-cell case---

238group latticecell

u-235 $11003.8393-5 \quad 579$ end

u-238 $11001.8917-2 \quad 579$ end

pu-239 $1006.5875-4579$ end

pu-240 $104.2323-5579$ end

pu-241 $107.0246-6579$ end

o-16 $1103.9235-2579$ end

'xe-135 $1100.4581-9579$ end

'sm-149 10 7.3667-8 579 end

zr $2 \quad 0 \quad 4.23-2 \quad 579$ end

h $3 \quad 0 \quad 4.783-2 \quad 579$ end

o-16 $3 \begin{array}{lll}0 & 2.391-2 & 579\end{array}$

b-10 $3004.7344-6579$ end

b-11 $3001.9177-5579$ end

end comp

triangpitch $1.27500 \quad 0.772 \quad 1 \quad 3 \quad 0.91642$ end

more data

limcen $=17000000$

ixtr $3=3$

$\mathrm{szf}=.75$ isn $=16$ eps $=.00001$ ptc $=.00001$

end more

end data

end

$=\operatorname{csasc} 1 \mathrm{x} \quad$ parm $=$ size $=1000000$

FMDP V2 S6 pin-cell case---

238group latticecell

u-235 $1103.8393-5 \quad 300$ end

u-238 $11001.8917-2 \quad 300$ end

pu-239 $1 \quad 0 \quad 6.5875-4300$ end

pu-240 $104.2323-5300$ end

pu-241 $107.0246-6300$ end

o-16 $103.9235-2300$ end

'xe-135 $100.4581-9300$ end

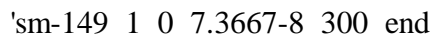

zr $2 \quad 0 \quad 4.23-2 \quad 300$ end

h $3 \quad 0 \quad 6.694-2 \quad 300$ end

o-16 $3 \begin{array}{llll}0 & 3.347-2 & 300 & \text { end }\end{array}$

b-10 $3 \quad 0 \quad 6.6262-6300$ end

b-11 $3002.6839-5300$ end end comp

triangpitch $1.27500 \quad 0.772 \quad 1 \quad 3 \quad 0.91642$ end more data

limcen $=17000000$

ixtr3=3

$\mathrm{szf}=.75$ isn $=16$ eps $=.00001$ ptc $=.00001$

end more

end data

end

$=\operatorname{csasc} 1 \mathrm{x} \quad$ parm $=$ size $=1000000$

FMDP V3 S1 pin-cell case---

238group latticecell

u-235 $11003.7843-41027$ end

u-236 $11008.6365-51027$ end

u-238 $1001.8327-21027$ end

np-237 $11002.4823-51027$ end

pu-238 $1006.7254-61027$ end

'np-239 $11001.8332-61027$ end

pu-239 $1 \quad 0 \quad 1.3111-4 \quad 1027$ end

pu-240 $103.6233-51027$ end

pu-241 $102.1701-51027$ end

pu-242 $110 \quad 4.7576-61027$ end

am-241 10 4.949-7 1027 end

am-242m $1 \quad 0 \quad 7.9194-91027$ end

am-243 $1106.6925-7 \quad 1027$ end

cm-242 $110 \quad 1.2582-7 \quad 1027$ end

cm-243 110 2.0629-9 1027 end

cm-244 $1001.2387-71027$ end

o-16 $103.9235-21027$ end

xe-135 $11009.4581-91027$ end

sm-149 $1 \quad 0 \quad 7.3667-8 \quad 1027$ end

zr $2004.23-2 \quad 579$ end

h $3 \quad 0 \quad 4.783-2579$ end

o-16 $3 \begin{array}{lll}0 & 2.391-2 & 579\end{array}$ end

b-10 $3 \quad 0 \quad 4.7344-6579$ end

b-11 $3 \quad 0 \quad 1.9177-5579$ end

end comp

triangpitch $1.27500 \quad 0.772 \quad 1 \quad 3 \quad 0.91642$ end more data

limcen $=16000000$

ixtr $3=3$

$\mathrm{szf}=.75$ isn $=16$ eps $=.00001$ ptc $=.00001$

end more

end data

end

$=\operatorname{csasc} 1 \mathrm{x} \quad$ parm $=$ size $=1000000$

FMDP V3 S3 pin-cell case---

238group latticecell

u-235 $11003.7843-41027$ end

u-236 $1100.6365-51027$ end

u-238 $1001.8327-21027$ end

np-237 100 2.4823-5 1027 end

pu-238 10 6.7254-6 1027 end

'np-239 $11001.8332-61027$ end

pu-239 10 ( 0 1.3111-4 1027 end 
pu-240 $103.6233-51027$ end pu-241 $102.1701-51027$ end pu-242 $1 \quad 0 \quad 4.7576-61027$ end am-241 $1004.949-71027$ end am-242m $1 \quad 0 \quad 7.9194-91027$ end am-243 $1 \quad 0 \quad 6.6925-7 \quad 1027$ end cm-242 $1 \quad 0 \quad 1.2582-7 \quad 1027$ end cm-243 110 2.0629-9 1027 end cm-244 $11001.2387-7 \quad 1027$ end o-16 $103.9235-21027$ end xe-135 $1100.4581-91027$ end sm-149 10 7.3667-8 1027 end zr $2 \quad 0 \quad 4.23-2 \quad 579$ end h $3 \quad 0 \quad 4.783-2579$ end o-16 $3 \begin{array}{lll}3 & 2.391-2 & 579\end{array}$ end

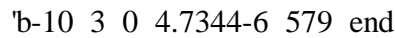
'b-11 $30 \begin{array}{lll}0 & 1.9177-5 & 579 \\ \text { end }\end{array}$ end comp

triangpitch $1.27500 \quad 0.772 \quad 1 \quad 3 \quad 0.91642$ end more data

limcen $=16000000$

ixtr $3=3$

$\mathrm{szf}=.75$ isn $=16$ eps $=.00001$ ptc $=.00001$

end more

end data

end

$=\operatorname{csasc} 1 \mathrm{x} \quad$ parm $=$ size $=1000000$

FMDP V3 S4 pin-cell case---

238group latticecell

u-235 $11003.7843-41027$ end

u-236 $11008.6365-51027$ end

u-238 $11001.8327-2 \quad 1027$ end

np-237 $10 \begin{array}{llll}2.4823-5 & 1027 & \text { end }\end{array}$

pu-238 $1 \quad 0 \quad 6.7254-61027$ end 'np-239 10 1.8332-6 1027 end pu-239 100 1.3111-4 1027 end pu-240 $103.6233-51027$ end pu-241 $102.1701-51027$ end pu-242 $11004.7576-6 \quad 1027$ end am-241 $11004.949-71027$ end am-242m $1 \quad 0 \quad 7.9194-91027$ end am-243 $1 \quad 0 \quad 6.6925-7 \quad 1027$ end cm-242 $1 \quad 0 \quad 1.2582-7 \quad 1027$ end cm-243 $11002.0629-91027$ end

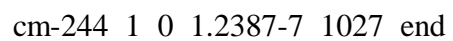
o-16 $103.9235-21027$ end 'xe-135 $1100.4581-91027$ end 'sm-149 $11007.3667-81027$ end zr $2 \quad 0 \quad 4.23-2 \quad 579$ end h $3 \quad 0 \quad 4.783-2 \quad 579$ end o-16 $300 \quad 2.391-2 \quad 579$ end b-10 $30 \begin{array}{lll}0 & 4.7344-6 & 579\end{array}$ end b-11 $3001.9177-5579$ end end comp triangpitch $1.27500 \quad 0.772 \quad 1 \quad 3 \quad 0.91642$ end more data

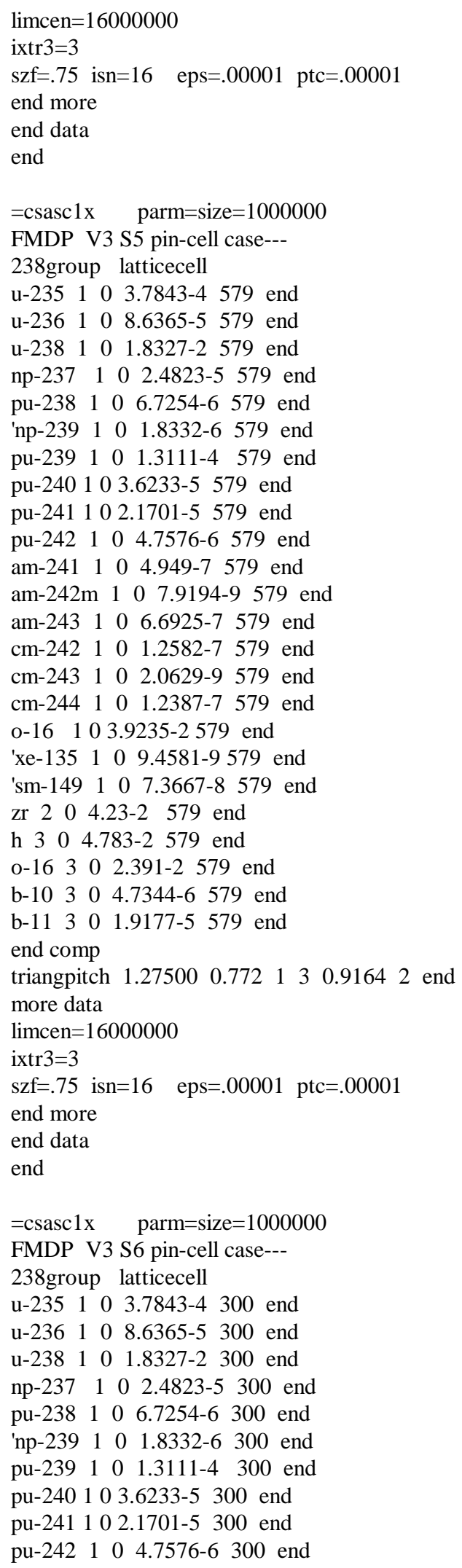


am-241 $11004.949-7 \quad 300$ end am-242m $1007.9194-9300$ end am-243 $1106.6925-7 \quad 300$ end cm-242 $10 \begin{array}{llll}1.2582-7 & 300 & \text { end }\end{array}$ $\mathrm{cm}-243 \quad 1 \quad 0 \quad 2.0629-9300$ end $\mathrm{cm}-244 \quad 1 \quad 0 \quad 1.2387-7300$ end o-16 $103.9235-2300$ end 'xe-135 $1100.4581-9300$ end

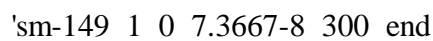
zr $2 \quad 0 \quad 4.23-2 \quad 300$ end h $3 \quad 0 \quad 6.694-2 \quad 300$ end o-16 $3003.347-2 \quad 300$ end b-10 $3 \quad 0 \quad 6.6262-6300$ end b-11 300 2.6839-5 300 end end comp triangpitch $\begin{array}{llllll}1.27500 & 0.772 & 1 & 3 & 0.9164 & 2\end{array}$ end more data

limcen $=16000000$

ixtr $3=3$

$\mathrm{szf}=.75$ isn $=16 \quad$ eps $=.00001 \mathrm{ptc}=.00001$ end more

end data

end

$=\operatorname{csasc} 1 \mathrm{x} \quad$ parm $=$ size $=1000000$

FMDP V4 S1 pin-cell case--238group latticecell

u-235 $11003.7843-41027$ end u-236 $11008.6365-51027$ end u-238 $11001.8327-2 \quad 1027$ end np-237 10 2.4823-5 1027 end pu-238 $1106.7254-61027$ end 'np-239 $1 \quad 0 \quad 1.8332-6 \quad 1027$ end pu-239 10 1.3111-4 1027 end pu-240 $103.6233-51027$ end pu-241 $102.1701-51027$ end rh-103 $1 \quad 0 \quad 1.8890-51027$ end xe-131 $11001.4255-5 \quad 1027$ end nd-143 $11002.6692-51027$ end pm-147 $1 \quad 0 \quad 6.1574-61027$ end cs-133 110 3.5974-5 1027 end tc-99 $11003.3320-51027$ end sm-152 $1 \quad 0 \quad 2.6842-61027$ end sm-151 110 3.0757-7 1027 end pu-242 $1004.7576-61027$ end am-241 $11004.9491-7 \quad 1027$ end am-242m 10 7.9194-9 1027 end am-243 $11006.6925-7 \quad 1027$ end cm-242 $1 \quad 0 \quad 1.2582-7 \quad 1027$ end cm-243 $11002.0629-91027$ end cm-244 $1001.2387-71027$ end o-16 $103.9235-21027$ end nd-145 $11001.9975-51027$ end eu-153 $11002.4801-61027$ end ag-109 $11002.2037-6 \quad 1027$ end eu-155 $1100.6857-81027$ end mo-95 10 3.3720-5 1027 end eu-154 10 5.1189-7 1027 end ru-101 $1 \quad 0 \quad 3.1134-51027$ end xe-135 $1100.4581-91027$ end sm-149 $1007.3667-81027$ end zr $2004.23-2 \quad 579$ end h $3 \quad 0 \quad 4.783-2 \quad 579$ end o-16 $3 \quad 0 \quad 2.391-2579$ end b-10 $3004.7344-6579$ end b-11 $3001.9177-5579$ end end comp triangpitch $1.27500 \quad 0.772 \quad 1 \quad 3 \quad 0.91642$ end more data limcen $=20000000$

ixtr $3=3$

$\mathrm{szf}=.75$ isn $=16$ eps $=.00001$ ptc $=.00001$

end more

end data

end

$=\operatorname{csasc} 1 \mathrm{x} \quad$ parm $=$ size $=1000000$

FMDP V4 S3 pin-cell case---

238group latticecell

u-235 $11003.7843-41027$ end

u-236 $11008.6365-5 \quad 1027$ end u-238 $100 \quad 1.8327-2 \quad 1027$ end np-237 $110 \quad 2.4823-51027$ end pu-238 $100.7254-61027$ end 'np-239 $1001.8332-6 \quad 1027$ end pu-239 $11001.3111-4 \quad 1027$ end pu-240 $103.6233-51027$ end pu-241 $102.1701-51027$ end rh-103 110 1.8890-5 1027 end xe-131 $1 \quad 0 \quad 1.4255-51027$ end nd-143 110 2.6692-5 1027 end pm-147 110 6.1574-6 1027 end cs-133 110 3.5974-5 1027 end tc-99 $1003.3320-51027$ end sm-152 $1 \quad 0 \quad 2.6842-6 \quad 1027$ end sm-151 110 3.0757-7 1027 end pu-242 $1 \quad 0 \quad 4.7576-61027$ end am-241 $1004.9491-7 \quad 1027$ end am-242m $1 \quad 0 \quad 7.9194-91027$ end am-243 $1106.6925-7 \quad 1027$ end cm-242 $11001.2582-7 \quad 1027$ end cm-243 110 2.0629-9 1027 end cm-244 110 1.2387-7 1027 end o-16 $103.9235-21027$ end nd-145 $11001.9975-51027$ end eu-153 110 2.4801-6 1027 end ag-109 10 2.2037-6 1027 end eu-155 110 9.6857-8 1027 end mo-95 110 3.3720-5 1027 end eu-154 $1005.1189-71027$ end ru-101 $11003.1134-51027$ end xe-135 $1100.4581-91027$ end sm-149 $1 \quad 0 \quad 7.3667-81027$ end zr $2 \quad 0 \quad 4.23-2 \quad 579$ end 
h $3 \quad 0 \quad 4.783-2579$ end

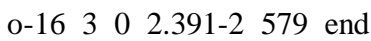

'b-10 $3004.7344-6 \quad 579$ end

'b-11 $3001.9177-5579$ end

end comp

triangpitch $1.27500 \quad 0.772 \quad 1 \quad 3 \quad 0.91642$ end

more data

limcen $=20000000$

ixtr $3=3$

$\mathrm{szf}=.75$ isn $=16$ eps $=.00001$ ptc $=.00001$

end more

end data

end

$=\operatorname{csasc} 1 \mathrm{x} \quad$ parm $=$ size $=1000000$

FMDP V4 S4 pin-cell case---

238group latticecell

u-235 $1103.7843-41027$ end

u-236 $11008.6365-51027$ end

u-238 $100 \quad 1.8327-2 \quad 1027$ end

np-237 $1102.4823-51027$ end

pu-238 $1006.7254-61027$ end

'np-239 $10 \begin{array}{llll}1.8332-6 & 1027 & \text { end }\end{array}$

pu-239 $11001.3111-4 \quad 1027$ end pu-240 $103.6233-51027$ end pu-241 $102.1701-51027$ end rh-103 $100 \quad 1.8890-51027$ end xe-131 $110 \quad 1.4255-5 \quad 1027$ end nd-143 1102 2.6692-5 1027 end pm-147 $1006.1574-61027$ end cs-133 $1003.5974-51027$ end tc-99 $1103.3320-51027$ end sm-152 $11002.6842-61027$ end sm-151 $1003.0757-7 \quad 1027$ end pu-242 $1004.7576-6 \quad 1027$ end am-241 10 4.9491-7 1027 end am-242m 10 7.9194-9 1027 end am-243 $1100.6925-7 \quad 1027$ end cm-242 $11001.2582-7 \quad 1027$ end cm-243 $11002.0629-91027$ end cm-244 $1 \quad 0 \quad 1.2387-7 \quad 1027$ end o-16 $103.9235-21027$ end nd-145 $110 \quad 1.9975-51027$ end eu-153 1102 2.4801-6 1027 end

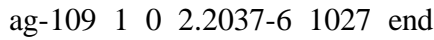
eu-155 $1100.6857-8 \quad 1027$ end mo-95 10 3.3720-5 1027 end eu-154 $11005.1189-7 \quad 1027$ end ru-101 $1 \quad 0 \quad 3.1134-51027$ end 'xe-135 100 9.4581-9 1027 end 'sm-149 $11007.3667-8 \quad 1027$ end zr $2004.23-2 \quad 579$ end h $3 \quad 0 \quad 4.783-2579$ end

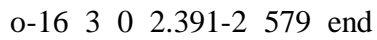
b-10 $3 \quad 0 \quad 4.7344-6579$ end b-11 $3001.9177-5579$ end end comp triangpitch $1.27500 \quad 0.772 \quad 1 \quad 3 \quad 0.91642$ end more data

limcen $=20000000$

ixtr $3=3$

$\mathrm{szf}=.75$ isn=16 eps $=.00001 \mathrm{ptc}=.00001$

end more

end data

end

$=\operatorname{csasc} 1 \mathrm{x} \quad$ parm $=$ size $=1000000$

FMDP V4 S5 pin-cell case---

238group latticecell

u-235 $11003.7843-4579$ end

u-236 $11008.6365-5 \quad 579$ end

u-238 $1001.8327-2579$ end np-237 $1002.4823-5579$ end pu-238 $100.7254-6579$ end 'np-239 $11001.8332-6579$ end pu-239 $1001.3111-4 \quad 579$ end pu-240 $103.6233-5579$ end pu-241 $102.1701-5579$ end rh-103 $1001.8890-5579$ end xe-131 $11001.4255-5579$ end nd-143 $1100 \begin{array}{llll}2.6692-5 & 579 & \text { end }\end{array}$ pm-147 $1 \quad 0 \quad 6.1574-6579$ end cs-133 $1103.5974-5579$ end tc-99 $1003.3320-5579$ end sm-152 $11002.6842-6579$ end sm-151 $11003.0757-7579$ end pu-242 $1004.7576-6579$ end am-241 $1004.9491-7 \quad 579$ end am-242m 10 7.9194-9 579 end am-243 $1100.6925-7 \quad 579$ end cm-242 $1001.2582-7579$ end $\mathrm{cm}-243 \quad 1 \quad 0 \quad 2.0629-9579$ end cm-244 $1001.2387-7579$ end o-16 $103.9235-2579$ end nd-145 $100 \quad 1.9975-5 \quad 579$ end eu-153 $11002.4801-6 \quad 579$ end ag-109 100 2.2037-6 579 end eu-155 $110 \quad 9.6857-8 \quad 579$ end mo-95 $1003.3720-5579$ end eu-154 100 5.1189-7 579 end ru-101 $1003.1134-5579$ end 'xe-135 $100.4581-9579$ end 'sm-149 $1007.3667-8 \quad 579$ end zr $2 \quad 0 \quad 4.23-2 \quad 579$ end h $3 \quad 0 \quad 4.783-2579$ end

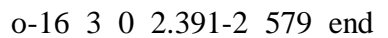
b-10 $3 \begin{array}{lll}0 & 4.7344-6 & 579\end{array}$ end b-11 $3 \quad 0 \quad 1.9177-5579$ end end comp triangpitch $1.27500 \quad 0.772 \quad 1 \quad 3 \quad 0.91642$ end more data limcen $=20000000$ ixtr3=3

$\mathrm{szf}=.75$ isn $=16$ eps $=.00001$ ptc $=.00001$ 
end more

end data

end

$=\operatorname{csasc} 1 \mathrm{x} \quad$ parm $=$ size $=1000000$

FMDP V4 S6 pin-cell case---

238group latticecell

$\begin{array}{lllll}\text { u-235 } & 1 & 0 & 3.7843-4 & 300\end{array}$ end

u-236 $11008.6365-5 \quad 300$ end

u-238 $1001.8327-2 \quad 300$ end

np-237 $1 \quad 0 \quad 2.4823-5300$ end

pu-238 $100.7254-6 \quad 300$ end

'np-239 $110 \quad 1.8332-6 \quad 300$ end

pu-239 $100 \quad 1.3111-4 \quad 300$ end

pu-240 $103.6233-5300$ end

pu-241 $102.1701-5300$ end

rh-103 $1 \quad 0 \quad 1.8890-5300$ end

xe-131 $100 \quad 1.4255-5300$ end

nd-143 $11002.6692-5300$ end

pm-147 $1 \quad 0 \quad 6.1574-6 \quad 300$ end cs-133 $1003.5974-5300$ end tc-99 $1 \quad 0 \quad 3.3320-5300$ end sm-152 $1 \quad 0 \quad 2.6842-6300$ end sm-151 $11003.0757-7 \quad 300$ end pu-242 $1004.7576-6300$ end am-241 10 4.9491-7 300 end am-242m 10 7.9194-9 300 end am-243 $1 \quad 0 \quad 6.6925-7 \quad 300$ end cm-242 $110 \quad 1.2582-7 \quad 300$ end cm-243 $11002.0629-9300$ end $\mathrm{cm}-244 \quad 1 \quad 0 \quad 1.2387-7300$ end o-16 $103.9235-2300$ end nd-145 $11001.9975-5 \quad 300$ end eu-153 $1100 \quad 2.4801-6 \quad 300$ end ag-109 $11002.2037-6 \quad 300$ end eu-155 $1100.6857-8 \quad 300$ end mo-95 $1003.3720-5300$ end eu-154 $11005.1189-7 \quad 300$ end ru-101 $1 \quad 0 \quad 3.1134-5300$ end 'xe-135 100 9.4581-9 300 end 'sm-149 $1 \quad 0 \quad 7.3667-8 \quad 300$ end zr $2 \quad 0 \quad 4.23-2 \quad 300$ end h $3 \quad 0 \quad 6.694-2 \quad 300$ end o-16 $3 \begin{array}{llll}0 & 3.347-2 & 300 & \text { end }\end{array}$

b-10 $3 \quad 0 \quad 6.6262-6 \quad 300$ end

b-11 30 2.6839-5 300 end

end comp

triangpitch $1.27500 \quad 0.772 \quad 130.91642$ end

more data

limcen $=20000000$

ixtr3=3

$\mathrm{szf}=.75$ isn $=16$ eps $=.00001$ ptc $=.00001$

end more

end data

end

$=\operatorname{csasc} 1 x \quad$ parm $=$ size $=1000000$
FMDP V4 S6 pin-cell case---

238group latticecell

u-235 $11003.8393-51027$ end

u-238 $110 \quad 1.8917-2 \quad 1027$ end

pu-239 $1 \quad 06.5875-41027$ end

o-16 104 4.1707-2 1027 end

xe-135 $1100.4581-91027$ end

sm-149 $1 \quad 0 \quad 7.3667-8 \quad 1027$ end

zr $2 \quad 0 \quad 4.23-2 \quad 579$ end

h $3 \quad 0 \quad 4.783-2 \quad 579$ end

o-16 $3 \quad 0 \quad 2.391-2 \quad 579$ end

b-10 $3 \quad 0 \quad 4.7344-6579$ end

b-11 $3001.9177-5579$ end

end comp

triangpitch $1.27500 \quad 0.77213 \quad 3 \quad 0.91642$ end

more data

limcen $=16000000$

ixtr $3=3$

$\mathrm{szf}=.75$ isn $=16$ eps $=.00001$ ptc $=.00001$

end more

end data

end

$=\operatorname{csasc} 1 \mathrm{x} \quad$ parm $=$ size $=1000000$

FMDP V7 S3 pin-cell case---

238group latticecell

u-235 $11003.8393-51027$ end

$\mathrm{u}-238 \quad 1 \quad 0 \quad 1.8917-2 \quad 1027$ end

pu-239 $1 \quad 06.5875-41027$ end

o-16 $104.1707-21027$ end

xe-135 $100 \begin{array}{llll}0.4581-9 & 1027 & \text { end }\end{array}$

sm-149 $1 \quad 0 \quad 7.3667-8 \quad 1027$ end

zr $2004.23-2 \quad 579$ end

h $3 \quad 0 \quad 4.783-2 \quad 579$ end

o-16 $3 \begin{array}{llll}0 & 2.391-2 & 579 & \text { end }\end{array}$

'b-10 $30 \begin{array}{llll}0 & 4.7344-6 & 579 & \text { end }\end{array}$

'b-11 $3001.9177-5579$ end

end comp

triangpitch $1.27500 \quad 0.772 \quad 1 \quad 3 \quad 0.91642$ end more data

limcen $=16000000$

ixtr $3=3$

$\mathrm{szf}=.75$ isn $=16$ eps $=.00001 \mathrm{ptc}=.00001$

end more

end data

end

$=\operatorname{csasc} 1 \mathrm{x} \quad$ parm $=$ size $=1000000$

FMDP V7 S4 pin-cell case---

238group latticecell

u-235 $11003.8393-51027$ end

u-238 $11001.8917-2 \quad 1027$ end

pu-239 $106.5875-41027$ end

o-16 $104.1707-21027$ end

'xe-135 10 9.4581-9 1027 end

'sm-149 $1007.3667-8 \quad 1027$ end

zr $20 \quad 4.23-2 \quad 579$ end 
h $3 \quad 0 \quad 4.783-2579$ end

o-16 $3 \begin{array}{llll}0 & 2.391-2 & 579 & \text { end }\end{array}$

b-10 $3 \begin{array}{lll}0 & 4.7344-6 & 579\end{array}$ end

b-11 $3 \quad 0 \quad 1.9177-5579$ end

end comp

triangpitch $\begin{array}{llllll}1.27500 & 0.772 & 1 & 3 & 0.9164 & 2\end{array}$ end

more data

limcen $=16000000$

ixtr $3=3$

$\mathrm{szf}=.75$ isn $=16$ eps $=.00001$ ptc $=.00001$

end more

end data

end

$=\operatorname{csasc} 1 \mathrm{x} \quad$ parm $=$ size $=1000000$

FMDP V7 S5 pin-cell case---

238group latticecell

u-235 $110 \begin{array}{llll}0.8393-5 & 579 & \text { end }\end{array}$

u-238 $1 \quad 0 \quad 1.8917-2 \quad 579$ end

pu-239 $106.5875-4579$ end

o-16 $1104.1707-2579$ end

'xe-135 $1100.4581-9579$ end

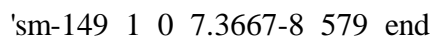

zr $2 \quad 0 \quad 4.23-2 \quad 579$ end

h $3 \quad 0 \quad 4.783-2 \quad 579$ end

o-16 $3 \quad 0 \quad 2.391-2 \quad 579$ end

b-10 $3004.7344-6579$ end

b-11 $3001.9177-5579$ end

end comp

triangpitch $\begin{array}{llllll}1.27500 & 0.772 & 1 & 3 & 0.9164 & 2\end{array}$ end

more data

limcen $=16000000$

ixtr $3=3$

$\mathrm{szf}=.75$ isn=16 eps $=.00001$ ptc $=.00001$

end more

end data

end

$=\operatorname{csasc} 1 \mathrm{x} \quad$ parm $=$ size $=1000000$

FMDP V7 S6 pin-cell case---

238group latticecell

u-235 $11003.8393-5 \quad 300$ end

u-238 $1 \quad 0 \quad 1.8917-2 \quad 300$ end

pu-239 $1 \quad 0 \quad 6.5875-4300$ end

o-16 $1104.1707-2 \quad 300$ end

'xe-135 $1100.4581-9300$ end

'sm-149 $1 \quad 0 \quad 7.3667-8 \quad 300$ end

$\begin{array}{lllll}\text { zr } 2 & 0 & 4.23-2 & 300 & \text { end }\end{array}$

h $3 \quad 0 \quad 6.694-2 \quad 300$ end

o-16 $3003.347-2 \quad 300$ end

b-10 $3 \begin{array}{llll}0 & 6.6262-6 & 300 & \text { end }\end{array}$

b-11 $3 \quad 0 \quad 2.6839-5300$ end

end comp

triangpitch $1.27500 \quad 0.772 \quad 1 \quad 3 \quad 0.91642$ end

more data

limcen $=16000000$

ixtr3=3

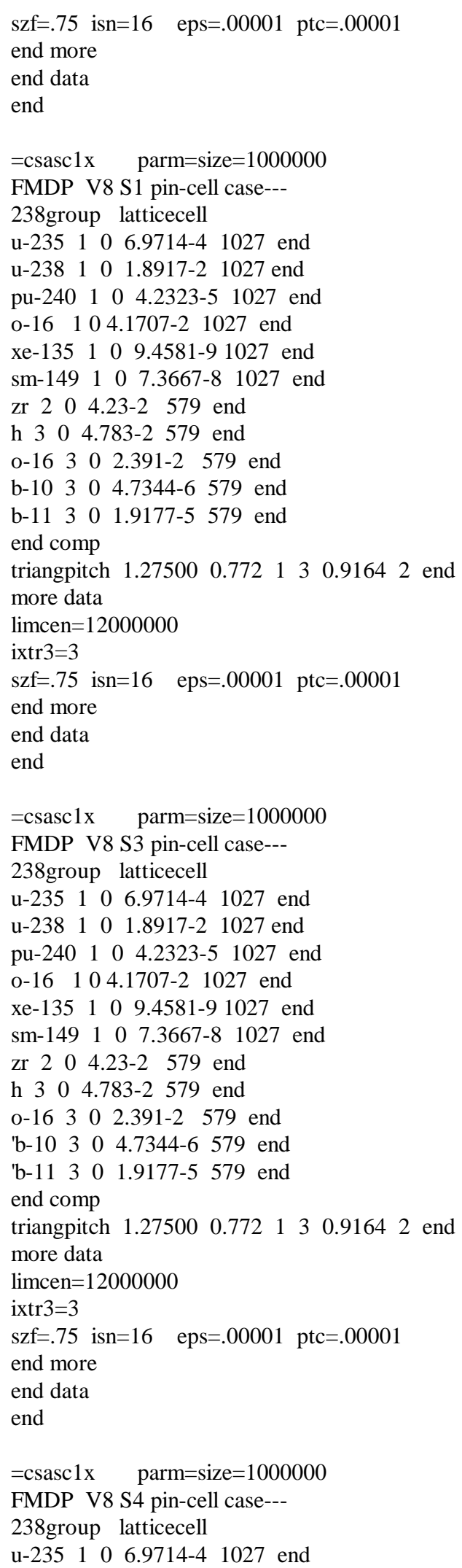


u-238 $1 \quad 0 \quad 1.8917-2 \quad 1027$ end pu-240 $1 \quad 0 \quad 4.2323-51027$ end o-16 $104.1707-21027$ end 'xe-135 100 9.4581-9 1027 end 'sm-149 10 7.3667-8 1027 end zr $2 \quad 0 \quad 4.23-2 \quad 579$ end h $3 \quad 0 \quad 4.783-2579$ end o-16 $300 \quad 2.391-2 \quad 579$ end b-10 $3004.7344-6579$ end b-11 $3001.9177-5579$ end end comp

triangpitch $1.27500 \quad 0.772 \quad 1 \quad 3 \quad 0.91642$ end more data

limcen $=12000000$

ixtr $3=3$

$\mathrm{szf}=.75$ isn $=16$ eps $=.00001$ ptc $=.00001$

end more

end data

end

$=\operatorname{csasc} 1 \mathrm{x} \quad$ parm $=$ size $=1000000$

FMDP V8 S5 pin-cell case---

238group latticecell

u-235 $11006.9714-4 \quad 579$ end u-238 $1 \quad 0 \quad 1.8917-2 \quad 579$ end pu-240 $11004.2323-5579$ end o-16 $104.1707-2579$ end 'xe-135 $11009.4581-9579$ end 'sm-149 $1007.3667-8 \quad 579$ end zr $2004.23-2 \quad 579$ end

h $3 \quad 0 \quad 4.783-2 \quad 579$ end o-16 $3 \begin{array}{llll}0 & 2.391-2 & 579 & \text { end }\end{array}$ b-10 $300 \quad 4.7344-6579$ end b-11 $3001.9177-5579$ end end comp triangpitch $1.27500 \quad 0.772 \quad 1 \quad 3 \quad 0.91642$ end more data

limcen $=12000000$

ixtr3=3

$\mathrm{szf}=.75$ isn $=16$ eps $=.00001$ ptc $=.00001$

end more

end data

end

$=\operatorname{csasc} 1 \mathrm{x} \quad$ parm $=\operatorname{size}=1000000$

FMDP V8 S6 pin-cell case--238group latticecell u-235 $1106.9714-4300$ end u-238 $1001.8917-2 \quad 300$ end pu-240 $1004.2323-5300$ end o-16 $1104.1707-2 \quad 300$ end 'xe-135 $1100.4581-9300$ end 'sm-149 $10 \quad 7.3667-8 \quad 300$ end zr $2004.23-2 \quad 300$ end h $3 \quad 0 \quad 6.694-2 \quad 300$ end o-16 $3 \quad 0 \quad 3.347-2 \quad 300$ end b-10 $3 \quad 0 \quad 6.6262-6300$ end b-11 $3 \quad 0 \quad 2.6839-5300$ end

end comp

triangpitch $1.27500 \quad 0.772 \quad 1 \quad 3 \quad 0.91642$ end

more data

limcen $=12000000$

ixtr $3=3$

$\mathrm{szf}=.75$ isn=16 eps=.00001 ptc $=.00001$

end more

end data

end

$=\operatorname{csasc} 1 \mathrm{x} \quad$ parm $=$ size $=1000000$

FMDP V9 S1 pin-cell case---

238group latticecell

u-235 $11003.8393-51027$ end

u-238 $110 \quad 1.8917-21027$ end

pu-241 1106 6.6577-4 1027 end

o-16 104 4.1707-2 1027 end

xe-135 $1100.4581-91027$ end

sm-149 $1 \quad 0 \quad 7.3667-8 \quad 1027$ end

zr $2 \quad 0 \quad 4.23-2 \quad 579$ end

h $3 \quad 0 \quad 4.783-2579$ end

o-16 3 0 $2.391-2 \quad 579$ end

b-10 $3004.7344-6579$ end

b-11 $3 \quad 0 \quad 1.9177-5579$ end

end comp

triangpitch $1.27500 \quad 0.772 \quad 1 \quad 3 \quad 0.91642$ end more data

limcen $=12000000$

ixtr $3=3$

$\mathrm{szf}=.75$ isn $=16$ eps $=.00001 \mathrm{ptc}=.00001$

end more

end data

end

$=\operatorname{csasc} 1 \mathrm{x} \quad$ parm $=$ size $=1000000$

FMDP V9 S3 pin-cell case---

238group latticecell

u-235 $11003.8393-5 \quad 1027$ end

u-238 $100 \quad 1.8917-21027$ end

pu-241 $1006577-41027$ end

o-16 $1104.1707-2 \quad 1027$ end

xe-135 $1100.4581-91027$ end

sm-149 $10 \begin{array}{llll}7.3667-8 & 1027 & \text { end }\end{array}$

zr $2004.23-2 \quad 579$ end

h $3 \quad 0 \quad 4.783-2 \quad 579$ end

o-16 $3 \begin{array}{llll}0 & 2.391-2 & 579 & \text { end }\end{array}$

'b-10 $30 \begin{array}{llll}0 & 4.7344-6 & 579 & \text { end }\end{array}$

'b-11 $3001.9177-5579$ end

end comp

triangpitch $1.27500 \quad 0.772 \quad 1 \quad 3 \quad 0.91642$ end

more data

limcen $=12000000$

ixtr $3=3$

$\mathrm{szf}=.75$ isn $=16$ eps $=.00001$ ptc $=.00001$

end more

end data 


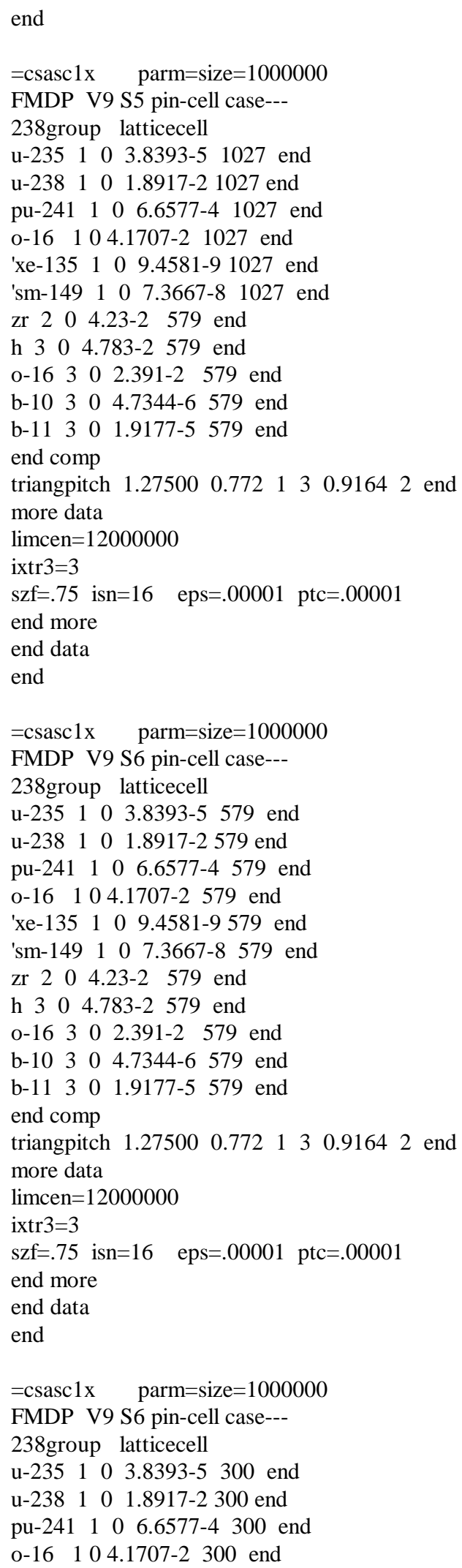

'xe-135 $10 \quad 0 \quad 9.4581-9300$ end 'sm-149 $1 \quad 0 \quad 7.3667-8 \quad 300$ end zr $2004.23-2 \quad 300$ end h $3 \quad 0 \quad 6.694-2300$ end o-16 $3 \begin{array}{llll}0 & 3.347-2 & 300 & \text { end }\end{array}$ b-10 $3 \quad 0 \quad 6.6262-6300$ end b-11 $3002.6839-5300$ end end comp triangpitch $1.27500 \quad 0.772 \quad 1 \quad 3 \quad 0.91642$ end more data limcen $=12000000$

ixtr $3=3$

$\mathrm{szf}=.75$ isn $=16$ eps $=.00001 \mathrm{ptc}=.00001$ end more end data end 



\section{INTERNAL DISTRIBUTION}

1. B. B. Bevard

2. S. M. Bowman

3. B. L. Broadhead

4-8. W. C. Carter (5)

9. E. D. Collins

10. B. S. Cowell

11. M. D. DeHart

12. M. E. Dunn

13. K. R. Elam

14. R. J Ellis

15. M. B. Emmett

16. I. C. Gauld

17. J. C. Gehin

18. N. M. Greene

19. S. R. Greene

20. O. W. Hermann

21-25. D. F. Hollenbach (5)

26. C. M. Hopper

27. O. W. Hermann

28. D. T. Ingersoll
29. H. T. Kerr

30. M. A. Kuliasha

31. L. C. Leal

32. S. B. Ludwig

33. G. E. Michaels

34. D. L. Moses

35. B. D. Murphy

36. L. M. Petrie

37-41. C. V. Parks (5)

42. L. M. Petrie

43-47. R. T. Primm (5)

48. B. T. Rearden

49. C. H. Shappert

50. T. E. Valentine

51. J. C. Wagner

52. R. M. Westfall

53. R. Q. Wright

54. Central Research Library Document Reference Section

55-56. ORNL Laboratory Records (2) for submission to OSTI

57. ORNL Laboratory Records - RC

\section{EXTERNAL DISTRIBUTION}

58. M. L. Adams, Department of Nuclear Engineering, Texas A\&M University, Zachry 129, College Station, TX 77843

59. R. E. Anderson, Los Alamos National Laboratory, P.O. Box 1663, NIS-6, MS J562, Los Alamos, NM 87545

60. W. L. Andrews, Defense Nuclear Facilities Safety Board, 625 Indiana Ave., Washington, DC 20004 
61. M. G. Bailey, U.S. Nuclear Regulatory Commission, MS O6-G22, Washington, DC 20555

62. J. B. Briggs, INEEL, P.O. Box 1625, MS-3855, Idaho Falls, ID 83402

63. T. Burns, Defense Nuclear Facilities Safety Board, 625 Indiana Ave., Washington, DC 20004

64. D. E. Carlson, U.S. Nuclear Regulatory Commission, MS O6-F18, Washington, DC 20555

65. I. Carron, Department of Nuclear Engineering, Texas A\&M University, 129 Zachry, College Station, TX 77843-3133

66. D. R. Damon, U.S. Nuclear Regulatory Commission, MS T8-H7, Washington, DC 20555

67. J. R. Davis, U.S. Nuclear Regulatory Commission, MS T8-A33, Washington, DC 20555

68. R. L. Dintaman, U.S. Department of Energy, DP-13, 19901 Germantown Rd., Germantown, MD 20874-1290

69. H. L. Dodds, University of Tennessee, Nuclear Engineering Dept., 214 Pasqua Engineering Bldg., Knoxville, TN 37922

70. T. W. Doering, Framatome Cogema Fuels, 1261 Town Center Drive, Las Vegas, NV 89124

71. J. D. Evans, U.S. Department of Energy, S-3.1/VISTA, 1000 Independence Ave., S.W., Washington, DC 22085

72. H. D. Felsher, U.S. Nuclear Regulatory Commission, MS T8-H7, Washington, DC 20555

73. P. Felsher, Rocky Flats Environment Technology Site, Bldg. T886B, P.O. Box 464, Golden, CO 80402-0464

74. J. R. Felty, Science Applications Int'l Corp., 2418 N. Dickerson St., Arlington, VA 22207

75. I. E. Fergus, U.S. Department of Energy, EH-22, 20300 Century Blvd., Germantown, MD 20874

76. E. K. Fujita, Argonne National Laboratory, 9700 South Cass Avenue, RA/208, Argonne, IL 60439-4842

77. A. S. Garcia, U.S. Department of Energy, Idaho Operations Office, 850 Energy Dr., MS 1154, Idaho Falls, ID 83401-1563

78. S. C. Keeton, Lawrence Livermore National Laboratory, P.O. Box 808 (L-128), Livermore, CA 94550

79. R. C. Little, Los Alamos National Laboratory, MS F663, P.O. Box 1663, Los Alamos, NM 87545

80. J. McKamy, U.S. Department of Energy, EH-34, 19901 Germantown Road, Germantown, MD 20874-1290

81. R. D. McKnight, Argonne National Laboratory, 9700 S. Cass Ave., Argonne, IL 604394842

82. T. P. McLaughlin, Los Alamos National Laboratory, P.O. Box 1663, Los Alamos, NM 87545

83. D. E.I. Mennerdal, E M Systems, Starvãgen 12, Täby, SWEDEN S-18357

84. D. C. Morey, U.S. Nuclear Regulatory Commission, MS T8-A33, Washington, DC 20555 
85. J. A. Morman, Argonne National Laboratory, 9700 S. Cass Ave., Bldg. 208, C237B, Argonne, IL 60439-4842

86. R. D. Mosteller, Los Alamos National Laboratory, MS J562, Los Alamos, NM 87545

87. K. A. Niemer, Duke Engineering \& Services, 400 S. Tryon St., WC26B, P.O. Box 1004, Charlotte, NC 28201-1004

88. H. Okuno, Japan Atomic Energy Research Institute, 2-4 Shirakara-shirane, Tokai-mura, Ibaraki-ken, JAPAN 319-1195

89. R. E. Pevey, University of Tennessee, Nuclear Engineering Dept., 214 Pasqua Engineering Bldg., Knoxville, TN 37922

90. M. Rahimi, U.S. Nuclear Regulatory Commission, M/S T7F3, Washington, DC 20555

91. P. T. Rhoads, Office of Fissile Materials Disposition, U.S. Department of Energy, MD4, 1000 Independence Avenue SW, Washington, DC 20585

92. B. Rothleder, U.S. Department of Energy, EH-31, 19901 Germantown Rd., Germantown, MD 20874-1290

93. M. K. Sheaffer, Lawrence Livermore National Laboratory, P.O. Box 808, L-634, Livermore, CA 94550

94. T. Taylor, Lockheed Martin Idaho Tech. Co., P.O. Box 1625, MS 3458, Idaho Falls, ID 83415-3458

95. C. Tripp, U.S. Nuclear Regulatory Commission, MS T8-A33, Washington, DC 20555

96. J. A. Thornton, Duke Engineering \& Services, 400 S. Tryon St., WC26B, P.O. Box 1004, Charlotte, NC 28201-1004

97. H. Toffer, Fluor Daniel Northwest, Inc., P.O. Box 1050, MSIN B4-44, 1100 Jadwin Ave., Richland, WA 99352-1050

98. E. F. Trumble, Westinghouse Safety Management Solutions, P.O. Box 5388, 1993 South Centennial Dr., Aiken, SC 29803

99. M. Wangler, Office of Site Operations, EM-70/CLV-1059, U.S. Department of Energy, 19901 Germantown Road, Germantown, MD 20874-1290

100. L. Wetzel, BWX Technologies, Inc., Naval Nuclear Fuel, P.O. Box 785, Lynchburg, VA 24505

101. B. H. White IV, U.S. Nuclear Regulatory Commission, MS O6-F18, Washington, DC 20555

102. Mark Williams, Louisiana State University, Baton Rouge, LA 70803-5820

103. R. E. Wilson, Safe Sites of Colorado, P.O. Box 464, Golden, CO 80402-0464

104 C. J. Withee, U.S. Nuclear Regulatory Commission, MS O6-G22, Washington, DC 20555 